SAND90-1984

Unlimited Release

Distribution

Printed May 1393

Category UC-721

\title{
Programmer's Manual for CAMCON:
}

\section{Compliance Assessment Methodology Controller}

\author{
R. P. Rechard \\ A. P. Gilkey ${ }^{1}$ \\ H. J. Iuzzolino ${ }^{2}$ \\ D. K. Rudeen ${ }^{1}$ \\ K. A. Byle ${ }^{1}$ \\ Performance Assessment Department \\ Sandia National Laboratories \\ Albuquerque, New Mexico 87185
}

\begin{abstract}
CAMCON, the Compliance Assessment Methodology CONtroller, is an analysis system that assists in assessing the compliance of the Waste Isolation Pilot Plant (WIPP) with applicable long-term regulations of the U.S. Environmental Protection Agency, including Subpart B of the Environmental Standards for the Management and Disposal of Spent Nuclear Fuel, High-Level and Transuranic Radioactive Wastes, 40 CFR 191 and 40 CFR 268.6, which is the portion of the Land Disposal Restrictions implementing the Resource, Conservative, and Recovery Act of 1976, as amended that states the conditions for disposal of hazardous chemical wastes. This manual provides an architectural overview of the CAMCON system. Furthermore this manual presents guideline: and presents suggestions for programmers developing the many different types of software necessary to investigate various events and physical prciesses of the WIPP. These guidelines include user interface requirements, minimum quality assurance requirements, coding style suggestions, and the use of numerous software libraries developed specifically for or adapted for the CAMCON system.
\end{abstract}

1. New Mexico Engineering Research Institute (NMERI), University of New Mexico, Albuquerque, NM

2. GeoCenters, Inc., Albuquerque, NM
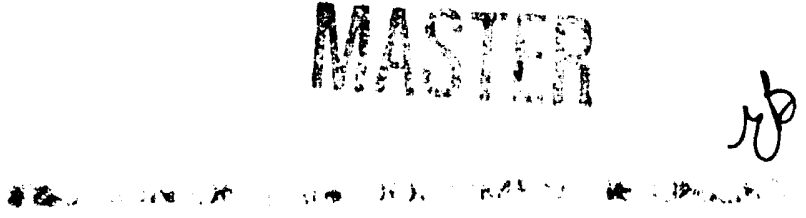
May 1993 
Software is fundamental to assessing the compliance of the Waste Isolation Pilot Plant (WIPP) with numerous environmental regulations: the task is impossible without it. The primary goals of this manual are to streamline software development for the CAMCON system and make future software modification easier. The high cost of code development and the higher cost of code maintenance and inhancements make these important goals. To meet these goals, this manual presents a set of development requirements and guidelines for software that will aid in producing well-structured (especially important for FORTRAN code) and sufficiently documented codes that are easily interfaced with other codes. The requirements are essentially those included in the software quality assurance (QA) procedures, documented elsewhere. As an aid to understanding this manual, the software QA procedures should be read first; although, a brief summary of the QA requirements is provided herein. The guidelines presented herein are essentially standard programming practices and are not intended to restrict individual programming styles. More importantly, this nianual describes the use of the numerous FORTRAN libraries routinely used by the CAMCON system software. However, some of the libraries change over time. The most current information on any library is available from on-line documentation (help files) on the Sandia WIPP computer.

\section{Related Documents}

Several reports describe various other aspects of the CAMCON system and are listed below (i.e., user's reference manual, software QA procedures, proposed concepts in the CAMCON system prior to development, and documentation for a FORTRAN library, SUPES).

Rechard, R. P., ed. 1992. User's Reference Manual for CAMCON: Compliance Assessment Methodology Controller, Version 3.0 . SAND90-1983. Albuquerque, NM: Sandia National Laboratories.

Rechard, R. P., P. J. Roache, R. L. Blaine, A. P. Gilkey, and D. K. Rudeen. 1991. Quality Assurance Proceduresfor Computer SoftwareSupportingPerformanceAssessmentsofthe WasteIsolation Pilot Plant. SAND90-1240. Albuquerque, NM: Sandia National Laboratories.

Rechard, R. P., 1989. Review and Discussion of Code Linkage and Data Flow in Nuclear Waste Compliance Assessments. SAND87-2833. Albuquerque, NM: Sandia National Laboratories.

Red-Horse,J.R., W.C. Mill-Curran, and D. P.Flanagan, 1990. SUPES Version 2.1: A Software Utilities Package for the Engineering Sciences . SAND90-0247. Albuquerque, NM: Sandia National Laboratories. 


\section{Acknowledgments}

Several of the ideas and concepts presented herein are based on work performed while two of the authors we members of Sandia's Engineering Sciences Center. While many of the specifics have changed or been enhanced, some of the basic ideas remain. The foresight of the members of the Engineering Sciences Center who contributed to these basic ideas is acknowledged.

The authors also appreciate the time and suggestions supplied by the technical reviewers: $\mathrm{M}$. E. Fewell, Sandia; R. L. Blaine, Ecodynamics, Inc., Albuquerque, NM; and J. D. Schreiber, SAIC, Albuquerque, NM. The text and illustrations were prepared by the artists, word processors, and production staff and edited by P. A. Oliver and D. L. Pulliam, of Tech Reps, Inc., Albuquerque, NM. 


\section{Contents}

\section{Introduction}

1.1 The WIPP and CAMCON …

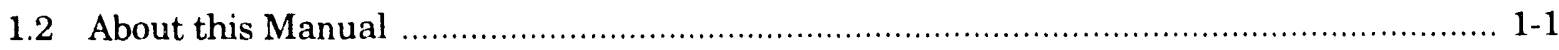

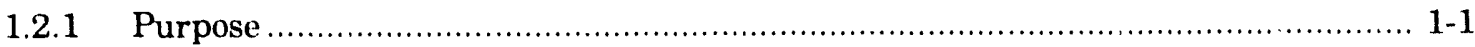

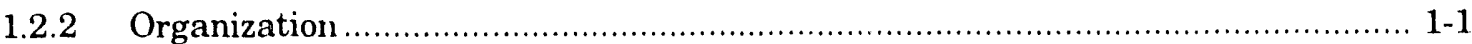

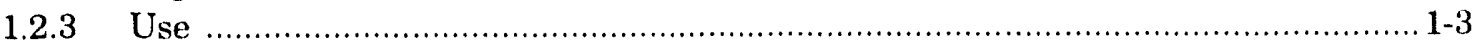

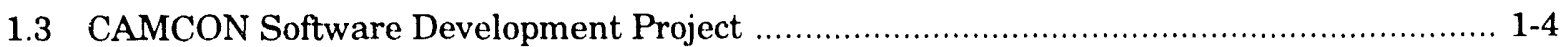

\section{The CAMCON System}

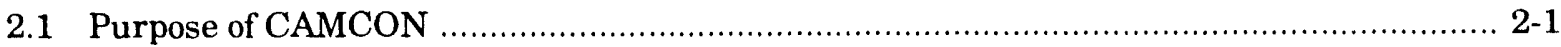

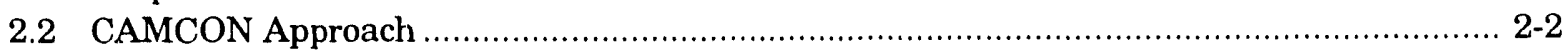

2.3 Software Management System and System Architecture ........................................ 2-3

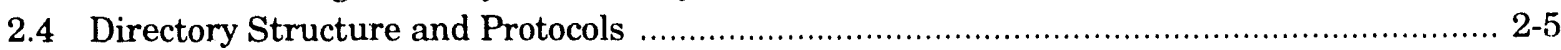

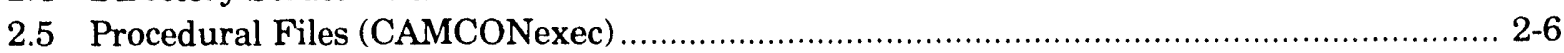

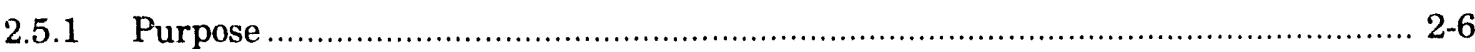

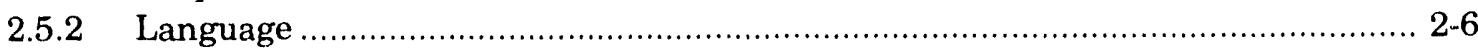

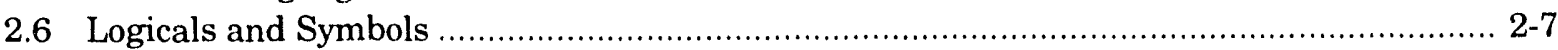

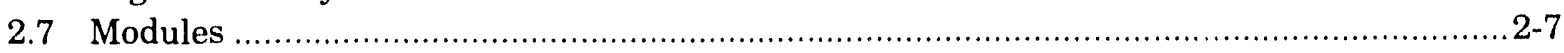

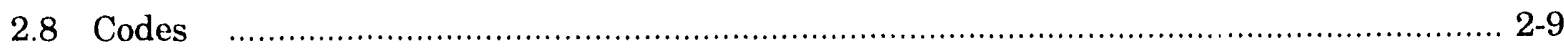

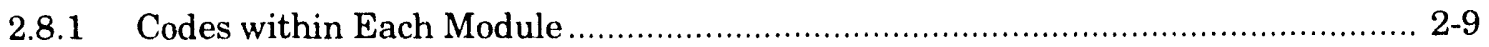

2.8.2 Code Directory Structure …................................................................... 2-10

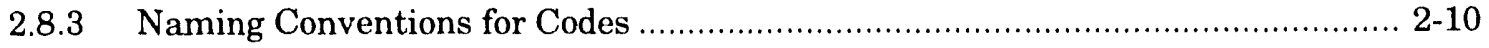

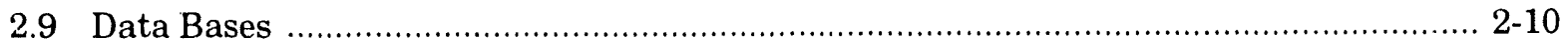

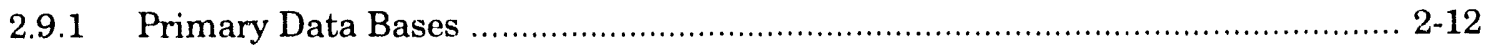

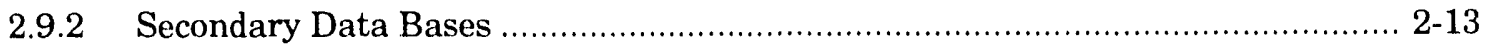

2.9.3 Computational Data Base, CAMDAT …...................................................... 2-15

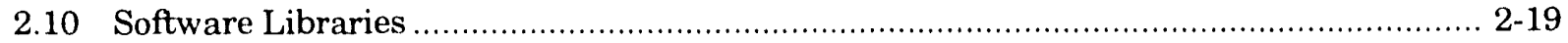

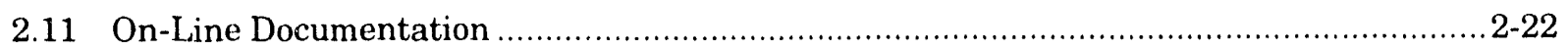

\section{Software Development Overview}

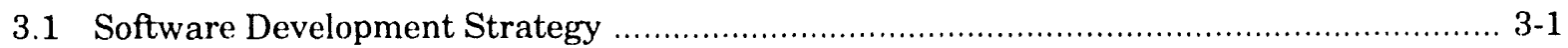

3.1.1 Multiple Passes through the Assessment …..................................................... 3-1

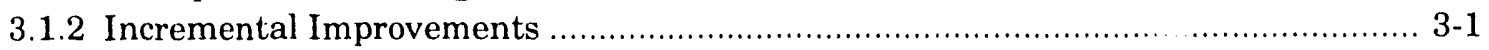

3.1.3 Prototyping the System and Selecting Software .......................................... 3-1

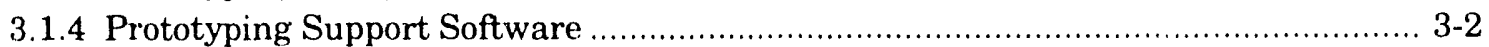

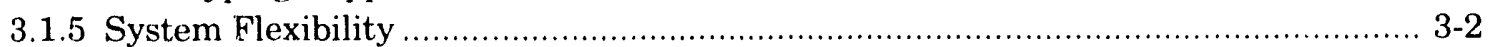

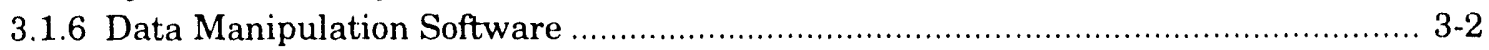

3.1.7 Close Links Between Developers and Analysts .................................................. 3-2

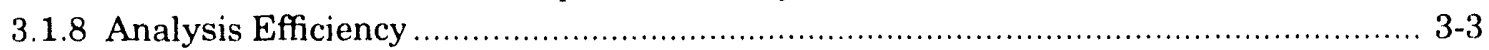

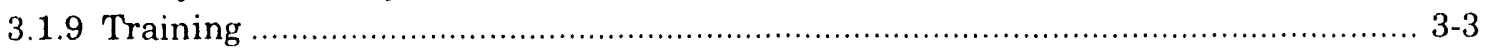

3.1.10 Selection of Primary Coding Language and Computer Operating System ................3-3 


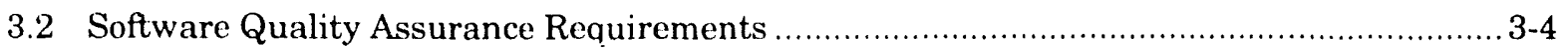

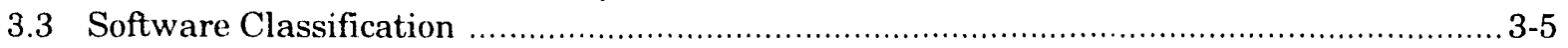

3.3.1 Description of the Four Software Classes .......................................................... 3-5

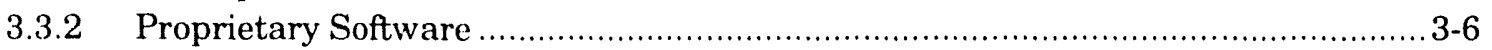

3.4 Software Development or Acquisition Procedures .......................................................... 3-9

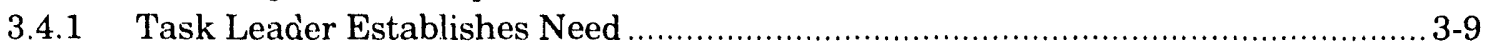

3.4.2 QA Coordinator Assigns a Software Sponsor ............................................. 3-9

3.4.3 Developer or Code Consultant Implements Development or Modifications ............ 3-9

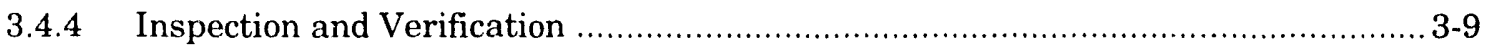

3.4.5 Software Sponsor Enters Software into CAMCON as Class X .............................. 3-9

3.4.6 Software Sponsor Enters Software into CAMCON as Class C ........................... 3-10

3.4.7 Software Sponsor Enters Software into CAMCON as Class A ............................. 3-11

3.4.8 Writing CAMCONexec Procedural Files for Software ..................................... 3-12

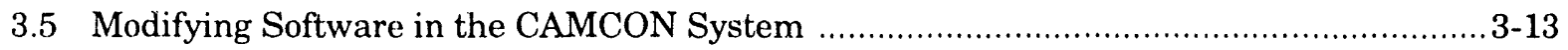

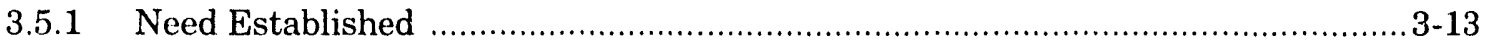

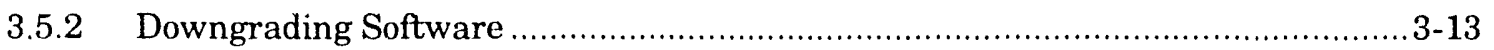

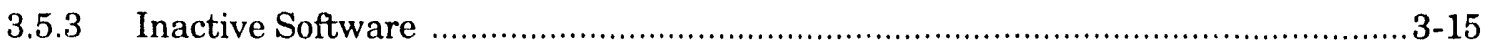

3.6 Replacing Software Already in the CAMCON System …......................................... 3-15

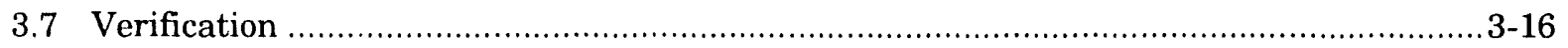

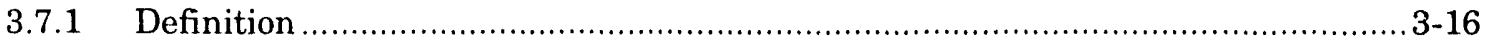

3.7.2 Examples of Verification Methods ….................................................................... 3-16

3.7.3 Appropriate Verification Method Dependent on Complexity, Type,

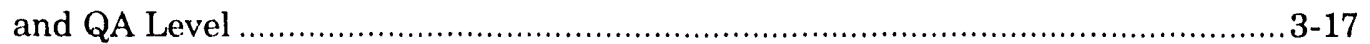

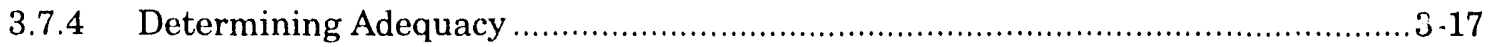

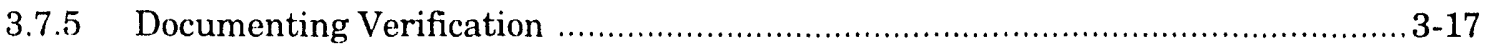

3.8 Single-Analysis Software and Analysis-Specific Modifications ...................................... 3-17

\section{User Interface Guidelines}

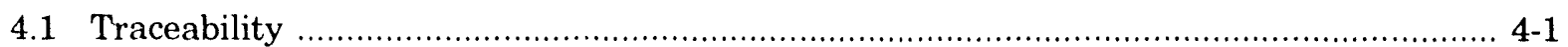

4.1.1 Softw are Name, PA Version ID, Date Stamp ................................................. 4-1

4.1.2 Author Names, Software Origin, Government Legal Disclaimer, and Proprietary Information .......................................................................... 4-3

4.1.3 Using CAMCON_LIB Routines to Meet Traceability QA Requirements

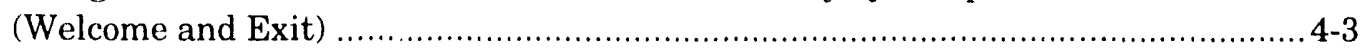

4.2 Files and Entering File Names .................................................................... 4-4

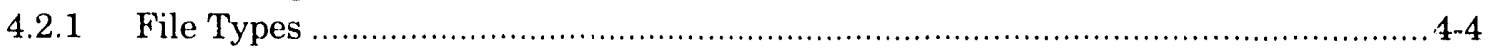

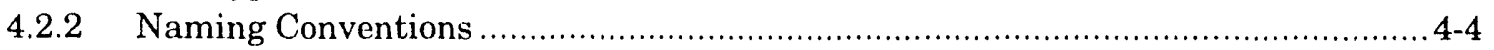

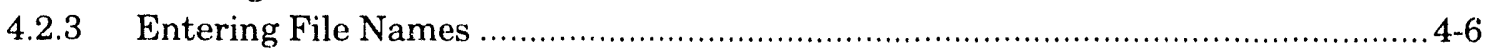

4.2.4 Example of File Reading ............................................................................. 4-6

4.3 Parameter Input .............................................................................................. $4-7$

4.3.1 Reading from Data Bases .......................................................................... 4-7

4.3.2 Reading from ASCII Input Files ............................................................. 4-8

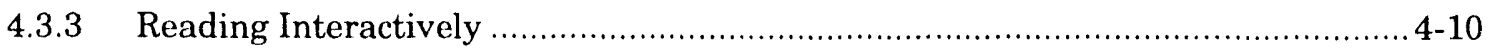

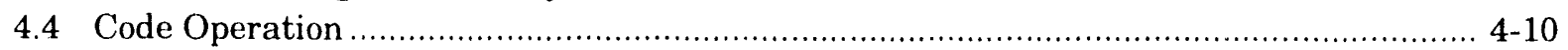

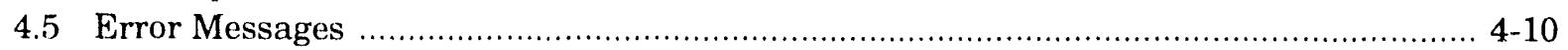

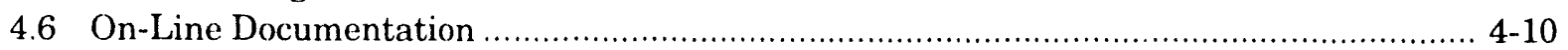

4.6.1 QA Requirements for On-Line Documentation ............................................... 4-10 


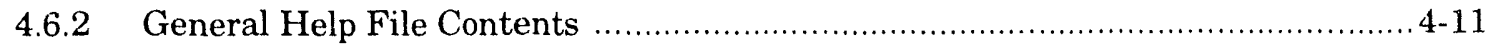

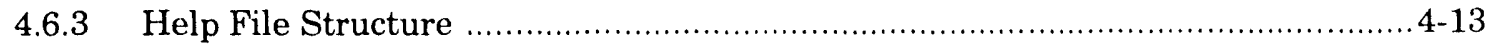

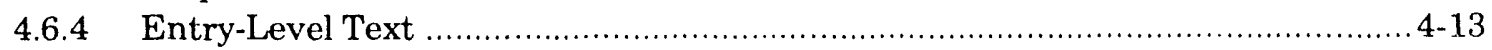

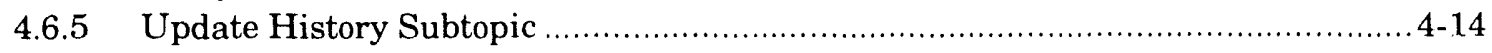

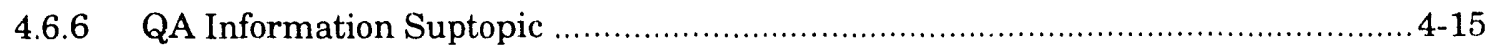

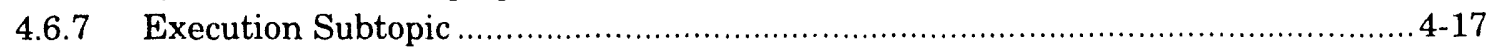

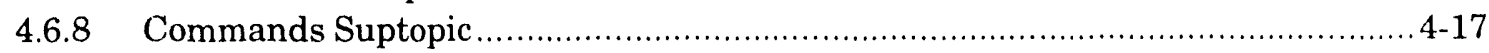

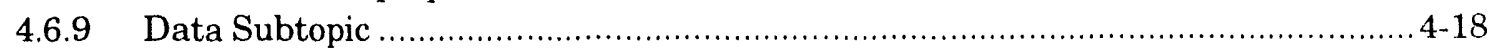

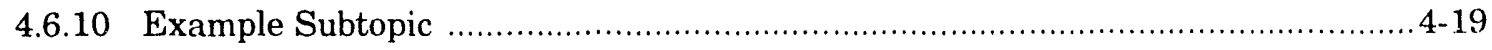

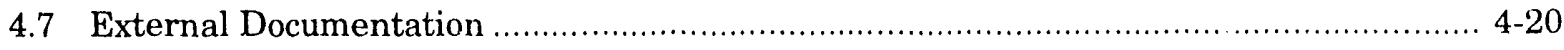

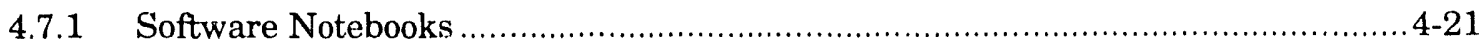

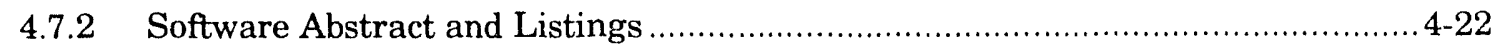

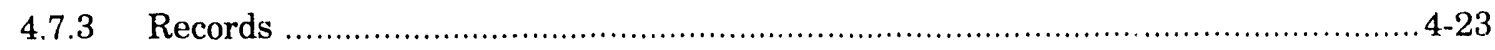

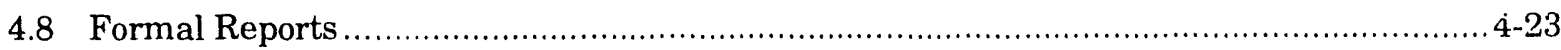

\section{General Programming Guidelines}

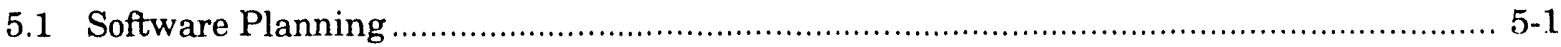

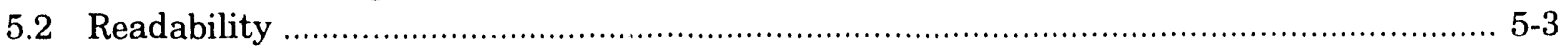

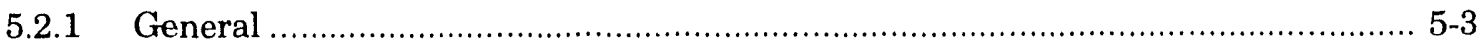

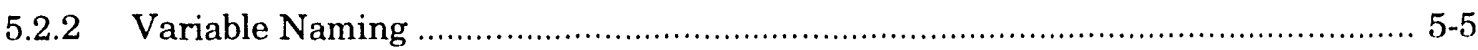

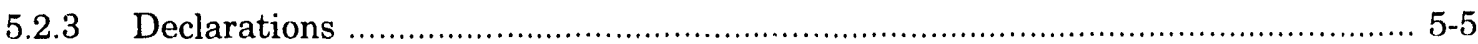

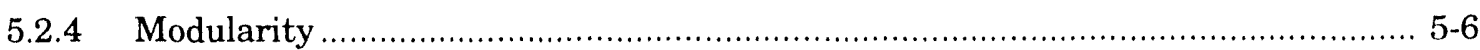

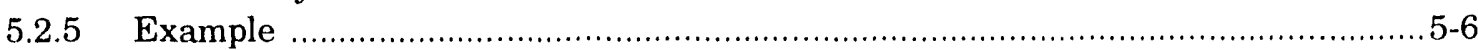

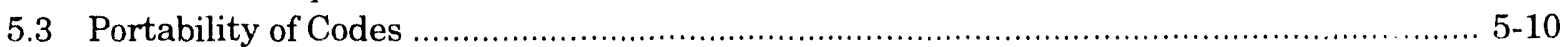

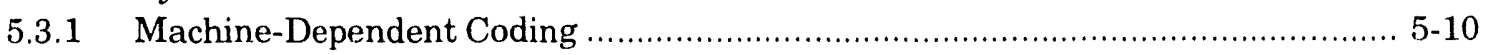

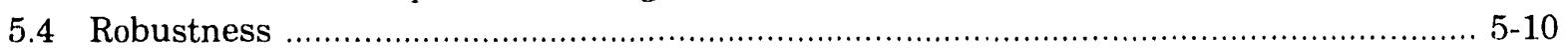

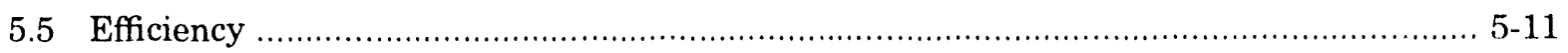

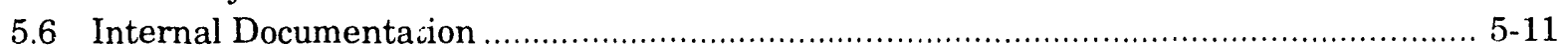

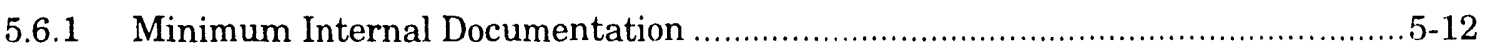

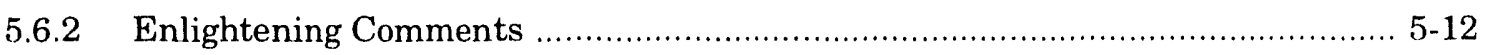

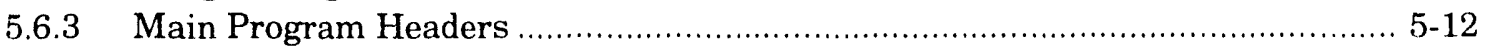

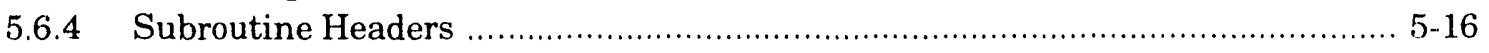

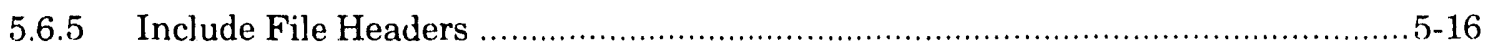

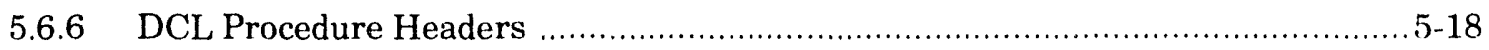

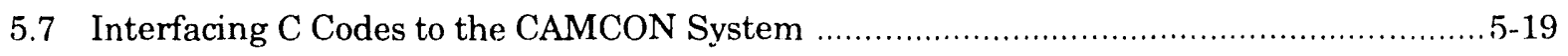

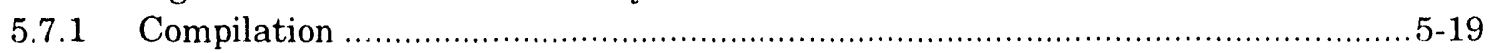

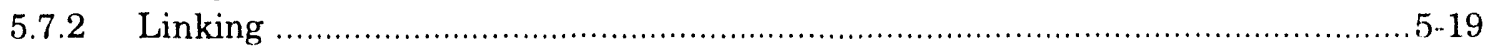

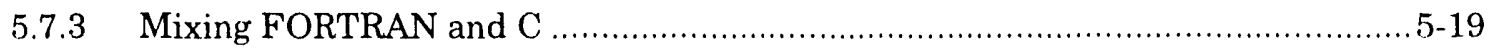

\section{CAMDAT_LIB: Computational Data Base Interface Library}

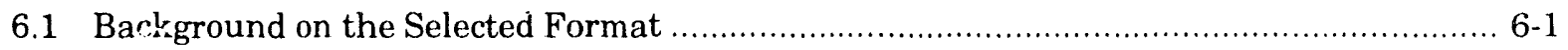

6.2 Correspondence Between Generic Computational Data Base
and Data Entities in CAMDAT File .................................................................. 6 .

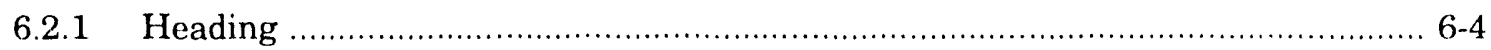

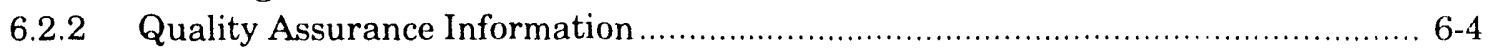




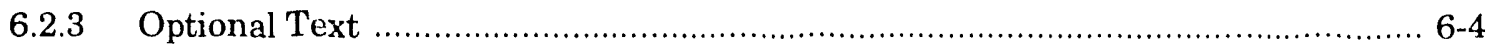

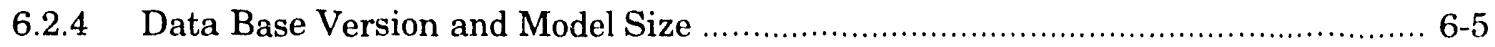

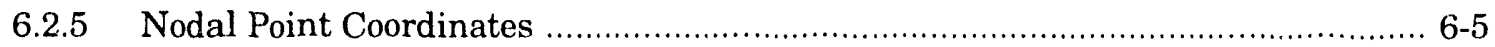

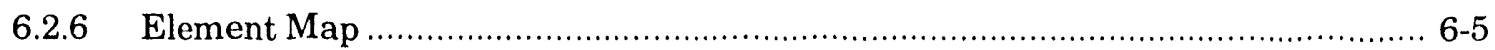

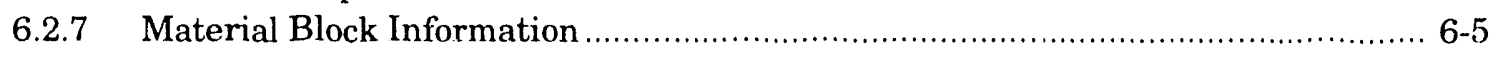

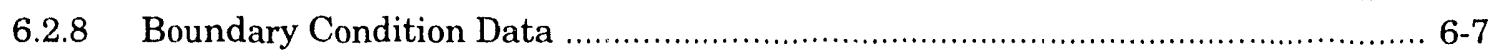

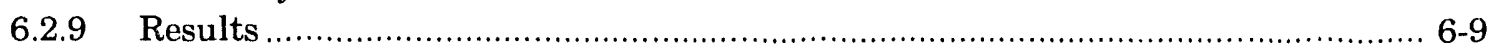

6.3 General Guidelines on the Use of CAMDAT_LIB Routines ..................................... 6-10

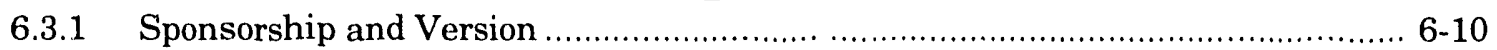

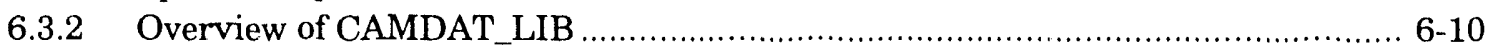

6.3.3 Error Messages ..................................................................................... 6-12

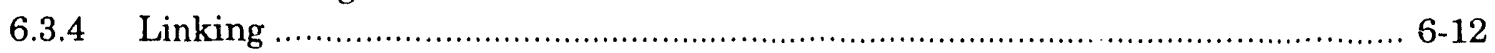

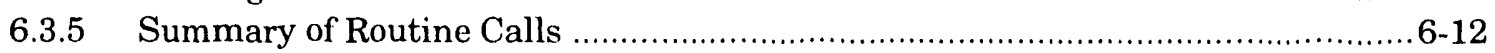

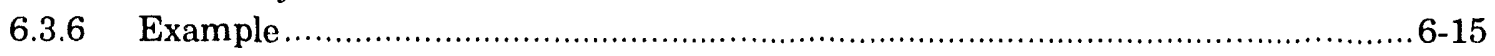

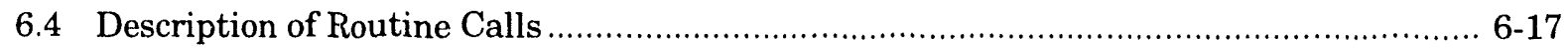

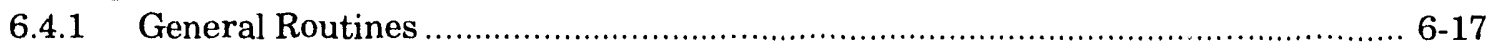

6.4.2 Detailed Descriptions of File Initialization Routines ...................................... 6-20

6.4.3 Detailed Descriptions of QA Information Routines .........................................6-34

6.4.4 Detailed Descriptions of Model Description Routines ......................................6-41

6.4.5 Detailed Descriptions of Analysis Results Routines ........................................6-69

\section{SDBREAD_LIB: Secondary Data Base Interface Library}

7.1 General Description of the Secondary Data Base ....................................................... 7-1

7.1.1 Secondary Data Base Structure and Similar Data Entities ............................... 7-1

7.1.2 Storage of Conceptual Model Stratigraphy …................................................ 7-1

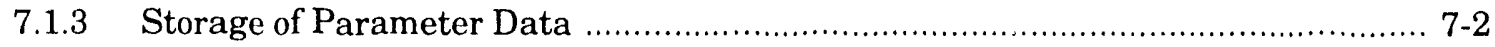

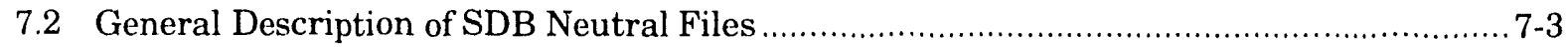

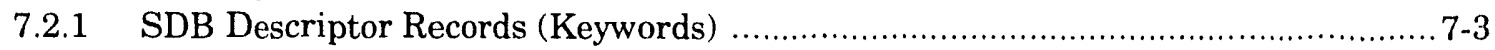

7.2.2 Format of SDB Descriptor Records ................................................................ $7-3$

7.2.3 Example of Descriptor Records .................................................................. 7-6

7.2.4 Identification of SDB Neutral Files …........................................................ $7-6$

7.3 General Guidelines on the Use of SDBREAD_LIB Routines ..................................... $7-7$

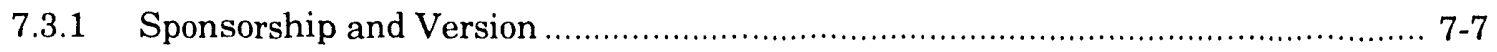

7.3.2 Overview of Capabilities and Method ….................................................... 7-7

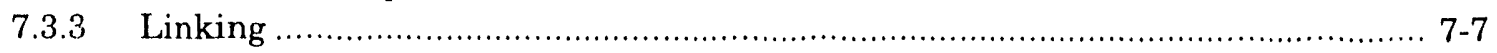

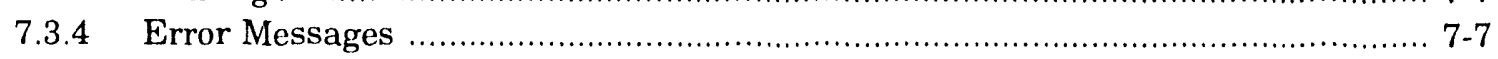

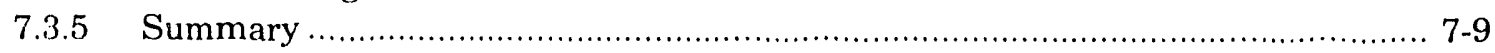

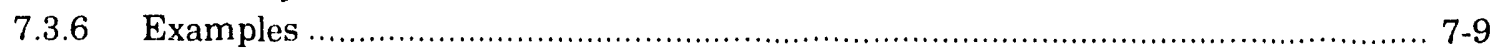

7.4 Description of Routine Calls ............................................................................ 7-13

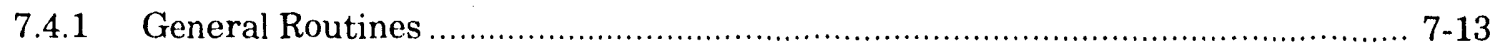

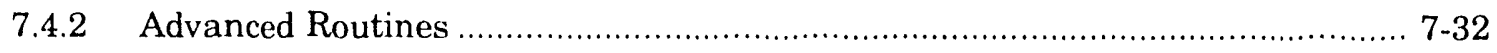

\section{CAMCON_LIB: Quality Assurance Routine Library}

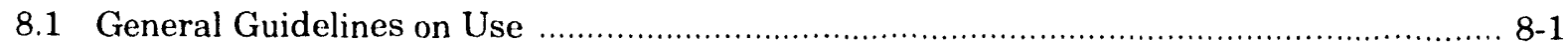

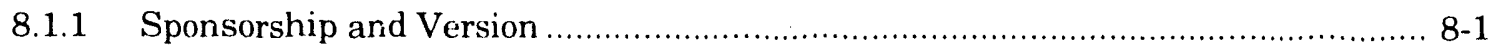




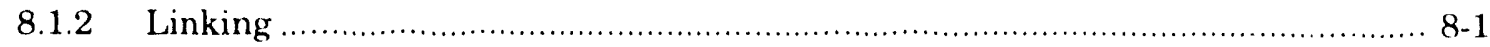

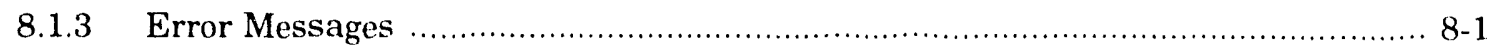

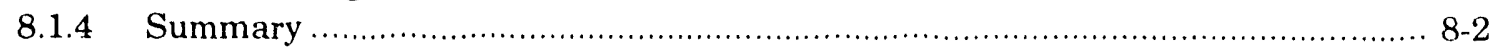

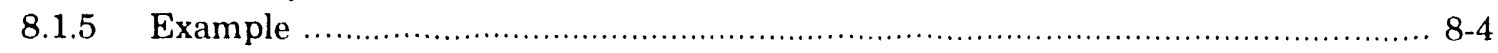

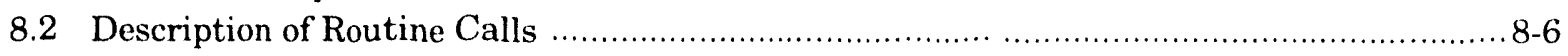

8.2.1 QA and User Interface Routines ........................................................... $8-6$

8.2.2 File Processing Routines ......................................................................... 8-22

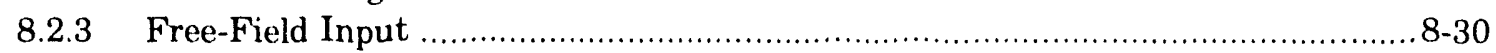

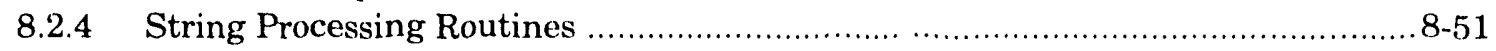

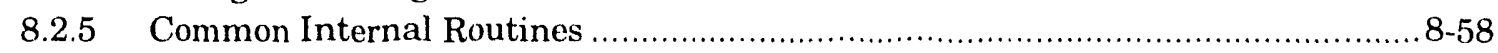

\section{CAMSUPES_LIB: Routines for Dynamic Memory Allocation} and Other FORTRAN Extensions

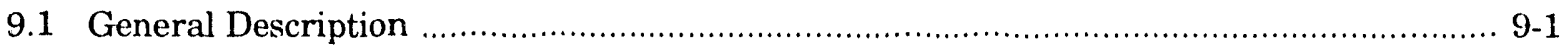

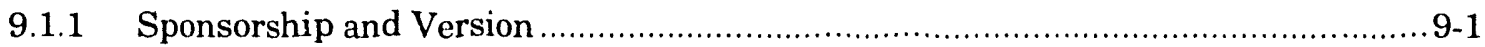

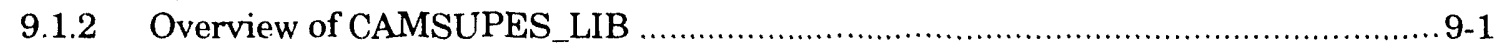

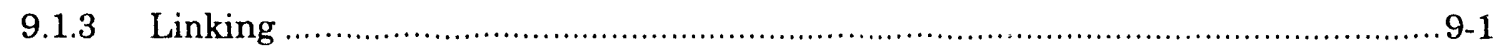

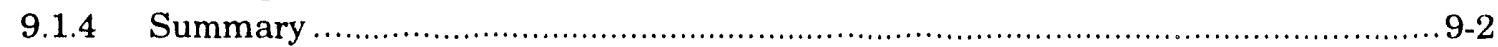

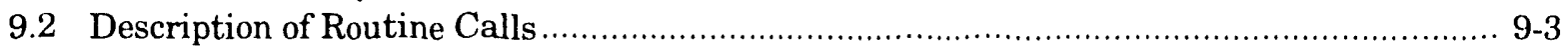

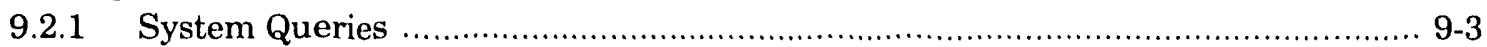

9.2.2 Memory Management Routines …................................................................. 9-11

9.2.3 Advanced Memory Management Routines ............................................... 9-23

9.2.4 Memory Management Debugging Routines .................................................... 9-34

\section{Graphics Libraries: PLT_LIB and HJIPLOT_LIB}

10.1 General Guidelines on the Use of PLT_LIB Routines .......................................... 10-1

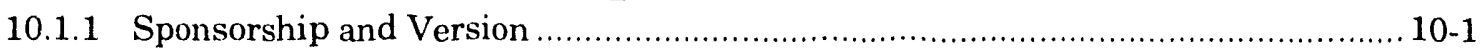

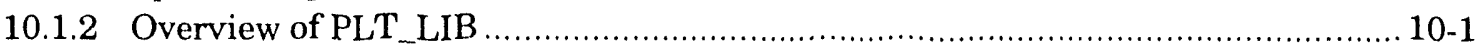

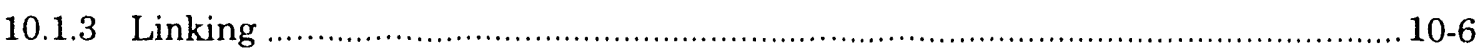

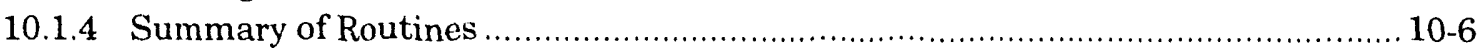

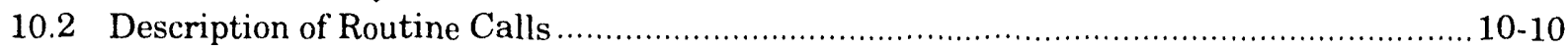

10.2.1 Plot Initialization and Control ….................................................... 10-10

10.2.2 Setting PLT_LIB Parameters ……................................................... 10-23

10.2.3 Hardware and Software Text .............................................................. 10-51

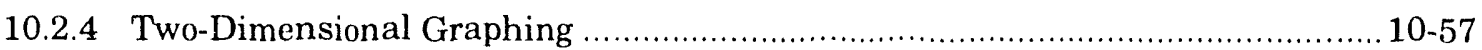

10.2.5 Device Coordinate Plotting Primitives ................................................... 10-71

10.2.6 Two- and Three-Dimensional Mapping Routines ........................................... 10-84

10.2.7 Clipping of Two- and Three-Dimensional Points, Lines, and Polygons .............10-117

10.2.8 Color Palette Routines ..............................................................10-128

10.2 .9 Obsolete Routines ....................................................................... 10-136

10.3 General Guidelines on the Use of HJIPLOT_LIB Routines ................................... 10-147

10.3.1. Sponsorship and Version ..............................................................10-147

10.3.2 Overview of HJIPLOT_LIB _........................................................ 10-147

10.3 .3 Linking .............................................................................. 10-147

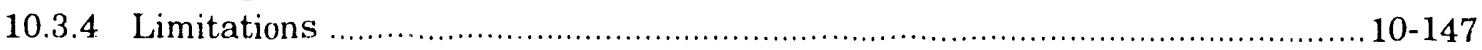




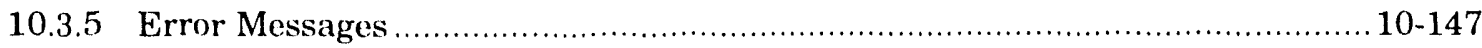

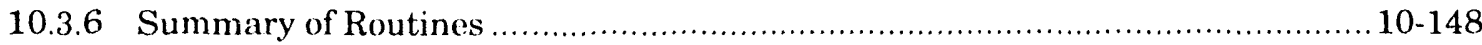

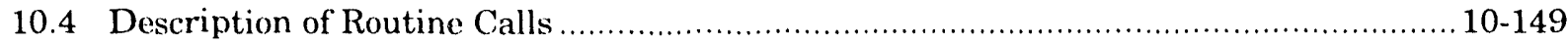

\section{Graphic Device Interfaces: SVDI and DVDI}

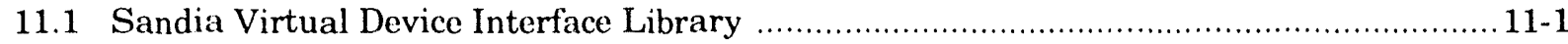

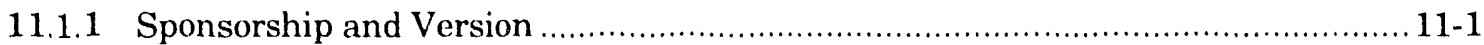

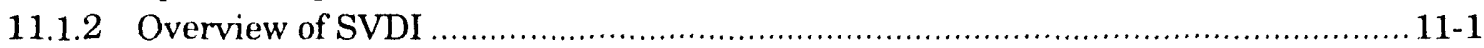

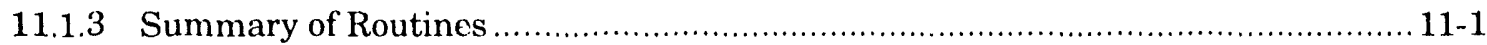

11.2 Escape Codes for SVDI-Supported Devices ….......................................................

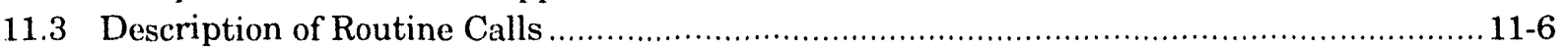

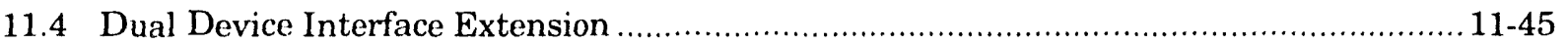

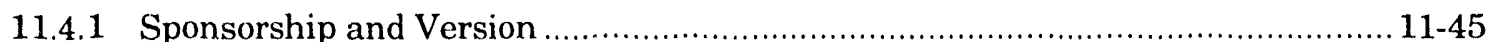

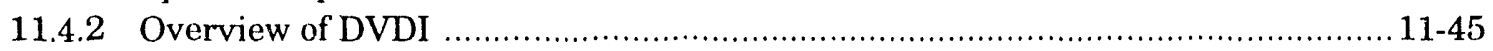

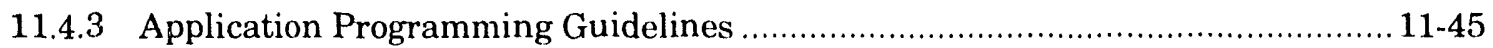

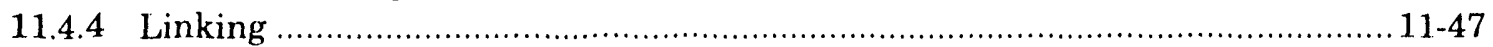

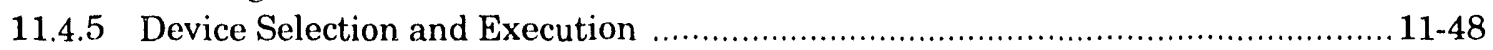

11.4.6 Adding a Device Driver ..................................................................................... 11-51

\section{Software Development Utilities}

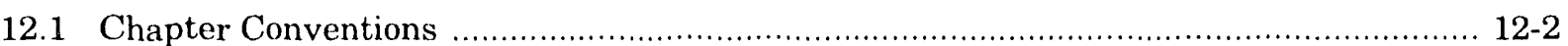

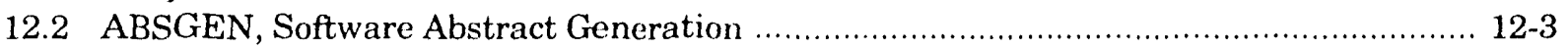

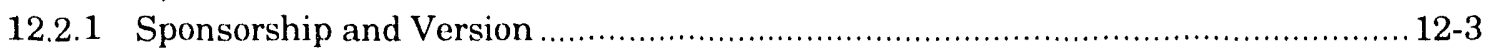

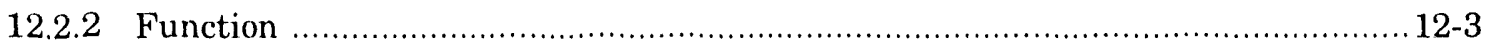

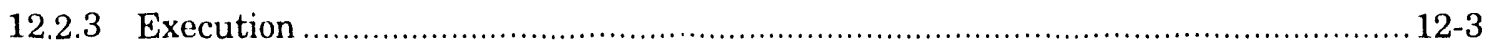

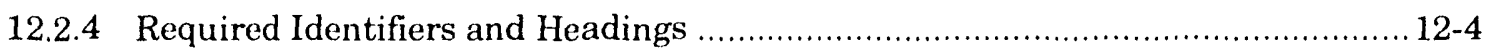

12.3 CHANGES, Error and Enhancement Reporting .................................................. 12-5

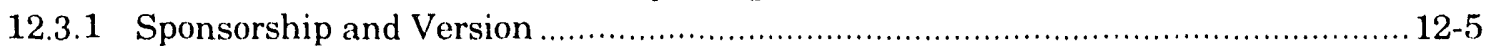

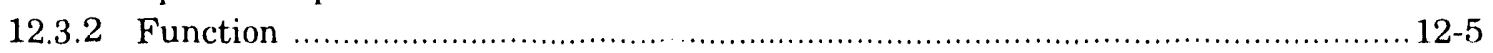

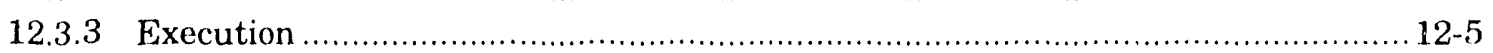

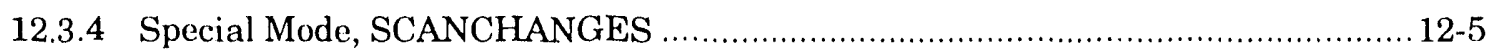

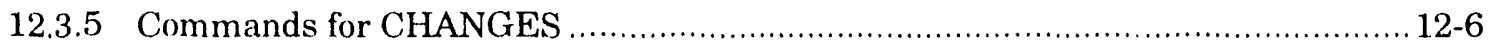

12.4 CREATE_PROGRAM, Compile and Link a Program .......................................... 12-9

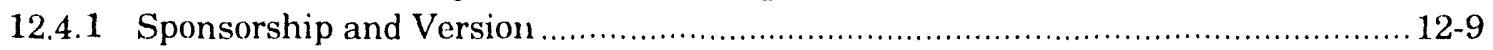

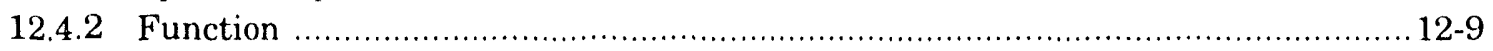

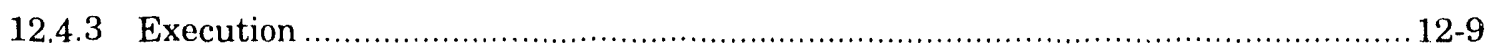

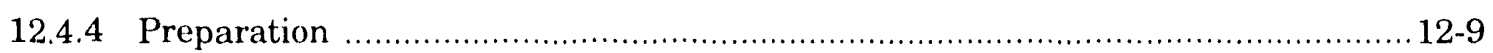

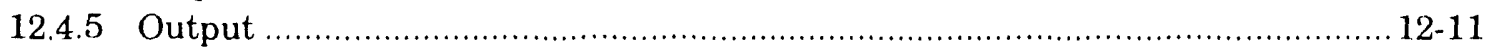

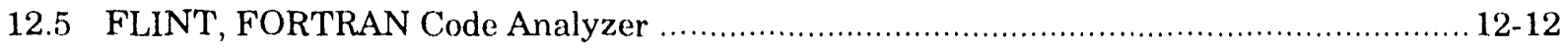

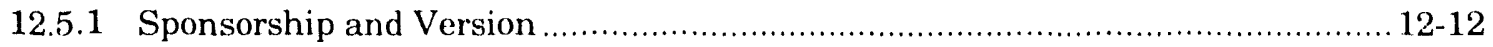

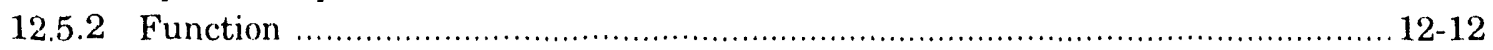

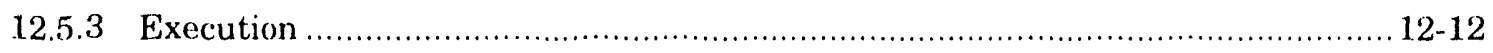

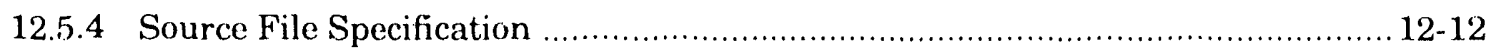

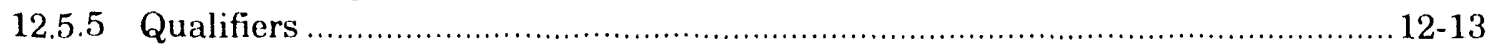

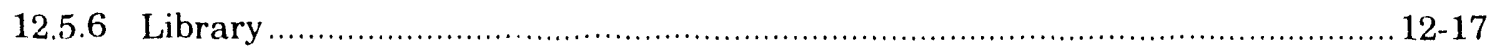


12.6 FORMRENUM, Format and Renumber ........................................................ 12-19

12.6.1 Sponsorship and Version ................................................................. 12-19

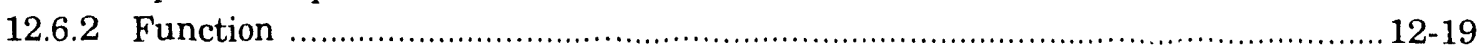

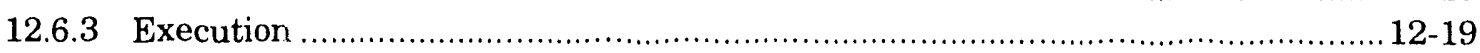

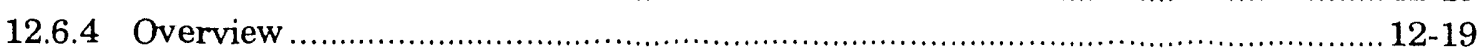

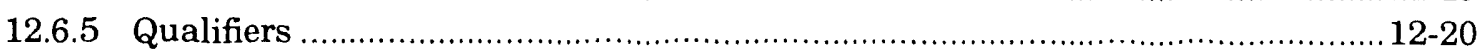

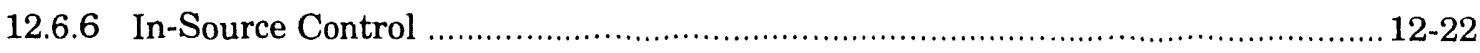

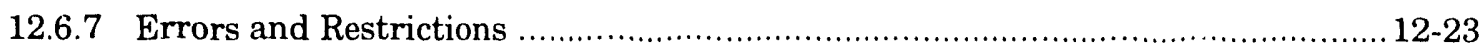

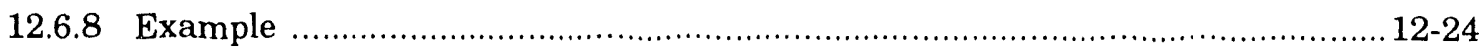

12.7 HLPCHK, Check Format and Content of Software Help File ................................... 12-26

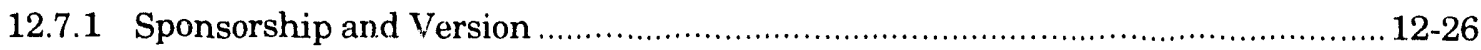

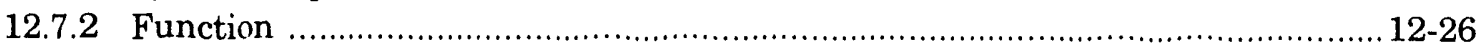

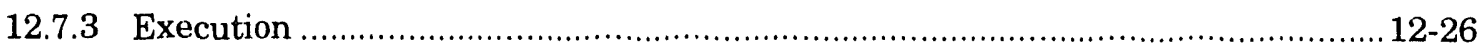

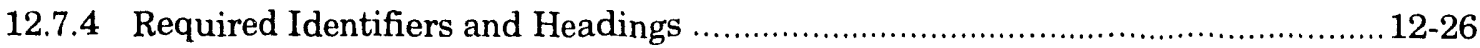

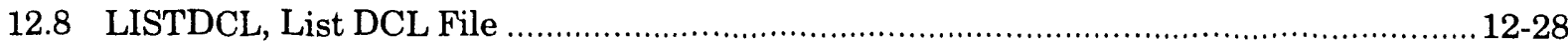

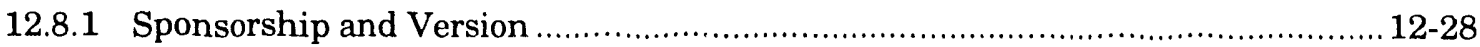

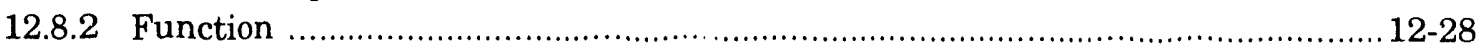

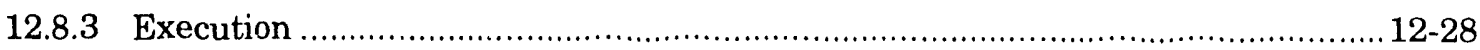

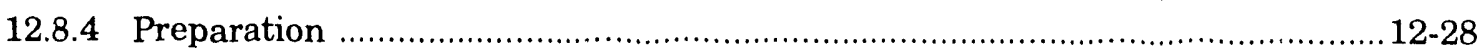

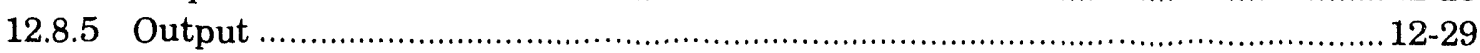

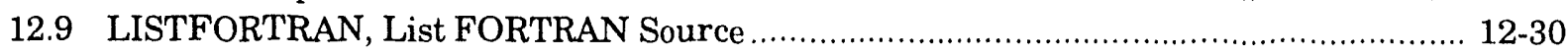

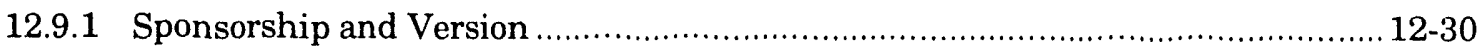

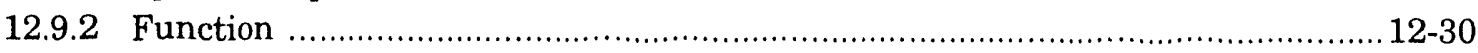

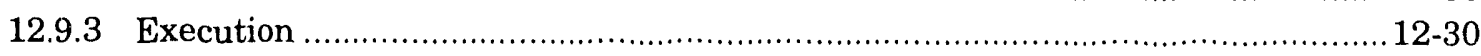

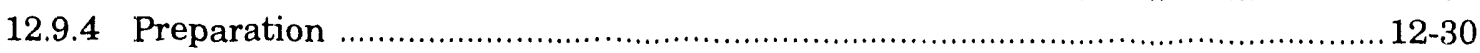

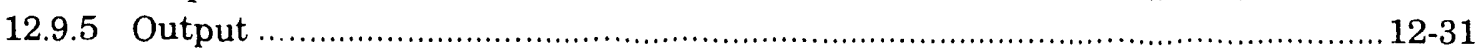

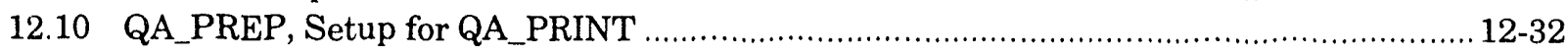

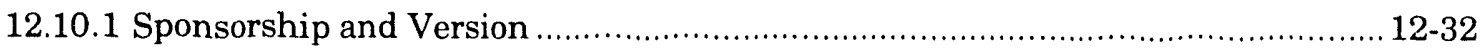

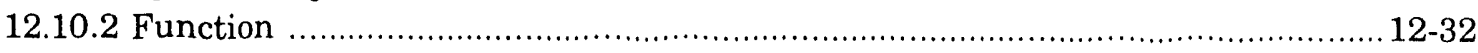

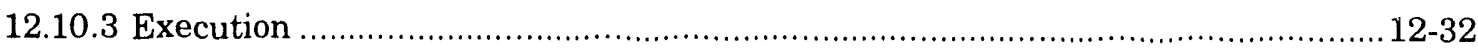

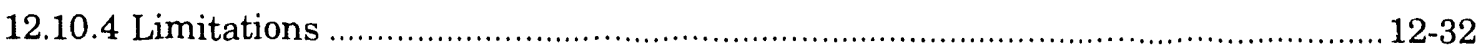

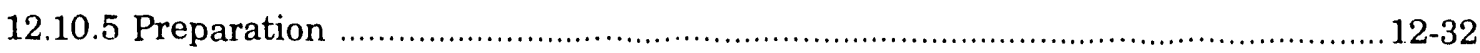

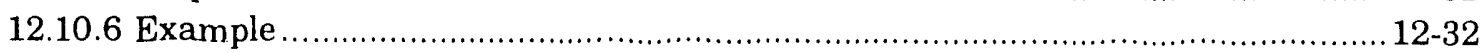

12.11 QA_PRINT, Quality Assurance Notebook Print Procedure …................................. 12-33

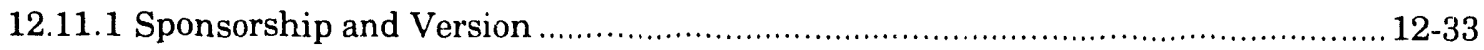

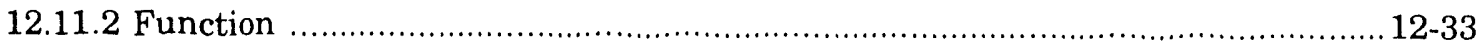

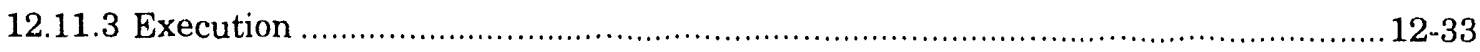

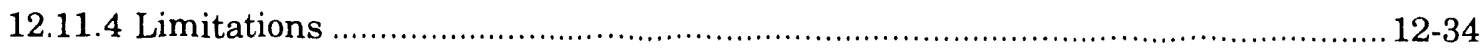

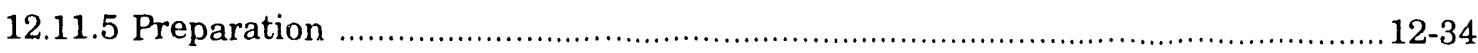

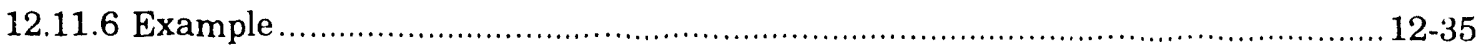

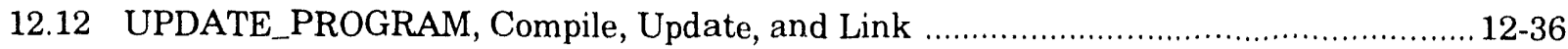

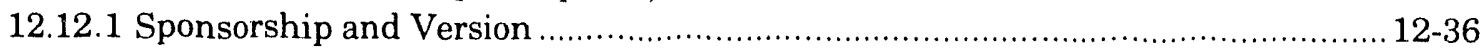

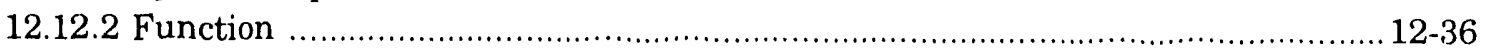

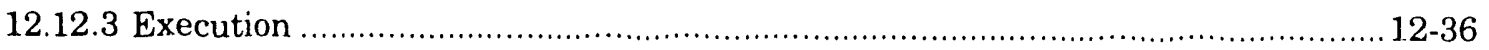

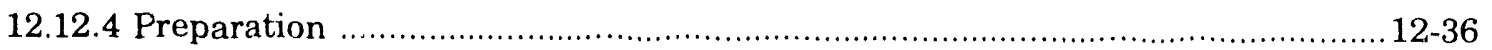

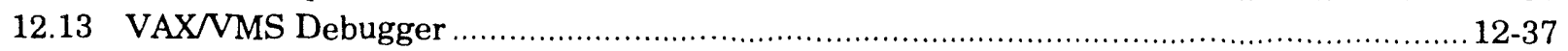

12.13.1 Sponsorship and Version ............................................................ 12-37

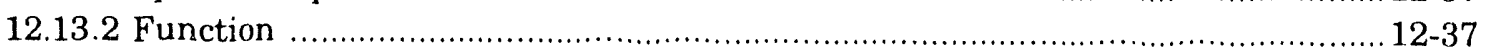


12.13.3 Reference

\section{References}

\section{Appendix A: Assessing the Performance of the WIPP}

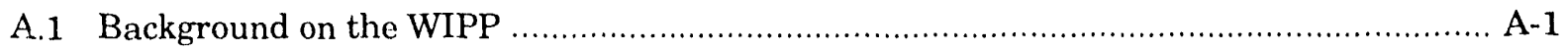

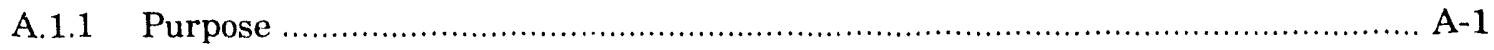

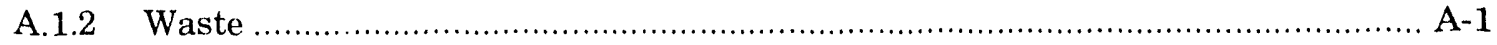

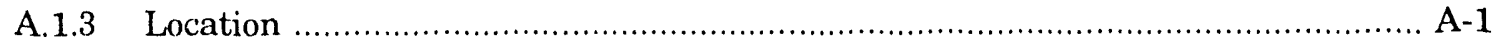

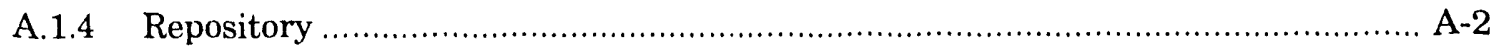

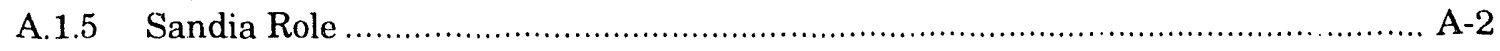

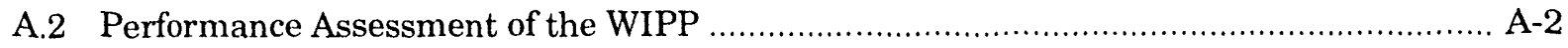

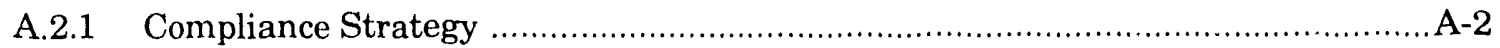

A.2.2 Six General Tasks of PA .................................................................

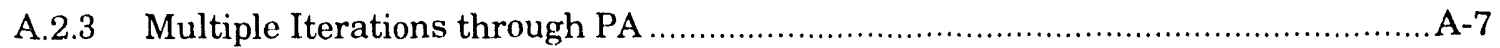

A.3 Overview of Consequence Modeling ............................................................ A-8

A.3.1 Model Subdomains in Consequence Modeling ......................................... A-8

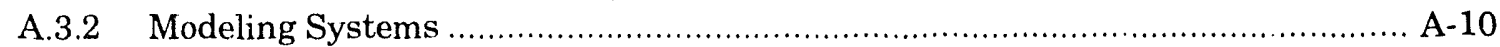

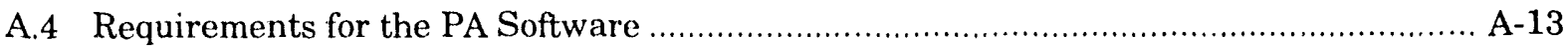

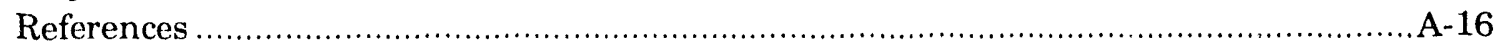

\section{Appendix B: CAMCON Systen Personnel and Software Sponsors}

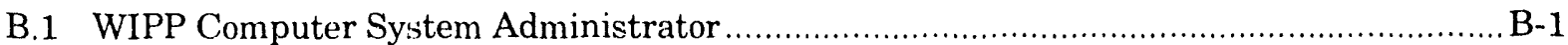

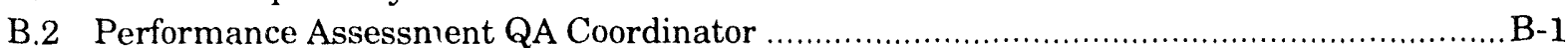

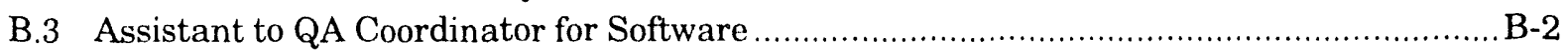

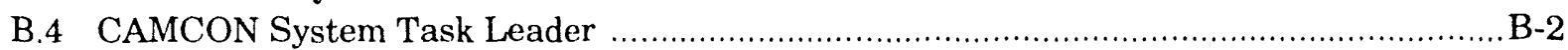

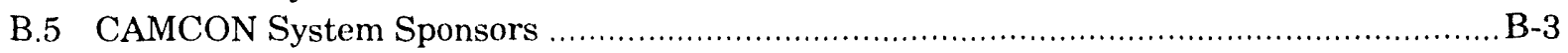

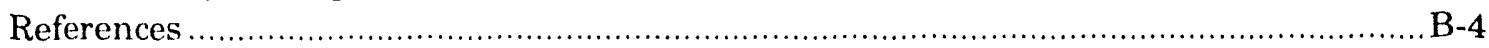

\section{Glossary}

Index 


\section{Figures}

1-1 CAMCON software development in the Sandia WIPP PA Department ...................... 1-5

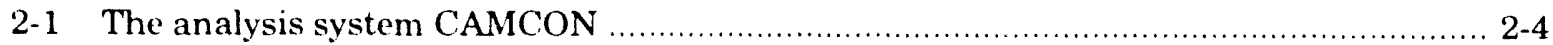

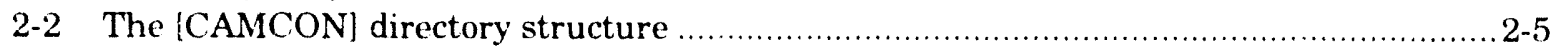

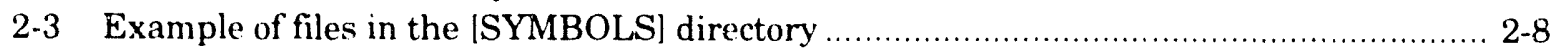

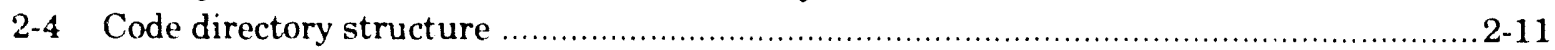

2-5 The investigator interprets data in the primary data base that is consistent with the use of a model parameter in the computational model .......................................2-14

2-6 The six FORTRAN CAMCON libraries provide interfaces to the PA analysts, secondary data base, computational data base, graphic devices, and computer memory

3-1a Steps followed to reach a Class X, C, or A software QA rating (part 1) ............................ 3-7

3-1b Steps followed to reach a Class X, C, or A software QA rating (part 2) ............................ 3-8

3-2 Steps followed for modifying Class $\mathrm{A}$ or $\mathrm{C}$ software ......................................................

6-1 Correspondence between generic computational data base and CAMDAT neutral

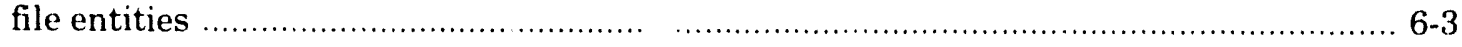

6-2 Storage of WIPP finite-difference mesh ... a CAMDAT file .......................................... 6-6

7-1 SDBREAD_LIB typically accesses three SDB neutral files exported from the

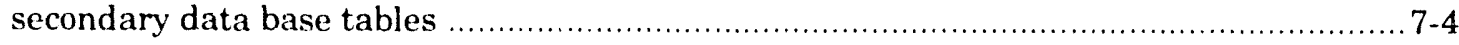

7-2. Example of data moving from the secondary data base to a CDB neutral file and the various possible identifiers for CAMCON SDB neutral files

11-1 Communication with graphic devices is accomplished through the Dual Virtual Device Interface (DVDI)

A-1 Sandia's method for assessing WIPP repository compliance involves six tasks

A-2 Major computational models (codes) and the physical features they simulate in the WIPP disposal system

A-3 Three model scales are typically used in compliance assessment ............................. A-9

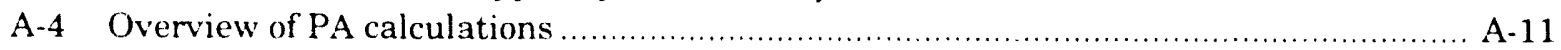

A-5 CAMDAT, a priniary component of the CAMCON system, is the behind-thescenes structure that supports the PA calculations depicted in Figure A-4

\section{Tables}

3-1 Relationship Between Software Class and Quality Assurance Requirements ............... 3-5

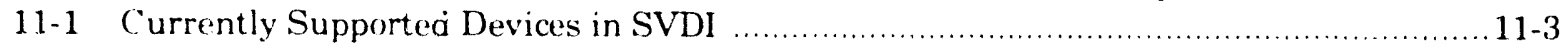

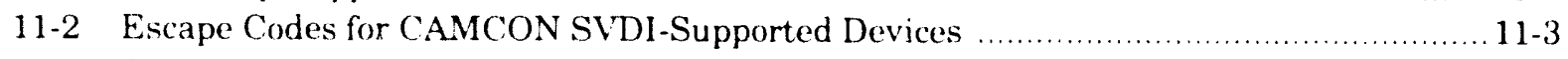

11-3 Communication Codes for the SVDI Routine, VDESCP ….................................... 11-47

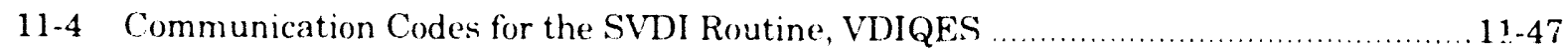

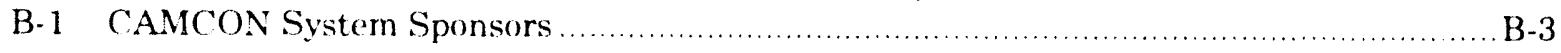




\section{Introduction}

\subsection{The WIPP and CAMCON}

The Waste Isolation Pilot Plant (WIPP) is a geologic repository being developed by the U.S. Department of Energy (DOE) as a research and development facility to demonstrate the safe storage, management, and disposal of defense-related transuranic (TRU) waste and, as such, must comply with several standards of the U.S. Environmental Protection Agency (EPA). The Compliance Assessment Methodology CONtroller, or CAMCON, is the analysis system used by the Performance Assessment (PA) Department of Sandia National Laboratories (Sandia) to assess the compliance of the WIPP with applicable lorg-term environmental regulations, including Subpart B of the EPA's Environmental Standards for the Management and Disposal of Spent Nuclear Fuel, High-Level and Transuranic Radioactive Wastes, 40 CFR 191 and 40 CFR 268.6, which is the portion of the Land Di osal Restrictions implementing the Resource Conservation and Recovery Act of 1976 (RCRA), as amended, that states the conditions for disposal of hazardous chemical wastes.

\subsection{About this Manual}

\subsubsection{Purpose}

This manual was written to assist programmers developing software for the CAMCON system. Specifically, the manual provides information on the following:

- An overview of the architecture of the CAMCON system

- Specific requirements for CAMCON software ${ }^{1}$

- Programming guidelines and desired program features for CAMCON software

- CAMCON libraries ${ }^{2}$

- Software development utilities available within the CAMCON system

- Background on assessing the compliance of the WIPP

\subsubsection{Organization}

The following is an overview of the organization and content of the remainder of this manual:

Chapter 2 The CAMCON System. This chapter presents an overview of the CAMCON system.

Chapter 3 Software Development Overview. This chapter provides an cverview of development strategy, verification, and placement of software into the CAMCON

1. Minimum quality assurance requirements and procedures for the software were first presented in Rechard et al., 1991. Current QA requirements are found in the PAP(12 (1993) and suhsequent revisions.

2. The most current information is available from on-line documentation. 
Chapter 4 User Interface Guidelines. This chapter discusses suggested software features for interfacing with the user (customer).

Chapter 5 General Programming Guidelines. This chapter discusses guidelines and desired internal features of software developed specifically for performance assessment and the CAMCON system.

Chapter 6 CAMDAT_LIB: Computational Data Base Interface Library. This chapter describes the computational data base, CAMDAT, and the calls to the routines in the CAMDAT_LIB library. CAMDAT_LIB contains subroutines that read from and write to a neutral file, which is a file to which codes in the CAMCON system are linked.

Chapter 7 SDBREAD_LIB: Secondary Data Base Interface Library. This chapter describes the secondary data base and the calls to the routines in the SDBREAD_LIB library. SDBREAD_LIB contains subroutines that read from several neutral files, which are files that store parameter values pertinent to performance assessment calculations.

Chapter 8 CAMCON_LIB: Quality Assurance Routine Library. This chapter provides a description of the calls to the routines in the CAMCON_LIB library, such as the QA headers required on output, which provide a common interface to the user.

Chapter 9 CAMSUPES_LIB: Routines for Dynamic Memory Allocation and Other FORTRAN Extensions. This chapter describes the calls to the routines in the CAMSUPES_LIB library, which primarily allows programmers to dynamically dimension arrays in FORTRAN.

Chapter 10 Graphics Libraries: PLT_LIB and HJIPLOT_LIB. This chapter describes Sandia graphics libraries used in CAMCON plot programs.

Chapter 11 Graphic Device Interfaces: SVDI and DVDI. This chapter briefly describes the operation of the SVDI and DVDI libraries for the major CAMCON plot programs.

Chapter 12 Software Development Utilities. This chapter describes twelve codes available to assist the software developer. 
Appendix A Assessing the Performance of the WIPP. This appendix provides background information on performance assessment.

Appendix B CAMCON System Personnel and Software Sponsors. This appendix lists individuals to contact within the PA Department concerning various aspects of the CAMCON system.

A glossary of terms is found at the back of this manual.

\subsubsection{Use}

This report is a reference programmer's manual. As such, it contains extensive descriptions of CAMCON software libraries, software development utilities, and programming requirements and guideline.s. However, the manual assumes

- Familiarity with computer programming in FORTRAN

- Familiarity with PA Department software quality assurance procedures

- Access to some currently existing CAMCON system software (but not necessarily an understanding of the software's purpose)

- A cursory understanding of the VAX/VMS operating system

Hence, this programming manual does not introduce a novice programmer to the CAMCON system. Furthermore, it does not describe the WIPP project or the mechanisms of performing a complete performance assessment (PA) except for the summary in Appendix A.

The following are some suggestions for using this manual based upon information sought and level of expertise:

1. If you are an experienced programmer on the WIPP project and are familiar with perforniance assessments and the CAMCON system

- Skim Chapters 2 and 3

- Read Chapters 4 and 5

- Use the Table of Contents and the Index to locate specific information to supplement the on-line documentation provided on the WIPP computer

2. If you are a new programmer seeking an introduction to the CAMCON system

- Refer to Appendix B for a list of people to contact for answering questions

- Browse through the current version of the CAMCON User's Manual (e.g., Rechard, 1992)

- Read Rechard et al. (1991) and the current revision of PAP02, Software QA Procedures for the PA Department

- Browse through a software notebook on any piece of software (contact the person listed in Appendix B) and/or access the CAMCON system on the WIPP computer and examine the on-line documentation for several pieces of software 
- Obtain a listing of a piece of CAMCON software for referenise

- Read Chapters 2 through 5 of this manual

3. If you are seeking a description of the CAMCON system

- Read the first few chapters of the current version of the CAMCON User's Manual (e.g., Rechard, 1992)

- Read Chapter 2 of this manual

- Skim Chapters 3, 4, and 5

- Skim Rechard (1989)

\section{If you are seeking information about performance assessments or the WIPP}

- Refer to Appendix A (or Rechard, 1989) for a cursory description of the mechanics of a performance assessment

- Refer to the extensive description in the Preliminary Performance Assessment for the Waste Isolation Pilot Plant for the current year (e.g., WIPP Performance Assessment Division, 1991) and the list of references for more detailed descriptions.

\subsection{CAMCON Software Development Project}

Although software development is potentially a large effort, most of the development of each piece of soft ware is best performed by one or two individuals because the development task is usually problemdriven, and quick responses to the needs of customers and software users are of paramount importance. Empowering only one or two individuals to respond to customer needs improves the response time.

Furthermore, the Sandia PA Department for the WIPP is not organized by software development categories, such as code definition, programming, or installation, but rather by modeling task assignments. These task assignments correspond to specific problem areas of the WIPP such as repository/ shaft/borehole modeling and Culebra groundwater flow and transport modeling. Consequently, many specific software development needs are often defined by a task leader who may or may not be the CAMCON system coordinator. In addition, the software development responsibilities are not assigned to individuals performing software development only, but rather to individuals who may be working on specific problem modeling areas and thus have several other demands placed on them not directly related to software development. Therefore, communication and coordination of software development does not generally occur along the hierarchical stencil set up for the department (Figure 1-1). Nonetheless, the software developer is responsible for coordinating the development with the CAMCON system task leader. The purpose of this manual is to assist in the software development and coordination tasks. Refer to Appendix B for current personnel assignments. 


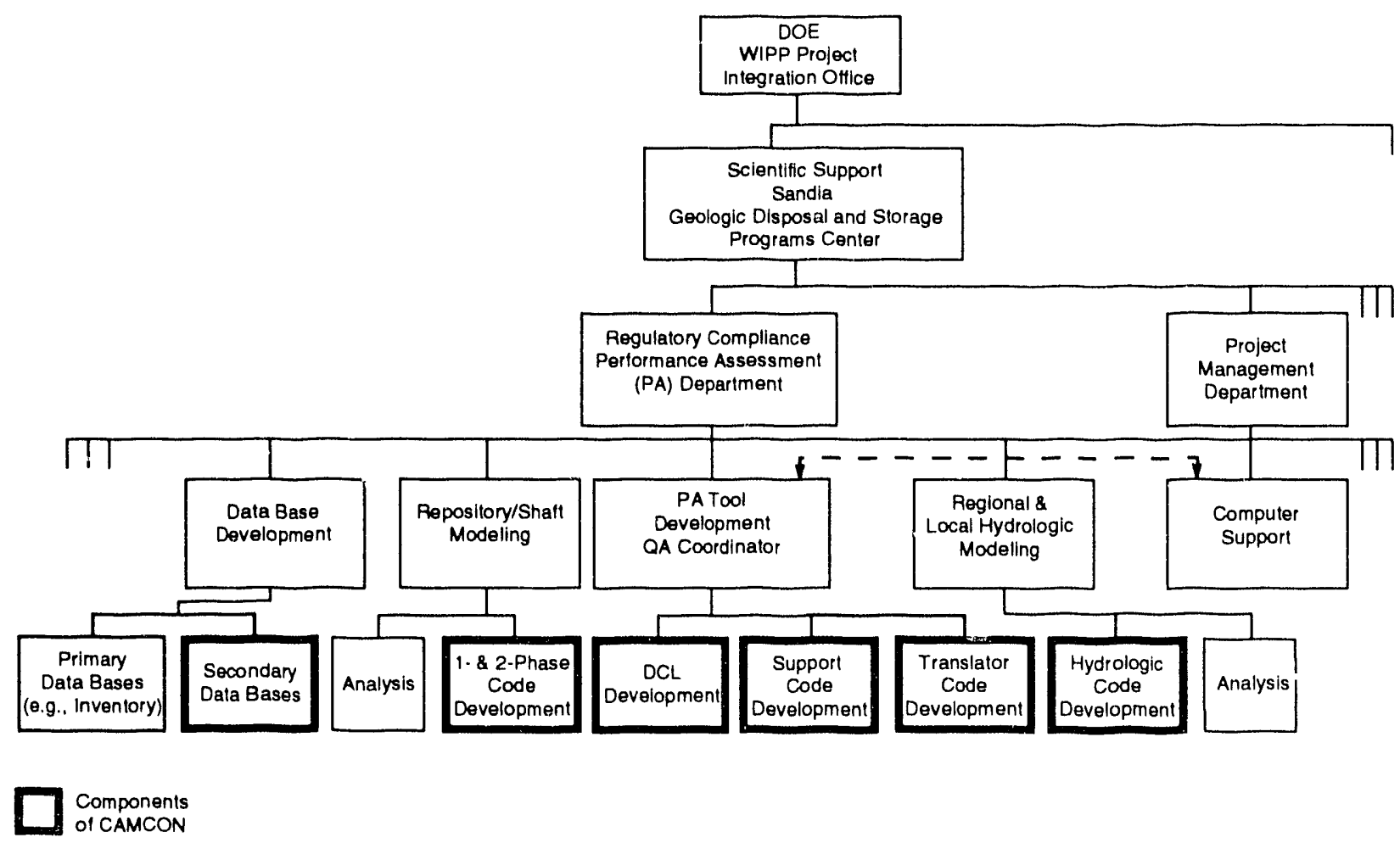

TRI-6334-219-1

Figure 1-1. CAMCON software development in the Sandia WIPP PA Department. 
Introduction

May 1993

1-6 


\section{The CAMCON System}

This chapter describes the purposes of the CAMCON system and, in general, how the system accomplishes its functions.

\subsection{Purpose of CAMCON}

A performance assessment (PA) is the process of assessing whether a system meets a set of performance criteria. For the WIPP, the system is a computational model (which, in turn, is a composite of several models) that represents a deep, geologic, repository disposal system for waste contaminated with transuranic radionuclides and hazardous chemical constituents; the assessment is performed by computer simulation; and the performance criteria are various long-term environmental metrics in U.S. government regulations (not short-term operational safety issues). CAMCON, the analysis system for running the PA calculations, has two important functions. First, it provides the analyst with the necessary tools and flexibility to build and execute all or some of the many different types of software necessary to investigate various events and physical processes of the WIPP and present the final output for comparison with the environmental regulations (e.g., the probabilistically based release limits in 40 $C F R$ 191). Specifically it is flexible in use so that it can be used to

- Characterize the WIPP

- Model scenarios and evaluate regulatory performance metrics

- Evaluate uncertainties

- Evaluate parameter importance on a disposal system or subsystem level

It is flexible in capabilities so that

- Many types of data can be used even with large uncertainty

- Many types of software can be used, including both codes that are computationally efficient and codes that accurately model various phenomena

For example, it allows an analyst to quickly identify available software and the necessary information for using individual codes, enabling the analyst to select the code(s) best suited for a particular study.

Second, several of CAMCON's procedures, utility programs, and even the directory structure assist in implementing software QA procedures (Rechard et al., 1991; PAP02, 1993). This is important because

- Repetitive calculations are needed for propagating uncertain'ties with $\mathrm{Mo}^{\mathrm{N}}$ e Carlo techniques

- There is a potential need to recreate simulations

- Software is constantly being refined to improve capabilities

For example, CAMCON:

- Has the ability to read model parameters from one central secondary data base to ensure the same data are used throughout the analysis and that data changes have to be made only in one place

- Semiautomatically links codes, reducing errors in keying in data by using a computational data base that stores all data and results in one location 
The CAMCON System

CAMCON Apprrach

- Has codes available to algebraically manipulate and plot intermediate (and final) results for careful scrutiny

- Serves as a software management system, providing rudimentary configuration control

- Stores FORTRAN libraries of commonly used subrot:íines

- Has on-line documentation for each code and library, consisting of a description of the code and its capability, summary of user commands, update history, and examples

\subsection{CAMCON Approach}

Several software analysis systems have been built to meet the general requirements of a PA. One approach is to build one code with numerous submodels (see, for example, VANDAL [Unsworth, 1989], SYVAC [Goodwin et al., 1987; Sherman et al., 1985; Dormuth and Sherman, 1981] or LISA [Saltelli, 1990; Bertozi, 1985; Saltelli, 1984|) or couple one analysis code with data preparation, Monte Carlo sampling, and results display (for example, NEFTRAN-S [Campbell et al., 1991]).

The Sandia PA Department for the WIPP chose to build an analysis "toolbox" (not one tool) by linking separate codes (either complex numerical or simple analytic codes) together. A performance assessment may or may not be rapid with the CAMCON system, however, because the difficulty and time required depend on the complexity and data requirements of the models (either analytic or numerical) chosen as components in the compliance evaluation. In a sense, the level of complexity of the models chosen determines how friendly the CAMCON system is to use. Hence, the CAMCON system can only approach the convenience of the other compliance assessment packages such as SYVAC (Sherman et al., 1985). Moreover, the use of individual codes in the CAMCON system requires that CAMCONexec, the executive package for CAMCON, be written using the computer system command language or using another language that makes extensive use of system routines.

However, these drawbacks are not always a shortcoming; the modular aspects of the CAMCON system and the WIPP compliance assessment methods make it easy for an analyst to

1. Directly make use of the interdisciplinary expertise residing in already existing codes as opposed to indirectly incorporating these concepts into one code,

2. Select the proper mathematical rigor from numerous existing codes, and

3. Use the same package for both the detailed examination of components as well as the overall disposal system (with the possible exception of substituting fast-running modules or modeling subdomains in the later analysis when desirable).

These points are especially important for nuclear waste repositories where the calculations are under intense scrutiny and sometimes elaborate procedures are necessary to justify that all steps taken in the analysis are defensible. For example, common engineering judgment in developing a simplified model, although frequently correct, is not always rigorously defensible; instead, detailed modeling must often 
precede simplification. Hence, a PA system that is capable of handling both detailed and simplified models is important and prompted the development of the CAMCON system.

\subsection{Software Management System and System Architecture}

The CAMCON system acts as part of a Software Management System (SMS) for software within the WIPP Project. The CAMCON system consists of the following:

- A repository for current versions readily available to Sandia personnel working on the WIPP Project but with "execute only" access

- Computerized help files on procedures for using the software that are readily accessible to users, sponsors, and auditors

Other conventional parts of a software management system, such as

- A cache system for storing previously used versions of software

- Storage for all pertinent documents and records required by the software QA procedures (e.g., software abstracts, formal external documentation, and change request forms)

are currently performed outside the CAMCON system.

The name CAMCON alludes to the concept of linking major codes together as a computational system, but it has many parts which make this function possible. The primary parts of the CAMCON system consist of (Figure 2-1):

1. Directory structure and protocols for storing software for rudimentary configuration control.

2. Executive package (a suite of procedural files and symbols to set up the computer environment) written in DEC (Digital Equipment Corporation) Command Language (DCL) for ready access and execution (either batch or interactively) of the computational and support modules .

3. Code modules broken down into:

- Seven computational modules (mesh generation, property assignment, etc.)

- One support module (e.g., plotting and algebraic manipulation) (eighth module)

- One utilities module for caching input files and results, listing programs, etc. (ninth module)

- A data base module containing soítware for storing and/or manipulating the secondary and computational data bases (tenth module)

4. The computational data base (CAMDAT) and secondary data base

5. A collection of frequently used subroutines in FORTRAN object libraries (e.g., plot libraries)

6. Help files for on-line documentation. 


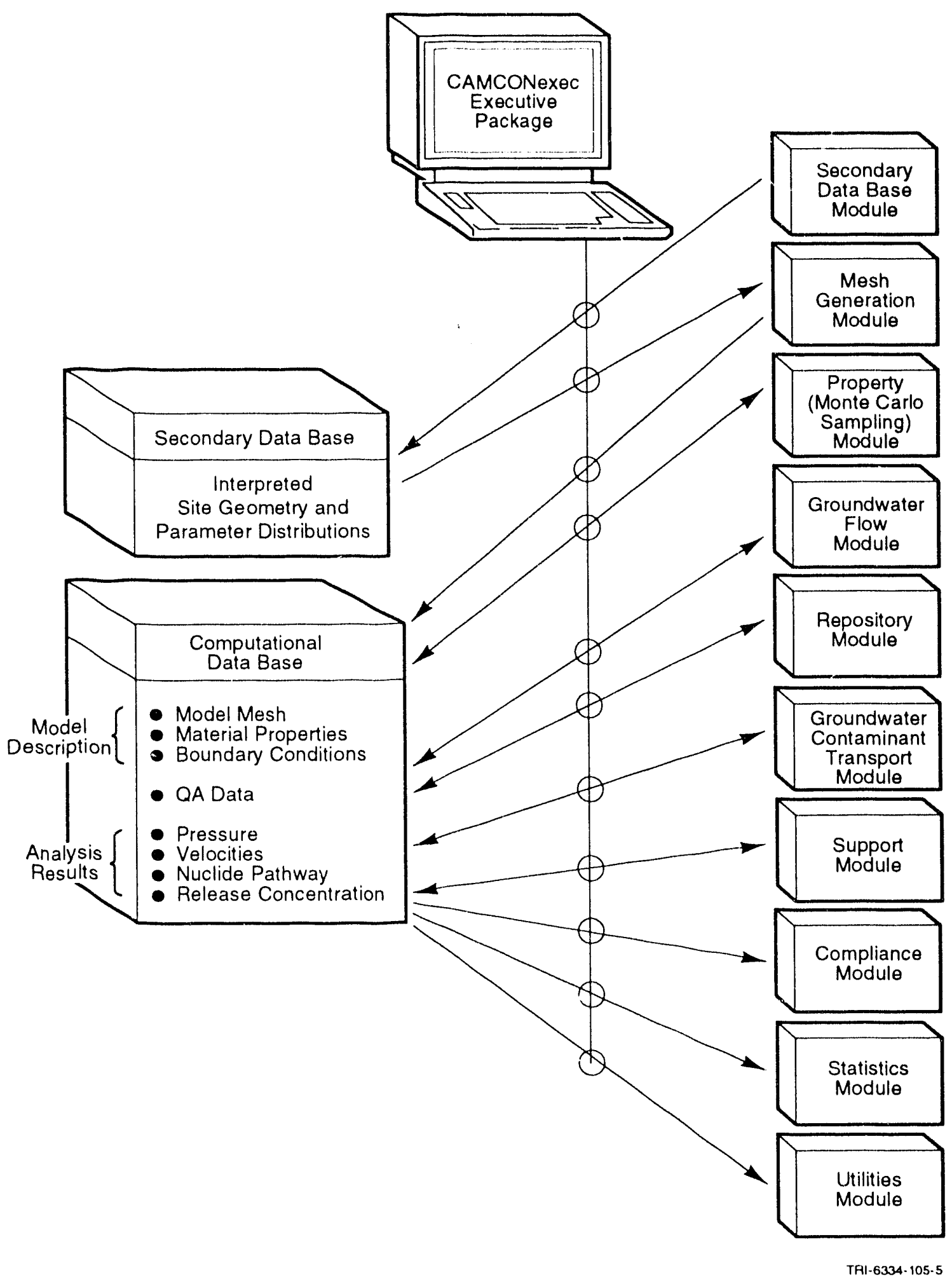

Figure 2-1. The analysis system CAMCON. The CAMCON system consists of (1) directory structures and protocols for storing software (not shown); (2) an executive package to access modules; (3) code modules consisting of seven computational modules, one support module, one utility module, and a data base module; (4) the computational data base (CAMDAT files) and secondary data base; (5) software libraries (not shown); and (6) help files for on-line documentation (not shown). (After Rechard, 1989, Figure 1.1.) 


\subsection{Directory Structure and Protocols}

The specific purpose of CAMCON's directory structure is to collect software essential to the performance assessment in one location. Except for the CAMDAT files generated during a simulation and the relational data base program, the CAMCON system resides under its own user account name (but owned by the CAMCONexec sponsor) on the WIPP VAX/VMS computer. The CAMCONexec sponsor is responsible for, among other things, directory setup and giving write access privileges to the software sponsors in the appropriate directories, where sponsor is generally defined as the individual responsible for ushering a particular piece of software through the quality assurance procedures of the PA Department and other adminsitrative tasks concerning the software (refer to the glossary for unfamiliar terms).

The main directory, [CAMCON], contains six categories of software divided into numerous code directories and five subdirectories that correspond to some of the CAMCON system parts (Figure 2-2):

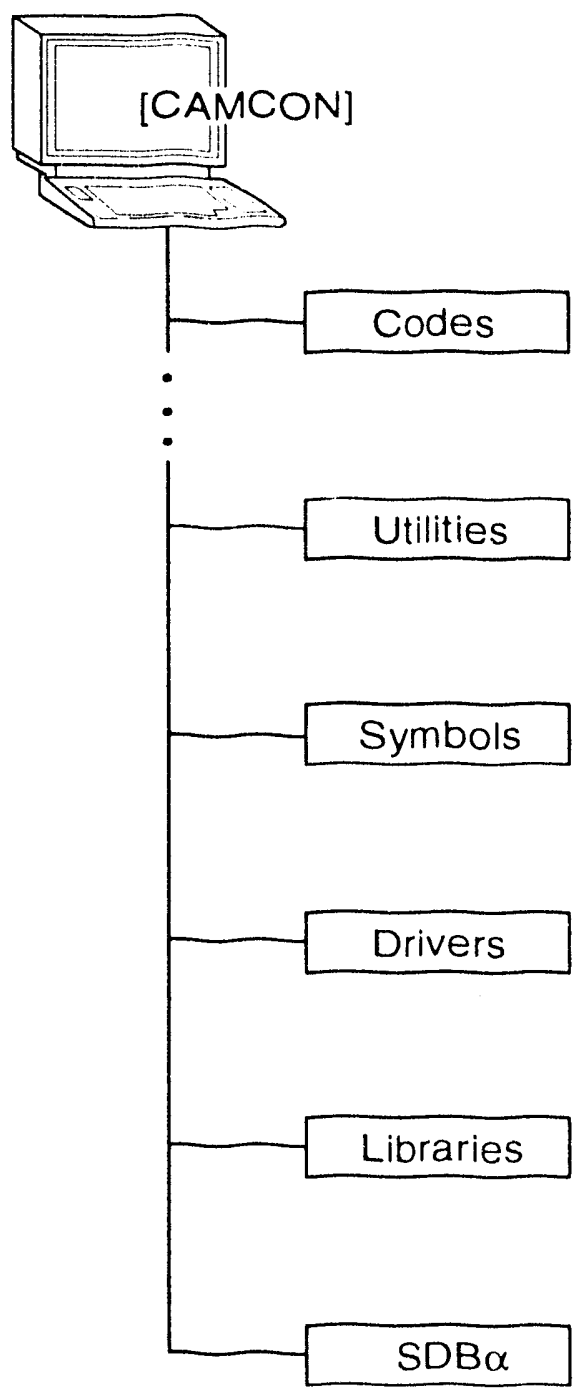

A code directory contains source files for a code. The CAMCON system has between 60 and 70 code directories that can be grouped into seven computational modules and one support module (see Section 2.7, "Modules").

The Utilities directory contains miscellaneous codes for the code developer, code sponsor, or analyst to help with simple tasks, e.g., LISTFOR for listing FORTRAN source cude.

The Symbols directory contains DCL command files that define logical symbols for the user (they build the CAMCON system "environment" for the user).

The Drivers directory contains the CAMCONexec executive package, comprised primarily of the DCL procedural files that link the computer codes of the CAMCON system.

The Libraries directory contains the program libraries such as COMCON_LIB, consisting of standard routines (e.g., QASETUP) used by most codes.

The SDBa directory contains the secondary data base files of parameters to use in the current $P A$ calculations, designated by TRI.6334.215.1 $\alpha$ (several SDB directories may be present).

Figure 2-2. The ICAMCON| directory structure. 
Except for the first category (codes), each category of software is stored in its own subdirectory. The first software category (codes) consists of all the codes in each of the ten modules. Although organizationally it might be desirable to have a code subdirectory which is then divided into ten modules and further subdivided for the individual codes, it was not desirable in a practical sense because the path name to the code quickly becomes long and un wieldy, a definite dra wback for the calls in the procedural files. There are ways around this difficulty but the easiest solution was not to add a detailed directory structure for the codes.

Furthermore, and more importantly, several of the codes do not fit in only one module category. Several of the codes have multiple capabilities, such as fluid flow and contaminant transport, and thus, the categories are more of a convenience when describing the codes to users, rather than an intrinsic property of the codes themselves. A notable exception to this general practice was the grouping of the codes from the utilities module into their own subdirectory since these were usually run outside the CAMCON executive package. The codes in the utilities module are described in Chapter 11, "Software Utilities."

\subsection{Procedural Files (CAMCONexec)}

\subsubsection{Purpose}

The purpose of the collection of CAMCON system procedural files, CAMCONexec, is to act as the interface between separate codes, automating many steps in the modeling task, and thus freeing the analyst from tedious and repetitious tasks (e.g., numerous Monte Carlo simulations). Furthermore, it helps to ensure quality results by logging file information. Specifically, CAMCONexec does the following:

- Acts as user interface with the codes permitting either interactive or batch operation (e.g., generation of "macros" for later submission in background)

- Automatically names transfer files between codes, including the Monte Carlo run number, to avoid misinterpretation

- Traces the calculations so that they can be repeated

- Builds cache files

Some tasks are not automated by CAMCONexec so that the analyst can have more flexibility in how an analysis is performed. For example, CAMCONexec does not set up a secondary data base or automatically dictate which modules are to be run in what order. Furthermore, CAMCONexec cannot eliminate all user errors (intermediate results should still be examined).

\subsubsection{Language}

Al though some routines do use C, CAMCONexec is mostly written in the operating system language, DEC Command Language (DCL), and is thus not directly portable to non-VMS systems. Even if a more 
general languare such as $\mathrm{C}$ had been used exclusively, the extensive need for access files and file attributes would have made the procedure quite specific to the VAX computer. CAMCON procedural files follow the same documentation requirements as all other software (e.g., FORTRAN), so they can

ie interpreted and translated to another computer system command language if necessary; although, • the amount of translation may be large if all the capabilities in the DCL procedural files are wanted.

Specific instructions to the CAMCONexec sponsor are given in Section 3.4.8, "Writing CAMCONexec Procedural Files for Software."

\subsection{Logicals and Symbols}

Defining "logicals" and "symbols" frequently results in a much more friendly environment for the analyst. Under VAXVMS, a logical is a name that can be used in place of a file specification (CAMCON\$ROOT is a iogical name for disk: [CAMCON]). Logicals define the paths to directories sioring the specific software such as codes and libraries. In contrast, a symbol is a name that represents a numeric, character, or logical value. A symbol can also be used as an abbreviation for a long command line, a command procedure, or to define a foreign command-a special symbol that can be used to execute a program by typing only the symbol name. Programs that use the command line to pass data (i.e., file names) must be defined as foreign commands. Most programs in CAMCON are defined as foreign commands

The command procedures that set the CAMCON system environment reside in the CAMCON\$ROOT: [SYMBOLS] directory. The main command fil, CAMCON SYMS.COM, under the ownership of the CAMCONexec sponsor, controls the environment set up by executing supplementary command files. The software sponsors are responsible for defining symbols and logicals for their soitware in the supplementary command files, sponsorname _SYMS.COM. (The sponsor's name is used instead of tne software name to reduce the number of supplementary command files since there are about ten sponsors versus over 80 pieces of software.) The last supplementary command file executed is PATCH_SYMS.COM, which can be modified by any analyst temporarily needing to redefine symbols or logicais. The analyst can define the (AMCON system environment by typing the system symbol CAMCON SYMS at any time or by placing the symbol in his or her LOGIN.COM file. (See Figure 23 for an example of files on the [SYMBOLS] directory.)

\subsection{Modules}

When describing CAMCON codes, the PA Department roups them into modules (categories) as a convenient means to describe their use to PA analysts. The ten code modules of the CAMCON system are the 11 secondary data base module, (2) mesh generation module, (3) property assignment module, 


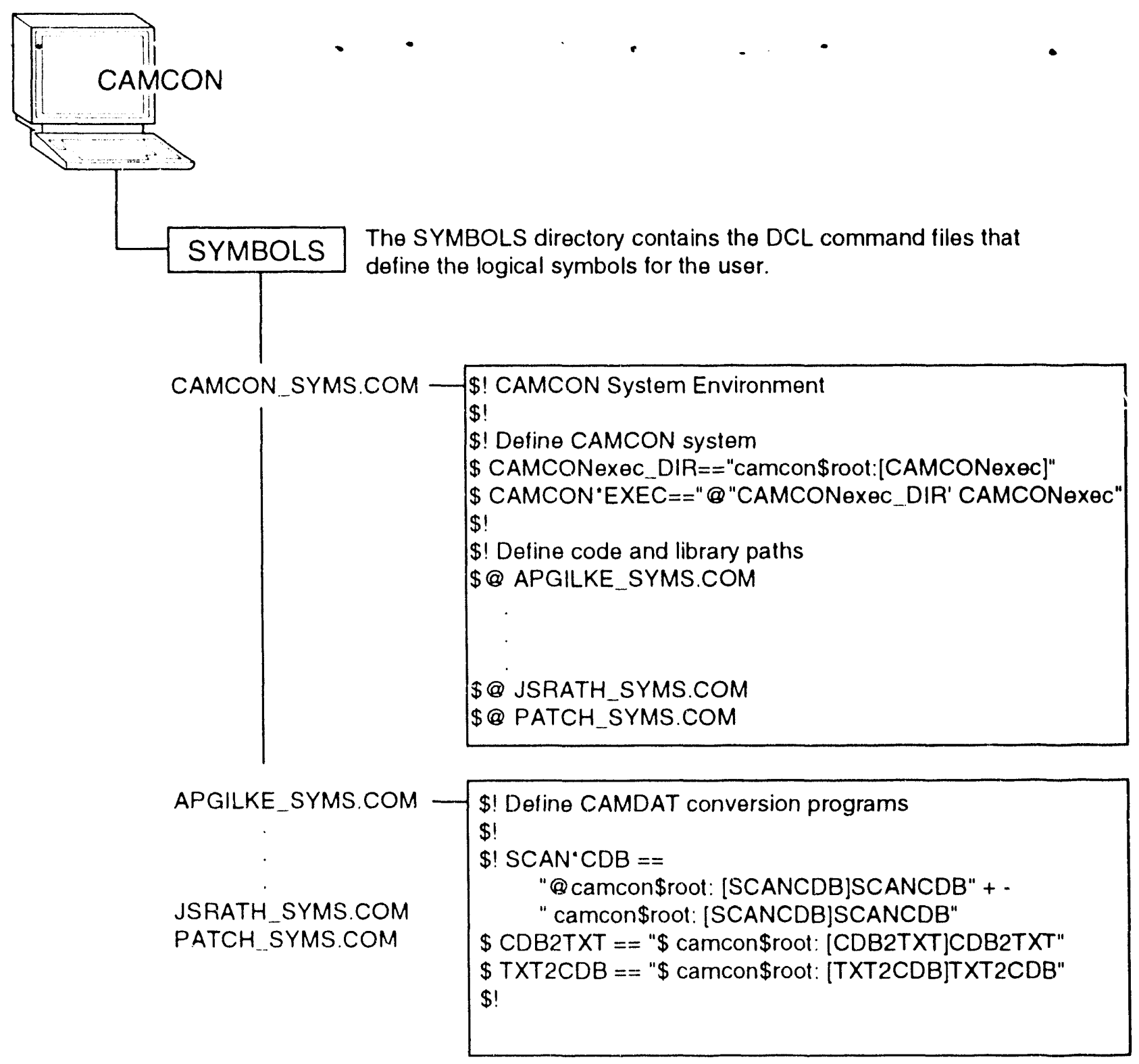

TRI-6334-271-0

Figure 2-3. Example of files in the |SYMBOLS| directory. This DCL fragment eliminates several conditional statements for clarity. Also, to facilitate moving the CAMCON system to other disks or computer systems, the full path name is not used. Rather, a system logical, CAMCON\$ROOT, is used to define the disk location. 
(4) groundwater flow module, (5) ground water contaminant transport module, (6) repository module, (7) support module, (8) compliance module, (9) statistics module, and (10) utilities module.

- The Secondary Data Base Module inputs and manipulates the data collected during disposal system characterizatiön.

- The Mesh Generation Module discretizes the model's domain (either the groundwater flow, ground water transport, or repository model's domain) to assess consequences of release scenarios with the consequences analysis models.

- The Property Assignment Module places initial conditions, boundary conditions, and all model parameters needed by consequent analysis models into a CAMDAT file.

- The Groundwater Flow Module establishes regional and local fluid flow conditions within and directly surrounding the disposal system.

- The Groundwater Contaminant Transport Module predicts radionuclide migration from the repository source to the disposal system boundary.

- The Repository Module develops a source term for transport calculations by incorporating the complex processes in the waste container, disposal room drifts, and shafts

- The Support Module contains codes that manipulate, list, and plot information in a CAMDAT file or codes to support other modules.

- The Compliance Module calculates various metrics (performance measures) to be used for evaluating compliance with environmental standards.

- The Statistics Module develops and plots regression models between various results (metrics) and parameters sampled.

- The Utilities Module contains codes that assist in the operation of the CAMCON system or specific codes within other modules and that do not interface with CAMDAT.

\subsection{Codes}

In most cases, a choice of computer codes is available within each module. For example, five codes are available in the ground water flow module; the selection depends upon the type of problem under consideration.

\subsubsection{Codes within Each Module}

The codes available within each module are described in on-line documentation and in the current version of the CAMCON user's manual (e.g., Rechard, 1992). 


\subsubsection{Code Directory Structure}

The directory structure for each code is arranged so that it can (1) store several active versions of a code, (2) store the help file directly with the source code files, (3) store the verification problems as a subdirectory to the source code files, and (4) store the previously working version of a code (desirable if the code is undergoing numerous modifications, i.e., a Class $\mathrm{X}$ or $\mathrm{C}$ code) as a subdirectory to the source code files (Figure 2-4). The previously working version is not necessarily the cached" (preliminary archive) version of the code, but remains on the system as a convenience to the analyst who may not be able to respond quickly to code changes, or who may discover a conflict (that needs to be corrected) with old and new capabilities. Having the old version remain on the system allows the analyst to continue working unaffected for a short period of time.

Only the software sponsor, responsible for ushering the code through the QA procedure, has write and delete privileges within the code directory. All other users have execute and read privileges only. In some cases, the read privilege is denied to all users, as in the case of proprietary codes or when desired by the software sponsor.

\subsubsection{Naming Conventions for Codes}

When several versions of the code exist within CAMCON (as frequently occurs), the software sponsor is responsible for defining which codes are available and the appropriate code designation. The usual designation for accessing these codes through CAMCON is as follows:

- Codename for current version

- NEW_codename for new version undergoing testing (source code resides in the sponsor's directory, not the CAMCON directory)

- OLDmmmyy_codename for previous working version (the three-letter month and two-digit year indicated by mmmyy are optional)

- A_codename when the version in the main code directory is something other than Class A software (e.g., the most recent version of a code is level $C$ but a previous version was level A)

- D_codename when it is necessary to temporarily activate an obsolete version of a code (e.g., when a customer asks that comparisons be made between an obsolete and new code. A D class code can never be the sole basis of an analysis (PAP002, 19931).

\subsection{Data Bases}

For the WIPP PA, the data bases are grouped into three general categories:

- Primary

- Secondary

- Computational

* Refer wo the Glessary for definitions of unfamiliar terminolegy 


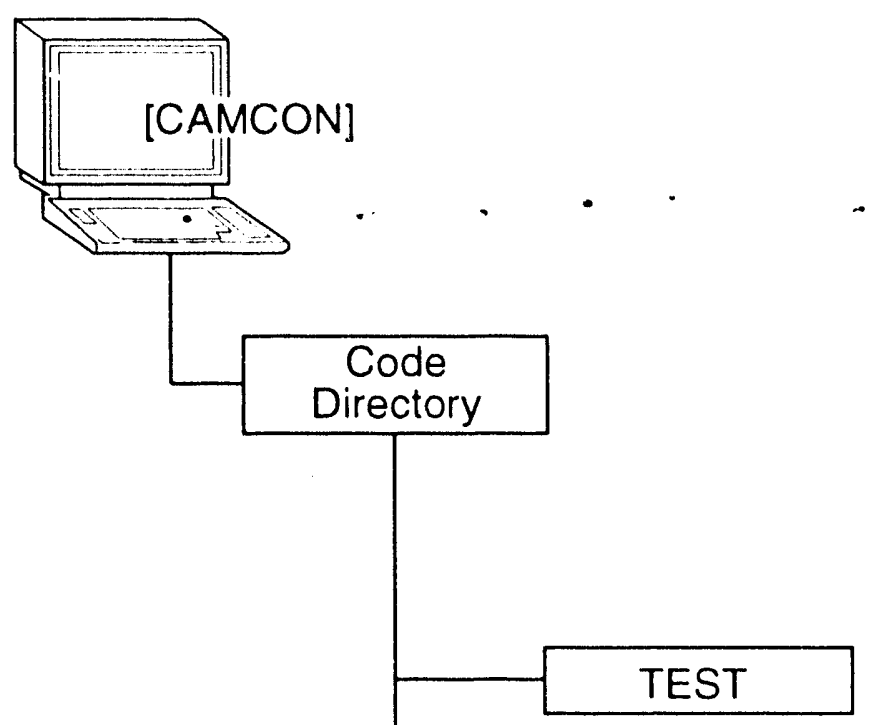

- The CODI: directory contains the following two file categories and up to four subdirectories:

-Help File

-Current Code Files

- The TTEST subdirectory contains input files (and any necessiry dalta base files) to verify the program.

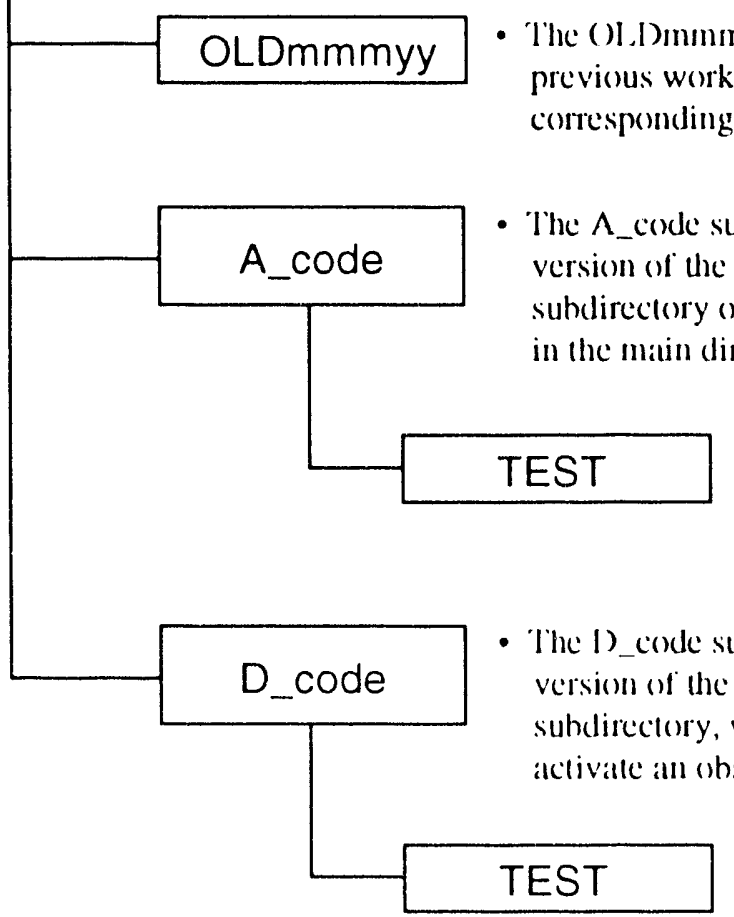

- 'The 'TLST' subdirectory contains the corresponding input files and necessary data base files 10 verify the $A$ version of the code.

The 1)_code subdirectory contains the (lass D) version of the code and llelp file and TTEST subdirectory, when necessiry to temporarily TEST

- The TIEST' subdirectory contains the correspending input files and necessary data hase files previously used to verify the code when al a level $A$ or $C$.

TRI-6342.267-1

Figure 2-4. Code directory structure. 
These categories correspond with the uncertainties in the data (e.g., measurement error-principally in the primary data base, conceptual model uncertainty-principally in the secondary data base, and computational model uncertainty-principally the models in the compliance assessment system). All the uncertainties combine to produce the uncertainty in the results. These three categories are discussed below.

\subsubsection{Primary Data Bases}

Requirements. Primary data bases contain the measured field, laboratory, and literature data gathered by investigators during disposal system and regional characterization (see Figure A-1 of this manual). Several requirements exist for the primary data base (Rechard et al., 1992b, Section 1.5; Rechard, 1989, p. 61):

- Contain high-quality data

- Serve as "memory" for the project or corporation

- Contain data applicable to the performance assessment

- Involve a minimum of subjective interpretation

- Possess a flexible, easily understood, and accessible structure

Furthermore, several general modeling difficulties affect the storage of data that must be addressed on a case-by-case basis. These include

- Spatial variability of parameters in a geologic medium

- Time varying parameters

The primary data base serves as a central repository of data for diverse disciplines and must be stored in a form readily understood by the various analysts. The data base must be readily accessible to the analysts, since varying conceptual models (embodied in the secondary data base) will be constructed from these data for the various scenarios as understanding about the disposal system changes.

Suggested Format. To accommodate these requirements and difficulties, a relational data base is suggested, but even within relational data bases, the contents of various tables can vary. Practical experience suggests that tables containing one parameter (e.g., hydraulic conductivity) or a group of closely related parameters (e.g., Young's modulus and Poisson's ratio) are best. The four proposed components of each relational table in the primary data base are

- Parameter location

- The parameter and units of measure

- Test conditions and supporting information

- References and miscellaneous information 
Primary data bases are not a formal part of the CAMCON system (nor are they shown in Figure 2-1) and are not discussed further. Rather, all codes in the CAMCON modules access data from a secondary data base, which contains model parameters derived from interpreted data extracted from one or more primary data bases.

\subsubsection{Secondary Data Bases}

Although text and general scientific information on the assumed important physical processes for the scenario are not stored, the secondary data base stores all parameters necessary to define the conceptual model of the disposal system for the hypothetical release scenarios. The data base is termed a "secondary" data base since the model parameters are a compilation of "primary" data (field measurements, lab experiments, and other measurements of mechanical, chemical, geologic, and hydrologic parameters) that have been interpreted by investigators and experts. The level of interpretation can range from objective interpolation (i.e., well-known statistical techniques that are easily reproduced by others) to subjective extrapolation (i.e., techniques that are not easily reproduced unless well documented). To bridge any remaining gaps in data, knowledge derived from literature, individual professional judgment, and expert panel judgment are sometimes used so that a complete conceptual model is available.

Figure 2-5 illustrates the relationship between the primary and secondary data bases.

Although the interpretation and selection of the property data is a mentally engaging process involving professional judgment, experience, and, at times, consensus building, data entry into computer models is a tedious, error-prone process, especially when done many times for different models in the performance assessment of the WIPP. Storing parameter data in one location offers several advantages:

- By isolating the data entry in one place, a task leader can exercise more effective control. For example, when a decision is made on the value of a parameter, the value has to be relayed to only one or two individuals who enter the data into the data base rather than to all analysts who are using the parameter in their models. Also, changes to data can be made without verifying that all analysts make the necessary changes in the applied models.

- The use of consistent units for parameters throughout an analysis can be more easily enforced.

- Elaborate checks on the reasonableness of the data can be made when entering data. These checks might be too time-consuming to build separately into all codes.

The parameters for the conceptual models are divided into four file groups (Figure 2-5):

- Parameter distributions

- Stratigraphy

- Well location data

- Typical dimensions of the facility (drawings) 


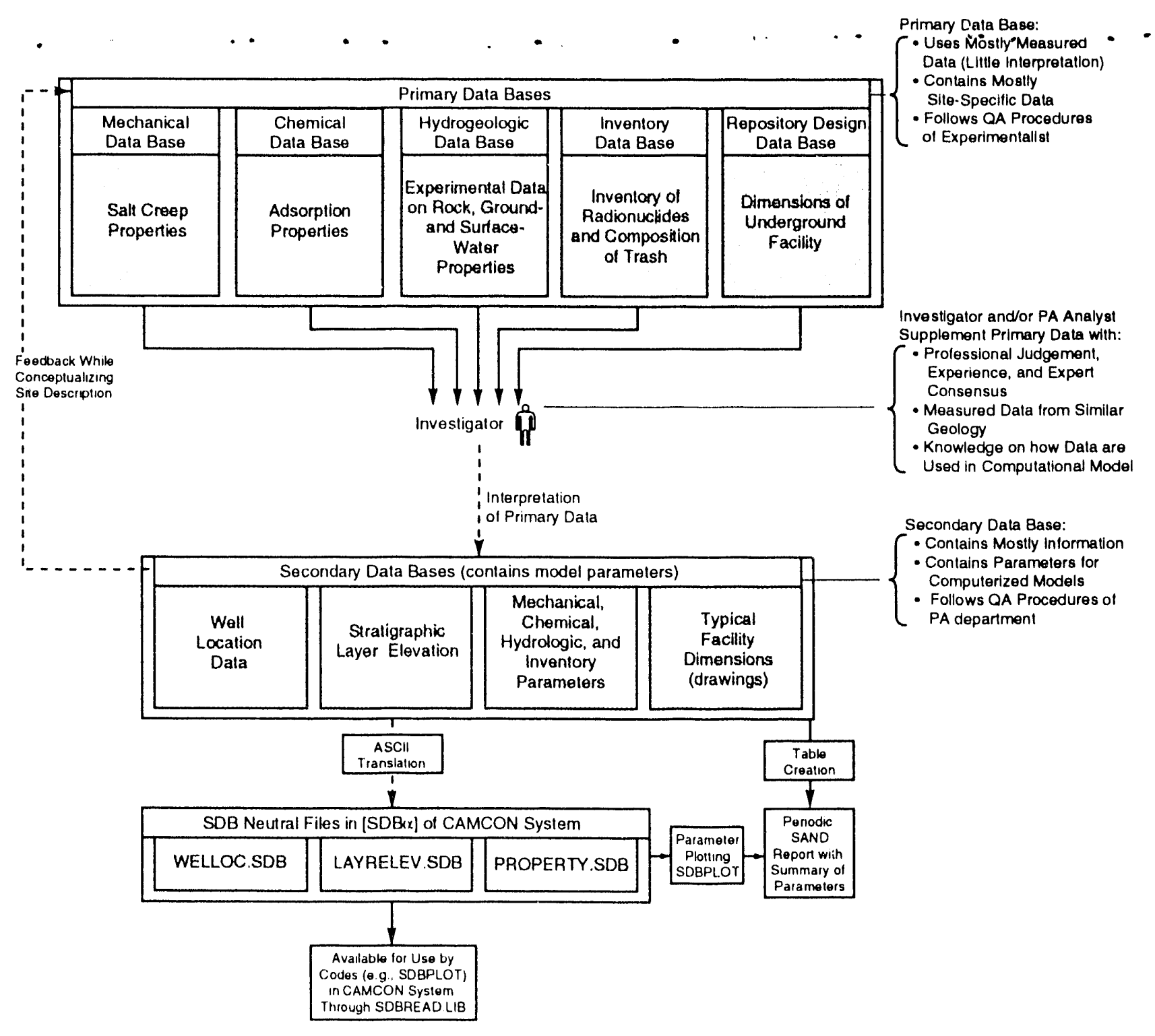

TA1-6334-20-2

Figure 2-5. The investigator interprets data in the primary data base that is consistent with the use of a model parameter in the computational model (after Rechard, 1989, Figure 5.1). This information is then stored in the PA secondary data base and made available to CAMCON codes via SDB neutral files and the SDBREAD_LIB routines. 
Currently, data for the WIPP are compiled using the INGRES ${ }^{\top M}$ relational data base software. The use of INGRES ${ }^{\text {TM }}$ is not a requirement since the CAMCON software reads an ASCII transfer file. In fact, $\mathrm{R}: \mathrm{BASE}^{\mathrm{TM}}$, a personal computer relational data base, was used while the secondary data base was small. The data base could also be linked to a graphical information system (GIS), such as Arclnfo ${ }^{\mathrm{TM}}$, if d.esired.

- The WंIPP project indirectly has a similar capability by moving data into the computational data base and then displaying the data spatially with the plotting package, BLOT (Rechard, 1992; Gilkey and Glick, 1989).

Some of the information stored in this data base is converted into neutral files and stored in the $[\mathrm{SDB} \alpha$ ] subdirectory of [CAMCON] (Figure 2-5). These files can then be read by software in the Property Assignment Module of the CAMCON system (see Figures 2-1 and 2-2). Initial software documents on the CAMCON system (e.g., Rechard et al., 1989) described in detail the formats used for writing the neutral files from the secondary data base. However, to facilitate rapid change in the file formats (sometimes necessary to accommodate new types of model parameters), codes now access the model parameters through the SDBREAD_LIB library mentioned in the previous section and fully described in Chapter 7 of this manual.

\subsubsection{Computational Data Base, CAMDAT}

The single most important method used to perform the complex tasks of the compliance assessment (i.e., linking codes, properly identifying Monte Carlı simulations, and controlling data flow) is requiring that all intermediate results of computer codes pass through a neutral file which is a part of the computational data base, or CAMDAT (Compliance Assessment Methodology Data Base). CAMDAT uses a neutral-file format so that a series of codes can be linked by a "zig-zag" connection rather than by the more common serial connection. For example, if the analyst wants to run a specific computational code, the analyst would use a data preparation code in the CAMCON system to first write the input file needed, execute the module, and then write the output file(i.e., results) to CAMDAT. These results could then be accessed by another module.

Neutral File Advantages. This method of using a neutral file offers six advantages, which are depicted on the next page and expanded in the paragraphs that follow:

- Only one plotting code is needed to display results

- Only one code is necessary for manipulating the data

- Analyses are easily changed to take advantage of a more precise code, a faster running code, or a subdomain of the model (a truly modular method)

- Order of applying codes is easily changed

- Coder interaction is feasible 


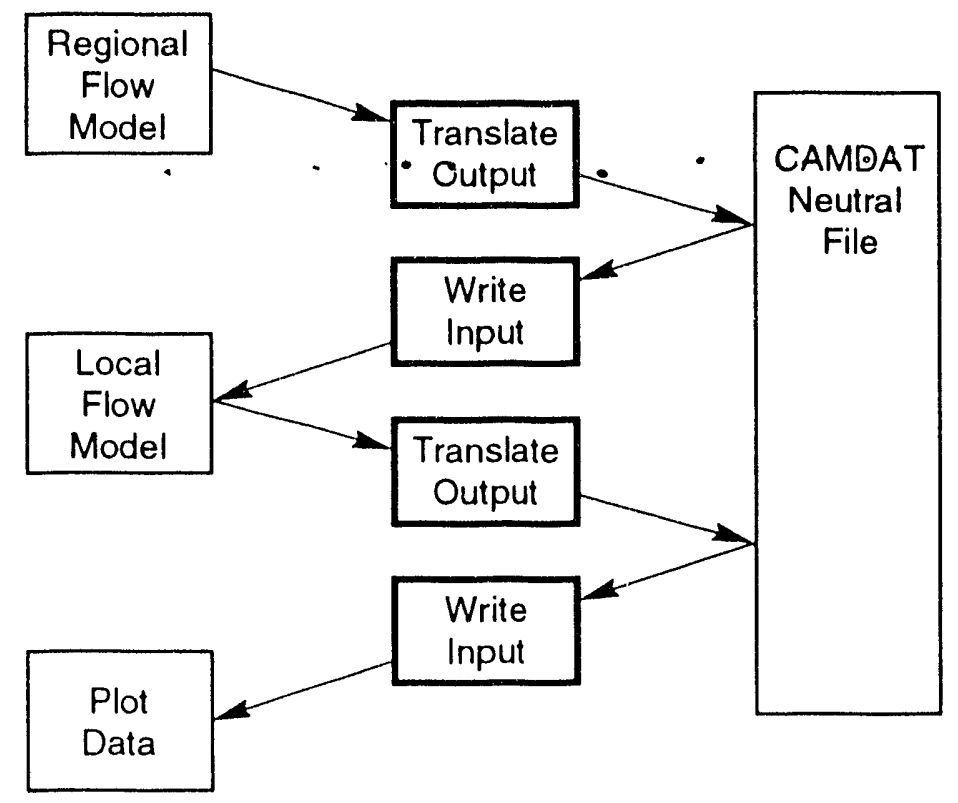

TRI.6334.29.2

- Design of CAMCON, system to automate performance analysis, is facilitated

- Assuring quality is straightforward because all data are in one location

One Plotting and One Data Manipulation Code. As implied above, a distinct advantage of coupling codes through the computational data base (CAMDAT) neutral file is that the codes in modules can be used at any time. Two codes of particular interest for analysis are the plotting and algebraic manipulation codes, BLOT and ALGEBRA, respectively (Gilkey and Glick, 1989; Gilkey, 1988; Rechard, ed., 1992). For example, BLOT can display data input, such as the spatial distribution of transmissivity, or intermediate results, such as flow velocity. Furthermore, parameters or results can be algebraically manipulated at any time by combining parameters to create code input (e.g., storativity from rock porosity, rock compressibility, and fluid compressibility) or to examine results (e.g., evaluating fluid flux across a boundary given the discretized areas and velocities).

Codes Easily Changed. Using a computational data base permits codes to be easily replaced because when swapping codes, completely new translators with well-defined goals can be written; a programmer need not patch up previously existing software. To elaborate, an experienced programmer can save time by not needing to first understand a previously written translator to substitute a code; instead, the programmer writes completely new translators between the computational data base and new code. 
The functional details of the translators will vary for each computational module of the compliance assessment. Potentially, a code module would be written so that it can read and write directly to the computational data base, entirely avoiding the need for a translator. When the code developer is responsible for both the physics and data manipulation, this approach is feasible; however, when the code is being developed by persons whose jobs vary between model physics, numerical solvers, and data and user interfaces, linking with pre- and post-processors as described below has worked well in practice.

In addition, for the WIPP, several major codes predate the computational data base. To avoid seriously complicating quality assurance by modifying coding, a translator is written that creates the ASCII input control cards by reading the data base and a supplemental program control file. Another translator is written to convert the ASCII output of the program module into the file format of the data base.

A distinct disadvantage of this approach is the limited ability to build a robust input translator to make use of all features of a general purpose code, especially if the code uses an archaic method of controlling features and lacks default values. However, only when a preexisting code is well understood have we modified a quality assured code (and quality assured again) to read the data base directly.

When examining the flow of data as described, it must be understood that the algorithm for assessing compliance of a disposal system has been made not only conceptually modular but modular in a practical sense also because the flexibility to replace codes is built in. Furthermore, if it makes sense to do so, the order of applying codes can easily be changed.

Code Iteration $\mathrm{Fe}_{\mathrm{e}}$ sible. Iterating between the results of two codes is more feasible, since the codes talk to each other through a data base. This greatly increases the flexibility of the performance evaluation algorithm. Apparent disadvantages to this are that processes may not be coupled, the computation may be inefficient, or numerical instability may occur; however, when these situations arise, the system will accommodate the introduction or development of a new code that does address these issues.

Controller Design Facilitated. Design of the executive package, CAMCONexec, to automate the analysis is facilitated when all information resides in one data base. By using a CAMDAT neutral file, building an executive package to couple the codes together, to control data flow carefully, and to run the codes is straightforward.

Assuring Quality More Easily. Assuring the repeatability and quality of the analysis is made easier when all data pass through one data base because information about all modifications of the data is recorded in a consistent format and stored in one location. These QA records are printed when results are plotted, a feature especially important when making numerous Monte Carlo runs (for propagating uncertainty of parameters) where results may vary only slightly and the analyst can easily misidentify and thus misinterpret a run. 
In summary, the advantages of the CAMCON system center around efficient use of software development resources, analyst efficiency, management of code development when codes are replaced, and quality assurance of the analysis.

Information Stored in CAMDAT. Three types of information are stored in a CAMDAT neutral file:

Computational Data Base (CAMDAT) Neutral File

- Quality assurance infurmation, such as a record of the code name, version identification, and the date on which each code writes to a CAMDAT file

- Input data, such as finite-element or finite-difference geometry data; geologic, hydrologic, and chemical parameters; and model boundary conditions

- Output data, such as fluid flow results, concentrations from source term or transport modules, and particle pathways

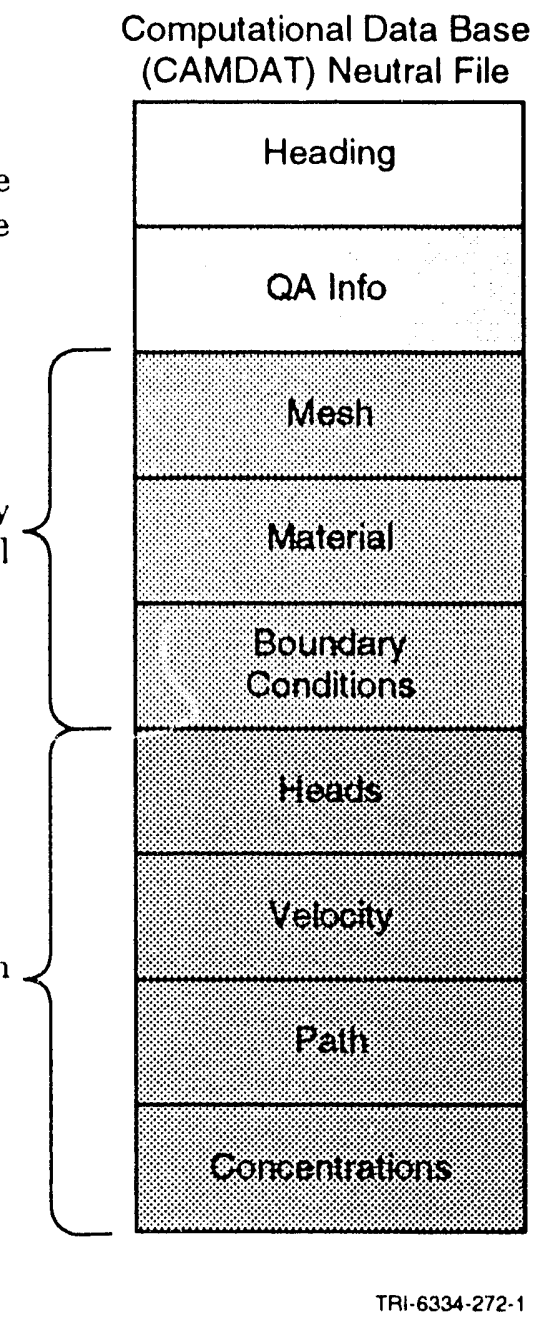

Besides the general requirement to store these three categories of information, other requirements on the data base file format include the following:

- Ability to handle input and output, multiple element types, and boundary conditions for both fluid and mechanics codes

- Storage of either finite element and finite difference mesh and results

- Storage of array lengths so that the reading program can dynamically allocate memory

- Efficient use of storage and rapid access of data are also desirable, but have been sacrificed somewhat to meet the other requirements 
Although the order of writing the data in a CAMDAT neutral file is conceptually correct in the preceding illustration, the actual manner of storage can constantly change as new requirements are placed on the data base; hence, codes do not directly access a data base neutral file but rather call subroutines which then access the data base. This groatly simplifies the process of changing the structure of the file. Thus, while earlier manuals on the CAMCON system had an extensive description of the manner of storage in a CAMDAT file, these descriptions have been replaced with descriptions of the calling subroutines in Chapter 6 of this manual.

A complicating factor of the computational data base is that several different model scales are necessary (see Appendix A): the regional, the repository, and the local. Furthermore, results from the regional, local, and repository models must be accessible to the other codes. To handle this problem, the computational data base is composed of separate files, all with the same format but with a file heading indicating the model scale. Furthermore, because uncertainty is propagated through the system by making numerous Monte Carlo runs, the number of files in CAMDAT is large. Currently, the CAMDAT is organized through the use of separate directories on the computer rather than through a relational data base system.

\subsection{Software Libraries}

A collection of subroutines has been placed in several object FORTRAN libraries within the CAMCON account, under the |LIBRARIES| subdirectory, to

- Simplify and speed up initial coding by reducing the amount of coding and subsequent errors

- Help to meet QA traceability standards

- Standardize the user interface

- Expand a code's capability

- Improve code maintainability (minimize the support effort)

- Isolate machine-dependent coding

Their use is strongly encouraged even though a programmer may dislike the use of "black boxes." The functions performed by these subroutines are usually quite simple, and source coding to understand the methods used is readily available in the software notebooks (see Section 4.7.1, "Software Notebooks"). 
The [LIBRARIES/ subdirectory is owned by the [CAMCON/ directory sponsor. The seven main libraries are:

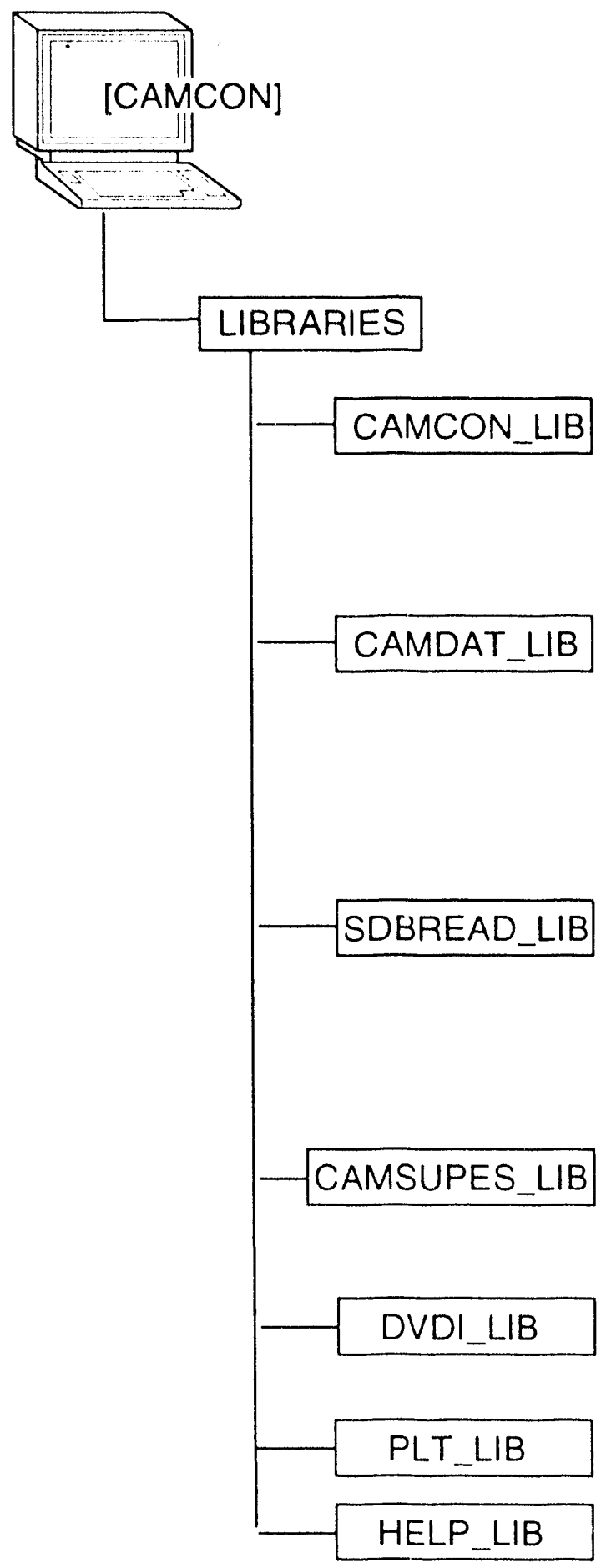

- The [LIBRARIES] subdirectory is owned by the [CAMCON] directory sponsor.

- CAMCON_LIB contains standard subroutines used by codes developed specifically for CAMCON, including subroutines that write standard headers to the output to meet software QA requirements and subroutines that give all the codes a standard user interface.

- CAMDAT_LIB contains the subroutines used to read from and write to the computational data base file, CAMDAT, to standardize the interface between the codes and CAMDAT. This standard interface permits the sponsor of the data base file to change the data structure to meet the changing needs of the compliance calculations without affecting the operation of individual codes.

- SDBREAD_LIB contains the subroutines used to read from and write to the secondary data base file which stores parameter values. These routines standardize the interface between the code and the secondary data bases such that any necessary changes on the data structure do not affect the operation of individual codes.

- CAMSUPES_LIB contains the subroutines for dynamic dimensioning of FORTRAN arrays, and thus isolates this machine-dependent coding.

- DVDI_LIB contains the Sandia graphic device interface drivers for the plotting codes.

-PLT_LIB contains the Sandia graphics library.

- HELP_LIB contains the help library for all of the codes within CAMCON. HELP_LIB is created from all the individual text help files, in the code and library directories and then is built as a standard VAX/VMS HELP file (DEC, 1989, 1990). 
Because the routines are intended to be reliable and maintainable, most.

- Are simple, not extremely sophisticated

- Do not necessarily maximize CPU speed

- Do not take advantage of extended system capabilities

- Are generaily independent of the operating system

- Isolate any system-dependent functions

Figure 2-6 illustrates the interfaces wrovided by the seven FORTRAN CAMCON libraries (CAMCON_LIB, CAMDAT_LIB, SDBREAD_LIB, PLT_LIB, HJTPLOT_LIB, CAMSUPES_LIB, and DVDI_LIB) to the PA analyst, secondary data base, computational data base, graphics devices, and computer memory, respectively:

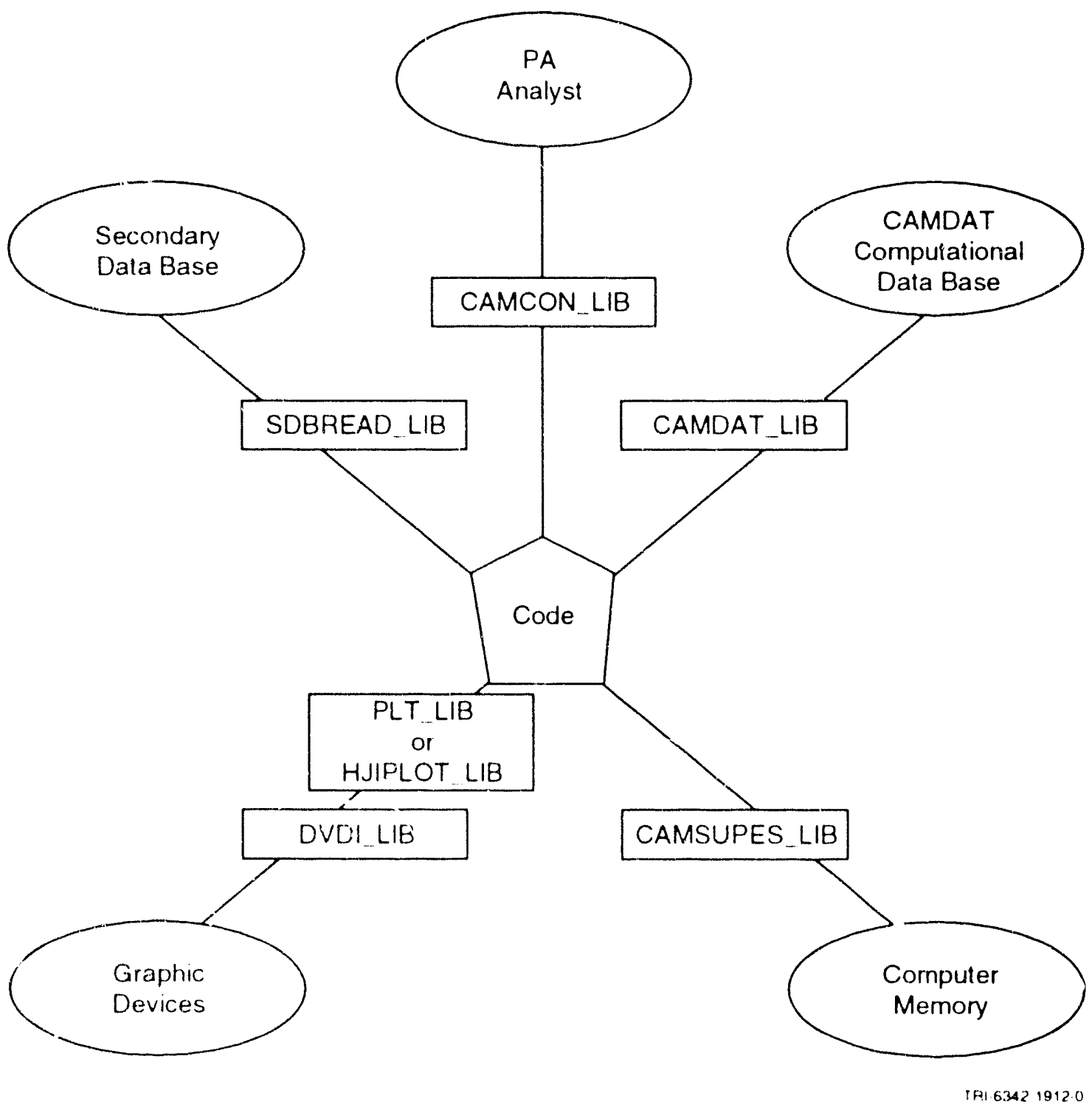

Figura 2-6. The six FORTRAN CAMCON libraries provide interfaces to the PA analysts, secondary data base, computational data base, graphic devices, and computer memory. 
The use or the CAMCON_LIB, CAMDAT_LIB, SDBREAD_LIB, CAMSUPES_LIB, and PLT_LIB are described in subsequent chapters of this manual.

\subsection{On-Line Documentation}

In the CAMCON system, on-line documentation is available for most pieces of software used. Online documentation, or the help file (a computer file generated by the software sponsors), allows users and auditors to retrieve basic information about each code quickly. Consequently, the software sponsor and software coordinator spend less time answering routine questions on code availability and capabilities. In the CAMCON system, the help file is structured as a VAX/VMS HELP library (DEC, 1988) and is located in the [LIBRARY] subdirectory of [CAMCON]. (The individual text about each piece of software that makes up the help library is stored in the same directory as the software.)

A detailed description of the help file is given in Section 4.6, "On-Line Docur tentation." 


\section{Software Development Overview}

\subsection{Software Development Strategy}

Software development for the CAMCON system is part of the complex interdisciplinary process alluded to in Chapter 2. What follows is a brief overview of the strategy used by the soft ware development team. Although planning had been done, it was often minimal in the early phase of the project. ${ }^{1}$ The demonstrated success of the soft ware of the PA project has greatly depended upon software development strategies rather than planning the details of implementing the CAMCON system.

\subsubsection{Multiple Passes through the Assessment}

Although not a software development strategy, a very important project strategy that greatly effects software development is the commitment by PA to perform periodic assessments of the WIPP even though it was not initially a customer requirement (see for example, Rechard, 1989, p. 49; BertramHowery and Hunter, ed., 1989; Marietta et al., 1989) For software development, the benefit has been great from iterating though the entire compliance assessment methodology several times followed by peer review by outside groups such as the Board of Radionuclide Waste Management of the National Academy of Sciences, even though sufficient time for detailed planning has been unavailable. The cycles have helped project managers and more importantly the participants themselves (PA analysts and code developers), allocate software development resources as well as plan for overall project needs. In short, the iterative nature and peer review ensures that the software development and enhancement process is a series of small steps repeated many times.

\subsubsection{Incremental Improvements}

A direct benefit of the periodic assessment cycles is that the software development or modification process is a series of small steps; consequently, software discrepancies and deficiencies are discovered and corrected within the overall WIPP analysis. More importantly, the software product can continue to improve based on experience, and we can incrementally institute quality assurance procedures rather than attempt to design the software perfectly the first time.

\subsubsection{Prototyping the System and Selecting Software}

A distinct disadvantage of the annual cycles is the lack of time for passing through the early planning phases of software development. A strategy that was initially adopted and has been used several times is to form two groups: one either to develop or adopt software that approximately meets the needs of the analysis but more importantly meets critical schedule times, and another group to more systematically develop software. The first group provides valuable information of technique and approaches, even

The proposal and planning document fer the CAMCON system was Rechard, laks. 
though the software is eventually discarded. Versions 1 and 2 of the CAMCON system followed this approach. Version 1 was a thrown-together version (which worked amazingly well due to the diligence of the developers), while Version 2 was developed in parallel and served as a foundation for further development.

\subsubsection{Prototyping Support Software}

For support software, often time is lacking for clear problem definition, etc., so this software begins as a prototype or rough draft from which the actual code is designed or greatly enhanced.

\subsubsection{System Flexibility}

As mentioned several times, the flexibility of the overall analysis system is a very important strategy adopted by PA. It has allowed the system to easily accommodate the study of new phenomena (e.g., gas generation from corrosion of waste containers) as the needs arose. This is possible even though each individual piece of software is not necessarily flexible.

\subsubsection{Data Manipulation Software}

ALGEBRA. Although actually a part of the CAMCON system, a code deserving special mention is the ALGEBRA code (Rechard, 1992; (Gilkey, 1988). AI,GEBRA algebraically manipulates data within a CAMDAT file. Its function is to transpose data for use by other codes. This code greatly diminished the planning necessary to have codes exchange results between each other - important when time is short and/or the usefulness of a code(s) is only being explored.

RELATE. Another code deserving special mention is the RELATE code. RELATE interpolates models, parameters, or results described at the nodes or elements of one mesh onto another mesh. This code greatly increased the flexibility in developing modeling scales.

\subsubsection{Close Links Between Developers and Analysts}

An important strategy has been the close association of the analysts with the developers. Because much of the software development is driven by events and discoveries of complex phenomena, this close association between analysts modeling the phenomena and the developer creating tools to aid in its analysis is essential. This close relationship also serves to ensure that the software does what it is supposed to do.

In several cases, the analyst and developer are one and the same. This situation can cause problems in that the apparent drudgery of plamning and prototyping is skipped over, and the soft ware is perceived as a tool to be used once toget at a solution. While a definite problem in short-term projects, the repetitive use of the same software in annual cycles tends o diminish this tendency. However, the WIPP PA 
Department must often respond rapidly to analyze perceived problems; hence, patches to codes can and do creep in. This can necessitate the formation of two groups, the users and developers, to allow progress in code enhancements, not just continual patches. Another option previously mentioned in Section 3.1.3 was forming two groups, one for early customer requirements and the other for long-term customer requirements.

\subsubsection{Analysis Efficiency}

Because thousands of runs through the system are necessary, computational efficiency is necessary. Thus, software development has centered not just on linking existing codes together or developing new codes with standard solvers, but has included supporting research and the adoption of new methods as a way to make dramatic improvements in the CAMCON system model. These tasks have not been under the supervision of the overall CAMCON system developer, but under a task leader, directly affected by the improvements to maintain a tight link between the developer and analysts.

\subsubsection{Training}

Throughout the project some informal training has occurred. This has included discussions of programming style, subroutine use, and QA requirements. As the project has grown, the need to present more formally thePADepartment's programming "culture" has precipikied the need for the programmer's manual.

\subsubsection{Selection of Primary Coding Language and Computer Operating System}

FORTRAN 77. As mentioned earlier, in the introduction, the CAMCON project is not individuals solely performing software development in development groups, but rather individuals performing other tasks such as analysis. The coding language of these individuals was predominantly FORTRAN. Furthermore, existing scientific and engineering software (SES) was predominantly FORTRAN; thus, it was selected. However, the selection of only one programming language is unnecessary since codes can be easily linked through the neutral files of CAMDAT.

Operating System. The primary criterion for selecting the operating system was the availability of an excellent debugging package for FORTRAN.

Mainframe Computer. As alluded to several times, assessment of a nuclear waste repository involves multidisciplinary codes that must be linked together as a system. The analysts on the project work together as a team, not as individuals with discrete parts of a problem. Although not necessarily required with today's networking capabilities, operation of the system is greatly facilitated by having all the software residing on one computer, since management and maintenance of the system is easier. Furthermore, vast amounts of dat a needed to be transferred among the team PA analysts. Also, the need 
for large amounts of disk storage space and memory was most easily met by a mainframe when the project began.

At the start of the CAMCON development project in January, 1988, there were indications that the $\mathrm{C}$ programming language and the UNIX operating system would become quite prevalent in the computer workplace. However, the project chose VAX/VMS (a non-UNIX operating system) and the corresponding DEC Command Language, DCL. The central reason for this selection was that although we were tempted to plan for the future general uses of the CAMCON system, we thought the monumental task of developing the extensive analysis system would tax the available resources, and hence, most tasks should be as simple as possible and use already existing and working computer systems at Sandia (as of January 1988). This approach has been very successful. Nevertheless, a small effort has been made to use FORTRAN or C (now that an ANSI standard for C has been set / Kernighan and Ritchie, 1988; ANSI C, 1989]) in place of DCL for interacting with the operating system to increase portability to different platforms.

\subsection{Software Quality Assurance Requirements}

\section{Note}

The software QA requirements and their implementation are explained in detail in Rechard et al. (1991) and officially issued as PAP02 ${ }^{1}$ (Performance Assessment Procedure number 2), as part of the Sandia National Laboratories WIPP Project Operating Procedures. ${ }^{2}$ Pertinent sections of the requirements are repeated here and thrr.ughout the programmer's manual for completeness but should not be considered as authoritative since changes in the QA procedures are likely to occur over time.

Herein, quality assurance, as related to computer software, means that the software user (PA analyst) has a reasonable degree of assurance that the software will correctly perform its stated capabilities when given the proper input. The WIPP PA software QA procedures (Rechard et al., 1991) accomplish this assurance by supplying a framework for controlling five aspects-traceability, retrievability, verification, peer review, and documentation-so that the analyst can depend upon software quality and consequently deliver quality work. The software $\mathrm{QA}$ procedures establish different minimum requirements for these properties for different classes and categories of codes based on the safety and programmatic risk associated with the task. This is a graded approach as endorsed by the WIPP Quality Assurance Program Description, or QAPD (QAPD, most recent).

'PAP(12 (1993) was originally designated QAP 2-2, Quality Assurance Precedure 2-2, prior to October 1992.

2The Quality Assurance Program Description (QAPD) (QAPD, most recent), originally designated Quality Assurance Program Plan (QAPP) (QAPP, 199()), is the primary QA decument for the WIPP project at Sandia with the PAP()2 elaborating on the hasic requirements. Another decument, QAP 19-1, Computer. Soltware Requirements, also has some bearing upen PAP(2, but its requirements are intended to be equivalent. Figure 1 of the QAPD describes the QA document hierarchy.

May 1993 


\subsection{Software Classification}

\subsubsection{Description of the Four Software Classes}

For QA purposes, software is identified by a letter that indicates its stage of development. The four software classes are A, C, D, and X (Table 3-1), which represent the following stages:

A - Adjudicated (full QA status)

C - Candidate (partial QA status, possibly undergoing continued refinement)

D - Dormant (obsolete software formerly in Class A or C)

$X$ - eXperimental (entry level, software in early stages of development or experimentation, no QA requirements)

Software developers and sponsors should strive to classify software to the highest possible QA level consistent with its importance within the WIPP project. Although new software can be entered at any level, a QA coordinator decides whether software is entered into Class C, D, or X, while a software review committee decides whether to classify soft ware as Class A. The various classes of software offer the user the following assurances:

Table 3-1. Relationship Between Software Class and Quality Assurance Requirements (Rechard et al., 1991, Table 2.1)

\begin{tabular}{|l|lllll}
\hline SA Requirement & A & C & D & X \\
\hline
\end{tabular}

\begin{tabular}{|c|c|c|c|c|}
\hline Status & Adjudicated & Candidate & Dormant & Experimental \\
\hline Source statements & Fixed & Fixed & Fixed & Not Fixed \\
\hline Direct user accessibility & Yes & Yes & $\mathrm{No}^{\mathrm{a}}$ & Nob \\
\hline Traceability & Yes & Yes & c & No \\
\hline Retrievability & Yes & Yes & c & No \\
\hline Verification & Yes & Yes & $\mathrm{c}$ & No \\
\hline \multicolumn{5}{|l|}{ Documentation } \\
\hline Internal & Yes & Yes & $\mathrm{c}$ & No \\
\hline Help File and Abstract & Yes & Yes & $\mathrm{c}$ & No \\
\hline Formal Reports & Yes & No & c & No \\
\hline Discrepancy Records & Yes & No & c & No \\
\hline
\end{tabular}

a Source code can be made available upon request.

b Software available to individuals within WIPP project, but usually only from software developer.

c QA properties of last $\mathrm{A}$ or $\mathrm{C}$ classification. 
Class $A$. Class A (Adjudicated) software has been evaluated by a software review committee. The software satisfies the QA requirements for traceability, retrievability, verification, peer review, and documentation. The software is available to any interested user within the WIPP project at Sandia. Any analysis where the consequences from discrepancies in the software (which affect analysis performance) are considered major in relation to meeting other expectations of the customer, such as time and cost, should use Class A software (e.g., final performance assessment of the WIPP as regards federal and state environmental or human safety regulations).

Class $C$. Class C (Candidate) software is a candidate for Class A, but currently satisfies only the traceability and retrievability requirements (as evaluated by the QA Coordinator). The adequacy of documentation and verification has not been formally evaluated. An up-to-date help file is maintained, a software abstract has been written, and required internal documentation exists. Furthermore, verification tests are available. However, external documentation and formal reports are in progress. Class $\mathrm{C}$ software is often undergoing continual refinement to adapt the software's capabilities to changing requirements. The software is available to any interested user within the WIPP project at Sandia.

Class $D$. Class D (Dormant or historical) software is software that was formerly classified as Class A or C. Class D software is archived because it is no longer being used (usually because a more sophisticated version has superseded its use). Class D software can be retrieved from a cache, if necessary. Results from Class D software may be compared with other software in benchmark comparisons, but it is not appropriate as the sole basis of an analysis.

Class $X$. Class X (eXperimental) software is in the early stages of development and has not been processed through any formal QA procedures. The primary reason for the Class $\mathrm{X}$ classification is to make the existence of the software known to potential users so that they can provide preliminary values for scoping calculations, calculations on short notice, or calculations where the results will not be used to decide the long-term WIPP performance. Usually, a user must obtain Class X software by contacting the software developer.

\subsubsection{Proprietary Software}

For proprietary software, all requirements of the software QA procedures apply within the constraints of the license agreement for the software. For example, internal documentation requirements will be waived when the source coding is not available. Thus, proprietary software can potentially reach Class $\mathrm{A}$. 


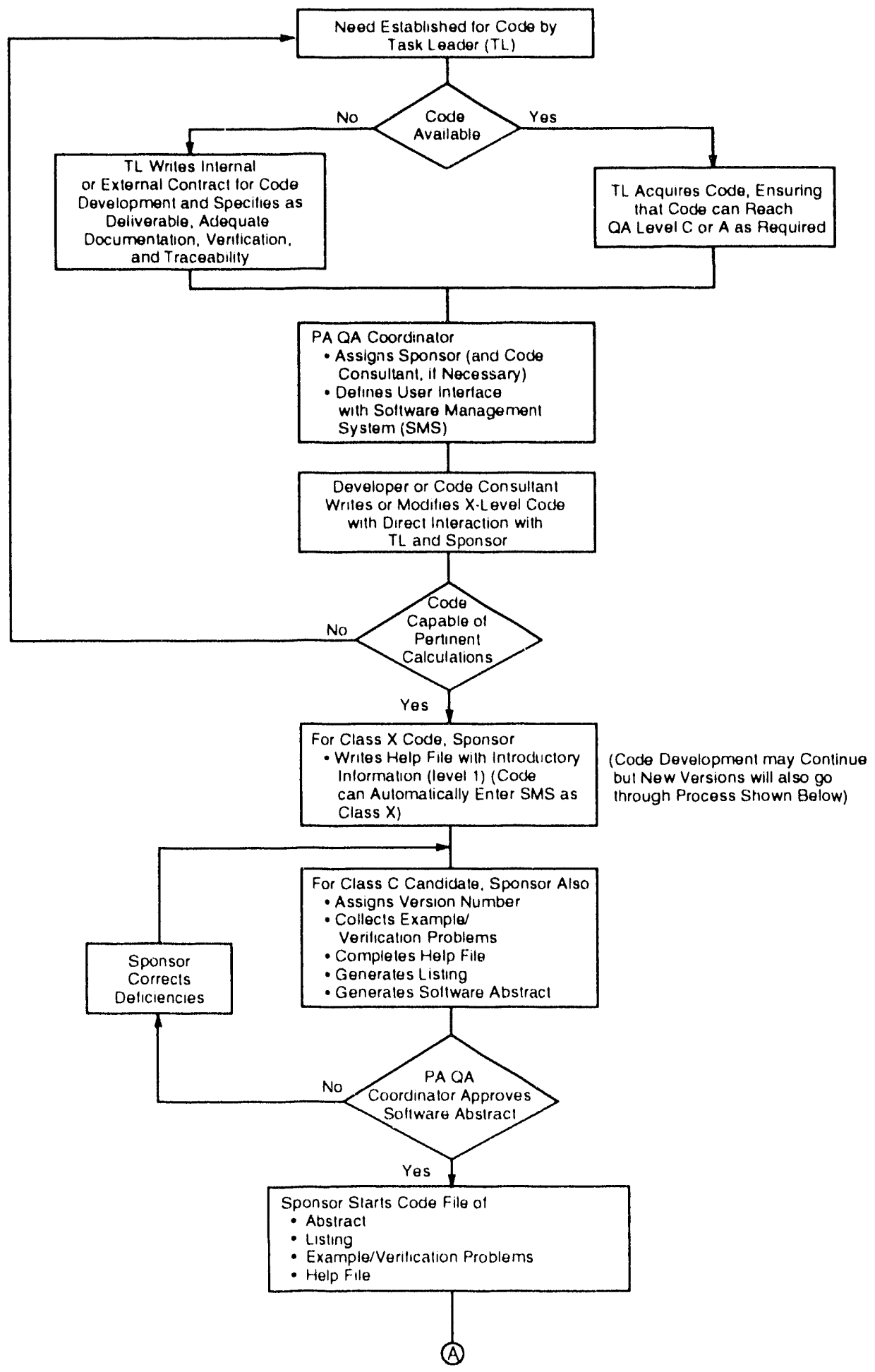

TRI. $6342 \cdot 591.0$

Figure 3-1a. Steps followed to reach a Class X, C, or A software QA rating (part 1). 
Software Development Overview

Software Classification

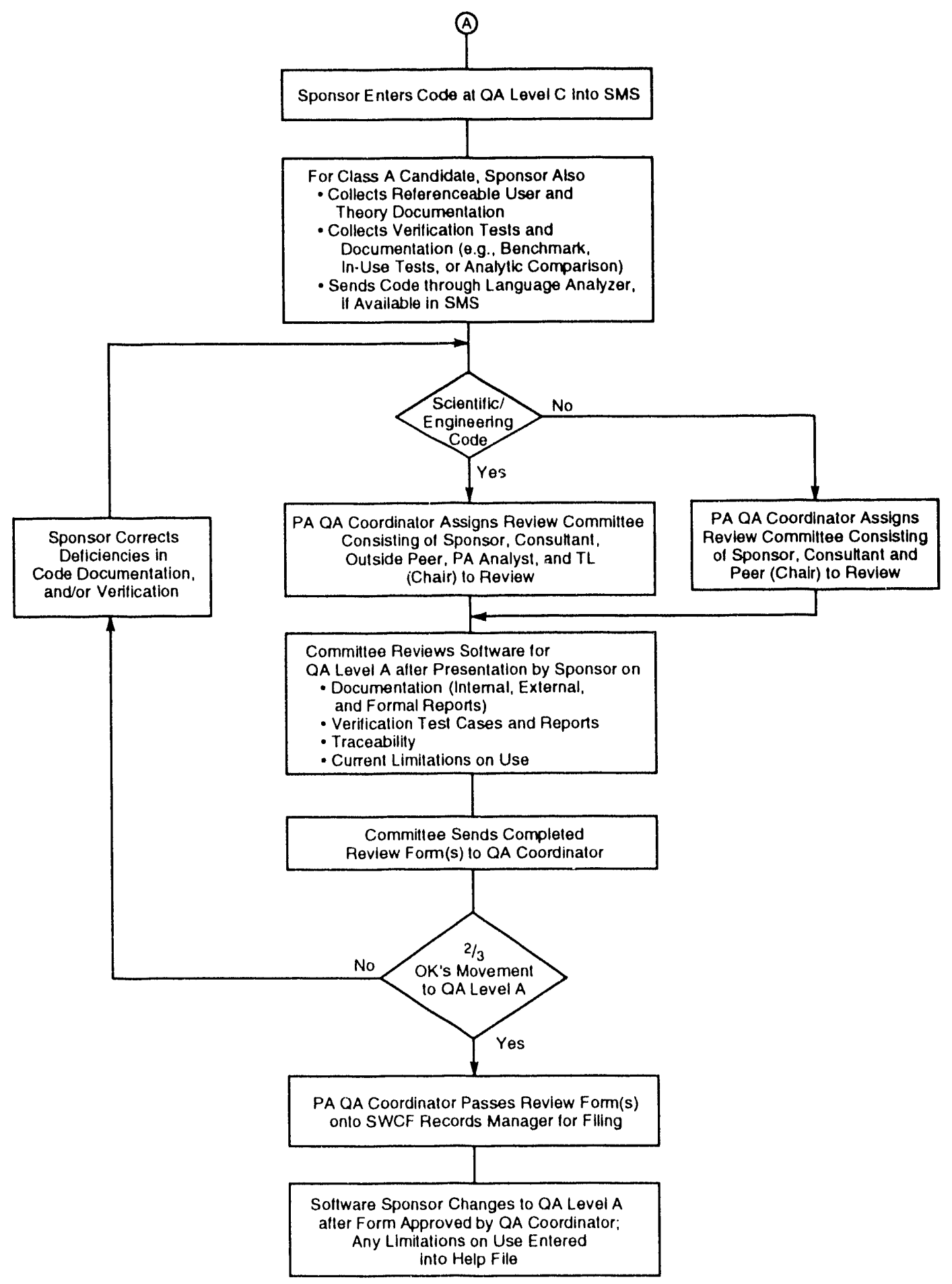

TRI-6342-591-0

Figure 3-1b. Steps followed to reach a Class X, C, or A software QA rating (part 2) (after Rechard et al., 1991. Figure 3.1). 


\subsection{Software Development or Acquisition Procedures}

The development or acquisition of software used by the PA Department follows the procedures described below and shown in Figure 3.1. This discussion comes from software QA procedures PAP02 (1993), but is supplemented with specific instruction.

\subsubsection{Task Leader Establishes Need}

When a modeling activity is identified, the modeling task leader (TL) for PA modeling establishes which software is needed to solve the problem. The software may already exist or may need to be developed. If the software already exists, the TL acquires the software, making sure that it can reach Class $\mathrm{A}$ or $\mathrm{C}$, as required. If the software does not already exist, then the TL contracts for the software to be developed, either with a Sandia organization or an external company, and specifies as deliverables adequate external and internal documentation, verification, and traceability.

\subsubsection{QA Coordinator Assigns a Software Sponsor}

After the need is established and the code is being acquired or developed, the QA coordinator assigns a sponsor for the code; if the software sponsor is unable, for any reason, to act as software consultant, then a software consultant is also assigned. The QA coordinator also defines the user interface and data flow for the software within the CAMCON system.

\subsubsection{Developer or Code Consultant Implements Development or Modifications}

The developer or code consultant writes or modifies the software (Class X), directly interacting with the TL for the PA modeling task and the QA coordinator. Software planning and programming guidelines are discussed in Chapter 5.

\subsubsection{Inspection and Verification}

Inspection and verification tests, as specified by the TL or developer, are performed to determine whether the software can run pertinent calculations. If it carmot, then the need for this software is again reviewed by the TL, following the process described above. If the software passes the test, then the software continues to be developed following the procedures below. More specifics on software verification are described in Section 3.7.

\subsubsection{Software Sponsor Enters Software into CAMCON as Class X}

Software development or modifications can continue without entering Class X software into the CAMCON system. However, inclusion of the software as Class $\mathrm{X}$ in the CAMCON system often serves 
to notify potential users of its existence, thereby encouraging its preliminary use and a discussion of its features. If the TL, users, or sponsor want the software to be used in this manner, the sponsor may enter the software into the CAMCON system, provided a help file is written that describes the name, purpose, authors, sponsor, and consultant(s) (i.e., the introductory information [Level 1] is completed in the help file). More specifics on entering a code into the CAMCON system are described in the following paragraphs.

\subsubsection{Software Sponsor Enters Software into CAMCON as Class C}

For a candidate Class $\mathrm{C}$ software, the software sponsor and software consultant must (1) assign a version number (see Section 3.3), (2) verify the software's capabilities (see Section 3.7), (3) complete the help file (see Section 4.6), (4) generate a software listing (see Section 4.7.1), and (5) generate the software abstract (see Section 4.7.1). After the QA coordinator has approved the abstract, the software sponsor starts a software notebook (see Section 4.7.1) for the soft ware that includes the (1) software abstract, (2) software listing, (3) example/verification problems, and (4) help file.

After the QA coordinator has approved the software abstract, the sponsor enters the software into CAMCON as Class $C$ with the appropriate fixed version number (see Section 4.1).

The specific instructions for a software sponsor are as follows (see also Section 3.4.8, "Writing CAMCONexec Procedural Files," for similar instructions for the CAMCONexec sponsor):

1. Create a subdirectory with the same name as the new program and the protections (S:RE, $\mathrm{O}: \mathrm{RWE}, \mathrm{G}: \mathrm{RE}, \mathrm{W}: \mathrm{RE}$ ) in the CAMCON\$ROOT directory.

2. Copy the optimized executable, help, and source files for the program to this directory. (The object files and debug version of the executable files should not be stored in the [CAMCON] directory.)

3. (New software sponsors only) Create a file in the CAMCON symbols subdirectory, CAMCON\$ROOT:ISYMBOLSI, with the namesponsor_user_name_SYMS.COM. Notify the CAMCONexec sponsor that this file has been created.

4. Define a symbol to run the code by adding a line to the file sponsor_user_name_SYMS.COM located in CAMCON\$ROOT:[SYMBOLS]. This symbol definition should refer to the CAMCON directory by its logical name, i.e., CAMCON\$ROO'T:[CODE_NAME]. (Also see Section 2.6, "Logicals and Symbols.") Because this DCL file is executed at login time by all CAMCON users, it is essential that this file be correct; hence, test your additions and changes.

5. Update the CAMCON help file, CHELP, with the command ADDCHELP code_name.HLP. (CHELP follows the standard VMS help format [DEC, 1988al.) (See Section 4.6, "On-Line Documentation.")

6. Mail the CAMCONexec sponsor the following information for use in developing a CAMCONexec interface with the new code: 
- A one-line description of the code. (This description will appear on-line with the other CAMCON codes when the CAMCON help option is selected.)

- A brief description of the code. (This description will appear after the code is selected from CAMCON.)

- A short description (for prompt) of all program $\mathrm{V} / \mathrm{O}$ files in the order they appear on the command line, including (Section 4.2.3)

- Usage (input/output, optional/required)

- Type (text string, CAMDAT data base file, ...)

- Default identifier (same as that in the CAMCON user's manual (Rechard, 1992) or help file) (Section 4.2.2)

(The parameters in the call to the CAMCON_LIB routine FILDFNAM contain this information.)

- If the code is typically or appropriately run in a sequence with other codes in the CAMCON system, this sequencing information should be provided.

7. Upon notice from the CAMCONexec sponsor that the interface to the code is complete, test the program from CAMCONexec and notify the CAMCONexec sponsor that the code performs as expected.

8. Notify users that the code is available by using the CHANGES utility (see Section 12.3).

\subsubsection{Software Sponsor Enters Software into CAMCON as Class A}

For a candidate for Class A software, the software sponsor and software consultant must perform the following tasks in addition to those required for Class $\mathrm{C}$ software: (1) collect referenceable user and theory documentation, (2) collect hardcopies of verification tests and documentation (e.g., benchmark test, in-use test, and analytic comparisons), and (3) run internally developed software through a language analyzer, if available in the CAMCON system, to identify hidden programming errors. This information should be added to the software notebooks (Section 4.7.1).

At this point, the software sponsor determines whether the software is ready to be considered for Class A. If the software is calculational SES software, then it must be reviewed by the software review committee. The software review committee consists of the software sponsor, software consultant (if applicable), peer outside of the PA Department, PA analyst, and modeling task leader. Two-thirds of the committee must approve the software for Class A based on the presentation by the sponsor regarding documentation, verification, traceability, and language analyzer result (if applicable).

The software review committee must prepare and send to the QA coordinator a review form that contains its decision. Any differences of opinion must be recorded on separate review forms (ask for Form PA002, current version, from the SWCF records manager). If the software does not progress to Class $\mathrm{A}$, the software is reviewed again, according to the procedure above. If the software review committee declares that the software is Class A but that limitations to use exist, then those limitations must be cited on the review form. 
Sellware Development Overview

Software Development or Acculusition Precedures

Non-SES software is also reviewed by a software review committee, though the committee usually is smaller consisting of the software sponsor, software consultant, and one peer, either inside or outside the PA Department. The QA coordinator assigns the peer reviewer to exercise the code. The software review committee then prepares a review form.

If the review form for either the SES or non-SES software states that the software is "Class A," then the QA coordinator passes the form to the SWCF records manager for filing. The code sponsor then changes the code identifier to QA Status A. The software sponsor must also note any cited limitations in the help file (Section 4.6).

\subsubsection{Writing CAMCONexec Procedural Files for Software}

The procedural files are normally written by the CAMCONexec sponsor, but the previous responsibility for providing the necessary information and testing resides with the software sponsor. (Parts of section 3.4 describe the steps and necessary information for placing soft ware in to the CAMCON system.)

After specifying the input and output information for a new code to be installed into CAMCONexec, the CAMCONExec sponsor should proceed as follows in generating procedural files:

1. Develop a procedural file that interfaces CAMCONexec with the new program by prompting the user for input according to CAMCONexec standards (Section 4.2, "Files and Entering File Names") and then invoking the program with these arguments. The easiest way to generate a procedural file is to find a prototype in the subdirectory CAMCON\$ROOT:[DRIVERS] and use it in conjunction with the code input/output information provided by the sponsor to develop a similar driver for the new code in a working directory outside [CAMCON].

- Name the procedural file code_DRIVER.COM.

- When the new code is part of a typical sequence, the sequence should have been provided to you by the software sponsor. The name of the next code in the sequence, if any, should be assigned to the symbol NEXT_PROGRAIM_DEFAULT in the procedural file. If the code is preceded in the sequence, the procedural file for the preceding program must be modified to assign the symbol NEXT_PROGRAM_DEFAULT.

- If the new CAMCON code is a general purpose utility (such as a graphics package) whose use should not modify the CAMCON user's default code selection, the procedural file should assign an asterisk $\left({ }^{*}\right)$ to the symbol NEXT_PROGRAM_DEFAULT.

2. If a sponsor is new, add a line to the file CAMCON_SYMS.COM in the subdirectory CAMCON\$ROOT:|SYMBOLS| referencing the sponsor's symbol file sponsor_user_name_SYMS.COM.

3. Test the new procedural file before installing it by executing CAMCON with the pathname to the directory containing the procedural file as a parameter, for example,

\$CAMCON disk:[directory.subdirectory]. 
4. Install the new procedural file by placing it in the subdirectory

CAMCON\$ROOT:[DRIVERS]. Also, add the procedural file name to the file DRIVERS.ALL by running the DCL file LISTDRIVERS.XCOM in the [DRIVERS] subdirectory.

5. Notify the code sponsor so that the interface can be tested further.

6. Upon notification from the sponsor that code testing is complete, the process can be completed by adding the code (and sponsor) to the SPONSORS.DAT file located in CAMCON\$ROOT:|CHANGES| and adding the code name and its one-line description to the DRIVERS.HLP file located in CAMCON\$ROOT:[DRIVERS]. Both of these files should be added to the curre:at CAMCON help with ADDCHELP.

Specific requirements of the software (including procedural files) for interfacing with the user are described in Chapier 4.

\subsection{Modifying Software in the CAMCON System}

Since most soft ware used tor evaluating the compliance of nuclear waste repositories is complex, the possibility of discovering limitations, inaccuracies, or errors always exists, even for Class A software versicns, which have satisfied the verification requirements. The mor "fication and potential downgrading of software follow the procedures described below and show: in Figure 3-2.

\subsubsection{Need Established}

The PA analyst (or other user) notifies the software sponsor that soft ware needs to be modified (eithe. an enhancement or error correction) through a change request. For corrections to Class A software, the change request must be specified on a formal request form (ask for Form PA003, current version, from the SWCF records manager). The software sponsor then discusses the modification with the QA coordinator, software consultant, PA analyst, and task leader for the modeling task, as necessary, to establish the priority and scope of interest in the modification. If the modification is for a PA calculation for evaluating WIPP repository compliance, or for an error correction, or if it can be easily implemented, then the software sponsor directs the code consultant to implement or direct the modification. If the modification is specific to a particular analysis that does not directly evaluate WIPP repository compliance, then the PA analyst may modify the code and document the modification as described in Section 3.8, "Single-Analysis Software," when reporting the results

\subsubsection{Downgrading Software}

If the changes are either minor errors being corrected, minor enhancements being added, or changes that are cosmetic or input/output specific, then a new revision of the software can maintain a Class $A$ rating, provided the decision is noted in the update isistory of the help file. The QA coordinator must cuncur with the software sponsor': assessment that the changes are cosmetic or $1 / O$-specific. The software revie' committee must concur with the assessment that the changes are minor. 


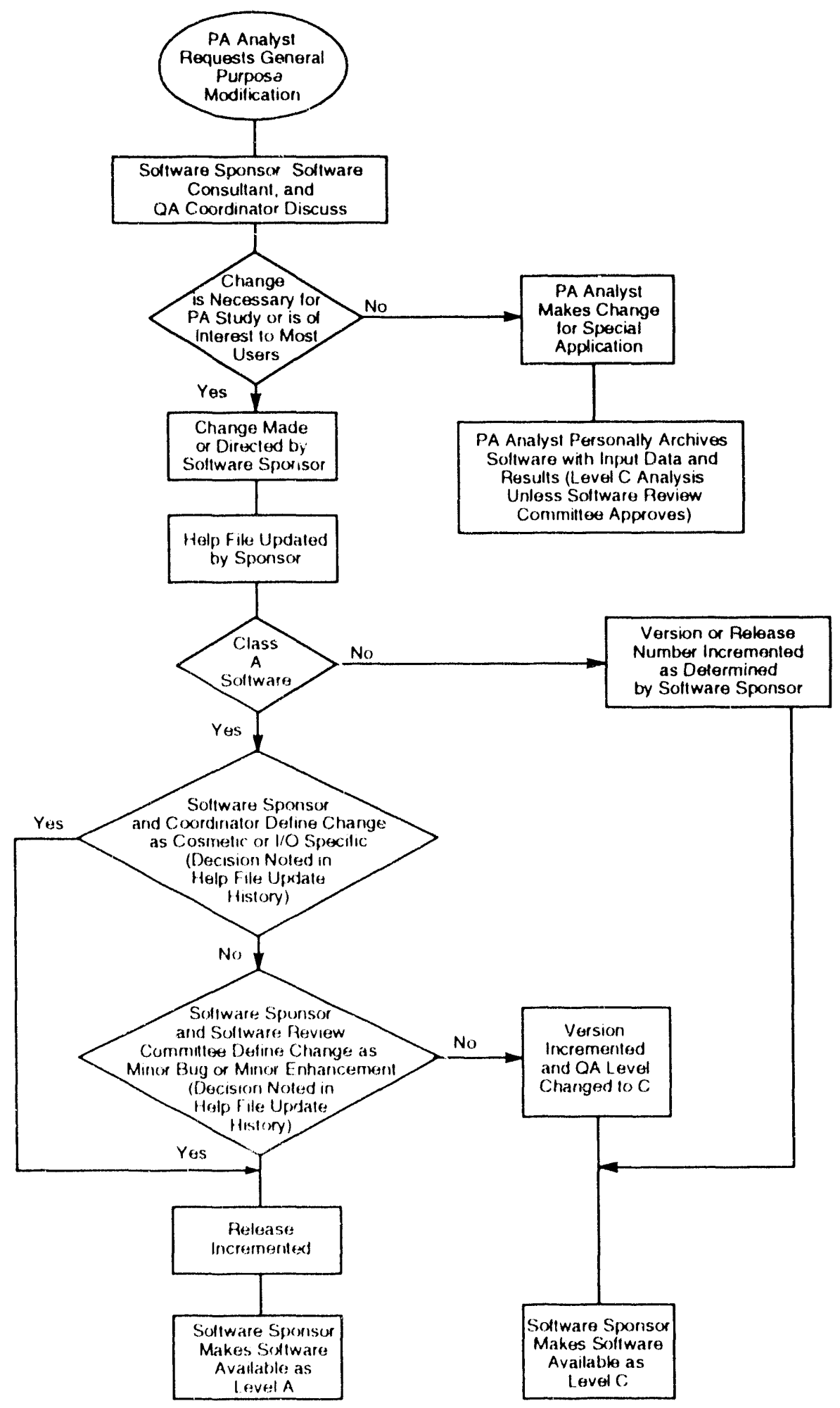

IFI. 6342265.0

Figure 3-2. Steps followed for modifying Class A or C software (Rechard et al., 1991, Figure 3-2). 
If significant capabilities are added or errors corrected in Class A software, the new software version is normally reclassified as Class $\mathrm{C}$ and reevaluated as described in Section 3.4, "Software Development or Acquisition Procedures."

For most modifications to Class $\mathrm{C}$ software, the new software version will retain the Class $\mathrm{C}$ rating, provided the help file is updated.

When retrieving an obsolete version of a Class $\mathrm{A}$ or $\mathrm{C}$ code (where obsolete means that a new version of the same class has replaced the cached version), the QA coordinator will downgrade the old version of a Class $\mathrm{A}$ or $\mathrm{C}$ code to Class $\mathrm{D}$ before allowing its use (e.g., benchmarking exercises). However, when a code that is not obsolete (e.g., a seldom-used code or version) is retrieved, the code can maintain its former class if a sponsor is assigned and the sponsor and QA coordinator jointly decide that the code can meet the current requirements for that class; otherwise, the QA coordinator must downgrade the code to Class D. Guidelines for changing the software ID are given in Section 4.1.1.

\subsubsection{Inactive Software}

Only operational software with a sponsor can be classified as Class A or C. Loss of operational status, loss of a sponsor, limited use, known significant errors, or a superior replacement are sufficient reasons for the QA coordinator to remove software from active use by purging the file from the currently operating version of the CAMCON system. For Class $A$ and $C$ software, purging can occur only after verifying that a cached version of the software is available.

\subsection{Replacing Software Already in the CAMCON System}

If the new version modifies the code command line in any way, the CAMCONexec sponsor must be notified of these changes so that a new interface can be developed and tested, as described above, before the new version is installed.

Once the interface is completed, the new version is installed as follows:

1. Create a directory named CAMCON\$ROOT:|code name.OLDmmmyy| (or other appropriate Name, e.g., A_, $_{-}$, Section 2.8.3)

2. Copy the current version into the "OLD" directory.

3. Copy the new version from your directory into CAMCON\$ROOT:|code_name|.

4. Purge the directories.

5. Add to your symbol definition file, OLD_codencme $==p$ at $h$.

6. Notify the CAMCON system users about the new version by using the CHANGES utility (see Section 12.3).

This procedure is preferred since it keeps a working executable version available to the CAMCON system at all times and provides an easily identifiable copy of the most recently used one. Note that some 
Software Development Overview

Verification

sponsors may choose to maintain, for example, the last three versions of the code, but at least one is expected to be available.

\subsection{Verification}

\subsubsection{Definition}

Verification of software is the process of illustrating through sufficient testing that the software satisfactorily performs its stated capabilities and providing a point of reference for testing of future modifications and testing the software on a new machine as appropriate. All Class $\mathrm{C}$ or A software must be verified.

\subsubsection{Examples of Verification Methods}

The following is a list of possible verification methods:

- Hand Calculation - the comparison of results obtained by implementing a solution by hand (to the results obtained by the software being verified where "by hand" includes the normal manual arithmetic operations outside a computational code as well as the use of spreadsheets).

- In-Use Test - the citation of widespread use of software (e.g., plotting packages) over a sufficient period of time to exercise most features of the software. In-use tests that previously "broke" a code may be retained as test cases.

- Comparison with Analytic Solution - the comparison of results obtained by analytically solving a mathematical model with those obtained by the software being verified.

- Benchmarking - the comparison of results made with one code with other codes that implement other solution techniques (e.g., numerical techniques) or mathematical formulations, where all codes are designed to solve comparable problems.

- Use in Sensitivity Analysis - the inclusion of a code in a sensitivity analysis. Sensitivity analysis as used here refers to the general measure of influence of a parameter on the variation in a result. The influence of a parameter can be measured based on equal percentage changes from a base case value or equal percentage changes of the total expected range of a parameter. While the latter is more common for WIPP calculations, both techniques require the analyst to gain vast experience with the code and thereby systematically test the capabilities of a code.

- Convergence Tests - the systematic refinement of spatial and/or temporal meshes of a numerically implemented mathematical model to determine whether zero, first, second, or higher order accuracy is observed. The observation can then be compared with the theoretically expected behavior to help evaluate whether the numerical procedure was correctly implemented.

- Other - depending upon the nature and complexity of the code, the software sponsor and/or software review committee may ask for additional specific verification tests. In this case, the nature of the test should be included in the test case documentation. 


\subsubsection{Appropriate Verification Method Dependent on Complexity, Type, and QA Level}

All the verification methods can be used on any piece of software, but the extent to which the software is tested is determined by the complexity of the software, the software type (SES or non-SES), and the software QA level (C and A). To elaborate concerning software complexity, adequate verification of simple code may be achieved (1) directly by comparing results against hand-calculated numbers or (2) indirectly by in-use tests and citing widespread use of the code. Verification of complex codes may require testing in stages (i.e., testing of individual modules) followed by (1) extensive numerical tests and comparison of results with analytic solutions, (2) bench marking the code against other verified software, or (3) using the code in sensitivity studies. Concerning software type, verification for non-SES codes can consist primarily of in-use test, while verifications for SES codes should include some comparisons with analytic solutions and possibly benchmarking with other codes. Finally, concerning software QA level, the number of individuals checking on the adequacy increases between Class $\mathrm{C}$ and Class $\mathrm{A}$ as noted below.

\subsubsection{Determining Adequacy}

Evaluating the adequacy of software verification is the responsibility of the software sponsor for Class $\mathrm{C}$ software. For Class A software, it is the responsibility of the software sponsor, the software review committee, and peers technically reviewing formal reports on the software.

\subsubsection{Documenting Verification}

Refer to Section 4.7, "External Documentation."

\subsection{Single-Analysis Software and Analysis-Specific Modifications}

Software written and intended solely for use within a single analysis not directly affecting WIPP performance with applicable federal and state regulations is exempt from requirements of PAP02 (1993), the software QA procedures, except that the documentation of the software shall be as described in Section 4.7, External Documentation.

Furthermore, changes to software that has been previously quality assured and changes that are specific to one analysis not directly affecting WIPP compliance issues are also exempt from these procedures except for the documentation requirements described below.

Error corrections, features, or capabilities added for two or more analyses or users, or added for an analysis directly affecting WIPP performance as determined by the task leader, are not considered analysis-specific modifications; these modifications shall be treated as new versions or revisions to existing software and shall follow the QA steps within PAP02 (1993). 
The individual using software written or modified for a single analysis shall ensure that the documentation included with the reported results contains the following basic elements:

- The software or modification must contain the minimum internal documentation required by PAP02 (1993).

- The report must list the code source or modified section of code. If the code listing is too lengthy, the individual must submit a hardcopy or microfiche listing (and possibly the listing on computer storage mer,.a) to the Sandia WIPP Central File (SWCF) and note the location and identification in the report.

- The report (or internal documentation) must describe the code input and data used.

- The report must describe the method of verification.

- The report must describe code validation as appropriate. 


\section{User Interface Guidelines}

This chapter describes suggested guidelines on how software should interact with the user, including suggestions on standard startup, manner of input and file names, error messages, etc., to provide a common user interface.

\subsection{Traceability}

For software to be maintained over time, it is important that users can identify the software and version used in a calculation to retrieve the correct copies of that software, if needed. The software QA procedures establish standards for tracing and retrieving software.

\subsubsection{Software Name, PA Version ID, Date Stamp}

To maintain the capabilities of tracing and retrieving software, the following information must be included on printed output, graphics output, and data bases that store results: the software name, the PA version identification (ID), and the date of the software that was used to generate the results. For software developed external to the PA Department, the original version ID accompanying the software (from which enhancements may have been added) may also be included. If several codes are used in sequence to produce the results, the data base that stores results must record the software name, PA version ID, input files, and date stamp for each code that writes data onto the data base.

The PA software identifier (ID) is of the form

\section{QVV.RRCO}

where

$\mathbf{Q}$ is the QA software classification of A, C, D, or X (see Section 3.3)

VV is a one- or two-digit integer designating the major version number (use a dash in the first position when the major version number is less than 10)

$\mathbf{R R}$ is a two-digit integer designating the release, or minor version number (use a zero in the first position when the revision number is less than 10)

C is the computer designator (for example, A for Alliant, C for CRAY, P for PC, V for VAX, and Z for Alpha)

$\mathrm{O}$ is the computer operating system (for example, V for VMS and U for UNIX)

The PA version ID changes as the software changes. Depending on the type of modification and the software classification, software identifiers are changed as follows:

Q Designator. The Q designator indicates the QA status of the software. As fully described in Section 3.4, "Software Development or Acquisition Procedures," the Q portion of the software ID usually starts 
at $\mathrm{X}$ to indicate the experimental nature of the software. The designator changes from $\mathrm{X}$ to $\mathrm{C}$ after required verification is performed (Section 3.7), required documentation is created (Section 4.7), and the software abstract (Form PA001 created by QA_PRINT [Chapter 12]) is approved by the QA coordinator (Section 3.4.6). The designator changes from $\mathrm{C}$ to $\mathrm{A}$ after favorable review by tine software review committee (Section 3.4.7) and the QA coordinator places the review form (PA003, current version) in the software notebook (Section 4.7.1) or gives it to the SWCF records manager.

The QA status can be downgraded because of major modifications, enhancements, or discovery of discrepancies as described in Section 3.5, "Modifying Software in the CAMCON System."

The Q and VV.RR portions of the software ID are not entirely independent of each other. Guidelines are provided under the next topic.

VV and RR Designators. The VV and RR designators respectively indicate major and minor changes to the software. For minor revisions, such as internal or external cosmetic changes, minor bug fixes, array size changes, and minor enhancements, the release designator $(\mathrm{RR})$ is incremented by one. In all cases for $\mathrm{C}$ and $\mathrm{A}$ software, an update history must be maintained in the on-line documentation (Section 4.6) and internal documentation (Section 5.6).

For major revisions that substantially change the software, such as adding a new capability, input changes that render the formal software documentation incompatible, adding a new and independent numerical algorithm, or correcting a major error, the major version designator (VV) is incremented by one and the release designator $(\mathrm{RR})$ returns to zero $(00)$.

As stated above, the $Q$ and VV.RR portions of the software ID are not entirely independent of each other. The decision as to whether a VV.RR change should accompany a $Q$ designator change (or vice versa) is the responsibility of the software sponsor, QA coordinator, and software review committee. Guidelines are as follows:

- $\mathrm{Q}$ and VV.RR Independent

- Class X or C software changes version or release (VV.RR)-provided the code remains at the X or $\mathrm{C}$ level, the $\mathrm{Q}$ and VV.RR designators are independent.

- Class A software changes release number (RR)-provided the software sponsor, QA coordinator, and the software review committee concur that the change is cosmetic, a minor bug fix, or a minor enhancement (see Section 3.5), class and Q designator remains A.

- Class A or C software is downgraded to D-when the software is downgraded to D (dormant) the VV.RR must not change.

- $Q$ and VV Dependent

- Software QA status upgraded from X to C-the VV.RR portion normally changes to 1.00 . However, an exception can occur. If an $\mathrm{X}$ code is used in many or highly visible PA calculations 
prior to upgrading, the QA coordinator and sponsor may jointly decide to change only the Q designator to maintain continuity with previous calculations.

- Class A software changes version number (VV)-software QA status and Q designator are downgraded to $\mathrm{C}$ level until formal reports are revised, verification tests performed, and a Class A rating approved by the software review committee.

- Class A software downgraded to Class X-although this has never occurred, in this case, the sponsor and QA coordinator will jointly decide whether to (a) rename the software and change the VV.RR portion to 1.00 or (b) retain the name and continue to increment VV.RR.

$C$ and $O$ Designators. These designators respectively identify the computer and operating system on which the software is executed. These designators change only when software is ported to another computer and/or operating system. The remainder of the software ID normally is unchanged unless the porting required substantial changes or other unrelated modifications where done in conjunction with the porting.

\subsubsection{Author Names, Software Origin, Government Legal Disclaimer, and Proprietary Information}

Printed output from PA software will display the author names, code origin, and either a government legal disclaimer on Sandia codes or proprietary information on external software (similar to requirements specified under internal documentation).

\subsubsection{Using CAMCON_LIB Routines to Meet Traceability QA Requirements (Welcome and Exit)}

To (1) properly identify the software and version for traceability, and (2) provide a uniform look to software developed specifically for the WIPP, a standard set of routines has been written and collected in CAMCON_LIB (Rechard et al., 1991, Table 4-1). Detailed explanations of the CALL statements are in Chapter 8, "CAMCON_LIB: Quality Assurance Routine Library."

Example of an Opening Section. The opening sections of most programs should be similar. The following code segment provides an example of the opening section. QASETUP places $\cap A$ information (program name, version ID, revision date, current date and time, code author, and code sponsor) into a common block for use by other subroutines and QABANNER prints code banner and lists credits (authors and origin of code). The routines QASETUP and QABANNER are located in CAMCON_LIB. Use of this library is described in Chapter 8.

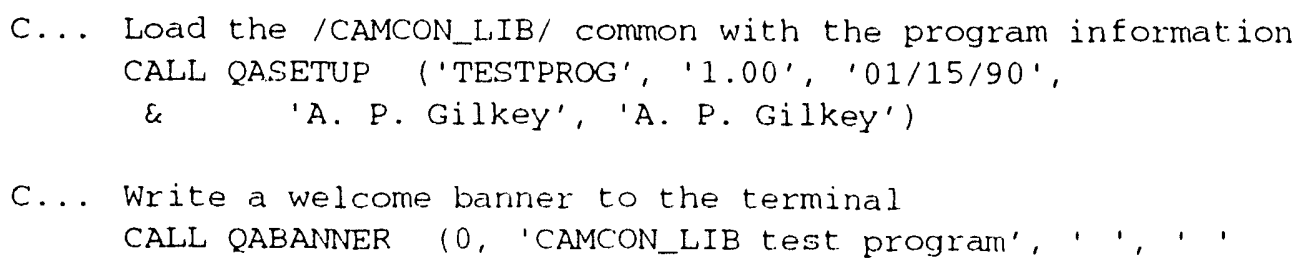


Closing Section. CAMCON_LIB also provides for a consistent and graceful exit from programs. The closing section should use the following guidelines:

- A normal program execution should

- print QA information onto the output file by calling QAPAGE

- provide timing information with a call to QACPUS

- terminate with an END in the main program, and STOP 'Normal codename completion'

- A code-trapped error should exit through a call to QAABORT, which returns an error status code to the operating system and prints "STOP" with an abort message.

\subsection{Files and Entering File Names}

\subsubsection{File Types}

Data moves in and out of the codes interactively and through files. The files consist of three file categories each consisting of several types

- Data bases

- computational

- secondary

- Input

- ASCII text

- binary data transfer file

- Output

- descriptive ASCII text

- data transfer file (binary or ASCII)

- debugging ASCII file

- $\log$ file of output normally sent to the terminal

\subsubsection{Naming Conventions}

Files generated by CAMCONexec procedural files (Section 3.4.8) follow a naming convention. However, all software must permit these default names to be overridden. The naming convention consists of five segments for the file name, two of which are required.

A default file name generated by the CAMCON executive program contains five segments as follows: nanle_Sxxxx_t_Rxxx.ext

1. The first segment (required), name, consists of the code name (or, in some cases, a problem descriptive name) for which the file is used as output.

2. The second segment (optional), $S x x x x$, identifies the scenario, where $x x x x$ is a numeric ID for the scenario agreed upon by PA analysts prior to the start of major calculations. Typically, the 
last two characters are used for the time of intrusion and scenario type while the leading characters vary:

$x_{1} \quad$ (e.g., type of transport; $1=$ single, 2 = dual)

$x_{1 \cdot 2 \cdot 2}$ (e.g., type of retardation; $0=$ no, $1=$ yes)

$x_{11-1}$ Time of intrusion $(1=1000 \mathrm{yrs}, 2=2000 \mathrm{yrs}$, etc.)

$x_{11} \quad$ Scenario type $(1=\mathrm{E} 1,2=\mathrm{E} 2,3=\mathrm{E} 1 \mathrm{E} 2)$

3. The third segment (optional), $t$, identifies whether the file type is regional, local, source, or cuttings scale model (R, L, S, or C, respectively) or general data grid (D).

4. The fourth segment (optional), $R x x x$, identifies the run number, where $x x x$ is the three-digit ID for the Monte Carlo run (vector).

5. The fifth segment (required), ext, is the file extension

CDB A Computational DataBase file (CAMDAT file).

DBG The ASCII output file containing code execution messages and results useful for DeBuGging.

INP The ASCII INPut control text files.

OUT The file as an ASCII QUTput text file.

SDB The files in the Secondary Data Bases.

TRN $\alpha \alpha \alpha$ The data TRaNsfer files used between the translator and main program modules (either binary or ASCII) without explanatory headings, where $\alpha \alpha \alpha$ is a threecharacter descriptor for data contained in the transfer file (for example, VEL for velocity, ICS for initial conditions, RIN for restart input, or ROT for restart output). Transfer files are typically tenporary and are used to transfer data between the translator and the main program.

The CAMCON user's manual (Rechard, 1992) describes the files needed, created, and/or used by the various codes in the CAMCON system modules. As an example, the data base file for the regional scale, scenario type $\mathrm{E} 1$ at 1000-year intrusion with retardation and dual transport, run 1 is as follows:

$$
\text { STAFF2D_S2111_R_R001.CDB }
$$

(VAX/VMS V5.0 and above permit file names and extensions with up to 39 characters each.)

By default, a code should only use the two required segments, name and ext, of the file name when run outside the CAMCON executive package. Furthermore, unless obvious alternatives exist, for default input data files, the name.ext segment should typically be one of the following:

CAMDAT.CDB

PROPERTY.SDB

Codename.INP

Codename.TRNaxxa

and for default output data files, the name.ext segment should typically be one of the following:

codename.CDB

codenanie.OUT 


\section{codename.TRN $x \times x$ \\ codename.DBG}

For some applications, the debug (.DBG) and output (.OUT) files are combined (.DBG or .OUT).

The other three segments ( $S x x \times x_{-} t_{-} R x x x$.) are used and generated by the CAMCONexec program, DRIVERS, when running inside the CAMCON executive package. Even here, the DCL procedures must allow the user to override the default names.

\subsubsection{Entering File Names}

Reading File Names on Command Line. As a ioption, read all names of files used by the code at the same time the code is executed ("command line read") (use FILDFNAM and FILRDNAMS in CAMCON_LIB). Dimension file names to at least 132 characters.

File Names not Supplied on Command Line. If running the program from the VMS prompt, read file names interactively if file names are not supplied on the command line. Often this is the only interactive input, although some programs allow the user to interactively enter the commands found in the user input file.

Read file names in the following standard order, if applicable:

- Required computational data base (CDB) files (input or output)

- Required secondary data base files (SDB) files (input or output)

- Required ASCII input files (INP)

- Required output file (OUT,TRN)

- Optional ASCII input files (INP)

- Optional output files (OUT,TRN,DBG)

A file name of CANCEL should specify that the file is not needed, and DEFAULT should force the use of the default file name. All of these capabilities are built into the CAMCON_LIB routines FILDFNAM and FILRDNAMS.

\subsubsection{Example of File Reading}

The following code segment calls several CAMCON_LIB routines to define and read files and to set up a diagnostic file (.DBG). The example as written will read file names from the command line and, if the program is being run from the VMS prompt, will interactively request files not specified on the command line or not found in the default or specified directory. 


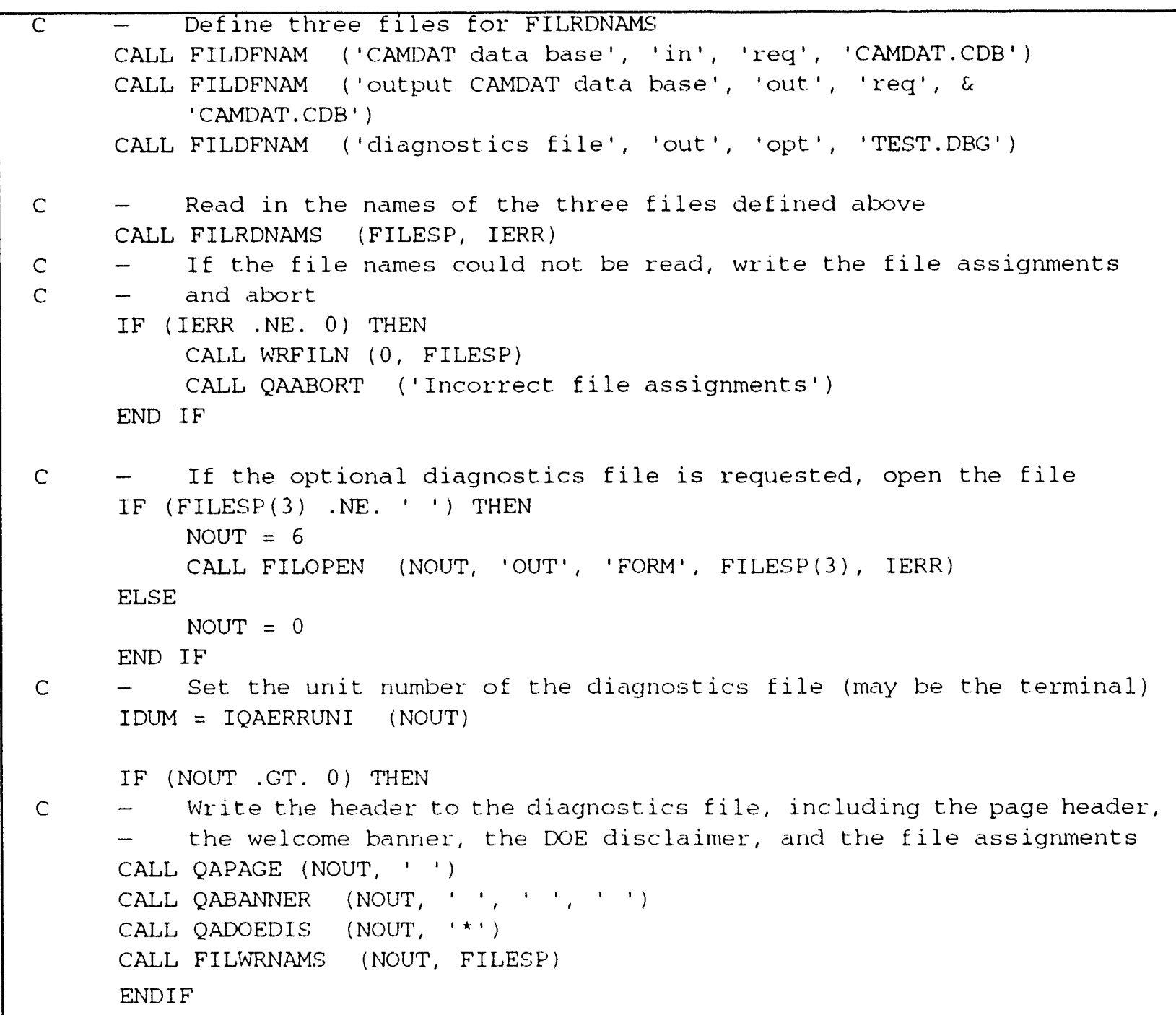

\subsection{Parameter Input}

Input for most CAMCON codes should occur by reading data either from the secondary or computational data bases or from ASCII text files. Normally, the mathematical model parameters (e.g., permeability $[k]$ ) enter via the data bases while numerical model parameters (e.g., solution time step) enter via the input text files. A few codes naturally require interactive input (e.g., plotting codes) and should also interactively accept input data. Guidelines for these forms of input are discussed below.

\subsubsection{Reading from Data Bases}

- Access the data bases through the libraries, CAMDAT_LIB and SDBREAD_LIB, mentioned in Chapter 2, and thoruughly described in Chapters 6 and 7, respectively.

- Use the standard naming conventions for parameters as the default name with the code, but permit the user to override the name. (See the CAMCON user's reference manual [Rechard, 1992] 
for a published list of default names or the secondary data base on-line documentation (help file) on the WIPP computer for the most current version.)

\subsubsection{Reading from ASCII Input Files}

- Although CAMCON codes may be written to run interactively, all should also be able to run using a user-friendly batch input file.

- Use keywords to identify blocks of parameter data.

- Signify the keyword by an asterisk $\left({ }^{*}\right)$ for the first level and, in the rare instance required, by two asterisks $\left(^{(* *)}\right)$ for the zeroth level; for example

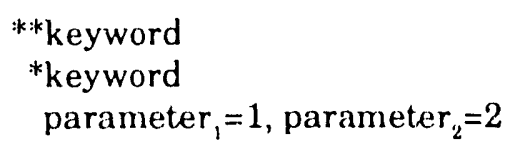

- Use parameter names that identify meanings of input values to make input easy to proofread.

- Program the code such that the keywords and parameters within the keyword data blocks can be entered in any order, making the data input file easy for the analyst to organize and understand.

- Omit the keywords if the screen instructions help organize parameter input when the codes are run interactively.

- Check input for plausibility and validity, checking that the values do not violate physical limits or code algorithm limits.

- Identify bad input and abort gracefully.

- Use the parsing routines FFRDFLDS and FFRDLINE in CAMCON_LIB to read a file record and thus permit a versatile input structure. (In these routines, lowercase al ways returns as uppercase unless enclosed by quotation marks or apostrophes. Furthermore, variables can be either numeric or alphanumeric; see Section 8.2.3, "Free-Field Input.")

- Assume that any ASCII character (not just ANSI standard FORTRAN) may appear in a character string.

The general instructions given to users for constructing input files are listed below and pointed out in the example on the facing page (Rechard, 1992). The reference numbers in the example correspond with the numbers from the list below (more specific details on the parsing routines are given in Chapter 8):

1. Precede the keyword identifying a data block with an asterisk $\left({ }^{*}\right)$.

2. Place keywords and their parameters anywhere in an 80-column record. (All 80 columns are scanned.) FFRDFLDS allows more than 80 characters; however, 80 is a convenient length for screen display.

3. Use an exclamation point (!) in any column to signal that a comment follows for the remainder of the record.

4. Delimit parameters with commas (,) or spaces. 
5. Separate a parameter from its value(s) with an equal sign.

6. Limit numerical data to 30 characters (E30.0).

7. Continue text onto the next record by using an ampersand (\&) at the end of the record to be continued.

8. Enclose character variables containing an ampersand (\&), exclamation point (!), delimiter characters (commas or equal signs or spaces-leading, embedded, or trailing), or those variables that are not to be converted to upper case, with an apostrophe (') or quotation marks (").

9. Use quotation morks to embed apostrophes and apostrophes to embed quotation marks within character variabies.

10. Except for character variables enclosed with apostrophes or quotation marks, case is unimportant for making comparisons within a code.

11. A field cannot be continued. Break records between fields, preferable before a parameter rather than between a paranieter and its value(s).

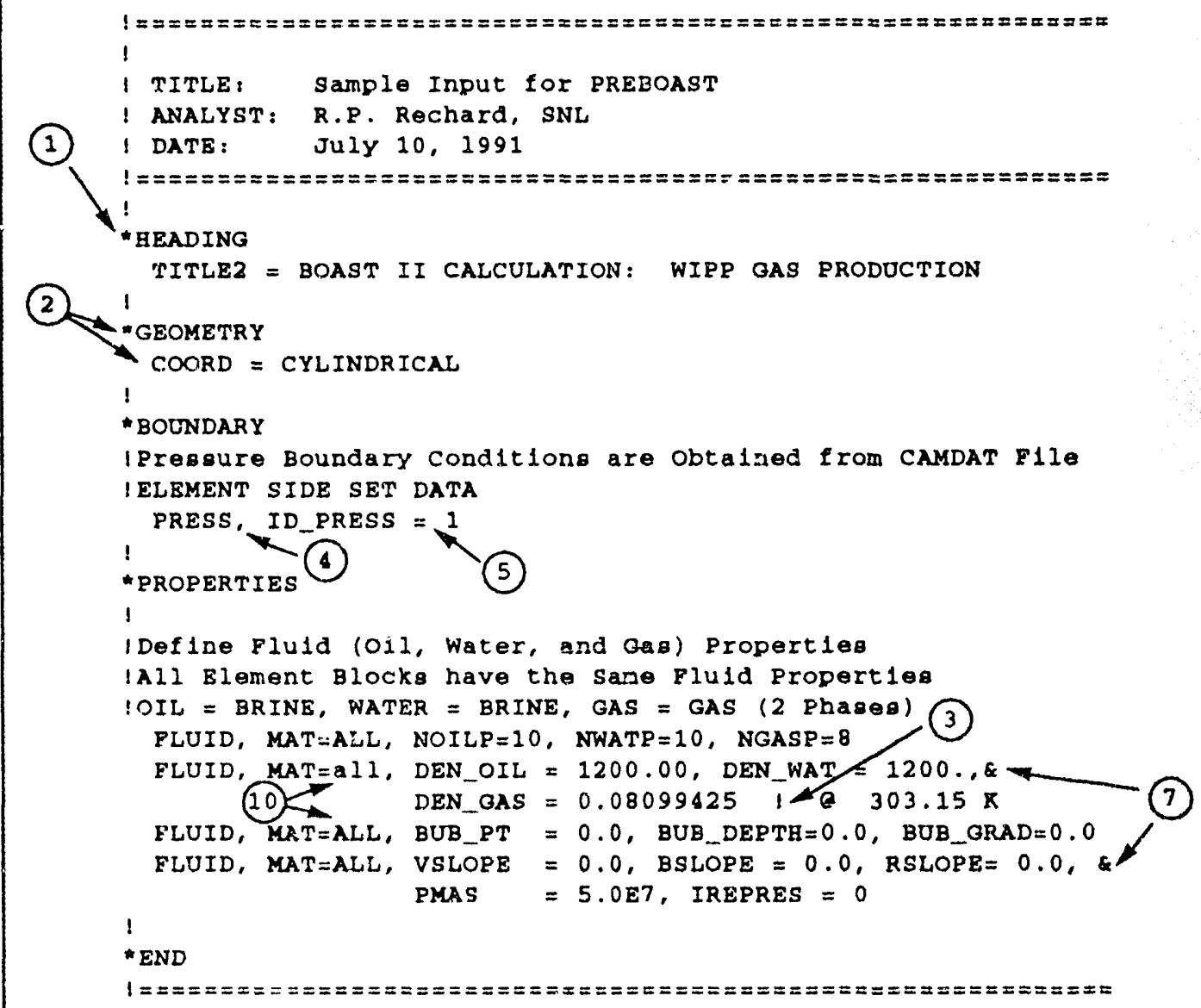


Uner Interface Guidelimes

Code Operatien

\subsubsection{Reading Interactively}

All the guidelines mentioned when reading from an input file are even more important when interactively reading data. For example, check the reasonableness of input and gracefully request the information again if errors are found rather than abruptly aborting with a terse read error message. Also, the FFRDFLDS and FFRDLINE subroutines of CAMCON_LIB work equally well interactively as parsers.

\subsection{Code Operation}

Make a code reasonably bomb-proof. Thus, things that are likely to cause errors (e.g., user input) should be checked. If a program aborts, it should do so gracefully with an informative error message for the user.

\subsection{Error Messages}

When writing error messages, distinguish between errors caused by traps deep in the program intended to avoid meaningless calculations and those caused by erroneous user input data. The error message for a program trap should tell the user to call the code sponsor with any relevant information (such as the subroutine name), while the error message for a user input error should tell the user what the problem is as clearly as possible and not include irrelevant information such as the subroutine name.

\subsection{On-Line Documentation}

\subsubsection{QA Requirements for On-line Documentation}

A software help file is required on-line documentation that must be developed and maintained for each piece of Class $\mathrm{C}$ and $\mathrm{A}$ software (e.g., FORTRAN library or code) used for assessing the performance of WIPP. Although a part of the external documentation discussed in Section 4.7, it is discussed separately because of the necessity for detail.

The help file shall contain the following (only the adequacy of the QA information will be formally evaluated by the QA coordinator for Class C software):

- All QA information necessary to complete the software abstract, i.e., a brief description of the purpose and capabilities of the software, the name(s) of the author(s), and the name of the code sponsor

- Update history of the software, including date, PA software ID, and description of modification

- Instructions for executing the software and necessary input and output files 


\subsubsection{General Help File Contents}

The general information available in a help file includes entry-level text (describing the title, purpose, authors, sponsor, company, and phone number), QA information, update history, and instructions for executing the code. Optional but desirable additions for codes include summary of input commands, data input from CAMDAT, and an example of an input file (refer also to ABSGEN and HLPCHK in Chapter 12):

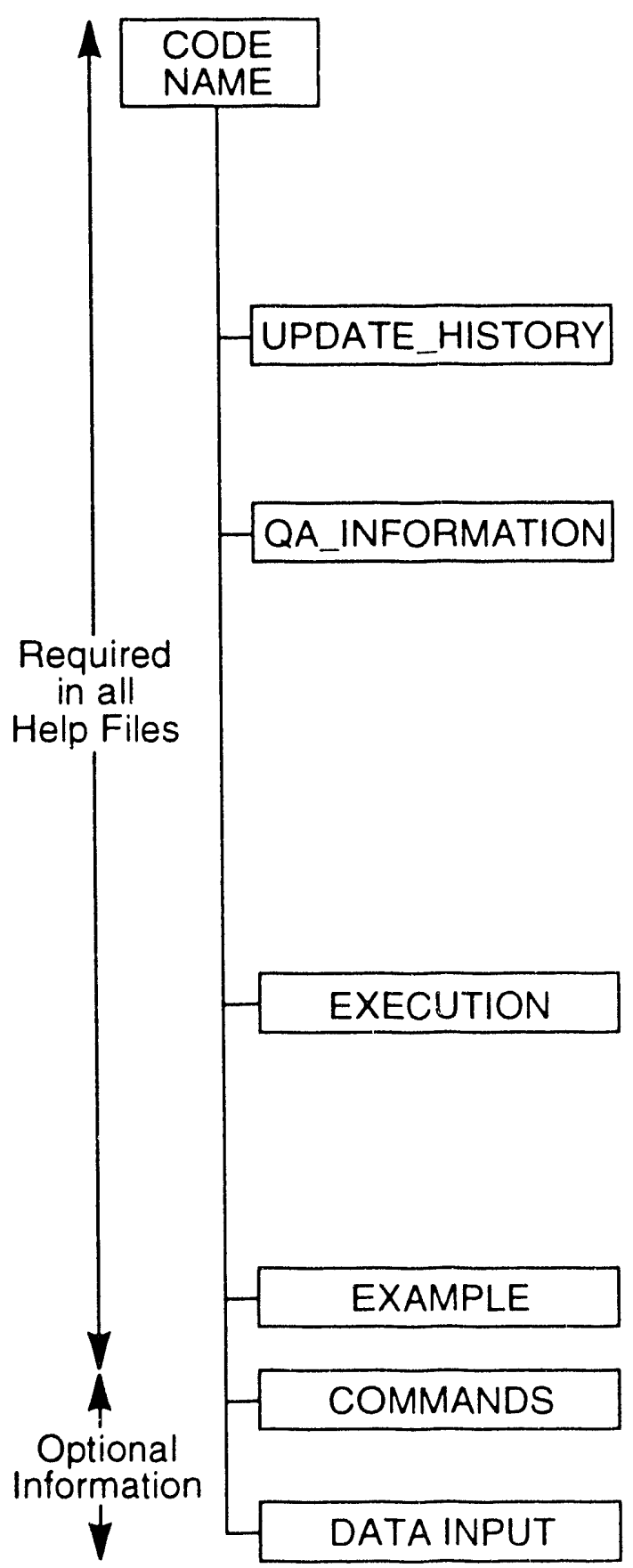

- The entry level text describes

- title

- purpose

- code author

- code sponsor and his or her company and telephone number

- code consultant and optionally ${ }^{*}$ his or her company and telephone number

- The UPDATE_HISTORY subtopic lists

- current version and date, and language

- dates, version number, and brief description of modifications made

- The QA_INFORMATION subtopic lists information necessary for completing the computer abstracts filed in the Sandia WIPP Central File (SWCF), i.e.,

- QA level and category

- limitations

- keywords

- origin

- parent program

- classification

- verification method

- documentation

- The EXECUTION subtopic lists

- symbol for executing the code (e.g., ALGEBRA)

- external files needed for execution listed in order requested by the code (input data base files, output data base files, input files, output files, optional input files, optional output files)

- other interactive input required for execution

- The EXAMPLE subtopic demonstrates the use of all or a subset of the commands summarized in COMMANDS.

- The COMMANDS subtopic summarizes the commands and corresponding parameters used in the input file.

- The DATA subtopic tabulates the data and its type normally passed between the program and data bases.

TR1.6334.218.0

"The primary contact for a code is the spensor and so this later information can be omitted if desired. 
Similarly, the help file for libraries includes entry level text, update history, QA information, and information on linking the library. Optional but desirable additions for libraries include a short description of routines available and examples of use:

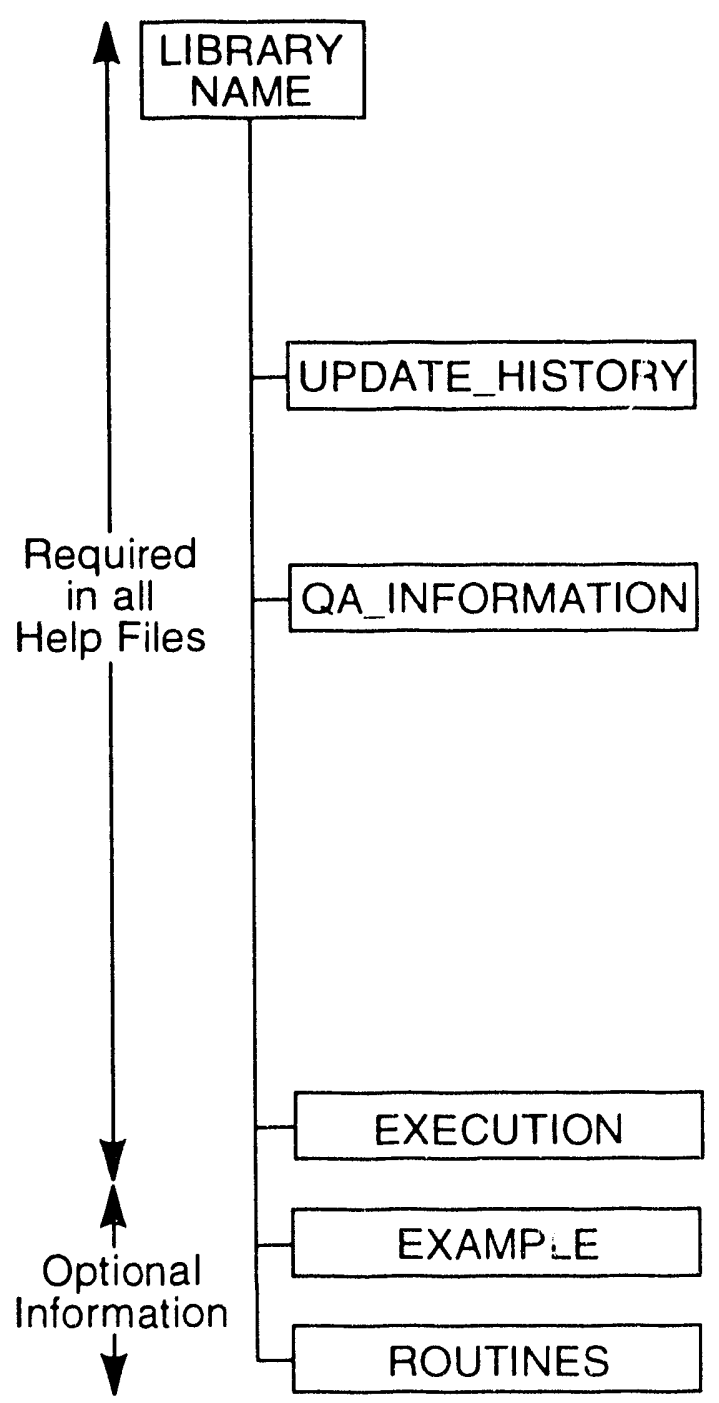

- The entry level text describes

- title

- purpose

- library author

- library sponsor and consultant, and his or her company and telephone number

- The UPDATE_HISTORY subtopic lists

- current version and date, and language

- dates, version number, and brief description of modifications made

-The QA_INFORMATION subtopic lists information necessary for completing the computer abstracts filed in the Sandia WIPP Central File (SWCF), i.e.,

- QA level and category

- limitaions

- keywords

- origin

- parent program

- classification

- verification method

- documentation

- The EXECUTION subtopic instructs the programmer on linking the library

- The EXAMPLE subtopic demonstrates the use of all or a subset of the routines summarized in ROUTINES

- The ROUTINES subtopic summarizes the routines, purpose, and parameters

TRI-6334-218-1

As mentioned earlier, some of the information found in the help file is used to create the software abstract, which is additional documentation required for all Performance Assessment software. The code ABSGEN (described in Chapter 12), which generates the Software Abstract, also expect several specific headings under each level identifier; these headings are listed in the following subsections.

Following is an input structure of the on-line documentation (help file on the VAX). Entries marked optional are not required by the software QA procedures but are still recommended. 


\subsubsection{Help File Structure}

The rules for entering text in help files provide a uniform look and facilitate generating software abstracts. These rules are listed below. The rule numbers correspond with the numbers on the example that follows the list. (Additional information is available in DEC documents [1988a] and in the descriptions of ABSGEN and HLPCHK in Chapter 12.)

1. Start all level numbers in column 1 .

2. Start all level identifiers in column 3 .

3. Start all headings in column 3 .

4. Start all subheadings in column 6 and subsubheadings in column 9 .

5. Use as many characters as necessary for identifiers and headings, but follow headings with a colon (:) and at least one white space (space, tab, new line).

6. Text for an identifier can be any number of lines with any number of characters per line, but delimit the text with a blank line.

Example:

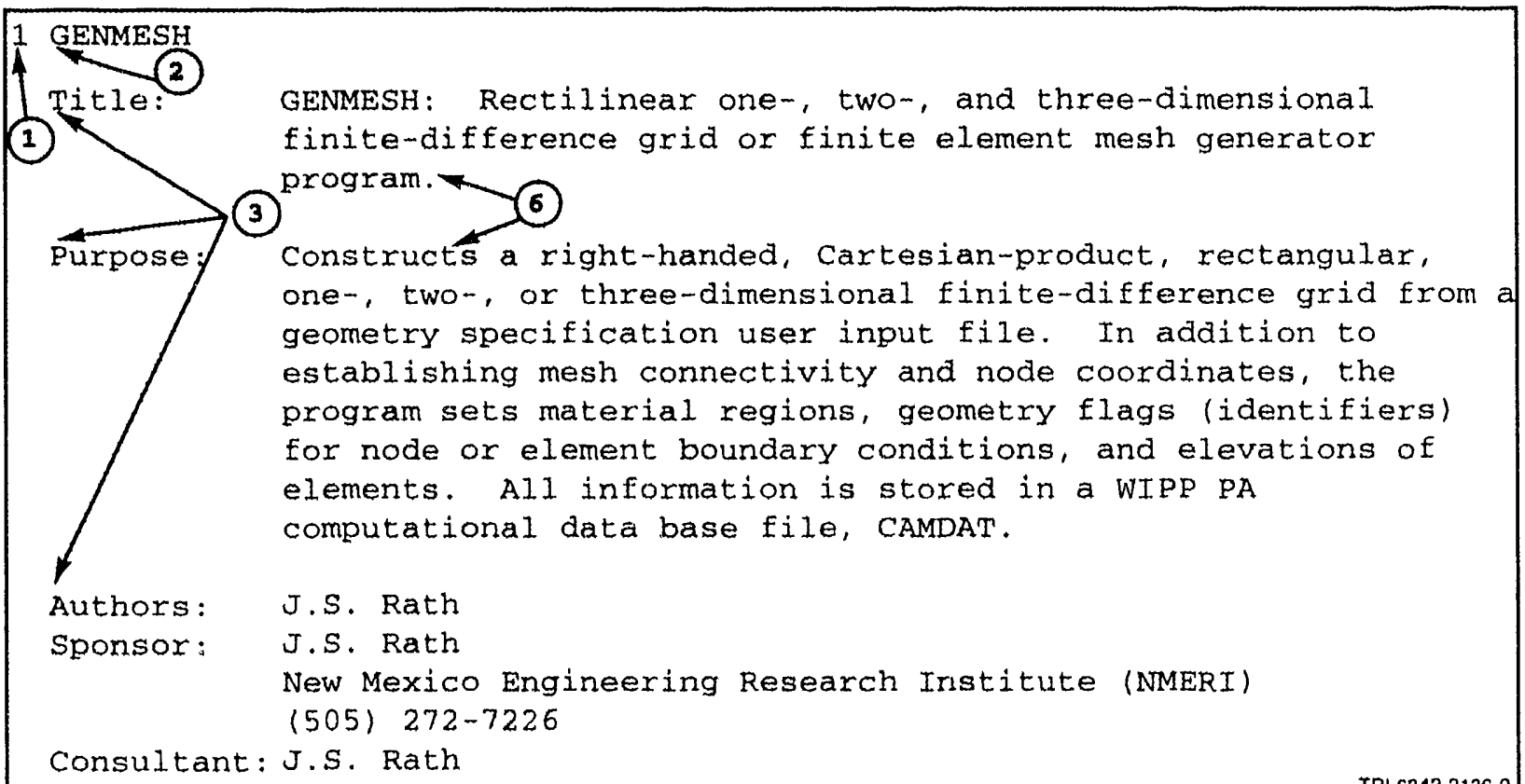

\subsubsection{Entry-Level Text}

The entry-level text in the help file describes the code name, title, purpose, code authors, CAMCON sponsor, and sponsor's and consultant's company and telephone number. The help file is designed so that the user immediately views level 1 information (title, purpose, authors, sponsor, company, and phone number) on the WIPP computer, and then can call up level 2 information (QA information, update history, execution instructions, summary of input commands, and an example input file) followed by other levels if necessary. Most of the level 1 and level 2 text is required by the QA procedures and by the software abstract (see PAP02, 1993). 


\section{Required Headings:Under CODE_NAME Identifier:}

\section{CODE NAME}

Title:

Purpose:

Authors:

Sponsor Name: (include SNL organization or company and phone number)

Consultant: (may be the same as Sponsor Name, and company and phone number can be omitted)

\subsubsection{Update History Subtopic}

The update history fields list the current version, date, language, and number of source lines. When modifications are made, the date, version number, and brief description of modifications are stored here (required text).

\section{Required Headings UnderUPDATE_HISTORYIdentifier:}

\section{UPDATE_HISTORY}

Version:

Language:

Date:

Examip!e:

2 UPDATE_HISTORY

\begin{tabular}{|c|c|c|}
\hline Version: & $\mathrm{A}-6.02 \mathrm{VV}$ & FORTRAN 77 \\
\hline Computer: & VAX/VMS 8810 & Total Lines: \\
\hline Date: & $07 / 02 / 92$ & $\begin{array}{ll}\text { Active Lines: } & 4496 \\
\text { Percent Comments: } & 47 \%\end{array}$ \\
\hline $07 / 02 / 92$ & $A-6.02 V V$ & $\begin{array}{l}\text { Improved routine GSEPEB. } \\
\text { Added a check so that material regions are not } \\
\text { overwritten by IJK-line defined element ranges. } \\
\text { Does not affect version } 6.01 \mathrm{~V} \text { because this is } \\
\text { an I/O specific change. }\end{array}$ \\
\hline $04 / 03 / 92$ & $\mathrm{~A}-6.01 \mathrm{VV}$ & $\begin{array}{l}\text { Added GENMAP routine which transforms } \\
\text { GENMESH's FD element numbers to CAMDAT element } \\
\text { numbers. No need to drop back to C-level } \\
\text { because the MAP }\left(^{\star} \text { ) array, which performs the }\right. \\
\text { transformation, was never used. This } \\
\text { modification is transparent to program flow } \\
\text { and logic. This change (rewriting the MAP(*) } \\
\text { array is output-specific and will be } \\
\text { integrated for use with other programs such as } \\
\text { BLOT, ALGEBRA, etc... }\end{array}$ \\
\hline $01 / 27 / 92$ & $\mathrm{~A}-6.00 \mathrm{VV}$ & A-level. \\
\hline $01 / 27 / 92$ & $\mathrm{C}-6.00 \mathrm{VV}$ & $\begin{array}{l}\text { Adapted to CAMDAT_LIB routines. Adapted for } \\
1-D \text { mesh/grids. Cosmetic changes made to HELP }\end{array}$ \\
\hline
\end{tabular}




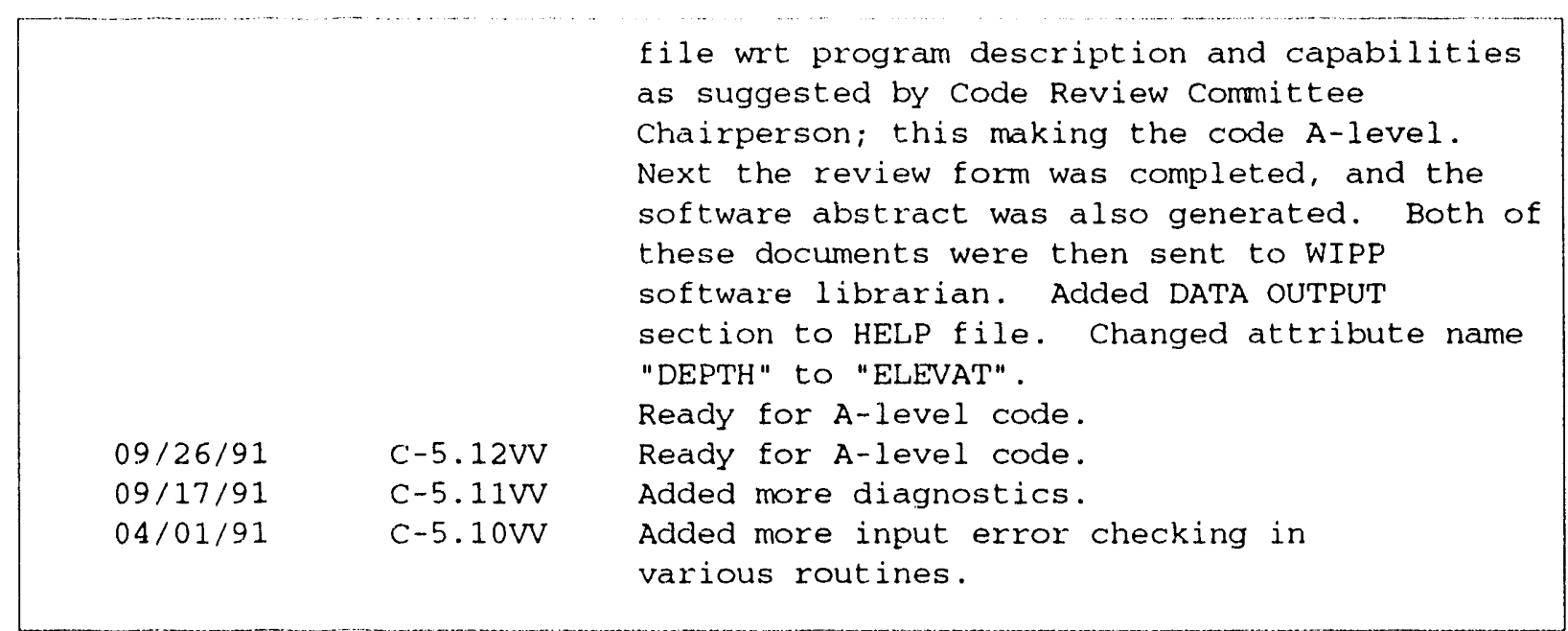

\subsubsection{QA Information Subtopic}

The QA information fields list data that are used to complete the soft ware abstract. The data include QA level, limitations, keywords, origin, parent program, classification, verification method, and documentation.

Required Headings UnderQA_INFOIdentifier:

2 QA_INFO

QA_Level:

(See UPDATE_HISTORY)

Limitations:

Keywords:

Origin:

Parent Program:

Classification:

Description:

Type:

Category:

CAMCON Module:

(e.g., Government owned, limited distribution)

(e.g., Scientific/engineering software (SES) or non-SES)

(e.g., Sandia internal software or external software)

(e.g., Mesh Generation)

Verification: (required)

Hand Calculation

In_Use Test

Comparison with Analytic Solution

Benchmarking

Use in Sensitivity Analysis

Convergence Tests

Other

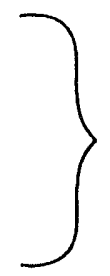

At least one of these seven entries is required (see Section 3.7, "Verification," for definitions).

Documentation:

User's Reference

Theoretical Reference

Verification/Sample Problems Reference

Other Reference

Primary Reference:

(required)

(optional)

(optional)

(optional)

(optional)

(required) 


\section{Example:}

2 QA_INFO

QA_Level:

See UPDATE_HISTORY

Limitations:

GENMESH is a Rectilinear 2D/3D FE/FD mesh/grid generator compatible with the WIPP CAMCON Computational Data base, CAMDAT. In addition to establishing the mesh connectivity, the program generates nodal point coordinates, material region information, Element Side set and/or Nodal point set boundary condition data. In addition, GENMESH automatically inserts the two attribute values, THICK, and DEPTH, into the output CAMDAT file.

Keywords :

Finite difference, Finite element, Grid generator, Mesh generator, Rectilinear, Performance Assessment, Waste Isolation Pilot Plant

Origin:

Sandia National Laboratories, Performance Assessment Department 6342, Albuquerque, NM 87185-5800

Parent Program(s): None Parent Reference(s): None Classification: Description: Type: Category: CAMCON Module: Government owned, limited distribution. non-SES Sandia internal software Mesh Generation

Verification: Hand Calculation In_Use Test

Documentation:

User's Reference: Rechard, R. P., ed. 1992. User's Manual for CAMCON: Compliance Assessment Methodology Controller, Version 3.0. SAND90-1983. Albuquerque, NM: Sandia National Laboratories.

Verification/Sample Problems Reference: Computer: VAX/VMS 8810 Node: Jimmy Directory: CAMCON\$ROOT: [GENMESH.A_LEVEL.TEST] Input Files: GENMESH_TEST_ID.INP, GENMESH_TEST_2D.INP, GENMESH_TEST_3D.INP, GENMESH_TEST_4.INP, GENMESH_TEST_5.INP, GENMESH_TEST_6.INP

Theoretical Reference: None

other Reference: None

Primary Reference:

Rechard, R. P., ed. 1992. User's Manual for CAMCON: Compliance Assessment Methodology Controller, Version 3.0. SAND90-1983. Albuquerque, NM: Sandia National Laboratories. 


\subsubsection{Execution Subtopic}

The execution fields list the computer symbol for executing the code (e.g., ALGEBRA), and external files needed for execution, listed in order as requested by the code (computational data base files, secondary data base files, input text files, output files, optional input files, optional output files [see Section 4.2.3, "Entering File Names")).

\section{Example:}

2 EXECUTION

Type "GENMesh" at the main menu of CAMCONexec or at a system prompt. Subsequent questions in the procedure file request names for up to three Eiles:

- Optional input control card data file \#1

(Two possibilities for entering data, one of which requires a file):

1a. Interactive (will generate a GENMESH input control card data file)

or

1b. Text file created independently or from previous interactive session <GENMESH. INP>

- Required output PRECAMDAT file

2. Name for binary PRECAMDAT file <PRECAMDAT.CDB>

- Optional output files

3. Name for diagnostic output file <GENMESH.DBG>

\subsubsection{Commands Subtopic}

The commands fields summarize the commands and the corresponding parameters used in the input file.

\section{Example:}

2 COMMANDS

Contents of GENMESH Input File. The GENMESH text file consists of eight keywords: (1) BOUNDARY_FLAGS, (2) ELEVATION_ELEMENT, (3) END,
(4) GRID_SPACING, (5) REGIONS
(6) SETUP_GRID,
(7) TITLE, and
(8) UNITS 
3 BOUNDARY_FLAGS

*BOUNDary_flags

The *BOUNDARY_FLAGS keyword sets boundary flags in the grid. Boundary conditions values are set in the program BCSET or in the pertinent analysis program. Two types of boundary conditions are available.

4 ELEMENT_SIDE_SETS

ELement_side=side_id, IRANGe=imın, imax, JRANGe=jmin, jmax, $k R A N G e=k m i n, k m a x$

1. side_id - integer identification for element side

2. nmin - minimum grid index in finite-difference grid

3. nmax - maximum grid index in finite-difference grid

The ELEMENT_SIDE boundary flag identifies a group of elements (or grid cells) with a unique ID. This feature is useful for surface boundary conditions for finite elements (e.g. fluid flux). Furthermore, the "nodes" of grid-centered finite-difference codes correspond to finite elements; hence, this type of boundary condition is used with SWIFT II.

\subsubsection{Data Subtopic}

The data subtopic tabulates the data and its type normally passed from/to the program to/from CAMDAT.

\section{Example:}

2 DATA_OUTPUT

GENMESH generates a virgin CAMDAT file, which contains:

3 NODAL_COORDINATES

Nodal coordinate data, stored for a node as:

(x) $\quad-1-D$ mesh (NDIM=1),

$(x, y) \quad-2-D$ mesh (NDIM=2), and

$(x, y, z)-3-D$ mesh (NDIM $=3)$.

For any 1-, 2-, or 3-dimensional rectilinear grid, nodes are numbered first in order of increasing $X$ (I-index), then $Y$ (J-index), and then $\mathrm{Z}$ ( $\mathrm{K}$-index). 
Thus for a 2-D grid that has $5 \mathrm{I}$-lines and $3 \mathrm{~J}$-lines (the 2-D mesh has 15 nodes and 8 elements), the GENMESH node numbering convention is:

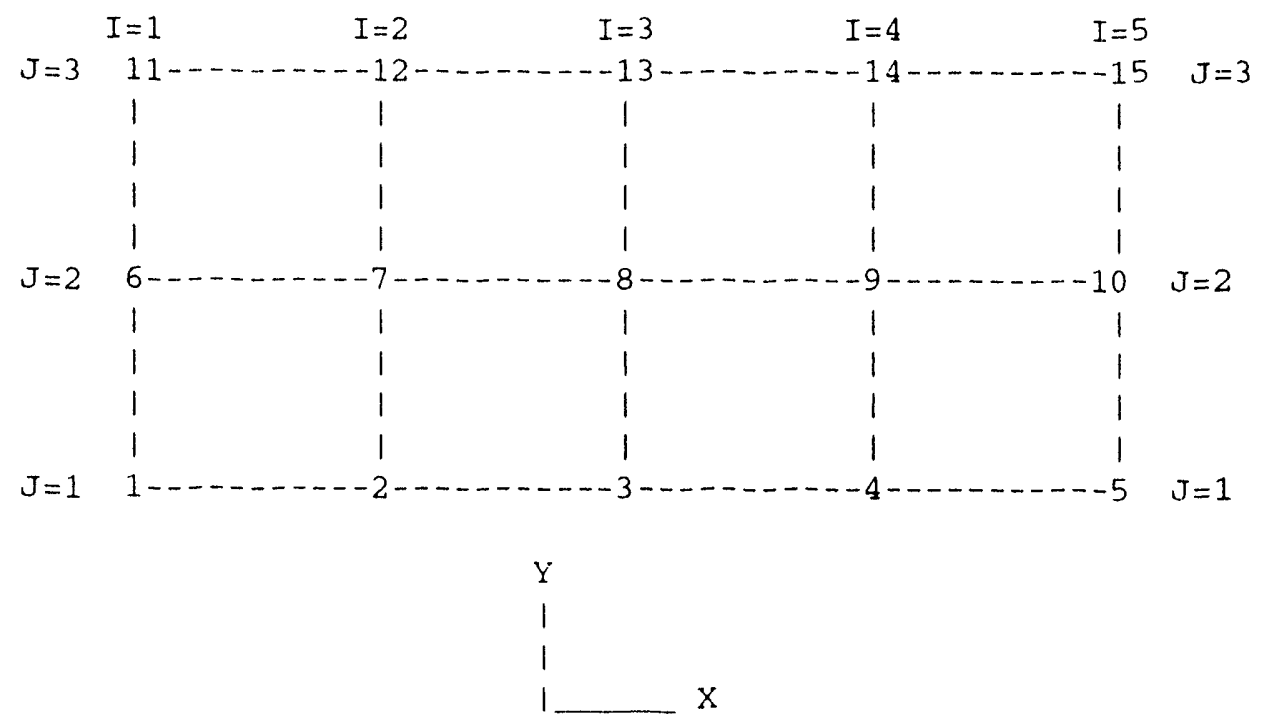

3 SUMMARY

Data GENMESH Transfers to CAMDAT

\begin{tabular}{|c|c|c|}
\hline \multicolumn{2}{|c|}{ Default Symbol } & Data Type \\
\hline$-\cdots---$ & $\ldots \ldots \ldots \ldots$ & $-\cdots-\cdots$ \\
\hline \multicolumn{3}{|l|}{ Nodal Coordinates } \\
\hline $\mathrm{X}$-coordinates & $\mathrm{X}$ & Coordinate \\
\hline Y-coordinates & Y & Coordinate \\
\hline Z-coordinates & $\mathrm{Z}$ & Coordinate \\
\hline Material Regioning & n./a. & Materials \\
\hline Element connectivity & n. $/ a$. & Nodes \\
\hline \multicolumn{3}{|l|}{ Element Attributes } \\
\hline Element thickness & THICK & Attribute \\
\hline Element centroid elevation & ELEVAT & Attribute \\
\hline Element $\mathrm{X}$-direction length & $D E L \_X$ & Attribute \\
\hline Element $Y$-direction length & DEL_X & Attribute \\
\hline Element $\mathrm{z}$-direction length & $D E L \_Z$ & Attribute \\
\hline \multicolumn{3}{|l|}{ Geometric Boundary Flags } \\
\hline Nodal point sets & $<$ no default $>$ & Node set \\
\hline Element side sets & $<$ no default $>$ & side set \\
\hline
\end{tabular}

\subsubsection{Example Subtopic}

The example fields demonstrate the use of all or a subset of the commands summarized in the commands subtopic. 


\section{Example:}

2 EXAMPLE

The following is an example input file for GENMESH:

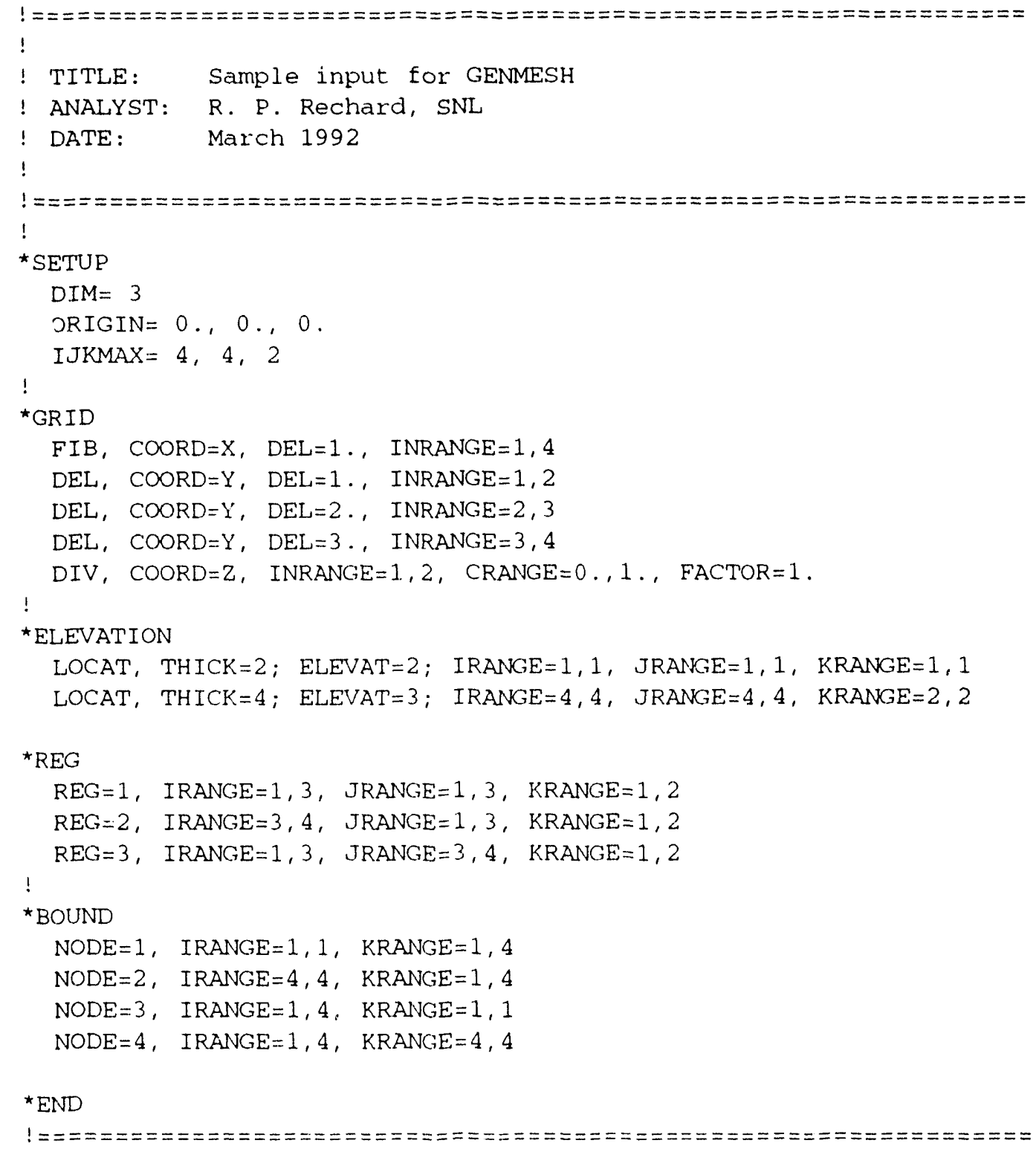

\subsection{External Documentation}

Before software can attain Class A or C status, the software must satisfy several requirements for external documentation as described below. 


\subsubsection{Software Notebooks}

The software notebouk is a collection of the information available on each piece of software in the CAMCON system that can be used by programmers, analysts, and QA personnel.

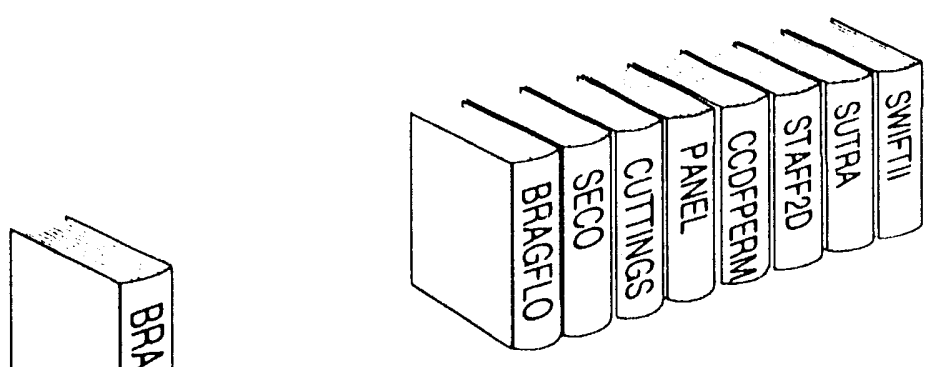

Contents - The motechook ustlally

contains the following headings and intomalton:

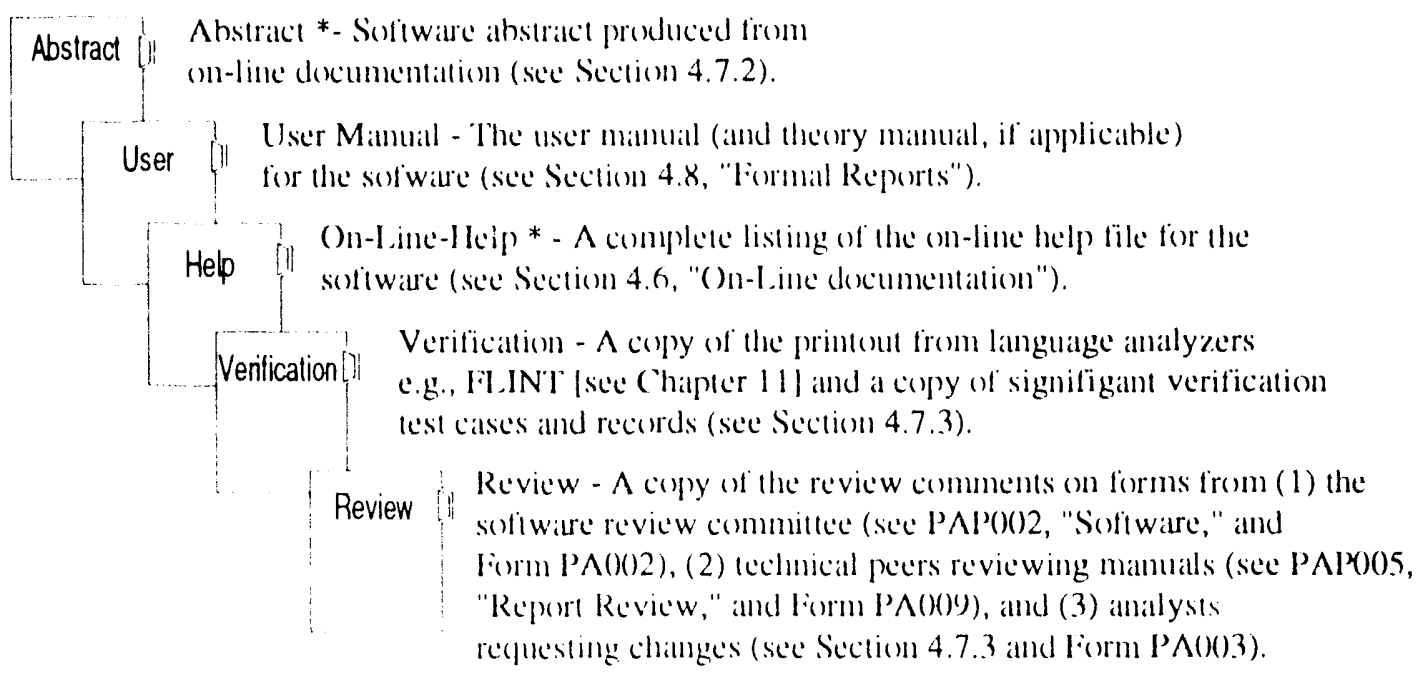

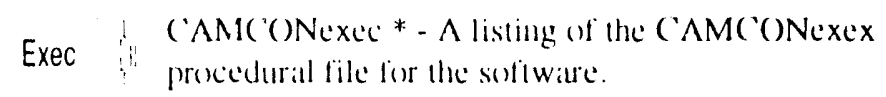
Source Sonre: ${ }^{*}-\Lambda$ listing of the source and included files.

TA1.6342-2138-0

\footnotetext{
I The ahstract can be printed using AlsSGEN (see Chapter 12).

2 The help, CAMCONexec: and source sections can be printed using QA_PREP and QA_PRINT (see Chapter 12).
} 
User Interlace Guidelines

External Decumentation

Some pieces of software may be too large to practically reproduce as hardcopy, or there may be proprietary restrictions on reproducing source code, etc. In these cases, a note in the appropriate section of the notebook should simply state why the information has been omitted and, if possible, where to find the information.

Responsibilities. The software sponsor of each piece of software(e.g., code, library, or DCL package) is responsible for providing all the information for the notebooks. The sponsor should use QA_PRINT (see Chapter 12) to help in producing uniform, consistent printouts for the abstract, help file, CAMCONexec procedural file, and source headings of the software notebook.

The QA coordinator or designated appointee helps the sponsor assemble and produce the notebooks to maintain some semblance of uniformity. The QA coordinator is also responsible for filing the notebooks with the Sandia WIPP Central Files (SWCF) records manager.

Updates. Each software notebook is usually updated sometime prior to periodic announced audits and whenever the sponsor or QA coordinator thinks the information has become so outdated as to be misleading or useless; it does not always provide the most current information. Rather, the on-line documentation is the most current document detailing the purpose, update history, QA information, execution, example problem, and data input to and output from data bases.

\subsubsection{Software Abstract and Listings}

Soft wa re Abstract. All Class C and A software will have a software abstract in the software notebook. Current abstracts must be produced (1) when major version numbers change and (2) when QA information in the on-line documentation (help file) changes, especially when the QA status changes. (See Chapter 12 for a discussion of ABSGEN, a program to generate this abstract from on-line documentation, or see QA_PREP and QA_PRINT below.) All software abstracts must be signed by the QA coordinator and be submitted to the SWCF.

Utilities. Two utilities exist to assist the FORTRAN code developer in satisfying QA requirements: QA_PREP and QA_PRINT. Both codes are defined as CAMCON system symbols and are available to all users of the WIPP computer. Their use is summarized below and fully described in Chapter 12 , "Software Development Utilities."

QA_PREP searches a specified directory and produces a file named ROUTINES.TXT. This file contains a listing of all *.FOR and *.INC files and identifies the main FORTRAN module.

QA_PRINT uses the file ROUTINES.TXT produced by QA_PREP to run FLINT the CAMCON implementation of the VMS-FORTRAN code analyzer), print the FLINT output, print the source code, print the CAMCONexec procedural file, and print the on-line documentation. 


\subsubsection{Records}

Two sets of records are maintained for software: verification records andchange records.

Verification Records. For Class C software, at least one test case (e.g., example problem)demonstrating that important capabilities of the software are correctly implemented must be in the help file. Other test cases including necessary input files (*.INP or *.TRN), secondary data base files (*.SDB). computational data base files $\left({ }^{*} . \mathrm{CDB}\right)$, and result files (*.OUT, *.DBG, *.TRN, or *.CDB) should be stored in the [code_name.TEST I subdirectory as thought appropriate by the software sponsor. A printout of the help file and |code_name.TEST| subdirectory contents, signed by the sponsor, should be

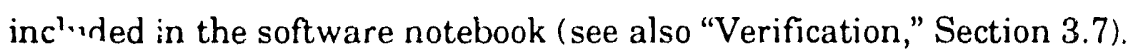

In addition to the focumentation for Class $\mathrm{C}$ verification, Class $\mathrm{A}$ software verification must include a summary statement of the (1) purpose, (2) types of the tests performed, and (3) results of the tests including any applicable performance criteria and all signed and dated by the sponsor. All this additional documentation must also be stored in the software notebook.

Change Records. An important aspect of improving the quality of software is the identification and correction of errors, inaccuracies, limitations, and enhancements of software capabilities. A software user should report all desired software enhancements and corrections to the software sponsor for approval (see also Section 3.5, "Modifying Software in the CAMCON System"). Corrections for Class A soft . re shall be submitted by means of a formal report form, the Change Request and Response Form (ask the SWCF records manager for form PA003, current version). In this case, the software sponsor must submit a formal reply about intended action in response to the reported software problem. The form must be filed in the software notebook.

\subsection{Formal Reports}

All Class A software will have formal reports. The reports describe what the software does (i.e., its purpose) and how to use the software (user's manual). All Class A software categorized as science and engineering software (SES) will also provide a theory manual that wescribes how the soft ware achieves its purpose, e.g., the theory and assumptions used in the software such as algorithms and numerical solution techniques.

Additional documentation, such as tutorials, sample problems, or programmer's manuals, is encouraged but not required.

The formal reports must be reviewed by the formal peer review and management review process described in Procedure PAP05, "Reporı Review Quality Assurance Procedures" (1993). If desired, the user's man tal, verification records, and theory manual (if necessary) can be combined in one document. Verification and change records can also be included in this document. (Verification documentation is discussed in Section 4.7 and changre records are discussed in Section 4.7.3.) 
User's Manual. The user's manual shall be sufficient to (1) enable a user with an adequate modeling and proper technical background to set up and run the program and (2) help the user resolve possible difficulties. The user's manual may be combined and published with other CAMCON system codes (e.g., Rechard, 1992). As a minimum, the user's manual will describe

- Input (e.g., variables, units, format, default values)

- Output (e.g., meaning of headers and appropriate units)

- One sample problem

Theory Manual. For Class A SES software, the theory manual will describe, as a minimum, the

- Class of equations solved and the corresponding assumptions and limitations

- Numerical solution techniques and related stability and accuracy with references

- Relationship between numerical and mathematical strategies (e.g., how boundary conditions are introduced) 


\section{General Programming Guidelines}

The primary goals of this chapter and the remaining chapters of this manual are to (1) ensure that the software developed for the CAMCON system does what is intended (reliability), (2) increase software readability and make future software modifications (i.e., enhancement and error correction) easier (reusability), and (3) streamline code development. These goals are primarily accomplished by providing code developers with style and development guidelines, discussed here, and development tools, which are discussed in the following chapters.

The high cost of software devslopment and the higher cost of software maintenance and enhancement make these important goals. We attempted to meet these goals by providing practical pointers to increase the chances that the software will be useful, i.e., do what it was intended to do and decrease the chances that the programmer will waste time. The essence of what is trying to be conveyed is summed up by the elusive word "style" - not a list of rules so much as an approach and an attitude. The guidelines presented herein are essentially good programming practices and are not intended to restrict individual programmers. ${ }^{1}$ When these programming practices are broken, some compensating merit should be found in the coding. However, at no point will someone check that a style guideline is followed (unlike the software QA requirements). (Even the examples provided do not follow all the guidelines presented.)

\subsection{Software Planning}

A critical phase in software development is the initial planning phase. Errors committed during this phase are often ruinous to the timely completion of the code, and have a much greater impact on the project than errors committed during code generation.

Although plans can go astray, plans certainly increase the chances of "success," i.e., the software doing what it is supposed to do by solving the correct problem and solving this problem correctly. With the maturing of the programming occupation, great focus has been placed on software development as a project within the usual life cycle of a project and the need for planning, in particular. Rather than paraphrase the insightful suggestions made in numerous texts and articles, the following discussion briefly mentions a few pointers pertinent to software development for the CAMCON system.

The planning for the CAMCON system as a whole can involve a group effort in defining goals. However, (1) because the CAMCON system is modularized and (2) because of the flexibility in interfacing codes in CAMCON, most software development projects can normally be planned and carried out by one

1. At the inception of the CAMCON project, these guidelines were informally presented in individual sessions with each programmer. 
person. What follows are suggestions not for the CAMCON system as a whole but for the individual codes. The scope of planning on this scale is generally similar to writing a report (Weiss, 1982, p. 16).

For the individual codes, good planning usually consists of the following:

1. Verify the need to program. Can the objective be reached by acquiring or purchasing already existing software? Is the objective too specialized or so ill-defined that it should be done by hand until the procedure is better defined or the needs are more general?

2. Define the subject and QA participants. Name the project and decide whether the code type is SES or non-SES. Who are the software sponsor and consultants?

3. Define the purpose. What are your primary specifications? What must be met before the customer considers the code a success? If possible, define the appropriate test cases to be run after completing the programming.

4. Identify strategic problems. What are the barriers to complete success (time, money, specifications)? What can be done to avert these obstacles?

5. Select the approach and scale. Is the code for one-time use, or is it considered a prototype, or must it serve as a solid foundation for future enhancements? Must the code interface with the CAMCON system or can it stand alone? How many developers are required?

6. Prepare a simple plan and estimate the production schedule.

- Define solution techniques. (The development of engineering software and the research into numerical algorithms which accompanies such development often precludes a detailed description of the techniques to be implemented in a code.)

- Plan program input, and presentation of results.

- Identify capabilities that may be included in a future version of the code.

- Plan the data storage, allocation, and management of computer memory.

- Divide code into proposed modules.

- Define interfaces between modules clearly. This includes unambiguous definition of all shared or possibly conflicting parameters, their units, and physical meaning.

7. Approve the plan. Present the plan to the customer (users) for approval.

These planning steps need not be formal. Even something quickly jotted down and informally discussed with the users may be sufficient for many software development tasks. However, this latter statement should not be taken as diminishing the importance of planning, only that the planning effort should be appropriate given the intended scope of the software development task and effort in generating the coding. 


\subsection{Readability}

This and the following sections deal primarily with increasing readability of software by improving programming style such that future modifications (i.e., enhancements and error corrections) are easier. The section generally consists of rules followed by specific coding examples in FORTRAN. As mentioned before, several books describe style issues in detail (e.g., Kernighan and Plauger, 1974). Here, we emphasize only style rules thought particularly important, parts of which were initially reported in Rottler et al. (1986).

QA standards have not been established in the areas of software maintainability (i.e., internal documentation, code structure, and code readability), portability (i.e., choice of language and extensions), and coding style because of the diverse needs of software used in performance assessment. The WIPP software is not aimed at general situations but instead is developed to solve specific problems requested or needed by DOE customers.

Nonetheless, in general, about $70 \%$ of software life cycle costs can be attributed to code maintenance, including code enhancements (40\%), bug fixes (15\%), and installation with new hardware/software (15\%)(Osborne, 1988). Consequently, these coding guidelines and the software libraries described in the subsequent chapters attempt to address the software properties and features mentioned above. The following guidelines are demonstrated in the complete subroutine example in Section 5.2.5.

\subsubsection{General}

The subroutine fragment on the next page points out some of the general guidelines for readability that are listed below:

- To increase general readability within the PA department, use the FORTRAN 77 language. An acceptable alternative is the ANSI $C$ language when the improved capability of this ianguage to interface with the operating system warrants.

- Make a routine read from top to bottom. Branch upward within a routine only to implement a loop.

- Avoid tricky or overly complex code in favor of simple, maintainable code. Do not sacrifice readability for efficiency unless efficiency is a real concern for the code segment in question.

- Do not use the old FORTRAN 66 constructs such as arithmetic IFs, computed GOTOs, and Hollerith data. Remove these constructs from old code when convenient.

- Do not patch or comment bad code; rewrite instead.

- Avoid using standard intrinsic function names in naming user-defined external functions. For example, do not use the function names SQRT, EXP, and LOG when naming an external function.

- Use ANSI upper- and lowercase letters consistently within a subroutine and preferably within a code. The ANSI FORTRAN standard specifies the use of uppercase letters in executable statements. However, complex numerical algorithms may be easier to read when array indices 


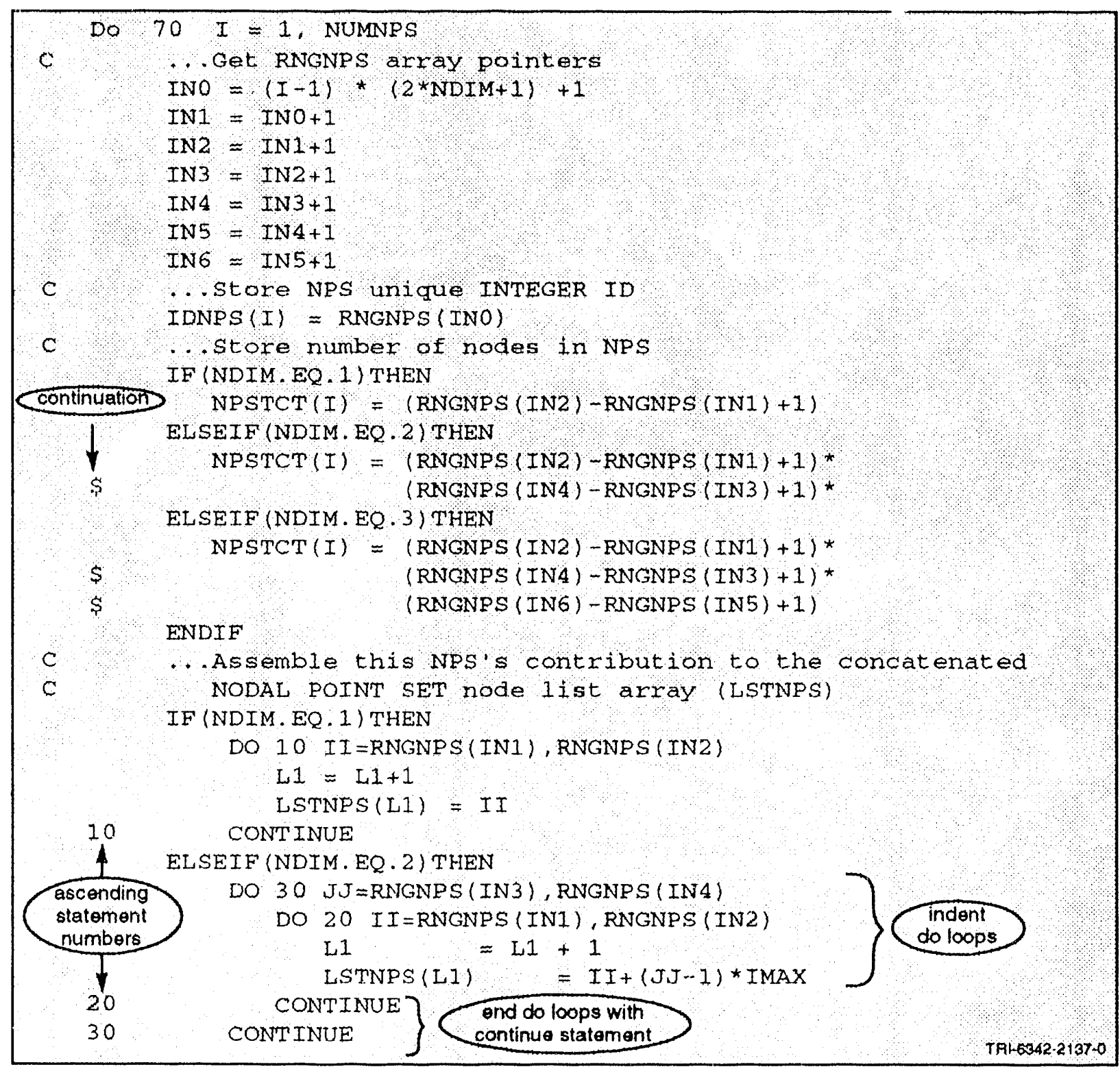

are lowercase while the matrices are uppercase and may justify an exception. Furthermore, the use of mixed-case letters for COMMENT statements and code output is encouraged to increase readability.

- Use a unique continuation character, such as $\$$ or $\&$, not one with another syntactic meaning. (The $\$$ is the only ANSI standard FORTRAN symbol without another syntactic meaning, but an acceptable non-ANSI standard FORTRAN symbol is the \&.

- Number statements always in ascending order within a routine. Make intervals between statement numbers large enough to allow one to insert new labeled statements. The programmer might consider using large numbers (e.g., 10000) for FORMAT and non-executable statements and small numbers for executable statements so that the statement labels convey some meaning. (A FORTRAN format and statement renumbering code, FORMRENUM, is available on the WIPP computer and is described in Chapter 12. Although FORMRENUM functions best on a compilable routine, it can help locate mismatched IF and DO block endings.) 
- Place FORMAT statements referenced only once in a routine at the point of reference. However, in working with frequently used FORMAT statements, the programmer may place them all in one location.

- Indent DO-loops and block IF constructs.

- End all DO-loops with the CONTINUE statement, and end nested DO-loops on separate labels.

- Avoid the use of the ASCII character code, CHAR(), when possible; the character in quotes is much more readable (e.g., ' versus CHAR(32) for specifying a blank).

- Use spaces between expressions and operation symbols in assignment statements to prevent crowding.

\subsubsection{Variable Naming}

- Although the FORTRAN 77 standard for the maximum length of variable names is six characters, it is still necessary to use descriptive names for constants and variables. Consequently, make them longer than six characters and use non-standard characters, suci as the underline, if necessary. For portability, consider making the first six characters uniquely identify the variable name.

- Use standard FORTRAN naming conventions for real data (A-H, O-Z) and integer data (I-N), but declare all variables.

- Avoid names that are unique by only one character.

- Purely mathematical functions should be defined using familiar notation.

- Names and array sizes should be the same in all occurrences of a named common.

- Only use double precision constants and variables when known to be necessary for numerical accuracy.

- Use variables for input/output init specifiers, not literals (e.g., PARAMETER (NOUTPUT=77); CALL IQAERRUNI (NOUTPUT)).

\subsubsection{Declarations}

- Declare all variables.

- Declare numerical constants in statements using the DATA statement or the PARAMETER statement. For example, the expression $0.5 * \mathrm{PI}$ is more suggestive than 1.570796. Other examples include the use of integer variables as array limits and DO-loop limits rather than the use of explicit integer constants, when appropriate.

- Be careful about machine precision when using numerical constants. Sometimes it is better to compute numbers to get the proper precision (e.g., $\mathrm{PI}=4.0 \mathrm{D}+00^{*} \mathrm{ATAN}(1.0 \mathrm{D}+00)$ ). 


\subsubsection{Modularity}

- Keep routine size to one to three pages of output to improve readability. (Ideally, it would be one page, but the comments in the header and the code often make this impractical.)

- Avoid excessive fragmentation (i.e., replacing short segments of code with calls to a routine that performs trivial tasks) unless done to isolate machine-specific coding.

- Link to the CAMCON_LIB, CAMSUPES_LIB, CAMDAT_LIB, and SDBREAD_LIB libraries. Whenever appropriate, use the routines from these libraries.

- Use dynamic memory when appropriate (see CAMSUPES_LIB). Fixed-length arrays may waste memory and put unnecessary constraints on the code.

- Place named COMMON blocks in include files, rather than placing in each subroutine, to facilitate making one change rather than many.

- Carefully organize COMMON block include files so that as few as possible are necessary in any one subroutine to avoid obscuring key information and reducing the readability.

\subsubsection{Example}

\begin{tabular}{|c|c|c|}
\hline & & $\begin{array}{l}\text { INE GAUSSU( A, NRANK， NRWDIM，B， MRHSV, } \\
\text { MCLDIM， INDXC， INDXR， IPIV ) }\end{array}$ \\
\hline$C$ & 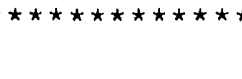 & 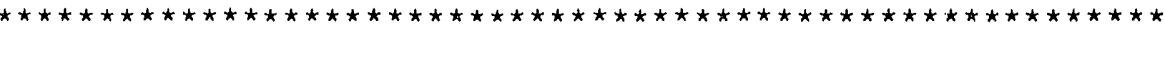 \\
\hline C & PURPOSE: & Linear equation solution by Gauss-Jordan elimination. \\
\hline $\mathrm{C}$ & & A is an input matrix of NRANK by NRANK elements, stored \\
\hline $\mathrm{C}$ & & in an array of physical dimensions NRWDIM by NRWDIM. \\
\hline $\mathrm{C}$ & & $B$ is an input matrix of NRANK by MRHSV containing the \\
\hline C & & MRhSV right-hand side vectors, stored in an array of \\
\hline $\mathrm{C}$ & & physical dimensions NRWDIM by MCLDIM. On output, $A$ is \\
\hline $\mathrm{C}$ & & replaced by its matrix inverse, and $B$ is replaced by the \\
\hline C & & corresponding set of solution vectors. \\
\hline C & & \\
\hline C & SOURCE: & Press, W.H., Flannery, B.P., Teukolsky, S.A., \\
\hline $\mathrm{C}$ & & Vetterling 'v.T., "Numerical Recipes: The Art of \\
\hline C & & Scientific Computing", Cambridge University Press, \\
\hline $\mathrm{C}$ & & 1987. \\
\hline C & & \\
\hline $\mathrm{C}$ & PROGRAMMER : & Jonathan S. Rath \\
\hline c & UPDATED: & 24-MAY-1993 JSR: Renamed variables for clarity. \\
\hline C & & 24-AUG-1992 JJSR: Added INDXC, INDXR, IPIV as arguments \\
\hline $\mathrm{C}$ & & 25-JUN-1992 JSR: Made everything DOUBLE PRECISION \\
\hline C & & 31-OCT-1991 JJSR: First id. \\
\hline $\mathrm{C}$ & & \\
\hline$C$ & CALLED BY: & MRQMIN \\
\hline C & CALLS : & QAABORT \\
\hline
\end{tabular}




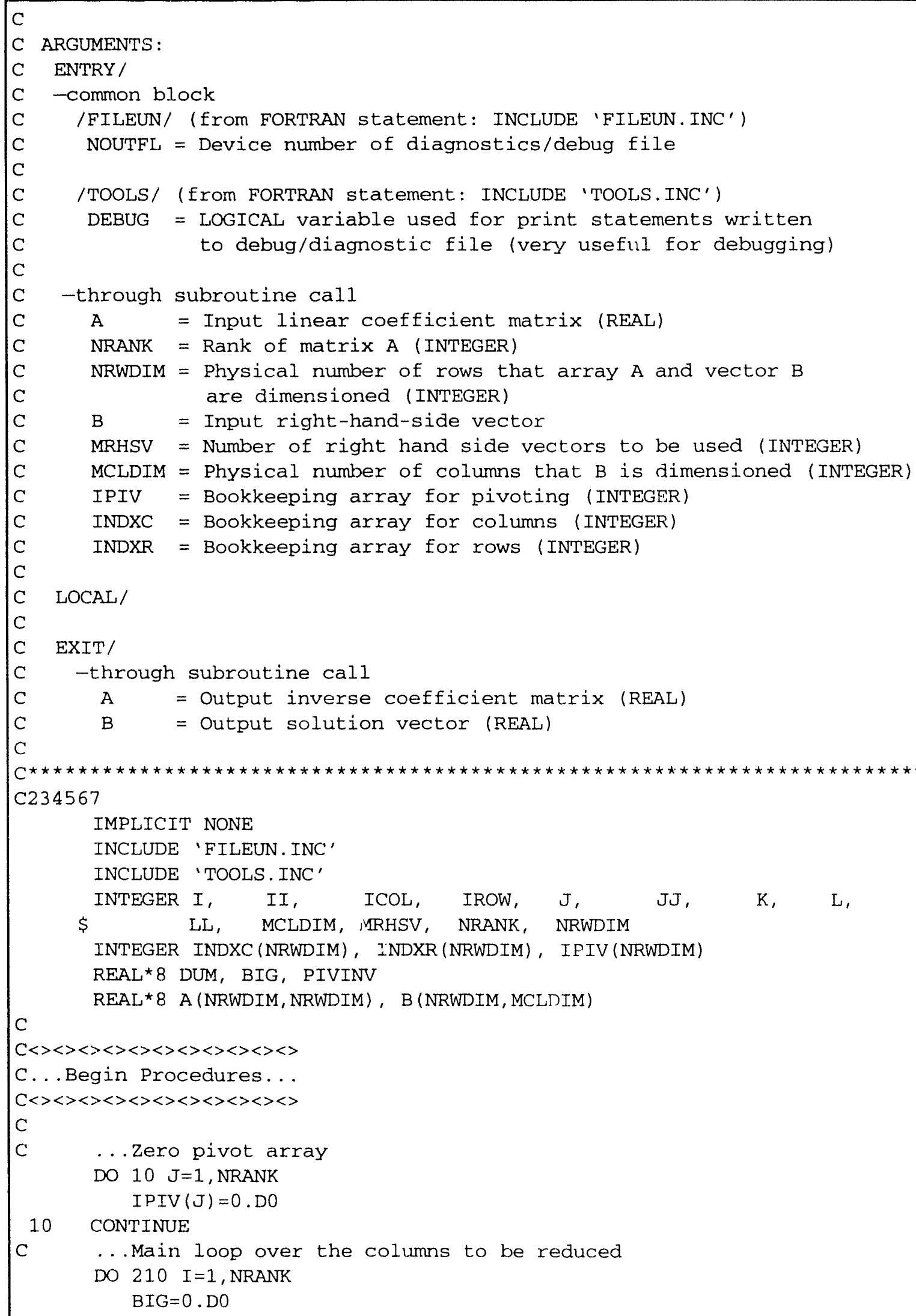




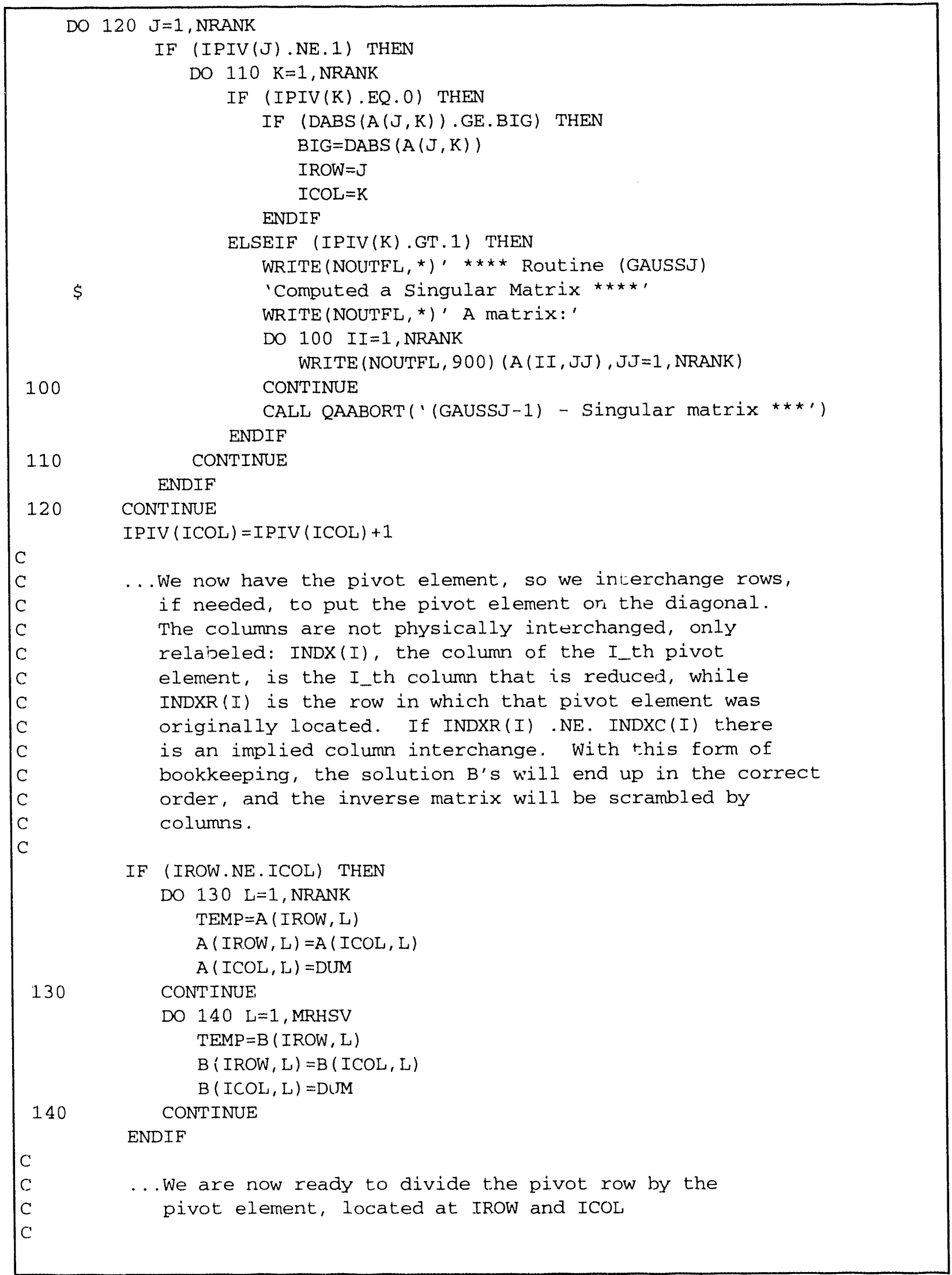




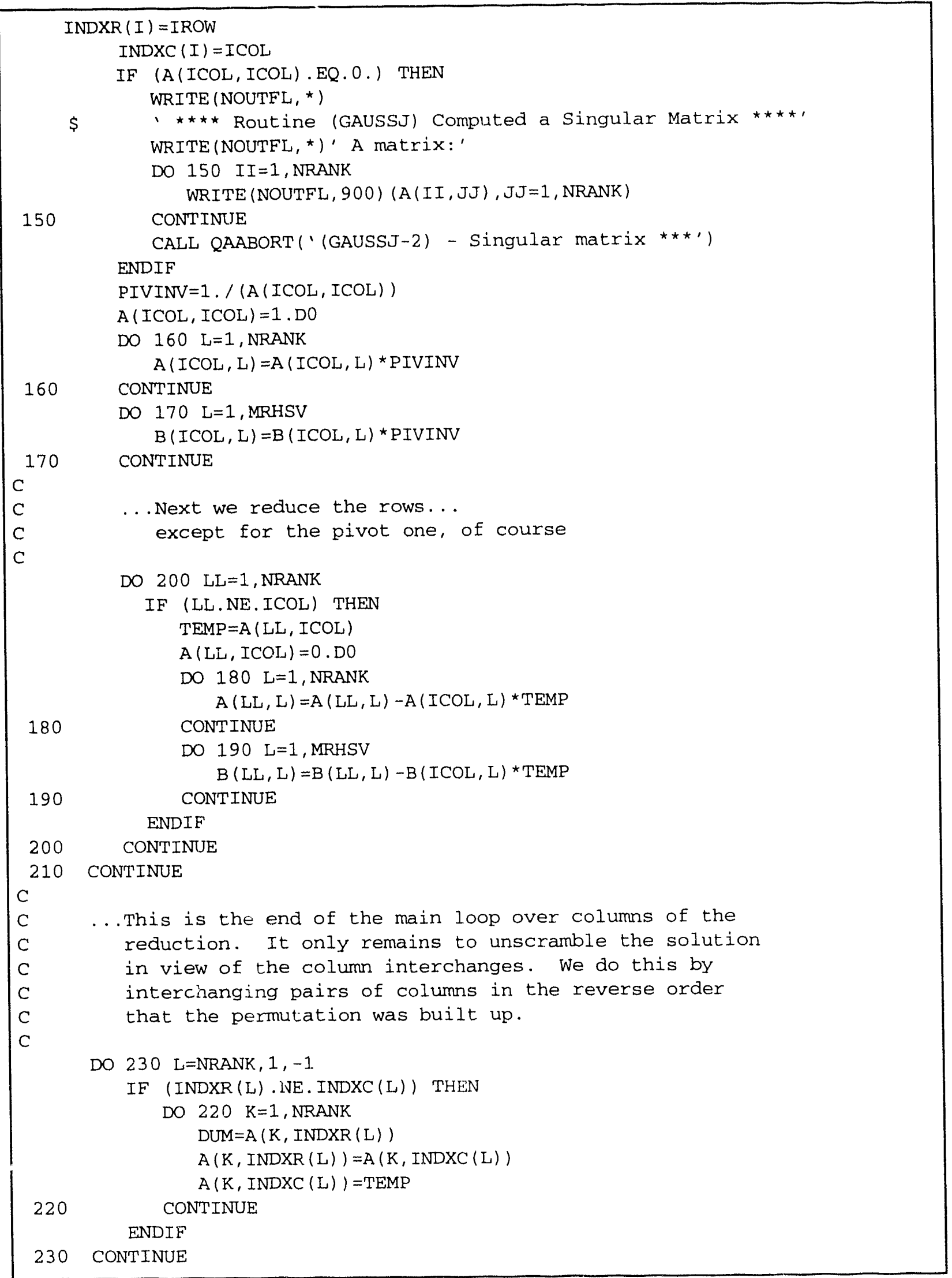




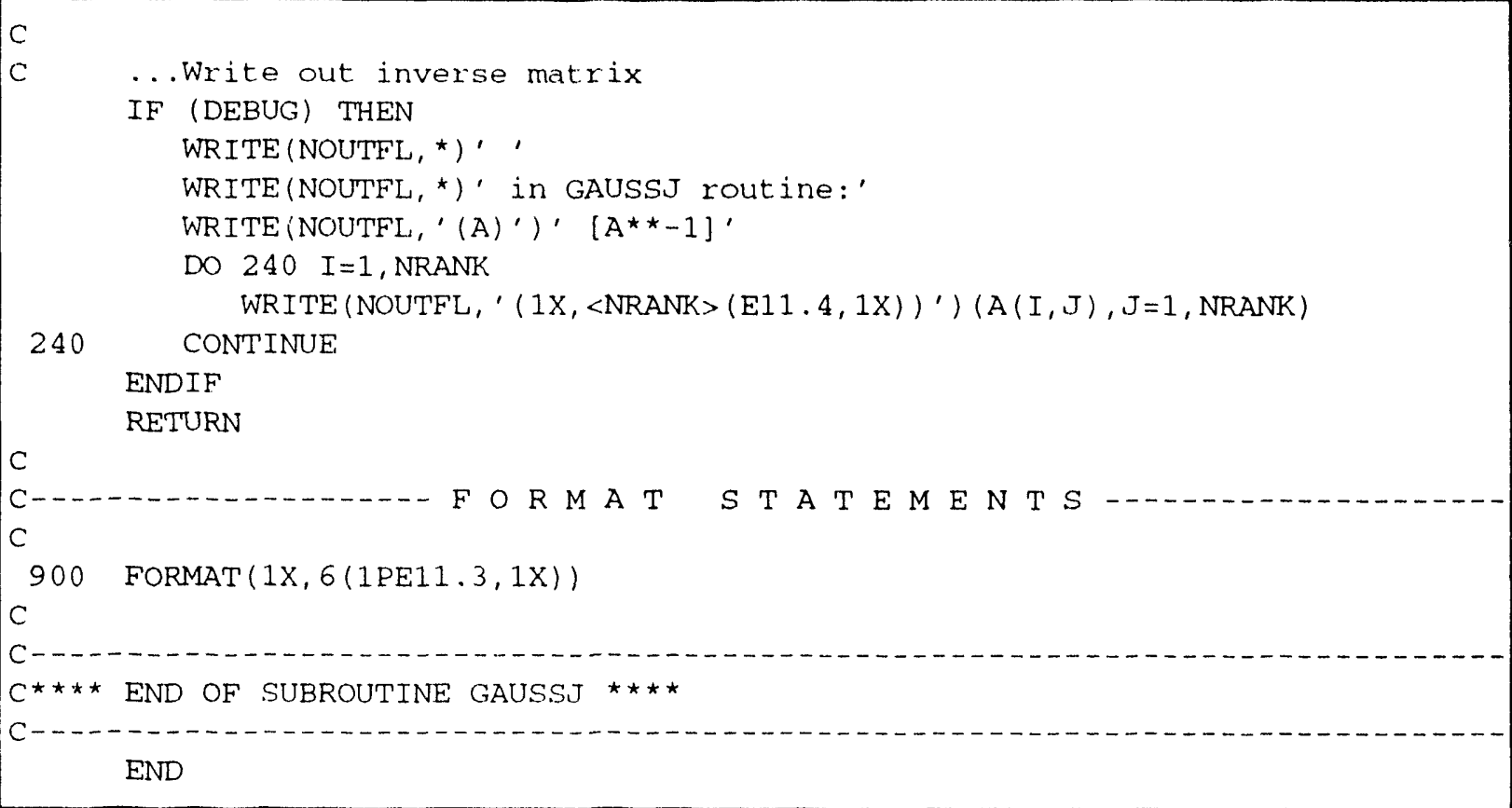

\subsection{Portability ố Codes}

- All FORTRAN code should be ANSI standard FORTRAN 77 unless nonstandard code is clearly desirable for readability.

- Use intrinsic library functions but reference by their generic names rather than their specific names. For example, use the generic name LOG in place of the specific name ALOG, DLOG, or CLOG, and the generic name SQRT in place of the specific name DSQRT or CSQRT. A table of generic function names and the corresponding specific names can be found in most FORTRAN manuals.

- Avoid character strings longer than 132 characters. Some compilers cannot process longer strings.

\subsubsection{Machine-Dependent Coding}

- All non-ANSI or processor-dependent coding should be clearly identified, either in the main program if generally applicable or in the header for a specific routine, and isolated in low-level routines if possible.

- Use the subroutines in the CAMCON_LIB and CAMSUPES_LIB libraries to perform common machine-specific functions (such as OPEN options, CPU time, time and date).

- Some computers (e.g. VAX) abort a program if an array in FORTRAN is dimensioned to zero elements. To prevent this problem, dimension an $\operatorname{ARRAY}(\mathrm{N})$ as $\operatorname{ARRAY}(\operatorname{MAX}(\mathrm{N}, 1))$.

- On the VAX, the CAMCON system version of ine code should be compiled with the NODEBUG /OPTIMIZE options and linked with the /NODEBUG/TRACE options. 


\subsection{Robustness}

- Software robustness: Coding that is less sensitive to misuse or error is an important consideration. Furthermore, the specific use of complex coding should be well documented. However, for calculation of complex, physical phenomena, nonlinear behavior must often be included and is a characteristic that can raise havoc when a programmer is trying to build a robust code. Consequently, a code that is not robust may fail to produce a solution for some parameters, but this lack of robustness is not a WIPP QA issue, provided the analyst is aware that he or she has not solved the problem for these conditions.

- Compare real variables using a tolerance test, not an equals (.EQ.) test unless testing for true zero or comparing integer values.

- Use vectorizable algorithms when possible.

- Initialize all variables with executable statements before use. Some machines do not zero memory prior to execution.

- Code numerical algorithms independently of physical units when possible.

- To prevent the program from aborting or printing meaningless error messages when printing a number that is too large for the FORMAT statement, include the "IOSTAT=IDUM" field in the WRITE statement.

\subsection{Efficiency}

- Keep coding simple to make it faster.

- Ensure that coding is correct and clear before trying to improve efficiency.

- Measure where code spends time before making decisions on efficiency.

- Search for a faster algorithm (or whole code), rather than tweaking code. The latter usually only makes it marginally faster while the former can make it orders-of-magnitude faster.

\subsection{Internal Documentation}

As has been stated, (e.g., Kernighan and Plauger, 1974, p. 117), the best internal documentation of a code is clear programming structure which follows the rules of the proceeding sections since the most reliable documentation of a program is the code itself. (Internal comments and external descriptions may differ from the code or be wrong, and thus be useless.) The following sections discuss first the minimum QA requirements, then examples and formats of enlightening comments embedded into the code and finally, the usual extensive descriptions added to the main program and subroutines to help future programmers more readily become familiar with the structure of a complex code. Internal comments can easily get out-of-date if a code undergoes continual revisions and require a personal commitment to keep the information accurate and up-to-date. 


\subsubsection{Minimum Internal Documentation}

As : minimum, all software developed at Sandia and classified as Class $\mathrm{A}$ or $\mathrm{C}$ will have internal documentation in the main program as listed below. ('This information will be added to external software for Classes $\mathrm{C}$ and $\mathrm{A}$ if the source code is available.)

- Software name and version identifier

- Brief description of software (similar to description on software abstract) indicating purpose, capabilities, and solution method(s)

- Documentation sources

- Name of author(s) and original source of software

- History of modifications, including name of modifier(s), version identifier, date and description of modification

- Government legal disclaimer if Sandia software, or proprietary information, if applicable

- Language and language extensions used

- Machine, operating system, and necessary supporting software, where operative

Much more internal documentation is desirable, and suggestions and guidelines are discussed more thoroughly in the following sections.

\subsubsection{Enlightening Comments}

- Write using structure, format, and meaningful labels and variable names rather than comment bad coding.

- Make sure romments agree with the code.

- Use extra spaces and good layout of coding on a page to convey meaning rather than relying solely on text comments.

- Only provide enlightening comments. Do not over-comment by highlighting the obvious to decrease the chance for discrepancies with actual coding. Although commenting a program before generating the code is a commendable practice (facilitates composition at the keyboard), make a check afterwards for comments highlighting the obvious.

- Avoid splitting short DO-loops and the block IF construct with comments.

\subsubsection{Main Program Headers}

Internal documentation at the beginning of the main program must contain the it $\epsilon$ ms required in the QA procedures: 
- Document software name, version identifier, name of authors, legal disclaimer, etc. (as described in Section 5.6.1, above).

Much more internal descriptive documentation at the beginning of the main program is also desirable.

- Document the meanings and types of variables.

- Document data storage in arrays, if practical.

- Document revisions made and date.

- Consider documenting the hierarchy of the subroutines (the tree structure output from FLINT [Chapter 12] can easily be copied into the code).

This last item is helpful for a programmer trying to modify an existing code, but the information is optional since it can usually be generated by the compiler or a FORTRAN code analyzer.

The following is an example of a header from the code MATSET:

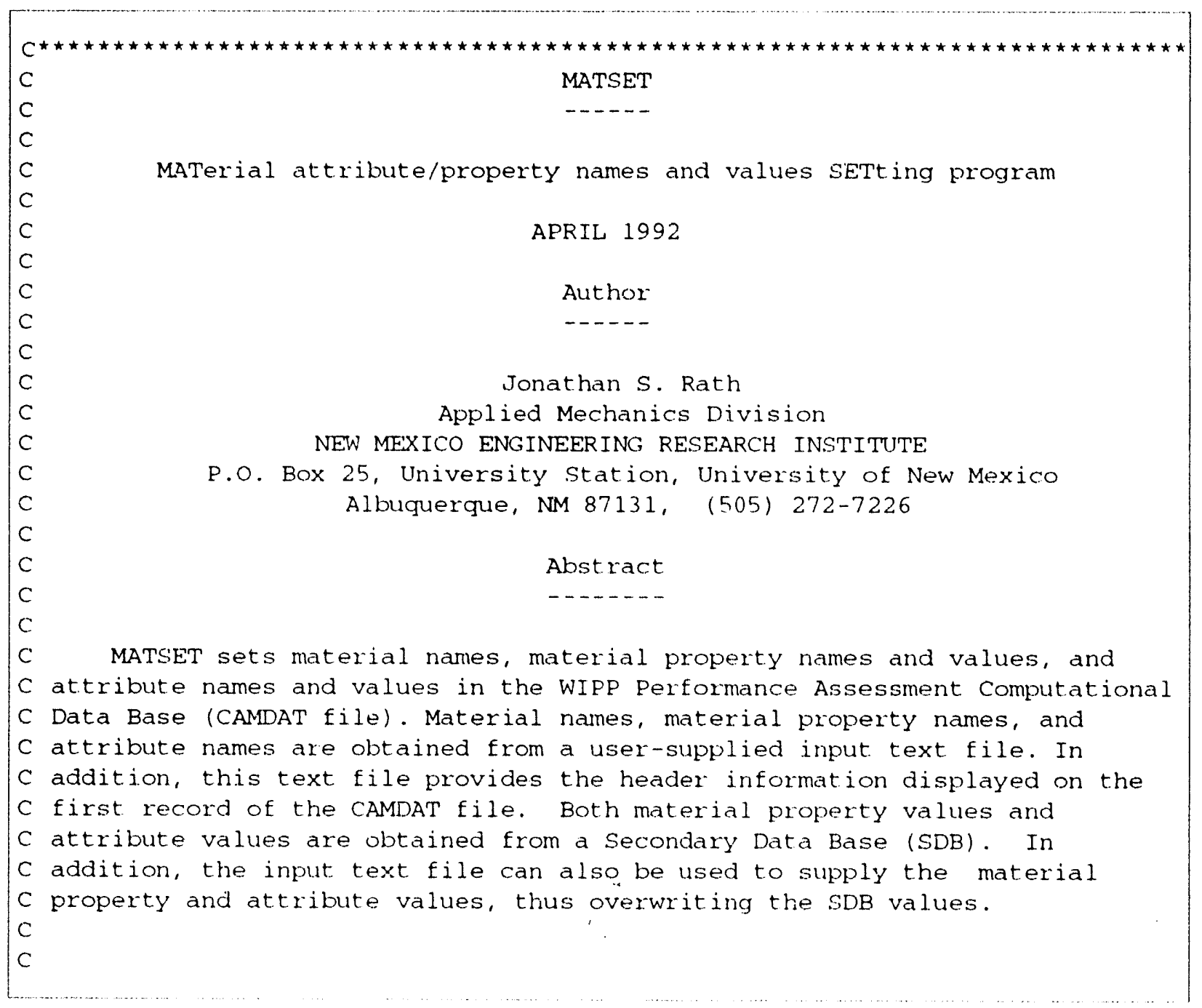




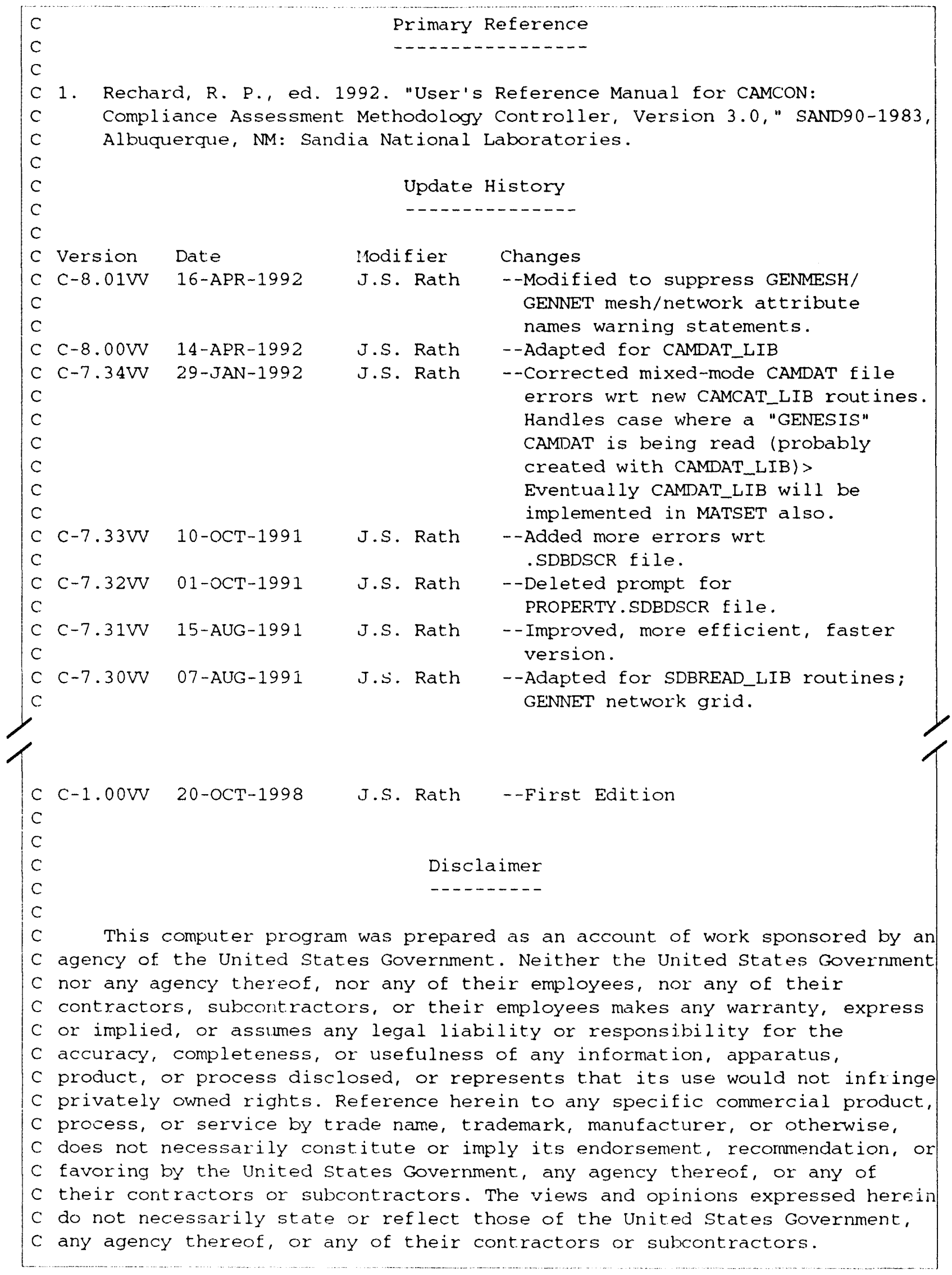




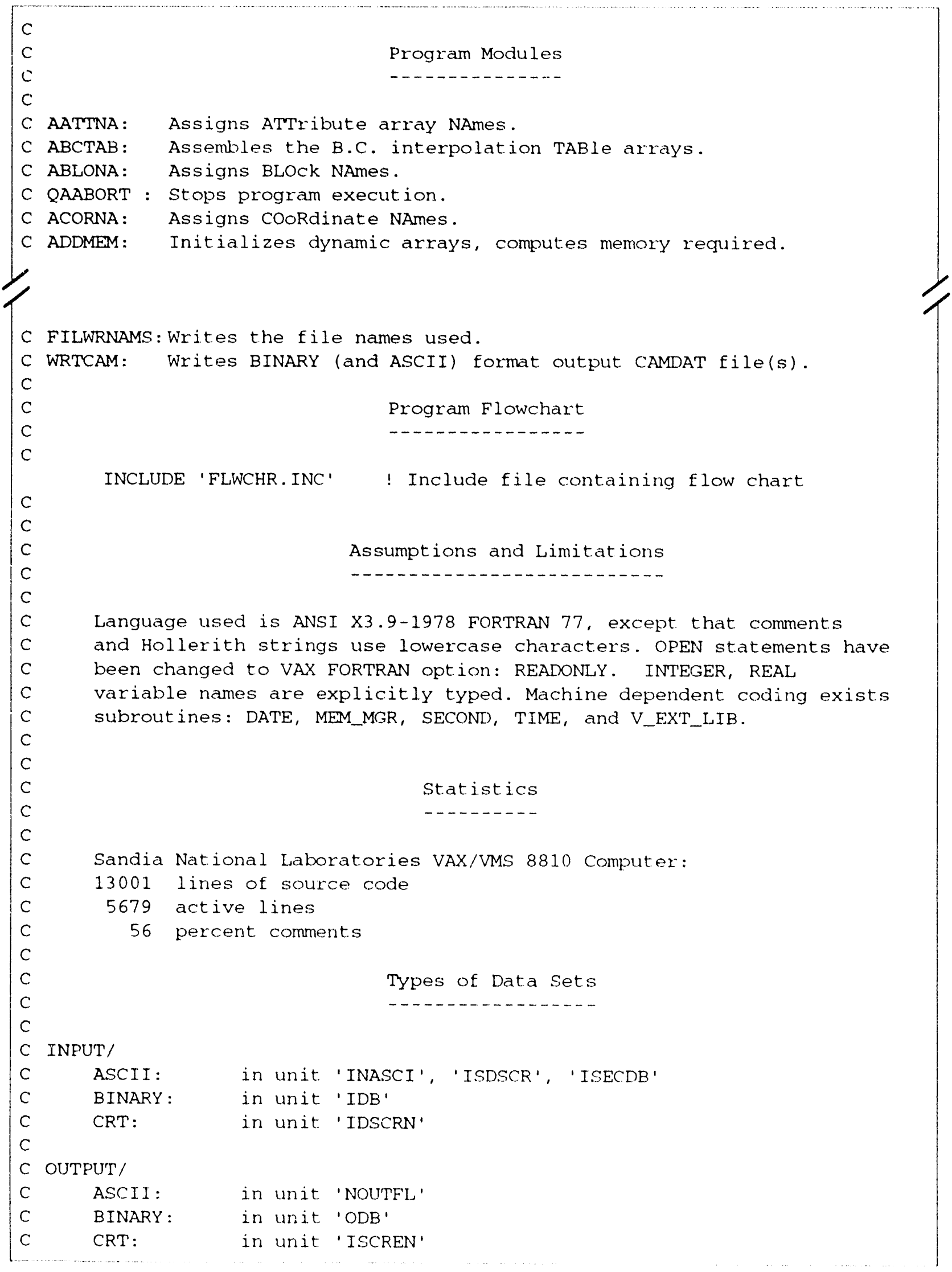




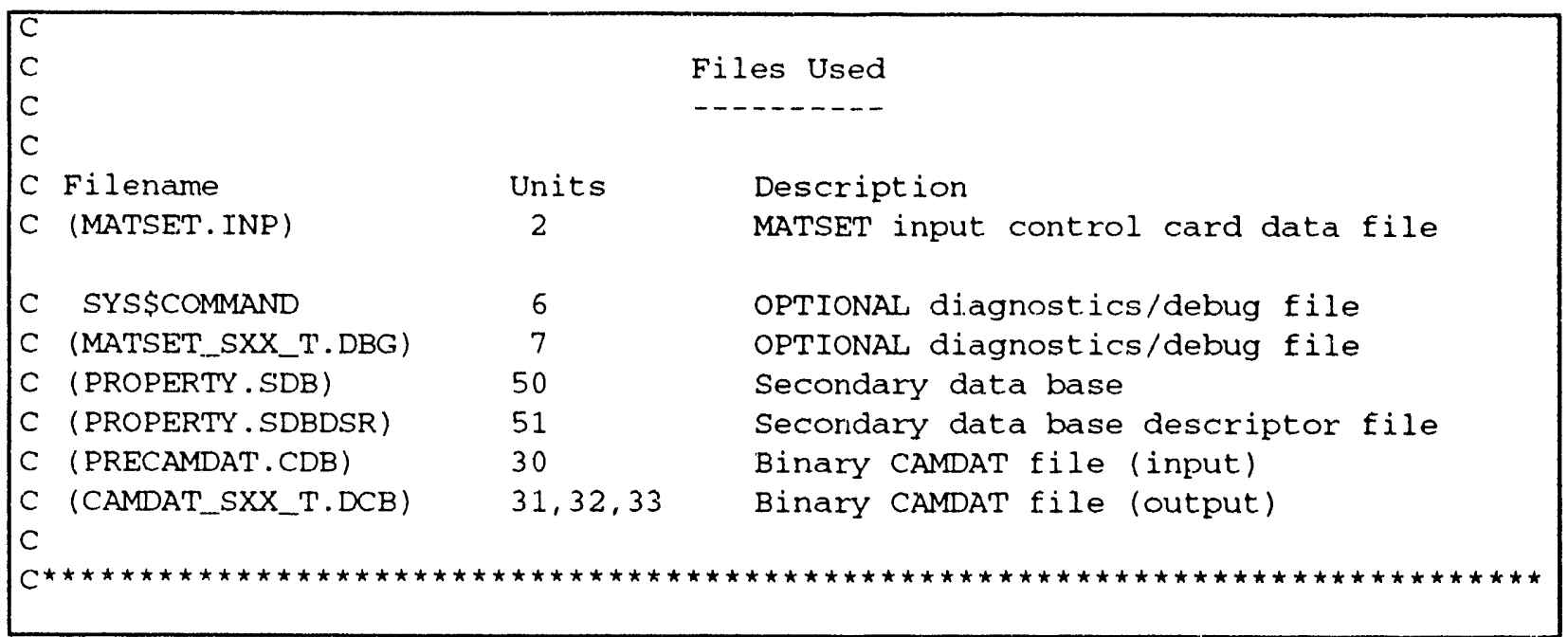

\subsubsection{Subroutine Headers}

Subroutine headers are similar to the descriptive header in the main program:

- Describe the function or purpose of the routine. This documentation should give a knowledgeable programmer enough information to use the routine without having to resort to deciphering the coding.

- Describe meaning and type of global variables used or modified (i.e., calling and common variables).

- Document author if different from the main program.

- Describe meaning and type of local variables.

- Document (by reference or otherwise) data storage in arrays.

- Document revisions made and date.

\subsubsection{Include File Headers}

Include files within the CAMCON environment are used as mechanisms for sharing information between FORTRAN files. Include files provide modularity by isolating key information and as such are a powerful tool for structural programming. The files should be named using "INC" as the extension (*.INC).

Headers of include files should provide descriptions of common blocks, variables, and arrays. These descriptions should be as detailed as explanations of these items found in the headers of FORTRAN files.

An example of an include file follows: 


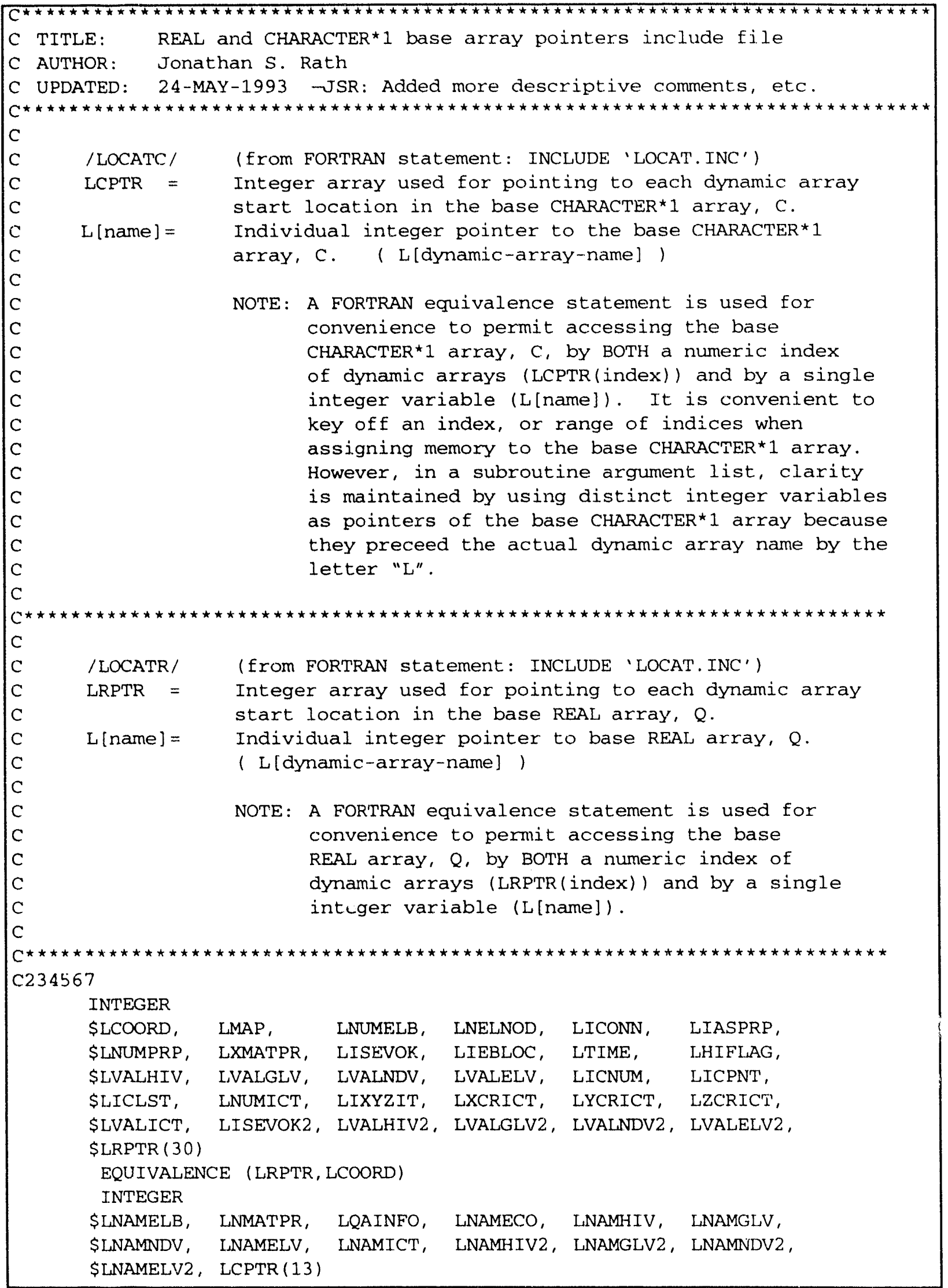




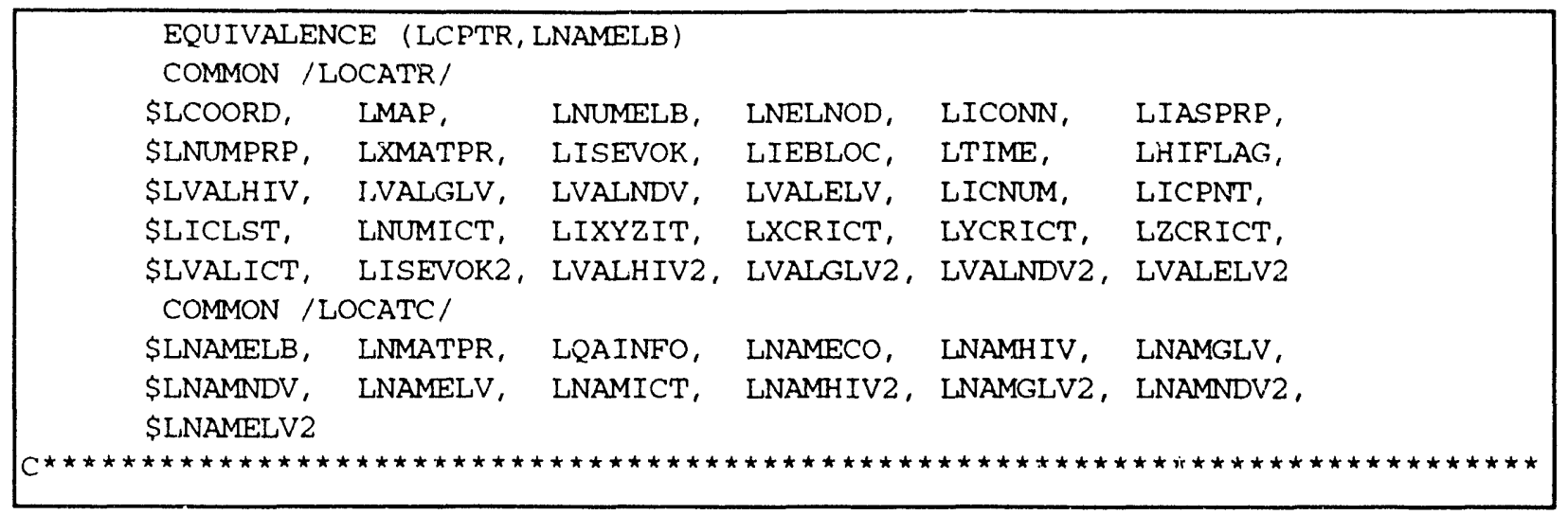

\subsubsection{Procedure Headers}

DCL procedure files should be named using the "COM" extension (*.COM). DCL headers are similar to subroutine headers and should include the following information:

- Describe the purpose of the procedure.

- Describe the usage of the procedure, including any parameters.

- Document the author of the procedure.

- Describe the meaning and type of global symbols used or modified.

- Comments should be delimited by using the standard VAX-DCL notation of $\$$ !.

The following is an example of a DCL procedure header:

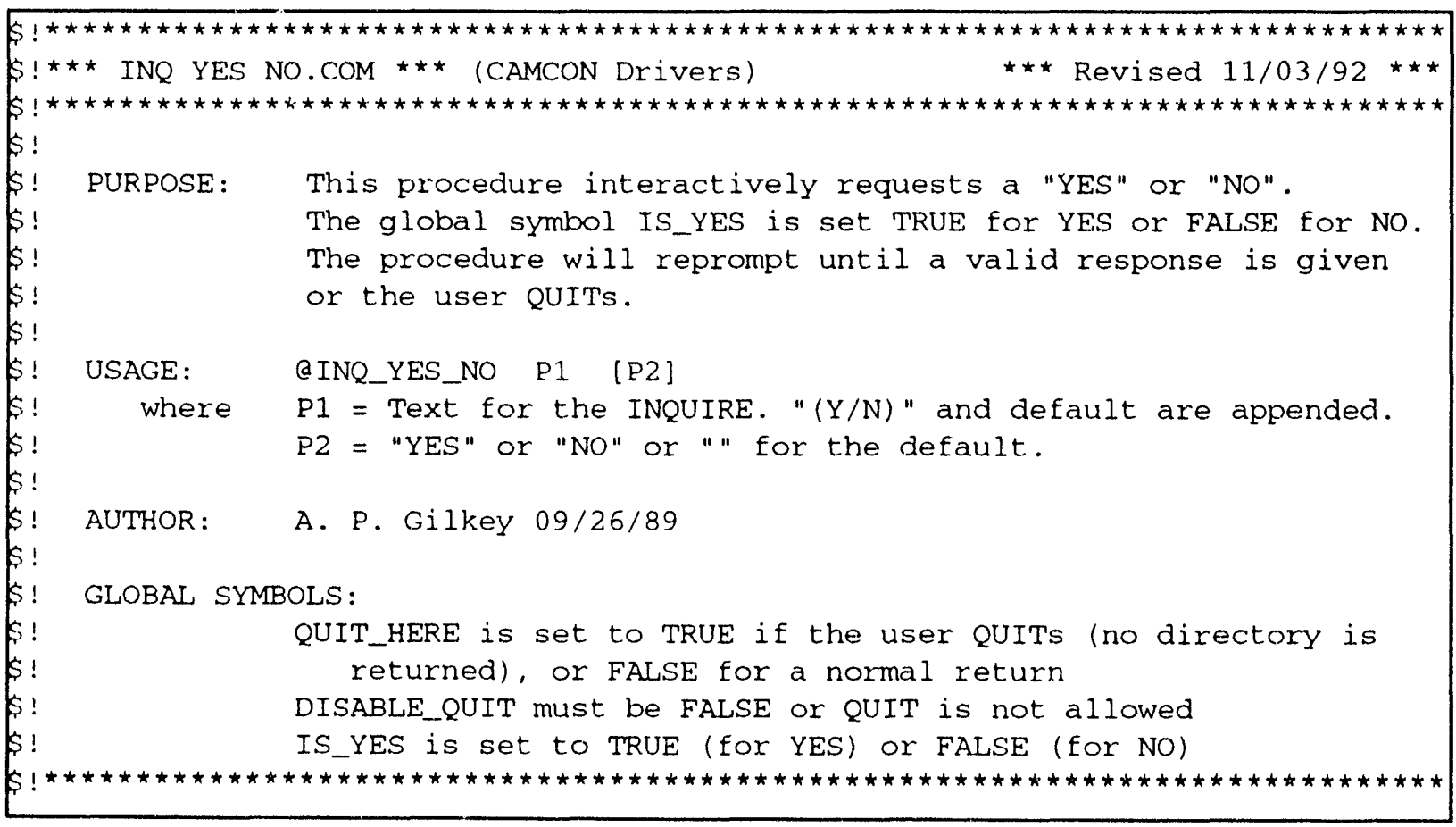




\subsection{Interfacing C Codes to the CAMCON System}

Occasionally, it is desirable to create utility modules using the ANSI C programming language when the increased capability to interface with the operating system warrants. The guidelines below are applicable to compiling and executing $\mathrm{C}$ codes on the WIPP VAX running VMS Version 5.4-3.

\subsubsection{Compilation}

Compile $\mathrm{C}$ code by typing at the system prompt $\mathbf{C C}$ cfile. A list of compiler options can be obtained by typing at the system prompt HELP CC.

\subsubsection{Linking}

Define the location of the $C$ run-time library, which can be found in SYS\$LIBRARY as \$DEFINE LNK\$LIBRARY SYS\$LIBRARY:VAXCRTL. This is currently done in CAMCON_SYMS.

Link the $\mathrm{C}$ code by typing at the system prompt LINK cfile.

Additional information can be found by typing at the system prompt HELP CC LINK.

\subsubsection{Mixing FORTRAN and $\mathrm{C}$}

VMS Version 5.4-3 supports mixed-language programming. VMS C is able to call or be called by routines written in other languages.

The details of passing variables are language dependent. When mixing FORTRAN and C,

- Use the VAXVMS-specific function, $\% \mathrm{REF}$, in the FORTRAN calling routine to pass variables by reference.

- Append a null character (i.e., CHAR(0)) on a string variable in the FORTRAN routine when this string variable will be passed to a $C$ routine.

- Remove null characters from a string variable passed from a $\mathrm{C}$ routine to a FORTRAN routine.

As an example, the following shows how the CAMCON.LIB FORTRAN routine, EXFILDAT (which passes the date/time stamp of a file) calls a $\mathrm{C}$ routine, CXFILDAT:

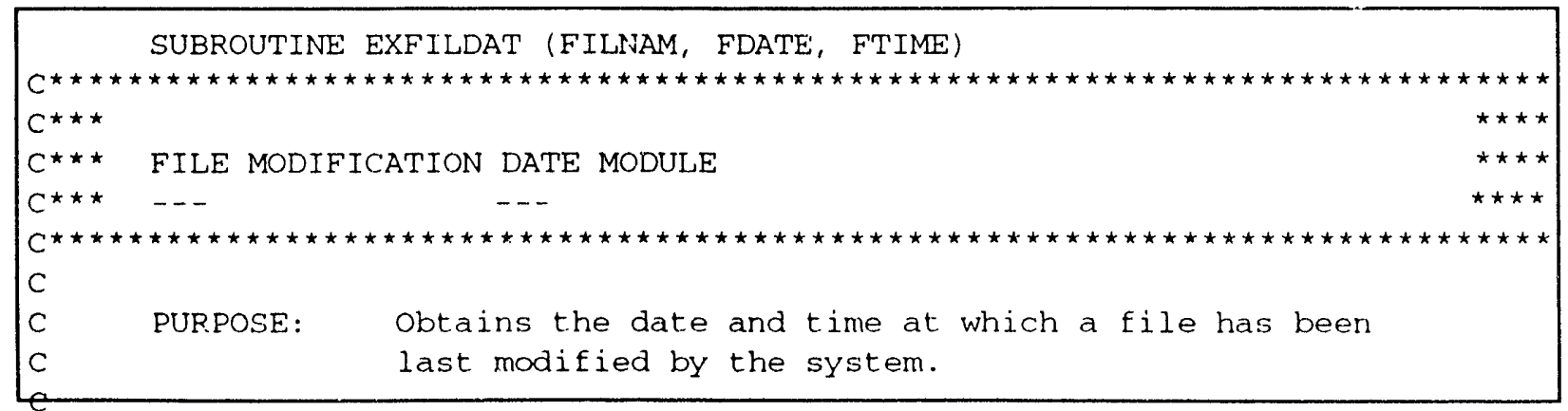




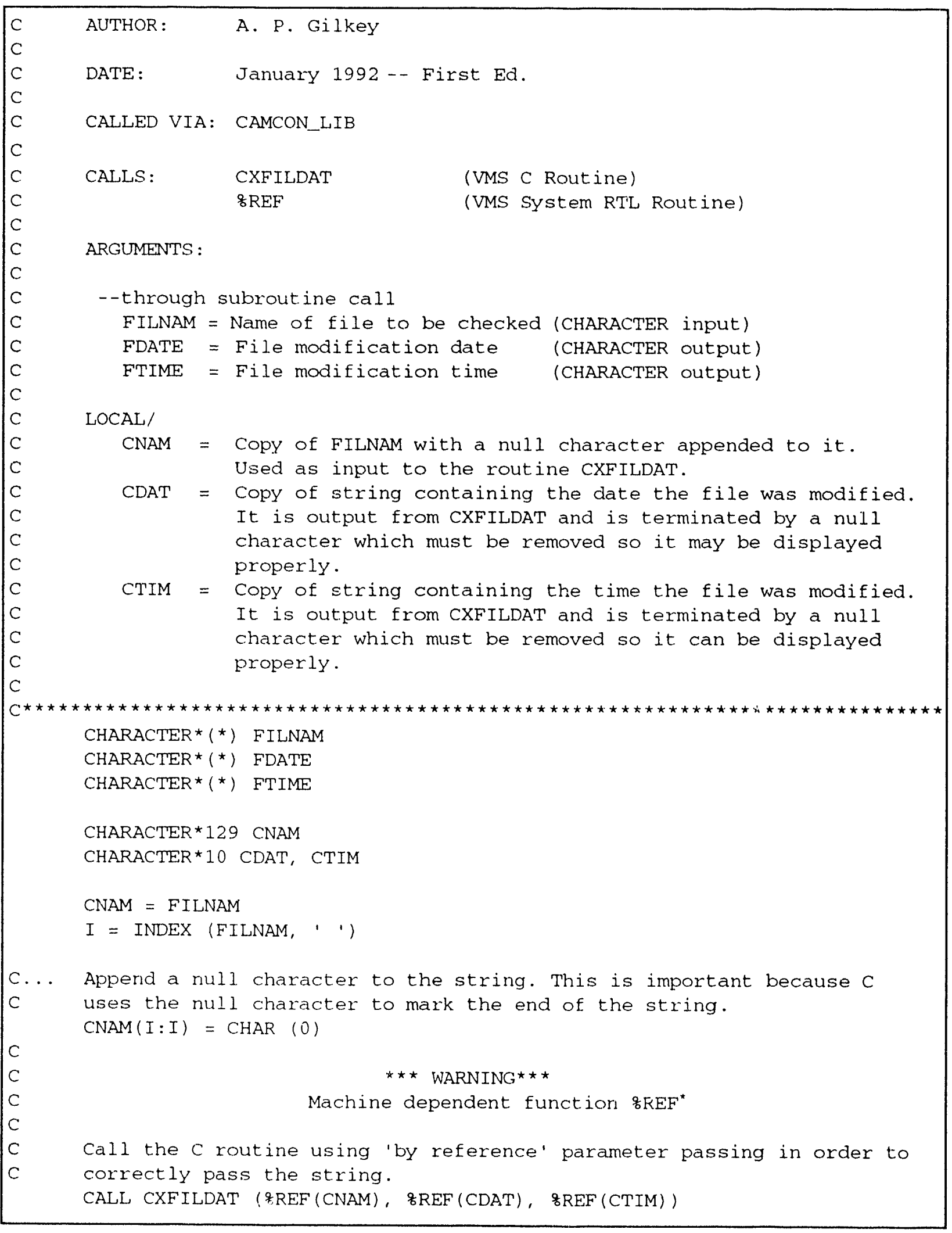

\footnotetext{
- See SDBREAD_LIB for a generic suhroutine that places this machine-dependent function in a low-level routine.
} 


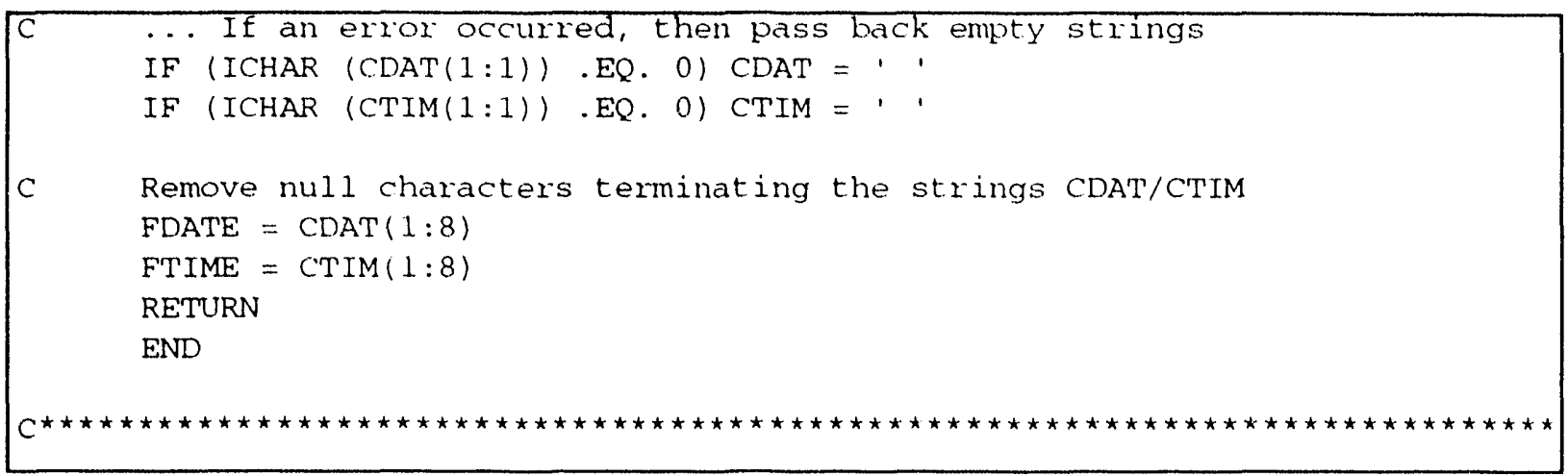


General Programming Guidiclines

Interfacing C Codes to the CAMCOiv System 


\section{CAMDAT_LIB: \\ Computational Data Base Interface Library}

This chapter describes the calls to the routines that read and write data to a computational data base (CAMDAT) neutral file, briefly discusses the history behind the selection of the CAMDAT file format, and describes suggested ways to use the CAMDAT neutral file subroutines to help the developer understand what can and cannot be done.

\subsection{Background on the Selected Format}

Storage of input and output requires a versatile file format that has been extensively tested. Although some projects (e.g., evaluation of transport contaminants or water rights disputes) have had some success with using a GIS package as the data base for storing and displaying results, WIPP PA rejected this approach because at the time

- Fast access (though not necessarily through a direct access file) directly to the data as stored (rather than through ASCII files) was essential since each file was projected to be as large as $5 \mathrm{MB}$ ( 10,000 blocks) (and one complete simulation might involve thousands of these files).

- Plotting capabilities, though extensive in GIS packages, did not have all the necessary capabilities, and because source code was unavailable, it limited the opportunity for modification.

- Adding arrays with a potentially large dimension to store time step information was awk ward and could hamper the usefulness.

The original file format chosen for CAMDAT was based on GENESIS (Taylor et al., 1987) and EXODUS (Mills-Curran etal., 1988), developed by Sandia National Laboratories, Engineering Analysis Department, for finite-element computer codes. This file format was originally chosen for the following reasons:

- It had demonstrated versatility in handling fluid and solid mechanics codes, multiple element types, and various boundary conditions.

- It could be easily modified to store both finite-element and finite-difference meshes and results.

- The file could be easily modified to store material properties.

- It stored data as unformatted binary for space efficiency.

- It had a file structure that defined the length of all records before the record was read, implying that the reading program could dynamically allocate memory for all arrays.

- It had powerful data manipulation and plotting codes already available (e.g., Gilkey, 1988).

A potential drawback was that it had a sequential file format, which, while simple and easy to use for programmers, made reading and writing a large data base file potentially cumbersome. 
Initial changes necessary to this file format were numerous and included storage of material properties, changing the storage of boundary condition information, expansion of QA information stored, and naming all entities stored.

Although the initial file format served the project well, ever-increasing types of information have needed to he stored. Hence, he data base is now accessed through a set of subroutines. The standard interface permits the sponsor of the data base file to change the structure to meet these changing needs and yet not significantly affect the operation of individual codes.

\subsection{Correspondence Between Generic Computational Data Base and Data Entities in CAMDAT File}

As mentioned previously and repeated here, basically three types of information are stored in a CAMDAT neutral file (Figure 6-1):

- Quality assurance information

- Model description data, such as finite-element or finite-difference geometry data; geologic, hydrologic, and chemical parameters; and model boundary conditions

- Analysis results data, such as fluid flow results, concentrations from source term or transport modules, and particle pathways

The correspondence of this general-to-specific information is discussed below and shown in Figure 6-1. The correspondence between the material property section and CAMDAT deserves special mention. When discussing parameters (or properties) in CAMDAT, we make a subtle distinction between two types of parameters: "material" and "attribute."

Material parameters Parameters or characteristics that vary only with material regions or not at all are termed material parameters or properties. These material parameters are defined for each material region of the mesh. Typical examples include material partition coefficient $\left(K_{d}\right)$ and solid density $\left(\rho_{s}\right)$.

Attribute parameters Parameters that have a rough characteristic range for each material but vary spatially and are thus different at each element in the computational mesh are termed attrib ute parameters. ('The properties are attributes of each element of the mesh.) Typical examples include hydraulic transmissivity, matrix porosity, and bulk density $\left(\rho_{\mathrm{b}}\right)$.

Although not all the computational codes recognize the same division of parameters, storage within the CAMDAT files is consistent and a translation from one type of description to the other is made in the preprocessor for the computational model (computer code). 


\section{Generic \\ Computational \\ Data Base File}

\section{CAMDAT \\ File Data Entities}
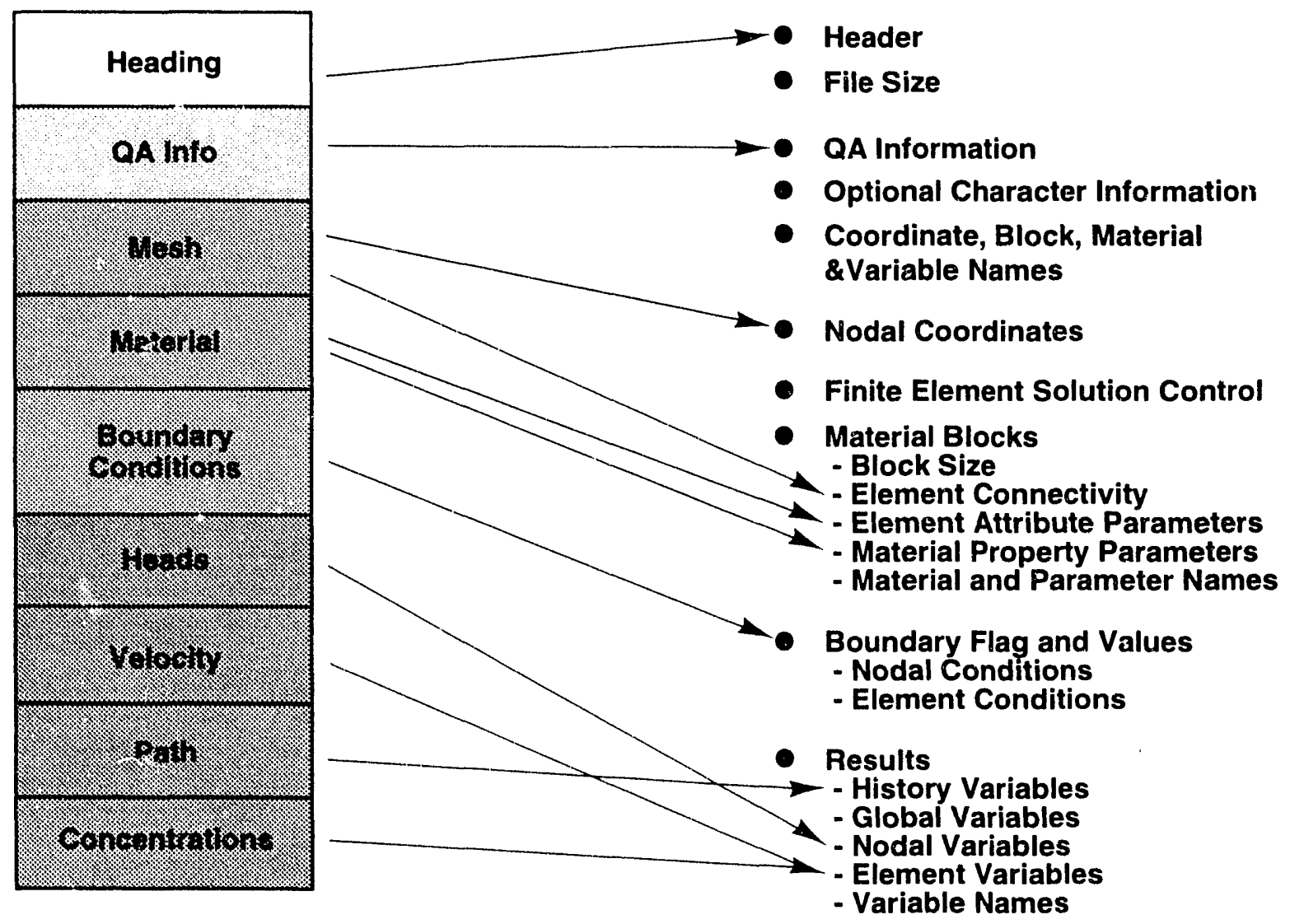

TRI-6334-44-1

Figure 6-1. Correspondence between generic computational data base file and CAMDAT neutral file entities. 
The following description of CAMDAT reiterates information pertinent to the WIPP project reported by Taylor et al. (1986) and Mills-Curran et al. (1988) concerning the GENESIS and EXODUS data base after which the computational data base is designed. The description uses the terminology of finite elements. For example, although the definition of the term "nodal point" changes between FE or nodecentered FD and body-centered FD analyses, a node is always described in FE analysis to be consistent.

\subsubsection{Heading}

The heading is an 80-character string placed on all printouts and plots from the computational data base. For the compliance evaluation, the following four groups of information are usually recorded on this line:

1. TITLE - descriptive, but brief title for stored data (CHARACTER*53)

2. IDSCNR - succinct scenario ID (CHARACTER*8)

3. SCLMTH - scale of the computer model (CHARACTER*8); choices are REGIONAL, SOURCE, LOCAL, or CUTTINGS

4. SIMRUN - the simulation run number (CHARACTER*8)

\subsubsection{Quality Assurance Information}

The first information necessary for this block of data is the number of quality assurance records (NQAREC)-a value which changes each time a code writes to the file. Following this information are the NQAREC arrays storing the eight-character name, version, date and time of all codes that write to the file (e.g., ALGEBRA C-3.11VV, 10/1/91, 09:30:00). This information is important for assuring that the calculations can be repeated-an important function of quality assurance procedures. Along with the HEADER information, the name, version, and date of each code that writes to the file are printed on all plots or printouts of data from the data base file.

\subsubsection{Optional Text}

CAMDAT can also store any number of records of optional text. The first item of information is the number of optional records (NXINFO). Following this item are the NXINFO records of optional text as CHARACTER $* 80$.

For the WIPP compliance evaluation, the random seed, SEED, (but stored as a character variable) used for the Mcnte Carlo sampling is stored as optional text.

In addition, for the WIPP, the translators for finite difference codes frequently require the number of elements (computational cells or grid blocks) in the $\mathrm{x}, \mathrm{y}$, and $\mathrm{z}$ directions. Although the information 
could be computed each time, the information (NX, NY, and NZ) is also stored here as character variables and then converted to numerical data.

Finally, an optional text record states the units used throughout the computations.

\subsubsection{Data Base Version and Model Size}

The data base version number defines the structure of the data base. Programs accessing the data file should abort if the data base version number is not compatible with the assumed version number in the code.

The following parameters define the size of the model:

NUMNOD - number of nodes (either FE or FD)

NDIM - number of coordinates (spatial dimensions) per node

NUMEL - total number of elements, including all element types

NELBLK - number of element blocks (for WIPP, NELBLK is the number of different material regions)

\subsubsection{Nodal Point Coordinates}

The mesh information is stored as nodal point coordinates $x, y, z$. The labels for the $x, y, z$-component must be specified.

\subsubsection{Element Map}

The element map contains the finite-difference element number for each CAMDAT element number. CAMDAT element numbering is explained in Section 6.2.7, and finite-difference element numbering is depicted in Figure 6-2.

\subsubsection{Material Block Information}

Element (or grid block) data are stored in blocks or groups. The block data refer to groups of elements representing different types of material or layers of strata surrounding the repository. Each block has the same material and element type. Grouping elements with similar material and element type is more space-efficient and permits vector processing of each element block. The local and global numbers of an element are implicitly defined by the order in which they appear in the file. Elements are globally numbered consecutively across all element blocks in the order the element blocks appear in the file. 
CAMDAT_LIB

Correspondence between Generic Computational Data Base and Data Entities in CAMDAT File

Element Block IDs and Names. Each block contains an integer block ID and a block name. By assigning a unique ID to the blocks, an analysis code can decipher the order in which materials or layers are stored in the computational data base.

Element Connectivity for a Material Block. For FE analysis, the nodes connected together to form each element are stored in the file. All elements in a material block must be of the same element type (i.e., contain the same number of nodes). No convention is specified in the data base, which implies that the codes must agree on conventions. For the codes used at Sandia, a counterclock wise ordering of nodes is used to define the connectivity of elements. In addition, for three-dimensional analysis, nodes are numbered first with increasing $x$, then increasing $y$, then $z$.

For FD analysis, the connectivity is unnecessary; however, to allow a general FE plotting package to display results for both FE and FD analysis (so that an analyst has to learn and use only one plotting package), a connectivity array is necessary for FD results as well, but the array is easy to generate.

Although not limited by CAMDAT, the node and element numbering used for WIPP FD grids is shown in Figure 6-2.

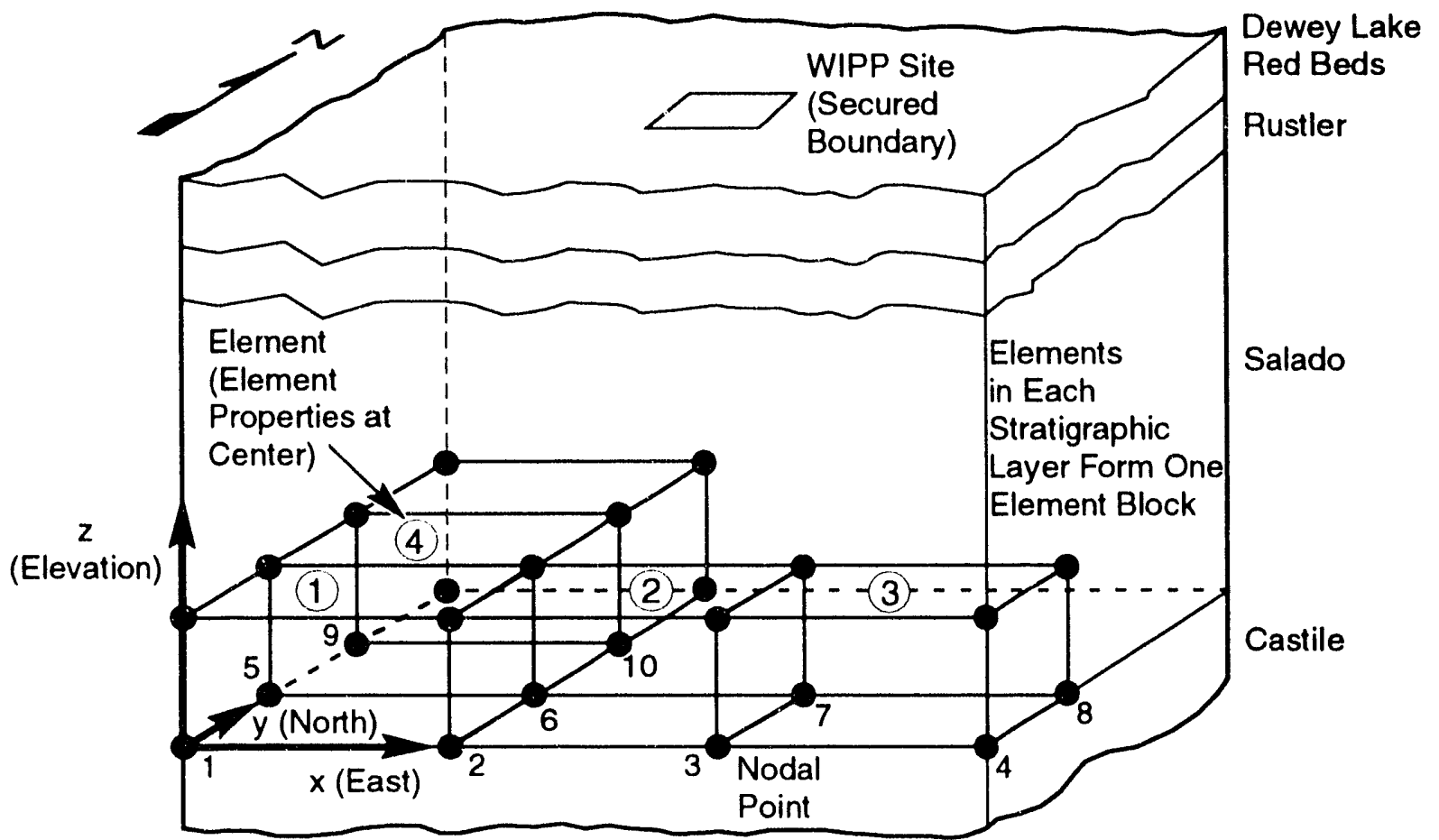

Figure 6-2. Storage of WIPP finite-difference mesh in a CAMDAT file. 
Attribute Parameters for each Element. Although some material properties are stored as block information, many are stored as element attributes to accommodate subtle changes of parameters within the same stratigraphic layer, if necessary. The attribute array stores information that varies with each element (e.g., transmissivity, porosity, brine density). Each attribute parameter has a name for identification. A particular attribute need not be defined in all material blocks.

Material Parameters for Material (Element) Blocks. The material parameterarray stores information pertaining to properties that vary only with each material (element) block (e.g., rock density, nuclide partition coefficients). Each material parameter has a name for identification. A particular material parameter need not be detined in all material blocks.

\subsubsection{Boundary Condition Data}

The file format can store boundary condition information (e.g., load or fluid pressure conditions) in two ways:

- Node sets

- Element side sets

The following parameters define the size of all boundary condition information:

NUMNPS - number of node sets

LNPSNL - length of the node sets node list

NUMESS - number of element side sets

LGELEL - length of the element side sets element list

LGNODL - length of the element side sets node list

Node Sets. A node set refers to a set of nodes (one or many) that has been identified with a unique ID (flag). Although a particular node may appear in any number of node sets, a node should appear only once in each node set. Each node in a set is assigned an individual factor (e.g., pressure or flux). All node sets are concatenated into one list; similarly, distribution factors for all nodes are concatenated. Consequently, besides the IDs, the node set lists and distribution factors; a pointer list and a counter list are required. Their descriptions follow:

- IDs AND NAMES FOR EACH NODE SET. Each node set has an ID (flag) and an eight-character identifier. Similarly to the material block ID, the node set ID is a unique integer label assigned by the user. The IDs are in an integer array of length NUMNPS. The names are in a CHARACTER*8 array of length NUMNPS.

- NUMBER OF NODES IN EACH NODE SET. The number of nodes in each node set is contained in an integer array of length NUMNPS. 
- POINTERS FOR LOCATING EACH NODE SET. An index for each node set which locates the beginning of the node set within the concatenated node list described below is contained in an integer array of length NUMNPS.

- CONCATENATED LIST OF NODES. The lists of nodes for all sets is concatenated into a single list. The count and pointer lists mentioned above are used to identify the nodes in any one particular node set. The concatenated list is contained in an integer array of length LNPSNL.

- FACTORS CORRESPONDING TO EACH NODE. Each node in the concatenated node list has a corresponding nodal factor stored in a similar concatenated list. Depending on how the analysis program interprets the data, these factors prescribe either the actual constraint conditions (e.g., pressure or flux) or the spatial distributions of the constraints (interpreted as multiplication factors in the latier case). The concatenated factor list is contained in a real array of length LNPSNL.

Element Side Sets. An element side set refers to a set of elements identified with a unique ID. This feature is useful for surface boundary conditions (e.g., heat or fluid flux).

Similarly to node sets, an element side should appear only once in each element side set. However, the element number may appear more than once within a side set if more than one side of an element is part of the set. Further, the nodes associated with a side set will generally appear several times within that side set's node list. Likewise, each side in a set is assigned an individual factor (e.g., flux). As before, all side sets are concatenated into one list; in the same way, the factors for all side sets are concatenated.

Consequently, the required information for storing element side sets is similar to that for storing node data (IDs, counts, pointers, nodal list, factor list) except that both the element side and the element side's associated nodes must be stored. Eight data components exist: one ID list, two count lists, two pointer lists, one element side list, one nodal list, and one factor list. Their descriptions follow.

For the WIPP, the finite element codes use arrays as described. The finite-difference codes need only a few of the arrays because nodes associated with an element are easily deciphered, since the mesh connectivity is regular. The requirements for element side sets follow:

- IDs AND NAMES FOR EACH ELEMENT SIDE SET. Each element side set has an ID (flag) and an eight-character identifier. The IDs are in an integer array of length NUMESS. The names are in a CHARACTER* 8 array of length NUMESS.

- NUMBER OF ELEMENT SIDES IN EACH ELEMENT SIDE SET. The number of element sides in each side set is contained in an integer array of length NUMESS. Similarly to node point sets, an element side should appear only once in each element side set.

- NUMBER OF NODES ASSOCIATED WITH EACH ELEMENT SIDE SET. The total number of element side nodes in each side set is contained in an integer array of length NUMESS. The number of nodes per element side is implied by the type of element (e.g., quadrilateral). 
- POINTERS FOR LOCATING ELEMENTS ASSOCIATED WITH SIDE SET. An index for locating each set of elements comprising the element side set within the concatenated list of elements for the side sets is contained in an integer array of length NUMESS.

- POINTERS FOR LOCATING NODES ASSOCIATED WITH SIDE SET. An index to locate the set of nodes associated with each side set within the concatenated list of all nodes is contained in an integer array of length NUMESS.

- CONCATENATED LIST OF ELEMENTS ENCOMPASSING ALL SIDE SETS. A concatenated list of elements encompassing all side sets is contained in an integer array of length LGELEL.

- CONCATENATED LIST OF NODES ENCOMPASSING ALL SIDE SETS. A concatenated list of nodes encompassing all side sets is contained in an integer array of length LGNODL.

- CONCATENATED LIST OF FACTORS FOR ELEMENTS COMPRISING SIDE SETS. Each element in the side sets has a corresponding factor. The factor list corresponds one-for-one to the concatenated list of elements (two bullets above); consequently the length of the real array is LGELEL. To maintain compatibility with the EXODUS data base (Mills-Curran et al., 1988), the array is padded with zeros to length of LGNODL.

\subsubsection{Results}

The final segment of the file are the results. This section of the file can vary greatly; hence, dynamic memory allocation is especially useful for reading element data. The variables are separated into two general classes: variables output at every time step ("history" variables) and variables output at designated time steps. This grouping allows an analyst to reduce the size of the output file. Variables output at designated time steps are further subdivided into three classes: global, element, and nodal. Specifically,

- History - output at every time step

- Global )

- Element $\}$ output at designated time steps

- Nodal $\}$

History and global variables refer to values of physical properties that apply to the large regions or the entire analysis, such as total energy or mass of the system. These properties can sometimes help indicate the accuracy of the analysis by checking whether energy or mass is conserved during the calculations.

Element variables refer to values of physical properties that apply to the finite element or the finite difference computational cell (grid block). An element variable may not be defined for every element block. The "element variable truth table" has an entry for each element variable, which consists of a logical value for each element block. The value is true if and only if the variable is defined in that element block. Most codes used in the WIPP performance assessment define each element variable for all element blocks.

Nodal variables refer to values of physical properties that apply to the computational FE or FD node. 


\title{
6.3 General Guidelines on the Use of CAMDAT_LIB Routines
}

\subsubsection{Sponsorship and Version}

\author{
Author/Sponsor: A. P. Gilkey \\ Version/Date: $\quad$ C-1.10VV/August 1992 \\ Language: $\quad$ FORTRAN 77
}

\subsubsection{Overview of CAMDAT_LIB}

The CAMDAT_LIB routines handle all computational data base (CDB) neutral file input and output. A data base neutral file can be accessed only through these routines; the CDB neutral file should not be accessed directly. The routine DBSETUP must be called to initialize the library before any other CAMDAT_LIB routines are called.

When creating or reading a CAMDAT file, the file must first be opened. The data base can be opened as an input data base or an output data base, but not both. The open routine requires a unit number, which is used in all subsequent routine calls to identify the data base file.

After the open routine is invoked, the routines that read or write (1) the QA information and (2) model description can be called in any order. However, these routines must be called before any routines that process the analysis results. Furthermore, for efficiency, all analysis results needed for a particular time step should be processed before proceeding to the next time step.

The CAMDAT_LIB routines manipulate the neutral file entities logically with little regard to how the data base file is organized physically. For example, the attribute names and values are handled as a logical item, even though currently the attribute values are stored with the property values and the attribute names are stored later with other names.

Output Data Base. An output data base file can be one of two kinds: a new data base being generated or a connected data base. All data base entities on a new data base must be specified fully with calls to data base output (DBO) routines (routines whose names begin with $\mathrm{DBO}$ ). If a particular data base entity does not exist, no action is necessary. For example, if there are no node sets, routine DBONDSET need not be called.

To modify an existing data base, an input data base can be connected to an output data base when the output data base is opened. The user must call data base output(DBO) routines only to specify items that are to be changed. By default, all QA and model description information is copied. No analysis results are copied unless requested. For example, to copy the QA and model description data with an added QA record, the user would call routines to perform the following tasks: open the input data base, open the output data base (connecting it to the input data base), add the QA record, then close the output data base. 
There are some restrictions on the order in which DBO routines can be called. The QA and model description routines can be called in any order, but the DBOHEAD rotitine must be called after these routines and before any analysis results routines are called. The first step in processing the analysis results is to define the types and names of all variables to be output. All the variables must be specified fully for the current time step before advancing to the next time step.

Input Data Base. When processing an input data base, the user need only retrieve information that will be used by the program. This does not include information that needs to be copied to a connected output data base; this occurs autornatically. When the data base is opened, it is scanned for all the sizing information that might be needed to read the data base. This information is available with a single call to DBISIZES.

The data base input (DBI) routines (routines whose names begin with $\mathrm{DBI}$ ) can be called in any order with some restrictions. Any QA and model description information needed should be read (and stored in memory if necessary) before proceeding to the analysis results. If an output data base is connected, DBOHEAD must be called before the input data base processes any analysis results. The user must also ensure that the current time step corresponds to the appropriate connected output data base time step if variables are to be copied.

The analysis results routines are most efficient if the user reads approximately the same variables for each time step and processes the time steps sequentially.

General Use Comments. Routines whose names begin with DBPdisplay useful summary information about an input or output data base. As a rule, do not write out the entire CAMDAT data base file in ASCII for debugging or other purposes. Instead, use the GROPE utility (Rechard, ed., 1992) to display any CAMDAT data base file entry.

The CAMDAT_LIB routines require the use of dynamic memory. The following lines must be included in any calling program:

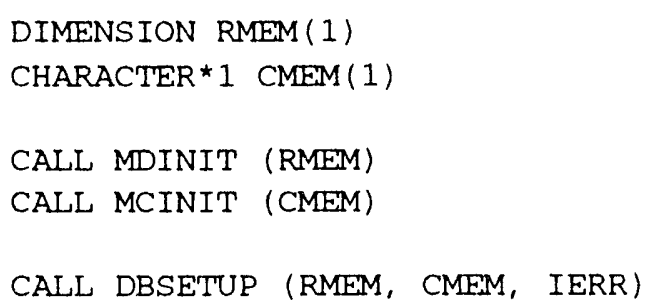

If the calling code also needs dynamic memory, it must use the same RMEM and CMEM arrays (see Section 9.2.2, "Memory Management Routines"). 
Some of the CAMDAT variable types (attributes, properties, elements) require what is called an existence (truth) table to describe where in the computational grid a particular variable is defined. A computational grid is broken down into blocks that are typically associated with specific materials. The existence table defines in which blocks a particular variable is defined. The existence table is either a singly or doubly dimensioned logical array where one of the dimensions is always a block number. For example, (1) ISATOK(b) is true if and only if the associated attribute exists in block b (see DBIATTR and DBOATTR), or (2) ISEVOK( $b, i)$ is true if and only if element variable i exists in block b (see DBIEVROK and DBOEVROK).

\subsubsection{Error Messages}

Almost all CAMDAT_LIB routines return an error flag. Zero indicates no error. A positive value is the FORTRAN I/O error status from an input or output operation (indicated by "I/O" in the error flag descriptions for specific routines). A value of -1 indicates an end of file was encountered. All other errors result in a negative value. Most other errors involve an invalid parameter, such as an invalid unit number or undefined variable name. Some routines check for dynamic memory errors. These errors may have occurred in the calling program before the routine was called. In general, a message is displayed when an error is flagged. Possible errors are discussed with each routine description.

\subsubsection{Linking}

The CAMDAT_LIB routines are an object library in the [LIBRARIES] subdirectory (see Section 2.10, "Software Libraries"). CAMDAT_LIB must be linked with libraries CAMCON_LIB and CAMSUPES_LIB. Link the three libraries with a code using the following command (logical names of libraries are assigned by each library sponsor):

LINK code_name ..., CAMDAT_LIB/LIB, CAMCON_LIB/LIB,CAMSUPES_LIB/LIB

A FLINT library for CAMDAT_LIB available for checking interfaces is in CAMCON\$ROOT:[LIBRARIES.CAMDAT_LIB]CAMDAT_LIB.LBT.

\subsubsection{Summary of Routine Calls}

CAMDAT_LIB includes the following routines:

\section{GeneralRoutines}

- DBERRUNI - define the unit number for the error messages

- DBFIXMEM - reset dynamic memory after M $\alpha$ COMP 


\section{File Initialization}

- DBSETUP - initialize the library

- DBIOPEN - open data base file for reading; must be called before any other DBI routine for the data base

- DBOOPEN - open data base file for writing; may be connected to an open input data base and has an associated scratch file; must be called before any other DBO routine for the data base

- DBICLOSE - close the input data base file

- DBOCLOSE - close the output data base file

- DBPDBNAM - print the data base file name

- DBISIZES - return the sizes of all QA and input data items on the data base file (e.g., the number of unique attributes, etc.); set with various $\mathrm{DBO}$ routines

- DBPSIZES - print the data base size information

- DBITITLE - return the data base file title

- DBOTITLE - set the data base file title

- DBPTITLE - print the title

- DBIVERS - return the data base file version; set by default to the current CAMDAT version

- DBPVERS - print the data base version

QAInformation

- DBIINFO

- DBOINFO

- DBPINFO

- DBIQAREC

- DBOQAREC -

- DBPQAREC return the information records set the information records print the information records

return the QA records

set the $Q A$ records

print the QA records

Model Description

- DBIATTR - return the values for the given attribute for all elements

- DBOATTR - set the values for the given attribute for all elements

- DBIATNAM - return the unique attribute names

- DBPAPNAM - print the attribute and property names

- DBIELBLK -

- DBOELBLK -

- DBPELBLK -

- DBOHEAD - return the element block names and ILs

set the element block names and IDs

print the element blocks summary

write the QA information and model description as copied from the connected data base being read or set with DBO routines 
- DBILINK - return the connectivity for all element blocks

- DBOLINK - set the connectivity for .11 element blocks

- DBIMAP - return the element order map

- DBOMAP - set the element order map

- DBINDSET - return the node sets

- DBONDSET - set the node sets

- DBPNDSET - print the node set summary

- DBINELB - return the number of elements per block; also returned by DBILINK

- DBIPROP - return the values for the given property for all element blocks

- DBOPROP - set the values for the given property for all element blocks

- DBIPRNAM - return the unique property names

- DBISDSET - return the side sets

- DBOSDSET - set the side sets

- DBPSDSET - print the side set summary

- DBIXYZ - return the coordinate names and the coordinates

- DBOXYZ - set the coordinate names and the coordinates

Analysis Results

- DBIEVROK - return the element variable truth table

- DBOEVROK - set the element variable truth table

- DBINVAR - return the number of variables; set with DBOVRNAM

- DBPNVAR - print the number of variables

- DBISTEP - advance to the specified time step and return the time

- DBOSTEP - set the current time step and advance to next time step

- DBINSTEP - return the number of time steps

- DBITIME - advance to the next time step and return the time

- DBOTIME - set the time for this time step

- DBIVAR - return the given variable for this time step

- DBOVAR - set the given variable for this time step

- DBIVRNAM - return the variable names of the given type

- DBOVRNAM - define the output variables

- DBPVRNAM - print the variable names

- DBINDVAR - define input variables as needed 


\subsubsection{Example}

The example below shows a series of FORTRAN code fragments containing CAMDAT_LIB calls that create a CAMDAT data base file:

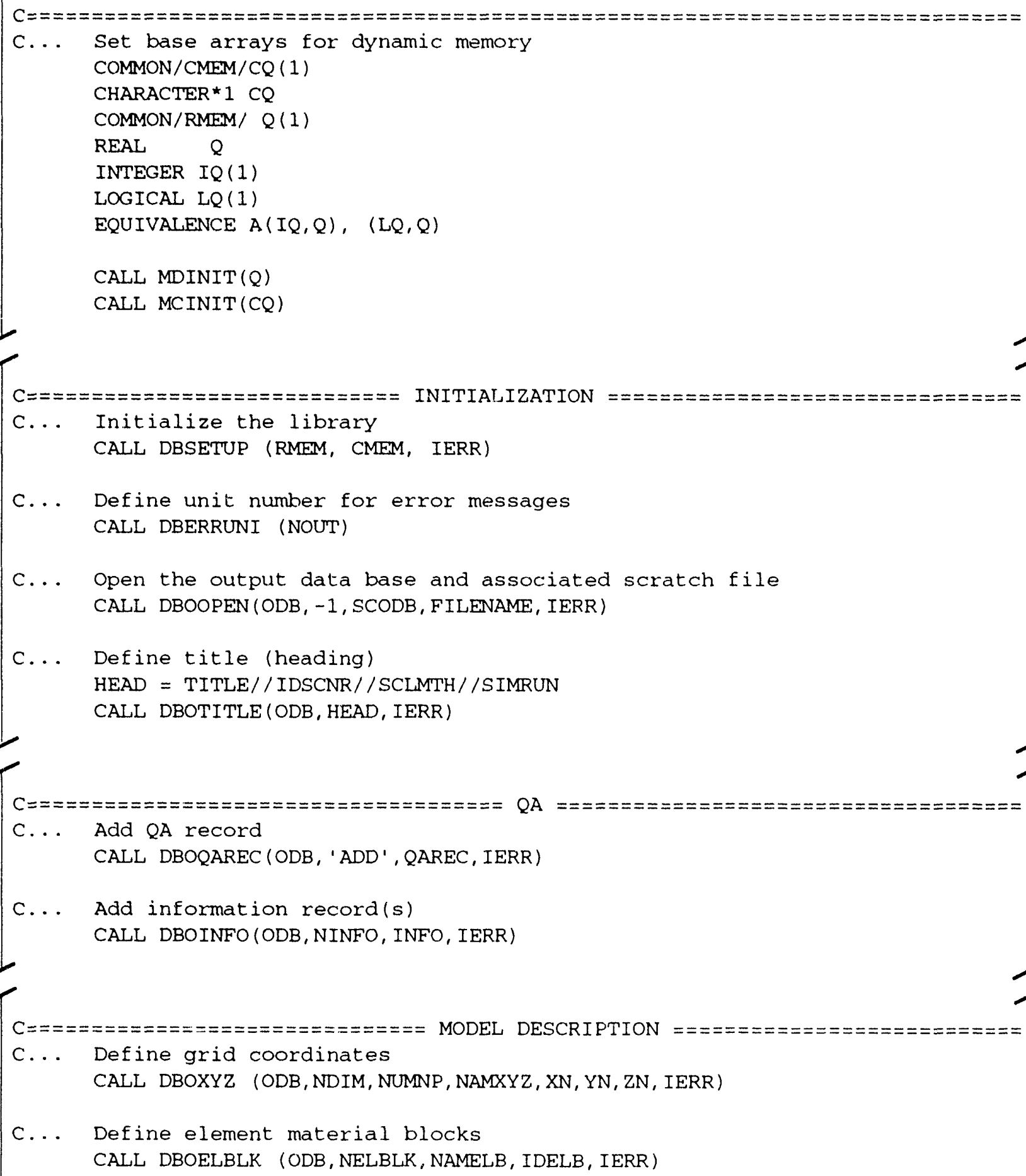




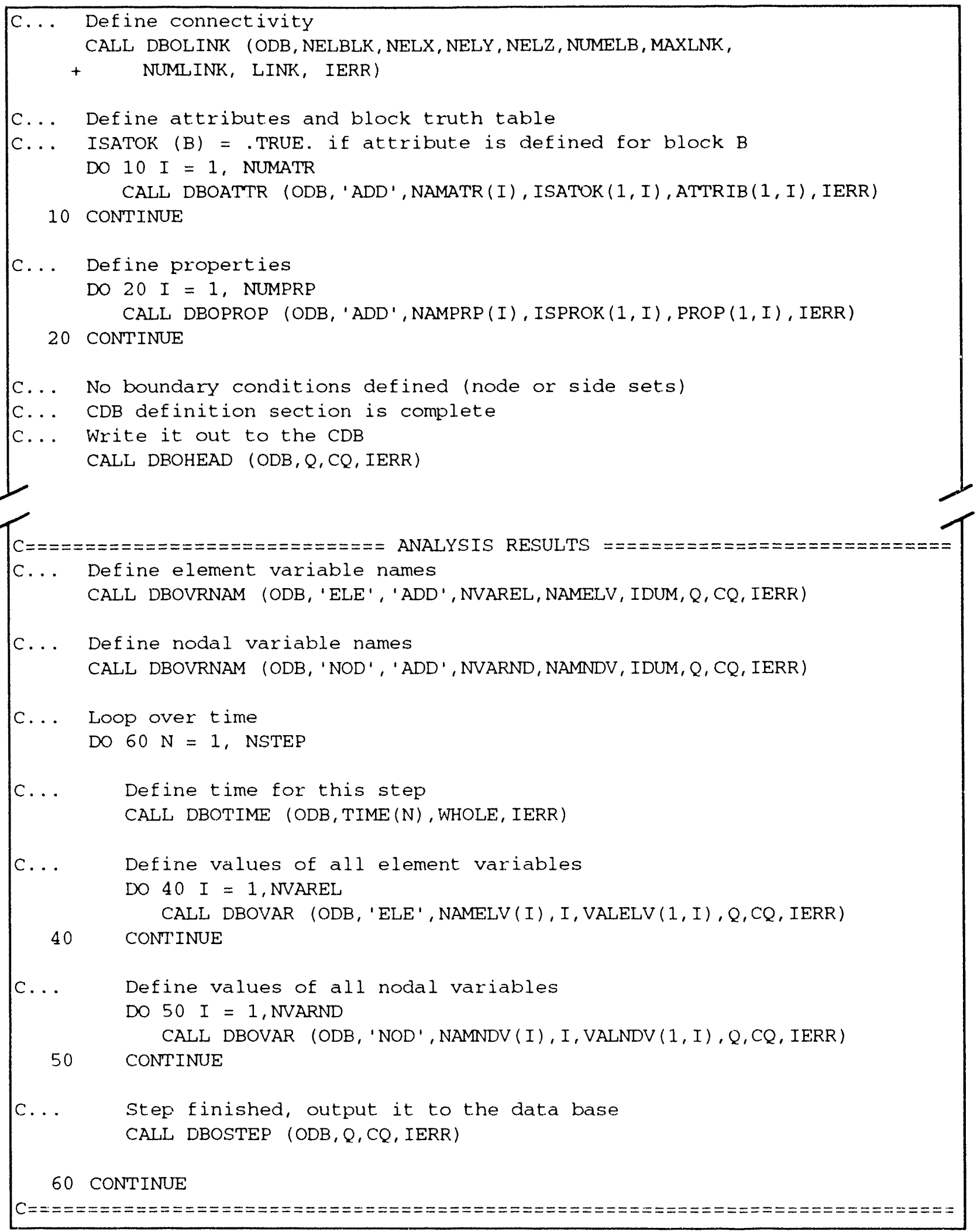




\subsection{Description of Routine Calls}

\subsubsection{General Routines}

Detailed descriptions of general CAMDAT_LIB routines are given on the following pages. The routines described in this section set up parameters for all CAMDAT_LIB system routines. They do not refer to a specific data base file. 


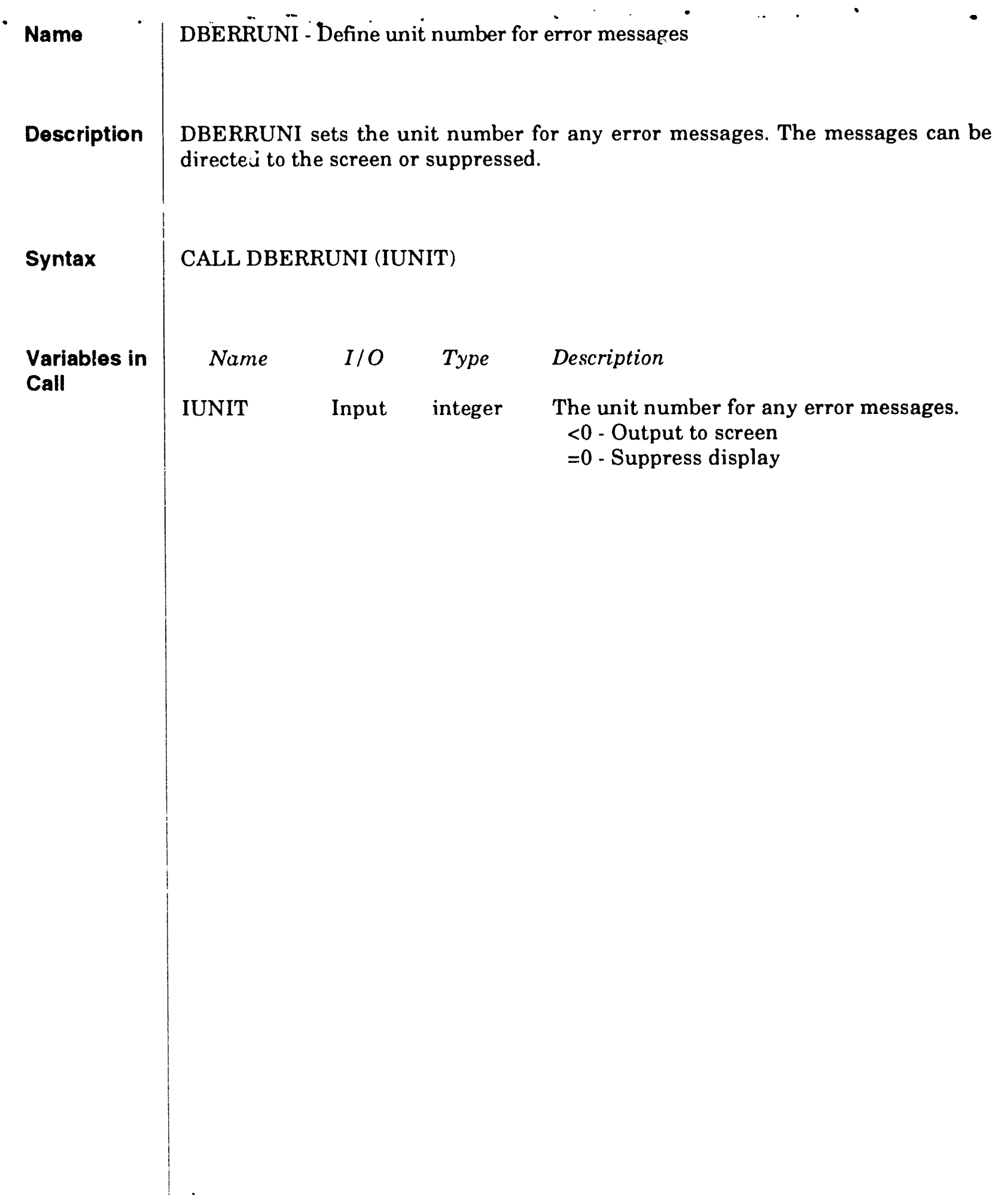




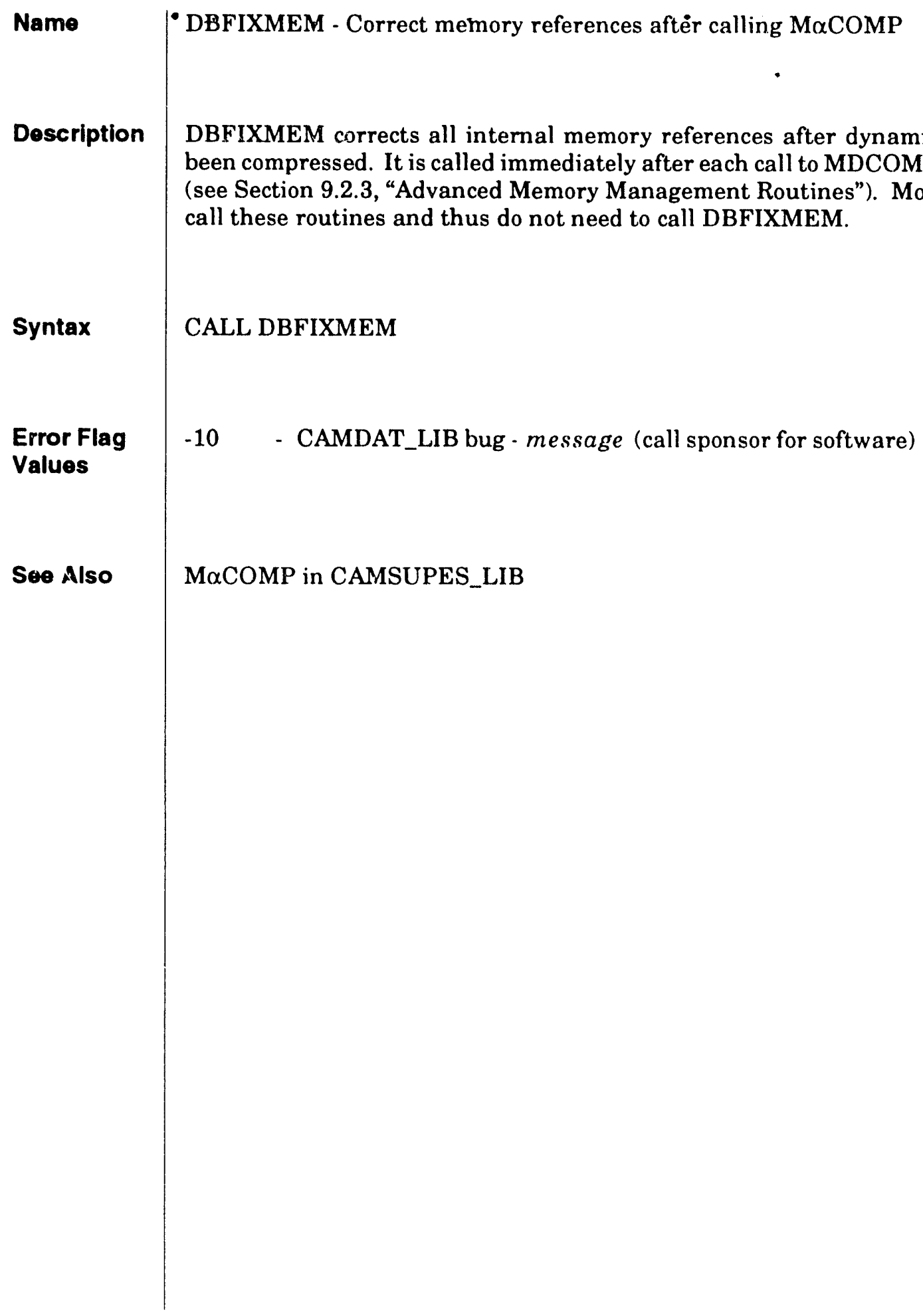


CAMDAT LIB

File Initialization

\subsubsection{Detailed Descriptions of File Initialization Routines}

Detailed descriptions of CAMDAT_LIB file initialization routines are given on the following pages. The routines described in this section are used to open, close, and initialize CAMDAT files. 


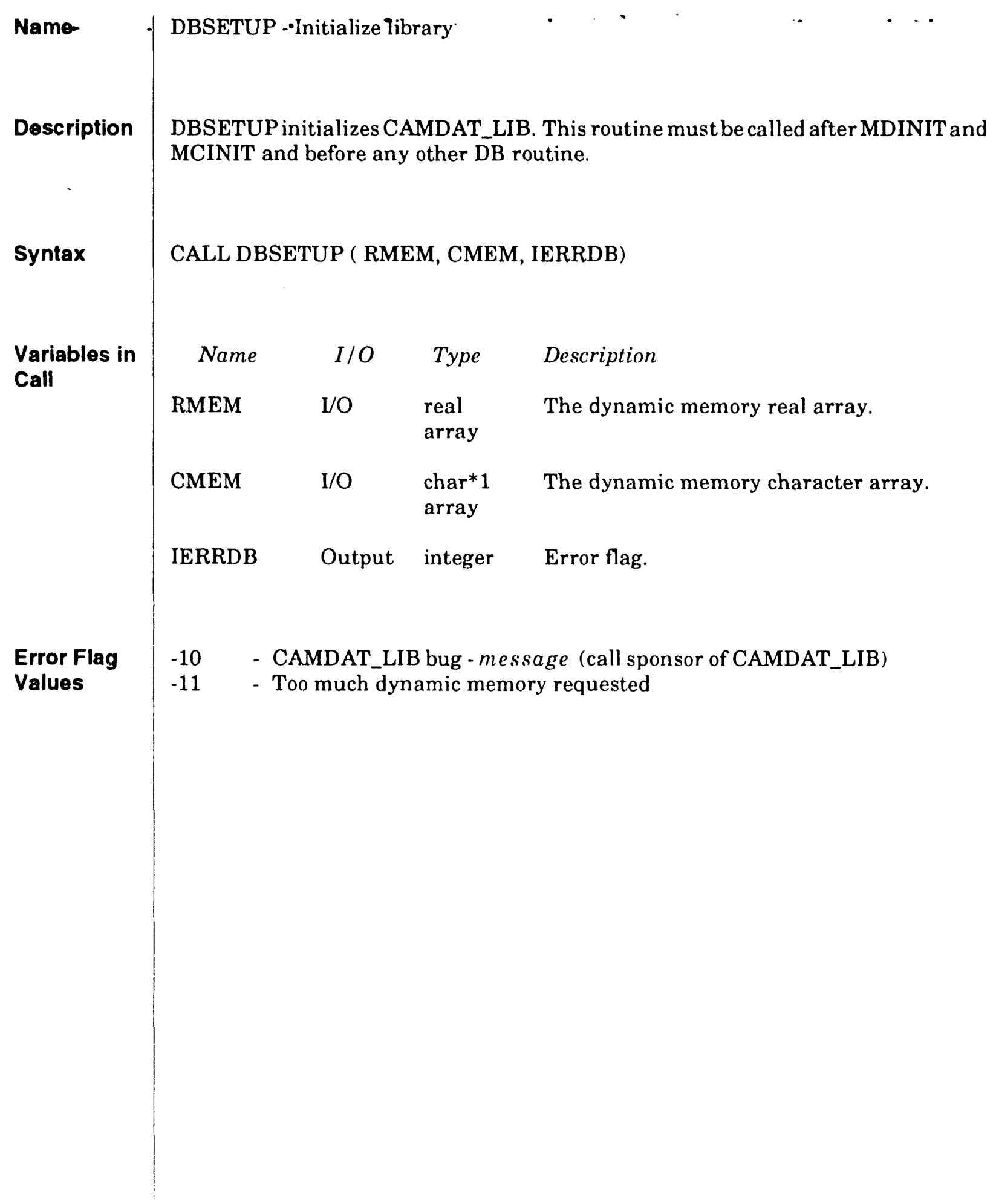




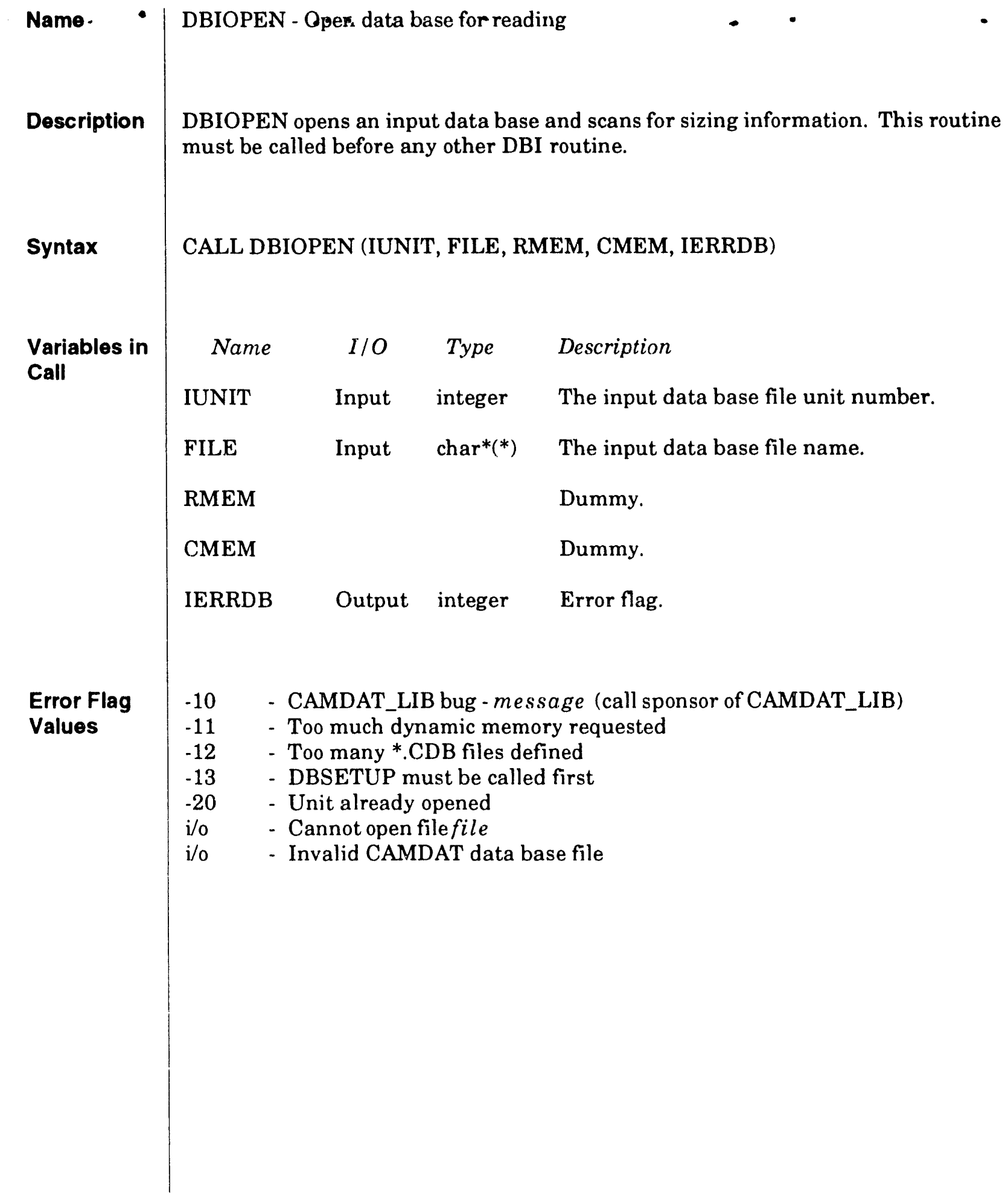




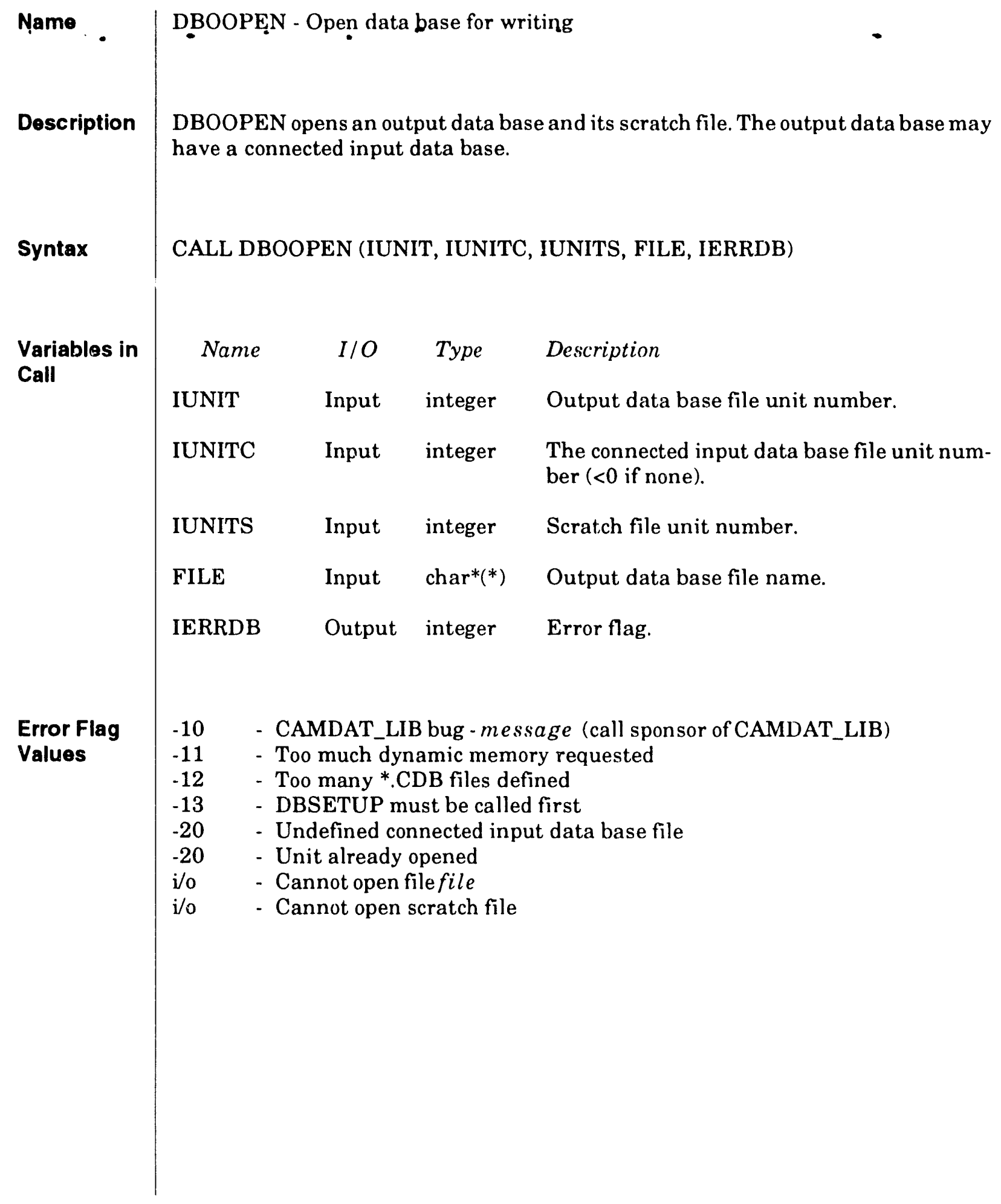




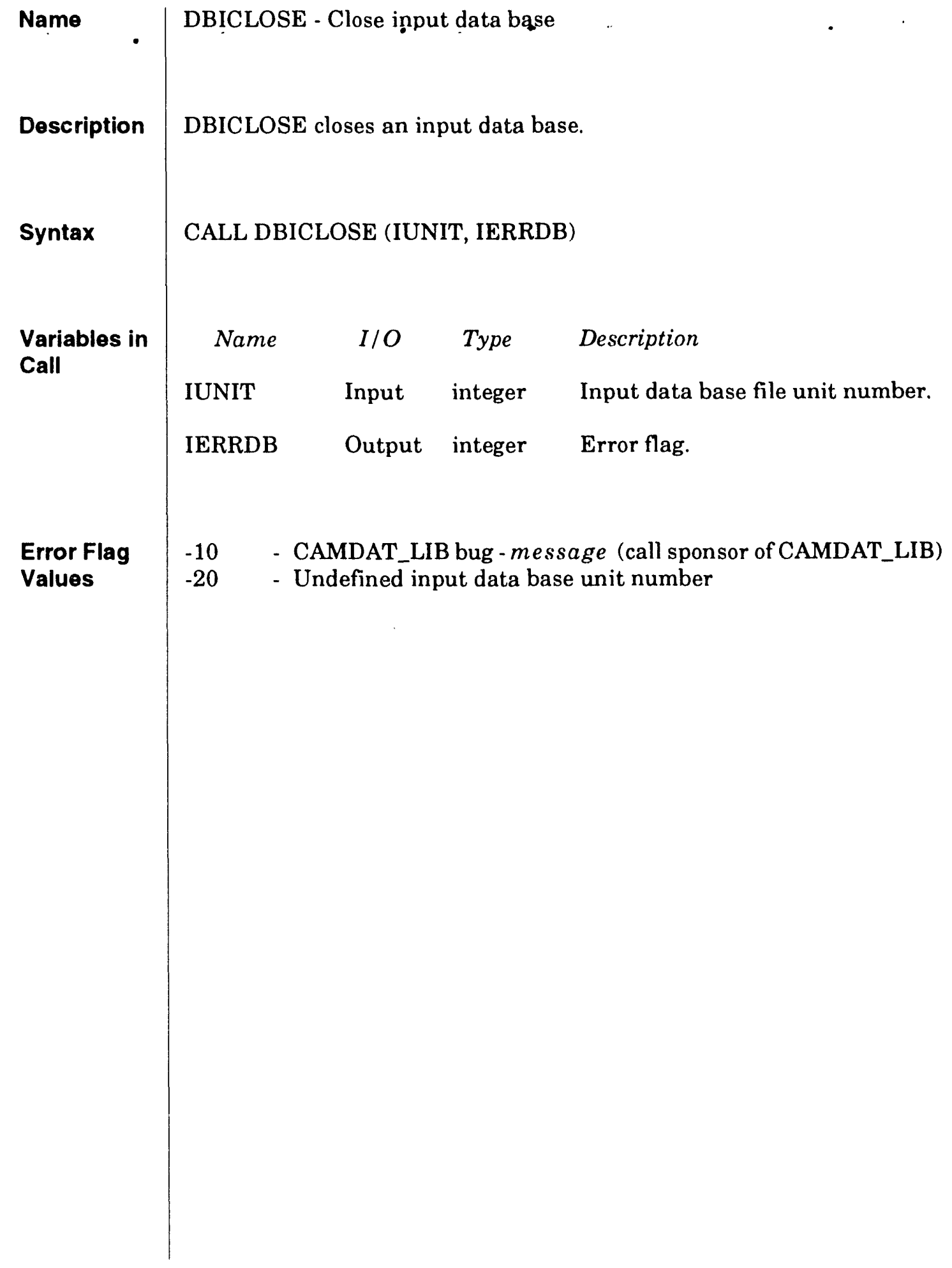




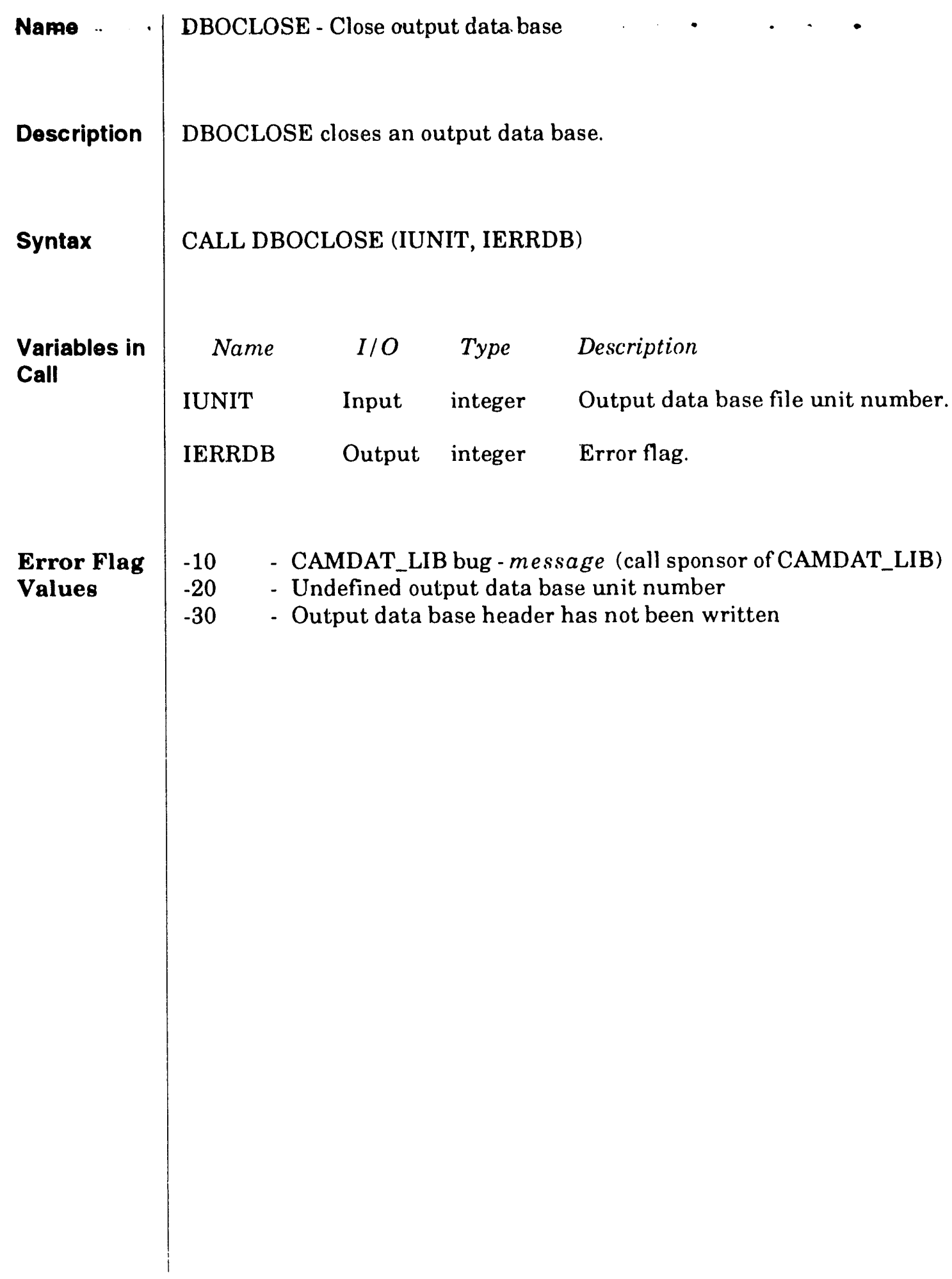




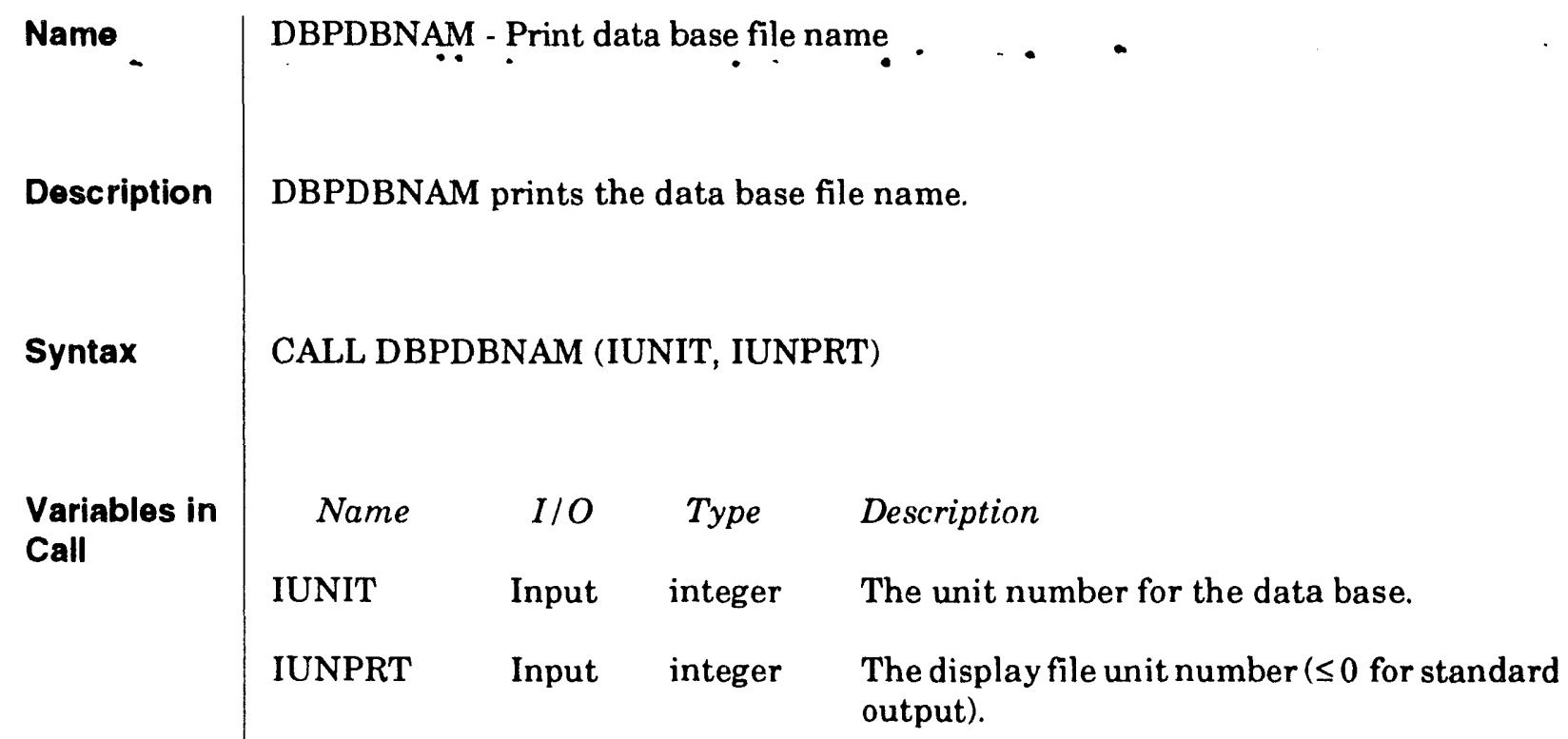


CAMDAT_LIB

DBISIZES

Subroutine

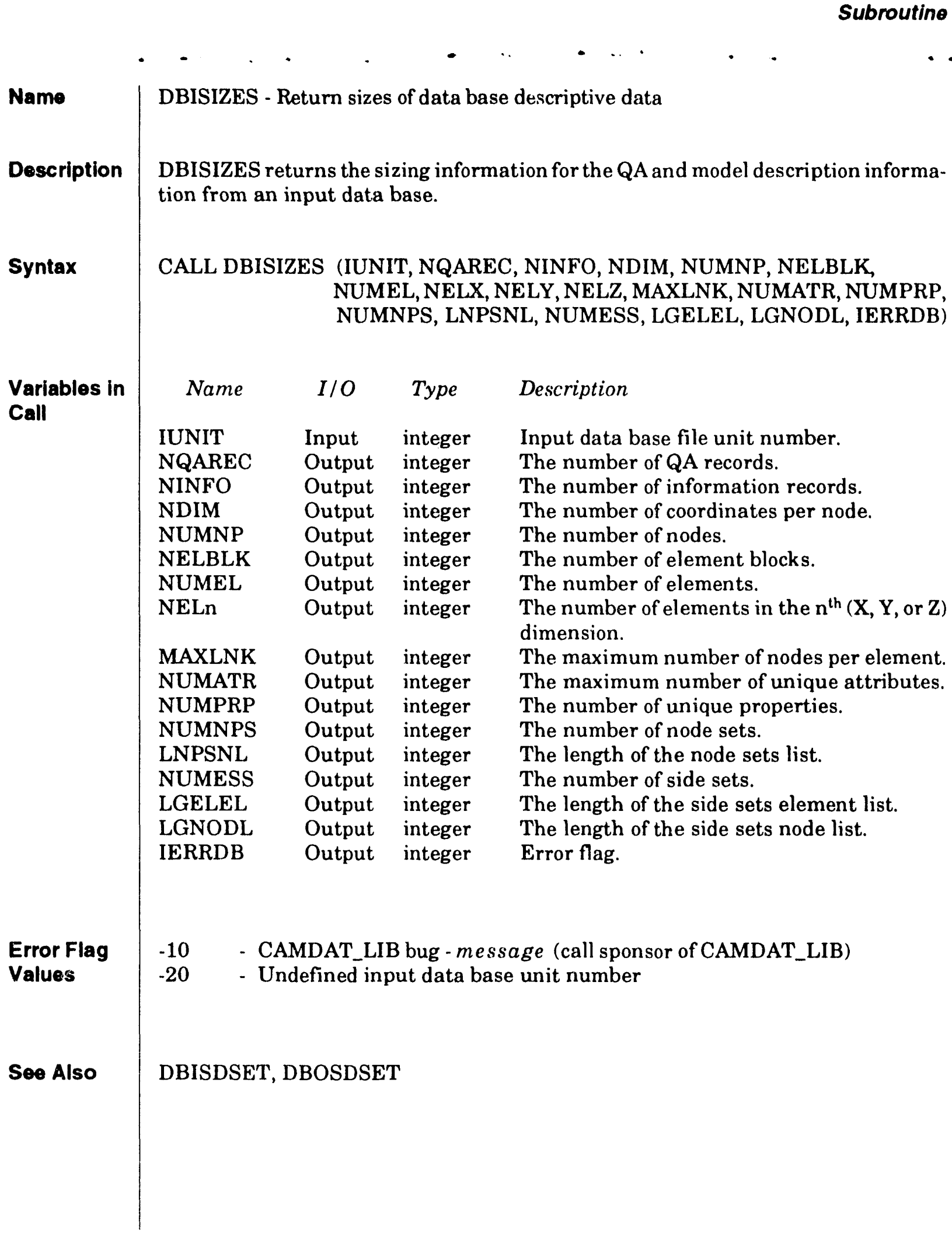


CAMDAT LIB

File Initialization

DBPSIZES

Subroutine

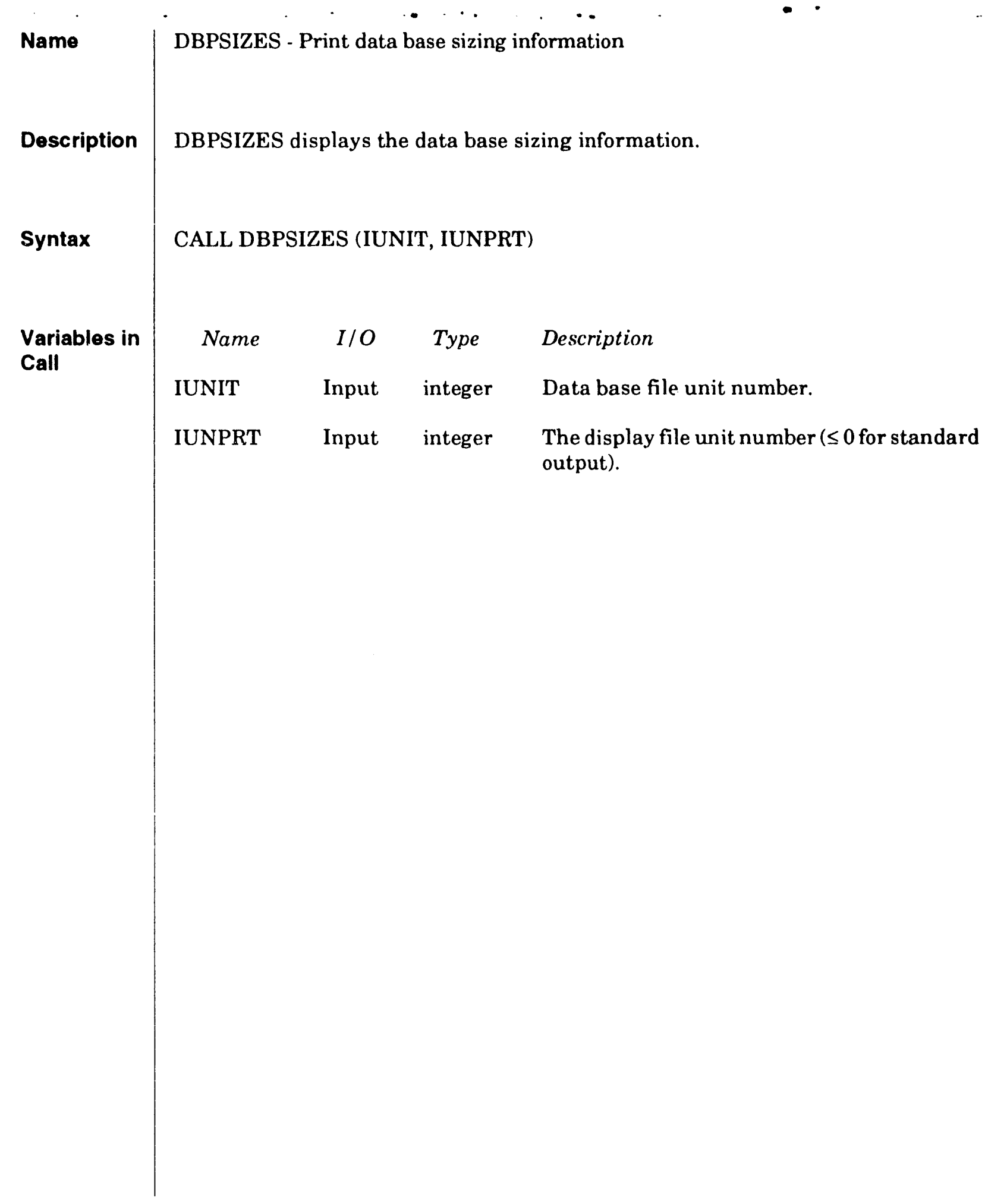




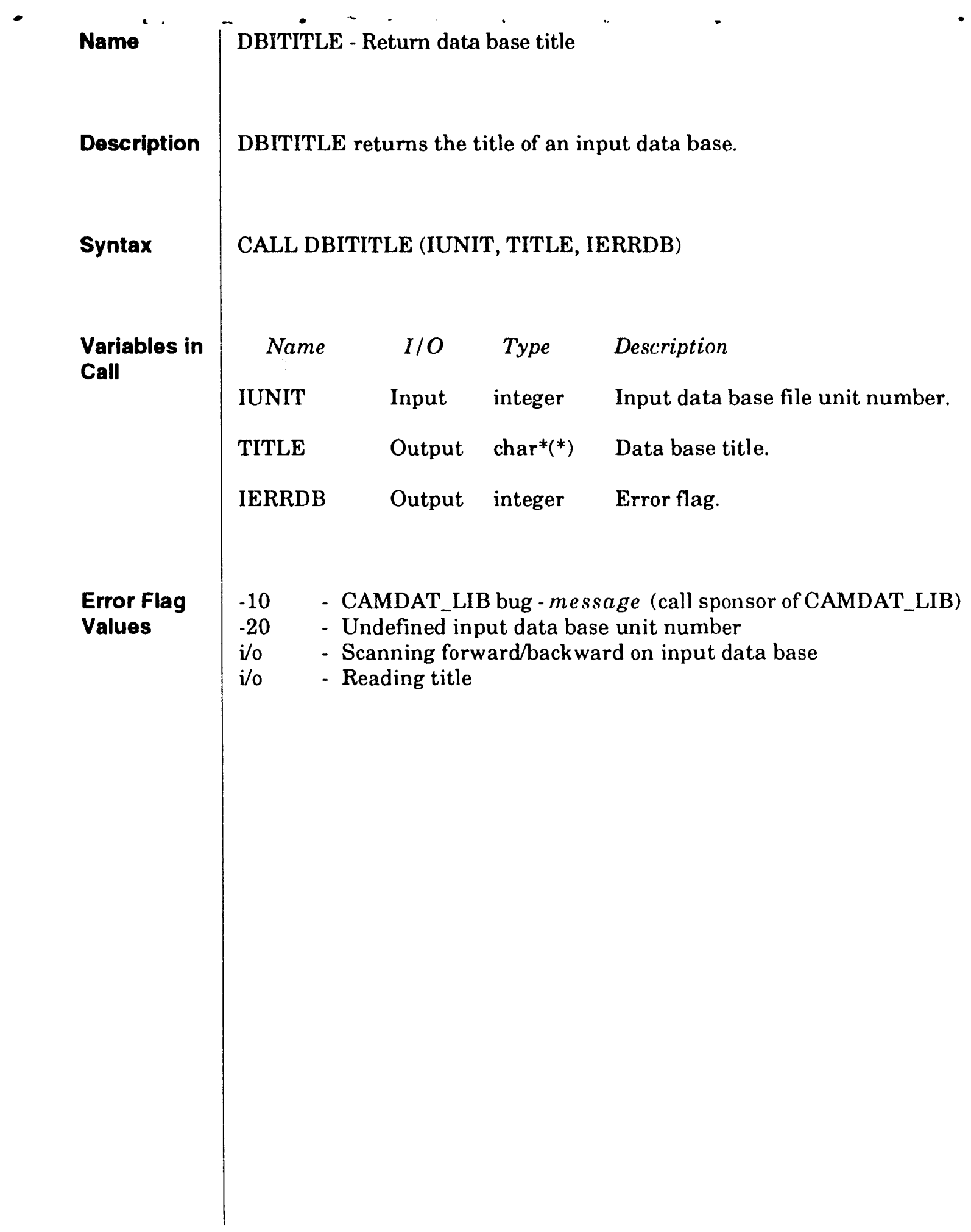




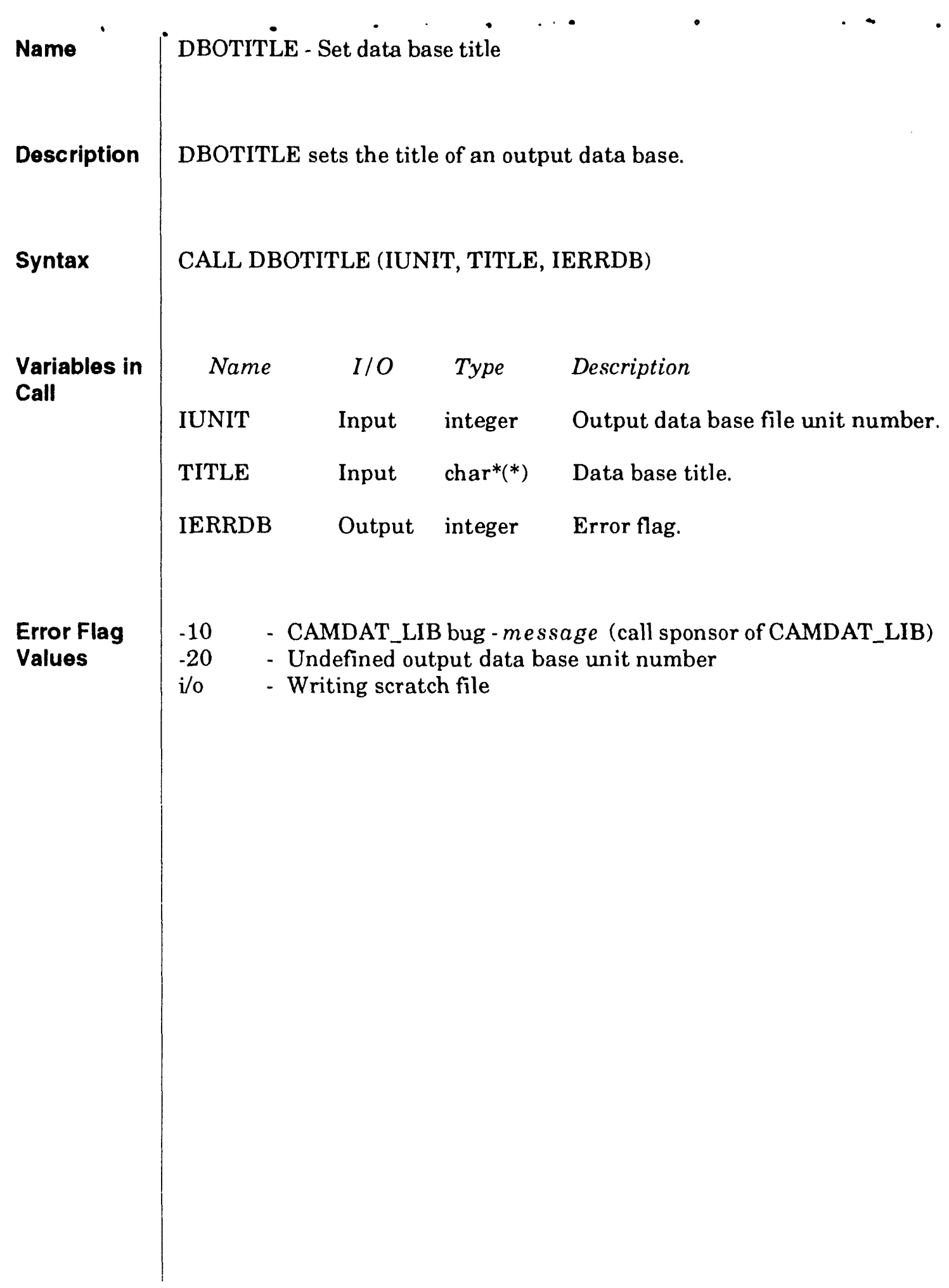




\begin{tabular}{|c|c|c|c|c|}
\hline Name & \multicolumn{4}{|c|}{ DBPTTCLE - Print data base title } \\
\hline Description & \multicolumn{4}{|c|}{ DBPVERS displays the data base title. } \\
\hline Syntax & \multicolumn{4}{|c|}{ CALL DBPTITLE (IUNIT, IUNPRT, TITLE) } \\
\hline \multirow{4}{*}{$\begin{array}{l}\text { Variables in } \\
\text { Call }\end{array}$} & Name & $I / O$ & Type & Description \\
\hline & IUNIT & Input & integer & Data base file unit number. \\
\hline & IUNPRT & Input & inieger & $\begin{array}{l}\text { The display file unit number ( } \leq 0 \text { for standard } \\
\text { output). }\end{array}$ \\
\hline & TITLE & Input & $\operatorname{char}^{*}(*)$ & Data base title. \\
\hline
\end{tabular}




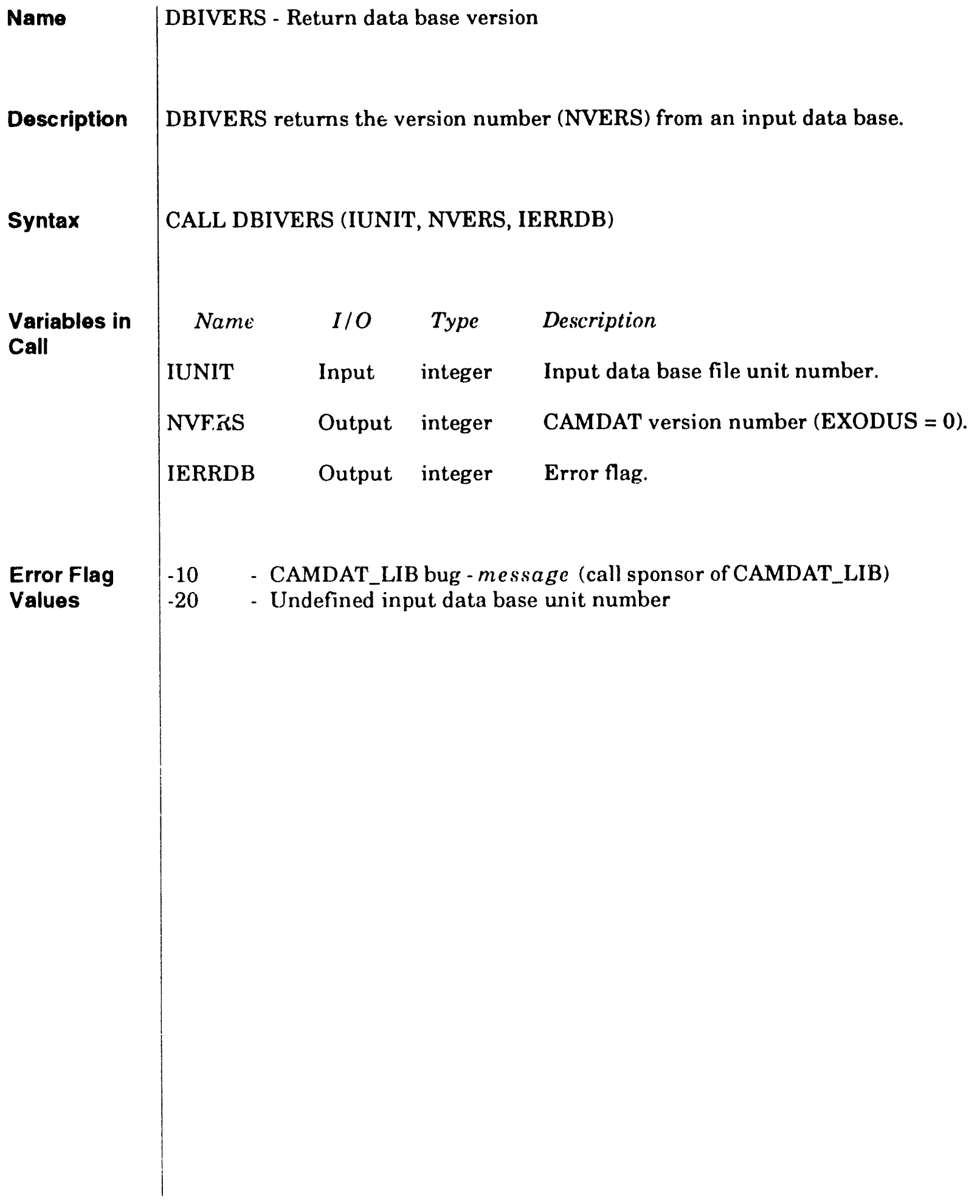




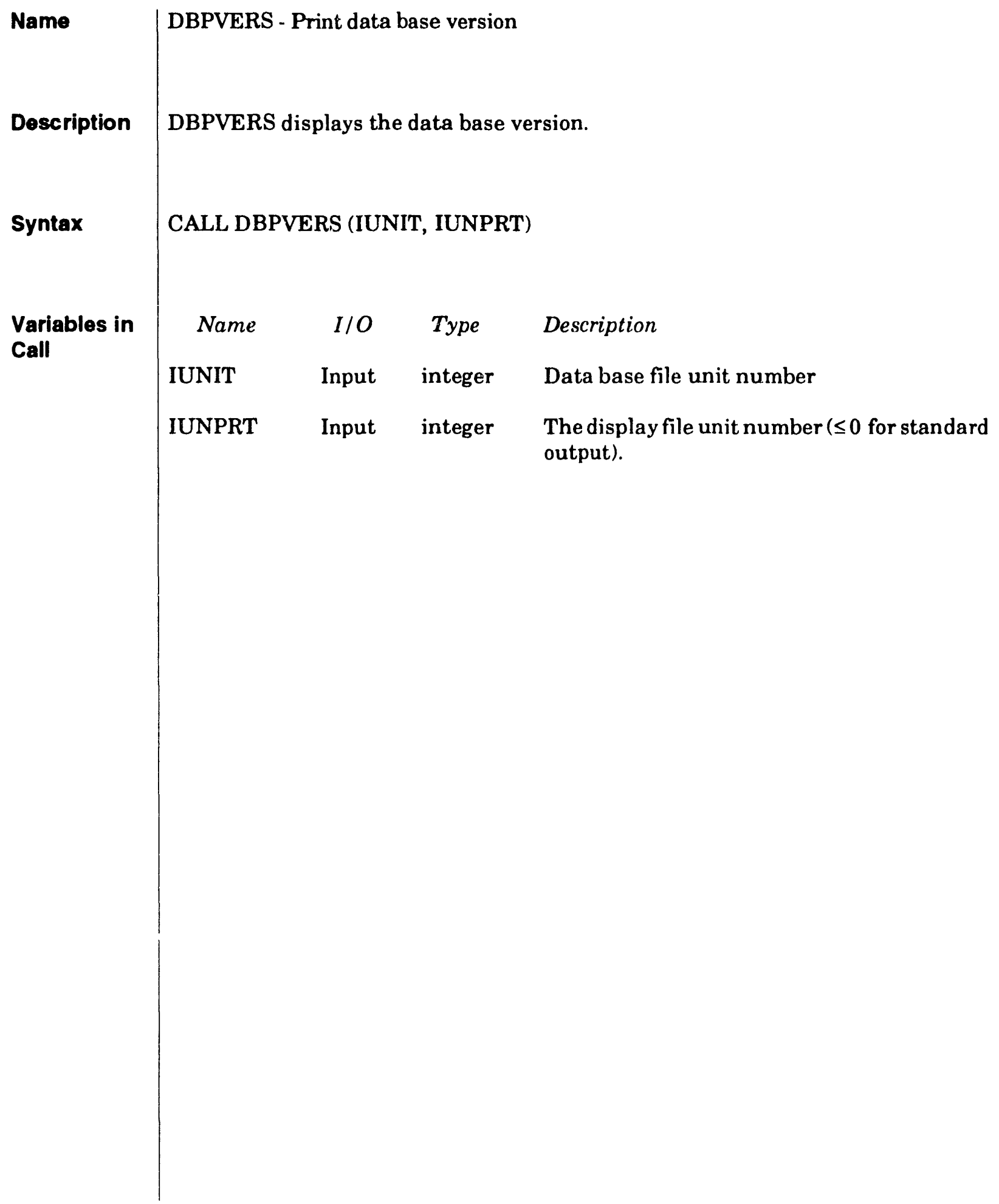


QA Information

\subsubsection{Detailed Descriptions of QA Information Routines}

Detailed descriptions of CAMDAT_LIB QA information routines are given on the following pages. The routines described in this section read and write quality assurance (QA) and optional information to the CAMDAT files. 


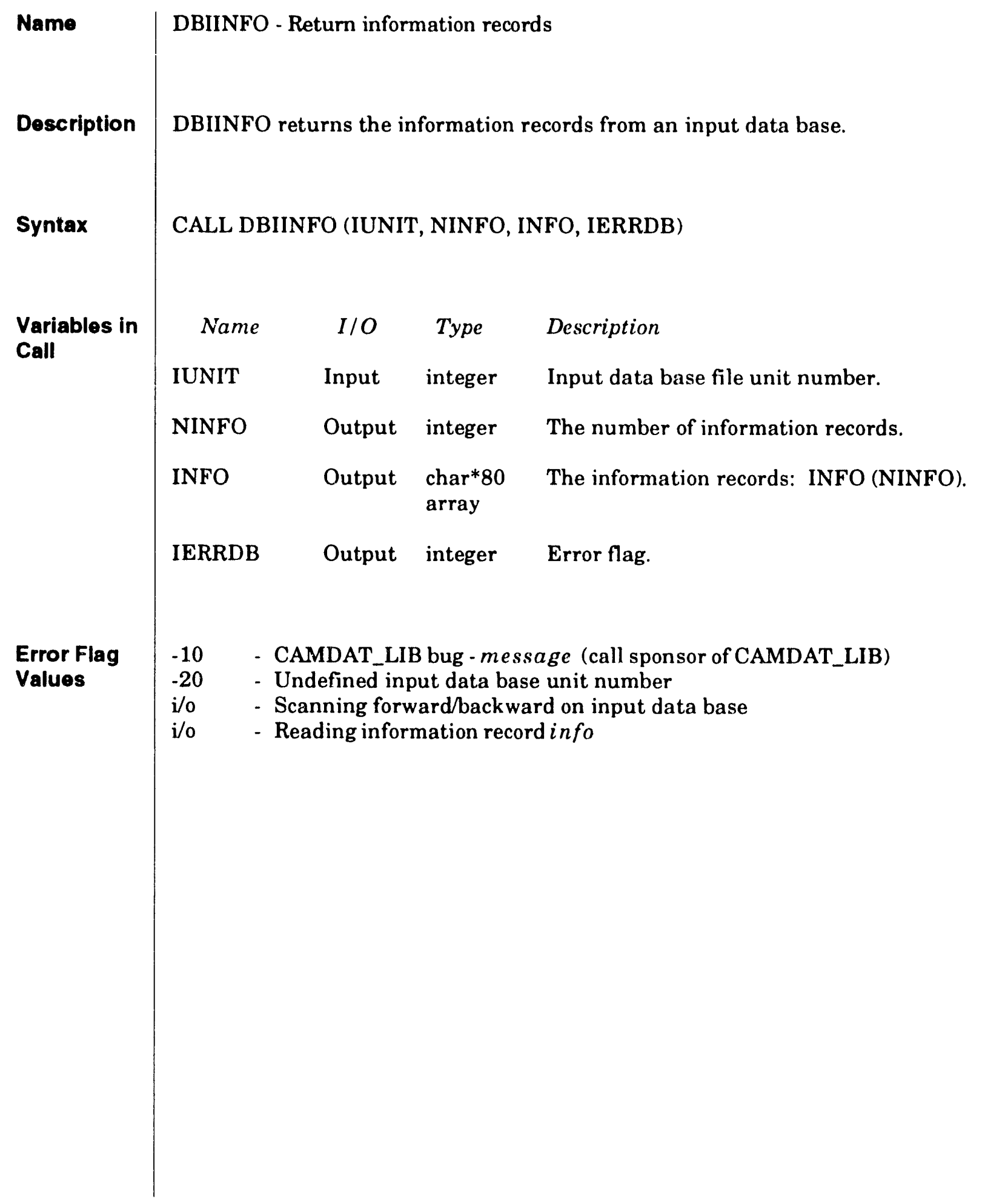




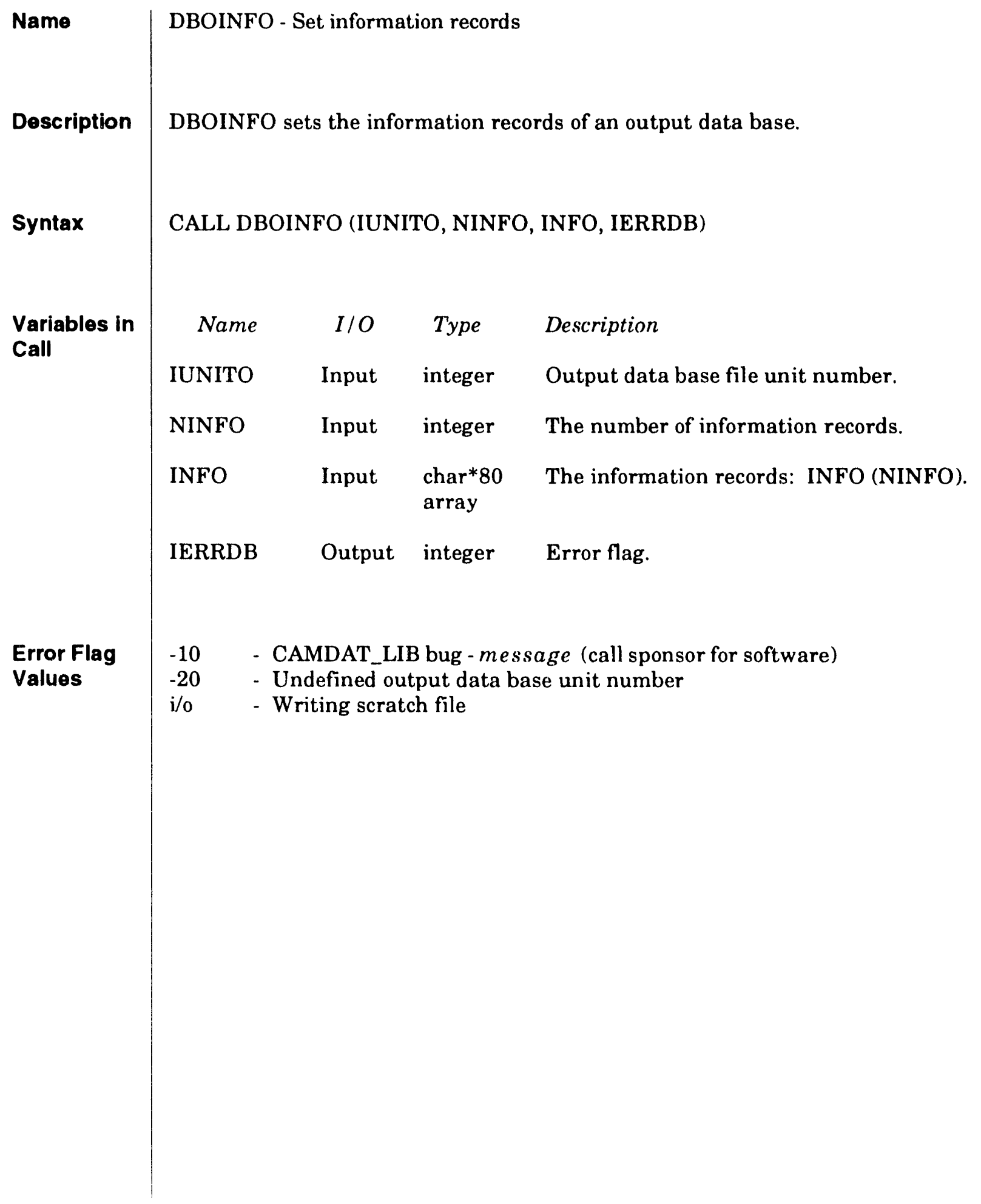




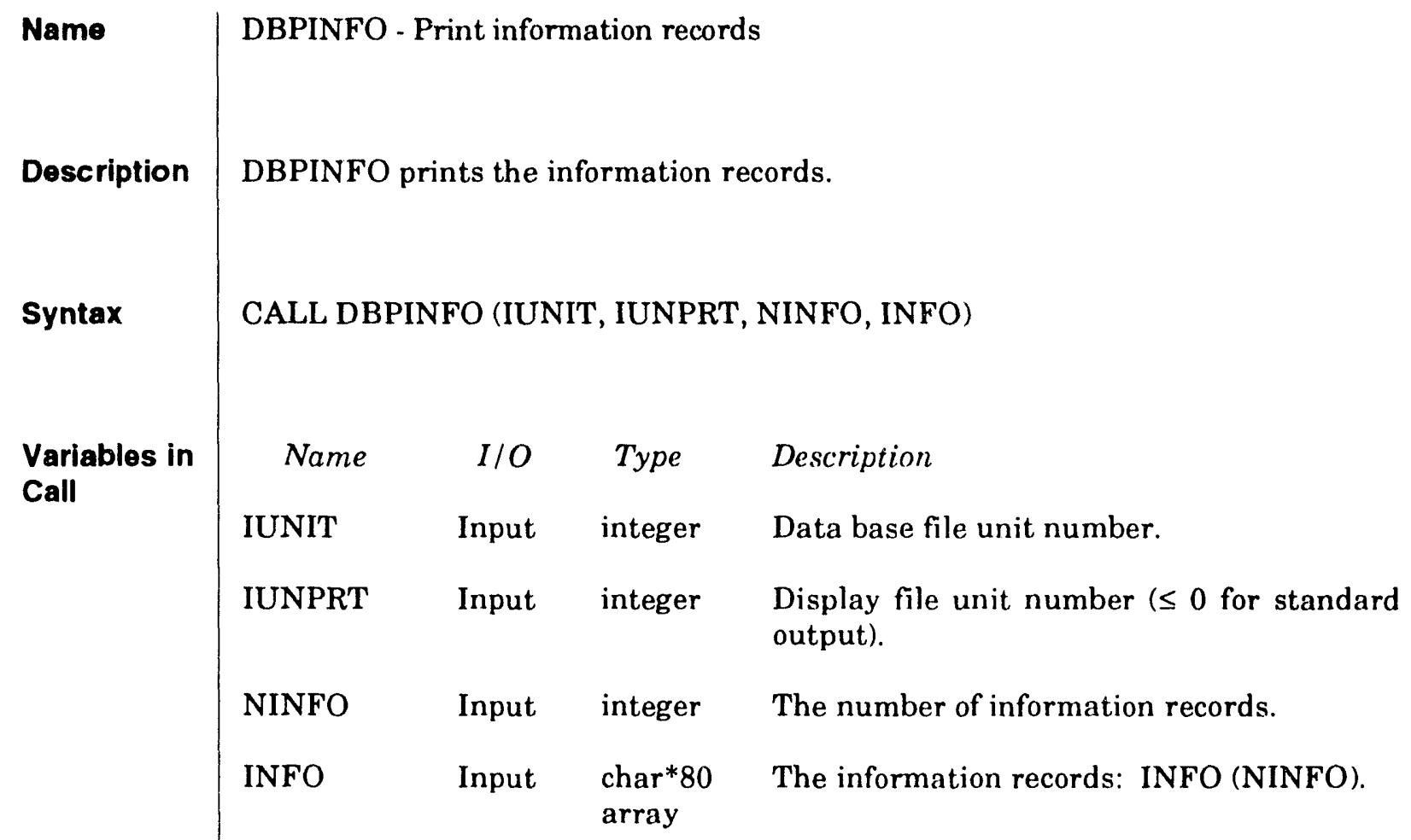




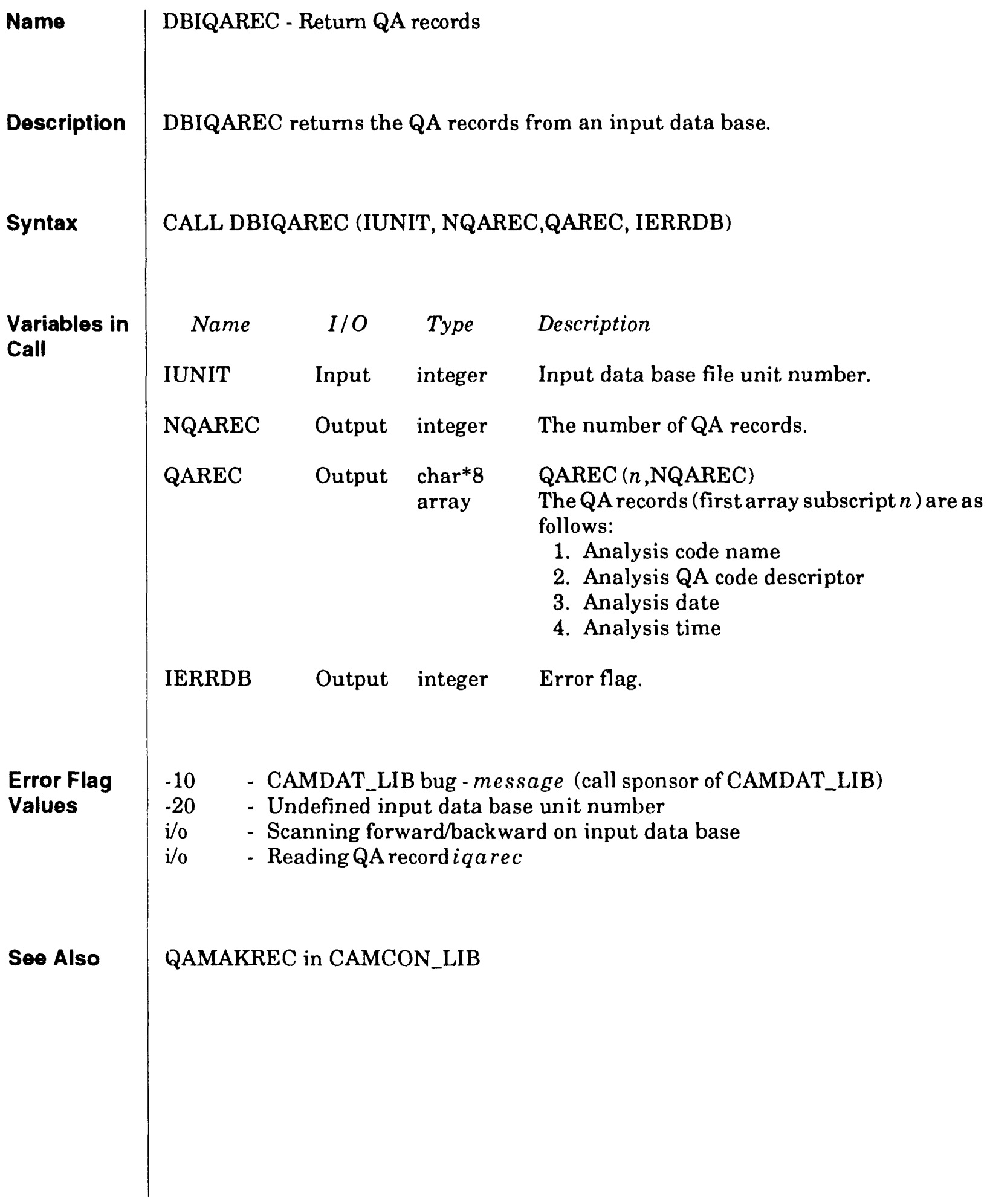




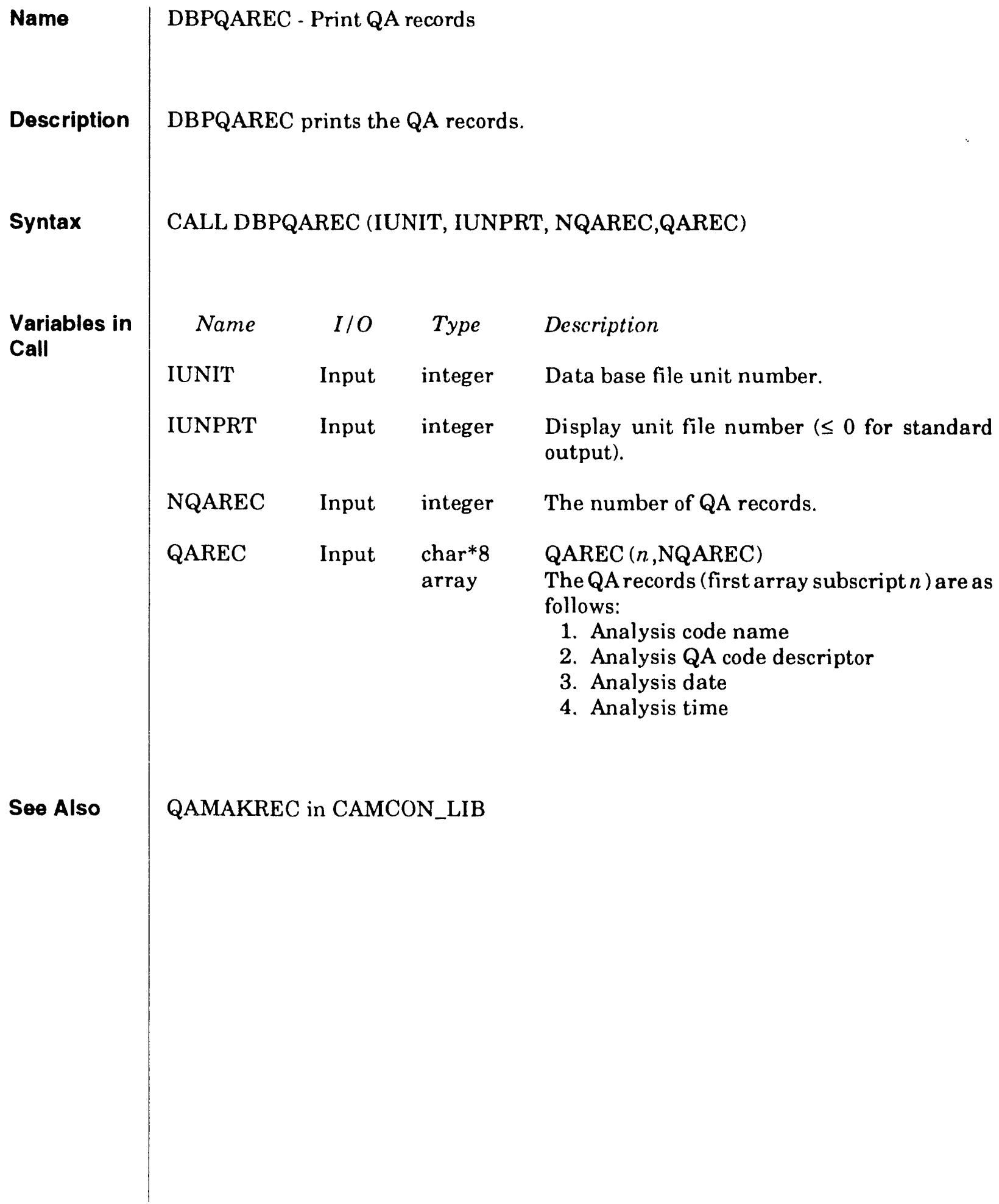




\subsubsection{Detailed Descriptions of Model Description Routines}

Detailed descriptions of CAMDAT_LIB model description routines are given on the following pages. The routines described in this section read or write information that describes the computational model. This includes nodal coordinates, element connectivity, element property attributes, element block properties, and boundary conditions as either parameter node sets or side sets. 


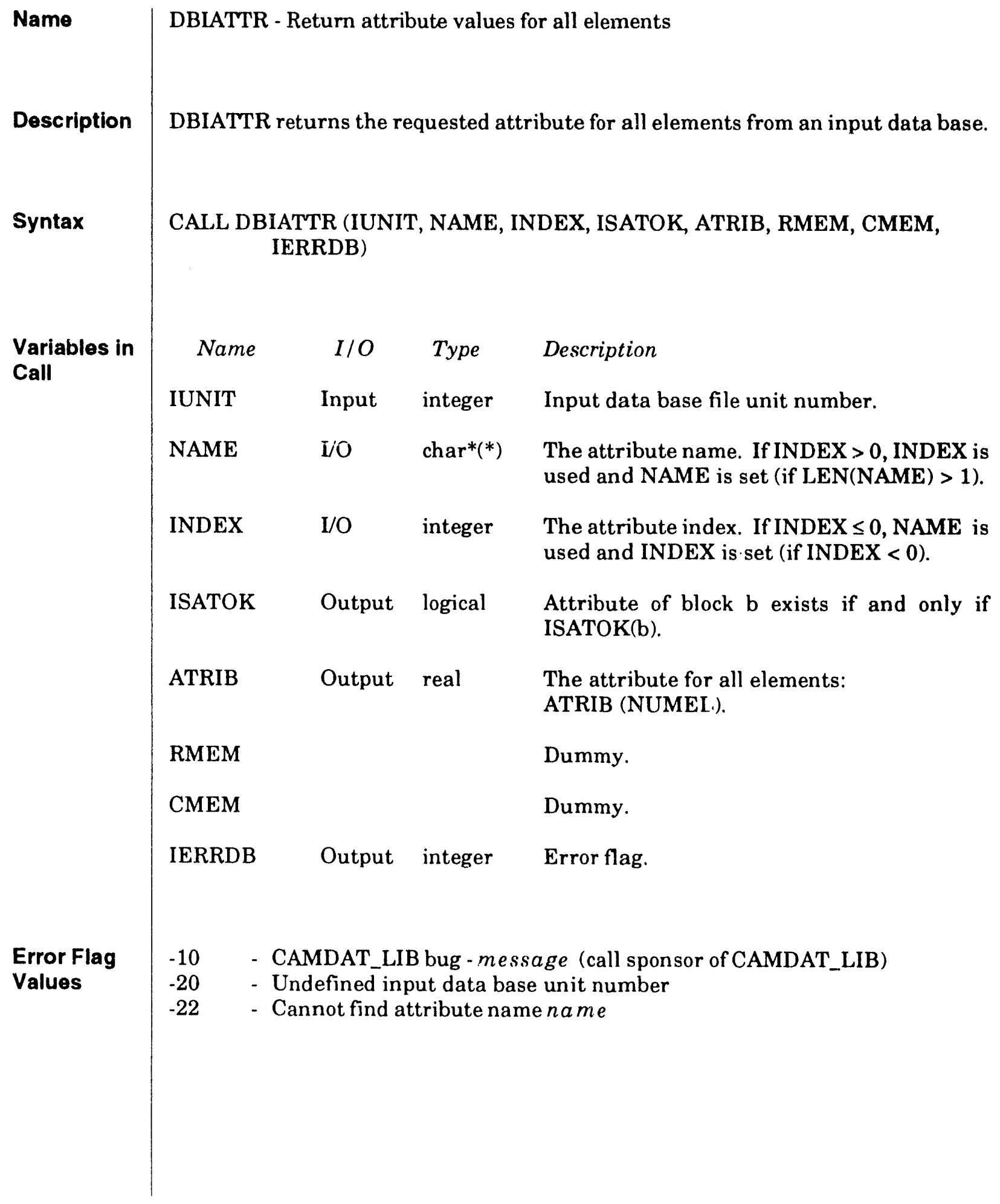




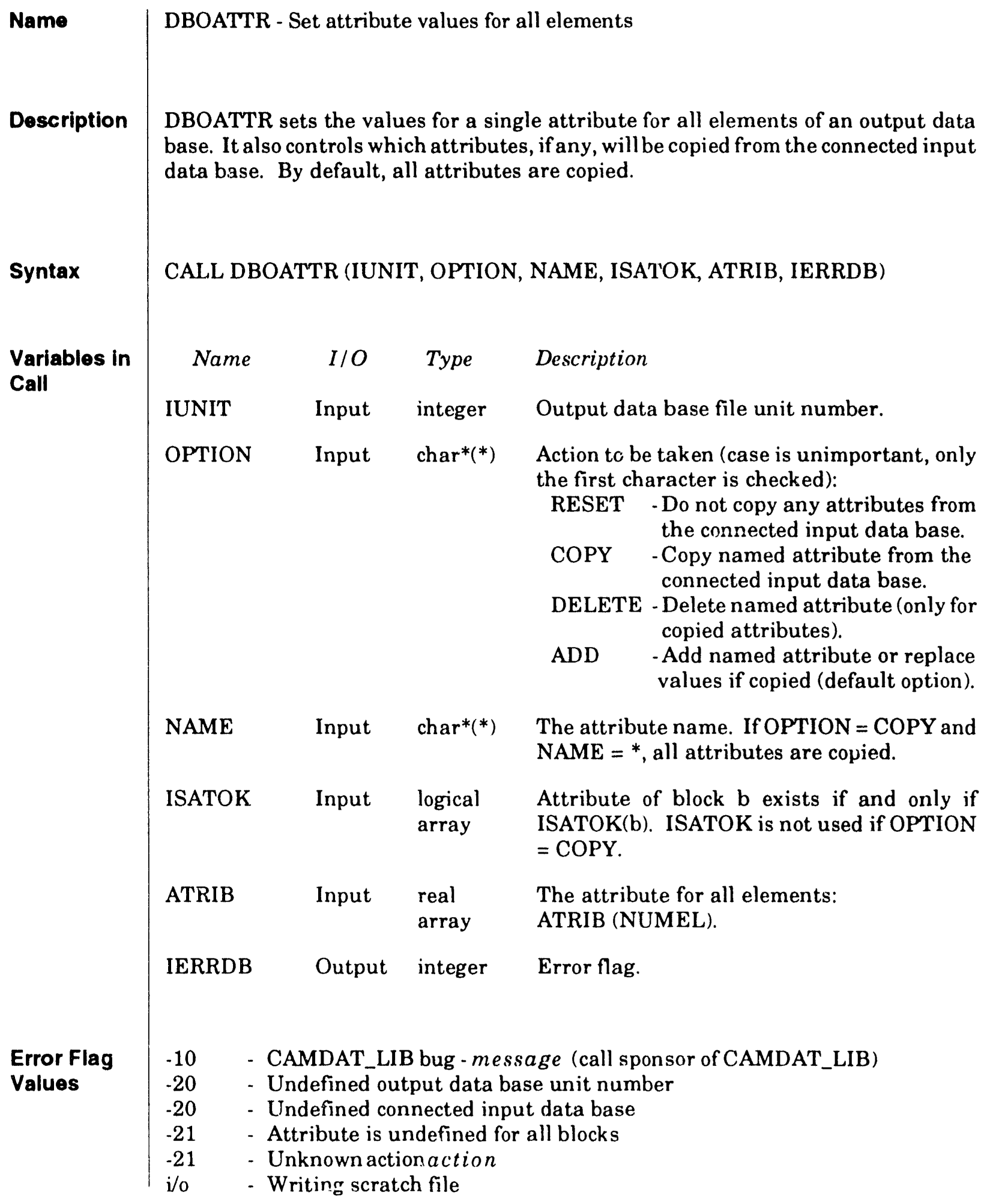




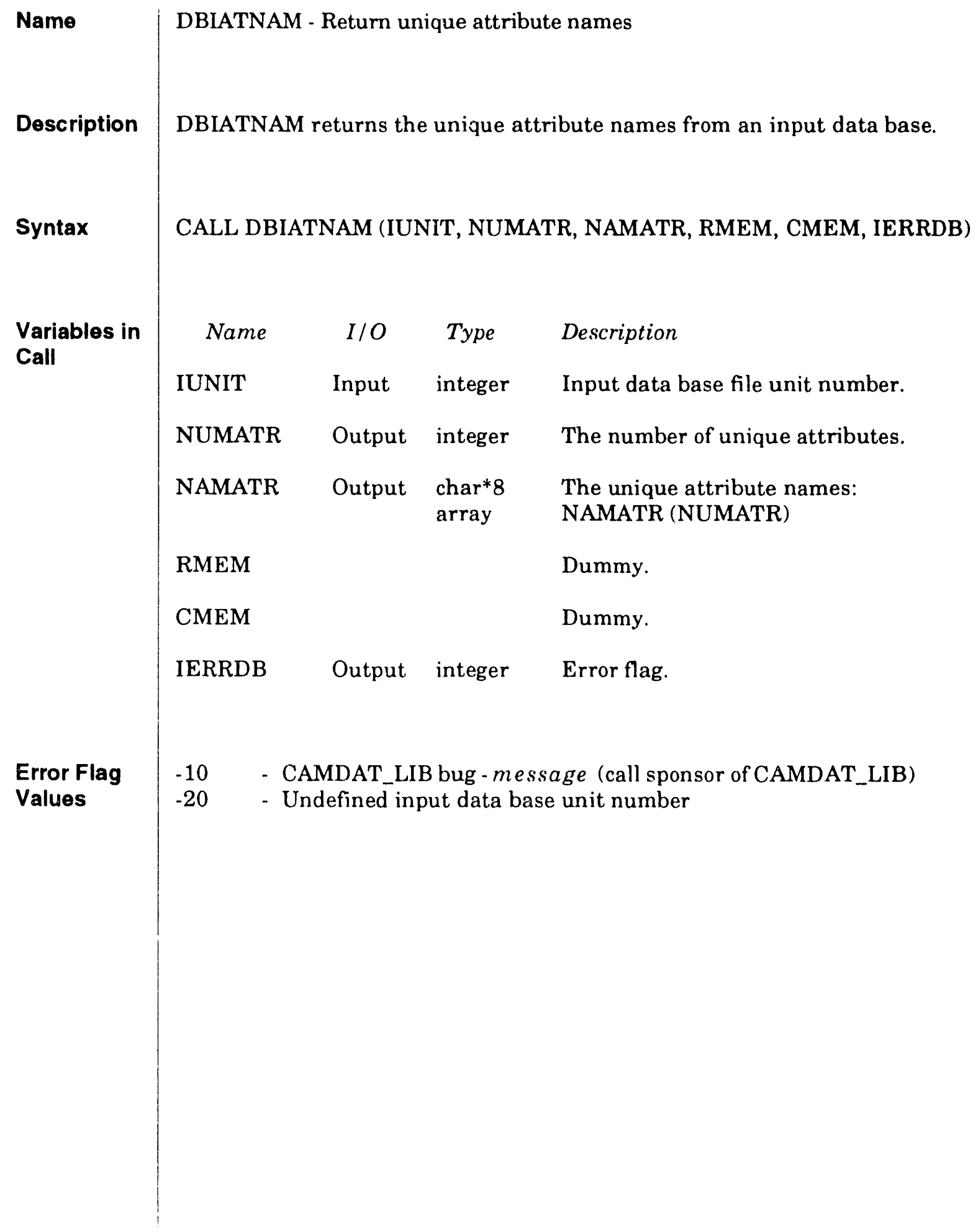




\begin{tabular}{|c|c|c|c|c|}
\hline Nam. a & \multicolumn{4}{|c|}{ DBPAPNAM - Print attribute and property names } \\
\hline Description & \multicolumn{4}{|c|}{ DBPAPNAM displays the attribute and property names. } \\
\hline Syntax & \multicolumn{4}{|c|}{ CALL DBPAPNAM (IUNIT, IUNPRT, NUMATR, NAMATR, NUMPRP, NAMPRP) } \\
\hline $\begin{array}{l}\text { Variables in } \\
\text { Call }\end{array}$ & Name & $I / O$ & Type & Description \\
\hline & IUNIT & input & integer & Data base file unit number. \\
\hline & I!JNPRT & Input & integer & $\begin{array}{l}\text { Display file unit number ( } \leq 0 \text { for standard } \\
\text { output). }\end{array}$ \\
\hline & NUMATR & Input & integer & The number of attributes. \\
\hline & NAMATR & Input & $\begin{array}{l}\text { char*8 } \\
\text { array }\end{array}$ & $\begin{array}{l}\text { The attribute names: } \\
\text { NAMATR (NUMATR) }\end{array}$ \\
\hline & NUMPRP & Input & integer & The number of properties. \\
\hline & NAMPRP & Input & $\begin{array}{l}\text { char*8 } \\
\text { array }\end{array}$ & $\begin{array}{l}\text { The property names: } \\
\text { NAMPRP (NUMPRP) }\end{array}$ \\
\hline
\end{tabular}




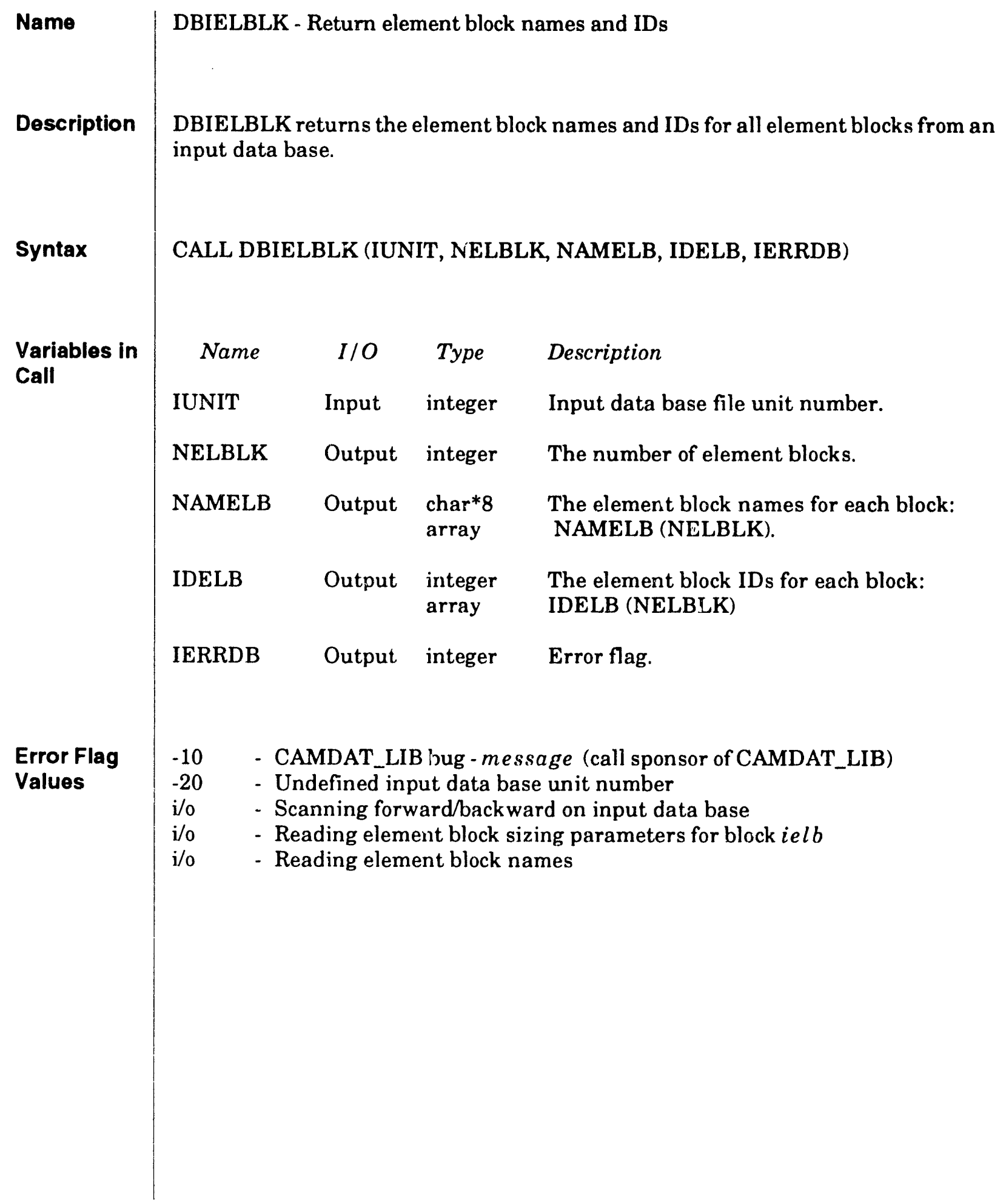




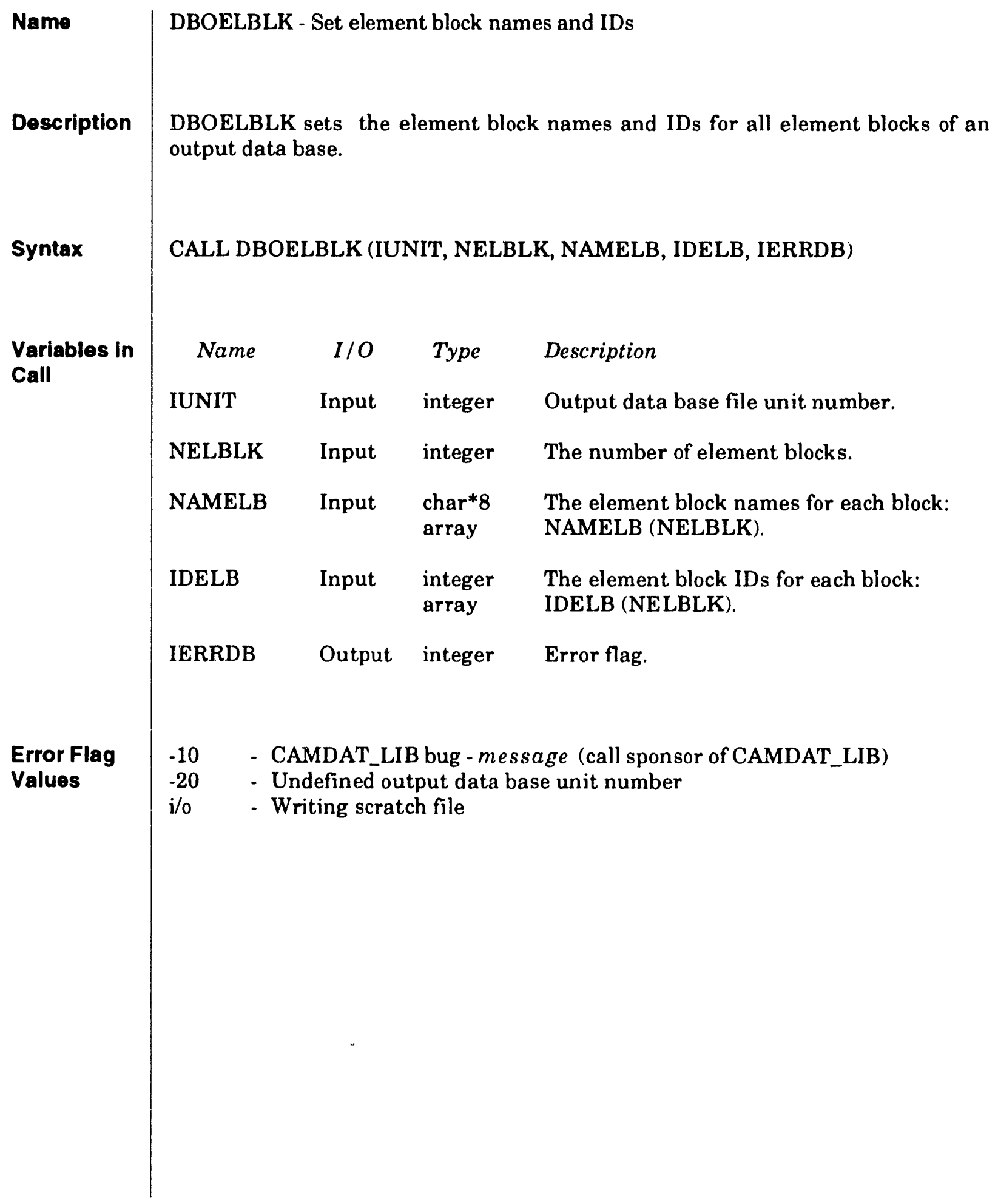




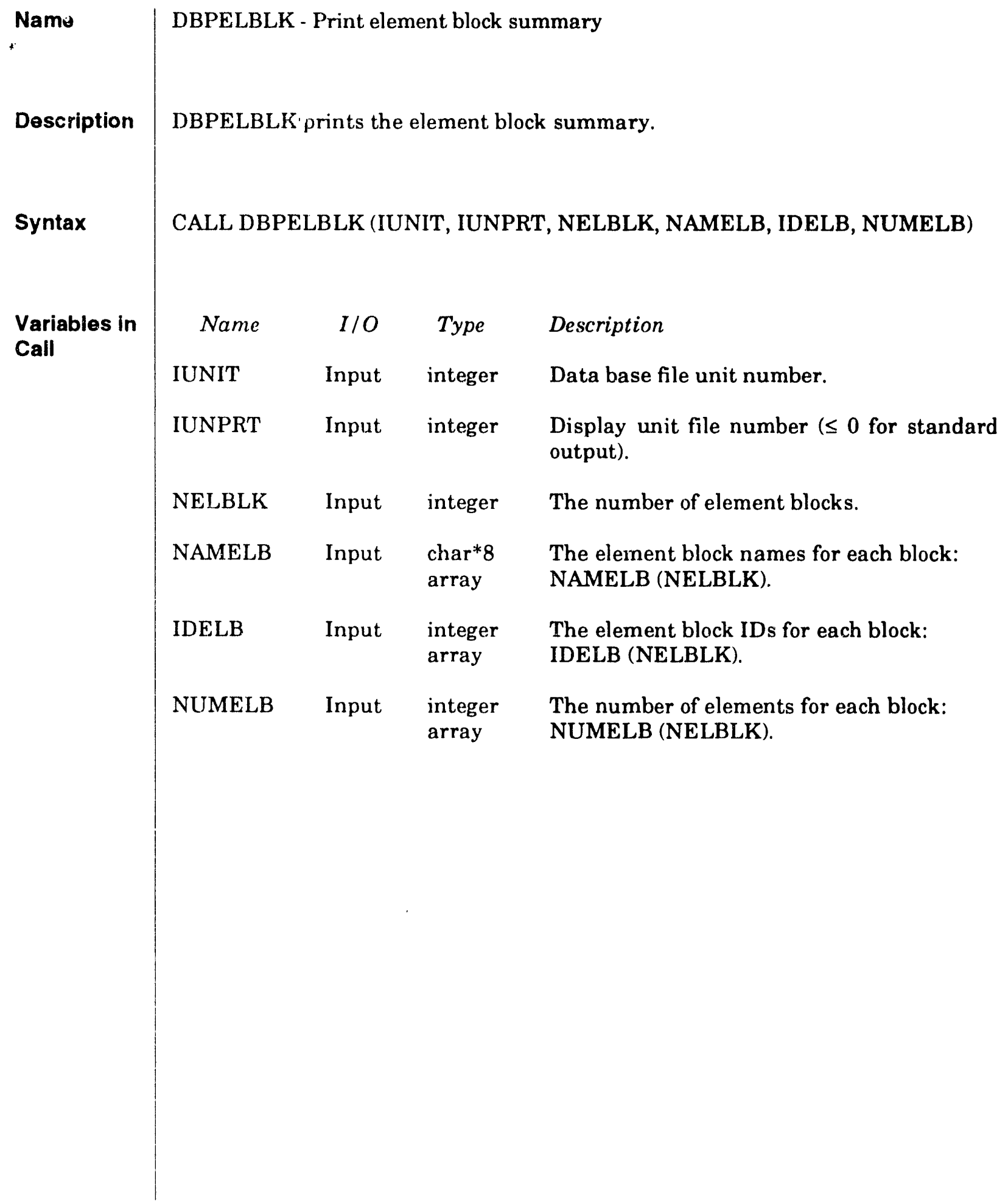




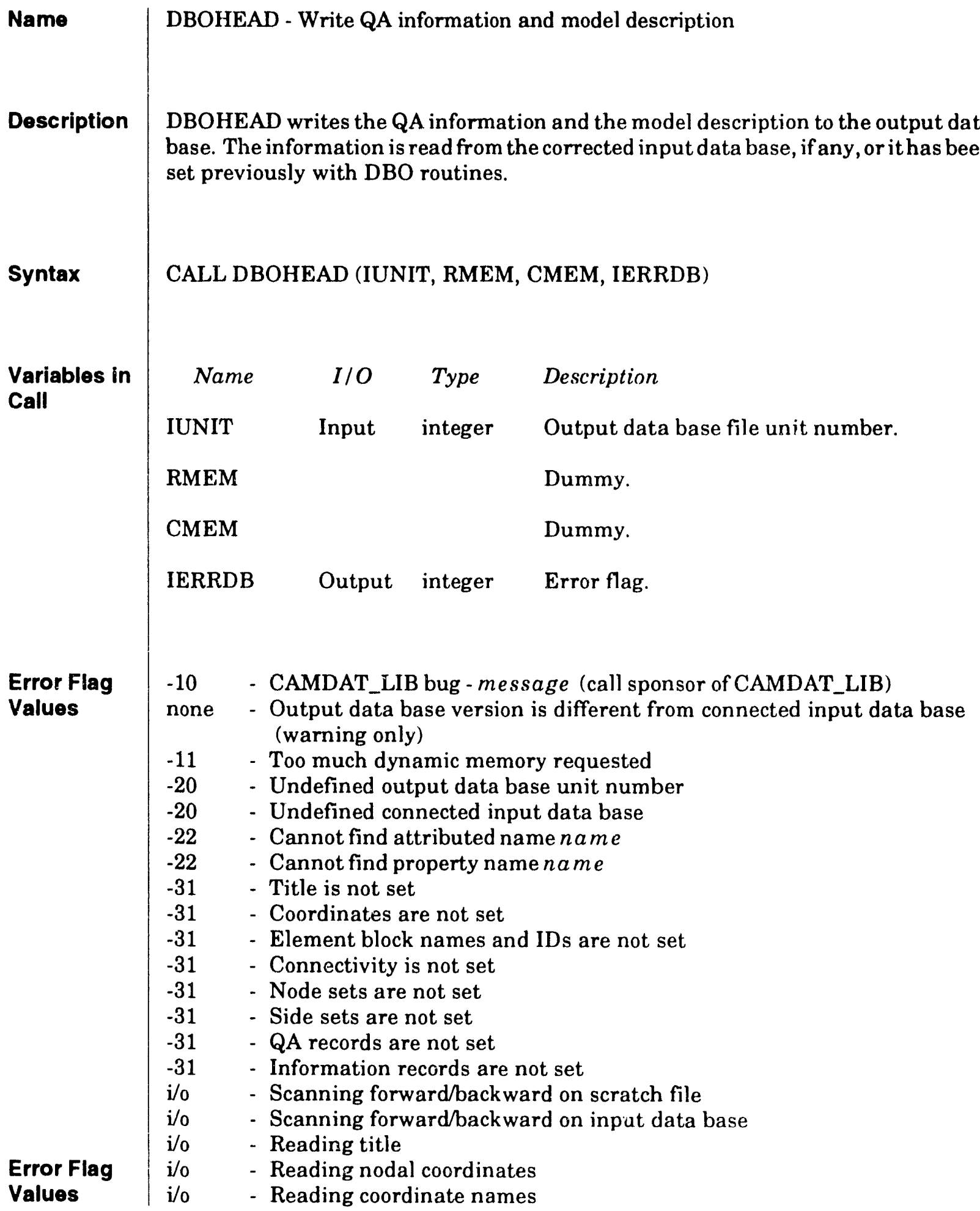




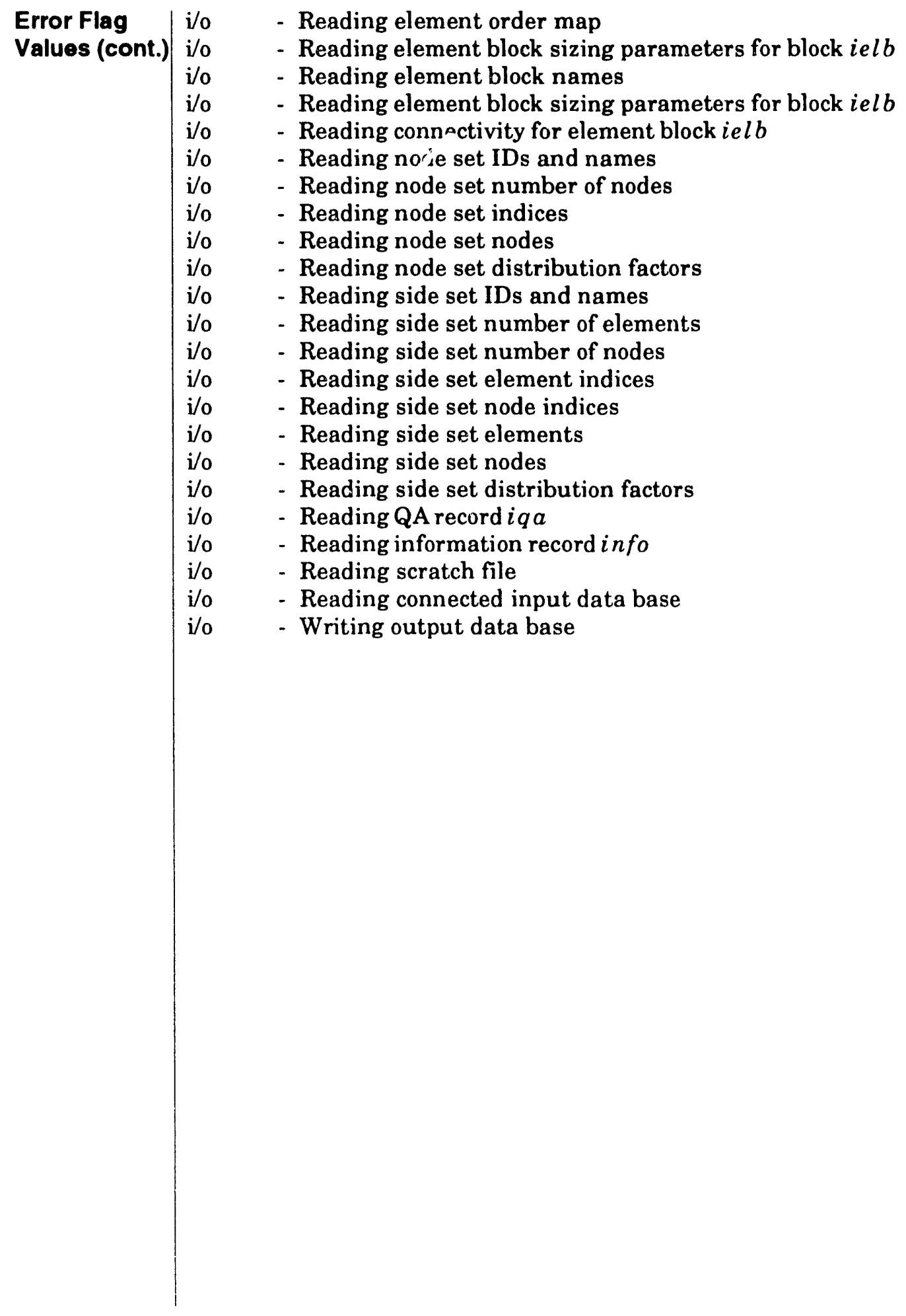




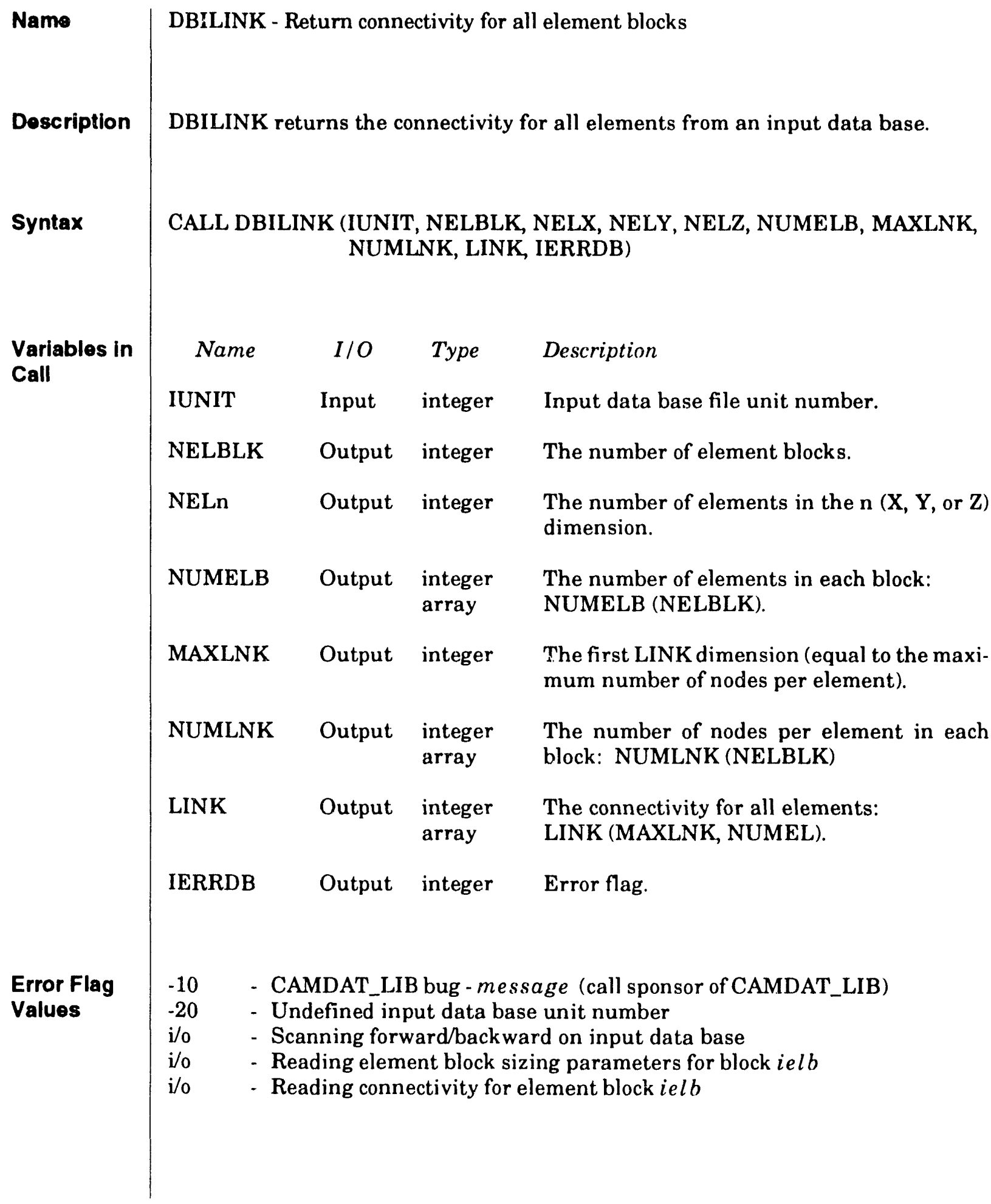




\begin{tabular}{|c|c|c|c|c|}
\hline Name & \multicolumn{4}{|c|}{ DBOLINK - Set connectivity for all element blocks } \\
\hline Description & \multicolumn{4}{|c|}{$\begin{array}{l}\text { DBOLINK sets the connectivity for all elements of an output data base. Note that } \\
\text { the number of elements in the } X, Y \text {, and } Z \text { dimensions are ignored at this time. }\end{array}$} \\
\hline Syntax & \multicolumn{4}{|c|}{$\begin{array}{l}\text { CALL DBOLINK (IUNIT, NELBLK, NELX, NELY, NELZ, NUMELB, MAXLNK, } \\
\text { NUMLNK, LINK, IERRDB) }\end{array}$} \\
\hline \multirow{9}{*}{$\begin{array}{l}\text { Variables in } \\
\text { Call }\end{array}$} & Name & $1 / O$ & Type & Description \\
\hline & IUNIT & Input & integer & Output data base file unit number. \\
\hline & NEBLK & Input & integer & The number of element blocks. \\
\hline & NELn & Input & integer & $\begin{array}{l}\text { The number of elements in the } n(X, Y \text {, or } Z) \\
\text { dimension. }\end{array}$ \\
\hline & NUMELB & Input & $\begin{array}{l}\text { integer } \\
\text { array }\end{array}$ & $\begin{array}{l}\text { The number of elements in each block: } \\
\text { NUMELB (NELBLK). }\end{array}$ \\
\hline & MAXLNK & Input & integer & $\begin{array}{l}\text { The first LINK dimension (at least the maxi- } \\
\text { mum number of nodes per element). }\end{array}$ \\
\hline & NUMLNK & Input & $\begin{array}{l}\text { integer } \\
\text { array }\end{array}$ & $\begin{array}{l}\text { The number of nodes per element in each } \\
\text { block: NUMLNK(NELBLK). If NUMLNK(1) } \\
\text { is }<0 \text {, MAXLNK is used for all blocks. }\end{array}$ \\
\hline & LINK & Input & $\begin{array}{l}\text { integer } \\
\text { array }\end{array}$ & $\begin{array}{l}\text { The connectivity for all elements: } \\
\text { LINK (MAXLNK, NUMEL). }\end{array}$ \\
\hline & IERRDB & Output & integer & Error flag. \\
\hline $\begin{array}{l}\text { Error Flag } \\
\text { Values }\end{array}$ & \multicolumn{4}{|c|}{$\begin{array}{ll}-10 & \text { - CAMDAT_LIB bug - message (call sponsor of CAMDAT_LIB) } \\
-20 & \text { - Undefined output data base unit number } \\
\text { i/o } & \text { - Writing scratch file }\end{array}$} \\
\hline
\end{tabular}




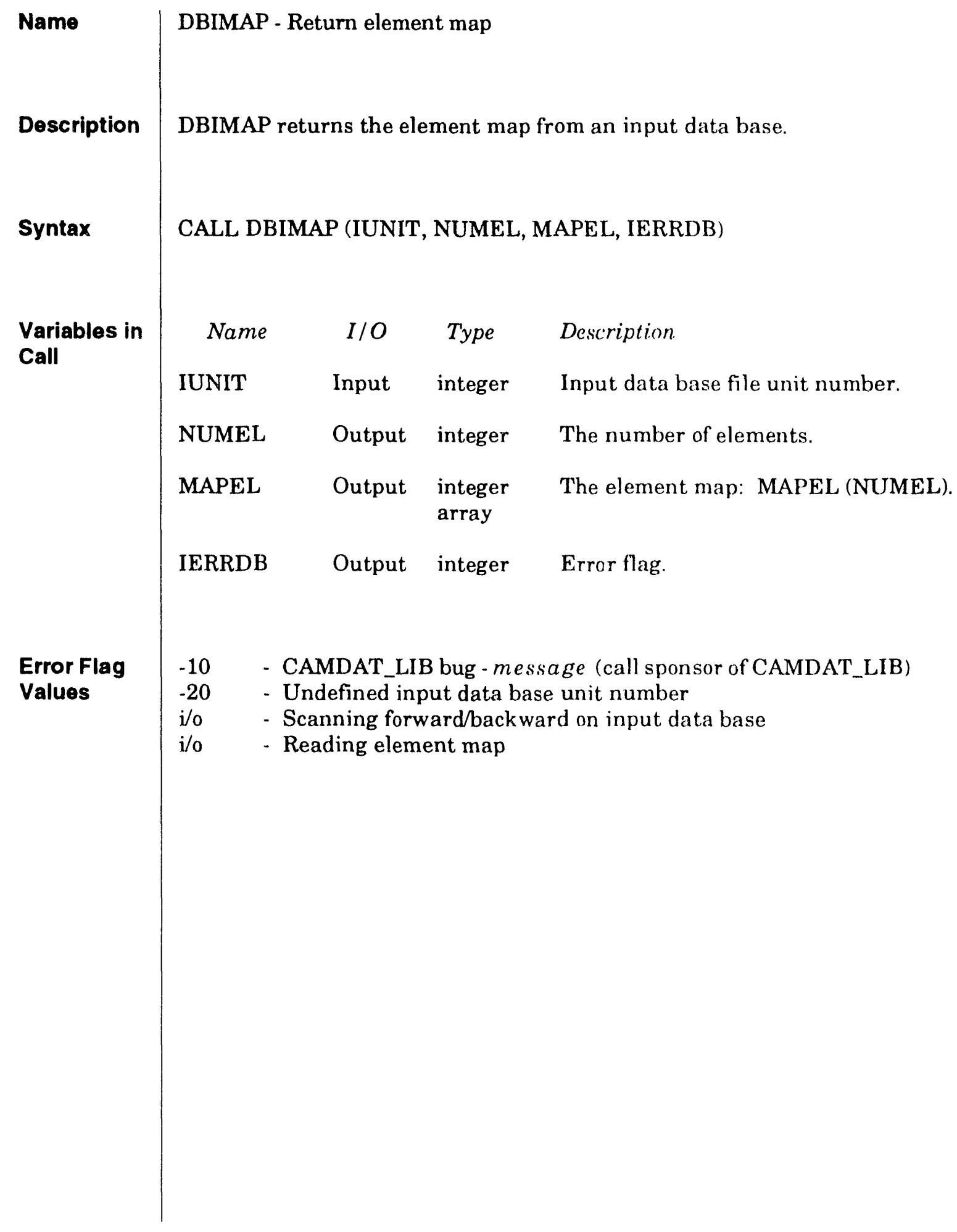




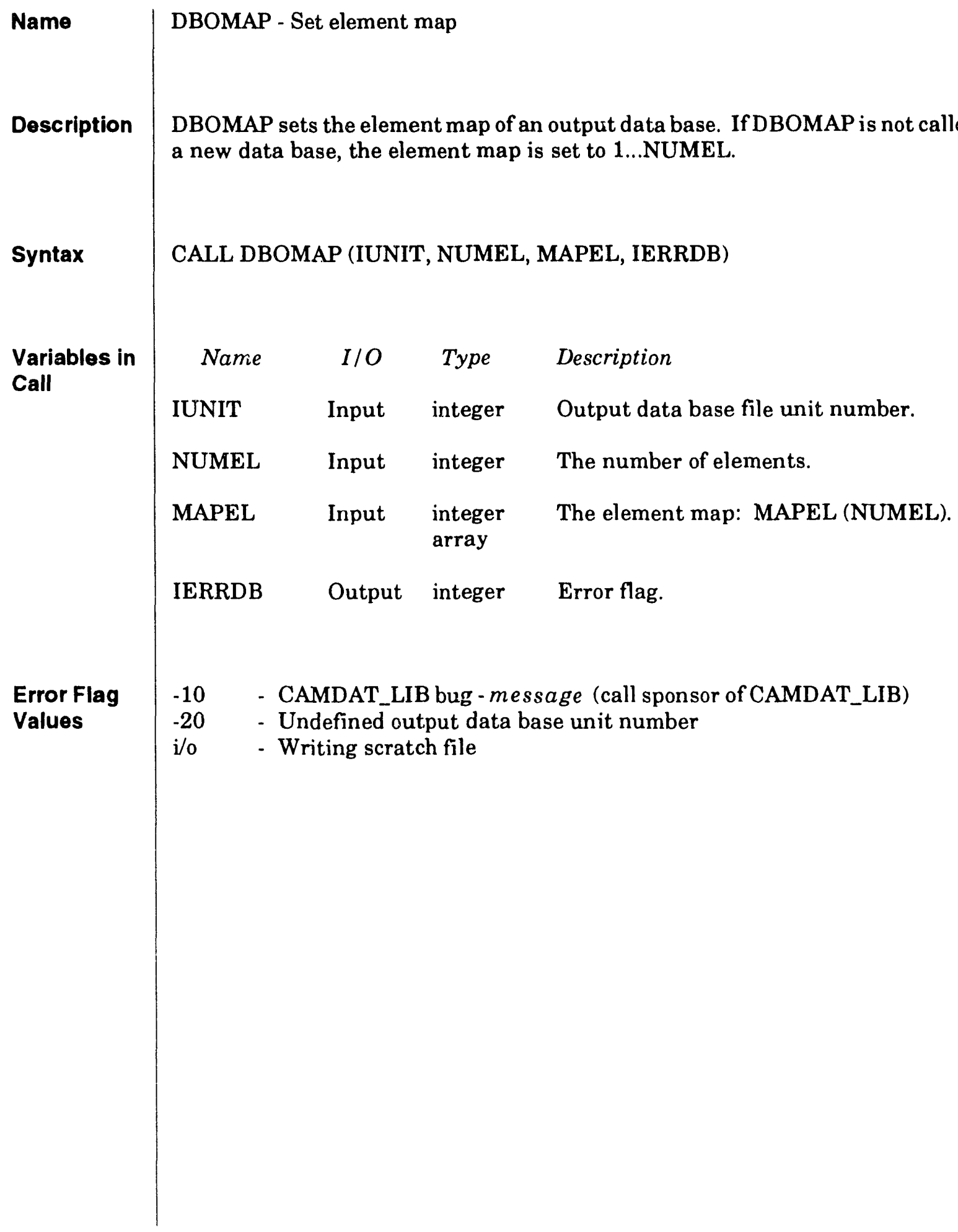




\begin{tabular}{|c|c|c|c|c|}
\hline Name & \multicolumn{4}{|c|}{ DBINDSET - Return node sets } \\
\hline Description & \multicolumn{4}{|c|}{ DBINDSET returns the node set information from an input data base. } \\
\hline Syntax & \multicolumn{4}{|c|}{$\begin{array}{l}\text { CALL DBINDSET (IUNIT, NUMNPS, NAMNPS, IDNPS, NNNPS, IXNNPS, } \\
\text { LTNNPS, FACNPS, IERRDB) }\end{array}$} \\
\hline \multirow{10}{*}{$\begin{array}{l}\text { Variables in } \\
\text { Call }\end{array}$} & Name & $I / O$ & Type & Description \\
\hline & IUNIT & Input & integer & Input data base file unit number. \\
\hline & NUMNPS & Output & integer & The number of node sets. \\
\hline & NAMNPS & Output & $\begin{array}{l}\text { char*8 } \\
\text { array }\end{array}$ & $\begin{array}{l}\text { The name for each node set: } \\
\text { NAMNPS (NUMNPS). }\end{array}$ \\
\hline & IDNPS & Output & $\begin{array}{l}\text { integer } \\
\text { array }\end{array}$ & $\begin{array}{l}\text { The node set ID for each node set: } \\
\text { IDNPS (NUMNPS). }\end{array}$ \\
\hline & NNNPS & Output & $\begin{array}{l}\text { integer } \\
\text { array }\end{array}$ & $\begin{array}{l}\text { The number of nodes for each set: } \\
\text { NNNPS (NUMNPS) }\end{array}$ \\
\hline & IXNNPS & Output & $\begin{array}{l}\text { integer } \\
\text { array }\end{array}$ & $\begin{array}{l}\text { The index of the first node for each set: } \\
\text { IXNNPS (NUMNPS). }\end{array}$ \\
\hline & LTNNPS & Output & $\begin{array}{l}\text { integer } \\
\text { array }\end{array}$ & The nodes for all sets: LTNNPS (LNPSNL). \\
\hline & FACNPS & Output & $\begin{array}{l}\text { real } \\
\text { array }\end{array}$ & $\begin{array}{l}\text { The distribution factors for all sets: } \\
\text { FACNPS (LNPSNL). }\end{array}$ \\
\hline & IERRDB & Output & integer & Error flag. \\
\hline $\begin{array}{l}\text { Error Flag } \\
\text { Values }\end{array}$ & \multicolumn{4}{|c|}{$\begin{array}{ll}-10 & \text { - CAMDAT_LIB bug - message (call sponsor of CAMDAT_LIB) } \\
-20 & \text { - Undefined input data base unit number } \\
\text { i/o } & \text { - Scanning forward/backward on input data base } \\
\text { i/o } & \text { - Reading node set IDs and names } \\
\text { i/o } & \text { - Reading node set number of nodes } \\
\text { i/o } & \text { - Reading node set indices } \\
\text { i/o } & \text { - Reading node set nodes } \\
\text { i/o } & \text { - Reading node set distribution factors }\end{array}$} \\
\hline
\end{tabular}




\begin{tabular}{|c|c|c|c|c|}
\hline Name & \multicolumn{4}{|c|}{ DBONDSET - Set node sets } \\
\hline Description & \multicolumn{4}{|c|}{ DBONDSET sets the node set information of an output data base. } \\
\hline Syntax & \multicolumn{4}{|c|}{$\begin{array}{l}\text { CALL DBONDSET (IUNIT, NUMNPS, NAMNPS, IDN'S, NNNPS, IXNNPS, } \\
\text { LTNNPS, FACNPS, IERRDB) }\end{array}$} \\
\hline \multirow{10}{*}{$\begin{array}{l}\text { Variables in } \\
\text { Call }\end{array}$} & Name & $1 / O$ & Type & Description \\
\hline & IUNIT & Input & integer & Output data base file unit number. \\
\hline & NUMNPS & Input & integer & The number of node sets. \\
\hline & NAMNPS & Input & $\begin{array}{l}\text { char*8 } \\
\text { array }\end{array}$ & $\begin{array}{l}\text { The name for each node set: } \\
\text { NAMNPS (NUMNPS). }\end{array}$ \\
\hline & IDNPS & Input & $\begin{array}{l}\text { integer } \\
\text { array }\end{array}$ & $\begin{array}{l}\text { The node set ID for each node set: } \\
\text { IDNPS (NUMNPS). }\end{array}$ \\
\hline & NNNPS & Input & $\begin{array}{l}\text { integer } \\
\text { array }\end{array}$ & $\begin{array}{l}\text { The number of nodes for each set: } \\
\text { NNNPS (NUMNPS). }\end{array}$ \\
\hline & IXNNPS & Input & $\begin{array}{l}\text { integer } \\
\text { array }\end{array}$ & $\begin{array}{l}\text { The index of the first node for each set: } \\
\text { IXNNPS (NUMNPS). }\end{array}$ \\
\hline & LTNNPS & Input & $\begin{array}{l}\text { integer } \\
\text { array }\end{array}$ & The nodes for all sets: LTNNPS (LNPSNL). \\
\hline & FACNPS & Input & $\begin{array}{l}\text { real } \\
\text { array }\end{array}$ & $\begin{array}{l}\text { The distribution factors for all sets: } \\
\text { FACNPS (LNPSNL). }\end{array}$ \\
\hline & IERRDB & Output & integer & Error flag. \\
\hline $\begin{array}{l}\text { Error Flag } \\
\text { Values }\end{array}$ & \multicolumn{4}{|c|}{$\begin{array}{ll}-10 & \text { - CAMDAT_LIB bug - message (call sponsor of CAMDAT_LIB) } \\
-20 & \text { - Undefined output data base unit number } \\
\mathrm{i} / \mathrm{o} & \text { - Writing scratch file }\end{array}$} \\
\hline
\end{tabular}




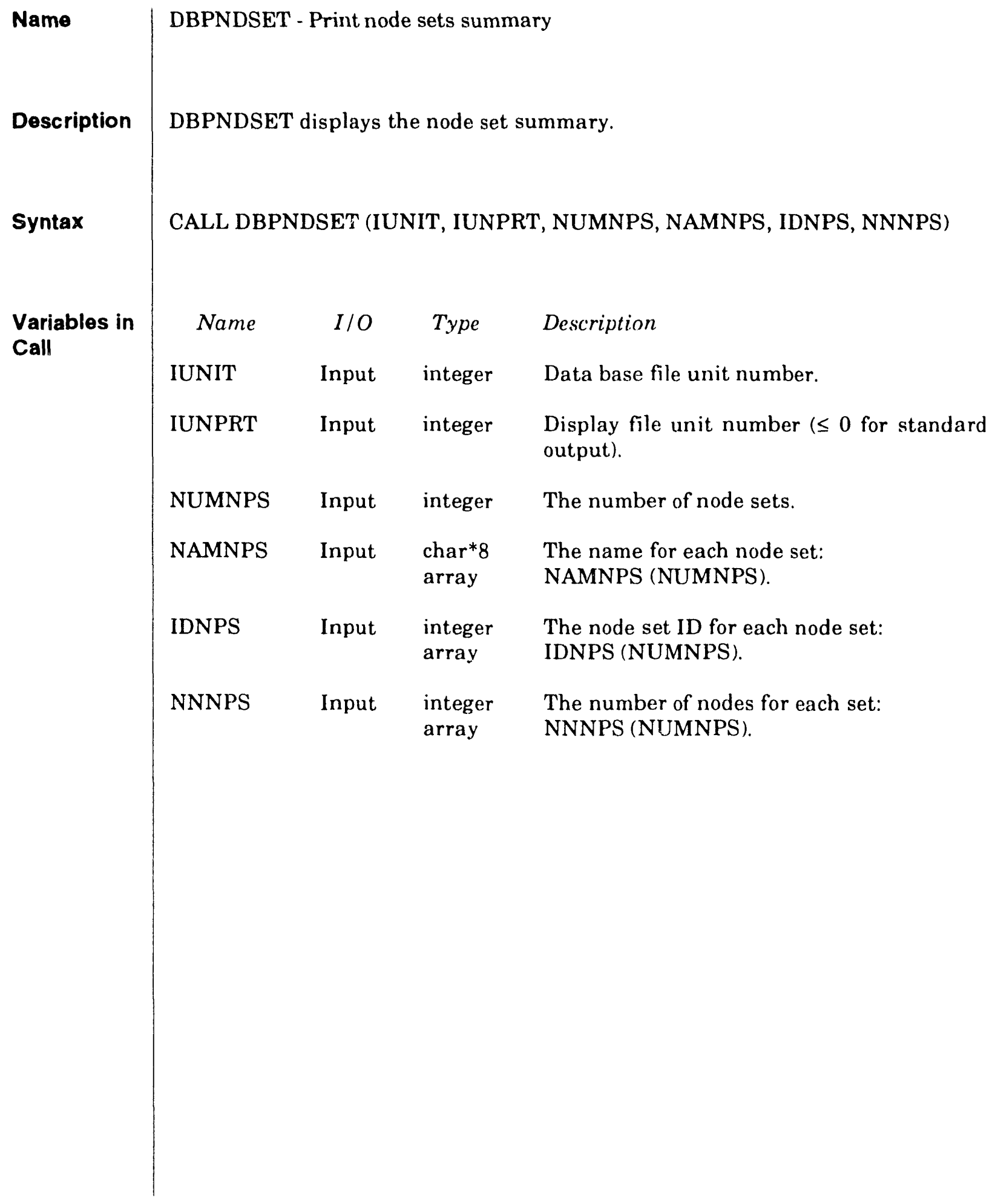




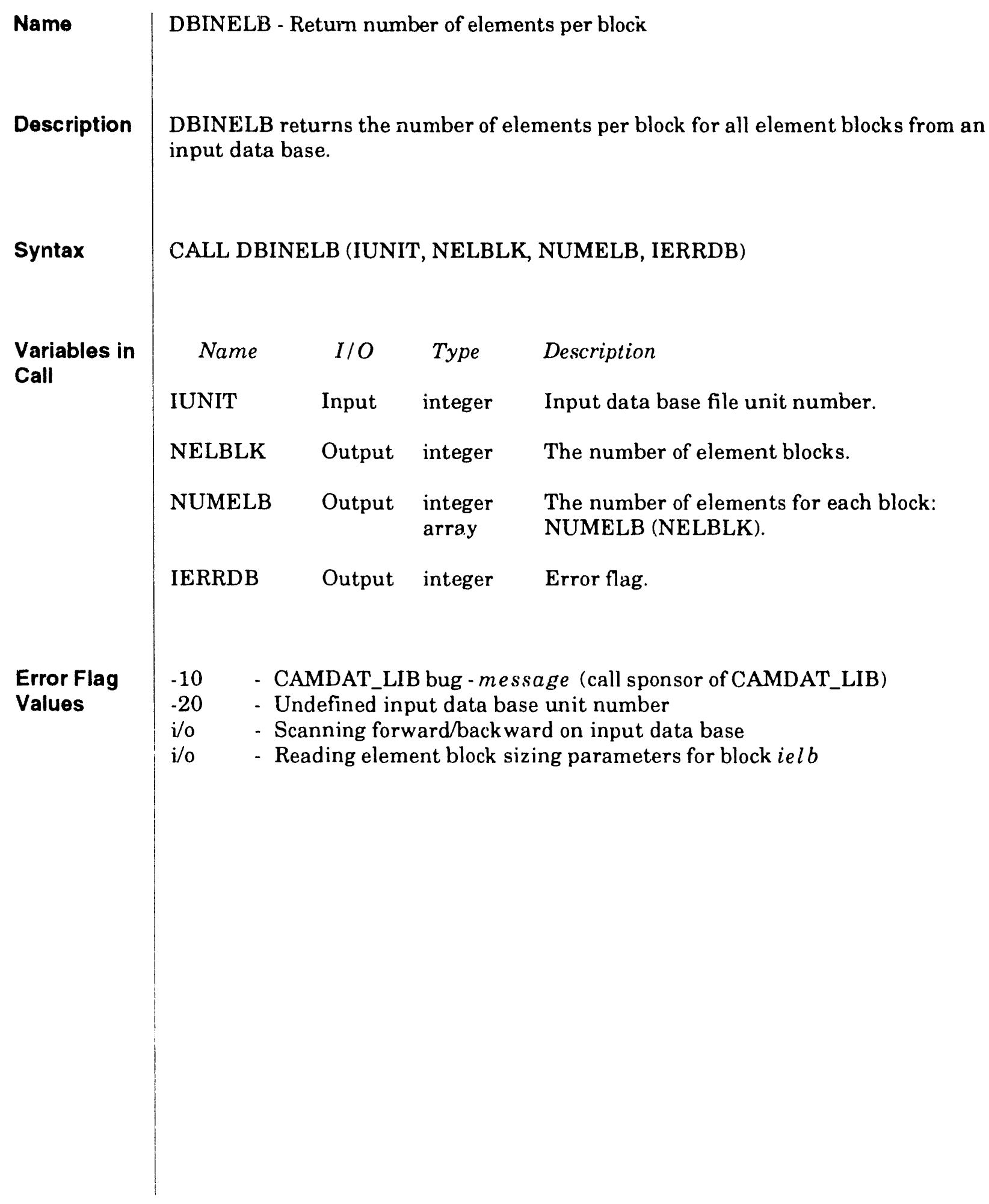




\begin{tabular}{|c|c|c|c|c|}
\hline Name & \multicolumn{4}{|c|}{ DBIPROP - Return property values for all element blocks } \\
\hline Description & \multicolumn{4}{|c|}{$\begin{array}{l}\text { DBIPROP returns the requested property for all element blocks from an input data } \\
\text { base. }\end{array}$} \\
\hline Syntax & \multicolumn{4}{|c|}{$\begin{array}{l}\text { 'ALL DBIPROP (IUNIT, NAME, INDEX, ISPROK, PROP, RMEM, CMEM, } \\
\text { IERRDB) }\end{array}$} \\
\hline \multirow{9}{*}{$\begin{array}{l}\text { Variables in } \\
\text { Call }\end{array}$} & Name & $I / O$ & Type & Description \\
\hline & IUNIT & Input & integer & Input data base file unit number. \\
\hline & NAME & $\mathrm{I} / \mathrm{O}$ & $\operatorname{char}{ }^{*}(*)$ & $\begin{array}{l}\text { The property name. If INDEX }>0 \text {, INDEX is } \\
\text { used and NAME is set (if LEN }(\text { NANE) }>1 \text { ). }\end{array}$ \\
\hline & INDEX & $\mathrm{I} / \mathrm{O}$ & integer & $\begin{array}{l}\text { The property index. If INDEX } \leq 0 \text {, NAME is } \\
\text { used and INDEX is set (if INDEX }<0 \text { ) }\end{array}$ \\
\hline & ISPROK & Output & $\begin{array}{l}\text { logical } \\
\text { array }\end{array}$ & $\begin{array}{l}\text { Property of block b exists if and only if } \\
\text { ISPROK(b). }\end{array}$ \\
\hline & PROP & Output & $\begin{array}{l}\text { real } \\
\text { array }\end{array}$ & $\begin{array}{l}\text { The property for all elements blocks: } \\
\text { PROP (NELBLK). }\end{array}$ \\
\hline & RMEi & & & Dummy. \\
\hline & CMEM & & & Dummy. \\
\hline & IERRDB & Output & integer & Error flag. \\
\hline $\begin{array}{l}\text { Error Flag } \\
\text { Values }\end{array}$ & $\begin{array}{l}-10 \\
-20 \\
-22\end{array}$ & \multicolumn{3}{|c|}{$\begin{array}{l}\text { - CAMDAT_LIB bug - message (call sponsor of CAMDAT_LIB) } \\
\text { - Undefined input data base unit number } \\
\text { - Cannot find property name name }\end{array}$} \\
\hline
\end{tabular}


Name
Description

Variables in
Call

\section{Error Flag} Values
DBOPROP - Set property values for all elements blocks

DBOPROP sets the values for a single property for all element blocks of an output data base. It also controls which properties, if any, will be copied from the connected input data base. By default, all properties are copied.

CALL DBOPROP (IUNIT, OPTION, NAME, ISPROK, PROP, IERRDB)

$\begin{array}{crc}\text { Name } & \text { I/O } & \text { Type } \\ \text { IUNIT } & \text { Input } & \text { integer } \\ \text { OPTION } & \text { Input } & \operatorname{char}^{*}(*)\end{array}$

\section{Description}

Output data base file unit number.

Action to be taken (case is unimportant; only the first character is checked):

RESET - Do not copy any properties from the connected input data base.

COPY - Copy named property from the connected input data base.

DELETE - Delete named property (only for copied properties).

ADD - Add named property or replace values if copied (default option).

NAME Input $\operatorname{char}^{*}\left({ }^{*}\right) \quad$ The property name. If OPTION $=$ COPY and NAME $=*$, all properties are copied.

ISPROK Input logical Property of block $b$ exists if and only if array ISPROK(b). ISPROK is not used if OPTION $=\mathrm{COPY}$.

PROP Input real The property for each element block: array PROP (NELBLK).

IERRDB Output integer Error flag.

-10 - CAMDAT_LIB bug - message (call sponsor of CAMDAT_LIB)

-20 - Undefined output data base unit number

-20 - Undefined connected input data base

-21 - Property is undefined for all blocks

-21 - Unknown actionaction

-23 - Cannot output properties on CAMDAT data base

i/o - Writing scratch file 


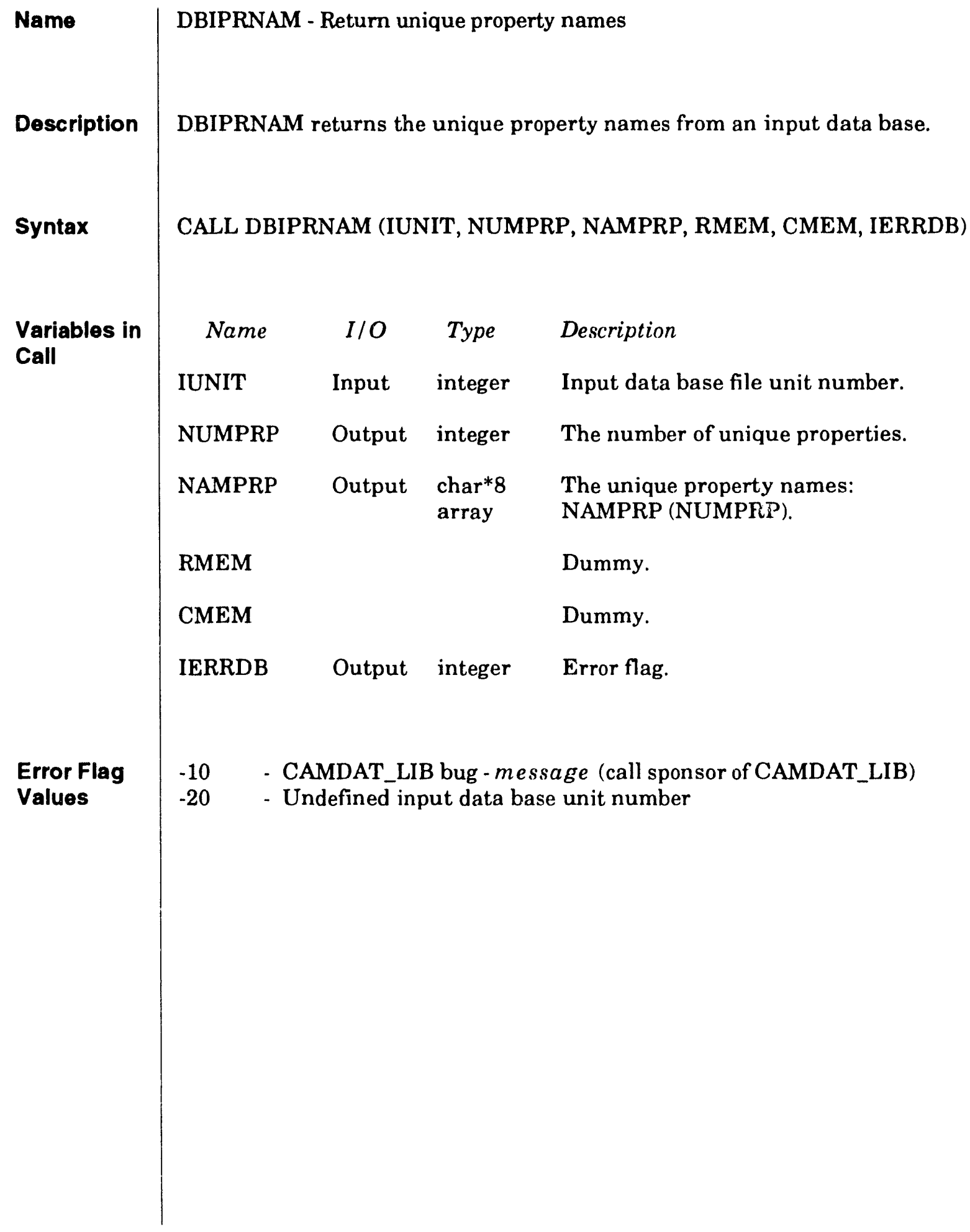




\begin{tabular}{|c|c|c|c|c|}
\hline Name & \multicolumn{4}{|c|}{ DBISDSET - Return side sets } \\
\hline Description & \multicolumn{4}{|c|}{ DBISDSET returns the side set information from an input data base. } \\
\hline Syntax & \multicolumn{4}{|c|}{$\begin{array}{l}\text { CALL DBISDSET (IUNIT, NUMESS, NAMES, IDS, NELEM, NNODES, IXELEM, } \\
\text { IXNODE, LTELEM, LTNODE, FACTOR, IERRDB) }\end{array}$} \\
\hline \multirow[t]{13}{*}{$\begin{array}{l}\text { Variables in } \\
\text { Call }\end{array}$} & Name & $I / O$ & Type & Description \\
\hline & IUNITI & Input & integer & Input data base file unit number. \\
\hline & NUMESS & Output & integer & The number of element side sets. \\
\hline & NAMES & Output & $\begin{array}{l}\text { char*8 } \\
\text { array }\end{array}$ & $\begin{array}{l}\text { The name for each side set: } \\
\text { NAMES (NUMESS). }\end{array}$ \\
\hline & IDS & Output & $\begin{array}{l}\text { integer } \\
\text { array }\end{array}$ & $\begin{array}{l}\text { The side set ID for each side set: } \\
\text { IDS (NUMESS). }\end{array}$ \\
\hline & NELEM & Output & $\begin{array}{l}\text { integer } \\
\text { array }\end{array}$ & $\begin{array}{l}\text { The number of elements for each set: } \\
\text { NELEM (NUMESS). }\end{array}$ \\
\hline & NNODES & Output & $\begin{array}{l}\text { integer } \\
\text { array }\end{array}$ & $\begin{array}{l}\text { The number of nodes for each set: } \\
\text { NNODES (NUMESS). }\end{array}$ \\
\hline & IXELEM & Output & $\begin{array}{l}\text { integer } \\
\text { array }\end{array}$ & $\begin{array}{l}\text { The index of the first element for each set: } \\
\text { IXELEM (NUMESS). }\end{array}$ \\
\hline & IXNODE & Output & $\begin{array}{l}\text { integer } \\
\text { array }\end{array}$ & $\begin{array}{l}\text { The index of the first node for each set: } \\
\text { IXNODE (NUMESS). }\end{array}$ \\
\hline & LTELEM & Output & $\begin{array}{l}\text { integer } \\
\text { array }\end{array}$ & $\begin{array}{l}\text { The elements for all sets: } \\
\text { LTELEM (LGELEL). }\end{array}$ \\
\hline & LTNODE & Output & $\begin{array}{l}\text { integer } \\
\text { array }\end{array}$ & The nodes for all sets: LTNODE(LGNODL). \\
\hline & FACTOR & Output & $\begin{array}{l}\text { real } \\
\text { array }\end{array}$ & $\begin{array}{l}\text { The distribution factors for all sets: } \\
\text { FACTOR (LGELEL). }\end{array}$ \\
\hline & IERRDB & Output & integer & Error flag. \\
\hline
\end{tabular}




\begin{tabular}{|c|c|c|c|c|}
\hline Name & \multicolumn{4}{|c|}{ DBOSDSET - Set side sets } \\
\hline Description & \multicolumn{4}{|c|}{ DBOSDSET sets the side set information of an output data base. } \\
\hline Syntax & \multicolumn{4}{|c|}{$\begin{array}{l}\text { CALL DBOSDSET (IUNIT, NUMESS, NAMES, IDS, NELEM, NNODES, } \\
\text { IXELEM, IXNODE, LTELEM, LTNODE, FACTOR, IERRDB) }\end{array}$} \\
\hline \multirow[t]{13}{*}{$\begin{array}{l}\text { Variables in } \\
\text { Call }\end{array}$} & Name & $I / O$ & Type & Description \\
\hline & IUNIT & Input & integer & Output data base file unit number. \\
\hline & NUMESS & Input & integer & The number of side sets. \\
\hline & NAMES & Input & $\begin{array}{l}\text { char*8 } \\
\text { array }\end{array}$ & $\begin{array}{l}\text { The name for each side set: } \\
\text { NAMES (NUMESS). }\end{array}$ \\
\hline & IDS & Input & $\begin{array}{l}\text { integer } \\
\text { array }\end{array}$ & $\begin{array}{l}\text { The side set ID for each side set: } \\
\text { IDS (NUMESS). }\end{array}$ \\
\hline & NELEM & Input & $\begin{array}{l}\text { integer } \\
\text { array }\end{array}$ & $\begin{array}{l}\text { The number of elements for each set: } \\
\text { NELEM (NUMESS). }\end{array}$ \\
\hline & NNODES & Input & $\begin{array}{l}\text { integer } \\
\text { array }\end{array}$ & $\begin{array}{l}\text { The number of nodes for each set: } \\
\text { NNODES (NUMESS). }\end{array}$ \\
\hline & IXELEM & Input & $\begin{array}{l}\text { integer } \\
\text { array }\end{array}$ & $\begin{array}{l}\text { The index of the first element for each set: } \\
\text { IXELEM (NUMESS). }\end{array}$ \\
\hline & IXNODE & Input & $\begin{array}{l}\text { integer } \\
\text { array }\end{array}$ & $\begin{array}{l}\text { The index of the first node for each set: } \\
\text { IXNODE (NUMESS). }\end{array}$ \\
\hline & LTELEM & Input & $\begin{array}{l}\text { integer } \\
\text { array }\end{array}$ & $\begin{array}{l}\text { The elements for all sets: } \\
\text { LTELEM (LGELEL). }\end{array}$ \\
\hline & LTNODE & Input & $\begin{array}{l}\text { integer } \\
\text { array }\end{array}$ & The nodes for all sets: LTNODE (LGNODL). \\
\hline & FACTOR & Input & $\begin{array}{l}\text { real } \\
\text { array }\end{array}$ & $\begin{array}{l}\text { The distribution factors for all sets: } \\
\text { FACTOR (LGELEL). }\end{array}$ \\
\hline & IERRDB & Output & integer & Error flag. \\
\hline
\end{tabular}




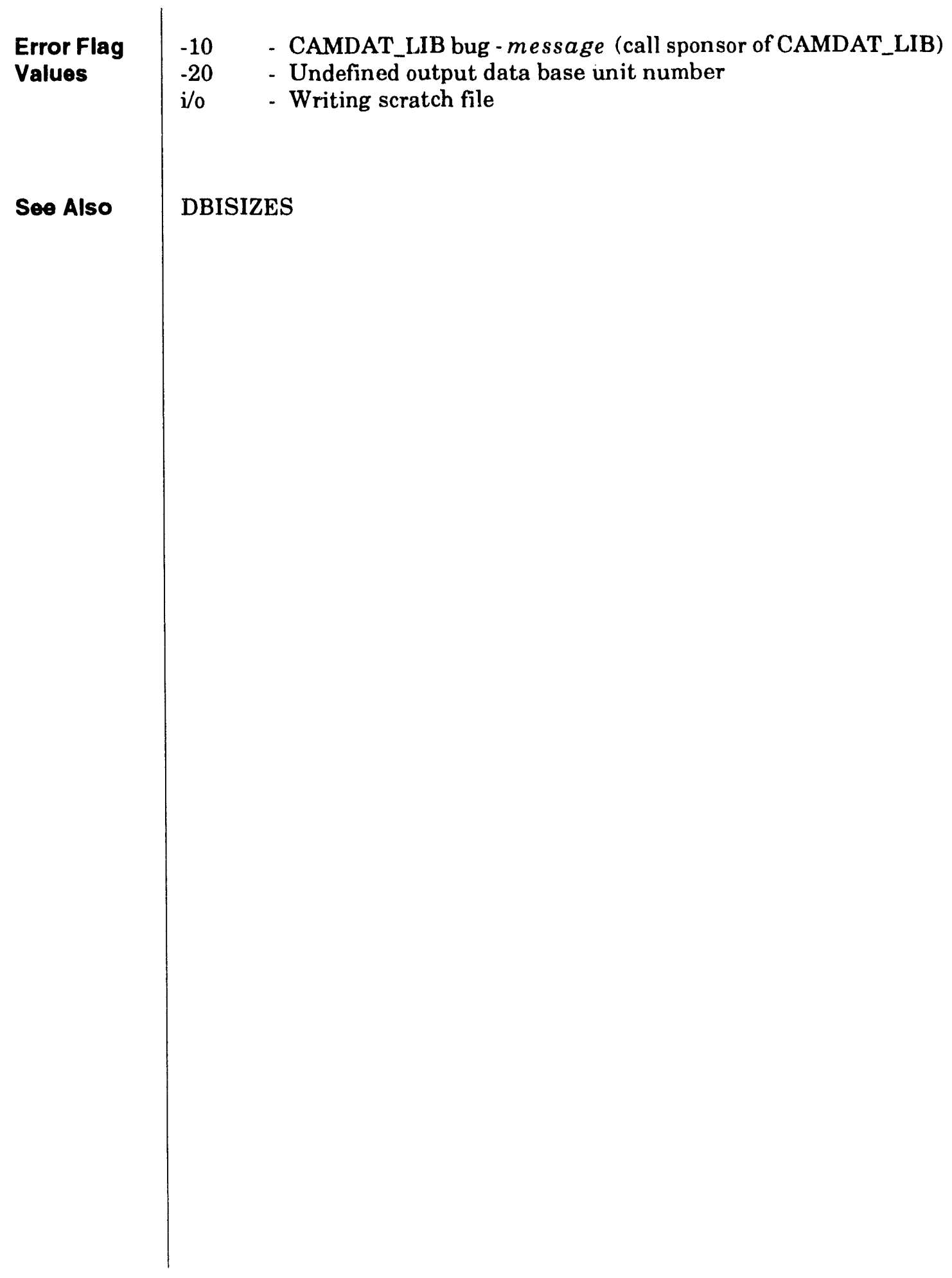




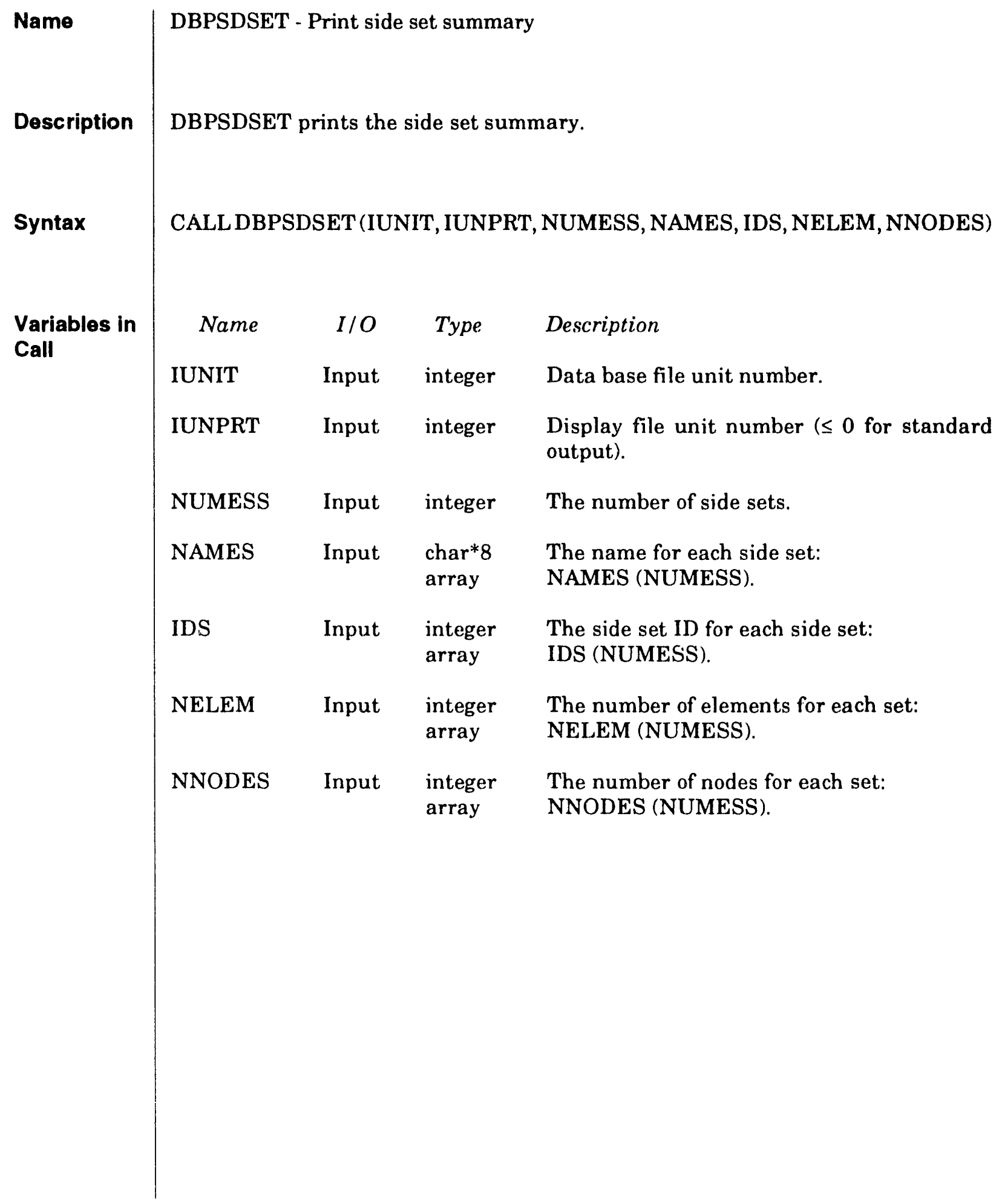




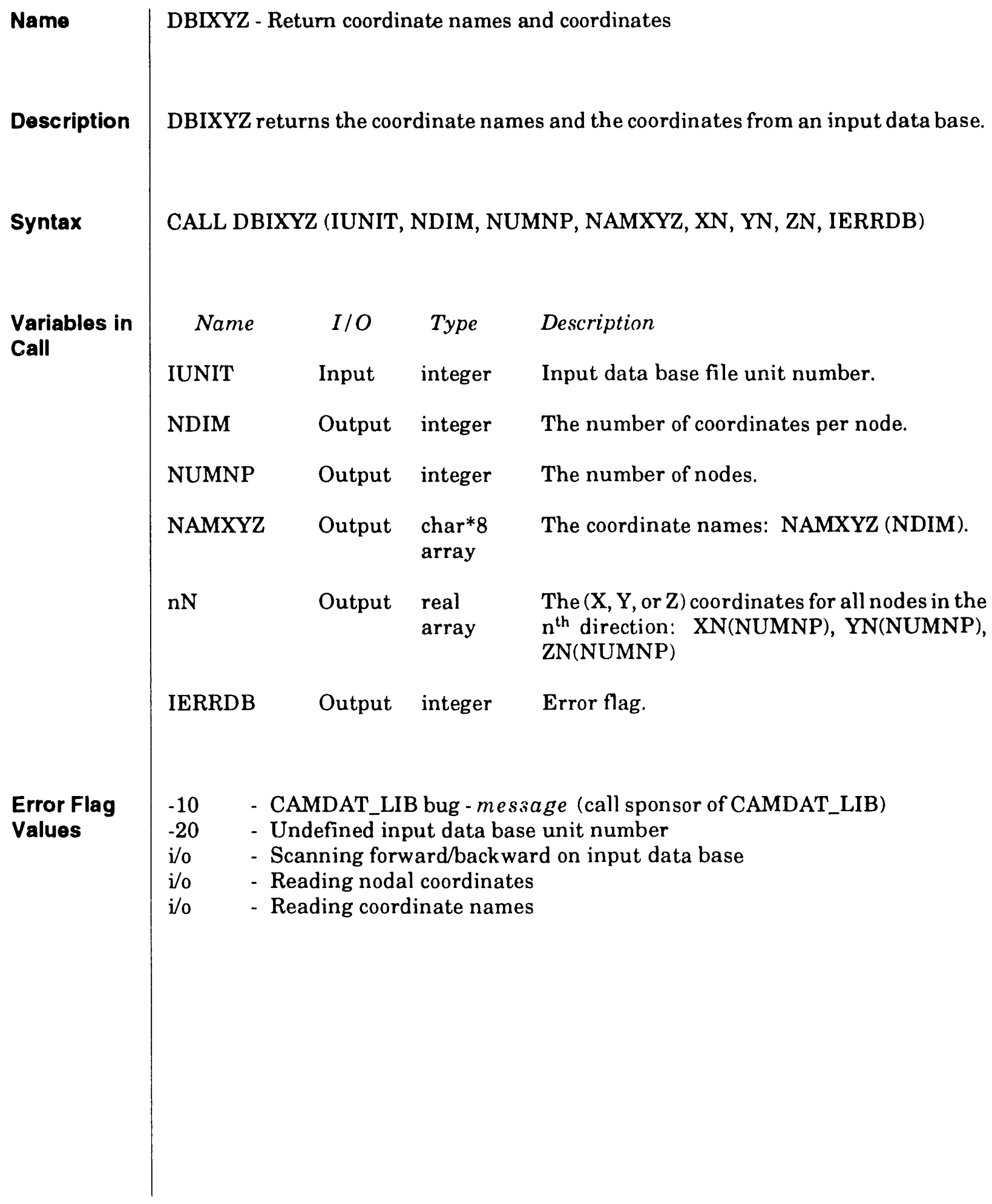




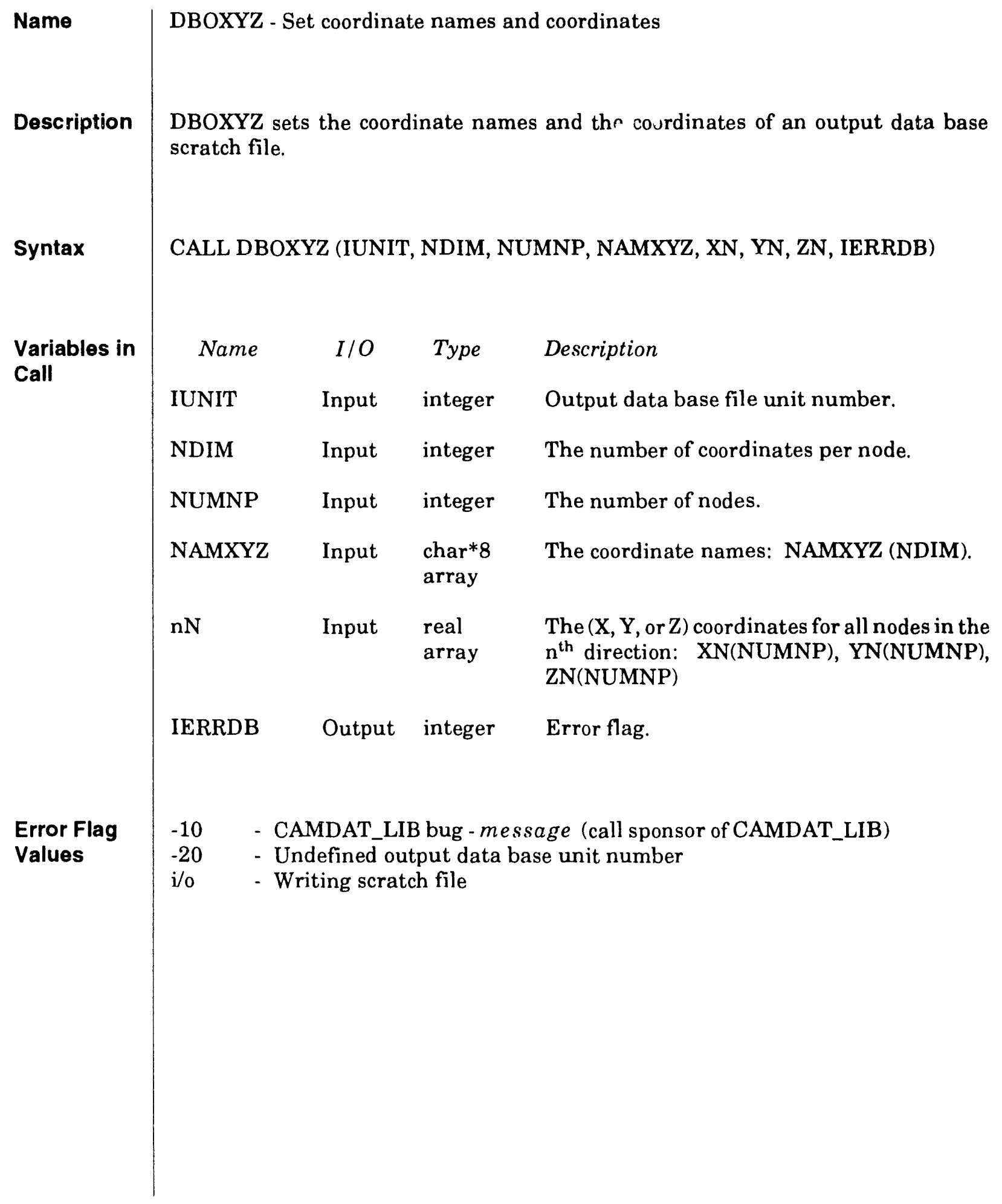




\subsubsection{Detailed Descriptions of Analysis Results Routines}

Detailed descriptions of CAMDAT_LIB analysis results routines are given on the following pages. This section describes functions that read and write analysis results data and related entities. These include simulation times and results variables (history, global, nodal, and elemental). 


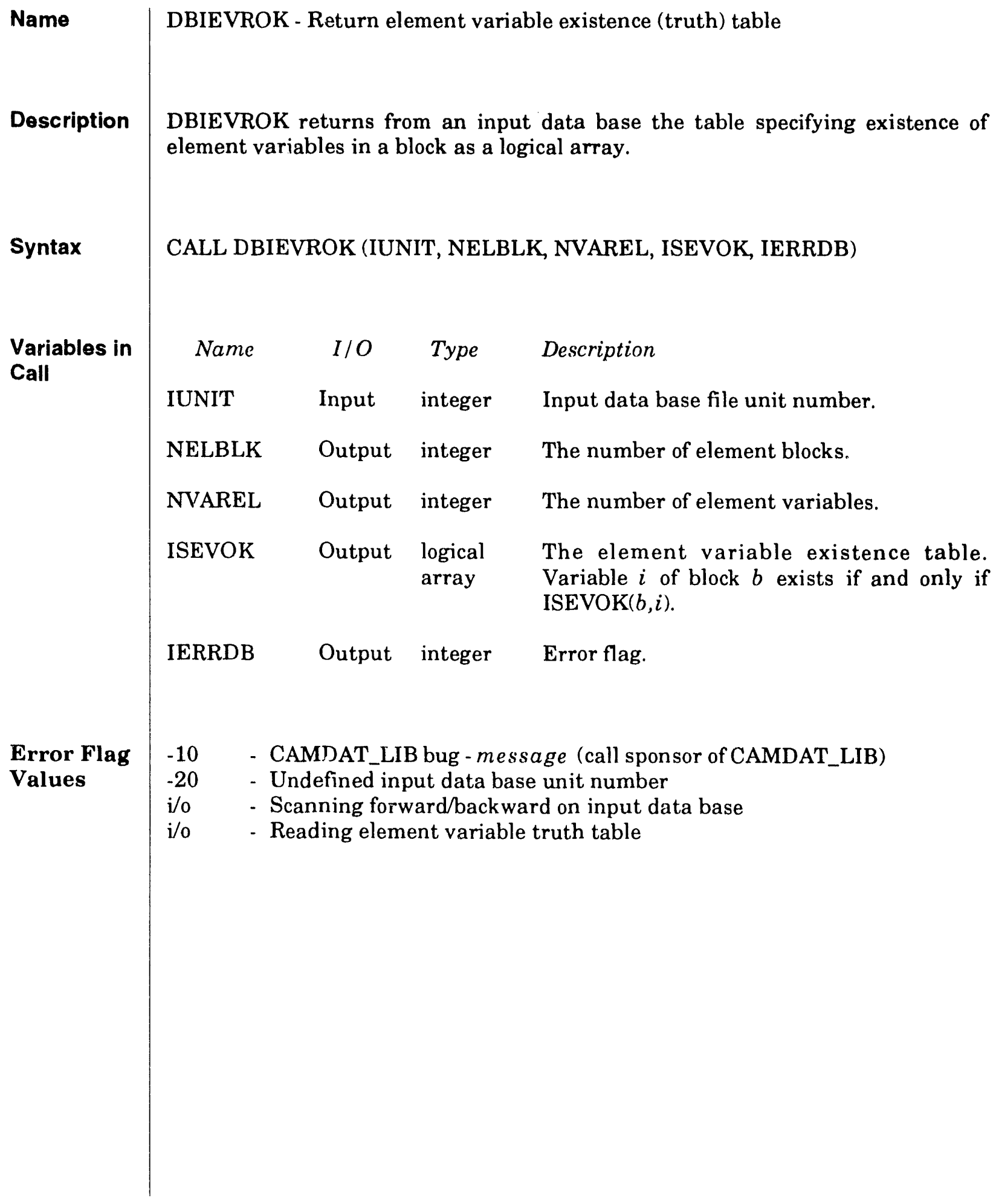




\section{DBOEVROK}

Subroutine

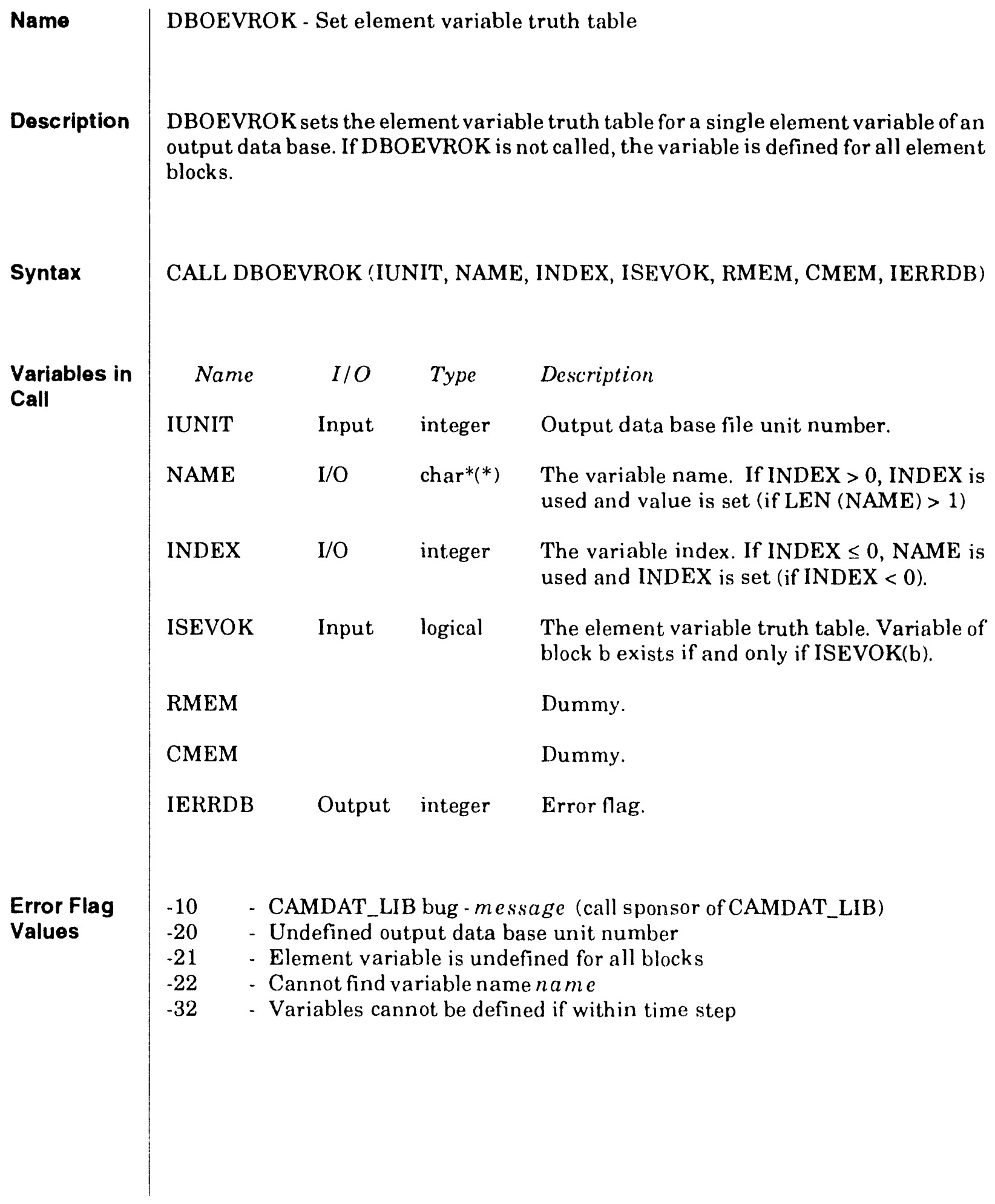




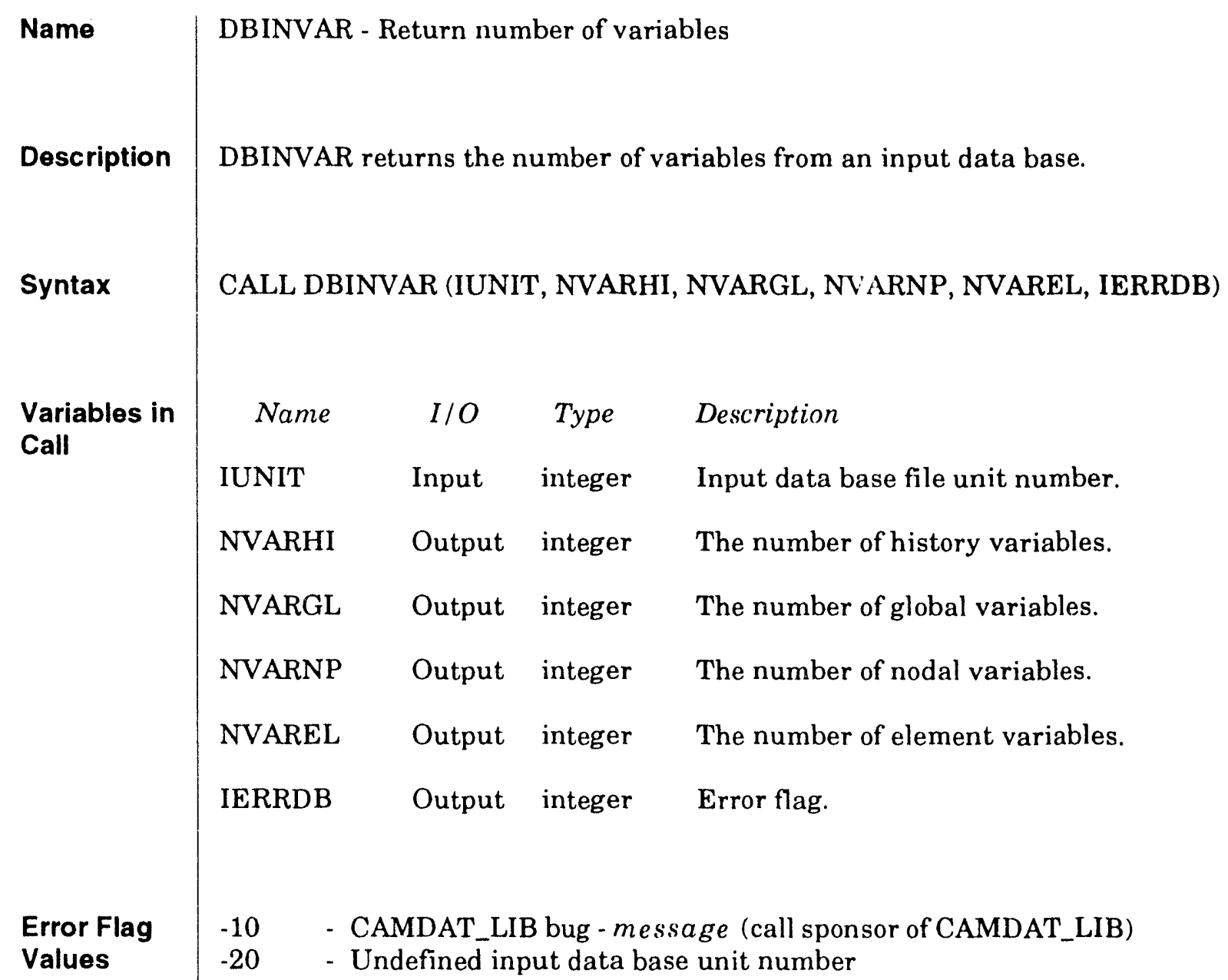




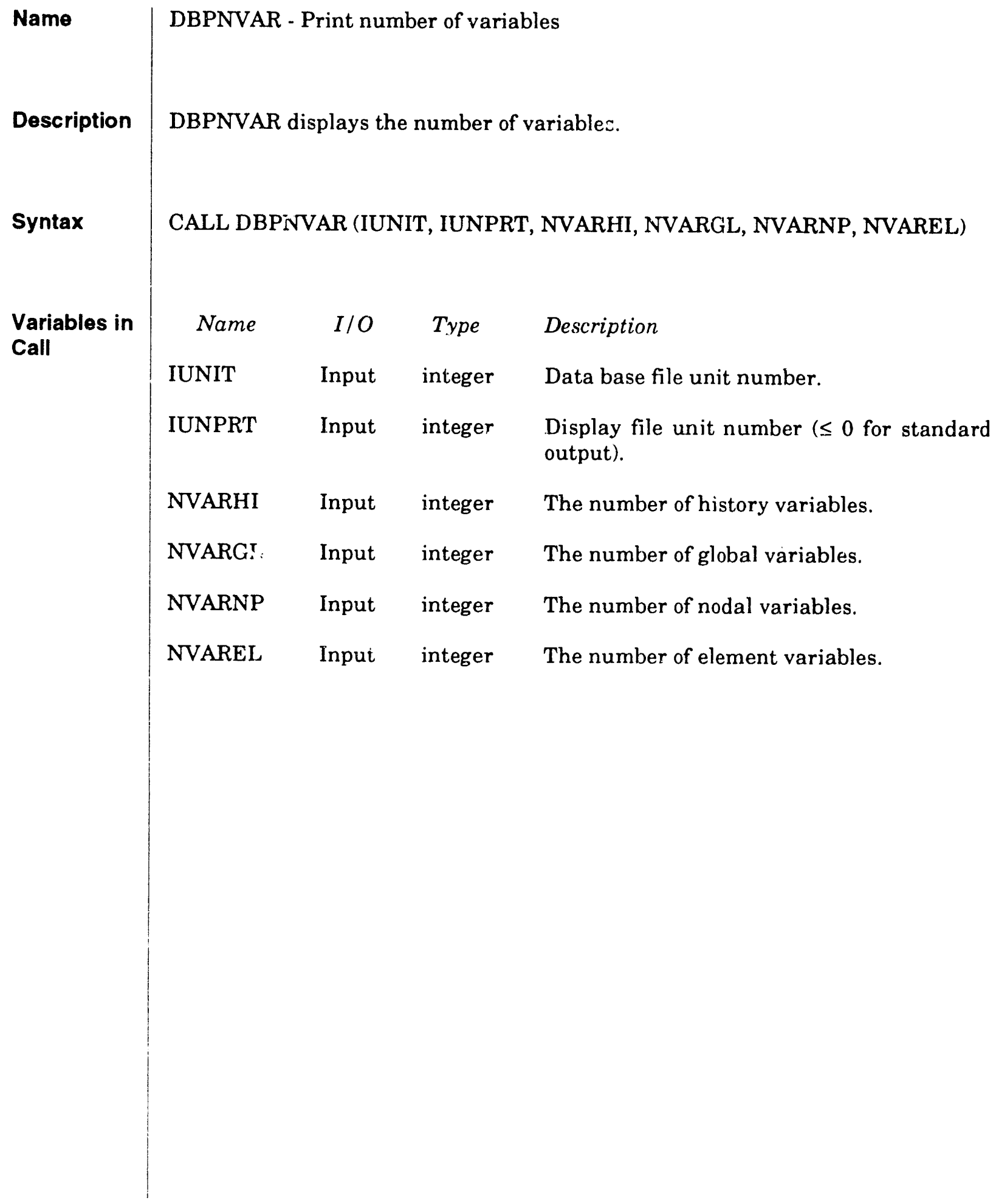




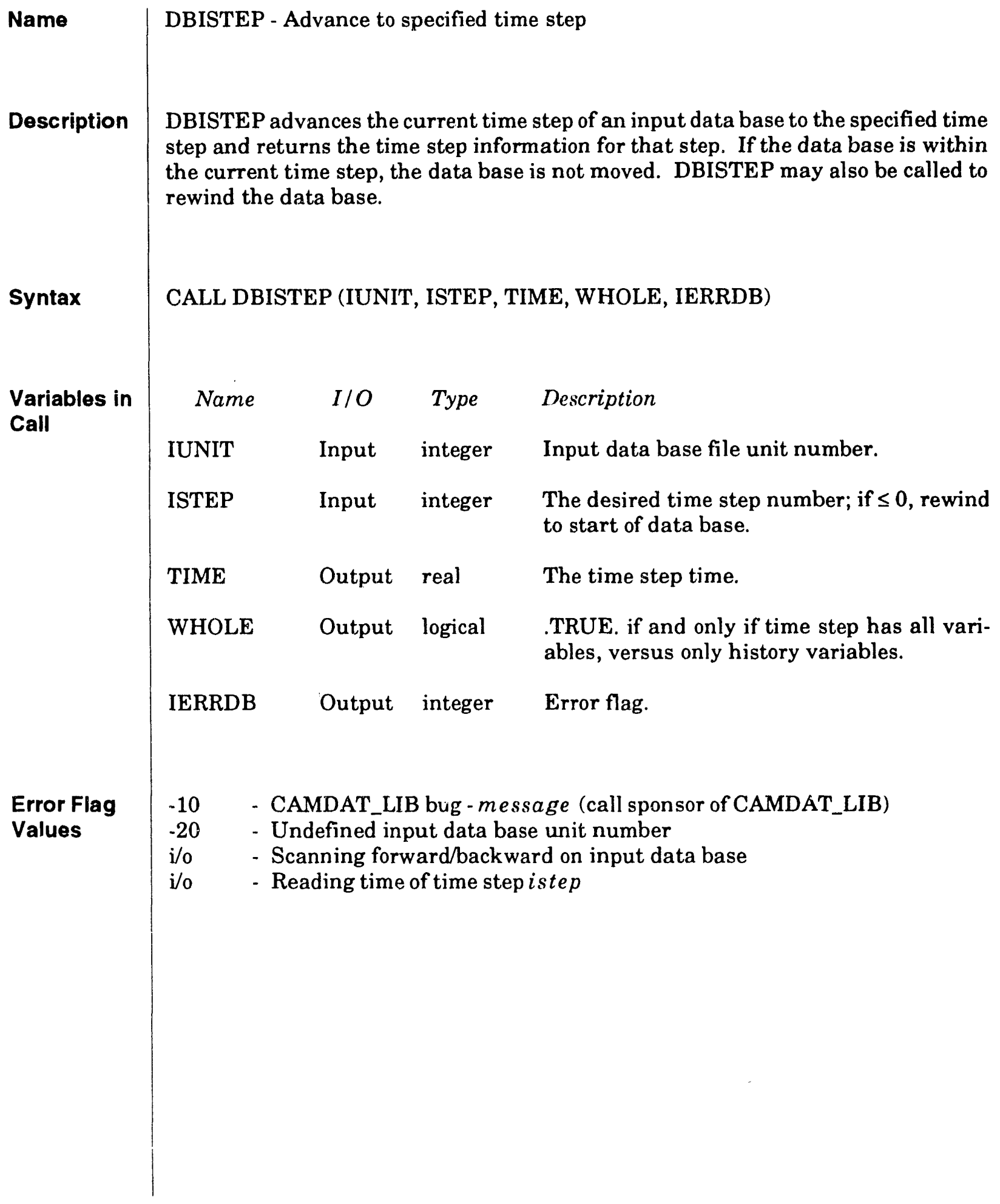




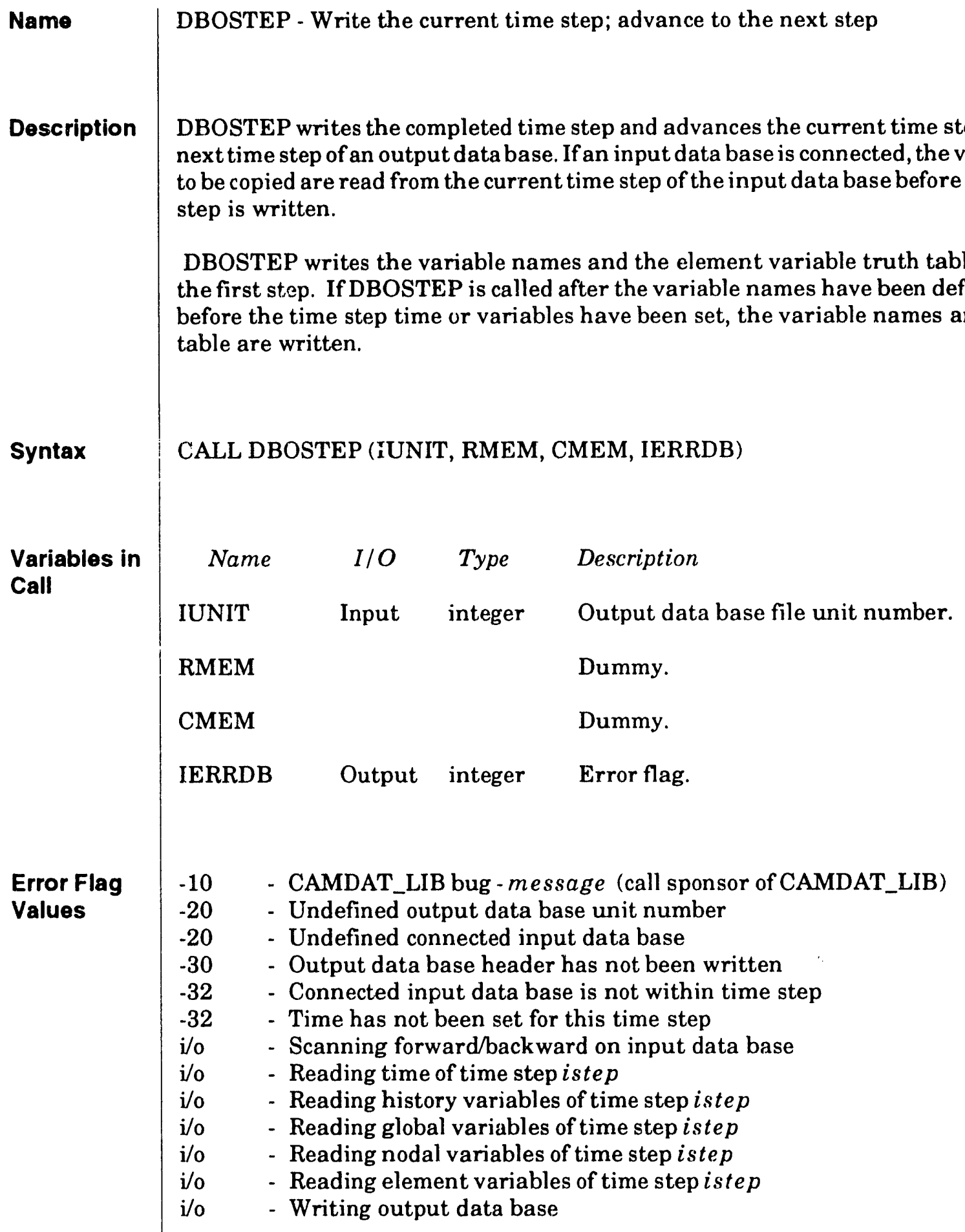




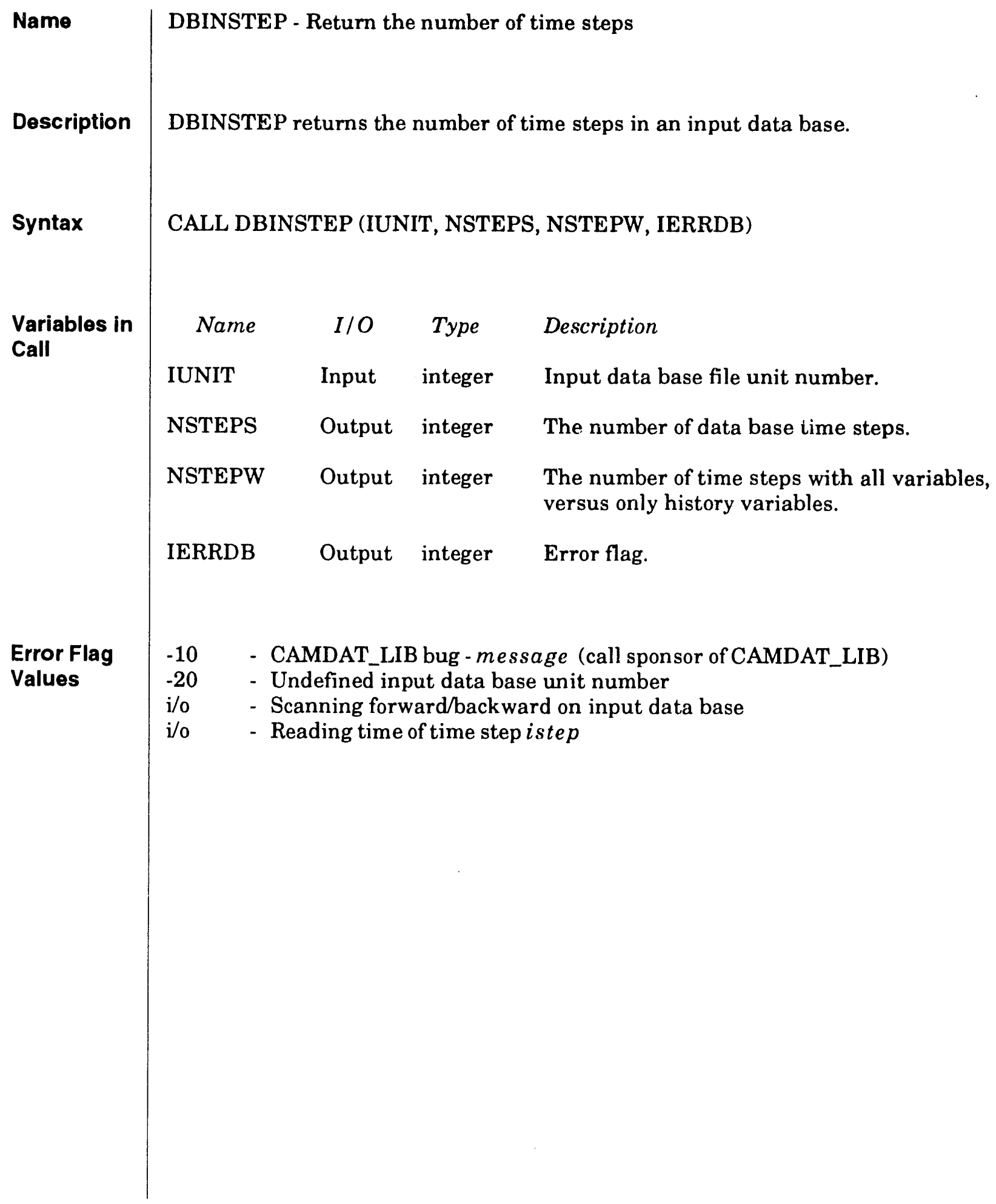




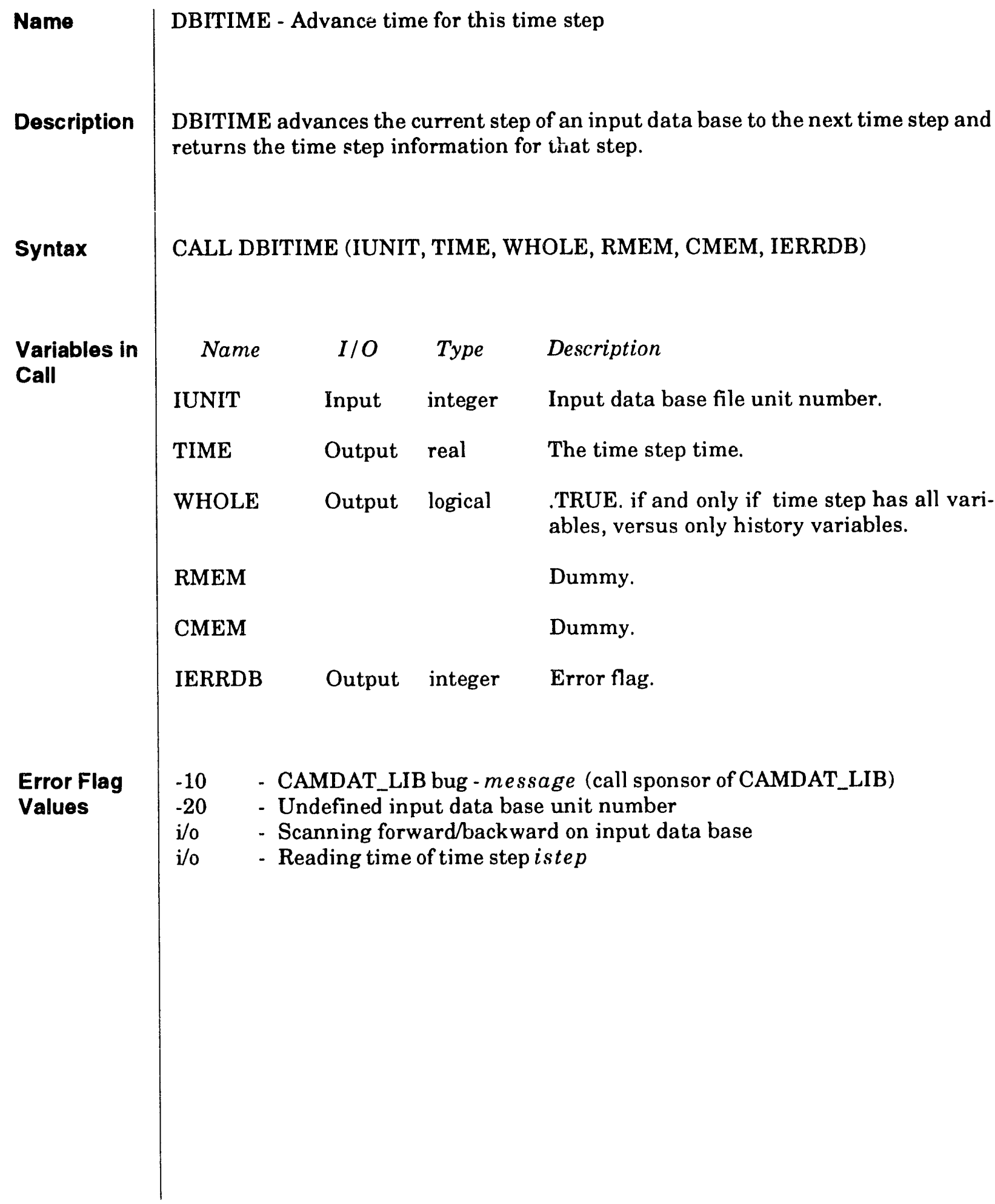




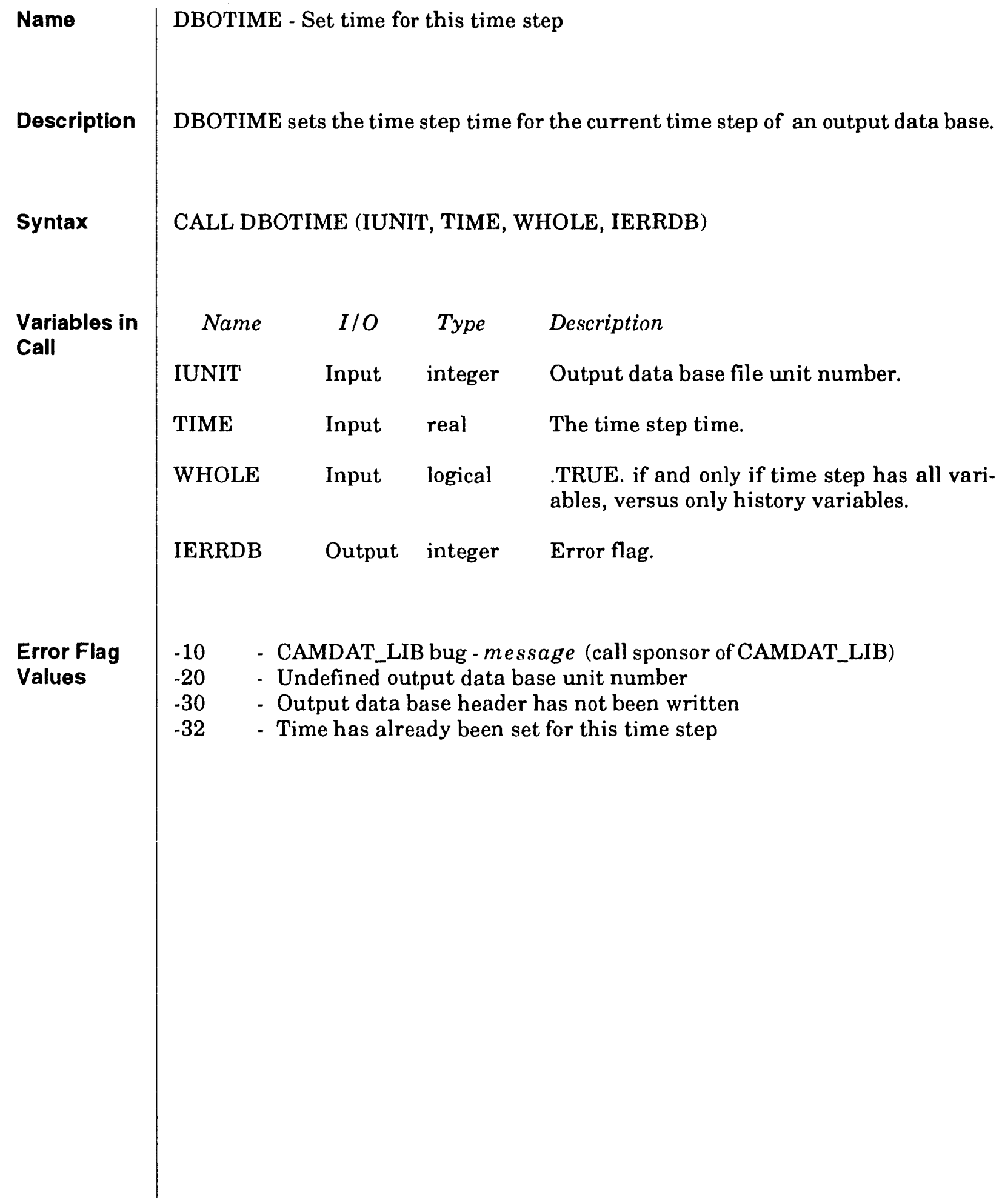




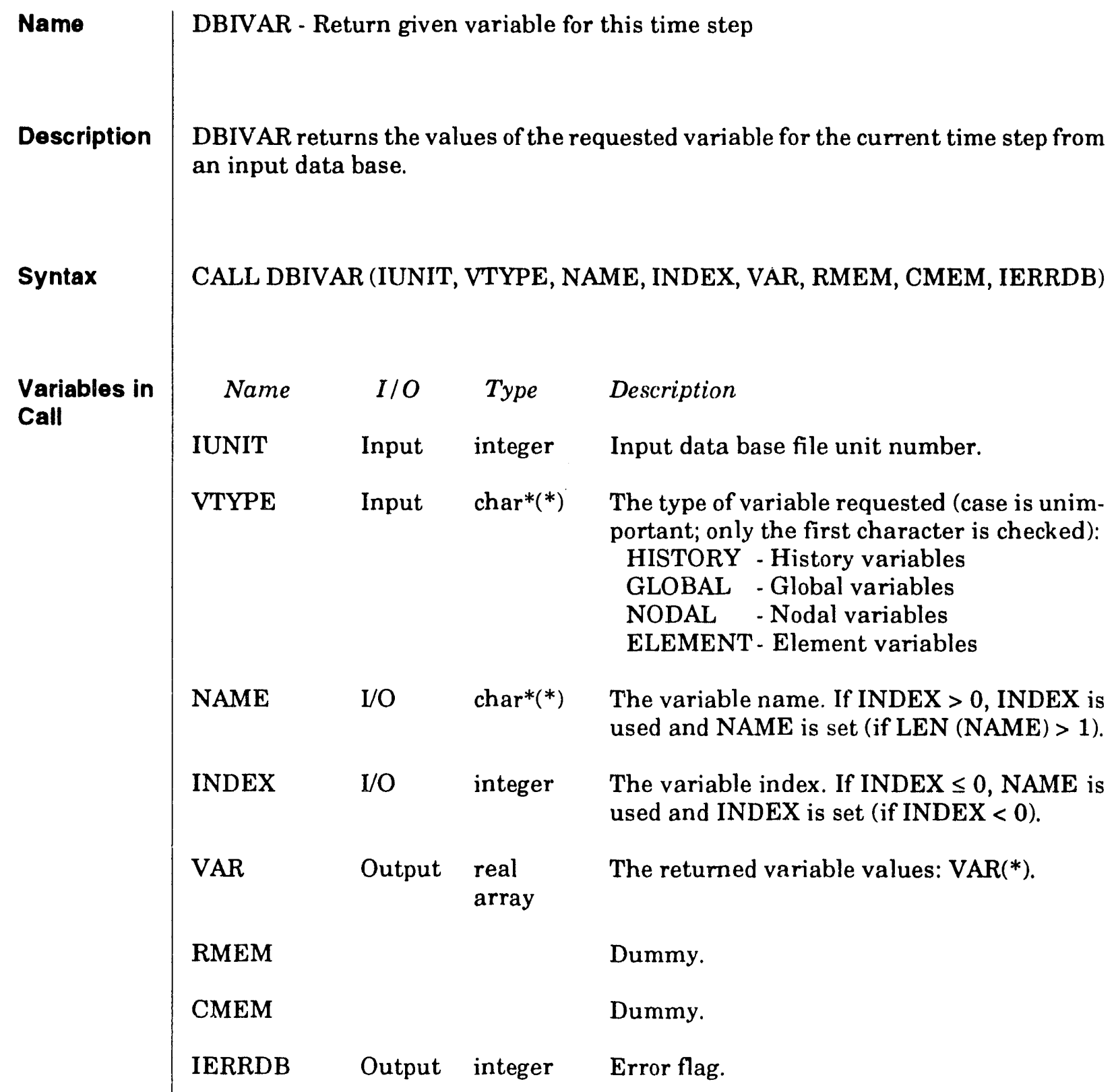




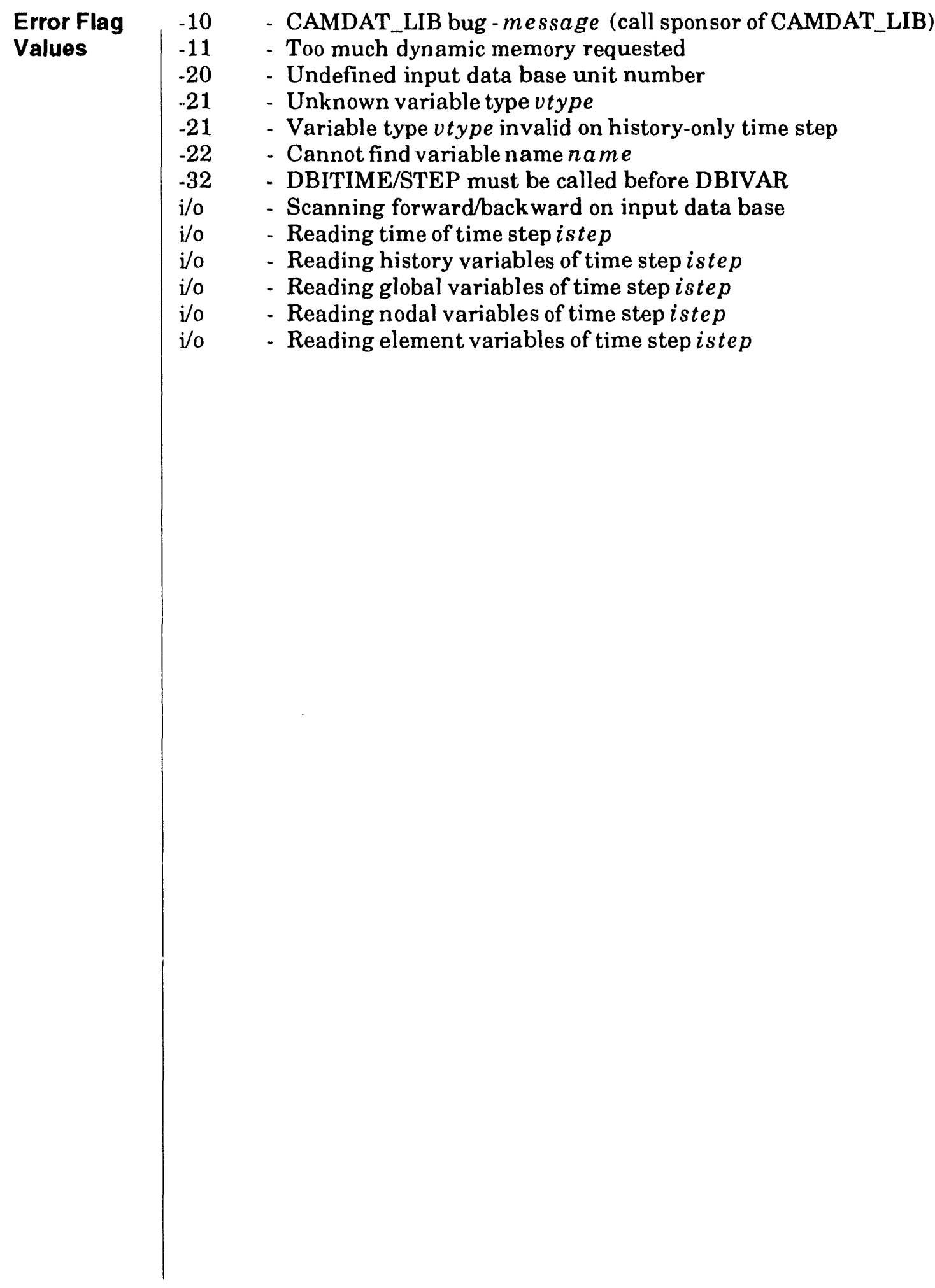




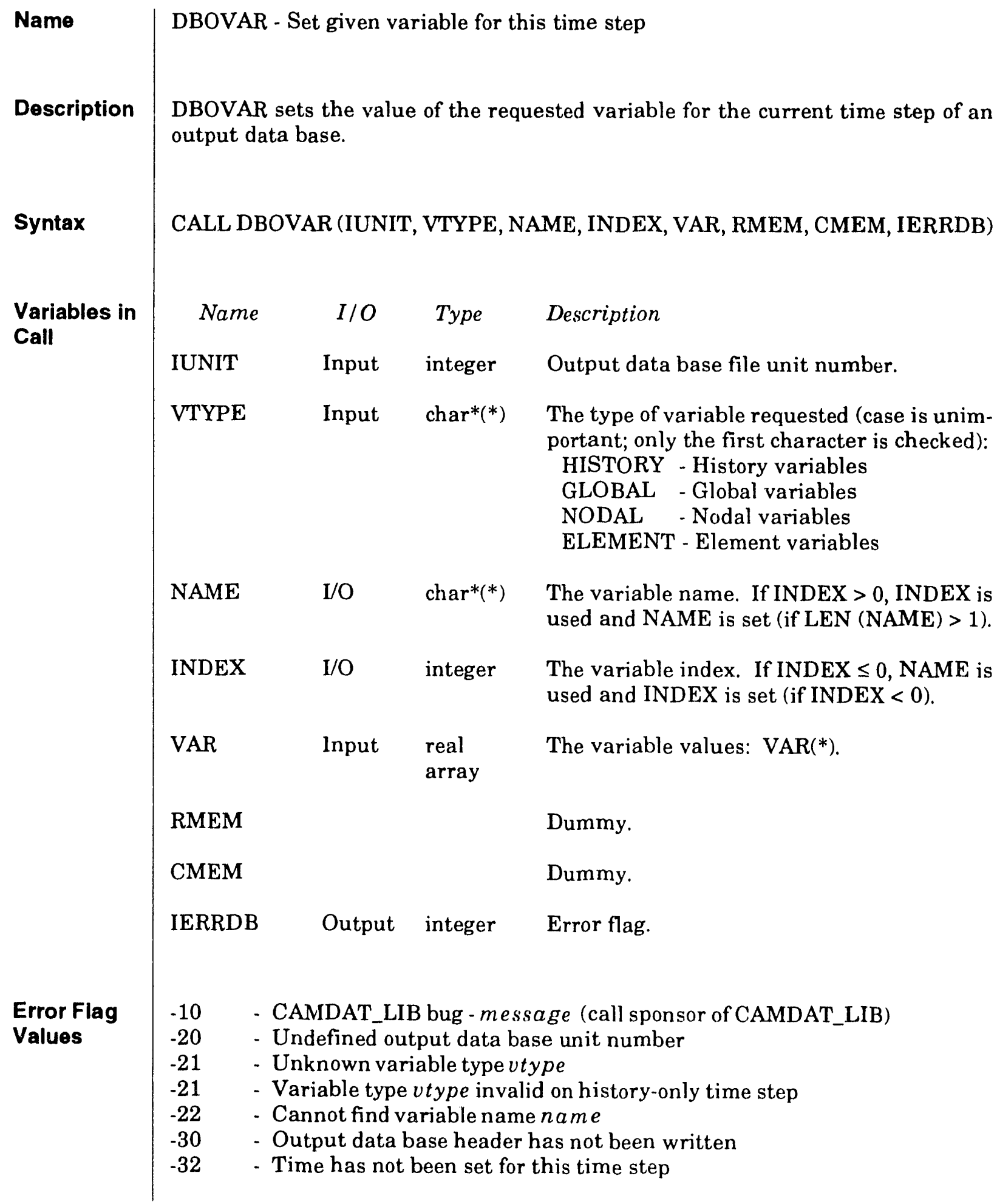




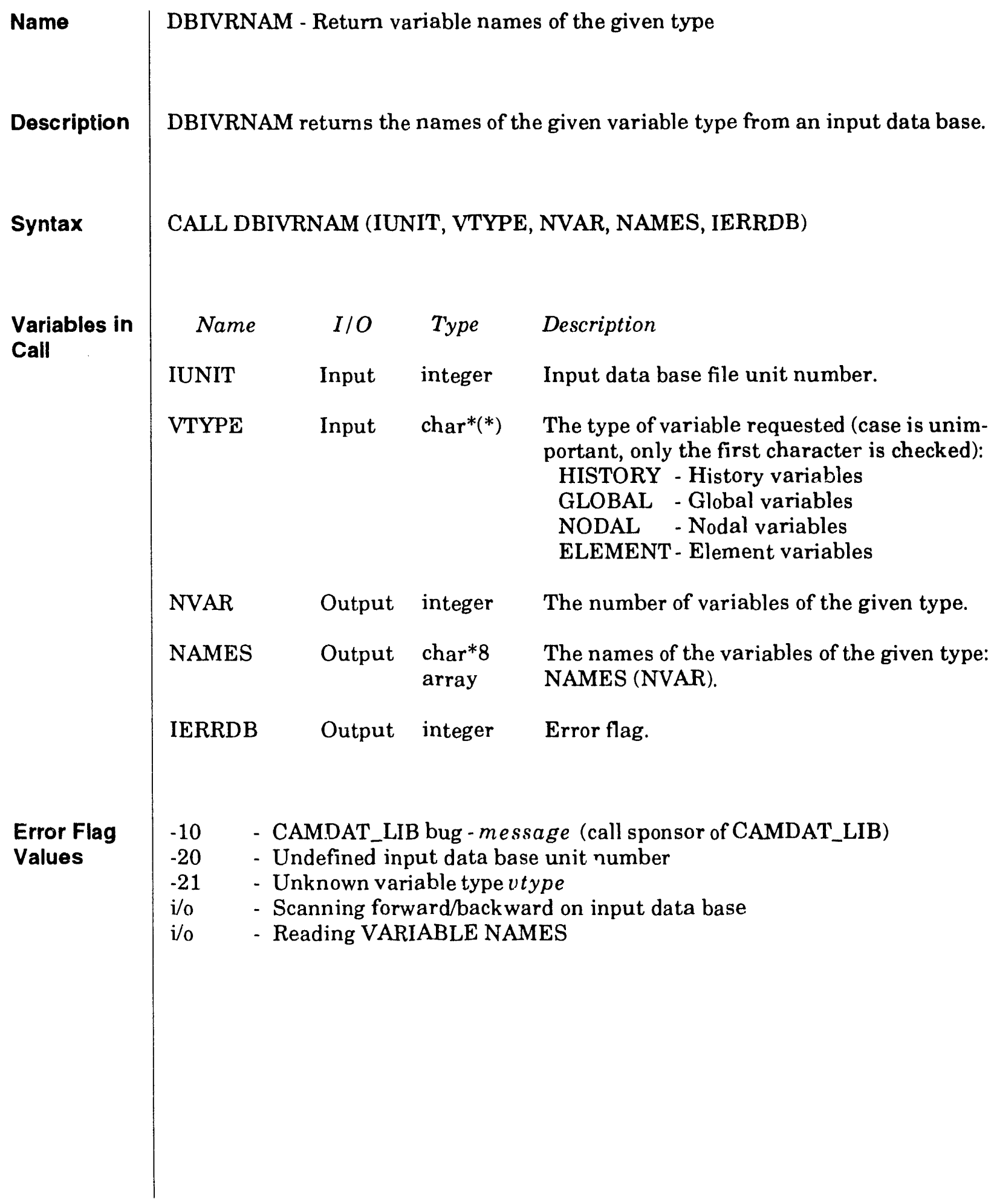




\section{Name \\ Description}

Syntax
Variables in
Call

\section{DBOVRNAM - Define output variables}

DBOVRNAM defines the names of the variables of a given type to be written to an output data base. It also controls whether any variables are to be copied from the connected input data base. By default, no variables are copied. DBOVRNAM may be called more than once for a given variable type, but all variables must be defined by this routine before DBOTIME or DBOVAR is called.

CALL DBOVRNAM (IUNIT, VTYPE, OPTION, NVAR, NAMES, LDUMMY, RMEM, CMEM, IERRDB)

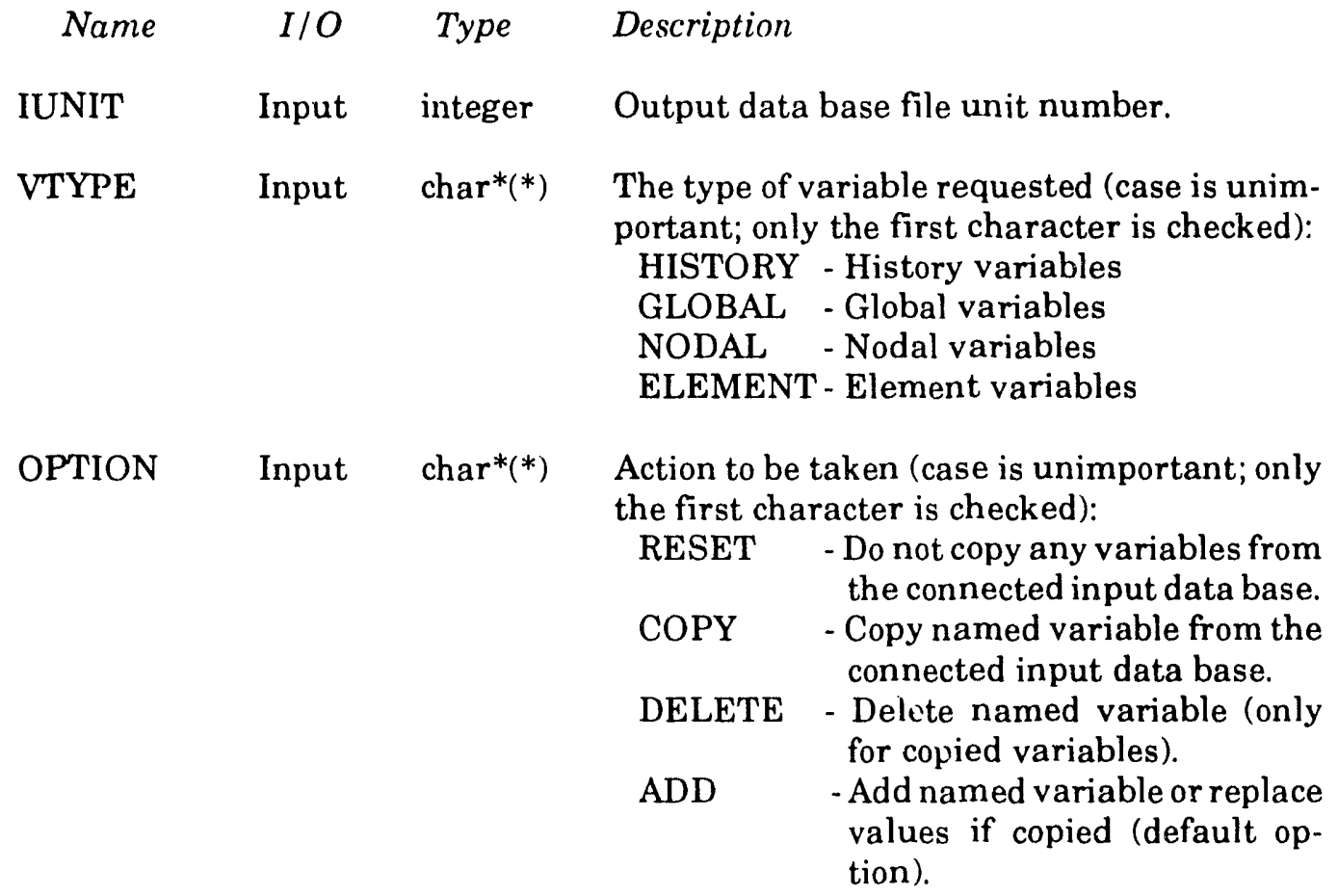

NVAR Input integer The number of variables of the given type.

NAMES Input char* $\left.{ }^{*}\right)$ The variable name: NAMES (NVAR). If array OPTION $=$ COPY and NAMES $(1)=*$, all attributes are copied.

LDUMMY None None

RMEM Durnmy.

CMEM Dummy.

IERRDB Output integer Error flag. 


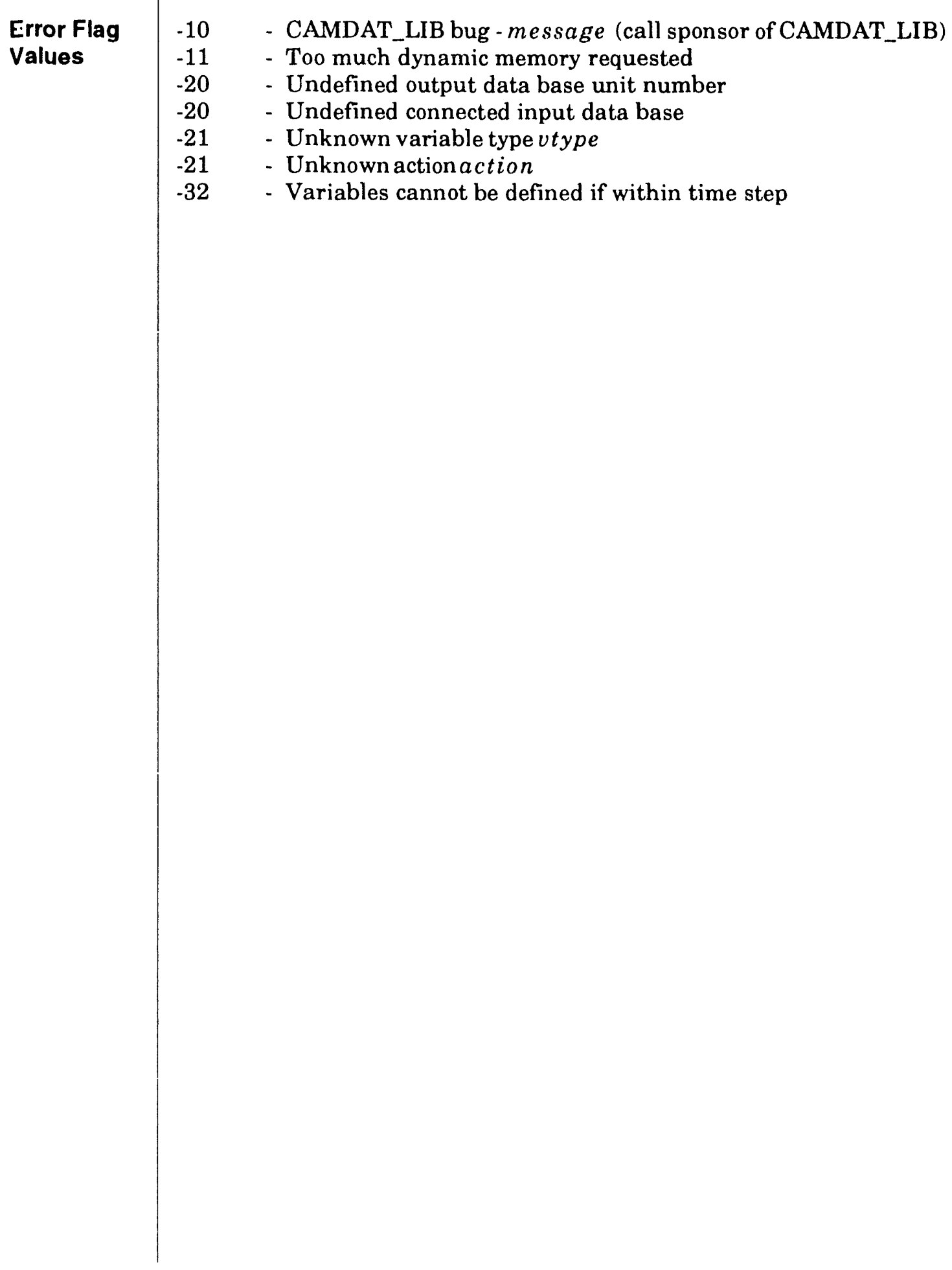




\begin{tabular}{|c|c|c|c|c|}
\hline Name & \multicolumn{4}{|c|}{ DBPVRNAM - Print variable names } \\
\hline Description & \multicolumn{4}{|c|}{ DBPVRNAM displays the variable names. } \\
\hline Syntax & \multicolumn{4}{|c|}{$\begin{array}{l}\text { CALL DBPVRNAM (IUNIT, IUNPRT, NVARHI, NAMEHV, NVARGL, NAMEGV, } \\
\text { NVARNP, NAMENV, NVAREL, NAMEEV) }\end{array}$} \\
\hline \multirow[t]{11}{*}{$\begin{array}{l}\text { Variables in } \\
\text { Call }\end{array}$} & Name & $I / O$ & Type & Description \\
\hline & IUNIT & Input & integer & Data base file unit number. \\
\hline & IUNPRT & Input & integer & $\begin{array}{l}\text { Display file unit number ( } \leq 0 \text { for standard } \\
\text { output). }\end{array}$ \\
\hline & NVARHI & Input & integer & The number of history variables. \\
\hline & NAMEHV & Input & $\begin{array}{l}\operatorname{char}^{*} 8 \\
\text { array }\end{array}$ & $\begin{array}{l}\text { The history variable names: } \\
\text { NAMEHV (NVARHI) }\end{array}$ \\
\hline & NVARGL & Input & integer & The number of global variables. \\
\hline & NAMEGV & Input & $\begin{array}{l}\text { char*8 } \\
\text { array }\end{array}$ & $\begin{array}{l}\text { The global variable names: } \\
\text { NAMEGV (NVARGL). }\end{array}$ \\
\hline & NVARNP & Input & integer & The number of nodal variables. \\
\hline & NAMENV & Input & $\begin{array}{l}\text { char*8 } \\
\text { array }\end{array}$ & $\begin{array}{l}\text { The nodal variable names: } \\
\text { NAMENV (NVARNP). }\end{array}$ \\
\hline & NVAREL & Input & integer & The number of element variables. \\
\hline & NAMEEV & Input & $\begin{array}{l}\text { char*8 } \\
\text { array }\end{array}$ & $\begin{array}{l}\text { The element variable names: } \\
\text { NAMEEV (NVAREL). }\end{array}$ \\
\hline
\end{tabular}




\begin{tabular}{|c|c|c|c|c|}
\hline Name & \multicolumn{4}{|c|}{ DBINDVAR - Define input variables as needed } \\
\hline Description & \multicolumn{4}{|c|}{$\begin{array}{l}\text { DBINDVAR defines which input variables are needed from an input data base. } \\
\text { Either the name or the variable index is used to specify a variable. This routine is } \\
\text { optional and is included to improve efficiency. }\end{array}$} \\
\hline Syntax & \multicolumn{4}{|c|}{$\begin{array}{l}\text { CALL DBINDVAR (IUNIT, VTYPE, ACTION, NAME, INDEX, RMEM, CMEM, } \\
\text { IERRDB) }\end{array}$} \\
\hline \multirow{4}{*}{$\begin{array}{l}\text { Variables in } \\
\text { Call }\end{array}$} & & $I / O$ & Type & Description \\
\hline & IUNIT & Input & integer & Data base file unit number. \\
\hline & VTYPE & Input & $\operatorname{char} *(*)$ & $\begin{array}{l}\text { Type of variable requested (case is unimpor- } \\
\text { tant; only the first character is used): } \\
\text { HISTORY - history variable(s) } \\
\text { GLOBAL - global variable(s) } \\
\text { NODAL - nodal variable(s) } \\
\begin{array}{ll}\text { ELEMENT - element variable(s) } \\
\text { * - all variables }\end{array}\end{array}$ \\
\hline & ACTION & Input & $\operatorname{char}{ }^{*}(*)$ & $\begin{array}{l}\text { Action to be taken: } \\
\begin{array}{ll}\text { RESET } & \text { - delete the named variable from } \\
& \text { the list of needed variables } \\
\text { DELETE } & \text { - delete the named variable } \\
\text { ADD } & \text { - add the named variable to the } \\
& \text { list of needed variables }\end{array}\end{array}$ \\
\hline
\end{tabular}

NAME Input/ char*(*) The variable name. If INDEX $>0$, INDEX Output rather than NAME is used to specify the variable. If INDEX $>0$ and the length of NAME $>1$, then NAME is set. If NAME is ' $*$ ' and INDEX $\leq 0$, then all variables of the type specified in VTYPE are added or deleted.

INDEX Input/ integer The variable index. If INDEX $\leq 0$, NAME Output rather than INDEX is used to specify the variable. INDEX is set if INDEX $<0$. 


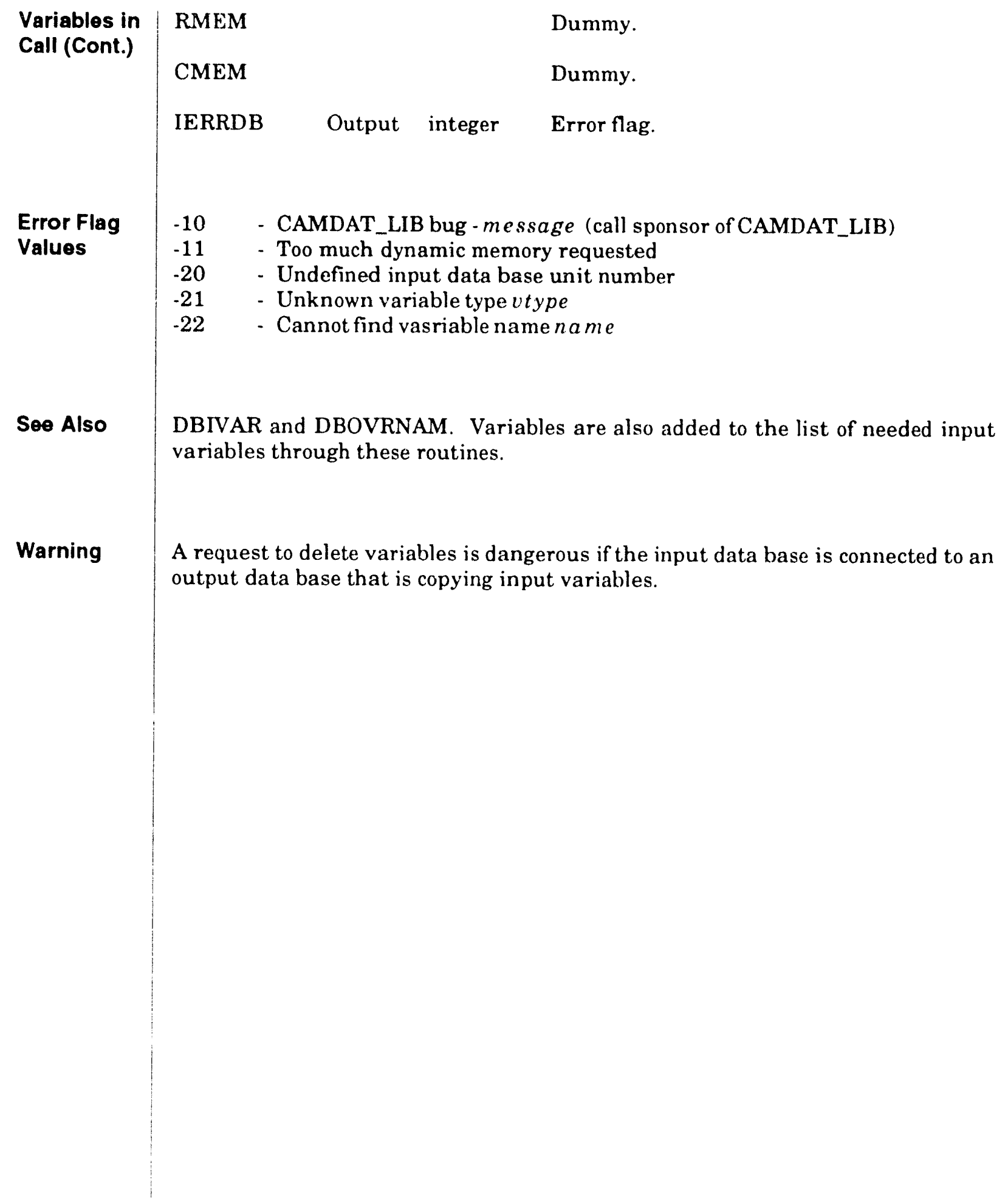


CAMDAT_LIB

Analysis Results 


\section{SDBREAD_LIB: \\ Secondary Data Base Interface Library}

This chapter describes the calls to the routines that read and write data from the neutral files, created from the secondary data base tables, storing parameters for chemical, mechanical, probabilistic, and hydrologic models. However, this chapter first provides a general description of the contents of the relational tables in the secondary data base.

\subsection{General Description of the Secondary Data Base}

The secondary data base stores the information that, defines the conceptual model of the disposal system. Although text and general scientific information on the assumed important physical processes for the scenario are not stored, all other data necessary to model the disposal system for a potential release scenario are stored in the data base; for instance, data gaps existing in the primary data base will have been filled in using objective interpolation or expert judgement (see also Section 2.9, "Data Bases").

The purpose of the secondary data base is to

- Provide consistent distributions among all the computational models used in the PA simulation.

- Place responsibility for maintaining the most current parameter values with only a few individuals.

- Provide a uniform interface for software used by PA analysts.

- Provide a uniform interface for investigators communicating with the PA Department.

\subsubsection{Secondary Data Base Structure and Similar Data Entities}

Because of the diverse types of information stored, the secondary data base consists of several separate tables (see Figure 2-5). The general scheme of the secondary data base tables is to place numeric variables needed for identifying and searching records at the beginning of a record, then value(s) of interest, then elaborating text. At present, the elaborating text in each of the tables consists primarily of QA information as follows:

- QA Information

- QA classification of the information

- Primary source of underlying data for parameter (INFOSR)

- Name of person making the change (CATALG)

- Date of last change (CHDATE)

\subsubsection{Storage of Conceptual Model Stratigraphy}

For WIPP, stratigraphic layer data from DOE, potash, and oil and gas wells were combined to form a secondary data base table. From this data base table, a conceptual (and mathematical) model of the 
stratigraphy is developed using the codes in the property assignment and mesh generation modules of the CAMCON system (see Rechard, 1992). The stratigraphic information is stored in two separate tables: well locations and layer elevations.

Well Locations. Information in the well-location table contains the following information (the usual identifier in the SDB neutral file, which is described in the next section, is shown in parenthesis):

- Identifier for information in each record

- Standard well ID for WIPP (IDWELL)

- Alternate well ID for WIPP (IDALT)

- Stored information

- Location in universal transverse mercator (UTM) coordinates (m) (XUTM, YUTM)

- Location in state planar coordinates ( $\mathrm{ft}$ ) (XSTPLAN, YSTPLAN)

- Location as section, township, and range (SECTION, TOWNSHP, RANGE)

Layer Elevations. The layer-elevation table contains the following information:

- Identifier for information in each record

- Material ID (IDMTRL) (e.g., stratigraphic layer)

- Well ID for WIPP (IDWELL)

- Indication of top or bottom of layer (IDLEVEL)

- Stored information

- Elevation above mean sea level of appropriate surface of material (m) (ELEVAT)

- Reference datum for elevation (DATUM)

- Measurement units (UNITS)

\subsubsection{Storage of Parameter Data}

The values and distributions of model parameters are stored in a separate table within the secondary data base. This information is used by the property assignment module of the CAMCON system (Rechard, 1992). The table contains the following information.

- Identifier for information in each record

- ID for material (IDMTRL)

- WIPP ID for well (IDWELL)

- ID for material parameter (IDPRAM)

- Stored information

- Type of distribution (DISTYP) 
- Mean parameter value of distribution (MEAN)

- Median parameter of distribution (MEDIAN)

- Lower limit of range (LOWRNG)

- Upper limit of range (HIRNGE)

- Measurement units in SI system (UNITS)

and depending on distribution type, one or all of the following information

- Data values for tabulated data and corresponding name of independent variable (PARAM)

- Probability, and corresponding parameter value of distribution (TABVALU)

- Other elaborating or pertinent information can include (but is not required)

- Value of additional information to the project

- Importance of parameter in individual applied models or within the total PA composite consequence or probability model

- Measurement error of data

- Measurement technique

\subsection{General Description of SDB Neutral Files}

For the tables of the secondary data base to be accessible to the routines in SDBREAD_LIB (and eventually, the codes in the Property Assignment Module of the CAMCON system (Rechard, 1992]), the relational tables are exported from the data base system. The exported files are written sequentially in an ASCII neutral file format.

\subsubsection{SDB Descriptor Records (Keywords)}

For the SDBREAD_LIB routines to perform properly, a description of the data entries must either be appended to the front of this ASCII neutral file or reside in a separate file. The SDBREAD_LIB routines use this description to decifer the contents of the neutral file. This approach is intended to eliminate the need to modify a code that accesses the SDB neutral files through the SDBREAD_LIB routines when the structure of the SDB neutral files changes (Figure 7-1).

When a separate file is used for the descriptor records, the default location is the same directory as the SDB neutral file [SDB $\alpha$ ], although the location can be changed. The default file name is the SDB neutral file name with the extension .SDBDSCR. For example, the descriptor file name for the SDB neutral file, PROPERTY.SDB, would be PROPERTY.SDBDSCR.

\subsubsection{Format of SDB Descriptor Records}

*TITLE=title <no default $>$

descriptive but brief title for stored data (CHARACTER*72). 


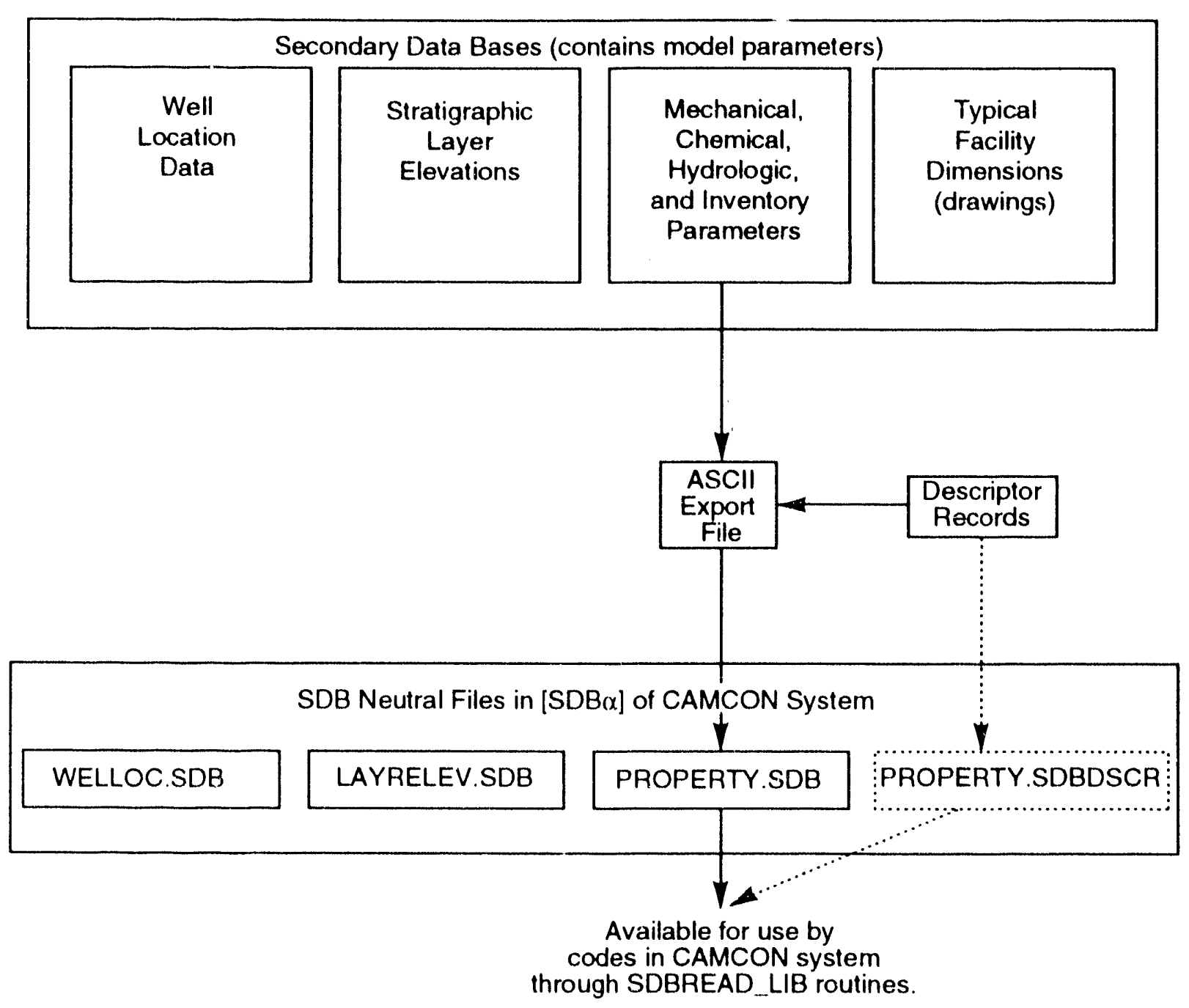

TRI.6334.284.0

Figure 7.1. SDBREAD_LIB typically accesses three SDB neutral files exported from the secondary data base tables. These SDB files require descriptor records to be appended to the file or reside in a separate file.

*SDBVERSION=QVV.RRT

The version of both the secondary data base and SDB neutral field is identified by the *SDBVERSION keyword.

The purpose of the version number is to maintain the capability of tracing the parameters when the PA secondary data base is the primary means of controlling parameter input. Each version of the SDB neutral file is marked with the secondary data base PA version identification (ID) and a QA level prefix. The PA version ID is of the form (Rechard et al., 1992, p. 22): 
QVV.RRT

where

$Q \quad$ is the SDB neutral file classification (A-Adjudicated, C-Candidate, X-experimental, T-temporary); equivalent to the lowest classification level of a parameter contained in the SDB neutral file; the fourth classification, D for dormant or historical, is used only when $100 \%$ of the parameters are historical.

$V V$ is a one- or two-digit integer designating the major version number of the secondary data base.

$R R$ is a two-digit integer designating the revision number of the secondary data base.

$T \quad$ is a two letter identifier designating the type of secondary data base (PR - property file, EL - elevation file, LC - well location file).

The PA version ID changes depending on the type of modification as follows:

- For minor revisions to entries in the secondary data base such as data entry corrections, the revision number $(R R)$ is incremented by one.

- For major revisions that substantially change several parameter distributions in the secondary data base, the major version number $(V V)$ is incremented by one and the revision number $(\mathrm{RR})$ returns to zero.

\section{Parameter Identification Records}

The SDBREAD_LIB routines assume that any descriptor record that does not start with an asterisked keyword (e.g., ${ }^{*} \mathrm{ENTRY}=$ nam $e_{1}$, nam $\left.e_{2}, \ldots\right)$ is a record identifying the fields required to describe a parameter. The parameter identification records give the name, column boundaries, formats, and expanded name for each field on the SDB neutral file. The column numbers that indicate the begin and end columns for the field are separated by a colon. All remaining information in the descriptor record is separated by one or more blanks. (If the expanded name contains any blanks, enclose the name in single quotes.) (See Section 7.2.4, "Example".)

* HEADERLINES $=n$

When the SDB neutral file contains additional miscellaneous header records (such as additional comments after the *ENDDSCR record but before the data records), then the number of such "header" records, $n$, must be specified in the descriptor file by HEADERLINES $=n$. Any number of these additional records may precede the data.

* ENTRY=name, name $_{2}, \ldots$

Although not required in the secondary data base, the SDB neutral file uses multiple records for model parameters that need more than three points to describe the probability density 
function (pdf) or cumulative distribution function (cdf). The *ENTRY keyword flags the names $\left(n a m e_{1}, n a m \epsilon_{2}, \ldots\right)$ that uniquely identify the distribution. If any of the names in the *ENTRY list change from one record to the next, the SDBREAD_LIB routines assume a new distribution is being described.

\section{* ENDDSCR}

The descriptor information is terminated by an *ENDDSCR record.

\subsubsection{Example of Descriptor Records}

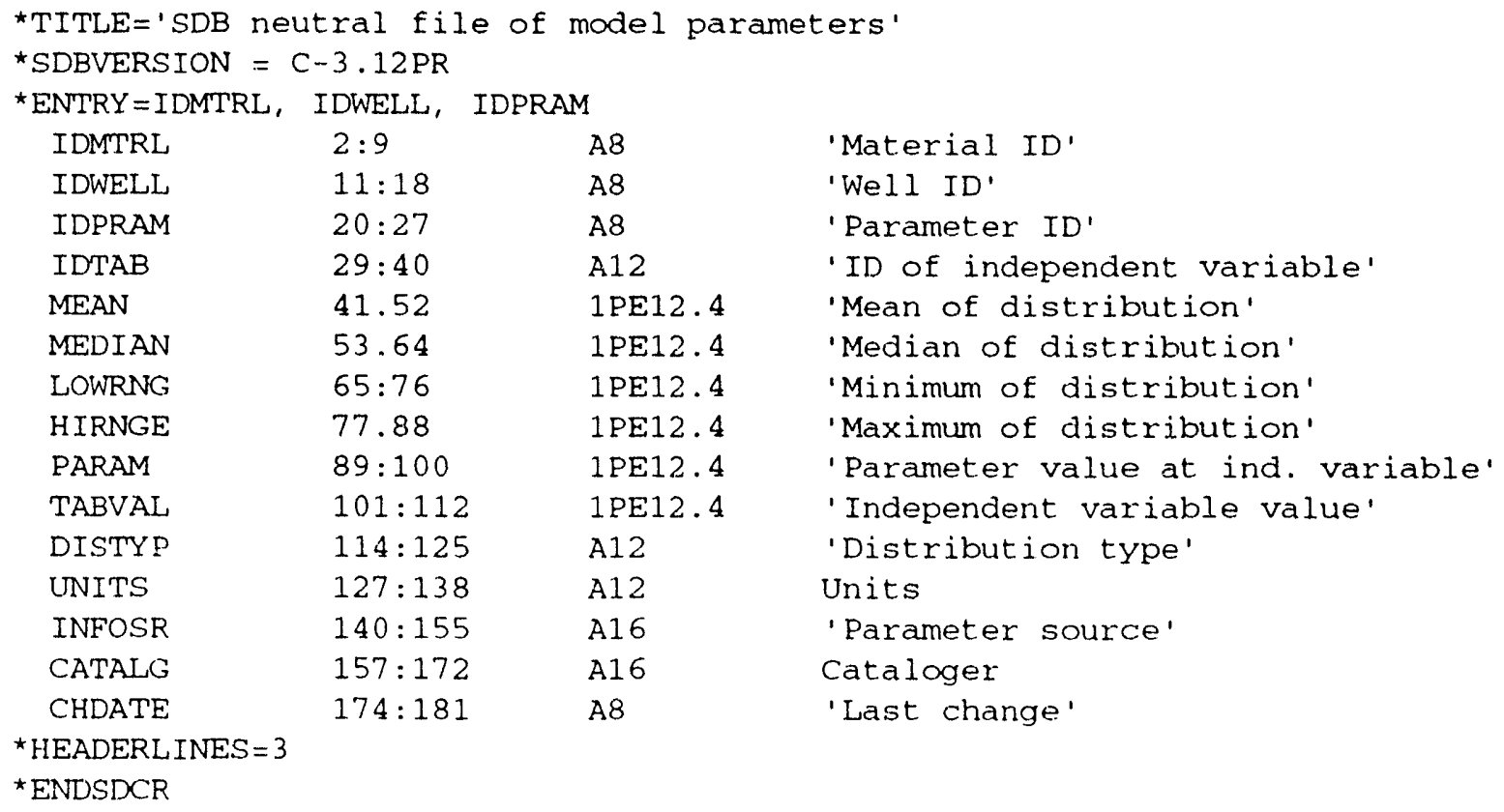

\subsubsection{Identification of SDB Neutral Files}

As explained in Section 7.2.2, the SDB neutral files are identified by the PA version identification (ID) number plus a QA-level prefix. The QA-level prefix identifies the lowest level of classification of the parameters in the file (excluding class D). The QA classification levels are A (Adjudicated), C (Candidate), X (eXperimental), and D (Dormant or historical). For example, if a file includes both A- and C-level parameters, then the QA-level prefix would be $C$. The D classification is never used as a QA-level ID for an SDB neutral file uniess $100 \%$ of the parameters are historical.

In general, parameters in an official SDB neutral file may not be changed. However, two types of parameter files are permitted as subsets of the SDB neutral file: a subset based on QA level (e.g., to generate an A-level only parameter file) and a subset that contains parameters changed by a PA analyst for the purpose of a comparison analysis. The latter subset is used by the PA analyst when he or she needs to compare different parameter values to better understand specific results; for this subset, the subset of modified parameters is designated "T" for temporary. 
The values and distributions of the parameters in the SDB neutral file with a $\mathrm{T}$ designation are the full responsibility of the PA analyst. If a QA designation standard $(\mathrm{A}, \mathrm{C}, \mathrm{X})$ is under consideration for this temporary SDB neutral file, then the parameter values and distributions must be adjudicated by the Analysis Review Workshop and/or described in documents that report on the analysis (Rechard et al., 1991, p. 37) (Figure 7.2).

\title{
7.3 General Guidelines on the Use of SDBREAD_LIB Routines
}

\subsubsection{Sponsorship and Version}

\author{
Author/Sponsor: H. J. Iuzzolino \\ Version:/Date: C-1.20VV/November 1992 \\ Language: $\quad$ FORTRAN 77
}

\subsubsection{Overview of Capabilities and Method}

SDBREAD_LIB enables a FORTRAN program to selectively read data from and write data to formatted SDB neutral files without being affected by changes in the number and column position of fields in the file. That is, the number and position of fields in the data base may be changed without having to modify the program reading the SDB neutral files as long as the lengths of the character-type data fields remain unchanged. One file is required by SDBREAD_LIB: a data base neutral file with several descriptor header records appended to the front. Alternatively, the descriptor header records may be in a separate file. The data base neutral file must have a fixed format with the data arranged in columns. The data may be integer, floating, and/or text.

Upon initialization of a data base neutral file, a format for reading or writing a line of data is constructed from the data in a descriptor file (described in Section 7.2.1). When reading, each record of the data base neutral file is read using this format. The data fields are compared to a retrieval condition, and if the condition is true, specified data are returned to the calling program. When writing, data sent by the calling program are converted to a text line using the format and are written to the data base.

\subsubsection{Linking}

The SDBREAD_LIB routines are in an object library in the [LIBRARIES j subdirectory (see Section 2.10, "Software Libraries"), CAMCON\$ROOT:(LIBRARIES.SDBREAD_LIBISDBREAD_LIB.OLB. The SDBREAD_LIB sponsor defines the logical symbol SDBREAD_LIB to point to this file. The link command has the following form:

\$LINK code_name ..., SDBREAD_LIB/LIB

\subsubsection{Error Messages}

In all cases, ERROR = TRUE if an error was encountered or FALSE if no error was encountered. 


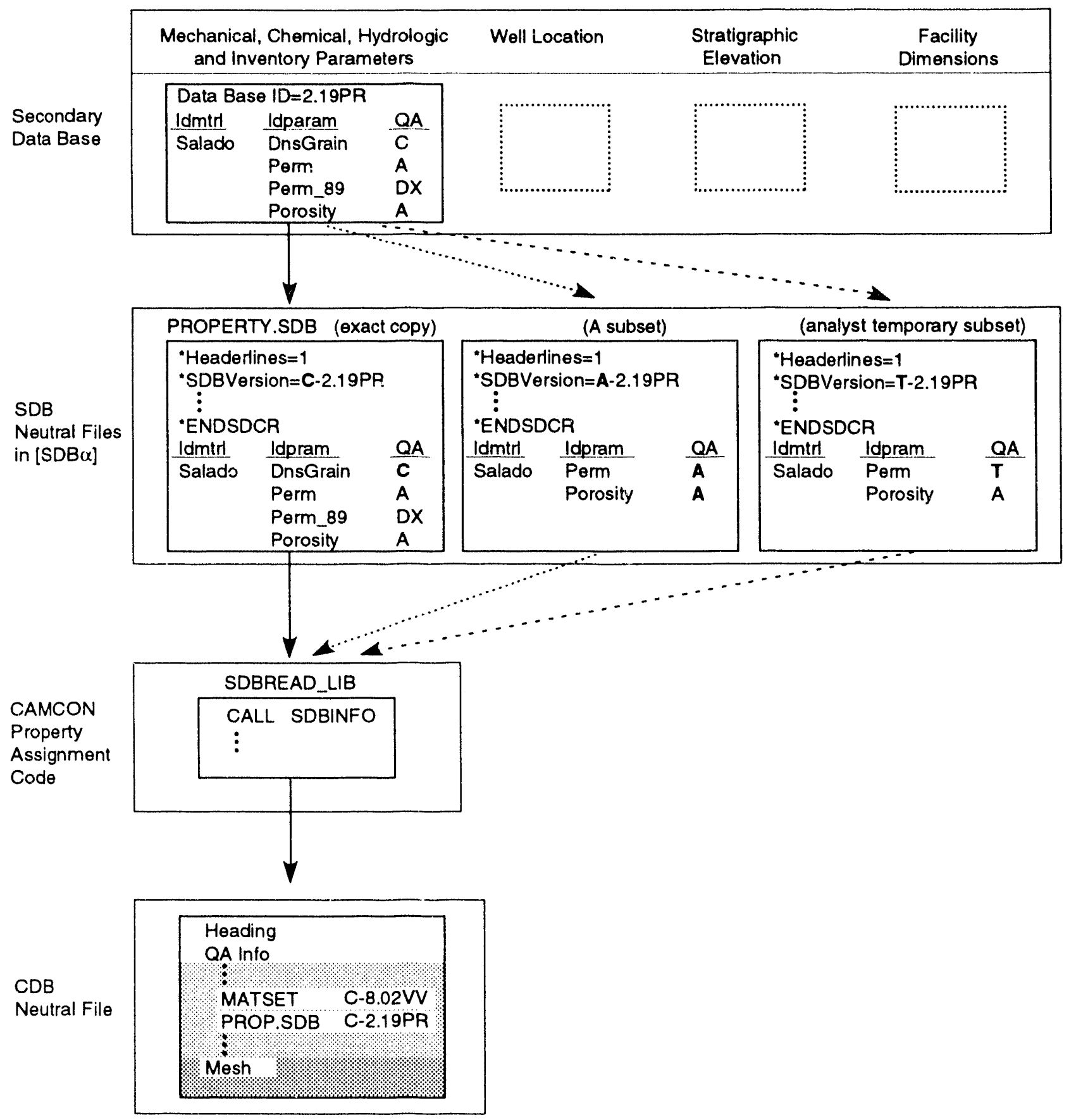

Figure 7.2. Example of data moving from the secondary data base to a CDB neutral file and the various possible identifiers for CAMCON SDB neutral files. 


\subsubsection{Summary}

The following routines are available for general use:

- SDBARES - Compare two text strings

- SDBCASES - Specify case sensitivity

- SDBCLOC - Get the location of a character variable

- SDBFRES - Compare two floating point numbers

- sDBGETF - Retrieve floating point data from a data base

- SDBGETL - Retrieve data of any type from a data base

- SDBIATR - Get the attributes of an identifier

- SDBILOC - Get the location of an integer variable

- SDBINFO - Get data base summary information

- SDBINIT - Prepare a data base for reading or writing

- SDBIRES - Compare two integers

- SDBNINFO - Get identifier names

- SDBNUMDB - Get data base FORTRAN unit number

- SDBPUTL - Write data to a data base

- SDBRLOC - Get the location of a real variable

- SDBSCMP - Compare the values in two text strings

- SDBSKHDR - Skip past the data base header

- SDBRWIND - Rewind the data base file

The following advanced routines are available but use requires an understanding of the internal algorithms of SDBREAD_LIB:

- SDBCONCR - Concatenate pieces of the retrieval condition

- SDBMOVRV - Move the retrieved values into common block SDBRTRVE

- SDBSETRC - Set pointers in the arrays needed to process the retrieval condition

- SDBSETRV - Set up the tables needed to process data retrieval

- SDBTSTRC - Test the retrieval condition on a record of data

\subsubsection{Examples}

The SDBREAD_LIB routines are called by a user-written program to read or write a data base. Up to ten data bases can be used in one program.

In simplest form, the user:

- Calls SDBINIT to initialize the reading or writing of each data base

- Puts the retrieval restrictions on the data into a character string

- Puts the names of the fields to be fetched into an array

- Puts the location(s) of storage for the retrieved variable(s) into an array

- Calls SDBGETL to get data that satisfies the retrieval condition from the data base

Subroutine SDBPUTL is used to write a new data base file. When writing a new data base, any header lines must be in the new data base file before SDBPUTL is called. 
In the preceding description, the retrieval condition may be a text string containing one or more expressions of the form ID OP VALUE separated by .AND. or .OR., where ID is the name of a data base identifier, $O P$ is a relational operator, and VALUE is a numeric or text value. ID must be specified in the same case (upper or lower) as it appears in the data base descriptor file. OP must be in the set I.EQ., .NE., .GT., .GE., .LT., .LE., .INCL., .EXCL.). The include and exclude operators (.INCL. and .EXCL.) are used only with text data. ID and VALUE must be of the same type (integer, floating point, or text). Parentheses may be used to group parts of the condition.

For example, the condition "IDMTRL .EQ. DrftSeal .AND. IDPRAM .EQ. DnsBlk" would cause the bulk density (DnsBlk) of the drift seal (DRftSeal) to be retrieved while the condition "IDMTRL .EQ. DrftSeal .OR. IDPRAM .EQ. DnsBlk" would cause all drift seal entries and all bulk density entries to be retrieved. The condition "IDPRAM .EQ. DnsBlk .AND. (MEDIAN .GT. 2000. .OR. MEDIAN .LT. 1000 )" would retrieve all entries with median values of bulk density either larger than 2000 or less than 1000 .

An example of using several of the SDBREAD_LIB routines follows.

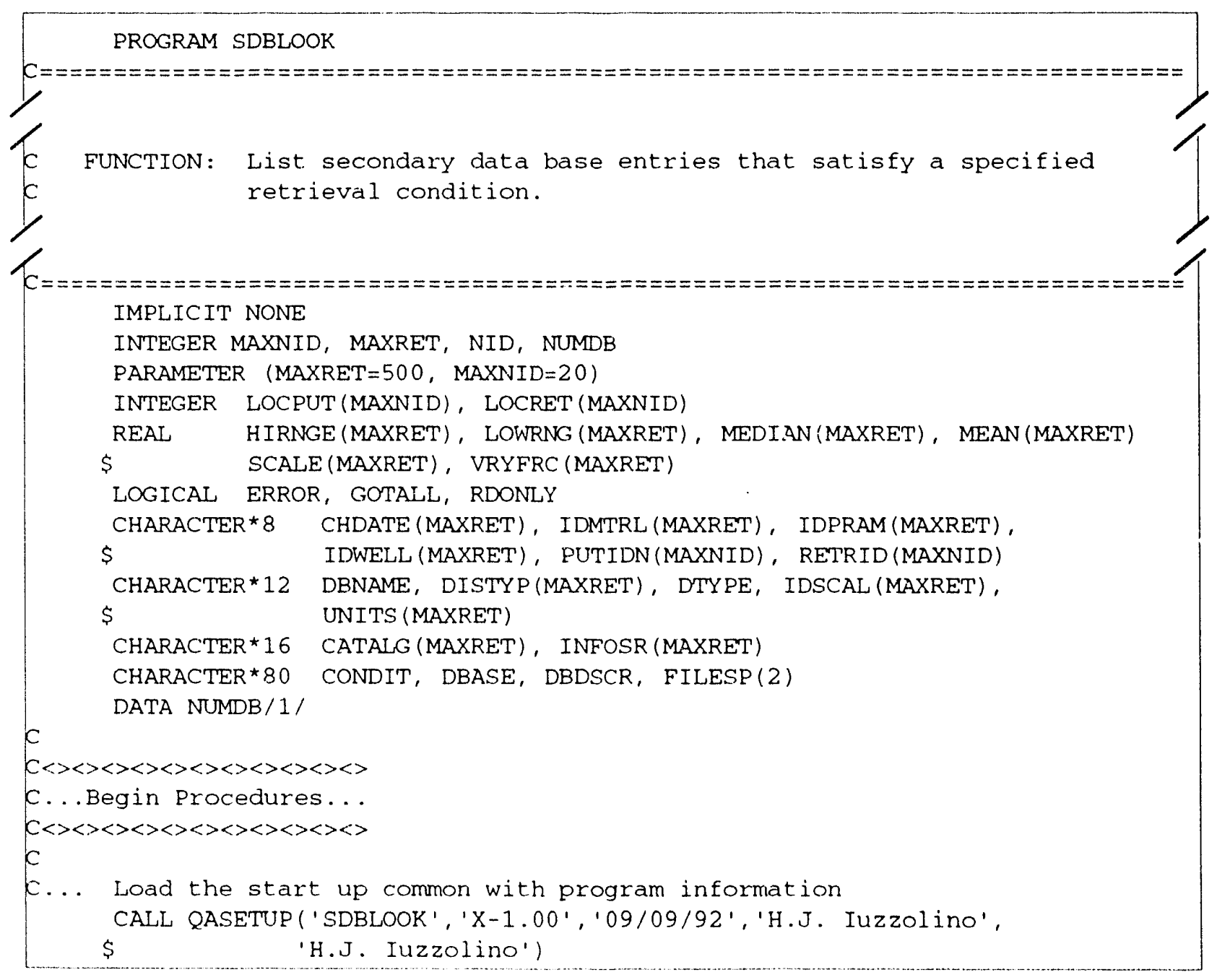




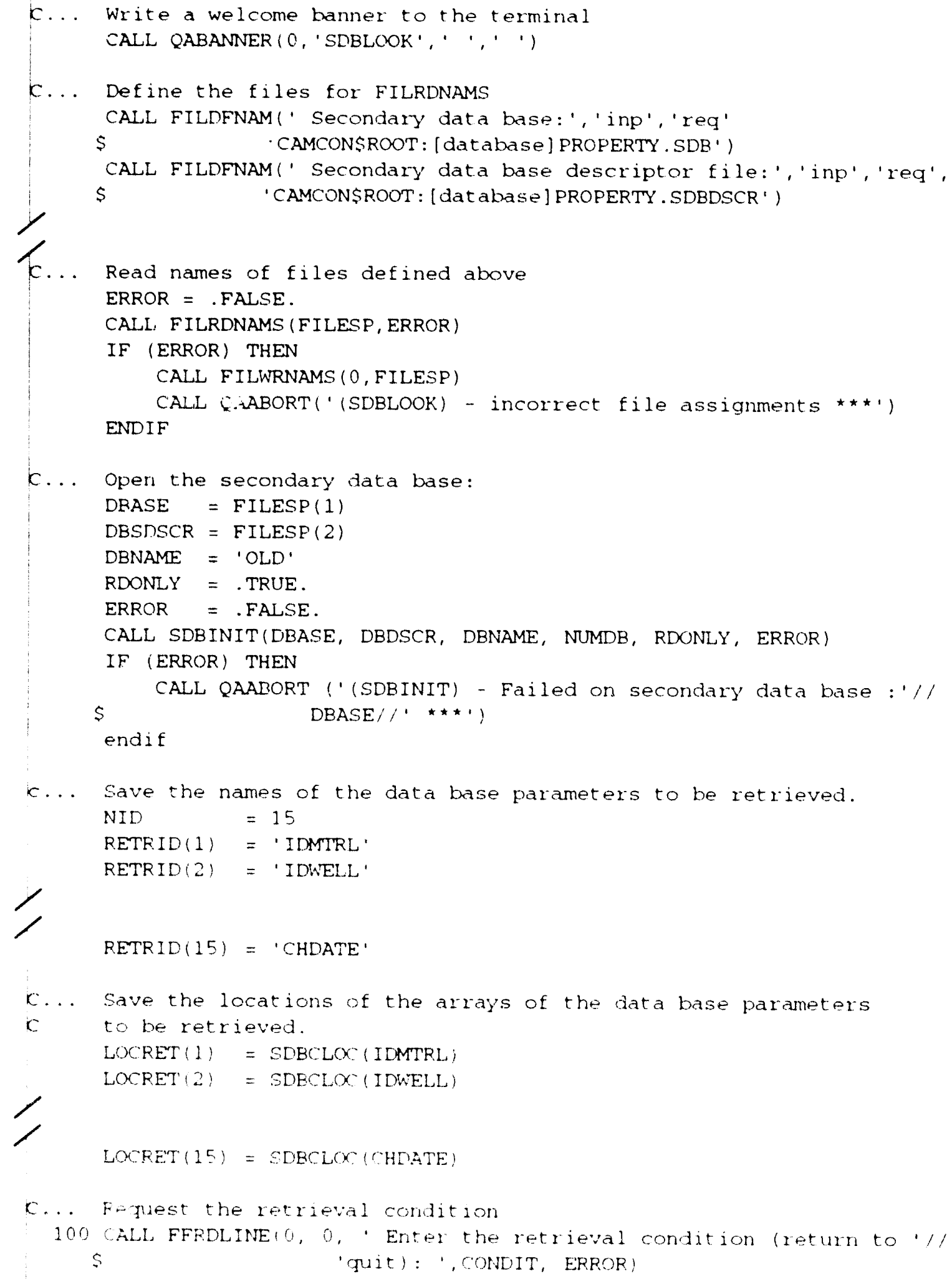




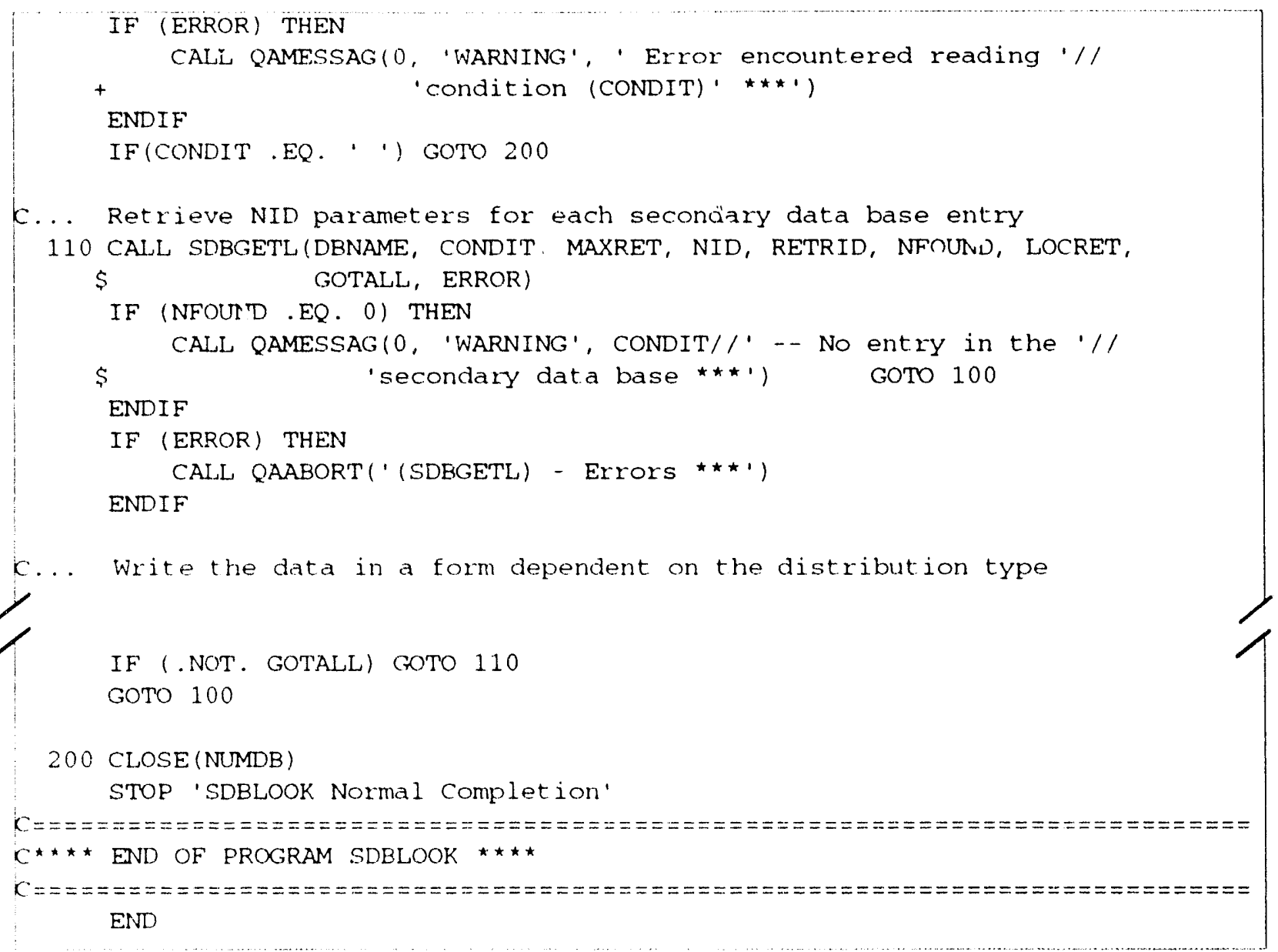




\subsection{Description of Routine Calls}

\subsubsection{General Routines}

Descriptions of general routines are given on the following pages. Lengths of character variables are arbitrary unless a length is specified in the "Variables in Call" paragraph of the routine descriptions. 


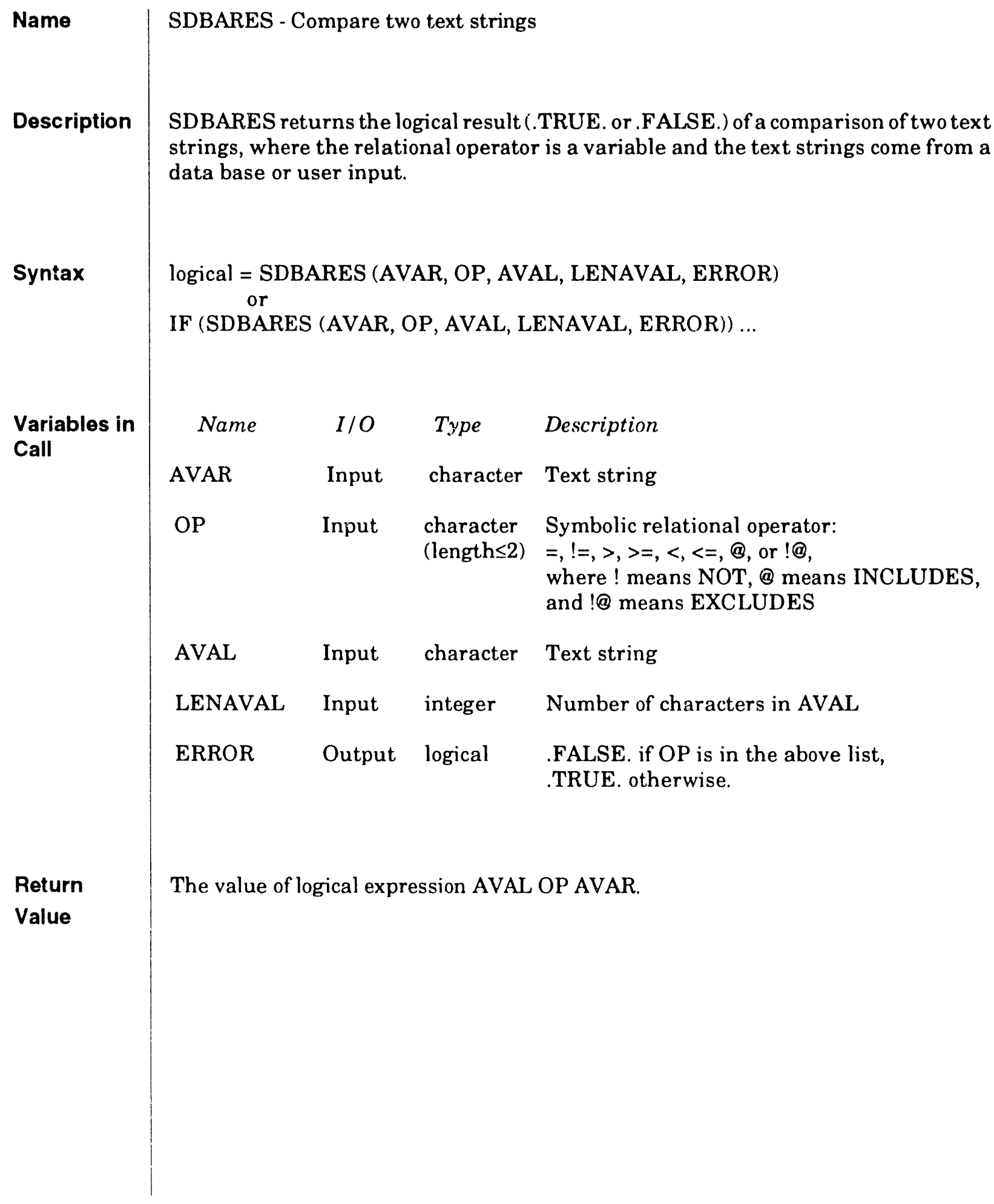




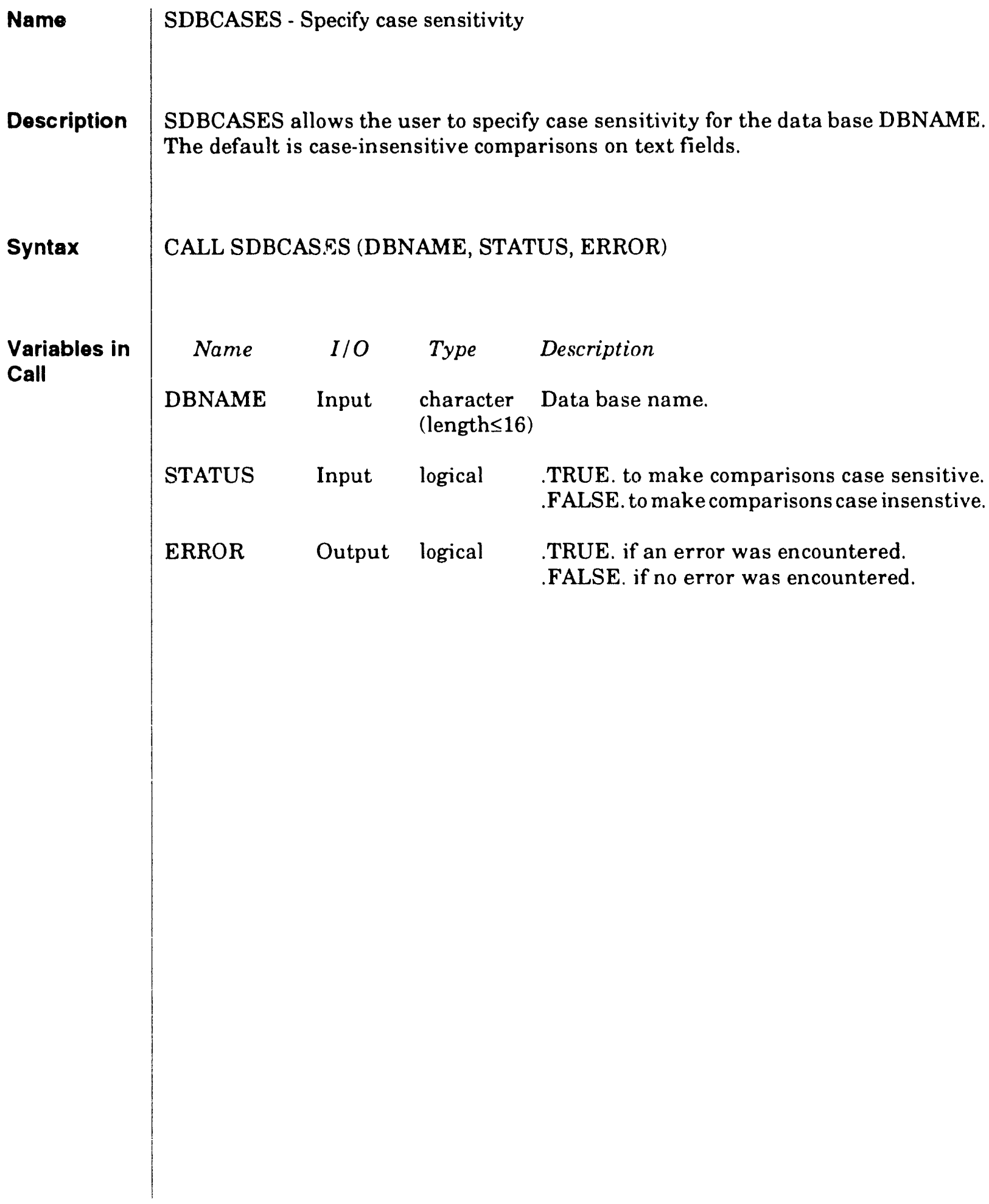




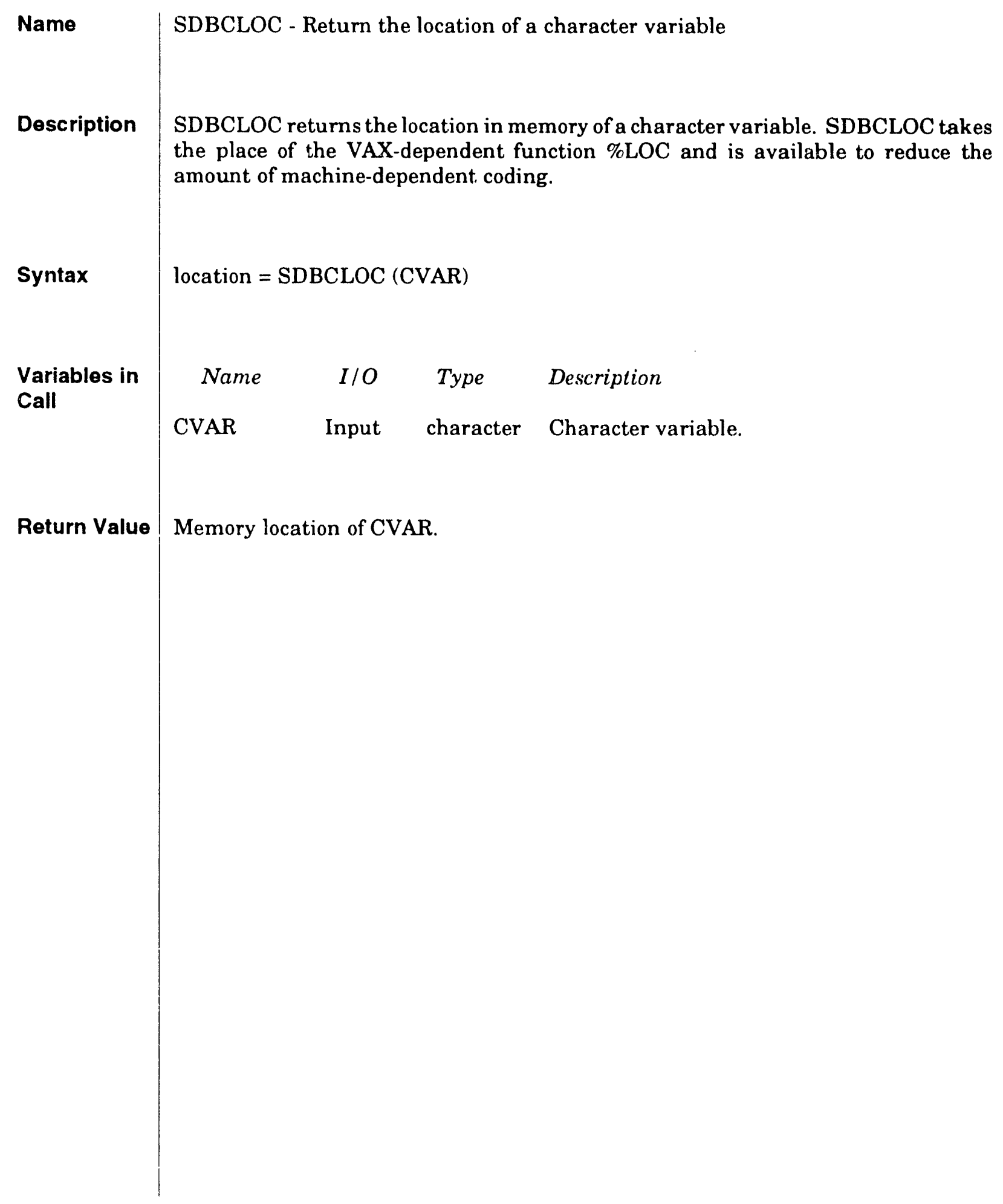




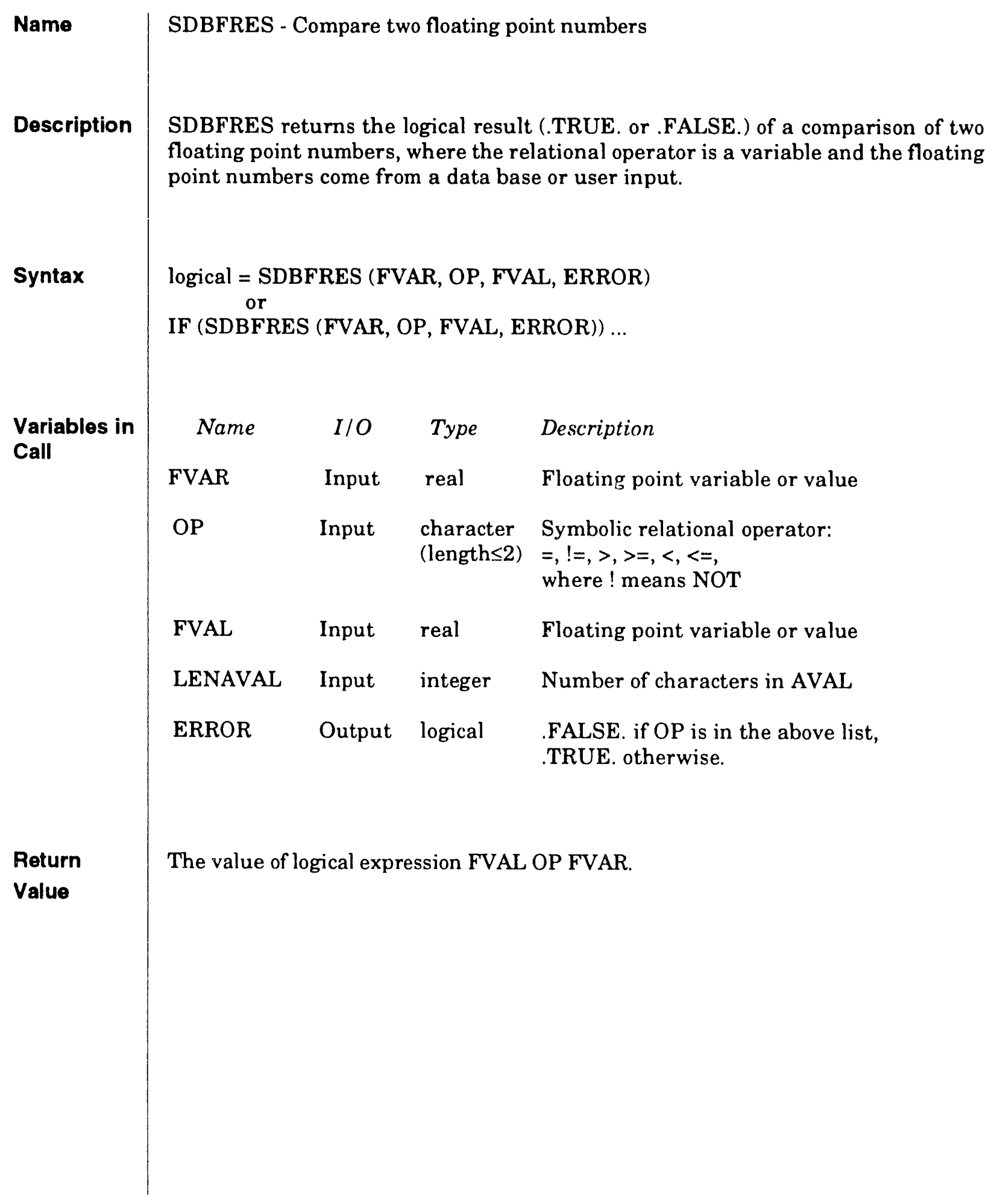




\begin{tabular}{|c|c|c|c|c|}
\hline Name & \multicolumn{4}{|c|}{ SDBGETF - Retrieve floating point (real) data } \\
\hline Description & \multicolumn{4}{|c|}{$\begin{array}{l}\text { SDBGETF returns up to MAXRET real values for each of the NID identifier names } \\
\text { in array RETRID provided retrieval condition COND is satisfied. }\end{array}$} \\
\hline Syntax & \multicolumn{4}{|c|}{$\begin{array}{l}\text { CALL SDBGETF (DBNAME, COND, MAXRET, NID, RETRID, NFOUND, VAL, } \\
\text { GOTALL, ERROR) }\end{array}$} \\
\hline \multirow[t]{10}{*}{$\begin{array}{l}\text { Variables in } \\
\text { Call }\end{array}$} & Name & $I / O$ & Type & Description \\
\hline & DBNAME & Input & $\begin{array}{l}\text { character } \\
\text { (length } \leq 16)\end{array}$ & The name of the data base. \\
\hline & COND & Input & character & The retrieval condition. \\
\hline & MAXRET & Input & integer & $\begin{array}{l}\text { Count of the maximum lines to be returned. I } \\
\text { there may be more data to be returned } \\
\text { GOTALL is set to.FALSE. }\end{array}$ \\
\hline & NID & Input & integer & Count of identifiers to be retrieved. \\
\hline & RETRID & Input & $\begin{array}{l}\text { character } \\
\text { array } \\
\text { (length } \leq 16)\end{array}$ & Names of identifiers to be retrieved. \\
\hline & NFフUND & Output & integer & $\begin{array}{l}\text { Count of lines of data that satisfied the re } \\
\text { trieval conditions. }\end{array}$ \\
\hline & VAL & Output & real array & $\begin{array}{l}\text { Values of identifiers that were retrieved. NID } \\
\text { times NFOUND values are stored in VAI } \\
\text { (NID values for each line). }\end{array}$ \\
\hline & GOTALL & Output & logical & $\begin{array}{l}\text { TRUE. if all lines on the data base were } \\
\text { checked. .FALSE. if more lines on the data } \\
\text { base need to be checked. }\end{array}$ \\
\hline & ERROR & Output & logical & $\begin{array}{l}\text { TRUE. if an error was encountered. } \\
\text {.FALSE. if no error was encountered. }\end{array}$ \\
\hline
\end{tabular}




\begin{tabular}{|c|c|c|c|c|}
\hline Name & \multicolumn{4}{|c|}{ SDBGETL - Retrieve data of any type } \\
\hline Description & \multicolumn{4}{|c|}{$\begin{array}{l}\text { SDBGETL returns up to MAXRET values for each of the NID identifier names in } \\
\text { array RETRID provided retrieval condition COND is satisfied. }\end{array}$} \\
\hline Syntax & \multicolumn{4}{|c|}{$\begin{array}{l}\text { CALLSDBGETL(DBNAME, COND, MAXRET, NID, RETRID, NFOUND, LOCVAL, } \\
\text { GOTALL, ERROR) }\end{array}$} \\
\hline \multirow{10}{*}{$\begin{array}{l}\text { Variables in } \\
\text { Call }\end{array}$} & Name & $I / O$ & Type & Description \\
\hline & DBNAME & Input & $\begin{array}{l}\text { character } \\
\text { (length } \leq 16)\end{array}$ & The name of the data base. \\
\hline & COND & Input & character & The retrieval condition. \\
\hline & MAXRET & Input & integer & $\begin{array}{l}\text { Count of the maximum lines to be returned. If } \\
\text { there may be more data to be returned, } \\
\text { GOTALL is set to.FALSE. }\end{array}$ \\
\hline & NID & Input & integer & Count of identifiers to be retrieved. \\
\hline & RETRID & Input & $\begin{array}{l}\text { character } \\
\text { array } \\
\text { (length } \leq 16)\end{array}$ & Names of identifiers to be retrieved. \\
\hline & NFOUND & Output & integer & $\begin{array}{l}\text { Count of lines of data that satisfied the re- } \\
\text { trieval conditions. }\end{array}$ \\
\hline & LOCVAL & Output & $\begin{array}{l}\text { integer } \\
\text { array }\end{array}$ & $\begin{array}{l}\text { Locations of arrays where retrieved values } \\
\text { are stored. NFOUND values are stored in } \\
\text { each array whose location is in LOCVAL. } \\
\text { There should be NID array locations in } \\
\text { LOCVAL. }\end{array}$ \\
\hline & GOTALL & Output & logical & $\begin{array}{l}\text { TRUE. if all lines on the data base were } \\
\text { checked. FALSE. if more lines on the data } \\
\text { base need to be checked. }\end{array}$ \\
\hline & ERROR & Output & logical & $\begin{array}{l}\text { TRUE. if an error was encountered. } \\
\text {.FALSE. if no error was encountered. }\end{array}$ \\
\hline Con & \multicolumn{4}{|c|}{ SDBGETL will skip over any data base header lines. } \\
\hline
\end{tabular}




\begin{tabular}{|c|c|c|c|c|}
\hline Name & \multicolumn{4}{|c|}{ SDBIATR - Return attributes } \\
\hline Description & \multicolumn{4}{|c|}{ SDBIATR returns the attributes of a data base identifier. } \\
\hline Syntax & \multicolumn{4}{|c|}{$\begin{array}{l}\text { CALL SDBIATR (DBNAME, IDENT, TYPE, NBYTES, FMT, KOLI, KOLF, } \\
\text { ERROR) }\end{array}$} \\
\hline \multirow{9}{*}{$\begin{array}{l}\text { Variables in } \\
\text { Call }\end{array}$} & Nume & $I / O$ & Type & Description \\
\hline & DBNAME & Input & $\begin{array}{l}\text { character } \\
\text { (length } \leq 16)\end{array}$ & The name of the data base. \\
\hline & IDENT & Input & $\begin{array}{l}\text { character } \\
\text { (length } \leq 16 \text { ) }\end{array}$ & The name of a data base identifier. \\
\hline & TYPE & Output & $\operatorname{char}^{*} 4$ & Type of identifier: CHAR, INT, or REAL. \\
\hline & NBYTES & Output & integer & $\begin{array}{l}\text { Length of the variable corresponding to } \\
\text { IDENT, in bytes (integer and real variables } \\
\text { always require } 4 \text { bytes). }\end{array}$ \\
\hline & FMT & Output & $\operatorname{char}^{*} 16$ & $\begin{array}{l}\text { Format for the variable corresponding to } \\
\text { IDENT, enclosed in parentheses. The format } \\
\text { is taken from the descriptor file. }\end{array}$ \\
\hline & KOLI & Output & integer & $\begin{array}{l}\text { Starting column on the data base for the } \\
\text { IDENT field. }\end{array}$ \\
\hline & KOLF & Output & integer & $\begin{array}{l}\text { Ending column on the data base for the IDENT } \\
\text { field. }\end{array}$ \\
\hline & ERROR & Output & logical & $\begin{array}{l}\text { FALSE. if there is no error; TRUE. if the } \\
\text { data base does not exist or if IDENT is not on } \\
\text { the data base. }\end{array}$ \\
\hline
\end{tabular}




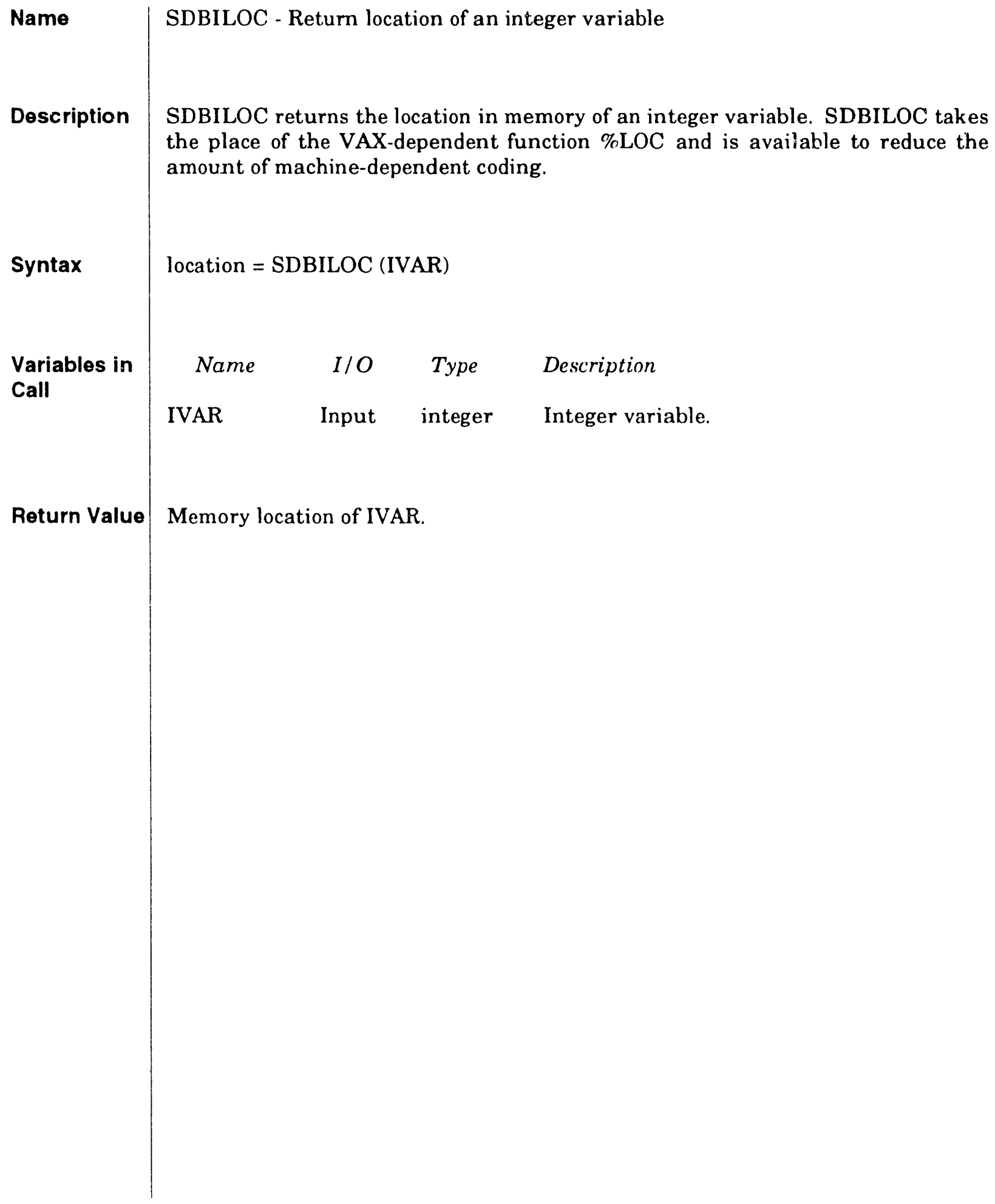




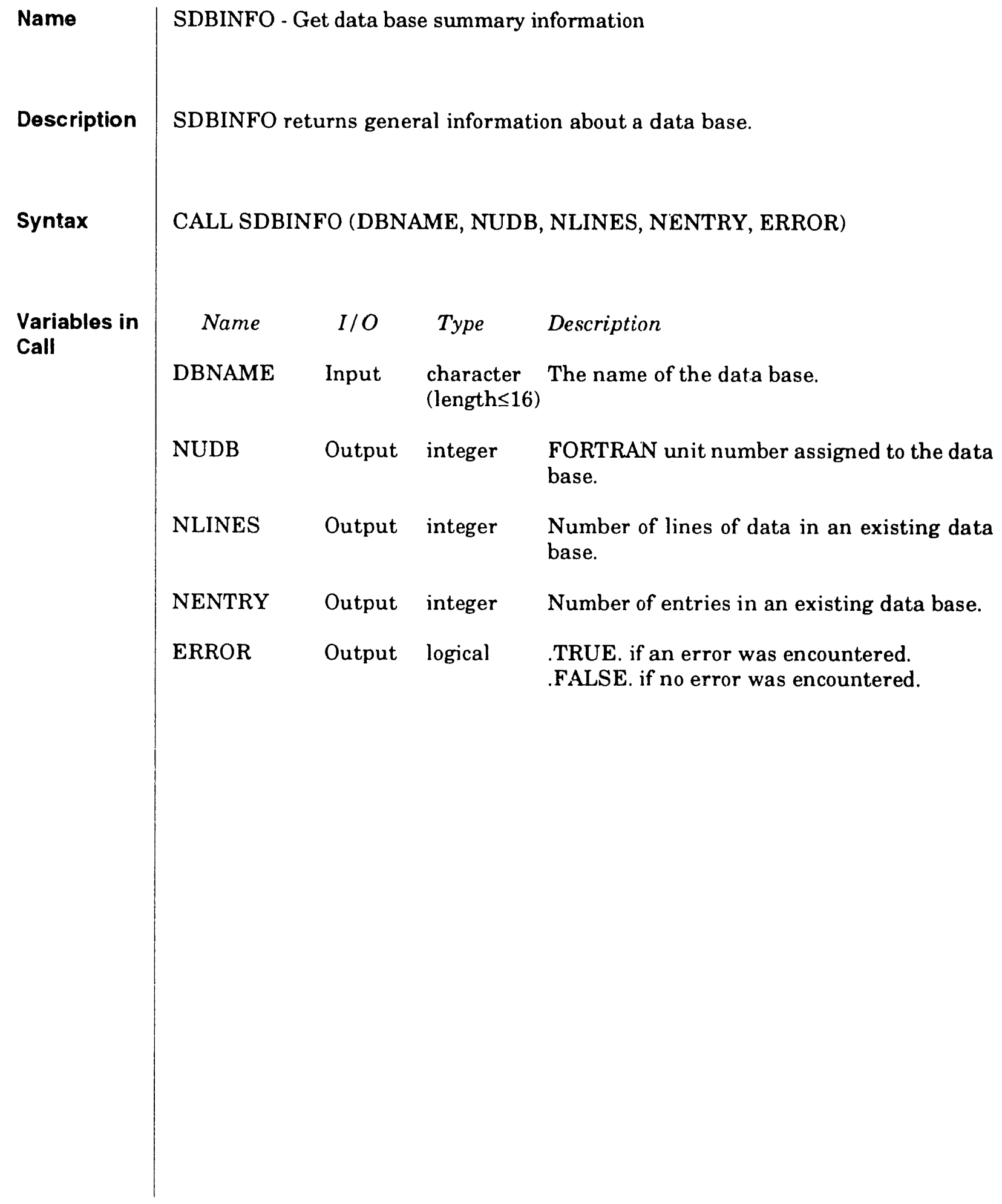




\begin{tabular}{|c|c|c|c|c|}
\hline Name & \multicolumn{4}{|c|}{ SDBINIT - Data base read/write initialization } \\
\hline Description & \multicolumn{4}{|c|}{$\begin{array}{l}\text { SDBINIT performs data base initialization for reading or writing. When writing a } \\
\text { new data base, the file must exist and contain header information before SDBINIT } \\
\text { is called. }\end{array}$} \\
\hline Syntax & \multicolumn{4}{|c|}{ CALL SDBINIT (DBASE, DBDSCR, DBNAME, UNITNUM, RDONLY, ERROR) } \\
\hline \multirow{7}{*}{$\begin{array}{l}\text { Variables in } \\
\text { Call }\end{array}$} & Name & $I / O$ & Type & Description \\
\hline & DBASE & Input & $\begin{array}{l}\text { character } \\
\text { (length } \leq 16 \text { ) }\end{array}$ & The path and file name of the data base. \\
\hline & DBDSCR & Input & character & $\begin{array}{l}\text { The path and name of the file describing the } \\
\text { data base neutral file (data base descriptor } \\
\text { file). Normally, DBDSCR is blank, indicating } \\
\text { the descriptor information is at the beginning } \\
\text { of the data base neutral file. }\end{array}$ \\
\hline & DBNAME & Input & $\begin{array}{l}\text { character } \\
\text { (length } \leq 16 \text { ) }\end{array}$ & The name of the data base. \\
\hline & UNITNUM & Input & integer & $\begin{array}{l}\text { User's choice of FORTRAN unit number for } \\
\text { the data base. If zero, the number is assigned } \\
\text { by SDBINIT as } 50+\text { the data base sequence } \\
\text { number. }\end{array}$ \\
\hline & RDONLY & Input & logical & $\begin{array}{l}\text { TRUE. if the data base should be opened as } \\
\text { read only. }\end{array}$ \\
\hline & ERROR & Output & logical & $\begin{array}{l}\text { TRUE. if an error was encountered. } \\
\text {.FALSE. if no error was encountered. }\end{array}$ \\
\hline
\end{tabular}




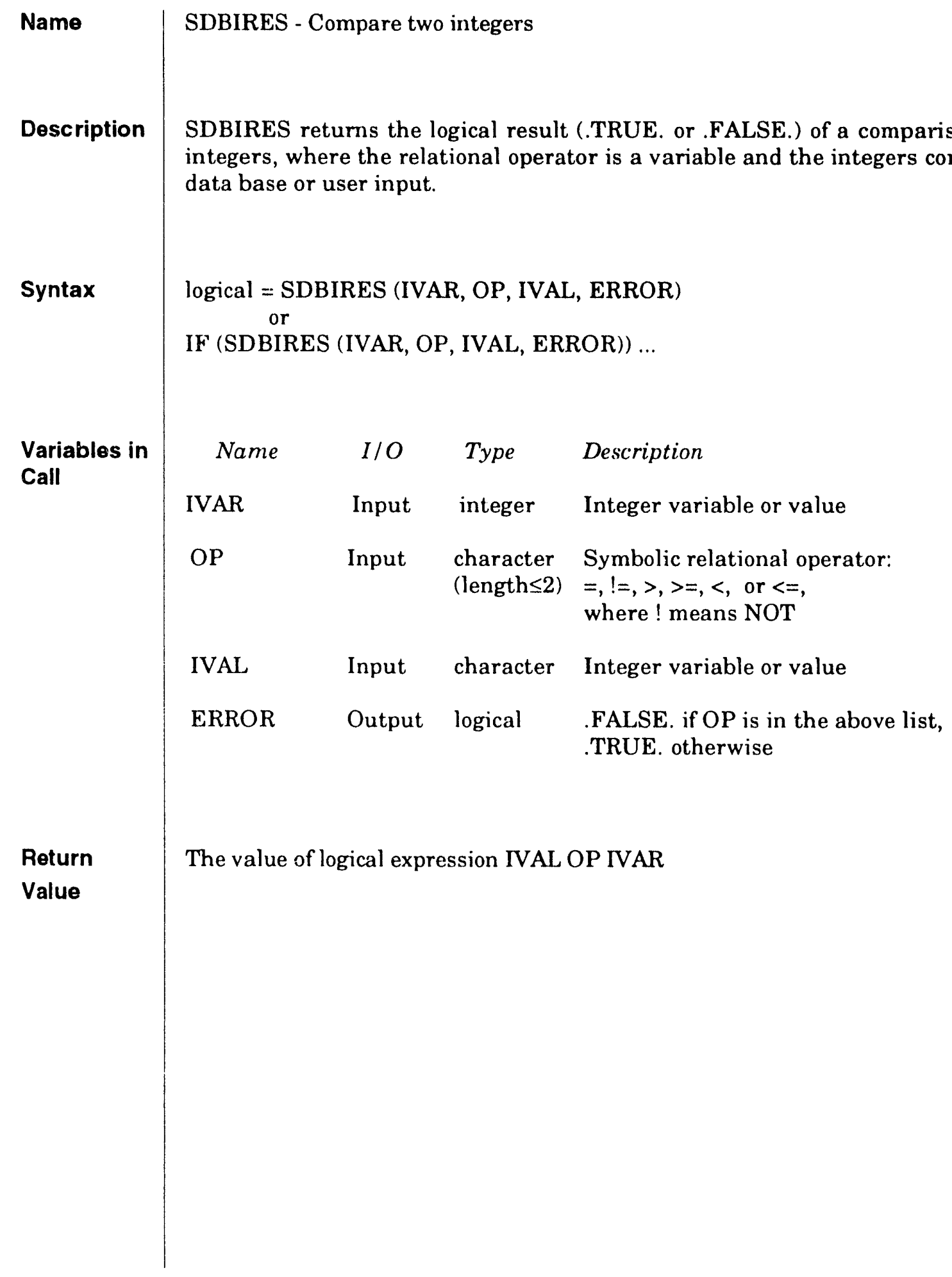




\begin{tabular}{|c|c|c|c|c|}
\hline Name & \multicolumn{4}{|c|}{ SDBNINFO - Get identifiers } \\
\hline Description & \multicolumn{4}{|c|}{ SDBNINFO returns identifier names contained in the data base descriptor file. } \\
\hline Syntax & \multicolumn{4}{|c|}{$\begin{array}{l}\text { CALL SDBNINFO (DBNAME, NUQID, UQID, MAXNIDH, NIDH, IDENT, } \\
\text { ERROR) }\end{array}$} \\
\hline \multirow{8}{*}{$\begin{array}{l}\text { Variables in } \\
\text { Call }\end{array}$} & Name & $I / O$ & Type & Description \\
\hline & DBNAME & Input & $\begin{array}{l}\text { character } \\
\text { (length } \leq 16 \text { ) }\end{array}$ & The name of the data base. \\
\hline & NUQID & Input & integer & $\begin{array}{l}\text { Count of identifiers needed to uniquely specify } \\
\text { an entry. }\end{array}$ \\
\hline & IVID & Input & $\begin{array}{l}\text { character } \\
\text { array } \\
\text { (length } \leq 16 \text { ) }\end{array}$ & $\begin{array}{l}\text { Array of identifiers needed to uniquely specify } \\
\text { an entry. }\end{array}$ \\
\hline & MAXNIDH & Input & integer & $\begin{array}{l}\text { Count of maximum number of identifiers to be } \\
\text { returned. }\end{array}$ \\
\hline & IIDH & Output & integer & $\begin{array}{l}\text { Count of identifiers defined in the data base } \\
\text { header. }\end{array}$ \\
\hline & 'DENT & Output & $\begin{array}{l}\text { character } \\
\text { array } \\
\text { (length } \leq 16 \text { ) }\end{array}$ & Array of identifier names. \\
\hline & ERROR & Output & logical & $\begin{array}{l}\text { TRUE. if an error was encountered. } \\
\text { FALSE. if no error was encountered. }\end{array}$ \\
\hline
\end{tabular}




\begin{tabular}{|c|c|c|c|c|}
\hline Name & \multicolumn{4}{|c|}{ SDBNUMDB - Return data base FORTRAN unit number } \\
\hline Description & \multicolumn{4}{|c|}{$\begin{array}{l}\text { SDBNUMDB returns the FORTRAN unit number NUMDB corresponding to the } \\
\text { data bare DBNAME. }\end{array}$} \\
\hline Syntax & \multicolumn{4}{|c|}{ CALL SDBNUMDB (DBNAME, NUMDB, ERROR) } \\
\hline \multirow{4}{*}{$\begin{array}{l}\text { Yariables in } \\
\text { Call }\end{array}$} & Name & $I / O$ & Type & Description \\
\hline & DBNAME & Input & $\begin{array}{l}\text { character } \\
\text { (length } \leq 16 \text { ) }\end{array}$ & The name of the data base. \\
\hline & NUMDB & Output & integer & $\begin{array}{l}\text { The FORTRAN unit number corresponding } \\
\text { to the data base specified by DBNAME. }\end{array}$ \\
\hline & ERROR & Output & logical & $\begin{array}{l}\text { TRUE. if an error was encountered. } \\
\text {.FALSE. if no error was encountered. }\end{array}$ \\
\hline
\end{tabular}


Name
Description

Syntax

Variables in Call
SDBPUTL - Write data

SDBPUTL writes values for identifiers IDENT(1) - IDENT(NID) for each of NLINES lines of data to a data base with name DBNAME.

CALL SDBPUTL (DBNAME, NID, IDENT, NLINES, LOCVAL, ERROR)

$\begin{array}{ccl}\text { Name } & \text { I/O } & \text { Type } \quad \text { Description } \\ \text { DBNAME } & \text { Input } & \begin{array}{l}\text { character The name of the data base. } \\ \text { (length } \leq 16)\end{array} \\ \text { NID } & \text { Input } & \text { integer } \quad \text { Count of identifiers to be written. } \\ \text { IDENT } & \text { Input } \quad \begin{array}{l}\text { character Array of identifier names. } \\ \text { array } \\ \text { (length } \leq 16)\end{array}\end{array}$

NLINES Input integer Count of lines of data to be written.

LOCVAL Input integer Array of locations of arrays to be written. array There should be NID array locations in LOCVAL. NLINES values are written from each array whose location is inLOCVAL.

ERROR Output logical .TRUE. if an error was encountered.

.FALSE. if no error was encountered.

\section{Comment \\ SDBPUTL will skip over any data base header lines.}




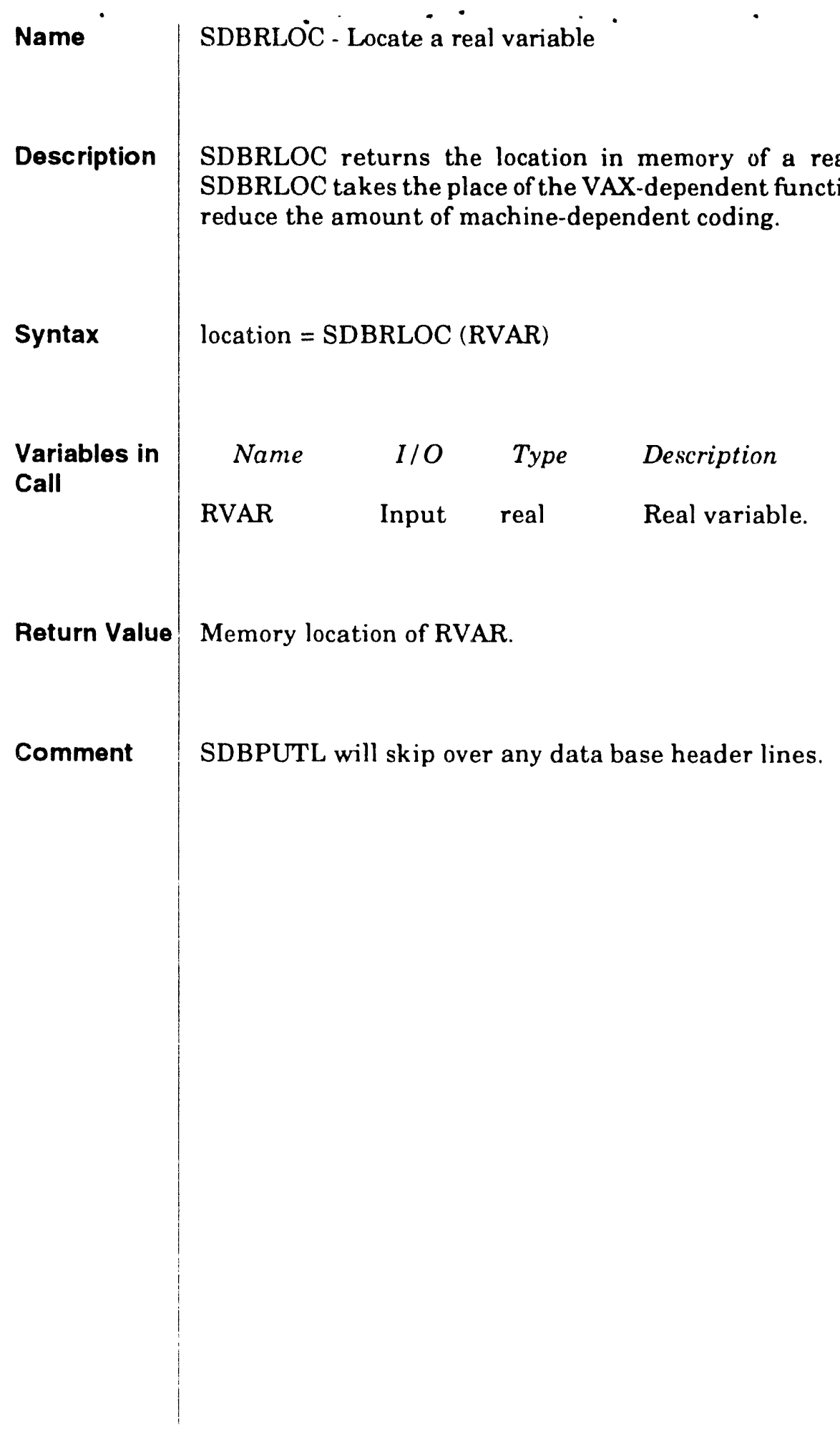




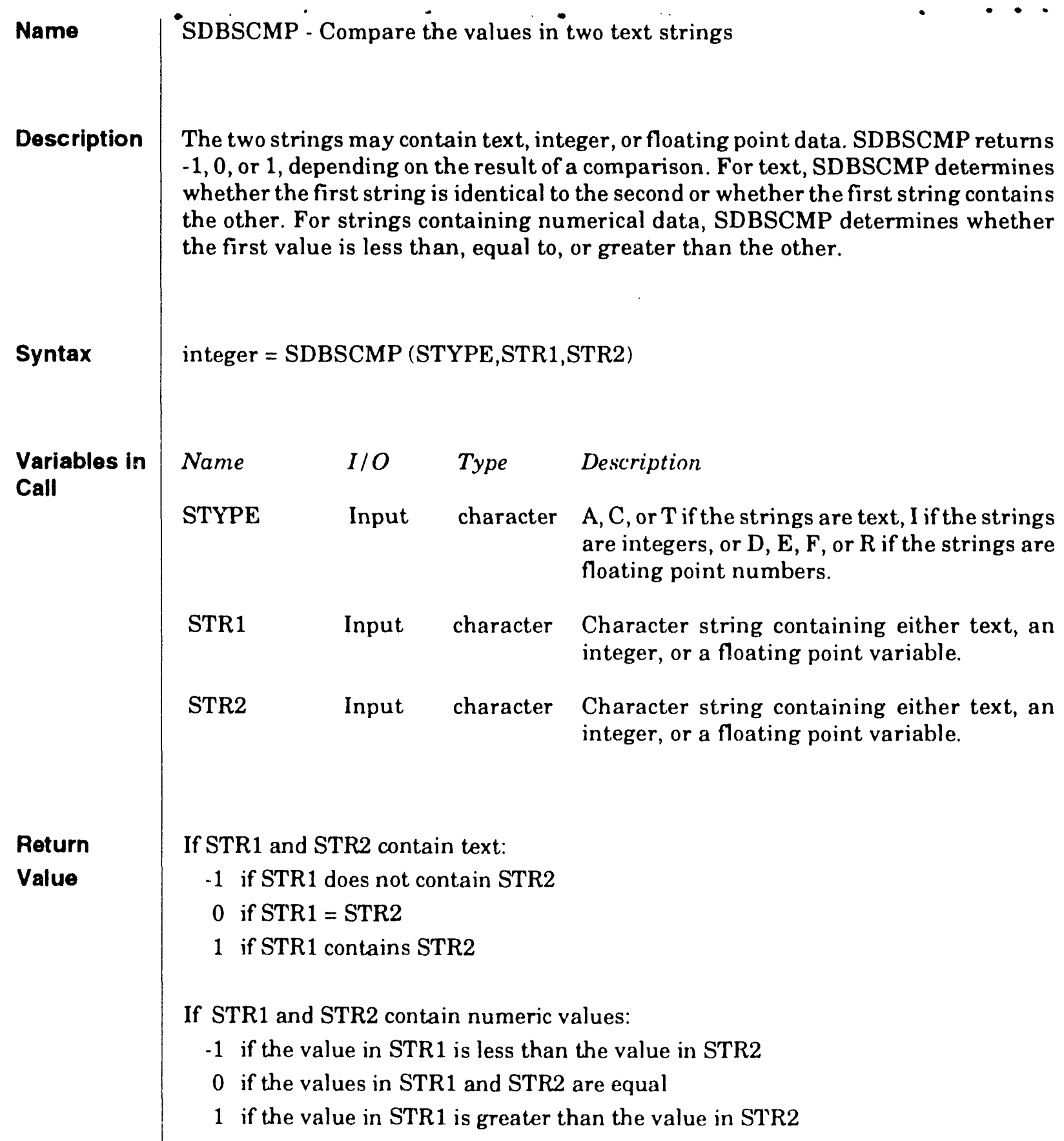




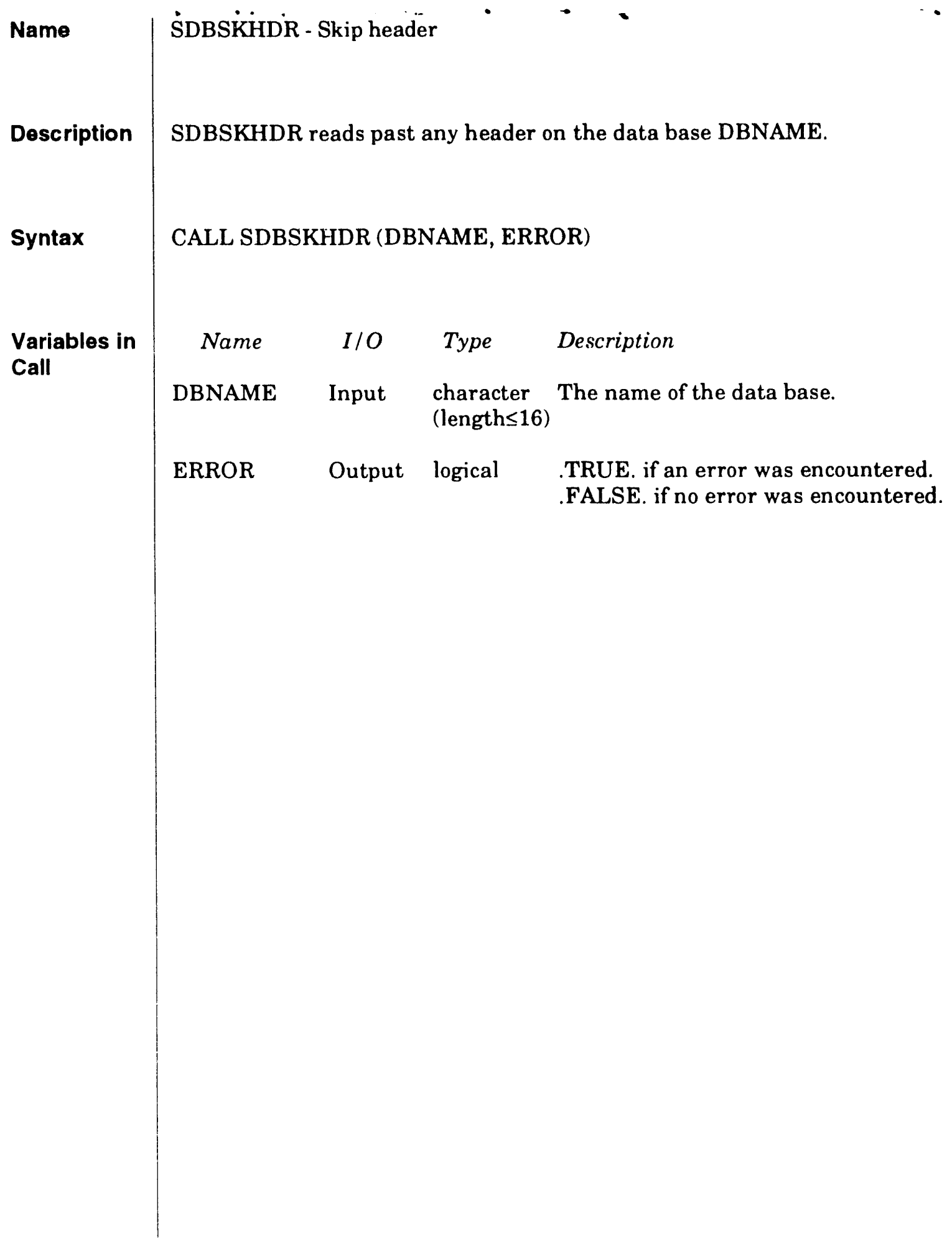




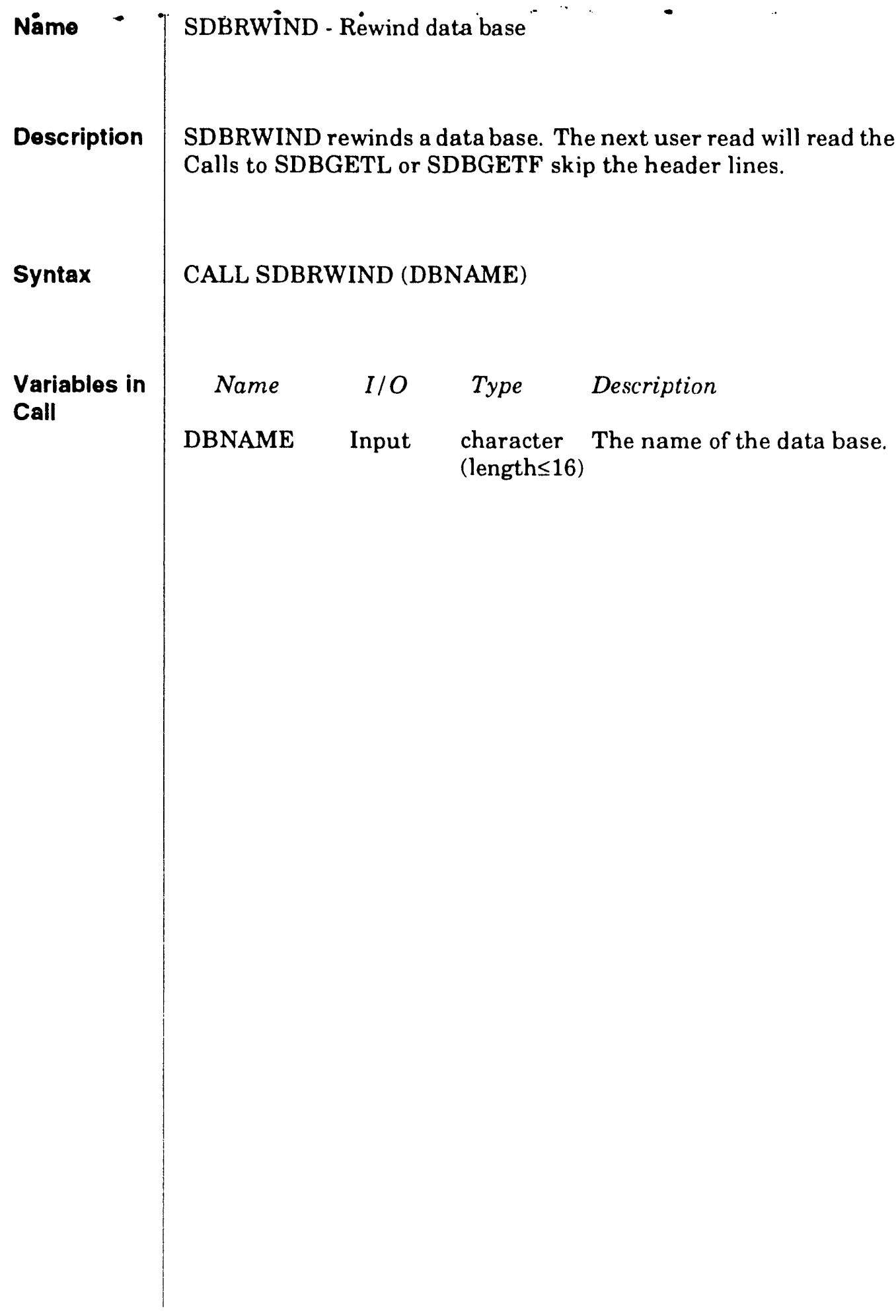


SDBREAD_LIB

Advanced Routines

\subsubsection{Advanced Routines}

Brief descriptions of specialized routines are given on the following pages. These routines perform tasks that, are necessary to data retrieval but are-too specialized to be very useful to most application programmers. 


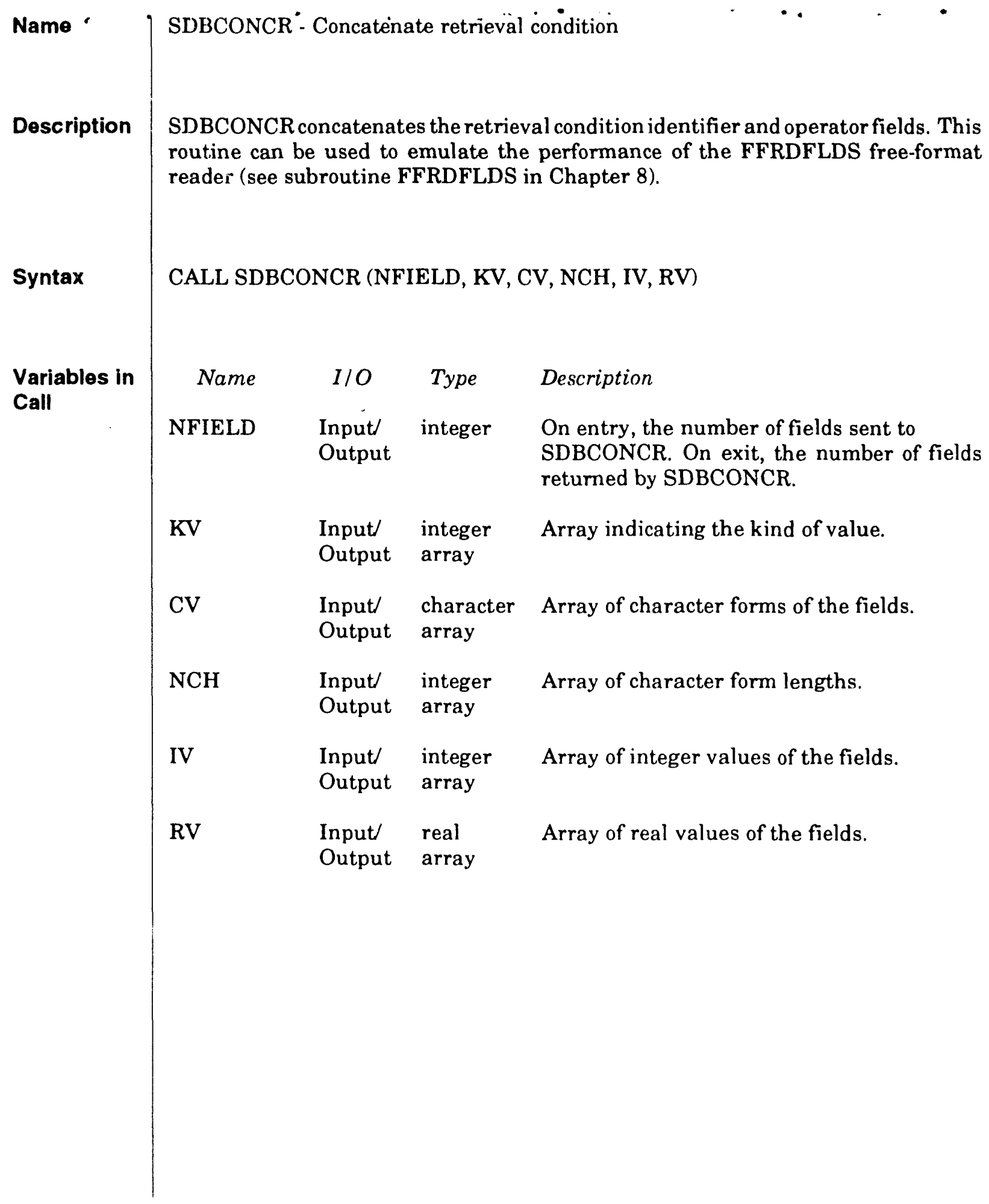




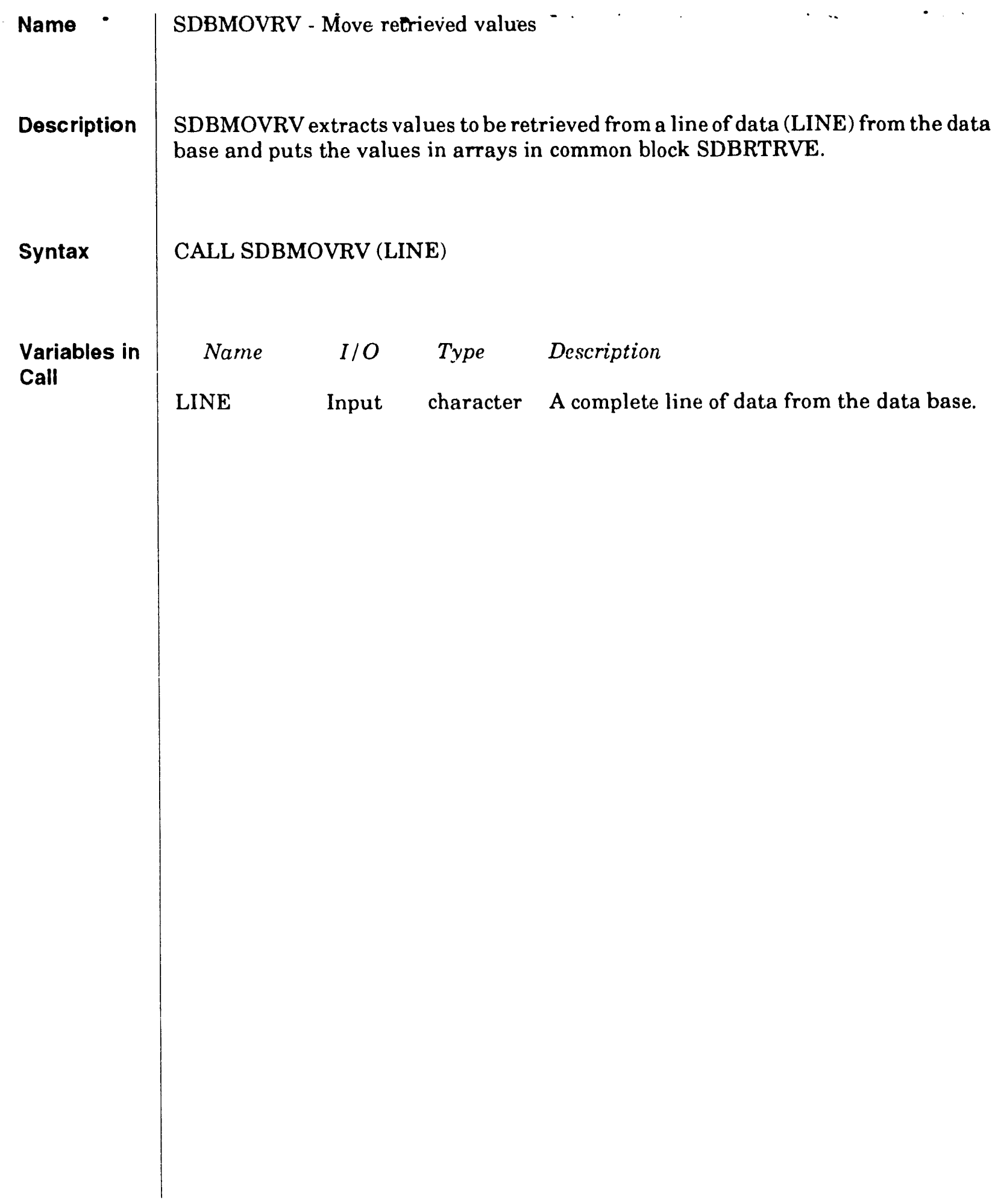




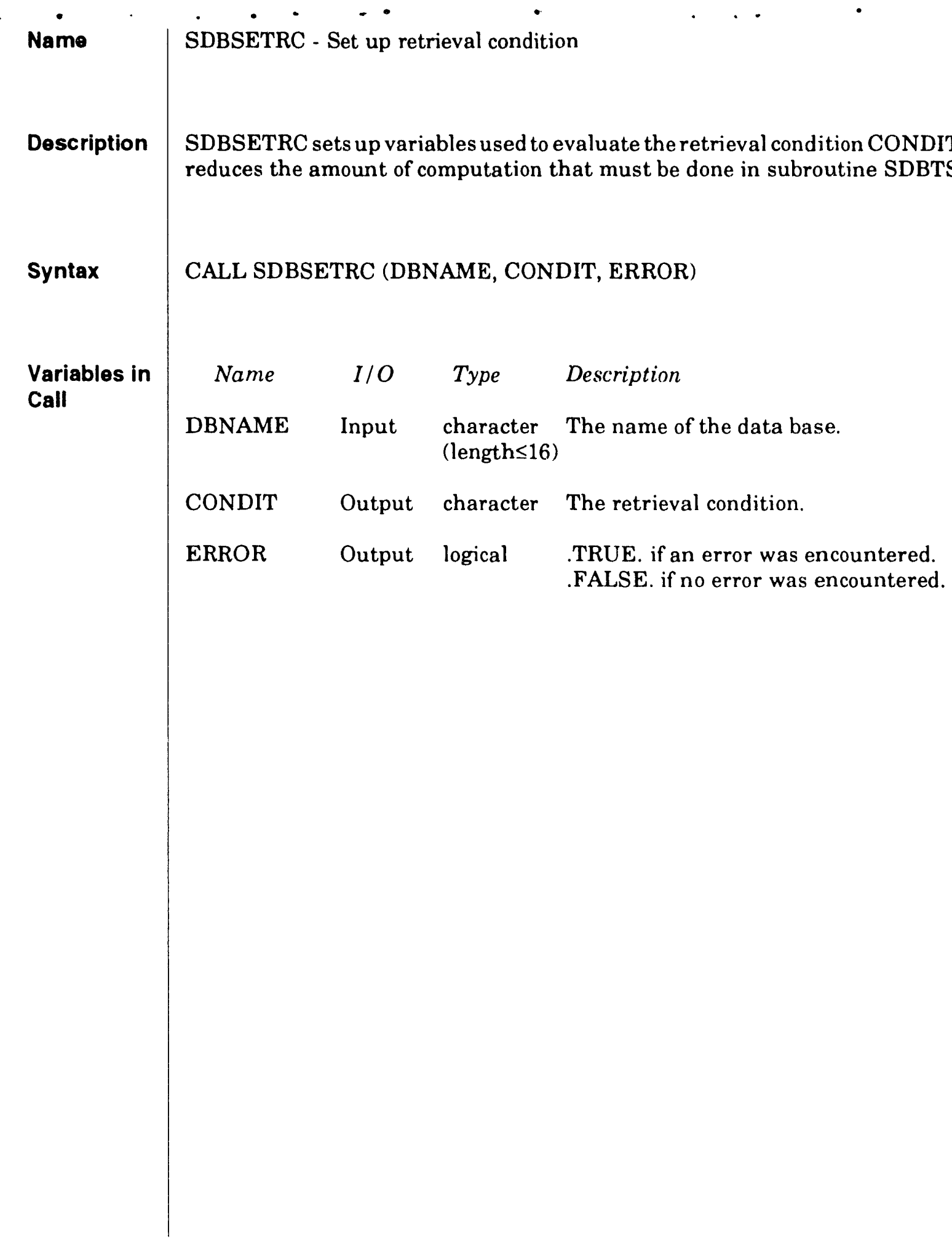




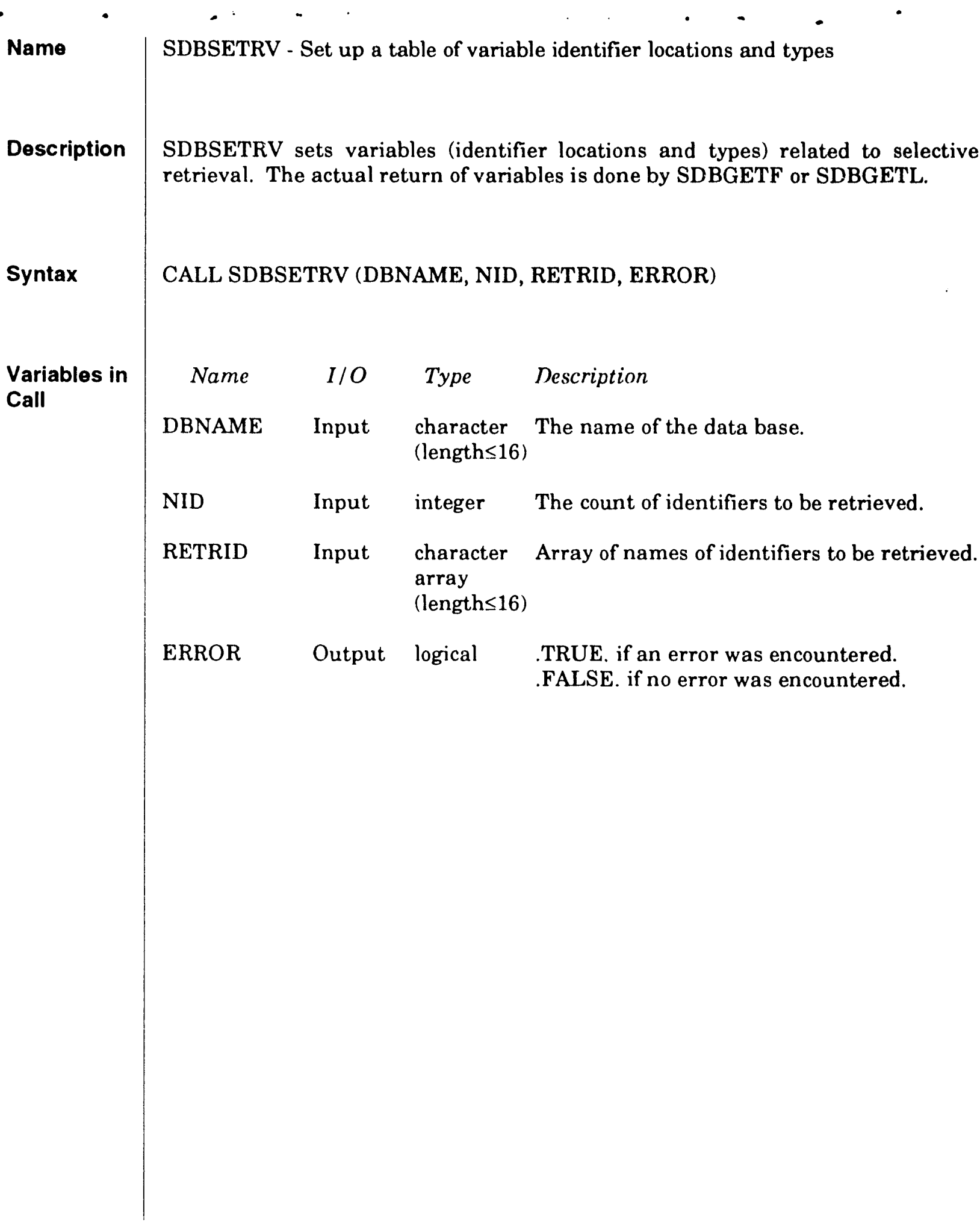


Name

Variables in

Call

SDBTSTRC - Test retrieval condition

SDBTSTRC tests the validity of the latest retrieval condition for the data base line contained in LINE.

CALL SDBTSTRC (DBNAME, LINE, ERROR)

$\begin{array}{cclc}\text { Name } & I / O & \text { Type } \quad \text { Description } \\ \text { DBNAME } & \text { Input } & \begin{array}{l}\text { character The name of the data base. } \\ \text { (length } \leq 16)\end{array}\end{array}$

LINE Input character A complete line of data from the data base.

ERROR Output logical .TRUE. if an error was encountered.

.FALSE. if no error was encountered. 
SDBREAD_LIB

Advanced Routines 


\section{CAMCON_LIB: \\ Quality Assurance Routine Library}

This chapter ciescribes the calls to quality assurance and numerous other routines repeatedly used in software developed specifically for the CAMCON system and collected into a FORTRAN library called CAMCON_LIR. The purpose of CAMCCN_LIB is to simplify coding and to standardize the user interface to all programs.

\subsection{General Guidısines on Use}

\subsubsection{Sponeorship and Version}
Authors:
A. F. Gilkey, J. S. Rath, D. K. Rudeen, D. W. Osborn, H. J. Iuzzolino, and W. C. Mills-Curran
Sponsor.
A. P. Gi'key
Version / Date:
C-2.00VV/ March 1.993
Language.
FORTRAN 77

\subsubsection{Linking}

CAMCON_LIB is intended to be used in conjunction with the CAMSUPES_LIB library, which includes dynamic memory waanagement and common machine-specific routines. As assigned by the library sponsor, the logical symbol for CAMCON_LIB is CAMCON_LIB; hence, to link this library to another code, type

\section{\$LIT̄K rode_rame, CAMCON_LIB/LIB, CAMSUPES_LIB/LIB}

A FLINT library for CAMCON_LIB is available for checking interfaces. It is in CAMCON\$ROOT:(LIBRARIES.CAMCON_LIB)CAMCON_LIB.LBT (see Section 12.5 for more information about the FLINT litrary).

\subsubsection{Error Mesranges}

CAMCON.LIB assumes that there is a diagnostic file where error messages are to be written. The user needs to set this file unit with the function IQAERRUNI. The user can retrieve this unit with IQAERRLNI (-1). If no file is defined or if this unit is 0 , output is written to the terminal or standard output device (SYS\$OUTPUT on the VAX) with a "WRITE $\left({ }^{*}, \ldots\right.$ " statement. 


\subsubsection{Summary}

The CAMCON_LIB routines are described in detail in the next section. The following is a summary of the CAMCON_LIB routines.

QA and User Interface Routines

- IQAERRUNI - Define diagnostics file unit

- QAABORT - Abort program and print error message to terminal and diagnostics file (if desired)

- QABANNER - Print code banner and list credits (authors and code origin)

- QABATCH - Return batch or interactive flag

- QAEIGLET - Print characters (e.g., code name) in big letters

- QACPUS - Print CPU seconds used

- Q.DOEDIS - Print standard DOE legal disclaimer

- QAFETCH - Return the program QA information

- QAMAKREC - Assemble QA record to identify code on CAMDAT file

- QAMAXERR - Check integer value against maximum

- QAMEMERR - Check dynamic memory error

- QAMESSAG - Print message of a specified type

- QAPAGE - Print QA information on output file (code name, software ID, and date)

- QAPRTSTR - Print message

- QASETUP Startup program and place code name, software ID, revision date, current date and time, software author, and software sponsor into a common block for use by other routines

File Processing Routines

- FILCMDLIN - Read and parse command line

- FILDFNAM - Define a file for use by FILRDNAMS

- FILECHO - Read formatted file and write listing

- FILOPEN - Open a file

- FILPARSE - Create file name from input and default names

- FILRDNAMS - Read the files defined by FILDFNAM

- FILWRNAMS - Write the file names read by FILRDNAMS

Free-Field Input Routines

- FFGETLIN - Return line read by free-field routine

- FFPAREQN - Equation parsing routine

- FFPARSE - Free-field parsing routine (called by FFRDFLDS)

- FFRDFLDD - Free-field input routine (double r 'ecisior'

- FFRDFLDG - Free-field input routine (double precision)

- FFRDFLDS - Free-field input routine (singie precision)

- FFRDKEY - Free-field heyword-parameter input routine

- FFRDLINE - Get literal input line

String Proces sing Routines

- ISTRFIND - Find string in list

- ISTRLEN - Return string length

- STRCMPRS - Remove extra blanks from string 
- STRLIMIT - Strip leading/trailing blanks

- STRPACK - Remove all blanks from string

- STRUPCASE - Convert string to upper case

Common Internal Routines

- FEELEM - Calculate element number associated with node

- FEIJK - Determine I,J,K index from node/element number

- FEMINMAX - Calculate min/max value

- FENENUM - Determine node/element number from I,J,K index

Obsolete Routines

- ABORT - Replaced by QAABORT

- BANNER - Replaced by QABANNER

- BATCH - Replaced by QABATCH

- BIGLET - Replaced by QABIGLET

- DEFFIL - Replaced by FILDFNAM

- DOEDIS - Replaced by QADOEDIS

- ECHO - Replaced by FILECHO

- ELEM - Replaced by FEELEM

- FFISTR - Replaced by FFPARSE

- FILDAT - Replaced by EXFILDAT (in CAMSUPES_LIB)

- FPARSE - Replaced by FILPARSE

- FREDBLD - Replaced by FFRDFLDD

- FREDBLG - Replaced by FFRDFLDG

- FREFLD - Replaced by FFRDFLDS

- FRELIN - Replaced by FFGETLIN

- GETINP - Replaced by FFRDLINE

- GETPRG - Replaced by QAFETCH

- IJK - Replaced by FEIJK

- ISTOUT - Replaced by IQAERRUNI

- LENSTR - Replaced by ISTRLEN

- LOCSTR - Replaced by ISTRFIND

- MAXERR - Replaced by QAMAXERR

- MEMERR - Replaced by QAMEMERR

- MINMAX - Replaced by FEMINMAX

- NENUM - Replaced by FENENUM

- OPNFIL - Replaced by FILOPEN

- PCKSTR - Replaced by STRPACK

- PRTMSG - Replaced by QAMESSAG

- PRTOUT - Replaced by QAPRTSTR

- QAPAGE - Replaced by QAPAGE

- QASSMB - Replaced by QAMAKREC

- RDCMDL - Replaced by FILCMDLIN

- RDFNAM - Replaced by FILRDNAMS

- SQZSTR - Replaced by STRCMPRS

- STRIPB - Replaced by STRLIMIT

- STRTUP - Replaced by QASETUP

- UPCASE - Replaced by STRUPCASE

- WRCPUS - Replaced by QACPUS

- WRFNAM - Replaced by FILWRNAMS 


\subsubsection{Exainple}

The opening sections of most programs should be similar. The following code segment provides an example of the opening section.

C... Load the/CAMCON_LIB/ common with the program information CALL QASETUP ('TESTPROG', 'X-1.00', '01/15/90'.

$\$$ 'A. P. Gilkey', 'A. P. Gilkey')

C... Write a welcome banner to the terminal CALL QABANNER 10, 'CAMCON_LIB test program', ' ,' ')

C... Define the files for FILRDNAMS

CALL FILDFNAM ('CAMDAT data base', 'in', 'req', 'CAMDAT.CDB')

CALL FILDFNAM ('output CAMDAT data base', 'out', 'req'. $\$$ 'CAMDAT.CDB')

CALL FILDFNAM ('diagnostics file', 'out', 'opt', 'TEST.DBG')

C... Read in the names of the files defined above CALL FILRDNAMS (FILESP, IERR)

C... If the file names could not be read, write the file assignments

C... and abort

IF (IERR . NE, O) THEN

CALL FILWRNAMS $(0$, FILESP)

CALL QAABORT ('Incorrect file assignments')

END IF

C... If the optional diagnostics file is requested, open the file IF (FILESP(3) .NE. '' ') THEN

NOUT $=6$

CALL FILOPEN (NOUT, 'OUT', 'FORM', FILESP(3), IERR) ELSE

NOUT $=0$

END IF

C... Set the unit number of the diagnostics file (may be the terminal) IDUM = IQAERRUNI (NOUT)

IF (NOUT .GT. 0) THEN

C... Write the header to the diagnostics file, including the page header,

C... the welcome banner, the DOE disclaimer, and the file assignments CALL QAPAGE (NOUT,' ')

CALL QABANINER (NOUT, $, \cdot, \cdot, \cdot$, )

CALL QADOEDIS (NOUT, **)

CALL FILWRNAMS (NOUT, FILESP)

ENDIF 
An example of a diagnostsics file header generated by this program segment is shown below.

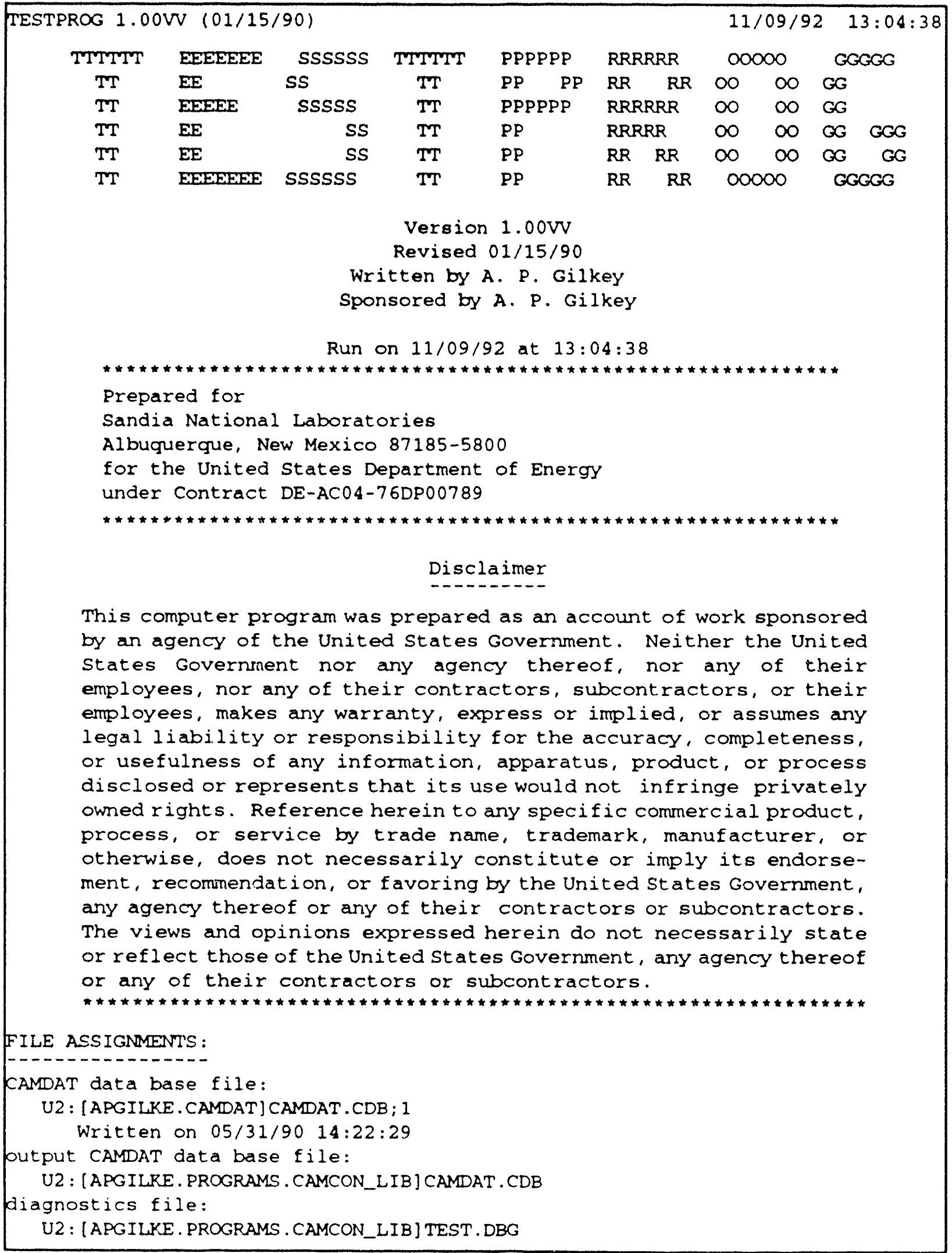


CAMCON_LIB

QA and User Interface

\subsection{Description of Routine Calls}

\subsubsection{QA and User Interface Routines}

Detailed descriptions of the ten routines for properly identifying the software and providing a uniform user interface are given on the following pages. 


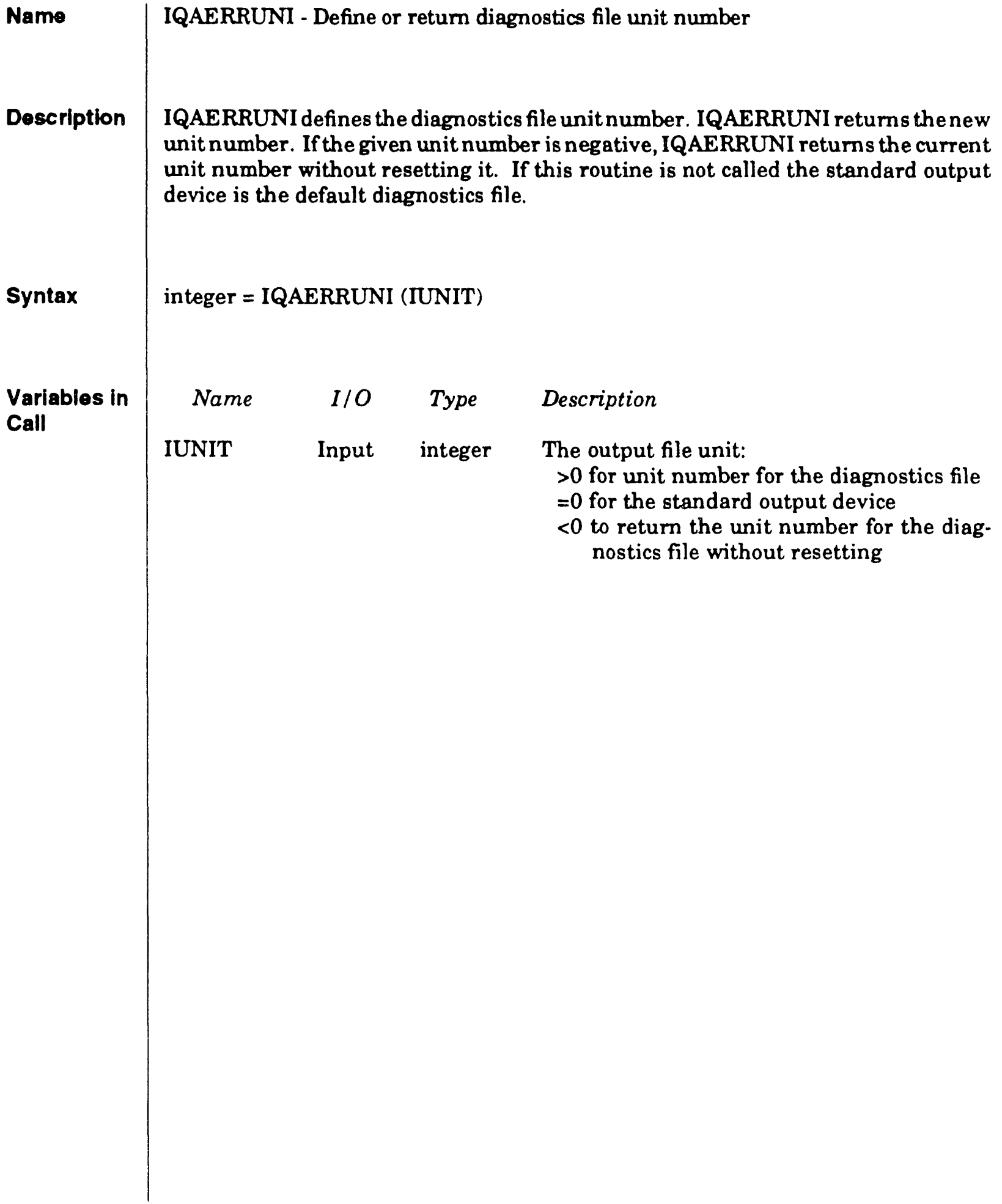




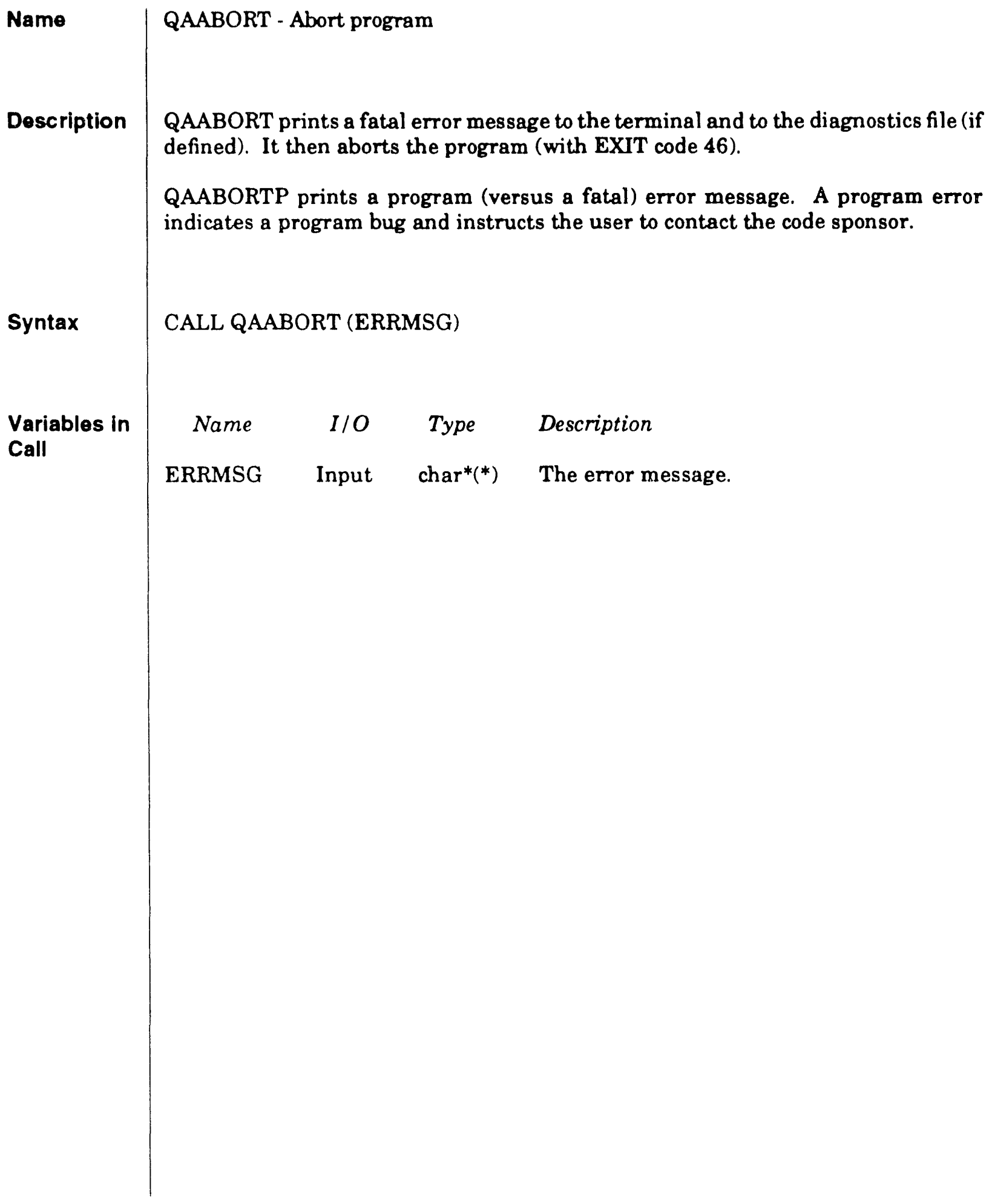




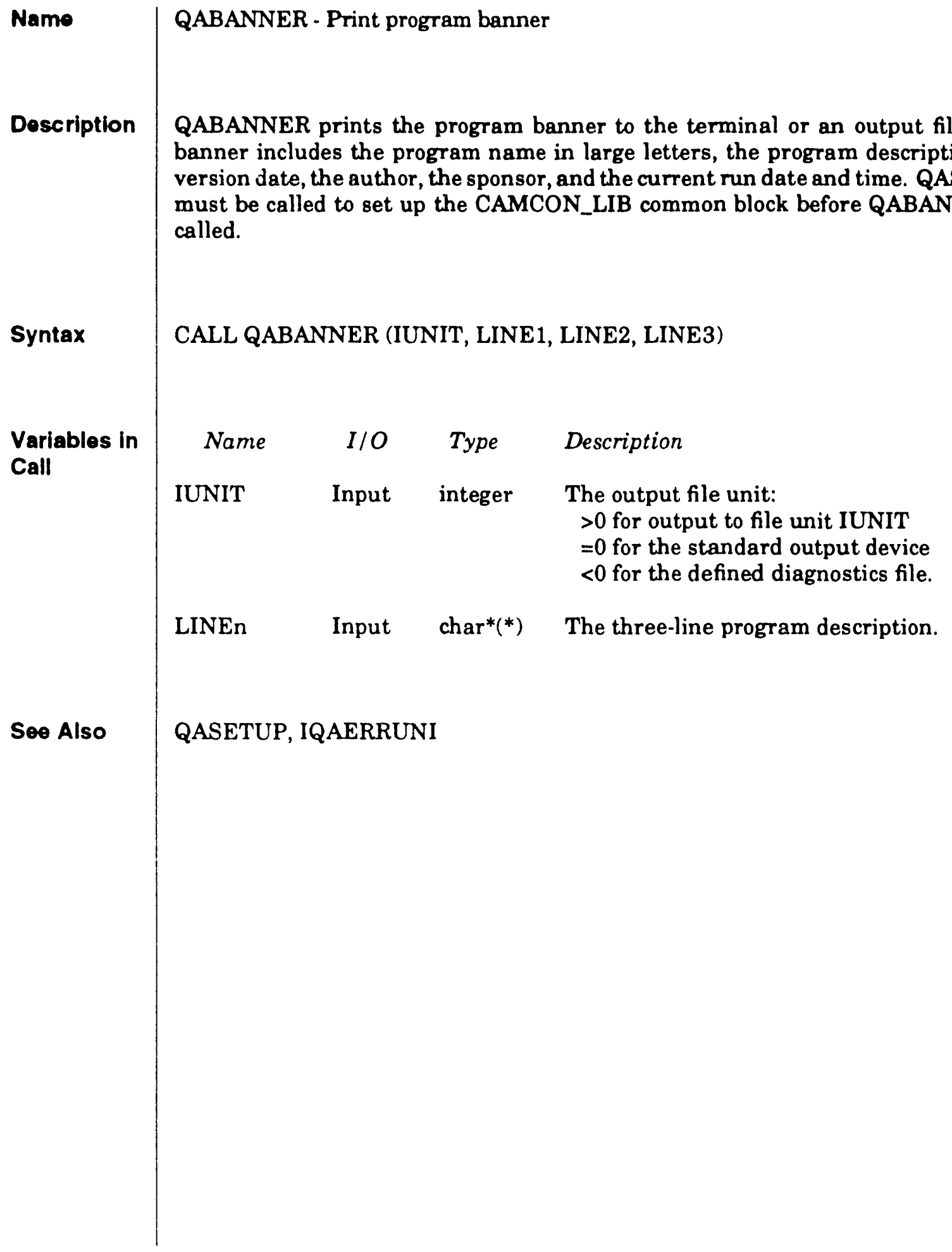


Name

Description

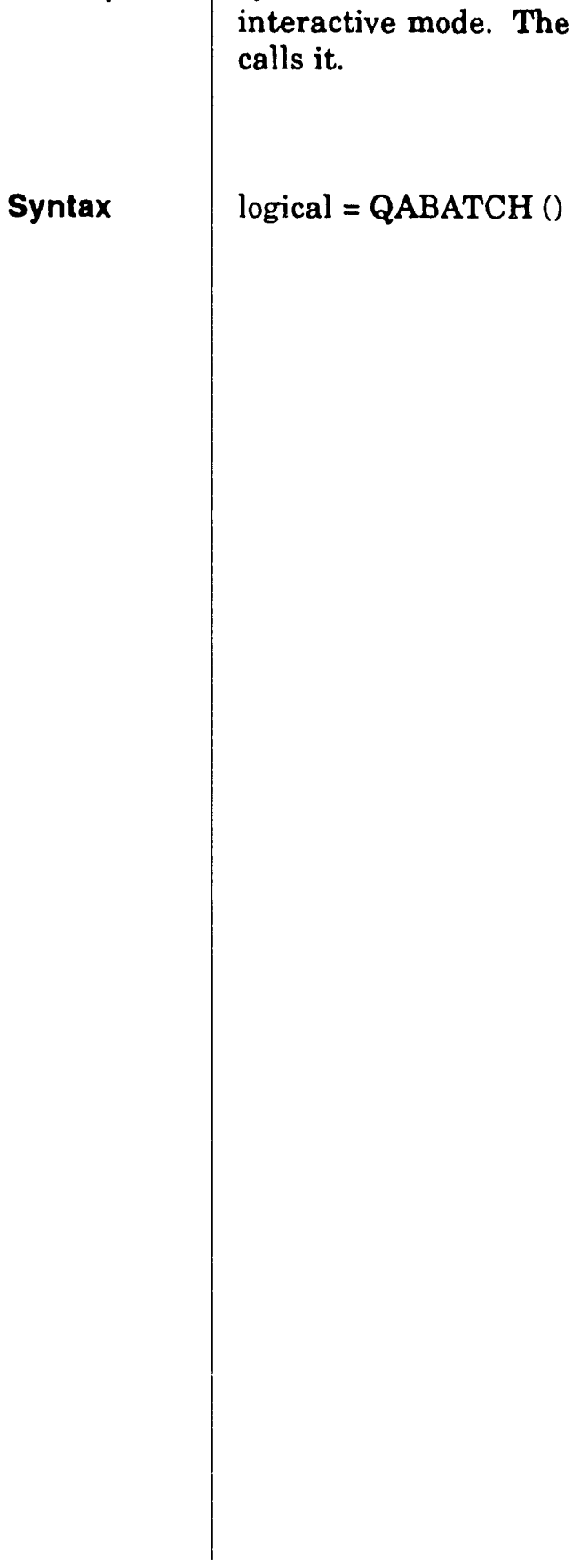
calls it.
QABATCH - Return batch versus interactive flag

QABATCH returns .TRUE. if the program is in the batch mode and .FALSE. if in the interactive mode. The function should be declared LOGICAL in any routine that 


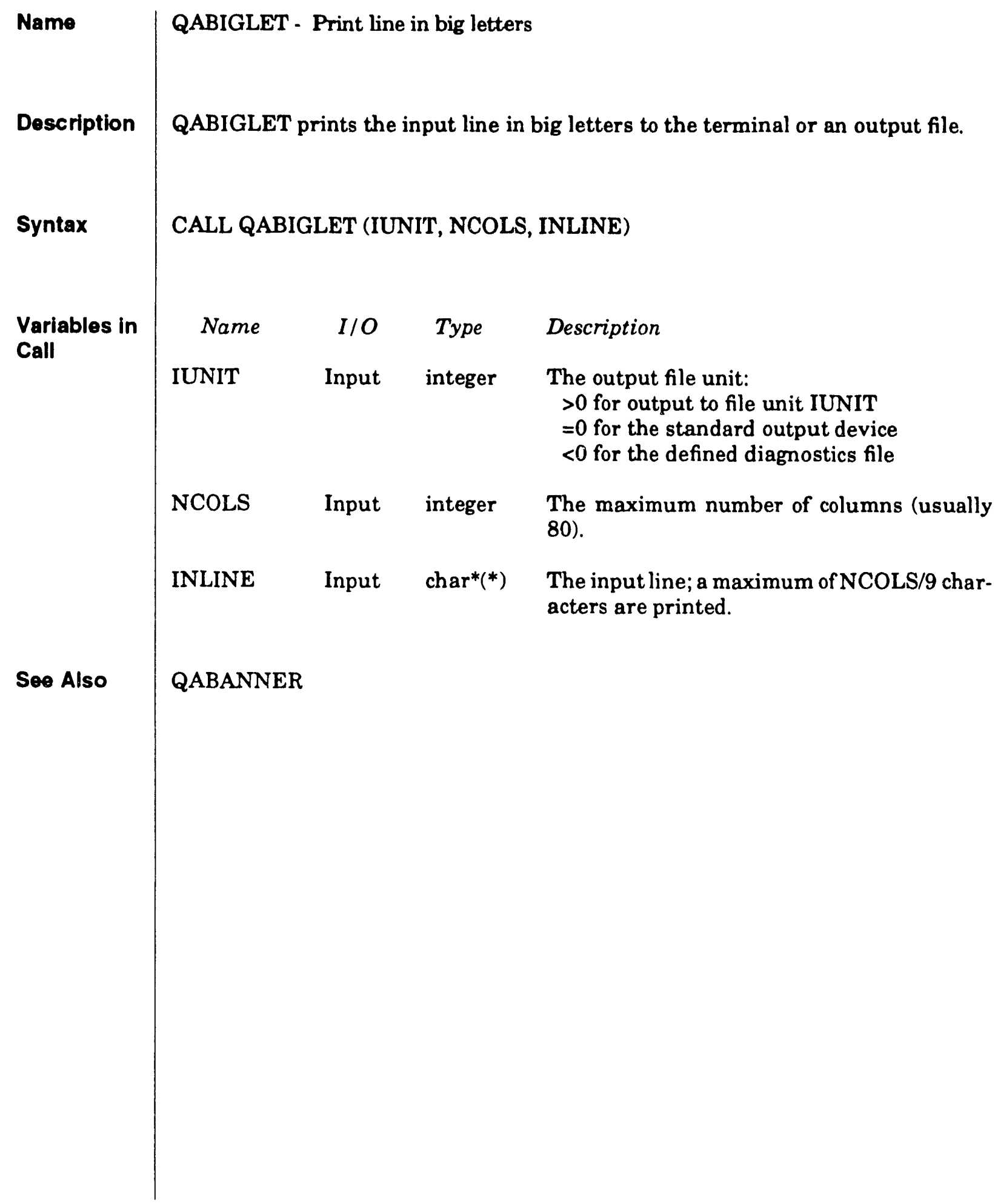




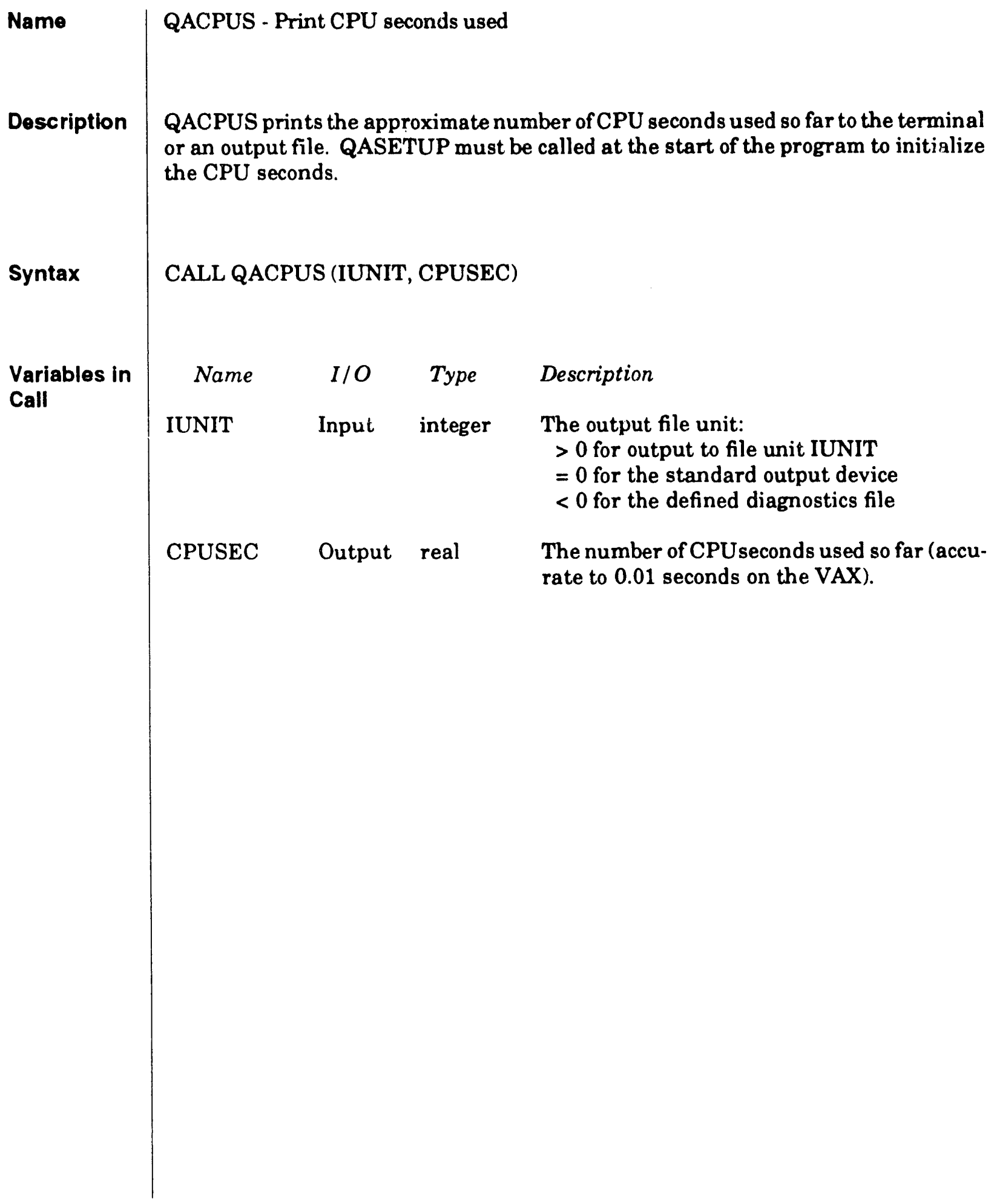




\begin{tabular}{|c|c|c|c|c|}
\hline Name & \multicolumn{4}{|c|}{ QADOEDIS - Print standard DOE disclaimer } \\
\hline Description & \multicolumn{4}{|c|}{$\begin{array}{l}\text { QADOEDIS prints the Sandia DOE contract statement and the DOE disclaimer to the } \\
\text { terminal or an output file. }\end{array}$} \\
\hline Syntax & \multicolumn{4}{|c|}{ CALL QADOEDIS (IUNOUT, CONTR) } \\
\hline \multirow{3}{*}{$\begin{array}{l}\text { Variables in } \\
\text { Call }\end{array}$} & Name & $I / O$ & Type & Description \\
\hline & IUNOUT & Input & integer & $\begin{array}{l}\text { The output file unit: } \\
>0 \text { for output to file unit IUNOUT } \\
=0 \text { for the standard output device } \\
<0 \text { for the defined diagnostics file }\end{array}$ \\
\hline & CONTR & Input & $\operatorname{char}^{*}(*)$ & $\begin{array}{l}\text { The DOE contract number. If CONTR is '*', } \\
\text { the contract number DE-ACO4-76DP00789 is } \\
\text { printed; if CONTR is ' ', the contract state- } \\
\text { ment is not printed. }\end{array}$ \\
\hline
\end{tabular}




\begin{tabular}{|c|c|c|c|c|}
\hline Name & \multicolumn{4}{|c|}{ QAFETCH - Return the program QA information } \\
\hline Description & \multicolumn{4}{|c|}{$\begin{array}{l}\text { QAFETCH returns the program QA information. QASETUP must be called to set } \\
\text { up the CAMCON_LIB common block before QAFETCH is called. }\end{array}$} \\
\hline Syntax & \multicolumn{4}{|c|}{ CALL QAFETCH (TPROG, TVERS, TDATE, TRDAT, TRTIM, TAUTH, TSPON) } \\
\hline $\begin{array}{l}\text { Variables in } \\
\text { Call }\end{array}$ & $\begin{array}{l}\text { Name } \\
\text { TPROG } \\
\text { TVERS } \\
\text { TDATE } \\
\text { TRDAT } \\
\text { TRTIM } \\
\text { TAUTH } \\
\text { TSPON }\end{array}$ & $\begin{array}{l}\text { I/O } \\
\text { Output } \\
\text { Output } \\
\text { Output } \\
\text { Output } \\
\text { Output } \\
\text { Output } \\
\text { Output }\end{array}$ & 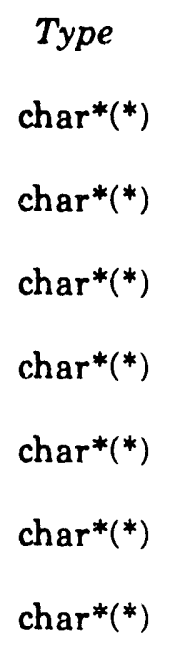 & $\begin{array}{l}\text { Description } \\
\text { Program name. } \\
\text { Program version number. } \\
\text { Program revision date (mm/dd/yy). } \\
\text { Program run date (mm/dd/yy). } \\
\text { Program run time (hh:mm:ss). } \\
\text { Program author. } \\
\text { Program sponsor. }\end{array}$ \\
\hline See Also & QASETUI & & & \\
\hline
\end{tabular}




\author{
Name \\ QAMAKREC - Assemble QA record for program \\ Description \\ QAMAKREC assembles a QA record for the program run. QASETUP must be called \\ to set up the CAMCON_LIB common block before QAMAKREC is called.

Syntax
Variables in
Call \\ CALL QAMAKREC (NQAREC, QAREC)

Name I/O Type Zescription \\ NQAREC $O$ integer \\ The number of QA records (incremented by \\ QAMAKREC). \\ QAREC IO $\operatorname{char}^{*} 8$ The QA records: QAREC $\left(n, \mathrm{NQA}^{\Gamma} \mathrm{EC}+1\right)$. \\ QAREC (n,NQAREC +1$)$ is set by \\ QAMAKREC. The $Q A$ resords (first array \\ subscript $n$ ) are as follows: \\ 1. Analysis code name \\ 2. Analysis QA code descriptor \\ 3. Analysis date \\ 4. Analysis time \\ See Also \\ QASETUP and in \\ CAMDAT_LIB: DBIQAREC, DBOQAREC, DBPQAREC
}




\begin{tabular}{|c|c|c|c|c|}
\hline Name & \multicolumn{4}{|c|}{ QAMAXERR - Check integer value against maximum } \\
\hline Description & \multicolumn{4}{|c|}{$\begin{array}{l}\text { QAMAXERR checks an integer value against a maximum value. It returns.TRUE. } \\
\text { if and only if the value is greater than the maximum. If the value is over the } \\
\text { maximum, an orror message is printed and QAABORT is called (if requested). The } \\
\text { function should be declared logical in any routine which calls it. }\end{array}$} \\
\hline Syntax & \multicolumn{4}{|c|}{ logical = QAMAXERR (DOABRT, INTNAM, MAXNAM, INTVAL, MAXVAL) } \\
\hline \multirow{6}{*}{$\begin{array}{l}\text { Variables in } \\
\text { Call }\end{array}$} & Name & $1 / O$ & Type & Description \\
\hline & DOABRT & Input & logical & $\begin{array}{l}\text { QAABORT is called when the check fails only } \\
\text { if DOABRT is .TRUE. }\end{array}$ \\
\hline & INTNAM & Input & $\operatorname{char}^{*}(*)$ & The description of the value being checked. \\
\hline & MAXNAM & Input & $\operatorname{char}^{*}(*)$ & The variable name of the maximum value. \\
\hline & INTVAL & L/O & integer & The value. \\
\hline & MAXVAL & input & integer & The maximum value. \\
\hline See Also & \multicolumn{4}{|l|}{ QAABORT } \\
\hline
\end{tabular}




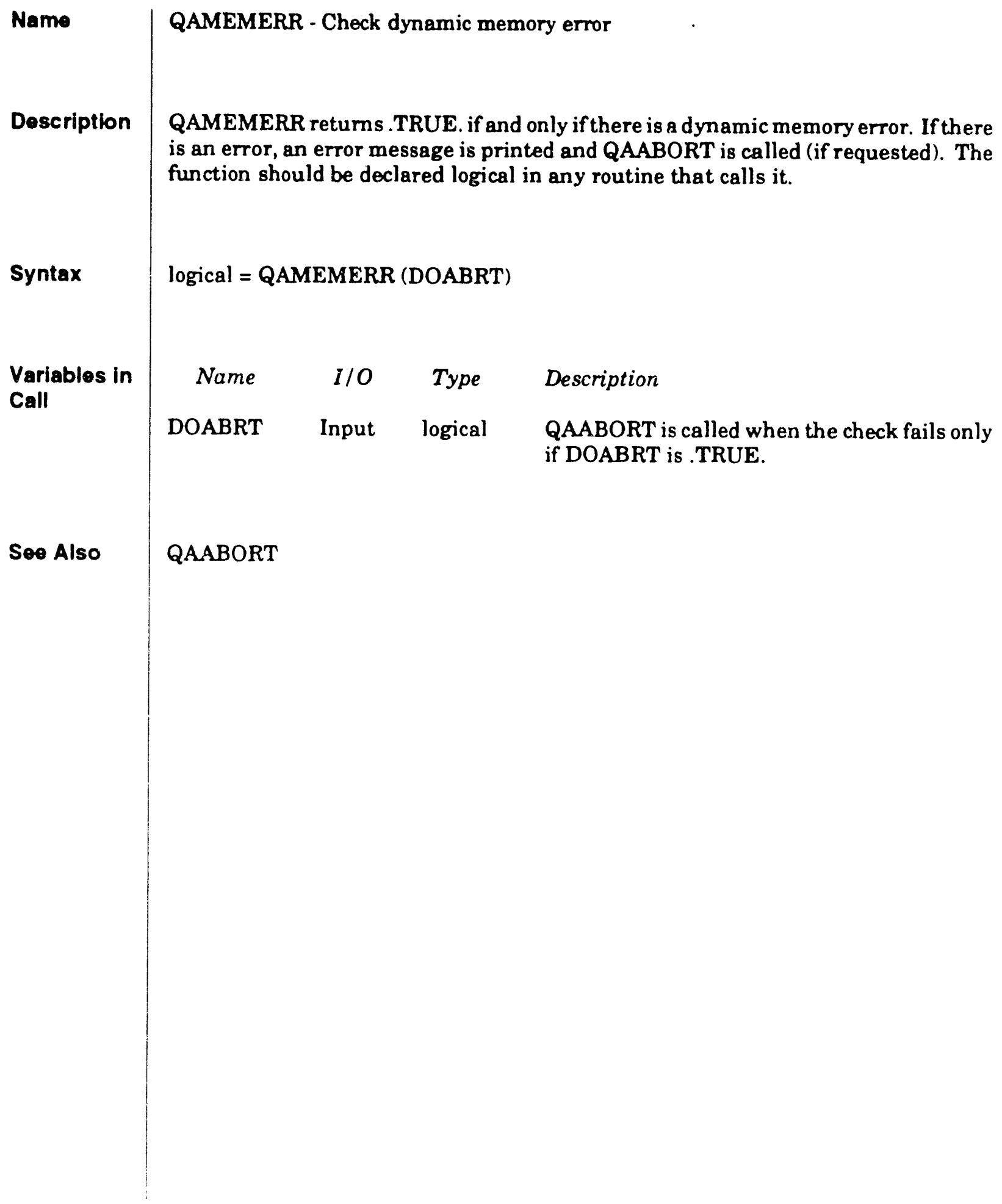




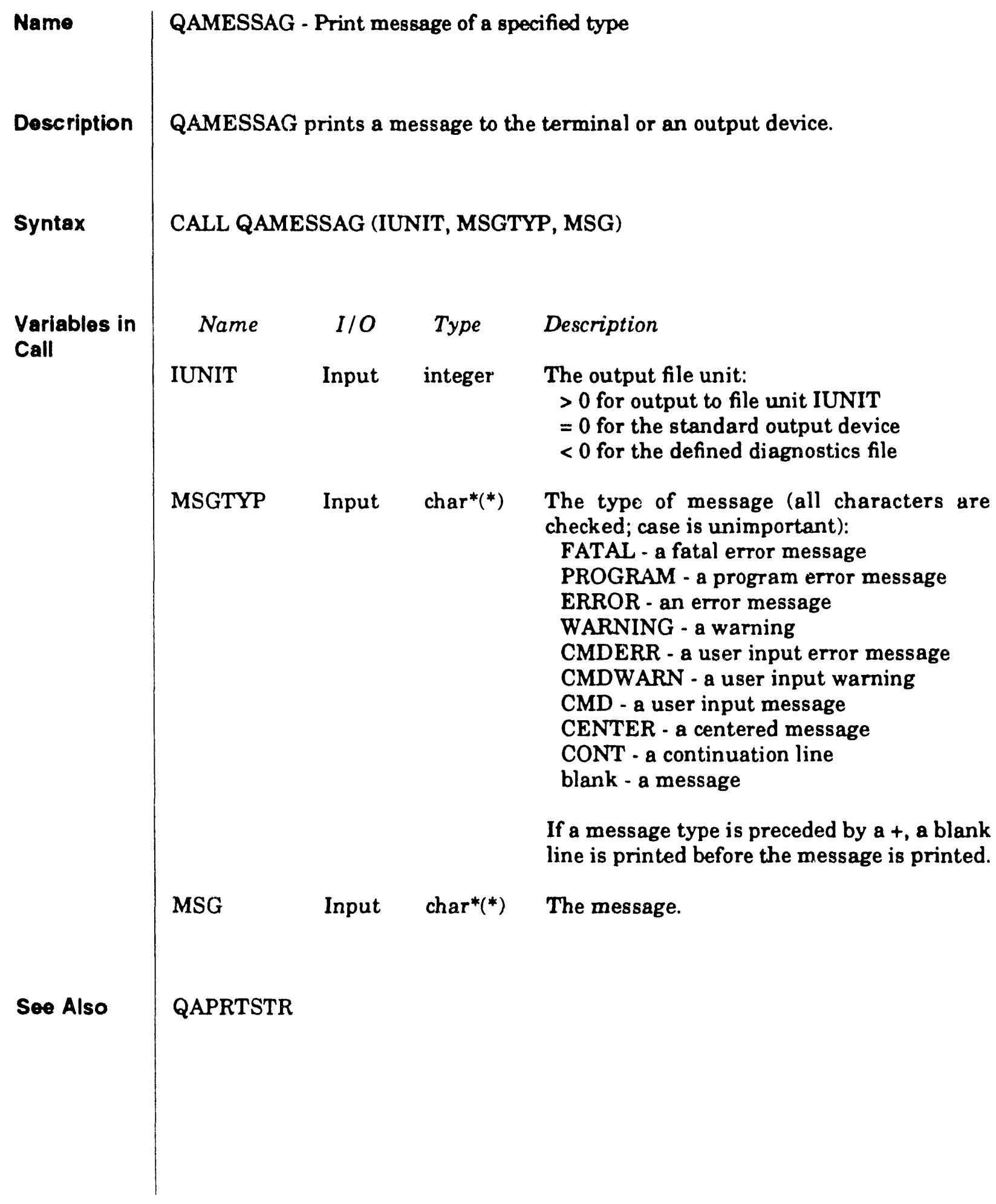




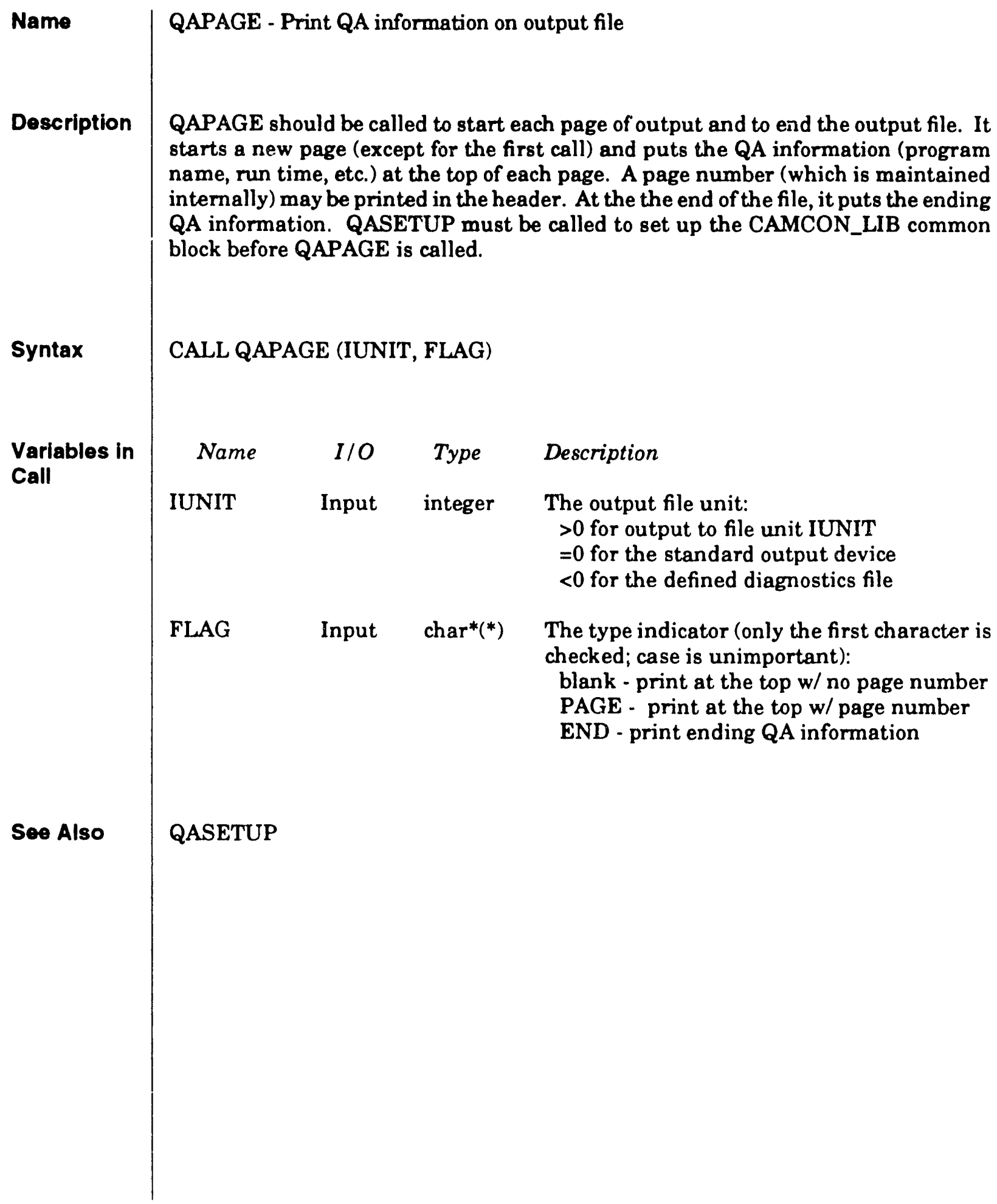




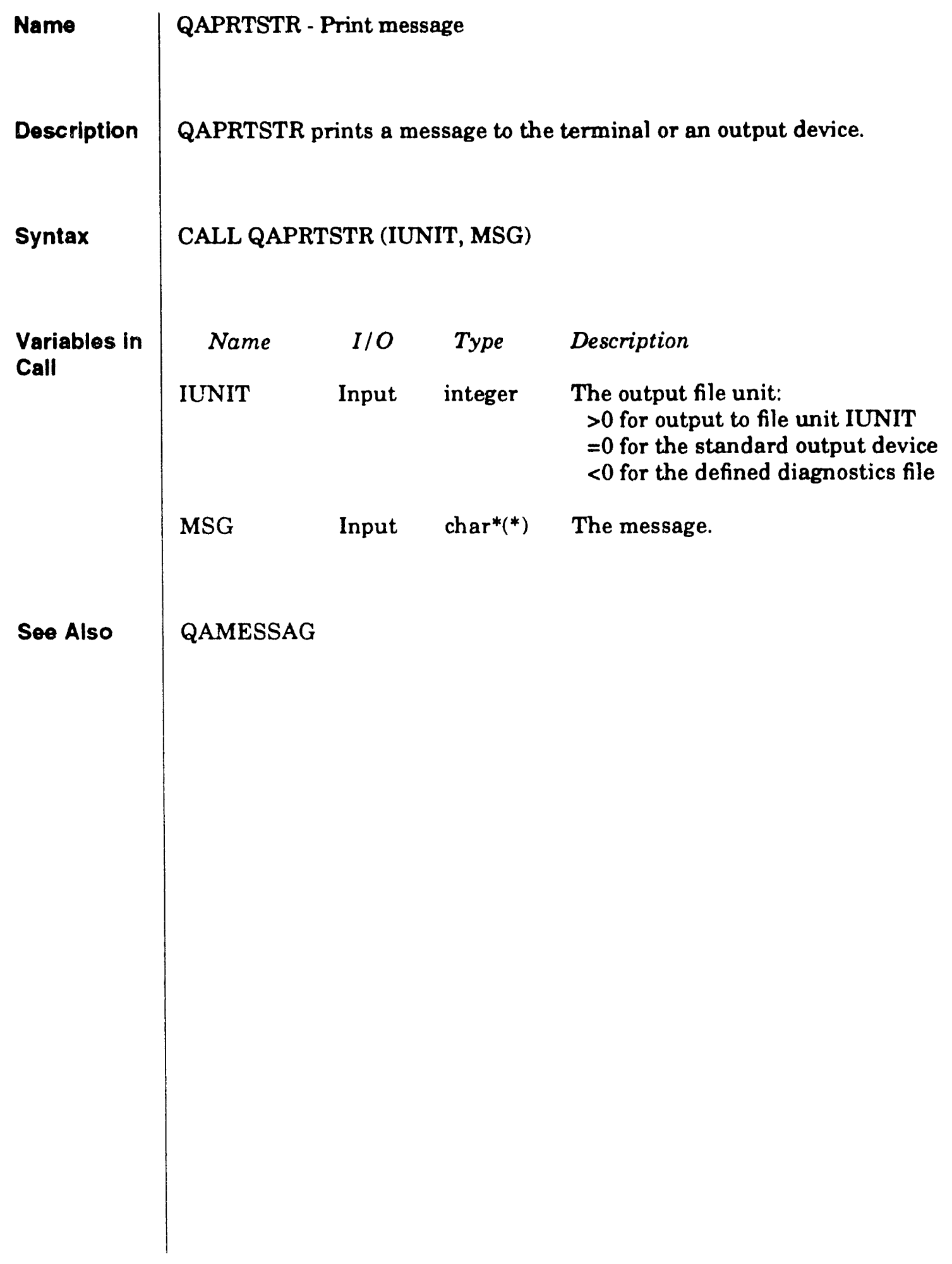




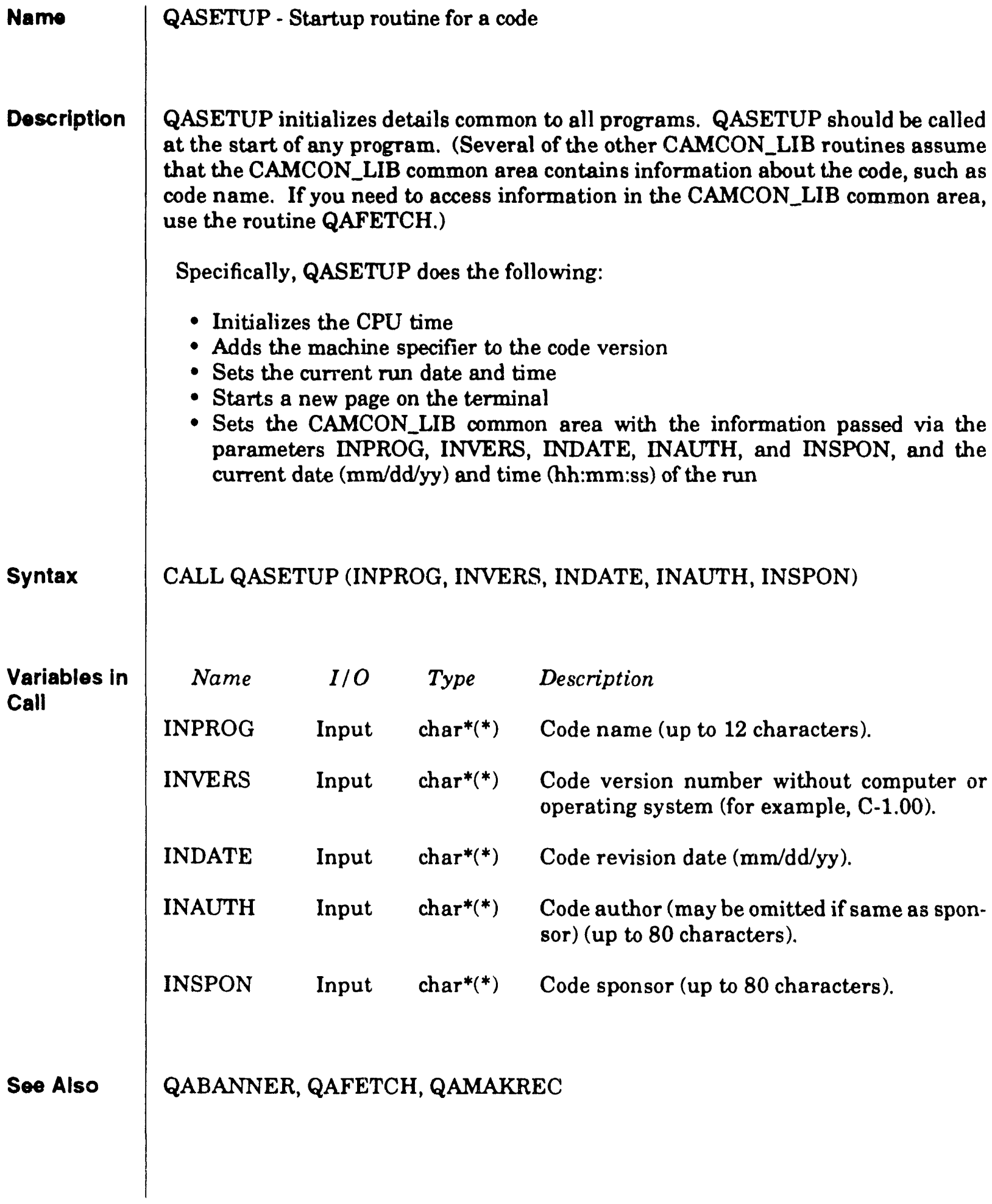


CAMCON_LB

File Processing

\subsubsection{File Processing Routines}

Detailed descriptions of the twelve routines for reading and writing file names and contents are given on the following pages. 


\section{FILCMDLIN}

Subroutine

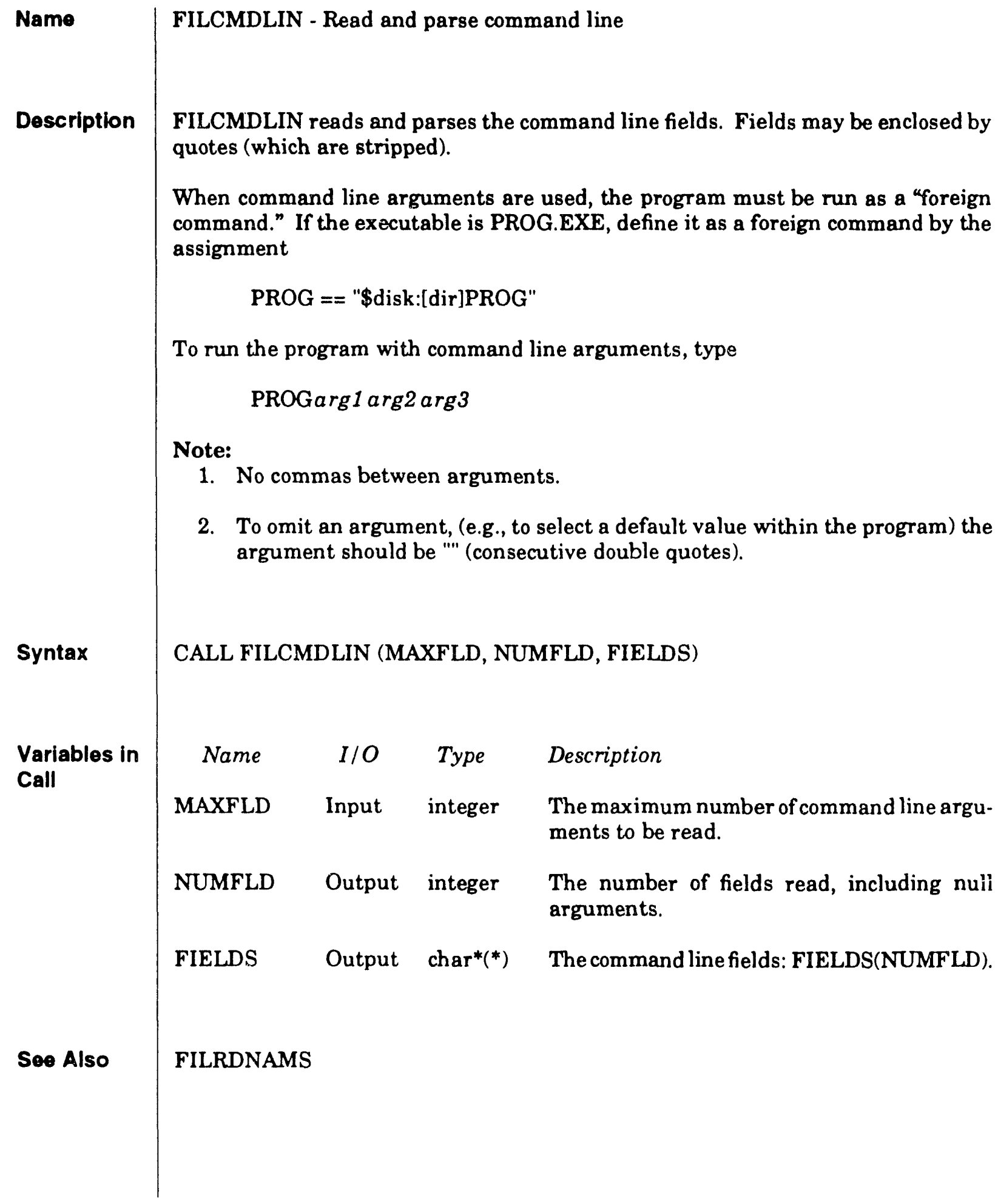




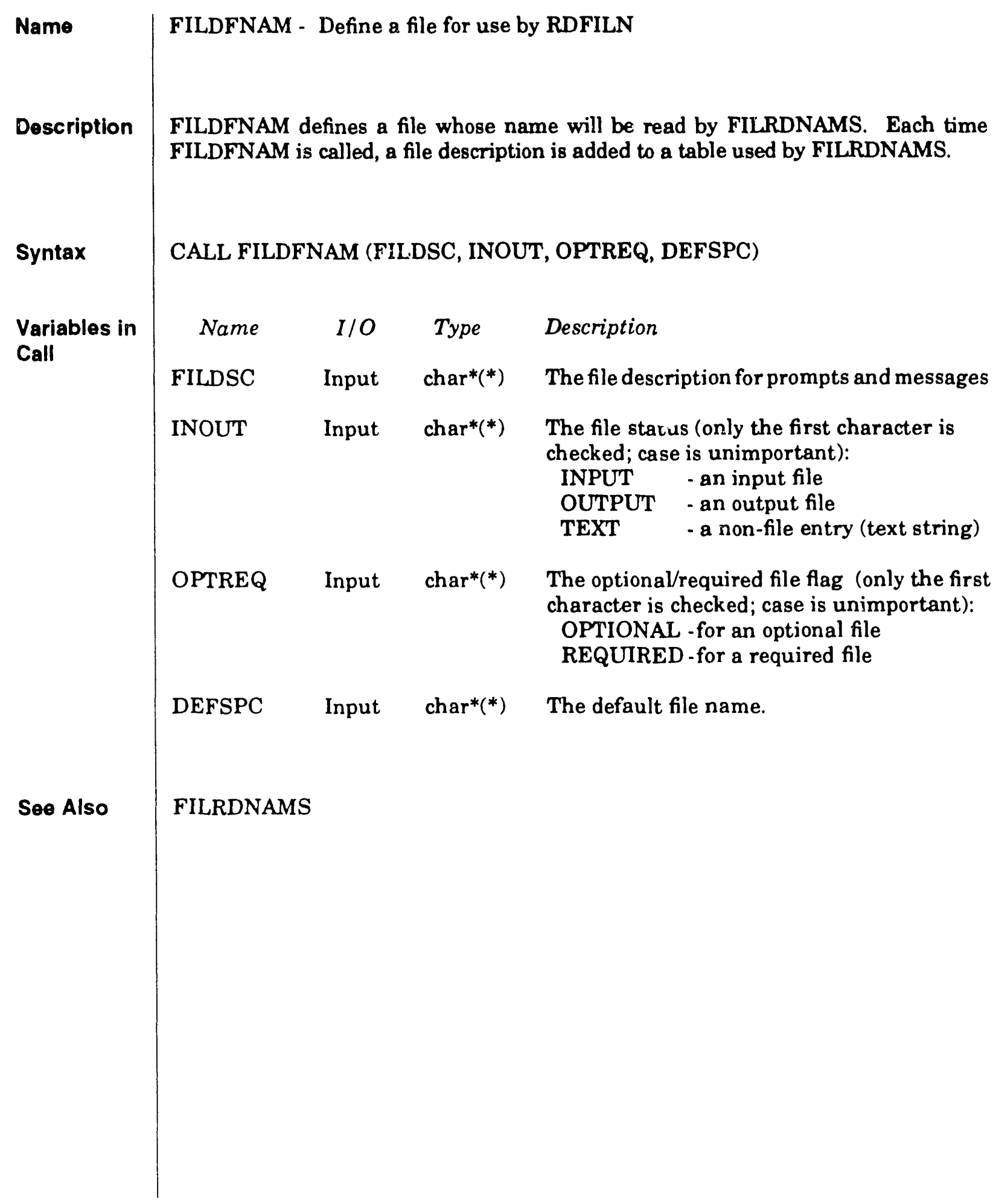




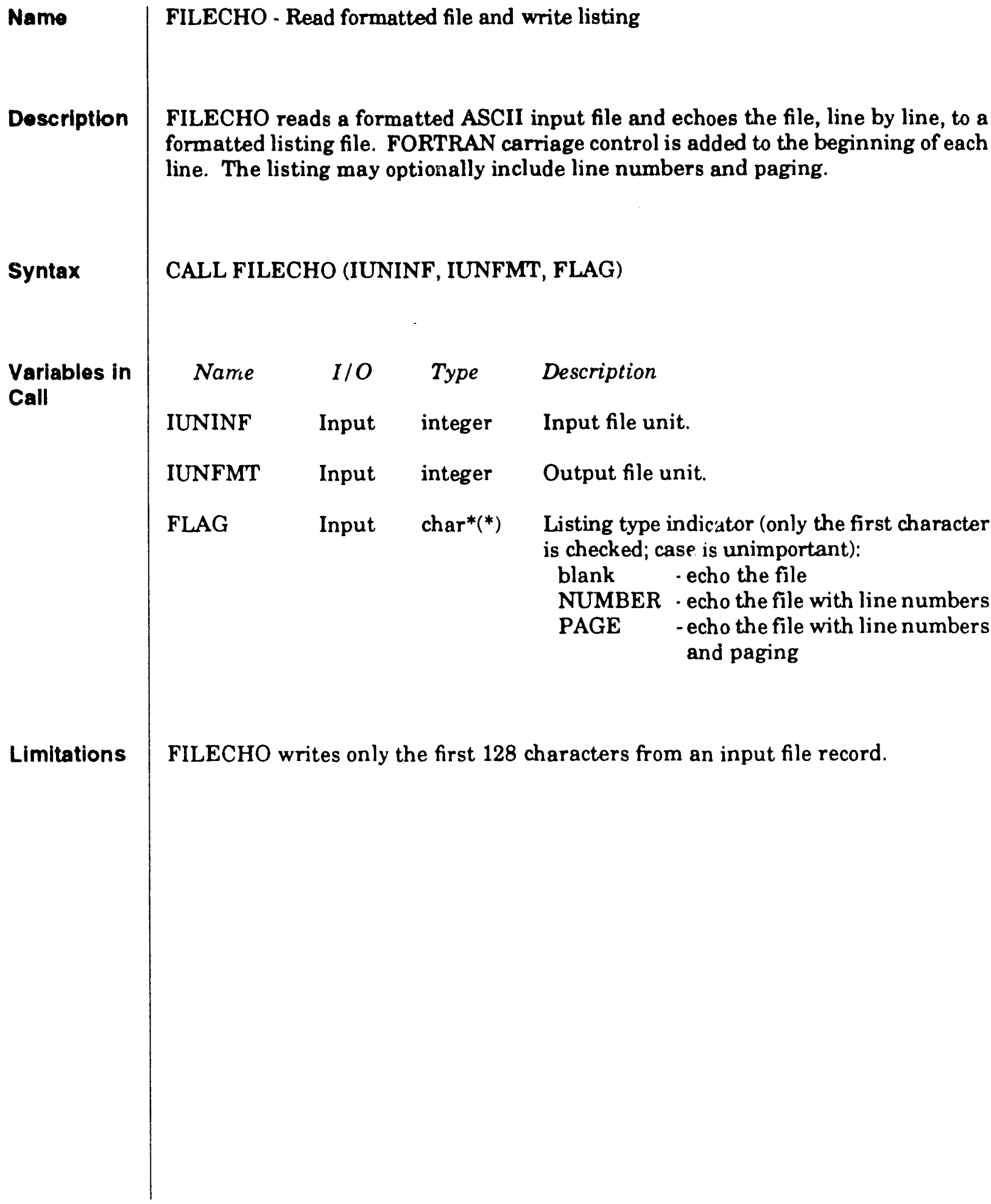




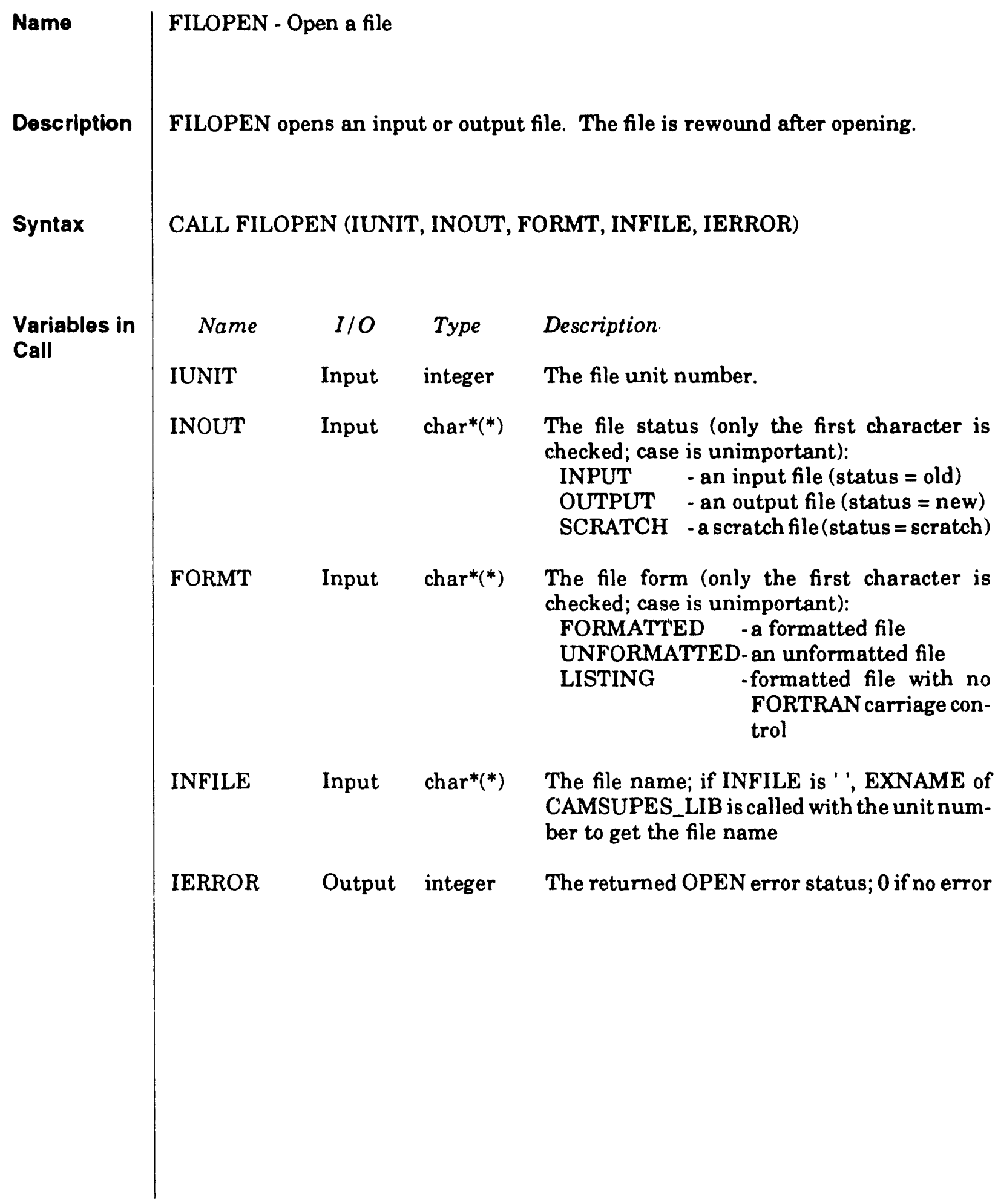




\begin{tabular}{|c|c|c|c|c|}
\hline Name & \multicolumn{4}{|c|}{ FILPARSE - Create a new file name from input and default } \\
\hline Description & \multicolumn{4}{|c|}{$\begin{array}{l}\text { FILPARSE takes an input file name and a default file name and creates an output } \\
\text { file name by substituting the input fields into the default file name. Either the file } \\
\text { name or a requested field are returned. }\end{array}$} \\
\hline Syntax & \multicolumn{4}{|c|}{ CALL FILPARSE (FIELD, INNAM, DEFNAM, OUTNAM) } \\
\hline \multirow{5}{*}{$\begin{array}{l}\text { Variables in } \\
\text { Call }\end{array}$} & Name & $1 / O$ & Type & Description \\
\hline & FIELD & Input & $\operatorname{char} *(*)$ & $\begin{array}{l}\text { The requested file fields (only the first } 3 \\
\text { characters are checked; case is unimportant): } \\
\text { blank - return the entire file name } \\
\text { DISK -return the disk name } \\
\text { DIRECTORY-return the directory name } \\
\text { NAME - return the base file name } \\
\text { EXTENSION-return the extension } \\
\text { VERSION - return the version }\end{array}$ \\
\hline & INNAM & Input & $\operatorname{char}{ }^{*}()$ & The input file name. \\
\hline & DEFNAM & Input & $\operatorname{char} *(*)$ & The default file name. \\
\hline & OUTNAM & Output & $\left.\operatorname{char} *^{*}\right)$ & The output file name or field (see FIELD). \\
\hline
\end{tabular}




\begin{abstract}
Name FILRDNAMS - Read the files defined by FILDFNAM

Description

FILRDNAMS requests the names of all files previously defined by FILDFNAM. The file names are read from the command line. Any file names not found on the command line are requested from the user (if running interactively). If the file is an input file, it must exist.

Some special inputs for the file names are allowed:

- CANCEL specifies that a file (which must be optional) is not needed

- INTERACTIVE specifies that the input will be entered interactively (if allowed)

- DEFAULT requests that the specified file (and all following files if this is the last entry on the command line) should use the default

Syntax

CALL FILRDNAMS (FILTBL, IERROR)

Variables in

Name

$I / O$

Type

Description

Call

FILTBL

Output

$\operatorname{char}^{*}(*)$

The returned file names.

array (" for an optional file that is not needed, INTERACTIVE for interactive).

IERROR Output integer Returned $>0$ if and only if the returned file names are not correct; if running interactively, this flag should always be 0 .

Soo Also

FILDFNAM, FILWRNAMS, FILCMDLIN
\end{abstract}




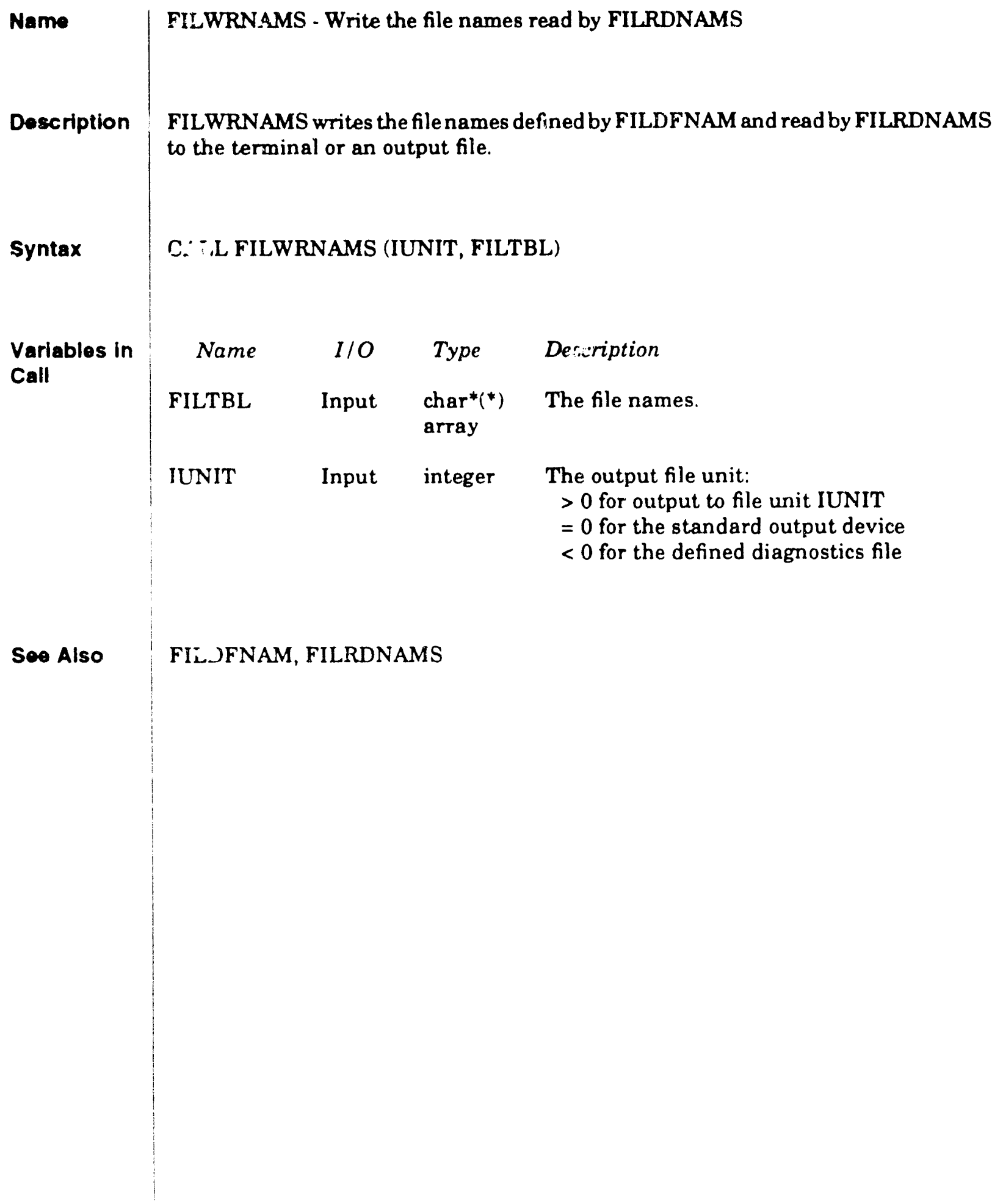


CAMCON_LIB

Free-Field Input

\subsubsection{Free-Field Input}

Syitax Rules. The syntax rules for the freefield input structure are listed below. This syntax describes how input records/strings are parsed into data fields. Both the end user and the applications programmer should clearly understand these few rules (see also Section 4.3, "Parameter Input").

1. A data field is any sequence of data characters within an input line. A data field is broken by (does not include) any non-data character or the end of the input line. A non-data character is a field separator, a comment indicator, or a continuation indicator. Any other character is a data character.

2. A field separator is a comma (,), an equal sign $(=)$, or a series of one or more spaces not adjacent to another separator. Tabs are treated as spaces.

3. An exclamation point (!) indicates a comment. All characters after and including the comment indicator are ignored.

4. An ampersand (\&) preceded by a separator indicates that the next input record/string will be treated as a continuation of the current line. All characters after and including the continuation indicator on the current line are ignored. Multiple records/strings that are "joined" by continuation indicators are treated is a single logical record.

5. A null field does not contain any data characters. A null field can be defined explicitly only by a field separator (spaces cannot act as a field separator for an explicit null field). Fields that are not defined on the input line are implicitly null.

6. Lowercase letters not contained in a quoted string are converted to uppercase.

7. A numeric field is a data field which adheres to an ANSI FORTRAN numeric format. A numeric field cannot be longer than 32 characters. A numeric field always defines a REAL (floating point) value; it also defines an INTEGER (fixed point) value if it adheres to a legal INTEGER format.

8. A quoted string is a data field in which the apostrophe (') or the quotation mark (") is the first nonblank character. An internal quote is indicated with two consecutive quote characters. If an end quotc character is not included, then the remainder of the record (excluding any trailing blanks) is treated as part of the quoted string. Within a quoted string, no character conversion to uppercase is performed. Delimiters (other than quotes) are treated as part of the string. Interpretation of data to numeric data will be performed, if possible.

9. A data field that does not begin with the quote character, but has a quote within the field (e.g., MOM'S) is not considered a quoted string. In this case, the internal quote is not a special character.

10. The maximum length of an input record (FFRDFLDS only) is 132 characters. Input strings to FFPARSE may be any length.

Some important points that are not obvious from the above rules are noted below.

- Spaces have no significance except when they act as field separators. 
- Only the first occurrence of a comment or continuation character is significant; subsequent characters are considered part of the comment.

- A blank line has no data fields.

- If no data characters appear after the last field separator, the field after that separator will not be counted.

- The free field clearly distinguishes between integer and real values. (Using Ivars = RVALUE( ) overrides this and permits users to input values with or without a decimal point, a capability that is sometimes useful.)

Free-Field Input Examples. The following two examplesillustrate the operation of the FFRDFLDS routines in the CAMCON_LIB library.

\section{Example 1}

Input record:

'Quoted strings ',quotes's, ' $50^{\prime}, !^{*},=^{\prime \prime \prime \prime}$ ! The rest is comment

Results returned from FFRDFLDS:

NFIELD $=5$

\begin{tabular}{|c|c|c|c|c|}
\hline I & KVALUE(I) & CVALUE(I) & RVALUE(I) & IVALUE(I) \\
\hline 1 & 0 & Quoted\#strings\#\# & $0.000 E+00$ & 0 \\
\hline 2 & 0 & QUOTES'S\#\#\#\#\#\#\#\# & $0.000 \mathrm{E}+00$ & 0 \\
\hline 3 & -1 & $\# \# \# \# \# \# \# \# \# \# \# \# \# \# \# \# \# \#$ & $0.000 \mathrm{E}+00$ & 0 \\
\hline 4 & 0 & $50 \# \# \# \# \# \# \# \# \# \# \# \# \# \#$ & $0.000 E+00$ & 0 \\
\hline 5 & 0 & 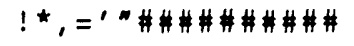 & $0.000 E+00$ & 0 \\
\hline 6 & -1 & 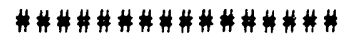 & $0.000 E+00$ & 0 \\
\hline
\end{tabular}

\section{Example 2}

The following example illustrates the use of the CAMCON_LIB free-field input parser in more detail. Shown below is a fragment of a typical input file, a table of values returned by the routine FFRDFLDS and a fragment of FORTRAN source code used to interpret those values. A couple of cautions are worth further discussion. It is important that the coding logic be able to distinguish between parameters and values (the equal sign has no special significance to the parser). For example, the string 'POROSITY' is used both as a parameter and a value in fields 4 and 5 . Poor coding logic such as a DOloop scanning all fields would pick up the second occurrence as a keyword. Care must also be exercised 
when picking the number of unique characters allowed for parameter abbreviation, particularly when new input parameters are added to existing code.

The free-field parser allows for simple programming for user as well as programmer convenience. In the CAMCON system, input values are often defined in data base files (e.g., PROPERTY.SDB or CAMDAT) and must be accessed by name. However, it sometimes becomes necessary to override them with a value. The KVALUE array provides this flexibility.

Another user convenience can be implemented by setting integer values with the RVALUE array rather than the IVALUE array. This allows the user to input integers with or without a decimal point. Using IVALUE forces the user to input integers without a decimal point.

Input Records:

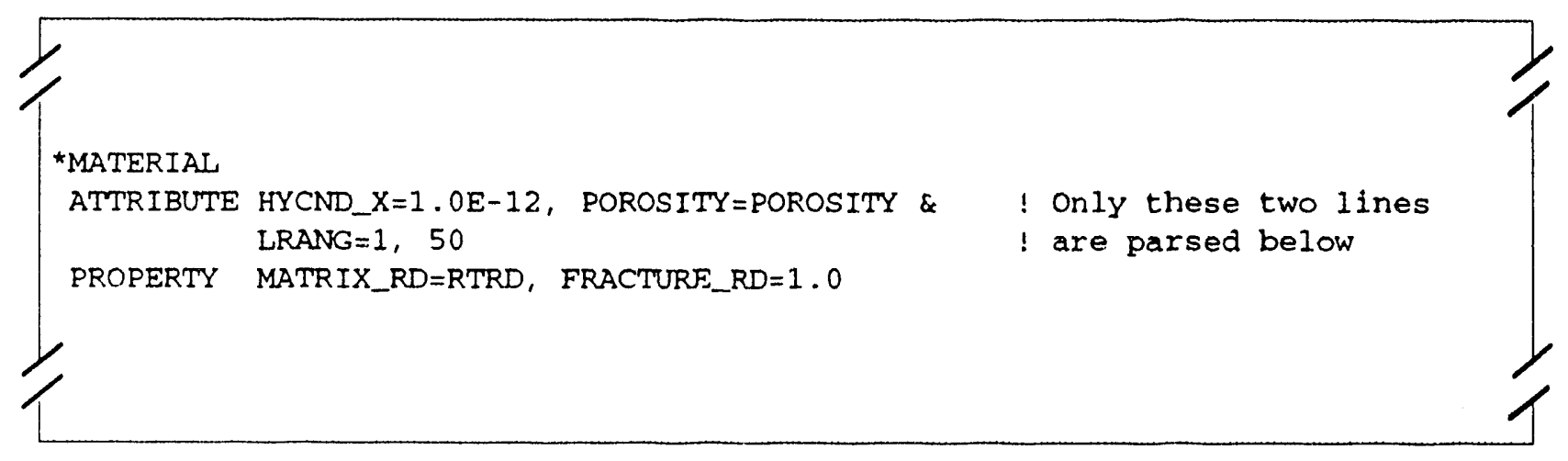

Record as parsed by FFRDFLDS:

NFIELD $=8$

$\begin{array}{llccc}\text { I } & \text { CVALUE(I) } & \text { KVALUE(I) } & \text { RVALUE(I) } & \text { IVALUE(I) } \\ 1 & \text { ATTRIBUTE } & 0 & 0 . & 0 \\ 2 & \text { HYCND_X } & 0 & 0 . & 0 \\ 3 & 1.0 E-12 & 1 & 1.0 E-12 & 0 \\ 4 & \text { POROSITY } & 0 & 0 . & 0 \\ 5 & \text { POROSITY } & 0 & 0 . & 0 \\ 6 & \text { LRANG } & 0 & 0 . & 0 \\ 7 & 1 & 2 & 1.0 & 1 \\ 8 & 50 & 2 & 50.0 & 50\end{array}$


FORTRAN code fragment used to read input record:

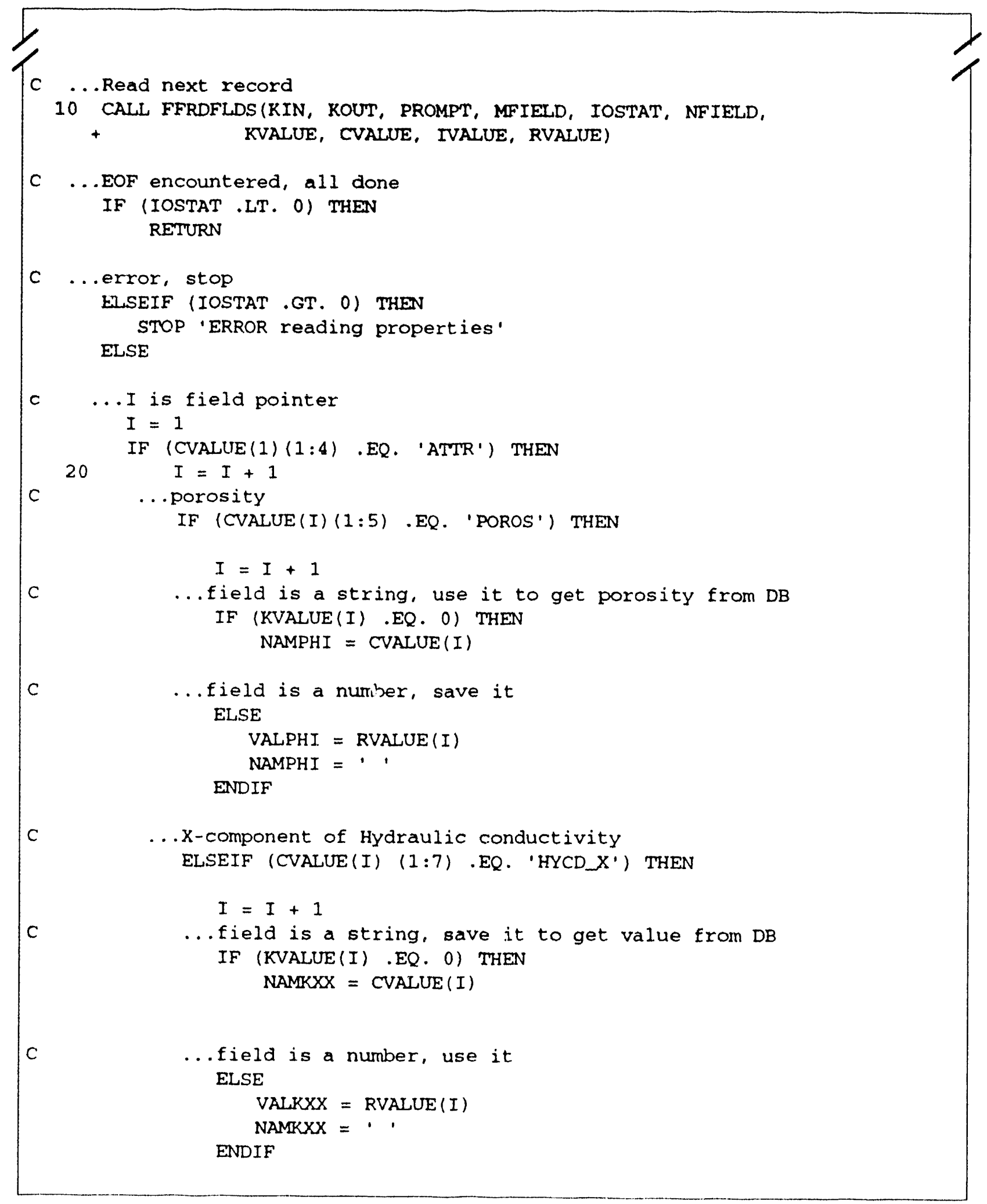




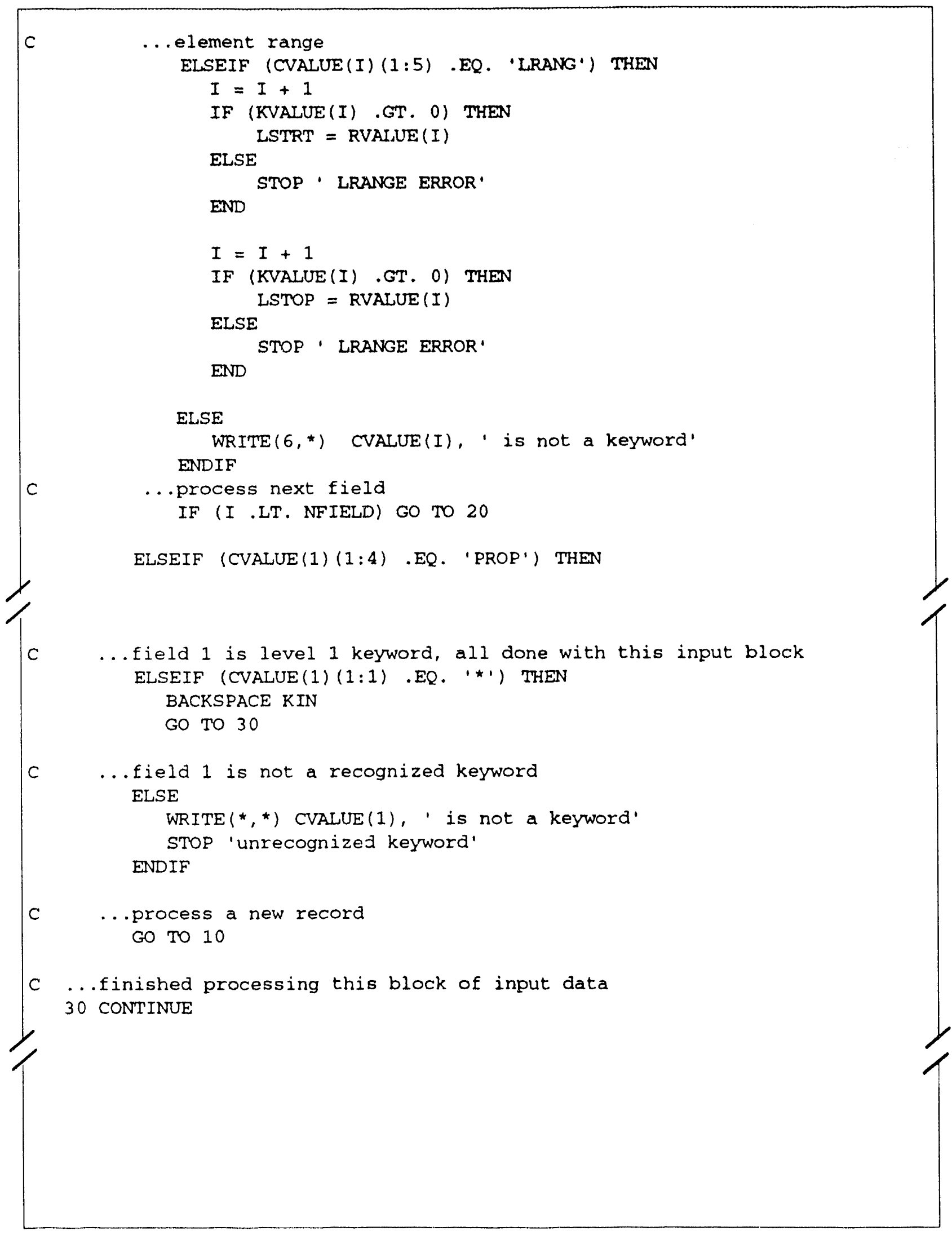


Free-Field Input Routines. The user interface to the CAMCON_LIB free field input system consists of two subroutines: FFRDFLDS and FFPARSE. Both routines perform parsing functions of strings. The main difference is that FFRDFLDS gets its input from a FORTRAN VO unit while FFPARSE gets its input from a character string. In fact, FFRDFLDS uses FFPARSE to perform parsing functions once FFRDFLDS has read a record. 


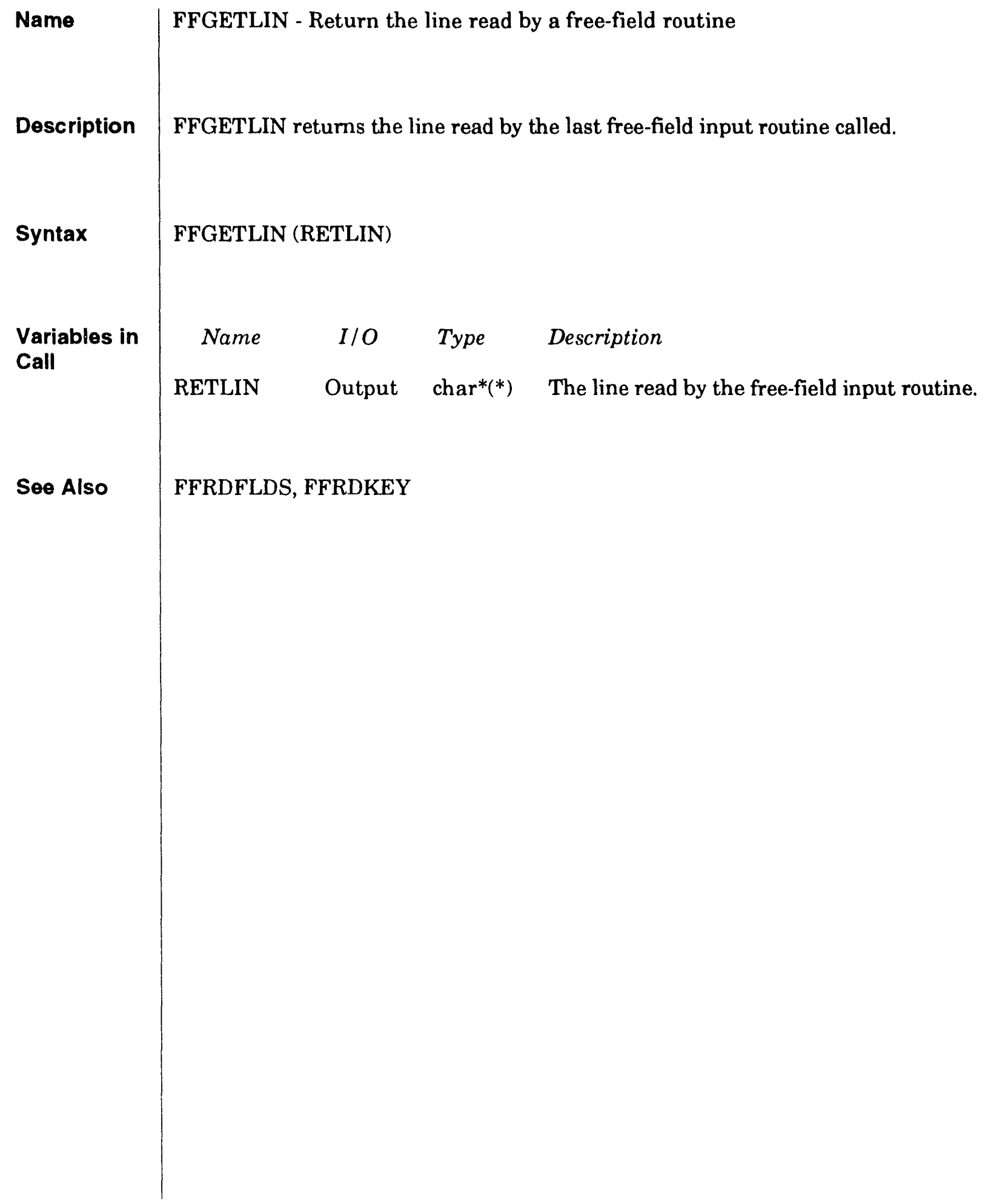




\begin{tabular}{|c|c|c|c|c|}
\hline Name & \multicolumn{4}{|c|}{ FFPAREQN - Equation parsing routine } \\
\hline Description & \multicolumn{4}{|c|}{$\begin{array}{l}\text { FFPAREQN converts a line to uppercase and parses the line into individual fields. A } \\
\text { field is one of the following: } \\
\text { A number - starts with } 0-9 \text { or a decimal point (.) and continues with } 0-9 \text {, a decimal } \\
\text { point (.), or an exponent (E, E+, or E-) } \\
\text { A name - starts with A-Z and continues with A-Z, } 0-9 \text {, or underscores (..) } \\
\text { A non-number or non-name character; }{ }^{* *} \text { is converted to } \\
\text {. }\end{array}$} \\
\hline Syntax & \multicolumn{4}{|c|}{$\begin{array}{l}\text { CALL FFPAREQN (STVERB, LINE, MAXFLD, IDCONT, NUMFLD, INTYP, CFIELD, } \\
\text { IFIELD, RFIELD) }\end{array}$} \\
\hline \multirow{10}{*}{$\begin{array}{l}\text { Variables in } \\
\text { Call }\end{array}$} & Name & $I / O$ & Type & Description \\
\hline & STVERB & Input & logical & $\begin{array}{l}\text { This variable is true if and only if the input line } \\
\text { starts with a command verb that must be } \\
\text { stripped from the equation line. }\end{array}$ \\
\hline & LINE & $\begin{array}{l}\text { Input/ } \\
\text { Output }\end{array}$ & $\operatorname{char} *(*)$ & $\begin{array}{l}\text { The input string, which is returned with the } \\
\text { command verb stripped if STVERB is true. }\end{array}$ \\
\hline & MAXFLD & Input & integer & Maximum number of data fields to be returned. \\
\hline & IDCONT & Input & integer & Continuation flag ( $0=$ no continuation). \\
\hline & NUMFLD & Output & & Number of data fields found. \\
\hline & INTYP & Output & integer & $\begin{array}{l}\text { Translation states of the data fields: } \\
\text { INTYP (MAXFLD) } \\
\begin{aligned}-1 & =\text { a null field } \\
0 & =\text { a nonnumeric field } \\
1 & =\text { a real numeric field } \\
2 & =\text { an integer numeric filed } \\
3 & =\text { a character string }\end{aligned}\end{array}$ \\
\hline & CFIELD & Output & $\operatorname{char}{ }^{*}(*)$ & $\begin{array}{l}\text { Character values of the data fields: } \\
\text { CFIELD (MAXFLD) }\end{array}$ \\
\hline & IFIELD & Output & integer & $\begin{array}{l}\text { Integer values of the data fields: } \\
\text { IFIELD (MAXFLD) }\end{array}$ \\
\hline & RFIELD & Output & real & $\begin{array}{l}\text { Floating-point values of the data fields: } \\
\text { RFIELD (MAXFLD) }\end{array}$ \\
\hline
\end{tabular}




\begin{tabular}{|c|c|c|c|c|}
\hline Name & \multicolumn{4}{|c|}{ FFPARSE - Free-field parse routine } \\
\hline Description & \multicolumn{4}{|c|}{$\begin{array}{l}\text { FFPARSE returns the parsed data field values defined in the input string. If a string } \\
\text { contains a continuation character, a flag is returned to the user indicating that another } \\
\text { string should be supplied to complete the logical record. }\end{array}$} \\
\hline Syntax & \multicolumn{4}{|c|}{$\begin{array}{l}\text { CALLFFPARSE(LINE, MFIELD, IDCONT, NFIELD, KVALUE, CVALUE, IVALUE, } \\
\text { RVALUE) }\end{array}$} \\
\hline \multirow{7}{*}{$\begin{array}{l}\text { Variables in } \\
\text { Call }\end{array}$} & Name & $I / O$ & Type & Description \\
\hline & LINE & Input & $\operatorname{char}^{*}(*)$ & $\begin{array}{l}\text { The input string containing the data to be } \\
\text { parsed. }\end{array}$ \\
\hline & MFIELD & Input & integer & $\begin{array}{l}\text { Maximum number of data fields to be re- } \\
\text { turned. }\end{array}$ \\
\hline & IDCONT & L/O & integer & $\begin{array}{l}\text { Continuation flag. } 0 \text { means no continuation. } \\
\text { On input, this flag indicates whether the } \\
\text { previous string contained a continuation indi- } \\
\text { cator. In this case, the current string will be } \\
\text { treated as part of the same logical record as } \\
\text { the previous string. }\end{array}$ \\
\hline & NFIELD & Output & integer & $\begin{array}{l}\text { Number of data fields found on this logical } \\
\text { record. }\end{array}$ \\
\hline & KVALUE & Output & integer & $\begin{array}{l}\text { Translation states of the data fields: } \\
\text { KVALUE(MFIELD). The value of each ele- } \\
\text { ment of this array is interpreted as follows: } \\
-1=\text { null field. } \\
0=\text { a nonnumeric field; only CVALUE } \\
\text { contains a specified value } \\
1=\text { a real numeric field; CVALUE and } \\
\text { RVALUE contain specified values } \\
2=\text { an integer numeric field; CVALUE, } \\
\text { RVALUE, and IVALUE contain } \\
\text { specified values }\end{array}$ \\
\hline & CVALUE & Output & $\operatorname{har}^{*}(*)$ & $\begin{array}{l}\text { Character values of the data fields: } \\
\text { CVALUE(MFIELD). }\end{array}$ \\
\hline
\end{tabular}




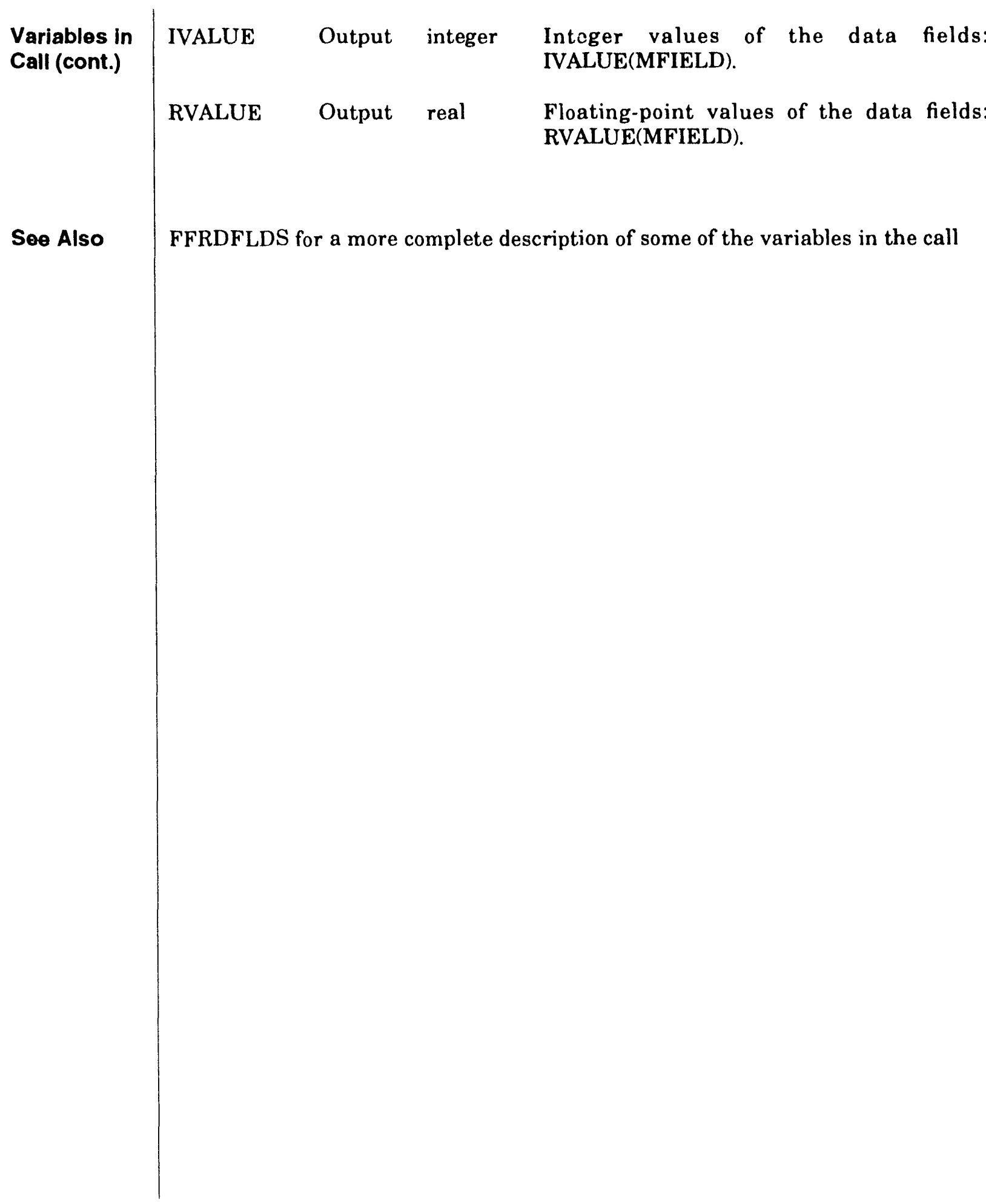




\begin{tabular}{|c|c|c|c|c|}
\hline Name & \multicolumn{4}{|c|}{ FFRDFLDD - Free-field input routine (double precision) } \\
\hline Description & \multicolumn{4}{|c|}{$\begin{array}{l}\text { FFRDFLDD is a special version of FFRDFLDS that returns double-precision values } \\
\text { (compiled with /NOG_FLOATING on the VAX). }\end{array}$} \\
\hline Syntax & \multicolumn{4}{|c|}{$\begin{array}{l}\text { CALL FFRDFLDD (KIN, KOUT, PROMPT, MFIELD, IOSTAT, NFIELD, KVALUE, } \\
\text { CVALUE, IVALUE, DVALUE) }\end{array}$} \\
\hline \multirow{8}{*}{$\begin{array}{l}\text { Variables in } \\
\text { Call }\end{array}$} & Name & $1 / O$ & Type & Description \\
\hline & KIN & Input & integer & Unit from which to read input. \\
\hline & KOUT & Input & integer & Unit to which to echo input. \\
\hline & PROMPT & Input & $\operatorname{char}{ }^{*}(*)$ & Prompt string. \\
\hline & MFIELD & Input & integer & Maximum number of data fields to be returned. \\
\hline & IOSTAT & Output & integer & $\begin{array}{l}\text { ANSI FORTRAN I/O status: } \\
\text { IOSTAT }<0 \text { End of File } \\
\text { IOSTAT }=0 \text { Normal } \\
\text { IOSTAT }>0 \text { Error }\end{array}$ \\
\hline & NFIELD & Output & integer & $\begin{array}{l}\text { Number of data fields found on this logical } \\
\text { record. }\end{array}$ \\
\hline & KVALUE & Output & integer & $\begin{array}{l}\text { Translation states of the data fields: } \\
\text { KVALUE(MFIELD). The value of each ele- } \\
\text { ment of this array is interpreted as follows: } \\
-1=\text { null field } \\
0=\text { a non-numeric field; only CVALUE } \\
\quad \text { contains a specified value } \\
1=\begin{array}{l}\text { a real or double-precision numeric field; } \\
\text { CVALUE and DVALUE contain } \\
\text { specified values } \\
2= \\
\text { an integer numeric field; CVALUE, } \\
\text { DVALUE, and IVALUE contain } \\
\text { specified values }\end{array}\end{array}$ \\
\hline
\end{tabular}




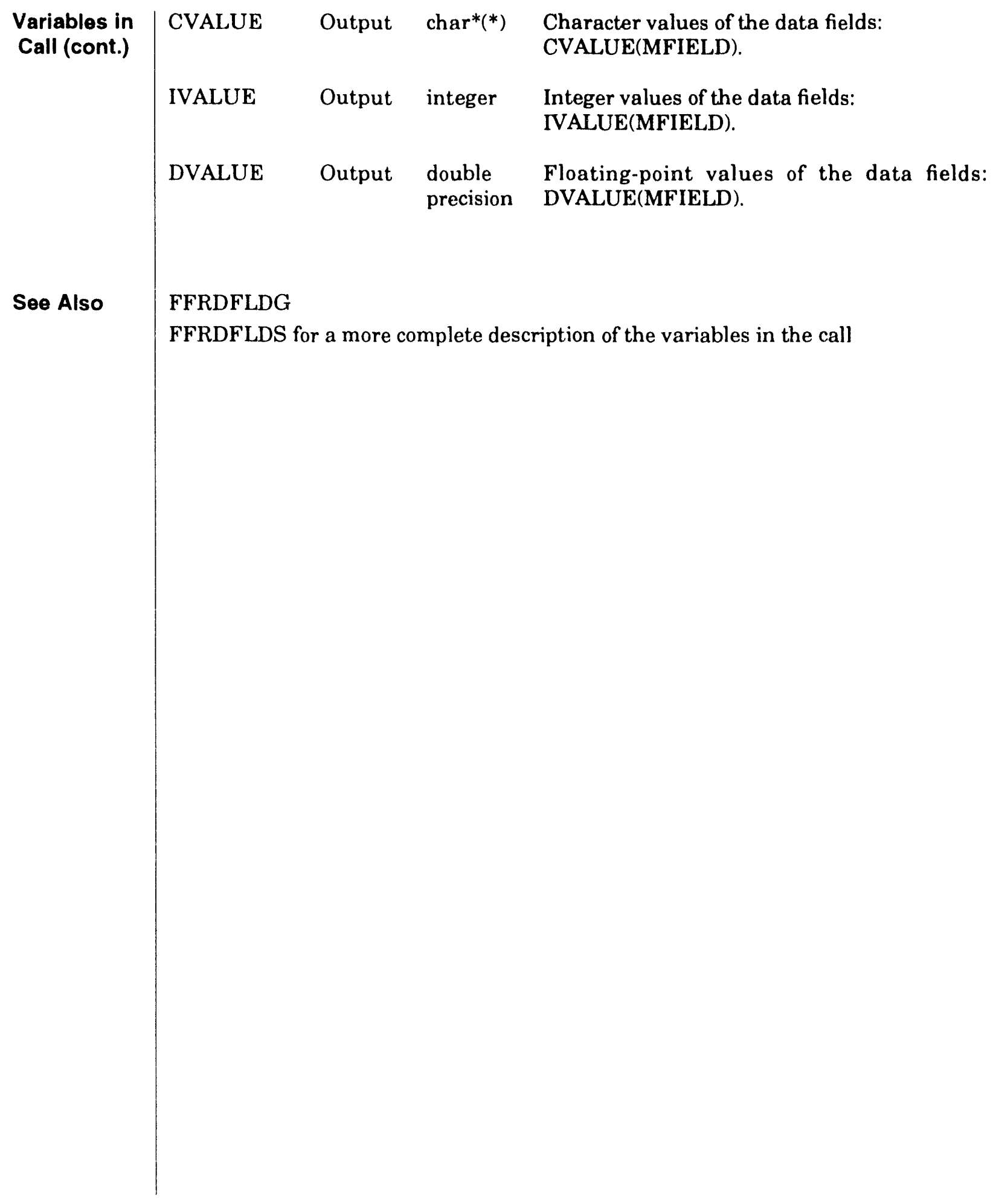




\begin{tabular}{|c|c|c|c|c|}
\hline Name & \multicolumn{4}{|c|}{ FFRDFLDG - Free-field input routine (double precision) } \\
\hline Description & \multicolumn{4}{|c|}{$\begin{array}{l}\text { FFRDFLDG is a special version of FFRDFLWS that returns double-precision values } \\
\text { (compiled with /G_FLOATING on the VAX). }\end{array}$} \\
\hline Syntax & \multicolumn{4}{|c|}{$\begin{array}{l}\text { CALL FFRDFLDG (KIN, KOUT, PROMPT, MFIELD, IOSTAT, NFIELD, KVALUE, } \\
\text { CVALUE, IVALUE, DVALUE) }\end{array}$} \\
\hline \multirow{8}{*}{$\begin{array}{l}\text { Variables in } \\
\text { Call }\end{array}$} & Name & $1 / O$ & Type & Description \\
\hline & $\mathrm{KIN}$ & Input & integer & Unit from which to read input. \\
\hline & KOUT & Input & integer & Unit to which to echo input. \\
\hline & PROMPT & Input & $\left.\operatorname{char} *^{*}\right)$ & Prompt string. \\
\hline & MFIELD & Input & integer & Maximum number of data fields to be returnfr! \\
\hline & IOSTAT & Output & integer & $\begin{array}{l}\text { ANSI FORTRAN I/O status: } \\
\text { IOSTAT }<0 \text { End of File } \\
\text { IOSTAT }=0 \text { Normal } \\
\text { IOSTAT }>0 \text { Error }\end{array}$ \\
\hline & NFIELD & Output & integer & $\begin{array}{l}\text { Number of data fields found on this logical } \\
\text { record. }\end{array}$ \\
\hline & KVALUE & Output & integer & $\begin{array}{l}\text { Translation states of the dota fields } \\
\text { KVALUE(MFIELD). The value u each ele } \\
\text { ment of this array is interpreted as follows: } \\
-1=\text { null field } \\
0=\text { non-numeric field; only CVALUE } \\
\text { contains a specified value } \\
1=\text { a real or double-precision num iric } \\
\text { field; CVALUE and DVALUE contain } \\
\text { specified values } \\
2= \\
\text { an inceger numeric field; CVALUE, } \\
\text { DVALUE, and IVALUE contain } \\
\text { S')ecified values }\end{array}$ \\
\hline
\end{tabular}




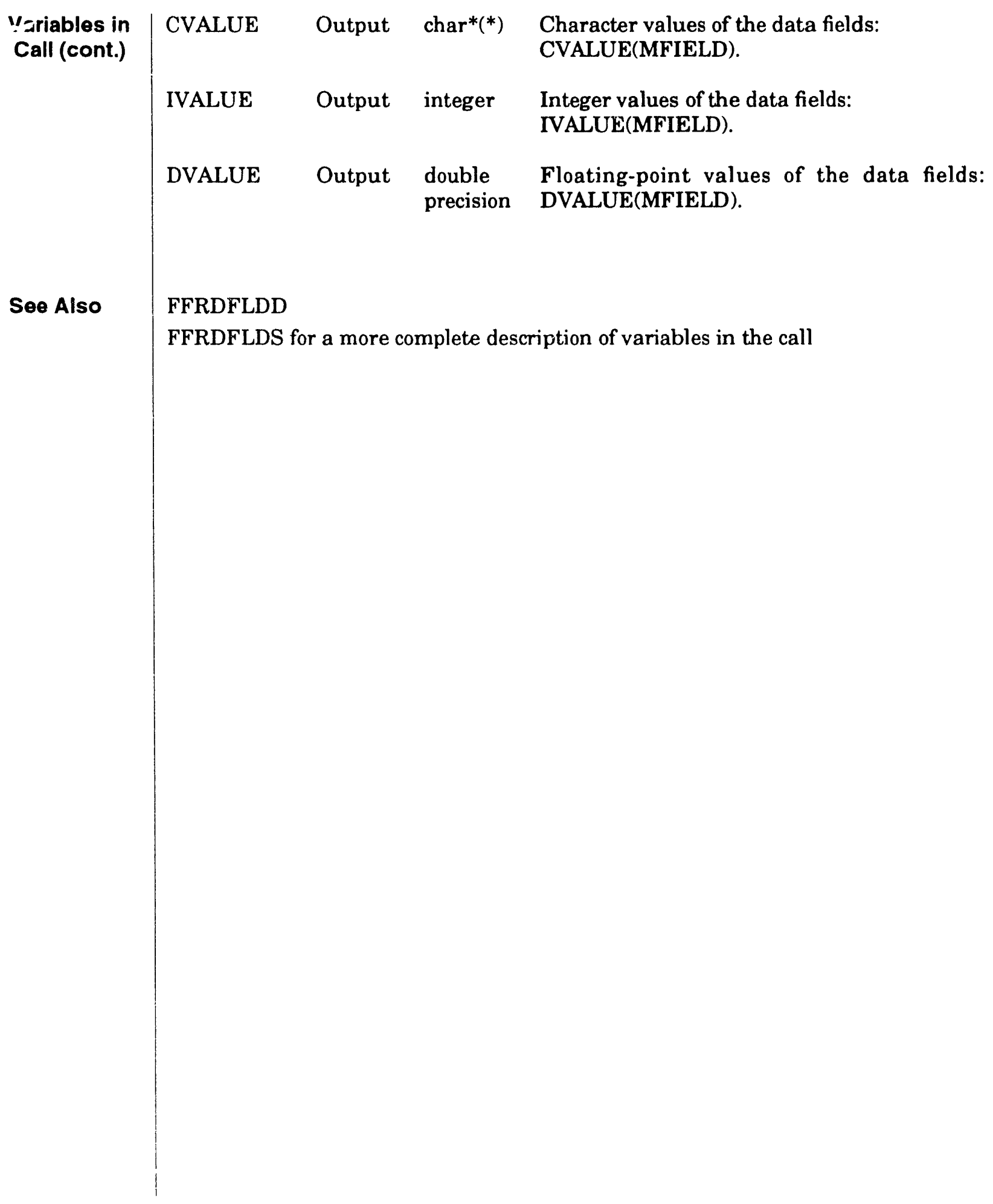




\begin{tabular}{|c|c|c|c|c|}
\hline Name & \multicolumn{4}{|c|}{ FFRDFLDS - Free-field input routine } \\
\hline Description & \multicolumn{4}{|c|}{$\begin{array}{l}\text { Input is prompted for, read, and echoed by FFRDFLDS using specified I/O units. } \\
\text { FFRDFLDS returns the parsed data fieid values defined on the next input record and } \\
\text { any continuation records. All I/O is accomplished by the utility routine FFRDLINE, } \\
\text { while the parsing is performed by FFPARSE. }\end{array}$} \\
\hline Syntax & \multicolumn{4}{|c|}{$\begin{array}{l}\text { CALL FFRDFLDS (KIN, KOUT, PROMPT, MFIELD, IOSTAT, NFIELD, KVALUE, } \\
\text { CVALUE, IVALUE, RVALUE) }\end{array}$} \\
\hline \multirow{4}{*}{$\begin{array}{l}\text { Variables in } \\
\text { Call }\end{array}$} & Name & $I / O$ & Type & Description \\
\hline & $\mathrm{KIN}$ & Input & integer & $\begin{array}{l}\text { Unit from which to read input. } \\
>0 \text { - read from the file whose unit is given; the } \\
\text { caller is responsible for opening/closing } \\
\text { this unit } \\
=0 \text { - read from the standard input device (ter- } \\
\text { minal or batch deck) and echo to the } \\
\text { standard outputdevice(terminal or batch } \\
\text { log) } \\
<0 \text { - read from the command line }\end{array}$ \\
\hline & KOUT & Input & integer & $\begin{array}{l}\text { Unit to which to echo input. } \\
>0 \text { - echo to the file whose unit is given; the } \\
\text { caller is responsible for opening/closing } \\
\text { this unit } \\
=0 \text { - do not echo other than to the standard } \\
\text { output device as described for KIN } \\
<0 \text { - echo to the standard output device }\end{array}$ \\
\hline & PROMPT & Input & $\operatorname{char}{ }^{*}(*)$ & $\begin{array}{l}\text { Prompt string. This string will be used to } \\
\text { prompt for data from an interactive terminal } \\
\text { and/or will be written as a prefix to the input } \\
\text { line for echo. If the string 'AUTO' is specified, } \\
\text { a prompt of the form 'n:', where " } n \text { " is the } \\
\text { current input line number (only lines read } \\
\text { under the AUTO feature are counted), will be } \\
\text { generated. }\end{array}$ \\
\hline
\end{tabular}




\begin{tabular}{|c|c|c|c|c|}
\hline \multirow[t]{7}{*}{$\begin{array}{l}\text { Variables in } \\
\text { Call (cont.) }\end{array}$} & MFIELD & Input & integer & $\begin{array}{l}\text { Maximum number of data fields to be returned. } \\
\text { The dimensions of each of the output arrays } \\
\text { described below must be greater than or equal } \\
\text { to this number. }\end{array}$ \\
\hline & IOSTAT & Output & integer & $\begin{array}{l}\text { ANSI FORTRAN I/O status: } \\
\text { IOSTAT }<0 \text { End of File } \\
\text { IOSTAT }=0 \text { Normal } \\
\text { IOSTAT }>0 \text { Error }\end{array}$ \\
\hline & NFIELD & Output & integer & $\begin{array}{l}\text { Number of data fields found on this logical } \\
\text { record. If this value is less than MFIELD, the } \\
\text { excess fields are implicitly defined as null fields. } \\
\text { If this value is greater than MFIELD, the extra } \\
\text { data fields are ignored. }\end{array}$ \\
\hline & KVALUE & Output & integer & $\begin{array}{l}\text { Translation states of the data fields: } \\
\text { KVALUE(MFIELD). The value of each ele- } \\
\text { ment of this array is interpreted as follows: } \\
-1=\text { null field } \\
0=\text { non-numeric field; only CVALUE } \\
\text { contains a specified value } \\
1=\text { a real numeric field; CVALUE and } \\
\text { RVALUE contain specified values } \\
2= \\
\text { an integer numeric field; CVALUE, } \\
\text { RVALUE, and IVALUE contain } \\
\text { specified values }\end{array}$ \\
\hline & CVALUE & Output & $\operatorname{char}{ }^{*}(*)$ & $\begin{array}{l}\text { Character values of the data fields: } \\
\text { CVALUE(MFIELD). The data will be left- } \\
\text { justified and either blank-filled or truncated. } \\
\text { The value in this array is set blank for a null } \\
\text { field. The character element size can be any } \\
\text { value set by the caller. }\end{array}$ \\
\hline & IVALUE & Output & integer & $\begin{array}{l}\text { Integer values of the data fields: } \\
\text { IVALUE(MFIELD). The value in this array is } \\
\text { set to zero for a null or non-INTEGER field. }\end{array}$ \\
\hline & RVALUE & Output & real & $\begin{array}{l}\text { Floating-point values of the data fields: } \\
\text { RVALUE(MFIELD). The value in this array is } \\
\text { set to zero for a null or nonnumeric field. }\end{array}$ \\
\hline
\end{tabular}




\begin{tabular}{|c|c|c|c|c|}
\hline Name & \multicolumn{4}{|c|}{ FFRDKEY - Free-field keyword parameter input routine } \\
\hline Description & \multicolumn{4}{|c|}{$\begin{array}{l}\text { FFRDKEY reads a record and parses the record into fields. The record should be in the } \\
\text { form: } \\
\qquad \text { keyword param }_{1}=\text { value }_{1}, \ldots, \text { value }_{n} \ldots \& \\
\qquad \text { param }_{n}=\text { value }_{1}, \ldots \text {, value } \\
\end{array}$} \\
\hline Syntax & \multicolumn{4}{|c|}{$\begin{array}{l}\text { CALLFFRDKEY (KIN, KOUT, PROMPT, IOSTAT, KEYWRD, MAXPRM, NUMPRM, } \\
\text { CPARAM, MAXVAL, NUMVAL, ITYVAL, CVALUE, IVALUE, RVALUE) }\end{array}$} \\
\hline \multirow{12}{*}{$\begin{array}{l}\text { Variables in } \\
\text { Call }\end{array}$} & Name & $I / O$ & Type & Description \\
\hline & KIN & Input & integer & Unit from which to read input. \\
\hline & KOUT & Input & integer & Unit to which to echo input. \\
\hline & PROMPT & Input & $\operatorname{char}^{*}(*)$ & The prompt string. \\
\hline & IOSTAT & Output & integer & $\begin{array}{l}\text { ANSI FORTRAN } 1 / O \text { status } \\
1000 \text { - number of parameters or values over- } \\
\text { runs maximum }\end{array}$ \\
\hline & KEYWRD & Output & $\operatorname{char} *(*)$ & The input keyword. \\
\hline & MAXPRM & Input & integer & Maximum number of parameters allowed. \\
\hline & NUMPRM & Outp'st & integer & $\begin{array}{l}\text { Number of input parameters } \\
<0-\text { no keyword or parameter (blank line) }\end{array}$ \\
\hline & CPARAM & Output & $\operatorname{char}{ }^{*}(*)$ & The input parameters: CPARAM (MAXPRM) \\
\hline & MAXVAL & Input & integer & Maximum number of values per parameter. \\
\hline & NUMVAL & OLtput & integer & $\begin{array}{l}\text { Number of input values for each parameter: } \\
\text { NUMVAL (MAXPRM) }\end{array}$ \\
\hline & ITYVAL & Output & integer & $\begin{array}{l}\text { Translation states of the parameter values: } \\
\text { ITYVAL (MAXVAL, MAXPRM) } \\
-1=\text { a null field } \\
0=\text { a non-numeric field } \\
1=\text { a real numeric field } \\
2=\text { an integer numeric field }\end{array}$ \\
\hline
\end{tabular}




\section{FFRDKEY}

\begin{tabular}{l|llll}
$\begin{array}{l}\text { Variables in } \\
\text { Call (cont.) }\end{array}$ & CVALUE & Output & char*(*) & $\begin{array}{l}\text { Character values of the parameter values: } \\
\text { CVALUE (MAXVAL, MAXPRM) }\end{array}$ \\
IVALUE & Output & integer & $\begin{array}{l}\text { Integer values of the parameter vaiues: } \\
\text { IVALUE (MAXVAL, MAXPRM) }\end{array}$ \\
RVALUE & Output & real & $\begin{array}{l}\text { Floating-point values of the parameter val- } \\
\text { ues: RVALUE (MAXVAL, MAXPRM) }\end{array}$
\end{tabular}

See Also

FFRDFLDS for a more complete description of some of the variables in the call 


\begin{tabular}{|c|c|c|c|c|}
\hline Name & \multicolumn{4}{|c|}{ FFRDLINE - Get literal input line } \\
\hline Description & \multicolumn{4}{|c|}{$\begin{array}{l}\text { All } \mathrm{V} \text { O for FFRDFLDS is done through this subroutine. This routine was intentionally } \\
\text { separated from FFRDFLDS so that the caller can obtain an unmodified line of input } \\
\text { (such as a problem title) via the same I/O stream. Applications that require a more } \\
\text { complex syntax than the free-field reader provides (e.g., algebraic operations) may find } \\
\text { FFRDLINE advantageous. }\end{array}$} \\
\hline Syntax & \multicolumn{4}{|c|}{ CALL FFRDLINE (KIN, KOUT, PROMPT, LINE, IOSTAT) } \\
\hline \multirow{4}{*}{$\begin{array}{l}\text { Variables in } \\
\text { Call }\end{array}$} & & $I / O$ & Type & Description \\
\hline & KIN & Input & integer & $\begin{array}{l}\text { Unit from which to read input. } \\
>0 \text { - read from the file whose unit is given; the } \\
\text { caller is responsible for opening/closing } \\
\text { this unit } \\
=0 \text { - read from the standard input device (ter- } \\
\text { minal or batch deck) and echo to the } \\
\text { standard outputdevice(terminal or batch } \\
\text { log) } \\
<0 \text { - read from the command line }\end{array}$ \\
\hline & KOUT & Input & integer & $\begin{array}{l}\text { Unit to which to echo input. } \\
>0-\text { ec } 20 \text { to the file whose unit is given; the } \\
\text { caller is responsible for opening/closing } \\
\text { this unit } \\
=0 \text { - do not echo other than to the standard } \\
\text { output device as described for KIN } \\
<0 \text { - echo to the standard output device }\end{array}$ \\
\hline & PROMPT & Input & $\operatorname{char} *(*)$ & $\begin{array}{l}\text { Prompt string. This string will be used to } \\
\text { prompt for data from an interactive terminal } \\
\text { and/or will be written as a prefix to the input } \\
\text { line for echo. If the string 'AUTO' is specified, } \\
\text { a prompt of the form 'n:', where "n" is the } \\
\text { current input line number (only lines read } \\
\text { under the AUTO feature are counted), will be } \\
\text { generated. }\end{array}$ \\
\hline
\end{tabular}




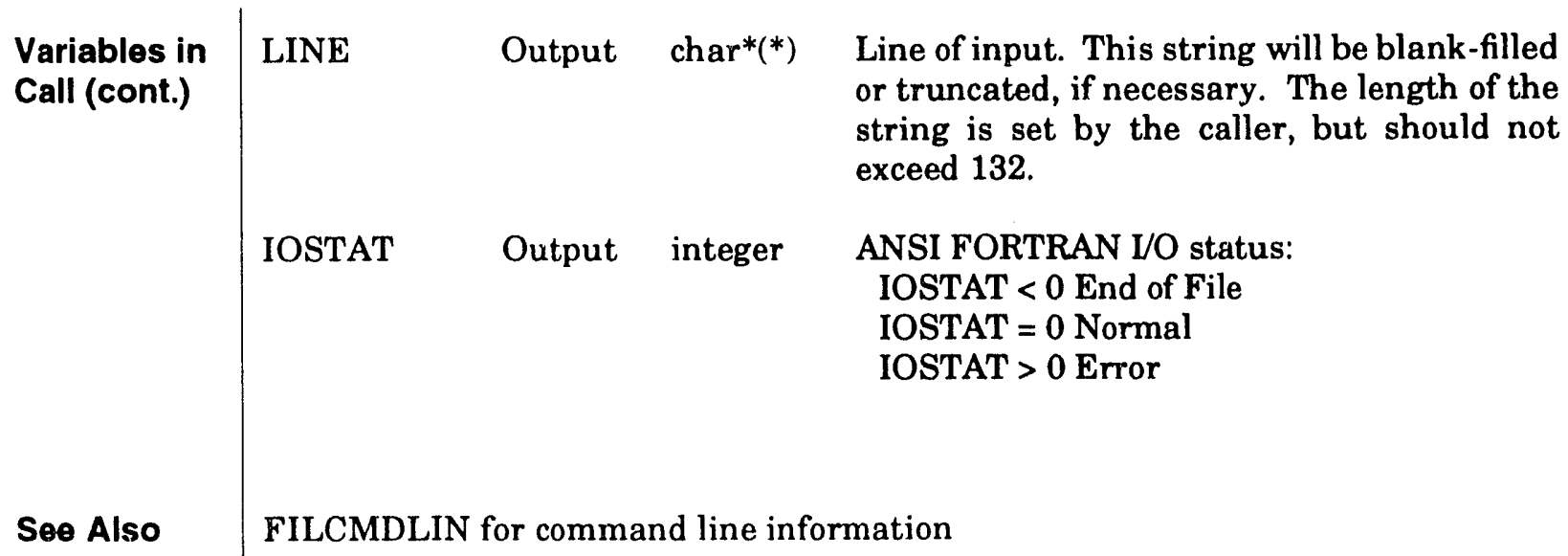




\subsubsection{String Processing Routines}

Detailed descriptions of the four routines for processing strings are given on the following pages. 


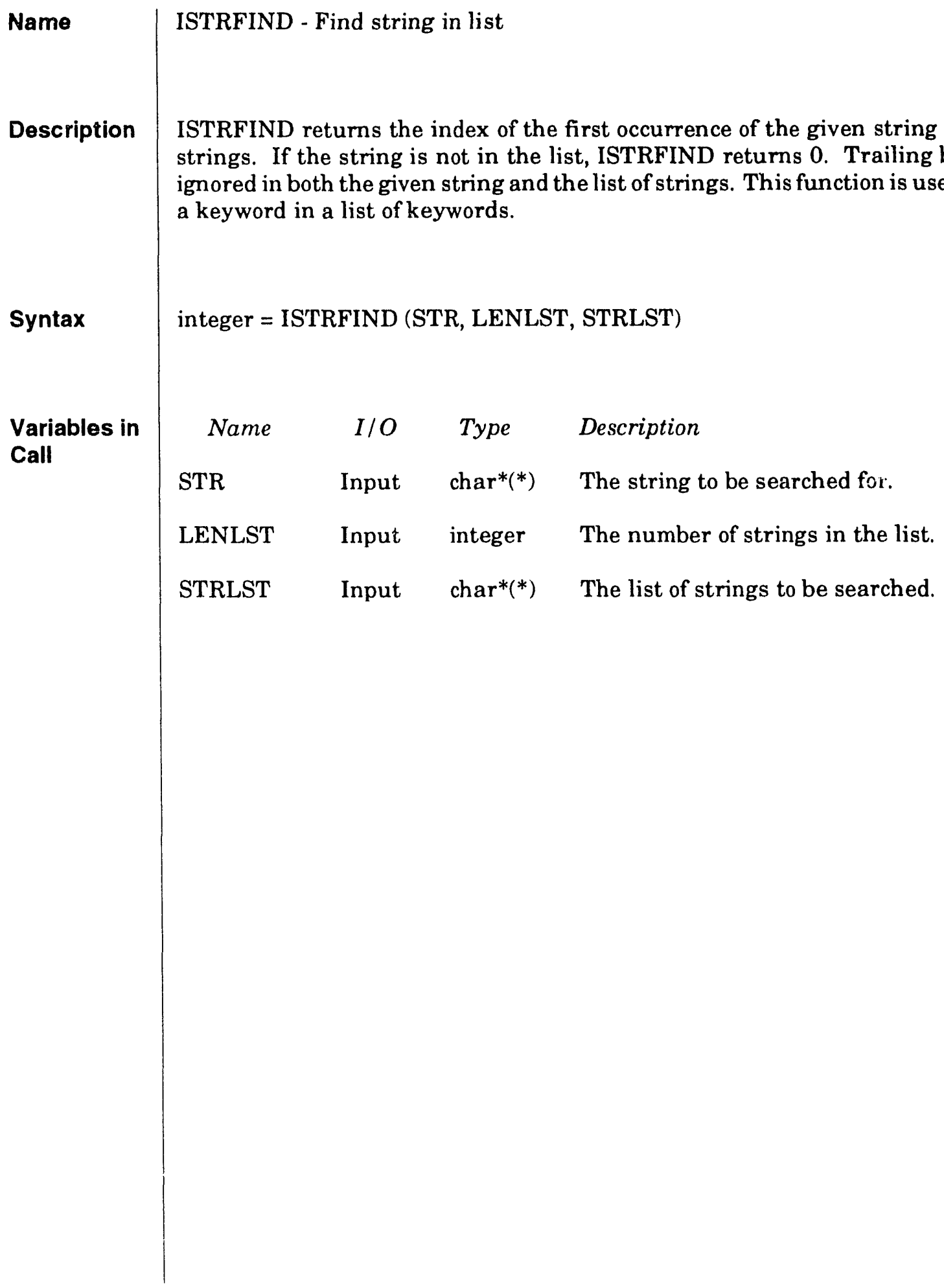




\begin{abstract}
Name
ISTRLEN - Return string length

Description

ISTRLEN returns the length of a given string excluding trailing blanks. There can be blanks embedded within the string. ISTRLEN returns a length of 0 for a blank string.

Syntax

integer = ISTRLEN (STRING)

Variables in

Call

Name

I/O Type

Description

STRING Input $\operatorname{char}^{*}\left({ }^{*}\right) \quad$ The string.

Comments

ISTRLEN differs from the FORTRAN LEN intrinsic function in that LEN returns the declared length of the string, but the ISTRLEN length does not include trailing blanks.
\end{abstract}




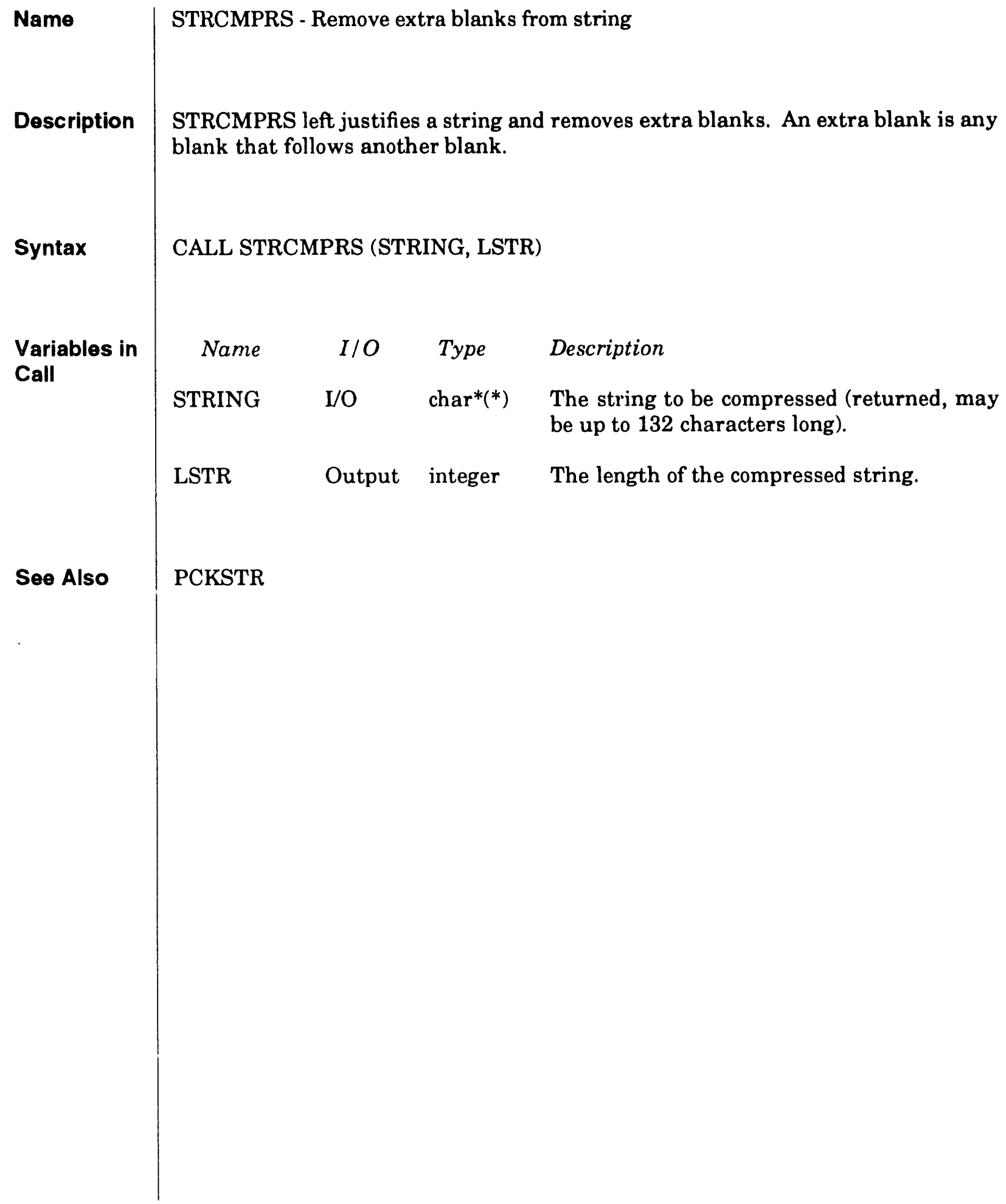




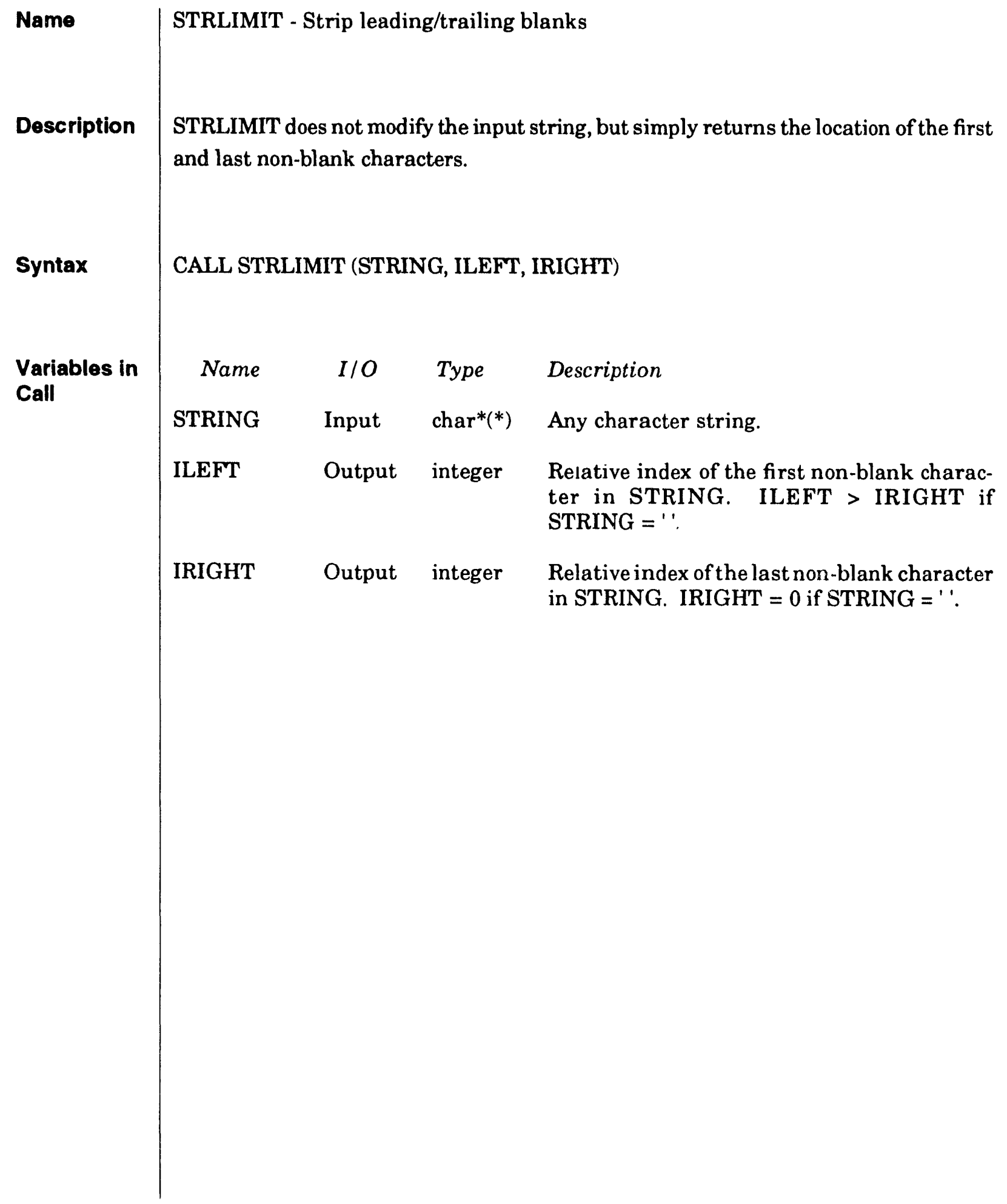




\begin{tabular}{|c|c|c|c|c|}
\hline Name & \multicolumn{4}{|c|}{ STRPACK - Remove all blanks from string } \\
\hline Description & \multicolumn{4}{|c|}{ STRPACK left justifies a string and removes all embedded blanks. } \\
\hline Syntax & \multicolumn{4}{|c|}{ CALL STRPACK (STRING, LSTR) } \\
\hline Variahles in & Name & $I / O$ & Type & Description \\
\hline & STRING & $\mathrm{I} / \mathrm{O}$ & $\operatorname{char}^{*}(*)$ & $\begin{array}{l}\text { The string, returned packed (may be up to } 132 \\
\text { characters long). }\end{array}$ \\
\hline & LSTR & Output & integer & The length of the packed string. \\
\hline Soe Also & \multicolumn{4}{|c|}{ STRCMPRS } \\
\hline
\end{tabular}




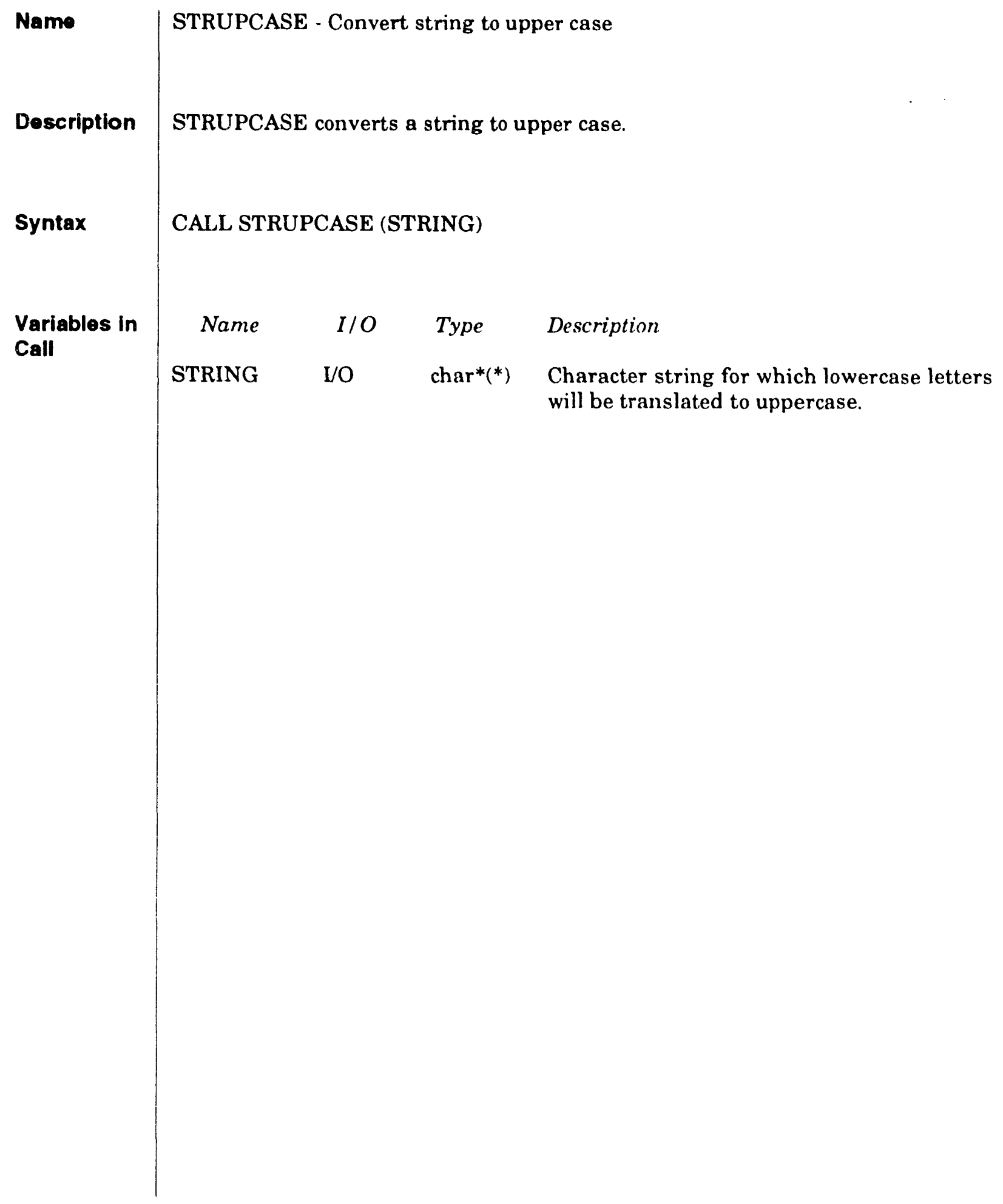


CAMCON_LIB

Common Internal Routines

\subsubsection{Common Internal Routines}

Detailed descriptions of seven routines commonly used with CAMCON system codes are given on the following pages. 
Name

Description

Syntax

Variables in

Call

Soe Also
FEELEM - Calculate element number associated with node

FEELEM uses routines FEIJK and FENENUM to calculate the element number associated with the given node number. Nodes and elements are assumed to be numbered first in order of increasing $\mathrm{X}$ ( $\mathrm{I}$-index), then $\mathrm{Y}$ ( $\mathrm{J}$-index), and then $\mathrm{Z}$ ( $\mathrm{K}$ index).

CALL FEELEM (NODE, NX, NY, NZ, IELEM)

Name I/O Type Description

NODE Input integer The node number of the first node and smallest node number in the connectivity list for the element.

Nn Input integer The number of nodes in the $\mathrm{n}(\mathrm{X}, \mathrm{Y}, \mathrm{Z}$ or I, J, $\mathrm{K})$ directions, respectively.

IELEM Output integer The corresponding element number.

FEIJK, FENENUM 
Name
Description

Description

FEIJK determines the I, J, $\mathrm{K}$ index for the given node or element number, assuming a two- or three-dimensional rectilinear grid where nodes and elements are numbered first in order of increasing $X(I$ index), then $Y(J$ index), and then $Z$ ( $K$ index).

CALL FEIJK (N, NX, NY, NZ, I, J, K)

Syntax

Variables in

Call

See Also

FEIJK - Determine I, J, K index from node/element number

Name I/O Type Description

$\mathbf{N}$

Input integer

The node or element number.

$\mathrm{Nn}$ Input integer For a node, the number of nodes in the $\mathrm{n}(\mathrm{X}, \mathrm{Y}$, $Z)$ direction; for an element, the number of elements in the $\mathrm{n}(\mathrm{X}, \mathrm{Y}, \mathrm{Z})$ direction (number of nodes minus one).

I, J, K Output integer For a node, the I, J, K index of the node; for an element, the first node and smallest node number in the connectivity list for the element (assumes WIPP connectivity convention). 


\begin{tabular}{l|l} 
Namo & $\begin{array}{l}\text { FEMINMAX - Calculate minimum/maximum value } \\
\text { Description }\end{array}$ \\
Syntax & FEMINMAX calculates the minimum and maximum value of the data. \\
Variables in \\
Call
\end{tabular} \mid $\begin{array}{cccc}\text { NALMINMAX (NPTS, PTS, VMIN, VMAX) } \\
\text { NPTS } & \text { Input } & \text { integer } & \text { The number of points. } \\
\text { PTS } & \text { Input } & \text { real array } & \text { The points } \\
\text { VMIN } & \text { Output } & \text { real } & \text { The minimum value of the points. } \\
\text { VMAX } & \text { Output } & \text { real } & \text { The maximum value of the points. }\end{array}$




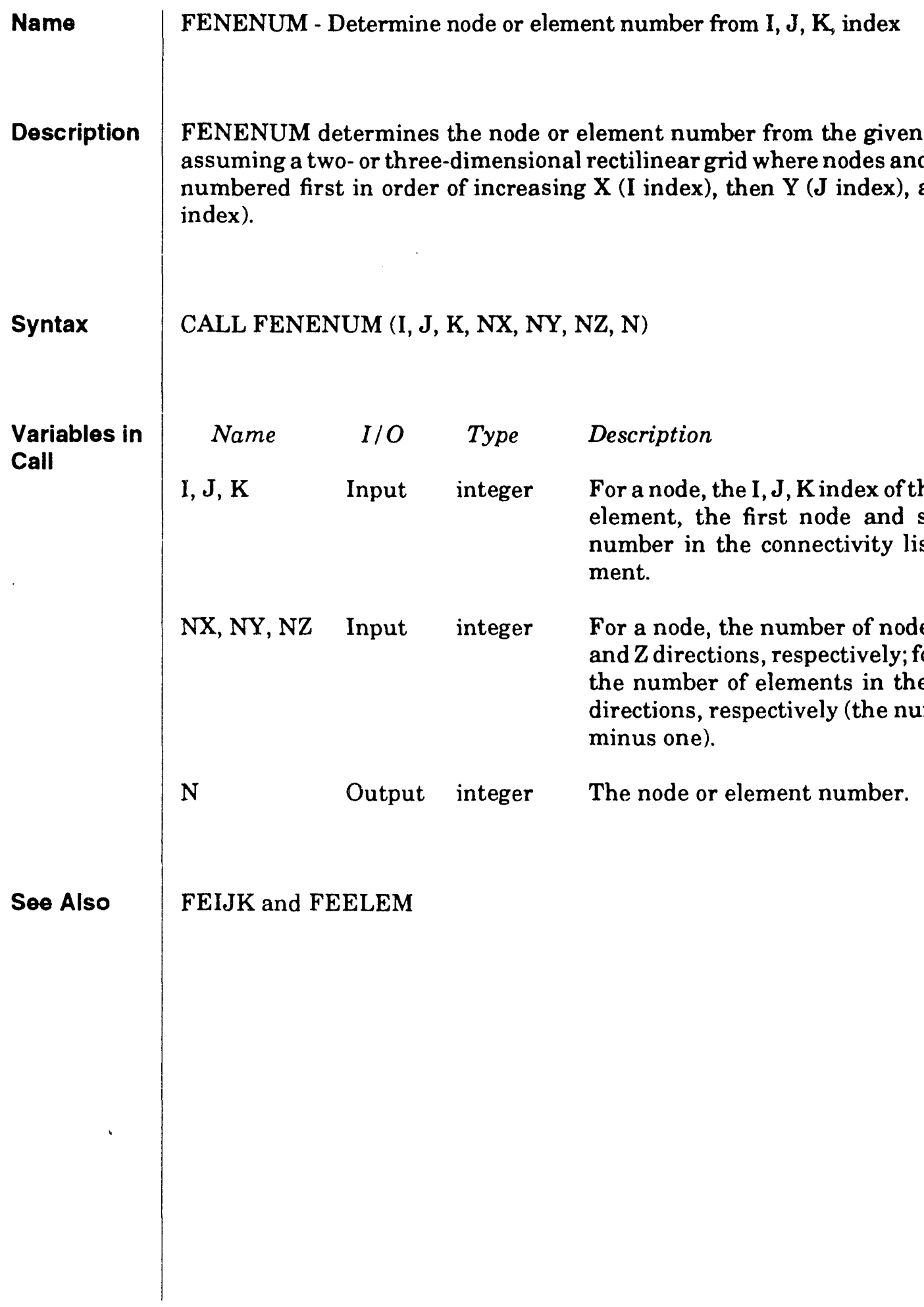




\section{CAMSUPES_LIB: Routines for Dynamic Memory Allocation and Other FORTRAN Extensions}

\subsection{General Description}

TheCAMSUPES_LIB library is a collection of routines that perform two frequently needed functions: (1) accessing computer-system-dependent parameters (e.g., the clock) and (2) allocating memory for arrays at run time for FORTRAN77.

\subsubsection{Sponsorship and Version}
Authors:
D. P. Flanagan, W. C. Mills-Curran, L. M. Taylor, J. R. Red Horse
Sponsor:
A. P. Gilkey
Version/Date:
C-2.10VV/March 1993
Version Base Case:
Language:
C-2.00VV equivalent to Version 2.1 acquired from authors May 1989
FORTRAN77

\subsubsection{Overview of CAMSUPES_LIB}

The routines were originally developed within another area of Sandia under the name SUPES (RedHorse et al., 1990; Flanagan et al., 1986) and adopted for use by the PA Department. Hence, the original documentation on the SUPES library (Red-Horse et al., 1990) provides the primary source of information on use of these routines. The original SUPÉS routines for parsing an input record have been modified and moved to CAMCON_LIB (Chapter 8).

\subsubsection{Linking}

The CAMSUPES_LIB routines are an object library in the [LIBRARIES] subdirectory (see Section 2.10, "Software Libraries"). To link a code with CAMSUPES_LIB, type (the logical name of the library is assigned by the library sponsor):

\$LINK code_name, ..., CAMSUPES_LIB/LIB

A FLINT library is available for checking interfaces. It is in CAMCON\$ROOT: [LIBRARIES.CAMSUPES_LIB] CAMSUPES_LIB.LBT. 


\subsubsection{Summary}

Library CAMSUPES_LIB has routines that provide: system queries, system-dependent utilities, and dynamic memory management. The following is a brief description of the CAMSUPES_LIB routines.

\section{System Queries}

- EXCPUS - Get accumulated processor time

- EXDATE - Get today's date

- EXFILDAT - Return last update date and time for a file

- EXNAME - Get unit file name or symbol value

- EXPARM - Get operating environment parameters

- EXTIME - Get time of day

\section{Memory Management}

- MDDEL / MCDEL - Delete dynamic array

- MDEROR / MCEROR - Print dynamic memory error summary

- MDFILL/ MCFILL - Enable data initialization

- MDFOFF / MCFOFF - Cancel data initialization

- MDGET / MCGET - Reserve memory blúck

- MDGIVE / MCGIVE - Release unallocated memory

- MDINIT / MCINIT - Initialize dynamic memory

- MDRSRV / MCRSRV - Define dynamic array

- MDSTAT / MCSTAT - Obtain dynamic memory statistics

Advanced Memory Management Routines

- MDCOMP / MCCOMP - Compress storage

- MDEFIX/ MCEFIX - Modify dynamic memory error count

- MDERPT / MCERPT - Dynamic memory error flag query

- MDEXEC / MCEXEC - Execute deferred memory requests

- MDFIND / MCFIND - Locate dynamic array

- MDLAST / MCLAST - Report last dynamic memory error

- MDLONG / MCLONG - Adjust dynamic array length

- MDMEMS / MCMEMS - Report storage information

- MDNAME / MCNAME - Rename dynamic array

- MDWAIT / MCWAIT - Enable deferred memory mode

Memory Management Debugging Routines

- MDDEBG / MCDEBG - Enable debug printing for dynamic memory

- MDLIST/ MCLIST - List dynamic memory storage tables

- MDPRNT/ MCPRNT - Print dynamic array

Detailed descriptions of these routines are provided on the pages that follow in this chapter. 


\subsection{Description of Routine Calls}

\subsubsection{System Queries}

This section prescribes the calling sequence for FORTRAN extension routines that are meant to be called directly from application programs.

The CAMSUPES_LB System Queries (FORTRAN extensions) provides a uniform interface to necessary operating system functions that are not included in the ANSI FORTRAN-77 standard. This package makes it possible to maintain many codes on different operating systems with a single point of support for system dependencies. Moreover, this maintenance is done by a single set of source files which should not only reduce the time involved in bookkeeping, but allow for standard procedures for building a CAMSUPES library. These routines provide very basic operating system support; they are not intended to implement clever features of a favorite system, to make FORTRAN behave like another language, or to improve execution efficiency.

Each module included in the CAMSUPES_LIB System Queries must satisfy the following criteria:

1. The routine must provide a service that is beneficial to a wide range of users.

2. This task cannot be accomplished by standard FORTRAN.

3. This capability must be generic to scientific computers. System query routines must be supportable on virtually any system. 


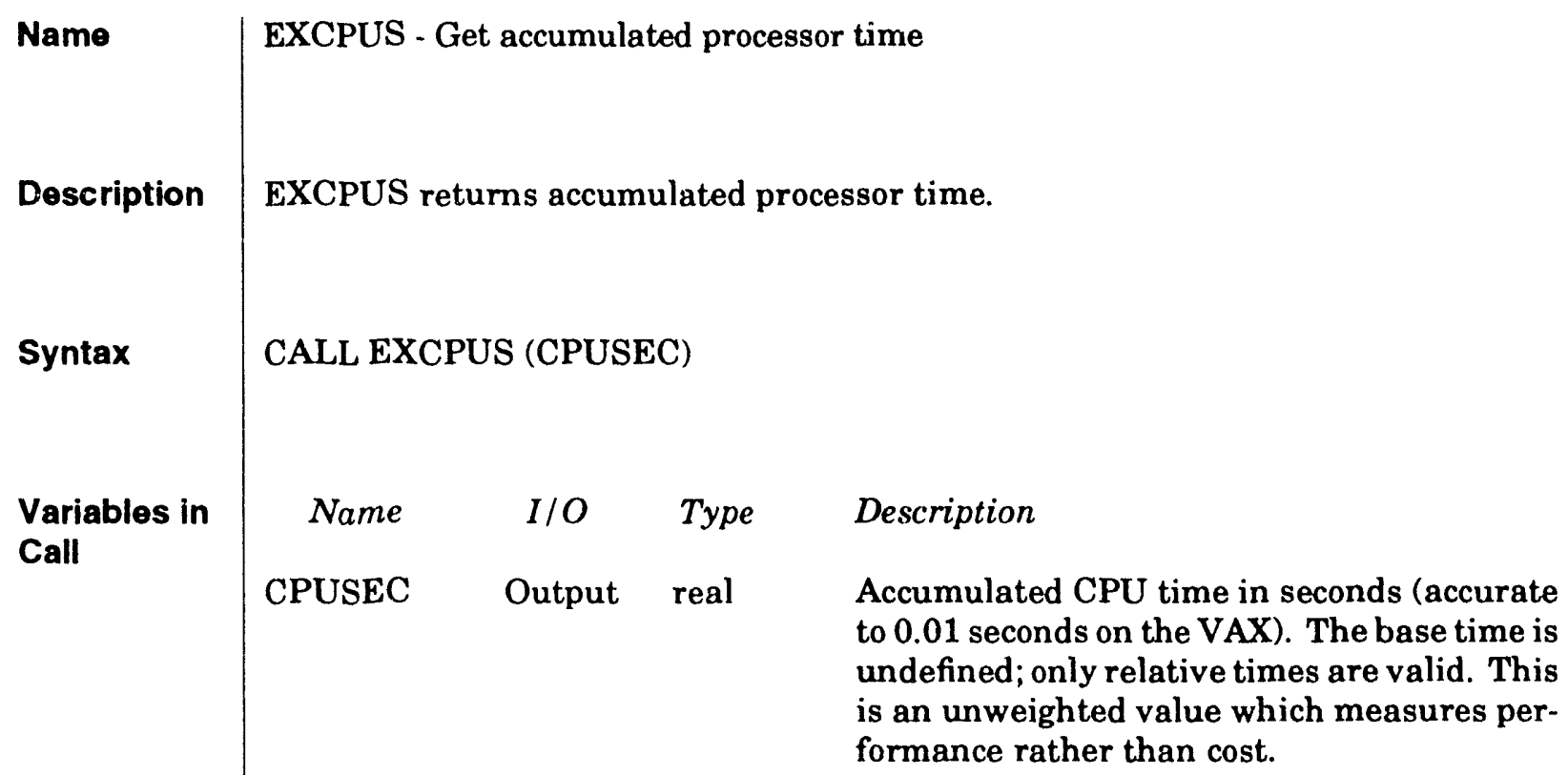




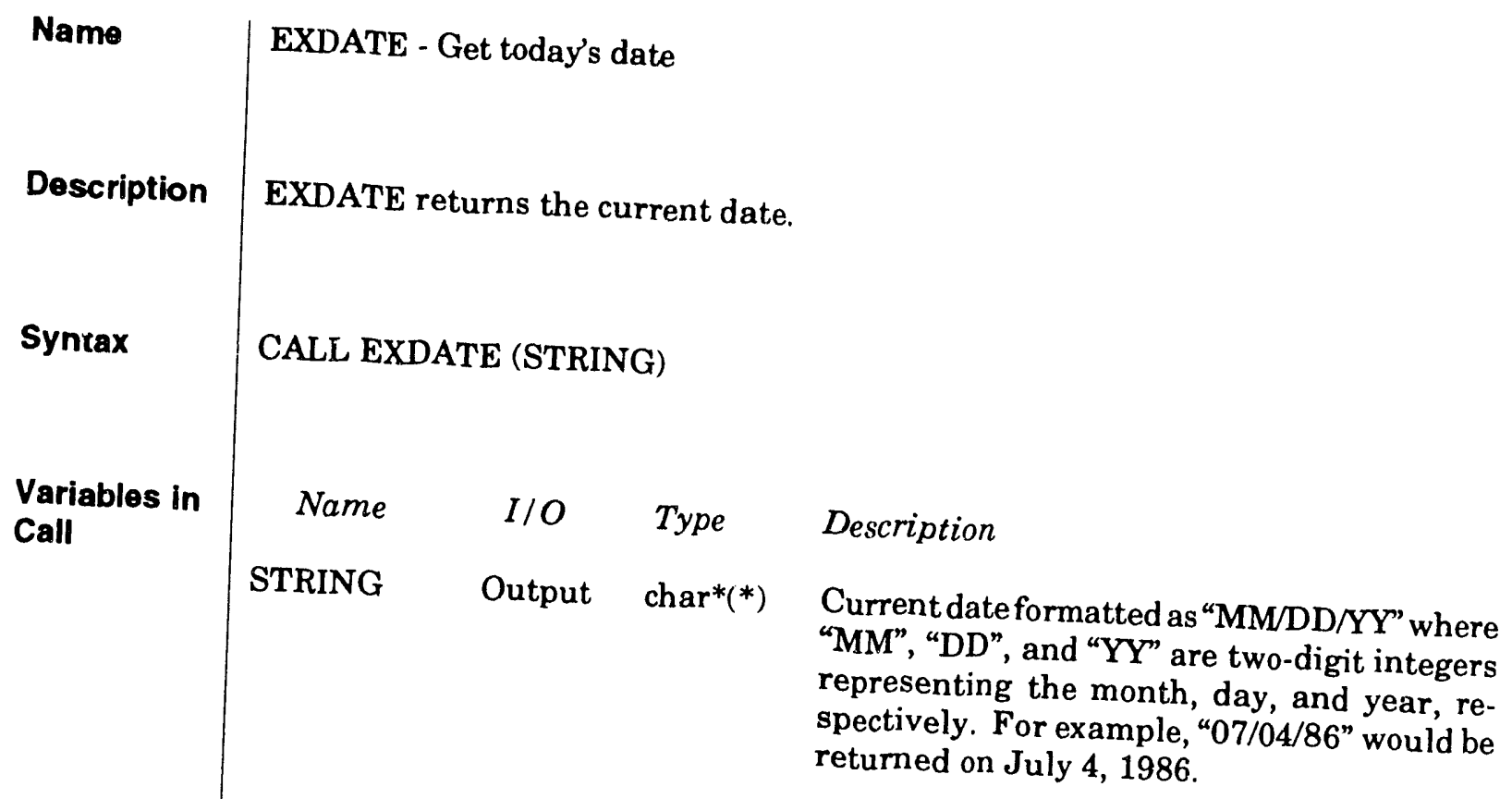




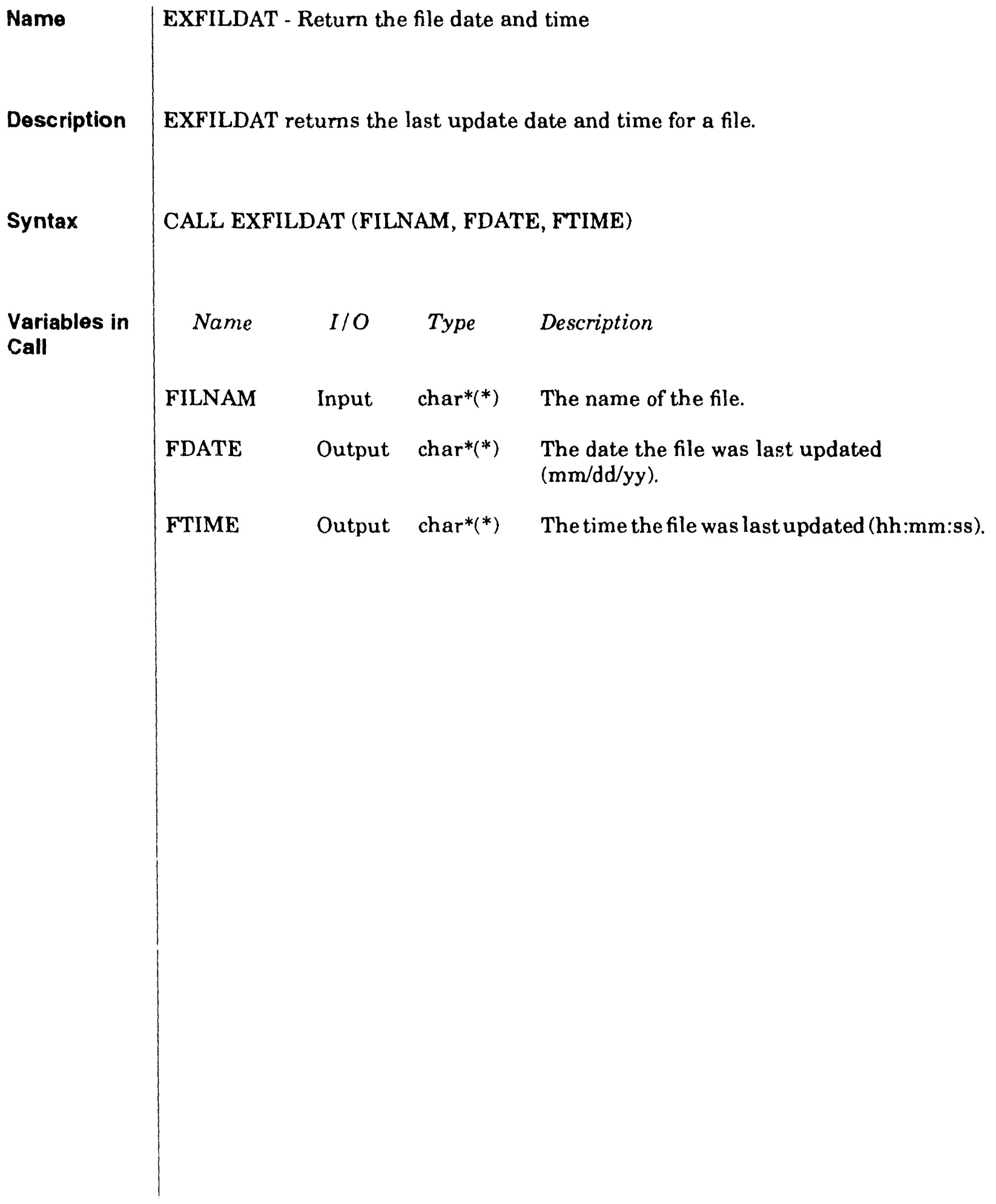


Name

Description

Syntax

Variables in

Call

EXNAME - Get unit file name or symbol value

EXNAME returns unit file name or symbol value. The unit file name or symbol must be set before the program is executed in a system-dependent fashion.

CALL EXNAME (IUNIT, NAME, LN)

$\begin{array}{ccc}\text { Name } & I / O & \text { Type } \\ \text { IUNIT } & \text { Input } & \text { integer }\end{array}$

Description

IUNIT Input integer Unit number if IUNIT $>0$, or symbol ID if IUNIT $<0$.

NAME Output $\operatorname{char}^{*}\left(^{*}\right)$ File name or symbol value obtained from the operating system. It is assumed that the unit/ file name or symbol/value linkage will be passed to this routine at program activation.

LN Output integer Effective length of the string returned in NAME. Zero indicates that no name or value was available.

This routine provides a standard interface for establishing execution time unit/file connection on operating systems that do not support pre-connection of FOR'TRAN I/O units. For example, on the VAX, the symbol is set up within a VAX DCL file before program execution as follows:

\section{\$ASSIGN/USER CAMDAT.CDB FOR010}

The returned string is used with the FILE specifier in an OPEN statement, as in the following example:

CALL EXNAME( 10, NAME, LN )

OPEN( 10, FILE=NAME (1:LN),... ) 

Comments
(continued)
The symbol mode of this routine provides a standard path through which to pass messages at program activation. The example below identifies the target graphics device for a VAX code that supports multiple devices. The global symbol is set up in a DCL file before program execution with

$$
\text { \$EXT02=="PST" }
$$

EXNAME can then retrieve and use the string as follows:

CALL EXNAME (-2, DEV, LD)

WRITE $\left(*,{ }^{*}\right)$ 'Running on hardcopy device ' /DEV(1:LD) 


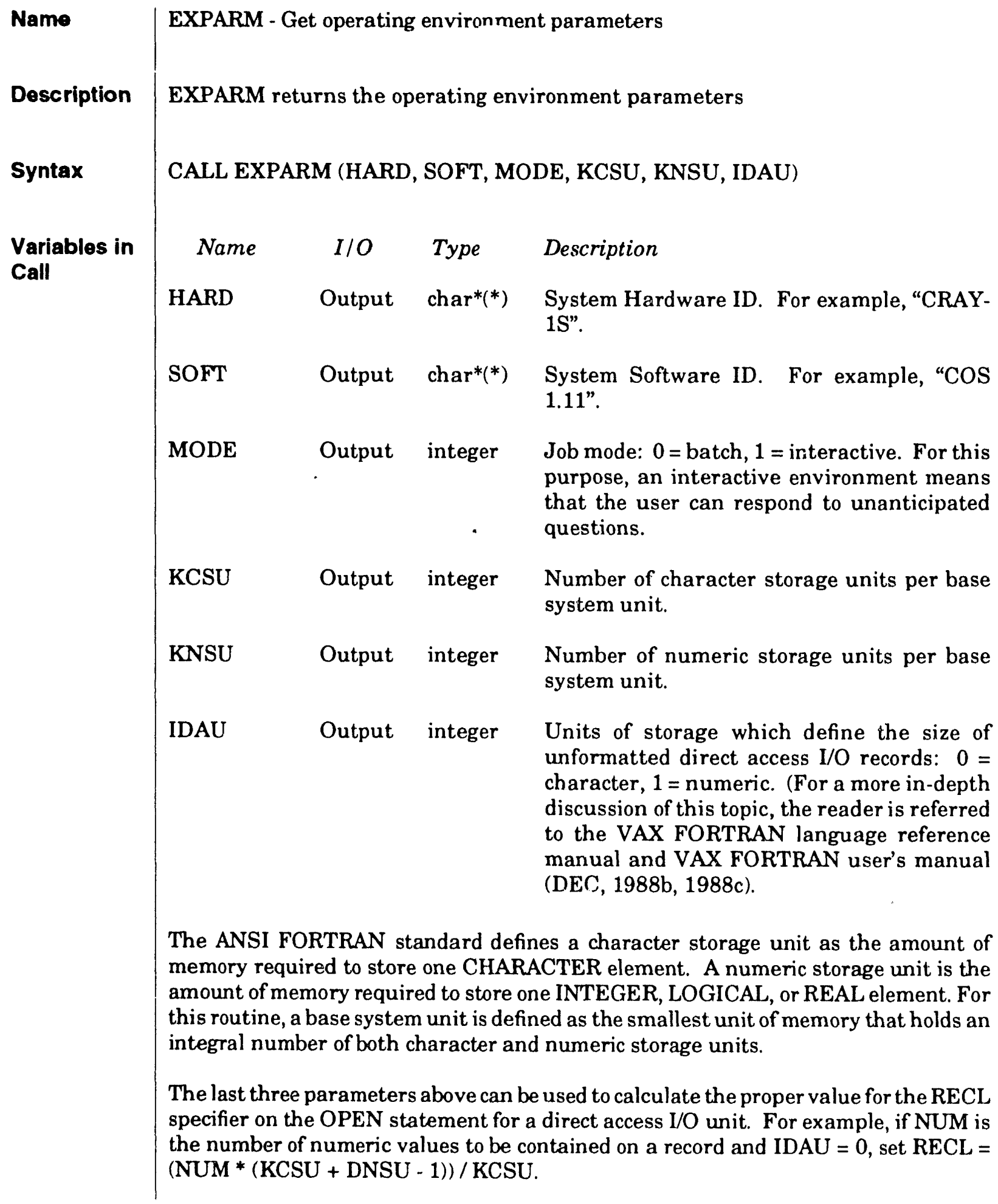




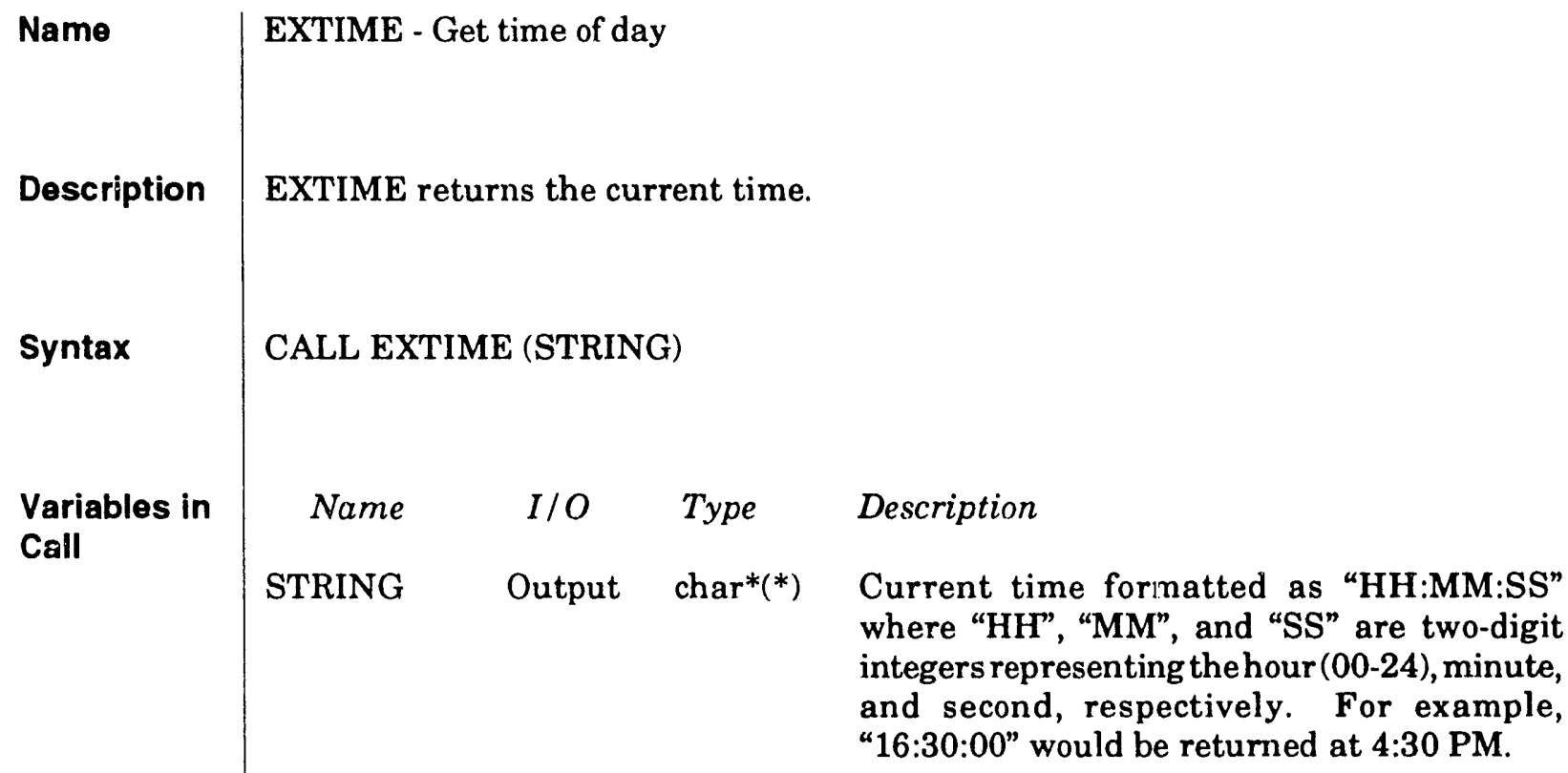




\subsubsection{Memory Management Routines}

The purpose of the memory management utilities is to allow an applications programmer to write standard, readable FORTRAN-77 code while employing dynamic memory management for REAL, INTEGER, LOGICAL and CHARACTER type arrays.

Because the array sizes in most programs are problem-dependent, a program's memory requirements are not known until the program is running. Since FORTRAN-77 does not provide for dynamic memory allocation, the programmer has to either predict the maximum memory requirement or use a machine-dependent request for memory. In addition, dynamic memory allocation is an error-prone exercise which tends to $\mathrm{m}$. $\mathrm{k}$ ke the source code difficult to read and maintain.

In CAMSUPES_LIB, the memory manager utilities are written in standard FORTRAN-77 and provide an interface that encourages readable coding and efficient use of memory resources. Machine dependencies are isolated through the use of the extension library (Section 9.2.1). All memory requests are in terms of numeric storage units for numeric data (integer, real, or logical) and character storage units for character data (ANSPL FORTRAN, 1978).

An important design feature of the memory manager is that the memory manager can be supported even when the system-dependent dynamic memory request routines are not implemented on a system. In this case, the memory manager will operate, allocating space from a user-supplied work array. This mode is described as dynamic allocation of static memory. Thus, modification of a user's application program is minimal on systems where dynamic memory is not implemented.

All user entry points to memory manager routines begin with either "MD" or "MC." In most cases, the " $\mathrm{MD}$ " routines are used for numeric data, while the "MC" routines are for character data. In some cases, however, the routines are interchangeable. These routines are documented as synonyms.

In this document, the term "M $\alpha$ " is used to refer simultaneously to both "MD" and "MC" routines. Thus, M $\alpha$ RSRV is a reference to both MDRSRV and MCRSRV subroutines.

General Information. In order to use the memory manager properly, the user must first understand the concept of using a base array with indexes for accessing memory address locations. At the core of this concept is FOR'RAN's convention of passing SUBROUTINE array references by address. The memory manager references all memory addresses relative to the addresses of user-supplied base arrays - one each for numeric and character data. A reference to memory is made in terms of a pointer to these base arrays. Specifically, the memory manager determines an indexing parameter by first determining the offset of the appropriate memory location relative to the address of the correct base array. The index is then computed in terms of the proper storage units (either character or numeric). Note that the resulting indexes may take on a wide range of values, including negative numbers. 
The base arrays must comply with the following rules (allocating memory for these arrays is explained below):

1. Numeric base arrays must be of type INTEGER, REAL, or LOGICAL. Modified word length storage arrays such as INTEGER*2 or REAL*8 (double precision) will result in invalid indexes with no error message.

2. Character base arrays must be declared CHARACTER*1.

The following FORTRAN statements define valid base arrays:

\section{DIMENSION NUMBAS (1)}

CHARACTER*1 CHRBAS (1)

Only one base array from each category (numeric and character) may be used in a program.

In order to use memory allocated by the memory manager, the user merely needs to pass the base array with the correct offsetting index to a subprogram. For example, for a base arrays NUMBAS and CHRBAS and indexes IP1 and IP2, a subroutine call would be:

\section{CALL SUBBIE ( NUMBAS(IP1), CHRBAS(IP2))}

Although the programmer is not restricted to using the allocated memory in subprograms only, the recommended usage for the memory manager is to allocate dynamic arrays in the main program and then pass them to subroutines.

To aliocate memory for a double precision array of $n$ elements, call MDRSRV with a length of $2 * n$. With in SUBBIE, define the array corresponding to NUMBAS (IP1) as DOUBLE PRECISION. Similar to allocating a CHARACTER* ${ }^{*}$ array of $n$ elements, call MCRSRV with a length of $c^{*} n$ and define the SUBBIE array corresponding to CHRBAS (IP2) as CHARACTER*c.

Basic Routines. The basic memory manager routines are those that are most commonly used and require little understanding of the internal workings of the utility. An example follows: 


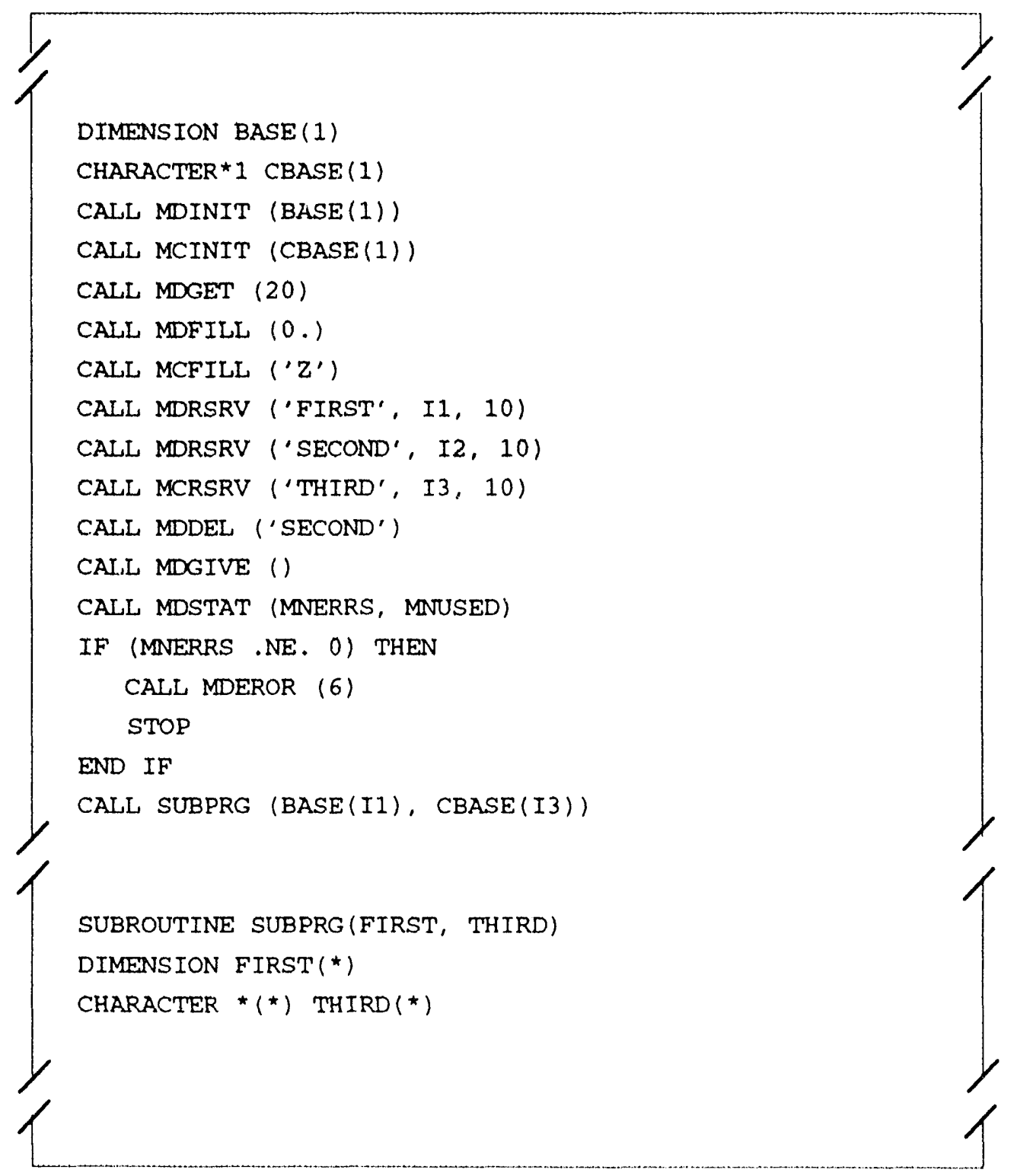




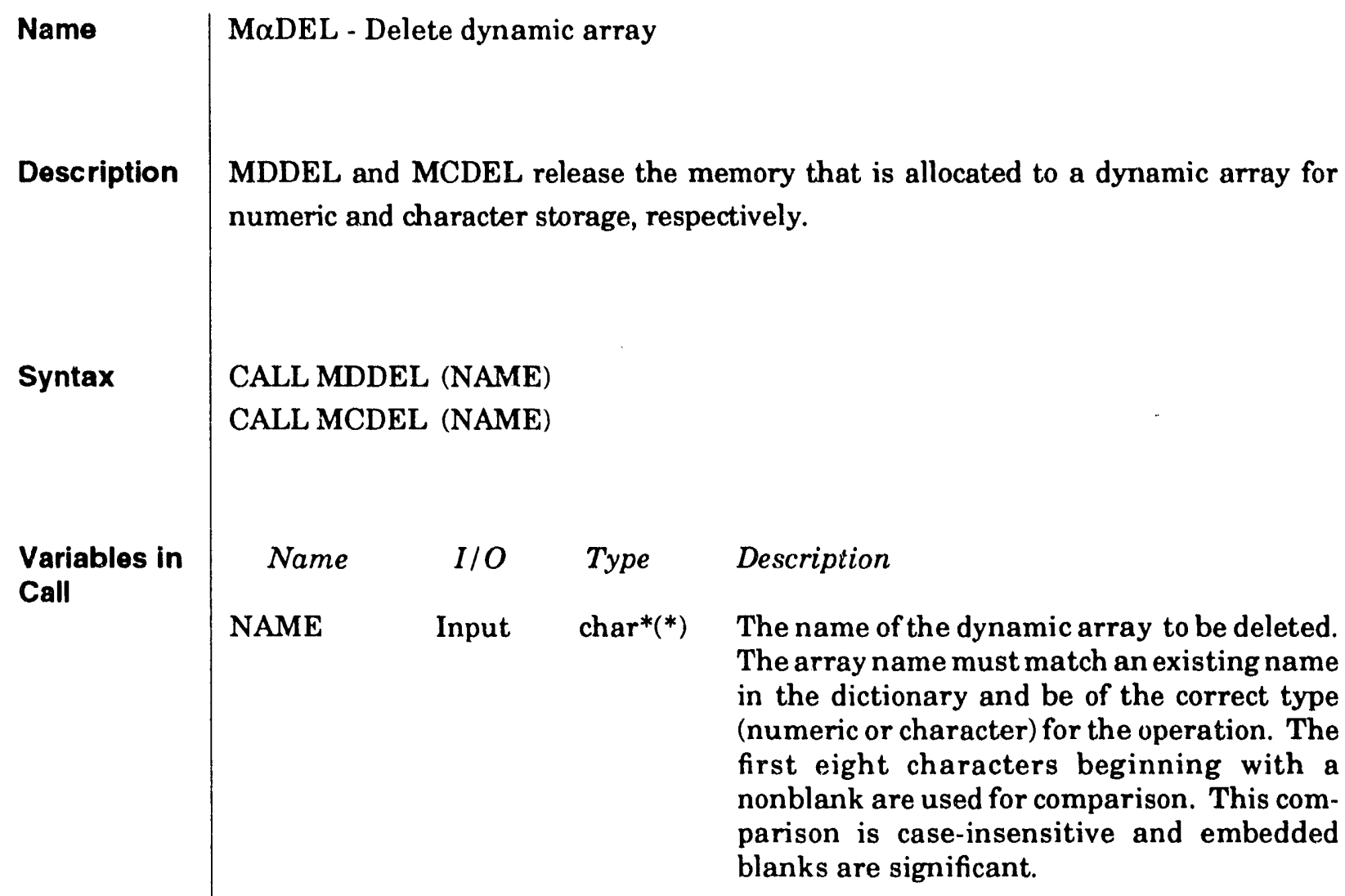




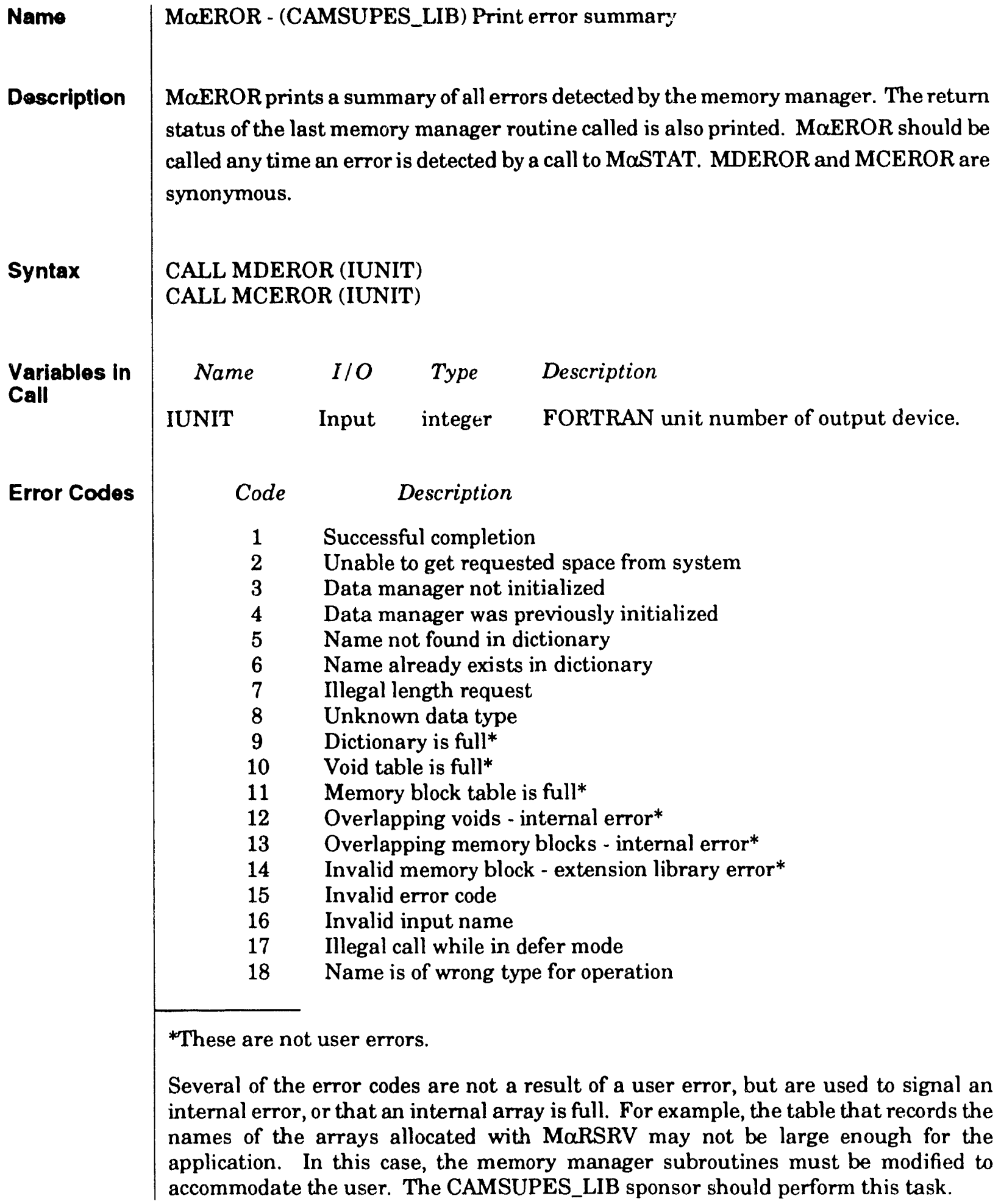




\begin{tabular}{|c|c|c|c|c|}
\hline Name & \multicolumn{4}{|c|}{ M $\alpha$ FILL - Enable data initialization } \\
\hline Description & \multicolumn{4}{|c|}{$\begin{array}{l}\text { M } \alpha \text { FII.L defines a fillinitialization pattern that is to be used for newly allocated storage. } \\
\text { MDFILL and MCFILL are in effect until cancelled by MDFOFF and MCFOFF, } \\
\text { respectively. MDFILL and MCFILL operate independently. }\end{array}$} \\
\hline Syntax & \multicolumn{4}{|c|}{$\begin{array}{l}\text { CALL MDFILL (NUMDAT) } \\
\text { CALL MCFILL (CHRDAT) }\end{array}$} \\
\hline \multirow{3}{*}{$\begin{array}{l}\text { Variables in } \\
\text { Call }\end{array}$} & Name & $I / O$ & Type & Description \\
\hline & NUMDAT & Input & integer & $\begin{array}{l}\text { The initialization datum for new storage allo- } \\
\text { cated with MDRSRV or extended with } \\
\text { MDLONG. The memory manager makes no } \\
\text { attempt to identify the type (INTEGER, REAL, } \\
\text { or LOGICAL) of either the initialization da- } \\
\text { tum or of a newly allocated array. Instead, the } \\
\text { bit of the initialization datum is stored with- } \\
\text { out interpretation. This pattern is then used } \\
\text { to initialize new storage. Since the internal } \\
\text { machine representation of REAL data is dif- } \\
\text { ferent from INTEGER data (or LOGICAL } \\
\text { data), the user may experience unexpected } \\
\text { results when dynamic memory is used as a } \\
\text { numeric type that is different from the type of } \\
\text { the initialization datum. }\end{array}$ \\
\hline & CHRDAT & Input & $\operatorname{char}^{*}(*)$ & $\begin{array}{l}\text { The initialization data for new storage allo- } \\
\text { cated with MCRSRV or extended with } \\
\text { MCLONG. Only the first character of } \\
\text { CHRDAT is used. }\end{array}$ \\
\hline
\end{tabular}


Name

Description

Syntax
M $\alpha$ FOFF - Cancel data initialization

MDFOFF and MCFOFF cancel the data initialization for numeric and character data, respectively. MDFOFF and MCFOFF operate independently.

CALL MDFOFF ()

CALL MCFOFF () 


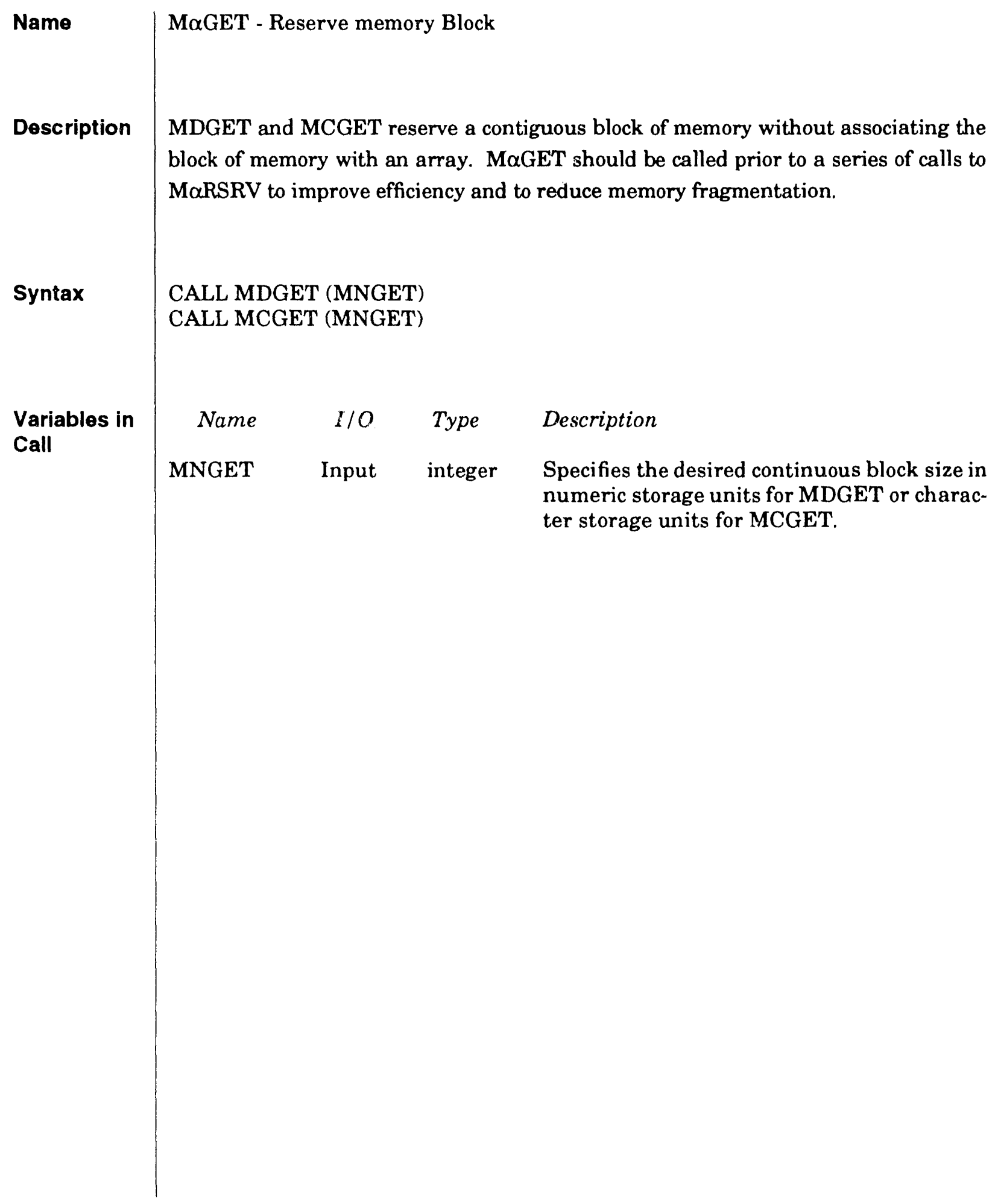


CAMSUPES_LIB

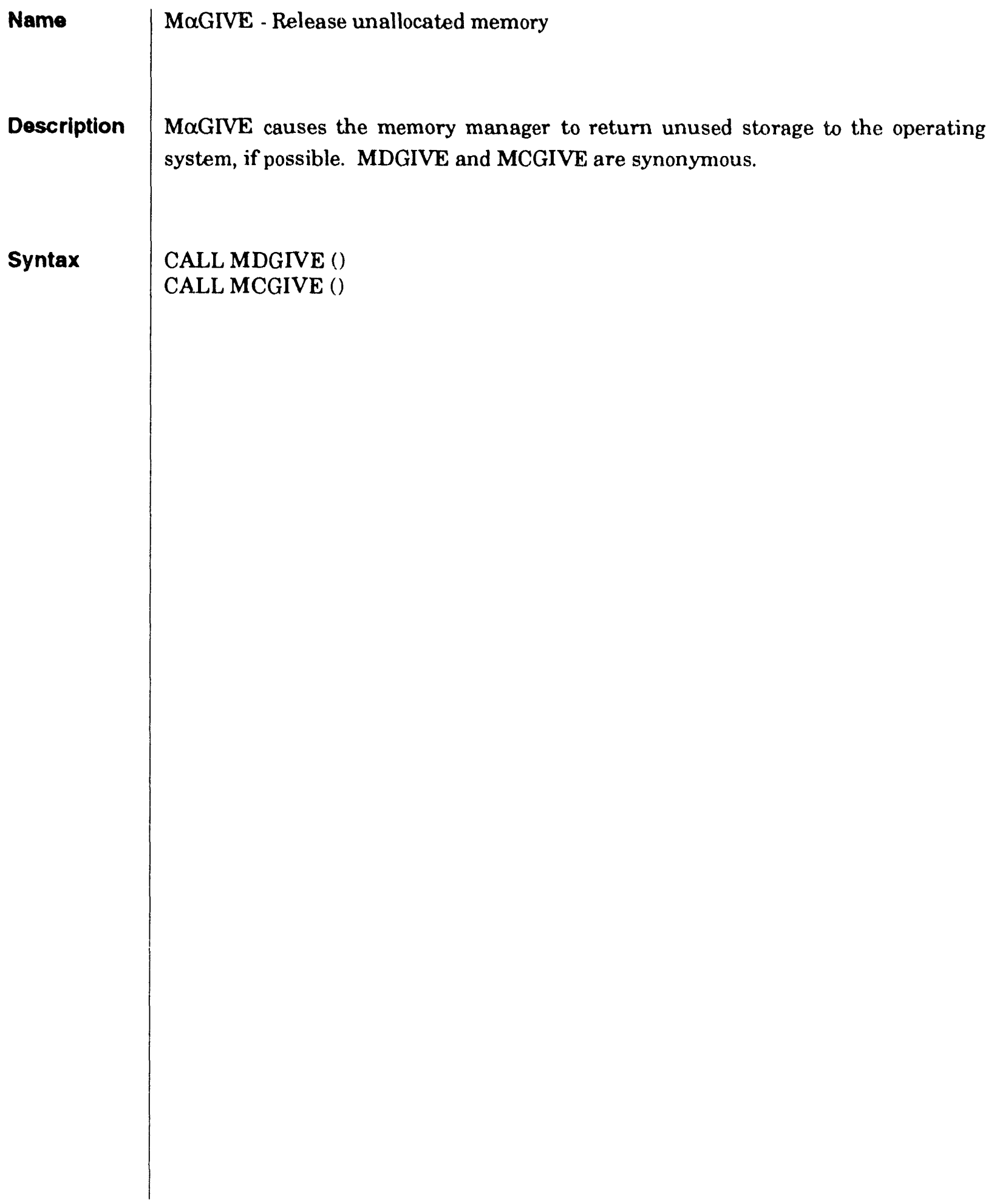




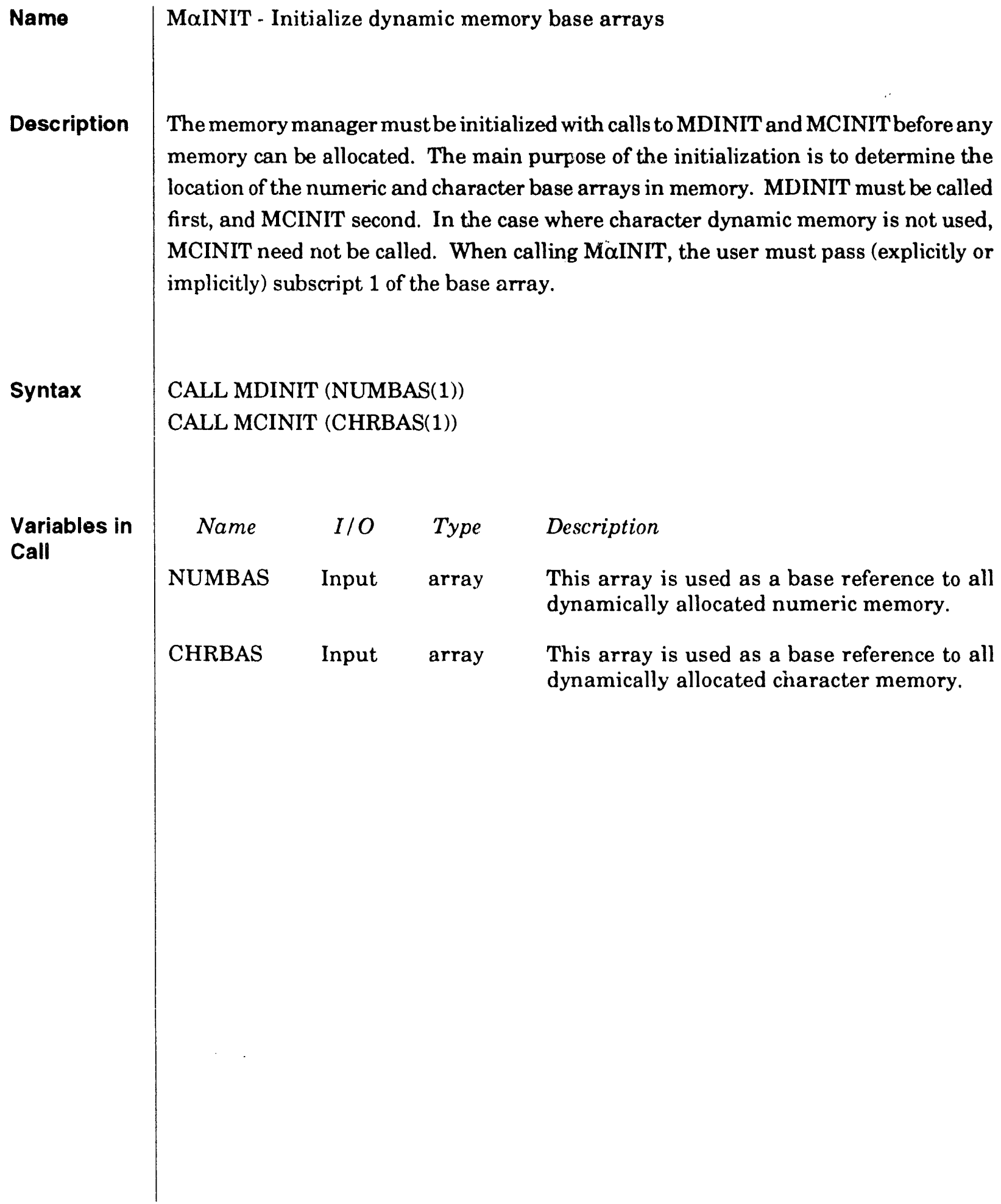




\section{Description}

Name of the new dynamic array. The memory manager will add this name to its internal dictionary; each array must have a unique name. The first eight characters beginning with a nonblank are us $\lrcorner \mathrm{d}$ for comparison. This comparison is case-insensitive and embedded blanks are significant.

Index to storage allocated to this dynamic array relative to the base array. The index for numeric data is to be used with the numeric array supplied to MDIN!T, and character data is to be used with the character array supplied to MCINIT.

Length to be reserved for the new array. Any nonnegative number is acceptable. A zero length does not cause any storage to be allocated and returns an index equal to one. The value of NEWLEN is in terms of numeric storage units for numeric data and character storage units for character data. 
Name

Description

\section{Syntax}

Variables in Call
M $\alpha$ STAT - Obtain statistics

M $\alpha$ STAT returns memory manager statistics. M $\alpha$ STAT provides a method for error checking, and thus should be used after other calls to the memory manager to assure no errors have occurred.

CALLMDSTAT(MNERRS, MNUSED)

CALL MCSTAT (MNERRS, MNUSED)

\begin{tabular}{|c|c|c|c|}
\hline Name & $I / O$ & Type & Description \\
\hline MNERRS & Output & integer & $\begin{array}{l}\text { Total number of errors detected by the memory } \\
\text { manager during the current execution. }\end{array}$ \\
\hline MNUSED & Output & integer & $\begin{array}{l}\text { Total number of storage units that are cur- } \\
\text { rently allocated to dynamic arrays. MDSTAT } \\
\text { returns the numeric storage in numeric stor- } \\
\text { age units, and MCSTAT returns the charac- } \\
\text { ter storage in character storage units. If any } \\
\text { storage has been requested in the deferred } \\
\text { mode and not yet allocated by the memory } \\
\text { manager(Section } 4.3 .8 \text { ), this storage is counted } \\
\text { as though it were actually allocated. }\end{array}$ \\
\hline
\end{tabular}




\subsubsection{Advanced Memory Management Routines}

The advanced routines are supplied to give added capability to the user who is interested in more sophisticated manipulation of memory. These routines are never necessary, but may be very desirable. 
M $\alpha$ COMP causes fragmented memory to be consolidated. Note that this may cause array storage locations to change. It is important to realize that all indexes must be recalculated by calling M $\alpha$ FIND after a compress operation. A call to M $\alpha$ COMP prior to $M \alpha G I V E$ will result in the return of the maximum memory to the system. MDCOMP and MCCOMP are synonymous.

Syntax

CALL MDCOMP ()

CALL MCCOMP () 
M $\alpha E F I X$

Subroutine

\begin{tabular}{|c|c|c|c|c|}
\hline Name & \multicolumn{4}{|c|}{ M $\dot{\alpha} E F I X$ - Modify error count } \\
\hline Description & \multicolumn{4}{|c|}{$\begin{array}{l}\text { M } \alpha \text { EFLX allows the error count for a particular error flag to be set to a specified value. } \\
\text { MDEFEX and MCEFIX are synonymous. }\end{array}$} \\
\hline Syntax & \multicolumn{4}{|c|}{$\begin{array}{l}\text { CALL MDEFIX (IFLAG, NERRS) } \\
\text { CALL MCEFIX (IFLAG, NERRS) }\end{array}$} \\
\hline \multirow{3}{*}{$\begin{array}{l}\text { Variables in } \\
\text { Call }\end{array}$} & Name & $I / O$ & Type & Description \\
\hline & IFLAG & Input & integer & $\begin{array}{l}\text { IFLAG specifies the number of the error flag } \\
\text { that will be set. See M } \alpha \text { EROR for a descrip- } \\
\text { tion of these flags. }\end{array}$ \\
\hline & NERRS & Input & integer & NERRS is the new value for the error count. \\
\hline
\end{tabular}




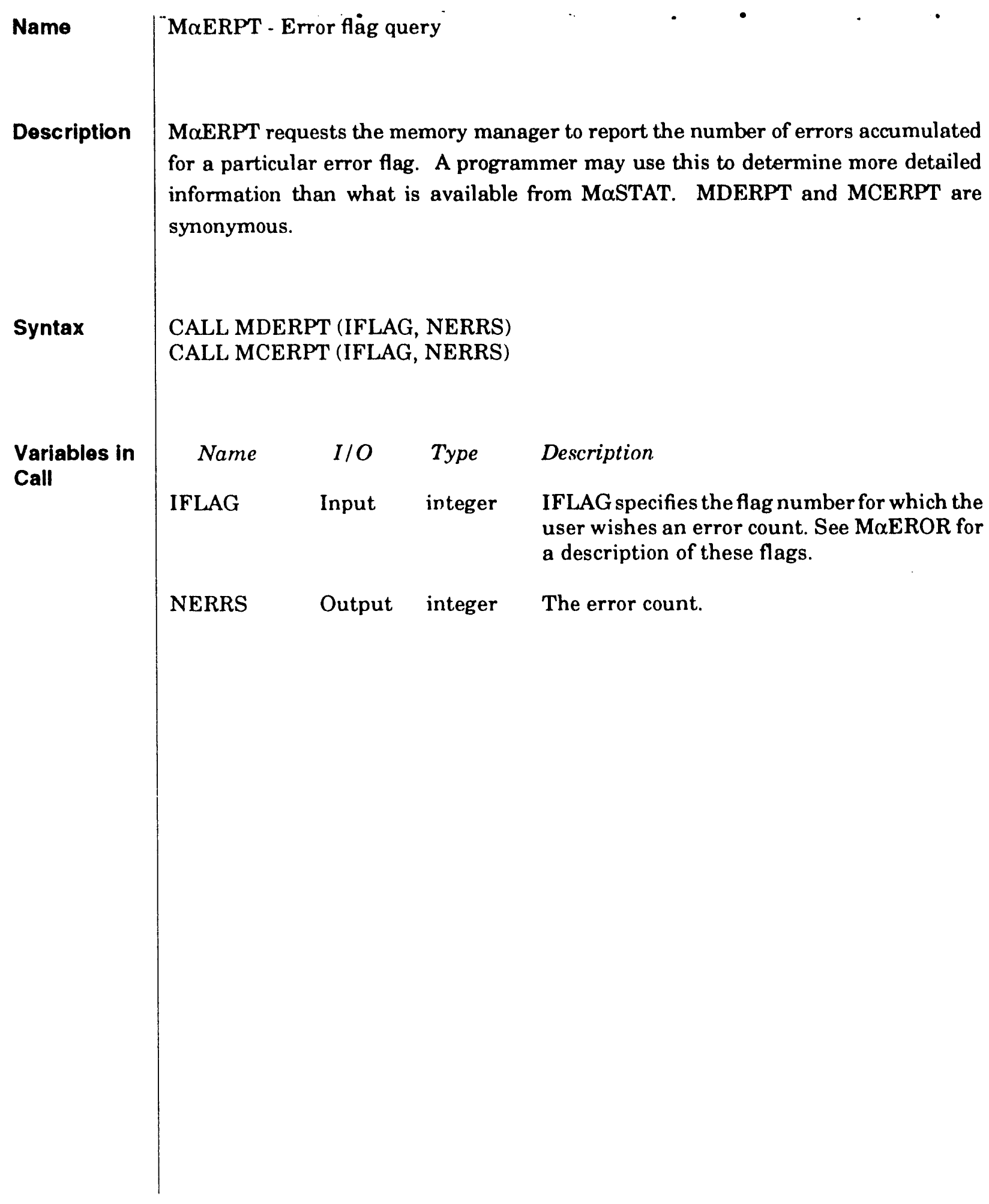




\begin{tabular}{|c|c|}
\hline Name & M $\alpha$ EXEC - Execute \\
\hline Description & $\begin{array}{l}\text { M } \alpha E X E C \text { causes all } \\
\text { to the operating syste } \\
\text { MDEXEC and MCEX } \\
\text { After returning from } \\
\text { updated. }\end{array}$ \\
\hline Syntax & $\begin{array}{l}\text { CALL MDEXEC () } \\
\text { CALL MCEXEC () }\end{array}$ \\
\hline Seo Also & M $\alpha$ WAIT \\
\hline
\end{tabular}




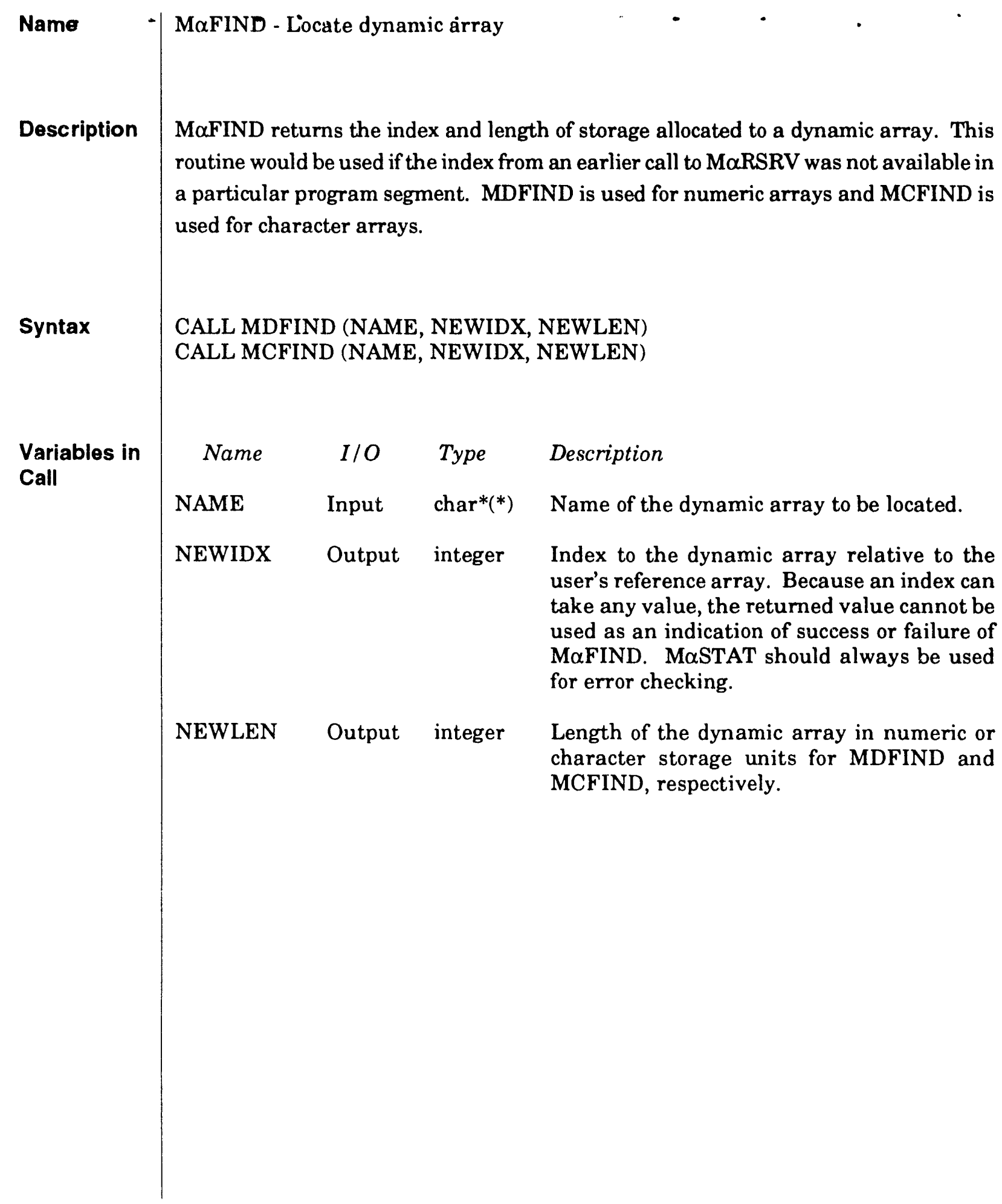




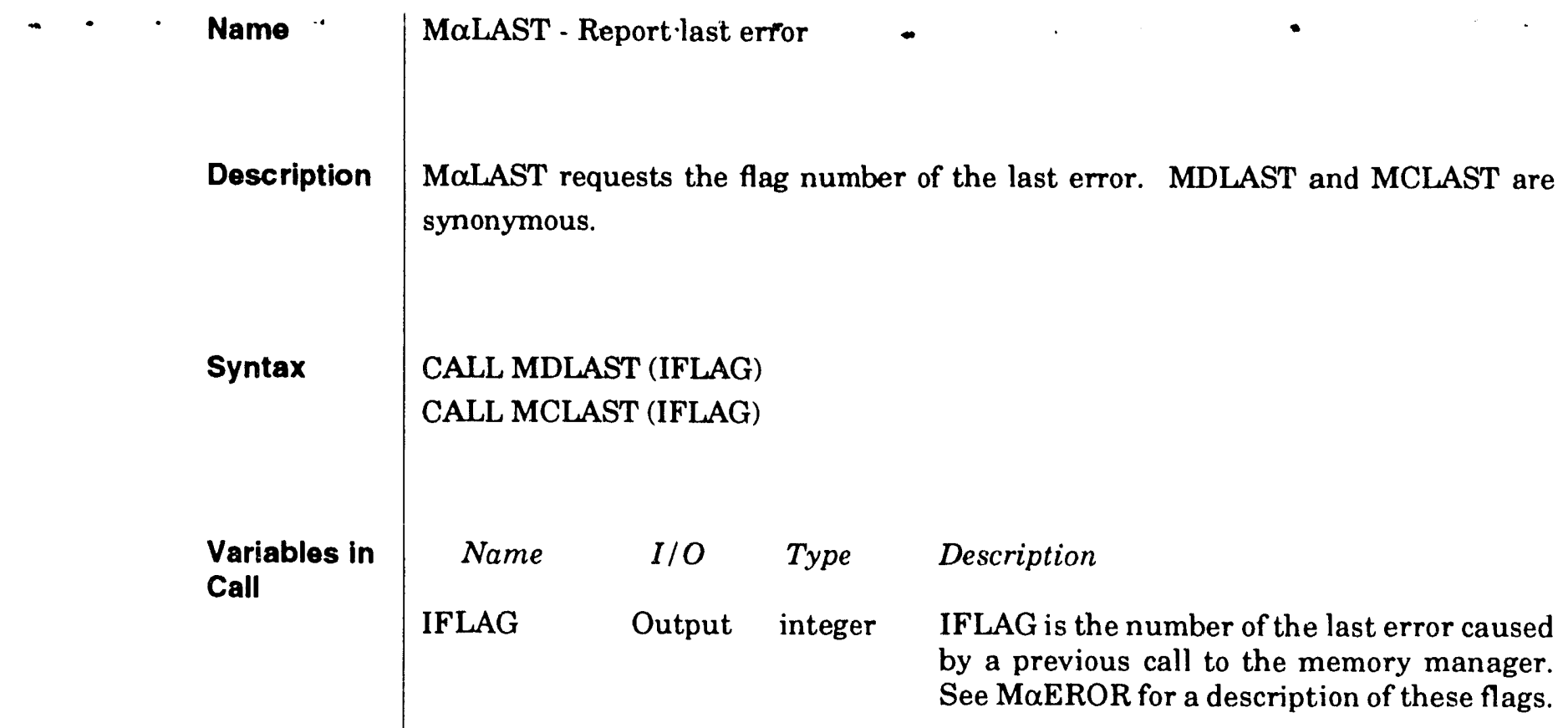




\begin{tabular}{|c|c|c|c|c|}
\hline Name & \multicolumn{4}{|c|}{ M $\alpha$ LONG - Adjust dynamic array length } \\
\hline Description & \multicolumn{4}{|c|}{$\begin{array}{l}\text { M } \alpha \text { LONG changes the length of a dynamic array. The memory manager will relocate } \\
\text { the array and move its data if storage cannot be extended at the array's current location. } \\
\text { The user should assume that M } \alpha \text { LONG invalidates the previous index to this array if } \\
\text { the array is extended. MDLONG is used for numeric arrays and MCLONG is used for } \\
\text { character arrays. }\end{array}$} \\
\hline Syntax & \multicolumn{4}{|c|}{$\begin{array}{l}\text { CALL MDLONG (NAME, NEWIDX, NEWLEN) } \\
\text { CALL MCLONG (NAME, NEWIDX, NEWLEN) }\end{array}$} \\
\hline $\begin{array}{l}\text { Variables in } \\
\text { Call }\end{array}$ & $\begin{array}{l}\text { Name } \\
\text { NAME } \\
\text { NEWIDX } \\
\text { NEWLEN }\end{array}$ & $\begin{array}{l}\text { I/O } \\
\text { Input } \\
\text { Output } \\
\text { Input }\end{array}$ & $\begin{array}{l}\text { Type } \\
\text { char*(*) } \\
\text { integer } \\
\text { integer }\end{array}$ & $\begin{array}{l}\text { Description } \\
\text { Name of the dynamic array to be shortened. } \\
\text { New index to the dynamic array. } \\
\text { New length for the dynamic array in numeric } \\
\text { storage units for MDLONG and in character } \\
\text { storage units for MCLONG. }\end{array}$ \\
\hline
\end{tabular}


Name

Description

Syntax

Variables in Call
M $\alpha$ MEMS - Report storage information

M $\alpha$ MEMS reports numeric or character storage information. This information may be useful for planning storage strategies during code development and for flow control during code execution.

CALL MDMEMS (NSUA, NSUD, NSUV, NSULV)

CALL MCMEMS (NSUA, NSUD, NSUV, NSULV)

$\begin{array}{cccl}\text { Name } & \text { I/O } & \text { Type } & \begin{array}{l}\text { Description } \\ \text { NSUA }\end{array} \\ \text { NSUD } & \text { Output } & \text { integer } & \begin{array}{l}\text { NSUA is the number of numeric/character } \\ \text { storage units currently allocated and not de- } \\ \text { ferred. }\end{array} \\ \text { NSUV } & \text { Output } & \text { integer } & \begin{array}{l}\text { NSUD is the number of numeric/character } \\ \text { storage units currently deferred. }\end{array} \\ & & \begin{array}{l}\text { NSUV is the amount of void space in numeric } \\ \text { or character storage units. Note that } \\ \text { MDMEMS and MCMEMS may be reporting } \\ \text { the same space for NSUV, but in different } \\ \text { units. }\end{array}\end{array}$

NSULV Output integer NSULV is the size of the largest void space in numeric or character storage units. Note that MDMEMS and MCMEMS may be reporting the same space for NSULV, but in different units. 


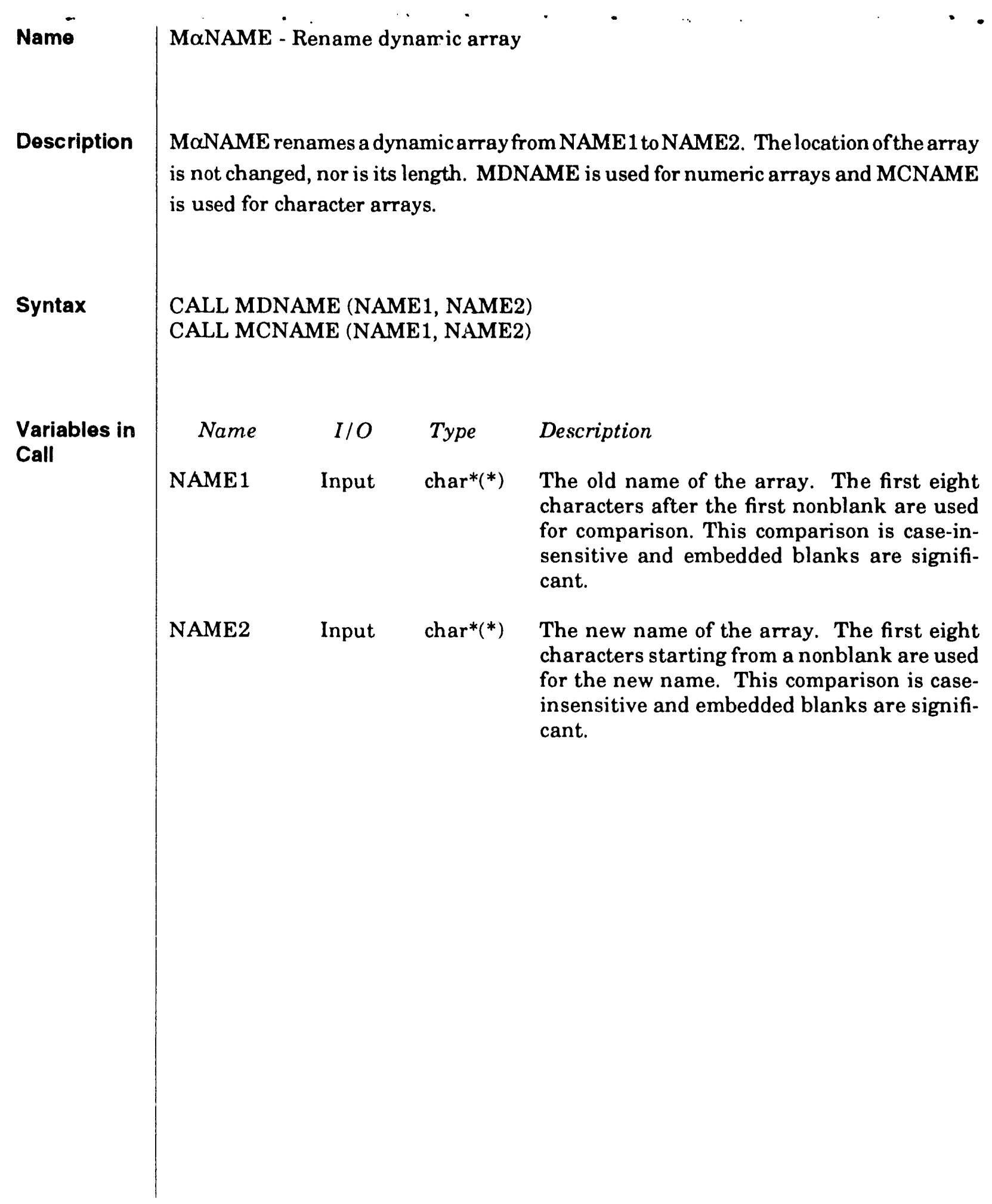


Name

Description

Syntax

See Also
MaWAIT - Enable deferred memory mode

M $\alpha$ WAIT enables the deferred memory mode of the memory manager. In this mode, any requests for additional memory with M $\alpha$ RSRV are satisfied only if a system call is not required. If a system call is required, the request for memory is deferred and will be satisfied when the deferred mode is cancelled by calling M $\alpha E X E C$ or a call to M $\alpha$ LONG requires a system call for memory for an existing array. MDWAIT and MCWAIT are synonymous.

Because the deferred mode may not actually provide array space at the time a call to M $\alpha R S R V$ is made, the base array pointer returned by M $\alpha$ RSRV may not be valid. In fact, for a deferred request, an invalid index is intentionally returned so that the requested array space cannot be erroneously used. When the deferred memory requests are eventually satisfied (by calling M $\alpha E X E C$ ), the indexes are automatically, asynchronously updated by the memory manager. Thus, upon return from M $\alpha E X E C$ the indexes used in MaRSRV will have a valid value.

The deferred mode is provided as a means of reducing the sometimes time-consuming calls to the operating system for new memory. A similar efficiency could be realized by judicious use of M $\alpha \mathrm{GET}$, but the deferred mode relieves the user of the burden of adding all memory requests before calling MoRSRV. The deferred mode is a sophisticated capability and should not be enabled if the user does not understand its implications.

The deferred mode must be used as follows:

1. The deferred mode begins with a call to M $\alpha$ WAIT.

2. Except for M $\alpha$ PRNT, all memory manager calls are permissible in the deferred mode.

3. Indexes returned by M $\alpha R S R V, M \alpha F I N D$, and M $\alpha$ LONG may not be assigned to other variables while the deferred mode is in effect.

4. Dynamic memory may not be accessed while the deferred mode is in effect.

5. The deferred mode is cancelled by calling M $\alpha$ EXEC.

CALL MDWAIT ()

CALL MCWAIT ()

M $\alpha$ EXEC 
CAMSUPES_LIB

Memory Management Debugging

\subsubsection{Memory Management Debugging Routines}

The routines in this section are designed to aid the programmer during development of a program, and probably would not be used during execution of a mature program, except as a response to a memory management error. 


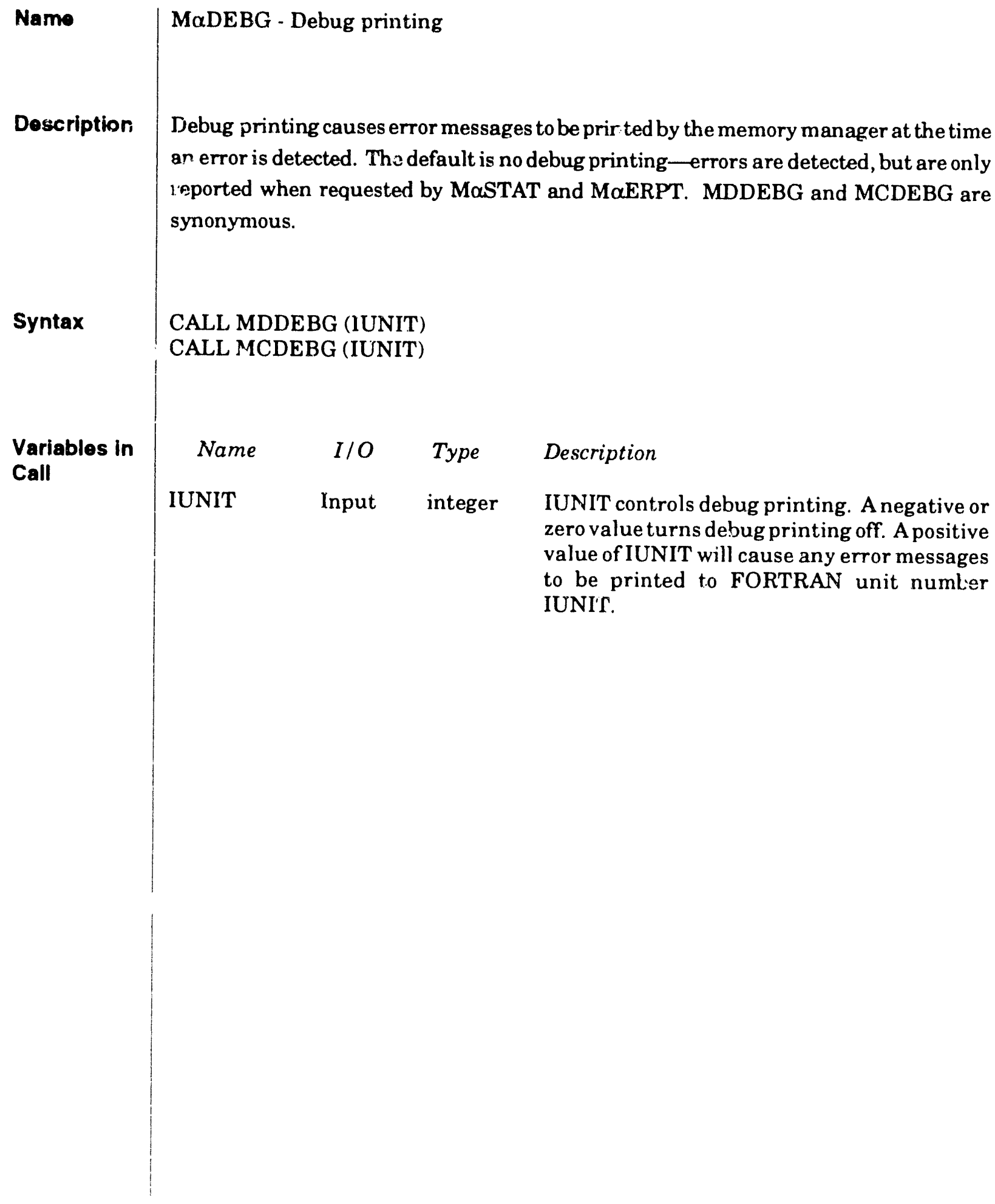




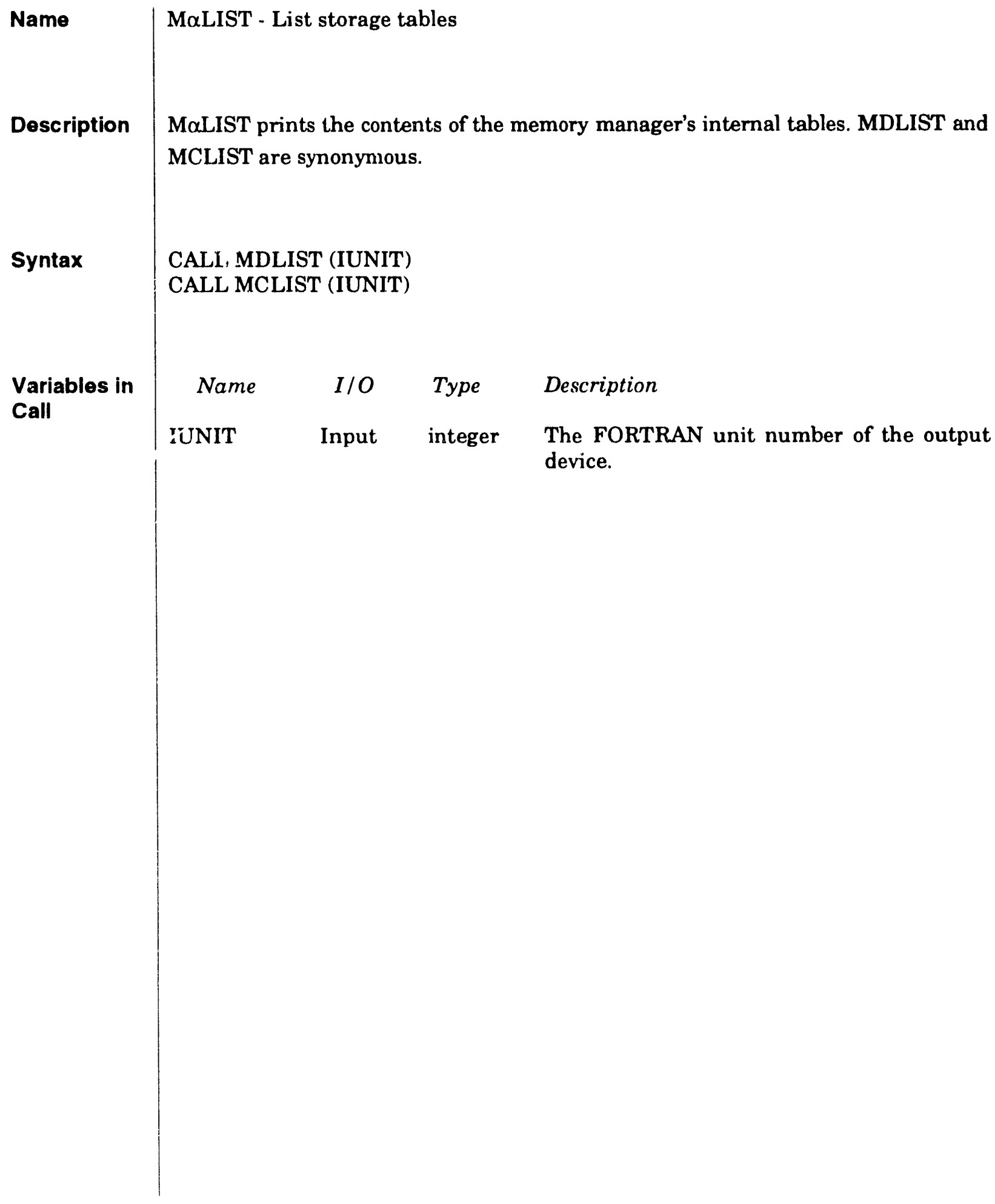




\begin{tabular}{|c|c|c|c|c|}
\hline Name & \multicolumn{4}{|c|}{ M $\alpha$ PRNT - Print dynamic array } \\
\hline Description & \multicolumn{4}{|c|}{$\begin{array}{l}\text { M } \alpha \text { PRNT prints the contents of an individual numeric and character array, respec- } \\
\text { tively. }\end{array}$} \\
\hline Syntax & \multicolumn{4}{|c|}{$\begin{array}{l}\text { CALL MDPRNT (NAME, IUNIT, TYPE) } \\
\text { CALL MCPRNT (NAME, IUNIT, NGRUP) }\end{array}$} \\
\hline \multirow[t]{5}{*}{$\begin{array}{l}\text { Variables in } \\
\text { Call }\end{array}$} & Name & $I / O$ & Type & Description \\
\hline & NAME & Input & $\operatorname{char}{ }^{*}(*)$ & The name of the array to be printed. \\
\hline & IUNIT & Input & integer & $\begin{array}{l}\text { The FORTRAN unit number of the output } \\
\text { device. }\end{array}$ \\
\hline & TYPE & Input & $\operatorname{char} *(*)$ & $\begin{array}{l}\text { TYPE indicates the date type of the data to be } \\
\text { printed; "R" for REAL, or "I" for INTEGER. } \\
\text { Note that this is not necessarily the declared } \\
\text { type of the base array. }\end{array}$ \\
\hline & NGRUP & Input & integer & $\begin{array}{l}\text { NGRUP controls the number of characters } \\
\text { that are grouped together without interven- } \\
\text { ing spaces. Since the storage is declared as a } \\
\text { CHARACTER* } 1 \text { array, NGRUP allows the } \\
\text { user to format the output consistent with } \\
\text { longer character strings. }\end{array}$ \\
\hline
\end{tabular}


CAMSUPES_LIB

Memory Management Debugging 


\section{Graphics Libraries: \\ PLT_LIB and HJIPLOT_LIB}

PLT_LIB is a general device-independent plotting package that uses SVDI (Sandia Virtual Device Interface) plot routines that perform basic graphic operations. PLT_LIB is described in Sections 10.1 and 10.2 .

HJIPLOT_LIB provides alternative mid-level plotting routines that also use SVDI plot primitives. HJIPLOT_LIB is described in Sections 10.3 and 10.4 .

The Sandia Virtual Device Interface (SVDI) is a library of graphics primitives designed to support graphics packages at Sandia National Laboratories. All routines with names that begin with VD are in the SVDI package. SVDI is described in Chapter 11.

\subsection{General Guidelines on the Use of PLT_LIB Routines}

\subsubsection{Sponsorship and Version}
Authors:
C. B. Selleck and P. M. Van Gulick
Sponsor:
A. P. Gilkey
Version / Date:
C-2.00VV/March 1993
Language:
FORTRAN 77

\subsubsection{Overview of PLT_LIB}

PLT_LIB is a collection of FORTRAN-callable routines that perform a variety of graphics tasks. PLT_LIB is divided into a number of modules. Each module contains subroutines related to the specific purpose of the medule. The current modules are:

- Plot initialization and control

- Routines to set various PLT_LIB parameters

- Hardware and software text routines

- Two-dimensional graphing routines

- Device coordinate plotting primitives

- Two-dimensional and three-dimensional mapping routines that map from user to device space with perspective and orthogonal projections

- Clipping of two-dimensional and three-dimensional points, lines, and polyguns

- Color palette routines

The PLT routines use three coordinate systems: the graph coordinate system, the eye coordinate system, and the device coordinate system. The graph coordinate system is the two- or three-dimensional right-hand system in which the user has defined the model to be plotted. For example, the mesh from 
PLT_LIB

General Guidelines

the CAMDAT data base would be in graph coordinates. The eye coordinate system defines how the graph coordinate system is viewed and is a right-hand coordinate system. The eye is at the origin of this system. The device coordinate system is the two-dimensional system that corresponds to the display device. The device coordinate system has its origin $(0.0,0.0)$ at the lower left corner of the device and goes from 0.0 to $X D M A X$ in the $\mathrm{x}$ direction and YDMAX in the y direction. XDMAX and YDMAX are dependent on the aspect ratio requested. The aspect ratio is the ratio of the $\mathrm{x}$ dimension to the $\mathrm{y}$ dimension, and the larger of these two must be 1.0 .

Initializing und Controlling PLT_LIB. The standard method for initializing PLT_LIB is to call the PLTINT routine. PLTINT sets an aspect ratio of 1.33 , which causes the device coordinate system to range from 0 to 1 in the $x$ direction and 0 to 0.75 in the $y$ direction. If the user wishes to set another aspect ratio, the routine PLTSTA may be used.

Terminal Input / Output. Before doing any terminal I/O, the user must call PLTFLU. This routine flushes the buffers, which ensures that the drawing on the screen is up-to-date and puts the terminal in alpha mode so the $\mathrm{I} / \mathrm{O}$ will go to the right place.

Interrupting Plotting. Several routines in PLT_LIB let a user interrupt plotting by typing CONTROL_C under VMS (i.e., pressing and holding the Control key and pressing the $\mathrm{C}$ key). The user may wish to use this capability of interrupting a plot, especially a long plot, in interactive plotting codes. The routines that normally take a long time to plot (e.g., axis labeling) have this capability. Also, the programmer of an interactive code can use PLTCANCEL, a logical function in PLT_LIB, to check whether the interrupt character was entered. A simplified example follows (an interactive program would also have calls to PLTCANCEL with false arguments at lower levels.

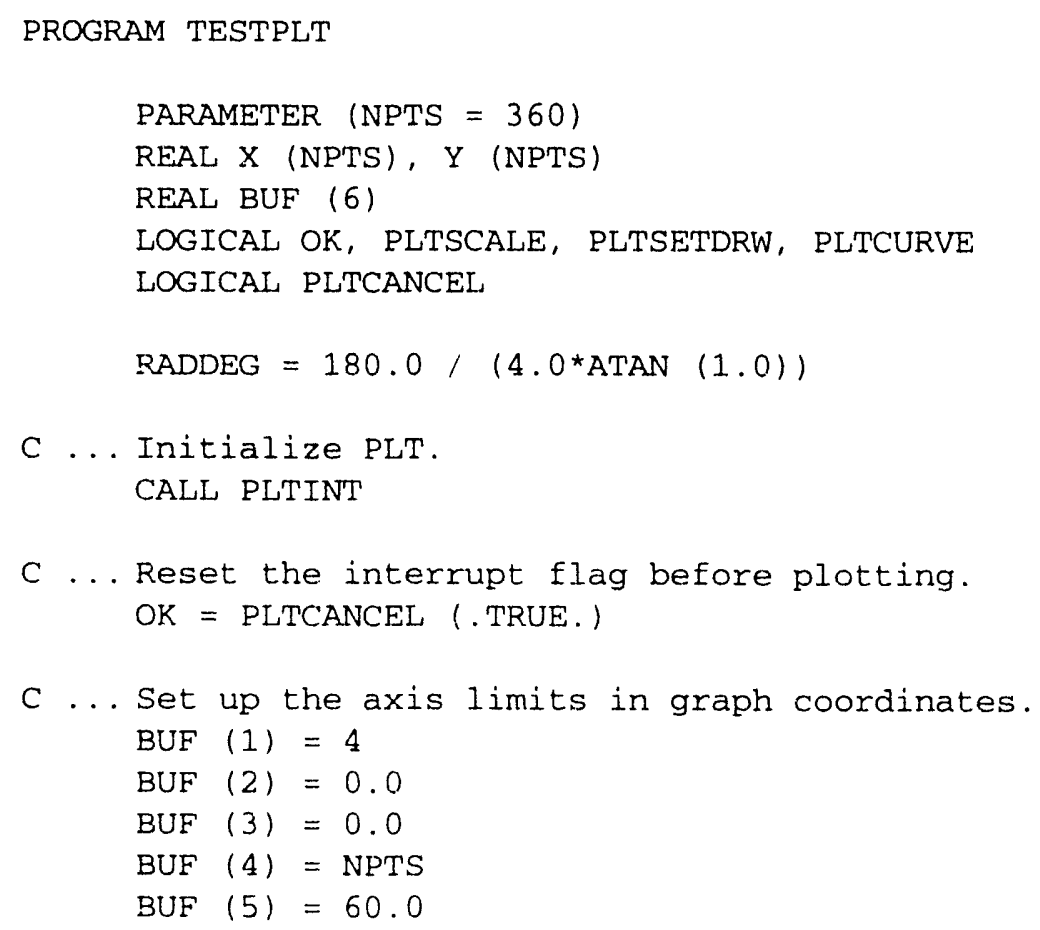




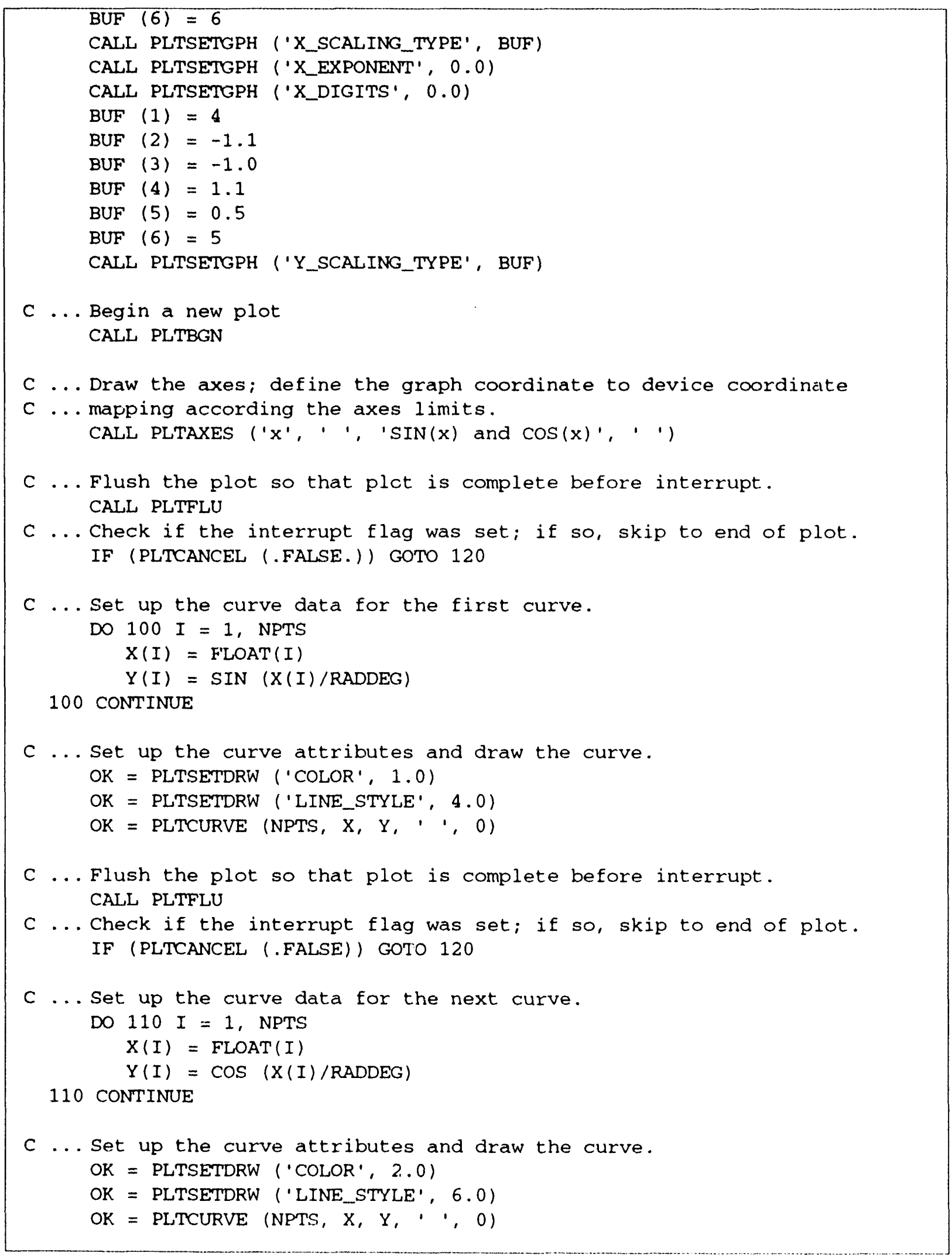


120 CONTINUE

C ... Terminate PLT.

CALL PLTEND

END

Terminating PLT_LIB. It is essential that the last PLT_LIB call be to PLT_END so that the output file is closed properly on batch devices.

Setting PLT_LIB Parameters. The PLTSETx routines (PLTSETGPH, etc.) are used to change the behavior of a particular routine and to change the basic graphics parameters, such as foreground color or line style. For instance, the routine PLTAXES draws a set of axes. All of the options associated with doing this, such as axis type, grid lines, etc., default to certain values. These values can be changed with the routine PLTSETGPH (set graph parameter). All subsequent calls to PLTAXES will use the new values. As another example, the routine PLTSETDRW (set basic graphics parameters) can be used to change the foreground color used when doing basic graphics primitives such as drawing lines.

Text Routines. Two types of characters are used by the text routines: hardware and software. The advantage of hardware characters is that they are displayed faster than software characters; however, hardware characters can be device dependent and so may not be readable on some devices. Also, some special escape symbols (see the list in Section 10.2.3) may not be available with hardware characters; in this case, the software characters are automatically displayed. The character type (i.e., hardware or software) and other text attributes are set in the routine PLTSETTXT.

Device Coordinate Plotting. The device coordinate plotting routines are the easiest way to plot and are helpful when a graph coordinate system is not defined (e.g., to outline the device plot window).

Two-Dimensional Graphing. The two-dimensional graphing routines are a straightforward means to plot a two-dimensional model. The user defines the world coordinate system be setting the viewing window with PLTSETGPH and then calling PLTAXES or PLTSCALE. A clipping window can also be defined (it defaults to the viewing window).

Mapping and Transformations. The mapping routines map from three dimensions to two dimensions and from two dimensions to two dimensions. These including modelling transformations (translate, rotate, and scale), viewing transformations (to locate the observer), projection transformations ( to do perspective or orthogonal projections), and viewport transformations (to define a rectangular portion of the device to draw on). Two-dimensional models that do not require these transformations can be plotted with the more efficient two-dimensional graphing routines.

In addition to the coordinate systems, a viewport is used. The viewport is a rectangular area of the device that defines where to draw the output. 
There are four types of transformation routines:

- Modelling - transforms the coordinate systems of objects (routines MPROTA, MPSCAL, and MPTRAN)

- Viewing - specifies a viewing position (routines MPPOLA and MPLOOK)

- Projection - transforms the eye coordinate system to device coordinates (routines MPPERS, MPWIND, MPORT2, and MPORT3)

- Viewport - defines a rectangular area of the device in which to display the results (routine MPVIEW)

There are drawing routines in addition to the transformation routines. The drawing routines map two- or three-dimensional points, vectors, or polygons to the device coordinates and then draw them on the device.

In a typical application, the user would call the mapping routines in the following order: projection, then viewing, and then modelling. That is, the user first defines the projection and the location of the eye, and after that, manipulates the object with the modelling routines. This order is not mandatory, however. The user can call the routines in any order provided the projection and viewing routines are called before the drawing routines.

All device coordinates are obtained as described below. The word "points" is used in this description, but the description is also true for vectors and polygons.

Two-dimensional:

First, the points are mapped by the model transformation. Then the points are clipped against any clip lines the user has specified with MPCLP2. Next these points are multiplied by the projection transformation. The viewing transformation does not make sense in two dimensions, so do not call a viewing routine in two dimensions. Finally, the points are mapped to the current viewport window and clipped against this same viewport window.

Three-dimensional:

First, the points are mapped by the model transformation. Then the points are clipped against any planes the user has specified with MPCLP3. Next these points are multiplied by the viewing transformation. Then the points are clipped against the near and far clipping planes. They are then multiplied by the projection transformation. Finally, the points are mapped and clipped to the current viewport.

Manipulating Output. The entire plot can be scaled, translated, and rotated. In addition, the user can specify a viewport in which to plot the data. The description of routine PLTSETMAP explains the options available here. Note that the entire plot (including the legend) is manipulated, not just the model being plotted. By default, all output is clipped to the full screen as defined by initialization calls, and no scaling, translation, or rotation is done. If the viewport aspect ratio is not the same as the aspect ratio specified in initializing PLT_LIB, the plot will be distorted. 


\subsubsection{Linking}

The PLT_LIB routines are in an object library in the [LIBRARIES] subdirectory (see Section 2.10, "Software Libraries"). PLT_LIB must be linked with the DVDI_LIB graphics interface. To link a code with PLT_LIB, type (the logical names of the libraries are assigned by the library sponsor):

\section{\$SELECT NULL}

\section{\$LINK code_name, ... , SHRPLT/LIB, CDRLIB/LIB/INCL = CDRABC}

A FLINT library is available for checking interfaces. It is in

CAMCON\$ROOT: [LIBRARIES.DVDI_LIB] PLT_LIB.LBT.

\subsubsection{Summary of Routines}

The PLT_LIB routines are described in detail in the next section. The following is a summary of the PLT_LIB routines.

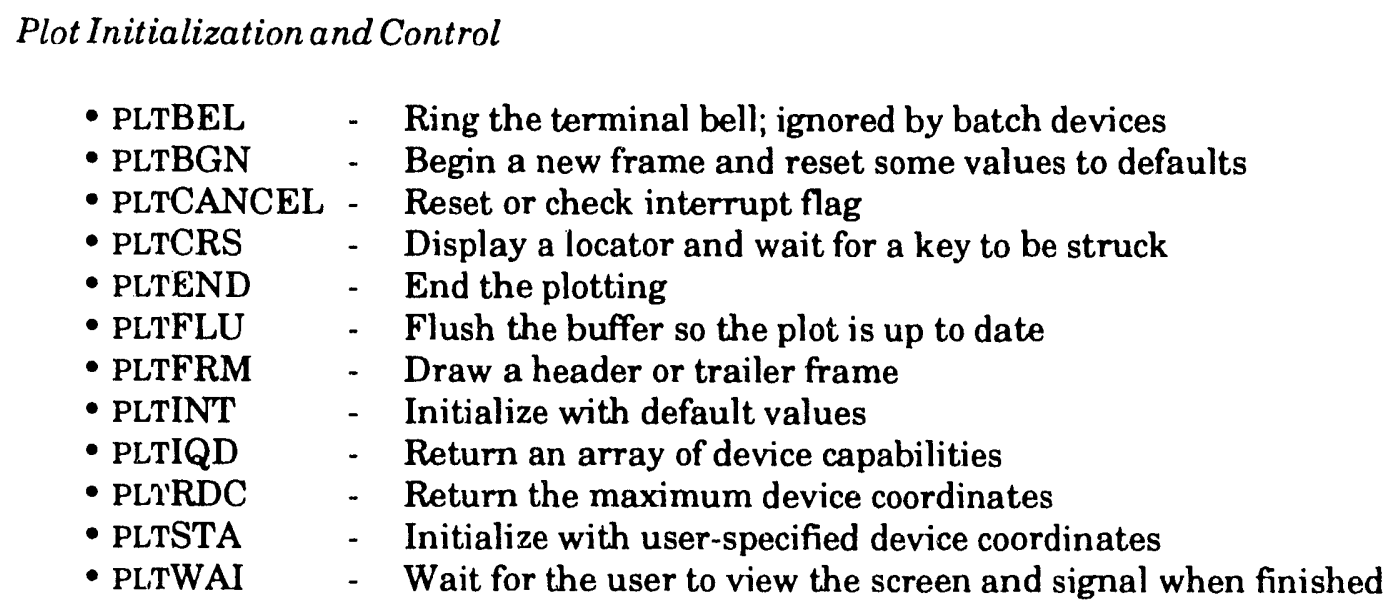

- PLTBEL - Ring the terminal bell; ignored by batch devices

- PLTBGN - Begin a new frame and reset some values to defaults

- PLTCANCEL - Reset or check interrupt flag

- PLTCRS - Display a locator and wait for a key to be struck

- PLTEND - End the plotting

- PLTFLU - Flush the buffer so the plot is up to date

- PLTFRM - Draw a header or trailer frame

- PLTINT - Initialize with default values

- PLTIQD - Return an array of device capabilities

- PLIRDC - Return the maximum device coordinates

- PLTSTA - Initialize with user-specified device coordinates

- PLTWAI - Wait for the user to view the screen and signal when finished

Routines to Set PLT_LIB Parameters

- PLTSETCOL - Set color parameters

- PLTGETCOL - Get color parameters

- PLTSETDRW - Set basic graphics parameters

- PLTGETDRW - Get basic graphics parameters

- PLTSAVDRW - Save basic graphics parameters

- PLTRESDRW - Restore basic graphics parameters

- PLTSETGPH - Set graph parameters

- PLTGETGPH - Get graph parameters

- PLTSAVGPH - Save graph parameters

- PLTRESGPH - Restore graph parameters

- PLTSETMAP - Set global mapping parameters

- PLTGETMAP - Get global mapping parameters

- PLTSAVMAP - Save global mapping parameters

- PLTRESMAP - Restore global mapping parameters

- PLTSETSYM - Set symbol parameters 
- PLTGETSYM - Get symbol parameters

- PLTSAVSYM - Save symbol parameters

- PLTRESSYM - Restore symbol parameters

- PLTSETTXT - Set text parameters

- PLTGETTXT - Get text parameters

- PLTSAVTXT - Save text parameters

- PLTRESTXT - Restore text parameters

Text Routines

- PLTTXTCEN - Plot text centered at the given location

- PLTTXTEND - Return the coordinates of the end of the last text output

- PLTTXTLEN - Return the length of the given character string using the current character size

- PLTTXTNXT - Return the coordinates of the beginning of the next line of text output

- PLTTXTSTR - Plot a character string

Two-Dimensional Graphing Routines

- PLTARROW - Draw an arrow on existing axes

- PLTAXES - Draw a set of axes and define the graph and clipping window

- PLTCIRCLE - Draw a circle on existing axes

- PLTCLIP - Convert and clip a vector within the clipping window

- PLTCURVE - Draw a curve with optional symbols on existing axes

- PLTD2G - Convert from device to graph coordinates

- PLTG2D - Convert from graph to device coordinates

- PLTINI - Return suitable numbers to use in axis scaling, inside the specified interval

- PLTINO - Return suitable numbers to use in axis scaling, outside the specified interval

- PLTPOLY - Draw a polygon on existing axes

- PLTSCALE - Define the graph window to fit curve points

- PLTSYMBOL - Draw a set of text symbols on existing axes

- PLTVECTOR - Draw a set of vectors on existing axes

Device Coordinate Plotting Primitives

- PLTARR - Draw an arrow

- PLTAXS - Draw a linear axis

- PLTDRW - Draw a vector from the current pen position to the specified position

- PLTMOV - Move the pen

- PLTPLY - Draw a filled polygon

- PLTPNT - Plot specified points

- PLTPTM - Plot specified points with masking

- PLTRXY - Return the current position of the pen

- PLTSYMCEN - Plot a character centered at the given locations

- PLTSYMMSK - Plot a symbol at each of the given points with masking

- PLTVCM - Draw vectors with masking

- PLTVCT - Draw vectors

Two-Dimensional and Three-Dimensional Mapping Routines

- MP2PG - Map two-dimensional polygons

- MP2PT - Map two-dimensional points

- MP2VC - Map two-dimensional vectors 


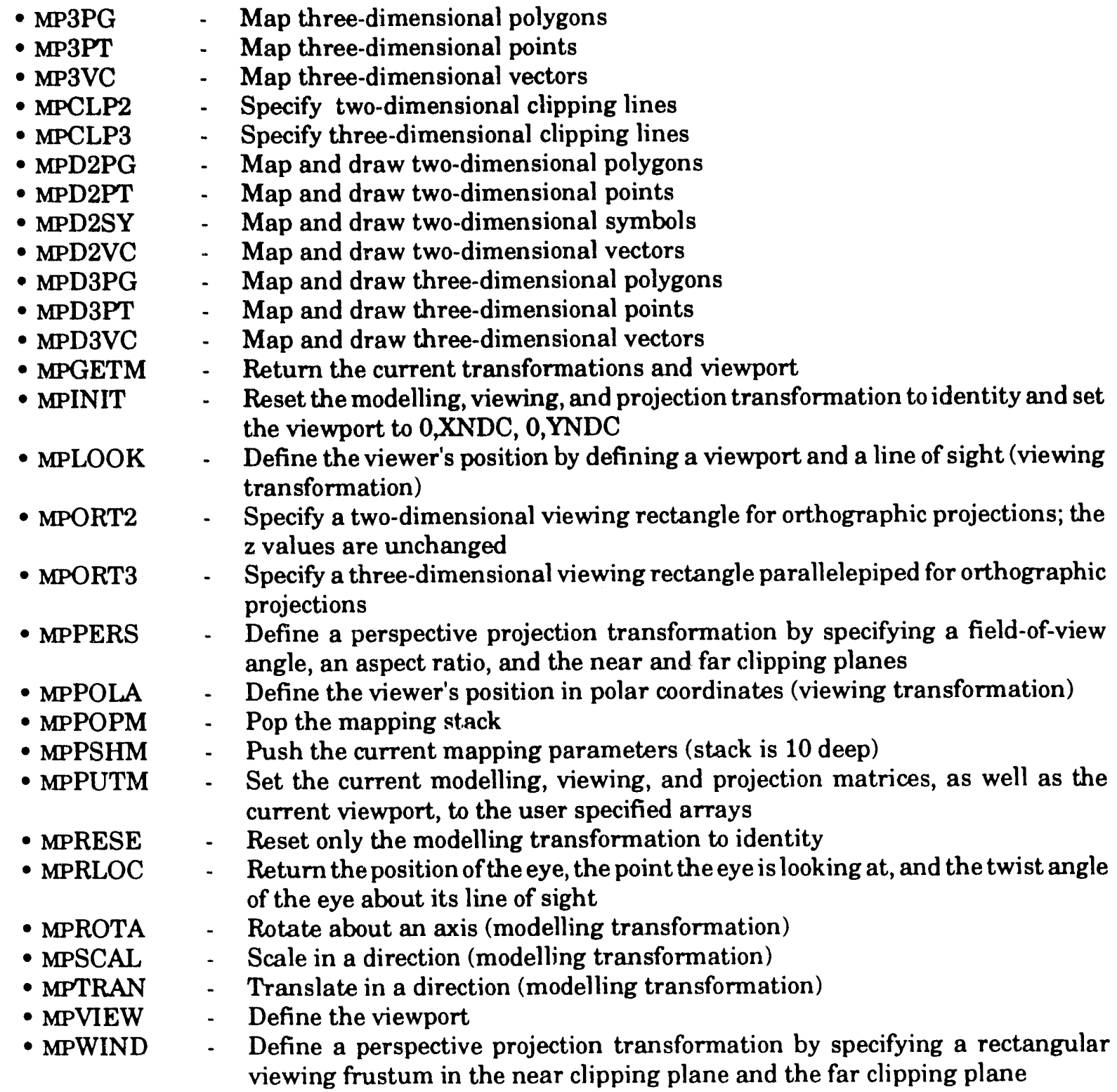

Clipping of Two-Dimensionaland Three-Dimensional Points, Lines, and Polygons

- PLTCG2 - Clip a two-dimensional polygon against a line

- PLTCP2 - Clip two-dimensional points against a line segment

- PLTCP3 - Clip three-dimensional points against a plane

- PLTCV2 - Clip two-dimensional vectors against a line segment

- PLTCV3 - Clip three-dimensional vectors against a plane

- PLTVWG - Clip a polygon against a viewport

- PLTVWP - Clip points against a viewport

- PLTVWV - Clip vectors against a viewport

- PLTZCP - Clip points against two clipping planes

- PLTZCV - Clip vectors against two clipping planes 
- PLTCNM - Return the color name for a given color index

- PLTICL - Return the color index for a given color name

- PLTIQC - Return the red, green, and blue intensity for the given color

- PLTISP - Map a number between 0 and 1 into the spectrum

- PLTPAL - Set the color and intensity of a palette color

- PLTCOL - Set a color to the specified red, green, and blue values

- PLTSPC - Set a range of colors in a specified interval of the spectrum

Obsolete Routines (These routines are still available but have been made obsolete by new routines.)

- PLTSTC - Replaced by PLTSETCOL

- PLTGTC - Replaced by PLTGETCOL

- PLTSTD - Replaced by PLTSETDRW

- PLTGTD - Replaced by PLTGETDRW

- PLTSVD - Replaced by PLTSAVDRW

- PLTRED - Replaced by PLTRESDRW

- PLTSTG - Replaced by PLTSETGPH, PLTSETDRW, and PLTSETSYM

- PitGTG - Replaced by PLTGETGPH, PLTGETDRW, and PLTGETSYM

- PLTSVG - Replaced by PLTSAVGPH, PLTSAVDRW, and PLTSAVSYM

- PLTREG - Replaced by PLTRESGPH, PLTRESDRW, and PLTRESSYM

- PLTSTM - Replaced by PLTSETMAP

- PLTGTM - Replaced by PLTGETMAP

- PLTSVM - Replaced by PLTSAVMAP

- PLTREM - Replaced by PLTRESMAP

- PLTSTT - Replaced by PLTSETTXT

- PLTGTT - Replaced by PLTGETTXT

- PLTSVT - Replaced by PLTSAVTXT

- PLTRET - Replaced by PLTRESTXT

- PLTSTV - Replaced by PLTSETDRW

- PLTGTV - Replaced by PLTGETDRW

- PLTSVV - Replaced by PLTSAVDRW

- PLTREV - Replaced by PLTRESDRW

- PLTSBM - Replaced by PLTSYMMSK

- PLTSYM - Replaced by PLTSYMCEN

- PLTXHE - Replaced by PLTTXTEND

- PLTXHL - Replaced by PLTTXTLEN

- PLTXHN - Replaced by PLTTXTNXT

- PLTXSE - Replaced by PLTTXTEND

- PLTXSL - Replaced by PLTTXTLEN

- PLTXSN - Replaced by PLTTXTNXT

- PLTXTC - Replaced by PLTTXTCEN

- PLTXTH - Replaced by PLTTXTSTR

- PLTXTS - Replaced by PLTTXTSTR

- PLTCUR - Replaced by PLTCURVE

- PLTGPH - Replaced by PLTAXES 
PLT LIB

Description of Routine Calls

\subsection{Description of Routine Calls}

\subsubsection{Plot Initialization and Control}

Detailed descriptions of the routines that initialize and control plotting are given on the following pages. 


\section{PLTBEL}

Subroutine

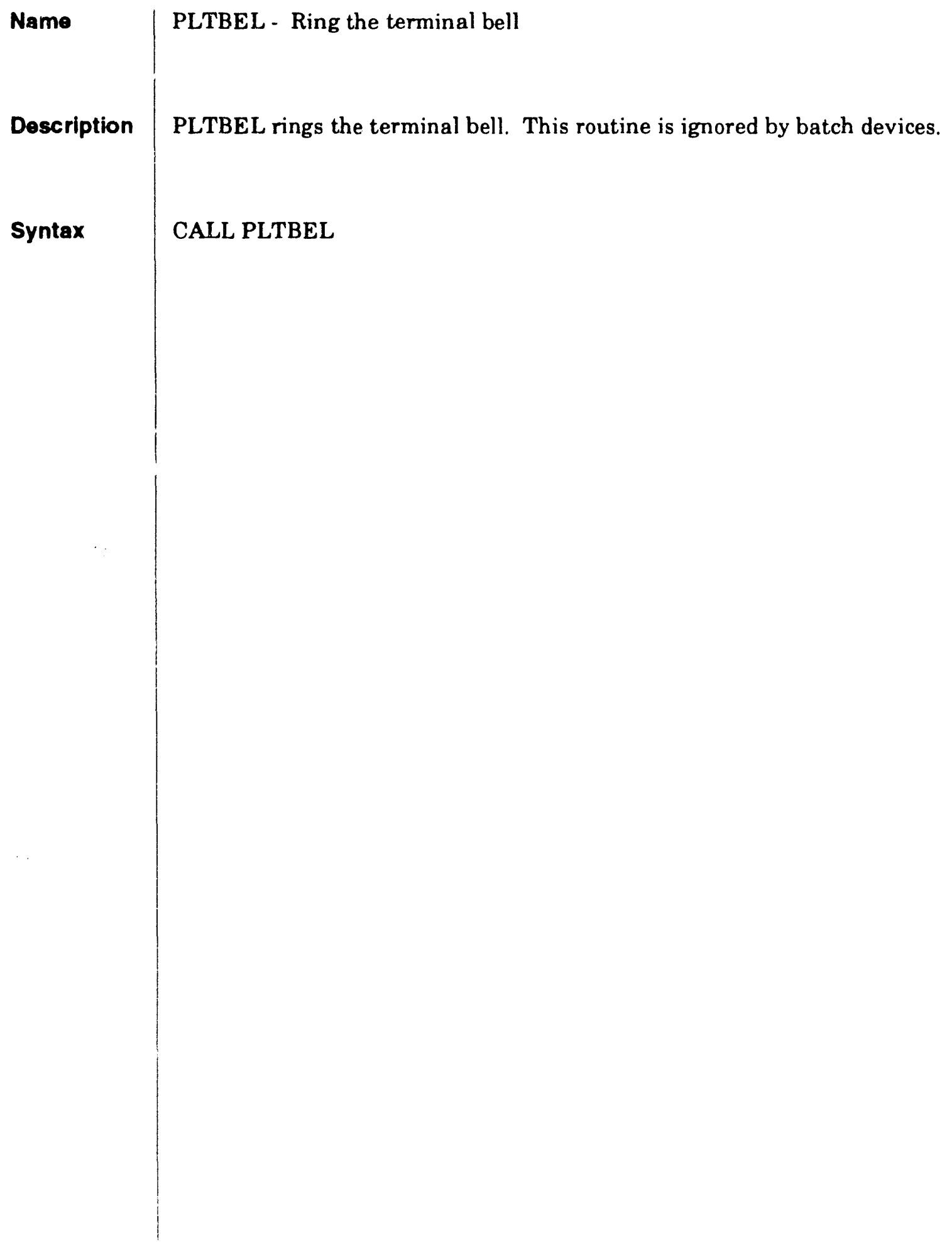




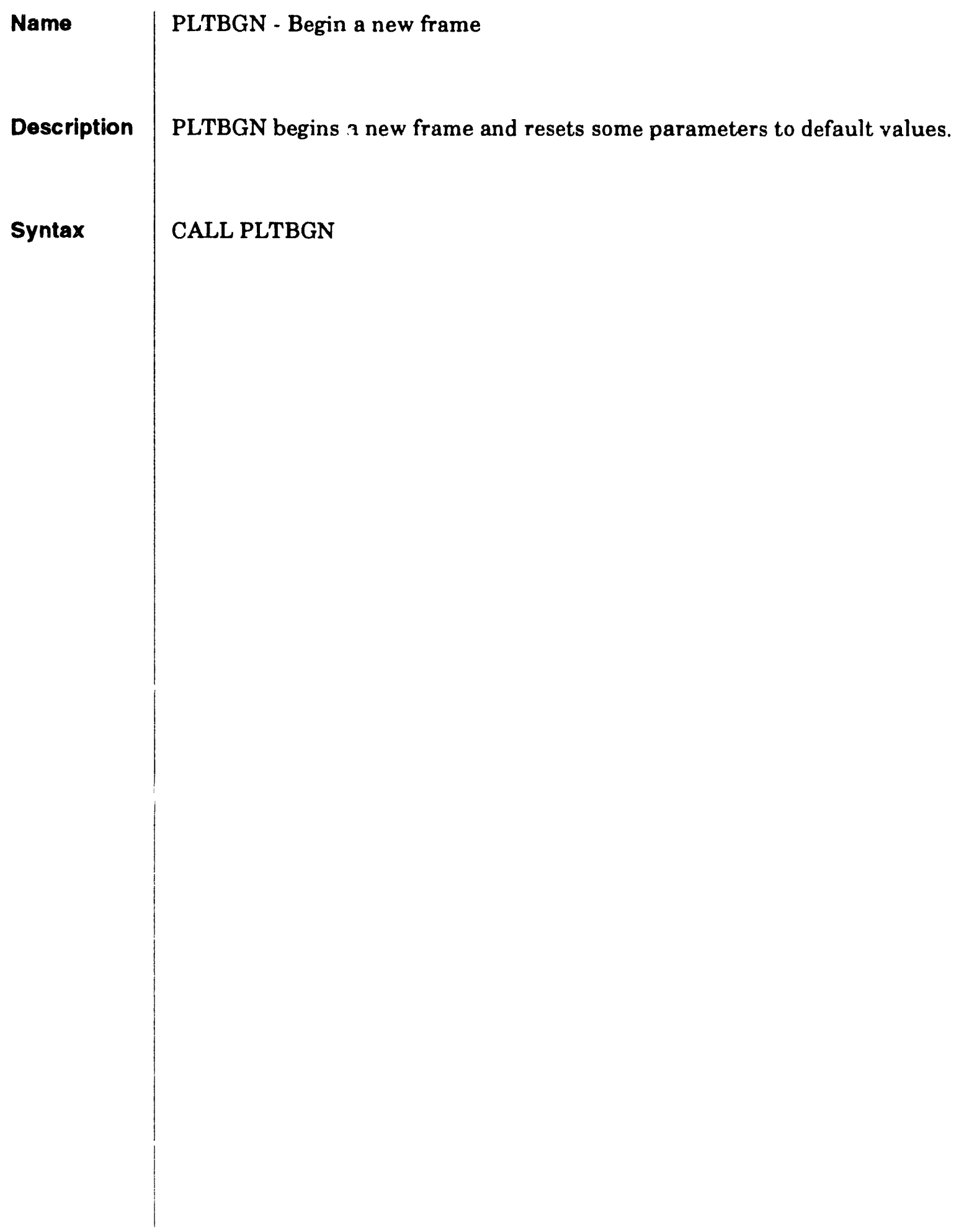




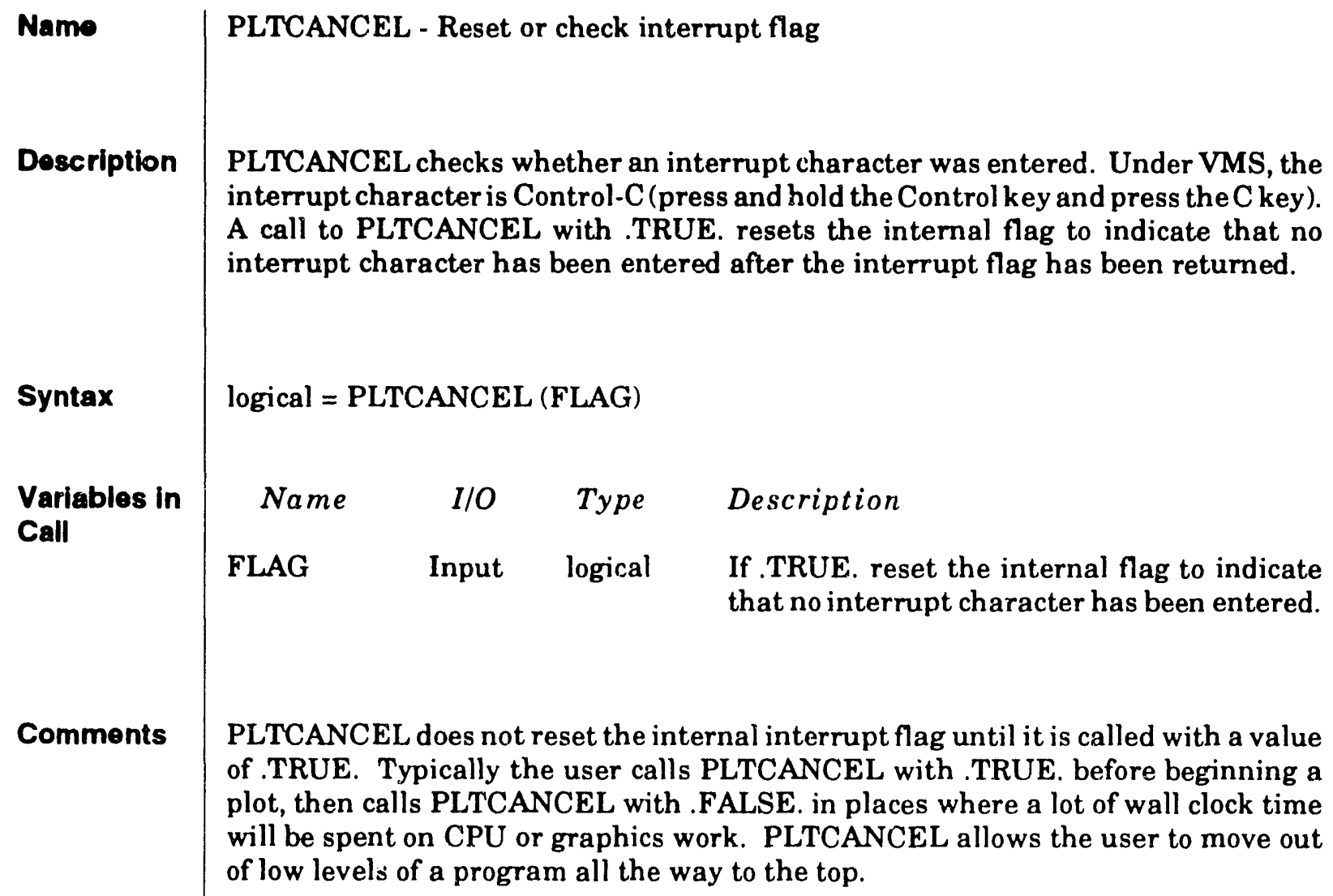


PLT_LIB

Description of Routine Calls

PLTCRS

Logical Function

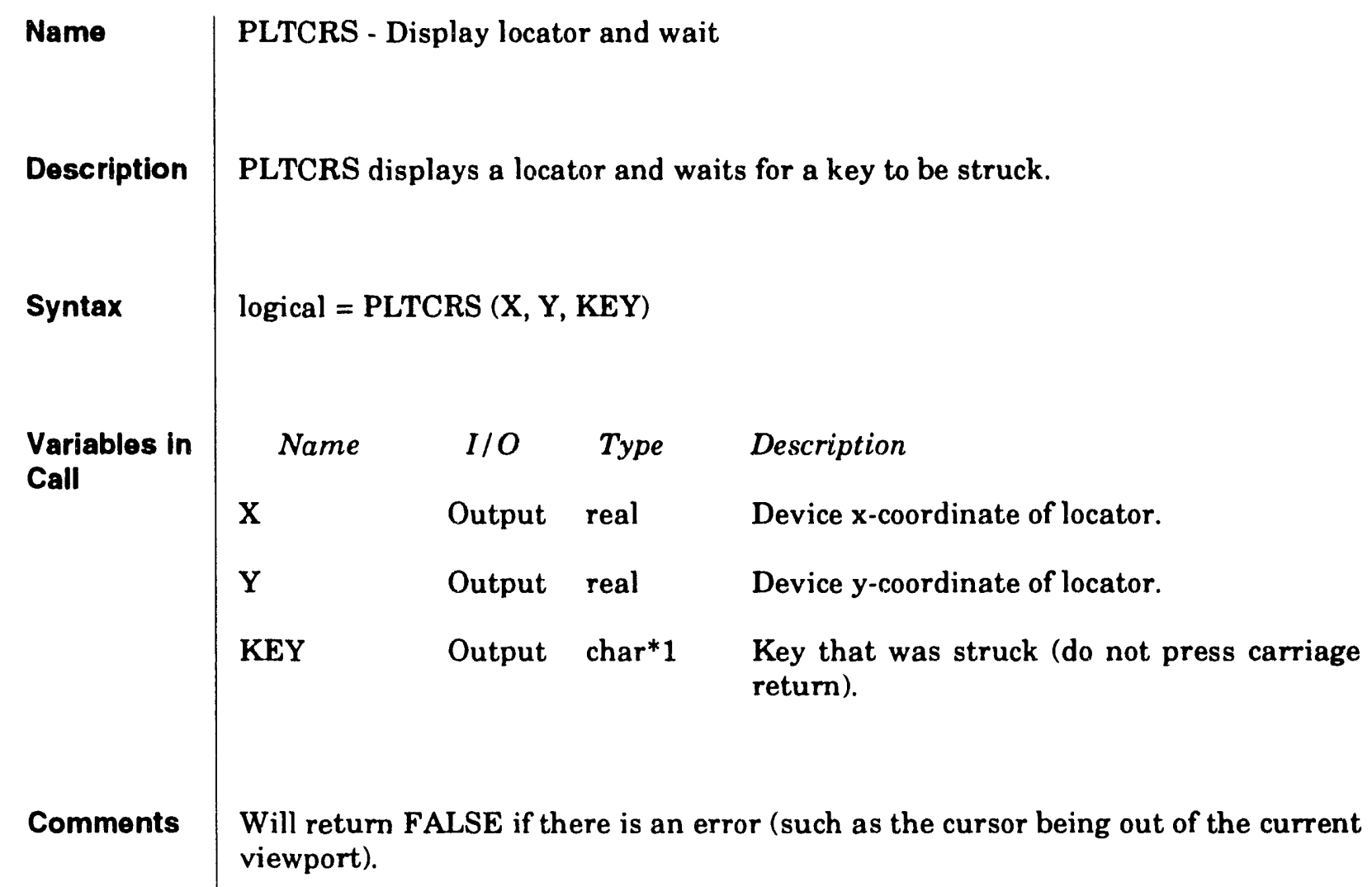




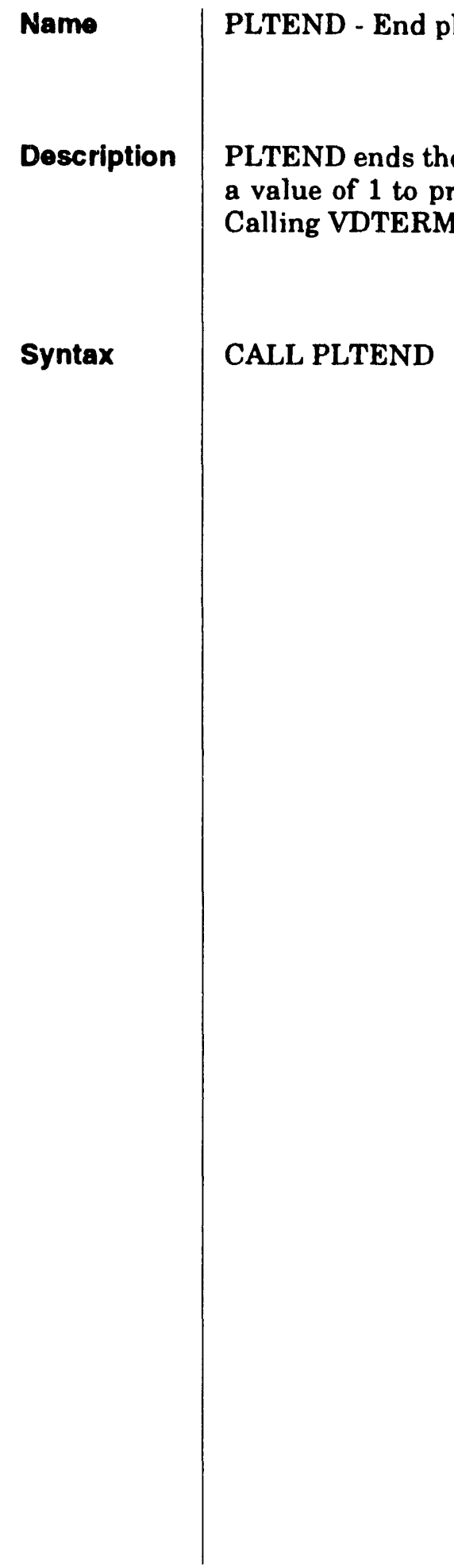


PLT_LIB

Description of Routine Calls

PLTFLU

Subroutine

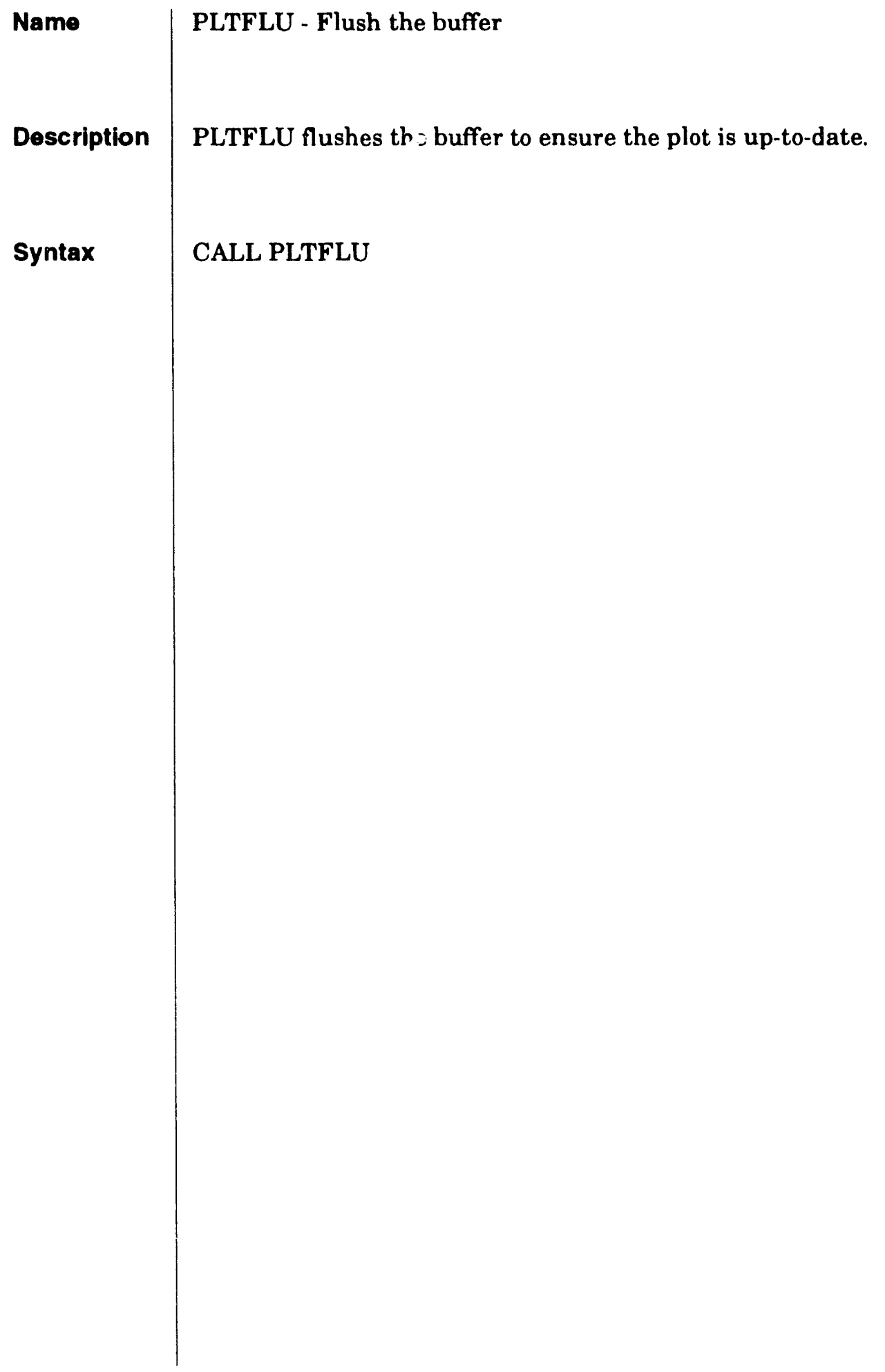




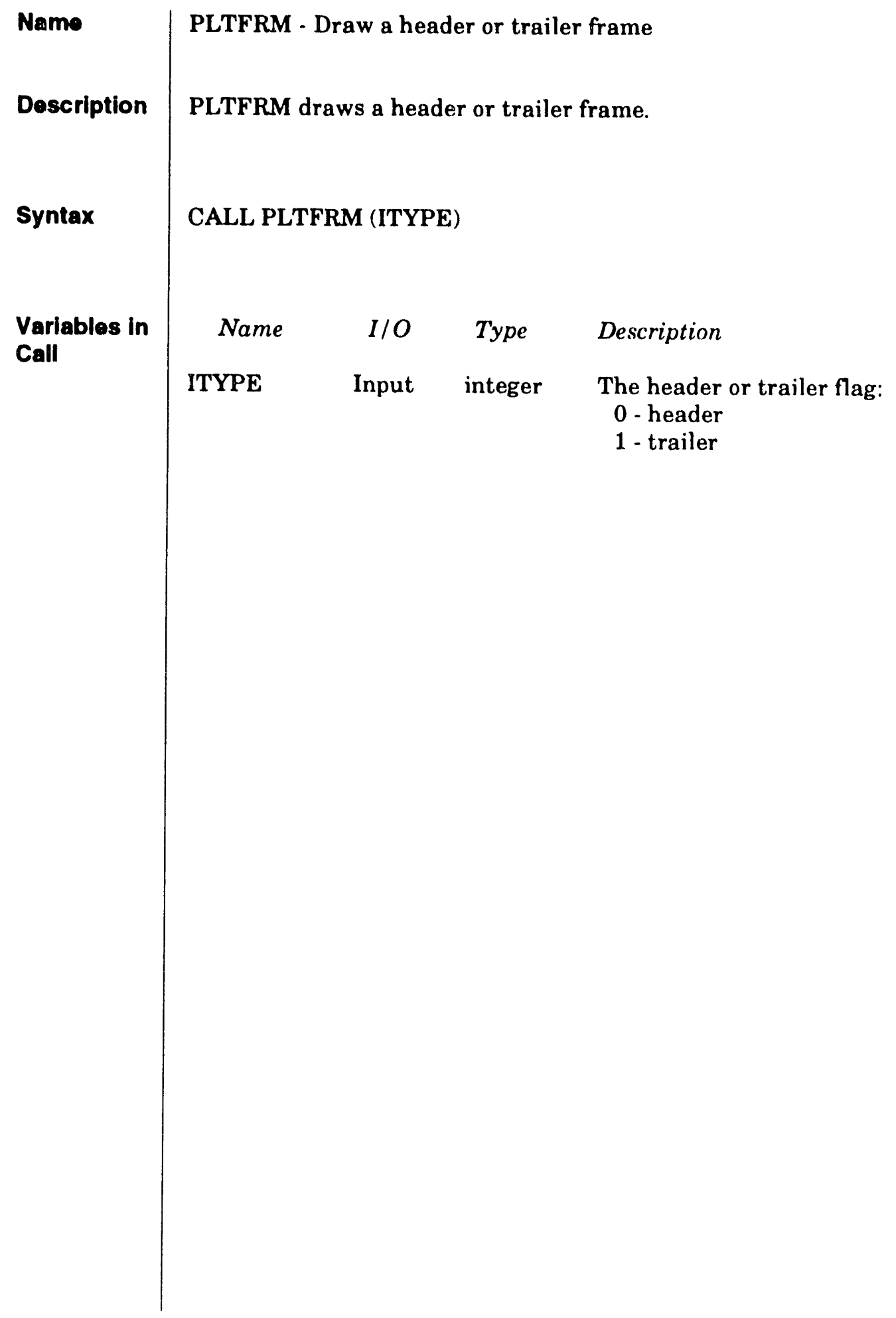




\begin{abstract}
Name PLTINT - initializes PLT_LIB

Description

PLTINT initializes PLT_LIB and must be called first if it is used. It can be called as many times as required. This routine calls the SVDI initialization routine VDINIT with an aspect ratio of 1.33 and a justification of 5 and then calls VDFRAM with a 0 to draw a header frame (on some devices).

Syntax

CALL PLTINT

Comments

PLTINT is the same as routine PLTSTA except that PLTINT calls VDINIT with device coordinates from 0 to 1 in the $x$ direction and from 0 to .75 in the $y$ direction, ASPECT $=1.0 / 0.75$, and JUSTIF $=5$, and then calls VDFRAM with a 0. PLTSTA was added to allow the user to set up his own device coordinates.
\end{abstract}

See Also

PLTSTA 


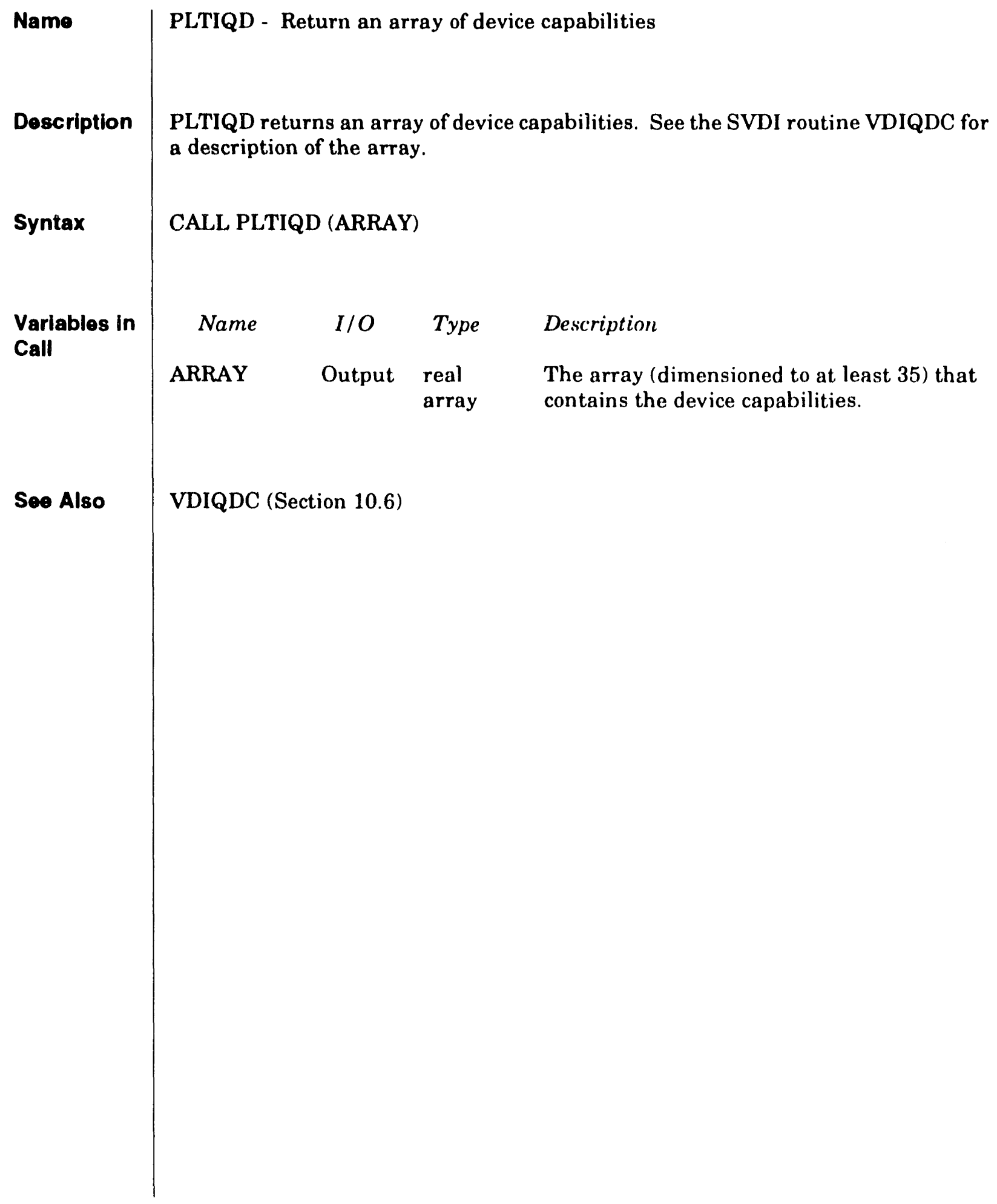




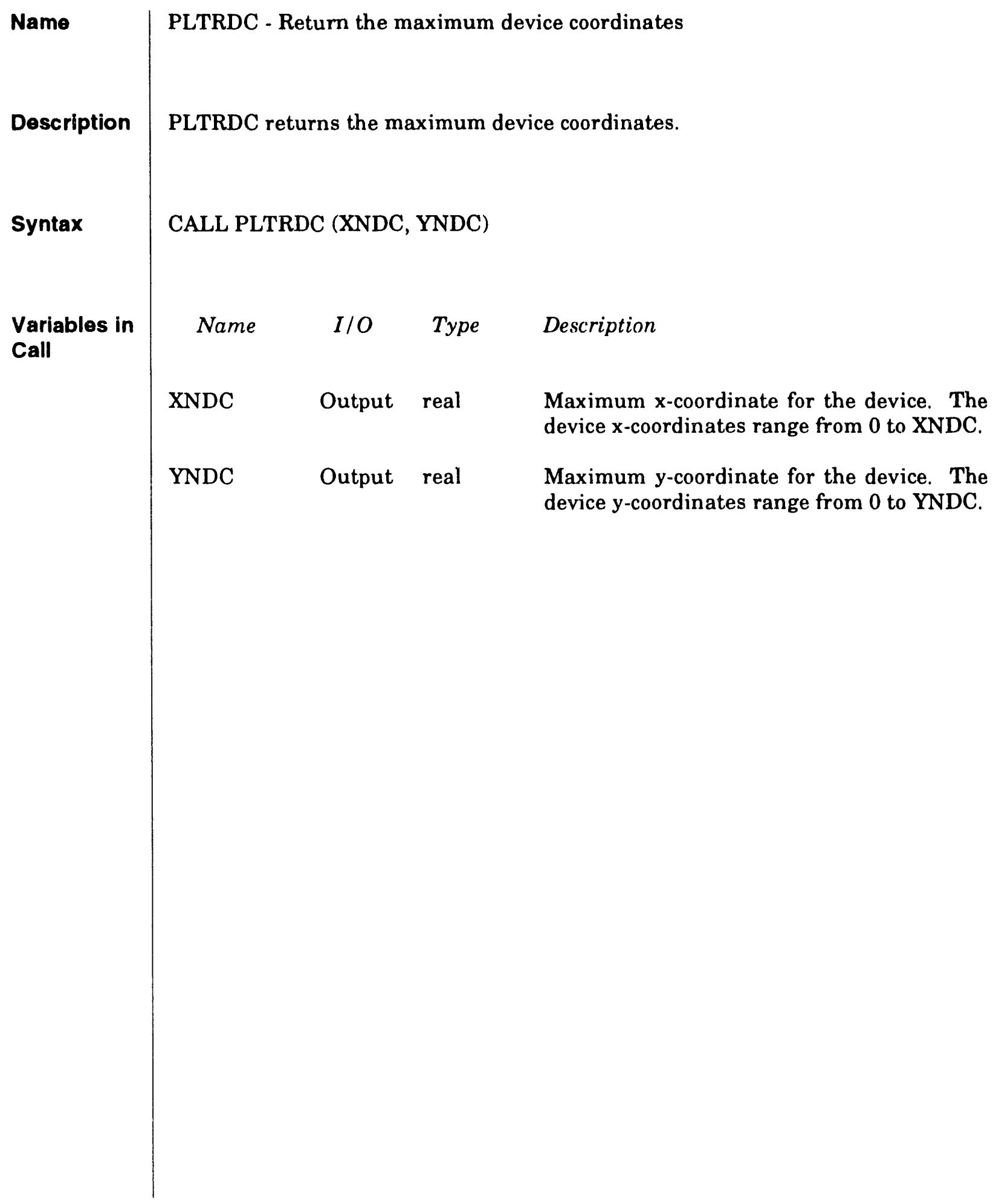


Name
Description

Syntax

Comments

See Also
PLTSTA - Initialize PLT_LIB

PLTSTA initializes PLT_LIB but does not call VDINIT and VDFRAM as PLTINT does. Either PLTSTA or PLTINT must be called before any other PLT_LIB routines. If PLTSTA is used, the user $m u$ st call VDINIT directly before the call to PLTSTA (the call to VDFRAM is optional). PLTSTA can be called as many times as required. After the first call, subsequent calls simply reinitialize PLT_LIB; VDINIT is called the first time only.

CALL PLTSTA

The user must call VDINIT before the call to PITSTA. The arguments to VDINIT are ASPECT and JUSTIF [CALL VDINIT (ASFECT, JUSTIF)]. ASPECT is the ratio of $\mathbf{x}$-dimension to $y$-dimension and JUSTIF is an integer as shown in the following table:

\section{9 \\ 456 \\ 123}

This table tells VDI how to justify the plot. For example, 7 means to justify at the upper left on the device surface, 5 means center vertically and horizontally, 3 means lower right, etc. For more information, see VDINIT in Chapter 11.

PLTINT (PLTINT is the same as PLTSTA except that the first time PLTINT is called, it calls VDINIT with ASPECT $=1.0 / 0.75$ and JUSTIF $=5$ and then calls VDFRAM with a 0 to draw a header frame on some devices.) 
PLT LIB

Description of Routine Calls

PLTWAI

subroutine

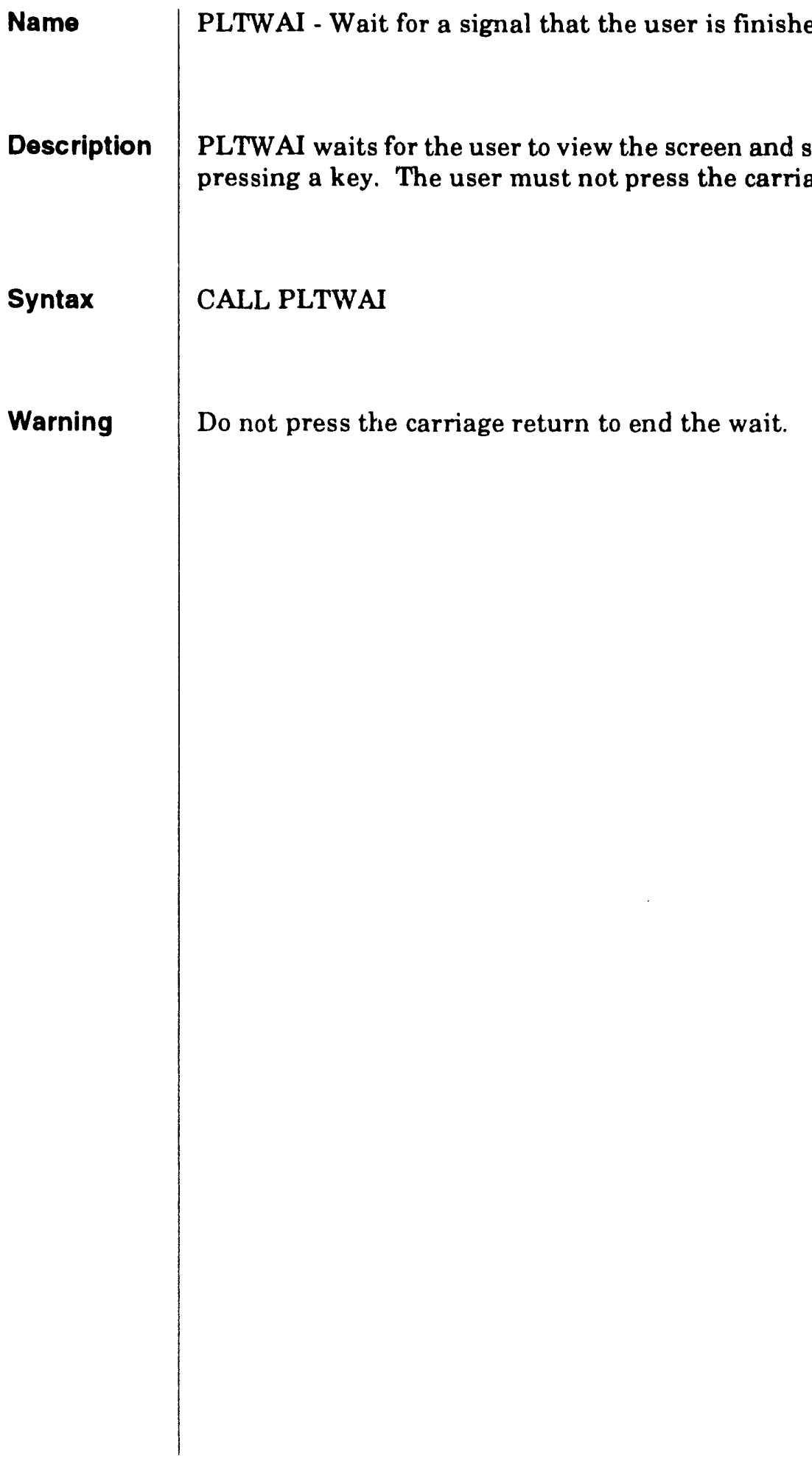


PLT LIB

\subsubsection{Setting PLT_LIB Parameters}

Detailed descriptions of the routines that set PLT_LIB parameters are given on the following pages. 


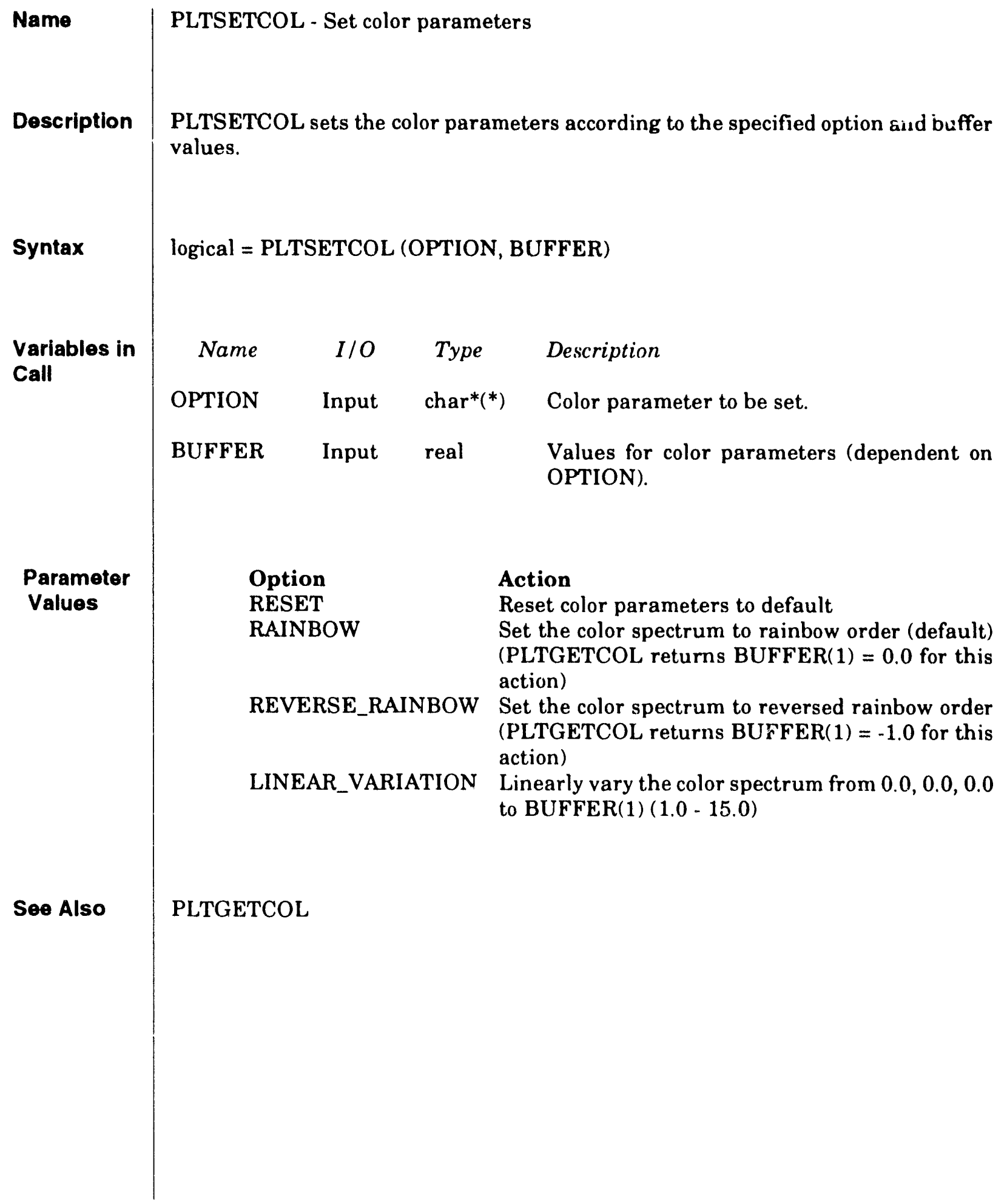




\section{PLTGETCOL}

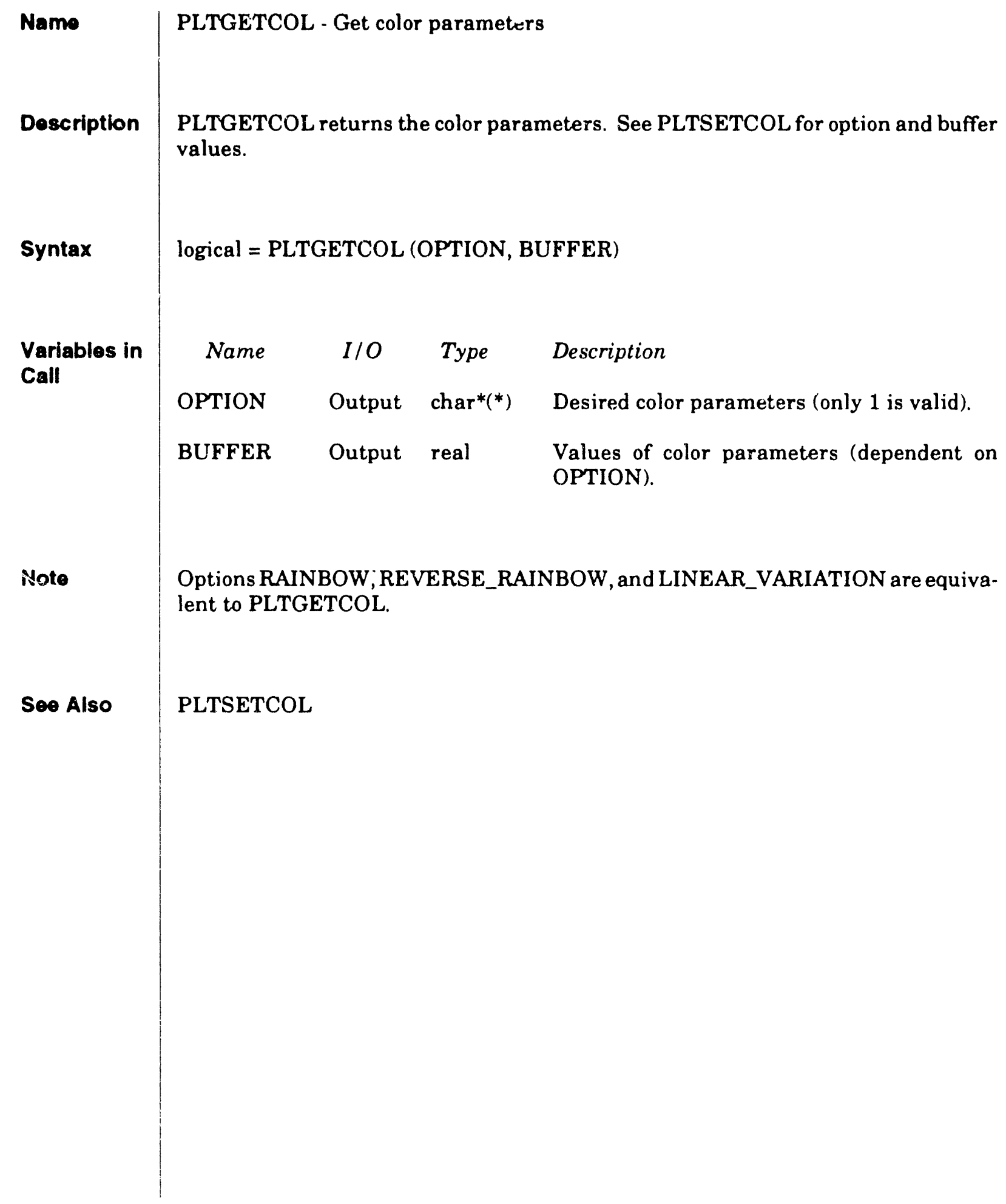




\begin{abstract}
\begin{tabular}{l|l} 
Name & PLTSETDRW - Set basic graphics parameters
\end{tabular}
Description

PLTSETDRW sets the basic graphics parameters such as color and line type according to the specified option and buffer values. The graphics buffer is flushed so that the changes are up-to-date.

Syntax

Variables in

Call
\end{abstract}

Parameter Values

Seo Also

\section{Action}

Reset device parameters to default

RESET

BACK_COLOR

COLOR

INTENSITY

LINE_STYLE

LINE_WIDTH

Set background color to BUFFER(1) (range 0.0 - 255.0)

Set foreground color used to draw all lines, points, and filled areas to BUFFER(1) (range $0.0-255.0$ )

Set intensity to BUFFER(1) (range $0.0-100.0$ ), where 0.0 is invisible

Set the line style based on BUFFER(1) as follows:

$0.0=$ no line

$1.0=$ solid

$2.0=$ dotted

$3.0=\operatorname{dot}$ dash

$4.0=$ short dash

$5.0=$ long dash

$6.0=$ medium dash

Set the line width to BUFFER(1) (range is $0.0-1000.0$, where 1000 is 0.01 in VDI space) 


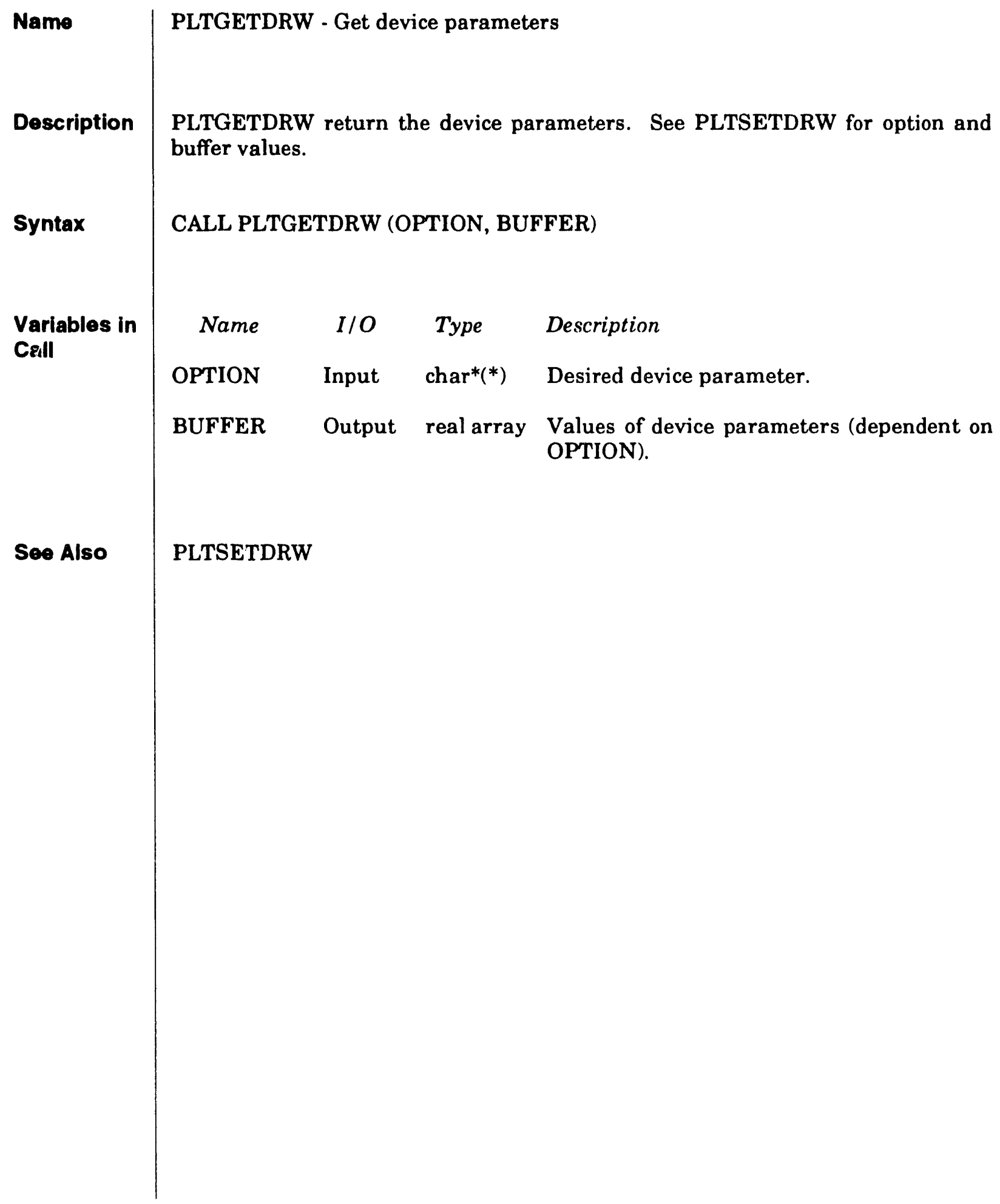




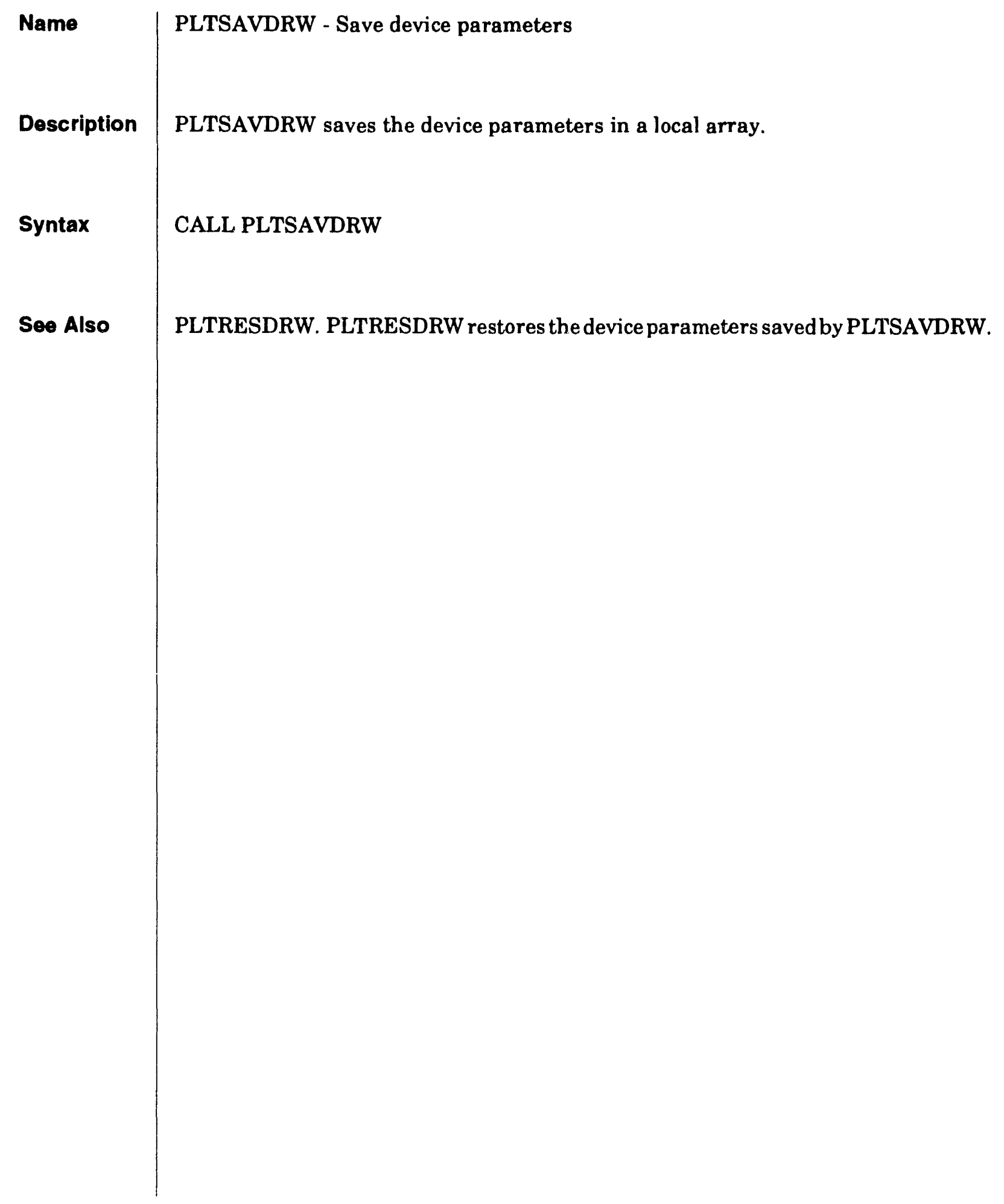


PLT_LIB

Setting PLT_LIB Parameters

PLTRESDRW

Subroutine

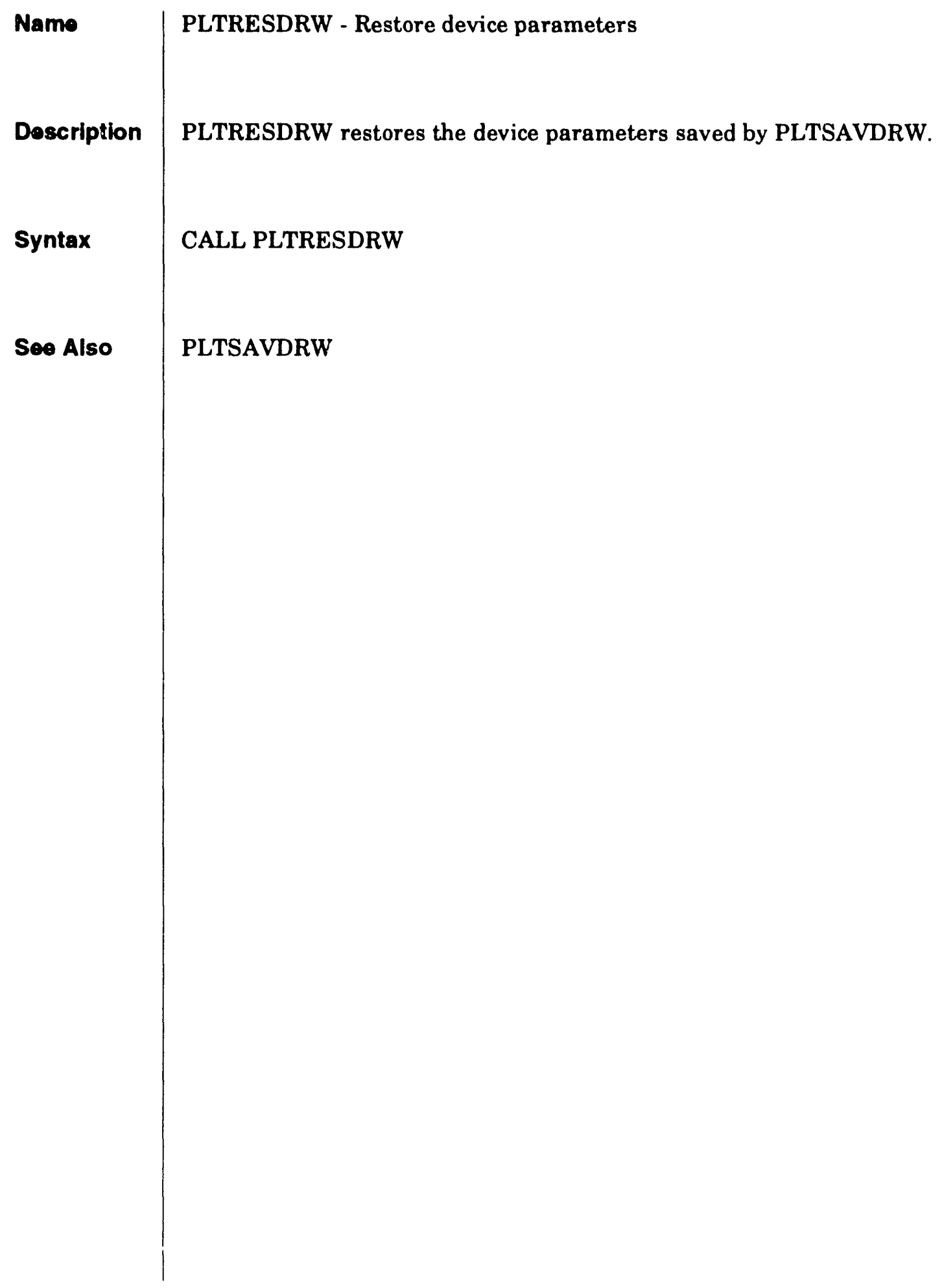




\begin{tabular}{|c|c|c|c|c|}
\hline Name & \multicolumn{4}{|c|}{ PLTSETGPH - Set graph parameters } \\
\hline Description & \multicolumn{4}{|c|}{$\begin{array}{l}\text { PLTSETGPH sets the graph parameters according to the specified option and buffer } \\
\text { values. }\end{array}$} \\
\hline Syntax & \multicolumn{4}{|c|}{ CALL PLTSETGPH (OPTION, BUFFER) } \\
\hline $\begin{array}{l}\text { Variables in } \\
\text { Call }\end{array}$ & $\begin{array}{r}\text { Name } \\
\text { OPTION } \\
\text { BUFFER }\end{array}$ & $\begin{array}{l}I / O \\
\text { Input } \\
\text { Input }\end{array}$ & $\begin{array}{l}\text { Type } \\
\text { integer } \\
\text { real array }\end{array}$ & $\begin{array}{l}\text { Description } \\
\text { Graph parameter to set. } \\
\text { Values for graph parameters (dependent on } \\
\text { OPTION). }\end{array}$ \\
\hline
\end{tabular}

Parameter Values

Option
RESET
X_ORIGIN
Y_ORIGIN
X_LENGTH
Y_LENGTH
X_AXIS_TYPE
Y_AXIS_TYPE
FRAME
AXIS_COLOR
AXIS_WIDTH
X_SCALING_TYPE

\section{Action}

Reset graph parameters to default Set $x$-origin of graph to BUFFER(1) Set y-origin of graph to BUFFER(1) Set length of $x$-axis to BUFFER(1) Set length of $y$-axis to BUFFER(1) Set $\mathrm{x}$-axis type based on BUFFER(1): $0=$ normal, $1=$ logarithmic

Set $y$-axis type based on BUFFER(1): $0=$ normal, $1=$ logarithmic Turn on graph framing if BUFFER(1) is 1 ; turn off if BUFFER(1) is 0

Set axis color to BUFFER(1) (range 0.0-255.0)

Set pen width of axes to BUFFER(1)

Set $x$-axis scaling type based on BUFFER(1) as follows:
BUFFER(1)
$=1.0$ - autoscale with PLTSCALE, differ- ent $x$ and $y$ scaling possible; PLTGETGPH will return BUFFER(2-5) as defined for BUFFER(1) $=3.0$
$=2.0$ - autoscale with PLTSCALE, same $x$ and y scaling forced; PLTGETGPH will return BUFFER(2-5) as defined for $\operatorname{BUFFER}(1)=3.0$




\section{Parameter Values (continued)}

\section{BUFFER(1) $=3.0 \quad-$ user defines scaling as follows: BUFFER(2) - minimum $x$ BUFFER(3) - maximum $x$ BUFFER(4) - number of major $x$ intervals BUFFER(5) - number of minor in- tervals on $x$-axis \\ $=4.0$ - user defines exact scaling as follows: \\ BUFFER(2) - minimum $x$ \\ BUFFER(3) - first nice $x$ \\ BUFFER(4) - maximum $x$ \\ BUFFER(5) - $x$ interval \\ BUFFER(6) - number of minor $x$ intervals}

Y_SCALING_TYPE Set $y$-axis scaling type based on BUFFER(1) as follows:

BUFFER(1)

$=1.0$ - autoscale with PLTSCALE, different $x$ and $y$ scaling possible; PLTGETGPH will return BUFFER(2-5) as defined for $\operatorname{BUFFER}(1)=3.0$

$=2.0$ - autoscale with PLTSCALE, same $x$ and y scaling forced; PLTGETGPH will return BUFFER(2-5) as defined for $\operatorname{BUFFER}(1)=3.0$

$=3.0$ - user defines scaling as follows:

BUFFER(2) - minimum y

BUFFER(3) - maximum y

BUFFER(4) - number of major $y$ intervals

BUFFER(5) - number of minor intervals on y-axis

$=4.0$ - user defines exact scaling as follows: BUFFER(2) - minimum y BUFFER(3) - first nice y BUFFER(4) - maximum y BUFFER(5) - y interval

BUFFER(6) - number of minor $y$ intervals

CLIPPING_WINDOW Set clipping window based on BUFFER(1) as follows:

$$
\begin{aligned}
& \text { BUFFER(1) } \\
& =0.0 \text { - clipping window is the same as the } \\
& \text { graph window (set by X/ } \\
& \text { Y_SCALING_TYPE) }
\end{aligned}
$$




\section{Parameter Values (continued)}

X_AXIS_SCI

Y_AXIS_SCI

X_EXPONENT

Y_EXPONENT

X_DIGITS

Y_DIGITS

Y_VERTICAL

X_NUMBER_SIZE

Y_NUMBER_SIZE

NUMBER_COLOR NUMBER_WIDTH X_LABEL_SIZE

Y_LABEL_SIZE

LABEL_COLOR LABEL_WIDTH GRID_COLOR
$=1.0$ - clipping window is defined with respect to the graph window as follows:

BUFFER(2) - amount to add to the $x$ minimum and subtract from the $x$ maximum (in graph units)

BUFFER(3) - amount to add to the y minimum and subtract from the $y$ maximum (in graph units)

$=2.0$ - clipping window is defined in graph units as follows:

BUFFER(2) - minimum $x$

BUFFER(3) - maximum $x$

BUFFER(4) - minimum y

BUFFER(5) - maximum y

Set type of notation on $\mathrm{x}$-axis based on BUFFER(1):

$0=$ normal, 1 = scientific

Set type of notation on y-axis based on BUFFER(1): $0=$ normal, $1=$ scientific

Set $x$-exponent to BUFFER(1); this exponent is the exponent labelled in scientific notation on the linear axis (ignored if scientific notation is not set); -999999.0 to let the code determine the exponent

Set y-exponent to BUFFER(1); this exponent is the exponent labelleci in scientific notation on the linear axis (ignored if scientific notation is not set); -999999.0 to let the code determine the exponent

Set number of decimal places in $\mathbf{x}$-axis numbers to BUFFER(1) (range 0-9)

Set number of decimal places in y-axis numbers to BUFFER(1) (range 0-9)

Set y number vertical or horizontal based on BUFFER(1) (1=vertical; otherwise horizontal)

Set character size of $x$-axis number to BUFFER(1); 0 turns off drawing the numbers

Set character size of $y$-axis number to BUFFER(1); 0 turns off drawing the numbers

Set color of axes number to BUFFER(1)

Set pen width of axes number to BUFFER(1)

Set character size of $x$-axis labels to BUFFER(1); 0 turns off drawing the ?abel

Set character size of $y$-axis labels to BUFFER(1); 0 turns off drawing the label

Set color for axes labels to BUFFER(1)

Set pen width of axes labels to BUFFER(1)

Set color for major grid to BUFFER(1) 


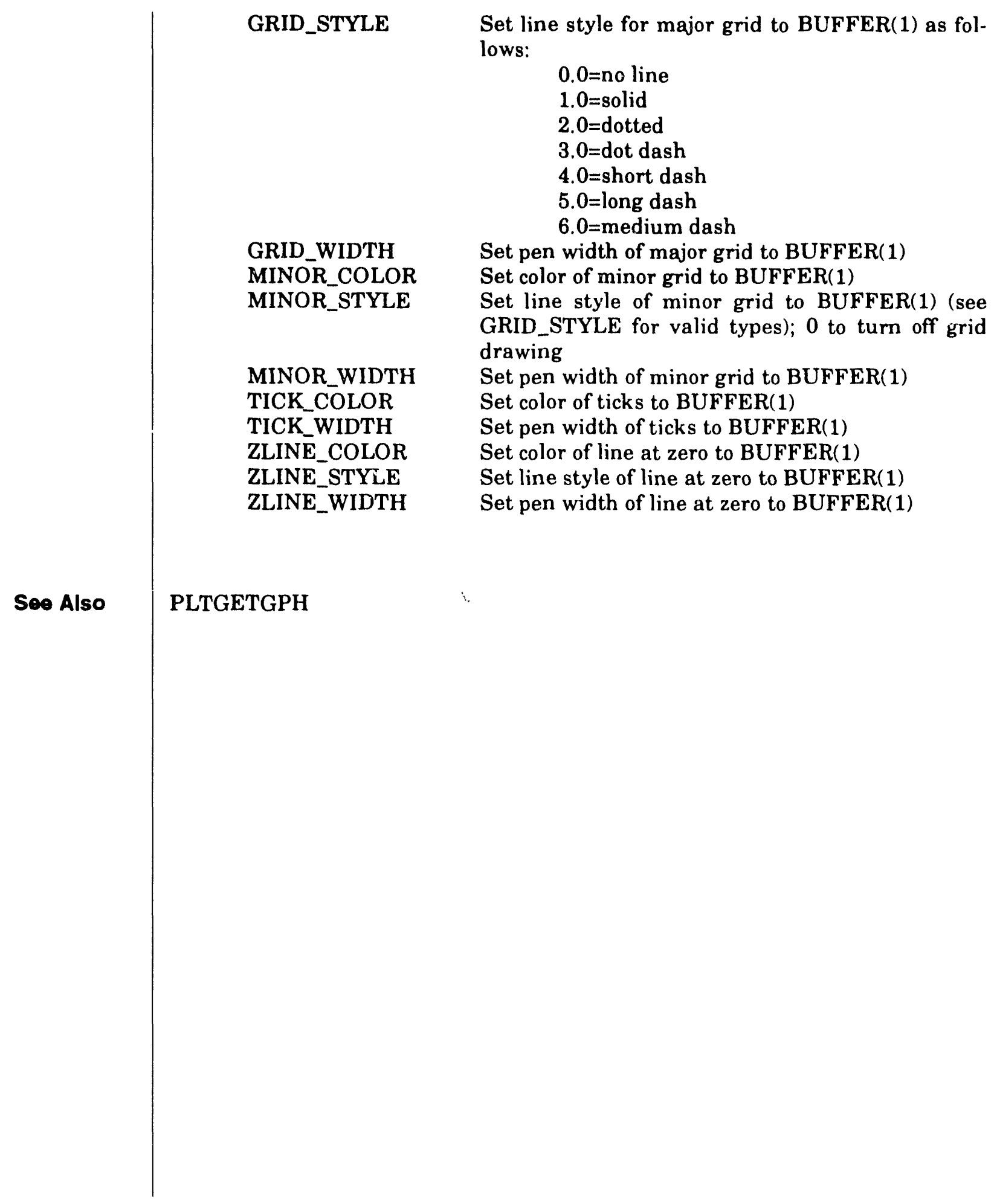


Name

Description

Syntax

Variables in

Call

See Also
PLTGETGPH - Get graph parameters

PLTGETGPH returns the graph parameters. See PLTSETGPH for option and buffer values. The only exception is when getting scaling type (SCALING_TYPE). Here BUFFER(1) is checked: if it equals user scaling (3.0) then BUFFER(2) BUFFER(9) are loaded with the user scaling; if it equals exact scaling (4.0), then BUFFER(2) - BUFFER(11) are loaded with the exact scaling.

logical $=$ PLTGETGPH $($ OPTION, BUFFER $)$

$$
\text { Name I/O Type Description }
$$

OPTION Input $\operatorname{char}^{*}\left({ }^{*}\right) \quad$ Desired graph parameter.

BUFFER Output real array Values for graph parameters (dependent on OPTION). 


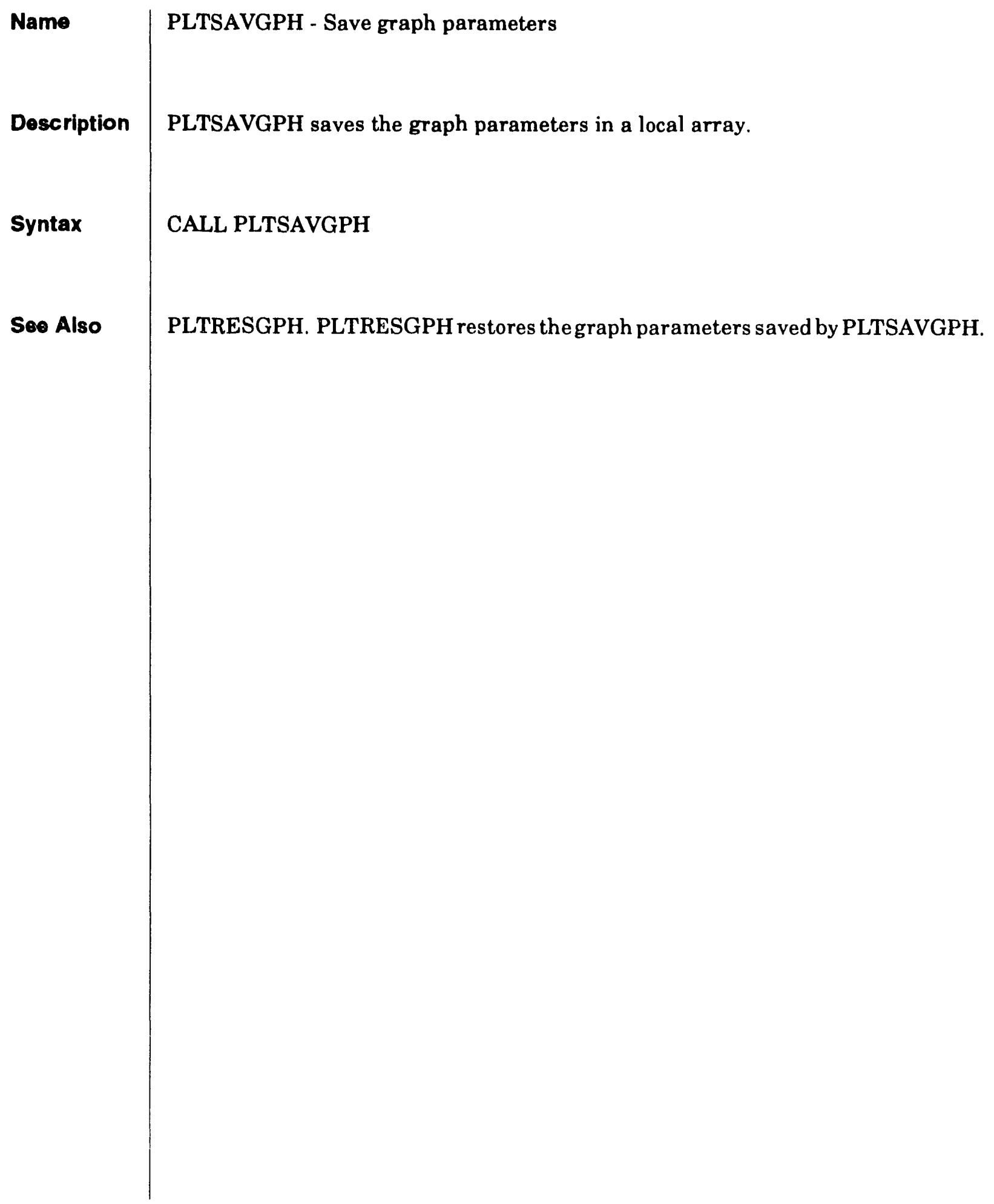




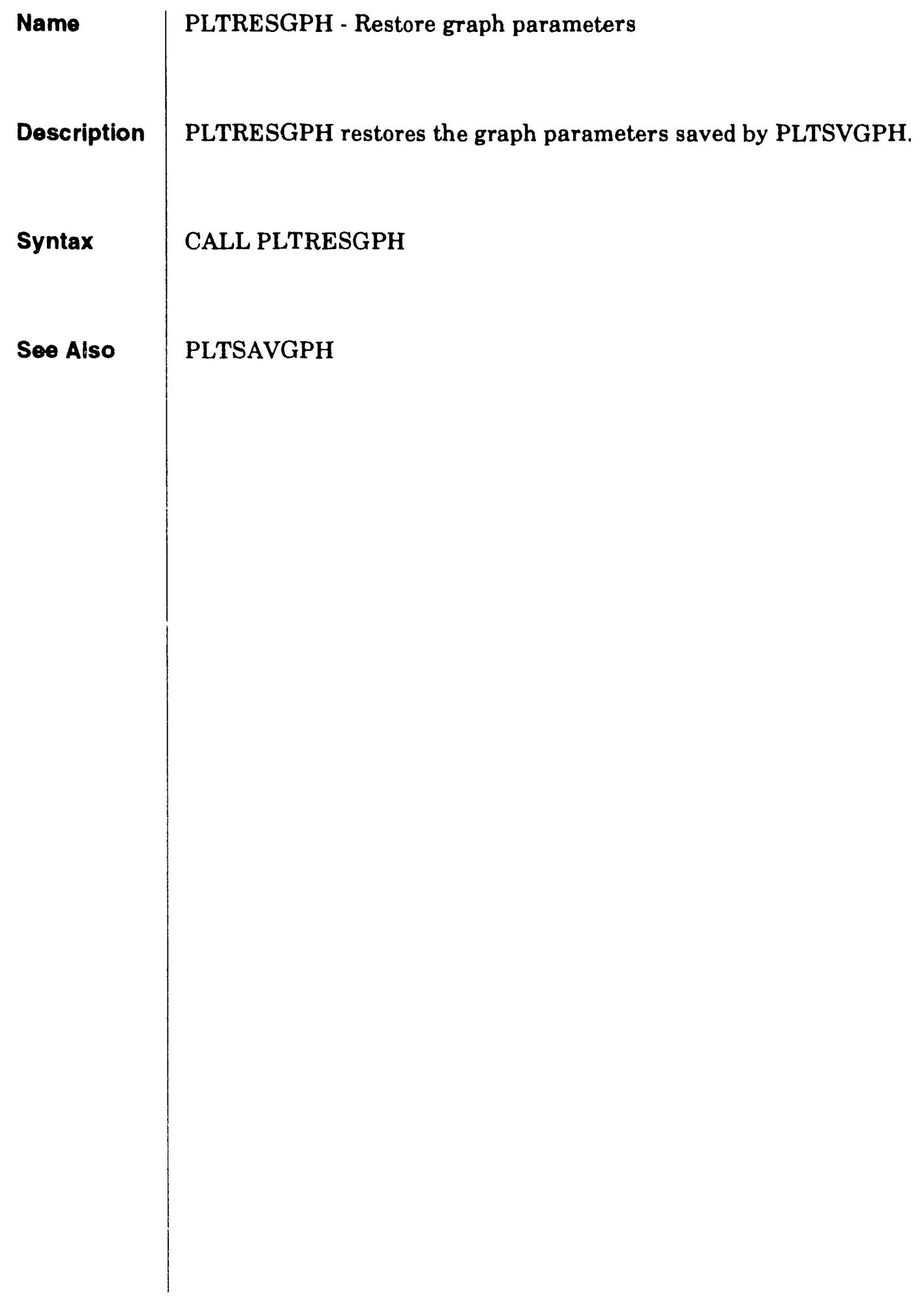




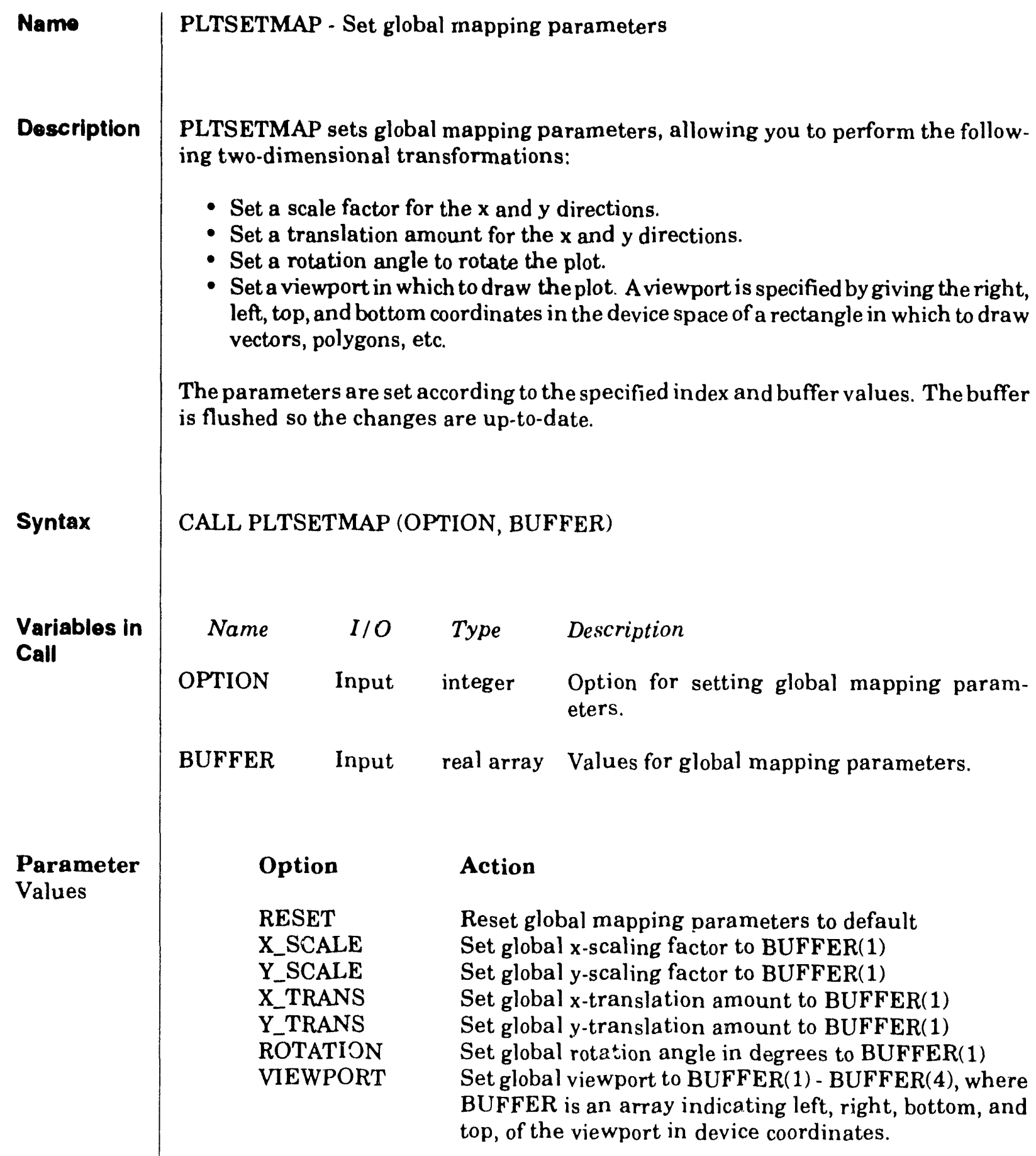




\begin{abstract}
Note
Caveats

Remember that the PLT coordinates are only mapped into this new coordinate system and viewport. The current position of the pen is still in PLT space even though the coordinates are mapped into some different device space.

- Hardware text cannot be rotated on some devices.

- The vectors, polygons, and text are clipped against the current viewport. Since the maximum $x$-coordinate can be larger than the maximum $y$-coordiante, you may have to scale the $x$-coordinate down if you rotate by 90 degrees, for example. It is recommended that you scale in the $x$ and $y$ directions by 1/ASPECT; this guarantees no distortion and makes sure everything still fits on the screen.
\end{abstract}

Comments

See Also

The global mapping routines have nothing to do with the mapping routines that begin with MP, except that output is funnelled through the global mapping routines. These global mapping routines allow the user to rotate, scale, and translate the final output from all plotting routines (except hardware-text routines). For instance, this allows the user to rotate a graph by 90 degrees so that the output is in portrait mode as opposed to landscape mode.

\section{PLTGETMAP}




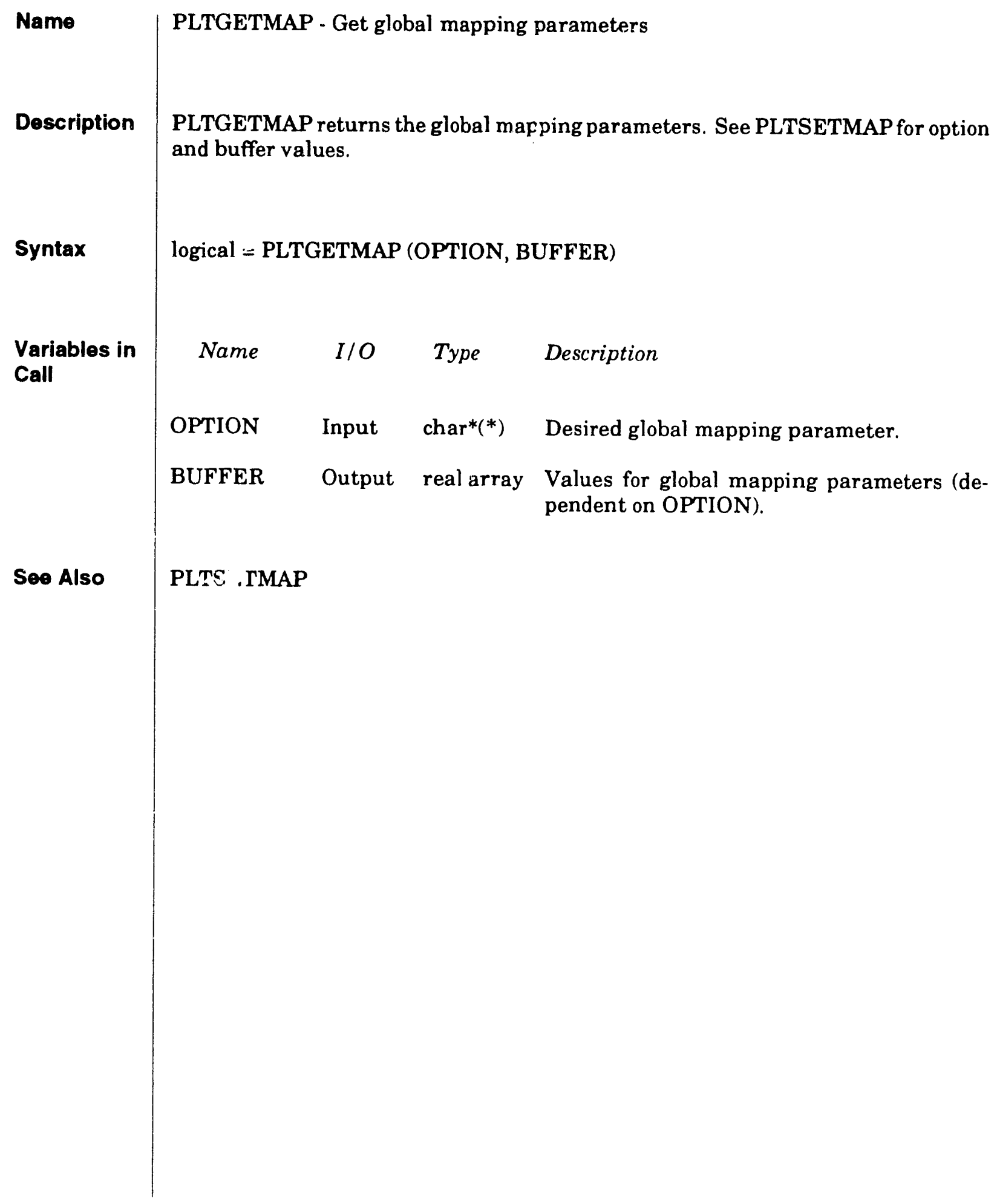




\begin{tabular}{l|l} 
Name & $\begin{array}{l}\text { PLTSAVMAP - Save global mapping parameters } \\
\text { Description }\end{array}$ \\
Syntax & PLTSAVMAP saves the global mapping parameters in a local array. \\
See Also & $\begin{array}{l}\text { PLTRESMAP. PLTRESMAP restores the global mapping parameters saved by } \\
\text { PSTSAVMAP. }\end{array}$
\end{tabular}




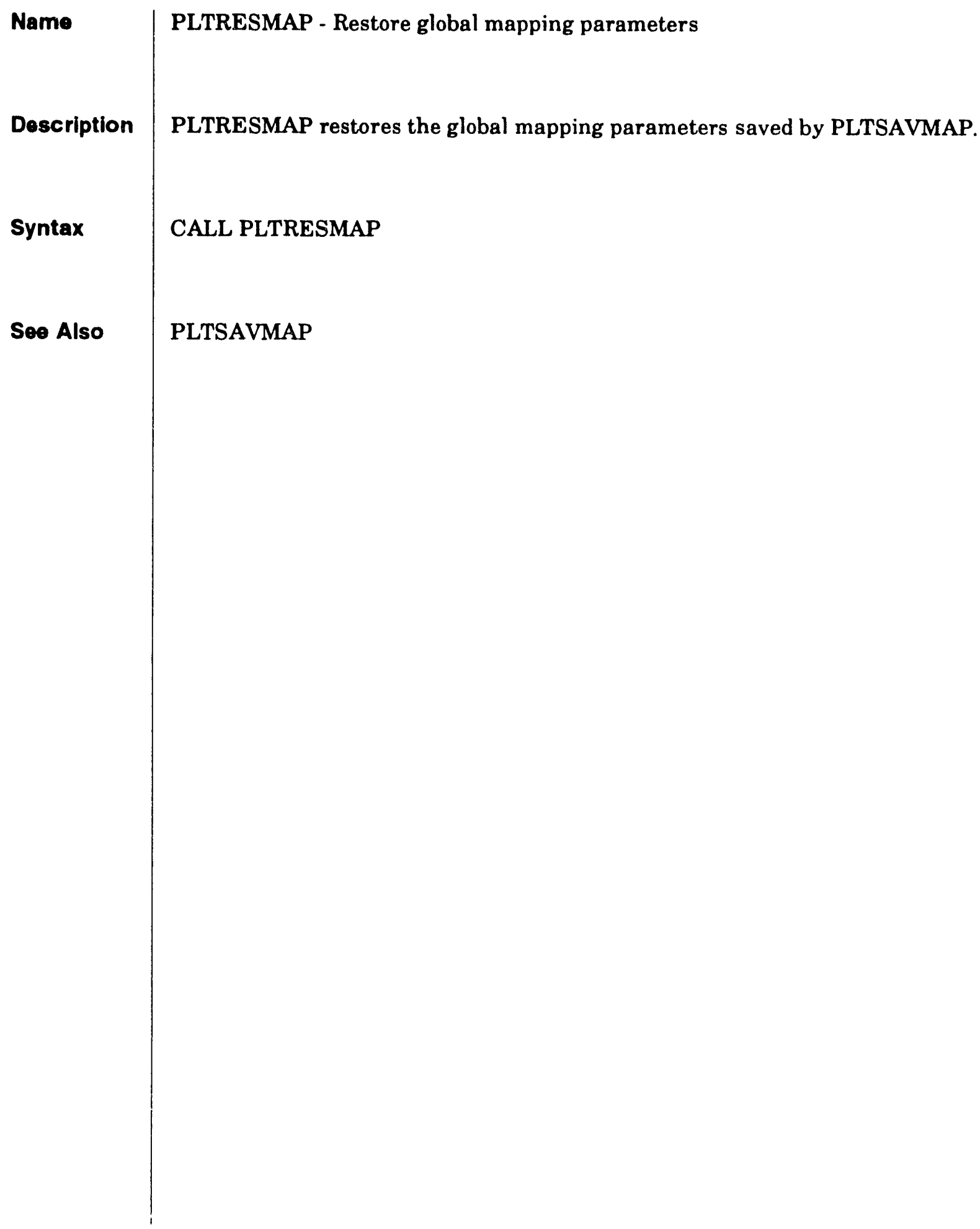




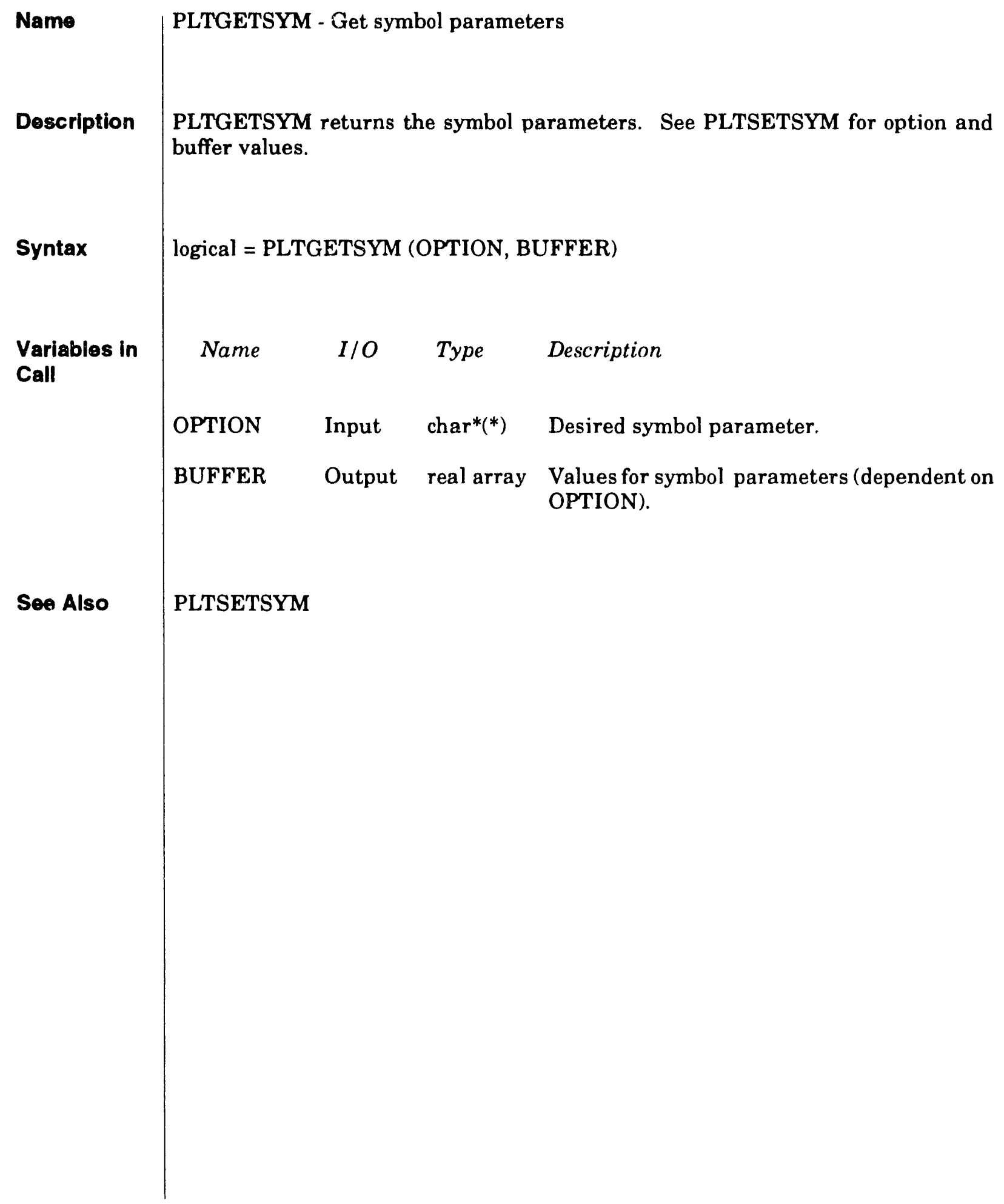




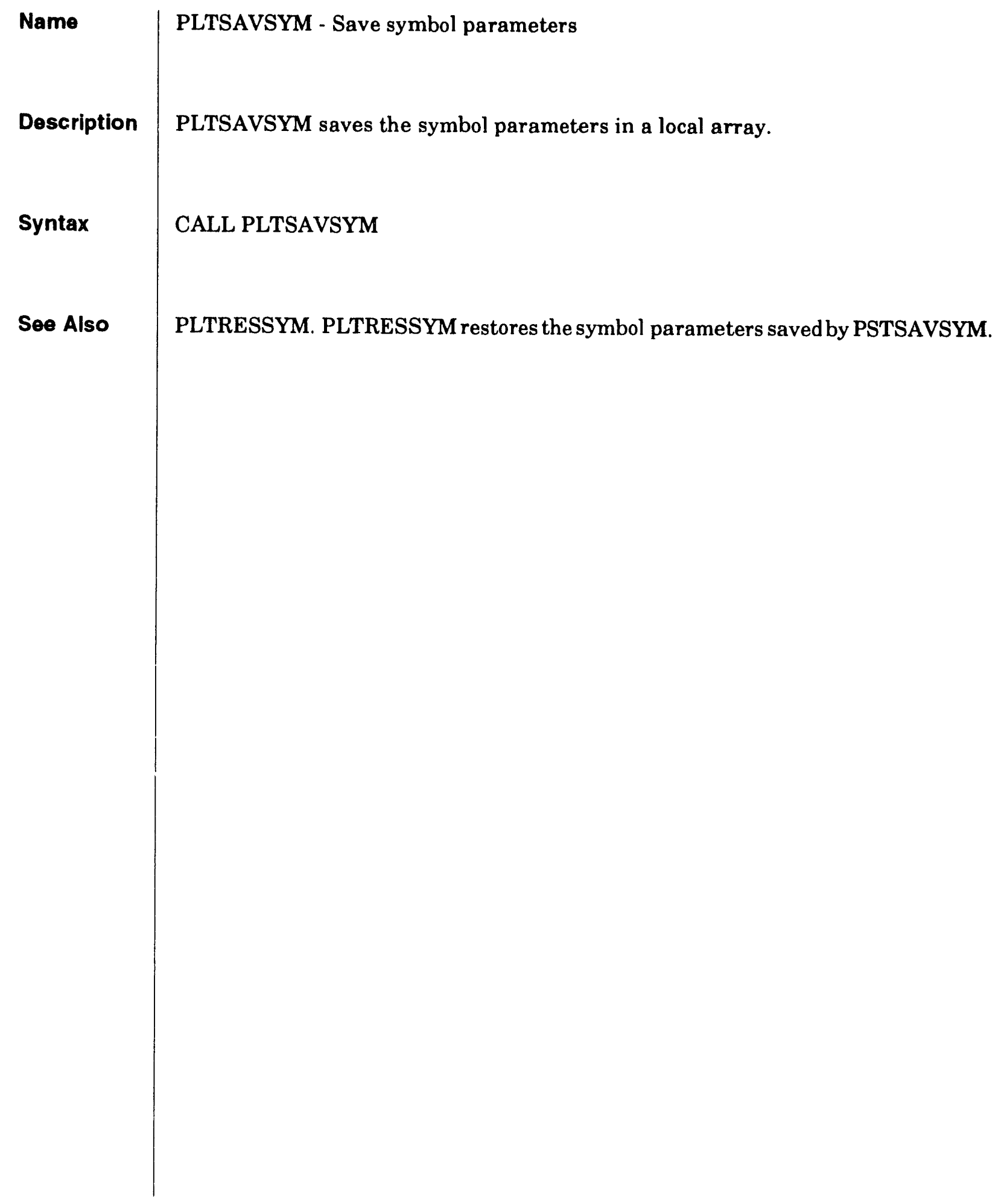




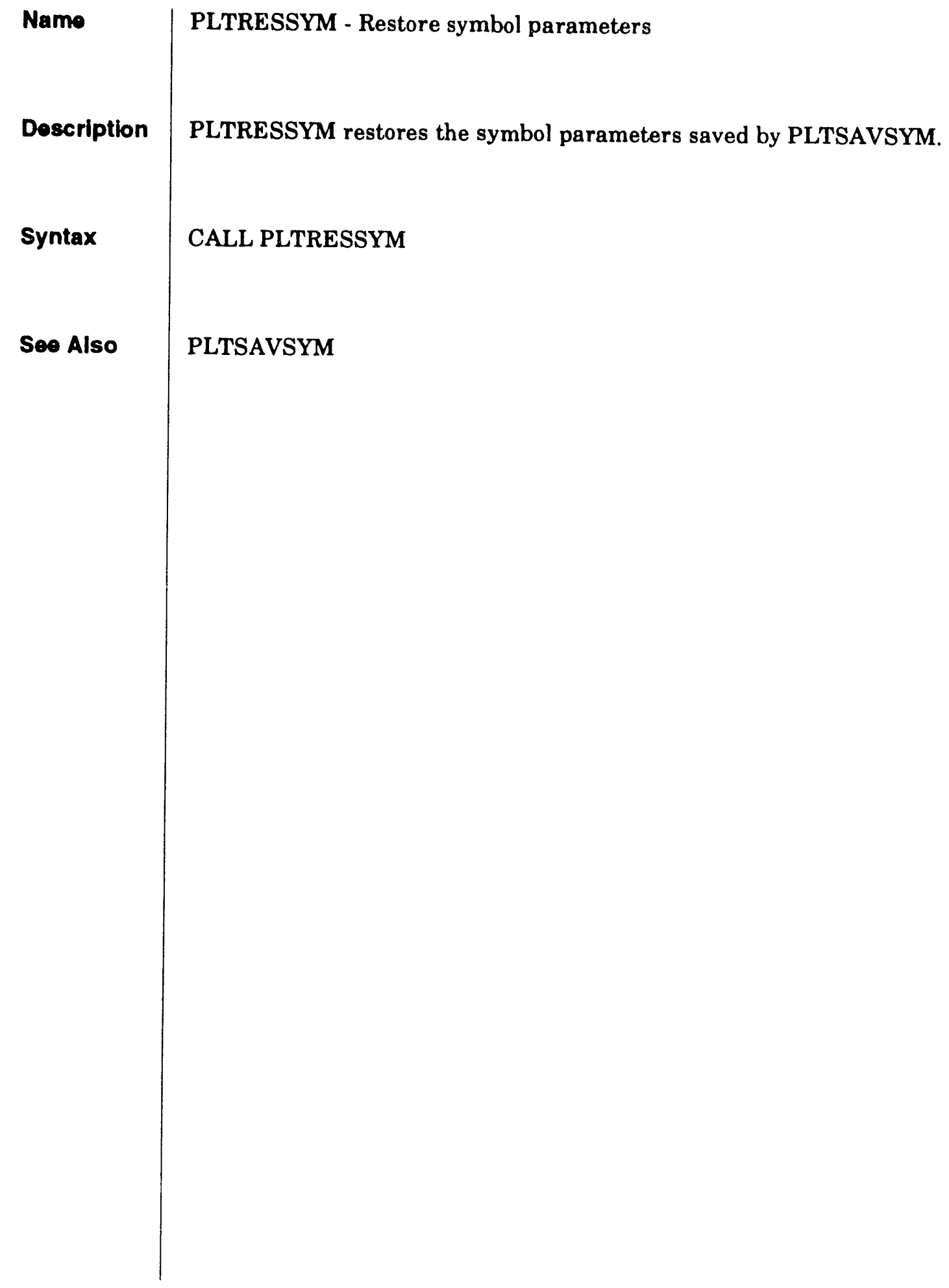




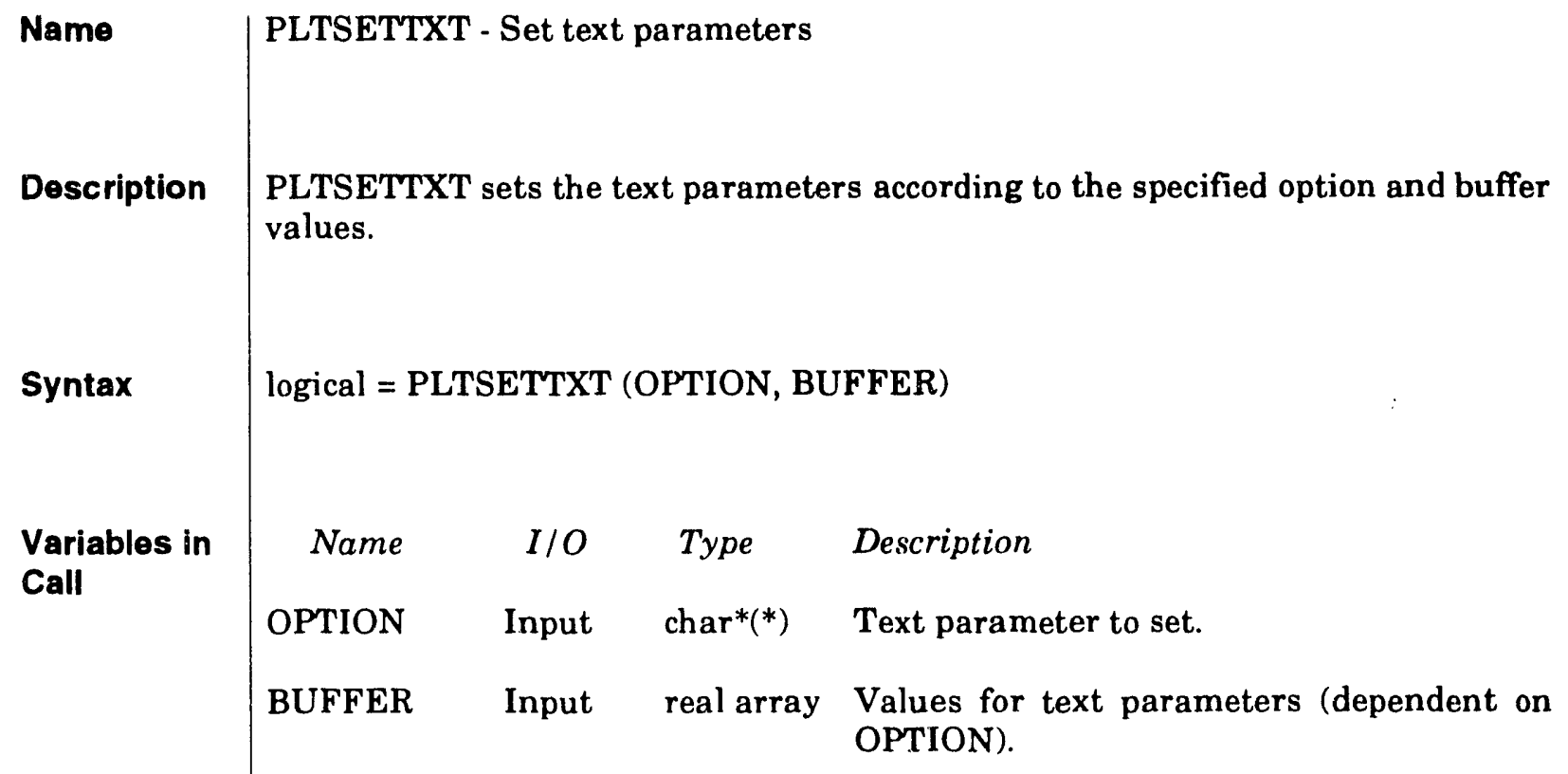

Option

RESET

SOFT_HARD

SOFT_FONT

HARD_FONT

TEXT_COLOR

TEXT_WIDTH

CHAR_SIZE

TEXT_ANGLE

CHAR_ANGLE

TEXT_WINDOW

LINE_SPACE
Action

Reset text parameters to default

Select software versus hardware characters based on BUFFER(1): 0 = software, 1 = hardware

Set the font for software text based on BUFFER(1) as follows:

$$
\begin{aligned}
& 0.0=\text { default } \\
& 1.0=\text { F.oman } \\
& 2.0=\text { stick } \\
& 3.0=\text { sans serif }
\end{aligned}
$$

Set the font for hardware text based on BUFFER(1) as follows: $0.0=$ default

Set the color for text to BUFFER(1)

Set the pen width for text to BUFFER(1)

Set character size to BUFFER(1)

Set angle for text to be written; BUFFER(1) is the angle in degrees

Set angle for characters to be written; BUFFER(1) is the angle in degrees

Set text window to BLFFER(1) - BUFFER(8); the buffer denotes the four points of the window in CCW Order

Set space between lines of text; BUFFER(1) contains a factor by which to multiply the y character size to obtain the linefeed size 


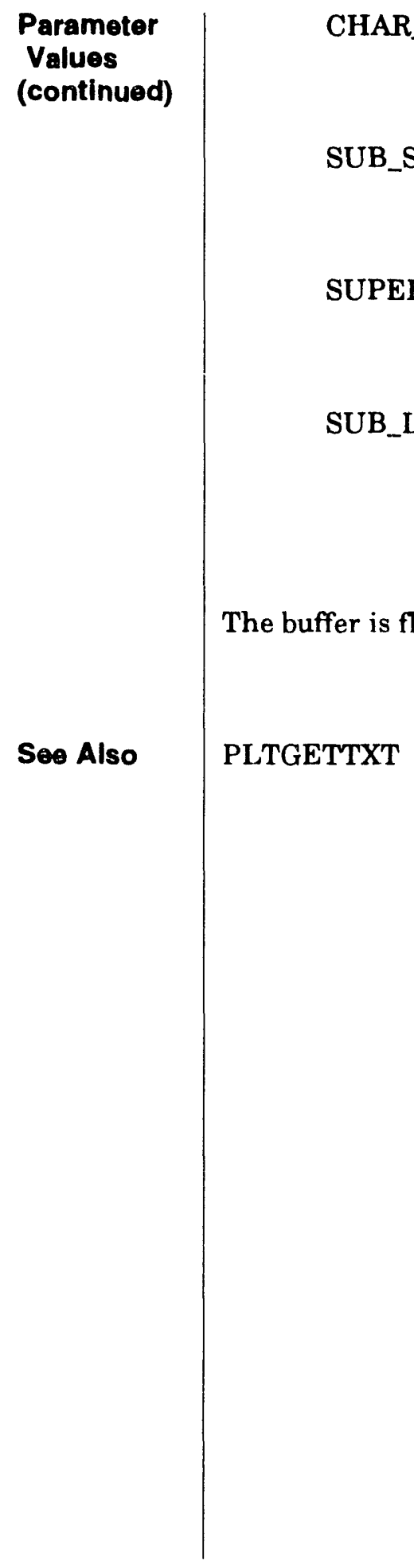


PLT_LIB

Description of Routine Calls

PLTGETTXT

Logical Function

\begin{tabular}{|c|c|c|c|c|}
\hline Name & \multicolumn{4}{|c|}{ PLTGETTXT - Get text parameters } \\
\hline Description & \multicolumn{4}{|c|}{$\begin{array}{l}\text { PLTGETTXT returns the text parameters. See PLTSETTXT for option and buffer } \\
\text { values. }\end{array}$} \\
\hline Syntax & \multicolumn{4}{|c|}{ logical = PLTGETTXT (OPTION, BUFFER) } \\
\hline Variables in & Name & $I / O$ & Type & Description \\
\hline & OPTION & Input & $\operatorname{char}{ }^{*}(*)$ & Desired text parameter. \\
\hline & BUFFER & Output & real array & $\begin{array}{l}\text { Values for text parameters (dependent on } \\
\text { OPTION). }\end{array}$ \\
\hline See Also & \multicolumn{4}{|c|}{ PLTSETTXT } \\
\hline
\end{tabular}




\begin{tabular}{l|l} 
Name & $\begin{array}{l}\text { PLTSAVTXT - Save text parameters } \\
\text { Description }\end{array}$ \\
Syntax & PLTSAVTXT saves the text parameters in a local array. \\
CALLTSAVTXT & \\
Soo Also & PLTRESTXT. PLTRESTXT restores the text parameters saved by PSTSAVTXT.
\end{tabular}


PLT_LIB

Description of Routine Calls

PLTRESTXT

Subroutino

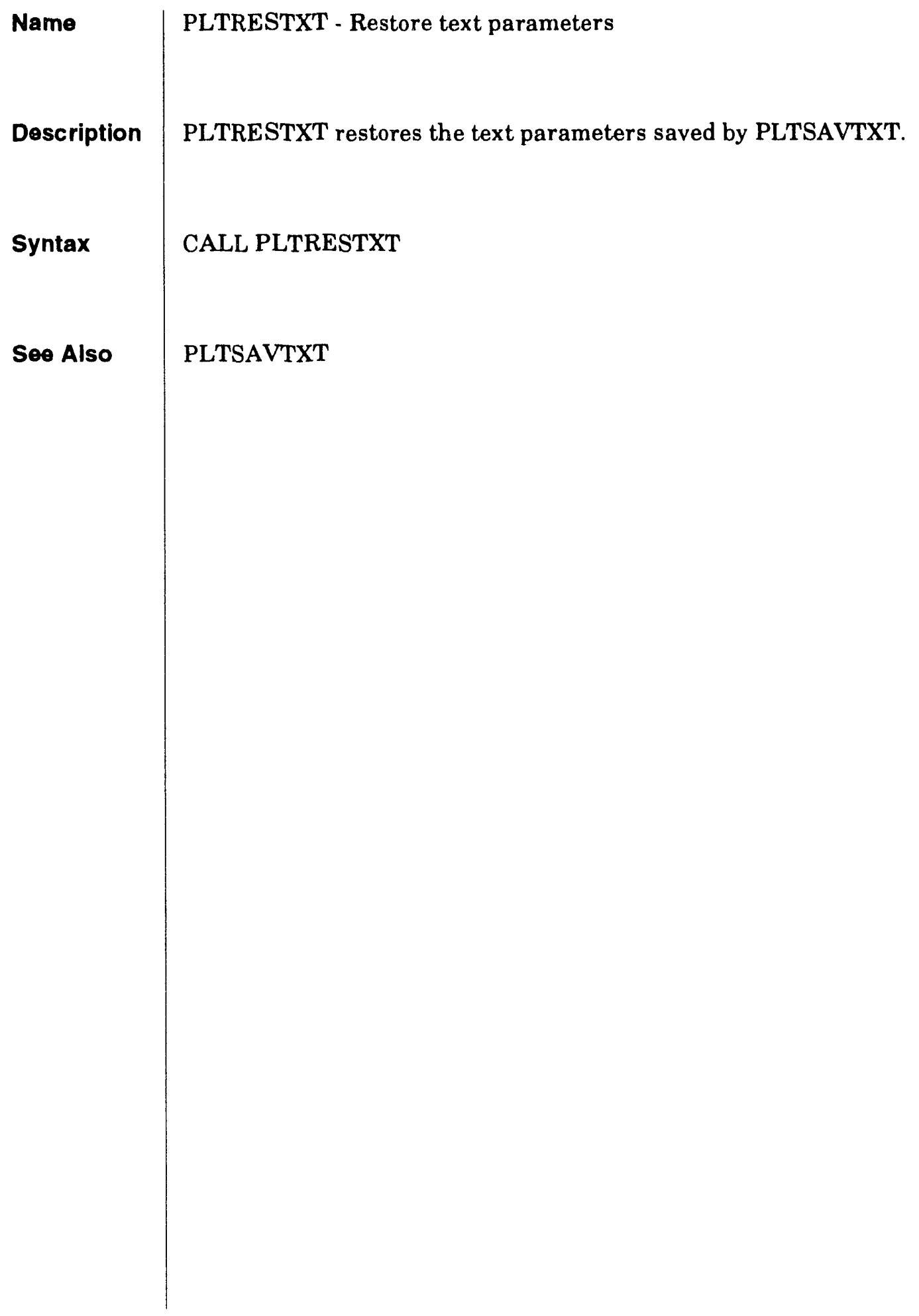




\subsubsection{Hardware and Software Text}

Detailed descriptions of the routines that plot hardware and software text are given on the following pages. The following escape characters may be used with the text plotting routines:

\begin{tabular}{|c|c|}
\hline $\begin{array}{l}\text { Escape } \\
\text { Character }\end{array}$ & $\begin{array}{l}\text { Action or Character } \\
\text { Generated on Plot }\end{array}$ \\
\hline ICR & - carriage return \\
\hline$\backslash L F$ & - line feed \\
\hline$\backslash \mathrm{CL}$ & - carriage return and line feed \\
\hline$\backslash \wedge$ & - superscript (no space required) \\
\hline 1- & - normal (no space required) \\
\hline$I_{-}$ & - subscript (no space required) \\
\hline$\backslash \mathrm{CLO}$ & - centered logo \\
\hline$\backslash \mathrm{CSQ}$ & - centered square \\
\hline$\backslash \mathrm{CDI}$ & - centered diamond \\
\hline$\backslash \mathrm{CCS}$ & - centered cross \\
\hline $\mathrm{ICX}$ & - centered X \\
\hline ICTR & - centered triangle \\
\hline$\backslash \mathrm{CCI}$ & - centered circle \\
\hline$\backslash \mathrm{CDO}$ & - centered dot \\
\hline \DDLINE & - dot-dash line \\
\hline$\backslash \mathrm{DLINE}$ & - dotted line \\
\hline$\backslash$ LDLINE & - long dash line \\
\hline$\backslash \mathrm{LO}$ & $-\log 0$ \\
\hline IMDLINE & - medium dash line \\
\hline \SDLINE & - solid line \\
\hline$\backslash S Q$ & - square \\
\hline$\backslash D I$ & - diamond \\
\hline $1 \mathrm{CS}$ & - cross \\
\hline$\backslash \mathrm{X}$ & $-\mathrm{X}$ \\
\hline ITR & - triangle \\
\hline$\backslash \mathrm{CI}$ & - circle \\
\hline$\backslash \mathrm{DO}$ & $-\operatorname{dot}$ \\
\hline$\backslash$ PLUSMIN & - plus or minus \\
\hline \GR & - start Greek font \\
\hline$\backslash \mathrm{ENG}$ & - return to normal font \\
\hline$\backslash \mathrm{LEQ}$ & - less than or equal to \\
\hline IGEQ & - greater than or equal to \\
\hline$\backslash N E Q$ & not equal to \\
\hline IPRIME & - prime \\
\hline \NLEQ & - not less than or equal to \\
\hline \NGEQ & - not greater than or equal to \\
\hline$\backslash \mathrm{LL}$ & - much less than \\
\hline$\backslash \mathrm{GG}$ & - much greater than \\
\hline$\backslash$ SUM & - summation \\
\hline$\backslash N L T$ & not less than \\
\hline$\backslash N G T$ & - not greater than \\
\hline IAPPROX & approximation sign \\
\hline 11 & - single backslash \\
\hline
\end{tabular}




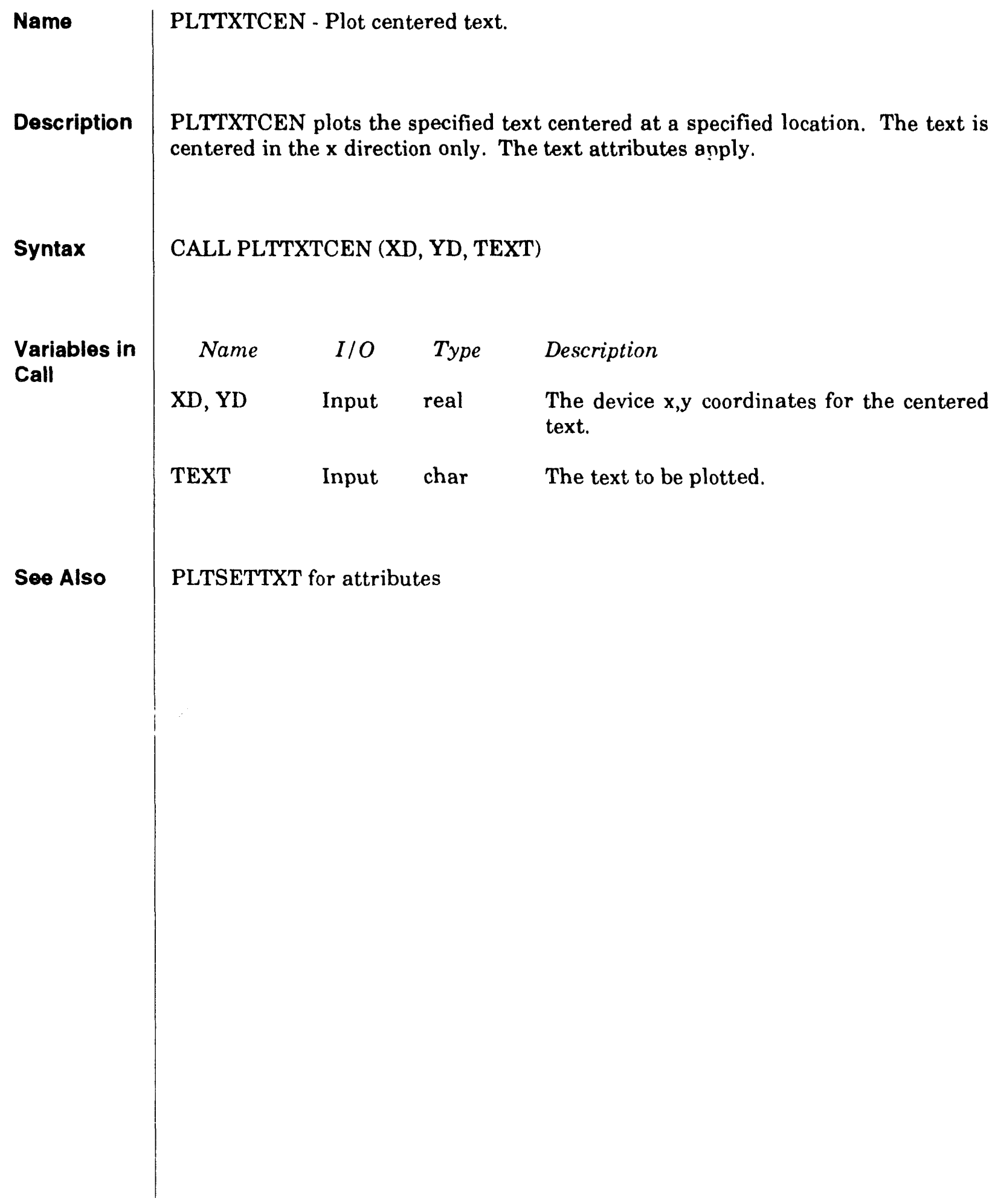




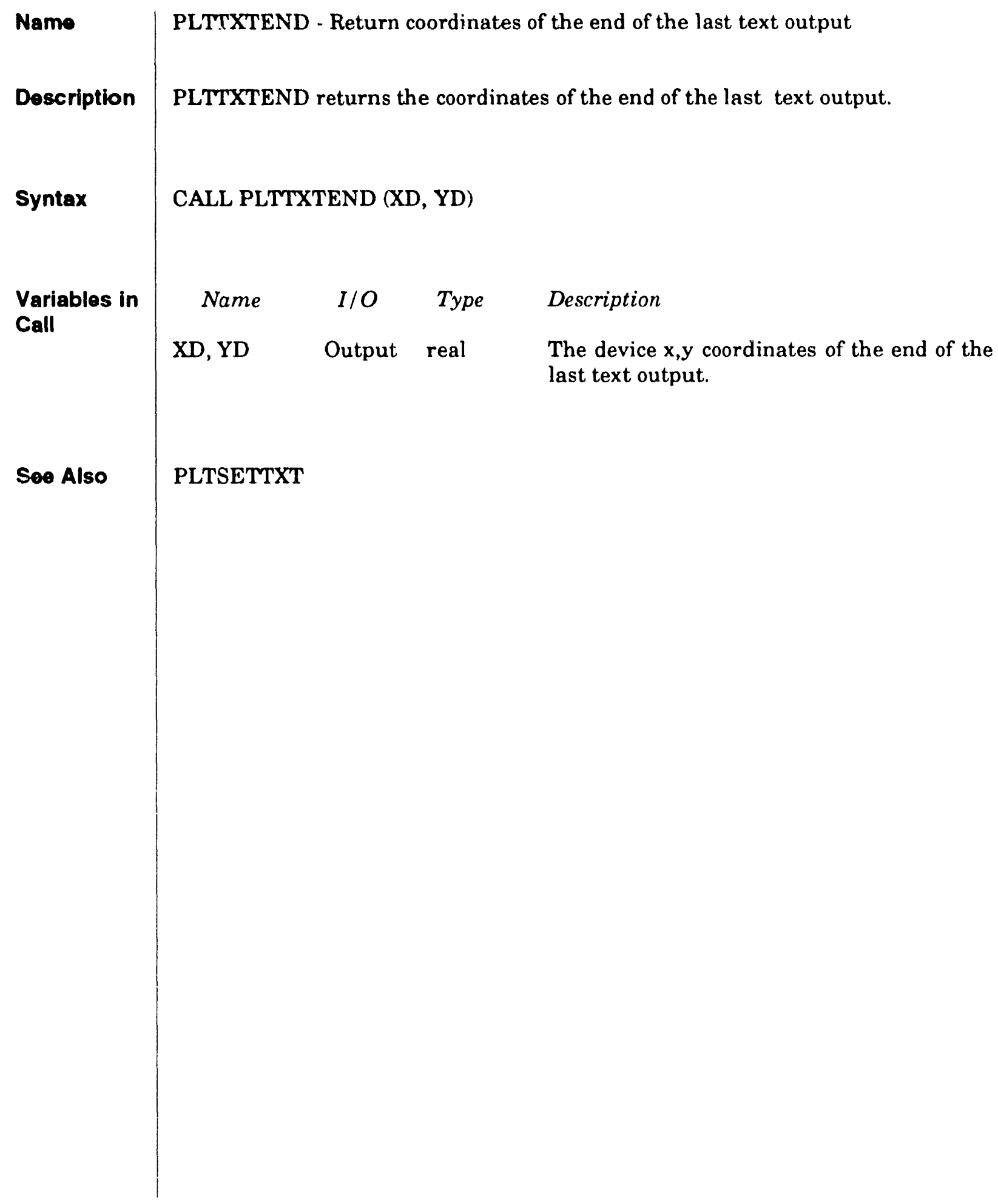




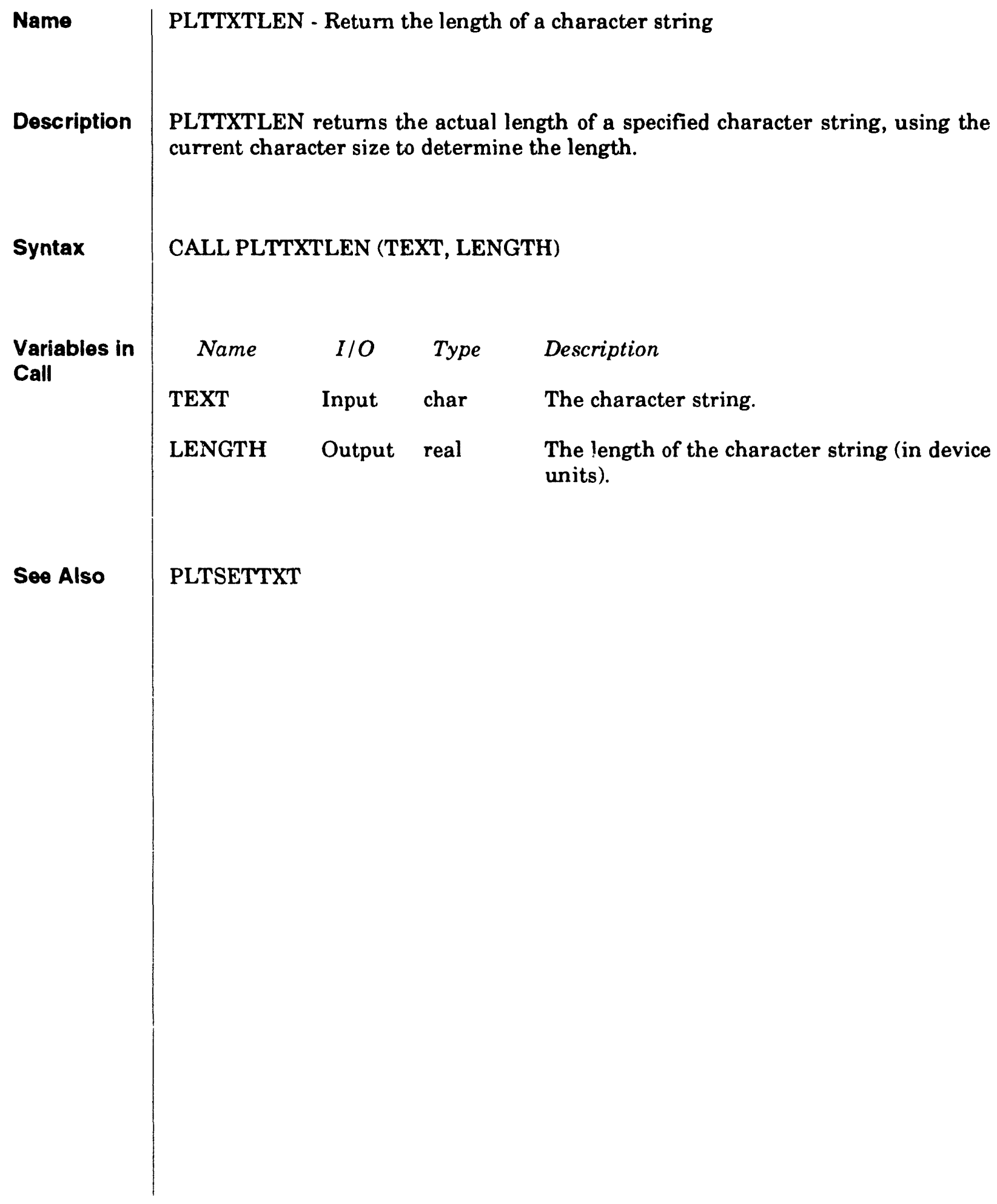


PLT_LIB

Hardware and Software Text

\section{PLTTXTNXT}

Subroutine

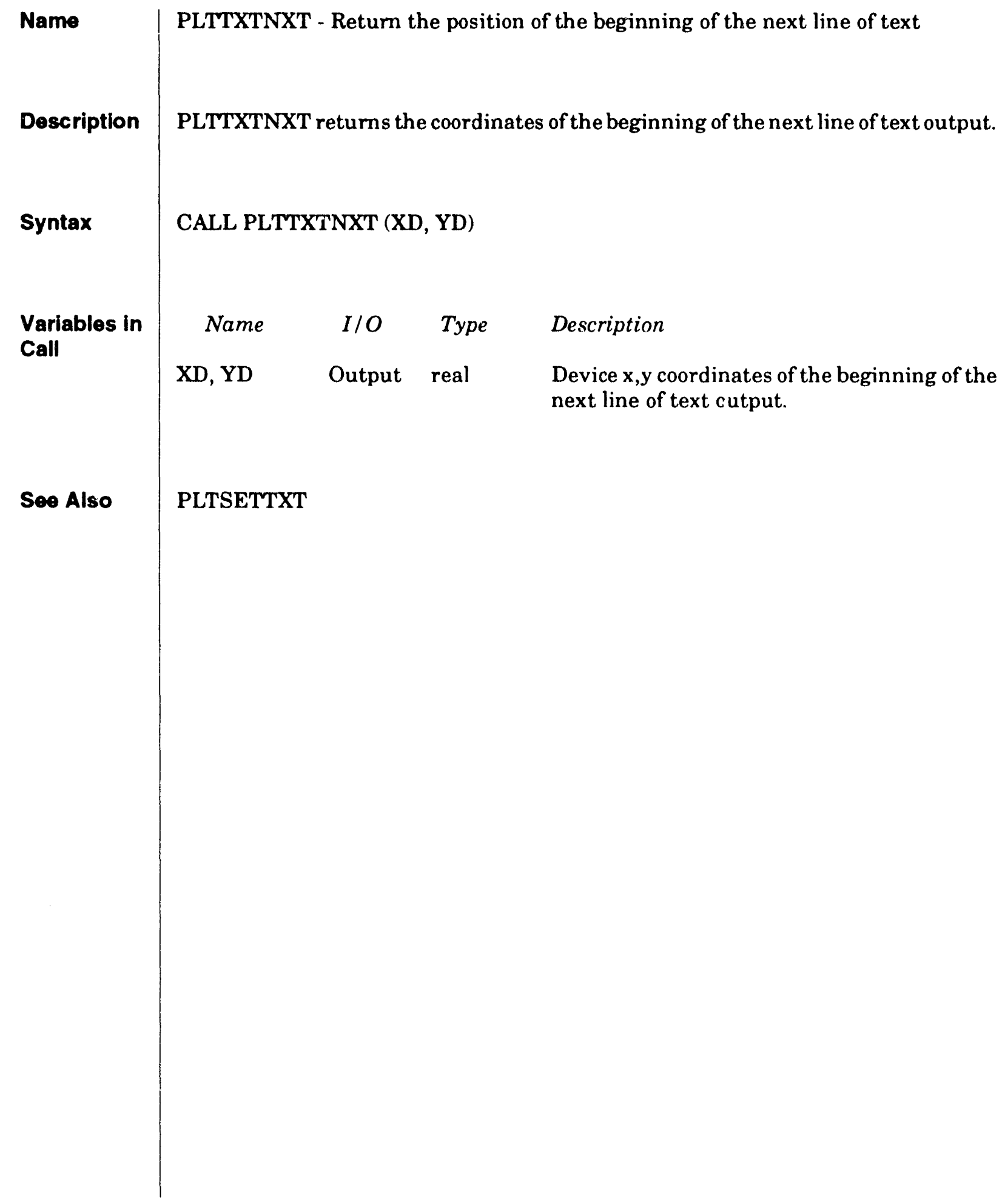




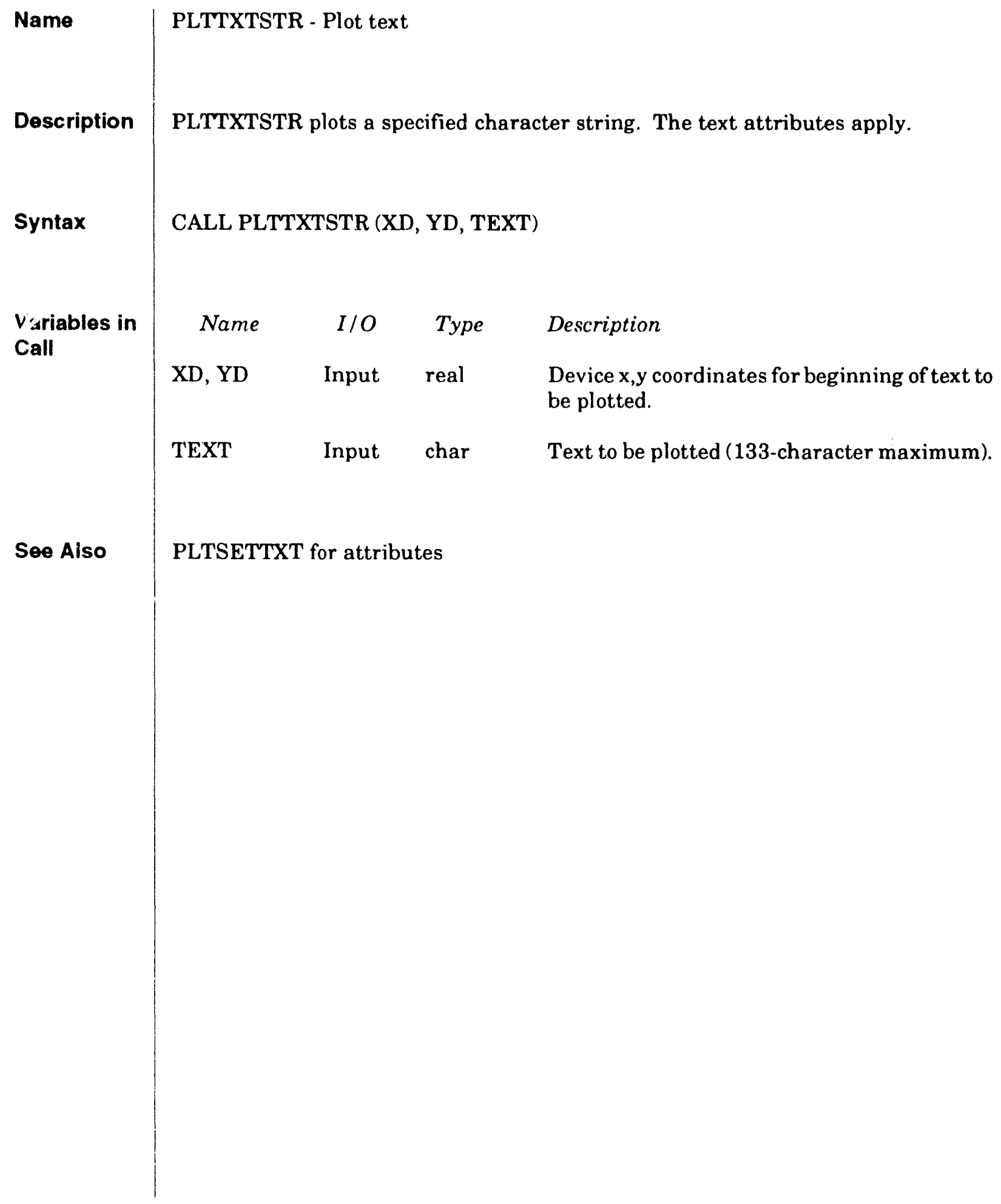




\subsubsection{Two-Dimensional Graphing}

Detailed descriptions of the routines used for two-dimensional graphing are given on the following pages. 
PLT_LIB

Description of Routine Calls

PLTARROW

Logical Function

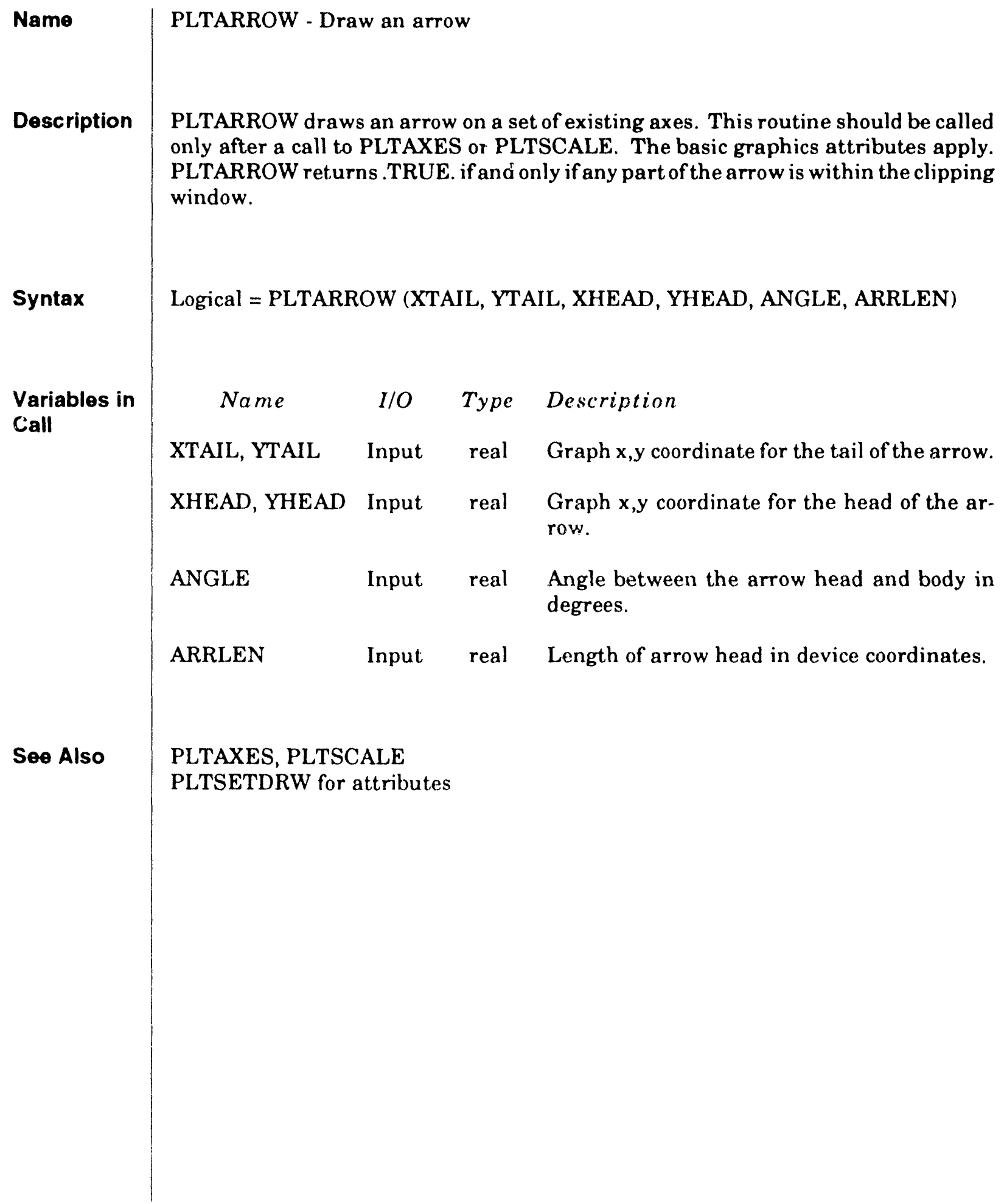




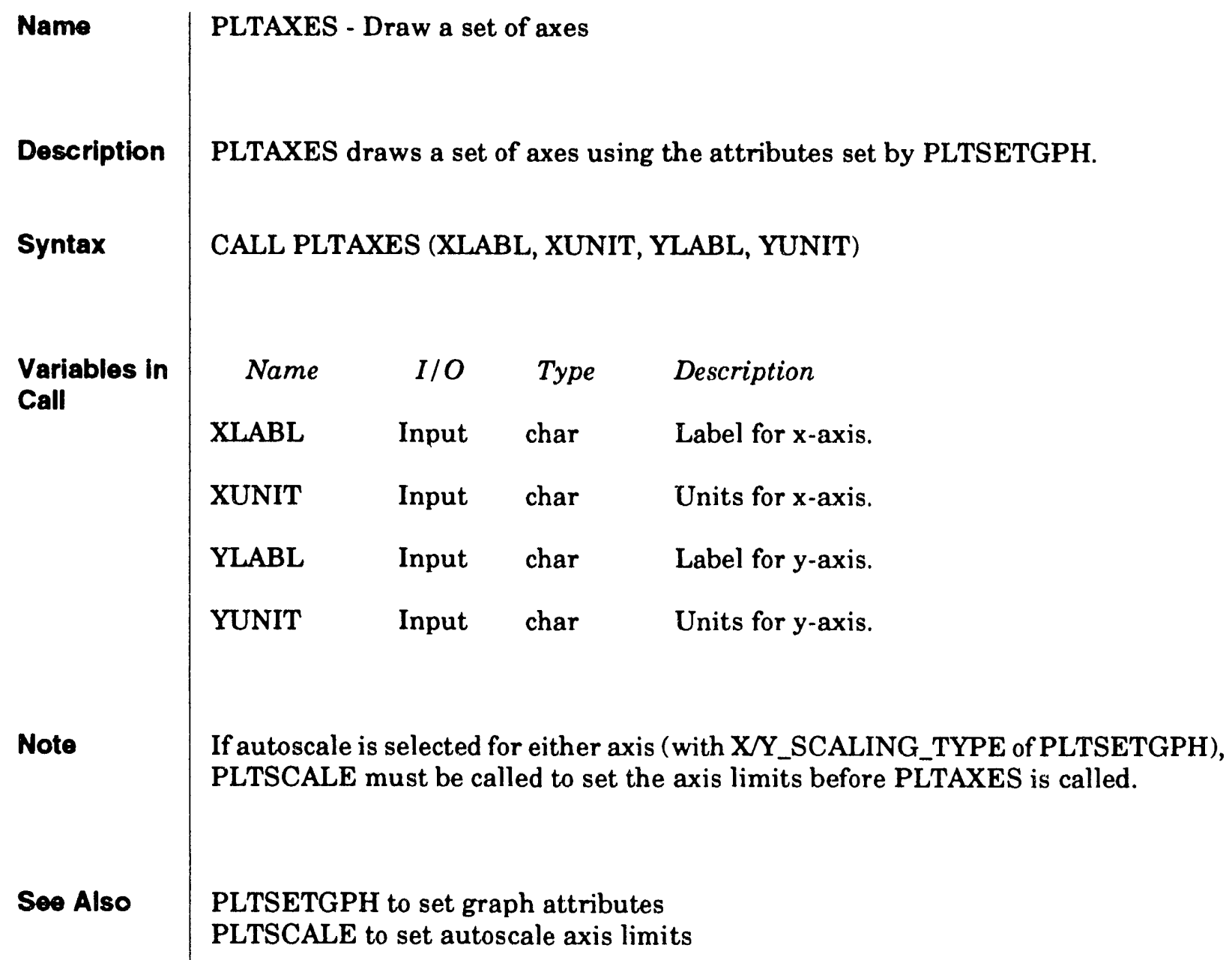




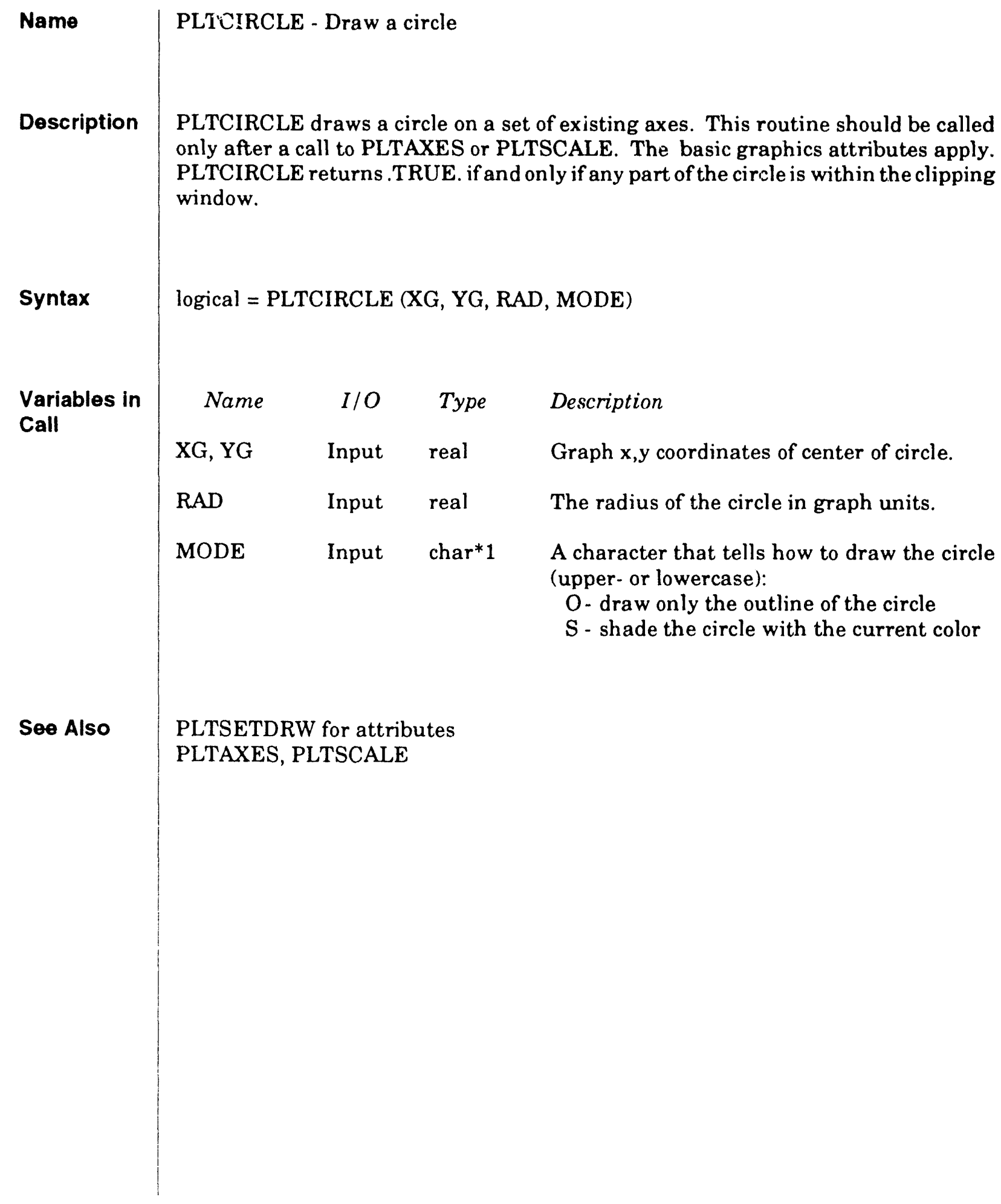




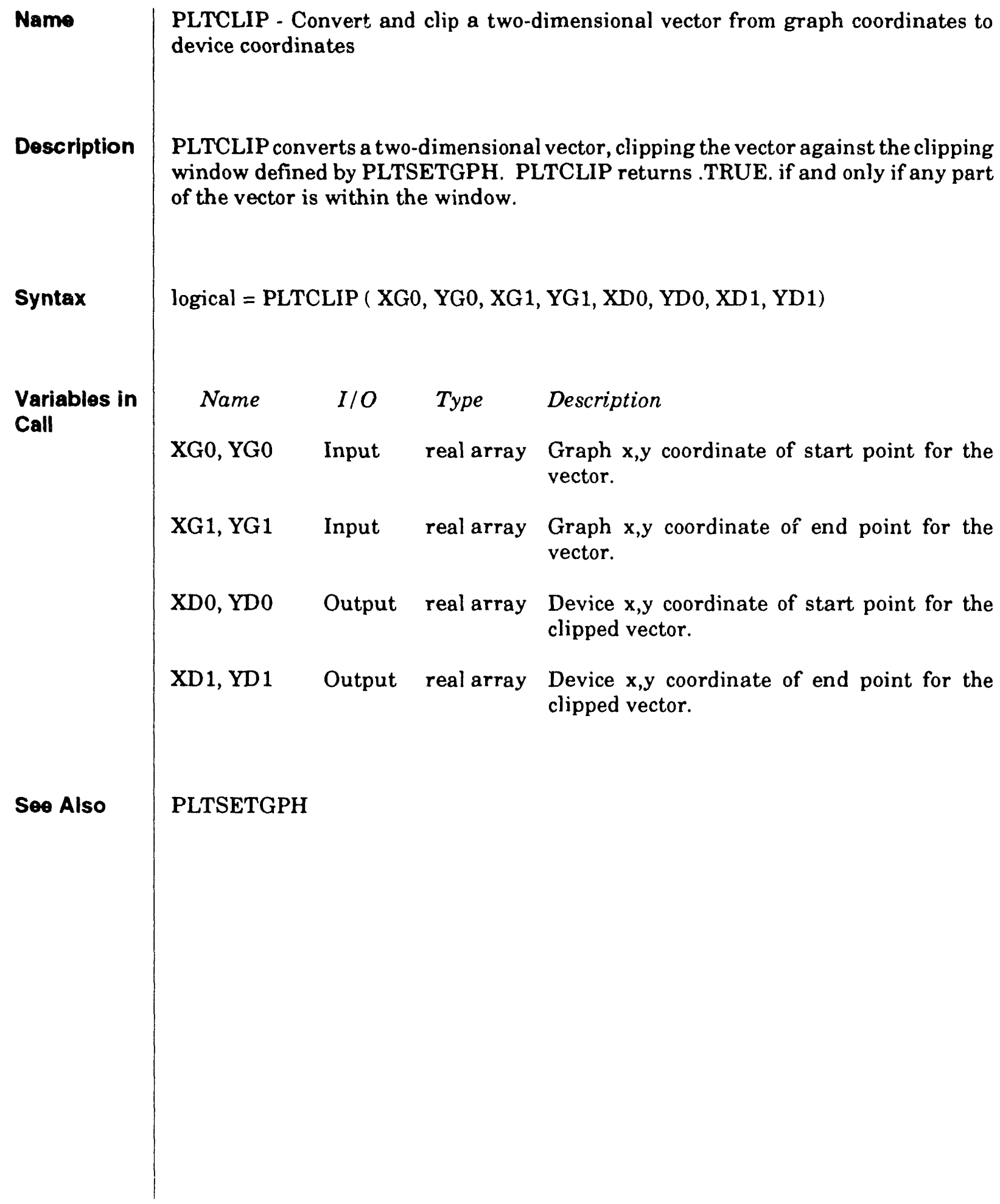




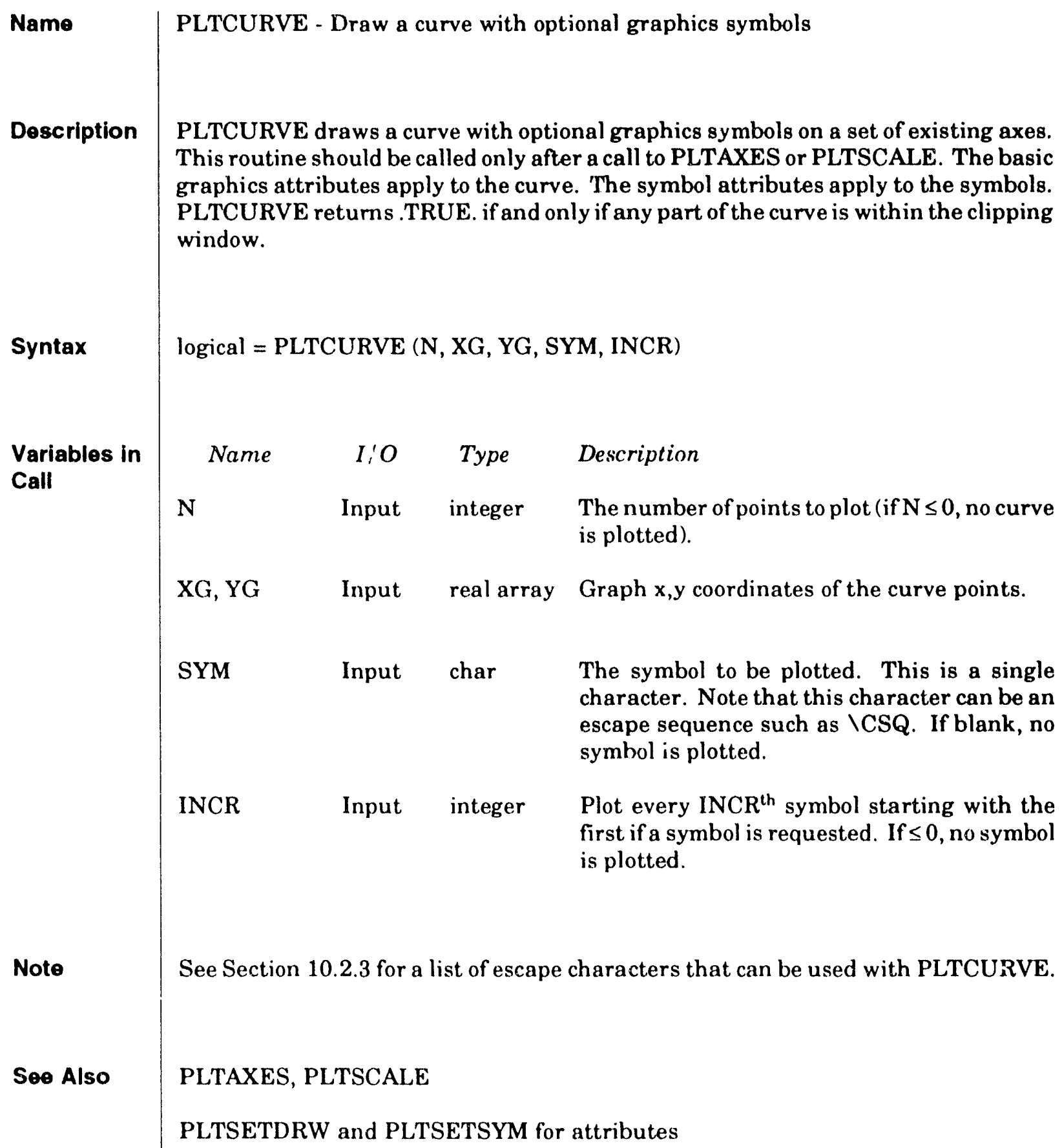




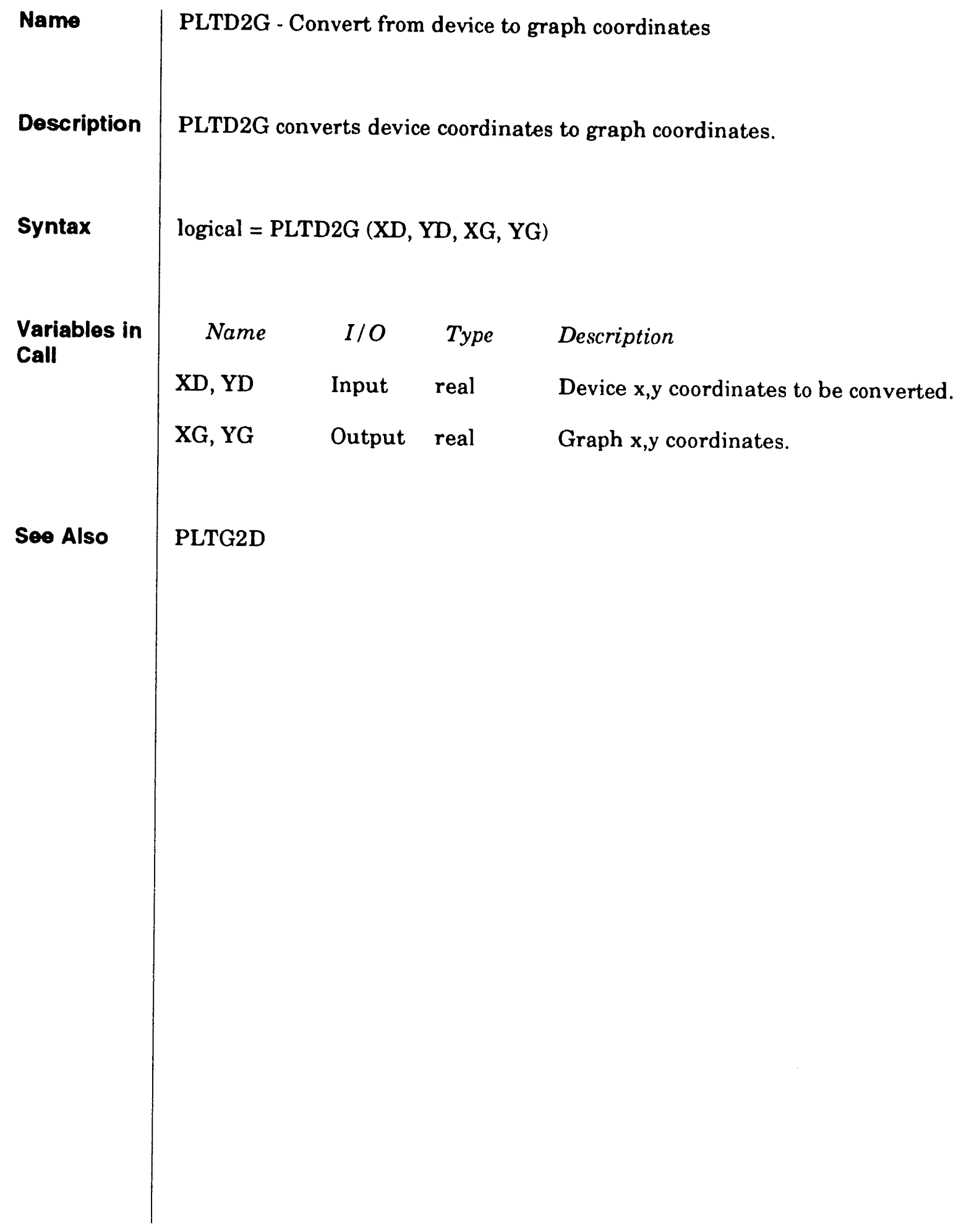




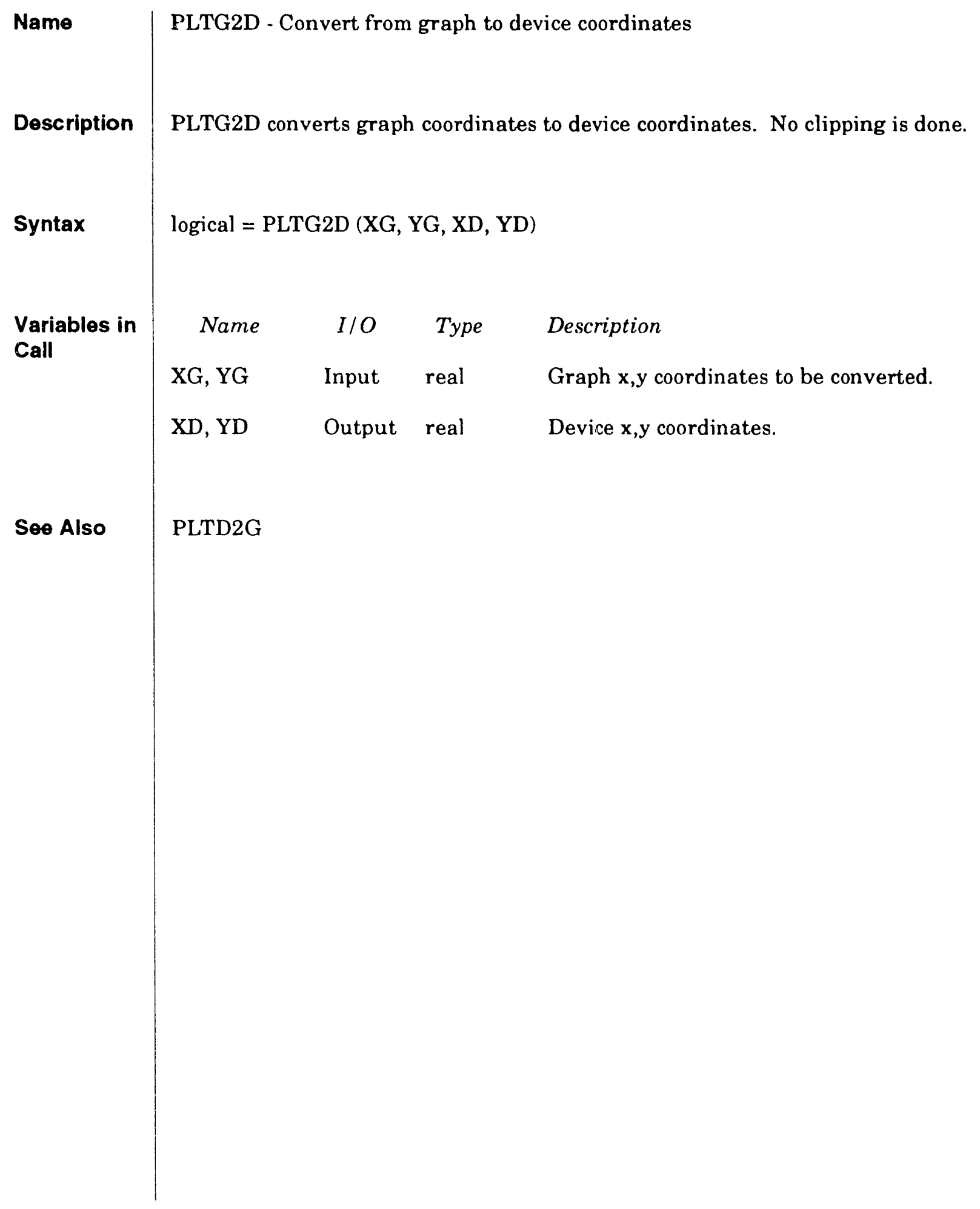


PLT LIB

\begin{tabular}{|c|c|c|c|c|}
\hline Name & \multicolumn{4}{|c|}{ PLTINI - Return appropriate number for axis scaling (within range) } \\
\hline Doscription & \multicolumn{4}{|c|}{$\begin{array}{l}\text { PLTINI returns appropriate numbers to use in axis scaling. These numbers are } \\
\text { inside the XMIN-to-XMAX interval so that XMIN }<\text { RSTART and REND }<\text { XMAX. } \\
\text { (PLTINO returns numbers that extend outside the XMIN-to-XMAX interval.) }\end{array}$} \\
\hline Syntax & \multicolumn{4}{|c|}{ CALL PLTINI (XMIN, XMAX, RSTART, REND, INTER, IEXP, NMIN) } \\
\hline \multirow{8}{*}{$\begin{array}{l}\text { Variables in } \\
\text { Call }\end{array}$} & Name & $1 / O$ & Type & Description \\
\hline & XMIN & Input & real & The minimum value for the interval. \\
\hline & XMAX & Input & real & The maximum value for the interval. \\
\hline & RSTART & Output & real & Starting value for scaled axis $\left(\times 10^{-I E X P}\right)$ \\
\hline & REND & Output & real & Ending value for scaled axis $\left(\times 10^{-1 E X P}\right)$ \\
\hline & INTER & Output & real & Interval for scaled axis $\left(\times 10^{-\mathrm{IEXP}}\right)$ \\
\hline & IEXP & Output & real & Exponent to be used in axis scaling. \\
\hline & NMIN & Output & integer & Number of minor intervals for scaled axis. \\
\hline
\end{tabular}

Soe Also PLTINO 


\begin{tabular}{|c|c|c|c|c|}
\hline Name & \multicolumn{4}{|c|}{ PLTINO - Return appropriate number for axis scaling (extends outside range) } \\
\hline Description & \multicolumn{4}{|c|}{$\begin{array}{l}\text { PLTINO returns apprupriate numbers to use in axis scaling. These number extend } \\
\text { outside the XMIN-to-XMAX interval so that RSTART }<\text { XMIN and XMAX }<\text { REND. } \\
\text { (PLTINI returns numbers inside the XMIN-to-XMAX interval.) }\end{array}$} \\
\hline Syntax & \multicolumn{4}{|c|}{ CALL PLTINO (XMIN, XMAX, RSTART, REND, INTER, IEXP, NMIN) } \\
\hline \multirow{8}{*}{$\begin{array}{l}\text { Variables in } \\
\text { Call }\end{array}$} & Name & $I / O$ & Type & Description \\
\hline & XMIN & Input & real & The minimum value for the interval. \\
\hline & XMAX & Input & real & The maximum value for the interval. \\
\hline & RSTART & Output & real & Starting value for scaled axis $\left(\times 10^{-\mathrm{IEXP}}\right)$. \\
\hline & REND & Output & real & Ending value for scaled axis $\left(\times 10^{- \text {IEXP }}\right)$. \\
\hline & INTER & Output & real & Interval for scaled axis $\left(\times 10^{-\mathrm{IEXP}}\right)$ \\
\hline & IEXP & Output & real & Exponent to be used in axis scaling. \\
\hline & NMIN & Output & integer & Number of minor intervals for scaled axis. \\
\hline
\end{tabular}




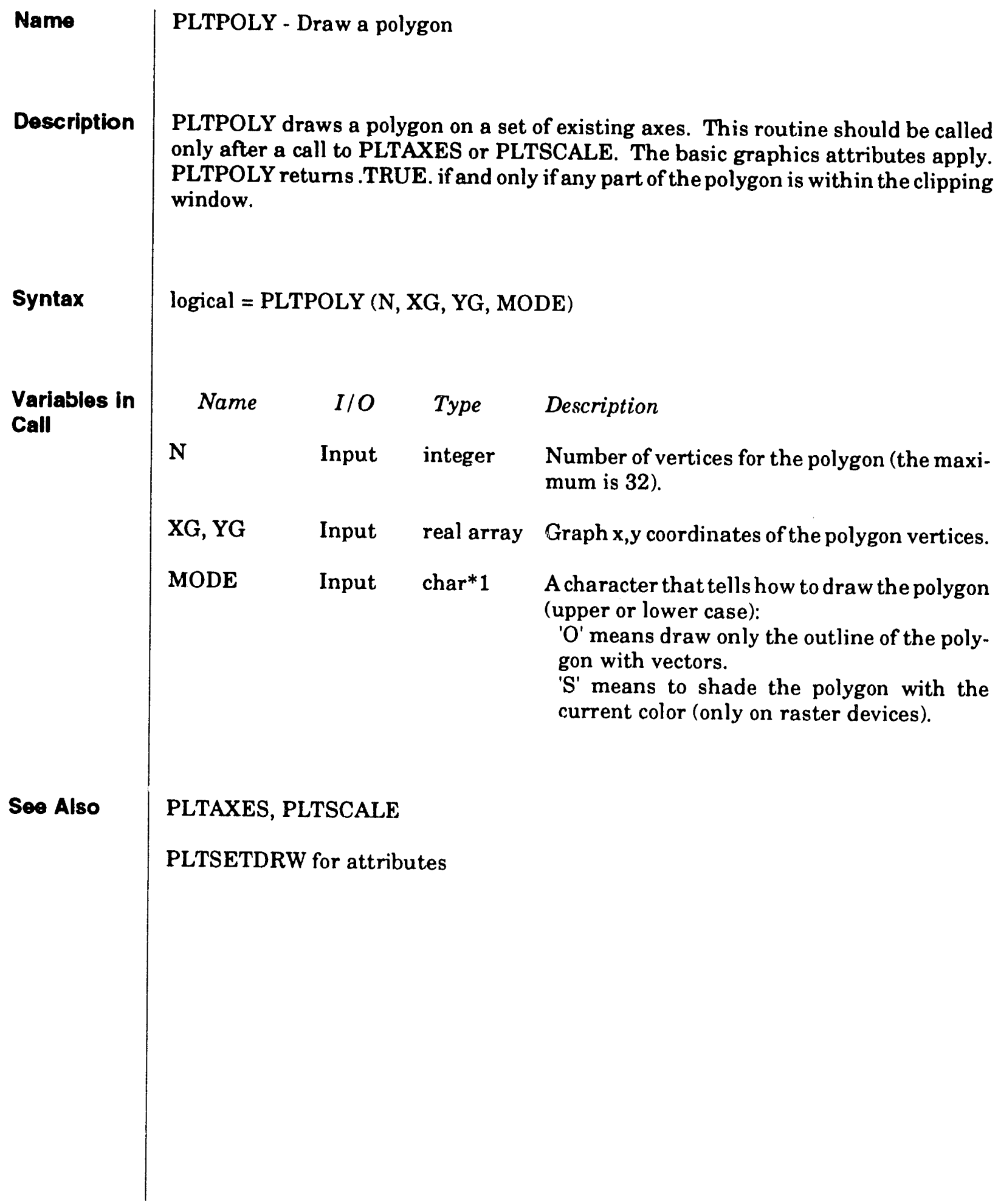




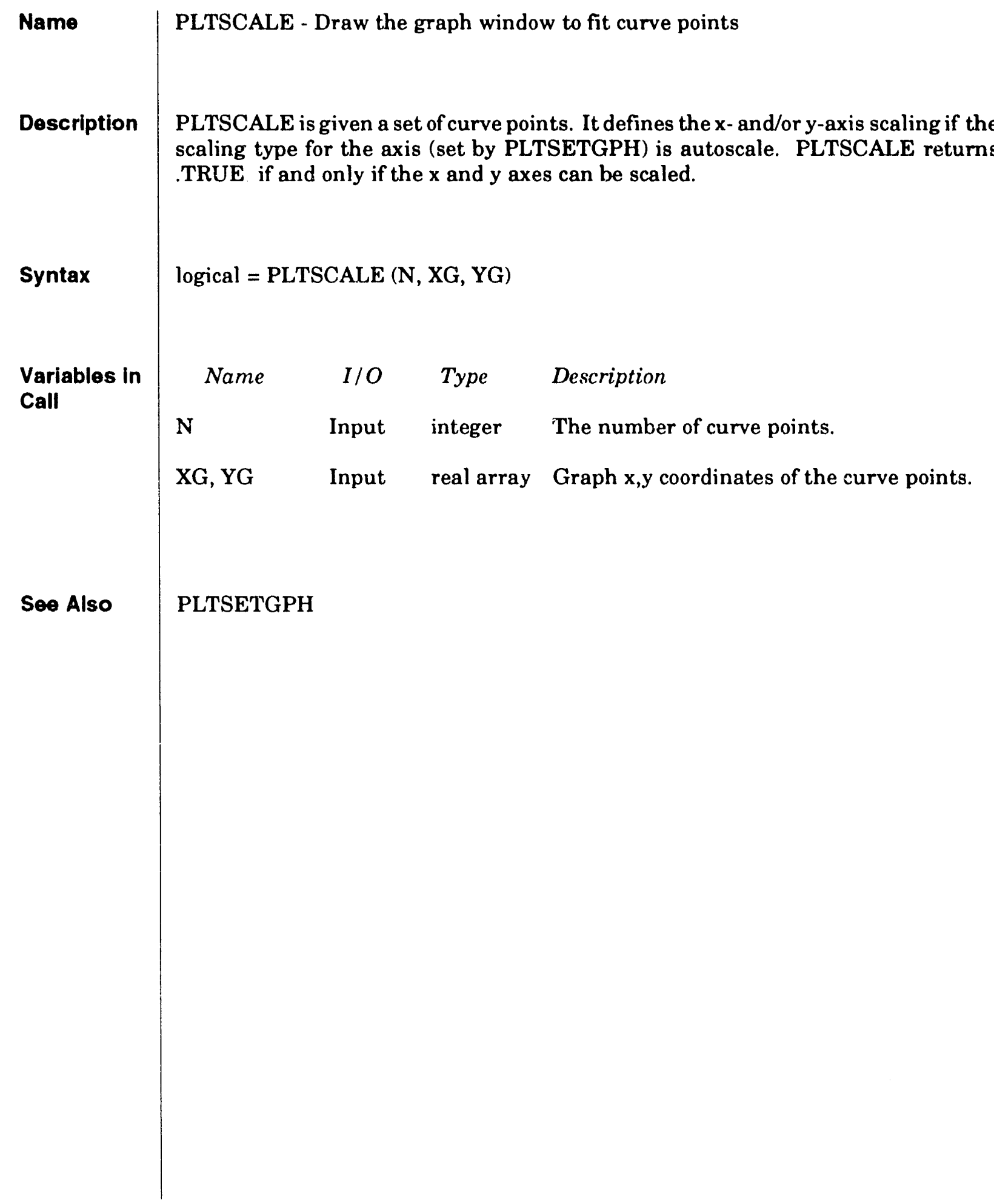




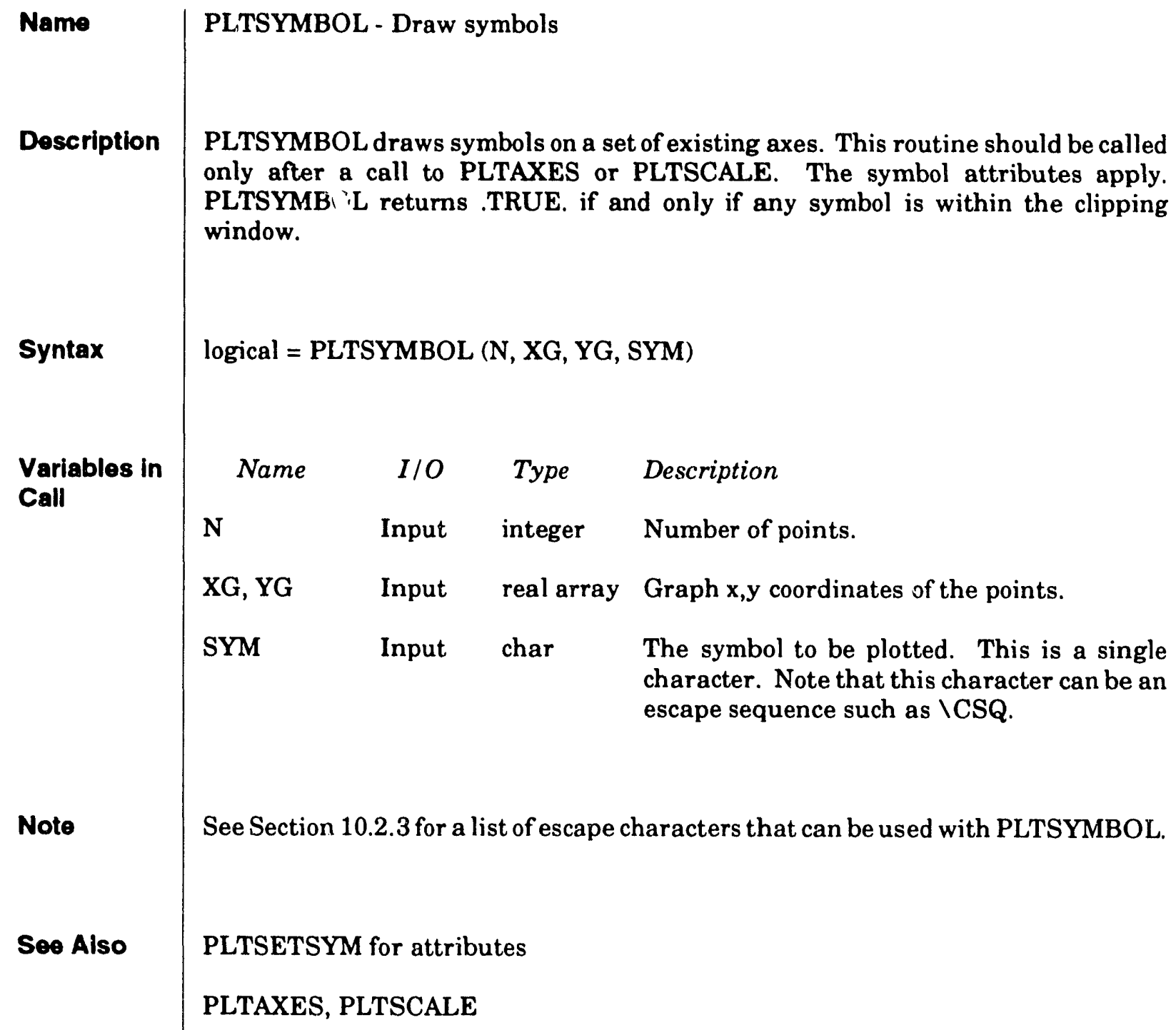




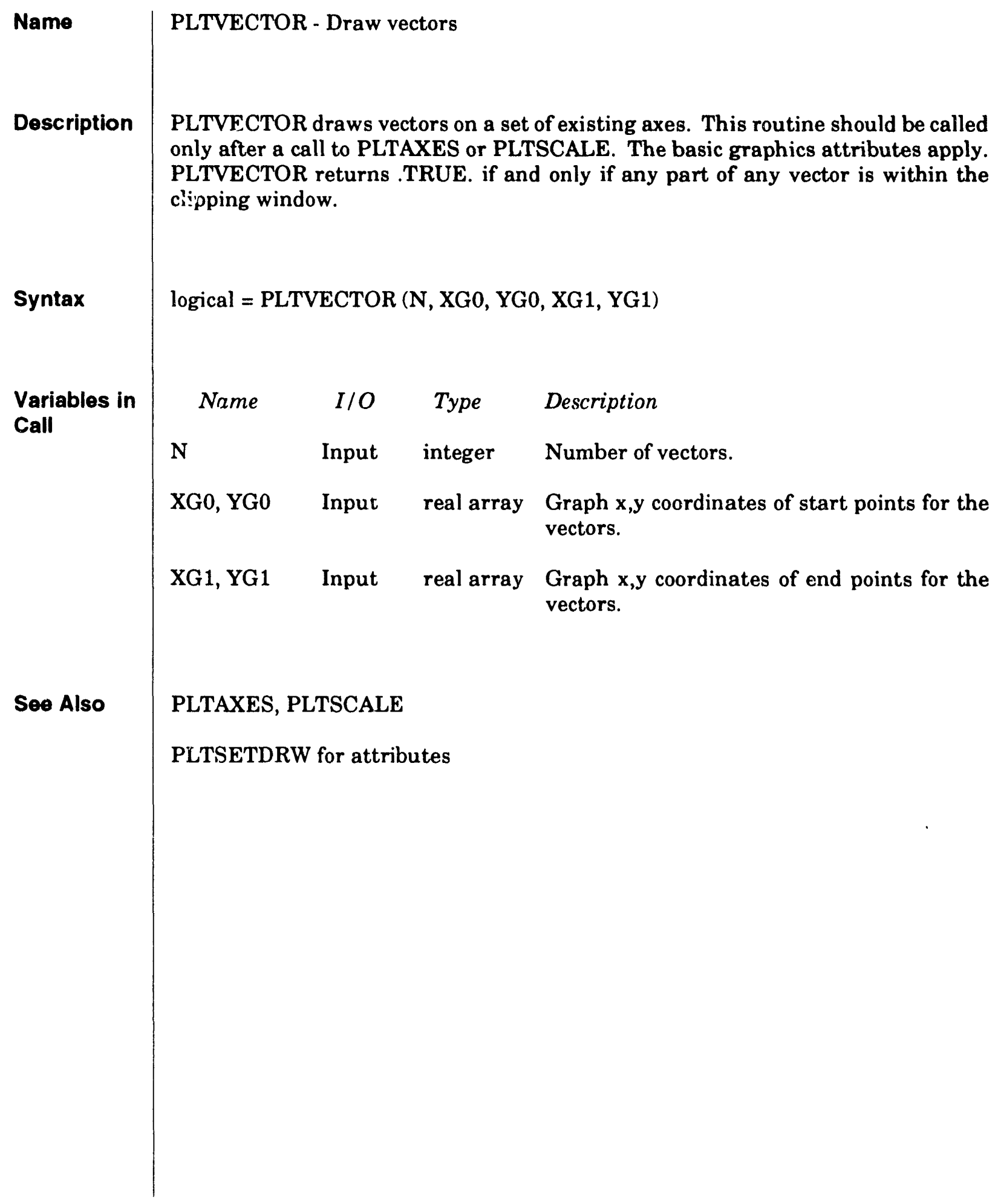


PLT_LIB

Device Coordinate Plotting Primitives

\subsubsection{Device Coordinate Plotting Primitives}

Detailed descriptions of the routines that provide device coordinate plotting primitives are given on the following pages. 


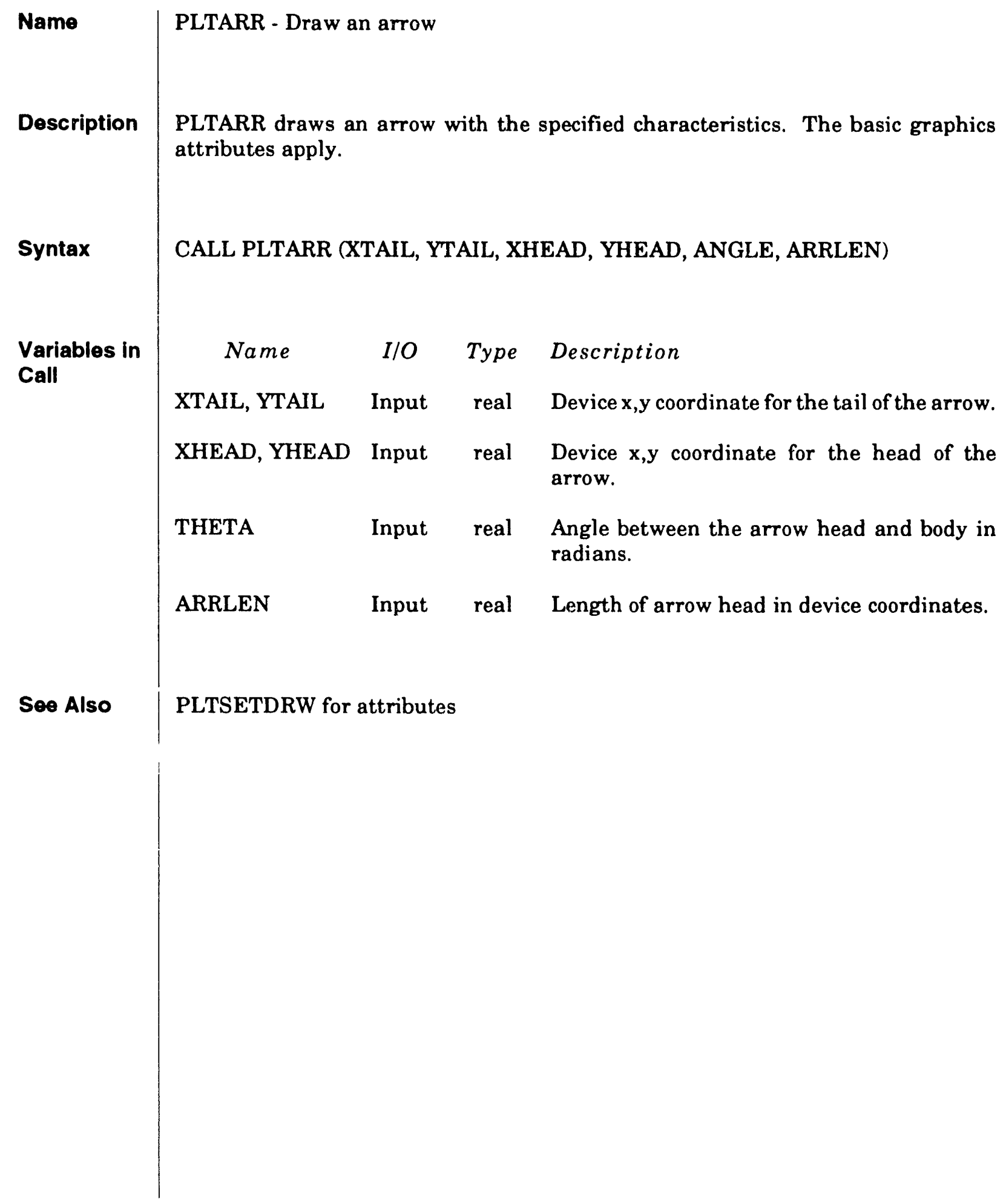


Name
Description

Syntax

Variables in Call
PLTAXS - Draw a linear axis

PLTAXS draws a linear axis (either $x$ or $y$, depending on TYPE). The axis attributes are set by PLTSETGPH.

CALL PLTAXS (X, Y, XLENG, YLENG, TYPE, XMIN, XMAX, XSTAR'T, NDEC, INTER, MININT, LABEL, UNITS, EXP)

\begin{tabular}{|c|c|c|c|}
\hline Name & $I / O$ & Type & Description \\
\hline $\mathrm{X}, \mathrm{Y}$ & Input & real & Origin of the axis in device \\
\hline XLENG & Input & real & Length of $x$-axis in devi \\
\hline YLENG & Input & real & $\begin{array}{l}\text { Length of } y \text {-axis in device coo } \\
\text { if frame around plot is desir }\end{array}$ \\
\hline TYPE & Input & char & Type of axis; either ' $x$ ' or ' $y$ '. \\
\hline XMIN & Input & real & Minimum number on axis. \\
\hline XMAX & Input & real & Maximum number on axis. \\
\hline
\end{tabular}

XSTART Input real Suitable number between XMIN and XMAX that tells where to start the first major interval.

NDEC Input integer Number of decimal places.

INTER Input integer The suitable interval between major ticks.

MININT Input integer Suitable number of minor intervals between minor ticks.

LABEL Input char Label for axis.

UNITS Input char Units on axis.

EXP Input real Exponent for axis.

See Also

PLTSETGPH to set graph attributes 
PLT_LIB

Description of Routine Calls

PLTDRW

Subroutine

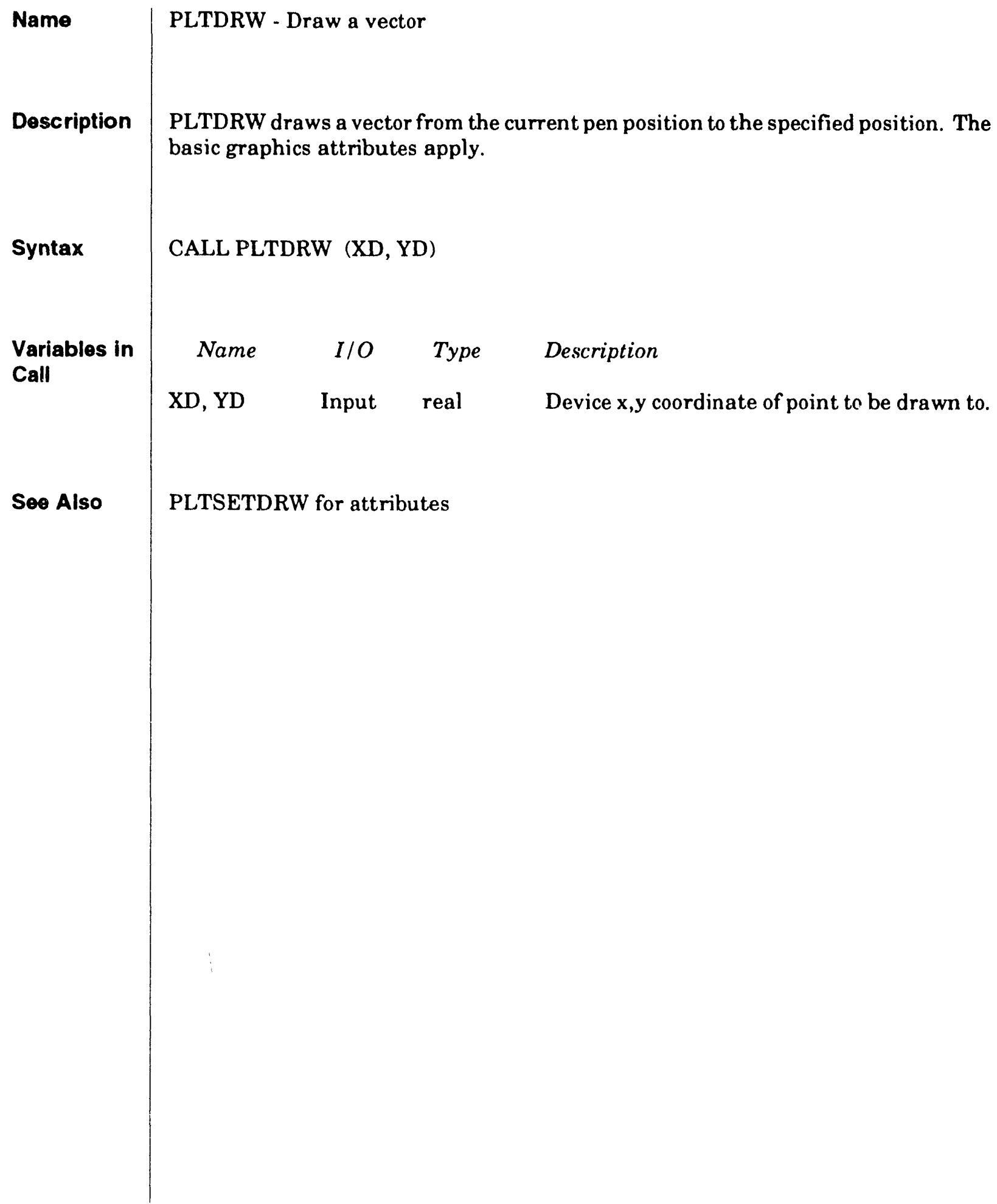




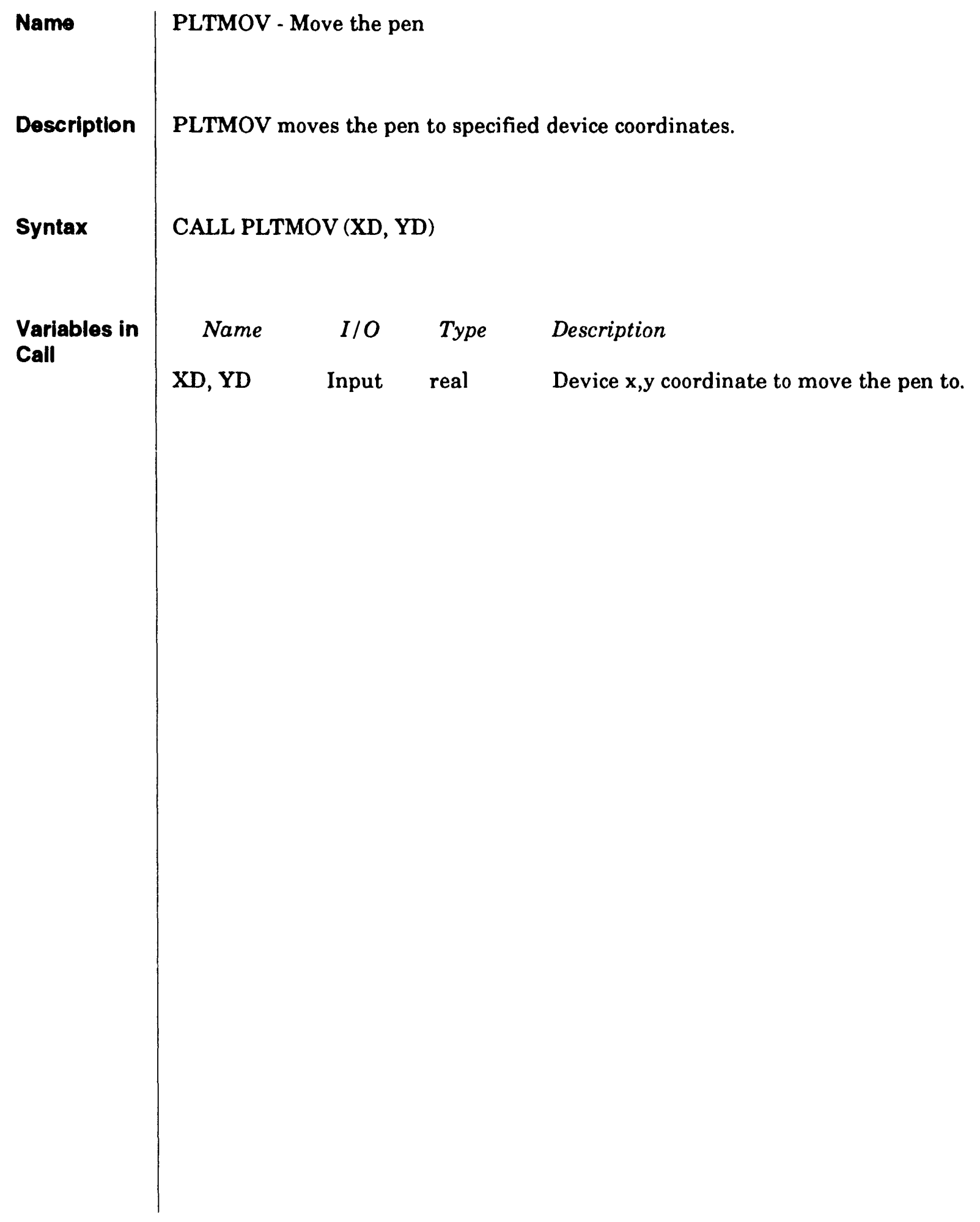


PLT_LIB

Description of Routine Calls

PLTPLY

Subroutine

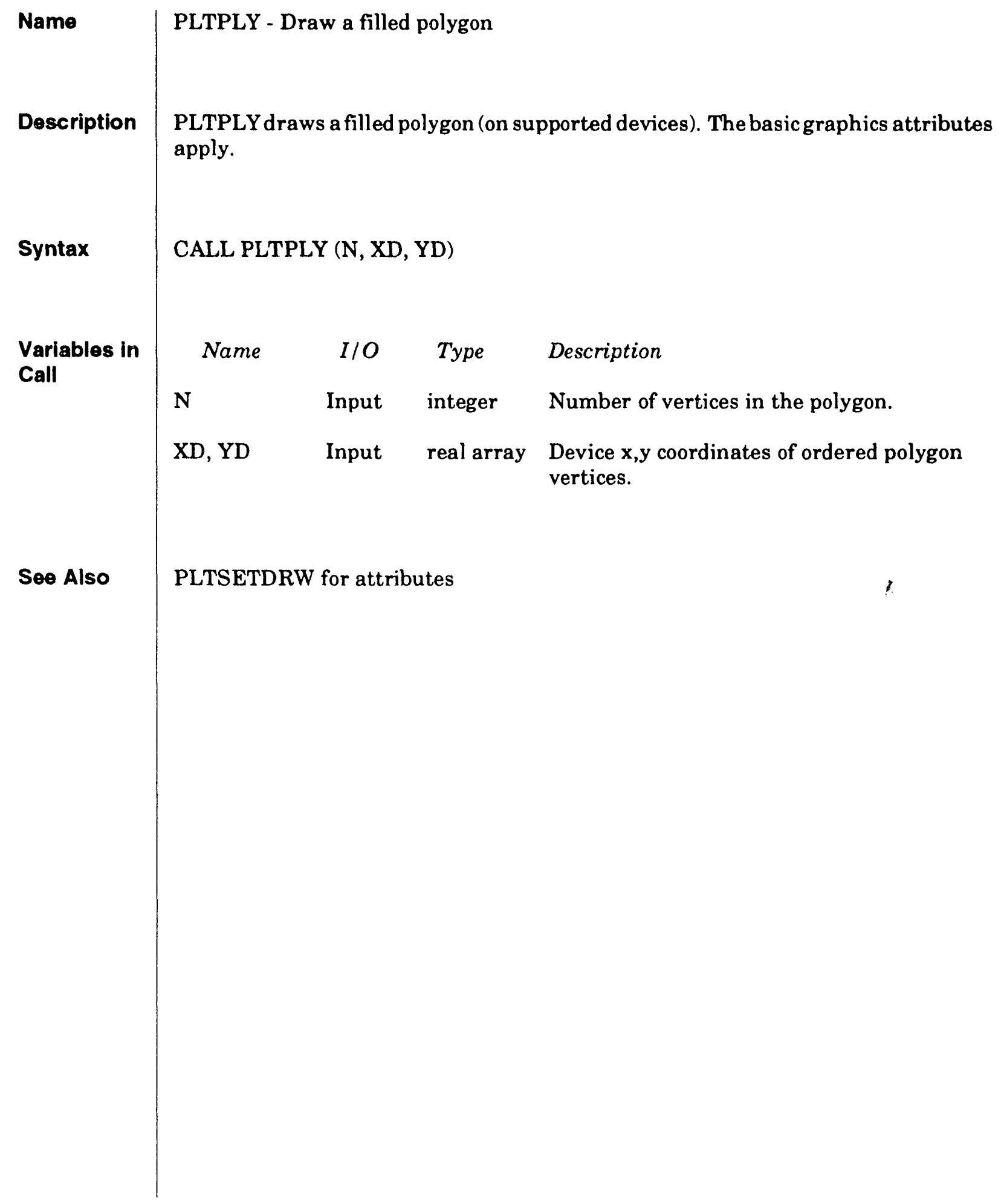




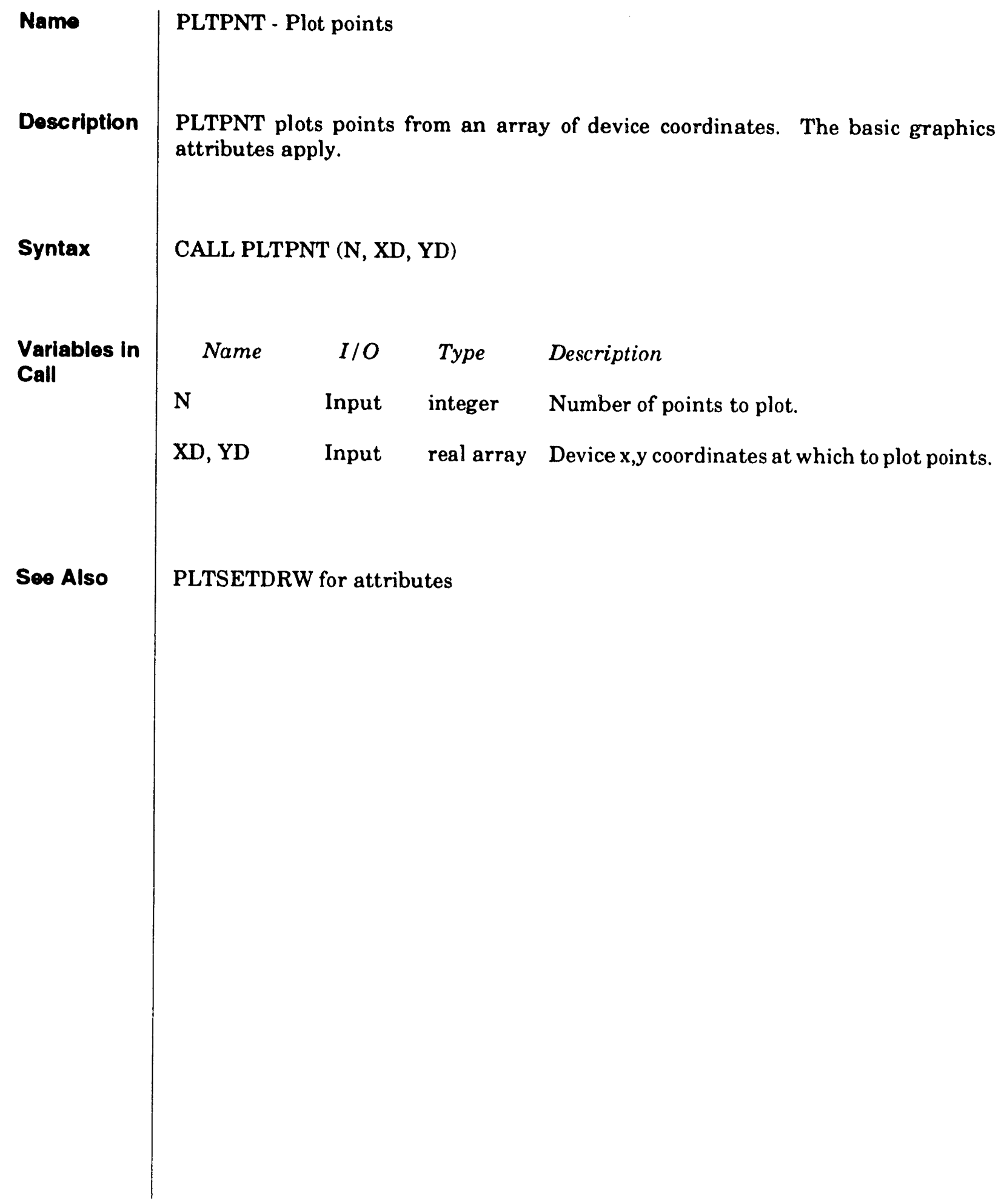




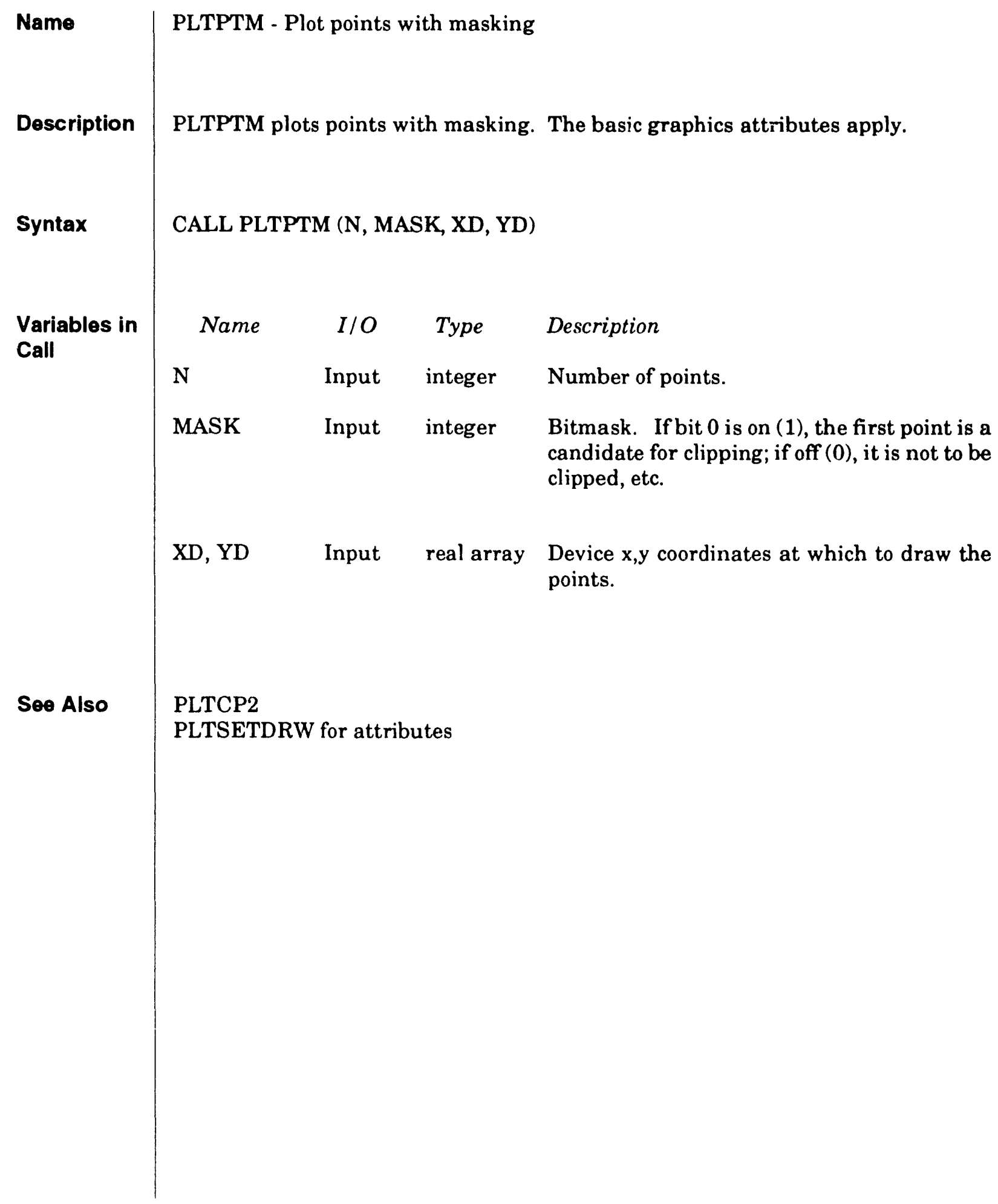




\section{PLTRXY}

subroutine

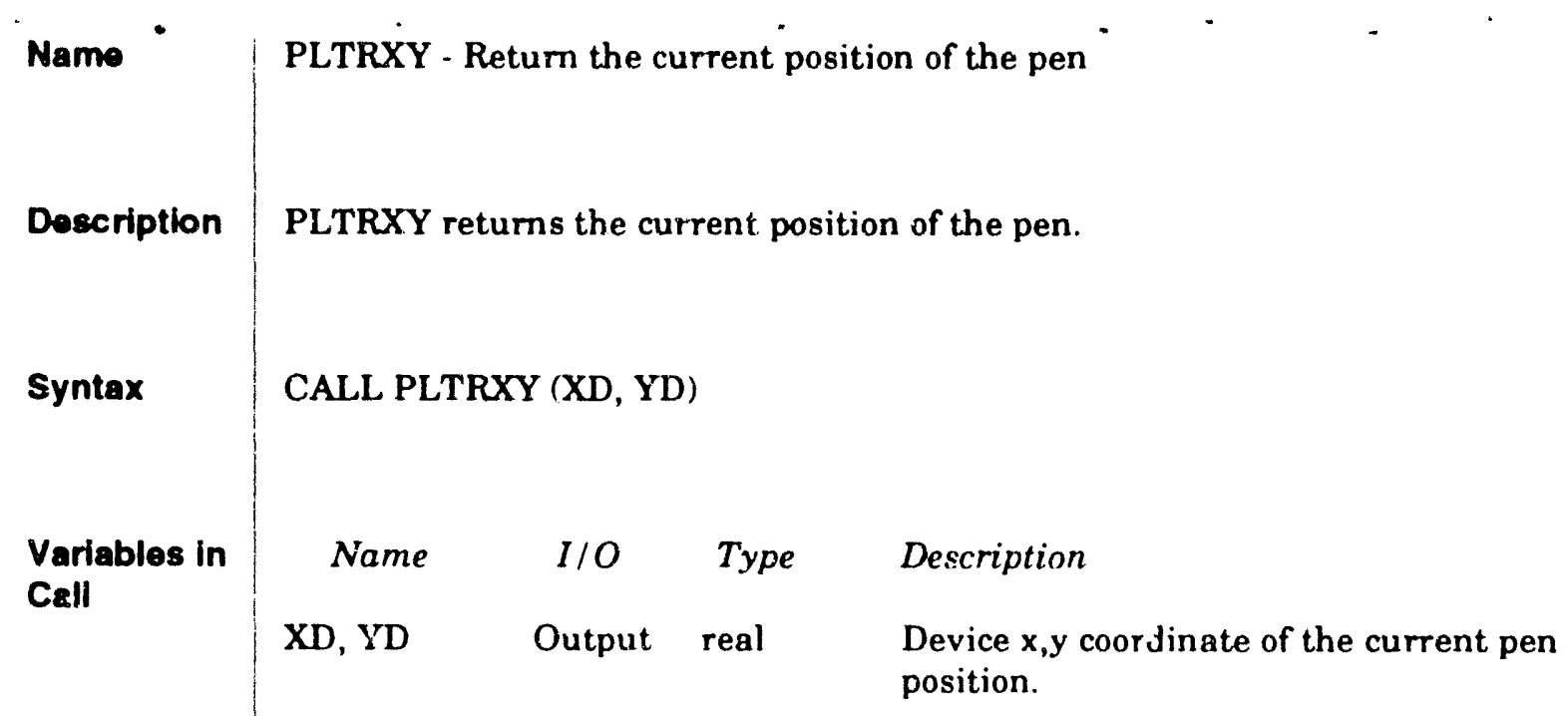




\begin{tabular}{|c|c|c|c|c|}
\hline Name & \multicolumn{4}{|c|}{ PLTSYMCEN - Plot a character centered at specified locations } \\
\hline Description & \multicolumn{4}{|c|}{$\begin{array}{l}\text { PLTSYMCEN plots a specified character centered at the specified locations. The } \\
\text { symbol attributes apply. }\end{array}$} \\
\hline Syntax & \multicolumn{4}{|c|}{ CALL PLTSYMCEN (N, XD, YD, SYM) } \\
\hline \multirow{4}{*}{$\begin{array}{l}\text { Variables in } \\
\text { Call }\end{array}$} & Name & $I / O$ & Type & Description \\
\hline & $\mathbf{N}$ & Input & integer & Number of points. \\
\hline & $\mathrm{XD}, \mathrm{YD}$ & Input & real & $\begin{array}{l}\text { Device } x, y \text { coordinates for the centers at which } \\
\text { the character is to be plotted. }\end{array}$ \\
\hline & SYM & Input & char & $\begin{array}{l}\text { The symbol to be plotted. This is a single } \\
\text { character. Note that this character can be an } \\
\text { escape sequence such as ICSQ. }\end{array}$ \\
\hline Note & \multicolumn{4}{|c|}{ See Section 10.2 .3 for a list of escape characters that can be used with PLTSYMCEN. } \\
\hline Soe A & \multicolumn{4}{|c|}{ PLTSETSYM for attributes } \\
\hline
\end{tabular}




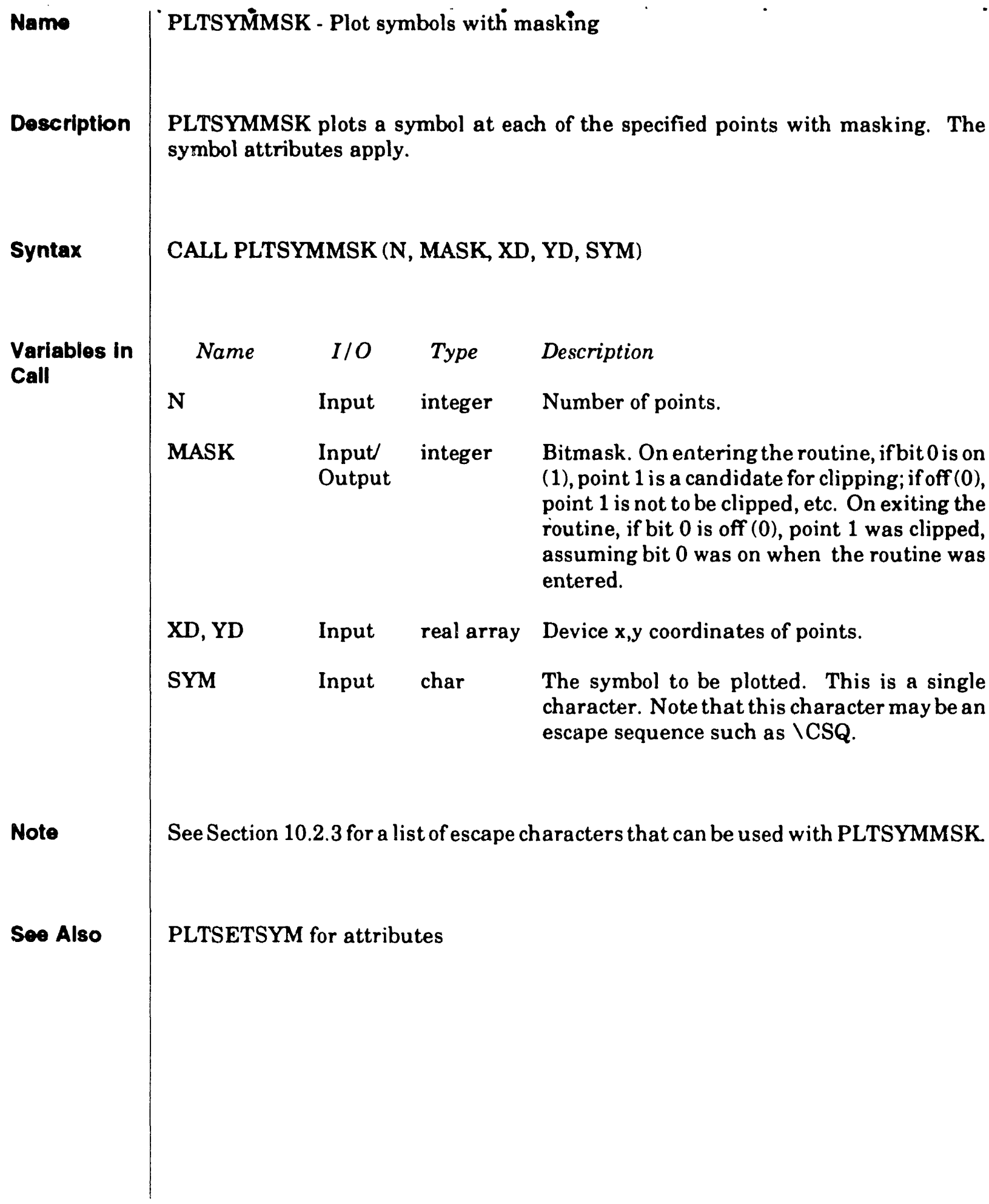




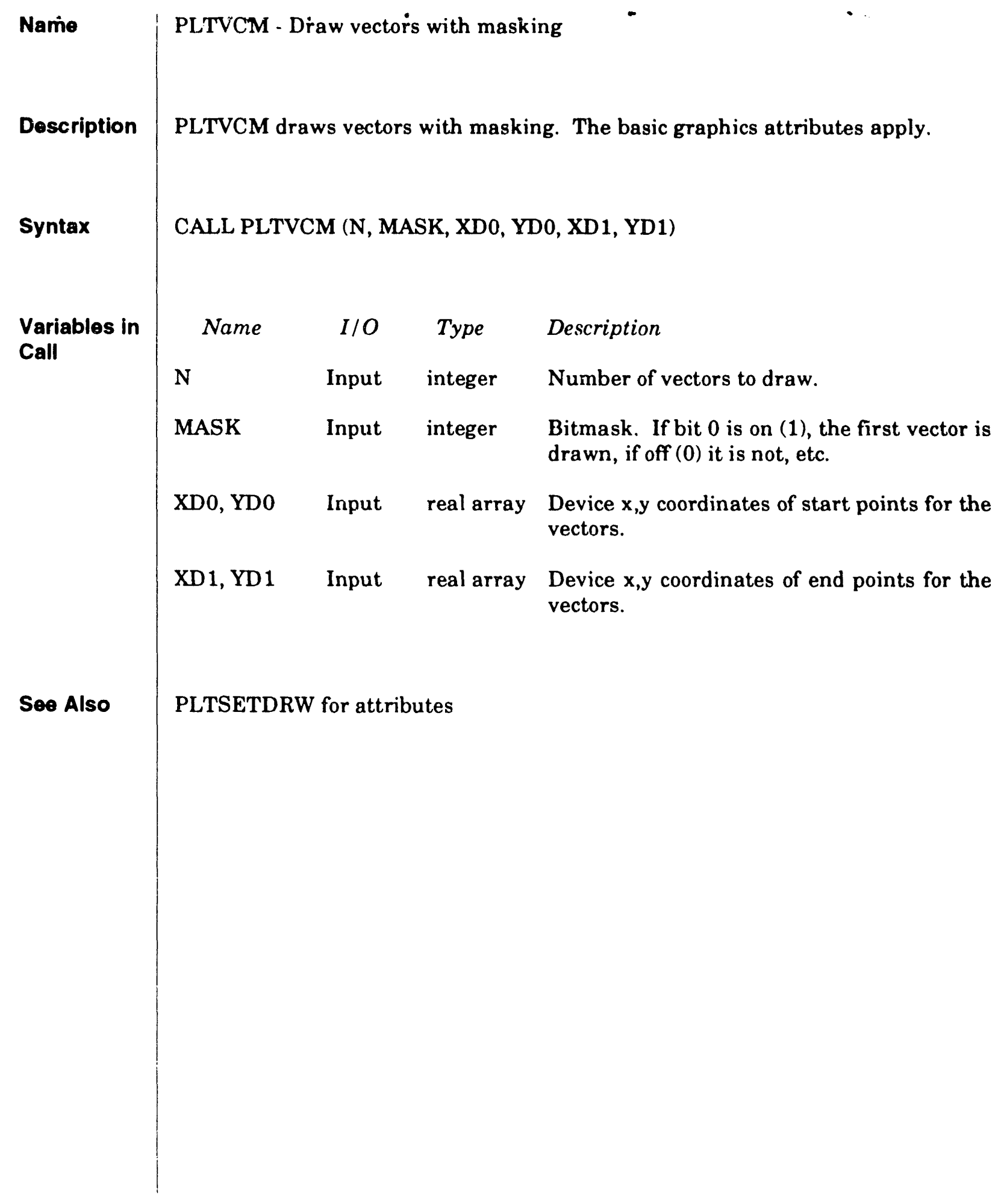




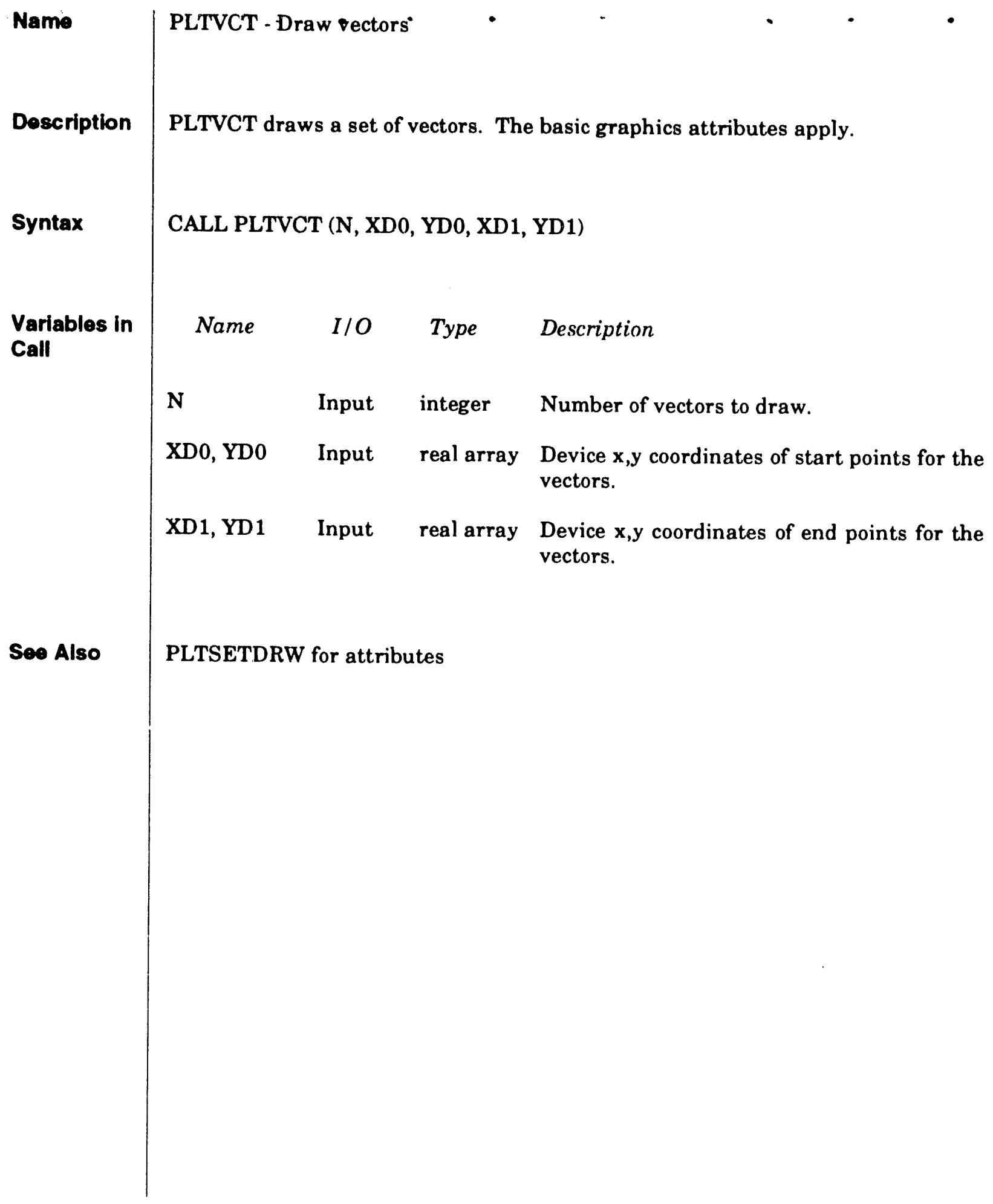


PLT_LIB

Description of Routine Calls

\subsubsection{Two- and Three-Dimensional Mapping Routines}

Detailed descriptions of the routines that provide two- and three-dimensional mapping are given on the following pages. 


\begin{abstract}
Name
Description
\end{abstract}

MP2PG - Mäp a tw̌o-dimeñsional polygon

Syntax

Variables in

Call

Comments

Seo Also

MP2PG maps a two-dimensional polygon.

CALL MP2PG (N, XG, YG, NM, XD, YD)

$\begin{array}{llll}\text { Name } & \text { I/O } & \text { Type } & \begin{array}{l}\text { Description } \\ \mathrm{N}\end{array} \\ \mathrm{XG}, \mathrm{YG} & \text { Input } & \text { integer } & \begin{array}{l}\text { Number of vertices for the polygon (the maxi- } \\ \text { mum is } 32) .\end{array} \\ \mathrm{NM} & \text { Input } & \text { real array } & \text { Graph } \mathrm{x}, \mathrm{y} \text { coordinates of the polygon vertices. } \\ \text { Output } & \text { integer } & \begin{array}{l}\text { On entering the routine, the maximum } \\ \text { number of ouput vertices that you have } \\ \text { allocated space for, i.e., the dimension of XD } \\ \text { and YD. On exiting the routine, the number } \\ \text { of resulting vertices (the maximum is } 32) .\end{array}\end{array}$

$\mathrm{XD}, \mathrm{YD}$ Output real array Device $\mathrm{x}, \mathrm{y}$ coordinates of mapped polygon vertices.

MP2PG uses whatever modelling and viewport information the user has set up with calls to the modelling, projection, and viewport routines. Note that no call to the viewing routines is necessary in two dimensions.

MPD2PG: MP2PG is the same as MPD2PG except that it doesn't draw the polygon, but returns the polygon vertices to the user. 


\begin{tabular}{|c|c|c|c|c|}
\hline Name & \multicolumn{4}{|c|}{ MP2PT - Map two-dimensional points } \\
\hline Description & \multicolumn{4}{|c|}{ MP2PT maps two-dimensional points. } \\
\hline Syntax & \multicolumn{4}{|c|}{ CALL MP2PT (N, XG, YG, XD, YD, MASK) } \\
\hline $\begin{array}{l}\text { Variables in } \\
\text { Call }\end{array}$ & $\begin{array}{l}\quad \text { Name } \\
\mathrm{N} \\
\mathrm{XG}, \mathrm{YG} \\
\mathrm{XD}, \mathrm{YD} \\
\text { MASK }\end{array}$ & $\begin{array}{l}I / O \\
\text { Input } \\
\text { Input } \\
\text { Output } \\
\text { Output }\end{array}$ & $\begin{array}{l}\text { Type } \\
\text { integer } \\
\text { real array } \\
\text { real array } \\
\text { integer } \\
\text { array }\end{array}$ & $\begin{array}{l}\text { Description } \\
\text { Number of points. } \\
\text { Graph } x, y \text { coordinates of points. } \\
\text { Device } x, y \text { coordinates of mapped points. } \\
\text { Bitmask. If bit } 0 \text { is off }(0) \text {, point } 1 \text { was clipped, } \\
\text { if on (1), it was not, etc. This array must be } \\
\text { dimensioned to the next higher integer after } \\
\text { N/32. }\end{array}$ \\
\hline
\end{tabular}

Comments

See Also
MP2PT uses whatever modelling and viewport information the user has set up with calls to the modelling, projection, and viewport routines. Note that no call to the viewing routines is necessary in two dimensions.

PLTPTM: You can use PLTPTM to plot these points.

MPD2PT: MP2PT is the same as MPD2PT except that it doesn't draw the mapped points; it returns them to the user. 


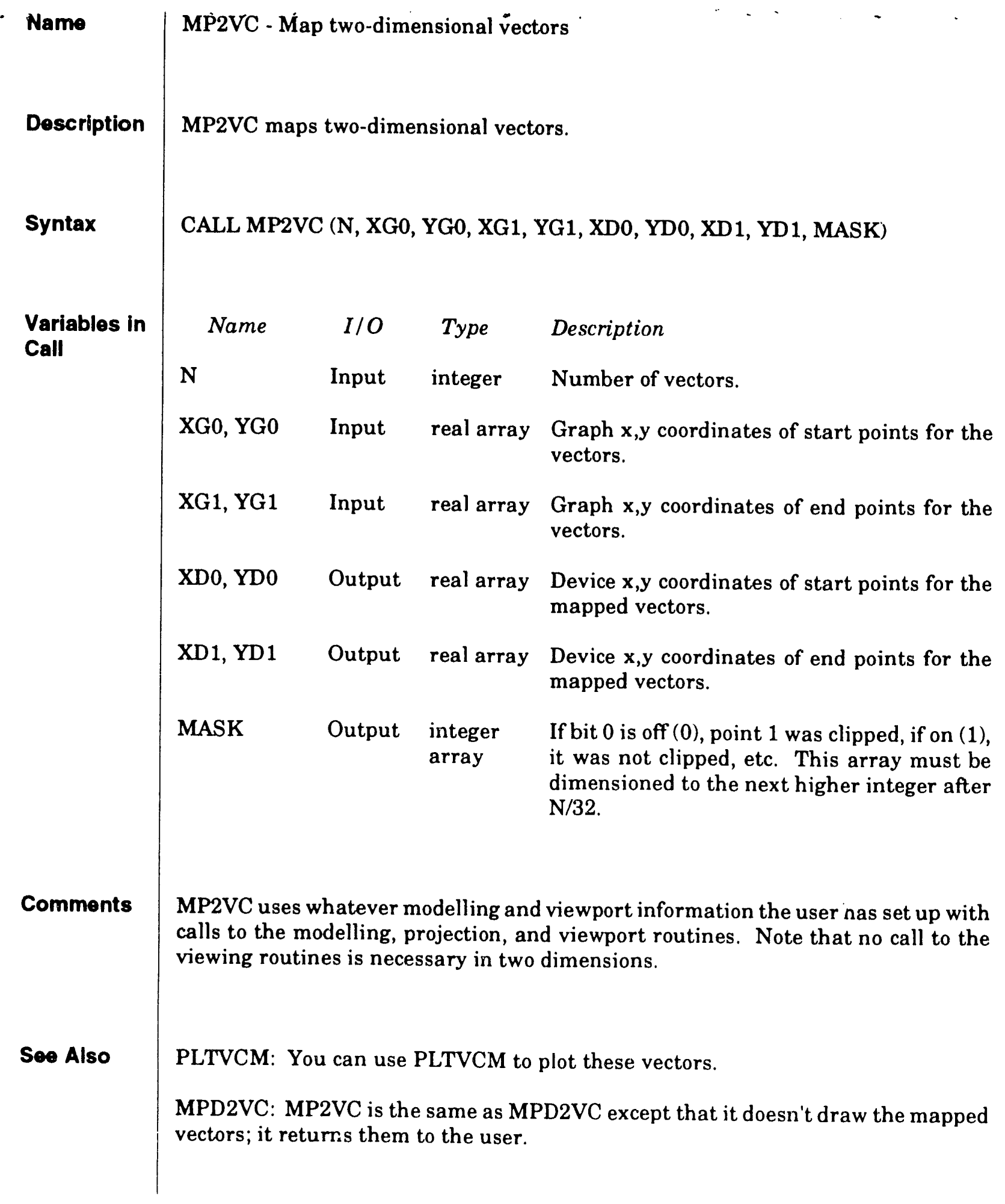


PLT LIB

Description of Routine Calls

MP3PG

Subroutine

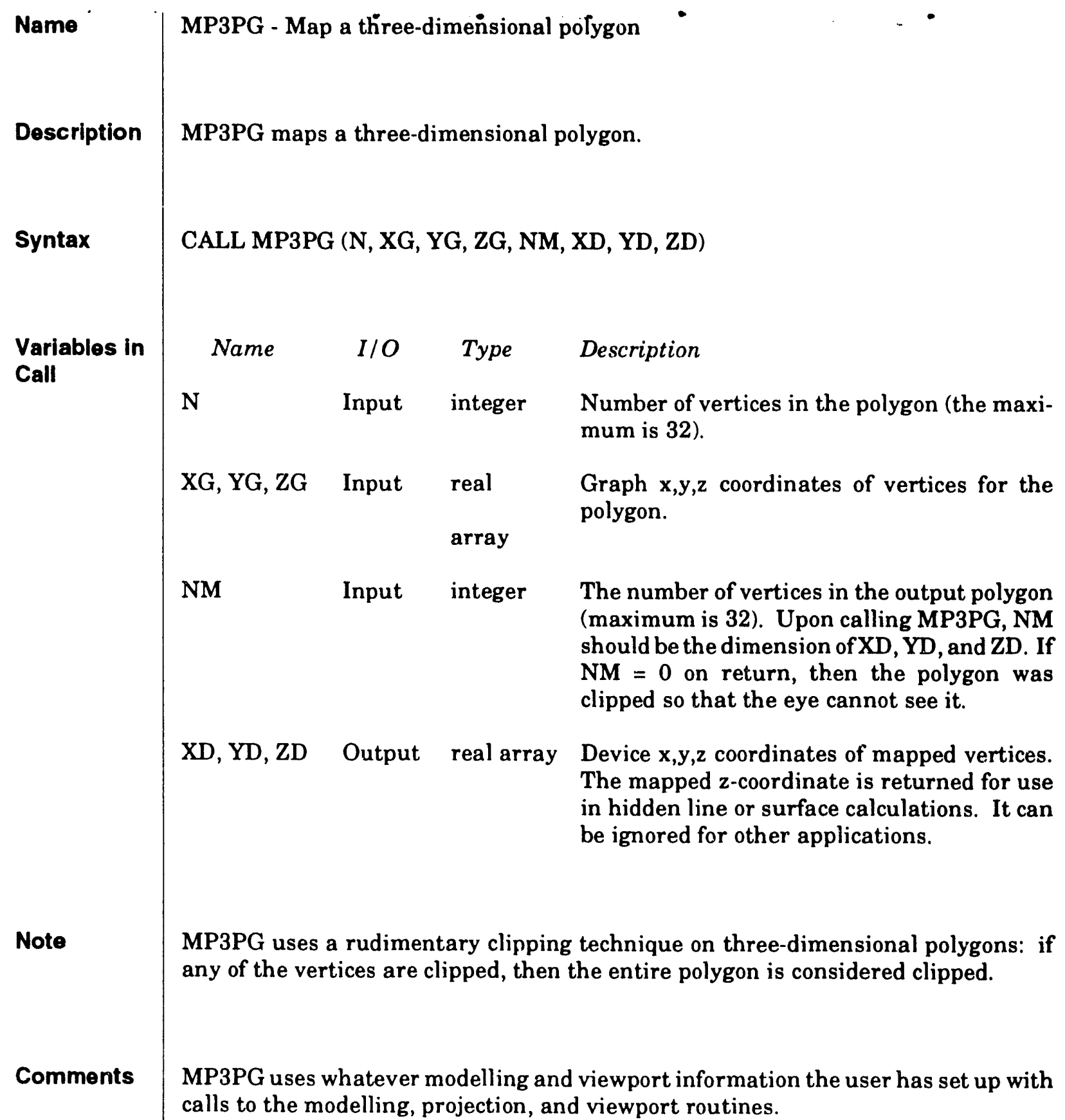
calls to the modelling, projection, and viewport routines. 


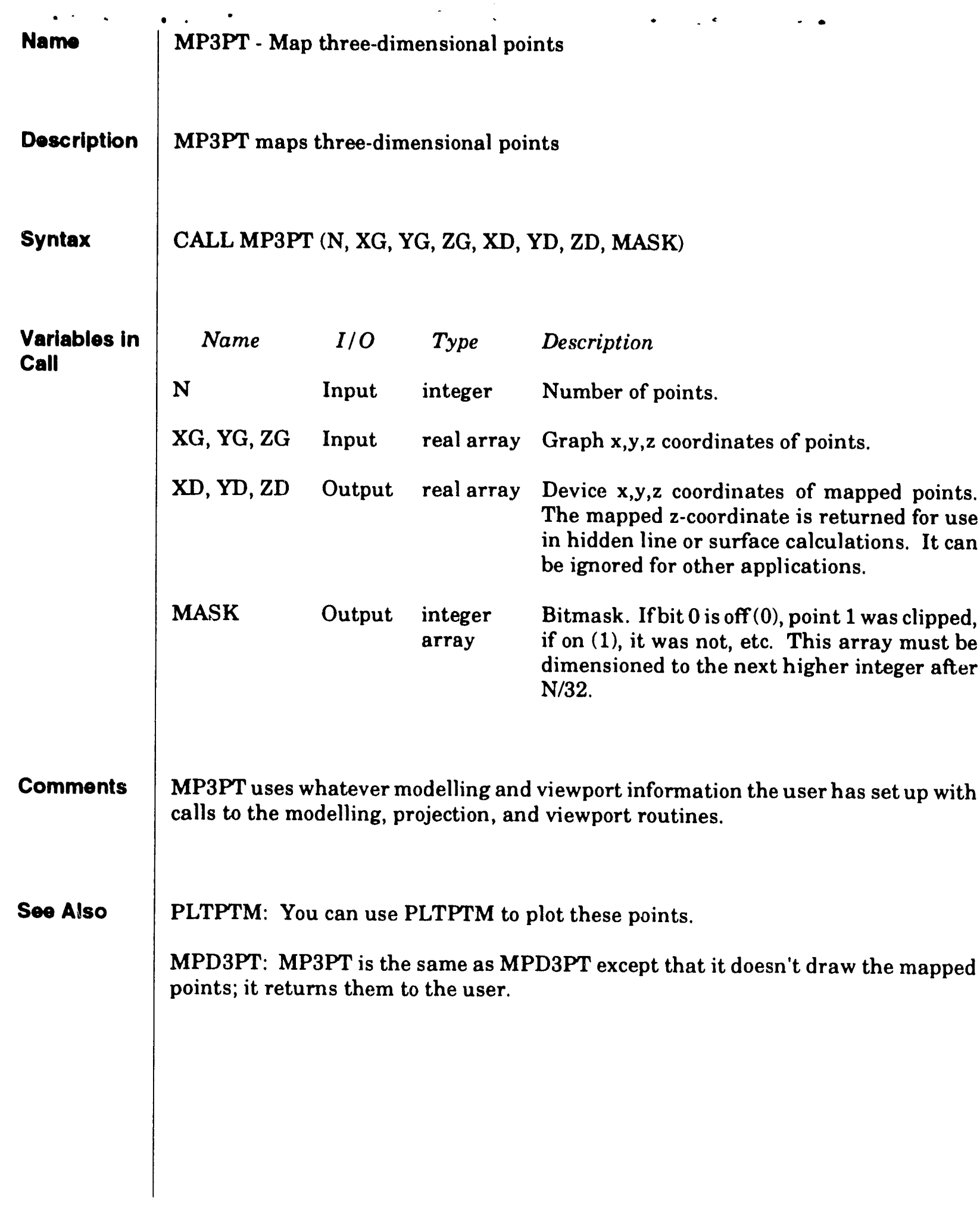


PLT_LIB

Description of Routine Calls

Name

Syntax

ariables in

Call

Comments

See Also
MP3VC - Map three-dimensional vectors

MP3VC maps three-dimensional vectors.

CALL MP3VC (N, XG0, YG0, ZG0, XG1, YG1, ZG1, XD0, YD0, ZD0, XD1, YD1, ZD1 MASK)

Name I/O Type Description

$\mathrm{N}$ Input integer Number of vectors.

XG0, YG0, Input real array Graph $x, y, z$ coordinates of start points for the ZG0 vectors.

XG1, YG1, Input real array Graph $x, y, z$ coordinates of end points for the ZG1 vectors.

$\mathrm{XD0}, \mathrm{YD} 0$, Output real array Device $\mathrm{x}, \mathrm{y}, \mathrm{z}$ coordinates of start points for the ZD0 mapped vectors.

XD1, YD1, Output real array Device $x, y, z$ coordinates of end points for the ZD1 mapped vectors.

MASK Output integer If bit 0 is off ( 0 ), point 1 was clipped, if on (1), array it was not clipped, etc. This array must be dimensioned to the next higher integer after $\mathrm{N} / 32$.

MP3VC uses whatever modelling and viewport information the user has set up with calls to the modelling, projection, and viewport routines.

PLTVCM: You can use PLTVCM to plot these vectors.

MPD3VC: MP3VC is the same as MPD3VC except that it doesn't draw the mapped vectors; it returns them to the user. 


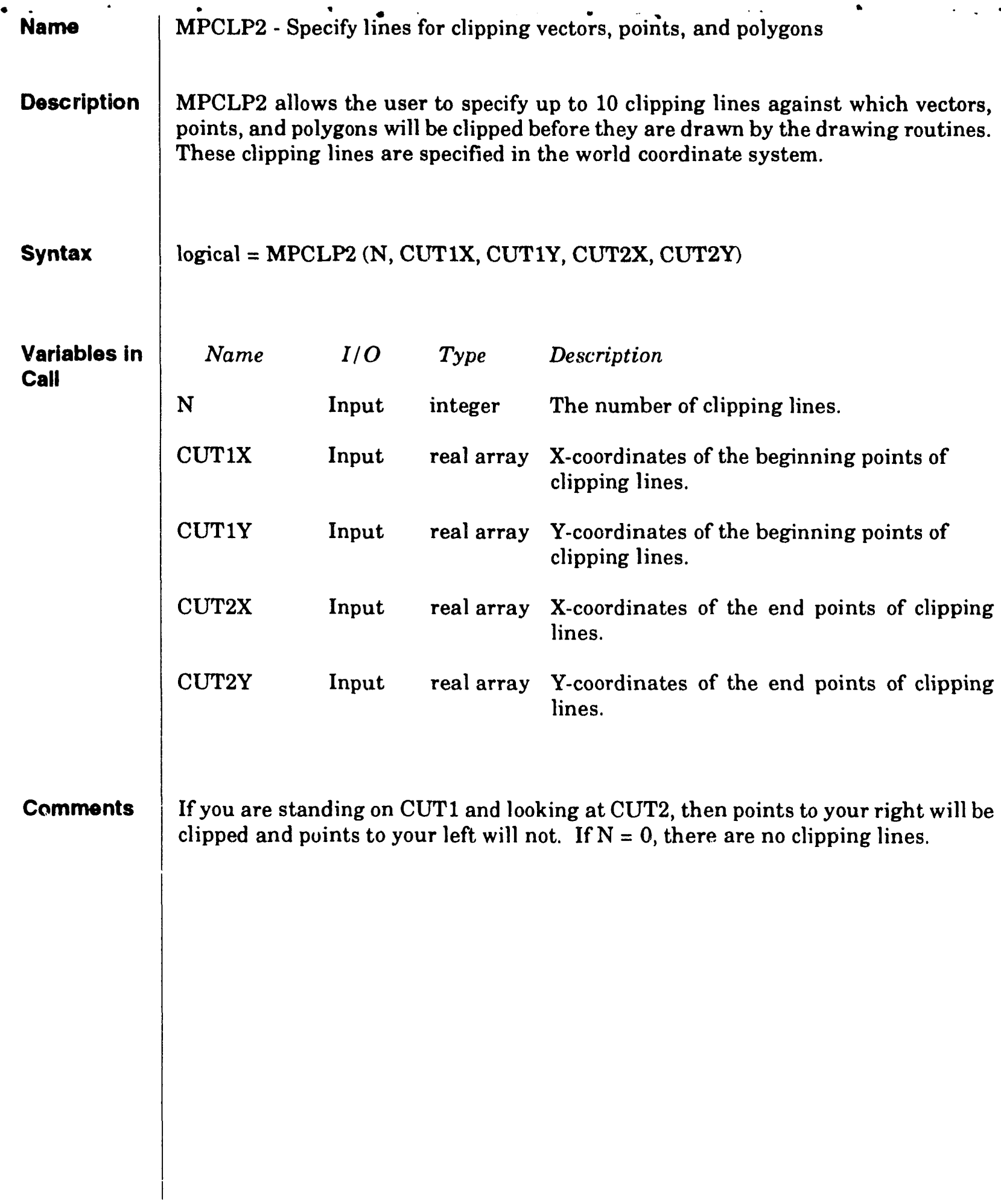




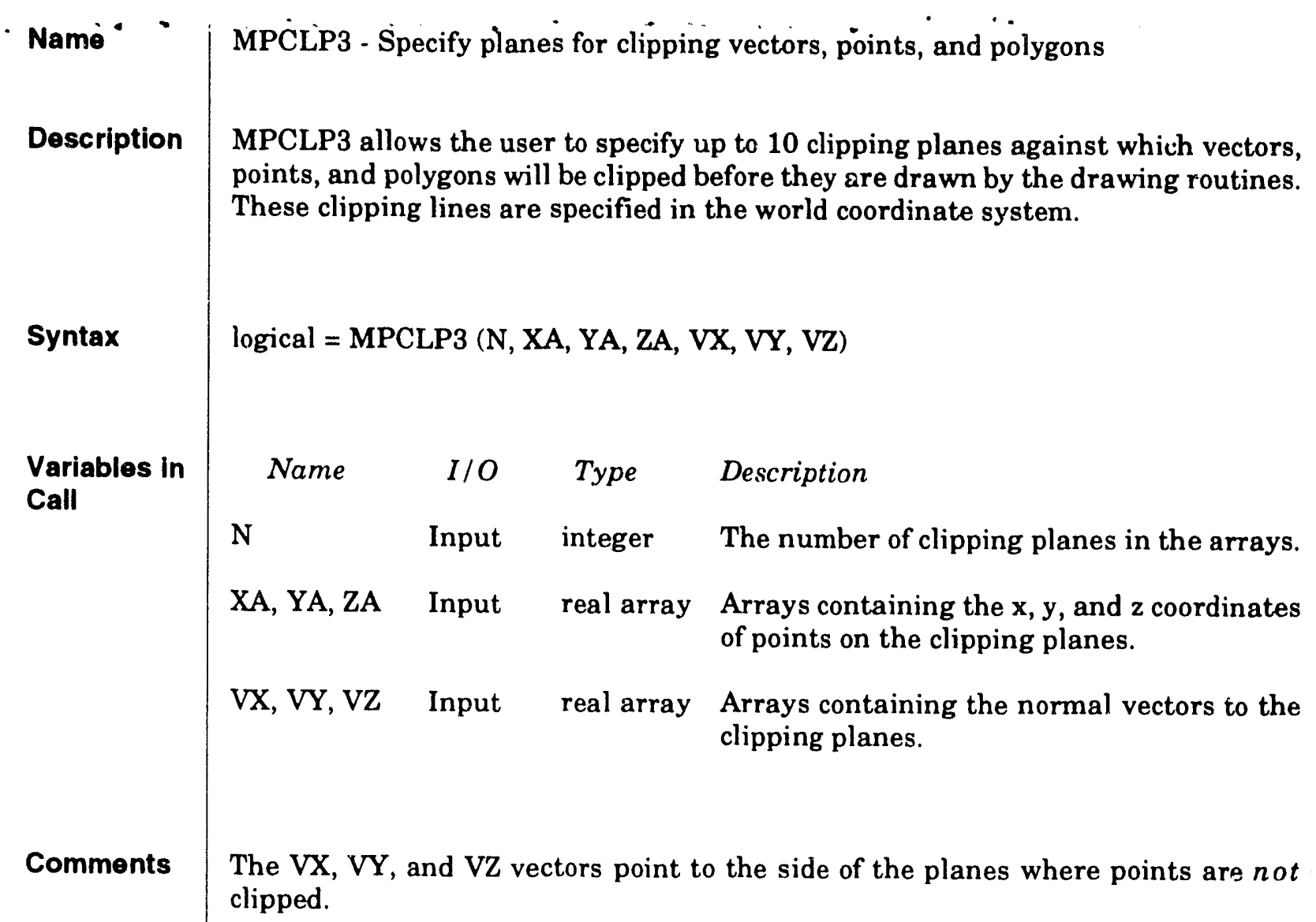




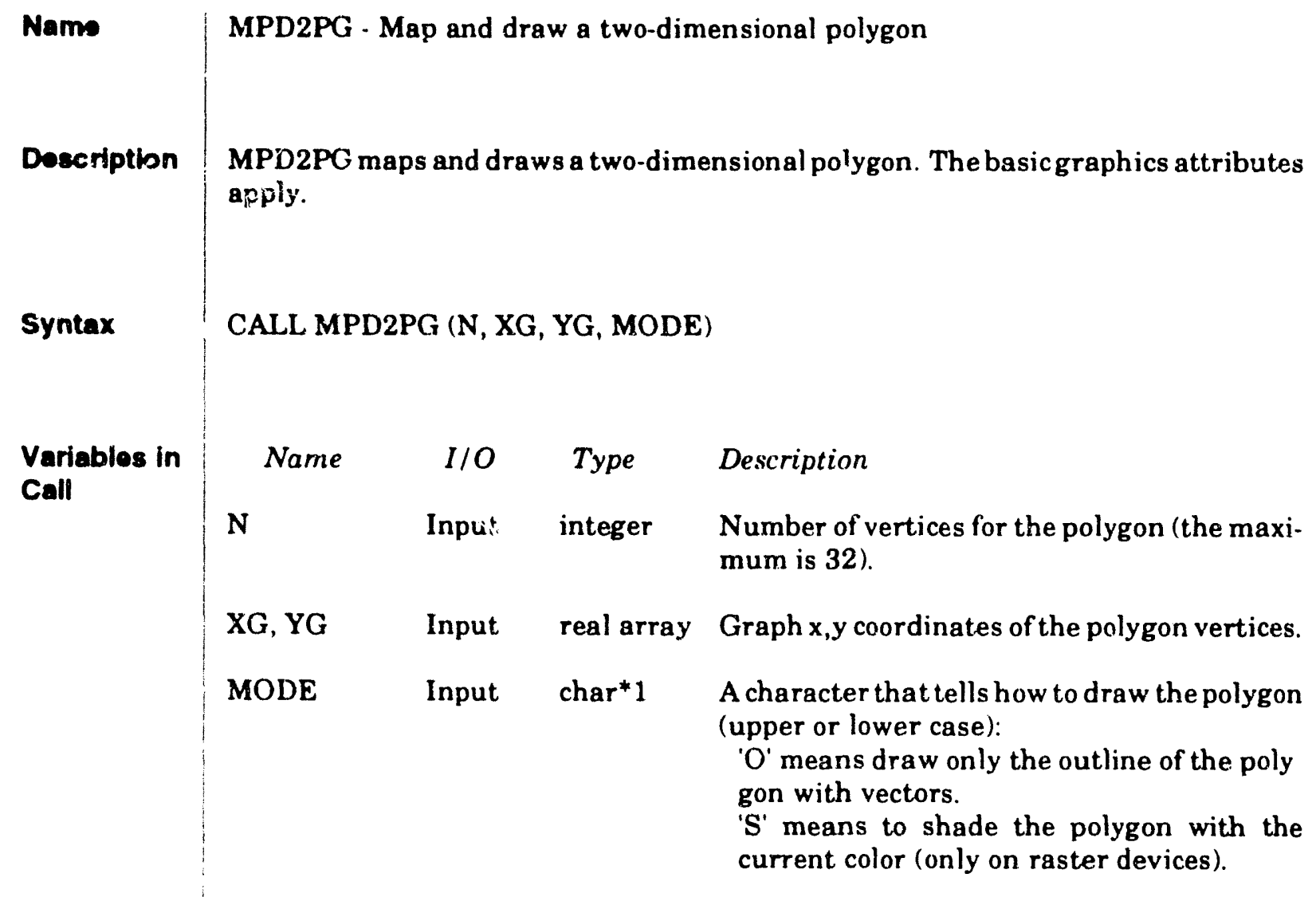
with calls to the modelling, projection, and viewport routines. Note that no call to the viewing routines is necessary in two dimensions.

\section{Sec Also}

MP2PG: MP2PG is the same as MPD2PG except that it doesn't draw the polygon, but returas the polygon vertices to the user.

PLTSETDRW for attributes. 
PLT LIB

Description of Routine Calls

MPD2PT

Subroutino

\begin{abstract}
\begin{tabular}{l|l} 
Name & MPD2PT - Map and draw two-dimensional points
\end{tabular}
Description MPD2PT maps and draws two-dimensional points. The busic graphics attributes apply.

Syntax

CALL MPD2PT (N, XG, YG)

Variables in

Call

Name I/O Type Description

$\mathrm{N} \quad$ Input integer Number of points.

XG YG Input real array Graph $x, y$ coordinates of the points.

Comments

MPD2PT uses whatever modelling and viewport information the user has set up with calls to the modelling, projection, and viewport routines. Note that no call to the viewing routines is necessary in two dimensions.
\end{abstract}

Seo Also

MP2PT: MP2PT is the same as MPD2PT except that it doesn't draw the mapped points; it returns them to the user.

PLTSETDRW for attributes. 


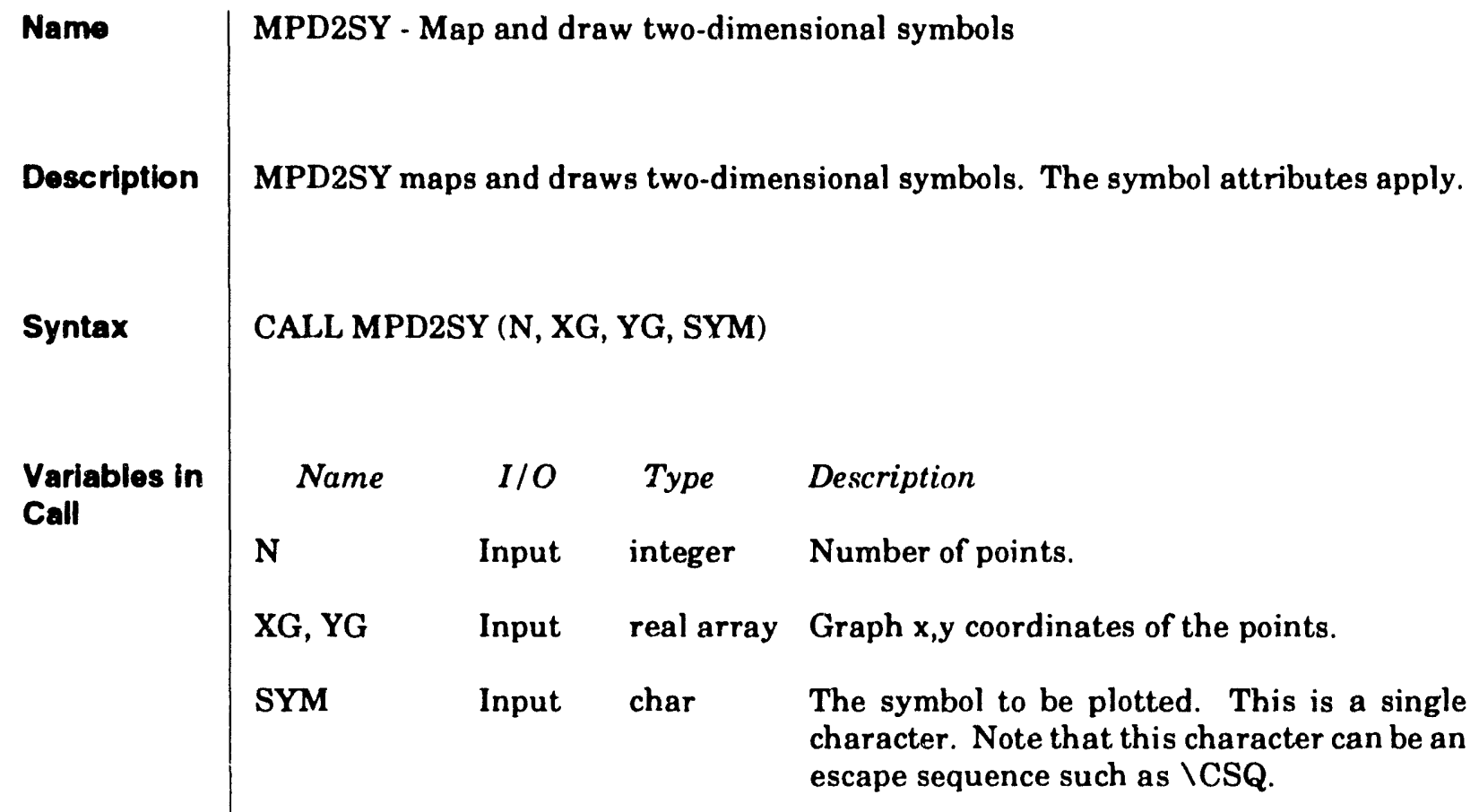

MPD2SY uses whatever modelling and viewport information the user has set up with calls to the modelling, projection, and viewport routines. Note that no call to the viewing routines is necessary in two dimensions.

Note

See Section 10.2.3 for a list of escape characters that can be used with MPD2SY.

PLTSETSYM for attributes 
PLT LIB

Description of Routine Calls

\begin{abstract}
\begin{tabular}{l|l} 
Name & MPD2VC - Map and draw two-dimensional vectors
\end{tabular}
Description

MPD2VC maps and draws two-dimensional vectors. The basic graphics attributes apply.

Syntax

CALL MPD2VC (N, XG0, YG0, XG1, YG1)

Variables in

Call

Name I/O Type Description

$\mathrm{N} \quad$ Input integer Number of vectors.

XG0, YG0 Input real array Graph $x, y$ coordinates of start points for the vectors.

XG1, YG1 Input real array Graph $x, y$ coordinates of end points for the vectors.

Comments

MP2VC uses whatever modelling and viewport information the user has set up with calls to the modelling, projection, and viewport routines. Note that no call to the viewing routines is necessary in two dimensions.
\end{abstract}

See Also

MP2VC: MP2VC is the same as MPD2VC except that it doesn't draw the mapped vectors; it returns them to the user.

PLTSETDRW for attributes. 


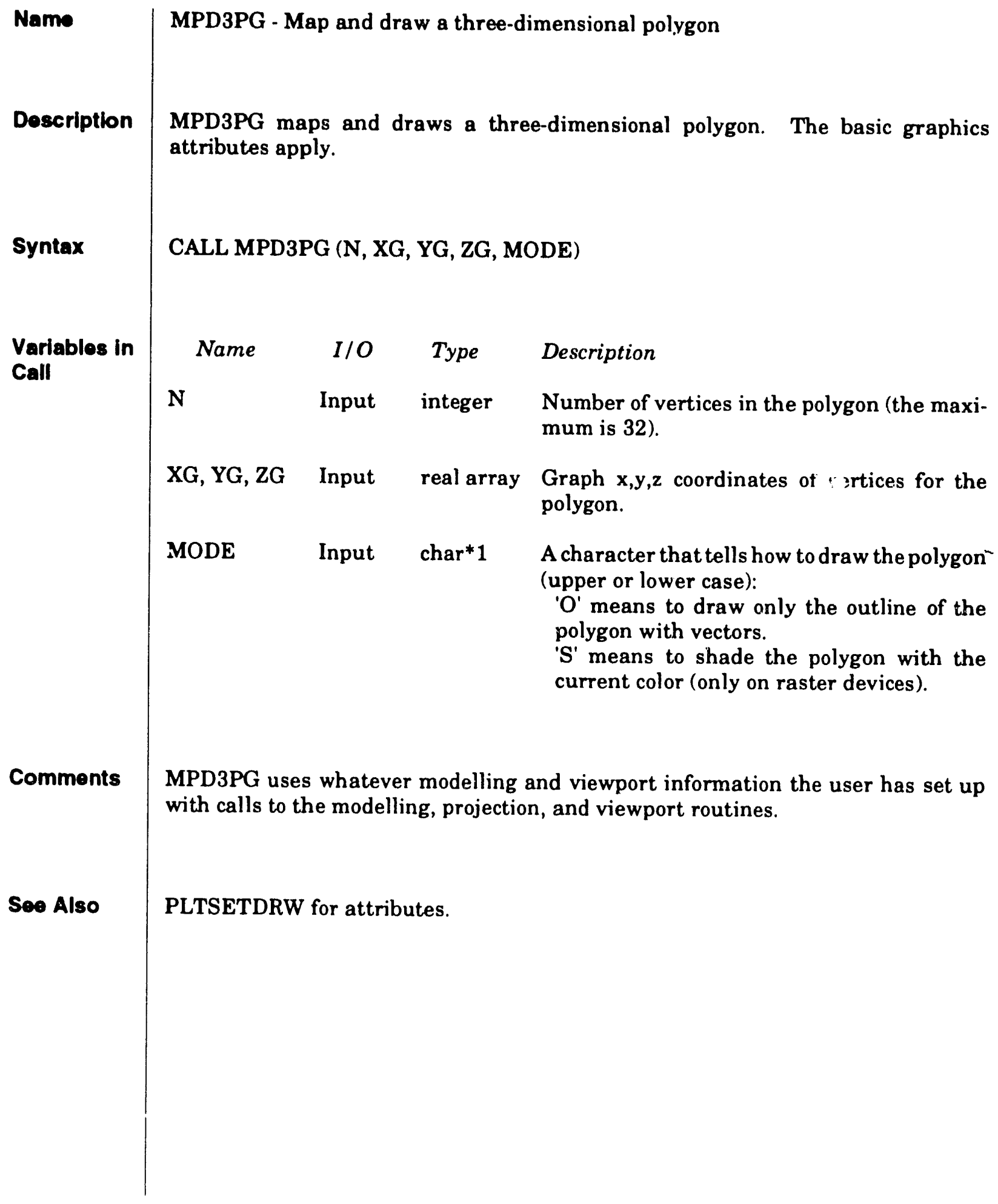




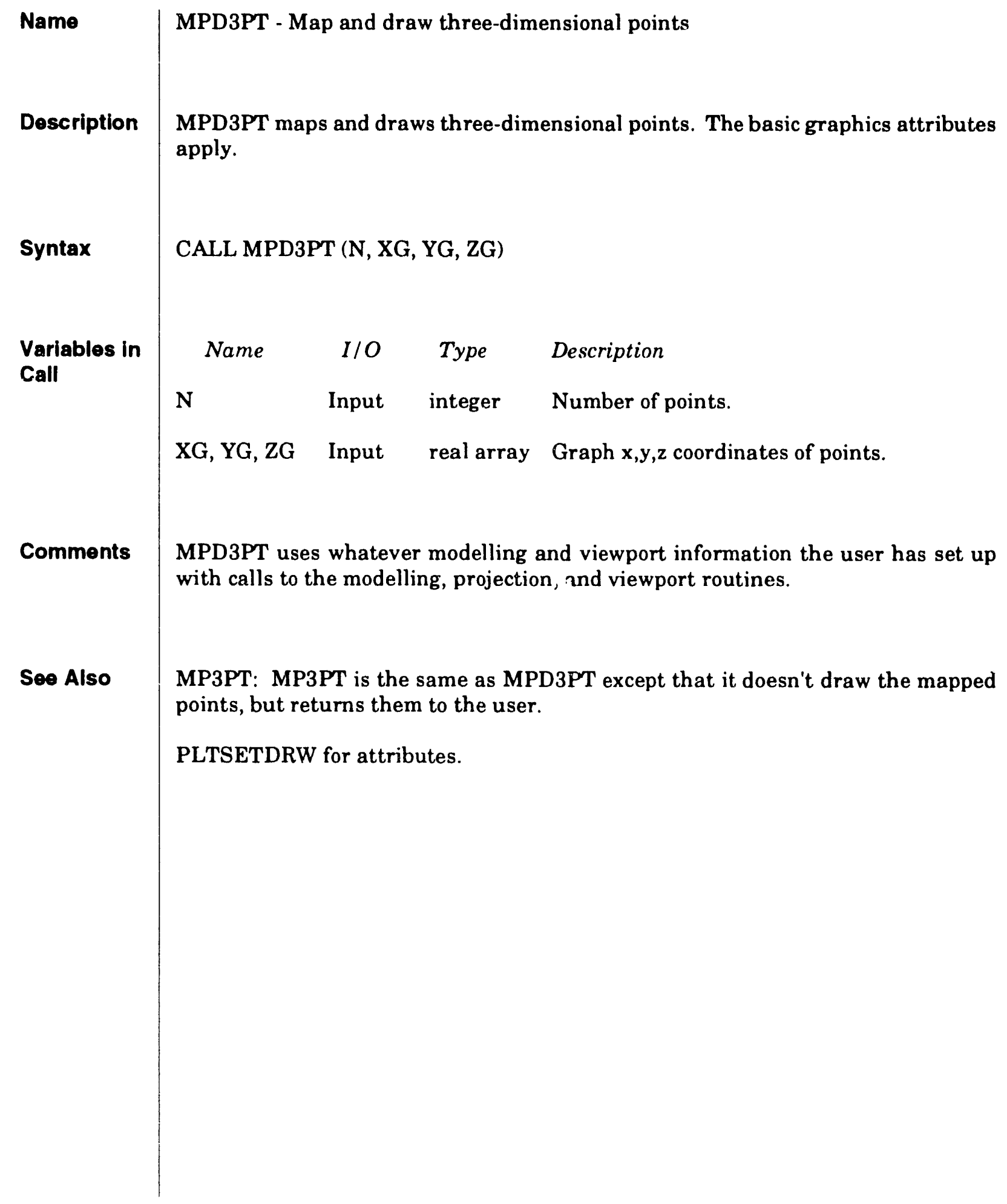




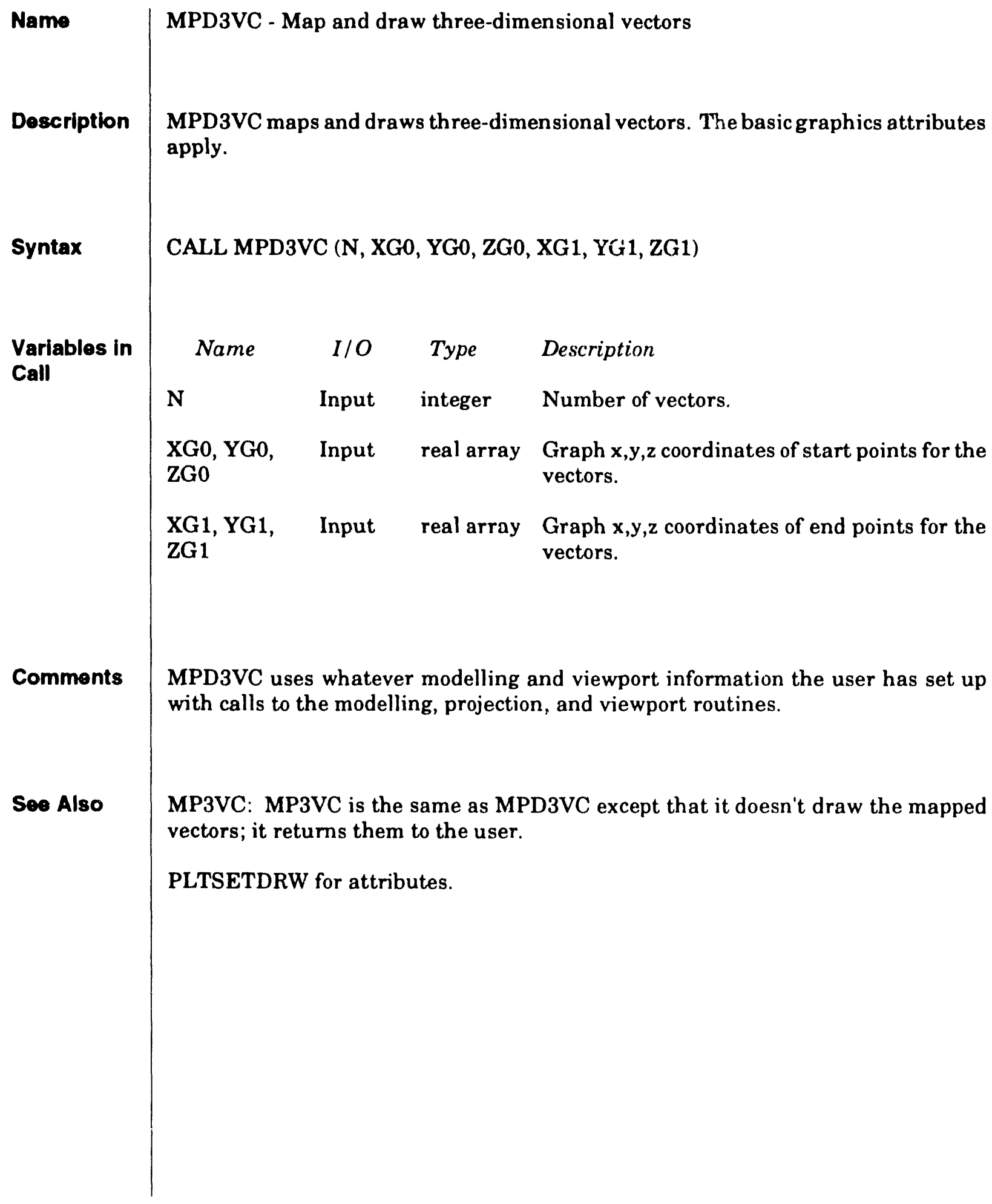




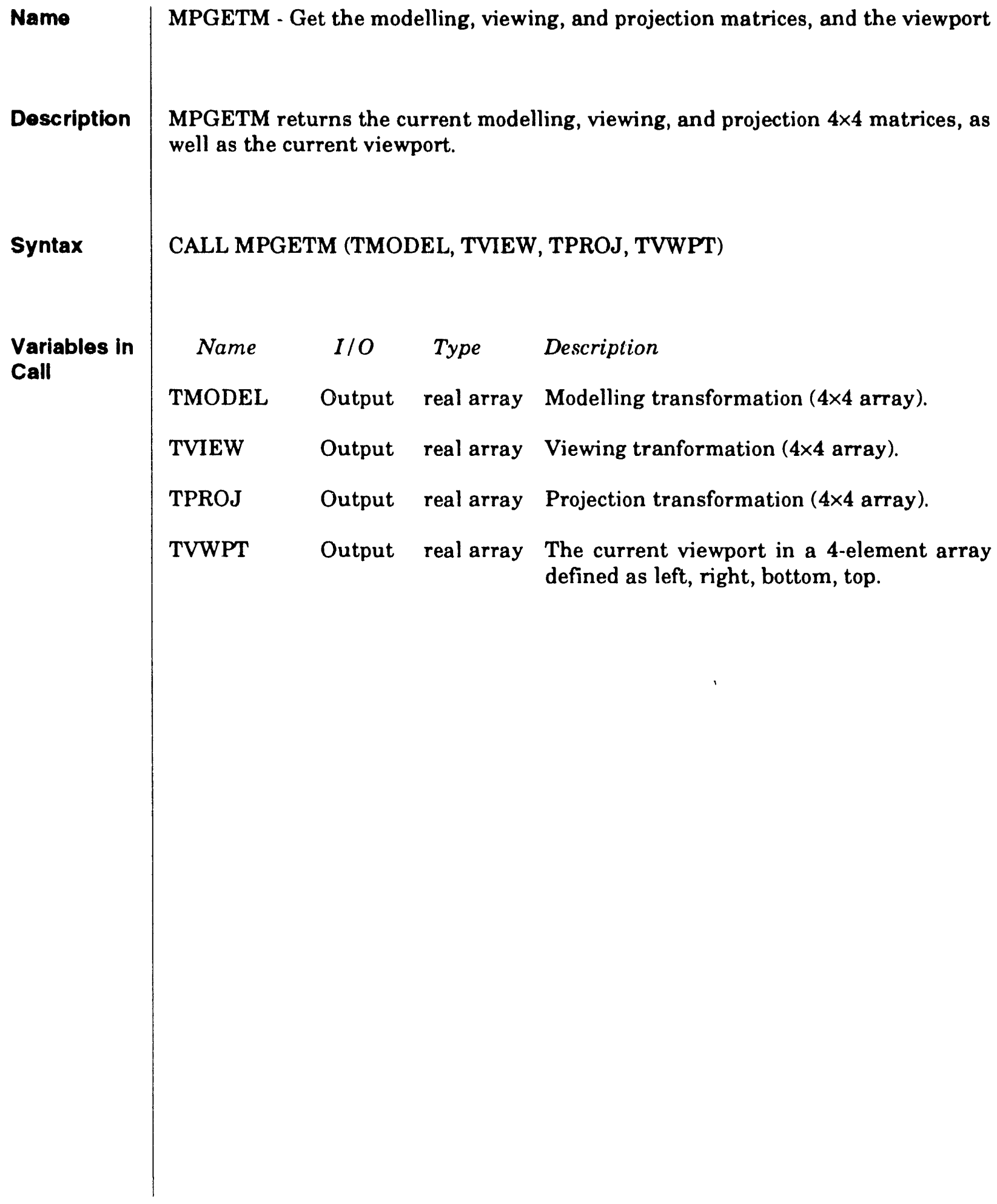




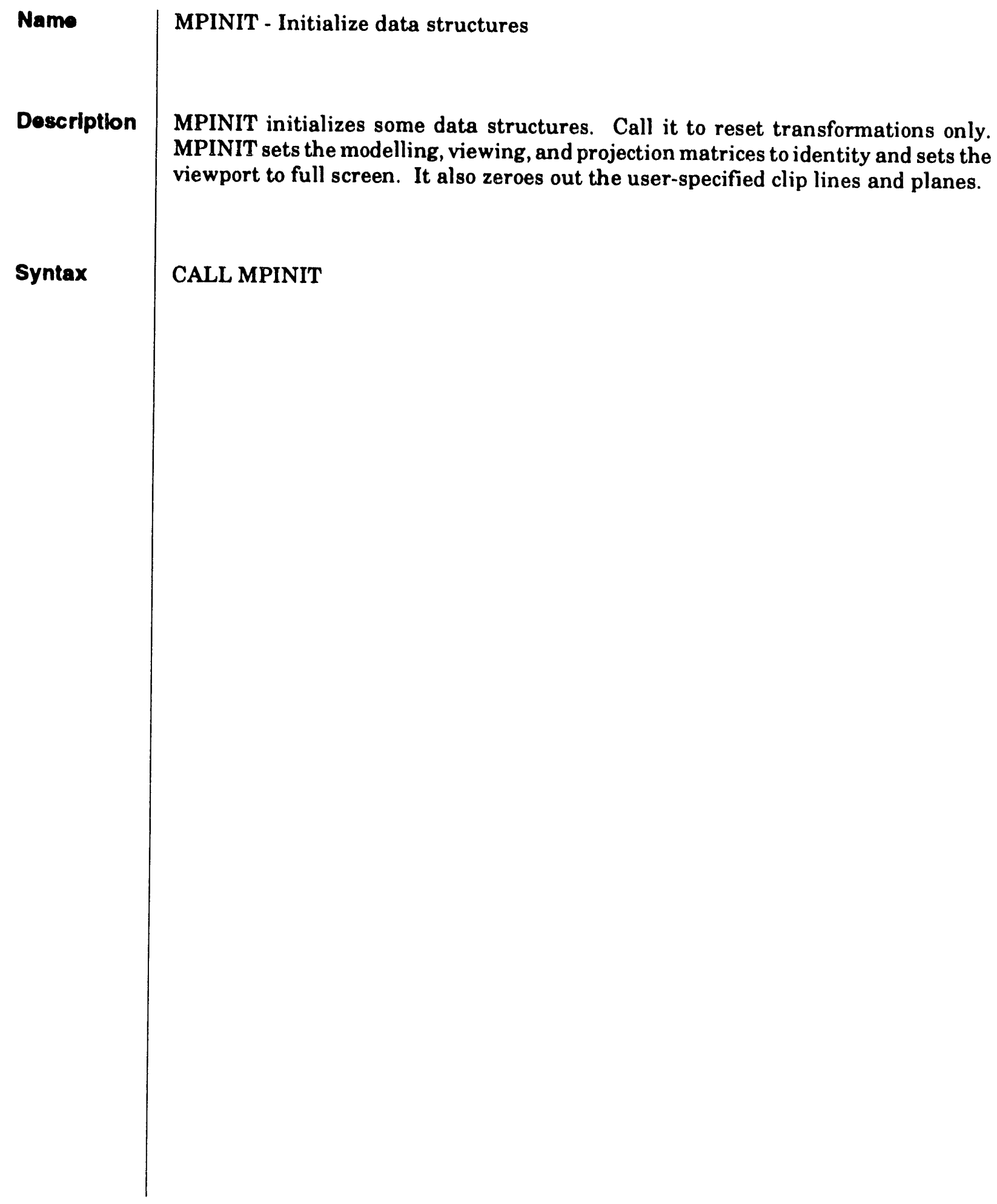


PLT LIB

Description of Routine Calls

\begin{abstract}
Name
MPLOOK - Define the viewing position using coordinates

Description

MPLOOK defines the viewing position, setting up the viewing matrix.

CALL MPLOOK (VX, VY, VZ, X, Y, Z, TWIST)

Variables in

Call

Name I/O Type Description

VX, VY, VZ Input real Coordinates for the location of the eye (viewpoint).

$\mathrm{X}, \mathrm{Y}, \mathrm{Z}$ Input real Coordinates for the point the eye is looking at (reference point).

TWIST Input real The angle of rotation in degrees about the eye system's z-axis (this axis is along a line from the viewpoint to the reference point).

This transformation is defined as follows:

TRANS (-VX, -VY, -VZ) × ROTY (THETA) × ROTX (PII) $\times$ ROTZ (-TWIST)

See the Silicon Graphics Graphics Library Programming Guide (McLendon, 1991), Appendix C.4, "Viewing Transformations," for more information.
\end{abstract}




\begin{tabular}{|c|c|c|c|c|}
\hline Name & \multicolumn{4}{|c|}{ MPORT2 - Define a two-dimensional orthographic projection transformation } \\
\hline Description & \multicolumn{4}{|c|}{$\begin{array}{l}\text { MPORT2 defines an orthographic projection tranformation, setting up the projec- } \\
\text { tion matrix. The function defines a two-dimensional clipping rectangle, and the } \\
\text { projection matrix is a two-dimensional map. The } z \text { values are unchanged. }\end{array}$} \\
\hline Syntax & \multicolumn{4}{|c|}{ logical = MPORT2 (LEFT, RIGHT, BOTTOM, TOP) } \\
\hline Variables in & Name & $I / O$ & Type & Description \\
\hline & LEFT & Input & real & $\begin{array}{l}\text { The } x \text {-coordinate of the left edge of the rect- } \\
\text { angle. }\end{array}$ \\
\hline & RIGHT & Input & real & $\begin{array}{l}\text { The } x \text {-coordinate of the right edge of the rect- } \\
\text { angle. }\end{array}$ \\
\hline & BOTTOM & Input & real & $\begin{array}{l}\text { The y-coordinate of the bottom edge of the } \\
\text { rectangle. }\end{array}$ \\
\hline & TOP & Input & real & $\begin{array}{l}\text { The y-coordinate of the top edge of the rect- } \\
\text { angle. }\end{array}$ \\
\hline Comments & \multicolumn{4}{|c|}{$\begin{array}{l}\text { The four values in the calling sequence are essentially the minimum and maximum } \\
\text { world coordinates that you wish to see. See the Silicon Graphics Graphics Library } \\
\text { Programming Guide (McLendon, 1991), Appendix C.6, "Orthographic Transforma- } \\
\text { tions," for more information. }\end{array}$} \\
\hline
\end{tabular}


Name

Description

Syntax

Variables in

Call
MPORT3 - Define a three-dimensional orthographic projection transformation

MPORT3 defines an orthographic projection transformation, setting up the projection matrix. The function defines a parellelepiped in the eye coordinate system.

logical = MPORT3 (LEFT, RIGHT, BOTTOM, TOP, NEAR, FAR)

\section{Name I/O Type Description}

LEFT Input real The $x$-coordinate of the left edge of the rectangle.

RIGHT Input real The $x$-coordinate of the right edge of the rectangle.

BOTTOM Input real The y-coordinate of the bottom edge of the rectangle.

TOP Input real The y-coordinate of the top edge of the rectangle.

NEAR Input real The location (distance from the eye) of the near clipping plane.

FAR Input real The location (distance from the eye) of the far clipping plane.

LEFT, RIGHT, TOP, and BOTTOM define the $x$ and $y$ clipping planes; NEAR and FAR define the near and far clipping planes. See the Silicon Graphics Graphics Library Programming Guide (McLendon, 1991), Appendix C.6, "Orthographic Transformations," for more information. 


\begin{tabular}{|c|c|c|c|c|}
\hline Name & \multicolumn{4}{|c|}{ MPPERS - Define a perspective projection transformation in angles } \\
\hline Description & \multicolumn{4}{|c|}{$\begin{array}{l}\text { MPPERS defines a perspective projection transformation, setting up the projection } \\
\text { matrix. }\end{array}$} \\
\hline Syntax & \multicolumn{4}{|c|}{ logical = MPPERS (FOVY, ASPECT, NEAR, FAR) } \\
\hline Variables in & Name & $I / O$ & Type & Description \\
\hline & FOVY & Input & real & $\begin{array}{l}\text { The field of view angle in degrees, in the } y- \\
\text { direction of the eye coordinate system. }\end{array}$ \\
\hline & ASPECT & Input & real & $\begin{array}{l}\text { The aspect ratio that determines the field of } \\
\text { view in the } x \text {-direction. }\end{array}$ \\
\hline & NEAR & Input & real & $\begin{array}{l}\text { The location (distance from the eye) of the } \\
\text { near clipping plane. }\end{array}$ \\
\hline & FAR & Input & real & $\begin{array}{l}\text { The location (distance from the eye) of the far } \\
\text { clipping plane. }\end{array}$ \\
\hline Comments & \multicolumn{4}{|c|}{$\begin{array}{l}\text { The aspect ratio is the ratio of } x \text { to } y \text {. This should match the aspect ratio for the } \\
\text { viewport. See the Silicon Graphics Graphics Library Programming Guide(McLendon, } \\
\text { 1991), Appendix C.5, "Perspective Transformations," for more information. }\end{array}$} \\
\hline
\end{tabular}




\begin{tabular}{|c|c|c|c|c|}
\hline Name & \multicolumn{4}{|c|}{ MPPOLA - Define the viewing position using angles } \\
\hline Description & \multicolumn{4}{|c|}{ MPPOLA defines the viewing position, setting up the viewing matrix. } \\
\hline Syntax & \multicolumn{4}{|c|}{ CALL MPPOLA (DIST, AZIM, INC, TWIST) } \\
\hline $\begin{array}{l}\text { Variables in } \\
\text { Call }\end{array}$ & Name & $I / O$ & Type & Description \\
\hline & DIST & Input & real & $\begin{array}{l}\text { The distance from the eye to the world spac } \\
\text { origin. }\end{array}$ \\
\hline & AZIM & Input & real & $\begin{array}{l}\text { The azimuthal angle in degrees in the } x- \\
\text { plane measured from the z-axis. This is } \\
\text { rotation about the y-axis. (Keep the right } \\
\text { hand rule in mind when you determine th } \\
\text { sign of the angle.) }\end{array}$ \\
\hline & INC & Input & real & $\begin{array}{l}\text { The incidence angle in degrees in the } y- \\
\text { plane measured from the z-axis. This is } \\
\text { rotation about the x-axis. (Keep the right } \\
\text { hand rule in mind when you determine the } \\
\text { sign of the angle.) }\end{array}$ \\
\hline & TWIST & Input & real & $\begin{array}{l}\text { The angle of rotation in degrees about the lin } \\
\text { of sight (right-hand rule applies). }\end{array}$ \\
\hline Comments & \multicolumn{4}{|c|}{$\begin{array}{l}\text { The line of sight is defined as the line between the eye and the world space origin } \\
\text { See the Silicon Graphics Graphics Library Programming Guide (McLendon, 1991) } \\
\text { Appendix C.4, "Viewing Transformations," for more information. This transforma } \\
\text { tion is defined as follows: }\end{array}$} \\
\hline
\end{tabular}

ROTY $(-A Z I M) \times$ ROTX $(-I N C) \times$ ROTZ $(-T W I S T) \times$ TRANS $(0,0,-$ DIST $)$ 


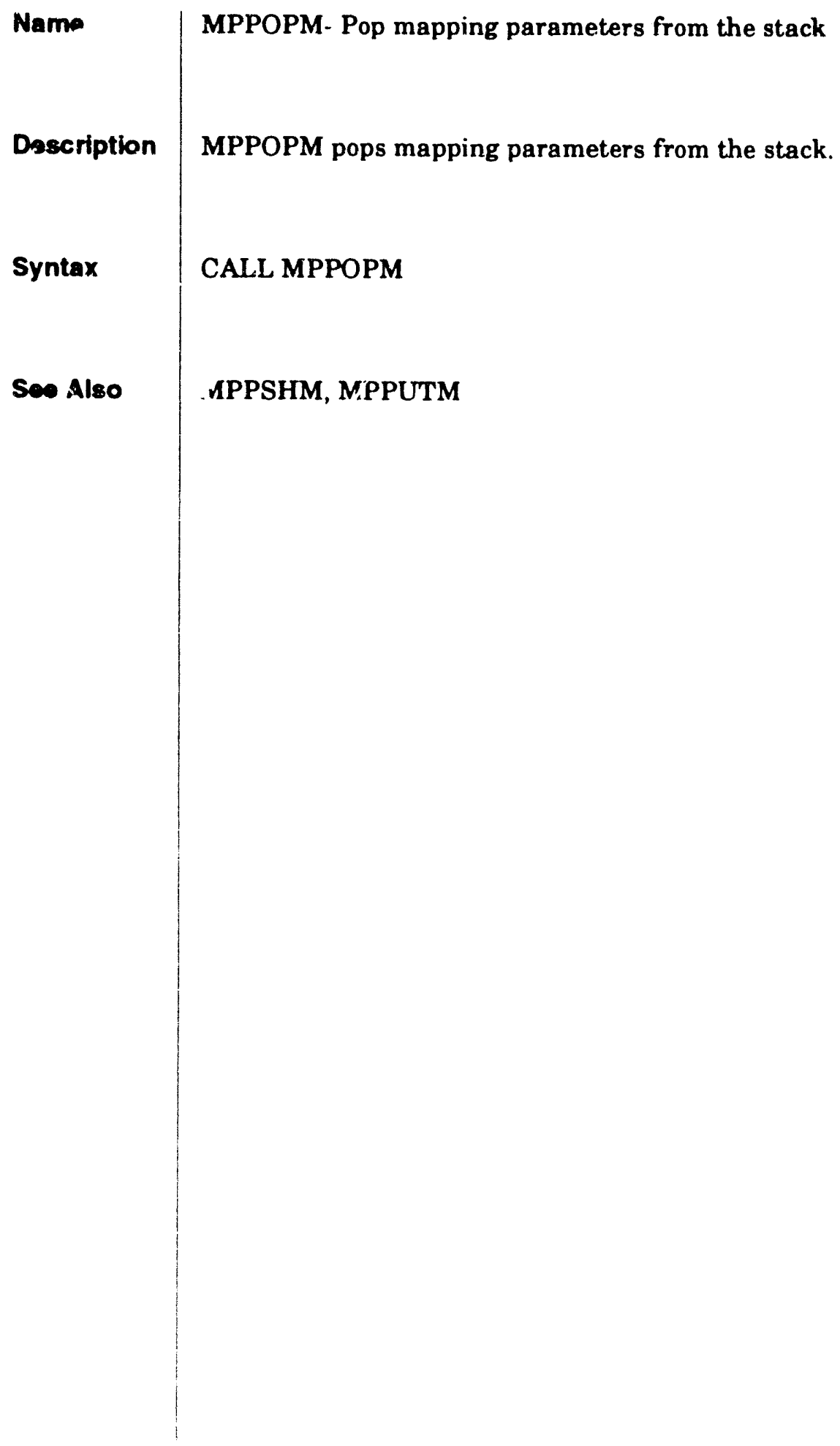


PLT_LIB

Description of Routine Calls

MPPSHM

Logical Function

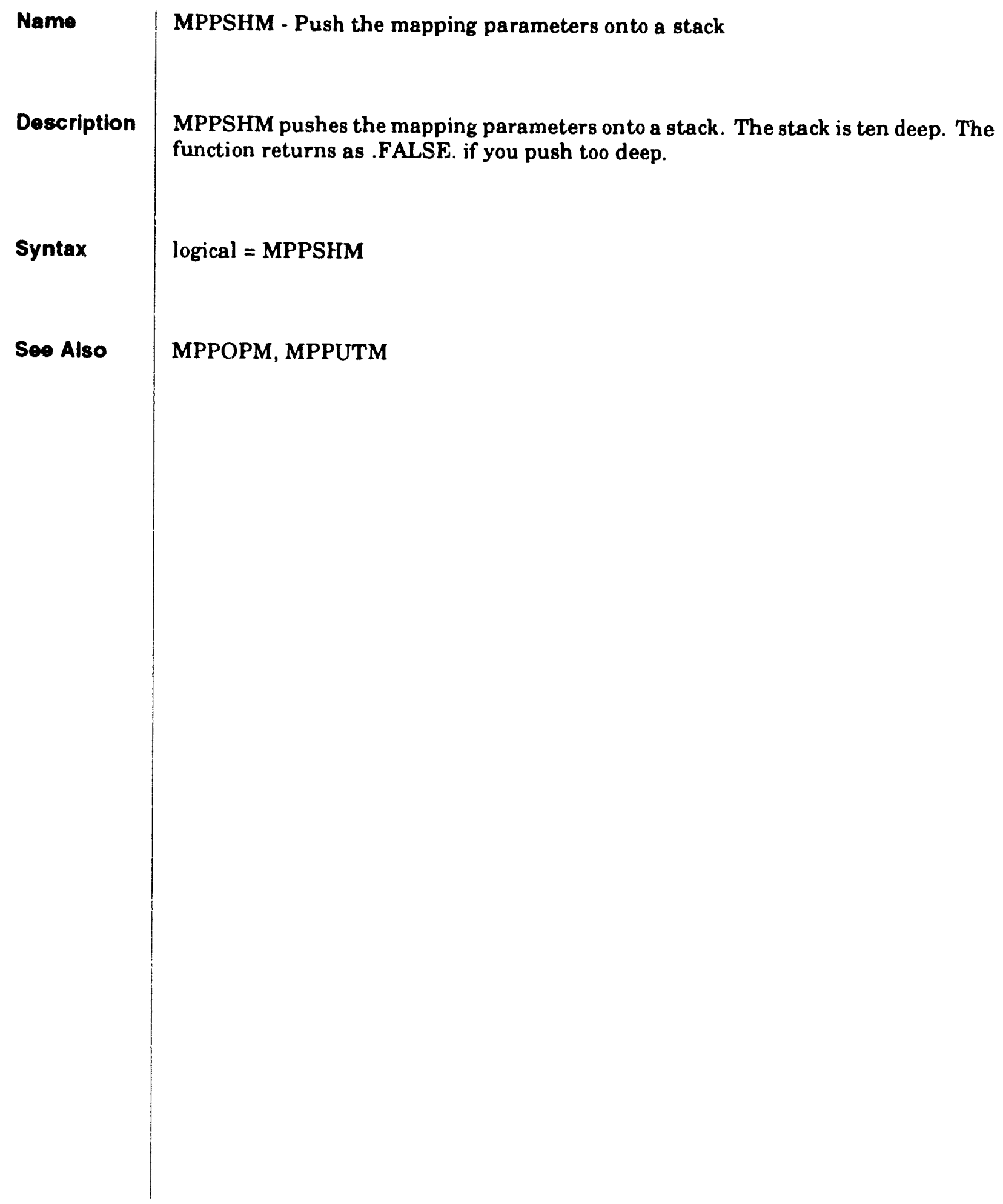




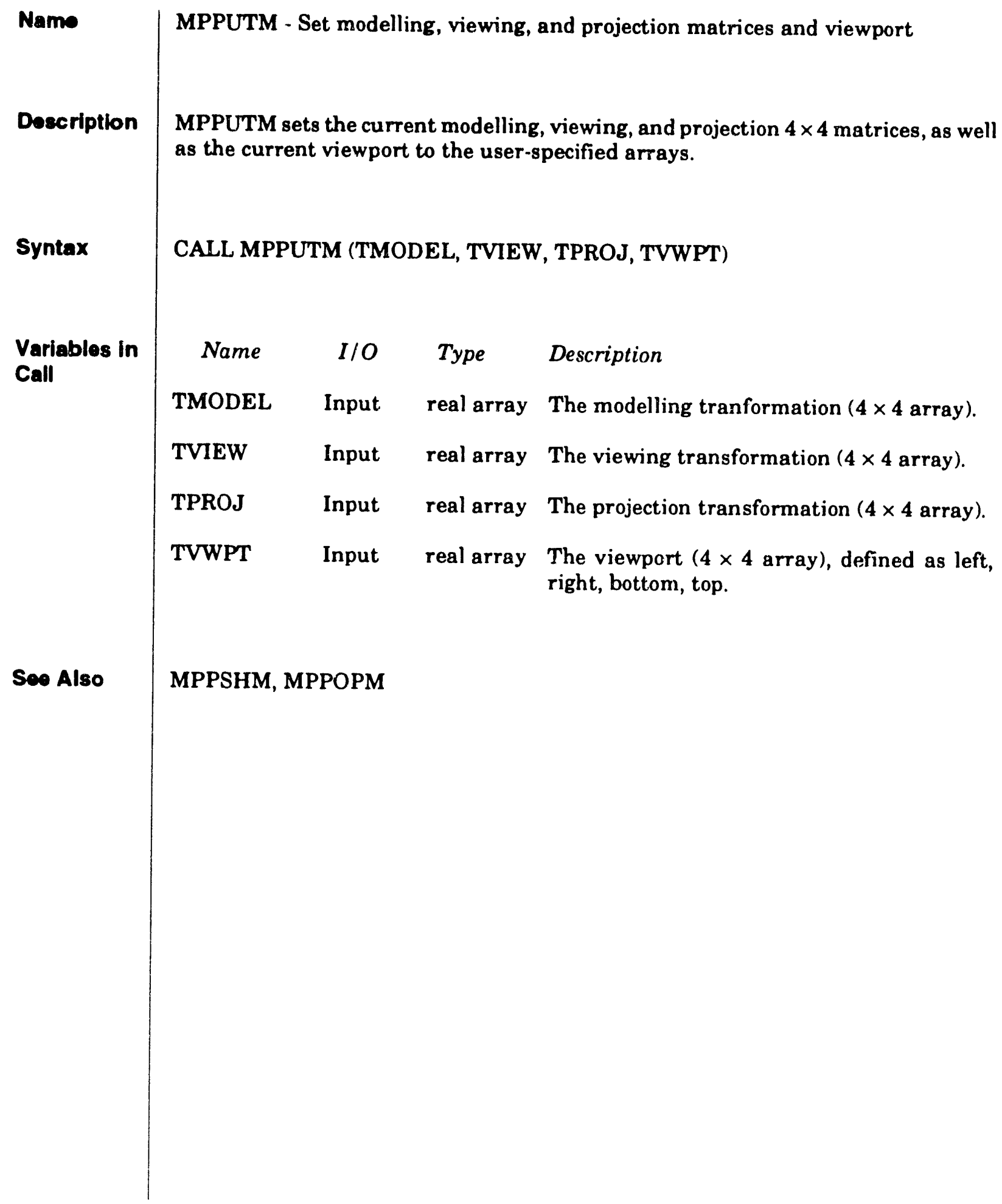


PLT_LIB

Description of Routine Calls

MPRESE

Subroutine

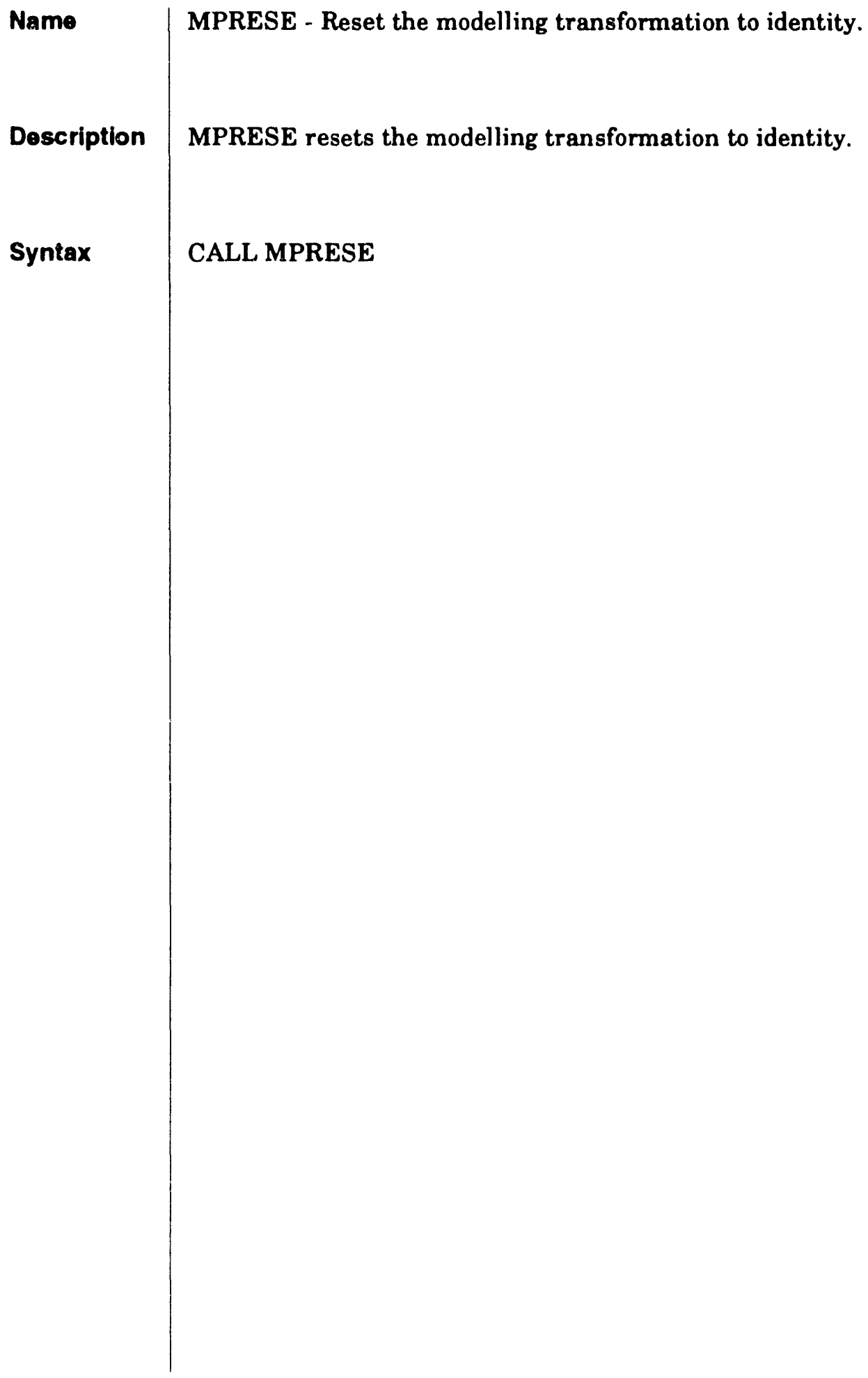




\section{MPRLOC}

\section{Subroutine}

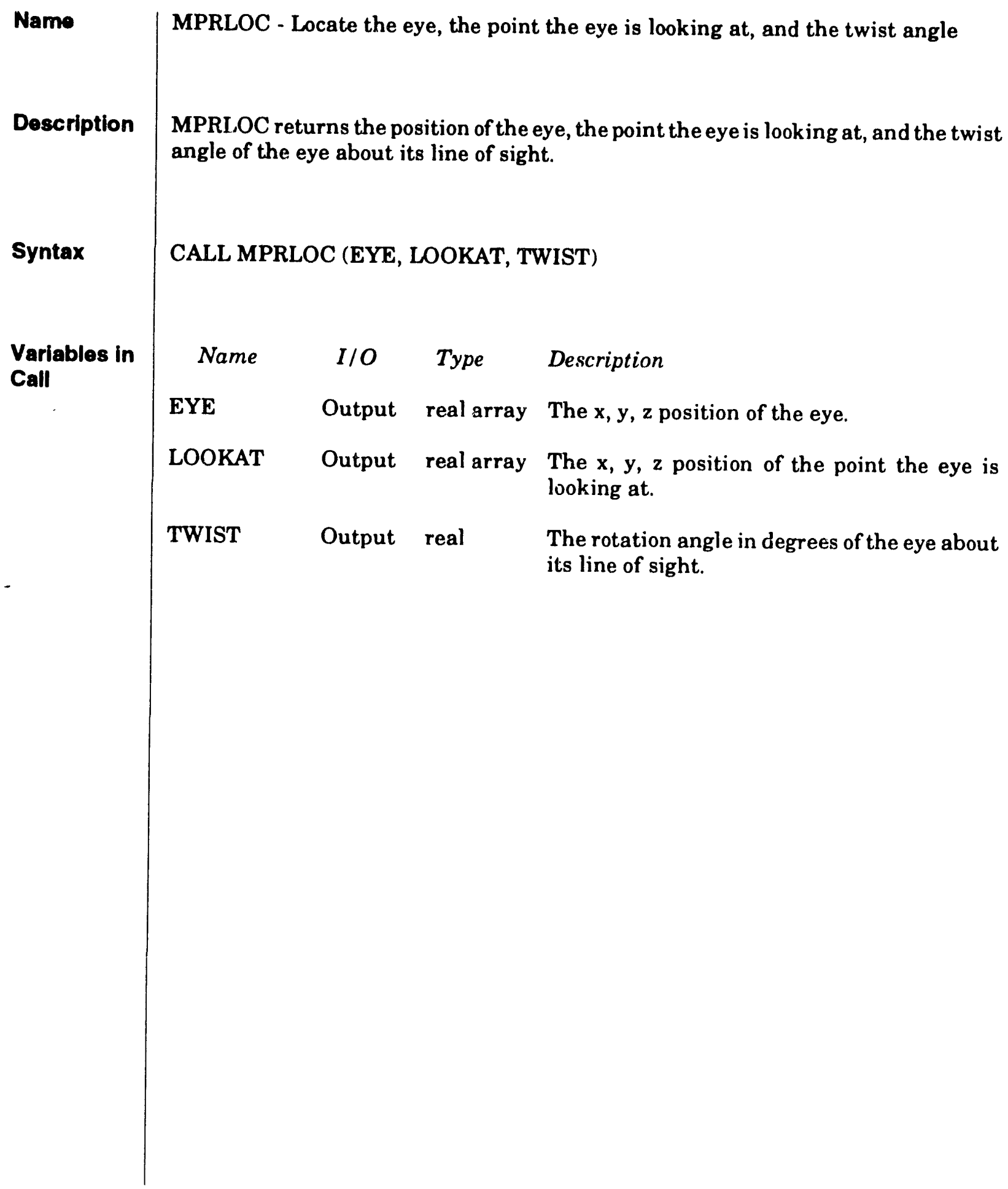




\begin{tabular}{|c|c|c|c|c|}
\hline Name & \multicolumn{4}{|c|}{ MPROTA - Rotate about an axis } \\
\hline Description & \multicolumn{4}{|c|}{ MPROTA rotates abor.c an axis. This function modifies the modelling matrix. } \\
\hline Syntax & \multicolumn{4}{|c|}{ logical = MPROTA (ANGLE, AXIS) } \\
\hline Variables in & Name & $I / O$ & Type & Description \\
\hline & ANGLE & Input & real & The amount to rotate in degrees. \\
\hline & & & & The axis to rotate about: "X" or "Y" or "Z". \\
\hline Note & \multicolumn{4}{|c|}{$\begin{array}{l}\text { The rotations are about the origin. See the Silicon Graphics Graphics Library } \\
\text { Programming Guide(McLendon, 1991), Appendix C.3, "Rotation," for more informa- } \\
\text { tion. }\end{array}$} \\
\hline
\end{tabular}




\begin{abstract}
\begin{tabular}{l|l} 
Name & MPSCAL - Scale in the $x, y$, and $z$ directions
\end{tabular}
Description

MPSCAL scales by X, Y, and Z. This subroutine modifies the modelling matrix.

Syntax

Variables in Call

Comment

CALL MPSCAL (X, Y, Z)

$\begin{array}{cccl}\text { Name } & \text { I/O } & \text { Type } & \text { Description } \\ \mathrm{X}, \mathrm{Y}, \mathrm{Z} & \text { Input } & \text { real } & \begin{array}{l}\text { The amount to scale in each direction. X, Y, or } \\ \mathrm{Z}>1 \text { to expand in that direction, }<1 \text { to shrink, } \\ \text { and }<0 \text { to mirror. }\end{array}\end{array}$

See the Silicon Graphics Graphics Library Programming Guide (McLendon, 1991), Appendix C.2, "Scaling and Mirroring," for more information.
\end{abstract}


PLT_LIB

Description of Routine Calls

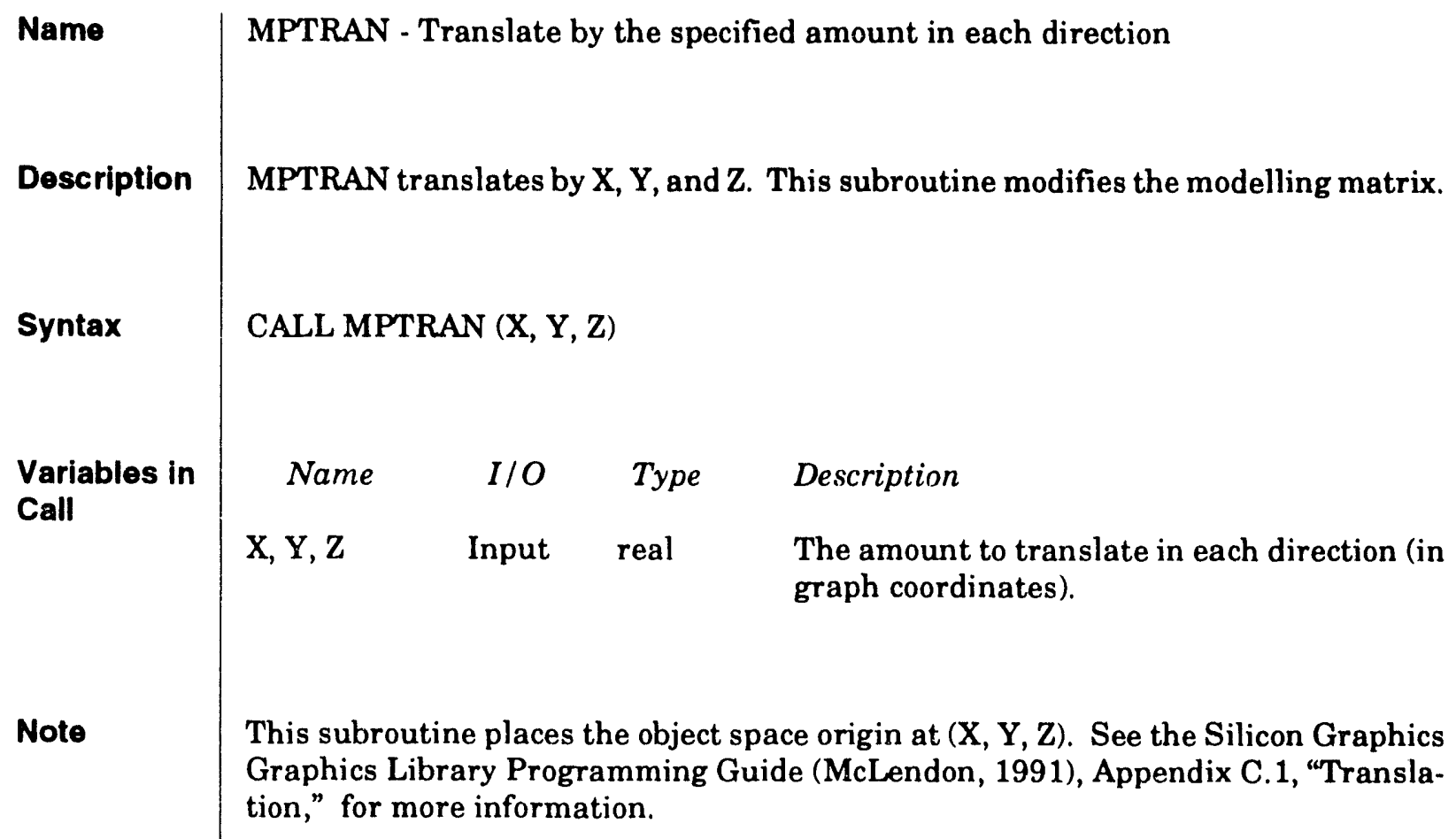




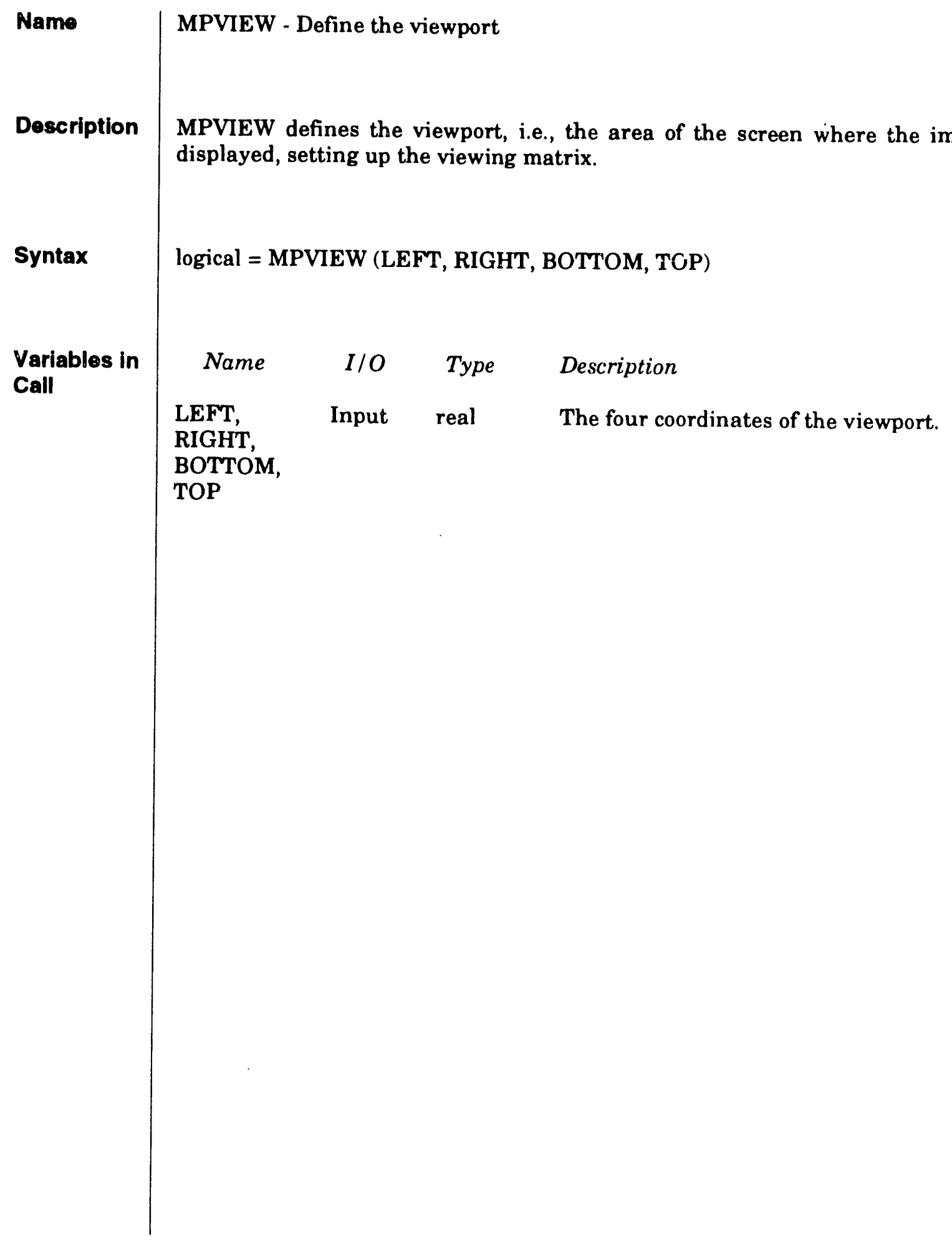




\begin{tabular}{|c|c|c|c|c|}
\hline Name & \multicolumn{4}{|c|}{ MPWIND - Define a perspective projection transformation in coordinates } \\
\hline Description & \multicolumn{4}{|c|}{$\begin{array}{l}\text { MPWIND defines a perspective projection transformation, setting up the projection } \\
\text { matrix. }\end{array}$} \\
\hline Syntax & \multicolumn{4}{|c|}{ logical = MPWIND (LEFT, RIGHT, BOTTOM, TOP, NEAR, FAR) } \\
\hline \multirow{7}{*}{$\begin{array}{l}\text { Variables in } \\
\text { Call }\end{array}$} & Name & $I / O$ & Type & Description \\
\hline & LEFT & Input & real & $\begin{array}{l}\text { The x-coordinate of the left edge of the view- } \\
\text { ing frustum. }\end{array}$ \\
\hline & RIGHT & Input & real & $\begin{array}{l}\text { The x-coordinate of the right edge of the view- } \\
\text { ing frustum. }\end{array}$ \\
\hline & BOTTOM & Input & real & $\begin{array}{l}\text { The y-coordinate of the bottom } \in \text { dge of the } \\
\text { viewing frustum. }\end{array}$ \\
\hline & TOP & Input & real & $\begin{array}{l}\text { The y-coordinate of the top edge of the viewing } \\
\text { frustum. }\end{array}$ \\
\hline & NEAR & Input & real & $\begin{array}{l}\text { The location (distance from the eye) of the } \\
\text { near clipping plane. }\end{array}$ \\
\hline & FAR & Input & real & $\begin{array}{l}\text { The location (distance from the eye) of the far } \\
\text { clipping plane. }\end{array}$ \\
\hline Comments & \multicolumn{4}{|c|}{$\begin{array}{l}\text { The viewing frustum is in the near clipping plane. See the Silicon Graphics Graphics } \\
\text { Library Programming Guide (McLendon, 1991), Appendix C.5, "Perspective Trans- } \\
\text { formations," for more information. }\end{array}$} \\
\hline
\end{tabular}




\subsubsection{Clipping of Two- and Three-Dimensional Points, Lines, and Polygons}

Detailed descriptions of the routines that provide clipping of two- and three-dimensional points, lines, and polygons are given on the following pages. 


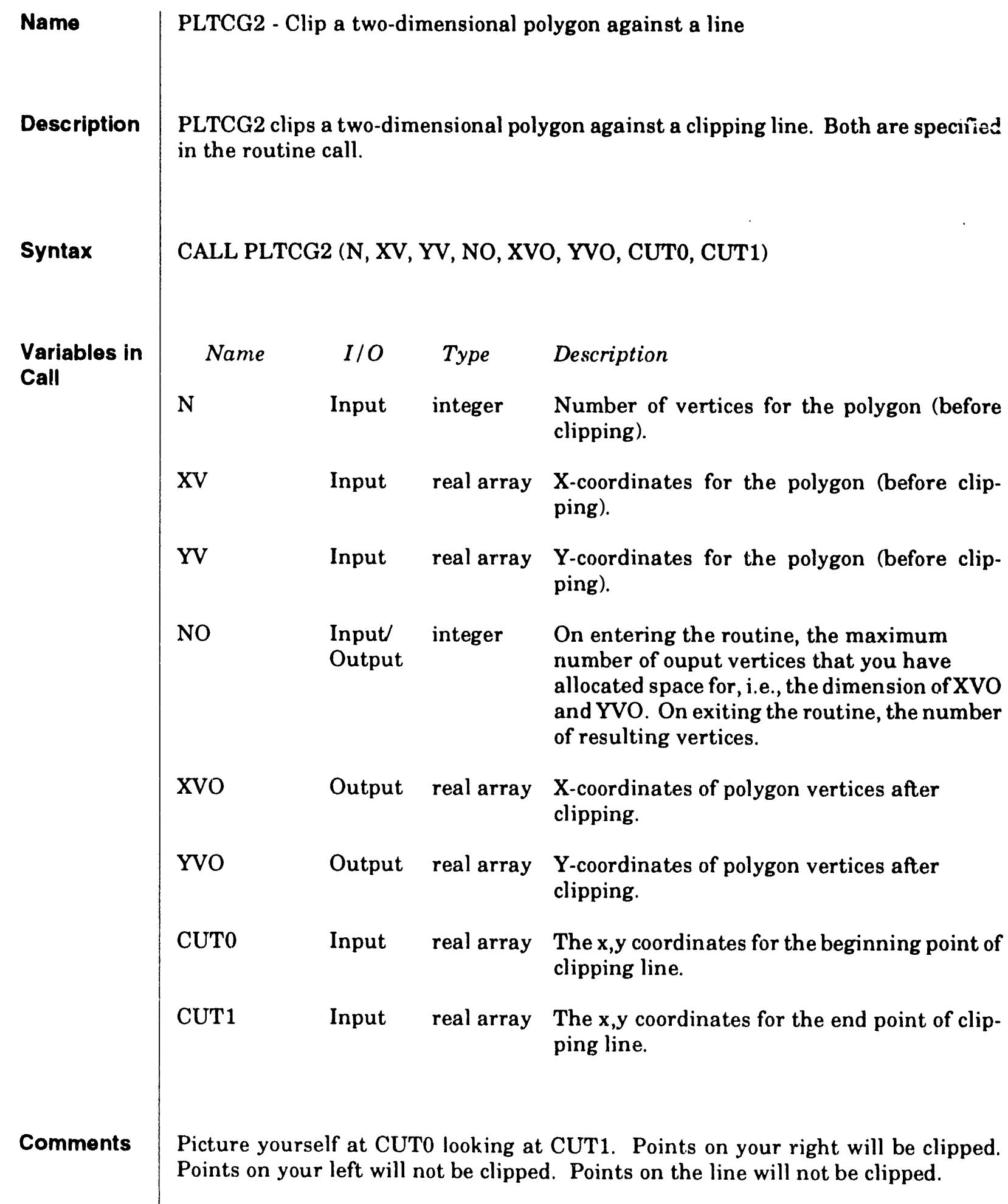


Name

Syntax
Variables in
Call

Comments
PLTCP2 - Clip two-dimensional points against a line

PLTCP2 clips two-dimensional points against a clipping line. Both the points and the clipping line are specified in the routine call.

CALL PLTCP2 (N, MASK, XA, YA, CUT0, CUT1)

\begin{tabular}{|c|c|c|c|}
\hline Name & $I / O$ & Type & Description \\
\hline $\mathrm{N}$ & Input & integer & Number of points. \\
\hline MASK & Input & $\begin{array}{l}\text { integer } \\
\text { array }\end{array}$ & $\begin{array}{l}\text { Bitmask. On entering the routine, if bit } 0 \text { is on } \\
(1) \text {, point } 1 \text { [XA( }(1), Y A(1)] \text { is a candidate for } \\
\text { clipping; if off }(0) \text {, point } 1 \text { is not to be clipped, } \\
\text { etc. On exiting the routine, if bit } 0 \text { is off }(0) \text {, } \\
\text { point } 1 \text { was clipped, assuming bit } 0 \text { was on } \\
\text { when the routine was entered. }\end{array}$ \\
\hline
\end{tabular}

XA Input real array $\mathrm{X}$-coordinates of points.

YA Input real array Y-coordinates of points.

CUT0 Input real array The $x, y$ coordinates for beginning point of clipping line.

CUT1 Input real array The $x, y$ coordinates for end point of clipping line.

Picture yourself at CUT0 looking at CUT1. Points on your right will be clipped. Points on your left will not be clipped. Points on the line will not be clipped. 
Name
Description

Syntax

Variables in Call
PLTCP3 - Clip three-dimensional points against a plane

PLTCP3 clips three-dimensional points against a plane. Both the points and the plane are specified in the routine call.

CALL PLTCP3 (N, MASK, XA, YA, ZA, PTIN, VECORT)

\section{Name I/O Type Description}

$\mathrm{N} \quad$ Input integer Number of points.

MASK Input/ integer Bitmask. On entering the routine, if bit 0 is on Output array or (1), point $1[\mathrm{XA}(1), \mathrm{YA}(1), \mathrm{ZA}(1)]$ is a candidate variable for clipping; if off $(0)$, point 1 is not to be clipped, etc. On exiting the routine, if bit 0 is off ( 0 ), point 1 was clipped, assuming bit 0 was on when the routine was entered.

$\mathrm{XA}$ Input/ real array X-coordinates of points; returned Output clipped.

YA Input/ real array Y-coordinates of points; returned Output clipped.

ZA Input/ real array Z-coordinates of points; returned Output clipped.

PTIN Input real array The $x, y, z$ coordinates of a point in the clipping plane.

VECORT Input real array The $x, y, z$ coordinates of a vector orthogonal to the clipping plane.

VECORT and PTIN define the clipping plane. VECORT points into the side of the plane where points are not to be clipped. 


\begin{abstract}
\begin{tabular}{l|l} 
Namo & PLTCV2 - Clip two-dimensional vectors against a line
\end{tabular}
Description

PLTCV2 clips two-dimensional vectors against a clipping line. Both the vectors and the clipping line are specified in the routine call.

Syntax

CALL PLTCV2 (N, MASK, XIN0, YIN0, XIN1, YIN1, XOUT0, YOUT0, XOUT1, YOUT1,CUT0, CUT1)

Variables in

Name I/O Type Description

Call

Conments

Input integer Number of vectors.

MASK Input integer Bitmask. On entering the routine, if bit 0 is on Output array (1), vector 1 is a candidate for clipping; if off $(0)$, vector 1 is not to be clipped, etc. On exiting the routine, if bit 0 is off $(0)$, vector 1 was clipped, assuming bit 0 was on when the routine was entered.

XINO, YINO Input real array Coordinates of start points for input vectors.

XIN1, YIN1 Input real array Coordinates of end points for input vectors.

XOUT0, Output real array Coordinates of start points for

YOUT'0 resulting (clipped) vectors.

XOUT1, Output real array Coordinates of end points for resulting

YOUT1 (clipped) vectors.

CUT0 Input real array The $x, y$ coordinates for the beginning point of the clipping line.

CUT1 Input real array The $x, y$ coordinates for the end point of the clipping line.
\end{abstract}




\begin{tabular}{|c|c|c|c|c|}
\hline Name & \multicolumn{4}{|c|}{ PLTCV3 - Clip three-dimensional vectors against a plane } \\
\hline Description & \multicolumn{4}{|c|}{$\begin{array}{l}\text { PLTCV } 3 \text { clips three-dimensional vectors against a plane. Both the vectors and the } \\
\text { plane are specified in the routine call. }\end{array}$} \\
\hline Syntax & \multicolumn{4}{|c|}{$\begin{array}{l}\text { CALL PLTCV3 (N, MASK, XIN0, YIN0, ZIN0, XIN1, YIN1, ZIN1, XOUT0, YOUT0, } \\
\text { ZOUT0, XOUT1, YOUT1, ZOUT1, PTIN, VECORT) }\end{array}$} \\
\hline \multirow[t]{8}{*}{$\begin{array}{l}\text { Variables in } \\
\text { Call }\end{array}$} & Name & I/O & Type & Description \\
\hline & MASK & $\begin{array}{l}\text { Input/ } \\
\text { Output }\end{array}$ & $\begin{array}{l}\text { integer } \\
\text { array or } \\
\text { literal }\end{array}$ & $\begin{array}{l}\text { Bitmask. On entering the routine, if bit } 0 \text { is on } \\
\text { (1), vector } 1 \text { is a candidate for clipping; if off } \\
(0) \text {, vector } 1 \text { is not to be clipped, etc. On exiting } \\
\text { the routine, if bit } 0 \text { is off }(0) \text {, vector } 1 \text { was } \\
\text { clipped, assuming bit } 0 \text { was on when the } \\
\text { routine was entered. }\end{array}$ \\
\hline & $\begin{array}{l}\text { XIN0, YIN0, } \\
\text { ZINO }\end{array}$ & Input & real array & Coordinates of start points for input vectors. \\
\hline & $\begin{array}{l}\text { XIN1, YIN1, } \\
\text { ZIN1 }\end{array}$ & Input & real array & Coordinates of end points for input vectors. \\
\hline & $\begin{array}{l}\text { XOUT0, } \\
\text { YOUT0, } \\
\text { zOUT0 }\end{array}$ & Output & real array & $\begin{array}{l}\text { Coordinates of start points for resulting } \\
\text { (clipped) vectors. }\end{array}$ \\
\hline & $\begin{array}{l}\text { XOUT1, } \\
\text { YOUT1, } \\
\text { ZOUT1 }\end{array}$ & Output & real array & $\begin{array}{l}\text { Coordinates of end points for resulting } \\
\text { (clipped) vectors. }\end{array}$ \\
\hline & PTIN & Input & real array & $\begin{array}{l}\text { The } x, y \text {, and } z \text { coordinates of a point in the } \\
\text { clipping plane. }\end{array}$ \\
\hline & VECORT & Input & real array & $\begin{array}{l}\text { The } x, y \text {, and } z \text { coordinates of a vector orthogo- } \\
\text { nal to the clipping plane. }\end{array}$ \\
\hline
\end{tabular}

Comments

VECORT and PTIN define the clipping plane. VECORT points into the side of the plane where points are not to be clipped. 


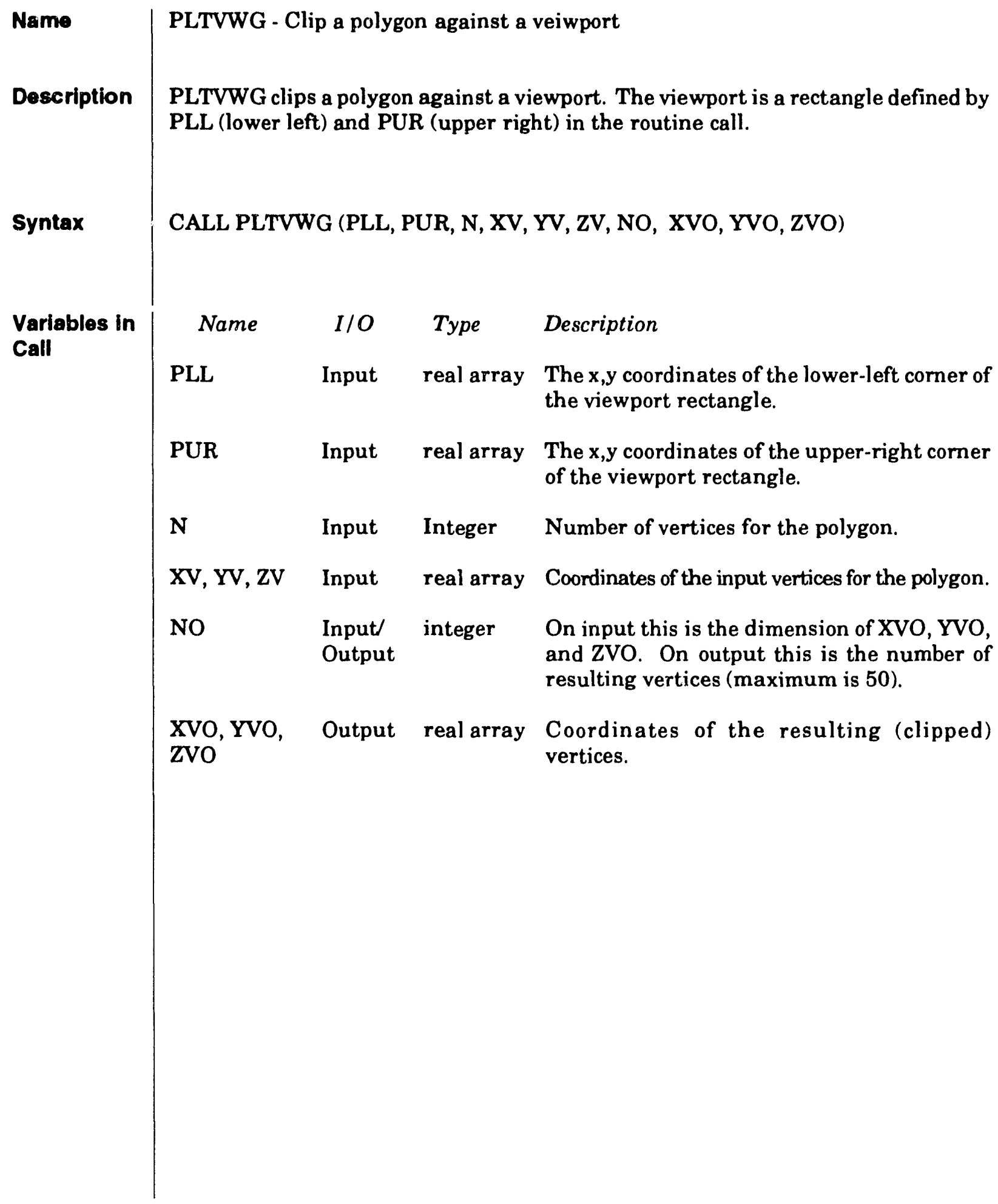


PLT_LIB

Description of Routine Calls

PLTVWP

Subroutine

\begin{tabular}{|c|c|c|c|c|}
\hline Name & \multicolumn{4}{|c|}{ PLTVWP - Clip points against a veiwport } \\
\hline Description & \multicolumn{4}{|c|}{$\begin{array}{l}\text { PLTVWP clips points against a viewport. The viewport is a rectangle defined by PLL } \\
\text { (lower left) and PUR (upper right) in the routine call. }\end{array}$} \\
\hline Syntax & \multicolumn{4}{|c|}{ CALL PLTVWP (PLL, PUR, N, MASK, X, Y) } \\
\hline \multirow{6}{*}{$\begin{array}{l}\text { Variables in } \\
\text { Call }\end{array}$} & Name & $I / O$ & Type & Description \\
\hline & PLL & Input & real array & $\begin{array}{l}\text { The } x, y \text { coordinates of the lower-left corner of } \\
\text { the viewport rectangle. }\end{array}$ \\
\hline & PUR & Input & real array & $\begin{array}{l}\text { The } x, y \text { coordinates of the upper-right corner } \\
\text { of the viewport rectangle. }\end{array}$ \\
\hline & $\mathbf{N}$ & Input & integer & Number of points. \\
\hline & MASK & $\begin{array}{l}\text { Input } \\
\text { Output }\end{array}$ & $\begin{array}{l}\text { integer } \\
\text { array }\end{array}$ & $\begin{array}{l}\text { On entering the routine, if bit } 0 \text { is on (1), } \\
\text { point } 1 \text { is a candidate for clipping; if off ( } 0) \text {, } \\
\text { point } 1 \text { is not to be clipped, etc. On exiting the } \\
\text { routine, if bit } 0 \text { is off }(0) \text {, point } 1 \text { was clipped, } \\
\text { assuming bit } 0 \text { was on when the routine was } \\
\text { entered. }\end{array}$ \\
\hline & $\mathrm{X}, \mathrm{Y}$ & $\begin{array}{l}\text { Input/ } \\
\text { Output }\end{array}$ & real array & $\begin{array}{l}\text { Coordinates of the points. These } \\
\text { are returned clipped. }\end{array}$ \\
\hline
\end{tabular}


Namo

Description

Syntax

Variables in Call
PLTVWV - Clip vectors against a veiwport

PLTVWV clips vectors against a viewport. The viewport is a rectangle defined by PLL (lower left) and PUR (upper right) in the routine call.

CALL PLTVWV (PLL, PUR, N, MASK, XV0, YV0, XV1, YV1)

\section{Name I/O Type Description}

PLL Input real array The $x, y$ coordinates of the lower-left corner of the viewport rectangle.

PUR Input real array The $x, y$ coordinates of the upper-right corner of the viewport rectangle.

N Input integer Number of vectors.

MASK Input/ integer Bitmask. On entering the routine, if bit 0 is on Output array (1), vector 1 is a candidate for clipping; if off $(0)$, vector 1 is not to be clipped, etc. On exiting the routine, if bit 0 if off $(0)$, vector 1 was clipped, assuming bit 0 was on when the routine was entered.

XV0, YV0 Input real array Coordinates of the start points for the Output vectors. These are returned clipped.

XV1, YV1 Input real array Coordinates of the end points for the Output vectors. These are returned clipped. 


\begin{tabular}{l|l} 
Name & $\begin{array}{l}\text { PLTZCP - Clip points against two clipping planes } \\
\text { Sescription }\end{array}$ \\
Variables in \\
Call \\
PLTZCP clips points against two clipping planes. Both the points and the planes are \\
their distances from the origin are ZNEAR and ZFAR.
\end{tabular}


Name

Description

Syntax

Variables in Call

PLTZCV - Clip vectors against two clipping planes

PLTZCV clips vectors against two clipping planes. Both the vectors and the planes are specified in the routine call. Both planes are perpendicular to the $\mathrm{z}$ direction and their distances from the origin are ZNEAR and ZFAR.

CALL PLTZCV (ZNEAR, ZFAR, N, MASK, XV0, YV0, ZV0, XV1, YV1, ZV1)

\begin{tabular}{|c|c|c|c|}
\hline Name & $1 / O$ & Type & Description \\
\hline JEAR & Input & real & Distance of the nearer plane from the origin. \\
\hline AR & Input & real & Distance of the further plane from the origin. \\
\hline & Input & integer & The number of vectors. \\
\hline ASK & $\begin{array}{l}\text { Input/ } \\
\text { Output }\end{array}$ & $\begin{array}{l}\text { integer } \\
\text { array }\end{array}$ & $\begin{array}{l}\text { Bitmask. On entering the routine, if bit } 0 \text { is on } \\
\text { (1), vector } 1 \text { is a candidate for clipping; if off } \\
(0) \text {, vector } 1 \text { is not to be clipped, etc. On exiting } \\
\text { the routine, if bit } 0 \text { is off }(0) \text {, vector } 1 \text { was } \\
\text { clipped, assuming bit } 0 \text { was on when the } \\
\text { routine was entered. }\end{array}$ \\
\hline
\end{tabular}

XV0, YVO, Input/ real array Coordinates of the start points of the vectors. ZVO Output These are returned clipped.

XV1, YV1, Input/ real array Coordinates of the end points of the vectors. ZV1 Output These are returned clipped. 
PLT_LIB

Description of Routine Calls

10.2.8 Color Palette Routines

Detailed descriptions of the routines that set the color palette and provide color information are given on the following pages. 


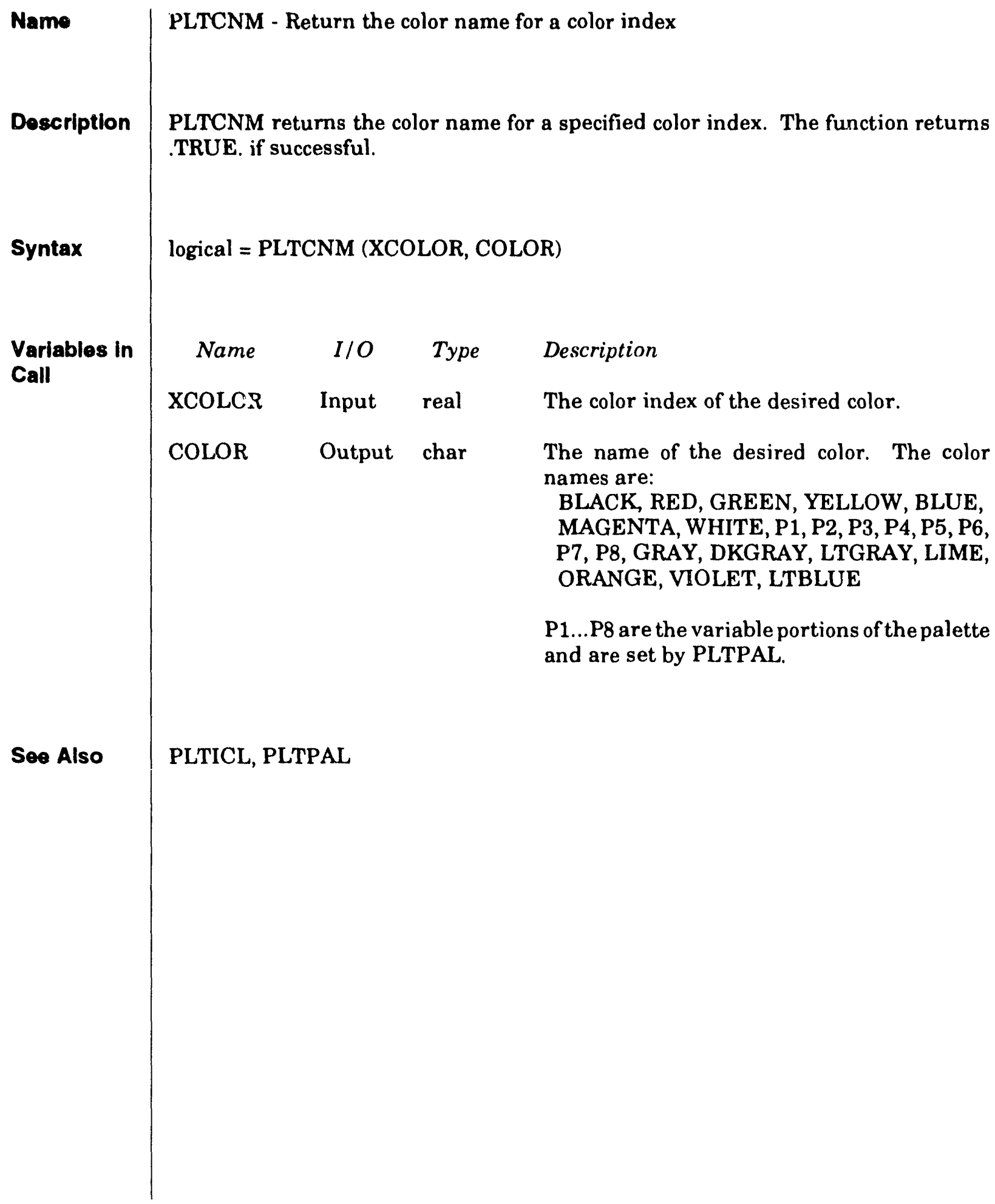




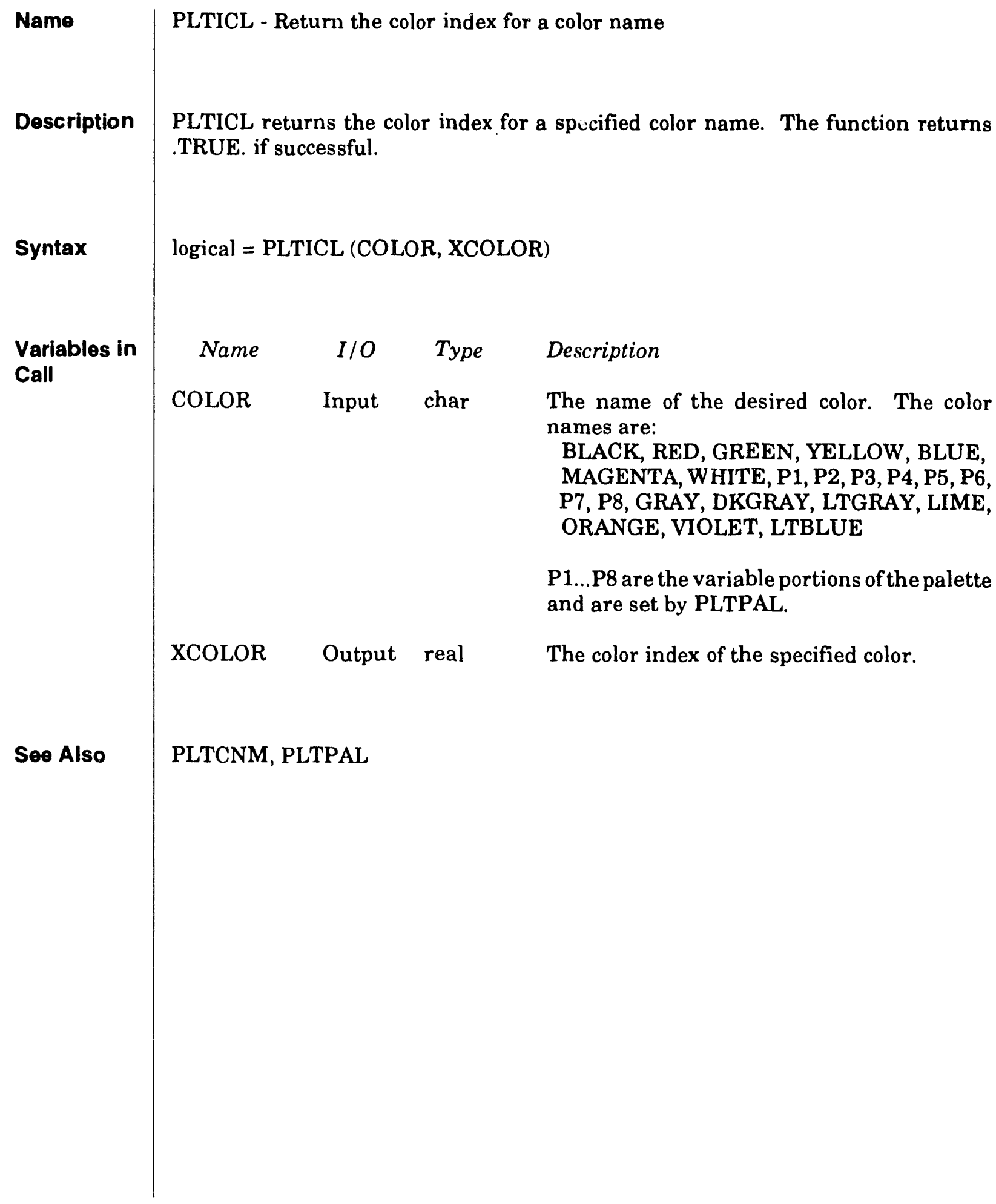




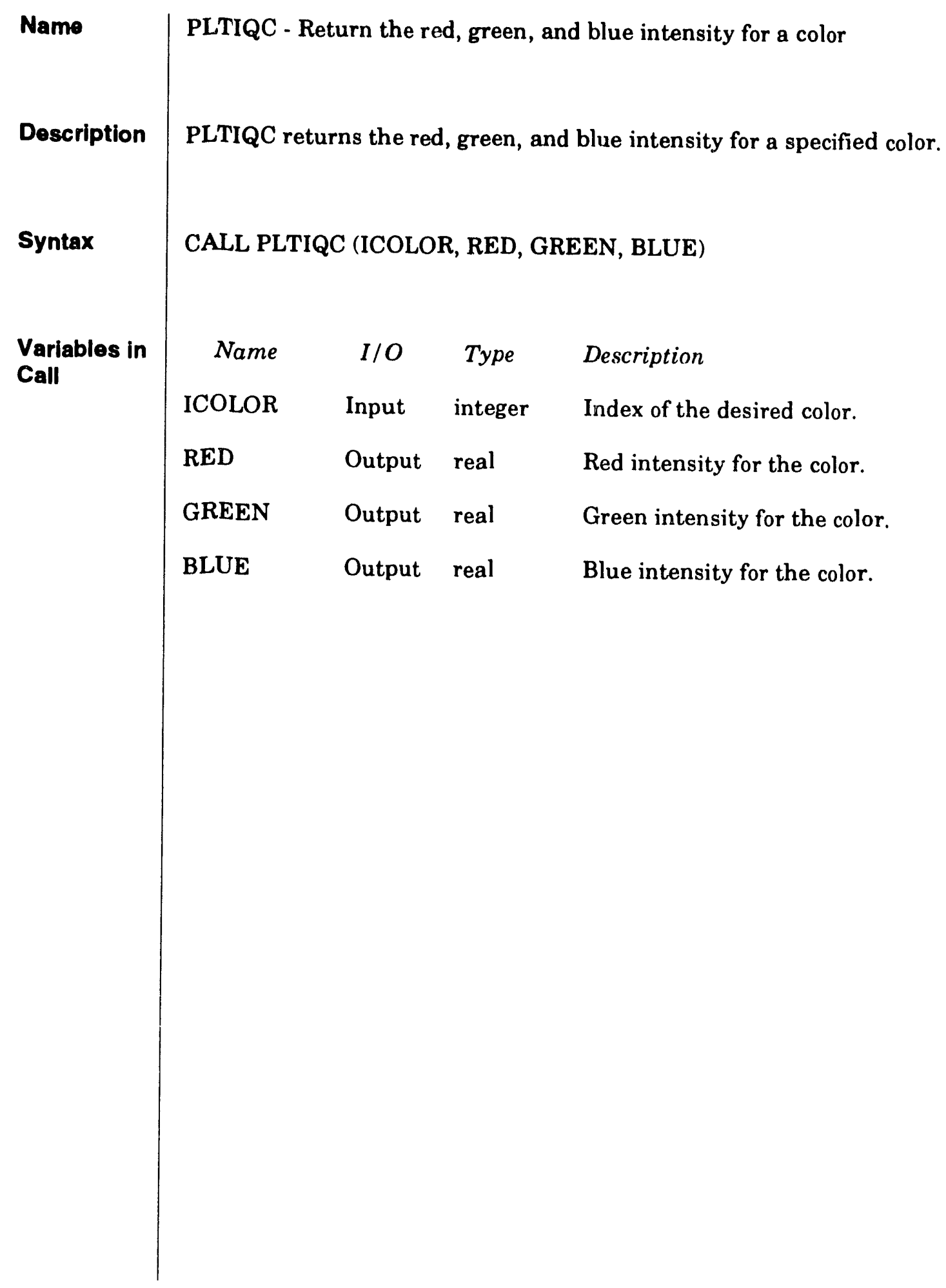




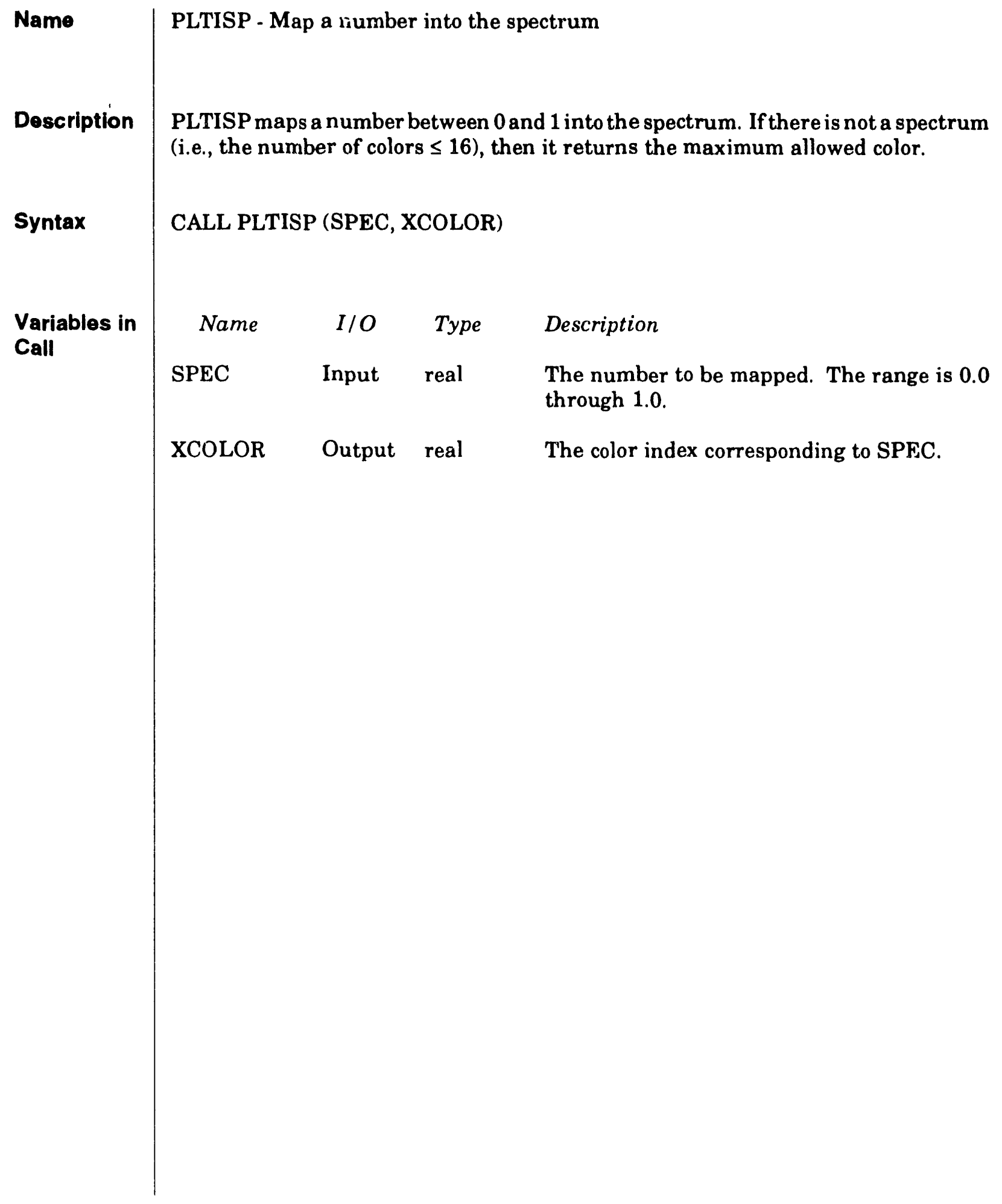




\section{PLTPAL}

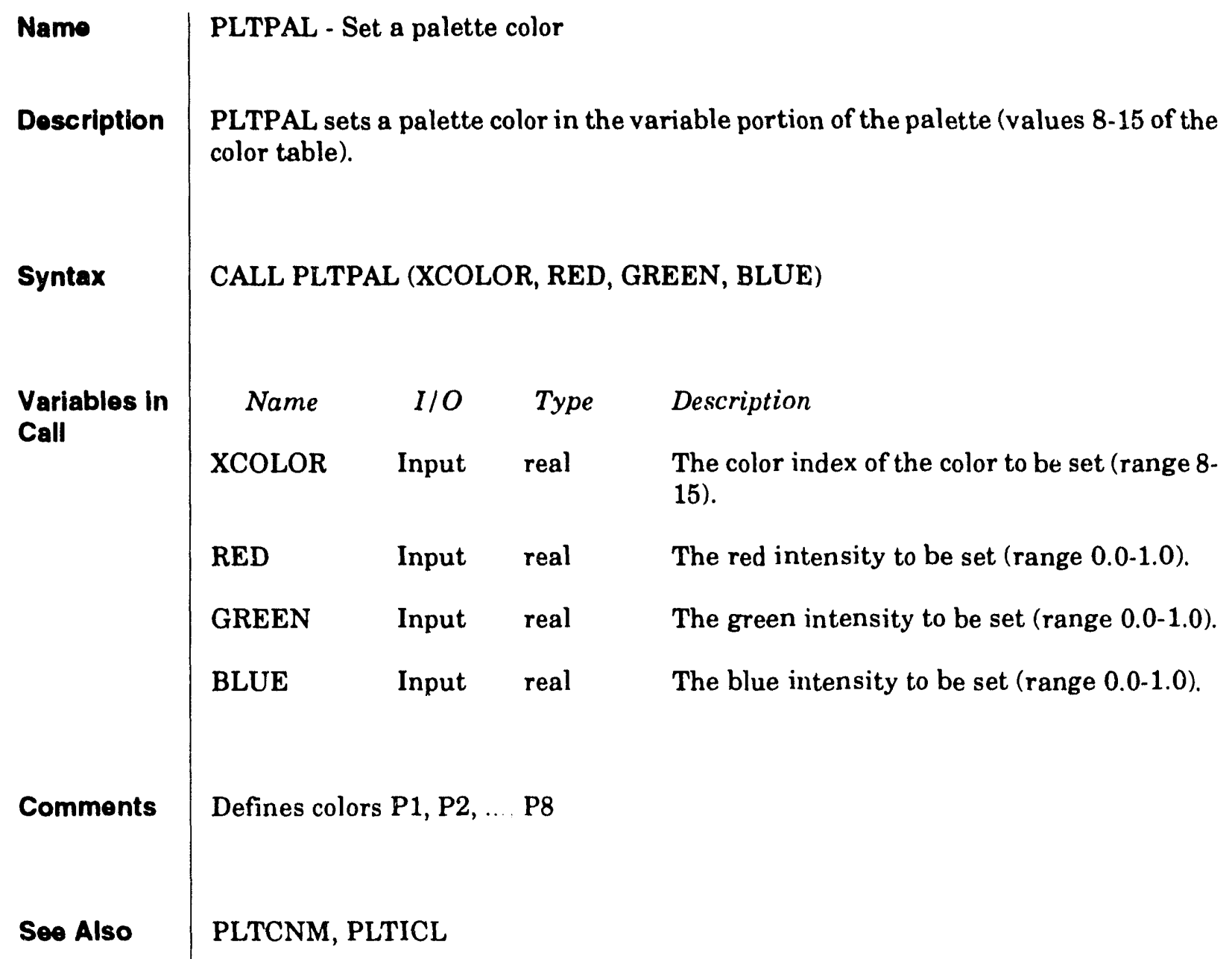




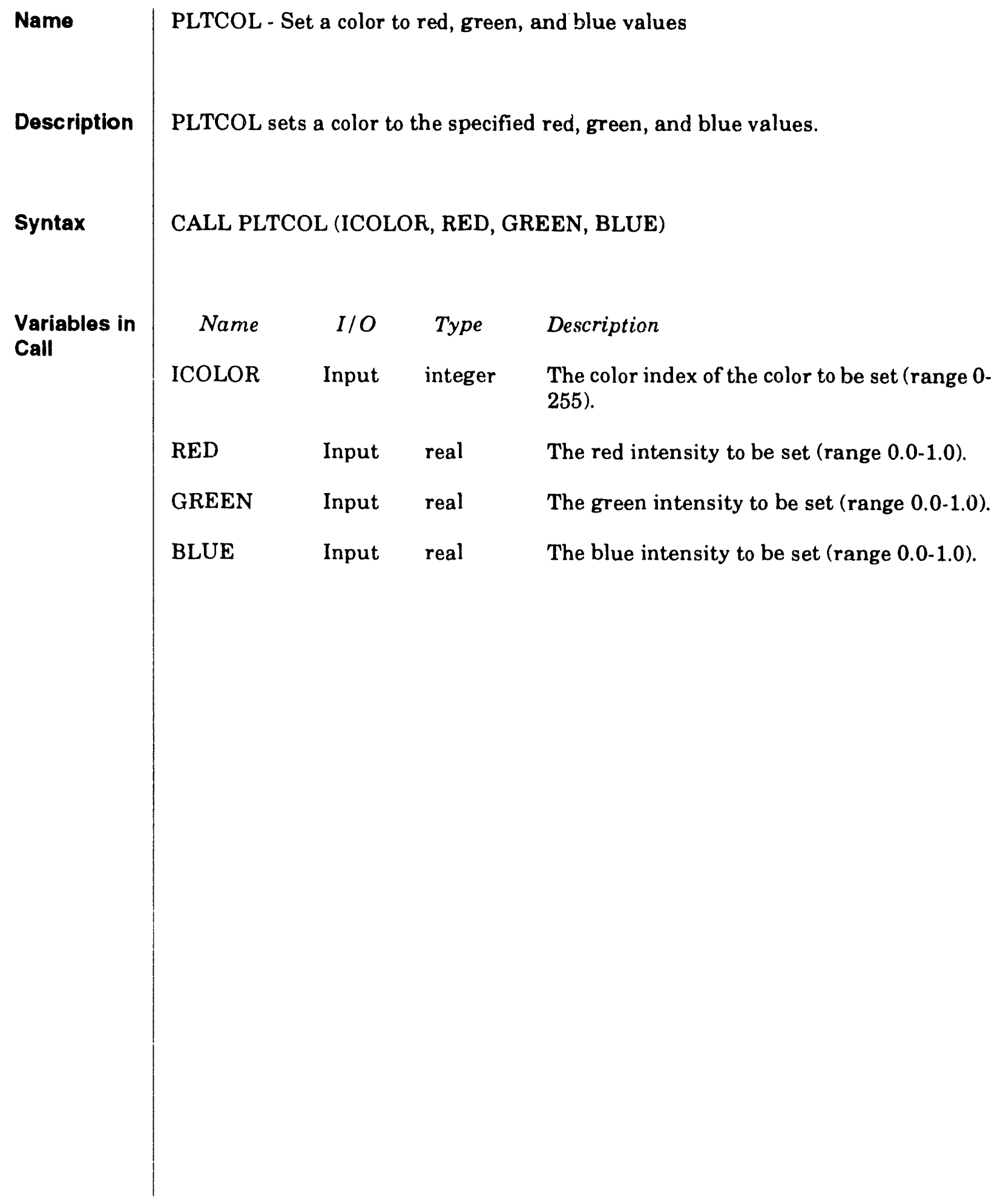




\begin{tabular}{|c|c|c|c|c|}
\hline Name & \multicolumn{4}{|c|}{ PLTSPC - Set up colors in the spectrum } \\
\hline Description & \multicolumn{4}{|c|}{$\begin{array}{l}\text { PLTSPC sets up colors in a specified interval of the spectrum. S1 and S2 are } \\
\text { normalized numbers between } 0 \text { and } 1 \text { that map to an index in the spectrum. }\end{array}$} \\
\hline Syntax & \multicolumn{4}{|c|}{ CALL FinSPC (S1, RED1, GREEN1, BLUE1, S2, RED2, GREEN2, BLUE2) } \\
\hline \multirow{9}{*}{$\begin{array}{l}\text { Variables in } \\
\text { Call }\end{array}$} & Nrame & $I / O$ & Type & Description \\
\hline & S1 & Input & real & $\begin{array}{l}\text { The starting value for the spectrum (range } \\
0.0-1.0 \text { ). }\end{array}$ \\
\hline & RED1 & Input & real & The red intensity at the starting value. \\
\hline & GREEN1 & Input & real & The green intensity at the starting value. \\
\hline & BLUE1 & Input & real & The blue intensity at the starting value. \\
\hline & S2 & Input & real & $\begin{array}{l}\text { The ending value for the spectrum (range } 0.0 \text { - } \\
1.0 \text { ). }\end{array}$ \\
\hline & RED2 & Input & real & $\begin{array}{l}\text { The red intensity at the end of the spectrum } \\
\text { interval. }\end{array}$ \\
\hline & GREEN2 & Input & real & $\begin{array}{l}\text { The green intensity at the end of the spectrum } \\
\text { interval. }\end{array}$ \\
\hline & BLUE2 & Input & real & $\begin{array}{l}\text { The blue intensity at the end of the spectrum } \\
\text { interval. }\end{array}$ \\
\hline
\end{tabular}


PLT_LIB

Description of Routine Calls

\subsubsection{Obsolete Routines}

The routines described in this section are still available but have been made obsolete by new routines. 


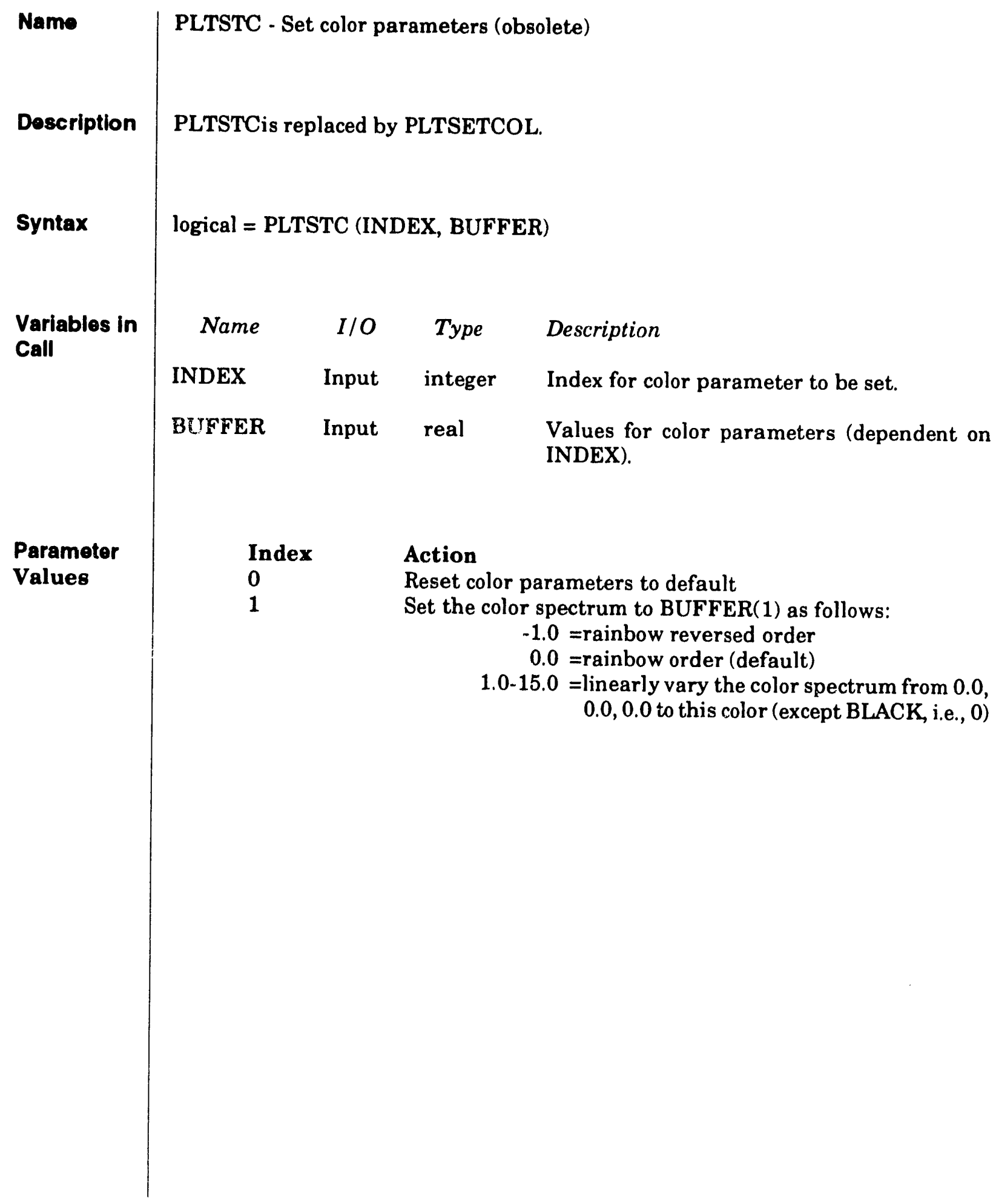




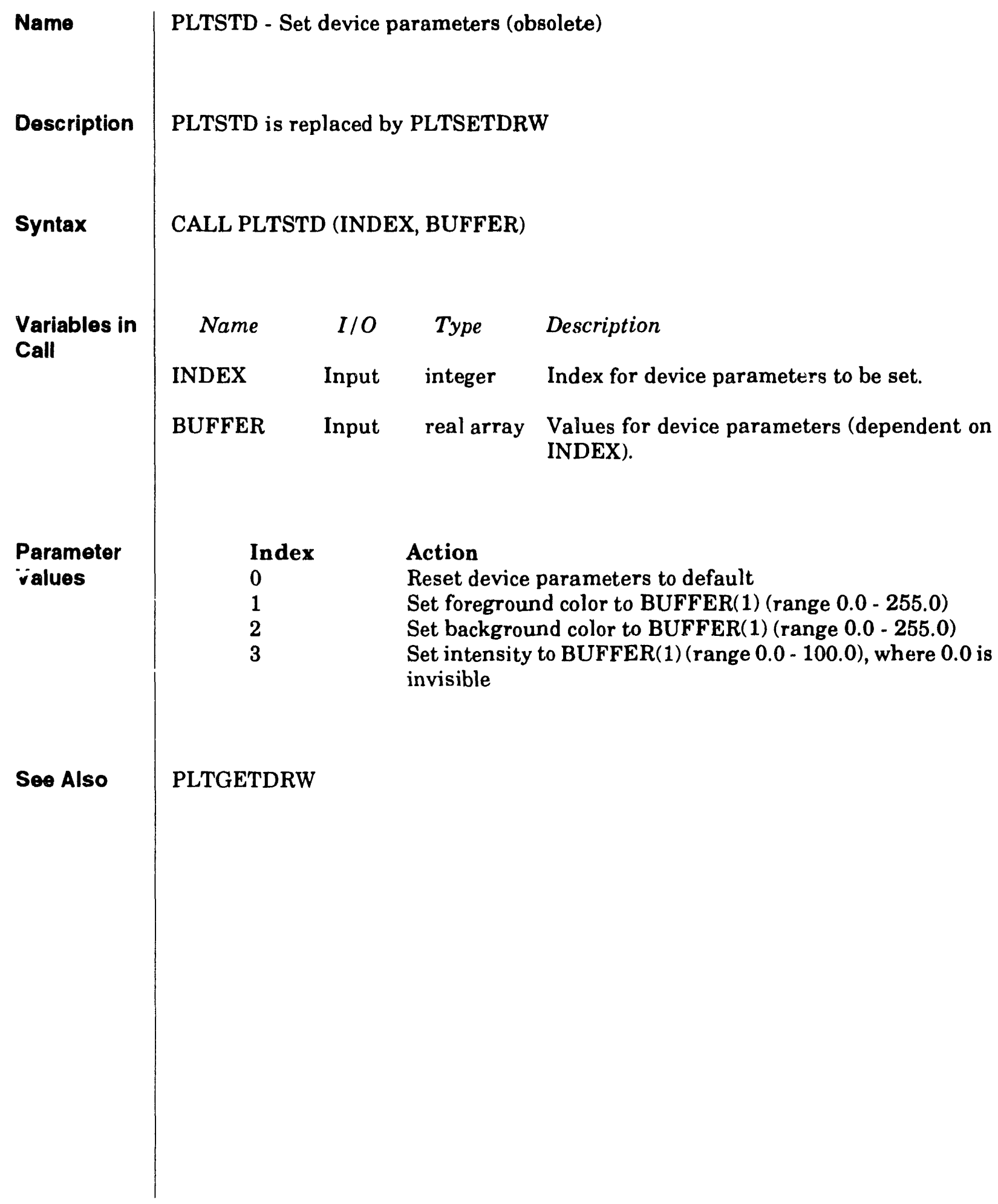




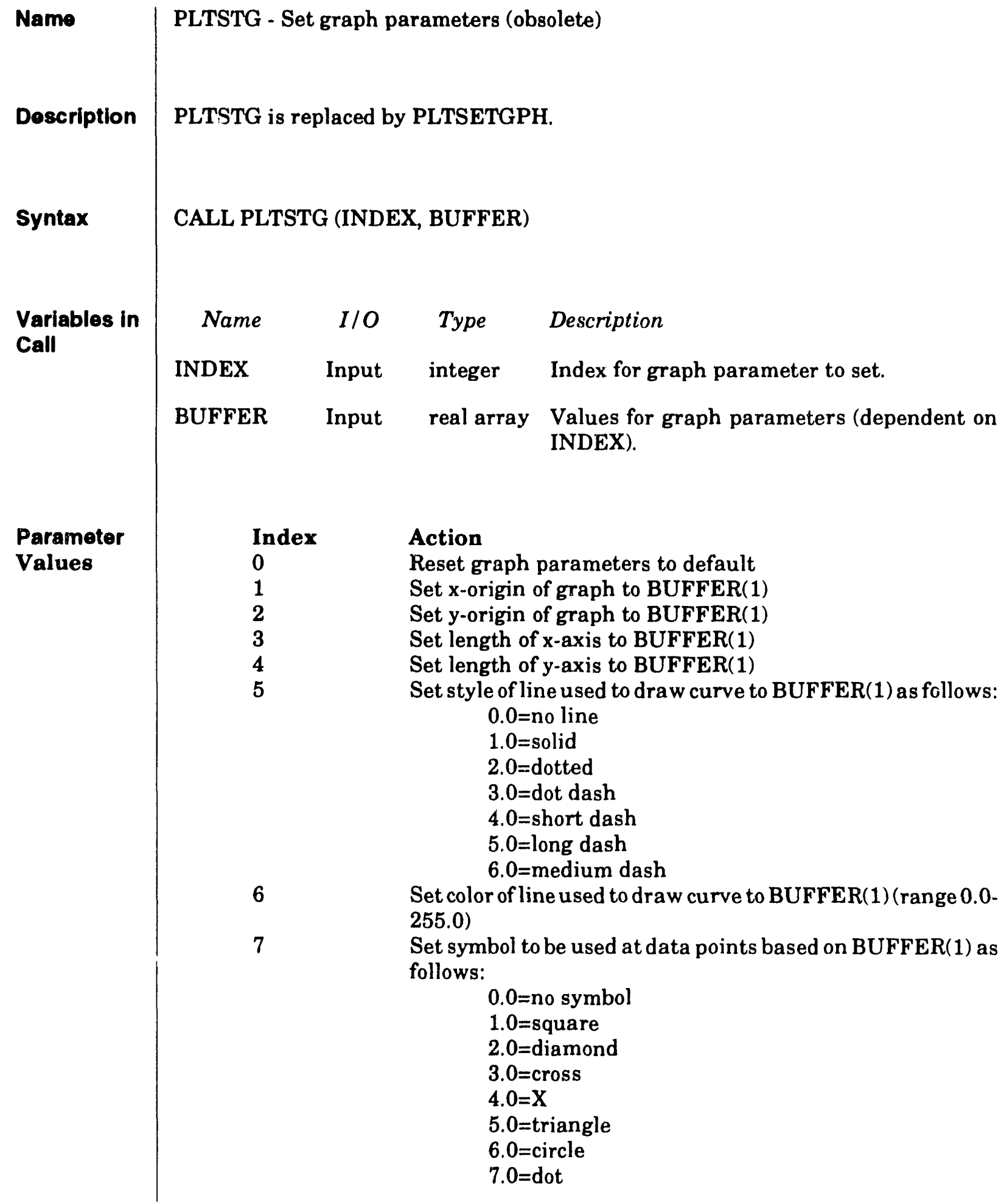




\section{Parameter Values (continued)}

8

9

11

$\operatorname{BUFFER}(1)=4.0$
Set symbol increment to BUFFER(1); if symbol plotting is turned on, plot only every $\mathbf{n}^{\text {th }}$ symbol starting with the first.

Set axis type based on BUFFER(1):

$1.0=$ normal $x$, normal $y$

$2.0=\log x$, normal $y$

$3.0=\log y$, normal $x$

$4.0=\log x, \log y$

Set axis color to BUFFER(1) (range 0.0-255.0)

Set scaling type based on BUFFER(1) as follows:

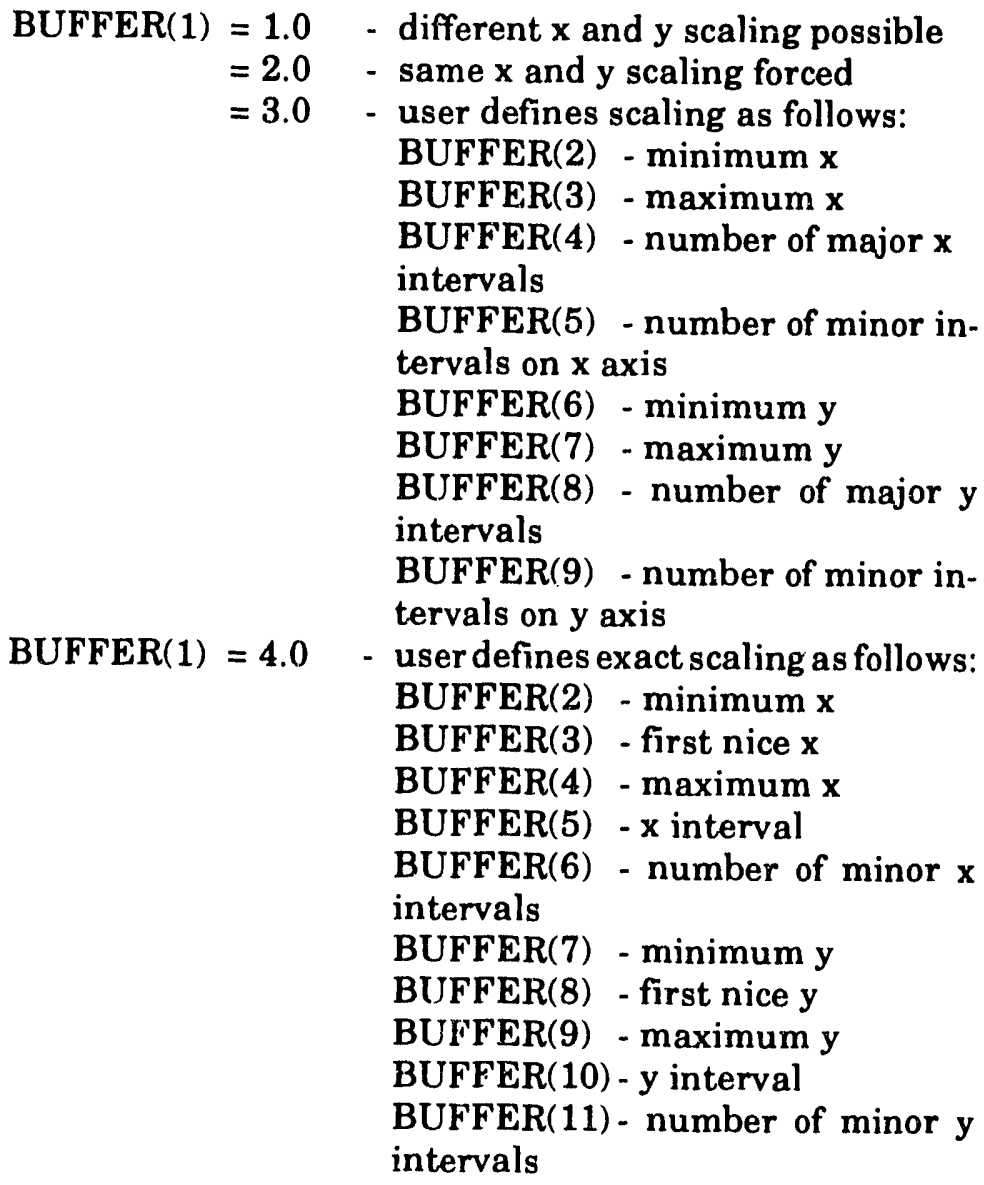

Turn on graph framing if BUFFER(1) is 1 ; turn off if BUFFER(1) is 0

Set $x$ exponent to BUFFER(1); this exponent is the exponent labelled in scientific notation on the linear axis (ignored if scientific notation is not set); -999999.0 to let the code determine the exponent 


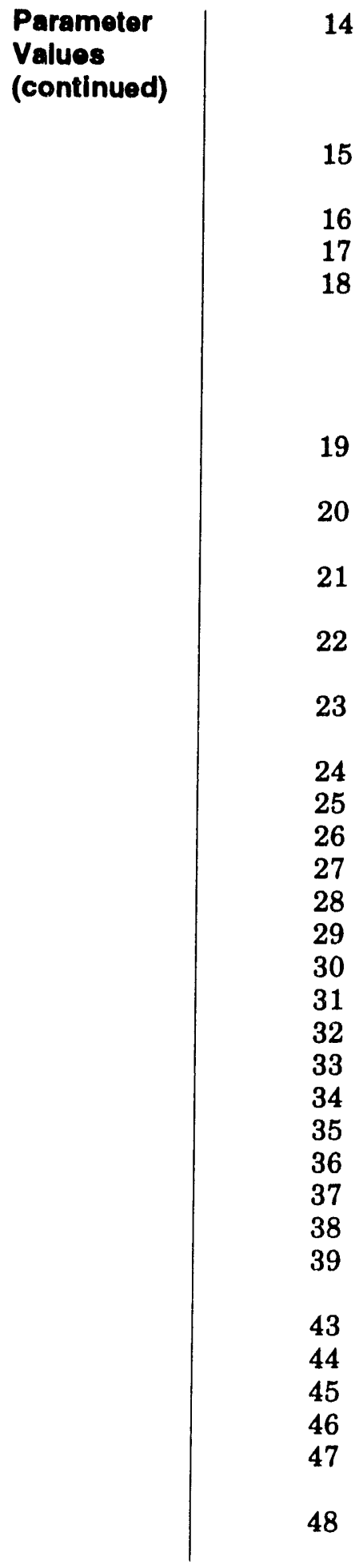

Set y exponent to BUFFER(1); this exponent is the exponent labelled in scientific notation on the linear exponent axis (ignored if scientific notation exponent value is not set); -999999.0 to let the code determine the exponent

Set line style for major grid to BUFFER(1) (see INDEX $=5$ for valid types); 0 to turn off grid drawing

Set color for major grid to BUFFER(1)

Set color for axis labels to BUFFER(1)

Set type of notation on axes based on BUFFER(1):

$$
\begin{aligned}
& 1.0=\text { scientific } x, \text { normal } y \\
& 2.0=\text { scientific } y, \text { normal } x \\
& 3.0=\text { scientific } x, \text { scientific } y \\
& 4.0=\text { normal } x, \text { normal } y
\end{aligned}
$$

Set number of decimal places in $x$-axis numbers to BUFFER(1) (range 0-9)

Set number of decimal places in y-axis numbers to BUFFER(1) (range 0-9)

Set $y$ number vertical or horizontal based on BUFFER(1) (1=vertical; otherwise horizontal)

"Set scaling for $x$-axis number to BUFFER(1); 0 turns off drawing the numbers

"Set scaling for $\mathrm{x}$-axis labels to BUFFER(1); 0 turns off drawing the label

"Set scaling for graph symbols to BUFFER(1)

Label minor $\mathrm{x}$ log ticks to BUFFER(1)

Label minor y log ticks to BUFFER(1)

Set map array for graph to BUFFER(1) - BUFFER(14)

Set pen width of axes to BUFFER(1)

Set pen width of curves to BUFFER(1)

Set pen width of axis number to BUFFER(1)

Set pen width of axis labels to BUFFER(1)

Set pen width of symbols to BUFFER(1)

Set pen width of ticks to BUFFER(1)

Set pen width of major grid to BUFFER(1)

Set pen width of minor grid to BUFFER(1)

Set pen width of line at zero to BUFFER(1)

Set line style of line at zero to BUFFER(1)

Set color of line at zero to BUFFER(1)

Set line style of minor grid to BUFFER(1) (see INDEX $=5$ for valid types); 0 to turn off grid drawing

Set color of minor grid to BUFFER(1)

Set color of symbols to BUFFER(1)

Set color of axes number to BUFFER(1)

Set color of ticks to BUFFER(1)

"Set scaling for y-axis numbers to BUFFER(1); 0 turns off drawing numbers

"Set scaling for y-axis labels to BUFFER(1); 0 turns off drawing the label 
See Also
Comment

\section{PLTGETGPH}

"INDEX $=22,23,24,47,48$ :

Axis labels, numbers, and symbols are scaled to some fraction of the average axis length $L(L=1 / 2$ ( $x$-axis length $+y$-axis length)). BUFFER(1), for these indices, allows the user to adjust labels, numbers, and symbols relative to this nominal size where

$$
\left\{\begin{array}{ccc}
1 & \text { is } & 0.5 \\
2 & \text { is } & 1.0 \\
10 & \text { is } & 1.5
\end{array}\right\} \times \text { the nominal size }
$$

For axis numbers, the nominal size is $\mathrm{L} / 50$.

For axis labels, the nominal size is $\mathrm{L} / 40$.

For symbols, the nominal size is $\quad \mathrm{L} / 120$. 


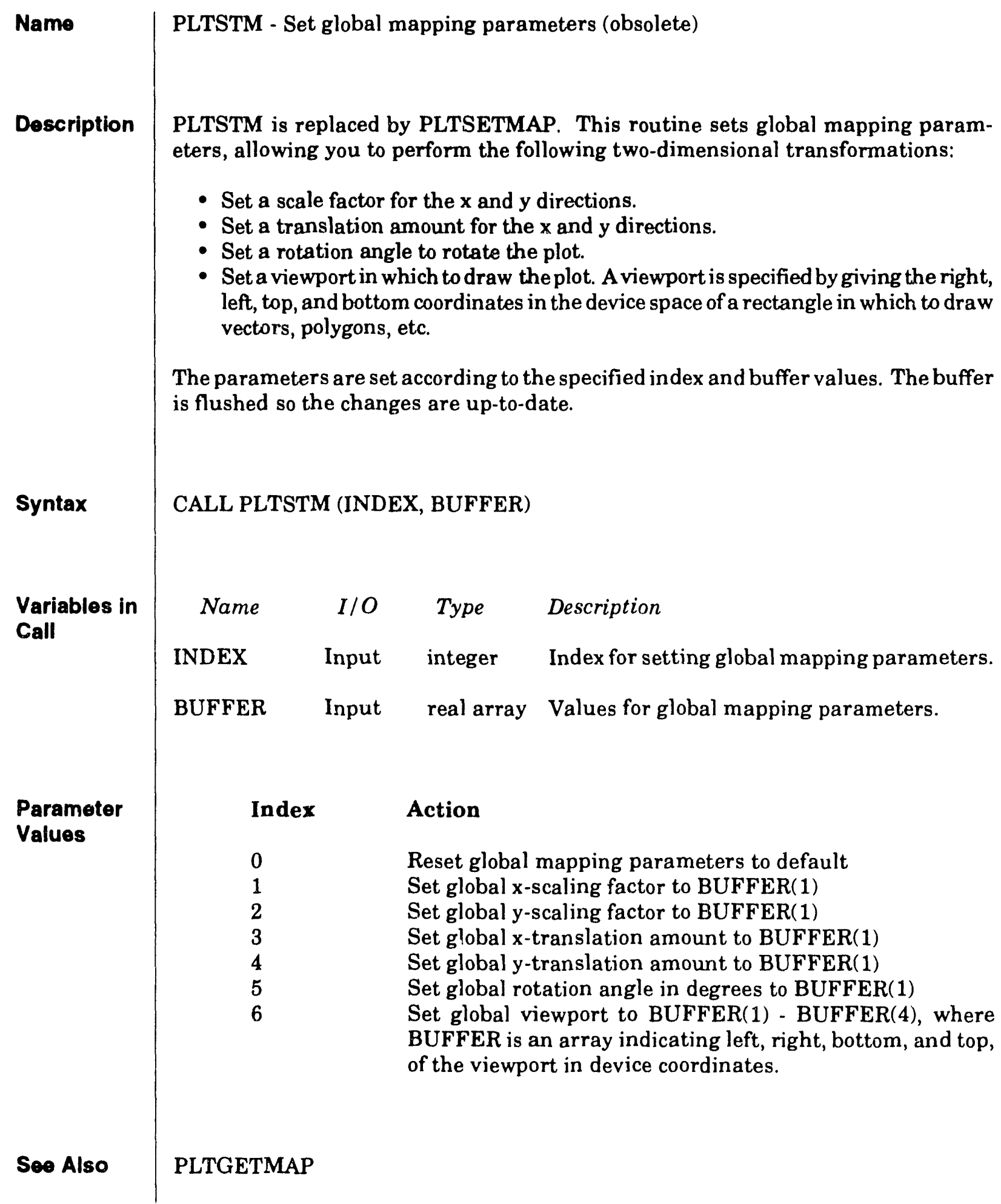


PLT_LIB

Description of Routine Calls

PLTSTT

Logical Function

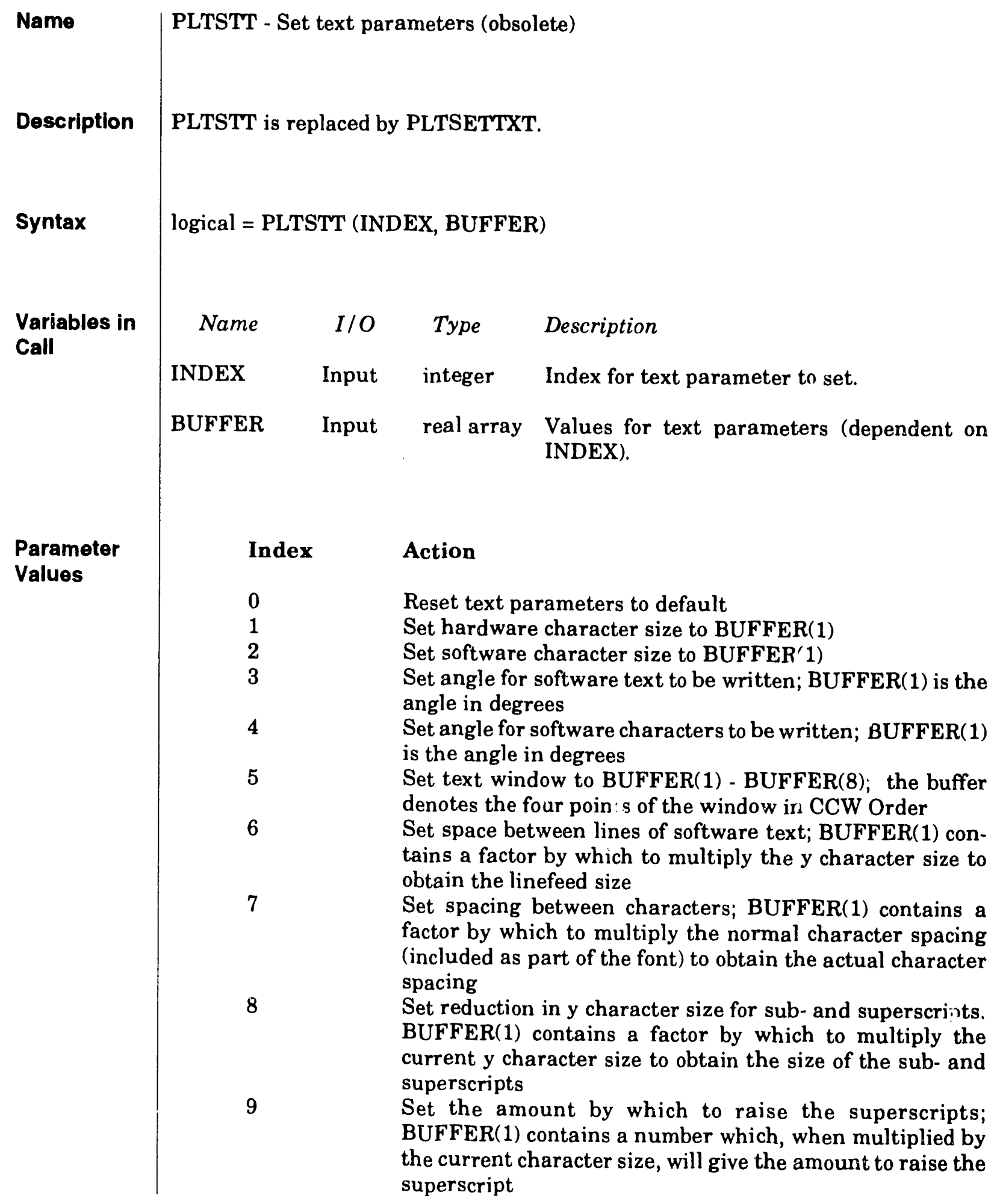




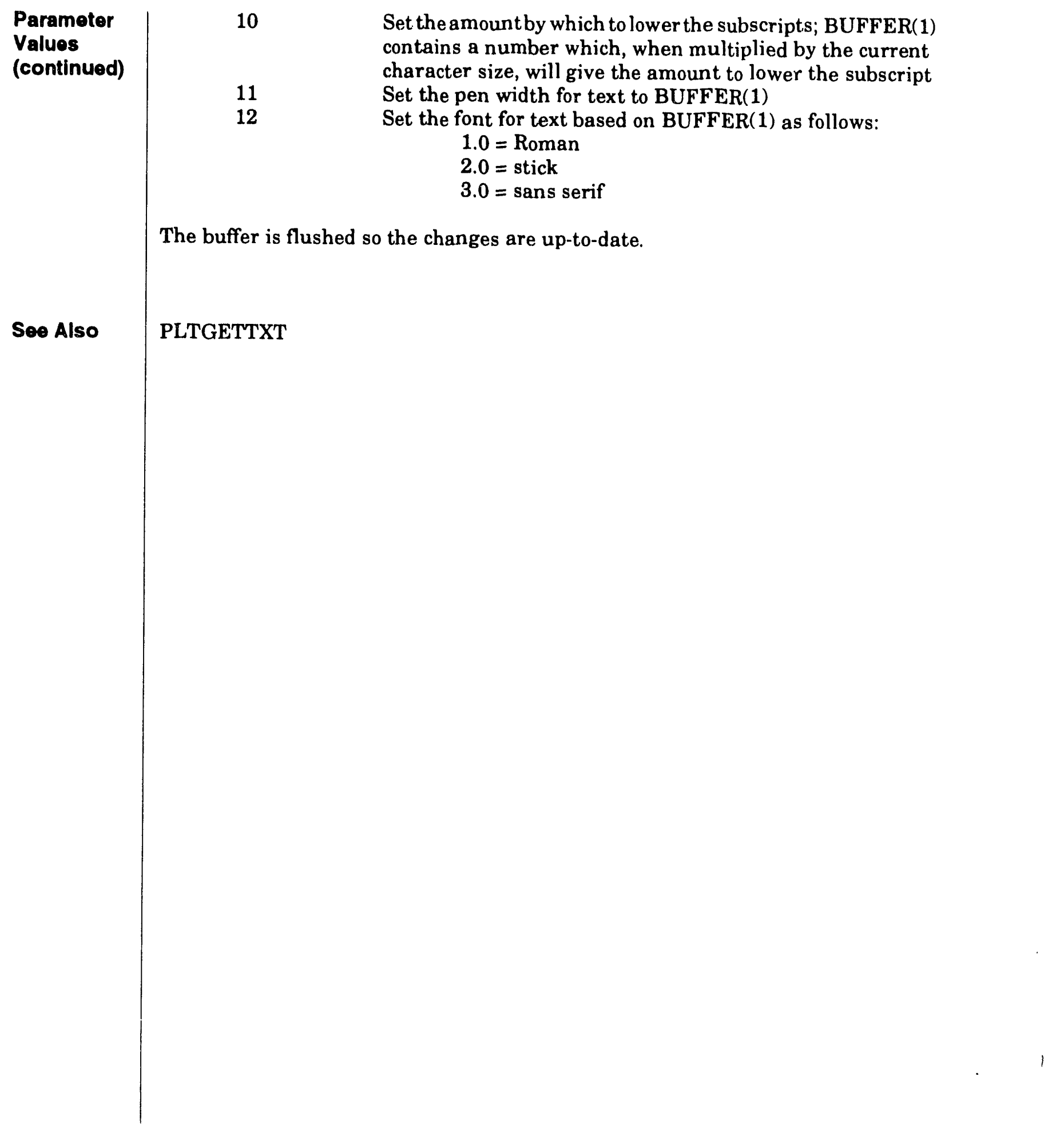




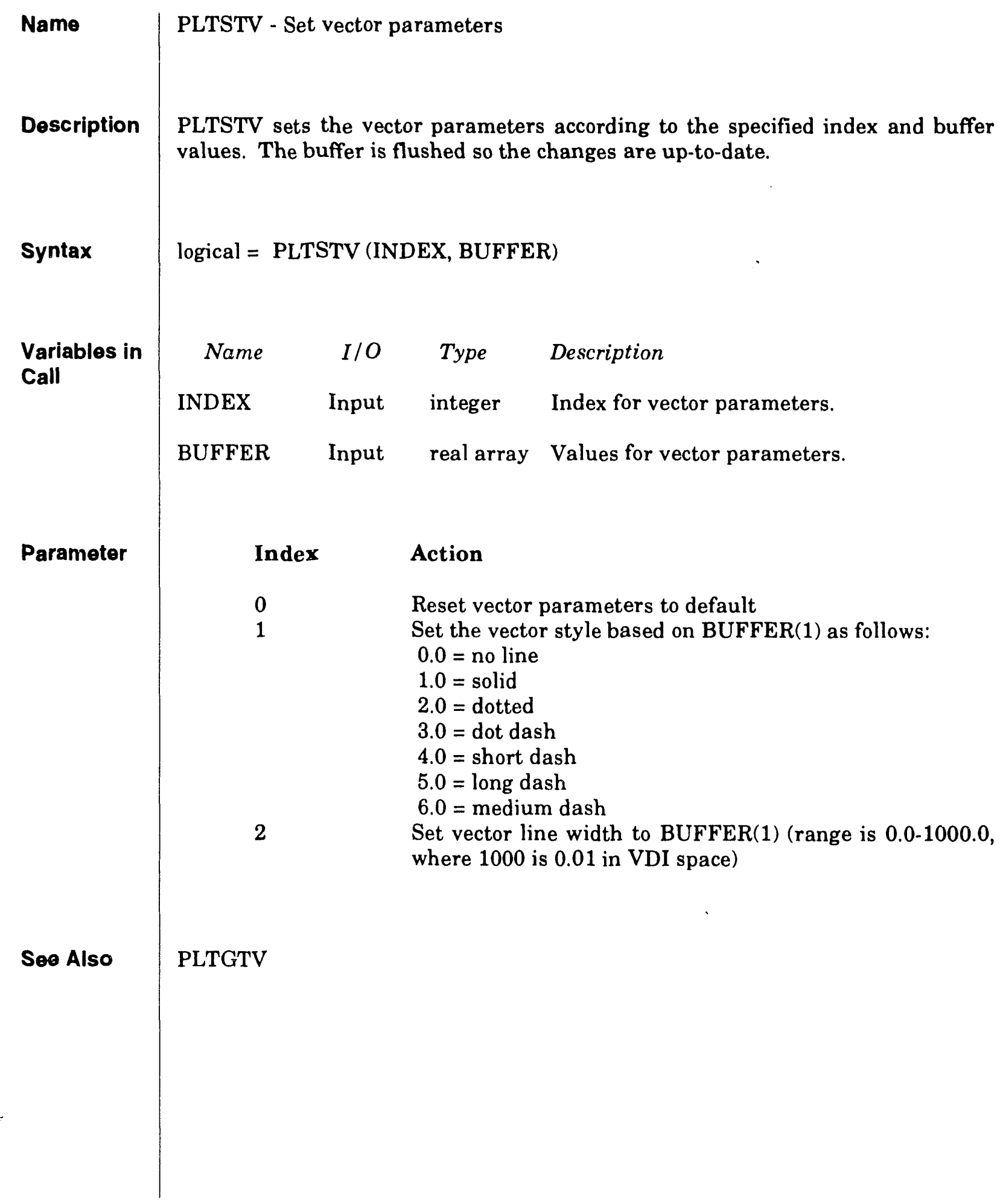




\subsection{General Guidelines on the Use of HJIPLOT_LIB Routines}

\subsubsection{Sponsorship and Version}

Author/Sponsor: H. J. Iuzzolino

Version/Date: C-2.00VV/December 1992

Language: $\quad$ FORTRAN 77

\subsubsection{Overview of HJIPLOT_LIB}

HJIPLOT_LIB provides numerous mid-level and high-level plot routines to FORTRAN and C programs. C routines should use call-by-address for INT and FLOAT variables. Text should be passed from $\mathrm{C}$ routines to FORTRAN as a two-word INT array: the string length and the string address.

HJIPLOT_LIB routines are called by a user-written program and cannot be executed without a userwritten main program.

\subsubsection{Linking}

The HJIPLOT_LIB routines are in an object library in file

CAMCON\$ROOT:[CAMCON.LIBRARIES.HJIPLOT_LIB]HJIPLOT_LIB.OLB.

CAMCON defines the symbol HJIPLOT_LIB to point to this file. The link command has the following form:

\$SELECT NULL

\$LINK code_name, HJIPLOT_LIB/LIB, SHRPLT/LIB, CDRLIB/LIB/INCL = CDRABC

\subsubsection{Limitations}

- The library must be linked with the Sandia VDI plot primitives.

- Procedure SELECT must be in:oked to select the terminal and hardcopy devices.

- Character rotation is unavailable at this time.

\subsubsection{Error Messages}

Error messages are writton to file PLOTERROR.MSG or to file FOR101.DAT. The error messages occur when illegal values are sent to the scaling routines. The illegal values are changed to legal values and processing continues. The error messages are as follows (the lowercase names represen ; numeric values):

$\begin{array}{lllll}\text { HPLQQQQ01: } & \text { ERROR: } & \text { VMIN=VMAX=vmin, } & \text { SETTING } & \text { VMAX=vmax } \\ \text { HPLQQQ001: } & \text { ERROR: } & \text { VMIN=VMAX=vmin, } & \text { SETTING } & \text { VMAX=vmax } \\ \text { HPLQQQ002: } & \text { ERROR: } & \text { VMIN=VMAX=vmin, } & \text { SETTING } & \text { VMAX=vmax }\end{array}$



HPLQQQ003:
ERROR: VMIN=0,
SETTING VMIN $=1 . E-6 *$ VMAX $=$ vmin
HPLQQQ003:
ERROR: VMAX $=0$,
SETTING VMAX $=1 . \mathrm{E} 6 * \mathrm{VMIN}=\mathrm{vmax}$
HPLQQQ003:
ERROR: VMIN=VMAX=vmin,
SETTING VMAX $=$ vmax

\subsubsection{Summary of Routines}

The HJIPLOT_LIB routines are described in detail in the next section. The following is a brief description of the HJIPLOT_LIB routines.

- HPLAHSAX - plots an arc-hyperbolic sine axis

- HPLAXIMODE - unpacks the axis mode value

- HPLAXIS - plots a linear axis at any angle

- HPLBOX - draws a rectangular box

- HPLDCLIMIT - returns plct frame limits in device coordinates

- HPLFIELDWD - returns the field width generated by a format

- HPLFNAHSIN - arc hyperbolic sine function

- HPLFNHSIN - hyperbolic sine function

- HPLGENFMT - generates a format suitable to print/plot each item in an array

- HPLINITPLOT - initializes the plotting with device aspect ratio and justification

- HPLINITPLT - initializes the plotting with device aspect ratio, justification, page mode, and font number

- HPLINTRP1 - performs linear interpolation

- HPLLENTEXT - returns the length of a string to the last nonblank, non-null character

- HPLLINE

- HPLLINED

- HPLLINEW

- HPLLOGAX

- HPLMARKER

- HPLMGDEVS

- draws a piecewise continuous line through points in world coordinates

- draws a piecewise continuous line through points in device coordinates

- draws a piecewise continuous line through points in world coordinates

- draws a logarithmic axis at any angle

- draws a centered marker

- HPLMULPLOT

- HPLMULPTRN

- initialize multiple graphics devices

- draws one or more curves on a frame using solid and dashed line patterns

- HPLNIDFD

- HPLNUMBER

- HPLPLOT

- HPLPLOTD

- HPLPLOTW

- HPLPOPVEC

- HPLPUSHVEC

- HPLQQQQU1

- HPLQQQ001

- HPLQQQ002

- HPLQQQ003

- HPLSCREEN

- HPLSYMBOL

- HPLWAITCR

- HPLWAITOFF

- HPLWAITON

- HPLXMAPDC

- HPLYMAPDC

- HPLXWTODC

- HPLYWTODC

- specify MULPLOT line pattern number

- returns the number of integer digits, fraction digits, and significant digits

- plots a number using a specified format

- draws or moves to a point with specified world coordinates

- draws or moves to a point with specified device coordinates

- draws or moves to a point with specified world coordinates

- restores the previous vector of plot attributes

- saves the current vector of plot attributes

- returns appropriate end points and tic spacing for an axis range

- returns appropriate end points and tic spacing for an axis range

- finds decade end points that bound minimum and maximum values

- finds round numbers that bound minimum and maximum values

- defines the bounds of the plot

- plots characters in a text string at a given angle

- holds the plot until the RETURN key is presseci

- turns off the WAITCR behavior of waiting for the RETURN key

- turns on the WAITCR behavior of waiting for the RETURN key

- maps world $x$-coordinates to device $x$-ioordinates

- maps world y-coordinates to device $y$-coordinates

- returns the device $x$-coordinate for a specified world $x$-coordinate

- returns the device $y$-coordinate for a specified world $y$-coordinate 


\subsection{Description of Routine Calls}

Detailed descriptions of the HJIPLOT_LIB routines are given on the following pages. 
Name

Description

Syntax

\section{Variables in} Call
HPLAHSAX - Plot an arc-hyperbolic sine axis

HPLAHSAX plots an arc-hyperbolic sine axis. This axis resembles a log axis but can include zero and negative numbers.

CALL HPLAHSAX (XDC, YDC, VI, VF, DCLEN, VLBL, U, MODE, ANGAX, ANGNM, LBL)

\begin{tabular}{lll}
\multicolumn{1}{c}{ Name } & I/O & Type \\
XDC & Input & real \\
YDC & Input & real \\
VI & Input & real \\
& & \\
VF & Input & real \\
DCLEN & Input & real \\
VLBL & Input & real \\
U & Input & real \\
& & \\
MODE & Input & integer
\end{tabular}

\section{Description}

Device $x$-coordinate for the start of the axis. Device $y$-coordinate for the start of the axis. World coordinate value for the start of the axis.

World coordinate value for the end of the axis. Axis length in device coordinate units.

Value of the smallest numbered tic mark. Scale factor. Recommended value is 0.2 times the world coordinate value of the smallest tic spacing.

Mode of axis numbering and tic style. The following are the values for MODE MOD 4 and the resulting tic modes:

0 tics are on both sides of the axis

1 tics are on the numbered side of the axis

2 tics are on the unnumbered side of the axis

3 only the tics are drawn

$\geq 4$ the numbering is suppressed

ANGAX Input real Axis angle (degrees) counterclockwise from the right horizontal.

Angle (degrees) of the axis numbering.

ANGNM Input real

LBL Input real
Axis label (FORTRAN character variable or constant). 


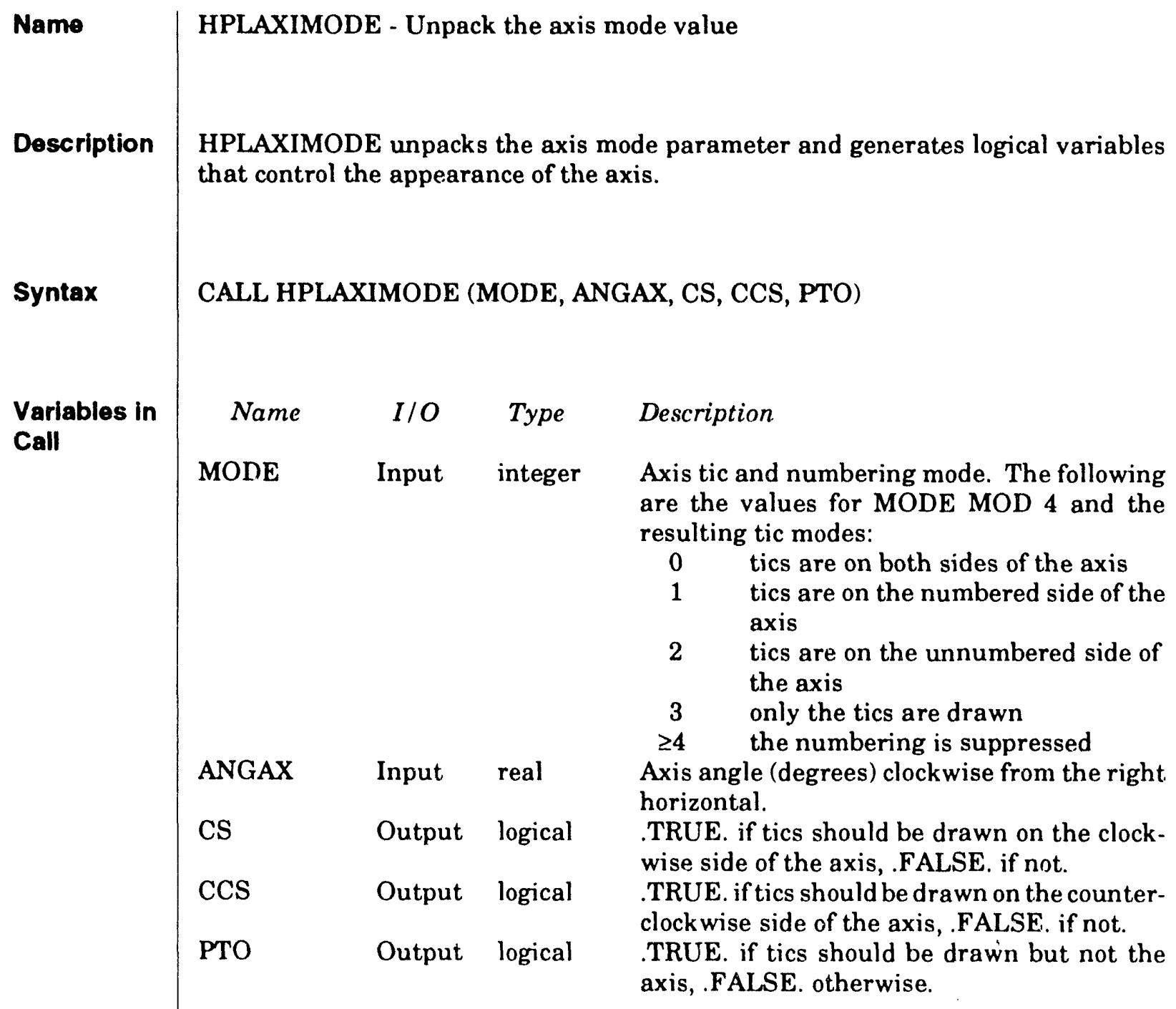

Although available for general use, HPLAXIMODE is intended for internal use within axis routines. 


\begin{tabular}{|c|c|c|c|c|c|}
\hline Name & \multicolumn{5}{|c|}{ HPLAXIS - Plot a linear axis at any angle } \\
\hline Description & \multicolumn{5}{|c|}{ HPLAXIS plots a linear axis at any angle. } \\
\hline Syntax & \multicolumn{5}{|c|}{$\begin{array}{l}\text { CALL HPLAXIS (XDC, YDC, VI, VF, VSC, VTIC, VLBL, MODE, FMT, ANGAX, } \\
\text { ANGNUM, LBL) }\end{array}$} \\
\hline $\begin{array}{l}\text { Variables in } \\
\text { Call }\end{array}$ & $\begin{array}{l}\quad \text { Name } \\
\text { XDC } \\
\text { YDC } \\
\text { VI } \\
\text { VF } \\
\text { VSC } \\
\text { VTIC } \\
\text { VLBL } \\
\text { MODE }\end{array}$ & $\begin{array}{l}I / O \\
\text { Input } \\
\text { Input } \\
\text { Input } \\
\text { Input } \\
\text { Input } \\
\text { Input } \\
\text { Input } \\
\text { Input }\end{array}$ & $\begin{array}{l}\text { Type } \\
\text { real } \\
\text { real } \\
\text { real } \\
\text { real } \\
\text { real } \\
\text { real } \\
\text { real } \\
\text { integer }\end{array}$ & $\begin{array}{l}\text { Descr } \\
\text { Devic } \\
\text { Devic } \\
\text { Worlc } \\
\text { axis. } \\
\text { Worlc } \\
\text { Scale } \\
\text { ordin } \\
\text { Tic sp } \\
\text { Spaci } \\
\text { coord } \\
\text { Axis t } \\
\text { are t } \\
\text { result } \\
0 \\
1 \\
2 \\
3 \\
\geq 4\end{array}$ & $\begin{array}{l}\text { tion } \\
\text { x-coordinate for the start of the axis. } \\
\text {-coordinate for the start of the axis. } \\
\text { oordinate value for the start of the } \\
\text { oordinate value for the end of the axis. } \\
\text { ctor: device coordinates per world co- } \\
\text { c } \\
\text { between in world coordinates. } \\
\text { ates. } \\
\text { and numbering mode. The following } \\
\text { values for MODE MOD } 4 \text { and the } \\
\text { g tic and numbering modes: } \\
\text { tics are on both sides of the axis } \\
\text { tics are on the numbered side of the } \\
\text { axis } \\
\text { tics are on the unnumbered side of } \\
\text { the axis } \\
\text { only the tics are drawn } \\
\text { the numbering is suppressed }\end{array}$ \\
\hline & FMT & Input & real & $\begin{array}{l}\text { Form } \\
\text { parer }\end{array}$ & $\begin{array}{l}\text { for the axis numbering, bounded by } \\
\text { heses. }\end{array}$ \\
\hline & ANGAX & Input & real & $\begin{array}{l}\text { Axis } \\
\text { the } r\end{array}$ & $\begin{array}{l}\text { igle (degrees) counterclockwise from } \\
\text { t horizontal. }\end{array}$ \\
\hline & $\begin{array}{l}\text { ANGNUM } \\
\text { LBL }\end{array}$ & $\begin{array}{l}\text { Input } \\
\text { Input }\end{array}$ & $\begin{array}{l}\text { real } \\
\text { real }\end{array}$ & $\begin{array}{l}\text { Num } \\
\text { from } \\
\text { Axis } \\
\text { const }\end{array}$ & $\begin{array}{l}\text { ring angle (degrees) counterclockwise } \\
\text { e right horizontal. } \\
\text { bel (FORTRAN character variable or } \\
\text { t). }\end{array}$ \\
\hline
\end{tabular}




\begin{tabular}{|c|c|c|c|c|}
\hline Name & \multicolumn{4}{|c|}{ HPLBOX - Draw a box } \\
\hline Description & \multicolumn{4}{|c|}{ HPLBOX draws a rectangular box, given the coordinates of two opposite corners. } \\
\hline Syntax & \multicolumn{4}{|c|}{ CALL HPLBOX (X1, Y1, X2, Y2) } \\
\hline $\begin{array}{l}\text { Variables in } \\
\text { Call }\end{array}$ & Name & $I / O$ & Type & Description \\
\hline & $\mathrm{X} 1$ & Input & real & $\begin{array}{l}\text { Device } x \text {-coordinate for one corner of the rect- } \\
\text { angle. }\end{array}$ \\
\hline & Y1 & Input & real & $\begin{array}{l}\text { Device y-coordinate for one corner of the rect- } \\
\text { angle. }\end{array}$ \\
\hline & $\begin{array}{l}\mathrm{X} 2 \\
\mathrm{Y} 2\end{array}$ & $\begin{array}{l}\text { Input } \\
\text { Input }\end{array}$ & $\begin{array}{l}\text { real } \\
\text { real }\end{array}$ & $\begin{array}{l}\text { Device } x \text {-coordinate for the opposite corner. } \\
\text { Device } y \text {-coordinate for the opposite corner. }\end{array}$ \\
\hline
\end{tabular}




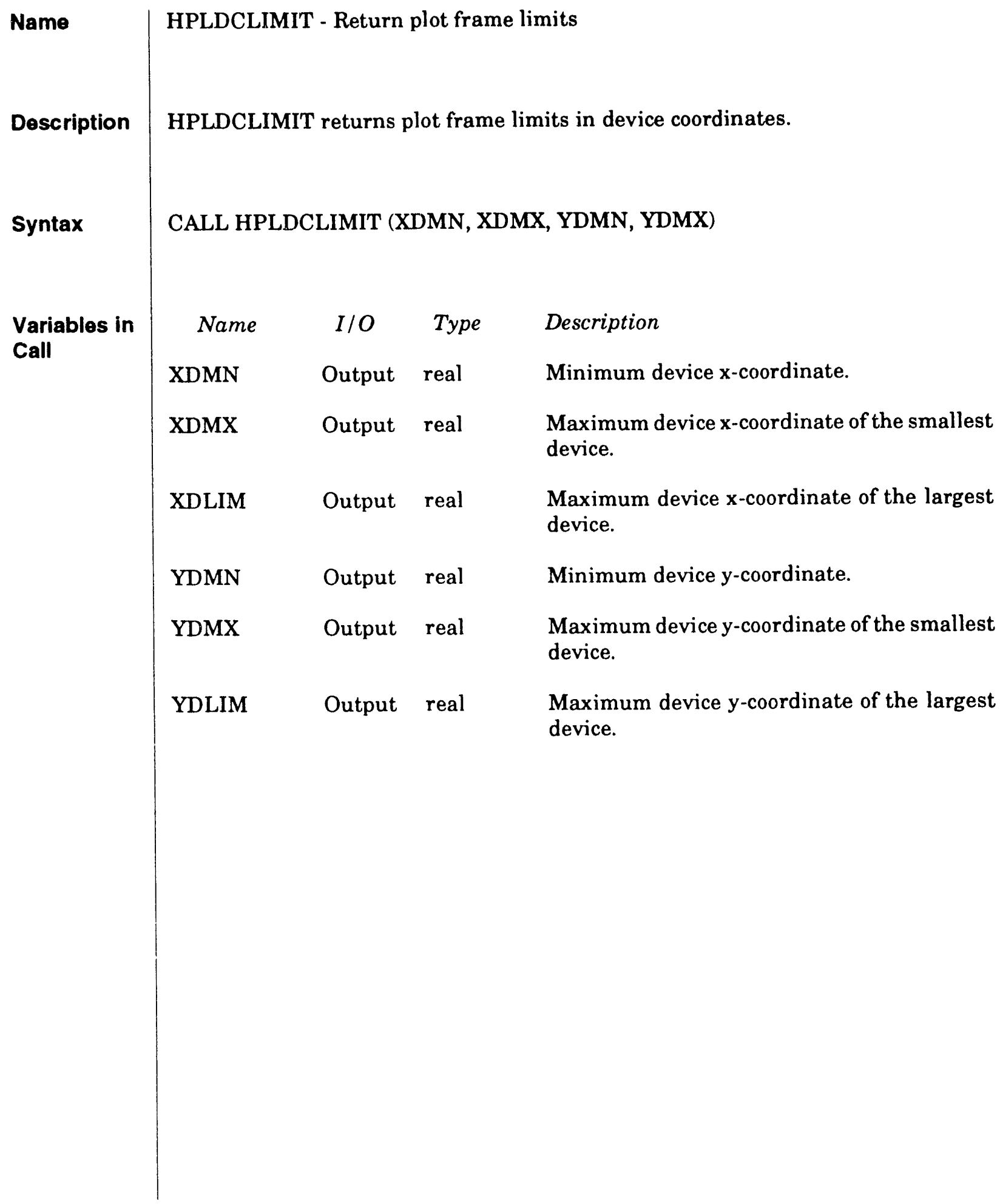




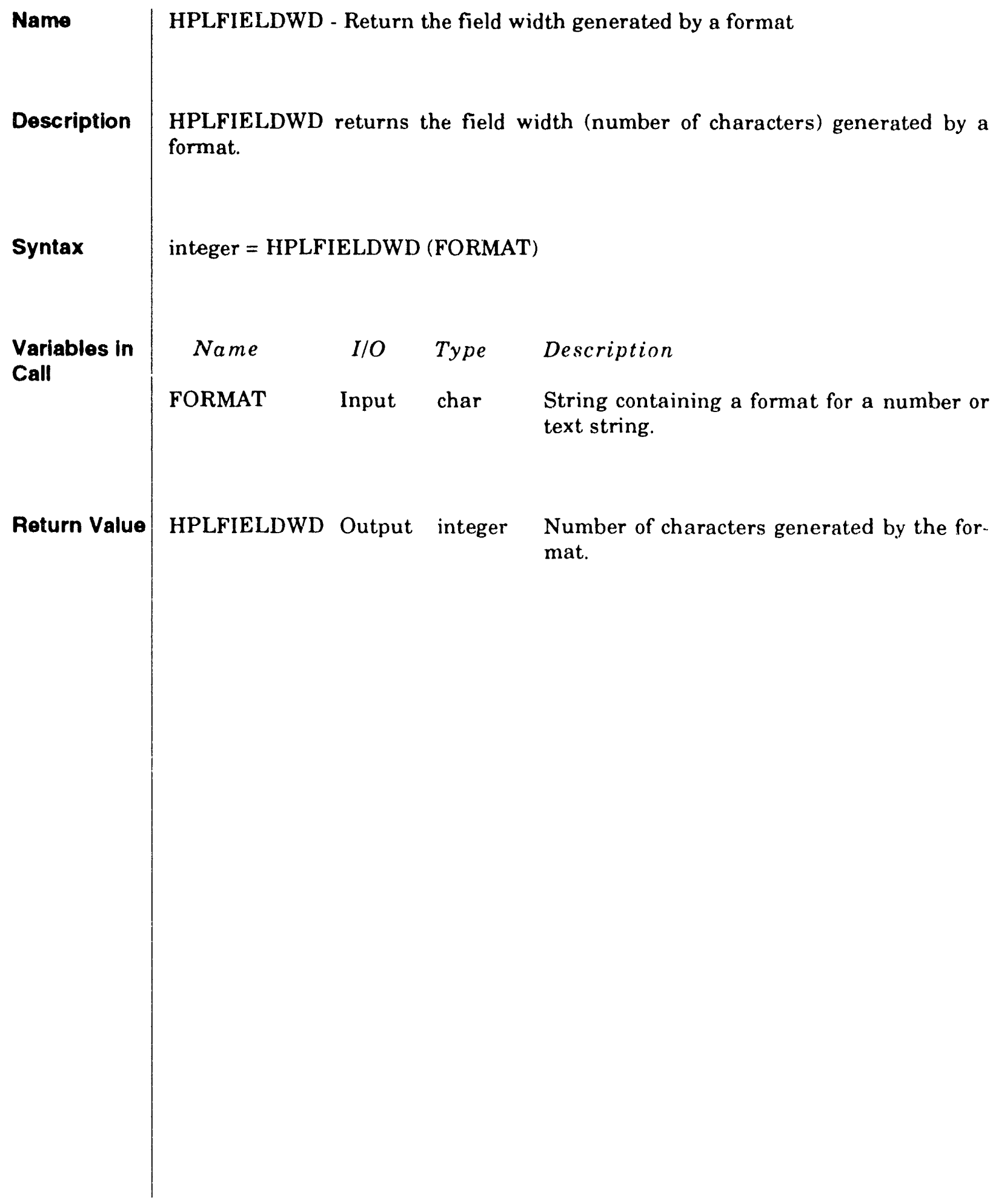




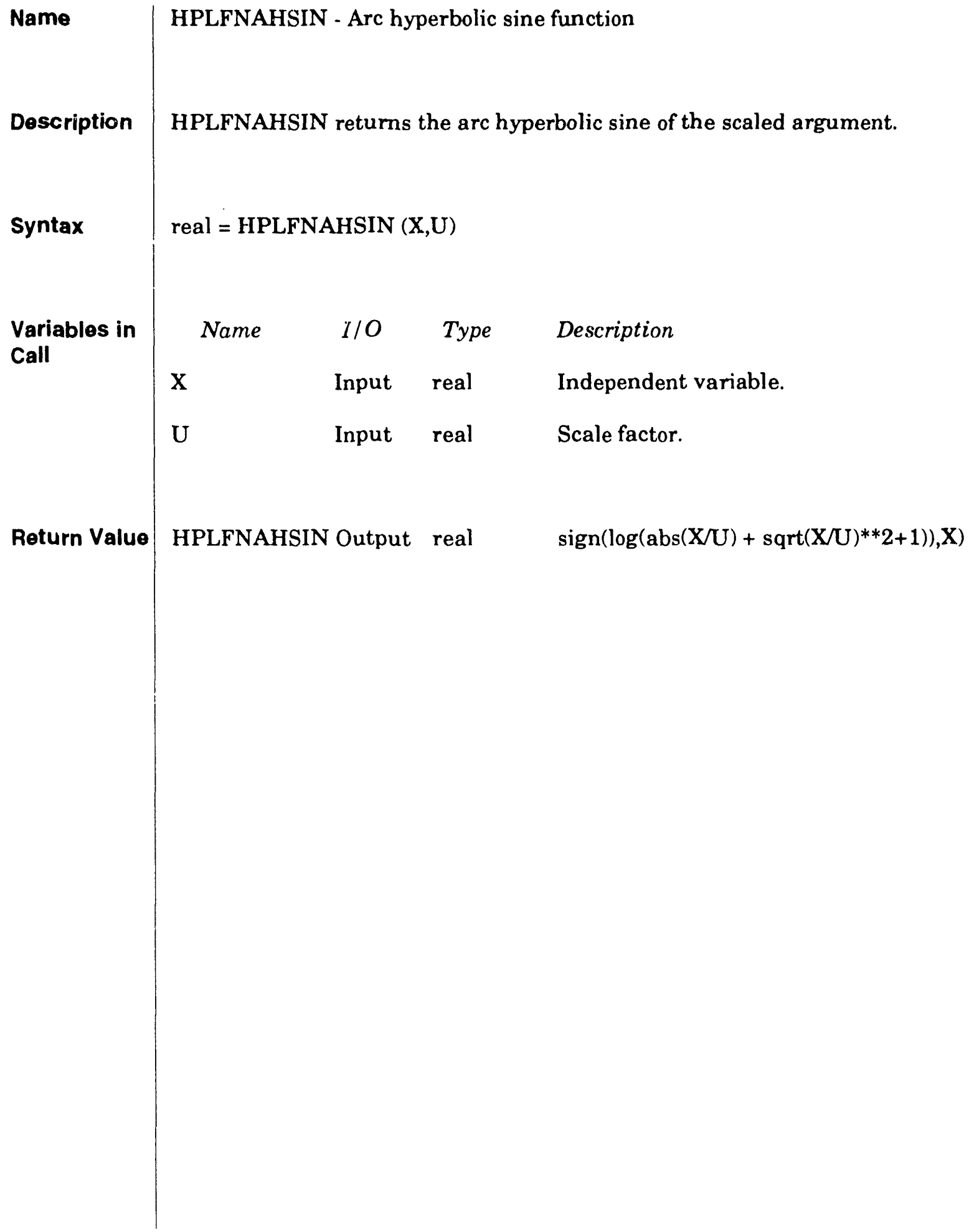




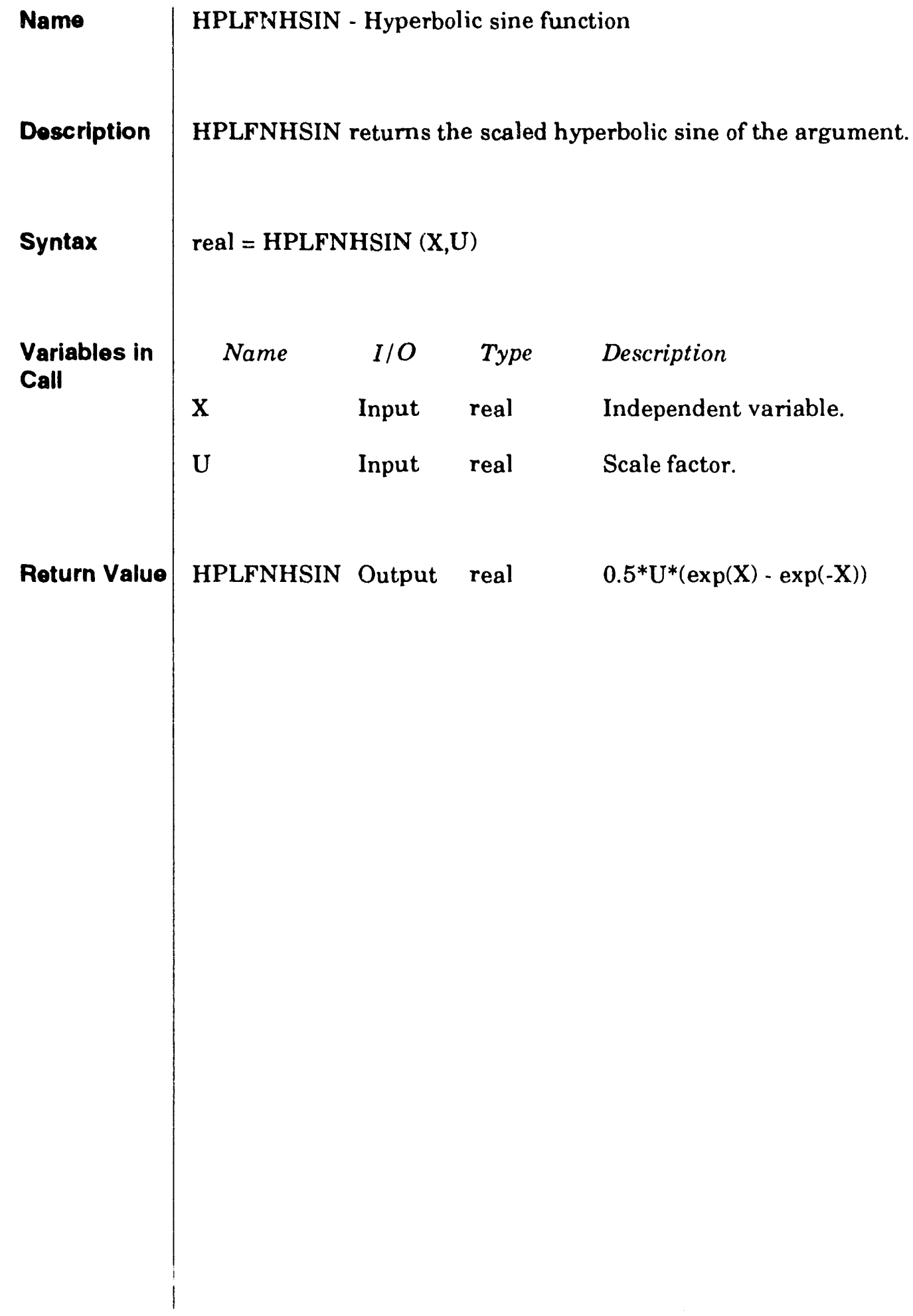


Name

Description

Syntax

Variables in Call
HPLGENFMT - Generate a format for printing items in an array

HPLGENFMT generates a format suitable to print/plot each item in an array.

CALL HPLGENFMT (FMT, ARRAY, NN)

\begin{tabular}{|c|c|c|c|}
\hline Name & $I / O$ & Type & Description \\
\hline FMT & Output & char & $\begin{array}{l}\text { Suitable format for printing each } \\
\text { ARRAY. The format is enclosed in } p \\
\text { ses. }\end{array}$ \\
\hline ARRAY & Input & $\begin{array}{l}\text { real } \\
\text { array }\end{array}$ & Array of FORTRAN real numbers. \\
\hline $\mathbf{N}$ & Input & integer & Number of numbers in ARRAY. \\
\hline
\end{tabular}




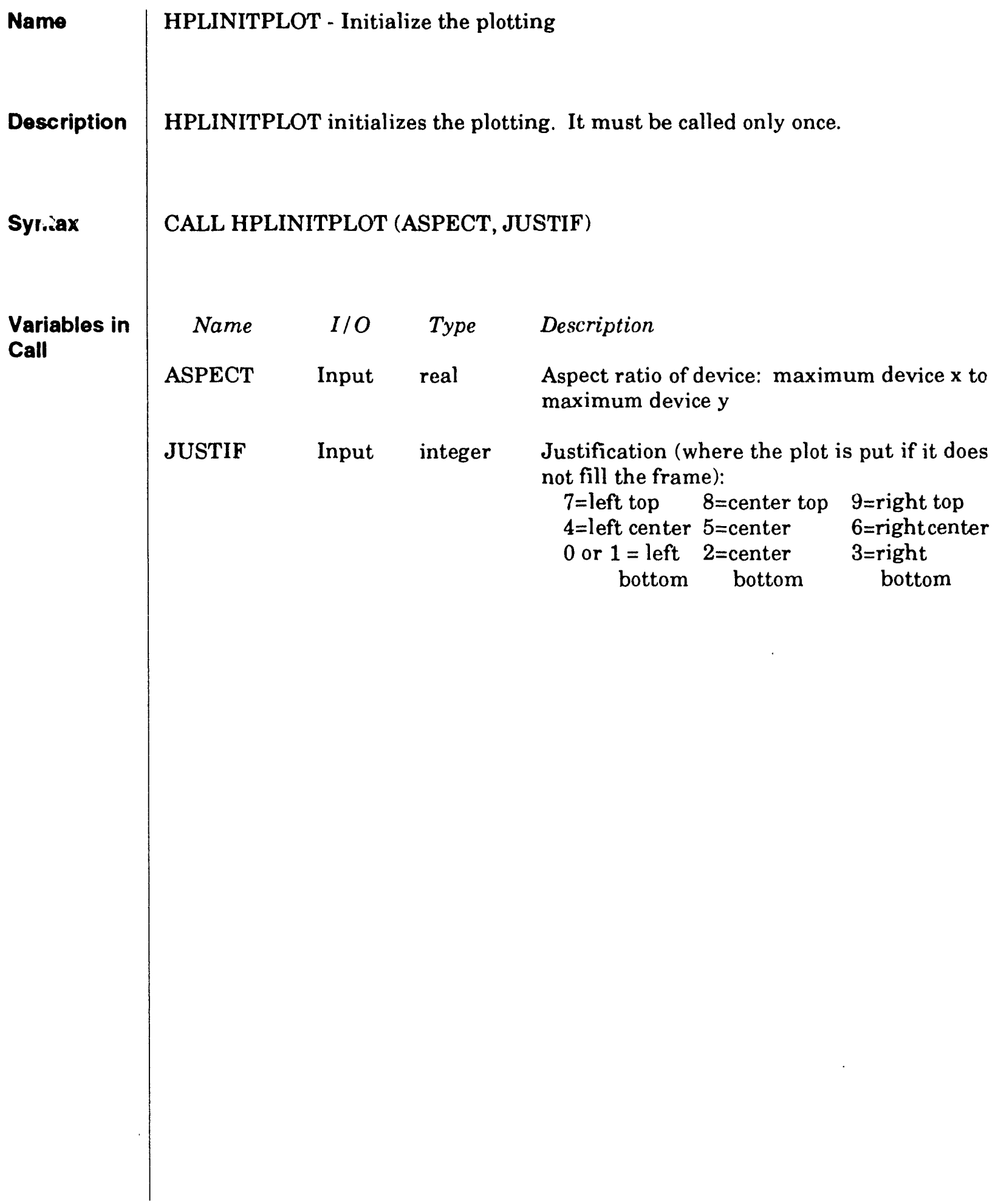




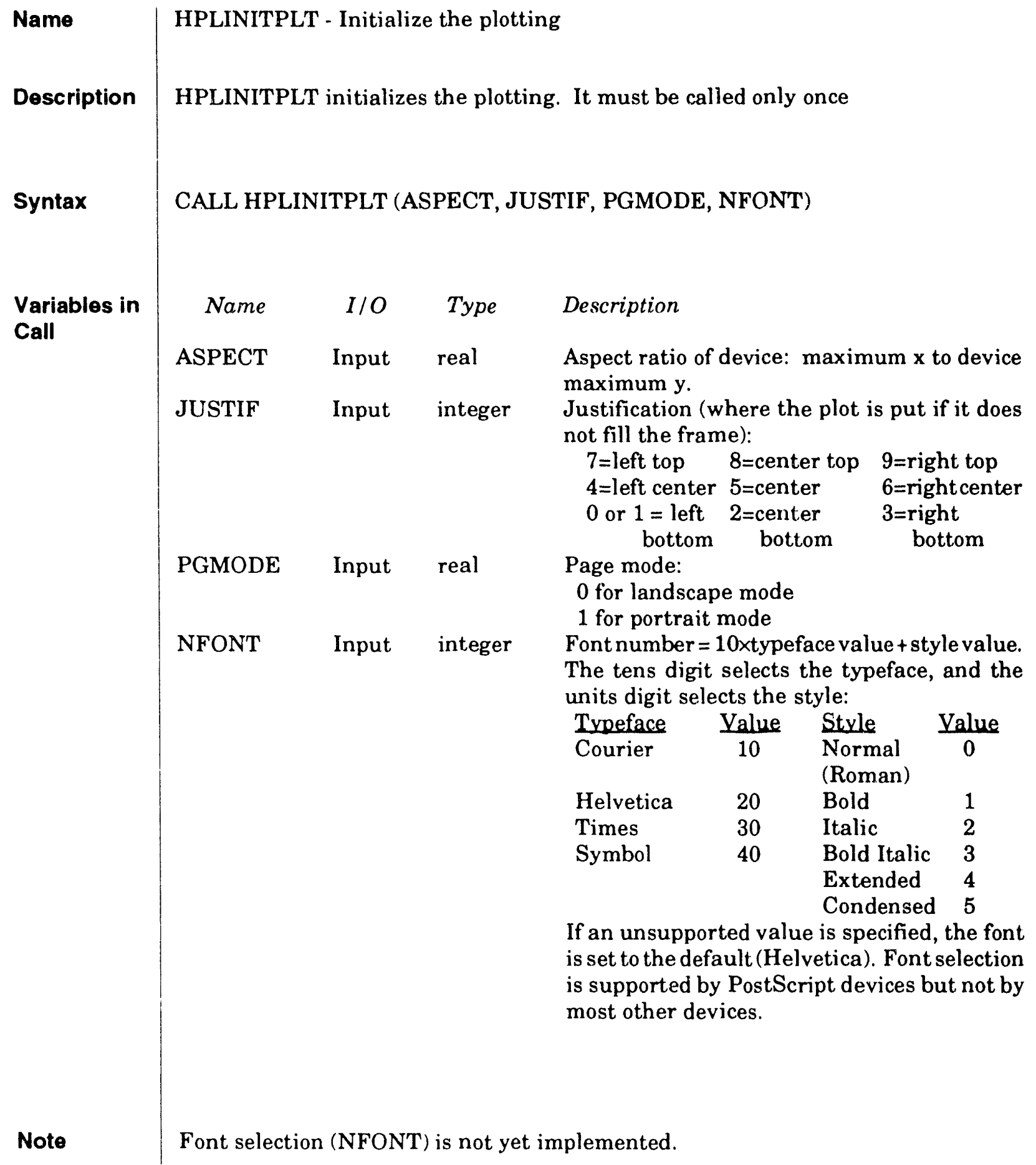




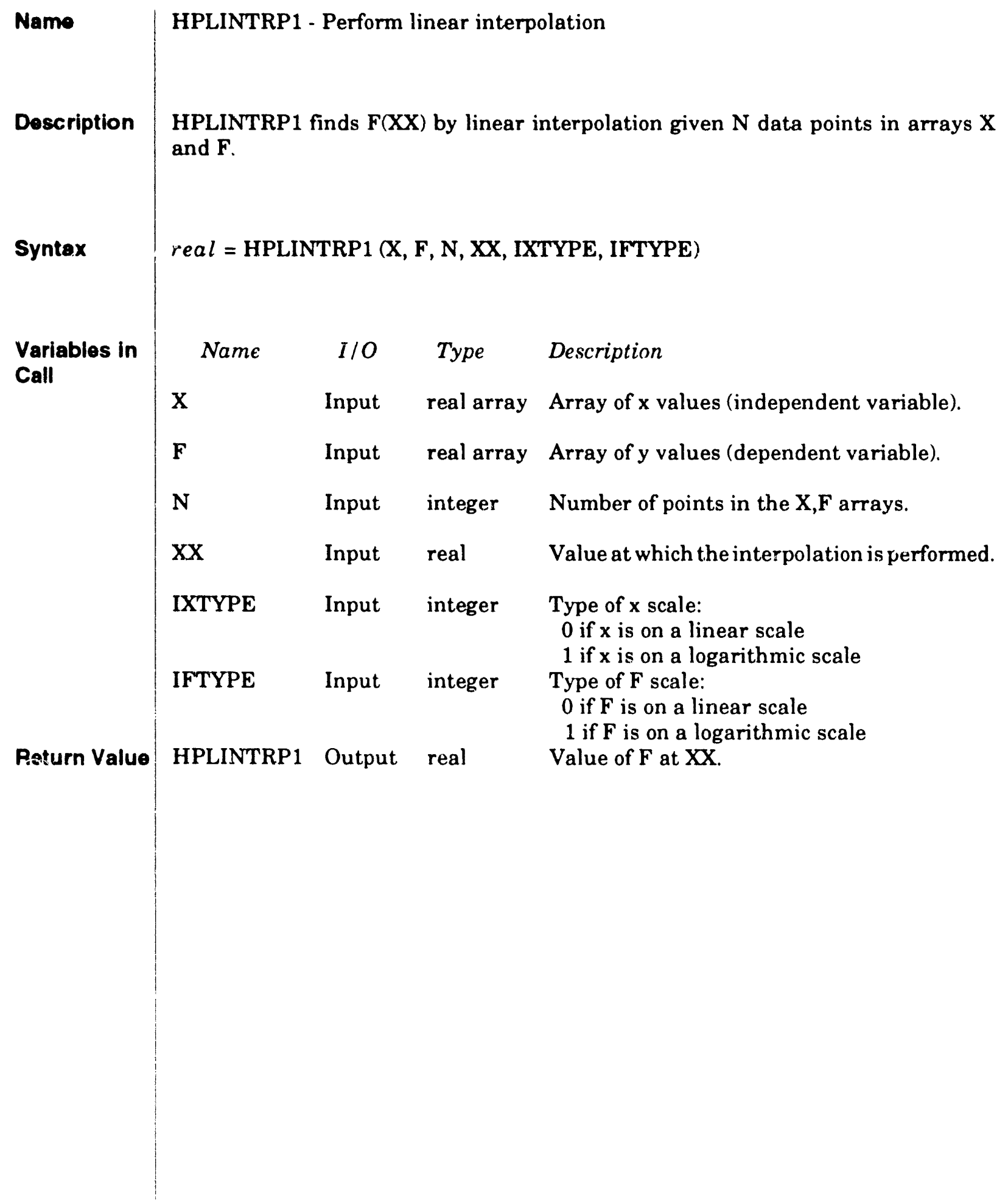




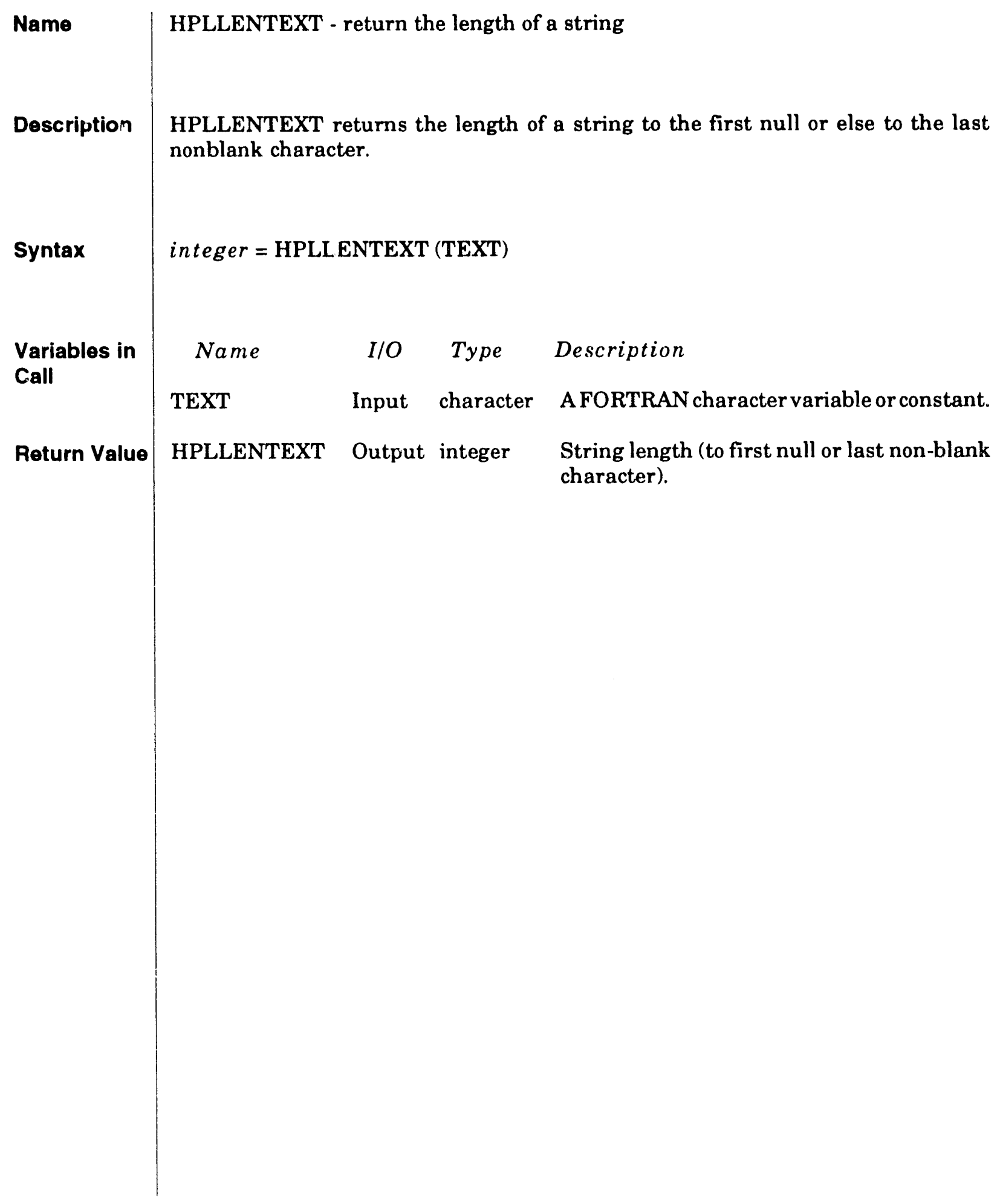




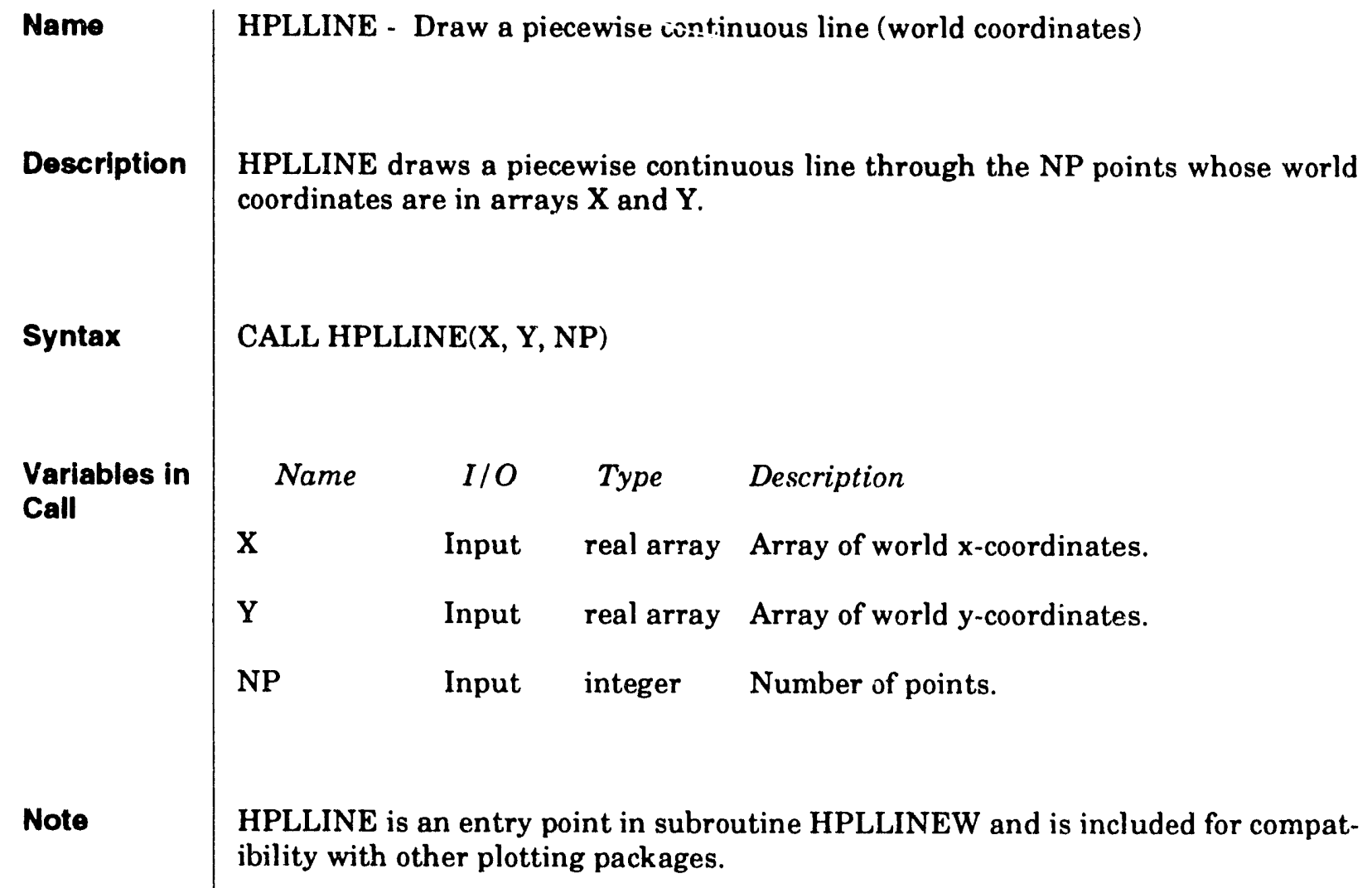




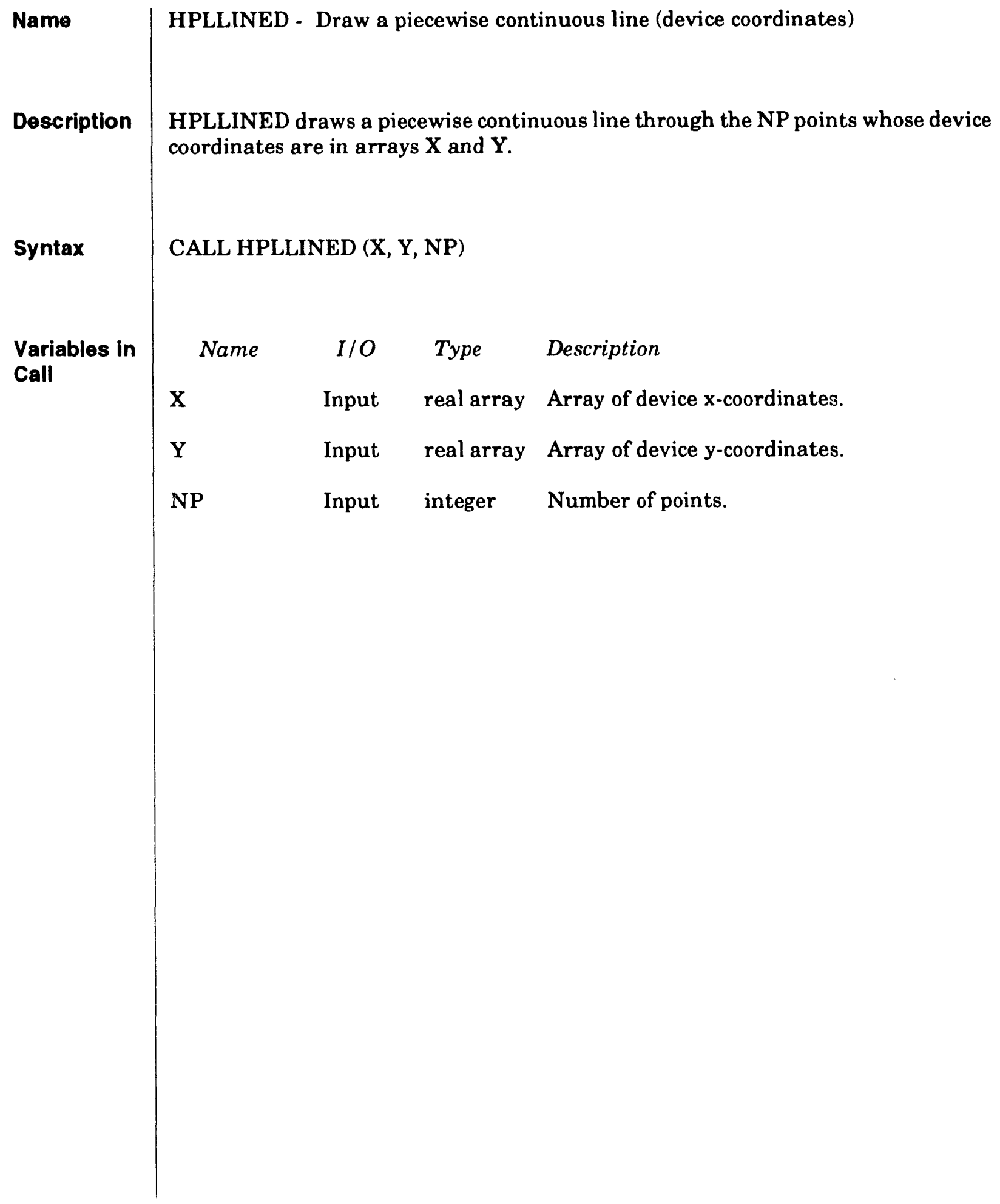




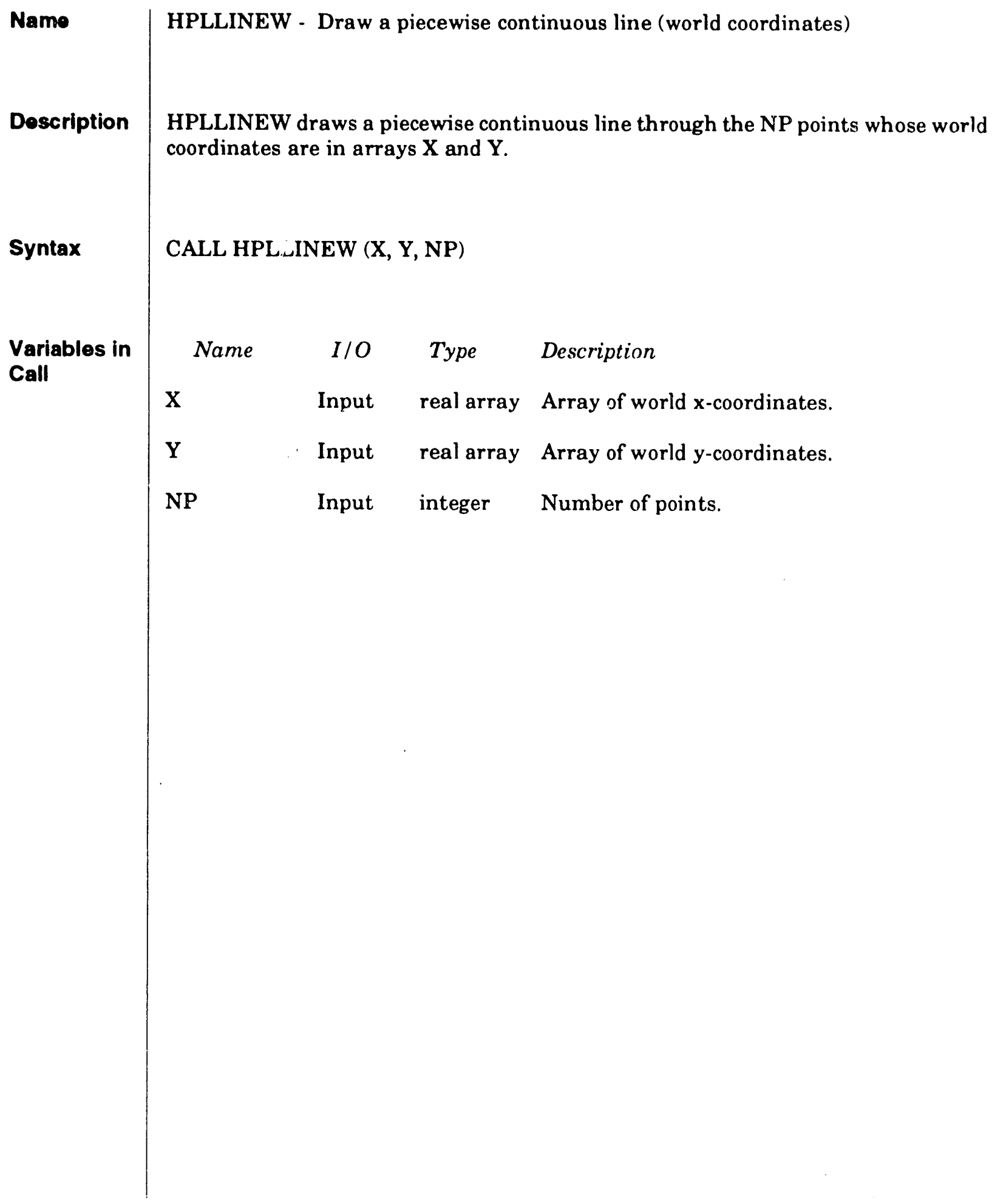




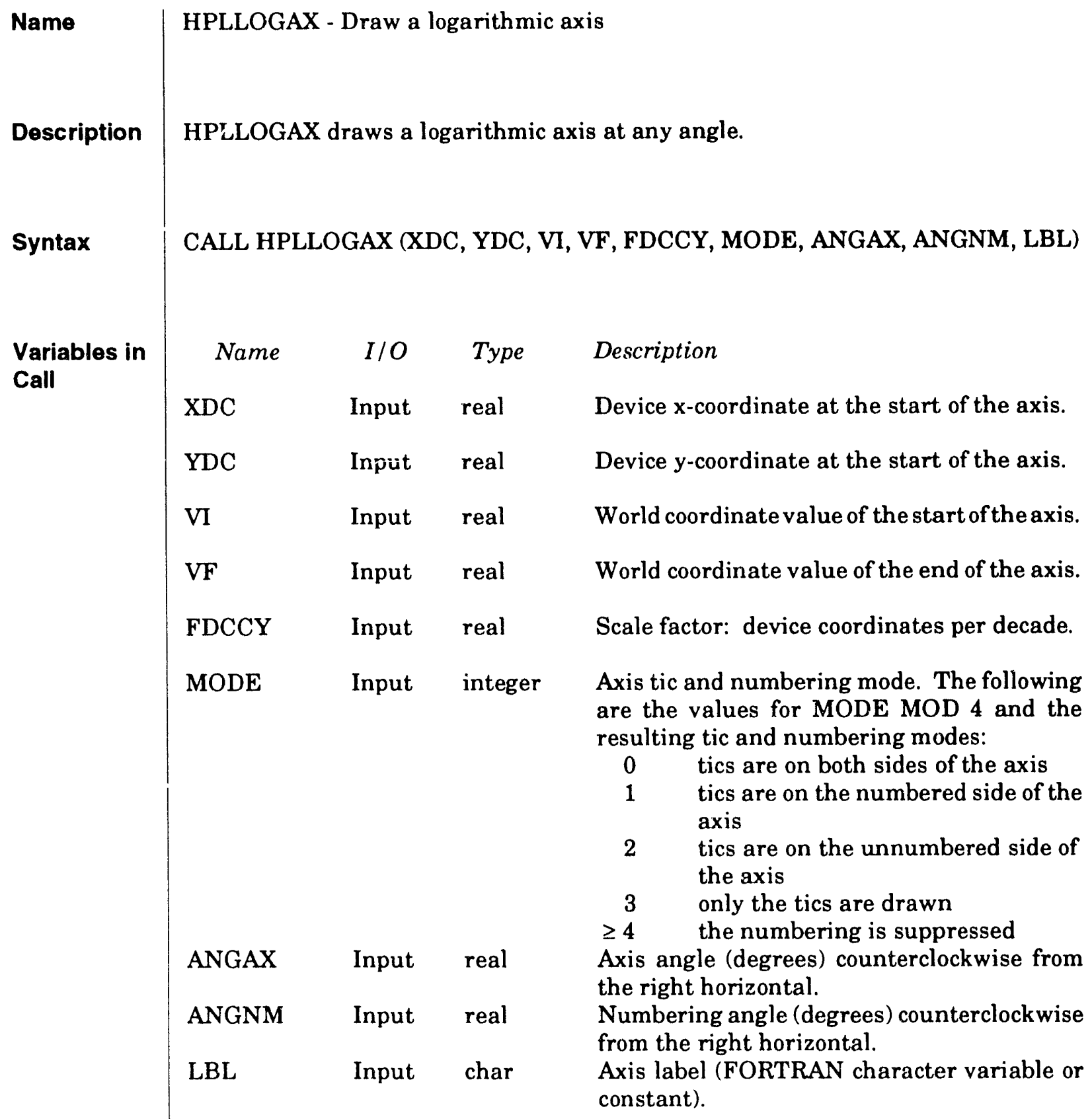




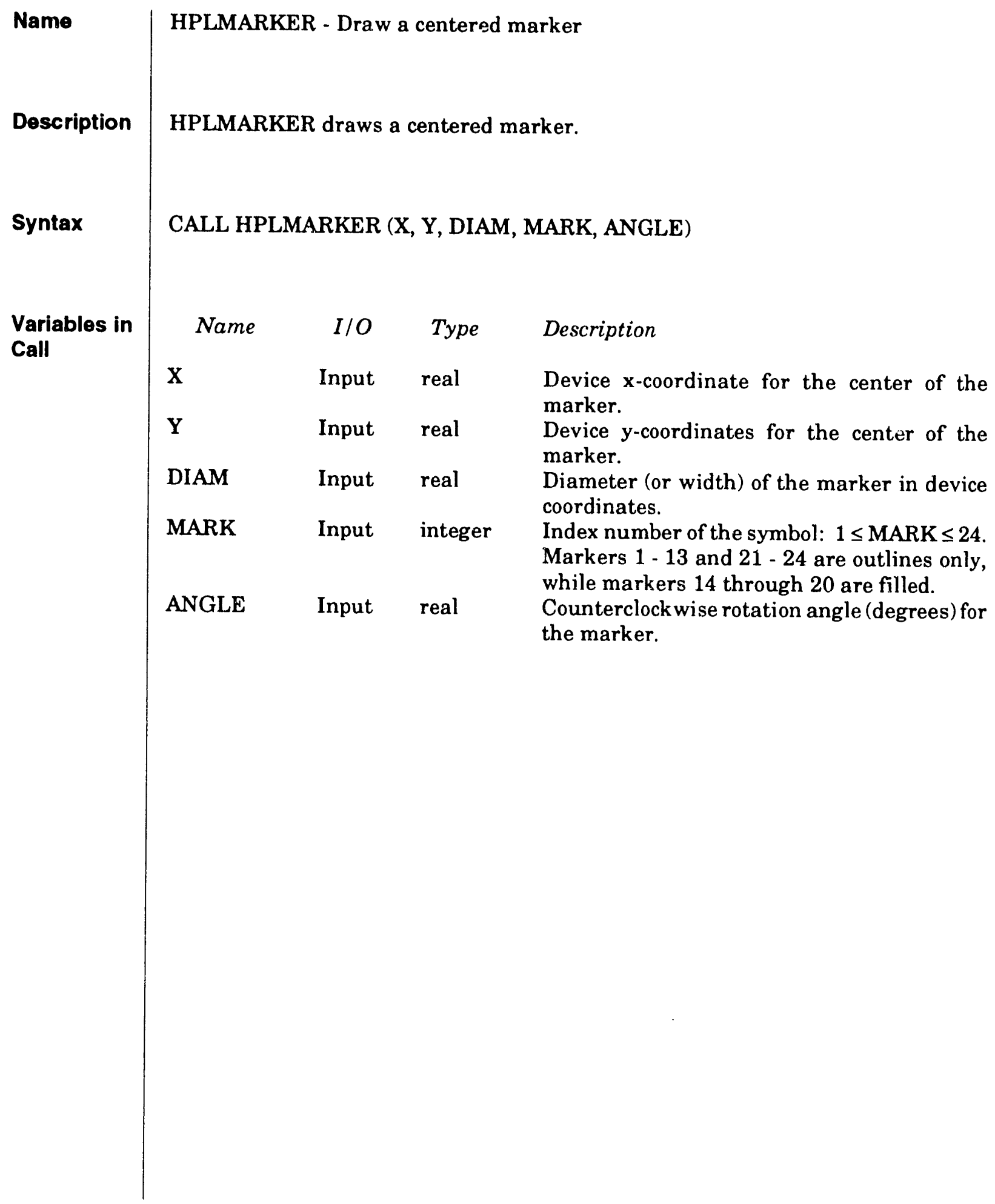




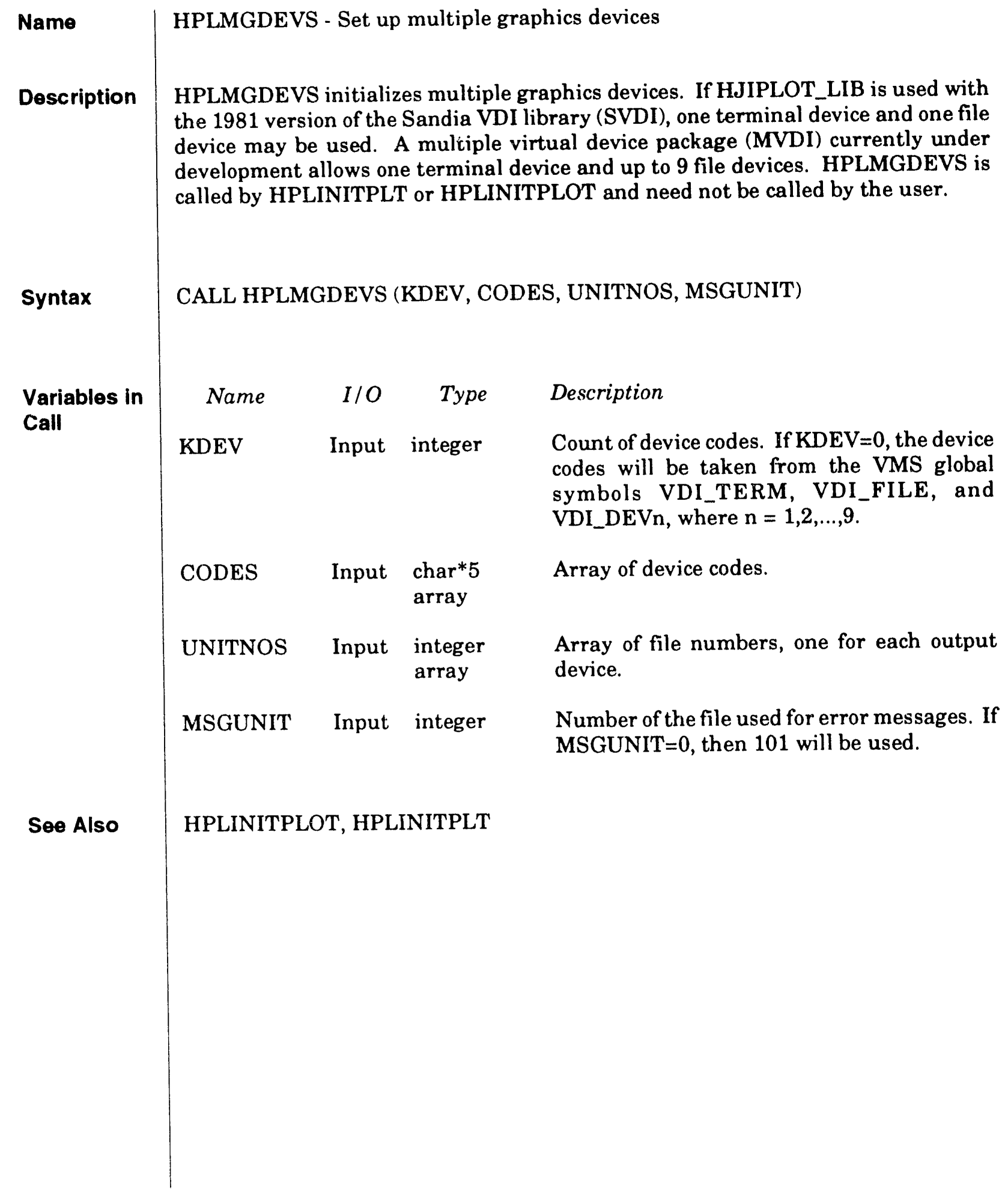


Name

HPLMULPLOT - Draw one or more curves on a frame using solid and dashed line patterns

HPLMULF'LOT draws axes around a frame and appends a curve to the frame on each call until the frame is advanced. The NP points whose coordinates are in arrays $\mathrm{X}, \mathrm{Y}$ are plotted as a solid line on the first call or as dashed lines on subsequent calls when the frame is not advanced. On each call an array of points can be appended to the plot as a set of special symbols.

Syntax

Variables in Call

CALL HPLMULPLOT (X, Y, NP, XS, YS, NPS, MODE, SCALES, ORGS, TITLES, AXLBLS, LEGEND)

\begin{tabular}{|c|c|c|c|c|}
\hline Name & $I / O$ & Type & \multicolumn{2}{|l|}{ Description } \\
\hline $\mathrm{X}$ & Input & $\begin{array}{l}\text { real } \\
\text { array }\end{array}$ & \multicolumn{2}{|c|}{$\begin{array}{l}\text { Array of device } x \text { coordinates of points to be } \\
\text { connected by a solid or dashed line. }\end{array}$} \\
\hline $\mathrm{Y}$ & Input & $\begin{array}{l}\text { real } \\
\text { array }\end{array}$ & \multicolumn{2}{|c|}{$\begin{array}{l}\text { Array of device y coordinates of points to be } \\
\text { connected by a solid or dashed line. }\end{array}$} \\
\hline NP & Input & integer & \multicolumn{2}{|c|}{ Number of points in $\mathrm{X}, \mathrm{Y}$ arrays. } \\
\hline $\mathrm{XS}$ & Input & $\begin{array}{l}\text { real } \\
\text { array }\end{array}$ & \multicolumn{2}{|c|}{$\begin{array}{l}\text { Array of device } x \text { coordinates to be plotted as } \\
\text { symbols. }\end{array}$} \\
\hline YS & Input & $\begin{array}{l}\text { real } \\
\text { array }\end{array}$ & \multicolumn{2}{|c|}{$\begin{array}{l}\text { Array of device y coordinates to be plotted as } \\
\text { symbols. }\end{array}$} \\
\hline NPTS & Input & integer & \multicolumn{2}{|c|}{ Number of points in XS, YS, arrays. } \\
\hline MODE & Input & integer & \multicolumn{2}{|c|}{ Bit pattern of plot modes. } \\
\hline & $\begin{array}{l}\text { MODF } \\
\text { Value }\end{array}$ & $\begin{array}{l}\text { the sum } \\
\text {-axis at } \\
\text {-axis at } \\
\text {-axis inc } \\
\text {-axis inc } \\
\text { inear } x-a\end{array}$ & $\begin{array}{l}\text { om } \\
\text { es to right } \\
\text { es up }\end{array}$ & $\begin{array}{l}\text { tions desired: } \\
\text { = } \\
\quad x \text {-axis at top } \\
\text { y-axis at right } \\
\quad x \text {-axis increases to left } \\
\text { y-axis increases down } \\
\text { logarithmic } x \text {-axis }\end{array}$ \\
\hline
\end{tabular}




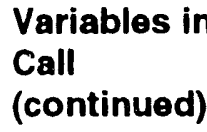

See Also linear $y$-axis

half frame

line plot

$\mathrm{X}$ array given

$Y$ array given advance frame after call
32 logarithmic $y$-axis

64 arc sinh $x$-axis

$128 \quad$ arc sinh $y$-axis

256 full frame

512 point plot

1024 X0, DX given

2048 Y0, DY given

4096 hold frame for another call

SCALES Input $\begin{aligned} & \text { real } \\ & \text { array }\end{aligned}$

ORGS

Input

real array

Axis scaling parameters:

$\mathrm{SC}(1)=\mathrm{x}$-axis length in device coordinates

$\mathrm{SC}(2)=\mathrm{x}$-axis initial value

$\mathrm{SC}(3)=\mathrm{x}$-axis final value

$\mathrm{SC}(4)=y$-axis length in device coordinates

$\mathrm{SC}(5)=y$-axis inital value

$\mathrm{SC}(6)=\mathrm{y}$-axis final value

$\mathrm{SC}(7)=$ minimum fraction of frame filled by $\mathrm{SC}(3)-\mathrm{SC}(2)$

$\mathrm{SC}(8)=$ minimum fraction of frame filled by $\mathrm{SC}(6)$ - SC(5)

$\mathrm{SC}(9)=$ scaling parameter for arc hyperbolic sine $x$-axis

$\mathrm{SC}(10)$ = scaling parameter for arc hyperbolic sine $y$-axis

Origin locations for various parts of the frame. $\operatorname{ORGS}(1),(2)=\mathrm{X}, \mathrm{Y}$ of bottom left corner of rectangle defined by axes

$\operatorname{ORGS}(3),(4)=\mathrm{X}, \mathrm{Y}$ of bottom left corner of main heading

ORGS(5), (6) = X,Y of top left corner of legends for graphs

$\operatorname{ORGS}(7),(8)=\mathrm{X}, \mathrm{Y}$ of bottom left corner of next frame

TITLES Input char*80 Three lines of the main heading.

array

AXLBLS Input char*80 Labels for the $\mathrm{x}$ and $\mathrm{y}$ axes.

array

LEGEND Input char Legend for the current graph. 
HPLMULPTRN

Subroutine

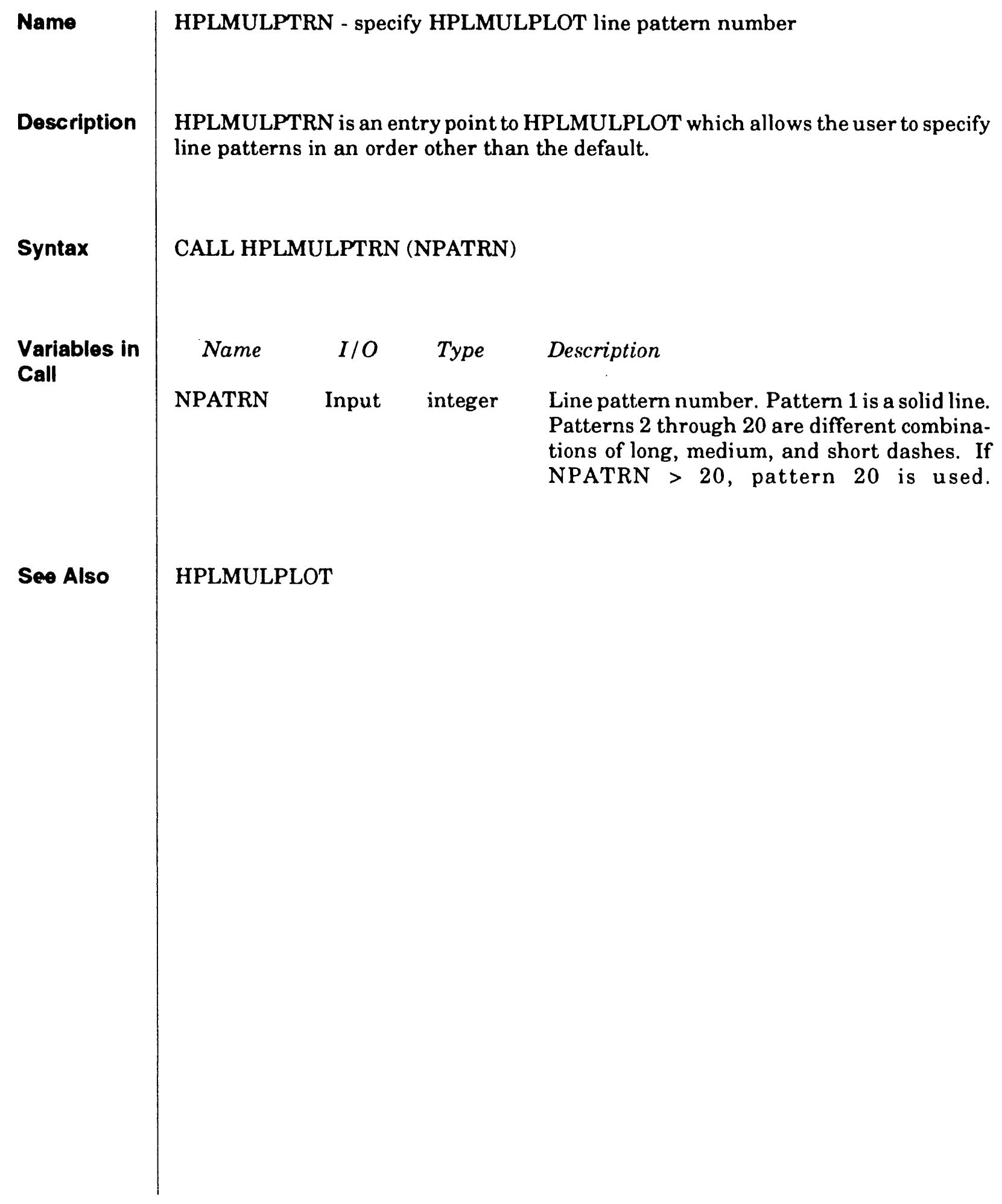




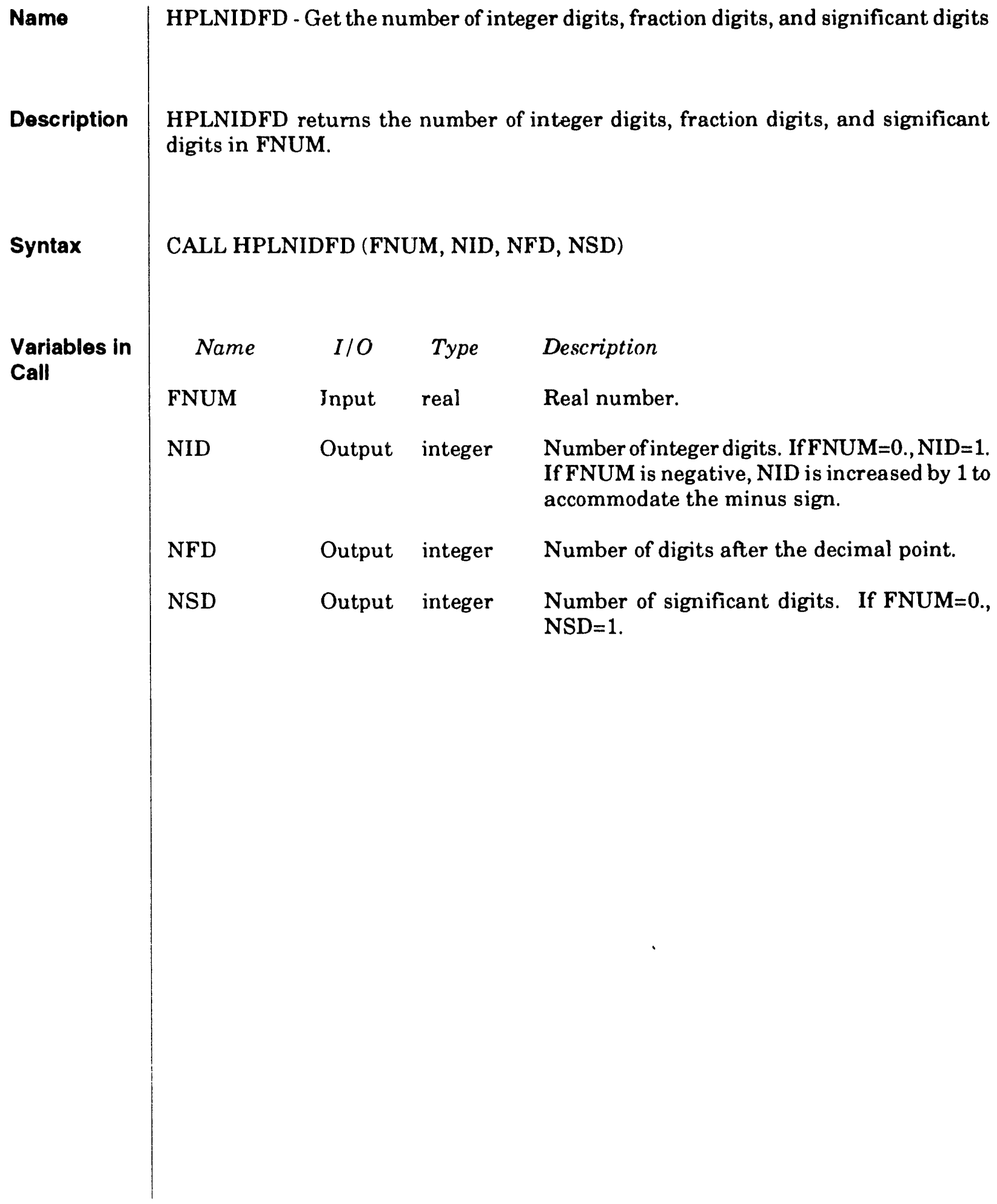




\begin{tabular}{|c|c|c|c|c|}
\hline Name & \multicolumn{4}{|c|}{ HPLNUMBER - Plot a number } \\
\hline Description & \multicolumn{4}{|c|}{ HPLNUMBER plots a number using format FMT. } \\
\hline Syntax & \multicolumn{4}{|c|}{ CALL HPLNUMBER (X, Y, HGT, VALUE, ANGLE, FMT) } \\
\hline \multirow{6}{*}{$\begin{array}{l}\text { Variables in } \\
\text { Call }\end{array}$} & Name & $I / O$ & Type & Description \\
\hline & $\mathrm{X}, \mathrm{Y}$ & Input & real & $\begin{array}{l}\text { Device } x \text { and } y \text { coordinates of the front bottom } \\
\text { corner of the rectangular area occupied by the } \\
\text { number. }\end{array}$ \\
\hline & HGT & Input & real & $\begin{array}{l}\text { Height of the number. The actual height } \\
\text { depends on the device and the aspect ratio. } \\
\text { Typically, HGT }=0.01 \text { produces fine print } \\
\text { (about } 0.05 \text { inch high) and HGT }=0.08 \text { pro- } \\
\text { duces digits about } 0.5 \text { inch high. }\end{array}$ \\
\hline & VALUE & Input & $\begin{array}{l}\text { real or } \\
\text { integer }\end{array}$ & $\begin{array}{l}\text { The value to be plotted. The value may be a } \\
\text { FORTRAN real or integer variable or con- } \\
\text { stant, but FMT must indicate the correct } \\
\text { type. }\end{array}$ \\
\hline & ANGLE & Input & real & $\begin{array}{l}\text { The rotation angle (degrees) measured coun- } \\
\text { terclock wise from the right horizontal direc- } \\
\text { tion. The nearest angle supported by the } \\
\text { graphics device will be used. }\end{array}$ \\
\hline & FMT & Input & char & $\begin{array}{l}\text { A character variable or constant holding the } \\
\text { format for the number, bounded by parenthe- } \\
\text { ses, e.g., FMT = '(F10.3)' for a real value or } \\
\text { FMT = '(I6)' for an integer value. }\end{array}$ \\
\hline
\end{tabular}




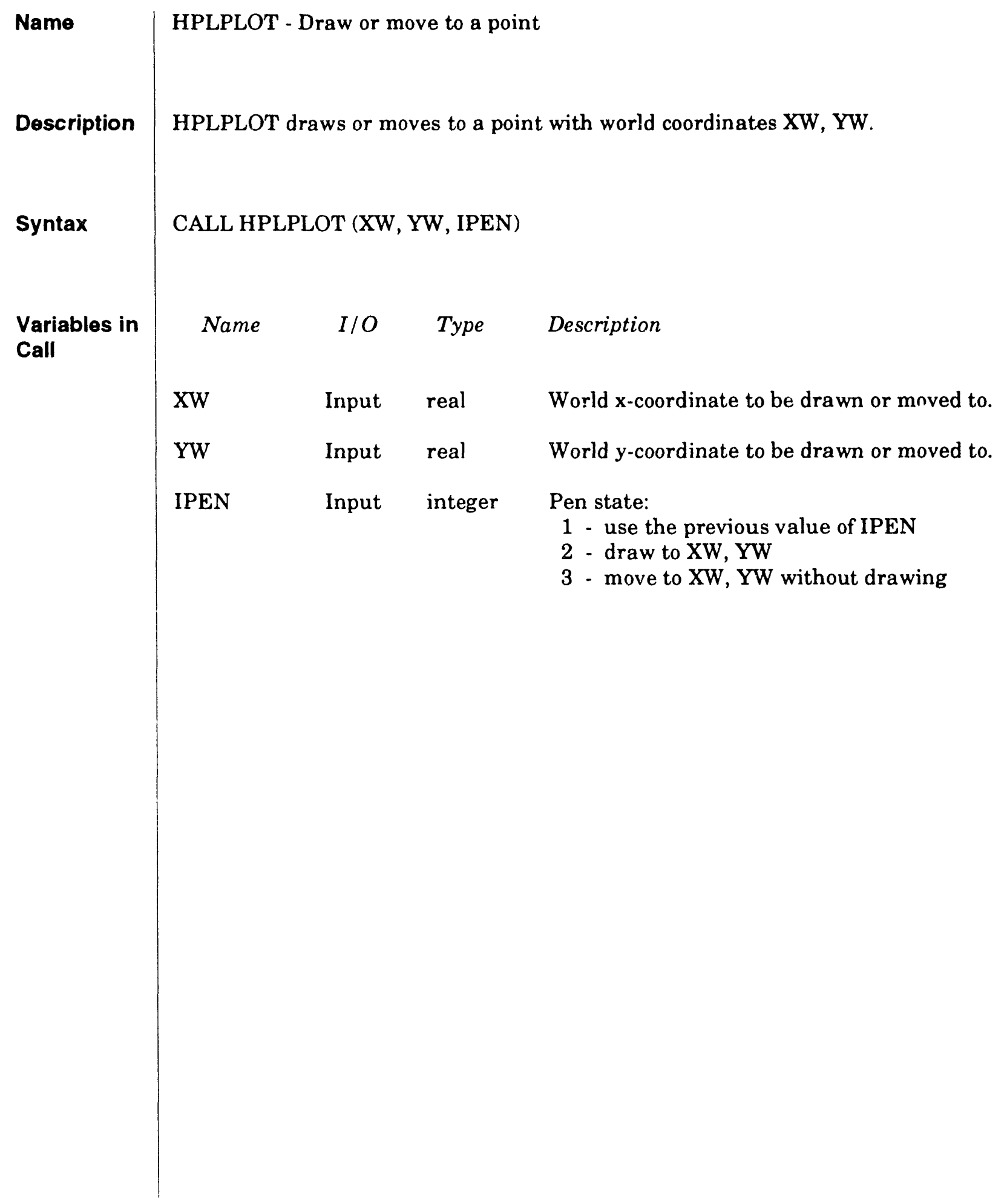


HJIPLOT_LIB

Description of Routine Calls

HPLPLOTD

Subroutine

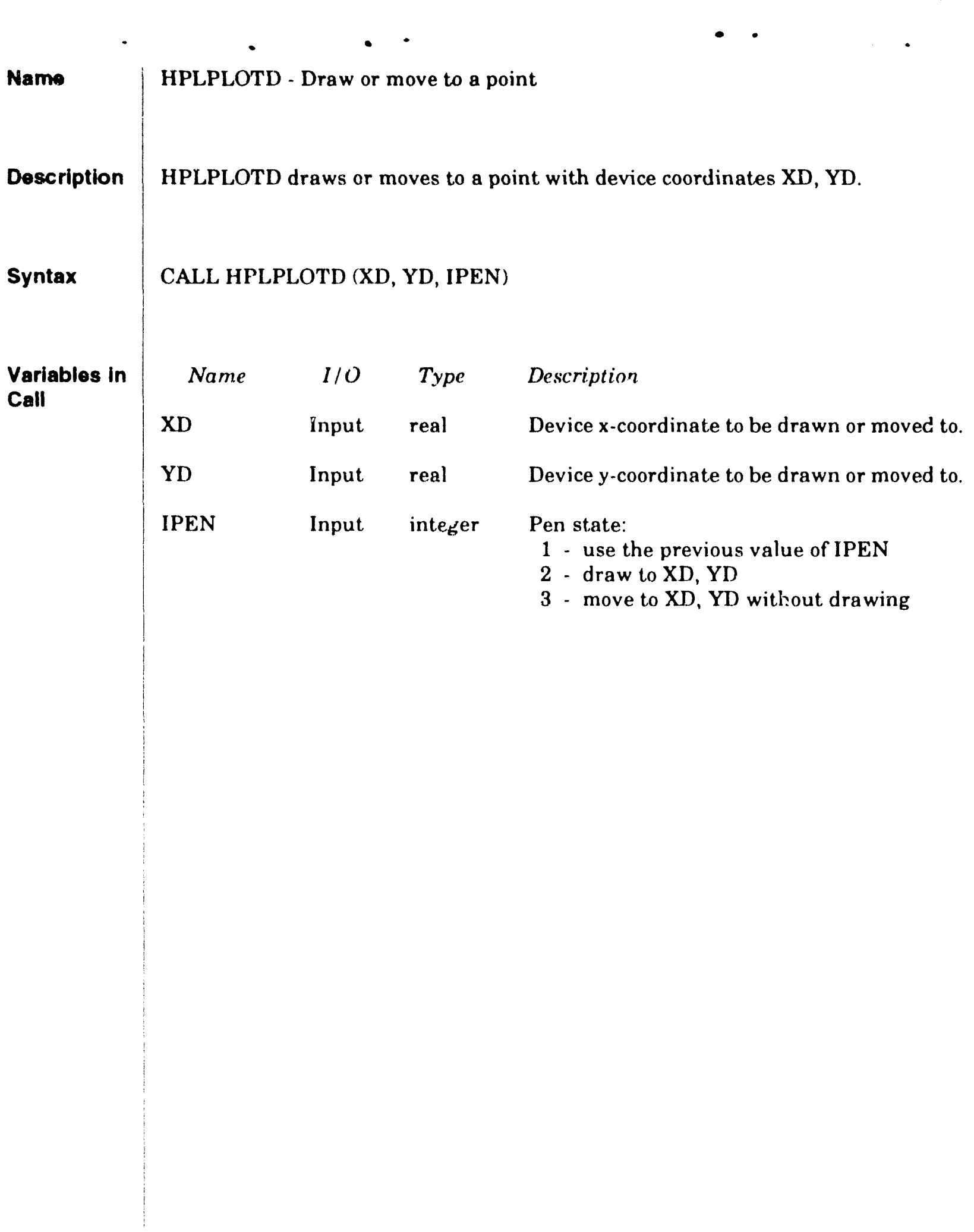




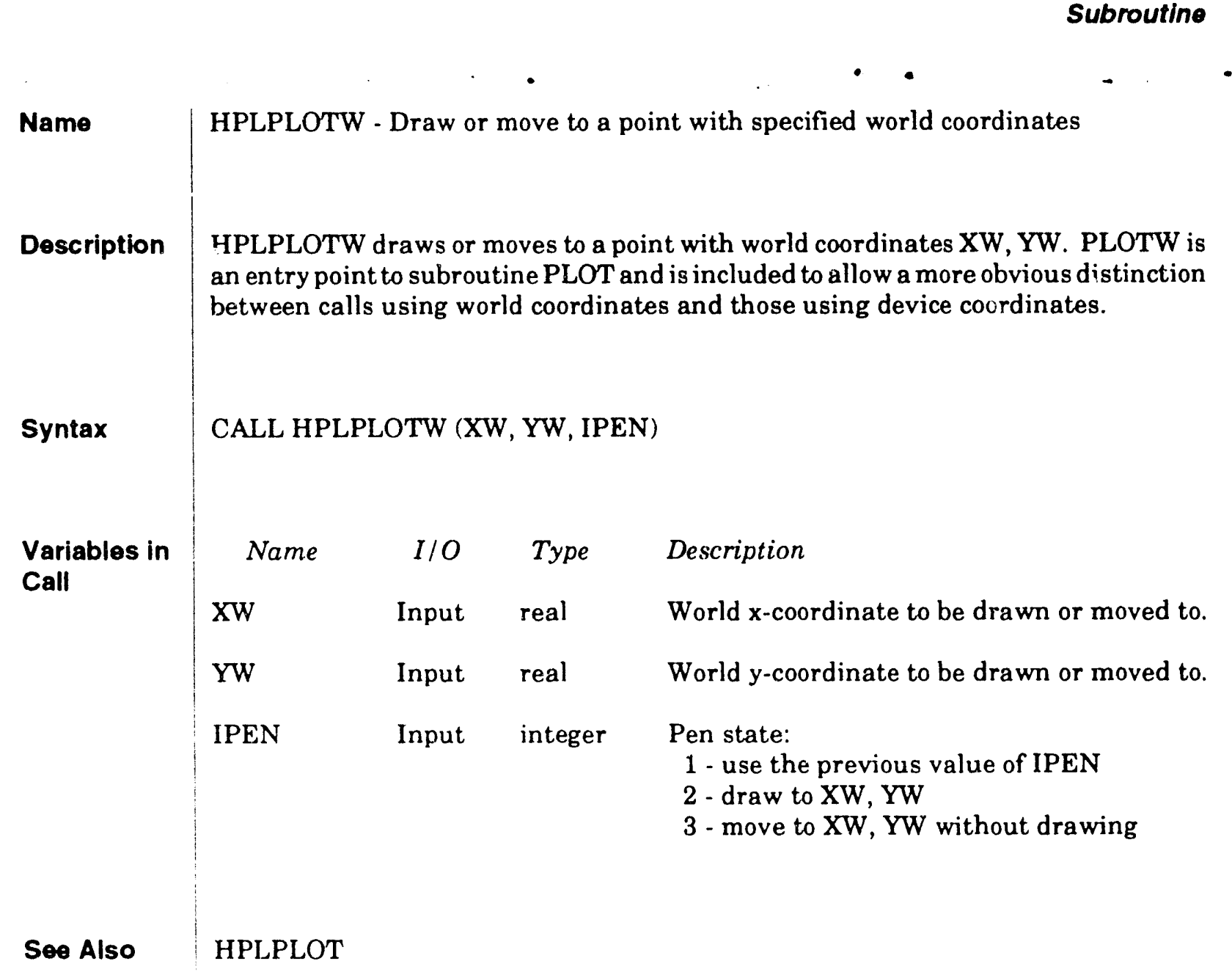


Description

Syntax

Soo Also
HPLPOPVEC restores the previous graphics attribute vector from a push-down stack. If HPLPOPVEC is called more times than HPLPUSHVEC, the initial graphics attribute vector will be restored. The graphics attribute vector contains the following data for each active graphics device:

$$
\begin{aligned}
\text { VECTOR }(1) & =\text { FOREGROUND COLOR } \\
(2) & =\text { BACKGROUND COLOR } \\
(3) & =\text { INTENSITY } \\
(4) & =\text { LINE STYLE } \\
(5) & =\text { LINE WIDTH } \\
(6) & =\text { CHARACTER BOX Y } \\
(7) & =\text { CHARACTER BOX X }
\end{aligned}
$$

CALL HPLPOPVEC

HPLPUSHVEC 


\section{Name \\ Description}

\section{Syntax}

Seo Also
HPLPUSHVEC - Save the current vector of plot attributes

HPLPUSHVEC saves the current graphics attribute vector on a push-down stack. The stack can hold the initial graphics attribute vector and up to nine changes. If the current attribute vector is the same as the previous vector, a counter is increased rather than adding to the stack. No additions can be made to the stack when it contains 10 vectors.

The graphics attribute vector contains the following data for each active graphics device:

$$
\begin{aligned}
\text { VECTOR }(1) & =\text { FOREGROUND COLOR } \\
(2) & =\text { BACKGROUND COLOR } \\
(3) & =\text { INTENSITY } \\
(4) & =\text { LINE STYLE } \\
(5) & =\text { LINE WIDTH } \\
(6) & =\text { CHARACTER BOX Y } \\
(7) & =\text { CHARACTER BOX X }
\end{aligned}
$$

CALL HPLPUSHVEC

HPLPOPVEC 


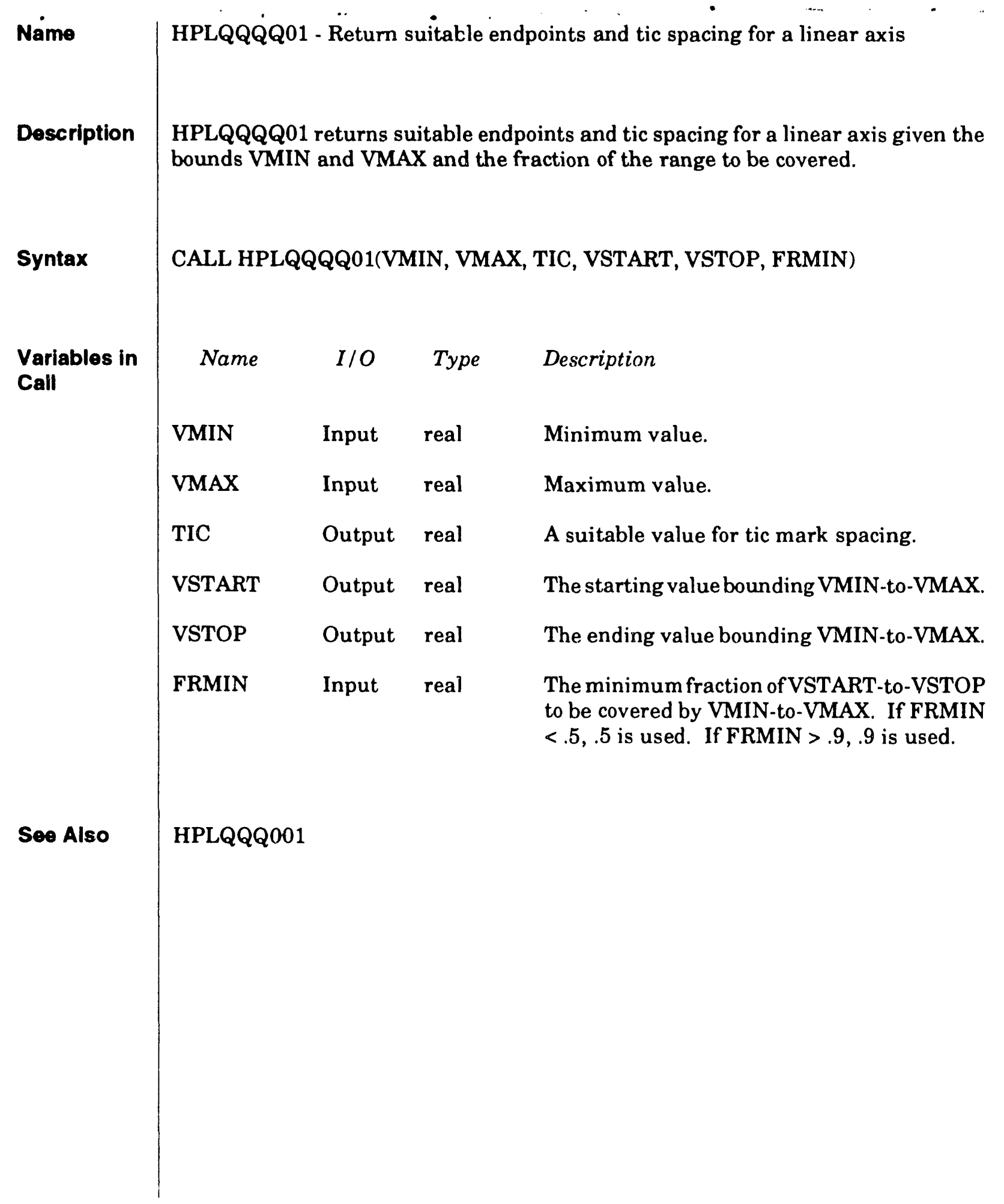




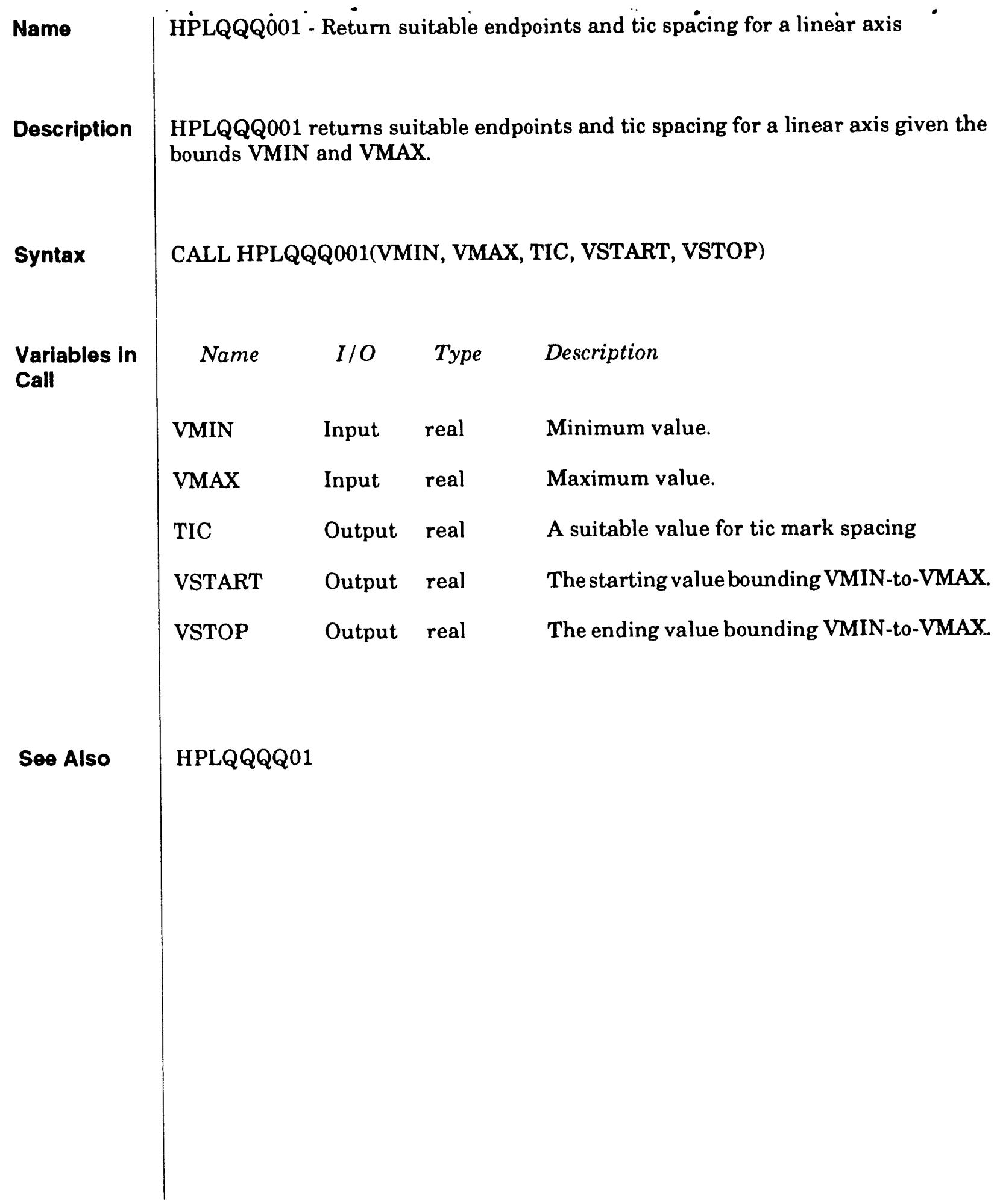




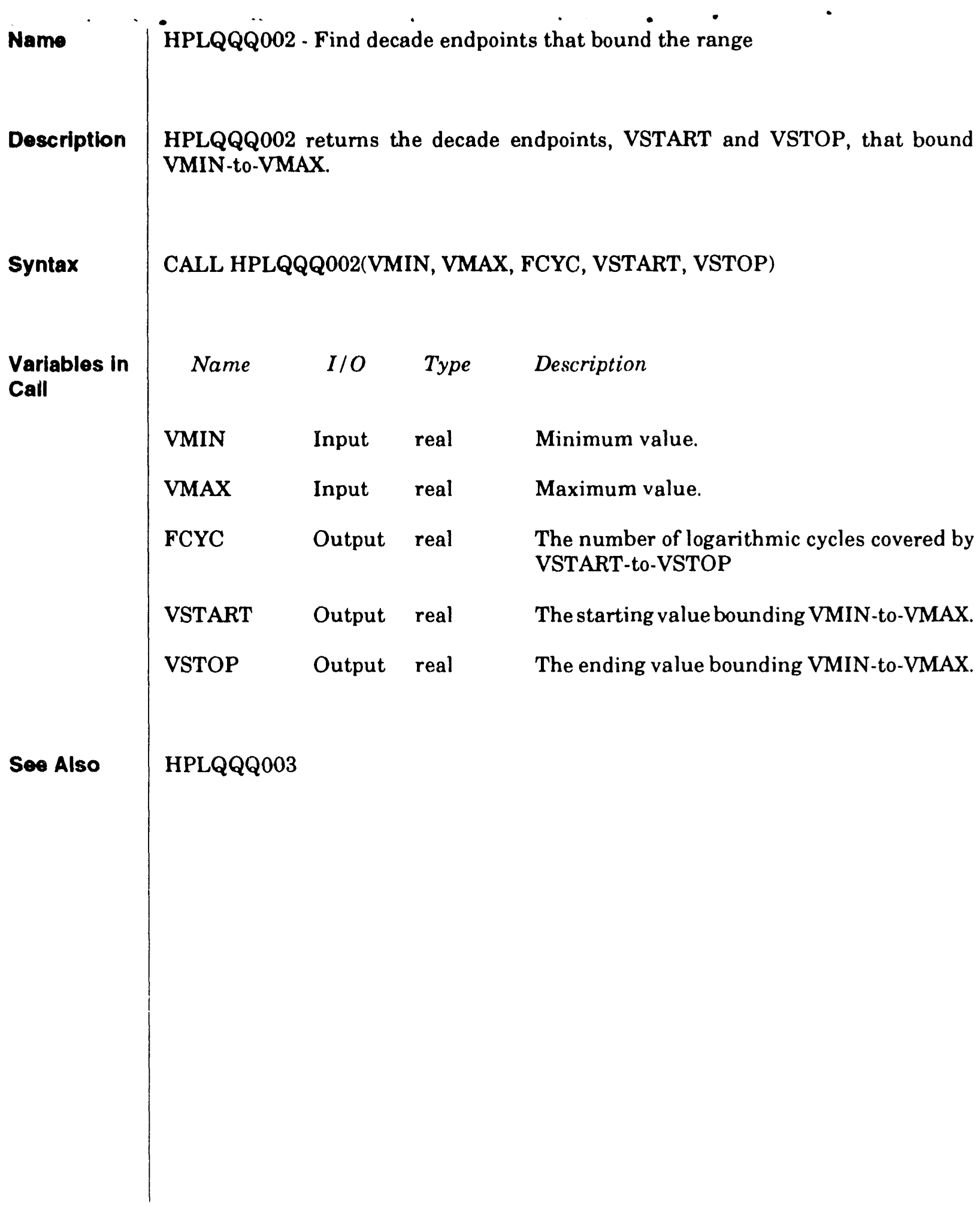


HPLQQQ003 returns round numbers (one nonzero digit) for the endpoints VSTART and VSTOP that bound VMIN-to-VMAX. VSTART and VSTOP are not necessarily the endpoints of a decade.

\section{Syntax}

Variables in Call

\section{See Also}

CALL HPLQQQ003(VMIN, VMAX, FCYC, VSTART, VSTOP)

$\begin{array}{rrrl}\text { Name } & \text { I/O } & \text { Type } & \text { Description } \\ \text { VMIN } & \text { Input } & \text { real } & \text { Minimum value. } \\ \text { VMAX } & \text { Input } & \text { real } & \text { Maximum value. }\end{array}$

VMAX Input real

FCYC Output real The number of logarithmic cycles covered by VSTART-to-VSTOP

VSTART Output real The starting value bounding VMIN-to-VMAX.

VSTOP Output real The ending value bounding VMIN-to-VMAX. 


\begin{tabular}{|c|c|c|c|c|}
\hline Name & \multicolumn{4}{|c|}{ HPLSCREEN - Define the bounds of the plot } \\
\hline Description & \multicolumn{4}{|c|}{$\begin{array}{l}\text { HPLSCREEN defines the bounds of the plot. Margins are set to about } 10 \% \text { of the } x \\
\text { and y extents. }\end{array}$} \\
\hline Syntax & \multicolumn{4}{|c|}{$\begin{array}{l}\text { CALL HPLSCREEN (XMIN, XMAX, YMIN, YMAX, XTYPE, YTYPE, XPRAM, } \\
\text { YPRAM) }\end{array}$} \\
\hline \multirow{9}{*}{$\begin{array}{l}\text { Variables in } \\
\text { Call }\end{array}$} & Name & $1 / O$ & Type & Description \\
\hline & XMIN & Input & real & Minimum world $\mathrm{x}$-coordinate to be displayed. \\
\hline & XMAX & Input & real & Maximum world $x$-coordinate to be displayed. \\
\hline & YMIN & Input & real & Minimum world y-coordinate to be displayed. \\
\hline & YMAX & Input & real & Maximum world y-coordinate to be displayed. \\
\hline & XTYPE & Input & integer & $\begin{array}{l}\text { X-coordinate type: } \\
0 \text { - linear } \\
1 \text { - logarithmic } \\
2 \text { - arc hyperbolic sine }\end{array}$ \\
\hline & YTYPE & Input & integer & $\begin{array}{l}\text { Y-coordinate type: } \\
0 \text { - linear } \\
1 \text { - logarithmic } \\
2 \text { - arc hyperbolic sine }\end{array}$ \\
\hline & XPRAM & Input & real & $\begin{array}{l}\text { Scaling parameter for arc hyperbolic sine } \\
\text { mapping of the } x \text {-axis. }\end{array}$ \\
\hline & YPRAM & Input & real & $\begin{array}{l}\text { Scaling parameter for arc hyperbolic sine } \\
\text { mapping of the y-axis. }\end{array}$ \\
\hline Soe Also & \multicolumn{4}{|c|}{ HPLXDCMAP, HPLYDCMAP } \\
\hline
\end{tabular}




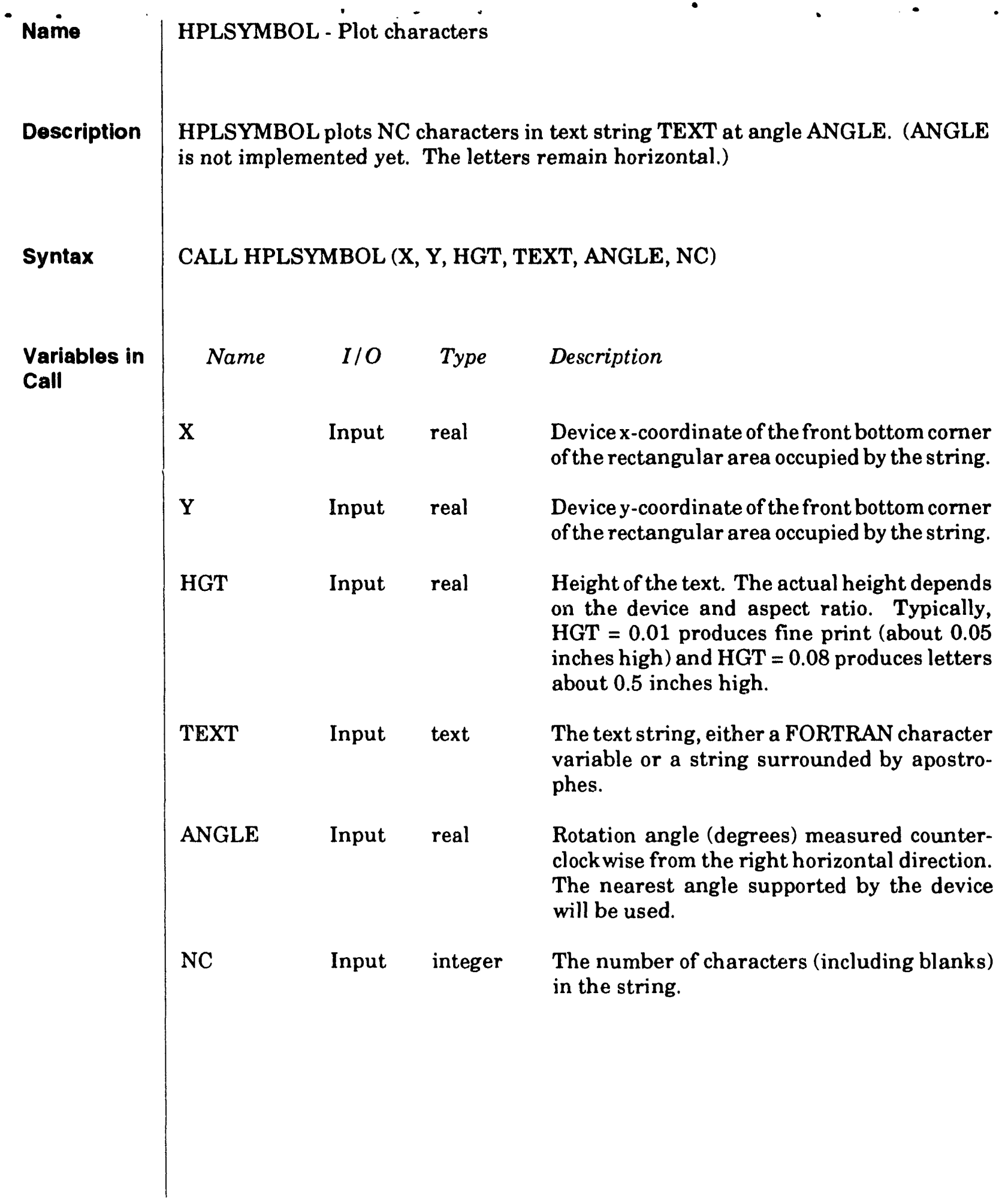




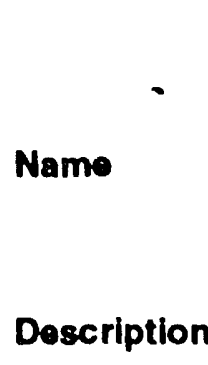

See Also

HPLWAITCR - Wait for Return key

HPLWAITCR holds the plot until the Return key is pressed. The message "Press RETURN to continue ${ }^{n}$ is displayed on the terminal screen. No message appears on hard copy.

\section{CALL HPLWAITCR}

HPLWAITOFF, HPLWAITON 


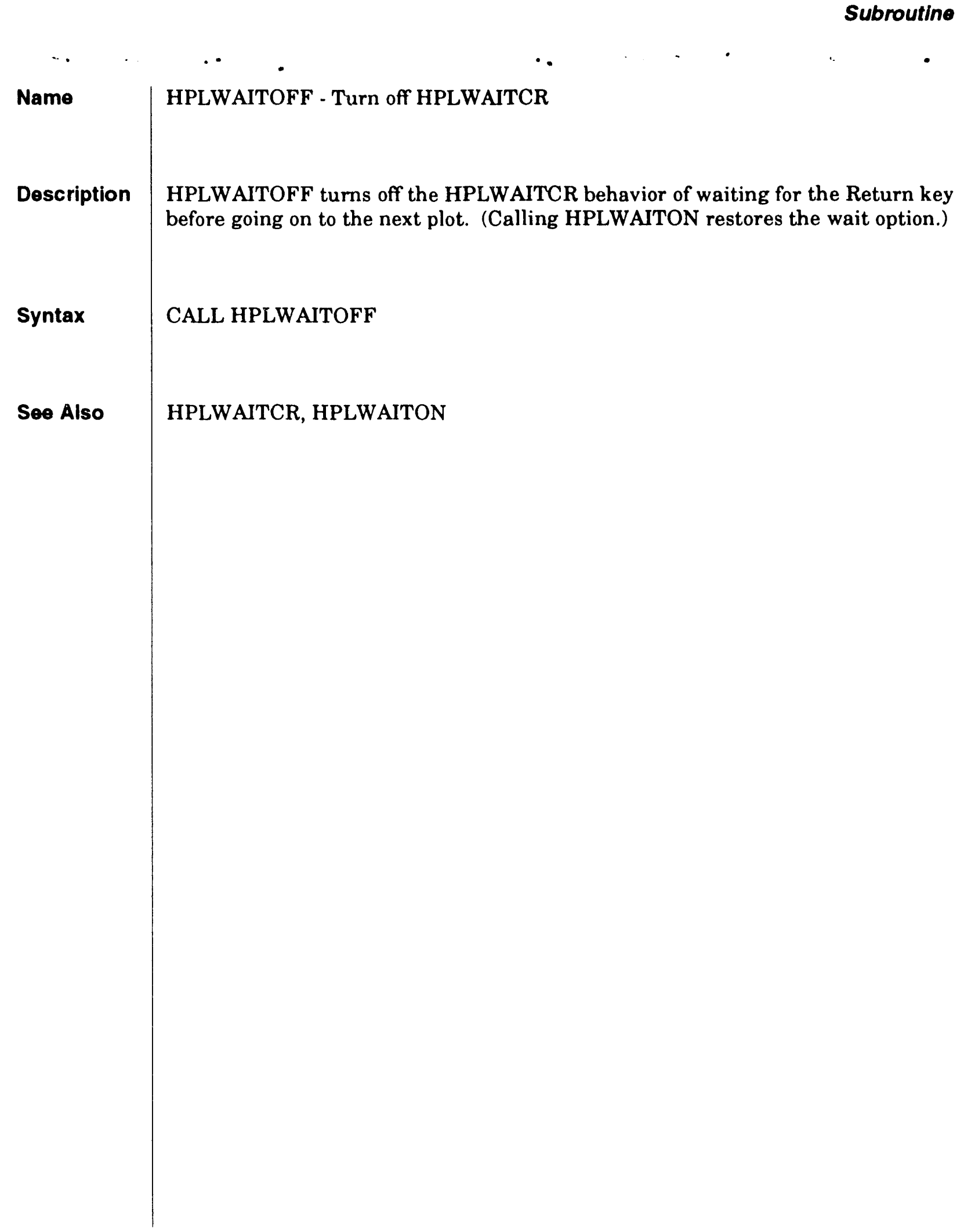


Name

Description

HPLWAITON turns on the HPLWAITCR behavior of waiting for the Return key before going on to the next plot. (Calling HPLWAITOFF turns off the wait option.)

HPLWAITON - Turn on HPLWAITCR

Soe Also

Syntax

CALL HPLWAITON

HPLWAITCR, HPLWAITOFF 


\begin{tabular}{|c|c|c|c|c|}
\hline Name & \multicolumn{4}{|c|}{ HPLXMAPDC - Map world x-coordinates to device $x$-coordinates } \\
\hline Description & \multicolumn{4}{|c|}{$\begin{array}{l}\text { HPLXMAPDC maps world } x \text {-coordinates XW1, XW2 to device } \mathrm{X} \text {-coordinates XD1, } \\
\text { XD2. The mapping is linear, logarithmic, or arc hyperbolic sine, depending on the } \\
\text { value of XTYP. }\end{array}$} \\
\hline Syntax & \multicolumn{4}{|c|}{ CALL HPLXMAPDC (XD1, XW1, XD2, XW2, XTYP, XPRAM) } \\
\hline \multirow{7}{*}{$\begin{array}{l}\text { Variables in } \\
\text { Call }\end{array}$} & Name & $I / O$ & Type & Description \\
\hline & XD1 & Input & real & Minimum device $x$-coordinate. \\
\hline & XW1 & Input & real & $\begin{array}{l}\text { Minimum world } x \text {-coordinate (corresponding } \\
\text { to XD1). }\end{array}$ \\
\hline & XD2 & Input & real & Maximum device $x$-coordinate. \\
\hline & XW2 & Input & real & $\begin{array}{l}\text { Maximum world } x \text {-coordinate (corresponding } \\
\text { to XD2). }\end{array}$ \\
\hline & XTYP & Input & integer & $\begin{array}{l}\text { Type of coordinate mapping: } \\
0 \text { - linear mapping } \\
1 \text { - logarithmic mapping } \\
2 \text { - arc hyperbolic sine mapping }\end{array}$ \\
\hline & XPRAM & Input & real & $\begin{array}{l}\text { Scaling parameter for the arc hyperbolic sine } \\
\text { mapping. }\end{array}$ \\
\hline Soe Also & \multicolumn{4}{|c|}{ HPLYMAPDC } \\
\hline
\end{tabular}




\begin{tabular}{|c|c|c|c|c|}
\hline Name & \multicolumn{4}{|c|}{ HPLYMAPDC - Map world y-coordinates to device y-coordinates } \\
\hline Description & \multicolumn{4}{|c|}{$\begin{array}{l}\text { HPLYMAPDC maps world y-coordinates YW1, YW2 to device y-coordinates YD1, } \\
\text { YD2. The mapping is linear, logarithmic, or arc hyperbolic sine, depending on the } \\
\text { value of YTYP. }\end{array}$} \\
\hline Synta: & \multicolumn{4}{|c|}{ CALL HPLYMAPDC (YD1, YW1, YD2, YW2, YTYP, YPRAM) } \\
\hline \multirow{7}{*}{$\begin{array}{l}\text { Variables in } \\
\text { Call }\end{array}$} & Name & $1 / O$ & Type & Description \\
\hline & YD1 & Input & real & Minimum device y-coordinate. \\
\hline & YW1 & Input & real & $\begin{array}{l}\text { Minimum world y-coordinate (corresponding } \\
\text { to YD1). }\end{array}$ \\
\hline & YD2 & Input & real & Maximum device $y$-coordinate. \\
\hline & YW2 & Input & real & $\begin{array}{l}\text { Maximum world y-coordinate (corresponding } \\
\text { to YD2) }\end{array}$ \\
\hline & YTYP & Input & integer & $\begin{array}{l}\text { Type of coordinate mapping: } \\
0 \text { - 'inear mapping } \\
1 \text { - logarithmic mapping } \\
2 \text { - arc hyperbolic sine mapping }\end{array}$ \\
\hline & YPRAM & Input & real & $\begin{array}{l}\text { Scaling parameter for the arc hyperbolic sine } \\
\text { mapping }\end{array}$ \\
\hline & \multicolumn{4}{|c|}{ HPLXMAPDC } \\
\hline
\end{tabular}




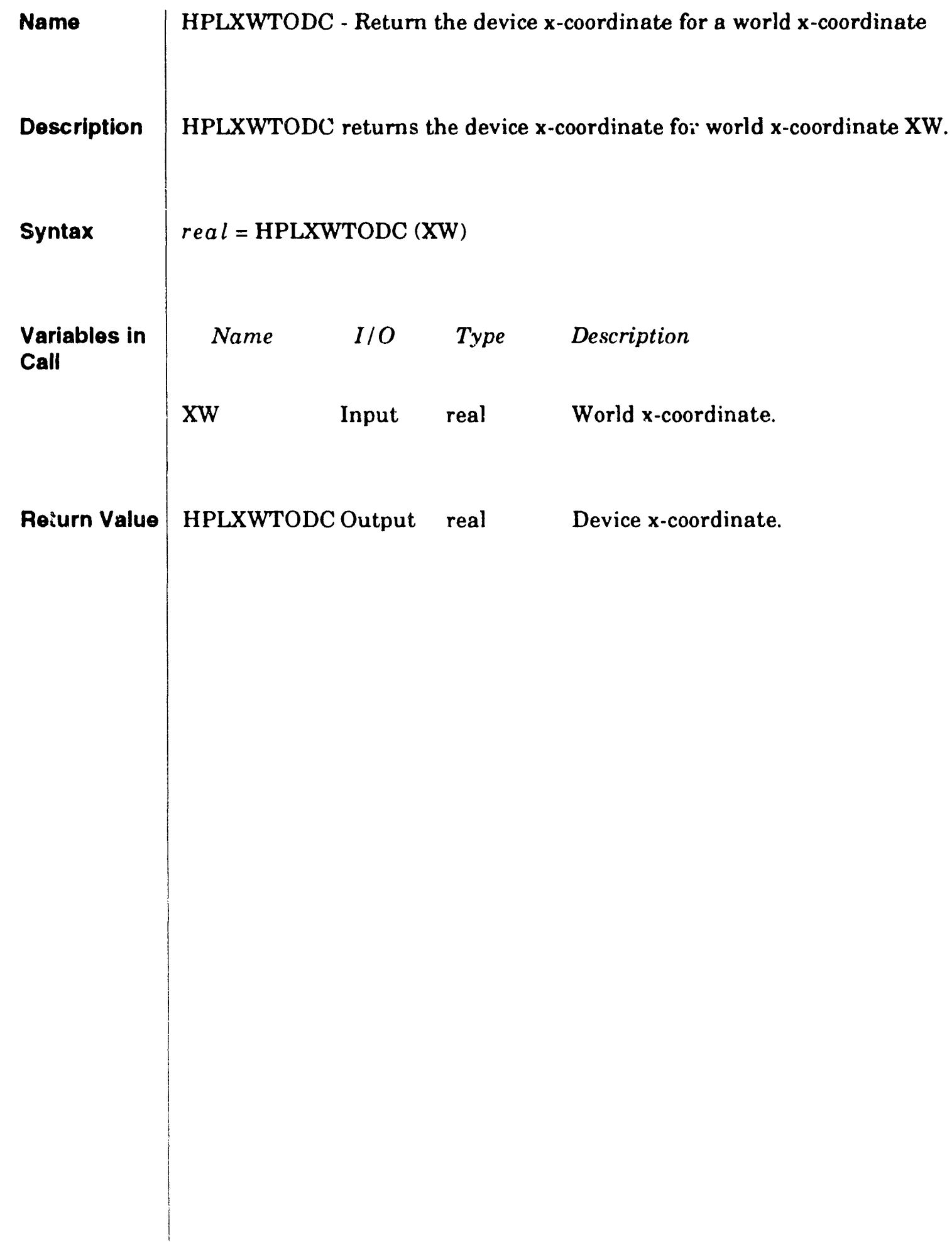




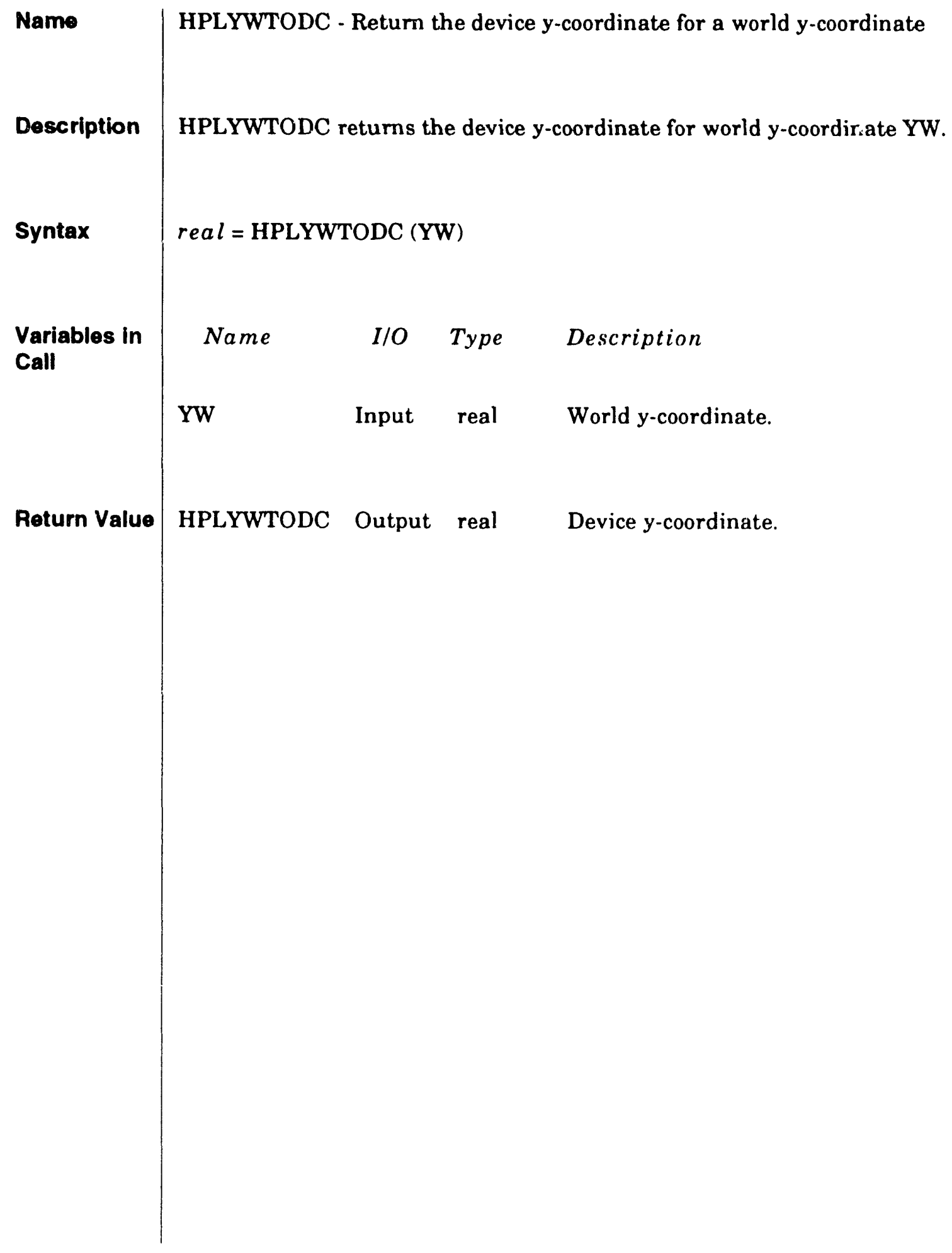


HJIPLOT_LIB

Description of Routıne Calls 


\section{Graphic Device Interfaces: SVDI and DVDI}

Major CAMCON system plot packages use the Sandia Virtual Device Interface (SVDI) and Dual Virtual Device Interface (DVDI) for communicating with a graphics device. The SVDI is a library of basic graphic commands designed to support higher level graphic packages such as PLT_LIB (see Chapter 10). The DVDI is an extension of the SVDI and permits communication with one or two graphics devices (either a terminal device and/or a hardcopy device) in one plotting session. The routines within SVDI are described in the following three sections. The use of DVDI is described in Section 11.4.

\subsection{Sandia Virtual Device Interface Library}

\subsubsection{Sponsorship and Version}

$\begin{array}{ll}\text { Authors: } & \text { R.W. Simmons, P. Watterberg, K. M. Erikson, D. L. Campbell, C. D. Brown, H. S. } \\ & \text { Lauson, J. P. Long, and P. L. Crotty } \\ \text { Sponsor: } & \text { H. J. Iuzzolino } \\ \text { Version /Date: } & \text { C-1.00VV/February } 1991 \\ \text { Language: } & \text { FORTRAN } 77\end{array}$

\subsubsection{Overview of SVDI}

The SVDI (Erikson and Simons, 1982) is a library of graphics primitives designed to support graphics packages at Sandia National Laboratories. SVDI consists of a library file of 30 routines for each of 9 terminal devices and 7 file devices. Library files are selected at run time for a terminal device or for a hardcopy device based on global DCL symbols VDI_TERM and VDI_FILE. (Both a terminal device and a hardcopy device can be selected using DVDI_LIB, as described in Section 11.4.)

SVDI provides the base graphics library for the PLT_LIB and HJIPLOT_LIB libraries. The PLT_LIB library provides substitute calls for all SVDI routines, and PLT_LIB users are encouraged to use the PLT_LIB versions instead of the SVDI routines. HJIPLOT_LIB users may use the SVDI routines directly, except VDINIT, since there are no substitutes in HJIPLOT_LIB. If any HJIPLOT_LIB routines are used, the user must call HPLINITPLOT or HPLINITPLT to initialize the plotting. If only SVDI routines are used, the SVDI subroutine, VDINIT, may be used to initialize the plotting.

\subsubsection{Summary of Routines}

The SVDI routines are described in detail in Section 11.3. The following is a summary of the SVDI routines. 
- VBERRH - error handling

- VDAABU - awaits any button

- VDABGL - awaits a button, then gets the keyboard locator

- VDAKGL - awaits a keyboard key, then gets the keyboard locator

- VDALOC - returns the keyboard locator

- VDBELL - rings the bell

- VDBUFL - flushes the buffer

- VDESCP - sets the escape code

- VDFRAM - draws header or trailer frame

- VDINIT - initializes SVDI

- VDIQCO - makes color table inquiry

- VDIQCP - makes current position inquiry

- VDIQDC - makes device capabilities inquiry

- VDIQES - makes escape inquiry

- VDIQND - makes NDC limits inquiry

- VDIQOS - makes output status inquiry

- vDLINA - draws a line

- vDLOGE - logs an error

- VDMOVA - moves the cursor

- VDNWPG - goes to new page

- VDPNTA - draws a point

- VDPOLY - draws a filled polygon

- VDSTBC - sets the background color

- VDSTCO - sets the color table

- VDSTCS - sets the character size

- VDSTFC - sets the foreground color

- VDSTFN - sets the hardware character font

- VDSTIN - sets the intensity

- VDSTLA - sets the initial locator posit ${ }^{\circ}$

- VDSTLS - sets the line style

- VDSTLW - sets the line width

- vDSTOS - sets the output status

- VDSTSA - sets the character string angle

- VDTERM - terminates SVDI

- VDTEXT - draws text

- VDWAIT - waits for the user

\subsection{Escape Codes for SVDI-Supported Devices}

An escape code provides access to device-dependent functions not directly supported by SVDI. Escape codes are accessed from within a FORTRAN routine by CALL VDESCP (ICODE, N, ARGS), where ICODE is an integer escape code, $\mathrm{N}$ is the number of arguments in ARGS ( $\mathrm{N} \geq 0$ ), and ARGS is a real array containing the arguments. The devices currently supported are shown in Table 11-1. Contact the SVDI sponsor to add or delete devices. Table 11-2 shows the escape codes for CAMCON SVDI-supported devices. Additional escape codes for DVDI are listed in Section 11.4. 
Table 11-1. Currently Supported Devices in SVDI

\begin{tabular}{cl}
\hline 3 Digit Code & Description \\
\hline Terminal Device Codes: & \\
T05 & Tektronix 4105 \\
T07 & Tektronix 4107 \\
V34 & Digital VT340 \\
V41 & Digital VT241 \\
& \\
Hardcopy Device Codes: \\
H75 & HP 7580 \\
PST & Postscript Level II
\end{tabular}

Table 11-2. Escape Codes for CAMCON SVDI-Supported Devices Syntax: CALL VDESCP (ICODE, N, ARGS)

\begin{tabular}{|c|c|c|c|}
\hline ICODE & $\mathbf{N}$ & ARGS & Meaning \\
\hline \multicolumn{4}{|c|}{ Device code $=\mathrm{H} 75$ : } \\
\hline 1400 & 2 & $\mathrm{XINCH}, \mathrm{YINCH}$ & $\begin{array}{l}\text { Size of paper in inches. Must be called before VDINIT. } \\
X=\text { side moved by roller drum, } Y=\text { side moved by pen. }\end{array}$ \\
\hline 1401 & 4 & $\begin{array}{l}\text { XNDC, YNDC, } \\
\text { ANGLE } \\
\text { TOLER }\end{array}$ & $\begin{array}{l}\text { Draw an arc of specified degrees and tolerence cen- } \\
\text { tered at XNDC, YNDC, using the current pen status. }\end{array}$ \\
\hline 1402 & $\begin{array}{l}1 \\
2\end{array}$ & $\begin{array}{l}\text { IRAD } \\
\text { TOLER }\end{array}$ & $\begin{array}{l}\text { Circle instruction. IRAD = radius in plotter units if } \\
\text { scaling is not in effect; otherwise use user units. } \\
\text { TOLER = chord tolerance in degrees or deviation } \\
\text { distance. }\end{array}$ \\
\hline 1403 & 1 & IFONT & $\begin{array}{l}\text { Designate alternate character set. IFONT }=0 \text { through } \\
5 \text { for fixed-font set and } 10 \text { through } 15 \text { for variable-font } \\
\text { set. Default }=0 \text {. }\end{array}$ \\
\hline 1404 & 1 & ANGLE & $\begin{array}{l}\text { Character chord angle instruction. ANGLE }=\text { angle in } \\
\text { degrees, values }>45 \text { are set to } 45 .\end{array}$ \\
\hline
\end{tabular}


Table 11-2. Escape Codes for CAMCON SVDI-Supported Devices Syntax: CALL VDESCP (ICODE, N, AR6S) (Continued)

\begin{tabular}{|c|c|c|c|}
\hline ICODE & $\mathbf{N}$ & ARIGS & Meaning \\
\hline \multicolumn{4}{|c|}{ Device code $=$ H75 (continued): } \\
\hline 1405 & 1 & IFONT & $\begin{array}{l}\text { Designate standard character set. IFONT }=0 \text { through } \\
5 \text { for fixed-font set and } 10 \text { through } 15 \text { for variable-font } \\
\text { set. Default }=0 \text {. }\end{array}$ \\
\hline 1406 & $\begin{array}{l}1 \\
2\end{array}$ & $\begin{array}{l}\text { WIDTH } \\
\text { HEIGHT }\end{array}$ & $\begin{array}{l}\text { Extra space instruction. WIDTH = spaces (positive } \\
\text { or negative) between characters. HEIGHT = spaces } \\
\text { (positive or negative) between lines. }\end{array}$ \\
\hline 1407 & 0 & & Select alternate character set. \\
\hline 1408 & 1 & TANANG & $\begin{array}{l}\text { Character slant instruction. TANANG = tangent of } \\
\text { the angle with the vertical. }\end{array}$ \\
\hline 1409 & 0 & & Select standard character set. \\
\hline 1410 & $\begin{array}{l}1 \\
2\end{array}$ & $\begin{array}{l}\text { IPENAC } \\
\text { IPEN }\end{array}$ & $\begin{array}{l}\text { Acceleration selectinstruction. IPENAC }=\text { pen acceler- } \\
\text { ation, } 1 \text { to } 4 . \text { IPEN }=\text { pen } 1 \text { through } 8 \text { to which } \\
\text { acceleration applies. Default }=\text { all pens. }\end{array}$ \\
\hline 1411 & $\begin{array}{l}1 \\
2\end{array}$ & $\begin{array}{l}\text { IPENFC } \\
\text { IPEN }\end{array}$ & $\begin{array}{l}\text { Force select instruction. IPENFC }=\text { pen force, } 1 \text { to } 8 \text {, } \\
\text { where force }=10+8 \times(\text { IPENFC }-1) \text { grams. IPEN }=\text { pen } \\
1 \text { through } 8 \text { to which force applies. Default = all pens. }\end{array}$ \\
\hline 1412 & $\begin{array}{l}1 \\
2\end{array}$ & $\begin{array}{l}\text { IVELOC } \\
\text { IPEN }\end{array}$ & $\begin{array}{l}\text { Velocity select instruction. IVELOC }=\text { pen velocity, } 1 \\
\text { to } 60 \mathrm{~cm} / \mathrm{sec} . \mathrm{IPEN}=\text { pen } 1 \text { through } 8 \text { to which velocity } \\
\text { applies. Default = all pens. }\end{array}$ \\
\hline 1413 & $\begin{array}{l}1 \\
2\end{array}$ & $\begin{array}{l}\mathrm{TP} \\
\mathrm{TN}\end{array}$ & $\begin{array}{l}\text { Tic length instruction. TP = percentage of } p 2 y-p i y \\
\text { above the } x \text {-axis or percentage of } p 2 x-p 1 x \text { to the right } \\
\text { of the } y \text {-axis. TN = percentage of } p 2 y-p 1 y \text { below the } x- \\
\text { axis or percentage of } p 2 x-p 1 x \text { to the left of the } y \text {-axis }\end{array}$ \\
\hline 1414 & 0 & & $\mathrm{X}$ tic instruction. \\
\hline 1415 & 0 & & $\mathrm{Y}$ tic instruction. \\
\hline
\end{tabular}


Table 11-2. Escape Codes for CAMCON SVDI-Supported Devices Syntax: CALL VDESCP (ICODE, N, AR6S) (Concluded)

\begin{tabular}{|c|c|c|c|}
\hline ICODE & $\mathbf{N}$ & ARGS & Meaning \\
\hline \multicolumn{4}{|c|}{ Device code = H75 (continued): } \\
\hline 1416 & 1 & $\mathrm{~N}$ & $\begin{array}{l}\text { Automatic pen pickup instruction. } \mathrm{N} \leq 32768 \text {. Only } \\
\text { the } 3 \text { least significant bits are used. } \\
\text { Bit } 0=1 \text { : pen lift after } 65 \text { seconds } \\
\text { Bit } 1=1 \text { : pen storage after } 65 \text { seconds } \\
\text { Bit } 2=1 \text { : delay retrieval } \\
\text { Default }=7 \text { (all three bits set) }\end{array}$ \\
\hline 1417 & 1 & $\mathrm{~N}$ & $\begin{array}{l}\text { Chord tolerance units instruction. Determines how to } \\
\text { interpret chord tolerance for circle and arc commands. } \\
\mathrm{N}=0 \text { for degree units, } \mathrm{N}=1 \text { for deviation units. }\end{array}$ \\
\hline 1418 & 0 & & Reset instruction. Sets plotter to defaults. \\
\hline \multirow[t]{3}{*}{1419} & 0 & & Input $\mathrm{P} 1$ and $\mathrm{P} 2$ instruction. Set scaling points. \\
\hline & 2 & P1X, P1Y & Omitting all sets P2X and P2Y $15 \mathrm{~mm}$ inside hard clip \\
\hline & 4 & $\mathrm{P} 2 \mathrm{X}, \mathrm{P} 2 \mathrm{Y}$ & $\begin{array}{l}\text { limits. Omitting last two causes } \mathrm{P} 2 \text { to move the same } \\
\text { distance as P1. }\end{array}$ \\
\hline 1420 & 4 & $\mathrm{X} 1, \mathrm{X} 2, \mathrm{X} 3, \mathrm{X} 4$ & $\begin{array}{l}\text { Input window instruction. Specifies } \mathrm{X} \text { and } \mathrm{Y} \text { coordi- } \\
\text { nates of opposite corners of the window. Values are in } \\
\text { NDC units. }\end{array}$ \\
\hline 1421 & 1 & IROTAT & $\begin{array}{l}\text { Rotate coordinate system. IROTAT }=0 \text { turns off } \\
\text { rotation. IROTAT }=90 \text { rotates coordinate system } 90 \\
\text { degrees about the plotter origin. }\end{array}$ \\
\hline 1422 & 4 & $\begin{array}{l}\text { XMIN, XMAX, } \\
\text { YMIN, YMAX }\end{array}$ & Scale instruction. Scales plotting areas to user units. \\
\hline
\end{tabular}

Device code $=$ T05:

$100 \quad 0$

Produce hardcopy of what is now on the screen and wait until complete.

Also uses T14 codes 1020-1025, 1030-1032, and 1505. 


\subsection{Description of Routine Calls}

Detailed descriptions of the SVDI routines are given on the following pages. 


\begin{tabular}{|c|c|c|c|c|c|c|}
\hline Name & \multicolumn{6}{|c|}{ VBERRH - Error handling } \\
\hline Description & \multicolumn{6}{|c|}{$\begin{array}{l}\text { VBERRH logs an error and continues or quits according to the severity of the error. } \\
\text { VBERRH calls VDLOGE to report the error before deciding whether or not to } \\
\text { terminate. }\end{array}$} \\
\hline Syntax & \multicolumn{6}{|c|}{ CALL VBERRH (ERRNUM, ERRSEV) } \\
\hline \multirow[t]{2}{*}{$\begin{array}{l}\text { Variables in } \\
\text { Call }\end{array}$} & $\begin{array}{l}\text { Name } \\
\text { ERRNUM }\end{array}$ & Input & $\begin{array}{l}\text { Type } \\
\text { integer }\end{array}$ & $\begin{array}{l}\text { Descr } \\
\text { Error } \\
\text { Value } \\
208 \\
\\
211 \\
212 \\
213 \\
401 \\
720 \\
721 \\
723 \\
\\
724 \\
725 \\
726 \\
727 \\
802 \\
901 \\
957\end{array}$ & $\begin{array}{c}\text { otion } \\
\text { number: } \\
\text { Severity } \\
5 \\
\\
6 \\
5 \\
5 \\
5 \\
5 \\
5 \\
\\
5 \\
5 \\
5 \\
5 \\
5 \\
5 \\
13 \\
13\end{array}$ & $\begin{array}{l}\text { Meaning } \\
\text { text contains an undefined } \\
\text { character } \\
\text { text string out of bounds } \\
\text { text string length }<1 \\
\text { text string length }>132 \\
\text { invalid attribute value } \\
\text { invalid justification sent to } \\
\text { VDINIT } \\
\text { invalid aspect sent to } \\
\text { VDINIT } \\
\text { invalid number of color table } \\
\text { indexes } \\
\text { invalid color table index } \\
\text { invalid color model } \\
\text { invalid device capability in- } \\
\text { dex } \\
\text { invalid color table entry } \\
\text { incorrect parameter count } \\
\text { job security level code is } \\
\text { undefined } \\
\text { attempt to run a classified } \\
\text { job using unclassified SVDI }\end{array}$ \\
\hline & ERRSEV & Input & integer & \multicolumn{3}{|c|}{$\begin{array}{cl}\text { Error } & \text { severity value: } \\
5 & \text { invalid parameter value } \\
6 & \text { invalid SVDI state } \\
10 & \text { operating system detected error } \\
11 & \text { security error - program may continue } \\
12 & \text { security error - may be fatal } \\
13 & \text { security error - fatal }\end{array}$} \\
\hline
\end{tabular}




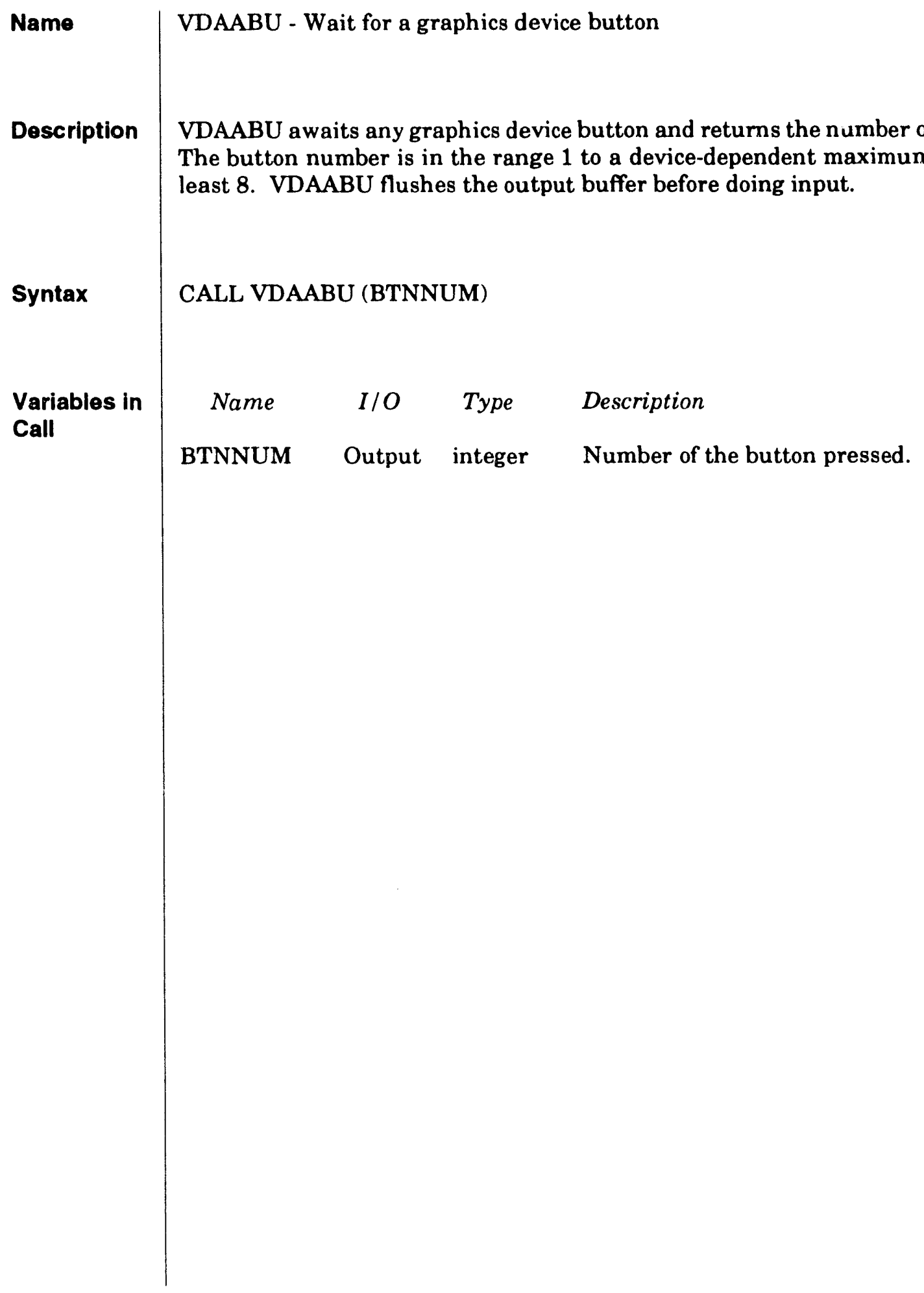




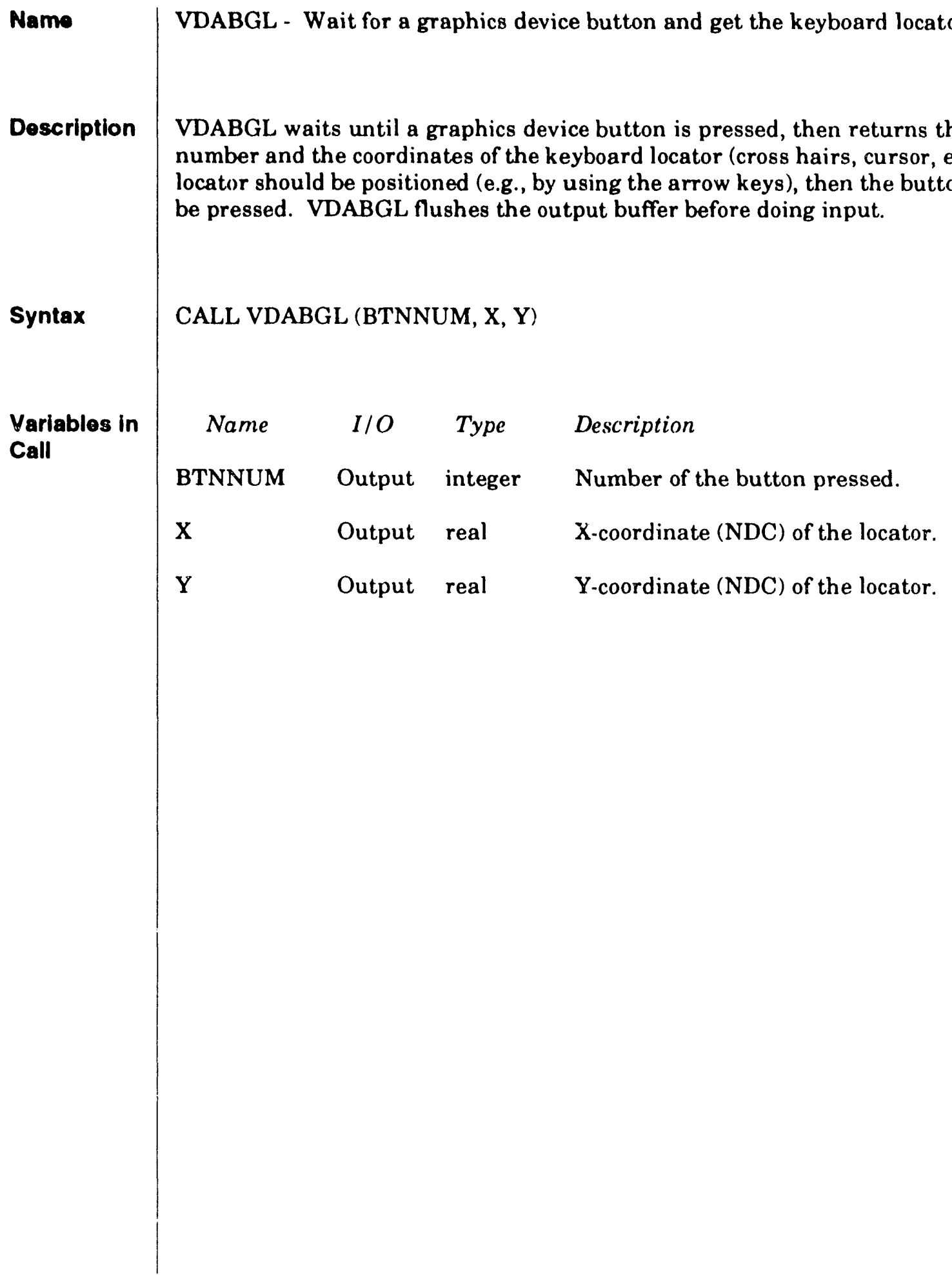


SVDI

Description of Routine Calls

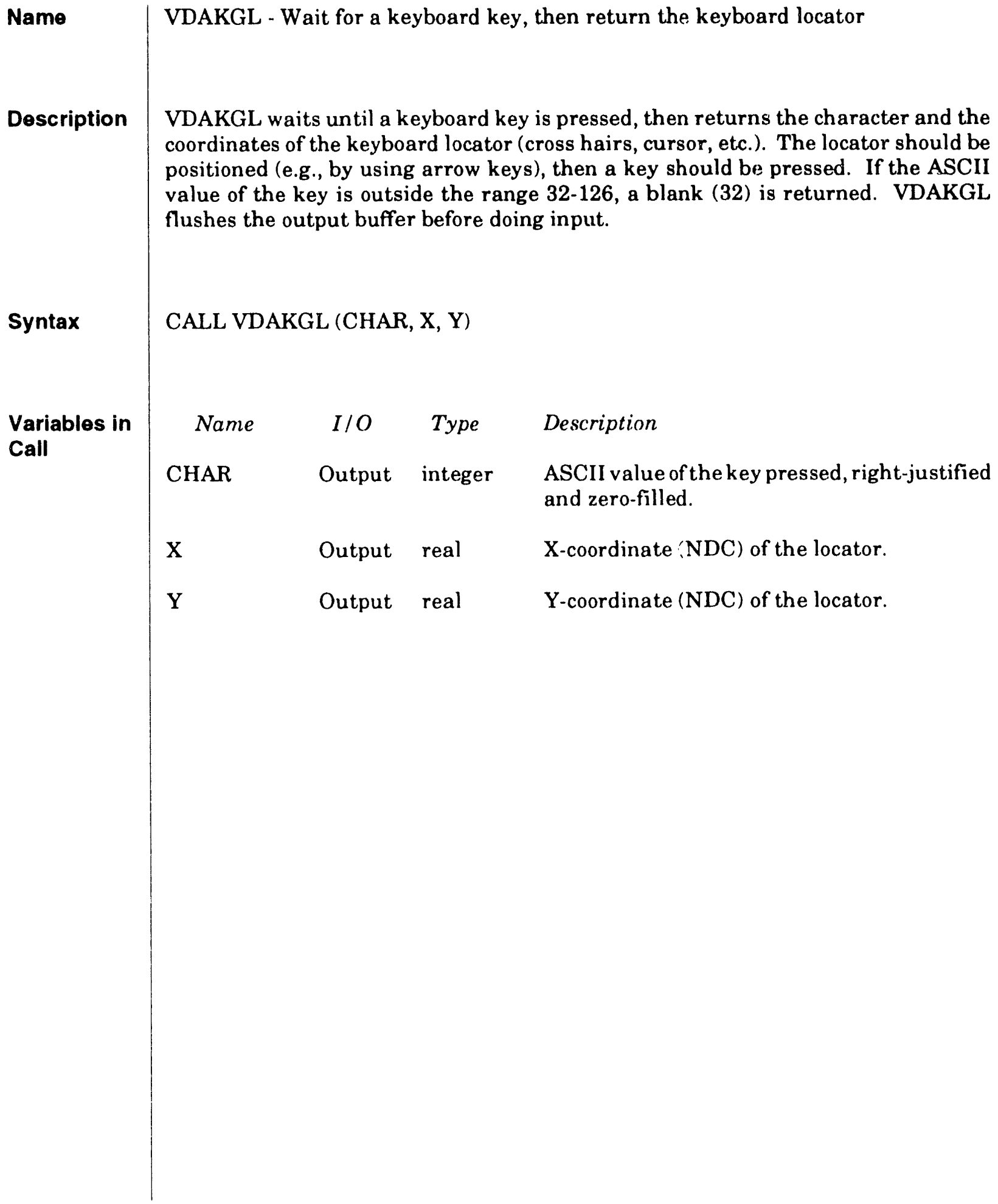




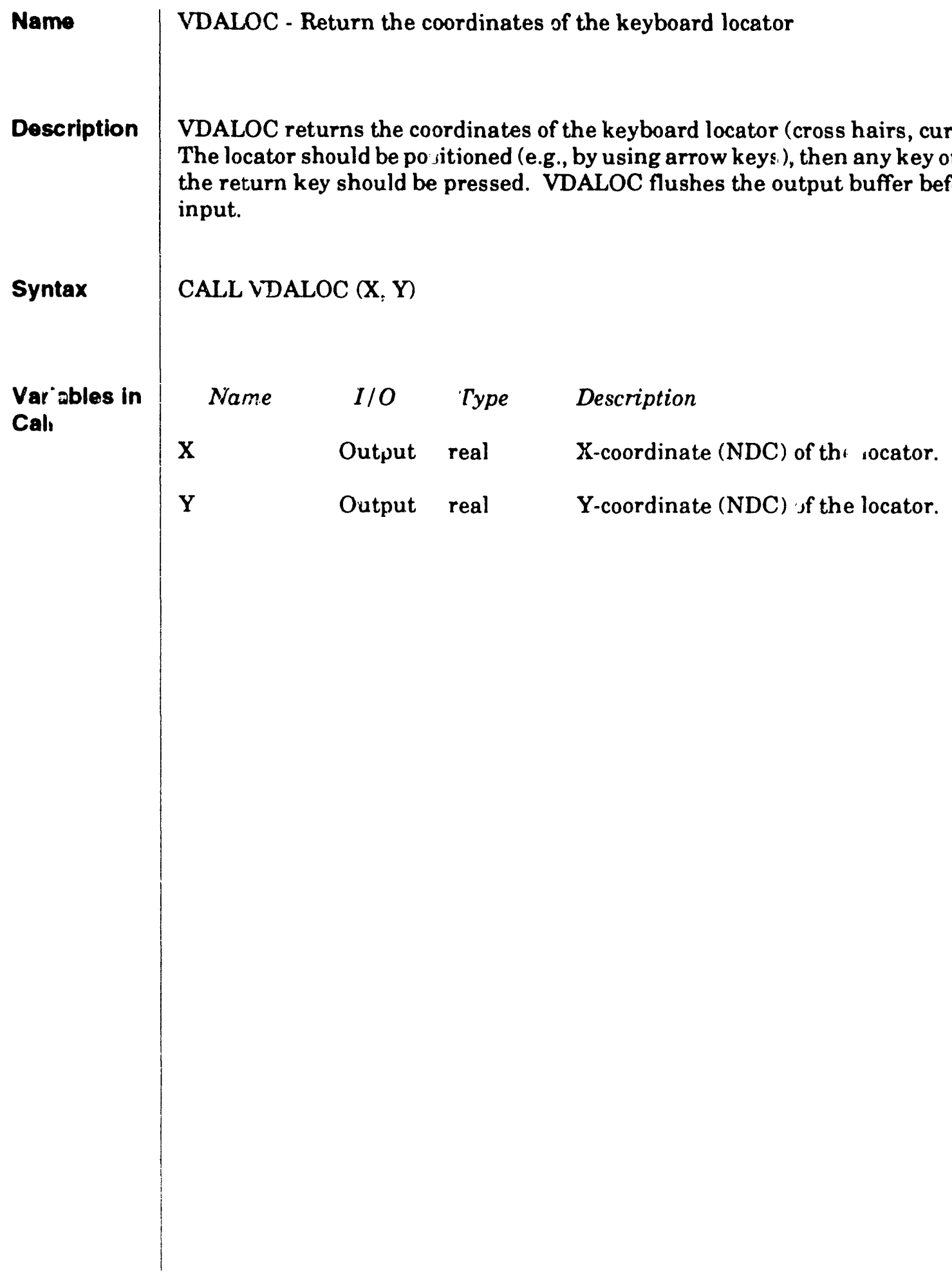




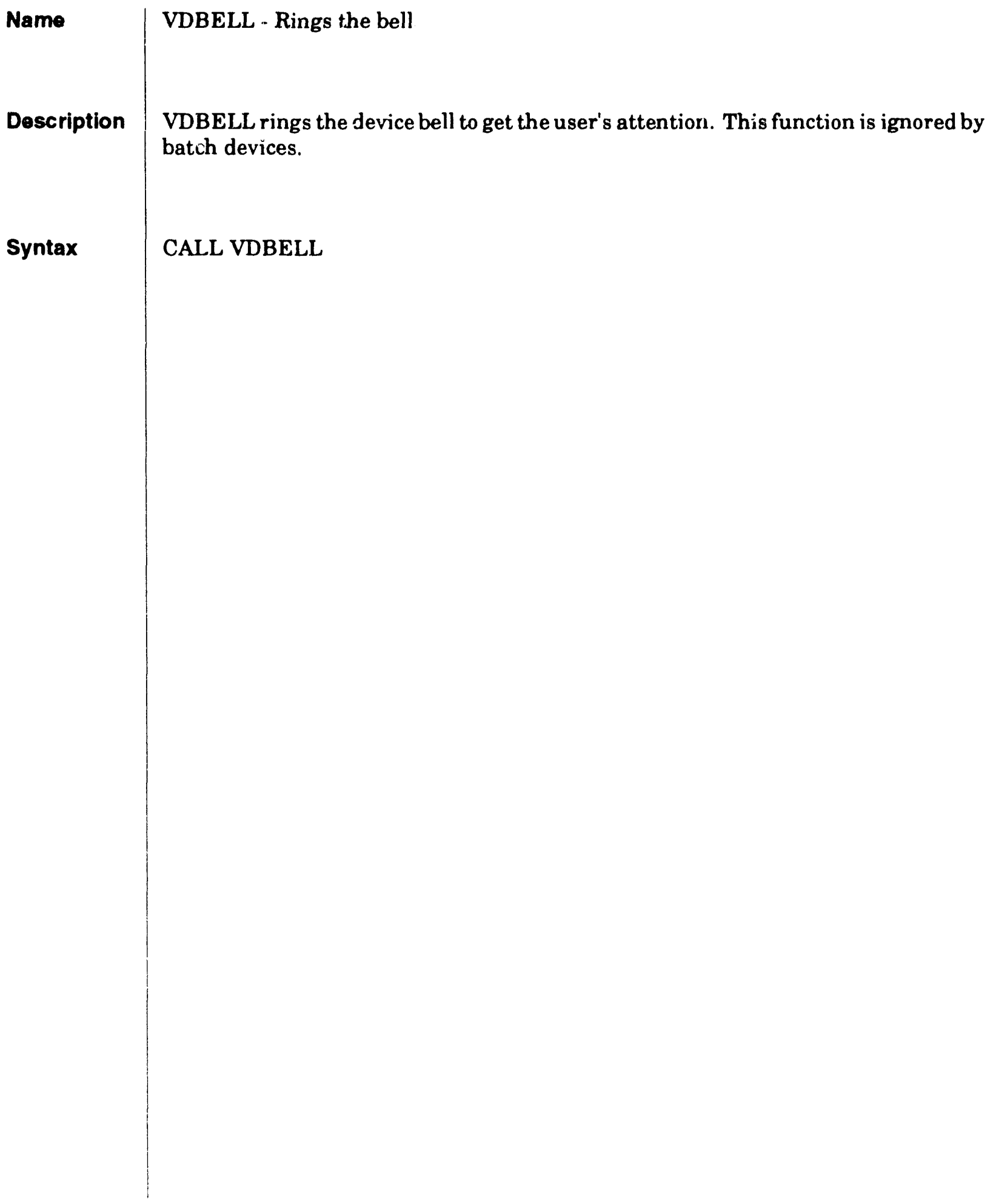




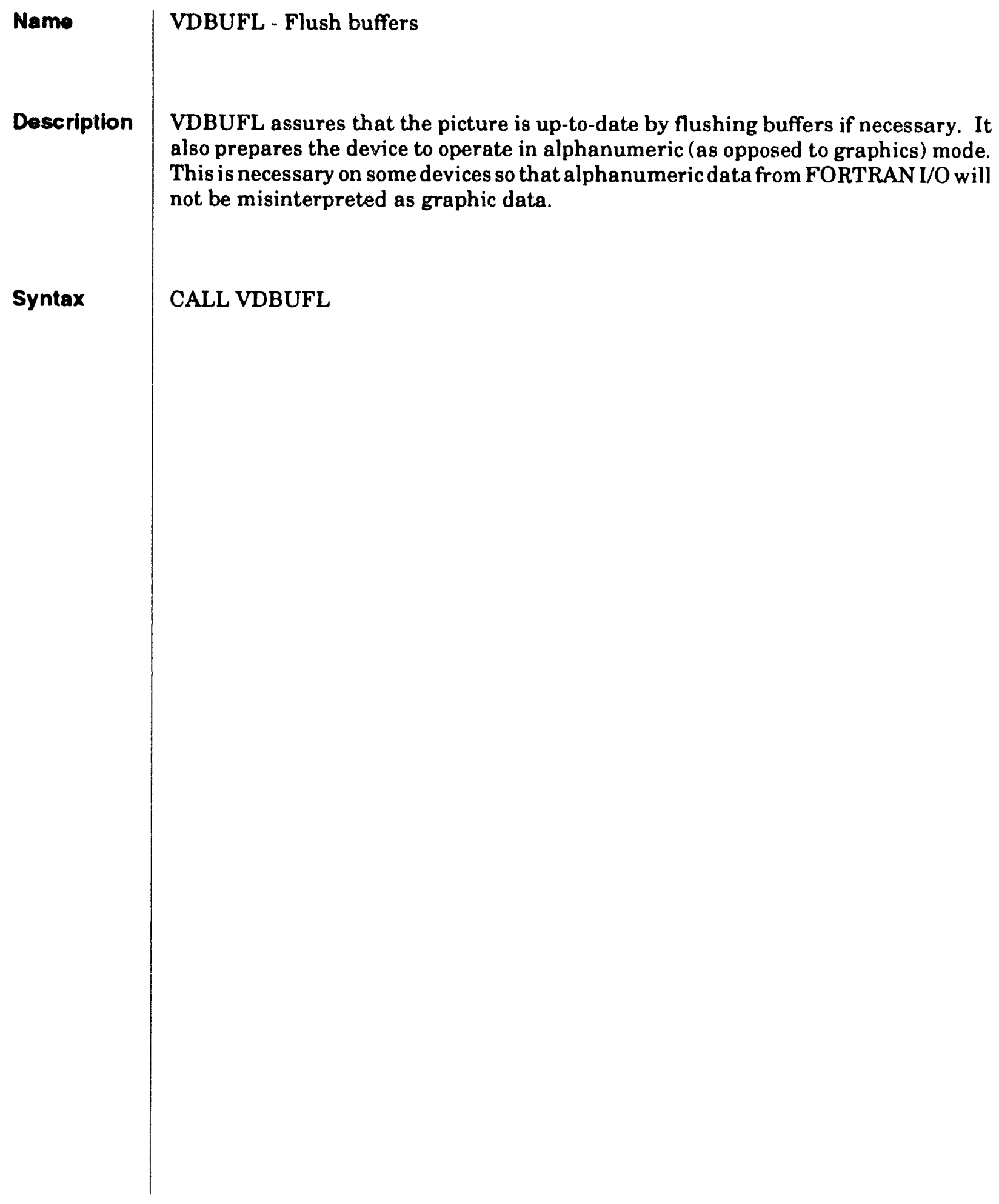


SVDI

Description of Routine Calls

VDESCP

Subroutine

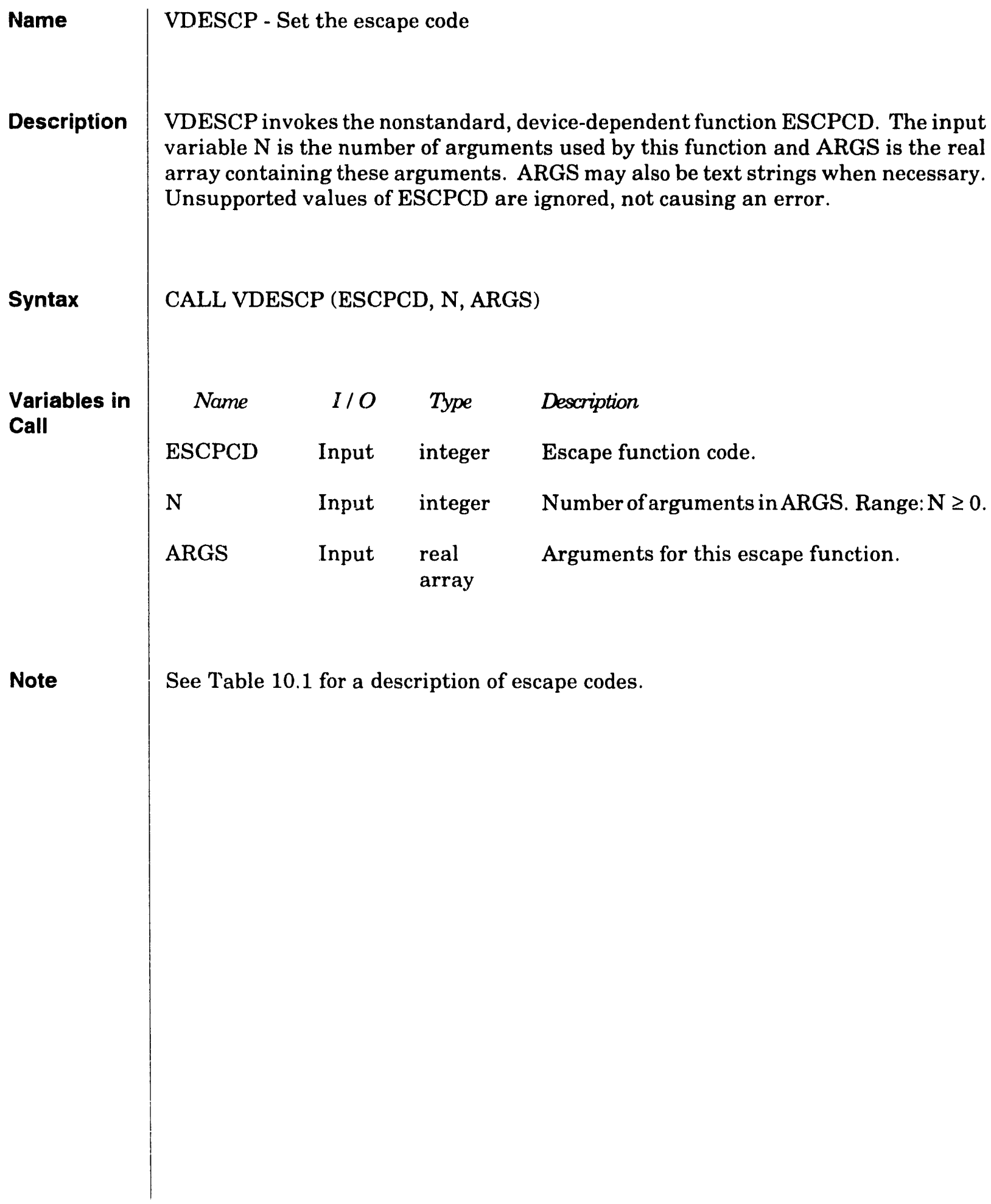




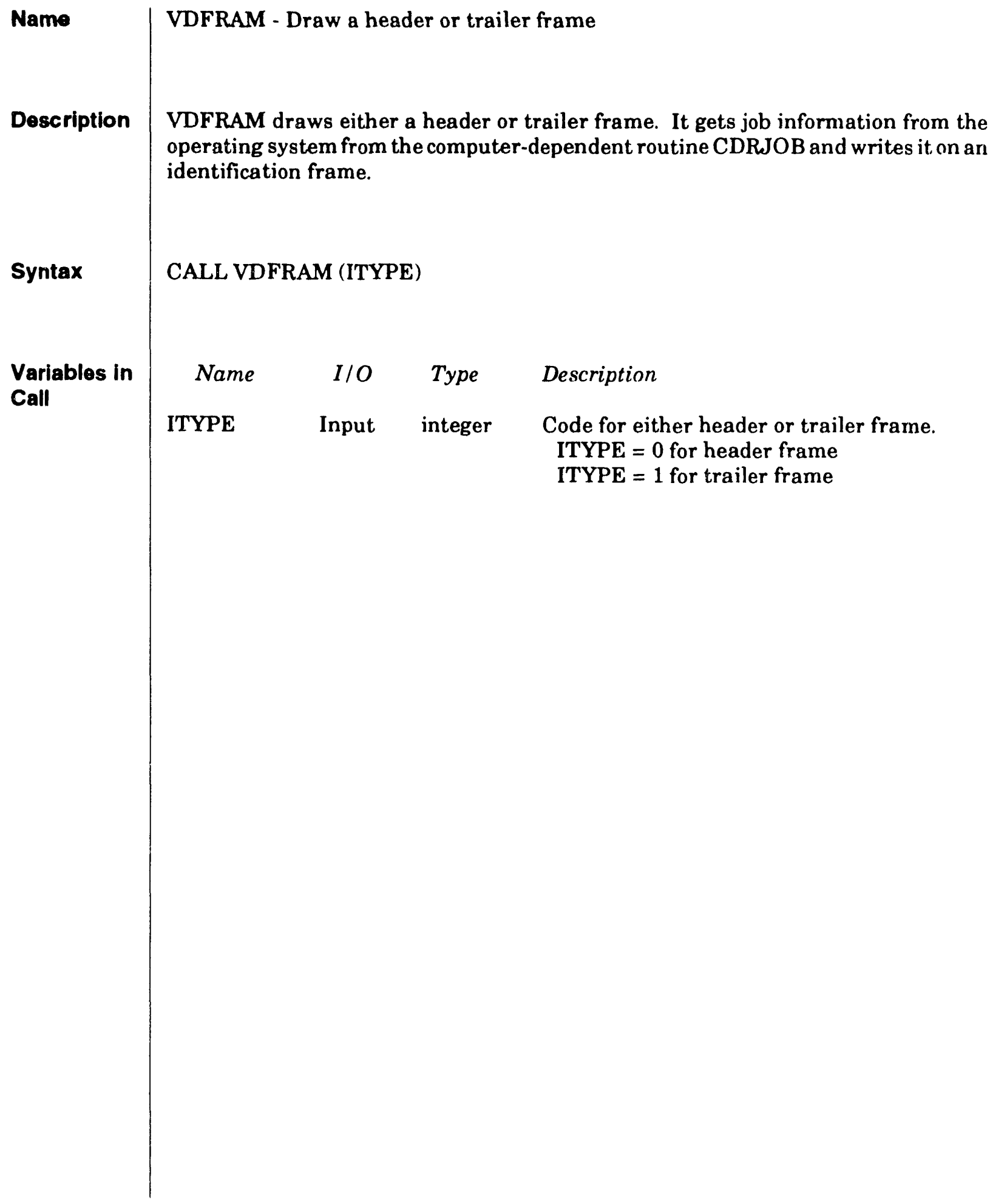


Name

Description

Syntax

Variables in Call

Comments

May 1993
VDINIT - Initialize SVDI

VDINIT initif izes SVDI. If only SVDI routines are used, this must be the first SVDI call (except cor calles to VDESCP). The color table, all attribute values, and the current position are set to the appropriate defaults for the device. All necessary input device initialization is done. The screen is cleared or paper advanced if necessary to guarantee a blank view surface for drawing.

CALL VDINIT (ASPECT, JUSTIF)

Name I/O Type Description

ASPECT Input real Specifies the ratio of the $x$-dimension to the $y$ dimension.

Range: ASPECT $>0.0$

Default: 0.0 (device dependent)

JUSTIF Input integer Justification of NDC space on the device.

Range: $0 \leq$ JUSTIF $\leq 9$

Default: 0 (device dependent)

The in put variable ASPECT specifies the ratio of the $x$-dimension to the $y$-dimension. Maximum NDC values (at least one of which will be 1.0) are computed to give the ASPECT specified. The default ASPECT $(0.0)$ is device-dependent and is equal to the aspect ratio of the physical device, except for variable aspect devices (such as drum plotters) which are assigned a default aspect of 1.0. The NDC rectangle is scaled until one dimension fills the corresponding dimension of the device.

The input variable JUSTIF determines where the rectangle is located on the device as shown below:

\section{9 \\ 456 \\ 123}

For example, JUSTIF $=7$ indicates that the NDC space will be upper-left justified on the device. 


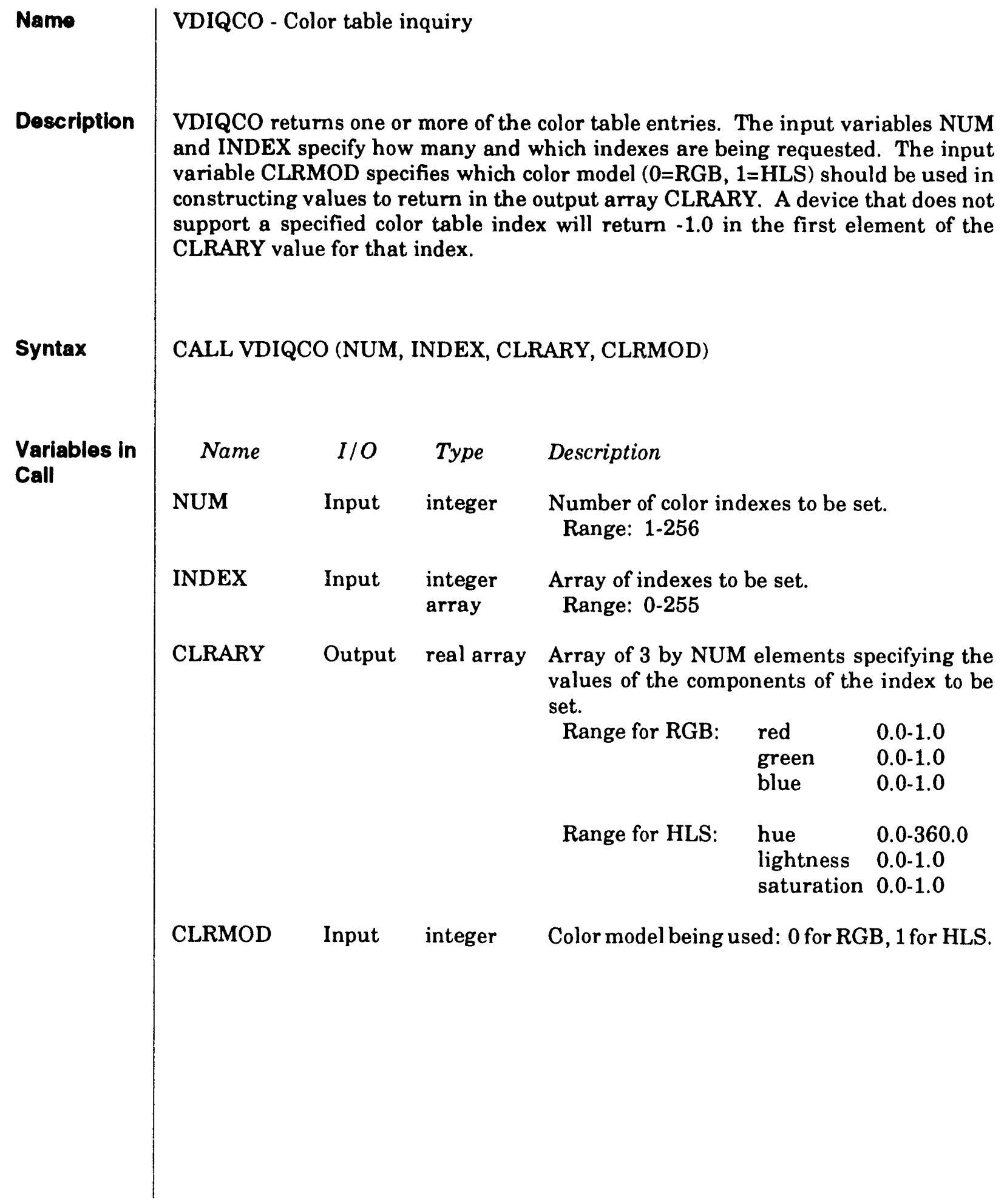




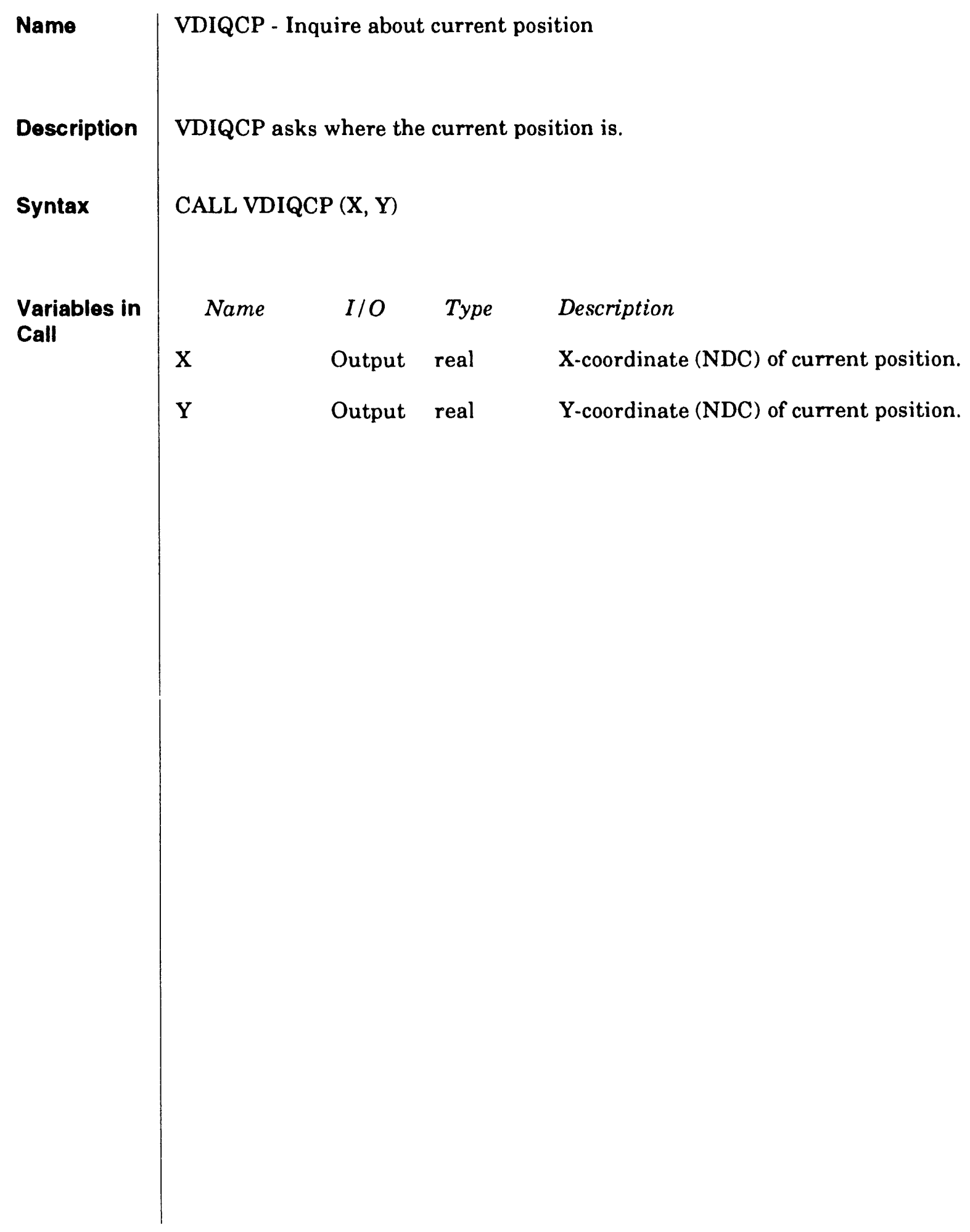




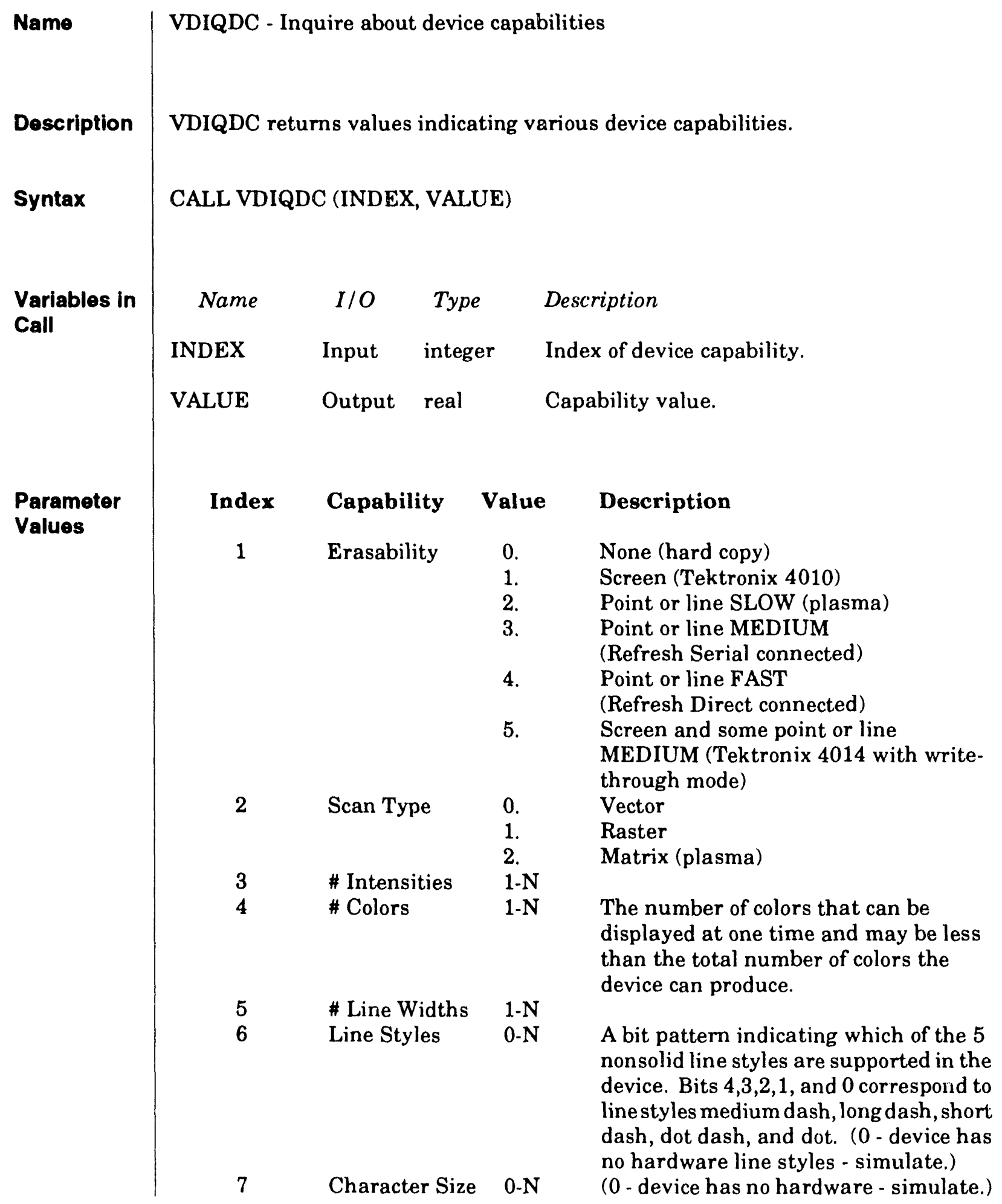




\begin{tabular}{|c|c|c|c|c|}
\hline $\begin{array}{l}\text { Parameter } \\
\text { Values } \\
\text { (continued) }\end{array}$ & $\begin{array}{l}18 \\
19 \\
20 \\
21 \\
22 \\
23\end{array}$ & $\begin{array}{l}\text { Input timing } \\
\text { X-dimension o } \\
\text { Y-dimension o } \\
\text { X-dimension o } \\
\text { if this dimensi } \\
\text { equal to the y- } \\
\text { Y-dimension o } \\
\text { Smallest line } \\
\text { Smallest poin } \\
\text { Smallest char } \\
\text { Header and tr } \\
\text { Device identif } \\
\end{array}$ & 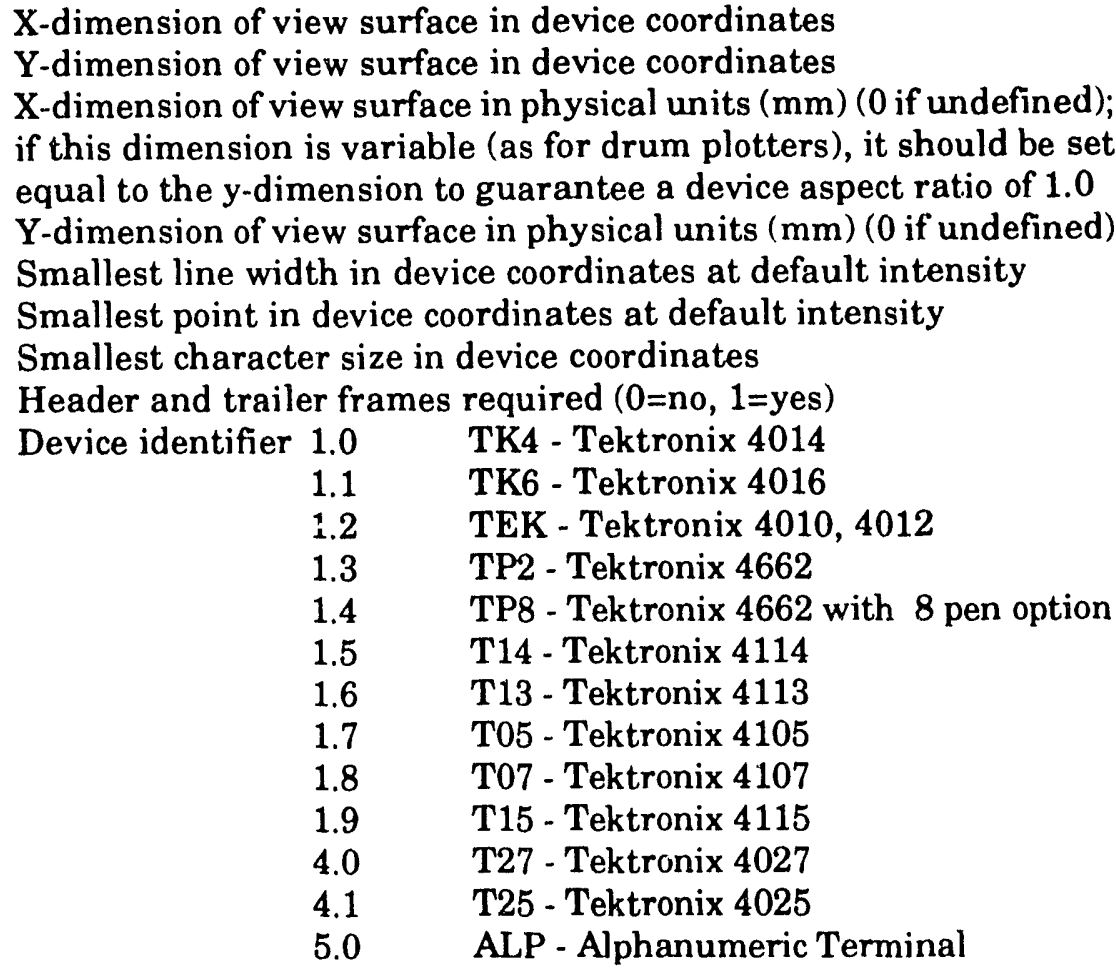 & $\begin{array}{l}\text { None } \\
\text { Synchronous only - program requests } \\
\text { input, then the user supplies it } \\
\text { Synchronous and asynchronous; } \\
\text { synchronous is the same as above; } \\
\text { asynchronous means the user can } \\
\text { provide input at any time; this input is } \\
\text { then saved by the system in an event } \\
\text { queue until the program calls for it } \\
\text { No timeout supported } \\
\text { Unreliable timing } \\
\text { Timeout with reliable timing } \\
\text { ce in device coordinates } \\
\text { ce in device coordinates } \\
\text { ce in physical units (mm) ( } 0 \text { if undefined); } \\
\text { le (as for drum plotters), it should be set } \\
\text { o guarantee a device aspect ratio of } 1.0 \\
\text { ce in physical units (mm) ( } 0 \text { if undefined) } \\
\text { ice coordinates at default intensity } \\
\text { oordinates at default intensity } \\
\text { device coordinates } \\
\text { required (0=no, } 1=y e s) \\
\text { TK4 - Tektronix } 4014 \\
\text { TK6 - Tektronix } 4016 \\
\text { TEK - Tektronix } 4010,4012 \\
\text { TP2 - Tektronix } 4662 \\
\text { TP8 - Tektronix } 4662 \text { with } 8 \text { pen option } \\
\text { T14 - Tektronix } 4114 \\
\text { T13 - Tektronix } 4113 \\
\text { T05 - Tektronix } 4105 \\
\text { T07 - Tektronix } 4107 \\
\text { T15 - Tektronix } 4115 \\
\text { T27 - Tektronix } 4027 \\
\text { T25 - Tektronix } 4025 \\
\text { ALP - Alphanumeric Terminal }\end{array}$ \\
\hline
\end{tabular}




\section{Parameter Values (continued)}

\section{Index Capability Value Description}

23 Device identifier (continued)

$\begin{array}{ll}\text { 8.0 } & \text { TST - Test Driver, Print VDI calls made } \\ 9.0 & \text { V25 - Digital VT125 } \\ 9.1 & \text { V40 - Digital VT240 } \\ 9.2 & \text { V41 - Digital VT241 } \\ 9.3 & \text { V34 - Digital VT340 } \\ 11.0 & \text { MET - SVDI Metafile } \\ 12.1 & \text { H75 - HP 7580 } \\ 12.3 & \text { H74 - HP 7475A } \\ 12.4 & \text { H50 - HP 7550 } \\ 21.0 & \text { QMS - QMS LASER PRINTER } \\ 21.1 & \text { QMS - QMS LASERGRAFIX - } \\ & \text { raster version } \\ 25 . & \text { T45 - Tektronix 4510 Rasterizer } \\ 27 . & \text { I10 - Imagen I10 laser printer } \\ 27.1 & \text { I30 - Imagen I30 laser printer } \\ 27.2 & \text { I30 - Imagen I30 - RASTER version } \\ 28 . & \text { BTK - Batch Tektronix Driver } \\ 29 . & \text { LN3 - DEC LN03 PLUS Laser printer }\end{array}$

Polygon support level
$0 . \quad$ no support
1. fills convex polygons
2. fills simple polygons (may be concave but not self-crossing)
3. full complex polygon fill support

Maximum number of points in a polygon (99999. if infinite)

Setable color table

$\begin{array}{ll}0 . & \text { no } \\ 1 . & \text { yes }\end{array}$

Device color palette size (1-N) - the number of different colors a device can produce (may be more than the device can display simultaneously) Direct color space size (0-N) - the number of colors available via direct RGB color specification (displayable simultaneously) Vector versus raster VDI
$0 . \quad$ SVDI
1. SVDI + Raster

Maximum character height (DC)

Maximum line width (DC)

Color versus monochrome (grayscale) device

$$
\begin{array}{ll}
0 . & \text { monochrome } \\
1 . & \text { color }
\end{array}
$$

Device pixel aspect - the ratio of the spacing of device pixels in $\mathbf{x}$ divided by the spacing in $y$ ( 1 for square pixels) 


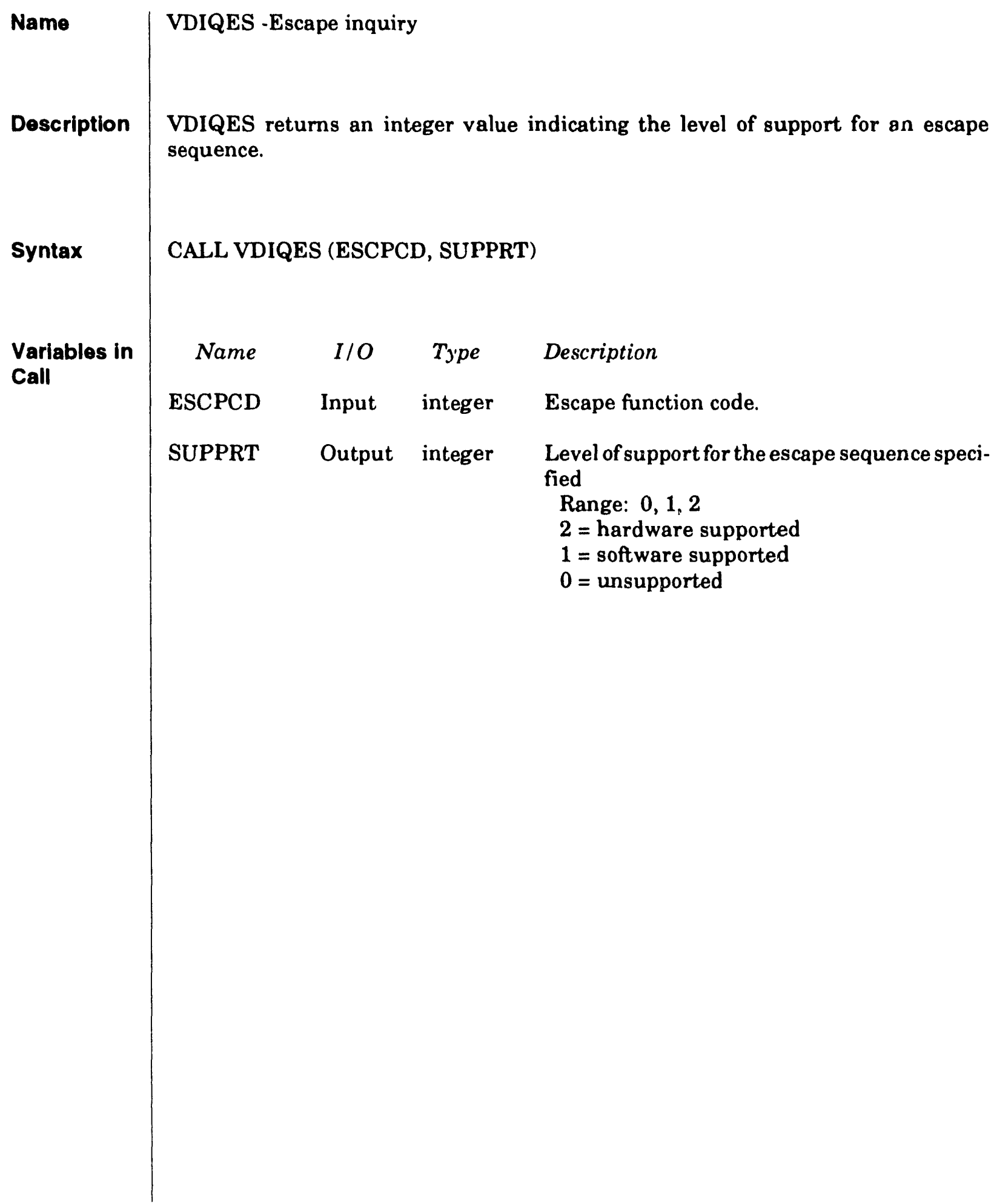




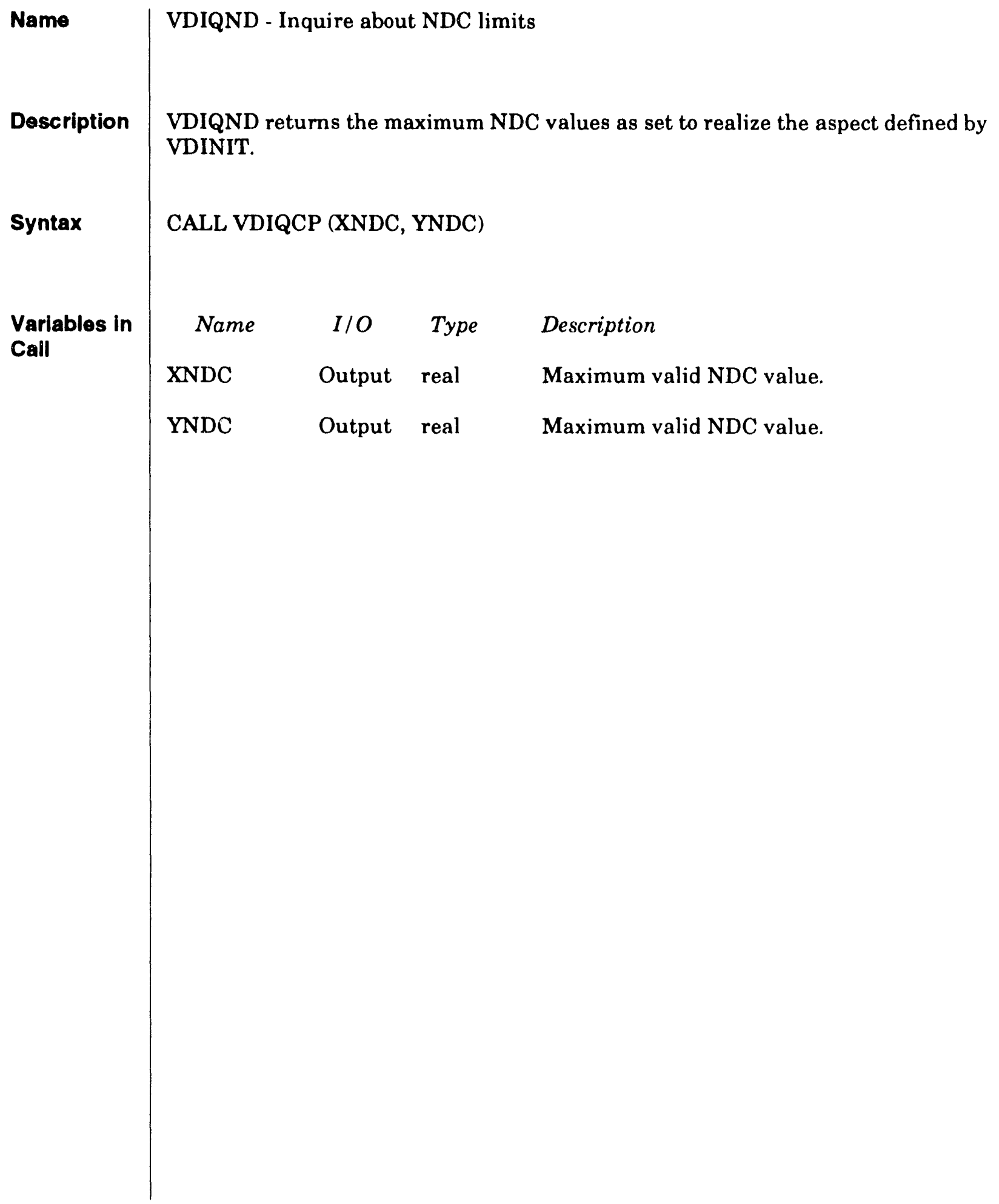




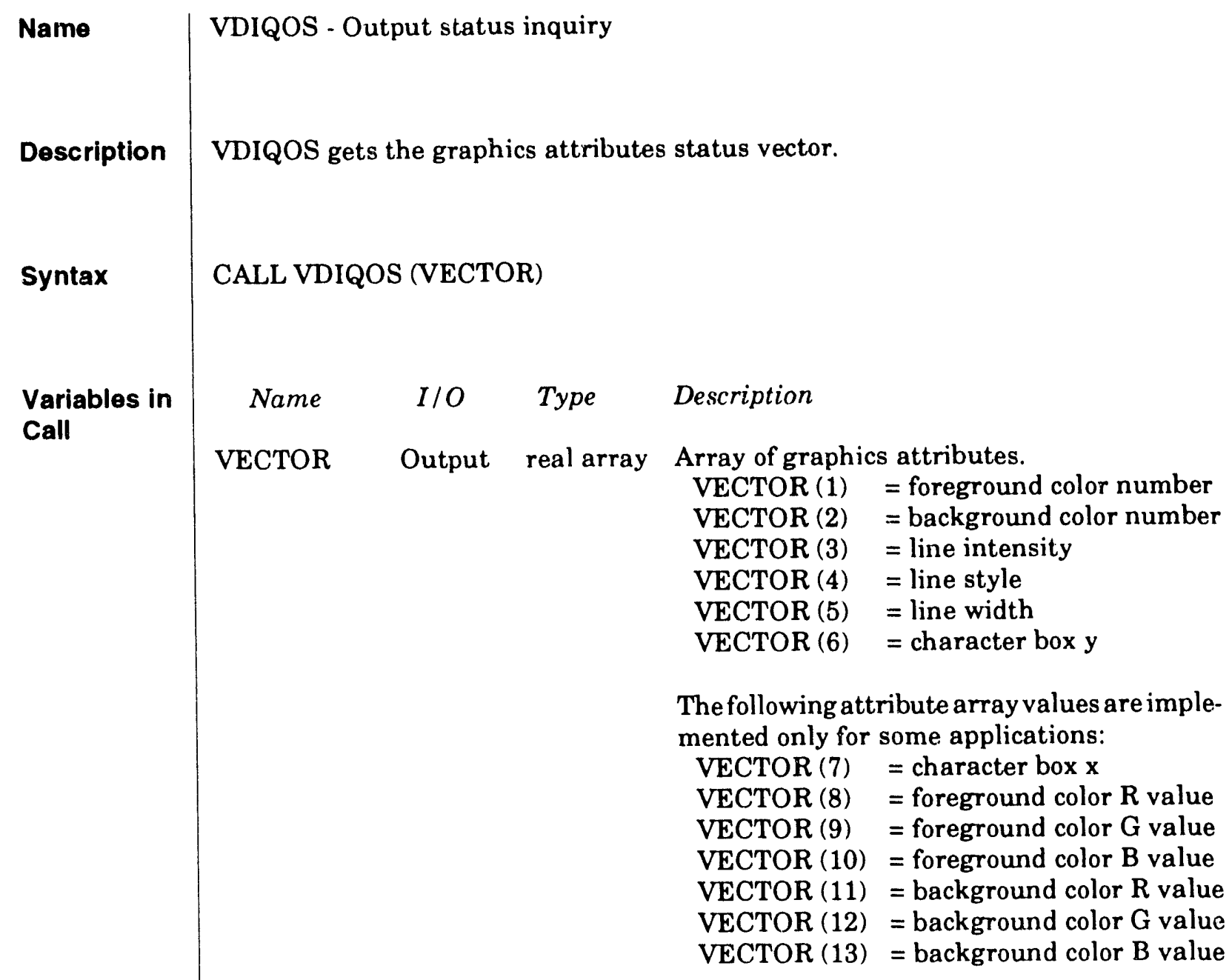




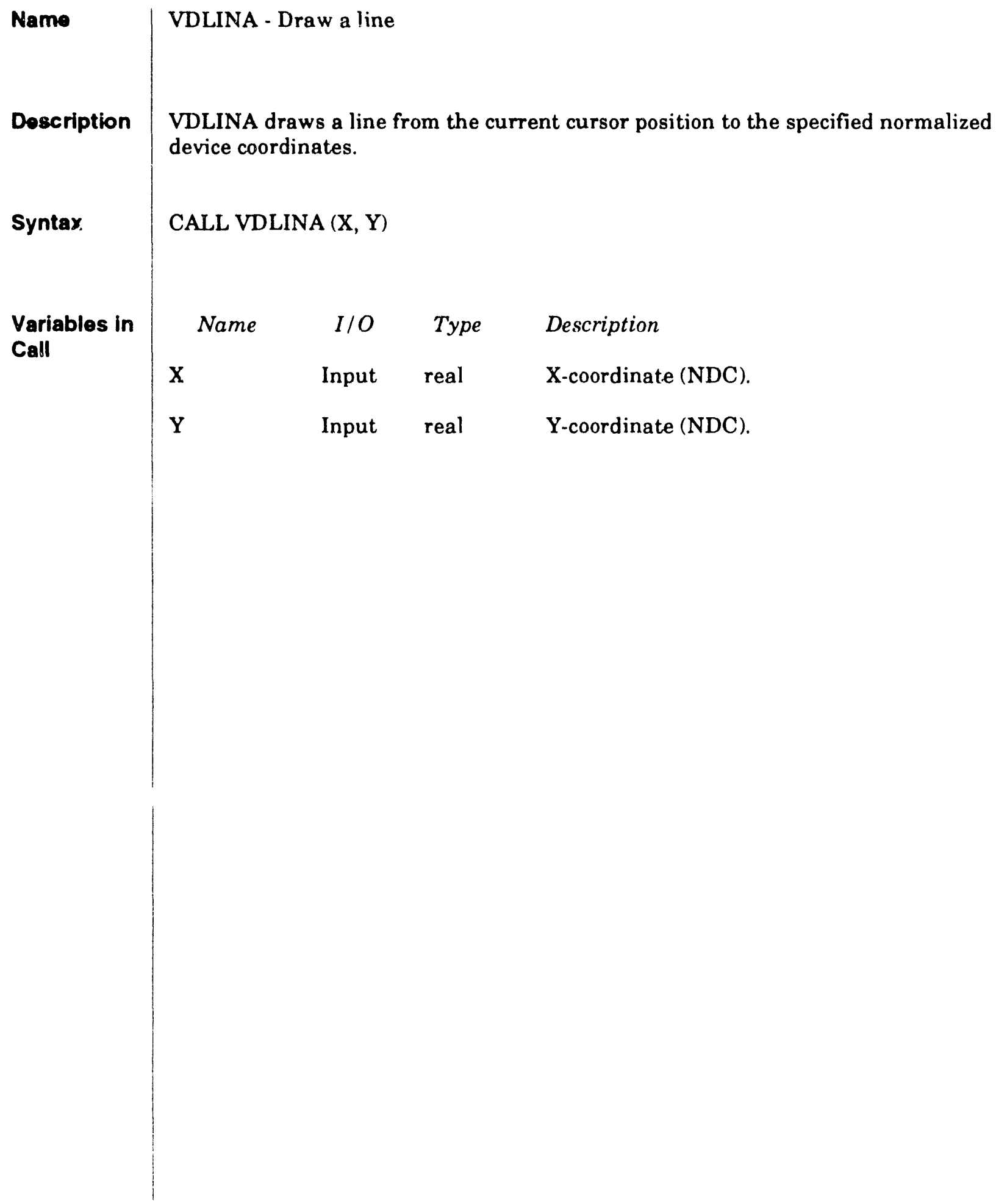




\begin{tabular}{|c|c|c|}
\hline Name & \multicolumn{2}{|c|}{ VDLOGE - Log an error } \\
\hline Description & \multicolumn{2}{|c|}{ VDLOGE logs an error an } \\
\hline Syntax & \multicolumn{2}{|c|}{ CALL VDLOGE (ERRNU } \\
\hline Variables in & Name & $I / O$ \\
\hline & ERRNUM & Input \\
\hline & ERRSEV & input \\
\hline $\begin{array}{l}\text { Parameter } \\
\text { Values }\end{array}$ & $\begin{array}{r}\text { ERRNUM } \\
\text { Value }\end{array}$ & Severity \\
\hline & 208 & 5 \\
\hline & 211 & 6 \\
\hline & 212 & 5 \\
\hline & 213 & 5 \\
\hline & 401 & 5 \\
\hline & 720 & 5 \\
\hline & 721 & 5 \\
\hline & 723 & 5 \\
\hline & 724 & 5 \\
\hline & 725 & 5 \\
\hline & 726 & 5 \\
\hline & 727 & 5 \\
\hline & 802 & 5 \\
\hline & 901 & 13 \\
\hline & 957 & 13 \\
\hline
\end{tabular}

\author{
Meaning \\ The text contains an undefined character \\ Text string out of bounds \\ Text string length less than 1 \\ Text string length exceeds 132 \\ Invalid attribute value \\ Invalid justification sent to VDINIT \\ Invalid aspect sent to VDINIT \\ Invalid number of color table indexes \\ Invalid color table index \\ Invalid color model \\ Invalid device capability index \\ Invalid color table entry \\ Incorrect parameter count \\ Job security level code is undefined \\ Attempt to run a classified job using unclassified \\ SVDI
}

\section{Meaning}

Invalid parameter value Invalid SVDI state Operating system detected error Security error - program may continue Security error - may be fatal Security error - fatal 


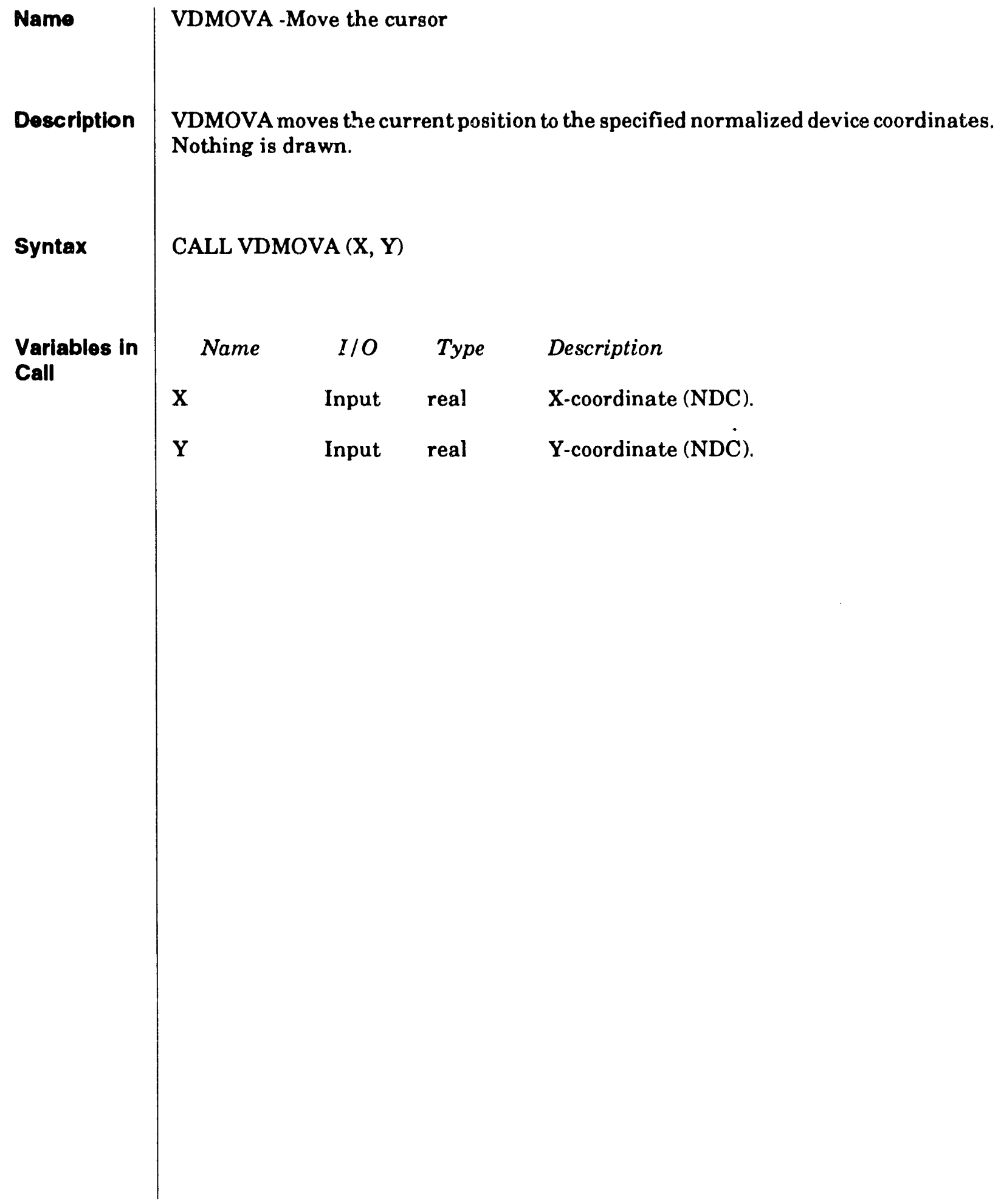


Name

Description

.

Syntax
VDNWPG - Advance to a new page

VDNWPG physically advances the medium or clears the screen, whichever is appropriate. It also floods the screen with the background color on devices that support this function. The current position is not changed.

\section{CALL VDNWPG}




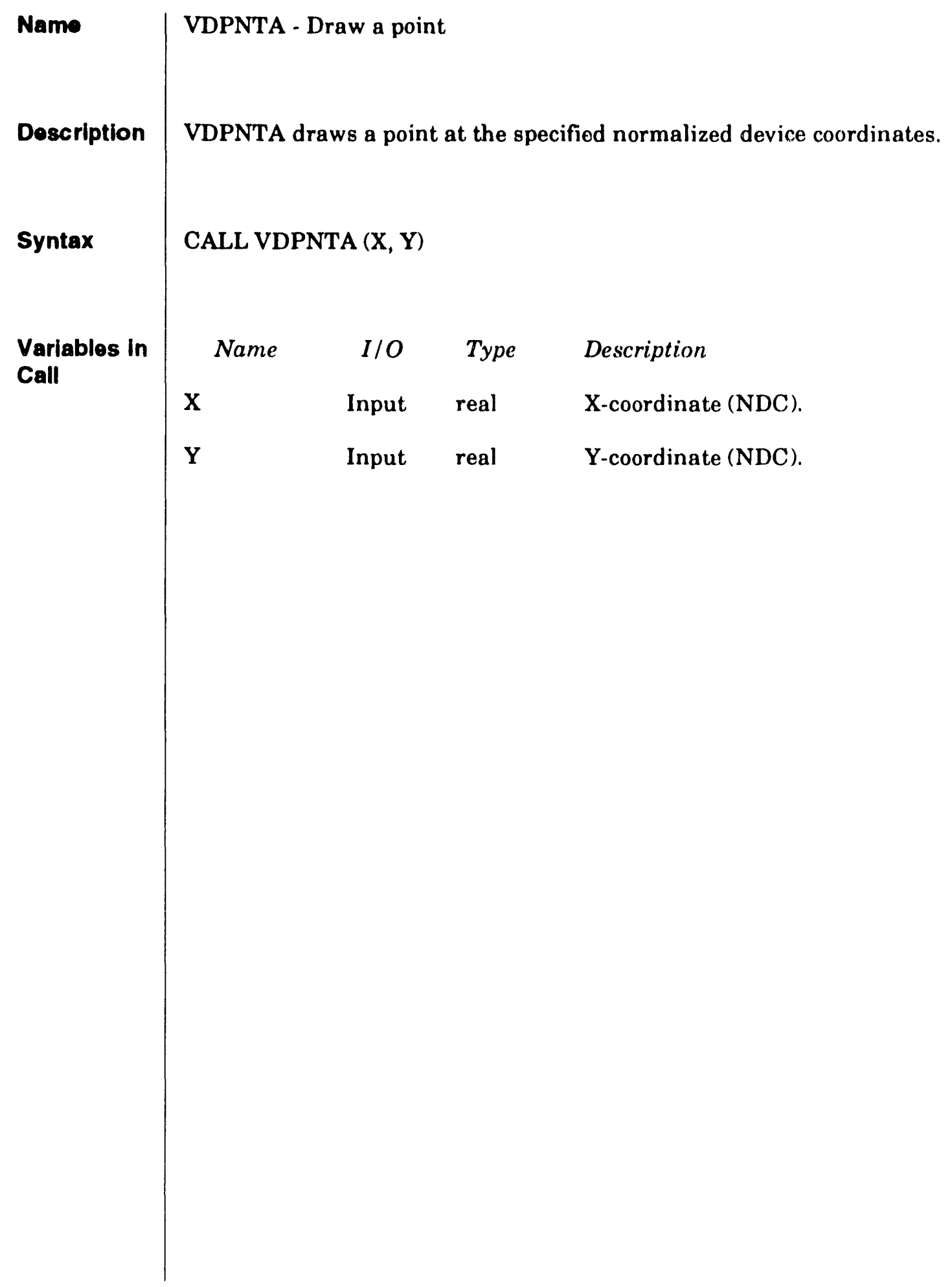




\begin{tabular}{|c|c|c|c|c|}
\hline Name & \multicolumn{4}{|c|}{ VDPOLY - Draw a filled polygon } \\
\hline Description & \multicolumn{4}{|c|}{$\begin{array}{l}\text { VDPOLY draws a polygon. On devices that support polygon fill, the polygon will be } \\
\text { filled with the current foreground color. VDI will close the polygon if the first and } \\
\text { last points are not the same. NPTS may not exceed } 508 \text {. }\end{array}$} \\
\hline Syntax & \multicolumn{4}{|c|}{ CALL VDPOLY (XARRAY, YARRAY, NPTS) } \\
\hline \multirow{4}{*}{$\begin{array}{l}\text { Variables in } \\
\text { Call }\end{array}$} & Name & $I / O$ & Type & Description \\
\hline & XARRAY & Input & $\begin{array}{l}\text { real } \\
\text { array }\end{array}$ & X-coordinate (NDC) array. \\
\hline & YARRAY & Input & $\begin{array}{l}\text { real } \\
\text { array }\end{array}$ & Y-coordinate (NDC) array. \\
\hline & NPTS & Input & integer & Number of points in (XARRAY, YARRAY) \\
\hline
\end{tabular}




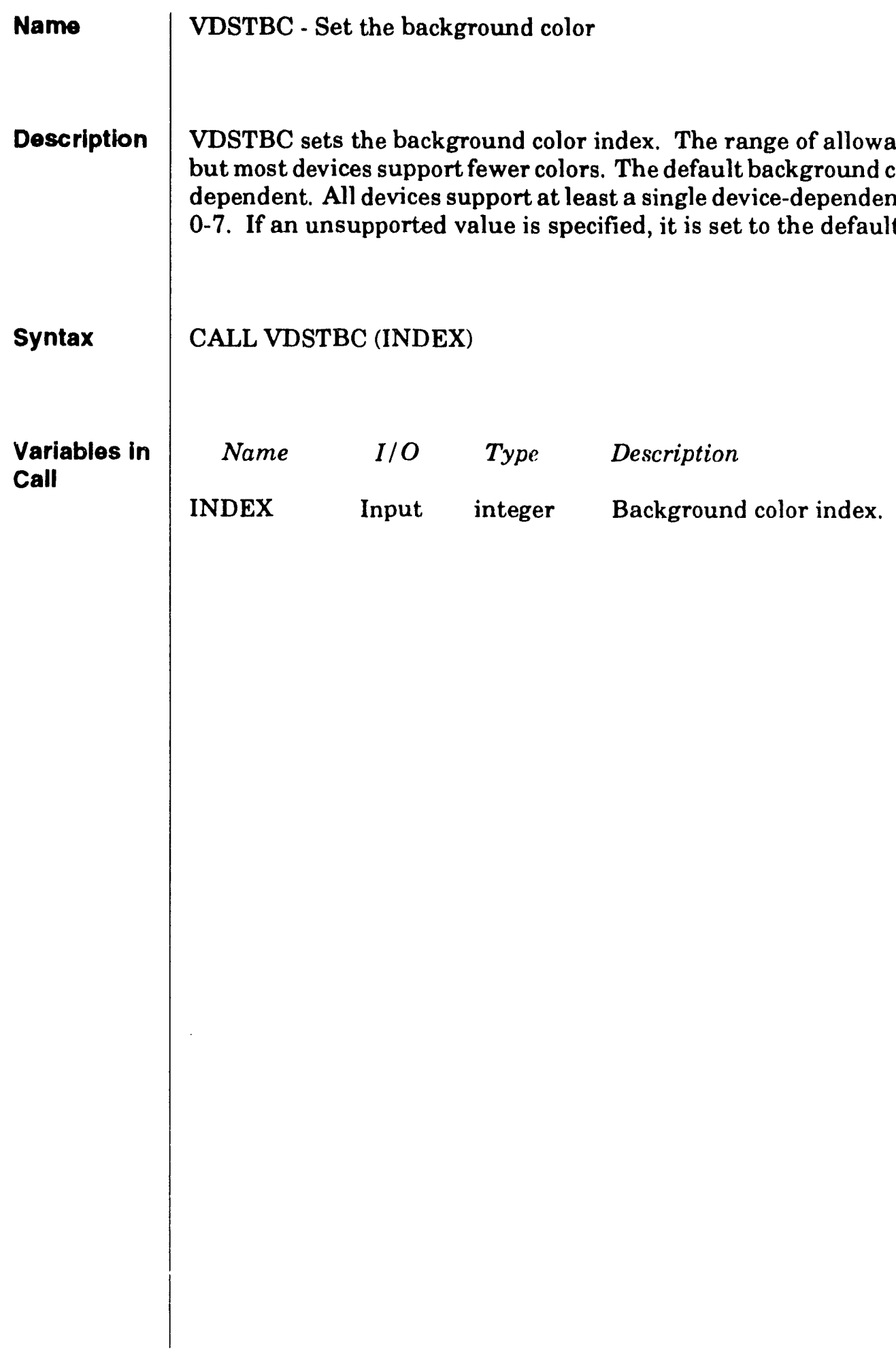




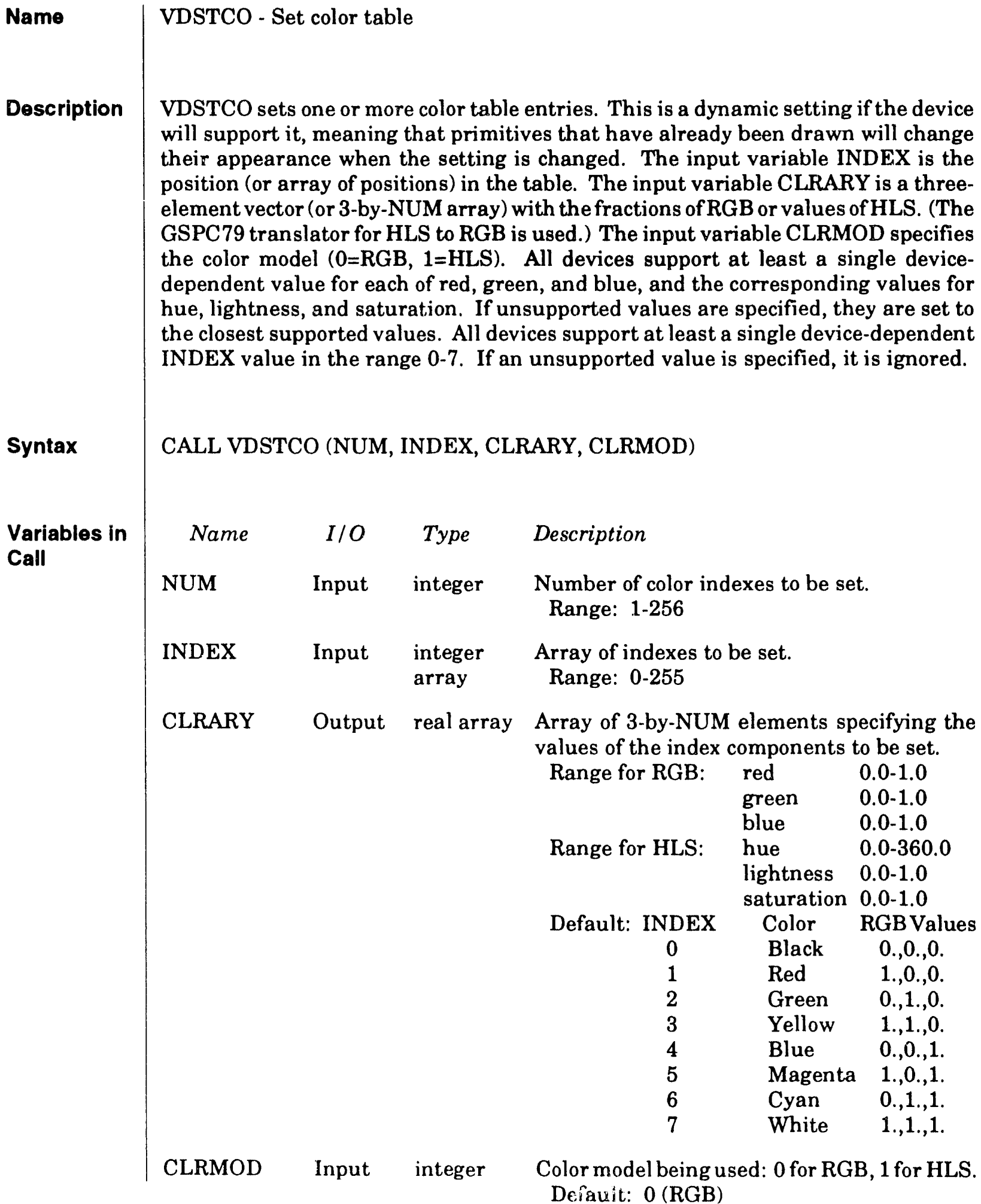




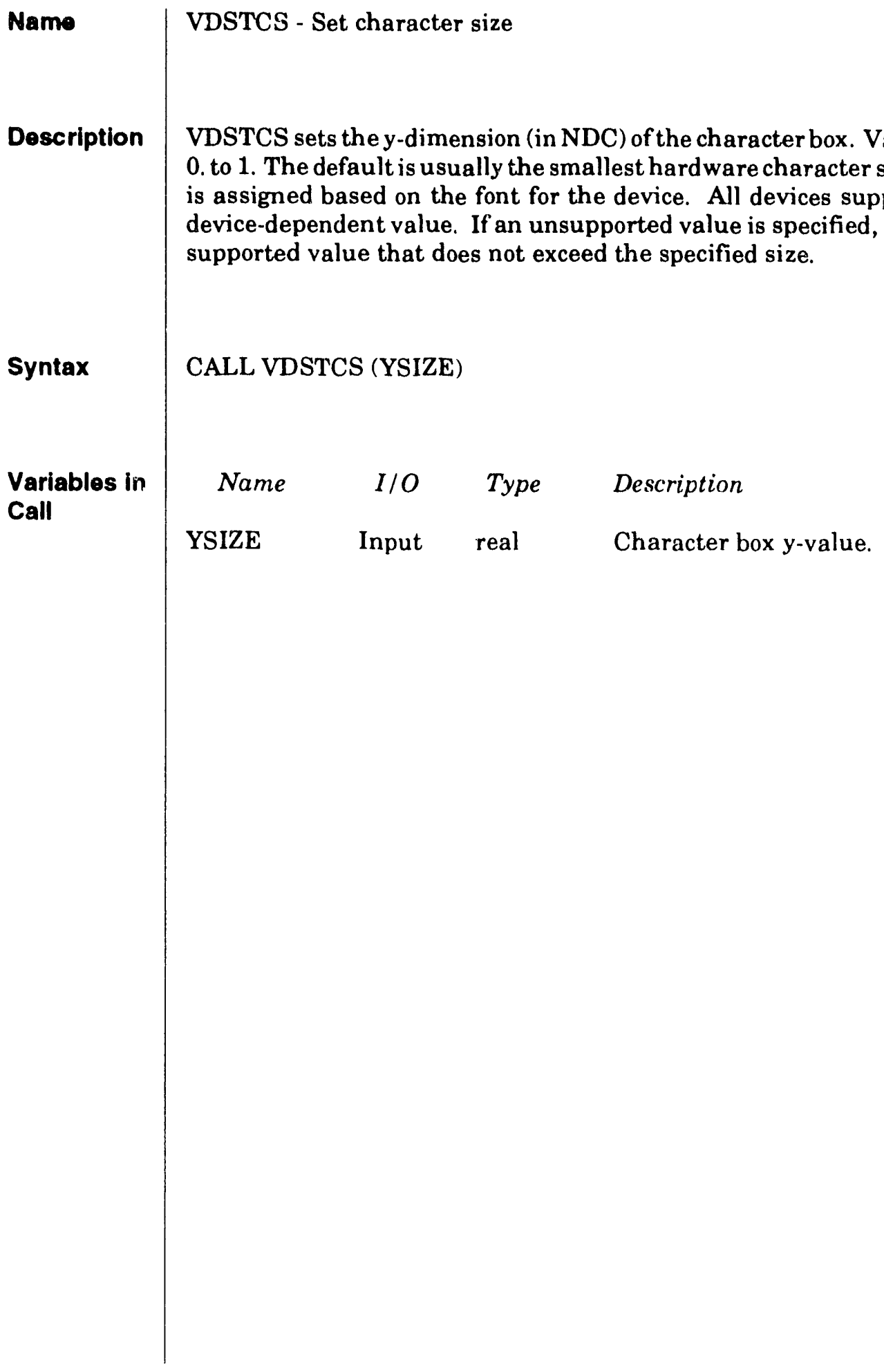




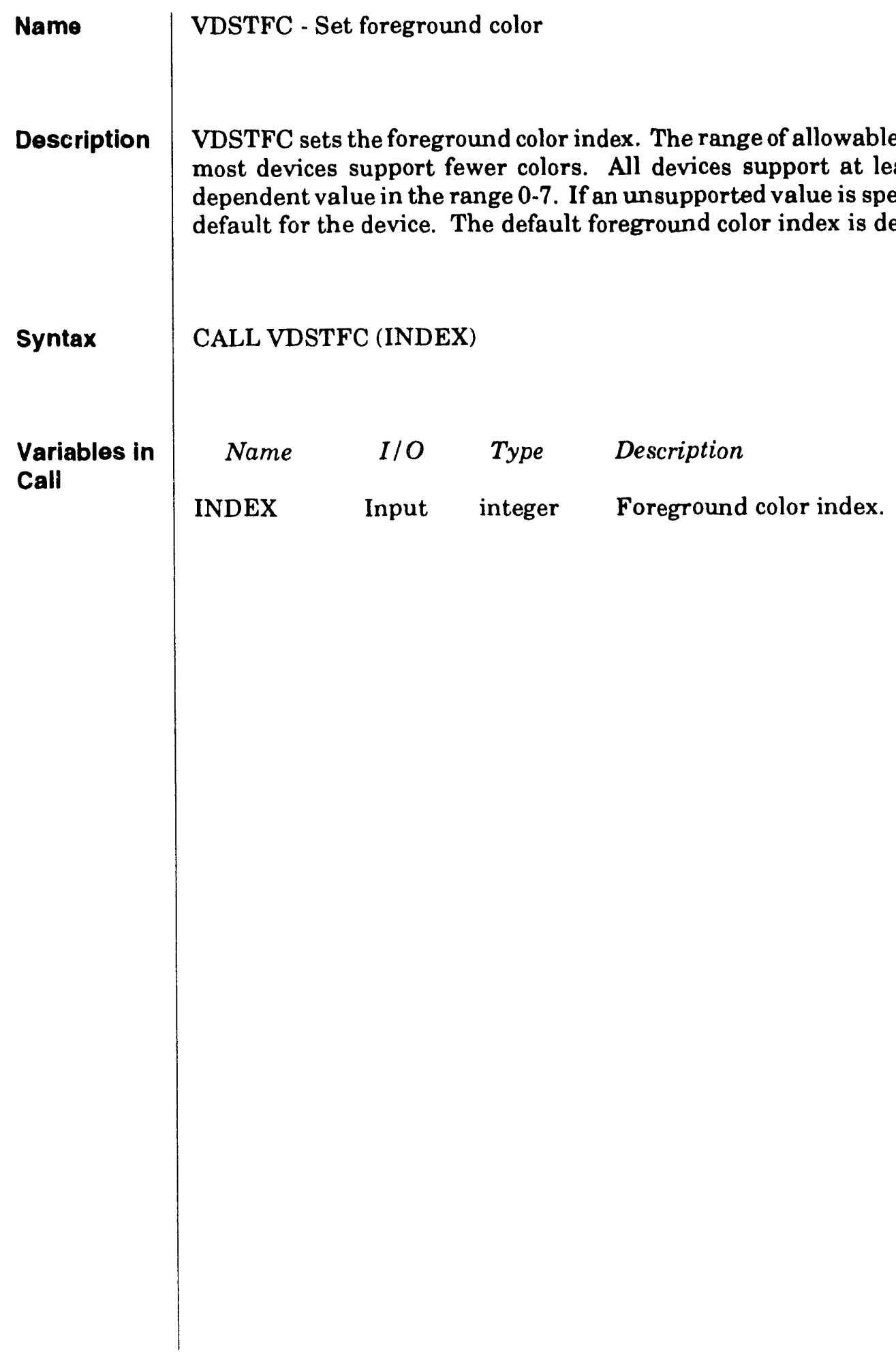




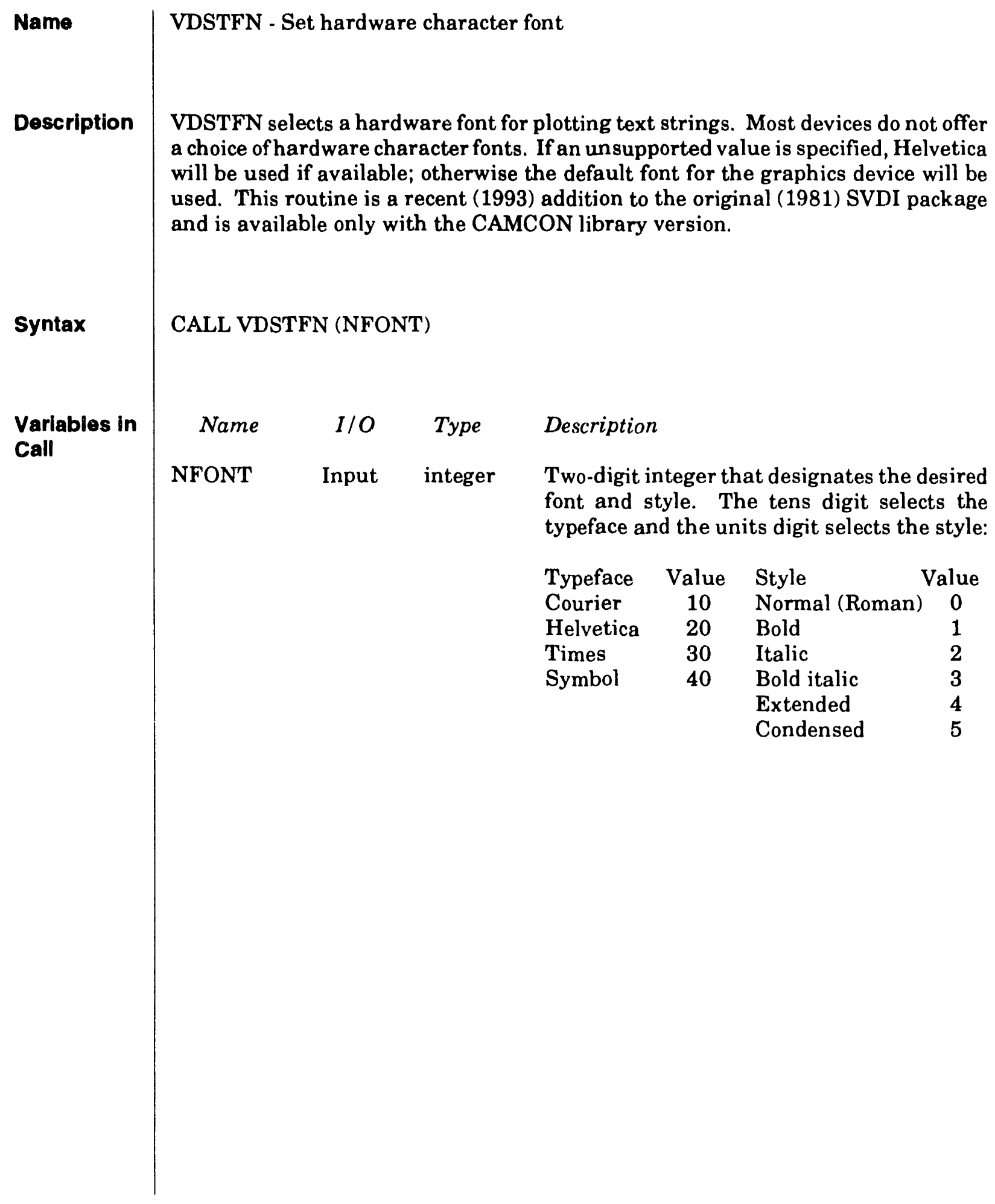




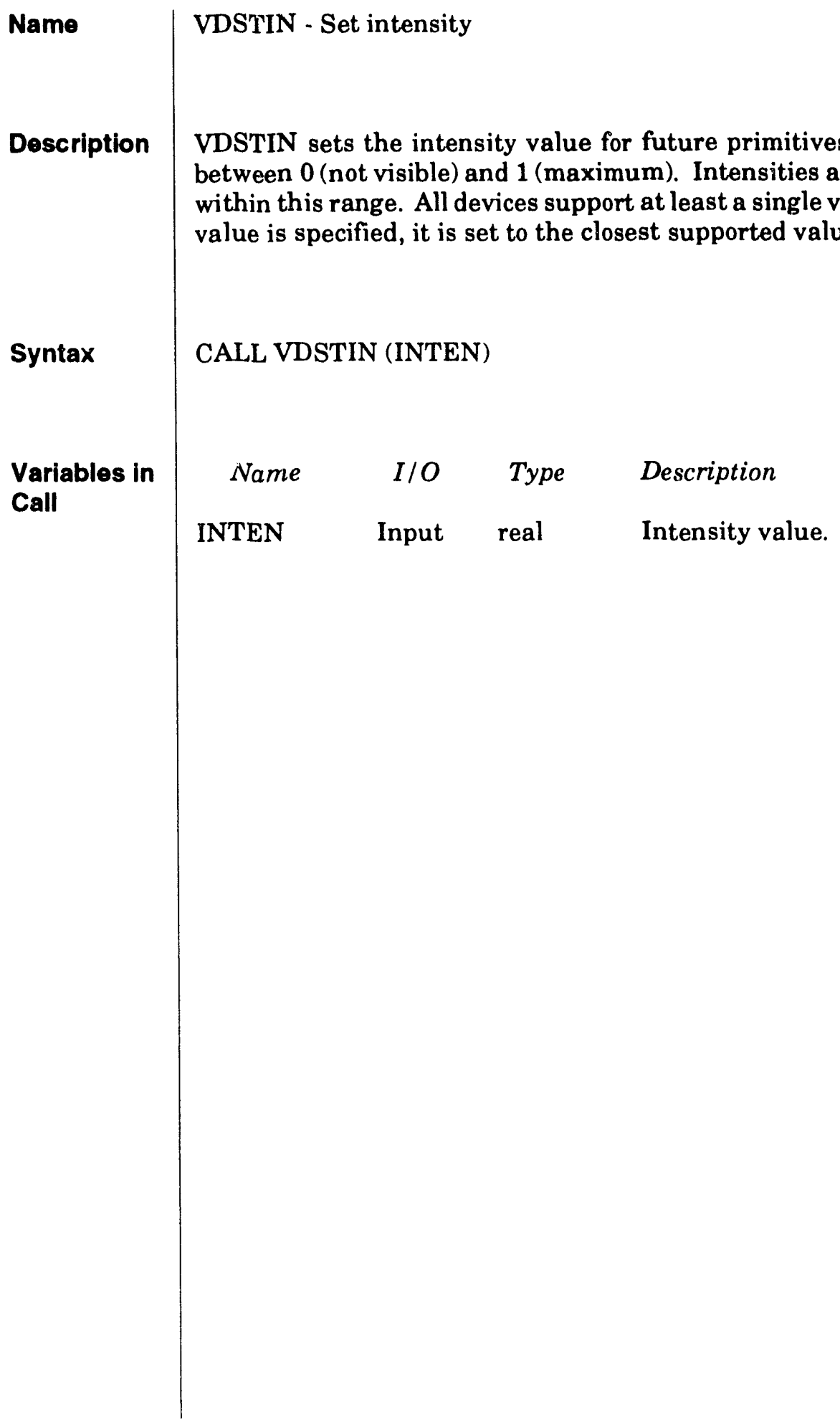




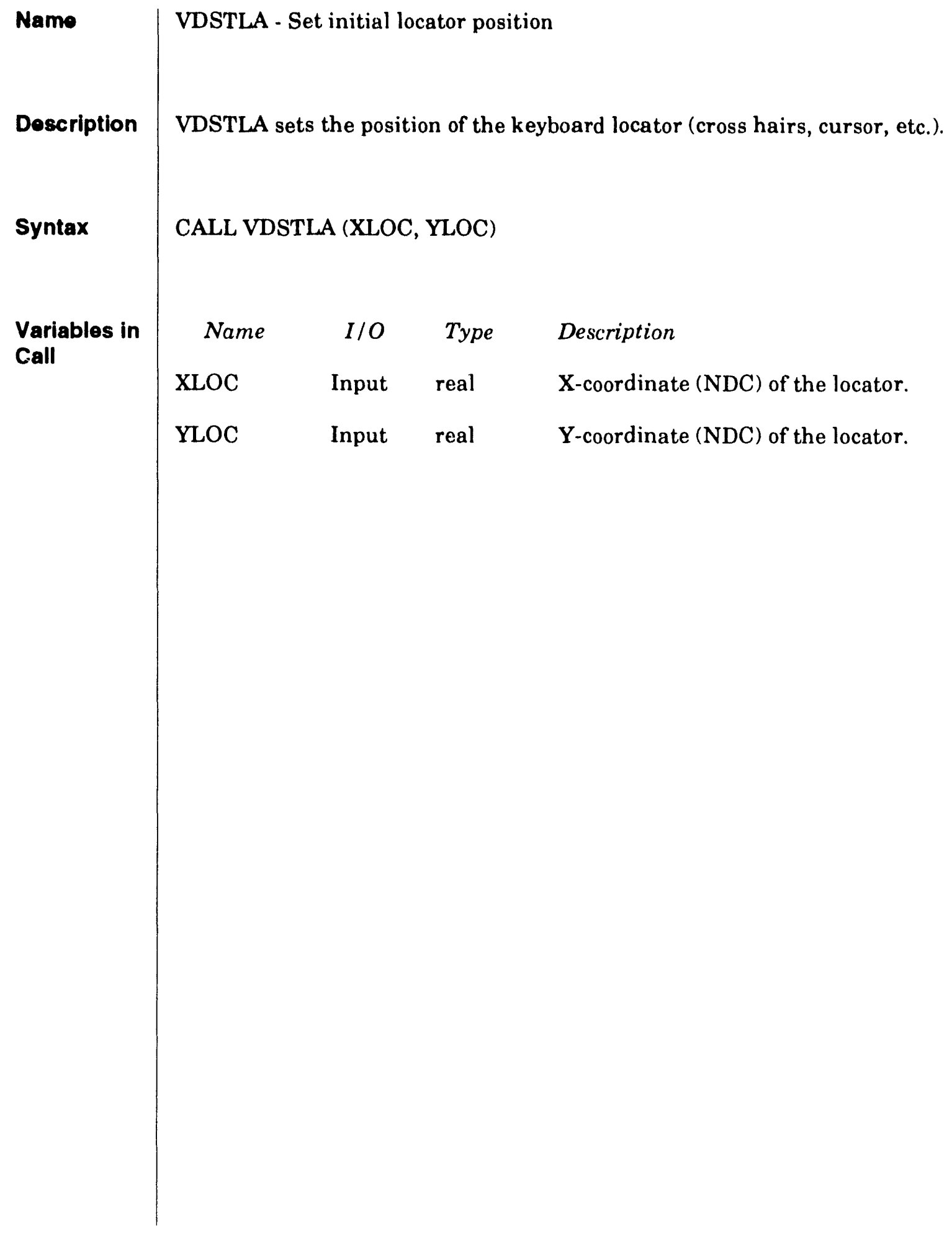


Description of Routine Calls

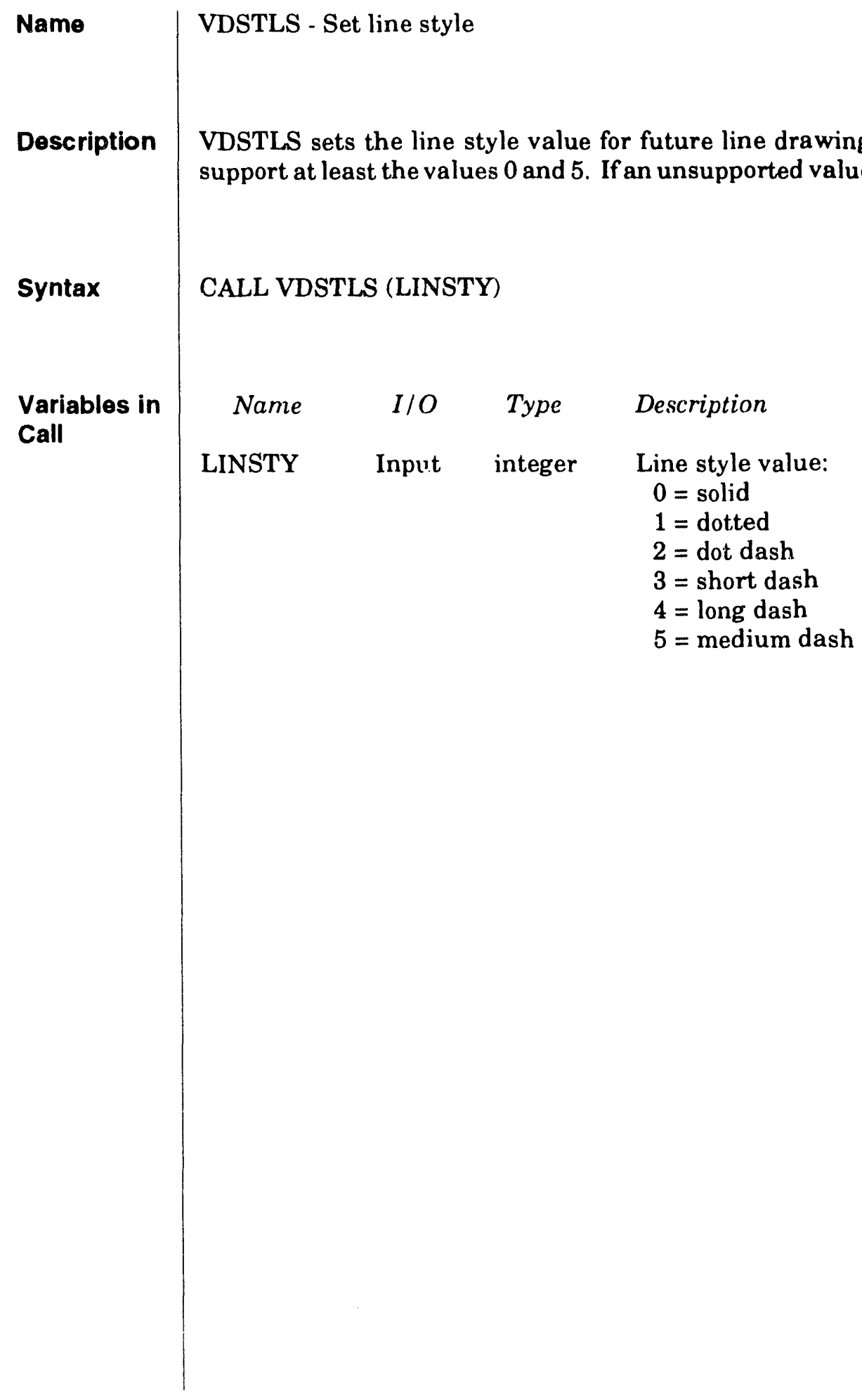




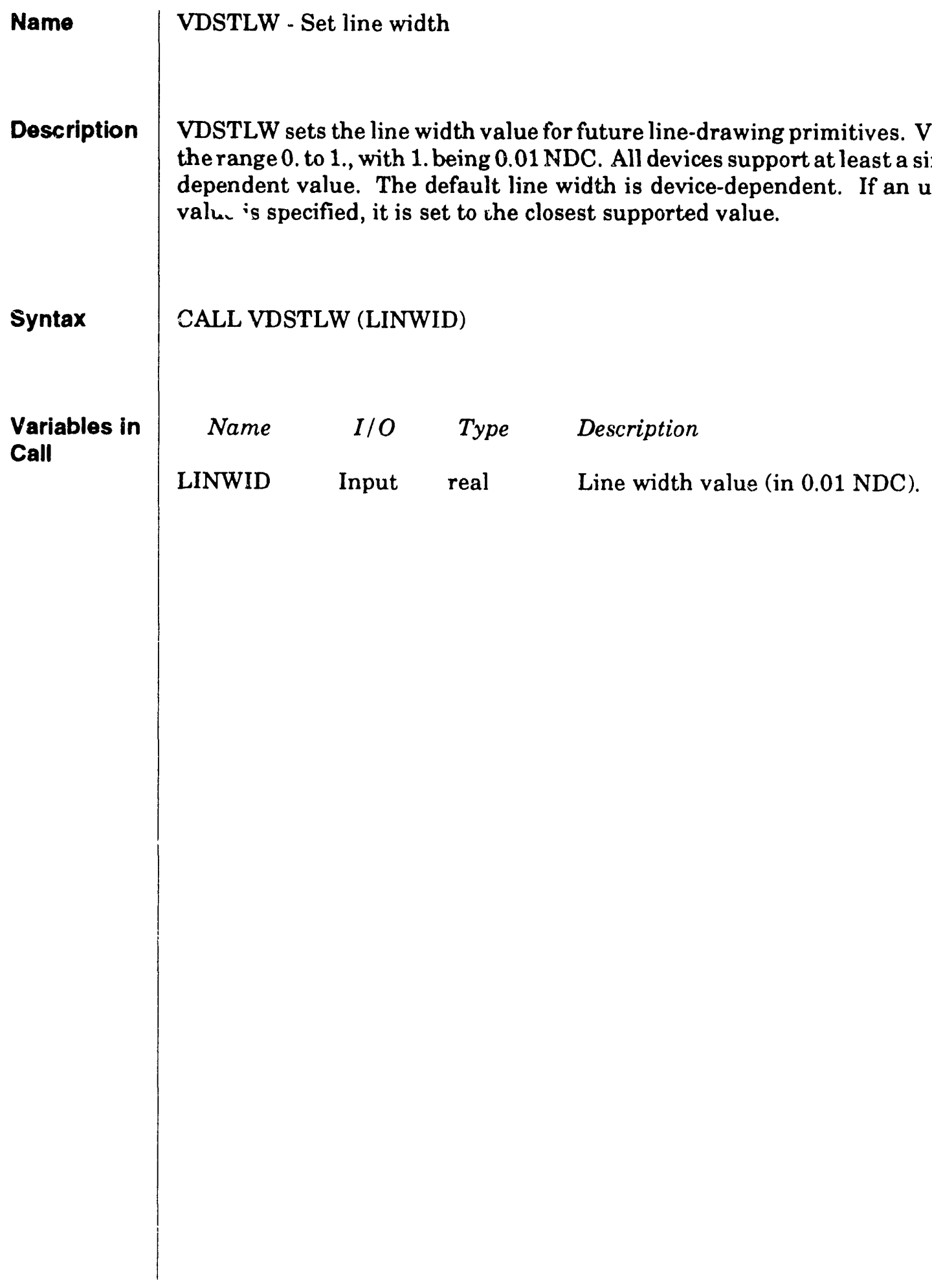




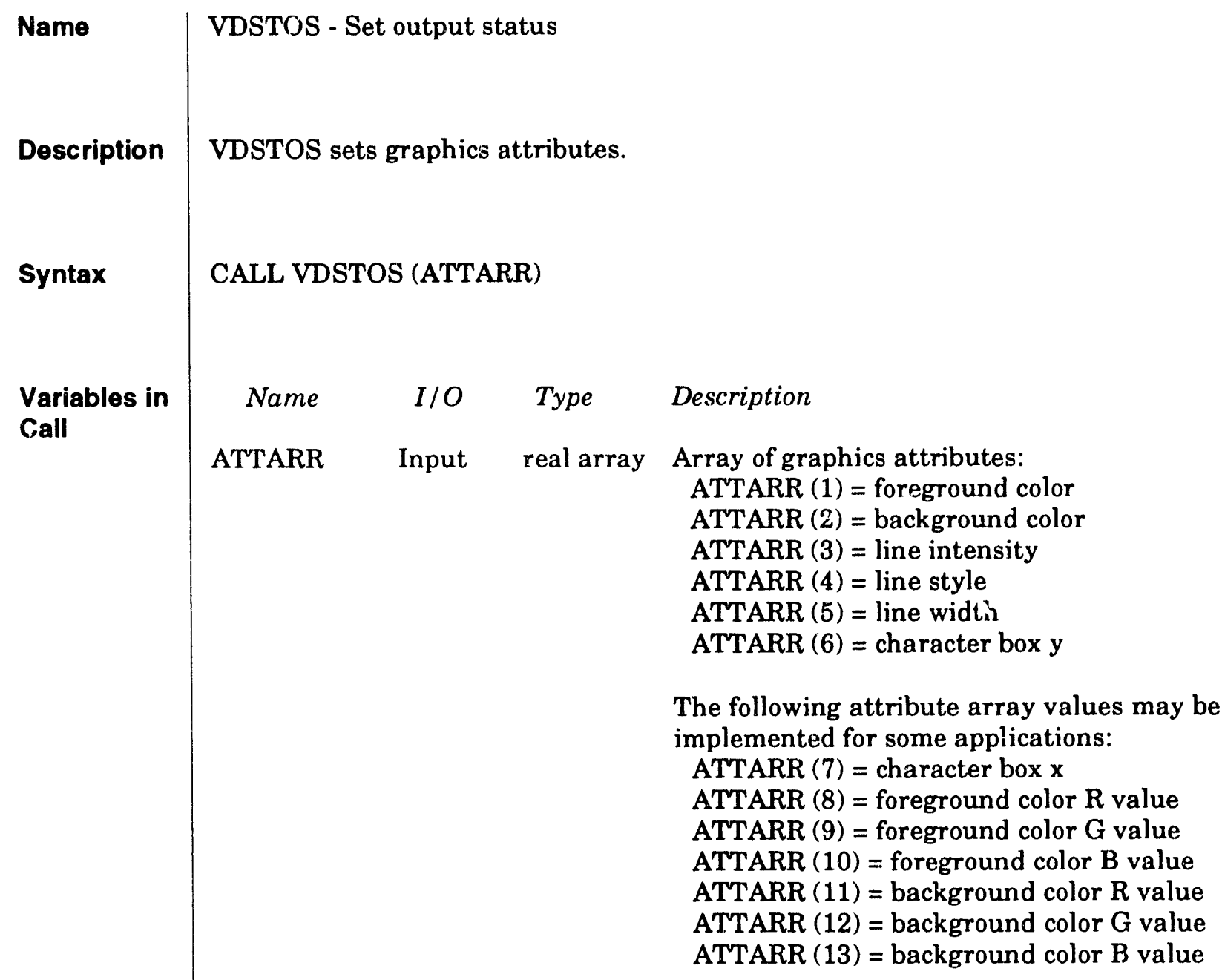




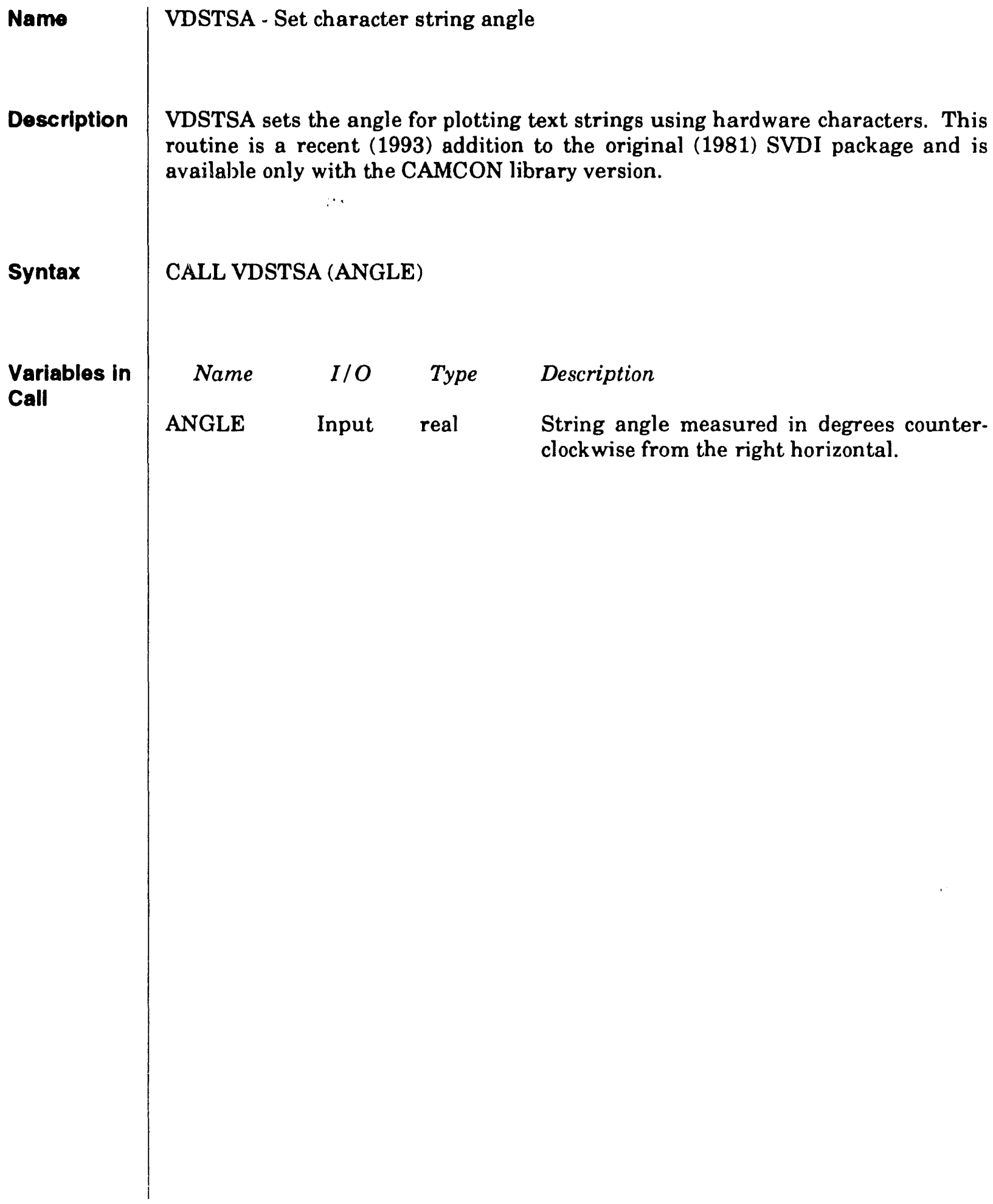


Name

Description

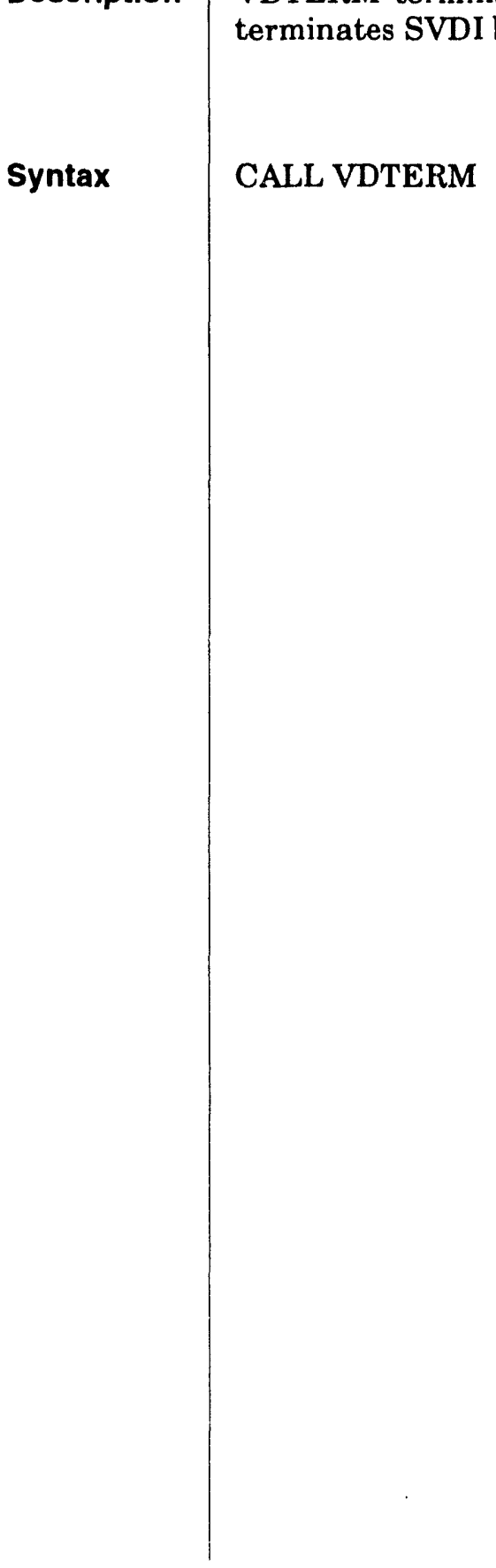

VDTERM - Terminate SVDI

VDTERM terminates SVDI. This should be the last SVDI call made, since it terminates SVDI by flushing buffers, etc.

\section{CALL VDTERM}




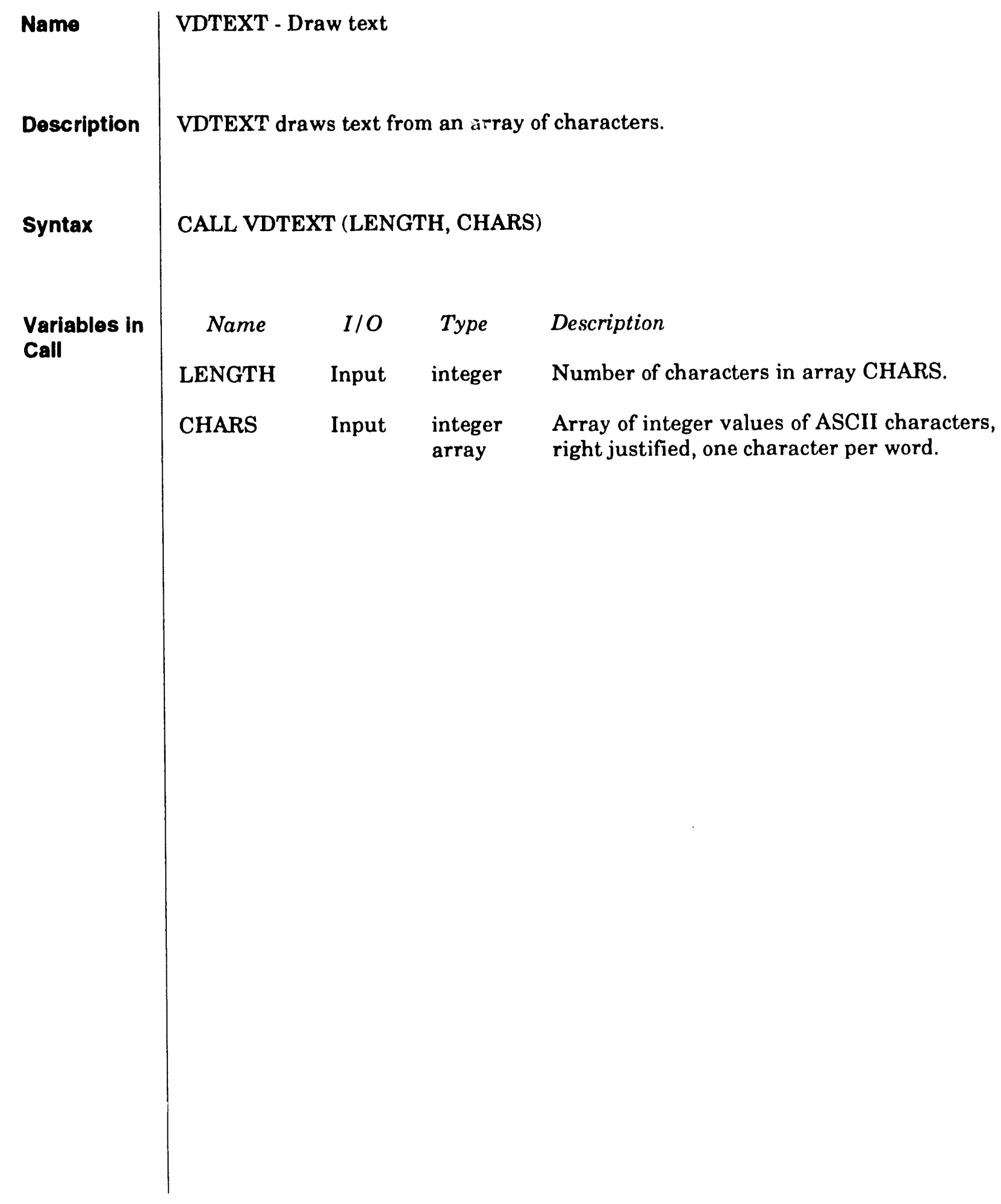


SVDI

Description of Routine Calls

VDWAIT

Subroutine

\begin{tabular}{l|l} 
Name & $\begin{array}{l}\text { VDWAIT - Wait for user } \\
\text { Description }\end{array}$ \\
$\begin{array}{l}\text { VDWAIT waits for the user to view the screen. The wait is ended by a signal from } \\
\text { the user, such as pressing any key. This function flushes the output buffers before } \\
\text { doing input. }\end{array}$
\end{tabular}

Syntax

CALL VDWAIT 


\subsection{Dual Device Interface Extension}

\subsubsection{Sponsorship and Version}
Author:
D. P. Flanagan
Sponsor:
A. P. Gilkey
Version/Date:
C-1.00VV/February 1991
Language:
DCL

\subsubsection{Overview of DVDI}

Major plot codes in the CAMCON system use the DVDI extension to SVDI. The primary purpose of the DVDI extension (although it is not limited to this purpose) is to allow a program to plot to an interactive terminal device and store all or selected plots in a graphics file for later printing on the appropriate hardcopy device. The graphics file created is not an exact copy of the terminal screen but rather a device-dependent copy that permits the plot image to be reproduced to the full capabilities of the hardcopy device; furthermore, the "device" may be either a specific piece of hardware (e.g., a brand name printer) or a graphics format (such as PostScript) that is used by a piece of hardware.

To accomplish its primary purpose, DVDI groups all devices into two categories: terminal devices and file devices (or "hardcopy"). The application program can choose one device from each category. A terminal device can be (but need not be) interactive; i.e., it can generate input as well as output and is usually tended by a user. A hardcopy device uses an intermediate graphics file and is never used interactively. Note that the category of a device depends upon the capabilities implemented in the available subroutine device driver, not the full advertised capabilities of the device (Figure 11-1).

Both device categories contain a null device, which is simply a set of dummy routines that satisfy calls from DVDI, but performs no functions. Hence, DVDI can run in a single device mode.

\subsubsection{Application Programming Guidelines}

DVDI Extensions. The extensions provided by the DVDI are programmed in three existing SVDI routines: VDESCP, VDIQES, and VDINIT (see Section 11.1, "Sandia Virtual Device Interface Library"). (Additional integer codes for the arguments have been provided to pass back required information, as described below.) This method of implementation means that DVDI is transparent to single-device graphics applications. Furthermore, programs that make use of dual devices should function (in single device mode) on systems where DVDI has not been installed.

It is important, however, for the application programmer to remember that the two devices do not communicate with each other. Consequently, if a device is made inactive, its state (cursor position, line style, color, etc.) may not be current with the active device. Therefore, when a device is reactivated, the application program must bring the reactivated device "up to date." 


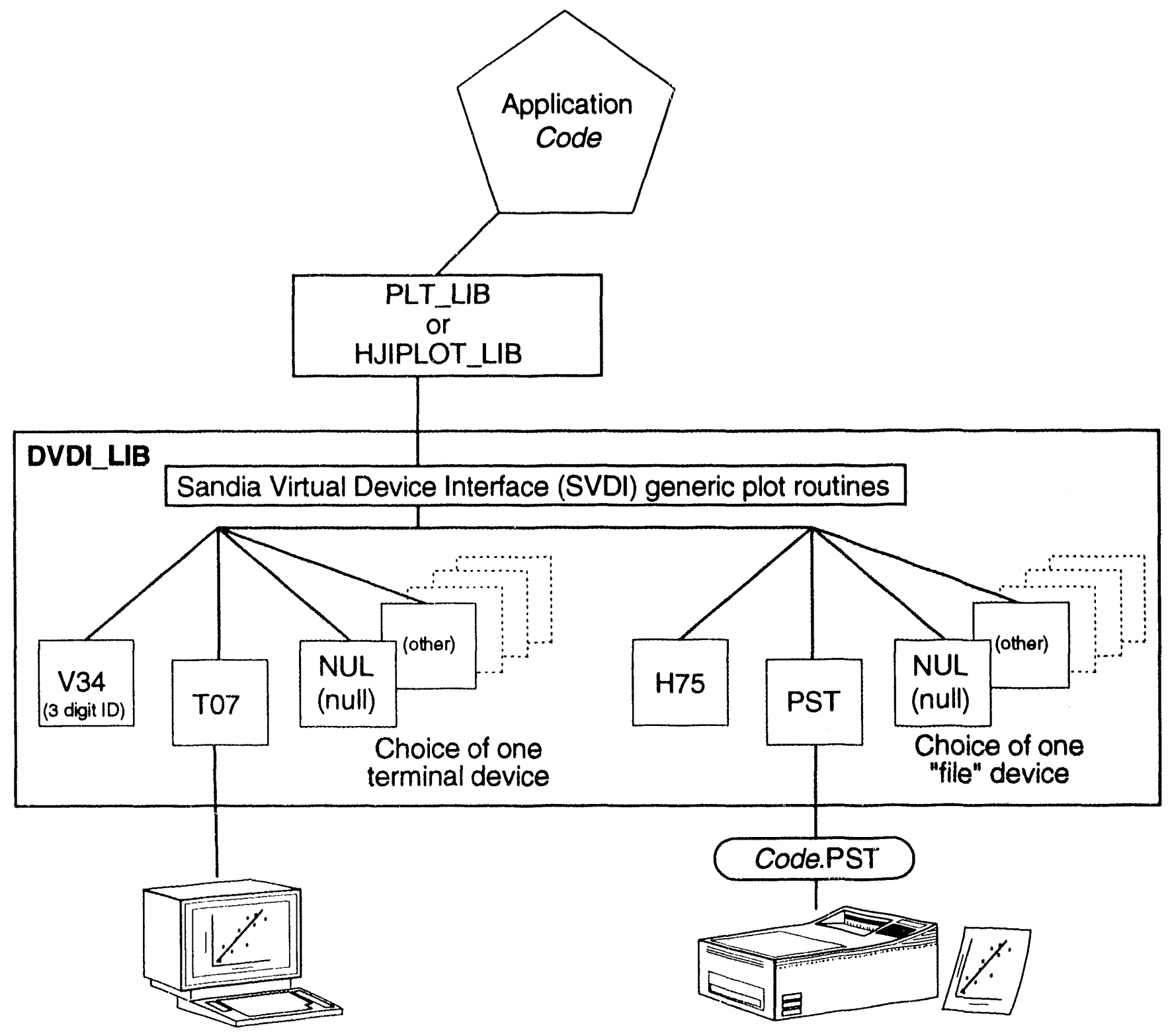

TRI.6342-2160-0

Figure 11-1. Communication with graphic devices is accomplished through the Dual Virtual Device Interface (DVDI).

Initialization by VDINIT. The modified SVDI routine, VDINIT, will determine whether either device is null, in which case that device is set to inactive (Off) for the duration of the program's execution, regardless of any calls to VDESCP. The VDINIT will initialize a currently active device only. Therefore, it is possible to initialize each device independently.

Communication Codes for VDESCP. An application program controls the status of the terminal and hardcopy devicas by calling VDESCP with the codes shuwn below (Table 11-3): Note that each call to VDESCP sets the status of both devices. Each device is initially active (On). 
Table 11-3. Communication Codes for the SVDI Routine, VDESCP Syntax: CALL VDESCP (ICODE, 0,0 )

\begin{tabular}{ccc}
\hline ICODE & Terminal & Hardcopy \\
\hline 10000 & Off & Off \\
10001 & On & Off \\
10002 & Off & On \\
10003 & On & On \\
\hline
\end{tabular}

Communication Codes for VDIQES. The application program can inquire as to the availability of DVDI, the terminal device, and the hardcopy device through VDIQES (Table 11-4). The routine returns the appropriate status in the argument ISTAT; a value of 1 indicates a vailable while a value of 0 indicates not a vailable. The meaning of the status returned by VDIQES for each DVDI escape code is given in Table 11-4. Note that these values reflect whether a device is available (i.e., not null) rather than the device's current state (i.e., On or Off).

Table 11-4. Communication Codes for the SVDI Routine, VDIQES Syntax: CALL VDIQES (ICODE, ISTAT)

\begin{tabular}{|c|c|}
\hline ICODE & Returns ISTAT $=1$ if and only if \\
\hline 10000 & DVDI implemented \\
\hline 10001 & Terminal device available \\
\hline 10002 & Hardcopy device available \\
\hline 10003 & $\begin{array}{l}\text { Terminal } a n d \text { hardcopy device } \\
\text { available }\end{array}$ \\
\hline
\end{tabular}

Input and Output. Duplex output through DVDI is straightforward: a call is made to the terminal device, if it is active, then a duplicate call is made to the hardcopy device, if it is active.

Input through DVDI is more subtle. DVDI merges input, including responses from inquiry functions, by calling the terminal device first. In other words, the terminal device has priority on input and is often called the "primary" device. It is possible to read or inquire from the hardcopy device only while the terminal device is inactive. Thus, the second device is often called the "secondary" device.

\subsubsection{Linking}

Most plot applications should be linked to shareable graphics libraries like PLT_LIB. These shareable images are already linked to the switchable dual device sets. Therefore, it should be rare that a programmer would need to link directly to the dual device drivers. 
When linking to DVDI, it is critical that both devices be selected to be null. Once this is done, an application is linked to the shareable DVDI graphics system simply by referencing the shareable graphics library. The following example links to the shareable PLT graphics library:

$\$$ SELECT NULL

\$ LINK code_narne, ..., SHRPLT/LIB, CDRLIB/LIB/INCL=CDRABC

\subsubsection{Device Selection and Execution}

The dual graphics devices are selected at image activation, i.e.,just prior to each execution. The DCL procedure, SELECT, controls device selection. It presents the user with a list or lists of appropriate devices, displays the default device selection(s), and prompts the user to make a selection. If the user simply hits the RETURN key, the default device selection will be retained. If the user enters "'" (two double quotes), the null device will be selected (see also Rechard, 1992, Appendix D).

The default devices are those devices specified in the last graphics program executed. The experienced user who typically plots to only one terminal type or hardcopy device may define the default for the devices by setting global symbols VDI_TERM (for the terminal) and/or VDI_FILE (for the hardcopy device) in his or her login file. For example:

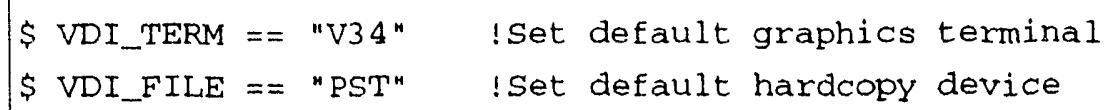

If the procedure executes successfully, it will set the logical name switches SHRVDT and SHRVDF to point to the appropriate terminal and hardcopy device images, respectively. Furthermore, the symbols VDI_TERM and VDI_FILE will contain the device IDs of the selections made.

When the user requests a plot to the graphics terminal, the plot appears immediately on the terminal. Requesting a plot to the hardcopy device, on the other hand, results in the creation of a file containing the commands necessary for the hardcopy device to roduce the plot. The device-dependent graphics file can be sent to the hardcopy device by using a PRINT command after the program has finished executing.

The default name of the graphics fiie is FOR077.DAT. It is suggested that the applications program or DCL file running the program rename the file to a more meaningful name. Most programs should use code_name.device_code (e.g., BLOT.PST).

Since device selection takes place before the application program is run, the program is conveniently run from a DCL file. The example of a DCL file shown below calls SELECT to select the devices, runs the given executable code, and renames the hardcopy file, if any. Note that it also assigns the codes of the selected devices to special symbols so they can be retrieved within the application program with the 
CAMSUPES_LIB routine EXNAME (see Chapter 9). The application program would retrieve these symbols as follows:

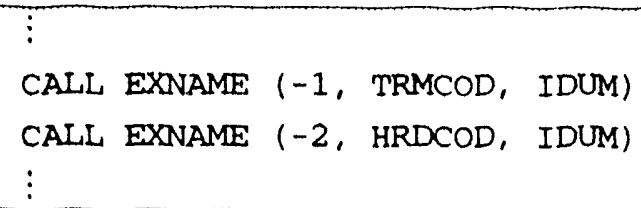

The following DCL fragment (with some added features) for running a generic application program is available in CAMCON\$ROOT:[CAMCON.LIBRARIES.DVDI_LIB]DVDI_DRIVER.COM.

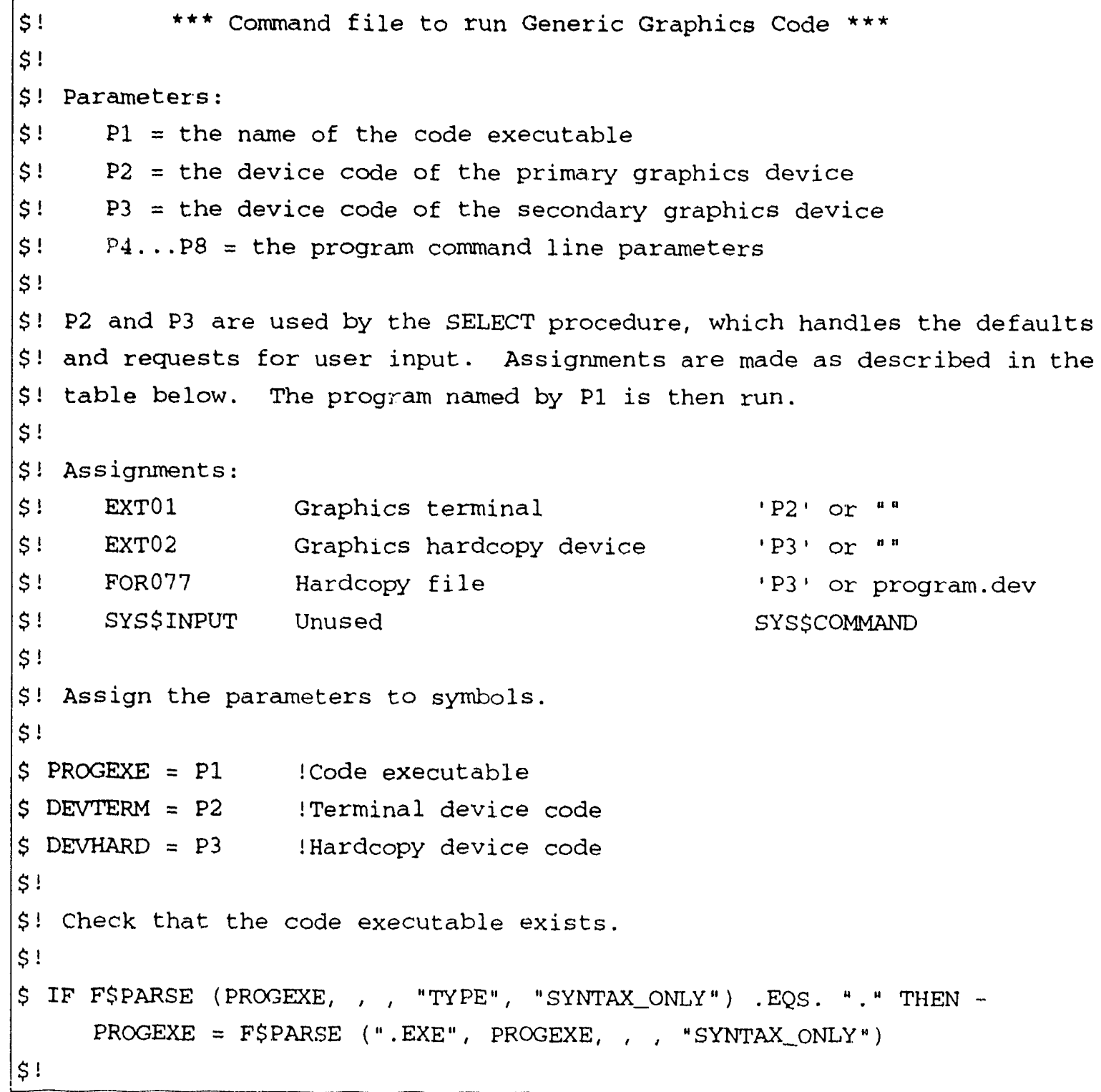




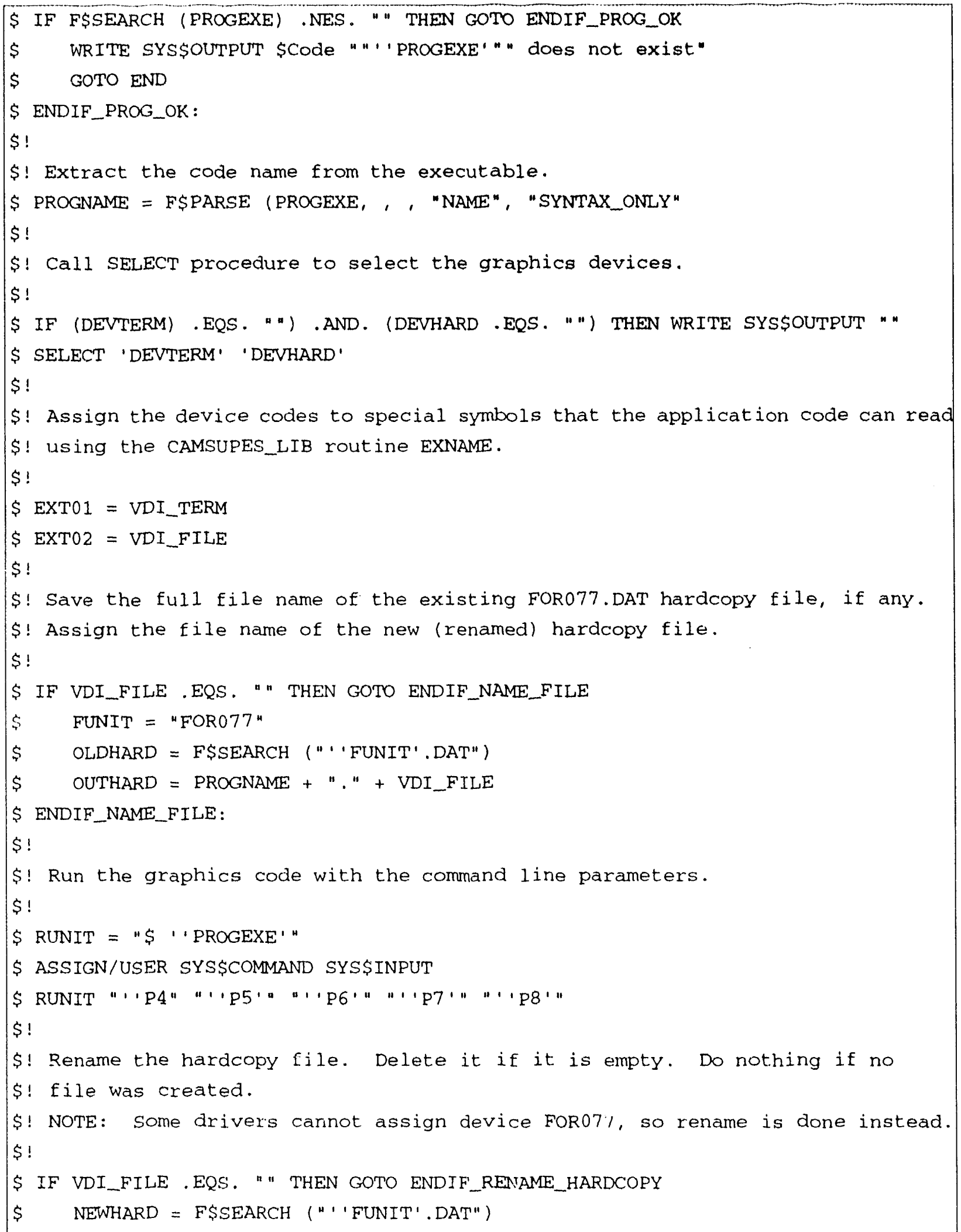




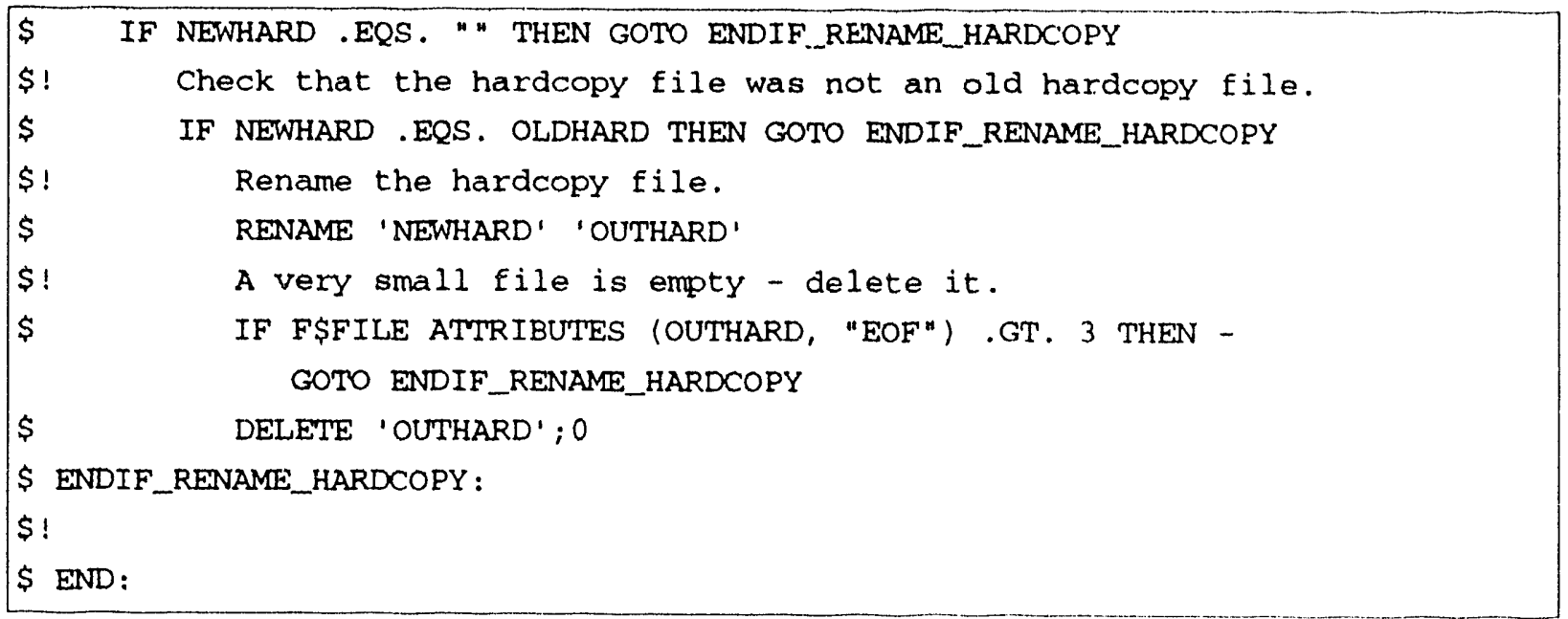

\subsubsection{Adding a Device Driver}

A device driver can be added at any time. The new device driver will automatically be available to all graphics applications using the shareable DVDI graphics system.

Normally, the CAMCONexec sponsor adds the device driver at the request of a user. All users have write privileges in the DVDI directory (SNL\$SHARE) so that an experienced user may add device drivers. The CAMCONexec sponsor needs to be notified when a driver is added. No other ope ti nis should be performed in this directory without the prior approval of the CAMCONexec sponsor.

The procedure for adding a device driver is straightforward, but the user should proceed carefully since all graphics applications will be affected. The user must first assign the device code of the desired device. A list of currently assigned device codes can be found by typing at the system prompt \$SHELP VDI DEVICES. Next, place the relocatable object library needed to add the driver in SYS\$VDI:VDIdev.OLB.

Next, ensure that your default directory is SNL\$SHARE. Finally, to add a terminal device driver for device trm, enter \$@SHRVDT trm.

To add a hardcopy device driver for device $h r d$, enter \$@SHRVDF $h r d$.

If the addition is successful, SHRVDT, SHRVDTtrm. EXE, and SHRVDTtrm. OLB are created. Also, the device code trm should appear in the SELECT prompt as an available terminal device. SHRVDF operates similarly. 
DVDI Graphics Interface 


\section{Software Development Utilities}

Twelve utility codes or procedural files are available to aid the software developer in meeting general coding guidelines or software QA requirements:

ABSGEN

CHANGES

CREATE_PROGRAM

FLINT

FORMRENUM

HLPCHK

LISTDCL

LISTFORTRAN

QA_PREP

QA_PRINT

UPDATE_PROGRAM

VAX/VMS DEBUG
A code that produces a software abstract from an ASCII text file structured as a VAX/VMS help file.

A code to allow users to report errors and propose enhancements to codes within the CAMCON system.

A code to compile and link a program.

A commercial code that can identify many subtle FORTRAN errors in both compiled and noncompiled codes. For example, it checks compatibility of calling sequence in routines and common blocks, variables used but not assigned or assigned but not used. FLINT can find an embarrassing number of dormant bugs in codes that have been running for months.

A code to format and renumber FORTRAN programs and identify subtle errors such as mismatched IF and DO block endings.

A code that checks the contents of the ASCII help file for a program.

A code to print listings for DCL code subroutines including both individual subroutine line members and total accumulated code line members. LISTDCL can be used for both programs consolidated into one large file or when subroutines and INCLUDE files are stored as separate files.

A code to print listings for FORTRAN code subroutines including both individual subroutine line members and total accumulated code line members. LISTFORTRAN can be used for both programs consolidated into one large file or when subroutines and INCLUDE files are stored as separate files.

A code that copies *.FOR an ${ }^{*}$.INC files from a directory and prepares them for input into QA_PRINT.

A DCL procedural file that runs FLINT, LISTFORTRAN, and LISTDCL with a set of standard options and parameters to produce uniform output for the software notebooks (see Section 4.6, "On-Line Documentarinn").

A code to compile a routine and link a program compiled by CREATE_PROGRAM.

A VAX/VMS debugging utility.

Details on the use of these codes are presented in the following sections, as well as a brief introduction to common commands used with the VAX/VMS DEBUG utility. 


\subsection{Chapter Conventions}

Throughout this chapter, the following organizational and typographic conventions are used:

- Descriptions of codes, and groups of keywords, commands, parameters, variables, and values are organized alphabetically.

- Data entered by the user (e.g., at the VAX/VMS system prompt, "\$”) are shown in boldface type (e.g., QA_PRINT).

- Unless otherwise indicated, data entered by the user must be followed by a $<$ RETURN>. For brevity, this is not shown in the text.

- All default values are enclosed within angle brackets, $<>$ (e.g., $<$ ROUTINES.TXT>).

- The essential portion of keywords and commands (i.e., the minimum number of characters required for the function to be recognized) appears in upper case (e.g., UPDATE_Program).

- The CAMCON system codes are case-insensitive and so the essential portion of a keyword does not have to be entered in upper case.

- Alphanumeric data for keywords and commands are shown in italics. 


\title{
12.2 ABSGEN, Software Abstract Generation
}

\author{
12.2.1 Sponsorship and Version \\ Name: $\quad$ ABSGEN, a code to extract data from software help files \\ Authors: $\quad$ J. S. Sandha, D. W. Osborn, and H. J. Iuzzolino \\ Sponsor: $\quad$ K. A. Byle \\ Version/Date: $\quad$ C-3.00VV/September 1992
}

\subsubsection{Function}

ABSGEN extracts QA information for software abstracts from CAMCON software help files and reformats it into a software abstract form acceptable for viewing, printing, or reading into a relational data base. The code searches for specific identifiers followed by a colon (:) and then extracts the text following the identifier. The program calls several plotting routines to draw the abstract form along with the appropriate text. The output of this program is a screen dump, plot file, or ASCII file.

ABSGEN is actually a DCL shell that calls two routines: HLP2ABS and ABS2RPT. HLP2ABS reads the help file specified and produces a temporary data file, codename.dat. ABS2RPT then reads this file and produces output at the sperified destination. Any temporary files created by ABS2GEN are automatically deleted prior to exiting the routine.

\subsubsection{Execution}

First, extract the CAMCON help file from CHELP by typing

\section{LIBRARY/EXTRACT - code_name/help/output = code_name.hlp CAMCON\$HELP}

where CAMCON\$HELP is a CAMCON system-defined symbol pointing to CAMCON\$ROOT:[LIBRARIES.HELP_LIB]CAMCON_HELP.HLP.

Second, at the system prompt type the following:

\section{ABSGEN code_name.hlp destination}

where code_name.hlp is the name of the CAMCON help file providing QA information for the software abstract and destination is a printing option. The destination options are

F - File; this option creates a file named codena me.rpt in the current directory

S - Screen; this option scrolls output directly to the terminal 
$\mathrm{P}$ - Printer; this option sends output to a printer defined by the CAMCONexec sponsor using the r.mbol ABSPRNTR.

Note: ABSGEN prompts the user for any arguments not provided on the command line.

\subsubsection{Required Identifiers and Headings}

ABSGEN expects the following identifiers and headings (see also Section 4.6, "On-Line Documentation"):

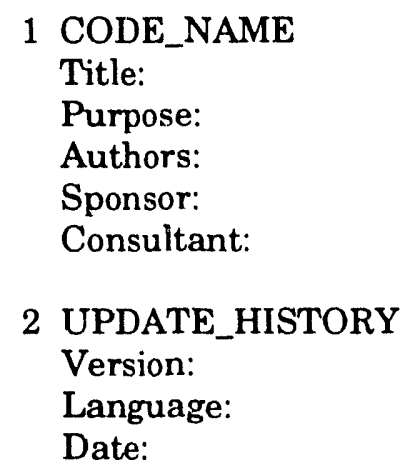

\section{QA_INFO}

QA_Level: see UPDATE_HISTORY

Limitations:

Keywords:

Origin:

Parent Program:

Classification:

Description:

Type:

CAMCON Module:

Verification:

Hand Calculation

In-Use Test

Comparison with Analytic Solutions

Comparison with Other Verified Programs

Parametric Sensitivity Analysis

Documentation:

User's Reference

Theoretical Reference

Verification/Sample Problems Reference

Other Reference

Primary Reference

The general instructions given to software sponsors for constructing help files are given in Section 4.6, "On-Line Documentation." 


\title{
12.3 CHANGES, Error and Enhancement Reporting
}

\subsubsection{Sponsorship and Version}

\author{
Name: $\quad$ CHANGES, CAMCON error and enhancement reporting system \\ Author/Sponsor: A.P. Gilkey \\ Version/Date: $\quad$ C-1.03VV/July 1992
}

\subsubsection{Function}

CHANGES allows users to report errors and propose changes to CAMCON software. An error/ enhancement report to a sponsor contains:

- A report number

- The name of the person who sent the report and when it was sent

- The software name and its sponsor

- The subject of the message (40 characters)

- A text message (up to 20 lines)

- An optional reply from the sponsor (up to 20 lines)

CHANGES also allows sponsors to broadcast reports to all users. These reports contain the same information as error reports, but they allow multiple replies from any user. This is useful for querying users about proposed changes.

Each report is assigned a report number that is printed with the report. This number is used to identify the report. A temporary (negative) number is assigned when a report is added. The permanent number is assigned upon exit. Since reports can be deleted, there may be gaps in the numbers.

The reports are maintained on a file that is not updated until the program is exited. If the program aborted or interrupted, all changes are lost.

\subsubsection{Execution}

At the CAMCONexec main menu or the system prompt, type CHANGES. A isting file, CHANGES.TXT, may be created by the user.

\subsubsection{Special Mode, SCANCHANGES}

SCANCHANGES displays "new" reports and replies that have been sent to the user. The user may reply to a new report or write the report to a file, but no command input is allowed. SCANCHANGES 
is a special mode of the CHANGES program. It can be run stand-alone to scan new reports. It is also run as a part of CHANGES before command input begins.

SCANCHANGES is run when setting up the CAIMCON system environment using CAMCON_SYMS, a summand that is usually part of the user's login file. Each user will have a file called SETUP.CHANGES created in the login directory. This file keeps track of when CHANGES was last run. When SCANCHANGES is run, each report and reply addressed to the last user (including broadcast reports) is checked against the time stamp of the last run. If it is a new report or reply, it is displayed to the user.

\subsubsection{Commands for CHANGES}

CHANGES recognizes the following user commands:

$\begin{array}{lll}\text { ADD } & \text { EDIT } & \text { QUIT } \\ \text { BROADCAST } & \text { EXIT } & \text { REPLY } \\ \text { DELETE } & \text { HELP } & \text { SPONSORS } \\ \text { DIRECTORY } & \text { PROGRAMS } & \text { VIEW }\end{array}$

These commands are described below.

After a command is issued, all input is prompied for with the default (if any) shown in angle brackets ( $>>$ ). Input may be in either lower or upper case. Most input can be abbreviated (i.e., the user need only enter enough characters to uniquely identify the item, but all characters given must be correct). Replying QUIT to most prompts will return to the CHANGES prompt, >. The sponsor name can be entered by the full name or the VAX system user name (either may be abbreviated).

\section{Add}

$\mathrm{ADD}$ sends a report to the appropriate software sponsor. The routine prompts for the name of the software (or the sponsor name for more general comments), the subject of the report, and the text file that contains the report text. The text may be entered interactively by omitting the name of a text file. The report will be displayed to the software sponsor the next time SCANCHANGES is run.

\section{Broadcast}

BROADCAST sends a report to all users. This is intended to help sponsors notify users of changes in software. The user (normally the software sponsor) is prompted for the name of the software, the subject of the report, and the text file that contains the report text. The text may be entered interactively by omitting the name of a text file. The report will be displayed to a user the next time SCANCHANGES is run. 


\section{DElete}

DELETẼ deletes a report. Only the user who sent the report or the sponsor the report is addressed to can delete the report. The routine prompts for a report identifier to select the report(s) to delete. The identifier may be

- The report number to select a specific report

- The software name to select all reports about that software

- Your user name to select all reports that you may delete

The user can optionally delete each selected report.

\section{Directory}

DIRECTORY displays a summary of the reports. The routine prompts for a report identifier to select the reports to view. The identifier may be

- The report number to select a specific report

- ALL to select all reports

- The software name to select all reports about that software

- The sponsor name to select all reports addressed to that sponsor

- BROADCAST to select all broadcast reports

The routine also prompts for a report number or date of the first report to display.

EDit

EDIT allows the user to edit a report or a reply. Only the user who entered a report or reply can edit it. The routine prompts for a report number of the report to edit.

\section{EXit}

EXIT updates the report file and exits the program.

Help

HELP lists a short description of each command.

\section{Programs}

PROGRAMS displays the names and sponsors for CAMCON software. The routine prompts for a sponsor name. If a sponsor name is entered, only the software with the selected sponsor is listed; otherwise all software is listed.

Quit

QUIT exits the program without updating the report file (no changes are recorded).

\section{Reply}

REPLY enters a reply to a report. Anyone can reply to a broadcast report. Only the sponsor the 
report is addressed to can reply to a non-broadcast report. 'The routine prompts for a report identifier to select the report(s). The identifier may be

- The report number to select a specific report

- The software name to select all reports about that software

- BROADCAST to select all broadcast reports

- Your user name to select all reports addressed to you (but not sent by you)

The user may optionally reply to each selected report. The routine prompts for the text for the reply. The reply is displayed to the author of the report the next time SCANCHANGES is run.

\section{Sponsors}

SPONSORS displays the sponsor names and system user names for all sponsors of CAMCON software.

\section{View}

VIEW displays the reports. The routine prompts for a report identifier to select the report(s) to view. The identifier may be

-A report number to select a specific report

- ALL to select all reports

- A software name to select all reports about that software

- BROADCAST to select all broadcast reports

The routine also prompts for a report number or date of the first report to display. Each selected report is displayed and the user may optionally reply to it (if possible) or send it to a listing file. 


\title{
12.4 CREATE_PROGraM, Compile and Link a Program
}

\author{
12.4.1 Sponsorship and Version \\ Name: $\quad$ CREATE_PROGRAM, a code to compile and link a program \\ Author/Sponsor: A.P. Gilkey \\ Version/Date: X-1.01VV/June 1992
}

\subsubsection{Function}

CREATE_PROGRAM compiles all *.FOR files in a directory, creates an object library, and links a program. A debug version may also be created in the sponsor's directory.

\subsubsection{Execution}

At the system prompt type the following (this code is not available through CAMCONexec [Rechard, 1992]):

\section{CREATE_Program}

If present in the directory, two optional files, ROUTINES.TXT and COMPILE_SYMBOLS.COM (explained below) are read.

\subsubsection{Preparation}

CREATE_PROGRAM works on a code that resides in its own directory. By default, the name of the directory is the name of the program (code_name in the following description). Although all source code for the program must reside in the directory, object libraries need not be in this directory.

ROUTINES.TXT File Contents. An optional file, called ROUTINES.TXT, may reside in the directory that lists the names of all INCLUDE files and all FORTRAN files used by the program (one file name per record). Each *.FOR file whose name is listed in ROUTINES.TXT is compiled. If ROUTINES.TXT does not exist, all *.FOR files in the directory are compiled. (See QA_PREP or QA_PRINT for information about preparing the ROUTINES.TXT file.)

COMPILE_SYMBOLS.COM Input Commands. An optional file, named COMPILE_SYMBOLS.COM, may reside in the directory that defines the symbols listed in the following paragraphs to customize the CREATE_PROGRAM and UPDATE_PROGRAM procedures. COMPILE_SYMBOLS is a standard DCL command file that will be executed in both procedures (with (aCOMPILE_SYMS). The symbols must be defined as DCL global (not local) symbols (either with == and quotes around the parameter, or alternately, with :== and no quotes around the parameter). Only symbols that differ from the default neer? to be assigned in the file. 
PROGNAME $==$ "code_name" <directory_name $>$

PROGNAME specifies the file name of the executable file(i.e., code name) without the extension. This defaults to the directory name (the lowest level name only).

MAINPROG =="code_name" <directory_name $>$

MAINPROG specifies the name of the program (in the FORTRAN program statement). MAINPROG should be set to double quotes, "'", for a nonexecutable (library) directory.

LIBRARIES $==$ "library" $<" C A M C O N \_L I B ">$

LIBRARIES specifies the libraries needed to link the program (not including code_name_LIB.OLB). Separate the library names by commas. Do not include the "/LIB" or the ".OLB" extension, but include any directory specifier.

BLOCK_DATA == "routine" <"">

BLOCK_DATA specifies the names of any BLOCK DATA routines that contain DATA statements. The BLOCK DATA routine must be named.

COMPILE_OPTIONS =="option $1 /$ option $_{2} / . . . "<" /$ LIST /NODEBUG/OPTIMIZE"> COMPILE_OPTIONS specifies the compile options for the standard version.

DEBUG_COMPILE_OPTIONS $==" o p$ tio $_{1} /$ option $_{2} / . .$. <"/LIST/DEBUG/NOOPTIMIZE">

DEBUG_COMPILE_OPTIONS specifies the compile options for the debug version.

\section{Example}

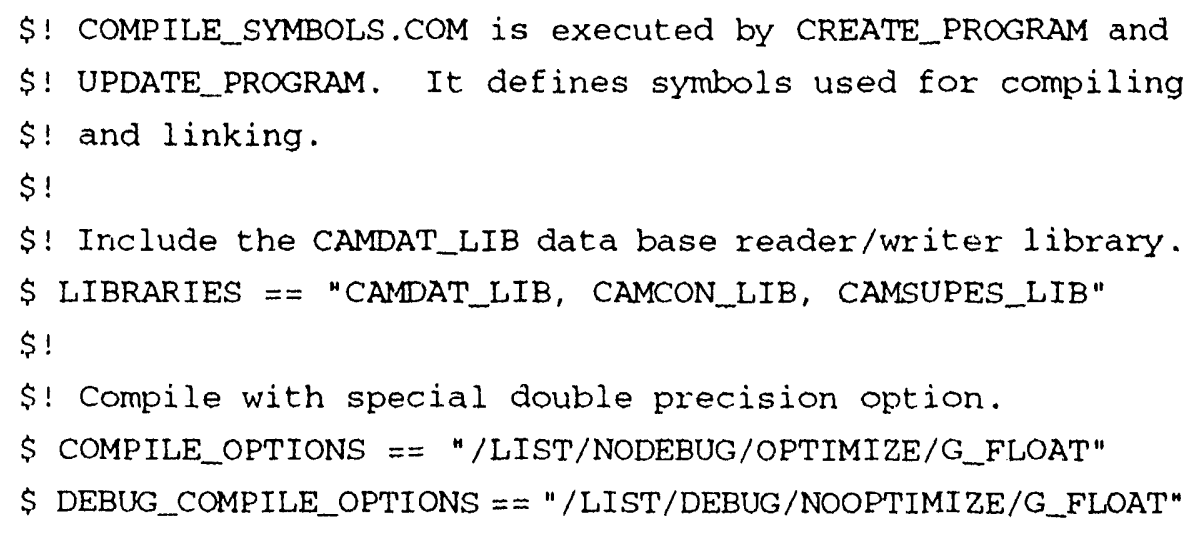




\subsubsection{Output}

As each file is compiled, a listing file is created and is then deleted if the compilation is successful. Thus, when CREATE_PROGRAM is completed, the only *.LIS files in the directory are for files with compilation errors. Also, an object library, code_name_LIB.OLB, consisting of all *.OBJ files in the directory, is created. The individual *.OBJ files are deleted.

If there were no compile errors, the program is then linked to create an executablefile,code_name.EXE. The options for linking are/TRACE/MAP. Use UPDATE_PROGRAM to recompile and relink individual routines.

If the symbol DEBUG_code_name is defined, a debug version of the code is created in the user's directory. All files are recompiled with debug options, an object library (code_name_LIB_DEBUG.OLB) is created, and an executable file (code_name_DEBUG.EXE) is linked with the options /DEBUG TRACE. The symbolDEBUG_code_name is defined similarly to the code_name symbol that runs the program, except that it points to the debug version of the code. Although the code_name symbol and the DEBUG_code_name symbol may be defined in SPONSOR_SYMS.COM in CAMCON\$ROOT:[SYMBOLS], the debug version source code is not in [CAMCON]; thus, the DEBUG symbol should point to the sponsor's working directory. An example of defining a DEBUG symbol is as follows:

\$DEBUG_code_name $==$ \$dis $k$ :Lirectory 1 code_name_DEBUG.EXE" 
Software Development Utilities

FLINT

\subsection{FLINT, FORTRAN Code Analyzer}

\subsubsection{Sponsorship and Version}

Name: $\quad$ FLINT: A FORTRAN source code analyzer

Author: $\quad$ Information Processing Techniques Corporation, Palo Alto, CA

Sponsor: $\quad$ A. P. Gilkey

Version/Date: $\quad$ C-2.71VV/August 1990

Version Base Case: C-2.71VV equivalent to version 2.71 acquired August 1990 with Sandia site license.

\subsubsection{Function}

Fortran-LINT is a commercial source code analyzer designed to simplify the debugging of FORTRAN programs.

\subsubsection{Execution}

At the system prompt, type the following (this code is not available through CAMCONexec):

FLINT[/qualifiers] $[$ source-file-spec.ext $[, \ldots]$

\subsubsection{Source File Specification}

The source-file-spec.ext parameter specifies one or more FORTRAN source codes to be analyzed. If a file type is not specified, FLINT uses a default file type appropriate with the /SYS switch setting. If no/SYS switch is present, the default type is *.FOR.

Temporary defaults are used in the file specification list. If a directory name is explicitly specified for a file specification, it becomes the default for any following file specifications with omitted directory names. Wild cards are permitted. File specifications may be separated by either commas or spaces.

FLINT interprets the file extension, ext, as follows:

\section{LBT files}

If a file with the extension '.LBT' is specified, it is taken to be a FINT library. FLINT libraries are used on /GLOBAL runs to allow call argument checking without having to re-parse all the sources each time.

\section{.TLB files}

If a file with the extension '.TLB' is specified, it is taken to be a text library. When FLINT comes across a text library include statement without a text library file name associated with it (e.g. 
INCLUDE '(\$FOOBAR)'), it searches all command-line text libraries in the order specified. If the module is not found, it then searches the user text library 'FORT\$LIBRARY', and then the system default library 'SYS\$LIBRARY:FORSYSDEF.TLB'.

\section{.TTB files}

If a file with the extension 'TTB' is specified, it is taken to be a tree table file. All source processing is bypassed, the symbol table is loaded from the tree table, and a call tree can be generated without having to re-parse the sources.

\subsubsection{Qualifiers}

Qualifiers (or "switches") for FLINT (/qualifier) are described as follows:

Source File Qualifiers. The following qualifiers control the interpretation of the input source files:

MLINES

DLINES processes source lines that begi : : vith a ' $\mathrm{D}$ ' (debug lines).

/EXTEND

/EXTEND extends source code line length from 72 columns to 132 columns.

FILES=file_spec

FILES specifies a list file that contains names of source files to be processed. These file names are treated as if they were included on the command line itself. The format of this list file is the same as the command line, except that switches are not allowed in the file. File specifications may be of any form, including wild cards, and may be separated by any white space.

$14<$ Default>

NOI4

By default, INTEGER and LOGICAL declarations are interpreted as INTEGER* 4 and LOGICAL*4. If the NOI4 switch is specified, INTEGER and LOGICAL declarations are interpreted as INTEGER*2.

Output File Qualifiers. The following qualifiers determine which output files will be generated by FLINT:

NIBRARY $=$ file_spec

/LIBRARY creates or updates the specified library. The default extension is .LBT. Interface information from subprograms can be stored in libraries to be used in analysis at a later date. No diagnostic output is given when creating or updating libraries, so the file should compile without errors before this switch is used. (Further information is available in Section 12.4.6.) 
IOUTPUT $=$ file_spec

/OUTPUT specifies the file to which the output from FLINT is directed. This switch is overridden by the/SPLIT switch. By default, the output is sent to SYS\$OUTPUT. (See also /SPLIT.)

ISPLIT=file_root

/SPLIT splits the output and redirects each stage of the analysis into its own file. The file names for output are built by adding various extensions to the base name file_root. Any extension on the file specification file_root is discarded. Output is redirected into the following files:

$$
\begin{aligned}
& \text { Analysis output } \rightarrow \text { file_root.LNT } \\
& \text { Symbol table (/XREF) } \rightarrow \text { file_root.XRF } \\
& \text { Call tree (/TREE) } \rightarrow \text { file_root.TRE } \\
& \text { Summary (/SUMMARY) } \rightarrow \text { file_root.SUM } \\
& \text { Statistics (/STAT) } \rightarrow \text { file_root.STT }
\end{aligned}
$$

By default, the output is sent to SYS\$OUTPUT. (See also /OUTPUT.)

\section{ISTATISTICS}

/STATISTICS produces statistics about the files (e.g., number of source lines, number of errors, etc.).

\section{ISUMMARY}

In conjunction with the /SPLIT switch, /SUMMARY creates a summary file showing only the more significant diagnostics. All error messages with a ' + ' after the type code in FLINT.ERR are listed in the summary file. (See also /SPLIT.)

\section{ITREE}

TREE produces a graphical representation of which routines call other routines. Only one/TREE may be specified per FLINT run. (See also/TRIM, /SQUISH,/GRAPHICS.)

\section{ITB=file_spec}

TTB creates a tree table file. The default extension is '.TTB'. The tree table stores all information required to generate a call tree for this run. This table can be used later to regenerate a call tree without having to reprocess the sources.

\section{IXREFERENCE}

XREFERENCE produce a cross reference as a part of the symbol table listing. 
Diagnostic Qualifiers. The following qualifiers control the diagnostics file generated by FLINT:

IANSI

/ANSI flags various extensions to the ANSI FORTRAN-77 standard. It has the same effect as $/$ PORT $=$ ANSI.

/FYI

FYI prints hints (for your information) which may be cause for concern.

\section{/GLOBAL}

/GLOBAL analyzes all subprograms as a group. If this switch is not specified, subprograms are processed on an individual basis and call interface checking is not performed. This switch also improves variable usage checking by allowing analysis results of one subprogram to be applied to another (such as usage of a variable passed as an actual argument).

\section{AMPLICIT}

IMPLICIT reports the use of implicit data typing.

\section{ANCLUDE}

/NCLUDE lists included files in the source listing.

\section{RISTING}

/LISTING produces a source listing with line numbers.

/PORTABHITY=(target, target,...$)$

/PORTABILITY prints warnings relating to problems the code might have while being ported to the specified compiler(s). The following is a list of valid compilers:

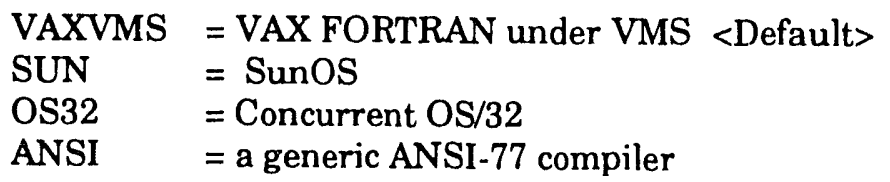

\section{ISUPPRESS $=(n, n, n, n, \ldots)$}

/SUPPRESS suppresses individual diagnostic messages. Arguments are generally the numbers of the messages to be suppressed. The forms the arguments may take are

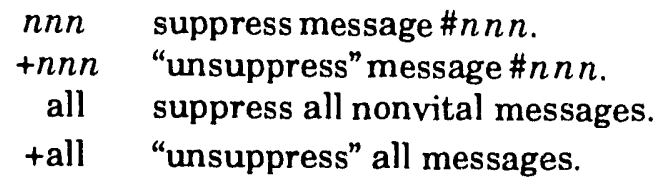

The message numbers are too numerous to repeat here. The usual mode of operation is to make a FLINT analysis without any suppression. If too many superfluous or 
meaningless messages are produced, redo the FLINT analysis with the meaningless message number suppressed.

/SYSTEM=opsys <VAXVMS>

/SYSTEM specifies the target compiler by system name. The following are valid operating systems:

$\begin{array}{ll}\text { VAXVMS } & \text { For VAX FORTRAN under VMS } \\ \text { SUN } & \text { For SunOS } \\ \text { OS32 } & \text { For Concurrent OS/32 }\end{array}$

NSAGE <Default>

NOUSAGE

/USAGE displays variable usage diagnostics. If the NOUSAGE switch is specified, variable usage checking is suppressed.

WARNINGS <Default>

NOWARNINGS

WARNINGS displays warning level diagnostics. If the /NOWARNINGS switch is specified, warning messages are suppressed.

Cross-Reference Table Qualifiers. The following qualifiers control the cross-reference tablegenerated with the XREFERENCE qualifier:

\section{ROCALSYMS <Default>} NOLOCALSYMS

/LOCALSYMS specifies that local symbols are to be included in the cross reference listing. If the NOLOCALSYMS switch is specified, local symbols are omitted from the cross reference.

\section{IPARAMETERS}

/PARAMETERS includes unused parameter declarations in the cross reference listing.

Call Tree Qualifiers. The following qualifiers control the call tree generated with the /TREE qualifiers:

/GRAPHICS $=x x: x x: x x: x x: x x$

The /GRAPHICS switch allows you to specify which graphic characters to use in producing the call tree. The five parameters are the hexadecimal codes for the following characters: 
Example: /GRAPHICS=C4:B3:C2:C3:C0

\section{ISQUISH}

/SQUISH compresses the call tree in the vertical direction. It is used primarily with the /GRAPHICS switch.

TRIM

TRIM t:ims the tree to prevent printing duplicate subtrees.

Miscellaneous Text Format Qualifiers. The following qualifiers set the format for all text files generated:

几PP $=n$

/LPP sets the number of lines per page. If set to 0 , there is no pagination. Either 0 or any positive number greater than 3 is valid. If output is being redirected, the page length is usually 60 lines. Otherwise it is usually 0 (no pagination).

\section{WIDTH $=n$}

/WIDTH specifies the output width in columns. Any value between 40 and 500 is valid. If output is being directed to SYS\$OUTPUT, the default width is 80 columns. Otherwise, it is 132 columns.

\subsubsection{Library}

A FLINT file can be produced for a library to allow software sponsors to check the interface between an application program and any libraries (e.g., CAMCON libraries). A FLINT library is created with the following command:

\section{FLINT/LIB $=$ code_name.LBT $\operatorname{src}_{1}$. FOR $\ldots \operatorname{src}_{\mathrm{n}}$. FOR}

All routines in the source files are added to the FLINT library filecode_name.LBT. If code_name.LBT already exists, it is updated; otherwise, code_name.LBT is created. The source files must be debugged before FLINT/LIB is issued because errors within the libraries are not reported.

In the case of imported libraries, the source for the library routines may not be available. The sponsor can make up a dummy source file that contains the calling parameter sequence with special FLINT options on the arguments. This file contains enough information to allow program sponsors to check the interface to the library. FLINT/LIB is run on the dummy source file as if it were a normal source file. 
The special options follow:

/s - an argument that is set in the routine

$/ r$ - an argument that is referenced in the routine

/i - an argument that is indeterminate

$/ \mathrm{v}$ - a parameter passed by value

/q - suppress error \#251 (scalar passed to array) and data type checking

$/ z$ - suppress all interface checking with regard to this argument

10 - optional argument

$\Lambda$ - the rest of the argument list from the preceding comma is optional

The following example is a dummy source file for two CAMSUPES_LIB routines:

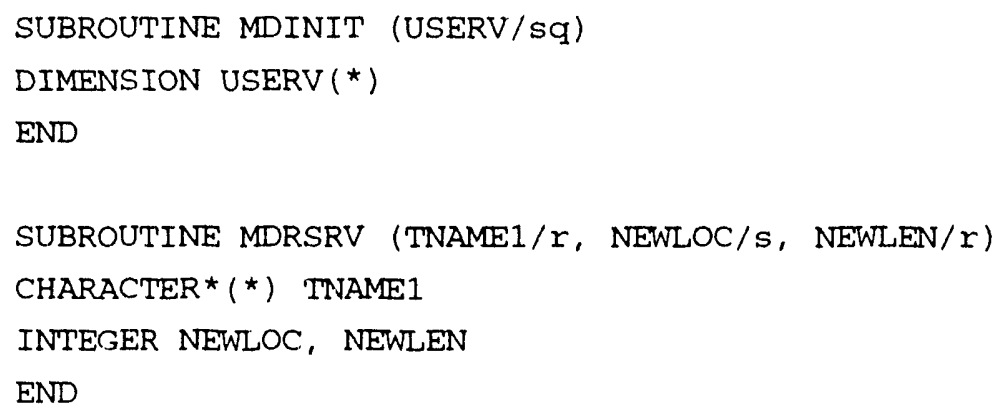




\subsection{FORMRENUM, Format and Renumber}

\subsubsection{Sponsorship and Version}

Name: $\quad$ FORMRENUM, a FORTRAN format and renumber program

Author/Sponsor: A. P. Gilkey

Version/Date: $\quad$ X-1.00VV/February 1990

\subsubsection{Function}

The main purpose of FORMRENUM is to create aesthetically pleasing code, but it is also useful for identifying some syntax errors (such as mismatched END IFs, duplicate labels, and misplaced continuation characters).

\subsubsection{Execution}

At the system prompt, type the following (this code is not available through CAMCONexec [Rechard, 1992]):

\section{FORMRENUM /qualifiers code_name.ext}

Thecode_name.ext parameter specifies the FORTRAN source file. If ext is omitted, FOR is assumed. The input file is replaced by an output file, but with a higher version number.

\subsubsection{Overview}

FORMRENUM formats and/or renumbers a FORTRAN source file. FORMRENUM handles standard FORTRAN and most VAX-specific FORTRAN constructs. The main advantage of FORMRENUM as opposed to other formatters is that the user can easily tailor FORMRENUM to conform to an individual programming style.

Formatting Features. If formatting is selected, FORMRENUM strips all leading blanks and tabs from the input source file and indents according to the options the user has specified.

Blocks of text within a DO-loop or an IF-THEN-ELSE structure are indented an optional number of spaces. VAX-specific constructs (e.g., the UNION structure) are also indented.

Continuation lines are indented either a specified amount from the current indentation or with respect to the first line of the statement (i.e., if the input continuation line is indented $n$ spaces from the first line, the continuation line is indented $n$ spaces from the current indentation). 
The current indentation should be zero at each END statement. If it is not, the statement is flagged with an error message and the current indentation is reset to zero.

Renumbering Features. If renumbering is selected, FORMRENUM renumbers all labels and all (correct) references to labels. Labels are renumbered in the order they are defined, not in the order they are referenced. Labels on FORMAT statements can be numbered differently from labels on other statements.

The VAX-specific DO-END DO construct can be changed to a DO-CONTINUE statement and labeled with a new label. The appropriate statement label is inserted in the corresponding DO statement. DO WHILE statements and their corresponding ElND DOs are left intact.

The label numbers are initialized at the start of the file and after each END statement. Multiple definitions of a label are flagged with an error message; labels that are not referenced are flagged with a warning.

Miscellaneous Features. Blanks and tabs are stripped from the end of each line. Only tabs at the beginning of a line are converted to blanks and only if formatting is selected. Other tabs are left intact. All labels are right-justified in the label field (if either formatting or renumbering is selected).

All continuation characters can be set to a user-supplied character. They can also be renumbered so that the first numeric continuation character is changed to a 1 , and the continuation character increments on each following line until the end of the statement. The character after 9 becomes \# (since 0 is not allowed), then cycles to 1 again.

FORMRENUM can format and renumber lines that start with a special comment sequence. This is useful for commenting out sections of code or handling machine-dependent code. If you are interested in thi, capability, please contact the FORMRENUM sponsor.

\subsubsection{Qualifiers}

FORMRENUM qualifiers ( $q$ ualifier) are listed below.

\section{Formatting Qualifiers}

/FORMAT <default>

/FORMAT specifies that the file should be formatted.

\section{NOFORMAT}

NOFORMAT specifies that the indentation should not be changed. The other formatting qualifiers have no meaning. 
ANDENT $=n<3>$

/NDENT sets the block indentation (for DO and IF-THEN-ELSE blocks) to $n$ characters. By default, $n$ is 3 .

ANDCONT $=n$ <block indentation set by /INDENT>

INDCONT sets the continuation line indentation to $n$ characters. If $n$ is less than zero, the continuation line is indented with respect to the first line of the statement. By default, $i$ is equal to the block indentation.

ANDFORMAT $=n<0>$

/INDFORMAT sets the FORMAT statement indentation to $n$ characters.

\section{RenumberingQualifiers}

/RENUMBER <default>

/RENUMBER specifies that the file should be renumbered. This is the default.

\section{NORENUMBER}

NORENUMBER specifies that the labels should not be changed. The other renumbering qualifiers have no meaning.

\section{MABSTMT $=n<100\rangle$}

/LABSTMT sets the starting label number for non-FORMAT statements to $n$. If $n$ is less than or equal to zero, non-FORMAT statement labels are not renumbered.

RABFORMAT $=n<10000>$

/LABFORMAT sets the starting label number for FORMAT statements to $n$. If $n$ is zero, FORMAT statement labels are numbered the same as non-FORMAT statement labels. If $n$ is less than zero, FORMAT statement labels are not renumbered.

$\Lambda N C=n<10>$

INC sets the statement label increment for FORMAT and other statements to $n$.

\section{NAXDO}

NAXDO changes the DO-END DO construct into a DO-CONTINUE construct with appropriate labeling. If this option is selected, the/LABSTMT option must be a positive number. By default, the DO-END DO constructs are left intact. 
Miscellaneous Qualifiers

NUMBER <default >

NUMBER specifies that numeric continuation characters should be renumbered.

\section{NONUMBER}

NONUMBER specifies that numeric continuation characters should notbe renumbered. If the /CONT option is specified, all continuation characters are set to the default continuation character.

\section{/CONT $=" \alpha "$}

/CONT sets the default continuation character to $\alpha$. All the non-numeric continuation characters are set to $\alpha$. Numeric continuation characters are set to $\alpha$ if the NONUMBER option is specified. If the /NUMBER option is active and $\alpha$ is numeric, all continuation characters are numbered consecutively. By default, the continuation characters are not changed.

\section{/COMMENT $=" \alpha "$}

/COMMENT sets the default comment character (in column 1) to $\alpha$. If $\alpha$ is not a blank, the comment character in all comment lines (including blank lines) is changed to $\alpha$. If $\alpha$ is a blank, lines that consist of only a comment character are changed to blank lines. By default, the comment characters are not changed.

\section{MAXCONT $=n<19$ (the FORTRAN standard) $>$}

MAXCONT sets the maximum number of continuation lines for a statement to $n$. If this limit is exceeded, the statement is flagged with an error message.

\subsubsection{In-Source Control}

The program can be controlled with commands inserted in the source file. This is a useful way of implementing special features, such as indenting a loop in a CONTINUE-GOTO structure or numbering sections of a file differently. However, these commands should be used with care.

The commands are comment lines and must start in column 1 . The formatting commands are valid only if the/FORMAT option is specified and the renumbering commands are valid only if the/RENUMBER option is specified.

\section{Formatting Commands}

\section{C\$\$SOFF}

C\$\$OFF turns off the formatting. 


\section{CSSSON}

$\mathrm{C} \$ \$ \$ O N$ turns on the formatting.

\section{Cs\$s+n}

$\mathrm{C} \$ \$ \$+n$ increments the current indentation by $n$ characters. If $n$ is omitted, $n$ is assumed to be the block indentation.

\section{css\$-n}

$\mathrm{C} \$ \$ \$-n$ decrements the current indentation by $n$ characters. If $n$ is omitted, $n$ is assumed to be the block indentation.

\section{Renumbering Commands}

\section{CSS\$R OFF}

$\mathrm{C} \$ \$ \mathrm{R}$ OFF turns off the label renumbering. References to renumbered labels continue to be changed.

\section{CSSSR ON}

$C \$ \$ R$ ON turns on the label renumbering.

\section{C\$\$\$R nstmt nfmt}

$\mathrm{C} \$ \$ \mathrm{R} n \mathrm{nstm} t \boldsymbol{n f m} \mathrm{t}$ sets the next label number for non-FORMAT statements to $n \mathrm{stm} t$ and for FORMAT statements to $n f m t$. If $n s t m t$ or $n f m t$ is zero, the respective label remains unchanged. If $n s t m t$ or $n f m t$ is less than zero, the respective statement labels are not renumbered. This command affects only the labels in the current program unit (subroutine, function, etc.). At the start of the next program unit, the labels are reset as specified by the /LABSTMT and/LABFORMAT qualifiers.

\subsubsection{Errors and Restrictions}

If any errors are encountered in the input file, an error message is displayed on the screen and inserted in the output file (normally above the source line ihat caused the error). A warning message is displayed on the screen only. An error message in the output file starts with "\$\$\$." It is automatically stripped from the file when FORMRENUM is rerun (although it is regenerated if the error is not corrected). Since error messages are not compilable, the source should be error-free before compilation.

Most errors flagged would cause compilation errors, but some are generated by formatting and renumbering. A line-too-long error may result if the formatted or renumbered line is longer than the input line. A label-too-long error results if a debug line (which has a $D$ in column 1) has a five-character generated label. 
The input line is limited to 132 characters. Characters input in columns number 73 and after are treated as statements characters, not comment characters. Therefore, input lines cannot include a sequence field and should not continue a character string over two lines. If an output line is too long (72 characters for code and 80 characters for comments), the line is flagged with an error message, but up to 132 characters are output.

The source file can be in upper or lower or mixed case. The keywords "GOTO," "ENDIF," and other VAX-specific END keywords can be written as two words (e.g., "GO TO"). Most other keywords cannot be split. Bizarre (but legal) programming practices, such as splitting keywords over lines, will confuse FORMRENUM.

\subsubsection{Example}

The following is an example of a source file:

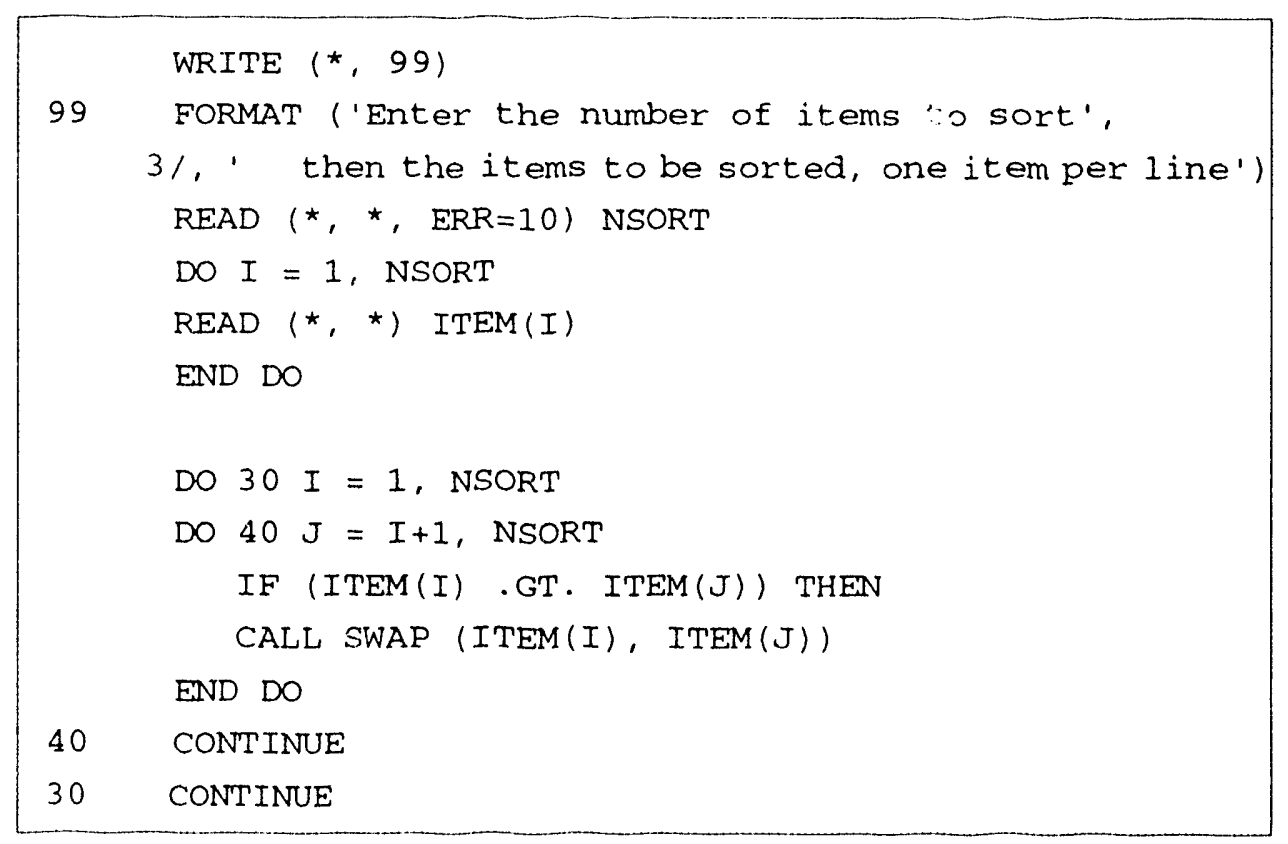


The following is the output source file after the FORMRENUM command has been issued with default qualifiers:

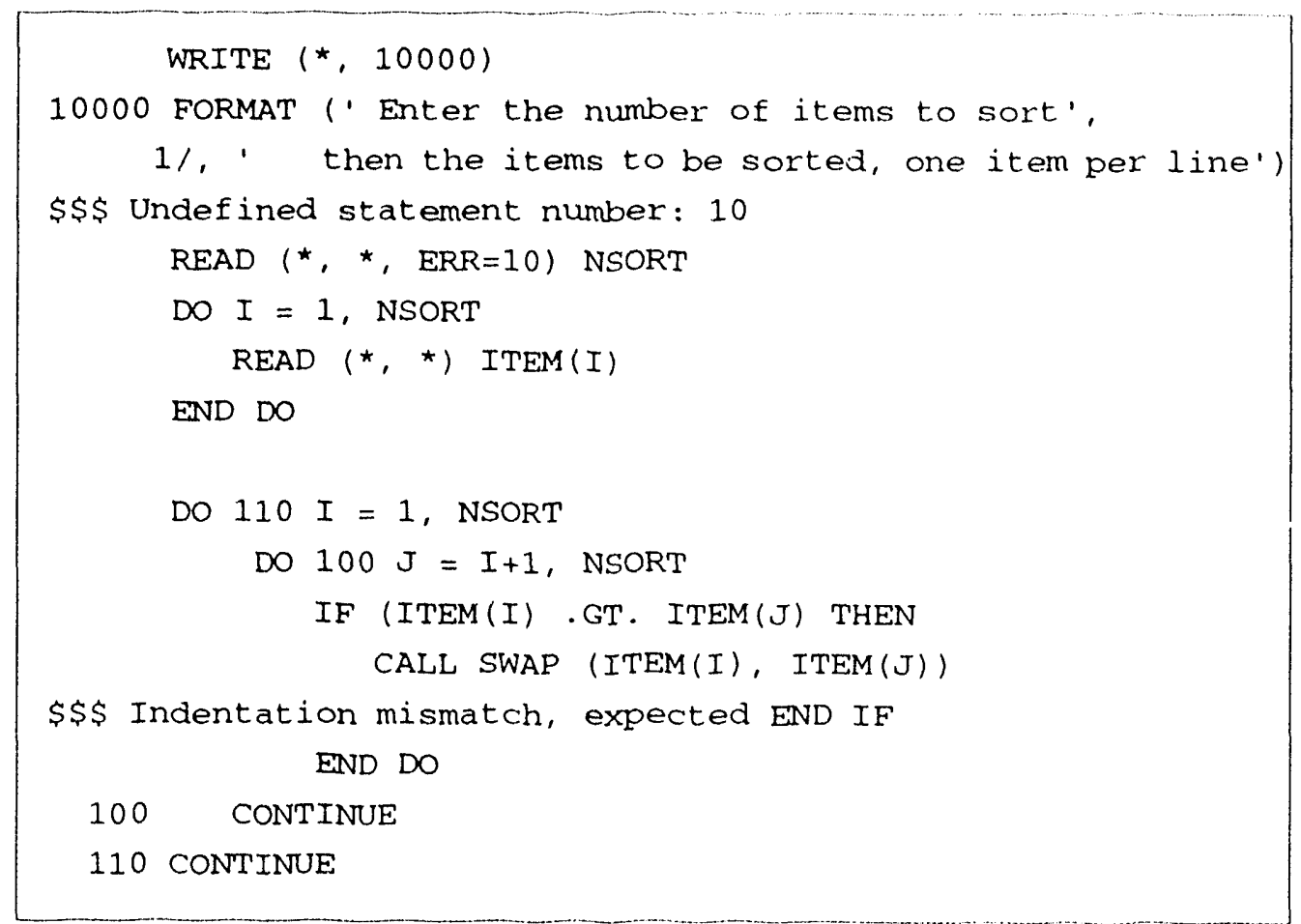




\subsection{HLPCHK, Check Format and Content of Software Help File}

\subsubsection{Sponsorship and Version}

Name: $\quad$ HLPCHK, a code to check the format and contents of a software help file

Author: $\quad$ J. S. Sandha and D. W. Osborn

Sponsor: $\quad$ K. A. Byle

Version/Date: $\quad$ C-2.01VV/September 1992

\subsubsection{Function}

HLPCHK is a code for checking the contents and format of a software help file. Its purpose is to ensure that the software help file contains the required identifiers and headers. If the identifier is not in the proper location, preceded and followed by the appropriate identifiers, the program displays an error message indicating that the identifier is out of sequence or missing.

\subsubsection{Execution}

At the system prompt, type the following:

\section{HLPCHK code_name.hlp}

where code_name. $h l p$ is the name of the CAMCON help file providing data for the software abstract. See ABSGEN for a description of how to extract code_name.hlp from the CAMCON help library.

\subsubsection{Required Identifiers and Headings}

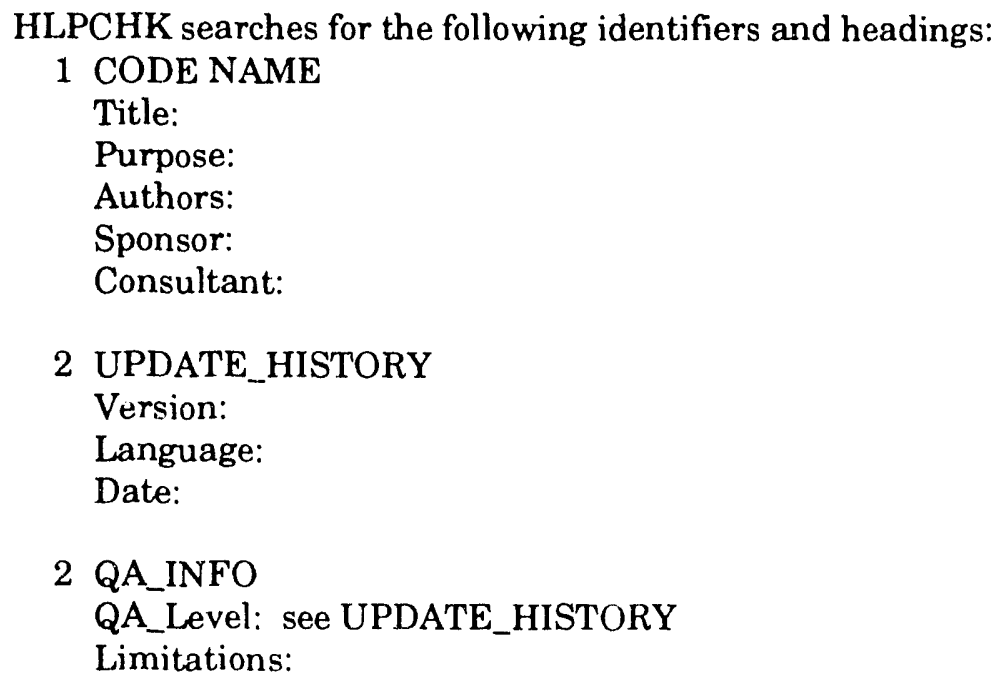


Keyword:

Origin:

Parent Program:

Classification:

Description: •

Type:

Verification:

Hand Calculation

In-Use Test

Comparison with Analytic Solutions

Comparison with Other Verified Programs

Parametric Sensitivity Analysis

Documentation:

User's Reference

Theoretical Reference

Verification/Sample Problems Reference

Other Reference

Primary Reference

2 EXECUTION:

2 EXAMPLE:

and either

2 COMMANDS

2 DATA INPUT

or

\section{a ROUTINES}

The general instructions given to software sponsors for constructing help files are given in Section 4.6, "On-Line Documentation." 


\subsection{LISTDCL, List DCL File}

\subsubsection{Sponsorship and Version}

Name: $\quad \cdots \quad$ LISTDCL, a code to create a listing of a DCL command file

Author/Sponsor: A. P. Gilkey

Version/Date: $\quad$ X-1.01VV/February 1991

\subsubsection{Function}

LISTDCL lists and prints a DCL source file in a standard format. The listing includes a count of the active DCL and comment lines.

\subsubsection{Execution}

At the system prompt type LISTDCL (this code is not available through CAMCONexec [Rechard, 1992]). The following items may follow LISTDCL on the command line; otherwise they will be prompted for:

- The name of the DCL file (default extension of .COM) or, if printing only, the listing file (default extension of .COMLIST)

- Whether a listing or printout is needed: <ALL>

- LIST to create listing only

- PRINT to print listing only

- ? to create listing and optionally print

- ALL to create listing and print

- If a printout is requested, the following information will be prompted for:

- The print queue (JOAN or ANNE) <JOAN>

- Thie print format per side ( 2 for two pages per side with border, 1 for one page per side with border, 0 for one page per side with no border) $\langle 0\rangle$

- Whether double-sided output is requestea $<\mathrm{NO}$, unless compressed $>$

- The printer tray (BOTTOM, MIDDLE, or TOP) <BOTTOM>

\subsubsection{Preparation}

A form feed forces a new page to begenerated. In addition, *NAME=routine_name line in the source code causes a new page to be generated and the routine_na me to be used as the routine name. The routine name is printed in the margin of the listing. This special line does not appear on the listing. 


\subsubsection{Output}

LISTDCL categorizes each input line and tallies the number of lines of each type for each routine and for all routines. The following counts are maintained and displayed:

- The total number of lines, including blank lines

- The number of lines with any DCL code

- The number of DCL statements (the same as above, but DCL continuation lines are not counted)

- The number of comment lines, including blank lines and DCL lines with trailing comments

- The number of comment lines with alphabetic characters, including DCL lines with trailing comments 


\subsection{LISTFORTRAN, List FORTRAN Source}

\subsubsection{Sponsorship and Version}

Name: $\quad$ LISTFERTRAN, a code to create a listing of a FORTRAiN source

Author/Sponsor: A. P. Gilkey

Version/Date: $\quad$ X-1.01VV/February 1991

\subsubsection{Function}

LISTFORTRAN lists and prints a FORTRAN source file in a standard format. The listing includes a count of the active FORTRAN and comment lines.

\subsubsection{Execution}

At the system prompt type LISTFORtran (this code is not available through CAMCONexec [Rechard, 1992]). The following items may follow LISTFORTRAN on the command line; otherwise they will be prompted for:

- The name of the FORTRAN file (default extension of .FOR) or if printing only, the listing file (default extension of .FORLIST)

- Whether a listing or printout is needed <ALL>:

- LIST to create listing only

- PRINT to print listing only

- ? to create listing and optionally print

- ALL to create listing and print

- If a printout is requested, the following information will be prompted for:

- The print queue (JOAN or ANNE) <JOAN>

- The print format ( 2 for two pages per side with border, 1 for one page per side with border, 0 for one page per side with no border) $<0>$

- Whether double-sided output is requested <NO, unless compressed $>$

- The printer tray (BOTTOM, MIDDLE, or TOP) <BOTTOM>

\subsubsection{Preparation}

A form feed forces a new page to be generated. Furthermore, LISTFORTRAN starts a new page whenever a PROGRAM, SUBROUTINE, FUNCTION, or ENTRY (if last statement was RETURN) statement is found. The routine name is printed in the margin of the listing.

In addition, $\mathrm{a}$ *NAME=routine_name line in the source code causes a new page to be generated and theroutine_name to be used as the routine name. An *END line causes a page break after the line. These special lines are useful for INCLUDE files and do not appear on the listing. 
If several routines are present in a single file and there are comments between the END and the routine start lines, the program must decide whether to associate these lines with the end of the last routine or the start of the current routine. Normally, they are associated with the start of the current routine unless one of the lines is a *NAME or *END line or a form feed.

\subsubsection{Output}

The program categorizes each input line and tallies the number of lines of each type for each routine and for all routines. The following counts are maintained and displayed:

- The total number of lines, including blank lines

- The number of lines with any FORTRAN code

- The number of FORTRAN statements (the same as above, but FORTRAN continuation lines are not counted)

- The number of comment lines, including blank lines and FORTRAN lines with trailing "!" comments 


\subsection{QA_PREP, Setup for QA_PRINT}

\subsubsection{Sponsorship and Version}

- Name:"

Author:

Sponsor:

Version/Date:
QA_PREP, a setup program for users of QA_PRINT •

D. W. Osborn

K. A. Byle

X-1.00VV/April 1992

\subsubsection{Function}

QA_PREP creates a file named ROUTINES.TXT, which is used as input into QA_PRINT.

\subsubsection{Execution}

At the system prompt, type the following (this code is not available through CAMCONexec [Rechard, 1992]):

\section{QA_PREP}

or

QA_PREPdirectory_name

QA_PREP will then scan the directory for all *.FOR and *.INC files and will produce the file ROUTINES.TXT as a result.

\subsubsection{Limitations}

QA_PREP assumes that there is only one main program file in the directory it is scanning.

\subsubsection{Preparation}

The software sponsor is required to provide only the directory that QA_PREP is to search.

\subsubsection{Example}

The following are examples of how to use QA_PREP:

\section{\$ QA_PREP CAMCON\$ROOT:[GENMESH]}

In the above example, QA_PREP will scan the directory CAMCON\$ROOT:[GENMESH] for all *.FOR and ${ }^{*}$.INC files. It will then build the file ROUTINES.TXT containing the result of its scan.

\section{\$ QA_PREP}

In this example, QA_PREP will ask the user the name of the directory to be scanned and then scan that directory for all *.FOR and *.INC files. It will then build the file, ROUTINES.TXT, containing the result of its scan. 


\subsection{QA_PRINT, Quality Assurance Notebook Print Procedure}

\subsubsection{Sponsorship and Version}

Nume: Q QA_PRINT, quality assurance notèbook print procedure

Author/Sponsor: J.S. Rath

Version/Date: $\quad$ C-1.06VV/April 1992

\subsubsection{Function}

QA_PRINT generates an $81 / 2 \times 11$ in. listing (hardcopy) of the source code, help file abstract, FLINT (FORTRAN code analyzer) output of the code, and the CAMCONexec driver for the software notebooks (see Section 4.7, "External Documentation").

\subsubsection{Execution}

At the system prompt, type QA_PRINT (this code is not available through CAMCONexec [Rechard, 1992]). Subsequent questions in the procedure file request the following information:

\section{Required}

- Name of the code to be printed in the QA format

- The program's source code directory (specify full directory path)

- File containing all the source code file names. In the CAMCON directories, this is typically called ROUTINES.TXT (see Section 12.11.5, below). Specify full directory path plus the file name if the file resides in a different directory than the source code. <ROUTINES.TXT>

- The program's help file (specify full directory path plus filename if file resides in a different directory than the source code). <PROGRAM.HLP>

Optional

- Request to cancel all print jobs. Typing NO_PRINTing in either upper or lower case will cancel all print jobs

In addition, by specifying all this information on the command line, the interactive mode may be bypassed. For more information on this enhancement, see Section 12.11.6, "Example." 


\subsubsection{Limitations}

QA_PRINT requires a file, ROUTINES.TXT, listing all files of the source code.

FLINT runs with the following options:

\$ FLINT /DLINES /FYI /GLOBAL/INCLUDE /LOCAL/NOWARNINGS /OUTPUT -

=FLINE /PARAMETERS /PORTABILITY=VAXVMS /SPLIT -

=FLINT /STATISTICS /SUMMARY /TREE

\subsubsection{Preparation}

Prior to executing QA_PRINT, the code sponsor must generate a file containing all source code file names, typically called ROUTINES.TXT. Proper generation of ROUTINES.TXT is essential for executing FLINT on the code and for generating a source code listing for the software notebooks.

If the user has been using the CAMCON_LIB routineCREATE_PROGRAM or UPDATE_PROGRAM, ROUTINES.TXT should already exist. However, the file is easy to generate. Either use QA_PREP or prepare the file manually as follows: Go to the source code directory and type the command:

\section{DIRECTORY/COLUMNS=1/OUTPUT=ROUTINES.TXT *.FOR,*.INC,*.CMN}

The above command generates a file with only one file name per record ( 1 column). Next, edit the ROUTINES.TXT file and remove all version identifiers (e.g., change SUBROUTINE.FOR;5 to SUBROUTINE.FOR). Also, remove all erroneous lines of text, such as blanks, directory names, total number of files, etc. In addition, check that the ROUTINES.TXT indeed contains all the types of files associated with their program, i.e., *.FOR, *.INC, *.CMN, etc.

The order that the source files are listed in ROUTINES.TXT is important. The ROUTINES.TXT file should first list FORTRAN files (usually *.FOR), followed by other files like INCLUDE files (usually *.INC or *.CMN), and any other types.

It is strongly recommended that the first FORTRAN file listed in ROUTINES.TXT be the main program (if you rename your main program file to AIMAIN.FOR, the above mentioned copy command will list files in ROUTINES.TXT in the correct order). 


\subsubsection{Example}

The following are QA_PRINT examples.

The first example uses the command line mode and assumes that the ROUTINES.TXT, GENMESH.SRC, and GENMESH.HLP files reside in the same directory as the source code (the dash is a record continuation mark).

\section{\$ QA_PRINT - GENMESH - CAMCON\$ROOT:[GENMESH.A_LEVEL] - ROUTINES.TXT - GENMESH.HLP}

The second example uses the command line mode and assumes that the user (code sponsor) is in the directory where the GENMESH source code exists. The example also shows how to use the fifth data field command line argument, NO_PRINTing, to execute QA_PRINT without sending the print jobs to the printer.

\section{\$ QA_PRINT GENMESH "'" "' "'" NO_PRINTing}




\subsection{UPDATE_PROGRAM, Compile, Update, and Link}

\subsubsection{Sponsorship and Version}

Name:

UPDATE_PROGRAM, $\infty$ code to compile and link a routine

Author/Sponsor:

A. P. Gilkey

Version/Date:

X-1.01VV/June 1992

\subsubsection{Function}

UPDATE_PROGRAM compiles the specified *.FOR file, updates the object library, and links the program. A debug version may also be updated in the sponsor's directory. CREATE_PROGRAM must be executed once before UPDATE_PROGRAM is executed.

\subsubsection{Execution}

At the system prompt, type the following (this code is not available through CAMCONexec):

\section{UPDATE_Programcode_name}

or

UPDATE_Program "code_name $e_{1}$ code_name $e_{2}, . .$.

where code_name is the name of the file to be compiled. As shown, multiple files can be specified for compilation by surrounding the file names in double quotes and separating them with commas.

\subsubsection{Preparation}

As UPDATE_PROGRAM compiles a specified source file(s), a listing file is created. Each listing file is deleted if the compile is successful. Also, the object file(s) are inserted or replaced in the object library named code_name_LIB.OLB. The individual *.OBJ files are deleted.

If there were no compile errors, the program is then linked to create an executablefile,code_name.EXE. The options for linking are "TRACE/MAP". If the symbol DEBUG_code_name is defined, a debug version of the code is also created. (Refer to CREATE_PROGRAM for an example of this symbol definition.) The given source file(s) are recompiled with debug options, the object library, code_name_LIB_DEBUG.OLB, is updated, and an executable file, code_name_DEBUG.EXE, is linked with the options /DEBUG/TRACE. 


\subsection{VAX/VMS Debugger}

\subsubsection{Sponsorship and Version}

- Name: $\quad$ VAXNMS Debugger

Author: $\quad$ Digital Equipment Corporation, Maynard, MA

Version/Date: $\quad$ VMS Version 5.4/June 19.90

\subsubsection{Function}

The VAX/VMS Debugger is an important tool for the CAMCON programmer. The Debugger is easy to implement and easy to use. The basic commands discussed here cover the majority of debugging situations.

\subsubsection{Reference}

More detail and more sophisticated commands can be found in the VMS Debugger Manual (DEC, 1988c).

\subsubsection{Execution}

To use the Debugger, the code must be compiled and linked with DEBUG options turned on as shown below. It has been found convenient to maintain two parallel libraries of object code-one for debugging, stored in the software sponsor's directory, and one for general use, stored in the [CAMCON] directory.

To compile source code, at the system prompt, type:

\section{FORTRAN/DEBUG/NOOP code_name.for}

To link the object code, at the system prompt, type:

\section{LINK/DEBUG code_name.obj, lib.olb/lib ...}

To execute, either build a symbol as follows:

code_name :=\$ \$dev:[path ...] code_name.exe and type code_name

or type:

\section{RUN dev:[path ...] code_name}


Execution will then take place in the Debugger automatically and will be user-controlled by typing commands at the Debug prompt, DBG>. The Debugger can be turned off by typing:

\section{RUN/NODEBUG code_name}

\subsubsection{Commands}

The most often-used commands are grouped under the following headings: Setting Break Points (SET BREAK), Executing (GO, STEP, EXIT, CTRL C), Listing Source Code (TYPE), Examining Values (EXAMINE), and Miscellaneous (SHOW CALLS and DEPOSIT).

Debugging commands can be abbreviated to the first set of unique characters. In the discussion below, these are shown with capital letters.

Setting Break Points

\section{SEt Break routine}

SET BREAK routine sets a break point at the beginning of routine; for example, SET B PREPRO.

\section{SEt Break \%LIne $n$}

SET BREAK \%LINE $n$ sets a break point at source line $n$ in the current routine; for example, SET B \%LI 122.

\section{SEt Break routind\%LI $n$}

SET BREAK routine $\backslash \%$ LI $n$ sets a break point at source line number $n$ in routine; for example, SET B PREPRO\\%LI 122.

\section{SEt Break routind\% $\mathrm{n}$ W WHEN (condition)}

SET BREAK routine $\backslash \% \mathrm{LI} n$ WHEN (condition) sets a break point at source line number $n$ in routine whencondition is true. The condition is written in the source code syntax, for example, SET B PREPRO \\%LI 122 WHEN (I .EQ. 20).

\section{SHow Break}

SHOW BREAK shows all defined break points.

\section{CANcel Break}

CANCEL BREAK cancels break points defined with the same format as SET BREAK, for example, CAN BR PREPRO\\%LI 122. 


\section{CANcol ALL}

CANCEL ALL cancels all break points.

\section{Executing}

Go (keypad: ,)

GO executes to the next break point, error, stop, or interrupt.

Stop (keypad: 0)

STEP executes a single line of code.

\section{Step $n$}

STEP $n$ executes $n$ lines of code.

Step/Nto (keypad: GOLD 0)

STEP/INTO steps into a subroutine. The default is to step over (i.e., execute the entire subroutine).

\section{Step/RETurn}

STEP/RETURN executes until a RETURN is encountered.

\section{Ctrl C}

Ctrl $\mathrm{C}$ interrupts the current execution and returns to the debug prompt.

EXIt

EXIT exits or quits the debug session.

Listing Source Code

Type

TYPE lists the current line of source code.

Typo n:m

TYPE $n: m$ lists lines $n$ to $m$ in the current routine.

\section{Type routinoln:m}

TYPE routine $\backslash n: m$ lists lines $n$ to $m$ in routine.

\section{SET SCope routine}

SET SCOPE routine sets the current routine for typing and examining. 
EXamine var, var $_{2} \cdots$

EXAMINE $v a r_{1}, v a r_{2}, \ldots$ lists the values of the variables $v a r_{1}, v a r_{2}$, etc. in their default format.

EXamine array (i:j, $n: m)$

EXAMINE array $(i, j, n: m)$ lists the values of array from $(i, n)$ to $(j, m)$.

\section{EXamine/ [BINARY, DECIMAL, HEXADECIMAL, OCTAL, ASCII, G_FLOAT, D_FLOAT]}

EXAMINE with any of the format types as a qualifier lists values in the designated format, overriding the default format.

Miscellaneous

SHow CAlls

SHOW CALLS lists the subroutine call sequences to the current position.

Deposit var=value

DEPOSIT var $=$ value resets the value of var.

For $i=m$ TO $n \mathrm{BY} j \mathrm{DO}(\quad)$

The FOR ... DO construct repeats the operation in parentheses; for example, FOR I = 1 TO 10 DO (DEPOSIT A(I)=0.).

\section{SPAWN command}

SPAWN command executes a DCL command and then returns to debug; for example, SPAWN TYPE GENMESH.INP.

\section{EValuate expression}

Evaluates a simple arithmetic expression of variables or array elements. 


\section{References}

ANS!C. 1989. Programming Language C. New York, NY: American National Standaras Institute, Inc.

ANSI FORTRAN. 1978. American National Standard Programming Language FORTRAN. ANSI X3.9-1978. New York, NY: American National Standards Institute, Inc.

Bertozzi, G., A. Saltelli, and H.C. Burkholder. 1985. "A Performance Assessment Code for Geological Fepositories of Radioactive Waste," International Topical Meeting on High-Level Nuclear Wa ste Disposal: Technology and Engineering, Pasco, WA, September 24, 1985. Columbus, OH: Battelle Press. 607-616.

Bertram-Howery, S.G., and R.L. Hunter, eds. 1989. Preliminary Plan for Disposal-System Characterization and Long-Term Performance Evaluation of the Waste Isolation Pilot Plant. SAND890178. Albuquerque, NM: Sandia National Laboratories.

Campbell, J. E., C. D. Leigh, and D. E. Longsine. 1991. NEFTRAN-S: A Network Flow and Cointaminart Transport Model for Statistical and Deterministic Simulations Using Personal Computers. SAND90-1987. Albuquerque, NM: Sandia National Laboratories.

DEC (Digital Equipment Corporation). 1988a. VMS Librarian Utility Manual, VMS Version 5.0, Programming Subkit, Vol. 2B. Maynard, MA: Digital Equipment Corporation. Lib-3 to Lib-10.

DEC (Digital Equipment Corporation). 1988b. VAX FORTRAN Language Reference Manual, VAX FORTRAN Version 5.0. Maj'nard, MA: Digital Equipment Corporation.

DEC (Digital Equipment Corporation). 1988c. VAX FORTRAN User's Manual, VAX FORTRAN Version 5.0. Maynard, MA: Digital Equipment Corporation.

DEC (Digital Equipment Corporation). 1990.VMS Debugger Manual, VMS Version 5.9. Maynard, MA: Digital Equipment Corporation.

Dormuth, KW. and G.R. Sherman. 1981.SYVAC: A Computer Program for Assessment of Nuclear Fuel Wa ste Management Systems, Incorporating Parameter Variability. AECL Report 5814. Pinawa, Manitoba, Canada:Atomic Energy of Canada Limited.

EPA. 1985. "40 CFR 191: Environmental Standards for the Management and Disposal of Spent Nuclear Fuel, High-Level and Transuranic Radioactive Wastes: Fina! Rule," Federal Register. Vol 50, no. 182, 38066-38089.

E ickson, K.M., and R.W. Simons. 1982. Functional Specification of the Sandia Virtual Dev "e Interface (SVDI). SAND8 L-1900. Albuquerque, NM: Sandia National Laboratories.

Flanagan, D. P., W. C. Mills-Curran, and L. M. Taylor. 1986. SUPES - A Software Utilities Package for the Engineering Sciences. SAND86-0911. Albuquerque, NM: Sandia National Laboratories.

Gilkey, A.P. 1988. ALSEBRA - A Program That Algebraically Manipulates the Output of a Finite Element Analysis (Exodus Version). SAND88-1431. Albuquerque, NM: Sandia National Laboratories. 
Gilkey, A.P., andJ. H. Glick. 1989.BLOT - A Mesh and Curve Plot Program for the Output of a Finite Element Analysis. SAND88-1432. Albuquerque, NM: Sandia National Laboratories.

Goodwin, B.W., T.H. Andres, P.A. Davis, D.M. Leneveu, T.W. Melnyk, G.R. Sherman, and D.M. Wuschke. 1987. "Post-Closure Environmental Assessment for the Canadian Nuclear Fuel Waste Management Program," Waste Management. Vol. 8, nos. 2-3, 241-272.

Helton, J. C., J. W. Garner, R. D. McCurley, and D. K. Rudeen. 1991. Sensitivity Analysis Techniques and Results for Performance Assessment at the Waste Isolation Pilot Plant. Contractor Report SAND90-7103. Albuquerque, NM: Sandia National Laboratories.

IAEA(International Atomic Energy Agency). 1989. Evaluating the Reliability of Predictions Made Using Environmental Transfer Models. Safety Series Report No. 100. Vienna, Anstria: International Atomic Energy Agency.

Kernighan, B. W. and P. J. Plauger. 1974. The Elements of Programming Style. New York, NY: McGraw-Hill.

Kernighan, B. W. and D.M. Ritchie. 1988. The C Programming Language. 2nd ed. Englewood Cliffs, NJ: Prentice Hall.

Marietta, M. G., S. G. Bertram-Howery, D. R. Anderson, K. F. Brinster, R. V. Guzowski, H. J. Iuzzolino, and R. P. Rechard. 1989. Performance Assessment Methodology Demonstration: Methodology Development for Evaluating Compliance with EPA 40 CFR 191, Subpart B for the Waste Isolation Pilot Plant. SAND89-2027. Albuquerque, NM: Sandia National Laboratories.

McLendon, P. 1991. Silicon Graphics Graphics Library Programming Guide. Document number 007-1210-040. Mountain View, CA: Silicon Graphics, Inc.

Mills-Curran, W.C., A.P. Gilkey, and D.P. Flanagan. 1988. EXODUS: A Finite Element File Format for Pre-and Post-Processing. SAND87-2957. Albuquerque, NM: Sandia National Laboratories.

Osborne, W.M. 1988. "Developers Must Design in Maintainability," IEEE Software. Vol. 5, no. 3, 104105.

Powers, D.W., S.J. Lambert, S.-E. Shaffer, L.R. Hill, and W.D. Weart, ed. 1978. Geological Characterization Report, Waste Isolation Pilot Plant (WIPP) Site, Southeastern New Mexico. SAND78-1596. Albuquerque, NM: Sandia National Laboratories. Vols. 1-2.

Rechard, R. P. 1989. Review and Discussio of Co e Linkage and Data Flow in Nuclear Waste Compliance Assessments. SAND87-2833. Albuquerque, NM: Sandia National Laboratories.

Rechard, R. P. 1991. "Overview of Calculat ions," Preliminary Comparison with 40 CFR Part 191, Subpart B fur the Waste Isolation Pilot Plan", December 1991. Volume 2: Probability and Consequence Modeling. SAND91-0893/2. Albuquerque, NM: Sandia Nationai Laboratories.

Rechard,R.P.ed. 1992.User's Reference Manual for CAMCON: Compliance Assessment Methodology Controller, Version 3.0. SAND90-1983. Albuquerque, NM: Sandia National Laboratories.

Rechard, R.P., P.J. Roache, R.L. Blaine, A.P. Gilkey, and D.K. Rudeen. 1991. Quality Assurance 
Procedures for Computer Software Supporting Performance Assessments of the Waste Isolation Pilot Plant. SAND90-1240. Albuquerque, NM: Sandia National Laboratories.

Rechard, R.P., D.K. Rudeen, and P.J. Roache. 1992a. Quality Assurance Procedures for Analyses and Report Reviews Supporting Performance Assessments of the Waste Isolation Pilot Plant. SAND910428. Albuquerque, NM: Sandia National Laboratories.

Rechard, R.P., K.M. Trauth, and R.V.Guzowski. 1992b.Quality Assurance Procedures for Parameter Selection and Use of Expert Judgment Panels Supporting Performance Assessments of the Waste Isolation Pilot Plant. SAND91-0429. Albuquerque, NM: Sandia National Laboratories.

Red-Horse, J.R., W.C. Mills-Curran, and D.P. Flanagan. 1990. SUPES Version 2.1: A Software Utilities Package for the Engineering Sciences. SAND90-0247. Albuquerque, NM: Sandia National Laboratories.

Rottler,J.S., A.P. Gilkey, and P.L. Hopkins. 1986. Guidelines for Computer Soft ware Development in Directorate 1500. SAND86-0378. Albuquerque, NM: Sandia National Laboratories.

Saltelli, A., G. Bertozzi, and D.A. Stanners. 1984. LISA: A Code for Safety Assessment in Nuclear Waste Disposal Program Description and User Guide. Luxembourg:Commission of the European Communities.

Saltelli, A., T. Homma, P. Prado, and C. Torres. 1990. "Development of the LISA Code," Proceedings of the International Symposium on Safety Assessment of Radioactive Waste Repositories, Paris, France, October 9-13, 1989. Paris, France: Organisation for Economic Co-operation and Development. 639-648.

Sherman, G.R., K.J. Hoffman, and D.C. Donahue. 1985. "The Program SYVAC, For Stochastic Assessment of Nuclear Fuel Waste Disposal," Waste Management '85: Waste Isolation in the U.S., University of Arizona, Tucson, AZ, March 24-28, 1985. Ed. R.G. Post. Tucson, AZ: University of Arizona. 403-408.

Taylor, L.M., D.P. Flanagan, and W.C. Mills-Curran. 1987. The GENESIS Finite Element Mesh File Format. SAND86-0910. Albuquerque, NM: Sandia National Laboratories.

Unsworth, M.D. 1989.VANDAL Technical Overview. DOE/RW/89.107. London, England:Department of the Environment.

Weiss, E. H. 1982. The Writing System for Engineers and Scientists. Englewood Cliffs, NJ: Prentice-Hall.

\section{WIPP Performance Assessment Procedures}

PAP02. 1993.Computer Software Supporting Performance Assessment of the Waste Isolation Pilot Plant. Albuquerque, NM: Sandia National Laboratories.

PAP05. 1993. Report Review Quality Assurance Procedures.. Albuquerque, NM: Sandia National Laboratories. 


\section{WIPP Quality Assurance Procedures}

QAPD. Sandia National LaboratoriesWIPP Quality Assurance Program Description. Department 6303, Current Revision. Albuquerque, NM: Sandia National Laboratories.

QAPP. 1990. Sandia National Laboratories Nuclear Waste Technology Department Waste Isolation Pilot Plant Quality Assurance Program Plan. Department 6340, Current Revision. Albuquerque, NM: Sandia National Laboratories. 


\section{Appendix A:}

Assessing the Performance of the WIPP

Assessing the compliance of the Waste Isolation Pilot Plant (WIPP) is reviewed here to provide the programmer with background on the demands placed on the users of CAMCON software and thereby help them better understand the customer's needs. In general, the discussion in this appendix focuses on the mechanics of the calculations (the "how") rather than on the more theoretical foundations (the "why"). If nothing else, this chapter strives to show the fundamental importance of software in the task of assessing the compliance of the WIPP with numerous regulations. The assessment task, in fact, would be impossible without this software.

An extensive description of (1) the WIPP can be found in Bertram-Howery and Hunter, eds., 1989, and (2) performance assessments (PA) can be found in Helton, 1991. In addition, the theoretical foundation for Sandia's approach to PA is thoroughly explained in reports by Tierney, 1991, and Helton et al., 1991.

\section{A.1 Background on the WIPP}

\section{A.1.1 Purpose}

The Department of Energy (DOE) was authorized by Congress in 1979 to build the Waste Isolation Pilot Plant(WIPP) as a research and development facility to safely manage, store, and eventually dispose of transuranic (TRU) waste generated by DOE defense programs (WIPP Act, 1979). Only after demonstrating compliance with the Environmental Protection Agency's (EPA) standard 40 CFR 191, Subpart B (EPA, 1985) and other laws and regulations (e.g., RCRA, 1976, and NEPA, 1969) will the DOE permanently dispose of TRU waste at the WIPP repository.

\section{A.1.2 Waste}

The TRU waste generated by nuclear weapon facilities consists of laboratory and production trash such as glassware, metal pipes, disposable laboratory clothing, cleaning rags, solidified sludges, etc. The trash is contaminated by $\alpha$-emitting, TRU elements (atomic numbers greater than uranium [92]) with half-lives greater than 20 years and curie contents greater than $100 \mathrm{nCi} / \mathrm{g}$ waste. Other radionuclide contaminants include uranium and several radionuclides with half-lives of less than 20 years. Approximately 60 percent of the trash may also be co-contaminated with RCRA (1976) hazardous waste such as lead (WEC, 1990).

\section{A.1.3 Location}

The WIPP site is located within a large sedimentary basin, the Delaware Basin, in southeastern New Mexico, an area of low population density, approximately $42 \mathrm{~km}(26 \mathrm{mi})$ east of Carlsbad. 


\section{A.1.4 Repository}

The repository is located in the Delaware Basin because the $600-\mathrm{m}(2,000$-ft)-thick Salado Formation of marine bedded salts eventually encapsulates the nuclear waste through salt creep. The bedded salts, consisting of thick halite and interbeds of minerals such as clay and anhydrides approximately 255 million years old ${ }^{1}$ (Late Permian Period), do not contain flowing water.

The repository level is located within these bedded salts about $658 \mathrm{~m}(2,160 \mathrm{ft})$ below the surface (381 $\mathrm{m}[1,250 \mathrm{ft}]$ above sea level). The WIPP repository is composed of a single underground disposal level connected to the surface by four shafts. The repository level consists of an experimental area at the north end and a disposal area at the south end.

\section{A.1.5 Sandia Role}

Besides the DOE project office in Carlsbad, NM, which oversees the project, the WIPP has two major participants (WIPP Strategy, 1989): (1) Sandia National Laboratories (Sandia) functions as scientific investigator/advisor, and (2) Westinghouse Electric Company (WEC) is responsible for the WIPP site operations. A past participant was Bechtel, Inc., who was responsible for repository design.

The specific tasks of Sandia are as follows:

- Characterizing the disposal system and surrounding region and responding to specific concerns of the State of New Mexico

- Assessing the performance of the WIPP (i.e., assessing regulatory compliance with 40 CFR 191 , Subpart B, except the Assurance Requirements)

- Performing analytic, laboratory, field experiments, and applied research to support disposal system characterization and performance assessment relevant to nuclear waste disposal in salt

- Providing ad hoc scientific and engineering support (e.g., supporting environmental assessments)

\section{A.2 Performance Assessment of the WIPP}

\section{A.2.1 Compliance Strategy}

A performance assessment (PA) is the general process of a ssessing whether a system meets a set of performance criteria. For assessments of long-term consequences of the full-scale Waste Isolation Pilot Plant (WIPP), the technique is performed by necessity with computer simulations. Hence, the system for the PA is a computational model that represents the WIPP's deep, geologic disposal system for waste

1. This time period reflects the revised 1983 geological time scale. 
contaminated with transuranic radionuclides and hazardous chemical constituents. Because it is not practical to build, modify, and solve a single, three -dimensional model of the WIPP that contains enough detail to simulate the phenomena, events, and features of the entire disposal system, several models (a composite function) are used to simulate the WIPP's major physical components.

For the WIPP, the performance criteria are various environmental metrics in government regulations. The Environmental Protection Agency's (EPA) Containment Requirements in 40 CFR 191 (Environmental Radiation Protection Standards for Management and Disposal of Spent Nuclear Fuel, High-Level and Transuranic Radioactive Waste) is a regulation that specifies criteria that must be met for the next $10,000 \mathrm{yr}$. Because complying with this regulation is a computationally demanding exercise, it is an excellent driver for the development of the modeling methodology and analysis tools used by Sandia National Laboratories. It is expected that, with minor modifications, these same tools can be used to assess compliance with other long-term environmental regulations.

Although 40 CFR 191 does not require that risk be evaluated, its requirements are risk-based. Also 40 CFR 191 explicitly recognizes the uncertainty in scientific explanation (i.e., that hypotheses can only be judged based on current knowledge and therefore must be continuously refined as knowledge increases). Consequently, Sandia's approach to evaluating WIPP compliance resembles a scenariobased, probabilistic risk assessment in which the performance metric-radioactive release specified in probabilistic terms-is a complementary cumulative distribution function (CCDF) (or probability of exceedance curve). In essence, the performance assessment can be viewed as the process that estimates this CCDF.

Sandia's methodology approach was developed simultaneously with both the EPA's standards and the Nuclear Regulatory Commission's regulations. The methodology has been used to evaluate many early options for deep geologic repositories (e.g., Chu et al., 1983; Campbell and Cranwell, 1988) and to perform feasibility studies with the international Nuclear Energy Agency for subseabed disposal in deep oceans (e.g., Marietta and Simmons, 1988). These exploratory computational tools provided guidance for the methodology currently used in evaluating long-term performance of the WIPP.

\section{A.2.2 Six General Tasks of PA}

A convenient way to mathematically describe a $\mathrm{PA}$ is by viewing the risk-based perforrnance criterion of the Containment Requirements in 40 CFR 191 as a set of ordered triples (Helton, 1993) that consists of answers to the following three questions:

- What can happen? (scenarios)

- What are the outcomes of these things happening? (consequences of scenarios)

- How likely are these things to happen? (probabilities of scenarios)

The answer to the first question is a systematic collection of features, events, and phenomena, which 
provides a set of similar occurrences that specify what may happen to the disposal system in the future, $S_{j}(x)$. Answering the sec $u n d$ question requires the use of a modeling system to estimate consequences of the $j$ th scenario occurring, $C\left(S_{j}(x), x\right)$. The answer to the third question is an estimate generated by a modeling system of how likely these scenarios are to happen, i.e.., the probability of the $j$ th scenario, $P\left(S_{j}(x), x\right)$. ACCDF displays the second and third elements, consequence and probability, of this ordered triple. Uncertainty in the calculation of the CCDF can arise from any of the three elements of the triplet. (scenario, consequence, and probability) or the underlying parameters $\left(\mathbf{x}=x_{1}, \ldots, x_{n}\right)$. The WIPP PA Department uses a Monte Carlo technique to propagate uncertainty through the PA and thereby quantitatively evaluate the uncertainty in the calculation. Although the predictive uncertainty cannot be evaluated quantitatively (e.g., through a parameter), it may be evaluated qualitatively by peer review (Rechard et al., 1992a; 1992b).

The theoretical foundations of CCDF construction are explained elsewhere (e.g., Tierney, 1991: Helton, 1993); however, the mechanical process of a PA consists of six general tasks. The components of these basic tasks are shown below:

1. WIPP disposal system and regional characterization through collection of data on waste properties, facility design and regional geology and hydrology

2. Scenario development, $S,(x)$, which identifies the features, events, and phenomena whereby contaminants might be released to the EPA-defined accessible environment, and scenario selection for modeling

3. Consequence analysis, which predicts contaminant amounts released, $C(S,(x), x)$, including evaluating the uncertainties associated with the predictions

4. Probability modeling, which provides estimates of the likelihood that the selected scenarios willoccur, $P(S,(x), x)$

5. Regulatory compliance assessment through construction of CCDFs and other performance metrics and comparison of them with long-term environmental regulations

6. Sensitivity analysis, which determines the parameters that most influence the performance metrics and thereby provide guidance to the WIPP project

The first two PA tasks listed above are referred to collectively as model conceptualization (Figure A1). Characterization of the disposal system and surrounding regional hydrology has been in progress since project inception in 1975 (e.g., Powers et al., 1978) and is nearing completion. Screening of events and processes that may affect performance of the system during the next 10,000 years is also nearly complete, and significant summary scenarios have been identified for consideration in consequence modeling (Guzowski, 1990; Helton, 1991) where scenario means a class or grouping of similar possible future conditions (histories) of the disposal system.

For Task 3, a probability model is developed to evaluate probabilities of detailed scenarios for analysis. Currently, the probabilities are evaluated assuming human intrusion (drilling for resources) is a Poisson process (Helton, 1991; Miller and Freund, 1977, p. 81). 


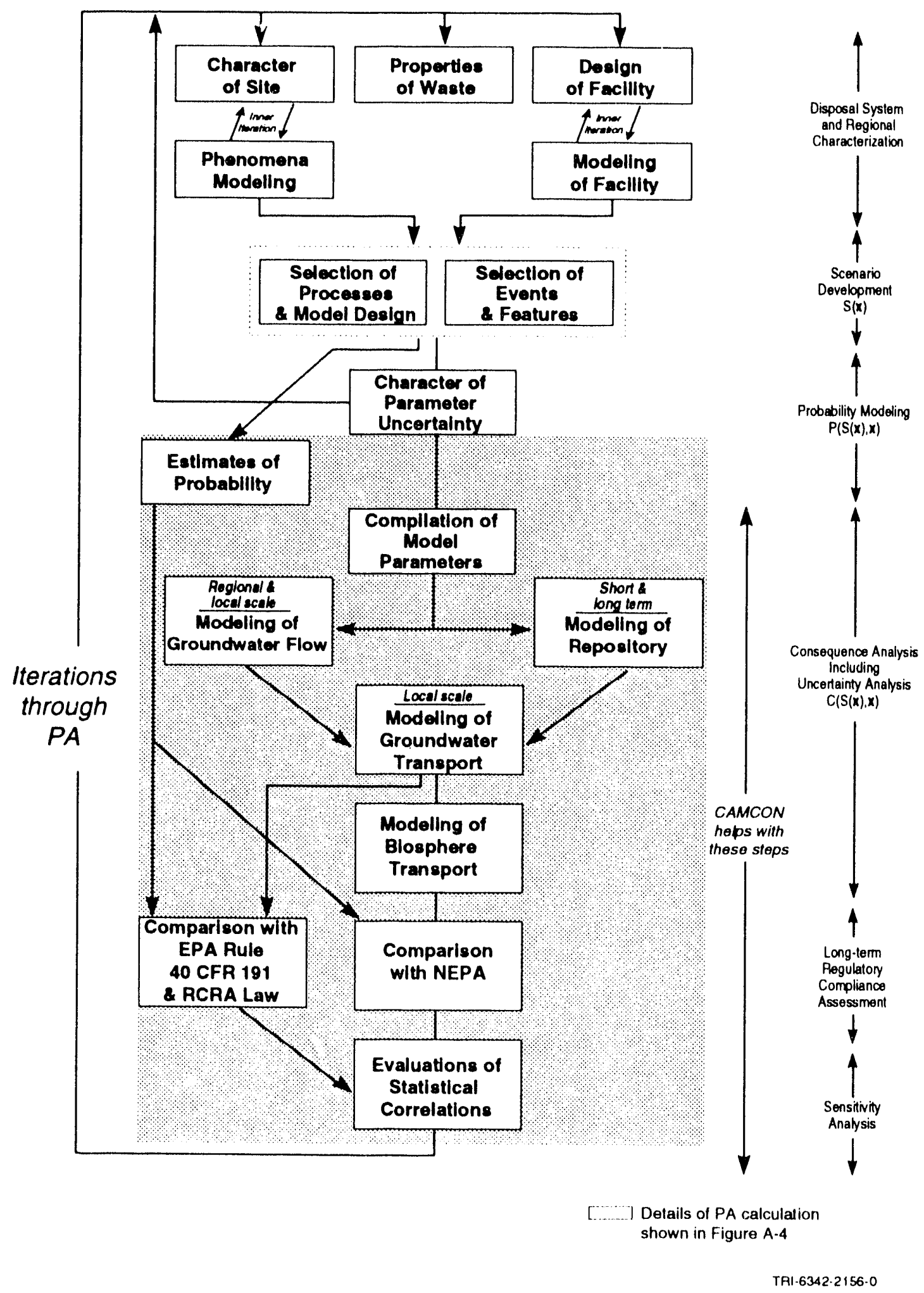

Figure A-1. Sandia's method for assessing WIPP repository compliance involves six tasks (after Rechard, 1989, Figure 3.1). 
A major portion of the methodology consists of simulating physical processes to estimate the amount of radionuclides released to the accessible environment following the hypothetical event that someone drills into the repository over the next 10,000 years. This process is referred to as consequence modeling and actually is a composite function of several models (Task 4) (Figure A-2). This task is more thoroughly described in the next section.

Several generic computational models are used to simulate major physical components of the WIPP disposal system (Figure A-2). Usually the codes are generic and implement a variety of mathematical models using several numerical solution techniques. Hence, some codes are used to model several different physical features of the WIPP and are repeated in several places. Furthermore, the need to benchmark results requires that several different codes be able to model similar features and phenomena; thus, some WIPP features show more than one code being used (refer to Rechard, 1992, for description of codes).

Because of imprecisely known parameters, uncertainty is incorporated into the performance assessment through a Monte Carlo analysis (part of Task 4), since Monte Carlo analysis can accommodate large uncertainty in input and large nonlinearity in the models. Monte Carlo analysis consists of first identifying the important parameters to vary and assigning ranges and distributions.

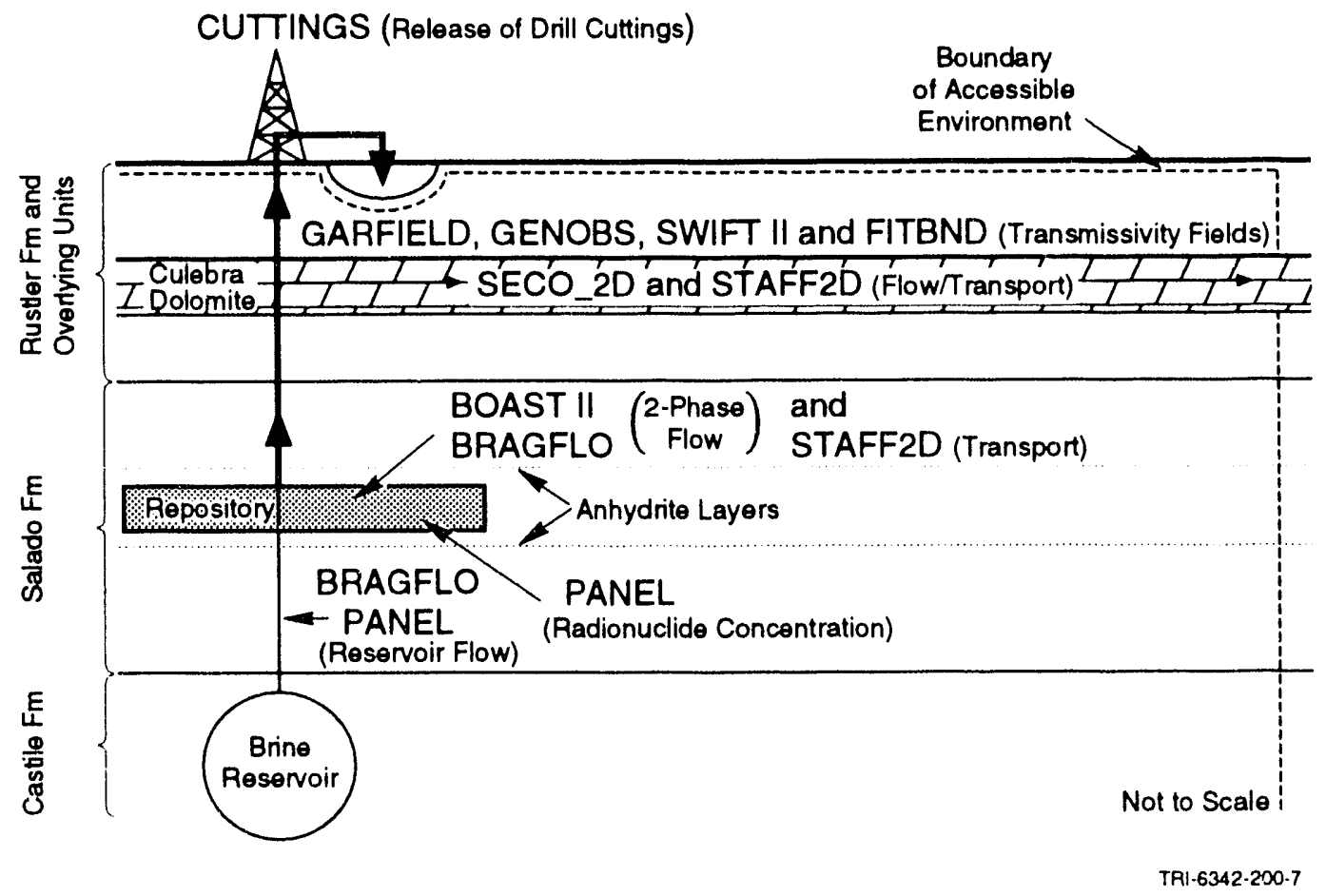

Figure A-2. Major computational models (codes) and the physical features they simulate in the WIPP disposal system (after Rechard, 1991, Figure 1-2). (Refer to User's Reference Manual for CAMCON [Rechard, 1992] for descriptions of codes listed.) 
Second, sample elements are generated from these distributions. In the WIPP performance assessment, Latin hypercube sampling (LHS) is used to minimize the number of sample elements needed to capture variability in the parameters adequately.

From the consequence results using Monte Carlo analysis, the final two tasks naturally follow. In Task 5, the results are compared with the regulations. In Sandia's approach to Task 5, estimated releases are combined into CCDFs (exceedance probability curves) as suggested in Appendix B, 40 CFR 191.

Task 6, performing sensitivity analyses, is a significant part of the WIPP performance assessment. Sensitivity analysis involves determining the contributions of an individual parameter to influence the consequences and its uncertainty. The analysis involves plotting scatter plots and developing regression models between the parameters and the various results. Two important purposes of the sensitivity analysis are (1) to help gain understanding and insight about the WIPP disposal system and (2) provide input so that decision makers can allocate data collection resources toward those parameters that most influence the results (e.g., CCDF), based on what is already known about the site. This thorough analysis also helps verify the correctness of the calculations.

\section{A.2.3 Multiple Iterations through PA}

The strategy of performing several PA iterations is beneficial because each iteration brings new information to the process. Initially, available data and information are used to develop preliminary scenarios, which are then analyzed with simple models to calculate bounding results. If these initial results are either indefensible or indecisive, better data, more complete conceptual models, and more complex computational models are used for subsequent calculations, which incorporate additional details of known phenomena, as appropriate. By repeating this process, defensible calculations are eventually constructed on which a final decision about radioactive waste disposal at the WIPP can be made. Sandia's PA Department has performed periodic PAs of the WIPP since 1989 (Marietta et al., 1989; Bertram-Howery et al., 1990; WIPP PA Division, 1991; WIPP PA Department, 1992).

Iterative refinement is not necessarily confined to the overall PA but can also occur in many subprocesses (Morgan and Henrion, 1990). Although iterations frequently occur during disposal system design and characterization, they are not limited to this step and can occur elsewhere within the PA process. A particularly important subprocess iteration for the PA is the assignment of parameters appropriate to the scale and detail of the models chosen for the PA analysis, given the facility design and what is known about phenomena at the site.

Multiple PA iterations achieve six goals (Rechard et al., 1992a; Rechard, 1989):

- The analysis team focuses on customer and stakeholder expectations and, through multiple iterations, can alter the process to satisfy a customer or stakeholder expectation; conversely, the customers and stakeholders are given several opportunities to learn about the PA process. This activity is especially important for decisions such as nuclear waste disposal, which must be made by more than one authority or agency over a period of years. 
- Analysts develop insight about the disposal system because different hypotheses about the system performance can be tested.

- Through periodic peer reviews, analysts receive scientific feedback from multidisciplinary teams.

- In some instances, analyses can be partially validated through multiple iterations (e.g., when critical questions are posed in early iterations and then validated in later iterations based on newly collected data).

- Multiple iterations of sensitivity analyses for both system-wide and individual components provide project managers and, more important, the participants themselves, with information to help more wisely allocate resources for data collection and either elaborate or simplify models.

- Large, long-term projects such as the WIPP can be divided into several parts with more easily agreed-upon constraints and schedules (i.e., the project becomes a series of small steps repeated several times-a useful technique to produce a quality product).

\section{A.3 Overview of Consequence Modeling}

The following discusses the calculations using the framework of the PA methodology. Task 4, consequence modeling, is emphasized. Prior to discussing the mechanics of the calculations, some general issues are discussed.

\section{A.3.1 Model Subdomains in Consequence Modeling}

A three-dimensional model with the necessary detail to analyze hydrologic processes (or transport) both far from and near to the repository for every scenario would take too long to build, modify, and solve. Hence, models are scaled to make an analysis tractable. However, disposal system analysis has an additional complication: the use of different model scales or subdomains that must pass pertinent results to each other. The modeling systems associated with the different subdomains are in addition to those required for evaluating different processes such as fluid flow or radionuclide transport.

Modeling Scales. For disposal systems, three scales are frequently used (Figure A-3):

- Repository

- Regional

- Local

The primary function of the repository scale model is to calculate migration of radionuclides (or other hazardous waste) from the consolidated waste-disposal rooms through the seals, drifts, and shafts. The time versus radionuclide concentration curves are then used as a source term for evaluating transport in the local scale models. The complexity of the physical processes affecting the repository requires that initial source-term calculations involving several codes modeling various coupled processes be carefully linked together. In other words, a microcosm of the overall compliance assessment is needed. For the WIPP project, the analysis of the repository scale has been divided into a cuttings model system and a repository/shaft/borehole modeling system, which are described later. 


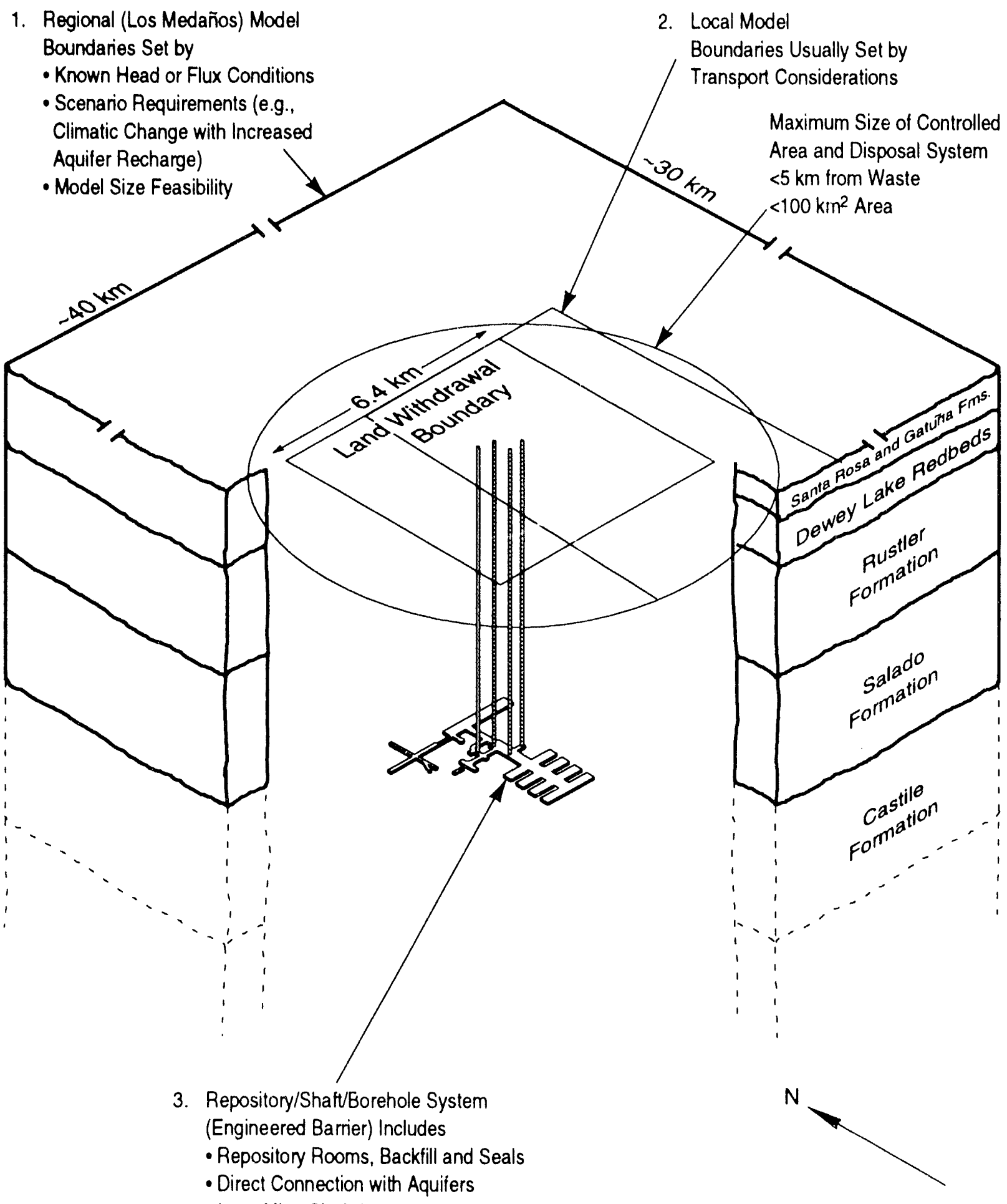
(e.g., Mine Shafts)

Not to Scale

TRI. $6330 \cdot 7 \cdot 8$

Figure A-3. Three model scales are typically used in compliance assessment (after Bertram-Howery and Hunter, eds., 1989, Figure II-1). 
The primary function of the regional scale model is to establish ground water flow patterns in a large region bounding the controlled area of the repository. The flow conditions (e.g., heads, fluxes, or brine densities) are then used to establish boundary conditions for the next scale model, the local model.

The function of the local scale model is to predict more precisely the flow field within the controlled area for transport calculations. The local scale model is completely embedded with the regional scale model. Two scales, a regional and a local scale, are used in the Culebra ground water modeling system in the analysis of the WIPP.

Setting Boundary Conditions. Embedding models can reduce unnecessary model complexity (operations per grid point per time step) and thereby model run times. However, when embedding models, an important process that affects the flow of data is setting boundary conditions. The process of embedding meshes as previously described involves interpolating from a coarse grid to a fine grid just the opposite of what is mathematically convenient. Simple or complex interpolation schemes can cause discontinuities in the fields, the simple by ignoring too much detail and the complex by fabricating more detail than is warranted from the information contained in the coarse mesh.

The development of interpolation programs is not trivial. Several questions must be answered:

- How will changes in vertical resolution of stratigraphy between the regional and local model be handled?

- How will time scales between the local and regional models be separated to permit different time steps, thereby increasing computational efficiency?

- How many surrounding grid points will be included in spatial interpolations?

\section{A.3.2 Modeling Systems}

As alluded to above, the WIPP disposal system was divided into three systems for modeling: Repository/Shaft/Borehole, Culebra Groundwater Flow and Transport, and Cuttings. Each of these modeling systems are analyzed in parallel. The results from these consequence modeling systems are combined with the results from the probability model to construct the complementary cumulative distribution functions (CCDFs) for the regulatory assessment and sensitivity analysis tasks. Besides the model and parameter selections, the general steps in each modeling system are(1) data preparation, either from the parameters or from results stored in the data bases, (2) simulation, and (3) examination of intermediate results (Figure A-4).

In the following discussions, typical code names from the CAMCON system are used as a way of identifying items in Figure A-4. The description of these codes is in the User's Reference Manual for CAMCON (Rechard, 1992). Other codes from the CAMCON modules identified on the edge of Figure A-4 may be used. Translators that may be necessary for severa! of the codes are not discussed or shown in Figure A-4. 


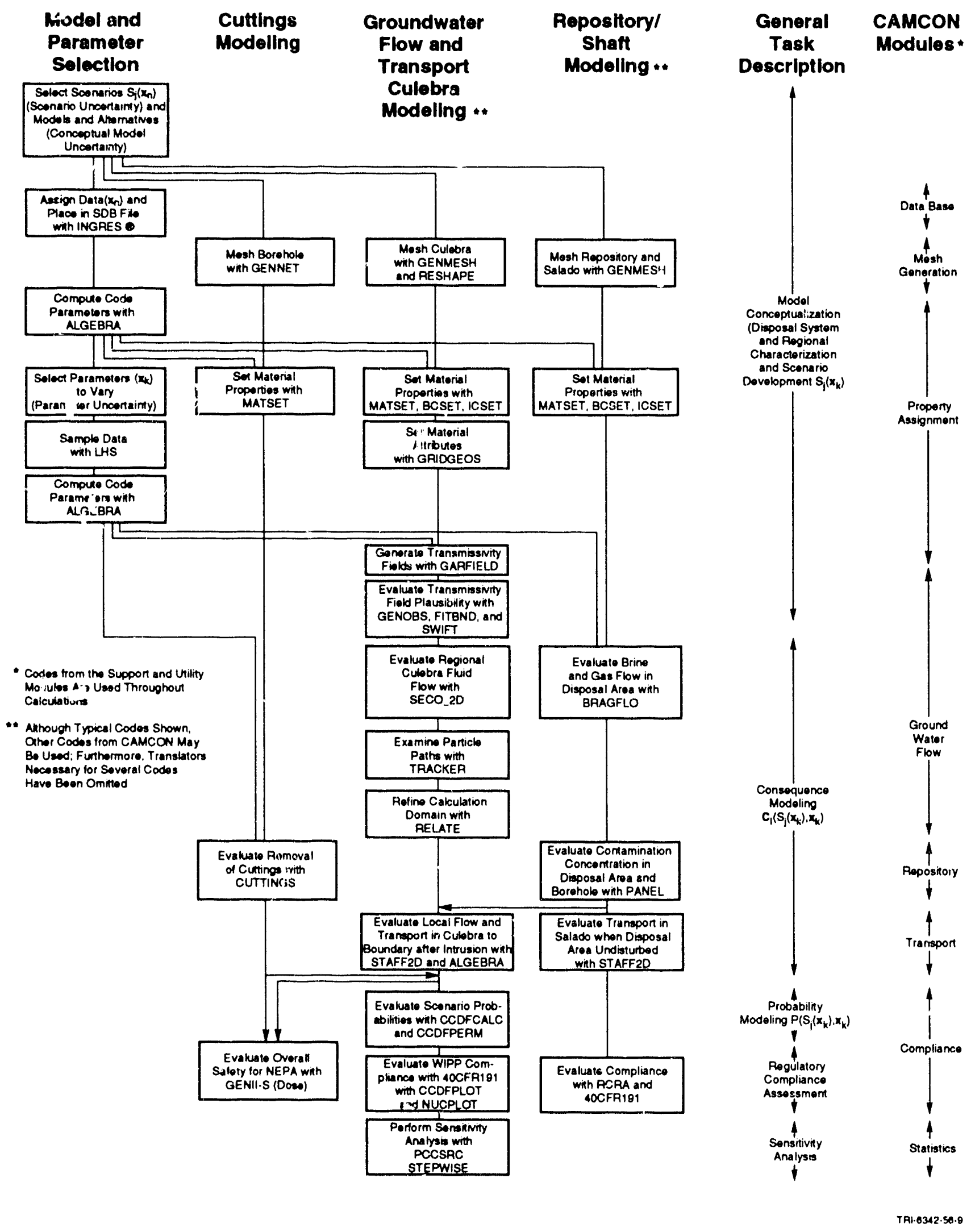

Figure A-4. Overview if PA calculations (after Rechard, 1991, Figure 1-3) (Refer to User's Reference Manual for CAMCON [Rechard, ed., 1992] for descriptions of codes listed.) 
Model and Parameter Selection. The calculations start with model and parameter selection. This can be a time-consuming process, but in short, the process involves compiling data and then developing conceptual, mathematical, and computational models if necessary. It is then followed by evaluating and selecting parameters to vary. Following these decisions, data are entered in the data base and are sampled. Once this critical step is completed, the analysts can begin the task of performing the calculations (Figure A-4).

Cuttings Modeling. The Cuttings Modeling System predicts the radionuclide release by transport of cuttings to the surface assuming intrusion by drilling sometime within the next 10,000 years. The mechanics of modeling the initial human intrusion by drilling into the repository is fairly simple. It involves input preparation such as the data base set up and extraction of pertinent data from the data base and sampled parameters. Then the CUTTINGS code is run for each sample element for each time.

Repository/Borehole Modeling. The repository/borehole modeling system models phenomena around the repository. These phenomena include gas generation from corrosion and microbiological degradation of the waste, brine movement around the waste over time, and the possible saturation of the waste by the brine reservoir following intrusion and creep closure. The two-phase numerical codes BRAGFLO and BOAST II and the one-phase analytic code PANEL model these phenomena.

Culebra Groundwater Flow Modeling. The Culebra Modeling System evaluates flow and transport in the Culebra Dolomite Member of the Rustler Formation following intrusion. Flow and transport are grouped into the same modeling subdivision because they model the same unit, the Culebra Dolomite Member at the Rustler Formation. However, the modeling ar,d number of simulations are different and are separated in this discussion.

The groundwater flow component of the Culebra modeling system is more complicated. It not only consists of a normal data-preparation steps, such as mesh generation (GENJMESH) and setting material properties (MATSET) and boundary conditions, but it also consists of evaluating the uncertainty of the transmissivity field of the Culebra (GENOBS, FITBND, and SWIFT). This can be followed by the analysis of the groundwater flow on the regional scale with SECO_2D.

Culebra Groundwater Transport Modeling. The second part of the Culebra modeling system is the evaluation of radionuclide transport from the intrusion borehole to the 5 -km boundary of the accessible environment and through the Culebra. The TRACKER code is used. The RELATE code is used to evaluate boundary conditions for fluid flow on a greatly decreased local mesh. STAFF2D (Huyakorn et al., 1991) can then be used to evaluate first flow and then transport on this loral two-dimensional domain. Currentity, no borehole model is used; rather, the radionuclide concentrations (mass flux only) from the repository/borehole modeling system are directly injected into the Culebra at a point directly above the center of tine disposal area. Following the STAFF2D simulations, a support program, ALGEBRA, is used to evaluate radionuclide transport across the $5-\mathrm{km}$ boundary of the accessible environment. 


\section{A.4 Requirements for the PA Software}

Based on the compliance assessment procedure just described, software to aid in PA calculations is needed to meet several requirements. Generally, the requirements can be grouped into two categories: built-in flexibility and built-in quality assurance (QA) features. Specifically, the software must

- Accurately link several distinct model components with little analyst intervention to eliminate tedious preparation of parameters (previous results from the chained analysis) (QA and flexibility)

- Trace the calculations so that they can be repeated (QA), in addition to software QA features on individual modules

- Propagate uncertainty by Monte Carlo analysis, uniquely identifying calculations to avoid misinterpretation (QA and flexibility)

- Permit an analyst to examine a large number of intermediate and final results (QA and flexibility)

- Allow interpolation between modeling scales (flexibility)

- Permit iteration between computer codes (flexibility)

- Aid in exploring phenomena modeled by codes (e.g., of the repository model) (flexibility)

- Be flexible in switching between codes to aid in scenario screening, model/model comparisons, sensitivity analysis, and fine-tuning of the system for final compliance assessment calculations (flexibility)

- Be computationally tractable

In other words, the variety of scenarios analyzed, the varying complexity of models used, and the need to switch codes when cycling through the procedure are characteristic of compliance assessments that require a flexible and versatile implementation. Furthermore, the number of repetitive computer runs, the need to properly identify runs, and the need to recreate runs are characteristics that require adequate quality assurance implementation.

Although several software analysis systems have been built to perform the numerous computer simulations necessary for a PA, the Sandia WIPP PA Department chose instead to build CAMCON, an analysis tool box that links together separate codes (either complex numerical or simple analytic codes) (Rechard, ed., 1992). This approach (1) directly makes use of the interdisciplinary expertise represented in pre-existing codes as opposed to indirectly incorporating these concepts into one comprehensive code, and (2) allows the same modeling system to be used for both detailed examination of the individual components and the overall disposal system (with the possible exception of substituting fast-running codes or modeling subdomains in the latter analysis when necessary). Both points are especially important for radioactive waste disposal where the calculations are under intense scrutiny and elaborate procedures are sometimes necessary to ensure that all steps taken in the analysis are defensible. A PA system that is capable of handling both detailed and simplified computational models is invaluable in this regard. 
A simplified "behind the scenes" view of the structure (CAMCON system) that supports the Repository/Shaft/Borehole Modeling System shown in Figure A-5 hints at the complexity of the flow of information in the PA calculations. Besides the flow of information, the parts of the CAMCON system shown are the secondary data base and numerous CAMDAT files. Basically, behind each of the modeling systems (Repository/Shaft/Borehole, Culebra Groundwater Flow and Transport, and Cuttings) are the numerous CAMDAT files storing the information for each of the sample (LHS) vectors. 


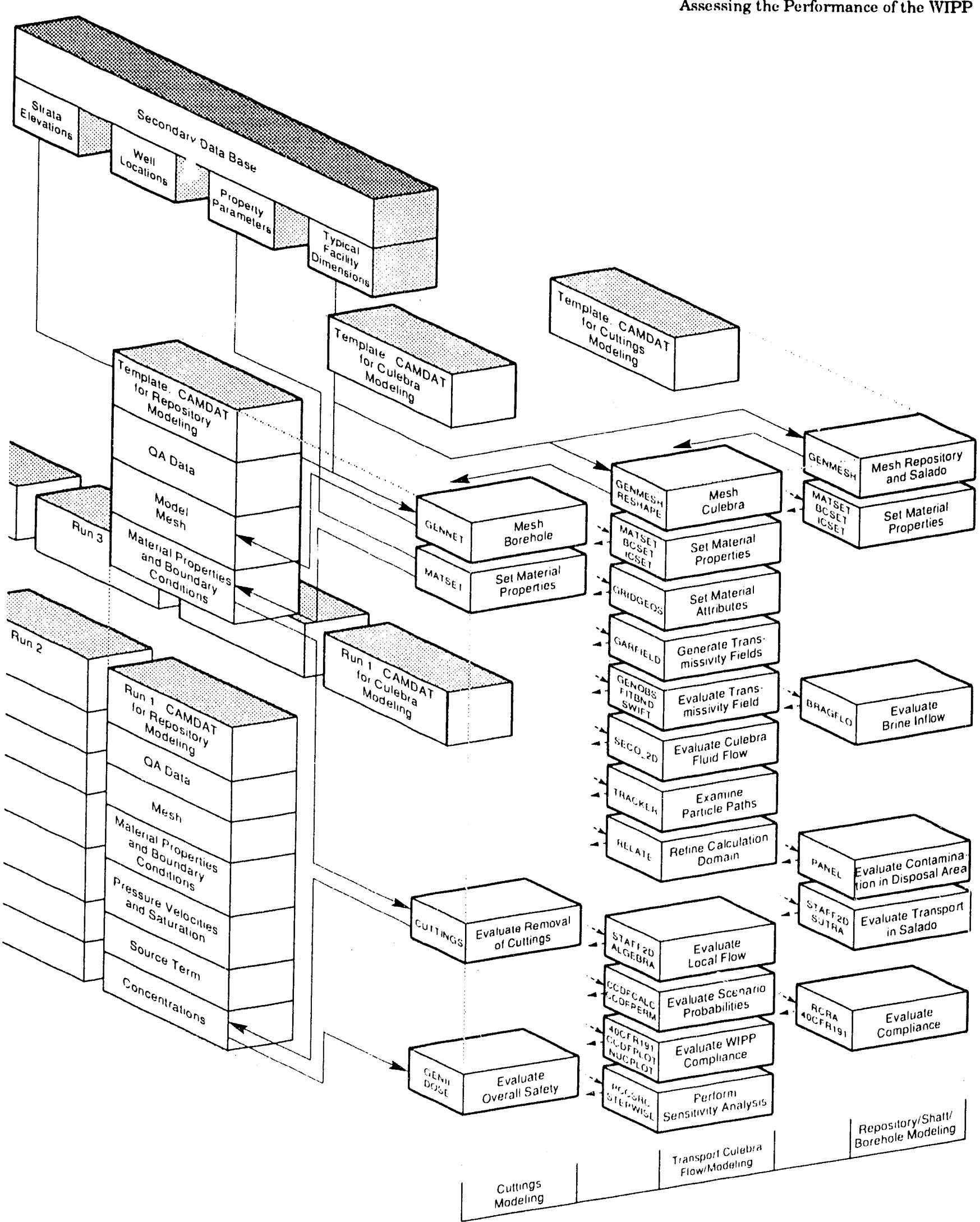

Figure A-5. CAMDAT, a primary component of the CAMCON system, is the behind-the-scenes structure that supports the PA calculations depicted in Figure A-4. 


\section{References}

Bertram-Howery, S. G., and R. L. Hunter, eds. 1989. Preliminary Plan for Disposal-System Characterization and Long-Term Performance Evaluation of the Waste Isolation Pilot Plant. SAND89-0178. Albuquerque, NM: Sandia National Laboratories.

Bertram-Howery, S. G., M. G. Marietta, R. P. Rechard, P. N. Swift, D. R. Anderson, B. L. Baker, J. E. Bean, Jr., W. E. Beyeler, F. F. Brinster, R. V. Guzowski, J. C. Helton, R. D. McCurley, D. K. Rudeen, J. D. Schreiber, and P. Vr aghn. 1990. Preliminary Com parison with 40 CFR Part 191, Subpart B for the Waste Isolation Pilot Plant, December 1990. SAND90-2347. Albuquerque, NM: Sandia National Laboratories.

Campbell, J. E., and R. M. Cranwell. 1988. "Performance Assessment of Radioactive Waste Repositories," Science. Vol. 239, no. 4846, 1389-92.

Chu, M. S. [Y.], N. R. Ortiz, K. K. Wahi, R. E. Pepping, and J. E. Campbell. 1983. An Assessment of the Proposed Rule (10CFR60) for Disposal of High-Level Radioactive Wastes in Geologic Repositories. Vol. 1. SAND82-2969, NUREG/CR-3111. Albuquerque, NM: Sandia National Laboratories.

EPA (U.S. Environmental Protection Agency). 1985. Environmental Standards for Management and Disposal of Spent Nuclear Fuel, High-Level and Transuranic Radioactive Wastes, Federal Register. Vol. 50, no. 182, 38066-38089.

Guzowski, R. V. 1990. Preliminary Identification of Scenarios That May Affect the Escape and Transport of Radionuclides from the Waste Isolation Pilot Plant, Southeastern New Mexico. SAND89-7149. Albuquerque, NM: Sandia National Laboratories.

Helton, J. C. 1991. "Performance-Assessment Overview," Preliminary Comparison with 40 CFR Part 191, Subpart B for the Waste Isolation Pilot Plant, December 1991. Volume 1: Methodology and Results. SAND91-0893/1. Albuquerque, NM: Sandia National Laboratories.

Helton, J. C. 1993. "Risk, Uncertainty in Risk, and the EPA Release Limits for Radioactive Waste Disposal," Nuclear Technology. SAND91-1255J. Vol. 101, no. 1, 18-39.

Helton, J. C., J. W. Garner, R. D. McCurley, and D. K. Rudeen. 1991. Sensitivity Analysis Techniques and Results for Performance Assessment at the Waste Isolation Pilot Plant. Contractor Report SAND90-7103. Albuquerque, NM: Sandia National Laboratories.

Huyakorn, P. S., H. O. White, Jr., and S. Panday. 1991. STAFF2D, Version 3.1: A Two-Dimensional Finite Element Code for Simulating Fluid Flow and Transport of Radionuclides in Fracturea Porous Media with Water Table Boundary Conditions. Herndon, VA: HydroGeologic, Inc.

Marietta, M. G., and W. F. Simmons. 1988. Feasibility of Disposal of High-Level Radioactive Wastes into the Seabed: Dispersal of Radionuclides in the Oceans: Models, Data Sets, and Regional Descriptions. SAND87-0753. Albuquerque, NM: Sandia National Laboratories.

Marietta, M. G., S. G. Bertram-Howery, D. R. Anderson, K. F. Brinster, R. V. Guzowski, H. [J.] Iuzzolino, and R. P. Rechard. 1989. Performance Assessment Methodology Demonstration: Methodology Development for Evaluating Compliance with EPA 40 CFR 191, Subpart B, for the Waste Isolation Pilot Plant. SAND89-2027. Albuquerque, NM: Sandia National Laboratories. 
Miller, I., and J. E. Freund. 1977. Probability and Statistics for Engineers. 2nd ed. Englewood Cliffs, NJ: Prentice-Hall.

Morgan, M. G., and M. Henrion. 1990. Uncertainty, A Guide to Dealing with Uncertainty in Quantitative Risk and Policy Analysis. Cambridge, UK: Cambridge University Press.

NEPA. 1969. National Environmental Policy Act of 1969. Public Law No. 91-190, 83 Stat. 852.

Powers, D. W., S. J. Lambert, S-E. Shaffer, L. R. Hill, and W. D. Weart, eds. 1978. Geological Characterization Report, Waste Isolation Pilot Plant (WIPP) Site, Southeastern New Mexico. SAND78-1596. Albuquerque, NM: Sandia National Laboratories. Vols. 1-2.

RCRA. 1976. Resource Conservation and Recovery Act of 1976. Public Law No. 94-580,90 Stat. 2795.

Rechard, R. P. 1989. Review and Discussion of Code Linkage and Data Flow in Nuclear Waste Compliance Assessments. SAND87-2833. Albuquerque, NM: Sandia National Laboratories.

Rechard, R. P. 1991. "Overview of Calculations," Preliminary Comparison with 40 CFR Part 191, Subpart B for the Waste Isolation Pilot Plant, December 1991. Volume 2: Probability and Consequence Modeling. SAND91-0893/2. Albuquerque, NM: Sandia National Laboratories.

Rechard, R. P., ed. 1992. User's Reference Manual for CAMCON: Compliance Assessment Methodology Controller, Version 3.0. SAND90-1983. Albuquerque, NM: Sandia National Laboratories.

Rechard, R. P., D. K. Rudeen, and P. J. Roache. 1992a. Quality Assurance Procedures for Analyses and Report Reviews Supporting Performance Assessments of the Waste Isolation Pilot Plant. SAND91-0428, Albuquerque, NM: Sandia National Laboratories.

Rechard, R. P., K. M. Trauth, and R. V. Guzowski. 1992b. Quality Assurance Procedures for Parameter Selection and Use of Expert Judgment Panels Supporting Performance Assessments of the Waste Isolation Pilot Plant. SAND91-0429. Albuquerque, NM: Sandia National Laboratories.

Tierney, M.S. 1991. Combining Scenarios in a Calculation of the Overall Probability Distribution of Cumulative Releases of Radioactivity from the Waste Isolation Pilot Plant, Southeastern New Mexico. SAND90-0838. Albuquerque, NM: Sandia National Laboratories.

U.S. DOE(Department of Energy). 1991. Recommended Strategy for the Remote-Handled Transuranic Waste Program. DOE/WIPP 90-058, Revision 1. Carlsbad, NM: Waste Isolation Pilot Plant.

WEC (Westinghouse Electric Corporation). 1990. Waste Isolation Pilot Plant No-Migration Variance Petition. DOE/WIPP 89-003, Rev. 1. Prepared for U.S. Department of Energy. Carlsbad, NM: Westinghouse Electric Corporation.

WIPPAct. 1979. Department of Energy National Security and Military Applications of Nuclear Energy Authorization Act of 1980. Title II - General Provisions: Sec. 213(a), Waste Isolation Pilot Plant, Delaware Basin, NM. Public Law No. 96-164, 93 Stat. 1259.

WIPP PA Department. 1992. Preliminary Performance Assessment for the Waste Isolation Pilot Plant, December 1992. Volume 1: Third Comparison with 40 CFR 191, Subpart B. SAND920700/1. Albuquerque, NM: Sandia National Laboratories. 
WIPP PA Division. 1991. Preliminary Comparison with 40 CFR Part 191, Subpart B for the Waste Isolation Pilot Plant, December 1991. Volume 1: Methodology and Results. SAND910893/1. Albuquerque, NM: Sandia National Laboratories.

WIPPStrategy. 1989. Waste Isolation Pilot Plant Compliance Strategy for 40 CFR Part 191. DOE-WIPP 86-013. Prepared by Sandia National Laboratories and Westinghouse Electric Corporation for the U.S. Department of Energy. Carlsbad, NM: WIPP Project Office. 


\section{Appendix B: \\ CAMCON System Personnel and Software Sponsors}

This appendix lists individuals within the PA Department that programmers may wish to contact to answer questions about the CAMCON system.

\section{B.1 WIPP Computer System Administrator}

The administrator and operators of the WIPP computer system are responsible for the day-to-day operation of the computer and implementing any major acquisition plans approved by the Sandia WIPP Project Manager.

A list of the current operators and how to contact them is obtained by typing CONTACT at the system prompt.

In addition to the system operators, a local system expert has been assigned by the PA Department to handle questions on PA Department computer equipment (e.g., PCs and printers), local computer equipment application software (e.g., spreadsheets), and the local area network.

PA Department Contact: Bruce L. Baker (BLBAKER)

Technadyne, Inc

505-766-9629

\section{B.2 Performance Assessment QA Coordinator}

The QA coordinator implements quality assurance tasks for software, data, analysis, document review, and expert judgment panels. Responsibilities related to software development for CAMCON are summarized below (see PAP02, 1993, or Rechard et al., 1991, for a complete list of responsibiiities for software and PAP03 PAP04, PAP05, and PAP06 [all 1993] for other responsibilities):

- Select software sponsors and software consultants

- Select software review committee

- Assist individuals in interpreting the meaning of QA procedures

- Approve content of software abstract

- Categorize software as to calculation type(Scientific/Engineering Software [SES] or non-SES) and source type (internal Sandia software or external software)

- Coordinate any assistants

QA Coordinator: $\quad$ Rob P. Rechard (RPRECHA)

Sandia National Laboratories

505-766-9629 


\section{B.3 Assistant to QA Coordinator for Software}

The primary responsibility of the assistant is to support the QA coordinator in the five main areas controlled by the QA procedures in the PA Department. The currently designated tasks for the software QA assistant are as follows

- Maintain software notebooks

- Send updates of software notebooks to WIPP QA chief

- Track and report status of CAMCON system software

- PA Department application software for local computer equipment (e.g., spreadsheets)

Contact: $\quad$ Kathleen A. Byle (KABYLE)

New Mexico Engineering Research Institute

505-766-9629

\section{B.4 CAMCON System Task Leader}

The CAMCON system task leader is responsible for coordinating the development of software tools for performance assessments. Several of the more prominent responsibilities are as follows:

- Set up and administer the CAMCON system (software management system described in PAP02, 1993, or Rechard et al, 1991)

- Periodically publish user's manuals summarizing codes within the CAMCON system (e.g., Rechard, 1992)

- Answer general questions on preferred programming practices (this manual)

- Define user interfaces and data flow in the CAMCON system for all software (e.g., coordinate development of translators) (this manual)

- Periodically publish the use of software libraries (this manual)

- Coordinate programmers who are developing software tools (utilities and support codes), thus directing CAMCON system support (this manual)

Contact: $\quad$ Rob. P. Rechard (RPRECHA)

Sandia National Laboratories

505-766-9629 


\section{B.5 CAMCON System Sponsors}

Table B-1 alphabetically lists the software and corresponding software sponsor reported within this programmer's manual. Other CAMCON system software is reported in the CAMCON User's Manual (Rechard, 1992). The sponsors are the best source of answers to detailed questions on the use of CAMCON system software; however, a general contact for programmers on the use of CAMCON system software within codes is as follows:

Contact:

J. S. Rath

New Mexico Engineering Research Institute

505-766-9629

Table B-1. CAMCON System Sponsors

\begin{tabular}{ll}
\hline Software & Sponsor \\
\hline ABSGEN & K. A. Byle ${ }^{1}$ \\
[CAMCON] Directories & A. P. Gilkey \\
CAMCONexec & A. P. Gilkey \\
CAMCON_LIB & A. P. Gilkey \\
CAMSUPES_LIB & A. P. Gilkey \\
CAMDAT_LIB & A. P. Gilkey \\
CHANGES & A. P. Gilkey \\
CREATE_PROGRAM & A.P. Gilkey \\
DVDI_LIB & A. P. Gilkey \\
FLINT & A. P. Gilkey \\
FORMRENUM & A. P. Gilkey \\
HLPCHK & K. A. Byle \\
HJIPLOT_LIB & H. J. Iuzzolino ${ }^{2}$ \\
LISTDCL & A. P. Gilkey \\
LISTFORTRAN & A. P. Gilkey \\
PLT_LIB & A. P. Gilkey \\
QA_PREP & K. A. Byle \\
QA_PRINT & J. S. Rath ${ }^{1}$ \\
SDBREAD_LIB & H. J. Iuzzolino \\
UPDATE_PROGRAM & A. P. Gilkey \\
& \\
\hline${ }^{1}$ New Mexico Engineering Research Institute, University of \\
New Mexico, Albuquerque, NM & \\
${ }^{2}$ Geo-Centers, Inc., Albuquerque, NM & \\
\hline & \\
\hline
\end{tabular}




\section{References}

Rechard, R. P.ed. 1992. User's Reference Manual for CAMCON: Compliance Assessment Methodology Controller, Version 3.0. SAND90-1983. Albuquerque, NM: Sandia National Laboratories.

Rechard, R.P., P.J. Roache, R.L. Blaine, A.P. Gilkey, and D.K. Rudeen. 1991. Qualiiy Assurance Procedures for Computer Soft ware Supporting Performance Assessments of the Waste Isolation Pilot Plant. SAND90-1240. Albuquerque, NM: Sandia National Laboratories.

\section{WIPP Performance Assessment Procedures:}

PAP02. 1993. Computer Software Supporting Performance Assessment of the Waste Isolation Pilot Plant. Albuquerque, NM: Sandia National Laboratories.

PAP03. 1993. Parameter Selection Quality Assurance Procedure. Albuquerque, NM: Sandia National Laboratories.

PAP04. 1993. Analysis Quality Assurance Procedures. Albuquerque, NM: Sandia National Laboratories.

PAP05. 1993. Report Review Quality Assurance Procedures. Albuquerque, NM: Sandia National Laboratories.

PAP06. 1993. Use of Expert Judgment Panel Quality Assurance Procedures. Albuquerque, NM: Sandia National Laboratories. 


\section{Glossary}

\section{Acronyms}

\begin{tabular}{|c|c|}
\hline ASCII & - American Standard Code for Information Interchange \\
\hline CAMCON & $\begin{array}{l}\text { - Compliance Assessment Methodology CONtroller-computational system for } \\
\text { assessing the performance of the WIPP }\end{array}$ \\
\hline CAMCONexec & $\begin{array}{l}\text { CAMCON executive package, a suite of procedural files that act as an inter- } \\
\text { face between separate codes within CAMCON }\end{array}$ \\
\hline CAMDAT & $\begin{array}{l}\text { Compliance Assessment Methodology DATaBase-computational data base } \\
\text { developed for the WIPP (extensive modification of EXODUS) }\end{array}$ \\
\hline CCDF & $\begin{array}{l}\text { Complementary Cum'slative Distribution Function-the performance measure } \\
\text { for spent nuclear fuel, HLW, and TRU repositories specified in } 40 \text { CFR } 191 \text {. }\end{array}$ \\
\hline $\mathrm{CDB}$ & - Computational Data Base (neutral file) \\
\hline $\mathrm{CH}$ & - Contact Handled (TRU waste) \\
\hline DCL & $\begin{array}{l}\text { - Digital Equipment Corporation Command Language for the VAX/VMS } \\
\text { operating system }\end{array}$ \\
\hline DOE & $\begin{array}{l}\text { U.S. Department of Energy, formed by DOE Organization Act (Public Law } \\
\text { 95-91, 91 Stat. 565), which replaced the Energy Research and Development } \\
\text { Agency (ERDA). The ERDA was formed by the 1974 Energy Reorganization } \\
\text { Act (Public Law 93-438), and replaced the Atomic Energy Commission (AEC), } \\
\text { formed in } 1946 \text { (August 1, 1946, } 60 \text { Stat. 755). }\end{array}$ \\
\hline DVDI & $\begin{array}{l}\text { Dual Virtual Device Interface-an extension to SVDI to allow two plot devices } \\
\text { to be active during a plotting session. }\end{array}$ \\
\hline EPA & - U.S. Environmental Protection Agency \\
\hline EXODUS & $\begin{array}{l}\text { Computational data base file format developed by Engineering Sciences } \\
\text { Center at Sandia (Mills-Curran et al., 1988) }\end{array}$ \\
\hline FD & - Finite difference numerical implementation of a mathematical model \\
\hline FE & - Finite element numerical implementation of a mathematical model \\
\hline HLW & $\begin{array}{l}\text { High-Level Waste, meaning (1) liquid wastes from reprocessing irradiated } \\
\text { reactor fuel (spent nuclear fuel), (2) solids into which the wastes have been } \\
\text { converted, and other highly radioactive material requiring permanent } \\
\text { isolation }\end{array}$ \\
\hline $\mathrm{A}$ & formance Assessment (see glossary definition) \\
\hline
\end{tabular}


QA

QAP

QAPP

RCRA

$\mathrm{RH}$

SDB

SVDI

SWCF

TRU

WIPP

40 CFR 191
- Quality Assurance (see glossary definition)

- Quality Assurance Procedure for the portion of the WIPP project conducted at Sandia

- Quality Assurance Program Plan for Sandia WIPP project

- Resource Conservation and Recovery Act of 1976 (Public Law 94-580) and subsequent amendments (e.g., HSWA-Hazardous and Solid Waste Amendments of 1984, Public Law 98-616).

- Remote Handled (TRU waste)

- Secondary Data Base (neutral file)

- Sandia Virtual Device Interface-a collection of subroutines that perform basic graphic operations with the capability to interface to several graphic devices.

- Sandia WIPP Central File-archives for WIPP QA records produced by Sandia National Laboratories

- Transuranic radionuclide waste-trash contaminated with more than $10 \mathrm{nCi}$ transuranic radionuclides (atomic number greater than 92) emitting $\alpha$ radiation with halflife greater than 20 years and an activity greater than 100 $\mathrm{nCi}$.

- Waste Isolation Pilot Plant-a deep geologic repository for TRU waste generated by the DOE nuclear weapons facilities authorized in Public Law 96-164, 93 Stat. 1259.

- Code of Federal Regulations, Title 40, Part 191, Environmental Radiation Protection Standards for Management and Disposal of Spent Nuclear 


\section{Definitions}

Accessible environment - "... (1) the atmospt ere, (2) land surfaces, (3) surface water, (4) oceans, and (5) all of the lithosphere that is beyond the controlled area" (40 CFR 191.12[k]) where "controlled area" means" (1) [a] surface location, to be identified by passive institutional controls, that encompasses no more than 100 square kilometers and extends horizontally no more than five kilometers in any direction fror 1 the outer boundary of the original location of the radioactive wastes in a disposal system; and (2) the subsurface underlying such a surface location $(\$ 191.12(\mathrm{~g})$ ).

Alternative conceptual models - Multiple working sets of hypotheses and assumptions of a system that are all scientifically acceptable (i.e., consistent with the purpose of the model, logically consistent with one another, in agreement with existing facts and observed data, and able to be tested).

Alternati ye models - Alternatives in model form that may exist at each stage of model development. Alternative conceptual models are often of the most concern.

Analysis - Analysis is the rational investigation of a natural or artificial phenomenon to discover principles that underlie the phen omenon. More formally, analysis is the five-step process of (1) defining (developing hypothesis and planning), (2) investigating, (3) validating, (4) reviewing, and (5) documenting the study of a system. This rational study often involves "teasing" the system "ap 'i.e., the division of the whole system into simpler components to gain understanding. $i_{\text {. }}$ wever it may also involve synthesis, i.e., the bringing together of diverse facts to comprehend the whole system. Consequently, analysis is at the heart of the method of scien. tific discovery and explanation. (See engineering analysis.)

Applied model - The analyst's application of the generic computational model to a particular system, using appropriate values for dimensions of the system components, parameters, and boli: lary and initial conditions. In a waste management, the system is a waste disposal site, and so this model is also referred to as a site-specific model.

Attribute parameters or properties - Parameters that have a rough characteristic range for each material but vary spatially and are thus differ at each element in the computational mesh. They are attributes of each node or element in the mesh (the numerical discretization of the material)

Benchmarking - The comparison of predictions of a particular problem or test case made with one applied model with predictions made with other numerical or analytical computational models, where all models were designed to solve comparable mathematical models. Verification (and occasirnally process validation) exercises may use benchmarkıng.

Cache - The process of storing information, data, and software necessary for the PA process at Sandia so that it can be retrieved in computer form and duplicated relatively easily. The stored information may eventually enter the official WIPP archives but not necessarily, and so the term archive is purposely avoided.

Class A Software - Class A (Adjudicated) software has been evaluated by the Software Review Committee. The software satisfies the quality assurance requirements for traceability, retrievability, documentation, and verification. 
Class C Software - Class C (Candidate) software is a candidate for Class A, but currently satisfies only the traceability and retrievability requirements (as evaluated by the QA Coordinator).

Class D Software - Class D (Dormant or Dead) software is obsolete, cached software that was formerly classified as Class $\mathrm{A}$ or $\mathrm{C}$, where obsolete means that a new version of the same class has replaced the cached version.

Class X Software - Class X (Experimental) software is currently being developed and has not been processed through any formal quality assurance procedures. The primary reason for the Class $\mathrm{X}$ classification is to make the existence of this software known to potential users. The software is available to any interested user within the WIPP Project at Sandia, but usually only from the developer.

Code - Software, but often implying SES software that groups together one or several different computational models.

Computational Data Base (CAMDAT) - The collection of neutral files, generated during a PA simulation, that link CAMCON codes together.

Computational model - The solution and implementation of the mathematical model. The solution may be either analytic (exact), numerical (approximate), or empirical. Conceivably, the implementation can bs either by hand or evaluated in a computer code. However because the latter is more frequent, the computational model is often called a computer model. Generic until site-specific data are used.

Computer model - See computational model.

Configuration management - A system of controls that prevents ambiguity about which version of software is used for a particular computation and that tracks, protects, and preserves requested modifications or corrections to these codes.

Conceptual model - The set of hypotheses and assumptions that postulates the description and behavior of a system. To be scientifically acceptable, the set of hypotheses must be (1) consistent with the purpose of the model, (2) logically consistent with one another, (3) in agreement with existing information and observed data, and (4) able to be tested (i.e., capable of being falsified by the collection of more data). At a minimum, these hypotheses and assumptions describe the following about the system, including whether it is time dependent: (1) the simplified physical arrangement of system components, $(2)$ the initial and boundary condition types, and (3) the nature of the relevant chemical, physical, cultural, or biological phenomena.

Customer - The customer is the recipient, purchaser, or beneficiary of the product, service, work, or other outcome of an ongoing process or project.

Data - Information that is purposely collected, organized, and used in preparing values for parameters of computational models in an analysis or used as the basis of a decision. As used here, data is a subset of general information.

Discrepancy - A term for a condition adverse to data, software, or analysis quality that refers to any of the following: errors, bugs, failures, malfunctions, deficiencies, defective items, and nonconformances with established standards. 
Disposal system - Any combination of engineered and natural barriers that isolate spent nuclear fuel or radioactive waste after disposal (40 CFR 191.12(a)). The natural barriers extend to the accessible environment.

Engineering analysis - An engineering analysis is similar to a general scientific analysis except that engineering analysis (1) has formal constraints on purposes (specifications) rather than the limits of human curiosity, (2) has formal constraints on resources (time and money) rather than the limits of human energy, (3) uses fairly accepted methods rather than novel approaches, and (4) seeks to clarify rather than to discover new knowledge (i.e., the study is likely to further understanding of a system through the application of already discovered knowledge). The term "engineering analysis" applies to the WIPP disposal system provided one understands the formal constraint on the analysis purpose, which is an analysis that is intended to investigate the entire system for the primary purpose of determining compliance with environmental regulations. (See analysis.)

Formal report - Formal reports are SAND reports (official reports issued by Sandia National Laboratories and other scholarly publications such as journal articles or books written by Sandia staff and contractors) that undergo the formal peer review procedure (PAP05).

Information - A collection of cognitive or intellective material. Information includes both observational data and communicated knowledge derived by inference and interpretation.

Management review-A review conducted by management to (1) assess the impact of a document or data on the project and (2) evaluate concurrence with broad corporate or government policy.

Material parameters or properties - Parameters or characteristics that vary only with material regions or not at all. Material parameters are defined for each material region for the mesh.

Mathematical model - The mathematical representation of a conceptual model (i.e., the coupled algebraic, differential, or integral mathematical equations that approximate the appropriate processes in a specified domain of the conceptual model).

Module - A grouping or category of codes in the CAMCON system; the module name describes the primary function provided in the performance assessment.

Neutral file - A data file that is stored in a common format and can be read or altered by either SDBREAD_LIB or CDBREAD_LIB. A neutral file is associated with either the secondary data base or the computational data base.

PA Analyst - The PA Department person selected by a task leader directing a task requiring analysis who designs and conducts the analysis of a PA problem. The analyst must ensure a quality analysis by (1) developing one or several conceptual models (working hypotheses) of a system, with the complexity level consistent with model purpose; (2) developing a mathematical description and implementing a solution and/or selecting the proper software; (3) providing approximate system parameters (data) and modeling features, such as discretization of values, types of initial conditions, and boundary conditions; (4) conducting convergence tests and interpreting results, including parameter sensitivity studies, (5) validating a conceptual model to the extent possible or reporting results from a plausible alternative conceptual model to properly state uncertainty, (6) co-authoring documents on the analysis with the task leader, (7) caching the computational model parameters and results, and (8) improving software quality by reporting suspected errors and suggested enhancements or acquiring/developing software when warranted (as required in the software QA procedures). 
Parameters - The underlying fundamental entities $\left(x=x_{1}, \ldots, x_{n}, \ldots, x_{n V}\right)$ of a computational model. As $\mathbf{x}$ changes, so does the model result. The individual parameters, $\mathbf{x}_{\mathbf{n}}$, are usually scalar quantities, but they may also be vectors or functions. Furthermore, the individual parameters are usually coefficients of the mathematical model, but they may also relate to scenario uncertainty or model form uncertainty. Two types of parameters are recognized in the CAMCON system: material parameters and attribute parameters.

Peer - A person with expertise comparable to that of the person whose work (software, data, or analysis) is being examined (or with expertise in a critical subset of the work) and who did not perform the original work.

Performance assessment (PA) - The process of assessing whether a system meets a set of performance criteria. For the WIPP, the system is a computational model (which, in turn, is a composite of several models) that represents a deep geologic repository disposal system for waste contaminated with transuranic radionuclides and hazardous chemical constituents; and the performance criteria are various long-term environmental metrics in U.S. government regulations (not short-term operational safety issues).

Primary Data Base - Stores the raw measured data of the experimentalist. In some cases, the data are interpreted.

Process validation - The supposition that the validation of a conceptual model and the corresponding mathematical and computer models at another sufficiently similar system can be used to bolster confidence that a system phenomenon can be sufficiently and accurately modeled at another system under study. For waste management systems, this includes (1) simulation of short-term laboratory experiments under controlled conditions and (2) analogy with a similar and previously validated conceptual and mathematical model. The complete validation of the conceptual model and corresponding mathematical and computational models require adequately explaining the phenomena under study with system-specific data.

QA coordinator - The PA Department Manager or the designated appointee within the Performance Assessment department who coordinates the implementation of the quality assurance tasks for data, software, analysis, and document review, and expert judgment panels.

Quality assurance (for a product) - Quality assurance is ensuring that the product does what it is supposed to do to meet the expectations of the customer. The customer expectation, as related to the performance assessment process, is that the analysis results present an adequate view of the WIPP performance based on currently available data and information.

Release - A unique issue of software that generally contains minor corrections to errors that existed in previous issues of the software. Documentation for a new release of software generally remains the same as the preceding release.

Repeatability - The ability to repeat any part or all of a product or service (e.g., analysis) through sufficient documentation to understand all nonobvious inputs and the basis of important decisions or conclusions. Repeatability may or may not include traceability and retrievability as requirements.

Retrievability - The ability to retrieve essential information in a reasonable period of time. For a large PA analysis, retrievability implies that the information can be retrieved in computer form from a cache. 
Scientific/engineering software (SES) - Software that models a physical process, often by the numerical solution of mathematical equations. Software that is used to establish the spatial variation of modeling parameters is also scientific/engineering software (SES). A significant distinction of SES is that validating the software is often subjective, difficult, and sometimes impossible.

Secondary Data Base - Stores interpreted data from the Primary Data Base for use as parameters for the PA simulation models. The information is stored in a tabular format within a relational data base system, under the control of the PA Department.

Site-specific model - See applied model.

Software - A set of computer operations specified in any language that can be translated unambiguously into machine language. Common synonyms are computer program, computer code, and operating system procedural files.

Software consultant - The PA department person selected by the QA coordinator to provide technical expertise in mathematical and numerical basis and application of software, occasionally shortened to "consultant."

Software sponsor - The PA department person selected by the QA coordinator to sponsor or guide a particular piece of software through the quality assurance process, often abbreviated to sponsor."

Stakeholder - A person, group, or agency who is not a customer but who nevertheless has a keen interest in a product or service and the recognized right to impact the process.

SWCF records manager - The Sandia WIPP Central Files (SWCF) records manager is chosen by the Sandia WIPP project manager or designated department manager. One of the SWCF records managers may be the Waste Management and Transportation (WMT) librarian. One of the SWCF records manager's many project tasks is to maintain quality assurance records in accordance with QAP 17-1 on Performance Assessment studies as required in the PA quality assurance procedure.

Task leader (TL) - The PA department person selected by the PA department manager and assigned responsibility for conducting the activities described in the periodically updated Sandia work plan for the WIPP project, which can include conducting an analysis, developing software, selecting data, and coordinating review and expert judgment panels. The TL is frequently called principal investigator or PI within Sandia, but TL was selected for use here so that this term cannot be confused with the PI in a formal contract with persons outside of Sandia.

Technical review - A review of a document by peers to (1) evaluate documents as to their applicability, correctness, and completeness, (2) evaluate whether the conclusions are reasonably supported by the evidence, results, and other facts, (3) generally verify compliance with common scientific, engineering, and industry practice and standards, and (4) evaluate the presentation of these points in the document.

Traceability - The ability to duplicate any part or all of a product or service exactly through records on (1) versions of all software tools used, (2) all analysis methods used, and (3) all required data and information. Traceability may or may not include retrievability as a requirement. 
Validation - The process of making valid by confirming, corroborating, substantiating, or supporting, where valid means of good authority, well founded, sound and to the point, and applicable to the subject or circumstances against which few objections can be fairly brought.

Validation of an (applied) model - The process of validating through sufficient testing (subjective) using system-specific observed data that a conceptual model and the corresponding mathematical and computational models explain a system with sufficient accuracy (subjective), consistent with the purpose of the model. In other words, model validation is the process of confirming that the applied model is solving the appropriate idealization of the system (correct applied model produced). Verification of a computational model precedes validation of an applied model. Model validation is an ongoing process.

Verification of a (computational) model - The process of verifying that a computational model appropriately solves and implements the mathematical model. In other words, model verification is the process of illustrating that the mathematical model is being solved appropriately (computational model correctly produced). Often the computational model is implemented as a code and verification of the computational model is closely connected to verification of the software. Given that the computational model is verified, the assumptions of the conceptual model underlying the mathematical and computational model using system-specific data (the applied model) should be validated.

Verification of software - The process of illustrating through sufficient testing (subjective) that the software satisfactorily performs its stated capabilities (subjective) and providing a point of reference for future modifications. The extent to which the software is tested is determined by the complexity of the software.

Version - A unique configuration of a code that differs in its capabilities from other configurations of the same code. 
Symbols

/ANSI

FLINT 12-15

/COMMENT

FORMRENUM 12-22

ICONT

FORMRENUM 12-22

/DLINES

FLINT 12-13

/EXTEND

FLINE 12-13

/FILES

FLINT 12-13

/FORMAT

FORMRENUM 12-20

/FYI

FLINT 12-15

/GLOBAL

FLINT 12-15

/GRAPHICS

FLINT 12-16

$/ 14$

FLINT 12-13

/IMPLICIT

FLINT 12-15

/INC

FORMRENUM 12-21

IINCLUDE

FLINT 12-15

/INDCONT

FORMRENUM 12-21

/INDENT

FORMRENUM 12-21

IINDFORMAT

FORMRENUM 12-21

ILABFORMAT

FORMRENUM 12-21

/LABSTMT

FORMRENUM 12-21

/LIBRARY

FLINT 12-13

/LISTING

FLINT 12-15

ILOCALSYMS

FLINT 12-16

/LPP

FLINT 12-17
/MAXCONT

FORMRENUM 12-22

/NOFORMAT

FORMRENUM 12-20

/NOI4

FLINT 12-13

NOLOCALSYMS

FLINT 12-16

/NONUMBER

FORMRENUM $12-22$

/NORENUMBER

FORMRENUM 12-21

NNOUSAGE

FLINT 12-16

/NOWARNINGS

FLINT 12-16

INUMBER

FORMRENUM 12-22

/OUTPUT

FLINT 12-14

/PARAMETERS

FLINT 12-16

/PORTABILITY

FLINT 12-15

/RENUMBER

FORMRENUM 12-21

/SPLIT

FLINT 12-14

ISQUISH

FLINT 12-17

/STATISTICS

FLINT 12-14

/SUMMARY

FLINT 12-14

/SUPPRESS

FLINT 12-15

/SYSTEM

FLINT 12-16

ITREE

FLINT 12-14

ITRIM

FLINT 12-17

ITTB

FLINT 12-14

/USAGE

FLINT 12-16

IVAXDO

FORMRENUM 12-21 


\section{/WARNINGS}

FLINT 12-16

/WIDTH

FLINT 12-17

IXREFERENCE

FLINT 12-14

[CAMCON] directory 2-5

[LIBRARIES] subdirectory 2-19

\section{A}

ABORT $8-8$

ABSGEN 12-3-12-4

Identifiers and headings $12-4$

Abstract generation 12-3-12-4

ADD

CHANGES $12-6$

Advance page 11-28

Advanced routines

CAMSUPES_LIB 9-2, 9-23

SDBREAD_LIB 7-9, 7-32

AHSAX 10-150

ALGEBRA 3-2

Analysis results 6-2, 6-14, 6-69

CAMDAT_LIB 6-14, 6-69

Analysis-specific modifications 3-17-3-18

Attribute parameters 6-2, 6-7

AXIS 10-151, 10-152

Axis scaling $10-65,10-66$

\section{B}

Background color 11-31

BANNER 4-3, 8-9

Basic plotting primitives

PLT_LIB 10-6, 10-7

BATCH $8 \cdot 10$

Bell 11-12

BIGLET 8-11

BI_OCK_DATA

CREATE_PROGRAM 12-10

BLOT 2-15

Bound for range $10-182$

Boundary condition data 6-7-6-9)

Bounds defined 10-183

BOX 10-153

BROADCAST

CHANGES $12-6$

C

C and FORTRAN

Mixing 5-19
$\mathrm{C}$ codes

Interfacing 5-19

Linking 5-19

C language $3-4,2-6$

C programming language 5-19

$\mathrm{C} \$ \$+\mathrm{n}$

FORMRENUM 12-23

C\$\$ \$-n

FORMRENUM 12-23

C\$\$\$FF

FORMRENUM 12-22

C\$\$ON

FORMRENUM 12-23

C.\$\$R

FORMRENUM 12-23

CAMCON approach $2-2-2-3$

CAMCON coordinator $1-4$

CAMCON defined 1-1

CAMCON system 2-1

CAMCON_LIB 10-1, 2-20-2-22, 4-3, 8-1

Common internal routines $8-58$

Error messages 8-1

File processing routines $8-22$

QA and user interface routines 8-6

Routine calls 8-6

String processing routines $8-51$

CAMCON_LIB free field input 8-35

CAMCONexec 2-6

CAMCONexec procedural files 3-12

CAMCONexec sponsor 2-5

CAMDAT 2-3, 2-15-2-22, 6-1-6-16

CAMDAT file format 6-1

CAMDAT_LIB 2-20-2-22, 6-1

Analysis results 6-14, 6-69

Execution 6-12

File initialization $6-20$

General routines 6-12, 6-17

Heading 6-4

Model description 6-13, 6-41

Overview 6-10-6-12

QA information 6-13, 6-34

Routine calls 6-17

CAMSUPES_LIB 2-20-2-22, 9-1-9-2

Advanced routines 9-23

Debugging routines 9-34

Memory management routines 9-11-9-13

System queries 9-3

CANCEL ALL

VAX/VMS debugger 12-39

CANCEL BREAK

VAX/VMS debugger $12-38$ 
Change records $4-23$

CHANGES 12-5-12-8

Character font $11-35$

Character size $11-33$

Character string angle 11-41

Character string length $10-54$

Check software help file 12-26-12-27

Class A software 3-5, 3-6, 3-11, 5-12

Class C software 3-5, 3-6, 3-10, 5-12

Class D software 3-5, 3-6

Class X software 3-5, 3-6, 3-9

Clipping

2D polygons $10-118$

2D vectors $10-121$

3D Vectors $10-122$

Points 10-91, 10-92, 10-119, 10-120, 10$124,10-126$

Points, lines, and polygons 10-117

Polygons 10-91, 10-92, 10-123

Vectors 10-91, 10-92, 10-125, 10-127

Clipping rout:nes

PLT_LIB 10-8, 10-117

Code Consultant 3-9

Code directory structure 2-10

Code operation 4-10

Code Review Committee 3-6

Code sponsor 3-9

Codes, computer 2-9

Color index 10-129, 10-130

Color intensity 10-131

Color name 10-129, 10-130

Color palette routines

PLT_LIB 10-9, 10-128

Color parameters

Getting 10-25

Color paramters

Setting 10-24, 10-137

Color spectrum 10-135

Color table 11-17, 11-32

Color values $10-134$

Commands subtopic 4-17

Comment identifier 4-8

Comments 5-12, 8-30

Common internal routines

CAMCON_LIB 8-3, 8-58

Compile program 12-9-12-11, 12-36

COMPILE_OPTIONS

CREATE_PROGRAM 12-10

Computational data base $2-3,2-15-2-22$

Computational Data Base Interface I ihrary 6-1

Computer codes 2.9
Conceptual model stratigraphy 7-1

Continuation indicator 8-30

Continuing statements 4-9

CREATE_PROGRAM 12-9-12-11

Curl C.

VAX/VMS debugger $12-39$

Current position 11-18

D

Data base model size $6-5$

Data base version 6-5

Data bases 2-10-2-19

Data ficld 8-30

Data subtopic 4-18

Date stamp 4-1

DBERRUNI 6-18

DBFIXMEM 6-19

DBIATNAM $6-44$

DBIATTR 6-42

DBICLOSE 6-24

DBIELBLK 6-46

DBIEVROK 6-70

DBIINTO 6-35

DBILINK 6-51

DBIMAP 6-53

DBINDSET 6-55

DBINELB 6-58

DBINSTEP 6-76

DBINVAR 6-72

DBIOPEN 6-21, 6-22

DBIPRNAM 6-61

DBIPROP 6-59

DBIQAREC. $6-38$

DBISDSET 6-62

DBISIZES 6-27

DBISTEP 6-74

DBITIME 6-77

DBITITLE 6-29

DBIVAR 6-79

DBIVERS 6-32

DBIVRNAM 6-82

DBIXYZ 6-67

DBOATTR 6-43

DBOCLOSE 6-25

DBOEL.BLK 6-47

DBOEVROK 6-71

DBOHEAD 6-49

DBOINIO 6-36

DBOLINK 6-52

DBOMAP 6-54

DBONDSET $6-56$ 
DBOOPEN 6-23

DBOPROP $6-60$

DBOQAREC 6-39

DBOSDSET 6-64

DBOSTEP 6-75

DBOTIME 6-78

DBOTITLE 6-30

DBOVAR 6-81

DBOVRNAM 6-83

DBOXYZ 6-68

DBPAPNAM 6-45

DBPDBNAM 6-26

DBPELBLK 6-48

DBPINFO 6-37

DBPNDSET 6-57

DBPNVAR 6-73

DBPQAREC $6-40$

DBPSDSET 6-66

DBPSIZES 6-28

DBPTITLE 6-31

DBPVERS 6-33

DBPVRNAM 6-85, 6-86

DCL $3-4$

DCL driver headers 5-18

DCLIMIT 10-154, 11-10

DEBUG_COMPILE_OPTIONS

CREATE_PROGRAM 12-10

Debugger $12-37-12-40$

Debugging routines

CAMSUPES_LIB 9-2, 9-34

DEC Command Language 3-4, 2-6

Declarations 5-5

DEFFIL 8-24

DELETE

CHANGES $12-7$

Delimiters 4-8

DEPOSIT

VAX/VMS debugger $12-40$

Developer 3-9

Device capabilities 10-19, 11-19

Device code 11-3-11-5

Device coordinates 10-20, 10-63, 10-64

Device driver 11-51

Device parameters $10-28$

Getting 10-27

Restoring 10-29

Saving $10-28$

Setting 10-26, 10-138

Device selection 11.48

Device $\mathrm{x}$-coordinate $10-190$

Device $y$-coordinate 10-191
Devices

DVDI 11-45-11-51

SVDI 11-2-11-5

Digits

Number of $10-172$

Directories 2-5

DIRECTORY

CHANGES $12-7$

Directory

Code 2-5

Drivers 2-5

Libraries 2-5

SDB 2-5

Symbols 2-5

Utilities $2-5$

Directory structure 2-5-2-6, 2-10

Documentation

External 4-20-4-23

Internal 5-11--5-18

On-line 4-10

DOEDIS 8-13

Downgrading software 3-13-3-15

Draw

Box 10-153

Centered marker 10-167

Logarithmic axis 10-166

Piecewise continuous line 10-163, 10-164, 10-165

To point 10-174, 10-175

Draw a line 11-25

Draw a point $11-29$

Draw a polygon 11-30

Draw text $11-43$

Drawing

2D points $10-94$

2D polygon 10-93

2D symbols $10-95$

2D vectors $10-96$

Arrows 10-58, 10-72

Curves 10-62, 10-68

Polygons 10-76

Vectors 10-74, 10-82, 10-83

Drivers 2-6-2-7

Dual Virtual Device Interface 11-1

DVDI 11-1

DVDI graphics interface 11-1

DVDI_LIB 2-20-2-22

E

ECIIO 8-25

EDIT

CHANGES $12-7$ 
Efficiency 5-11

ELEM 8-59

Element block ID 6-6

Element block name 6-6

Element connectivity 6-6

Element side sets 6-7, 6-8

Enhancement reporting 12-5-12-8

Entering file names 4-6-4-19

Error handling 11-7

Error messages 10-6, 4-10, 6-12

Error reporting 12-5-12-8

Escape code 11-14

Escape codes 11-2-11-5

Escape inquiry 11-22

EXAMINE

VAX/VMS debugger $12-40$

Example subtopic 4-19

EXCPUS 9-4

EXDATE 9-5

Executing

VAX/VMS debugger 12-39

Execution fields 4-17

Execution of CAMDAT_LIB 6-12

Execution subtopic 4-17

EXIT

CHANGES $12-7$

VAX/VMS debugger 12-39

EXNAME $9-7$

EXPARM 9-9

External documentation 4-20-4-23

EXTIME 9-10

\section{F}

FFISTR 8-39

Field separator 8-30

FILDAT 9-6

File exter.

File initialization 6-20

CAMDAT_LIB 6-13, 6-20

File names, entering 4-6-4-19

File naming conventions 4-4-4-19

Fille processing routines

CAMCON_LIB 8-2, 8-22

File reading 4-6

File types 4-4-4-19

Find end of text 10-53

Find next line 10-55

Finite-difference mesh 6-6

FLINT 12-12-12-18

Call tree qualifiers $12-16$

Cross-reference table qualifiers $12-16$
Diagnostic qualifiers $12-15$

Output file qualifiers $12-13$

Source file qualifiers $12-13$

Text format qualifiers $12-17$

Flush buffer 10-2, 10-16

Flush buffers 11-13

FOR ... DO

VAX/VMS debugger $12-40$

Foreground color 11-34

Formal reports 4-23

Format utility 12-19-12-22

FORMRENUM 12-19-12-22, 5-4

Formatting commands $12-22$

Formatting qualifiers $12-20$

Renumbering commands $12-23$

Renumbering qualifiers $12-21$

FORTRAN and C

Mixing 5-19

FORTRAN code analyzer 12-12-12-18

FORTRAN language 3-3, 5-10

FPARSE 8-27

Frame limits 10-154, 11-10

Free field input

CAMCON_LIB 8-30

Free-ficld input 8-30-8-35

Free-field input routines

CAMCON_LIB 8-2

FREFLD 8-37, 8-41, 8-43, 8-45, 8-46

G

General routines 6-17

CAMDAT_LIB 6-12, 6-17

SDBREAD_LIB 7-9, 7-13

Generate a format 10-158, 11-14, 11-16

Generic computational data base $6-2$

GENFMT 10-158, 11-14, 11-16

GETINP 8-49

GETPRG 8-14

Getting

Color parameters 10-25

Device parameters $10-27$

Global mapping parameters 10-39

Graph parameters $10-34$

Text parameters 10-43, 10-48

Global mapping parameters $10-40$

Getting 10-39

Restoring 10-41

Saving 10-40

Setting 10-37, 10-143

Go

VAX/VMS debugger $12-39$ 
Graph coordinates 10-63, 10-64

Graph parameters

Getting 10-34

Restoring 10-36

Saving 10-35

Setting 10-30, 10-139

Graphics application interface 11-45

Graphics library 10-1

Graphing

Two-dimensional $10-57$

Graphing routines

PLT_LIB 10-7

Grid block information 6-5

H

Hardware text $10-51$

Header

Main program 5-12-5-16

Header frame 10-17, 11-15

Headers

DCL Driver 5-18

Include file 5-16

Subroutine 5-16

Heading, CAMDAT_LIB 6-4-6-5

HELP

CHANGES $12-7$

Help file 2-22, 4-11-4-19

Help file structure 4-13

HELP_LIB 2-20-2-22

HJIPLOT_LIB 10-1, 10-147-10-148

HJIPLOT_LIB routines 10-148, 11-1

HLP2CHK 12-26-12-27

Identifiers and headings $\quad 12-26$

\section{I}

IJK $8-60$

Inactive software 3-15

Include file headers 5-16

Initialize plotting 10-159, 10-160, 10-176, 10-177

Initialize SVDI 11-16

Initializing PLT_LIB 10-18, 10-21

INITPLO'T 10-159

INITPLT 10-160

Input data base 6-11

Inquiry

Color table 11-17

Current Position 11-18

Device capabilitics $11-19$

Escape 11-22

NDC limits 11-23
Output status 11-24

Inspection 3-9

Intensity $11-36$

Interfacing $C$ codes 5-19-5-21

Internal documentation 5-11-5-18

INTRP1 10-161

ISTOUT 8-7

K

Keyboard coordinates 11-11

Keyboard locator 11-37

$\mathbf{L}$

Layer elevations 7.2

LAYRELEV.SDB 7-2

Legal disclaimer 4-3

Length of string 10-162

LENSTR 8-53

LENTEXT 10-162

LIBRARIES

CREATE_PROGRAM 12-10

Libraries

CAMDAT_LIB 6-1

Main 2-20

Library

FLINT 12-17

Line style 11-38

Line width 11-39

Linear interpolation 10-161

LINED 10-164

LINEW 10-163, 10-165

Link program 12-36

Linking $C$ codes 5-19

Linking librarics 10-1, 10-147, 11-1, 11-45, $11-47,6-12,7-7,8-1,9-1$

Linking SDBREAD_LIB routines 7-7

List DCL filc 12-28-12-29

List FORTRAN source 12-30-12-31

LISTDCL 12-28-12-29

LISTFORTRAN 12-30-12-31

LOCSTR 8-52

Log an error 11-26

LOGAX 10-166

Logicals $2-7$

Lowercase letters $8-30$

M

Machine-dependent coding 5-10

Main program headers 5-12-5-16

MAINPROG 
CREATE_PROGRAM 12-10

Manipulating output $10-5$

MAP $10-4,10-5$

Map

X-coordinates $10-188$

Y-coordinates $\quad 10-189$

Mapping 10-4

2D points $10-86,10-94$

2D polygon $10-93$

2D polygons $10-85$

2D symbols $10-95$

$2 \mathrm{D}$ vectors $10-87,10-96$

3D points $10-89$

3D polygons $10-88$

$3 \mathrm{D}$ vectors $10-90$

Mapping routines

PLT_LIB 10-7, 10-84

MARKER 10-167

Masking 10-78, 10-81, 10-82

Material block information 6-5-6-7

Material parameters 6-2, 6-7

MAXERR 8-16

Maximum device coordinates $10-20$

MCCOMP 9-24

MCDEBG 9-35

MCDEL 9-14

MCEFIX 9-25

MCEROR 9-15

MCERPT 9-26

MCEXEC 9-27

MCFILL 9-16

MCFIND 9-28

MCFOFF 9-17

MCGET 9-18

MCGIVE 9-19

MCINIT 9-20

MCLAST 9-29

MCLIST 9-36

MCLONG $9-30$

MCMEMS 9-31

MCNAME 9-32

MCPRNT 9-37

MCRSRV 9-21

MCSTAT 9-22

MCWAIT 9-33

MDCOMi $9-24$

MDDEBG 9-35

MDDEL 9-14

MDEFIX 9-25

MDEROR 9-15

MDERPT 9-26
MDEXIEC 9-27

MDFILL 9-16

MDFIND 9-28

MDFOFF 9-17

MDGET 9-18

MDGIVE 9-19

MDINI'T 9-20

MDLIST 9-36

MDLONG 9-30

MDMEMS 9-31

MDNAME 9-32

MDPRNT 9-37

MDRSRV 9-21

MDSTAT 9-22

MDWAIT 9-33

MEMERR 8-17

Memory management

CAMSUPES_LIB 9-2

Memory manágement routines

CAMSUPES_LIB 9-11

Mesh

Finite difference 6-6

MINMAX 8-61

Mixing FORTRAN and C 5-19

Model description 6-2, 6-13, 6-41

CAMDAT_LIB 6-13, 6-41

Modelling transformations $10-5$

Modifying software 3-13-3-15

Modularity 5-6

Modules of the CAMCON system 2-7-2-9

Move

To point 10-174, 10-175

Move the cursor 11-27

Move the pen 10-75

MP2PG 10-85

MP2PT $10-86$

MP2VC $10-87$

MP3PG $10-88$

MP3РГ 10-89

MP3VC 10-90

MPCLP2 10-91

MPCLP3 10-92

MPD2PG 10-93

MPD2PT $10-94$

MPD2SY 10-95

MPD2VC 10-96

MPD3PG 10-97

MPD3PT 10-98

MPD3VC. 10-99

MPGETM 10-100

MPINIT 10-101 
MPLOOK 10-102

MPORT2 10-103

MPORT3 10-104

MPPERS 10-105

MPPOLA 10-106

MPPOPM 10-107

MPPSHM 10-108

MPPUTM 10-109

MPRESE 10-110

MPRLOC 10-111

MPROTA $10-112$

MPSCAL 10-113

MPTRAN 10-114

MPVIEW 10-115

MPWIND 10-116

\section{$\mathbf{N}$}

Name of software 4-1

Naming conventions 2-10, 4-4-4-19

NDC limits 11-23

NENUM 8-62

Neutral file 2-15

Neutral-file format 2.15

New frame 10-12

NIDFD 10-172

Nodal point coordinates $6-5$

Node point sets 6-7

Node sets 6-7

Non-SES software 3-12

Null field 8-30

NUMBER 10-173

Numbering statements 5-4

Numeric field 8-30

Numerical data 4-9

\section{O}

On-line documentation 2-22, 4-10

OPNFIL, 8-26

Output data base 6-10

Output status 11-24, 11-40

\section{$\mathbf{P}$}

Palette color 10-133

Parameter data 7-2

Farameter input 4-7

Parsing routines 4-8

PCKSTR 8-56

Pen position 10-79

PLOT 10-174

Plot
Arc-hyperbolic sine axis $\quad 10-150$

Characters 10-184

Linear axis 10-151, 10-152

Number 10-173

PLOTD 10-175

PLOTS 10-176, 10-177

Plotting

Characters $10-80$

Graphs 10-59, 10-60, 10-73

Points 10-77, 10-78

Symbols 10-81

Text 10-52, 10-56

Plotting package 10-1

Plotting text $10-51$

PLT_LIB 10-1-10-9

Clipping routines $10-117$

Color palette routines $10-128$

Mapping routines $10-84$

Setting parameters 10-23, 10-136

Two-dimensional graphing $10-57$

PLT_LIB routines 10-6

PLTARR 10-58, 10-72

PLTAXS 10-73

PLTBEL 10-11

PLTBGN 10-12, 10-13

PLTCG2 10-118

PLTCLIP 10-1, 10-6

PLTCNM 10-129

PLTCOL 10-134

PLTCOLOR 10-9

PLTCP2 10-119

PLTCP3 $10-120$

PLTCRS 10-14

PLTCUR 10-62, 10-68

PLTCV2 10-121

PLTCV3 10-122

PLTD2G 10-63

PLTDRW 10-74

PLTEND 10-15

PLTEXT 10-1

PLTFLU 10-2, 10-16

PITTFRM 10-17

PLTG2D 10-64

PLTGPH 10-59, 10-60

PLTGRAPII 10-7

PLTGTC 10-25

PI.TGTD 10-27

PLTGTG 10-34

PLTGTM 10-39

PITGTT 10-43, 10-48

PLTICL. 10-130 


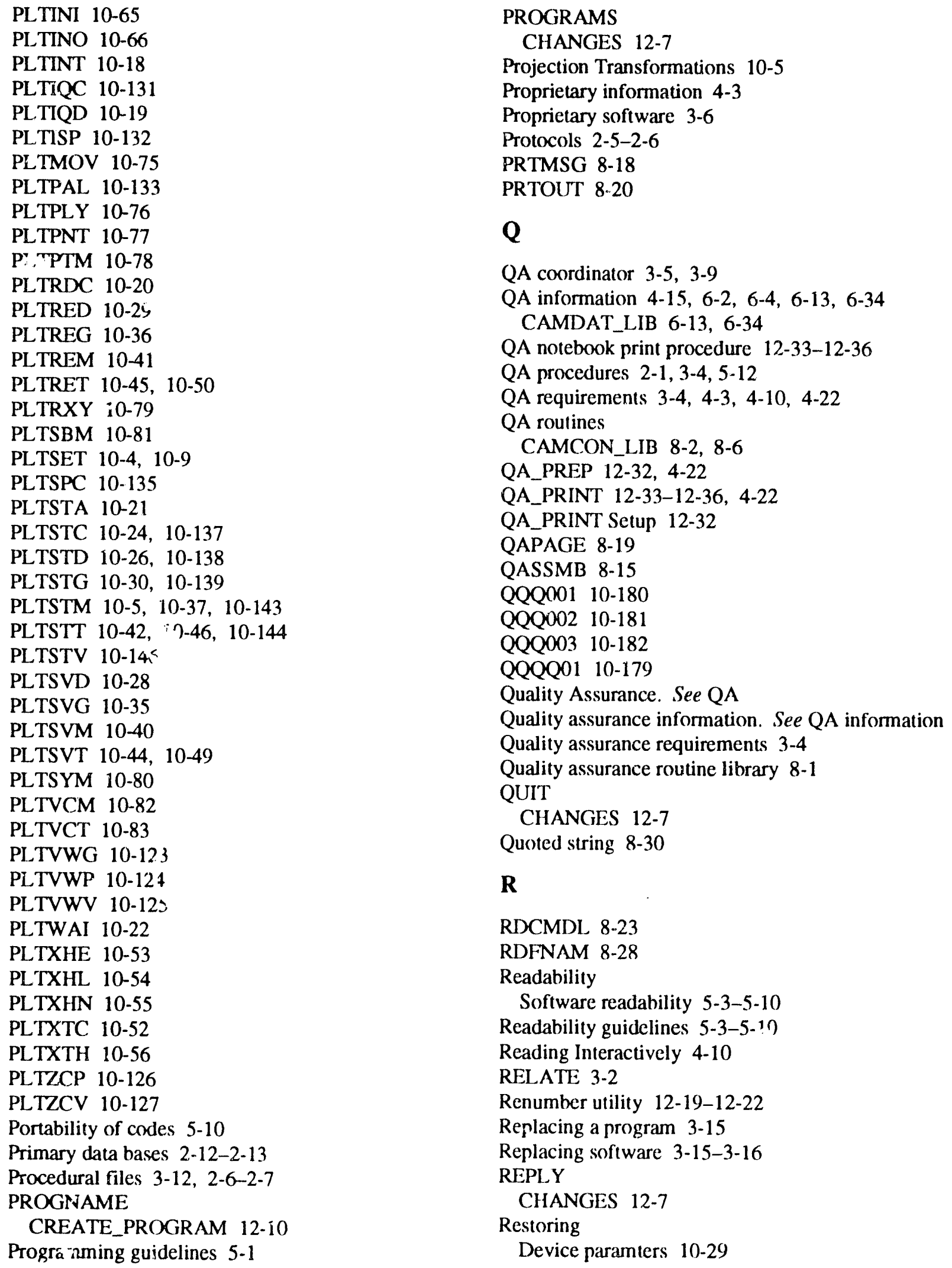


Global mapping parameters $10-41$

Graph parameters $10-36$

Text parameters 10-45, 10-50

Return end points 10-179, 10-180, 10-181

Return tic spacing $10-179,10-180$

Robustness 5-11

\section{$\mathbf{S}$}

Sandia Virtual Device Interface 11-1

Sandia virtual device interface $10-1$

Saving

Device parameters $10-28$

Global mapping parameters $10-40$

Graph parameters 10-35

Text parameters $10-44,10-49$

Saving parameters $10-28,10-40$

SCANCHANGES $12-5-12-6$

Scientific and engineering software 3-3

SCREEN 10-183

SDBARES 7-14

SDBCASES 7-15

SDBCLOC 7-16

SDBCONCR 7-33

SDBFRES $7-17$

SDBGETF 7-18

SDBGETL 7-19

SDBIATR 7-20

SDBILOC. $7-21$

SDBINFO 7-22

SDBINIT 7.23

SDBIRES 7-24

SDBMOVRV 7-34

SDBNINFO 7-25

SDBNUMDB 7-26

SDBPUTL 7-27

SDBREAD_LIB 2-20-2-22, 7-1

Advanced routines 7-32

General routines 7-13

Linking 7-7

Parameter data 7-2

Required files $\%-7$

Routine calls 7-13

Use 7-9

SDBRLOC 7-28

SDBRWIND 7-31

SDBSCMP 7-29

SDBSETRC 7-35

SDBSETRV 7-36

SDBSKHDR $7-30$

SDB TSTRC 7-37

Secondary data base $2-3,2-13-2-15,7-1-7-3$
Secondary data base interfac

SES software 3-3, 3-11

Set background color 11-31

SET BREAK

VAX/VMS debugger 12-38

Set character font 11-35

Set character size 11-33

Set character string angle 11-41

Set color table 11-32

Set foreground color 11-34

Set intensity 11-36

Set keyboard locator 11-37

Set line style 11-38

Set line width 11-39

Set output status $11-40$

SET SCOPE

VAX/VMS debugger $12-39$

Setting

Color parameters 10-24, 10-137

Device parameters 10-26, 10-138

Global mapping parameters $10-37,10-143$

Graph parameters 10-30, 10-139

Text parameters 10-42, 10-46, 10-144

Vector parameters $10-146$

Selting parameters

PLT_LIB 10-6, 10-23, 10-136

Setting PLT_LIB parameters $10-6$

SHOW BREAK

VAX/VMS debugger $12-38$

SHOW CALLS

VAX/VMS debugger $12-40$

Single-analysis software 3-17-3-18

Software abstract 4-22

Software acquisition procedures $3-9-3-13$

Software classification 3-5-3-6

Software development procedures 3-9-3-13

Software development strategy 3-1-3-4

Software development utilities 12-1

Software libraries 2-19-2-22

Software management system 2-3-2-6

Software name 4-1

Software notebook 4-21-4-22

Software planning 5-1-5-2

Software review committee 3-11

Software robustness 5-11

Software sponsor 3-9

Software text 10-51

SPAWN

VAX/VMS debugger $12-40$

Spectrum mapping 10-132

Sponsor 3-9 
Definition 2-5

SPONSORS

CHANGES $12-8$

SQZSTR 8-54

Statement numbering 5-4

Step

VAX/VMS debugger $12-39$

Stratigraphy $7-1-7-2$

String 8-30

String processing routines

CAMCON_LIB 8-2, 8-51

STRIPB 8-55

STRTUP 8-21

Subroutine headers 5-16

SVDI 10-1, 11-1

SVDI Devices 11-'2-11-5

SVDI routines 11-6

SWCF records manager 3-10

SYMBOL 10-184

Symbols 2-7

Syntax conventions 4-8

Syntax rules 8-30

System architecture 2-3

System queries

CAMSUPES_LIB 9-2, 9-3

\section{$\mathbf{T}$}

Task leader 3-9

Terminal bell 10-11

Terminate SVDI $11-42$

Text parameters

Getting 10-43, 10-48

Restoring 10-45, 10-50

Saving 10-44, 10-49

Setting 10-42, 10-46, 10-144

Text plotting 10-51

Theory manual 4-24

Three-dimensional coordinates $10-5$

TL 3-9

Traceability $4-1-4-3$

Trailer frame 10-17, 11-15

Transformations $10-4$

Two-dimensional coordinates $10-5$

Two-dimensional graphing 10-57

PLT_LIB 10-57

TYPE

VAX/VMS debugger 12-39

$\mathbf{U}$

UPCASE $8-57$
Update history 4-14

Update program 12-36

UPDATE_PROGRAM 12-36-12-37

User interface 4-1

User interface routines

CAMCON_LIB 8-2, 8-6

Utilities

FLINT 12-12-12-18

FORMRENUM 12-19-12-22

LISTDCL $12-28$

LISTFOR 12-30-12-31

QA_PREP 12-32

QA_PRINT 12-33-12-36

UPDATE_PROGRAM $\quad 12-36$

V

Variable Names 5-5

VAX/VMS debugger 12-37-12-40

Examining variable values $12-40$

Executing 12-39

Listing source code 12-39

Miscellaneous commands $12-40$

Setting break points $12-38$

VBERRH 11-7

VDAABU $11-8$

VDABGL 11-9

VDAKGL 11-10

VDALOC 11-11

VDBELL 11-12

VDBUFL 11-13

VDESCP 11-2, 11-14

VDFRAM 11-15

VDINIT 11-16

VDIQCO $11-17$

VDIQCP $11-18$

VDIQDC 11-19

VDIQES 11-22

VDIQND 11-23

VDIQOS 11-24

VDLINA 11-25

VDLOGE 11-7, 11-26

VDMOVA 11-27

VDNWPG 11-28

VDPNTA $11-29$

VDPOLY $11-30$

VDSTBC 11-31

VDSTCO 11-32

VDSTCS 11-33

VDSTFC 11-34

VDSTFN 11-35

VDSTIN 11-36 
VDSTLA $11-37$

VDSTLS 11-38

VDSTLW 11-39

VDSTOS $11-40$

VDSTSA 11-41

VDTERM 11-42

VDTEXT 11-43

VDWAIT $11-44$

Vector parameters

Setting 10-146

Verification 3-9, 3-16-3-17

Verification records $4-23$

Version ID 4-1

VIEW

CHANGES $12-8$

Viewing Transformations $10-5$

Viewport 10-4, 10-123, 10-124, 10-125

Viewport transformations $10-5$

W

Wait for graphics device 11-8, 11-9

Wait for kcy 10-14, 10-22

Wait for keyboard 11-10

Wait for return 10-185, 10-186, 10-187

Wait for user 11-44

WAITCR 10-185, 10-186, 10-187

Well locations 7.2

WRCPUS 8-12

WRFNAM 8-29

$\mathbf{X}$

XMAPDC $10-188$

XWTODC. $10-190$

Y

YMAPDC 10-189

YWTODC 10-191 


\section{DISTRIBUTION LIST}

(Send Distribution list changes to M.M. Gruebel, Dept. 6342, Sandia National Laboratories, PO Box 5800, Albuquerque, NM 87185-5800)

\section{Federal Agencies}

US Department of Energy (6) Office of Civilian Radioactive Waste Management

Attn: Deputy Director, RW-2

Associate Director, RW-10/50 Office of Program and Resource: Management Office of Contract Business Management

Director, Analysis and Verification

Division, RW-22

Associate Director, RW-30 Office of Systems and Compliance

Associate Director, RW-40

Office of Storage and

Transportation

Director, RW-4/5

Office of Strategic Planning and International Programs Office of External Relations

Forrestal Building

Washington, DC 20585

US Department of Energy

Albuquerque Operations Office

Attn: National Atomic Museum Library

PO Box 5400

Albuquerque, NM 87185

US Department of Energy (2)

Office of Environmental Restoration and Waste Management

Attn: EM-1

$$
\text { C. Frank, EM-50 }
$$

Washington, DC 20585

US Department of Energy (3)

Office of Environmental Restoration and Waste Managemet:t

Attn: M. Frei, EM-34, Trevion II

Director, Waste Management Projects

Wastuington, DC 20585-0002

US Department of Energy

Office of Environmental Restoration and Waste Management

Attn: J. Lytle, EM-30, Trevion II

Washington, DC 20585-0002
US Department of Energy

Office of Environmental Restoration and Waste Management

Attn: S. Schneider, EM-342, Trevion II

Washington, DC 20585-0002

US Department of Energy (3)

WIPP Task Force

Attn: G.H. Daly

S. Fucigna

B. Bower

12800 Middlebrook Rd., Suite 400

Germantown, MD 20874

US Department of Energy (4)

Office of Environment, Safety and Health

Attn: R.P. Berube, EH-20

C. Borgstrum, EH-25

R. Pelletier, EH-231

K. Taimi, EH-232

Washington, DC 20585

US Department of Energy (6)

WIPP Project Integration Office

Attn: S. Alcorn

W.J. Arthur III

J. Coffey

L.W. Gage

P.J. Higgins

D.A. Olona

PO Box 5400

Albuquerque, NM 87115-5400

US Department of Energy (2)

WIPP Project Integration Satellite Office

Attn: R. Batra

R. Becker

PO Box 3090, Mail Stop 525

Carlsbad, NM 88221-3090

US Department of Energy (10)

WIPP Project Site Office (Carlsbad)

Attn: A. Hunt (4)

V. Daub (4)

J. Lippis

K. Hunter

PO Box 3090

Carlsbad, NM 88221-3090 
US Department of Energy

Research \& Waste Management Division

Attn: Director

PO Box E

Oak Ridge, TN 37831

US Department of Energy (2)

Idaho Operations Office

Fuel Processing and Waste

Management Division

785 DOE Place

Idaho Falls, ID 83402

US Department of Energy

Savannah River Operations Office

Defense Waste Processing

Facility Project Office

Attn: W.D. Pearson

PO Box A

Aiken, SC 29802

US Department of Energy (2)

Richland Operations Office

Nuclear Fuel Cycle \& Production

Division

Attn: R.E. Gerton

825 Jadwin Ave.

PO Box 500

Richland, WA 99352

US Department of Energy

Office of Geologic Disposal

Yucca Mountain Project Office

Attn: Associate Director, RW-20

PO Box 98608

Las Vegas, NV 89193-8608

US Department of Energy (3)

Nevada Operations Office

Attn: J.R. Boland

D. Livingston

P.K. Fitzsimmons

2753 S. Highland Drive

Las Vegas, NV 89183-8518

US Department of Energy (2)

Technical Information Center

PO Box 62

Oak Ridge, TN 37831

US Department of Energy

Los Alamos Area Office

528 35th Street

Los Alamos, NM 87544
US Department of Energy (2)

Chicago Operations Office

Attn: J.C. Haugen

9800 South Cass Avenue

Argonne, IL 60439

US Department of Energy (3)

Rocky Flats Area Office

Attn: W.C. Rask

G. Huffman

PO Box 928

T. Lukow

Golden, CO 80402-0928

US Department of Energy

Dayton Area Office

Attn: R. Grandfield

PO Box 66

Miamisburg, OH 45343-0066

US Department of Energy

Attn: E. Young

Room E-178

GAO/RCED/GTN

Washington, DC 20545

US Bureau of Land Management

Carlsbad Office

101 E. Mermod

Carlsbad, NM 88220

US Bureau of Land Management

New Mexico State Office

PO Box 1449

Santa Fe, NM 87507

US Environmental Protection Agency (2)

Radiation Protection Programs

Attn: M. Oge

ANR-460

Washington, DC 20460

US Environmental Protection Agency, Region 6

Attn: C. Byrum, 6T-ET

1445 Ross Ave.

Dallas, TX 75202

US Geological Survey (2)

Water Resources Division

Attn: C. Peters

4501 Indian School NE

Suite 200

Albuquerque, NM 87110 
US Nuclear Regulatory Commission

Division of Waste Management

Attn: H. Marson

Mail Stop 4-H-3

Washington, DC 20555

US Nuclear Regulatory Commission (4)

Advisory Committee on Nuclear Waste

Attn: D. Moeller

M.J. Steindler

P.W. Pomeroy

W.J. Hinze

7920 Norfolk Ave.

Bethesda, MD 20814

Defense Nuclear Facilities Safety Board

Attn: D. Winters

625 Indiana Ave. NW

Suite 700

Washington, DC 20004

Nuclear Waste Technical Review Board Attn: Library (2)

1100 Wilson Blvd.

Suite 910

Arlington, VA 22209-2297

Energy and Science Division

Office of Management and Budget

Attn: K. Yuracko

725 17th Street NW

Washington, DC 20503

\section{State Agencies}

New Mexico Bureau of Mines

and Mineral Resources

Socorro, NM 87801

New Mexico Energy, Minerals and Natural

Resources Department

Attn: Librarian

2040 South Pacheco

Santa Fe, NM 87505

New Mexico Energy, Minerals and Natural

Resources Department

New Mexico Radioactive Task Force (2)

(Governor's WIPP Task Force)

Attn: A. Lockwood, Chairman

C. Wentz, Policy Analyst

2040 South Pacheco

Santa Fe, NM 87505
Bob Forrest

Mayor, City of Carlsbad

PO Box 1569

Carlsbad, NM 88221

Carlsbad Department of Development

Executive Director

Attn: C. Bernard

PO Box 1090

Carlsbad, NM 88221

New Mexico Environment Department

Secretary of the Environment (3)

Attn: J. Espinosa

PO Box 968

1190 St. Francis Drive

Santa Fe, NM 87503-0968

New Mexico Environment Department

Attn: P. McCasland

WIPP Project Site Office

PO Box 3090

Carlsbad, NM 88221-3090

New Mexico State Engineer's Office

Attn: M. Chudnoff

PO Box 25102

Santa Fe, NM 87504-5102

Environmental Evaluation Group (5)

Attn: R. Neill

7007 Wyoming Blvd. NE, Suite F-2

Albuquerque, NM 87109

\section{Advisory Committee on Nuclear Facility Safety}

John F. Ahearne

Executive Director, Sigma Xi

99 Alexander Drive

Research Triangle Park, NC 27709

James E. Martin

109 Observatory Road

Ann Arbor, MI 48109

WIPP Panel of National Research Council's Board on Radioactive Waste Management

Charles Fairhurst, Chairman

Department of Civil and

Mineral Engineering

University of Minnesota

500 Pillsbury Dr. SE

Minneapolis, MN 55455-0220 
John O. Blomeke

3833 Sandy Shore Drive

Lenoir City, TN 37771-9803

John D. Bredehoeft

Western Region Hydrologist

Water Resources Division

US Geological Survey (M/S 439)

345 Middlefield Road

Menlo Park, CA 94025

Rodney C. Ewing

Department of Geology

University of New Mexico

Albuquerque, NM 87131

B. John Garrick

PLG, Inc.

4590 MacArthur Blvd.

Suite 400

Newport Beach, CA 92660-2027

Leonard F. Konikow

US Geological Survey

431 National Center

Reston, VA 22092

Jeremiah O'Driscoll

505 Valley Hill Drive

Atlanta, GA 30350

Chris G. Whipple

ICF Kaiser Engineers

1800 Harrison St.

Oakland, CA 94612-3430

National Research Council (3)

Board on Radioactive

Waste Management

RM HA456

Attn: P.B. Myers (2)

G.J. Grube

2101 Constitution Ave.

Washington, DC 20418

Performance Assessment Peer Review Panel

G. Ross Heath

College of Ocean and Fishery Sciences, HN15

583 Henderson Hall

University of Washington

Seattle, WA 98195
Thomas H. Pigford

Department of Nuclear Engineering

4159 Etcheverry Hall

University of California

Berkeley, CA 94720

Thomas A. Cotton

JK Research Associates, Inc.

4429 Butterworth Place NW

Washington, DC 20016

Robert J. Budnitz

President, Future Resources Associates, Inc. 2000 Center Street, Suite 418

Berkeley, CA 94704

C. John Mann

Department of Geology

245 Natural History Bldg.

1301 West Green Street

University of Illinois

Urbana, IL 61801

Frank W. Schwartz

Department of Geology and Mineralogy

The Ohio State University

Scott Hall

1090 Carmack Rd.

Columbus, $\mathrm{OH} \mathbf{4 3 2 1 0}$

\section{National Laboratories}

Argonne National Laboratory (2)

Attn: A. Smith

D. Tomasko

9700 South Cass, Bldg. 201

Argonne, IL 60439

Battelle Pacific Northwest

Laboratory (2)

Attn: S. Bates

R.E. Westerman

MSIN P8-4!

Battelle Boulevard

Richland, WA 99352

Idaho National Engineering

Laboratory (2)

Attn: H. Loo

R. K linger

Mail Stop 5!08

Idahc Falls, ID 83403-4000 
Los Alamos National Laboratory (5)

\section{Attn:}

B. Erdal, CNC-11

M. Ennis, HS- 12

Miail Stop J900

S. Kosiewicz, EM-7

Mail Stop J595

L. Soholt, EM-13

Mail Stop M992

J. Wenzel, HS-12

Mail Stop K 482

PO Box 1663

Los Alamos, NM 87545

Oak Ridge National Laboratory

Transuranic Waste Manager

Attn: D.W. Turner

Bldg. 3047

PO Box 2008

Oak Ridge, TN 37831-6060

Pacific Northwest Laboratory

Attn: B. Kennedy

PO Box 999

Richland, WA 99352

Savannah River Plant (2)

Attn: R.G. Baxter Bldg. 704-S

K.W. Wierzbicki

Bldg. 703-H

Aiken, SC 29808-0001

Westinghouse-Savannah River Technology

Center (4)

Attn: N. Bibler

J.R. Harbour

M.J. Plodinec

G.G. Wicks

Aiken, SC 29802

\section{Corporations/Members of the Public}

Battelle Memorial Institute

Attn: R. Root

J. Kircher

505 Marquette NW

Suite 1

Albuquerque, NM 87102

Benchmark Environmental Corp.

Attn: C. Frederickson

4501 Indian School NE

Suite 105

Albuquerque, NM 87110
City of Albuquerque

Public Works Department

Utility Planning Division

Attn: W.K. Summers

PO Box 1293

Albuquerque, NM 87103

Deuel and Associates, Inc.

Attn: R.W. Prindle

7208 Jefferson NE

Albuquerque, NM 87109

Disposal Safety, Inc.

Attn: B. Ross

1660 L Street NW, Suite 314

Washington, DC 20036

Ecodynamics (2)

Attn: P. Roache

R. Blaine

PO Box 9229

Albuquerque, NM 87119-9229

EG \& G Idaho (3)

1955 Fremont Street

Attn: C. Atwood

C. Hertzler

T.I. Clements

Idaho Falls, ID 83415

Geomatrix

Attn: K. Coppersmith

100 Pine St., Suite 1000

San Francisco, CA 94111

Golder Associates, Inc. (2)

Attn: R. Kossik

I. Miller

4104 148th Avenue NE

Redmond, WA 98052

INTERA, Inc.

Attn: A.M. LaVenue

1650 University Blvd. NE, Suite 300

Albuquerque, NM 87102

INTERA, Inc.

Attn: J.F. Pickens

6850 Austin Center Blvd., Suite 300

Austin, TX 78731

INTERA, Inc.

Attn: W. Stensrud

PO Box 2123

Carlsbad, NM 88221 
INTERA, Inc.

Attn: W. Nelson

101 Convention Center Drive

Suite 540

Las Vegas, NV 89109

IT Corporation (2)

Attn: R.F. McK inney

J. Myers

Regional Office

Suite 700

5301 Central Avenue NE

Albuquerque, NM 87108

John Hart and Associates, P.A.

Attn: J.S. Hart

2815 Candelaria Road NW

Albuquerque, NM 87107

John Hart and Associates, P.A.

Attn: K. Lickliter

1009 North Washington

Tacoma, WA 98406

MAC Technical Services Co.

Attn: D.K. Duncan

8418 Zuni Road SE

Suite 200

Albuquerque, NM 87108

Newman and Holtzinger

Attn: C. Mallon

$1615 \mathrm{~L}$ Street NW

Suite 1000

Washington, DC 20036

$\mathrm{RE} / \mathrm{SPEC}$, Inc. (2)

Attn: W. Coons

4775 Indian School NE

Suite 300

Albuquerque, NM 87110

RE/SPEC, Inc.

Attn: J.L. Ratigan

PO Box 725

Rapid City, SD 57709

Reynolds Electric and Engineering Company, Inc.

Attn: E.W. Kendall

Building 790

Warehouse Row

PO Box 98521

Las Vegas, NV 89193-8521
Science Applications International

Corporation (SAIC)

Attn: H.R. Pratt

10260 Campus Point Drive

San Diego, CA 92121

Science Applications International

Corporation (2)

Attn: D.C.Royer

C.G. Pflum

101 Convention Center Dr.

Las Vegas, NV 89109

Science Applications International

Corporation (2)

Attn: M. Davis

J. Tollison

2109 Air Park Road SE

Albuquerque, NM 87106

Science Applications International

Corporation (2)

Attn: J. Young

D. Lester

18706 North Creek Parkway, Suite 110

Bothell, WA 98011

Southwest Research Institute

Center for Nuclear Waste Regulatory Analysis

(2)

Attn: P.K. Nair

6220 Culebra Road

San Antonio, TX 78228-0510

Systems, Science, and Software (2)

Attn: E. Peterson

Box 1620

P. Lagus

La Jolla, CA 92038

TASC

Attn: S.G. Oston

55 Walkers Brook Drive

Reading, MA 01867

Tech Reps, Inc. (6)

Attn: J. Chapman

C. Crawford

D. Marchand

T. Peterson

J. Stikar

D. Scott

5000 Marble NE, Suite 222

Albuquerque, NM 87110 
Tolan, Beesun \& Associates

Attn: T.I.. Toilan

$2320 \mathrm{~W}$. 15th Avenue

Kennewick, WA 99337

TRW Environmental Safety Systems (2)

Attn: I. Sacks, Suite 800

L. Wildman, Suite 1300

2650 Park Tower Drive

Vienna, VA 22180-7306

Sanford Cohen and Associates

Attn: J. Channell

7101 Carriage Rd NE

Albuquerque, NM 87109

Westinghouse Electric Corporation (5)

Attn: Library

C. Cox

L. Fitch

B.A. Howard

R.F. Kehrman

PO Bcx 2078

Carlsbac, NM 88221

Westinghouse Hanford Company

Attn: D.E. Wood, MSIN HO-32

PO Box 1970

Richland, WA 99352

Western Water Consultants

Attn: P.A. Rechard

PO Box 4128

Laramie, WY 82071

Western Water Consultants

Attn: D. Fritz

1949 Sugarland Drive \#134

Sheridan, WY 82801-5720

P. Drez

8816 Cherry Hills Road NE

Albuquerque, NM 87111

David Lechel

9600 Allende Rd. NE

Albuquerque, NM 87109

C.A. Marchese

PO Box 21790

Albuquerque, NM 87154

Arend Meijer

3821 Anderson SE

Albuquerque, NM 87108
D.W. Powers

Star Route Box 87

Anthony, TX 79821

Shirley Thieda

PO Box 2109, RR 1

Bernalillo, NM 87004

Jack Urich

c/o CARD

144 Harvard SE

Albuquerque, NM 87106

\section{Universities}

University of California

Mechanical, Aerospace, and

Nuclear Engineering Department (2)

Attn: W. Kastenberg

D. Browne

5532 Boelter Hall

Los Angeles, CA 90024

University of California

Mine Engineering Department

Rock Mechanics Engineering

Attn: N. Cook

Berkeley, CA 94720

University of Hawaii at Hilo

Business Administration

Attn: S. Hora

Hilo, HI 96720-4091

University of New Mexico

Geology Department

Attn: Library

Albuquerque, NM 87131

University of New Mexico

Research Administration

Attn: H. Schreyer

102 Scholes Hall

Albuquerque, NM 87131

University of Wyoming

Department of Civil Engineering

Attn: V.R. Hasfurther

Laramie, WY 82071

University of Wyoming

Department of Geology

Attn: J.I. Drever

Laramie, WY 82071 
University of Wyoming

Department of Mathematics

Attn: R.E. Ewing

Laramie, WY 82071

\section{Libraries}

Thomas Brannigan Library

Attn: D. Dresp

106 W. Hadley St.

Las Cruces, NM 88001

Hobbs Public Library

Attn: M. Lewis

509 N. Ship Street

Hobbs, NM 88248

New Mexico State Library

Attn: N. McCallan

325 Don Gaspar

Santa Fe, NM 87503

New Mexico Tech

Martin Speere Memorial Library

Campus Street

Socorro, NM 87810

New Mexico Junior College

Pannell Library

Attn: R. Hill

Lovington Highway

Hobbs, NM 88240

Carlsbad Municipal Library

WIPP Public Reading Room

Attn: L. Hubbard

$101 \mathrm{~S}$. Halagueno St.

Carlsbad, NM 88220

University of New Mexico

General Library

Government Publications Department

Albuquerque, NM 87131

\section{NEA/Performance Assessment Advisory Group (PAAG)}

P. Duerden

ANSTO

Lucas Heights Research Laboratories

Private Mail Bag No. 1

Menai, NSW 2234

AUSTRALIA
Gordon S. Linsley

Division of Nuclear Fuel Cycle and Waste

Management

International Atomic Energy Agency

PO Box 100

A-1400 Vienna, AUSTRIA

Nicolo Cadelli

Commission of the European Communities

200, Rue de la Loi

B-1049 Brussels, BELGIUM

R. Heremans

Organisme Nationale des Déchets Radioactifs

et des Matiéres Fissiles

(ONDRAF)

Place Madou 1, Boitec 24/25

B-1030 Brussels, BELGIUM

J. Marivoet

Centre d'Etudes de l'Energie Nucléaire

(CEN/SCK)

Boeretang 200

B-2400 Mol, BELGIUM

P. Conlon

Waste Management Division

Atomic Energy Control Board (AECB)

PO Box 1046

Ottawa, Ontario KIP 559, CANADA

A.G. Wikjord

Manager, Environmental and Safety

Assessment Branch

Atomic Energy of Canada Limited

Whiteshell Nuclear Research Establishment

Pinewa, Manitoba ROE ILO

CANADA

Teollisuuden Voima Oy (TVO) (2)

Attn: Timo Äikäs

$$
\text { Jukka-Pekka Salo }
$$

Annankatu $42 \mathrm{C}$

SF-00100 Helsinki Suomi, FINLAND

Timo Vieno

Technical Research Centre of Finland (VTT)

Nuclear Er rgy Laboratory

PO Box 208

SF-02151 Espoo, FINLAND 
Division de la Sécurité et de la Protection de l'Environment (DSPE)

Commissariat á l'Energie Atomique

Agence Nationale pour la Gestion des

Déchets Radioactifs (ANDRA)

Attn: Gérald Ouzounian

M. Claude Ringeard

Route du Panorama Robert Schuman

B. P. No. 38

F-92266 Fontenay-aux-Roses Cedex

FRANCE

Claudio Pescatore

Division of Radiation Protection and Waste

Management

OECD Nuclear Energy Agency

38, Boulevard Suchet

F-75016 Paris, FRANCE

M. Dominique Greneche

Commissariat á l'Energie Atomique

IPSN/DAS/SASICC/SAED

B.P. No. 6

F-92265 Fontenay-aux-Roses Cedex

FRANCE

Robert Fabriol

Bureau de Recherches Géologiques et

Miniéres (BRGM)

B.P. 6009

45060 Orléans Cedex 2, FRANCE

P. Bogorinski

Gesellschaft für Reaktorsicherheit (GRS)

$\mathrm{MBH}$

Schwertnergasse 1

D-5000 Köln 1, GERMANY

R. Storck

GSF - Institut für Tieflagerung

Theodor-Heuss-Strabe 4

D-3300 Braunschweig, GERMANY

Ferrucio Gera

ISMES S.p.A

Via del Crociferi 44

I-00187 Rome, ITALY

Hiroyuki Umeki

Isolation System Research Program

Radioactive Waste Management Project

Power Reactor and Nuclear Fuel

Development Corporation (PNC)

1-9-13, Akasaka, Minato-ku

Tokyo 107, JAPAN
P. Carboneras Martinez

ENRESA

Calle Emilio Vargas, 7

R-28043 Madrid

SPAIN

Tönis Papp

Swedish Nuclear Fuel and Waste Management Co.

Box 5864

S 10248 Stockholm

SWEDEN

Conny Hägg

Swedish Radiation Protection Institute (SSI)

Box 60204

S-104 01 Stockholm

SWEDEN

J. Hadermann

Paul Scherrer Institute

Waste Management Programme

CH-5232 Villigen PSI

SWITZERLAND

J. Vigfusson

HSK -Swiss Nuclear Safety Inspectorate

Federal Office of Energy

CH-5232 Villigen-HSK

SWITZERLAND

D.E. Billington

Departmental Manager-Assessment Studies

Radwaste Disposal R\&D Division

AEA Decommissioning \& Radwaste

Harwell Laboratory, B60

Didcot Oxfordshire OXII ORA

UNITED KINGDOM

P. Grimwood

Waste Management Unit

BNFL

Sellafield

Seascale, Cumbria CA20 IPG

UNITED KINGDOM
Alan J. Hooper
UK Nirex Ltd
Curie Avenue
Harwell, Didcot
Oxfordshire, OX11 ORH
UNITED KINGDOM 
Jerry M. Boak

Yucca Mountain Project Office

US Department of Energy

PO Box 98608

Las Vegas, NV 89193

Seth M. Coplan (Chairman)

US Nuclear Regulatory Commission

Division of High-Level Waste Management

Mail Stop 4-H-3

Washington, DC 20555

\author{
A.E. Van Luik \\ INTERA/M\&O \\ The Valley' Bank Center \\ 101 Convention Center Dr. \\ Las Vegas, NV 89109
}

\section{NEA/Probabilistic System Assessment Group (PSAG)}

Shaheed Hossain

Division of Nuclear Fuel Cycle and Waste

Management

International Atomic Energy Agency

Wagramerstrasse 5

PO Box 100

A-1400 Vienna, AUSTRIA

Alexander Nies (PSAC Chairman)

Gesellschaft für Strahlen- und

Institut für Tieflagerung

Abteilung für Endlagersicherheit

Theodor-Heuss-Strasse 4

D-3300 Braunschweig, GERMANY

Eduard Hofer

Gesellschaft für Reaktorsicherheit (GRS)

$\mathrm{MBH}$

Forschungsgelände

D-8046 Garching, GERMANY

Andrea Saltelli

Commission of the European Communities

Joint Resarch Centre of Ispra

I-21020 Ispra (Varese), ITALY

Alejandro Alonso

Cátedra de Tecnologia Nuclear

E.T.S. de Ingenieros Industriales

José Gutiérrez Abascal, 2

E-28006 Madrid, SPAIN
ENRESA (2)

Attn: M. A. Cuñado

F. J. Elorza

Calle Emilio Vargas, 7

E-28043 Madrid, SPAIN

Pedro Prado

CIEMAT

Instituto de Tecnologia Nuclear

Avenida Complutense, 22

E-28040 Madrid, SPAIN

Nils A. K jellbert

Swedish Nuclear Fuel and Waste Management

Company (SK B)

Box 5864

S-102 48 Stockholm, SWEDEN

Björn Cronhjort

Royal Institute of Technology

Automatic Control

S-10044 Stockholm, SWEDEN

Richard A. Klos

Paul-Scherrer Institute (PSI)

CH-5232 Villingen PSI, SWITZERLAND

Nationale Genossenschaft für die Lagerung

Radioaktiver Abfälle (2)

Attn: C. McCombie

F. Van Dorp

Hardstrasse 73

CH-5430 Wettingen, SWITZERLAND

N. A. Chapman

Intera Information Technologies

Park View House

14B Burton Street

Melton Mowbray

Leicestershire LE13 IAE

UNITED KINGDOM

Daniel A. Galson

Galson Sciences Ltd.

35, Market Place

Oakham

Leicestershire LE15 6DT

UNITED KINGDOM

David F. Hodgkinson

Intera Information Technologies

45 Station Road, Chiltern House

Henley-on-Thames

Oxfordshire RG9 IAT

UNITED KINGDOM 
Brian G.J. Thompson

Department of the Environment: Her

Majesty's Inspectorate of Pollution

Room A5.33, Romney House

43 Marsham Street

London SWIP 2PY

UNITED KINGDOM

Intera Information Technologies

Attn: M.J.Apted

3609 South Wadsworth Blvd.

Denver, CO 80235

US Nuclear Regulatory Commission (2)

Attn: R. Codell

N. Eisenberg

Mail Stop 4-H-3

Washington, DC 20555

Battelle Pacific Northwest Laboratories

Attn: P.W. Eslinger

MS K 2-32

PO Box 999

Richland, WA 99352

Center for Nuclear Waste Regulatory Analysis (CNWRA)

Southwest Research Institute

Attn: B. Sagar

PO Drawer 28510

6220 Culebra Road

San Antonio, TX 78284

\section{Geostatistics Expert Working Group (GXG)}

Rafael L. Bras

R.L. Bras Consulting Engineers

44 Percy Road

Lexington, MA 02173

Jesus Carrera

Universidad Politècnica de Cataluña

E.T.S.I. Caminos

Jordi, Girona 31

E-08034 Barcelona, SPAIN

Gedeon Dagan

Department of Fluid Mechanics and Heat

Transfer

Tel Aviv University

PO Box 39040

Ramat Aviv, Tel Aviv 69978

ISRAEL
Ghisiain de Marsily (GXG Chairman)

University Pierre et Marie Curie

Laboratorie de Geologie Applique

4 , Place Jussieu

T.26 - 5e etage

75252 Paris Cedex 05, FRANCE

Alain Galli
Centre de Geostatistique
Ecole des Mines de Paris
35 Rue St. Honore
77035 Fontainebleau, FRANCE

Christian Ravenne

Geology and Geochemistry Division

Institut Francais du Pétrole

$1 \& 4$, Av. de Bois-Préau B.P. 311

92506 Rueil Malmaison Cedex

FRANCE

Peter Grindrod

INTERA Information Technologies Ltd.

Chiltern House

45 Station Road

Henley-on-Thames

Oxfordshire, RG9 IAT, UNITED

KINGDOM

Alan Gutjahr

Department of Mathematics

New Mexico Institute of Mining and

Technology

Socorro, NM 87801

C. Peter Jackson

Harwell Laboratory

Theoretical Studies Department

Radwaste Disposal Division

Bldg. 424.4

Oxfordshire Didcot Oxon OXII ORA

UNITED KINDGOM

Rae iriackay

Department of Civil Engineering

University of Newcastle Upon Tyne

Newcastle Upon Tyne NEI 7RU

UNITED KINGDOM

Steve Gorelick

Department of Applied Earth Sciences

Stanford University

Stanford, CA 94305-2225

Peter Kitanidis

60 Peter Coutts Circle

Stanford, CA 94305 
Dennis McLaughlin

Parsons Laboratory, Room 48-209

Department of Civil Engineering

Massachusetts Institute of Technology

Cambridge, MA 02139

Shlomo P. Neuman

College of Engineering and Mines

Department of Hydrology and Water

Resources

University of Arizona

Tucson, AZ 85721

Yoram Rubin

Department of Civil Engineering

University of California

Berkeley, CA 94720

\section{Foreign Addresses}

Studiecentrum Voor Kernenergie

Centre D'Energie Nucleaire

Attn: A. Bonne

SCK /CEN

Boeretang 200

B-2400 Mol

BELGIUM

Atomic Energy of Canada, Ltd. (3) Whiteshell Research Establishment

Attn: M.E. Stevens

B.W. Goodwin

D. Wushke

Pinewa, Manitoba ROE $1 \mathrm{LO}$

CANADA

Juhani Vira

Teollisuuden Voima Oy (TVO)

Annankatu $42 \mathrm{C}$

SF-00100 Helsinki Suomi

FINLAND

Jean-Pierre Olivier

OECD Nuclear Energy Agency (2)

38, Boulevard Suchet

F-75016 Paris

FRANCE

D. Alexandre, Deputy Director

ANDRA

31 Rue de la Federation

75015 Paris

FRANCE
Claude Sombret

Centre D'Etudes Nucleaires

De La Vallee Rhone

CEN/VALRHO

S.D.H.A. B.P. 171

30205 Bagnols-Sur-Ceze, FRANCE

Bundesministerium fur Forschung und

Technologie

Postfach 200706

5300 Bonn 2, GERMANY

Bundesanstalt fur Geowissenschaften

und Rohstoffe

Attn: M. Langer

Postfach 510153

3000 Hanover 51, GERMANY

Gesellschaft fur Reaktorsicherheit (GRS) (2)

Attn: B. Baltes

W. Muller

Schwertnergasse 1

D-5000 Cologne, GERMANY

Institut fur Tieflagerung (2)

Attn: K. Kuhn

Theodor-Heuss-Strasse 4

D-3300 Braunschweig, GERMANY

Physikalisch-Technische

Bundesanstalt

Attn: P. Brenneke

Postfach 3345

D-3300 Braunschweig, GERMANY

Shingo Tashiro

Japan Atomic Energy Research Institute Tokai-Mura, Ibaraki-Ken

319-11, JAPAN

Netherlands Energy Research Foundation (ECN)

Attn: L..H. Vons

3 Westerduinweg

PO Box 1

1755 ZG Petten, THE NETHERLANDS

Johan Andersson

Swedish Nuclear Power Inspectorate

Statens Kärnkraftinspektion (SKI)

Box 27106

S-10252 Stockholm, SWEDEN 
Fred Karlsson

Svensk Karnbransleforsorjning

AB SK B

Box 5864

S-102 48 Stockhoim, SWEDEN

Nationale Genossenschaft für die Lagerung

Radioaktiver Abfälle (2)

Attn: S. Vomvoris

P. Zuidema

Hardstrasse 73

CH-5430 Wettingen, SWITZERLAND

AEA Technology

Attn: J.H. Rees

D5W/29 Culham Laboratory

Abington

Oxfordshire OXI4 3DB, UNITED

KINGDOM

AEA Technology

Attn: W.R. Rodwell

O44/A31 Winfrith Technical Centre

Dorchester

Dorset DT2 8DH, UNITED KINGDOM

AEA Technology

Attn: J.E. Tinson

B4244 Harwell Laboratory

Didcot, Oxfordshire OXII ORA

UNITED KINGDOM

D.R. Knowles

British Nuclear Fuels, plc

Risley, Warrington

Cheshire WA3 6AS, 1002607

UNITED KINGDOM

\section{Internal}

1

20

1502

1511

6000

6115

6115

6119

6119

6121

6121

6233

6300

6302

6303
A. Narath
O.E. Jones
J.C. Cummings
D.K. Gartling
D.L. Hartley
P.B. Davies
R.L. Beauheim
E.D. Gorham
Staff (14)
J.R. Tillerson
Staff (7)
J.C. Eichelberger
D.E. Ellis
L.E. Shephard
S.Y. Pickering

6303

6305

6306

6312

6313

6331

6341

6342

6342

6343

6343

6345

6345

6347

6348

6348

6351

6352

6352

6400

6613

6613

6613

6622

6641

7141

7151

7613-2

W.D. Weart

S.A. Goldstein

A.L. Stevens

F.W. Bingham

L.S. Costin

P.A. Davis

Sandia WIPP Central Files (100)

D.R. Anderson

Staff (30)

V. Harper-Slaboszewicz

Staff (3)

R.C. Lincoln

Staff (9)

D.R. Schafer

J.T. Holmes

Staff (4)

R.E. Thompson

D.P. Garber

S.E. Sharpton

N.R. Ortiz

R.M. Cranwell

R.L. Iman

C. Leigh

M.S.Y. Chu

R.E. Luna, Acting

Technical Library (5)

Technical Publications

Document Processing for DOF/OSTI (10)

8523-2 Central Technical Files 

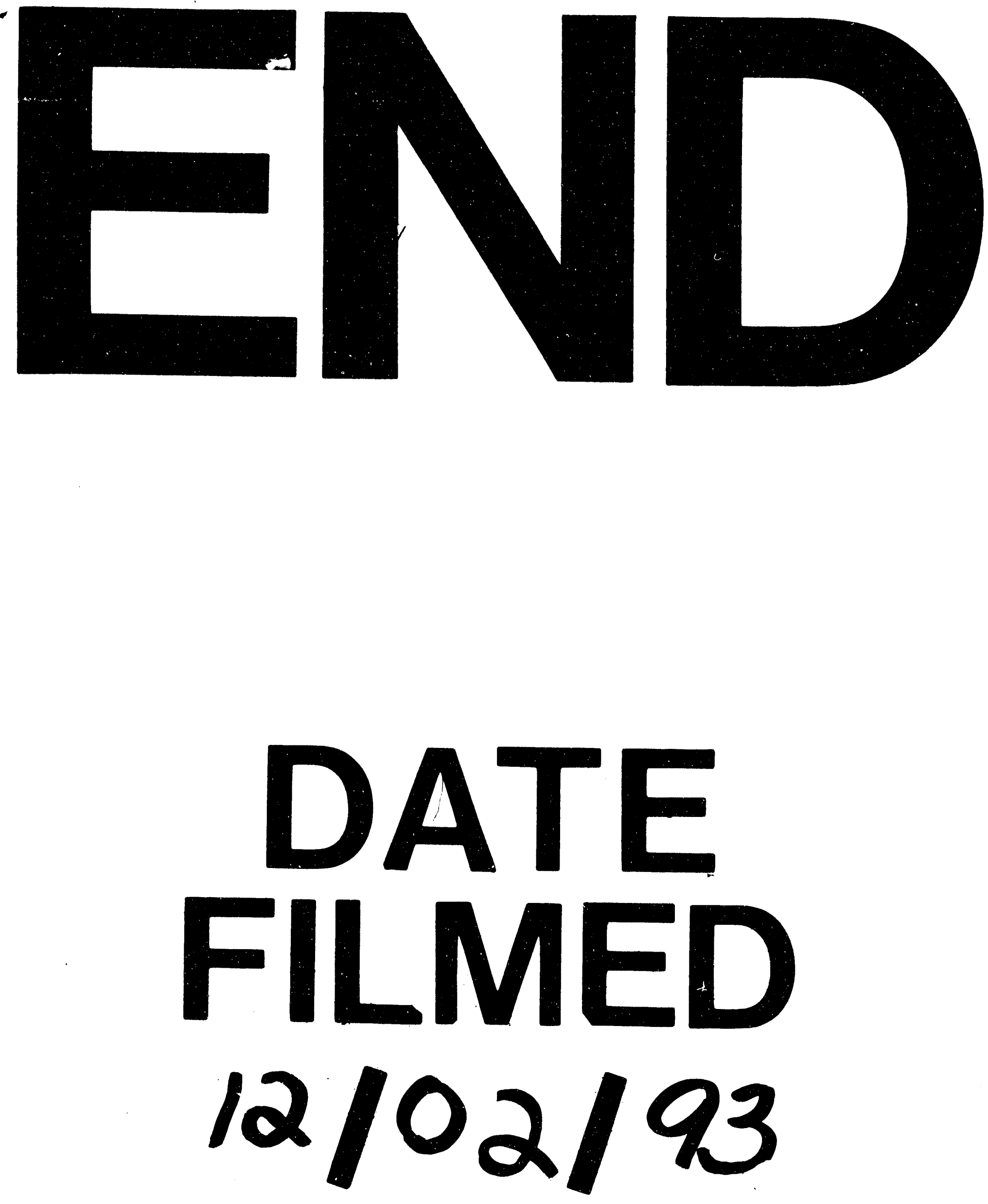
